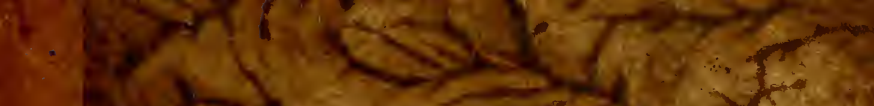

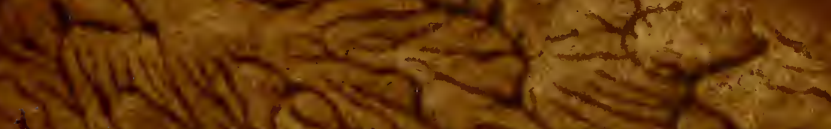

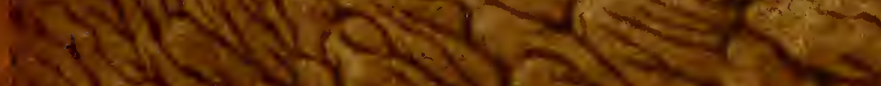

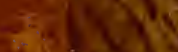

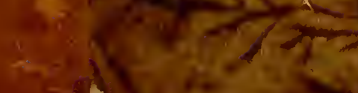

3.

1)

sy

-46 -

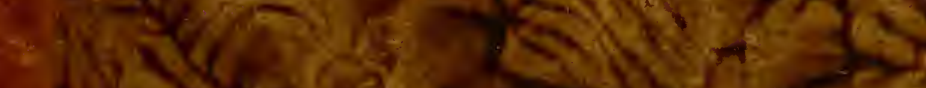
whar

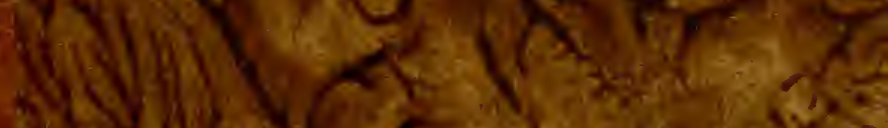

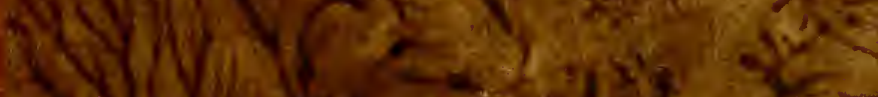

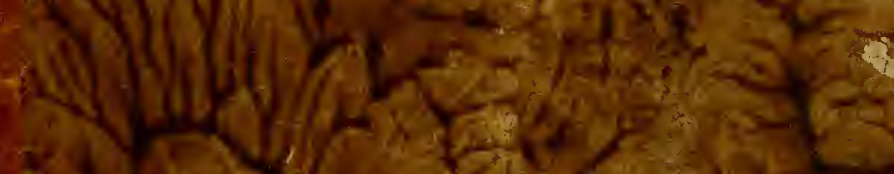

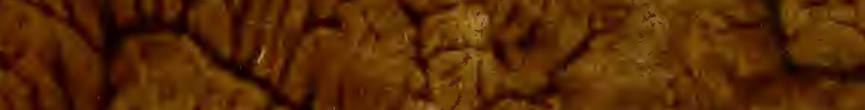
1)

arear an

undir ands? S. W. S.m.

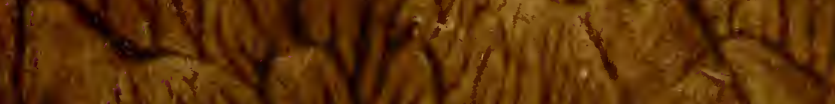
(4)

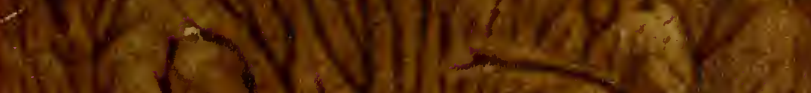

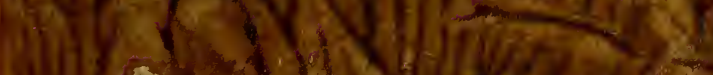




$$
\begin{array}{r}
88416 \\
303 \%
\end{array}
$$


acheinear 1878. 



\section{$\mathrm{ZOOLO}$ G I S C H E}

\section{B E Y T R $\ddot{A} G E$ \\ Z U R}

X I. I I. A U S G A B

DES LINNÉISCHEN

NATURS YSTEMS

VON

JOHANN AUGUST DONNDORFF.

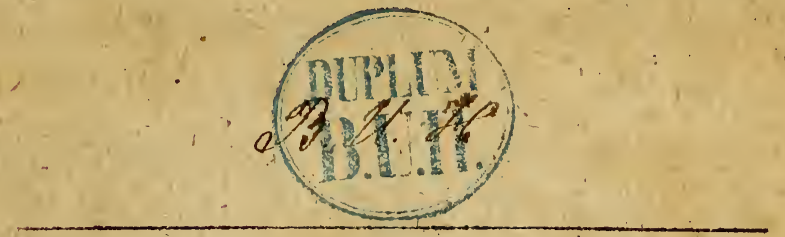

ERSTER BAND.

D I E S ÄU G T H I E R E.

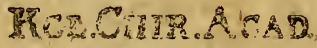

$$
\text { Lie ipzig, }
$$

in der Weidmannfchen Buchhandlung.

$$
1792 .
$$





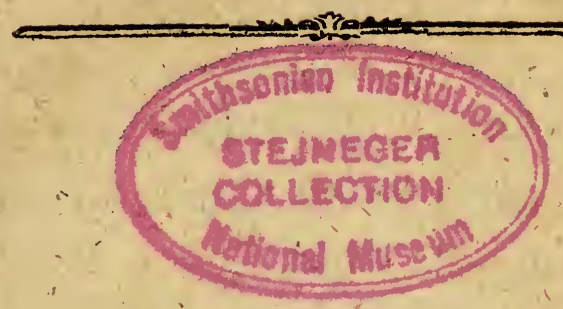

Vorrede.

Jinelins Verdiente um die dreyzehnte Ausgabe des Linneifchen Naturfyftems find zu bekannt, -und zu entfchieden, als dafs es nöthig wäre, noch das mindefte darüber zu fagen. Mit wie viel neuen Thiergattungen, die dem nordifchen Plinius noch unbekanne waren, ift fie nicht bereichert, und wie vieles ift nicht darin nach neuern Entdeckungen und Bemerkungen der bewährteften $\mathrm{Na}$ turforfcher berichtiget! Es war aber nicht die Abficht des grofsen Zoologen, bey jeder Gattung alle Synonymen zu bemerken, oder 
oder alle davon redonde Stellen anzuführen, und fie konnte "es auch nach feinem Plan nicht feyn. Ja es find feit der Zeit, da der erfte Band die Preffe verliefs, fchon hier und da wieder viele neue Entdeckungen gemacht worden, und mehrere Schriften in diefem Fache herausgekommen.

Ich habe daher gleich feit der Ericheinung der erftern Theile des erfen Bandes zu meinem eigenen Vergnügen Nachträge dazu gefammelt, alle mir vorgekommene Synonymen bemerkt, ältere und nevere Syfteme, Reifebefchreibungen, periodifche Schriften, Ueberfetzungen, u. d. gl. wo diefes oder jenes Thiers gedacht wurde, meinem Exemplar beygefügr, und diefe Arbeit für mich beym Nachfchlagen und Vergleichen der Quellen bald von reellem Nutzen befunden. Ich fiel daher auf den Gedanken, es dürffe vielleicht mehrern Freunden der, Zoologie nicht unangenehm feyn, wenn ich diefe, blofs zu meinem Vergnügen gefammelten Beytrăge dem Druck übergäbe.

Frey. 


\section{Vorrede.}

Freylich múfste hiebey zugleich der Gedan: ke mit entftehen, dafs es ein grofser Unterfchied fey, etwas zu feinem Privatgebrauch żu fammeln, oder dem Públiko mit einer folchen Arbeit unter die Augen zu treten. Bis jetzt verkenne ich auch die, im letztern Fall damit verbundenen Schwierigkeiten nicht, bin auch bey weitem weder fo ftolz, dafs ich mir einbilden follte, etwas vollftändiges liefern zu können, noch fo undelikat, dafs es mir gleichviel gelten könnte, die Arbeit möchte ausfallen oder vom vernünfrigen Publiko (denn von diefem ift allemal die Rede) aufgenommen werden, wie fie wolle. Aber ich bin auch eben fo gewifsüberzeugt, dafs Niemand, er fey wer er wolle, etwas vollitändigges in diefem Fache zu liefern vermögénd ift, und dafs den Liebhabern des Studiums der Zoologie, in deren Händen das Linneifche Syftem ift und feyn mufs, kein ganz unangenehmer Dienft damit geleiftetwerden könne, die wefentlichften Quellen zu diefer Wiffenfchaft hier faft alle bey-

$$
\text { a } 3
$$

fam- 
fammen $z u$ finden; und das faft mit einem Blicke zu überfehen, was zum Ganzen gehört, und im vorkommenden Fall nur mit Mühe zufammengebracht werden kann.

Ich habe daher meinen Plan weiter verfolgt, aus meinen Kollektaneen ein eigenes Werk bearbeitet, noch mehrere Materialien mic Fleifs dazu gefammelt, folche gehörig geordnet, und übergebe hiemit dem Publico den eriten Band davon, der fich blos über die Säugthiere erftreckt, nach folgender Einrichtung:

1) Habe ich bey jedem Gefchlecht, und bey jeder Gattung der Thiere, alle mir bekannte, deutfche und andere Synonymen, die ihnen fowohl von Schrifttellern als im gemeinen Leben gegeben werden, bemerkt, dabey die fo oft gefchehene Verwechfelung der Namen berichtiget, und die Urfach diefer oder jener Benennung beygefügt. Von ausländifchen Synonymen, deren im Werke felbft gröfstentheils noch nicht gedacht wor- 


\section{Vorrede.}

worden, habe ich am Ende ein befonderes Verzeichnifs angehängt.

2) Habe ich bey jeder Gattung alle mir irgend bekannte ältere und neuere Schriftfteller angefühirt, die fich im Syftem felbft nicht finden, und doch entweder zur $\mathrm{Na}$ tưrgefchichte des Thiers überhaupt wiffenswürdige Beyträge geliefert, oder auch blos auf die Verbreitung deffelben, Nutzen, Aberglauben, u. d. gl. Beziehung haben. Hiebey habe ich fowohl auf aus - als vaterländifche Schriften, Originale und Ueberfetzun: gen, auf periodifche Schriften, Reifebefchreibungen, u. f. w. Rückficht genommen; un jeden Liebhaber der Naturgefchichte, wenn er auch kein Gelehrter von Profeffion ift, in den Stand zu fetzen, dafs er von jedem Thier, mit deffen Gefchichte er be-, kannt zu feyn wünfcht, das Nöthige ohne Schwierigkeit auffinden kann. Freylich find hier auch zuweilen folche Schriften mit unter gelaufen, die dem Zóologen als Zoolo- 
gen fehr entbehrlich feyn möchten. Aber fie konnten doch um des Ganzen willen nicht wegbleiben. So würde ich z. E. MER KLE INS Thierreich gewifs nicht mit angeführt haben, wenn ich nicht geglaubt liätte, dafs es wegen der vielen darin enthalcenen naturhiftorifchen Vorurtheile, und ältern abergläubifchen Meinungen mit bemerkt werden mufste. Auch K a A T S Ausrottung graufaner. Thiere; L E M M E R Y Mategiablescicon, u. d. gl. m. würde ich weggelaffen haben, wenn ich fie nicht entweder wegen eigner Synonymen, oder wegen befonderer Cyftematifchen Eintheilungen, oder auch wohl wegen mancher Bemerkungen mit anzufuthren für nöthig gefunden hätte. Wenn von einem in einer fremden Sprache verfarsten Buche deutfhe Ueberfetzungen vorhanden waren, fo habe ich mehrentheils beydes, z. E. Pallas fpicileg. zool. und $N a-$ turgefchichte nerkzourdiger Thiere; - und wenn der letztern mehrere waren, auch diefe $z$. E. v́on Kleins quuads. difp. die unter dem

Titel: 
Titel: Claffification und kurze Gefcbicbte der vierfülsigen Thiere von Behn zuLübeck 1760 . in 8. als auch die von dem Verf. felbnt ver. anftaltete, und hernach von Reyger; unter. dem Titel: Natürliche Ordnung wnd vermebrtc Hifforie der vierfïssigen Thiere, in eben dem Jahre zu Danzig in 4 . herausgegebene Ueberfetzung $\mathrm{u}$. d. gl. m. angeführt. Vom Büffon habe ich mich der deutfchen Ueberfetzung in 8. welche feit $177^{2}$. von Martini, und nachhero von Otto weiter beforgt und mit vielen Zufätzen bereichert, int, bedient. Schriften, die im Syftem felbft fchon ftehen, habe ich meines Wiffens nirgends wiederhohlt, es fey denn, dafs ich eine andere Ausgabe, oder eine andere Ueberfetzung zur Hand gehabt hätte. So if z. E. von Egedeas Befclor. von Grönland im Syftem die franzöfirche Ueberfetzung vom J. 1763. allegirt; ich aber habe die deittche von eben diefem Jahr, die aus derfelben verfertiget worden, zur Hand gehabt, und habe fie, da fie fich vielleicht in noch mehrern 
Händen als jene befinder, mit angeführt. Eben dies findet auch bey Cranz Hiftorie von Grönland, und in mehrern Fällen Statt. Auch habe ich wohl der Berichtigung halber manchmal hin und wieder eine im Syftem fchon befindliche Stelle alsdenn wiederhohlt, wenn durch einen Druckfehler ein unrech. ter Band, eine unrechte Seitenzahl, oder eine unrechte Kupfertafel citirt war. Möglich wäre es indeffen wohl, dafs auch einmal ein überfüffiges Citatum von der Art mit eingefchlichen wäre, und das wird hoffentlich bey der Menge von Citaten, die ich beygebracht habe, wohl verzeihlich fevn. Von Zimnnermanns geogr. Zool. habe ich die deutfche Ausgabe vom Jahr 1778. genutzt, weil diefe vom Verfaffer vermehrt und verbeffert worden ift; und daher von ihm felbft als Originalfchrift ausgegeben wird, die mit dem lateinifchen Werk nun einen gleichen Plan hat. Von Pennants aretifcher Zoologie habe ich die deutfche Ueberfetzung von Zimmermann vor mir gehabt. Verfchiede- 


$$
\text { Vorrede. }
$$

ne Werke älterer und mittlerer Naturforfcher, als die von Hill, Houttyns, Argenfold, Edroards, Hughes, Lawfon, u. f. w. habe ich nicht, oder doch nur felten angeführt; man findet fie aber alle in Erxlebens Manmalibus, auf die ich allemal hingewiefen habe, und ich kann vorausfetzen, dafs dies Buch fich in den Händen eines jeden Żoologen befindet.

Hiernächft gründen fich meine Beyträge überhaupt auf die Schriften eines Adanfon, Agricola, Aldrovmd, Alpin, Anderfon, Baldaeus, Bankroft, Barrere, Batfch, Bechftein, Beckniann, Bergen, Berkel, Blumnenbach, Bock, Boddnert, Bonnet, Borousky, Bojsmann, Boswell, Boullaye, Browne, Burgsdorf, de la Caille, Camper, Carver, Cetti, Chardin, Charleton, Charlervoix, Charras, Choify, Condamine, Cook, Cubn, Dampier, Dapper, Daubenton, Demanet, Derham, Dodart, Dobrizboffer,' Döbel, Eberbard, Ebert, Ellis, Eraleben, Fabriz, Falk, Falkner, Fermin, Fifcher, Forskäl, Forfter,

Frific, 
Frifch, Funke, Gatterer, le Gentil, Georgi, Geoffroy, Gesner, Gilii, Gmelin, Goeze, Graumann, Gronov, Gumilla, Hacquet, du. Halde, Halle, Hartfink, Hafjelquift, Hane en, Hawkesworth, Heppe, Herder, Herrnann, Högftröm, Höpfner, Höft, Horrebow, Hubn, Hunne, Hupel, Fablonsky, Fonfton, Ifert, Irves, Kä̈mpfer, Kircber, Klïgel, Knorr, Knox, Köbler, Kolbe, Krafcheninnikov, Krünitz, Labat, Laet, Leenn, Leppechin, Leske, Leffeps, Lichtenberg, Lichtenftein, Lininé, Low, Ludolf, Ludovici, Marcgrav, Marnnol, Martens, Martini, Mayer, Mekel, Mellin, Merrem, Meyer, Molina, Moskati, Miiller, Murr, Neuboff, Niebubr, Nieremberg, Oednann, Olaffen, Olearius, Pallas, Paw, Penzant, Perrault, Pbilipps, Pbipps, Pifo, Plattner, Pokok, Pyrard, Rajus, Ridinger, Rocbow, Römer, Rozier, Rytfcbkow, Rzaczynsky, Sander, Scheffer, Schneider, Scblözer, Scbönfeld, Scböpf, Schreber, Scbroenkfeld, Seba, Severin, Sbam, Sibbald, Stuellie, Sömnering, Spallanzani, Sparrmann, Sprengel, Steller, Strablenberg, Taube, Tavernier, Thevet, Tremarek, Uno 
von Troil, Ulloa, le Vaillant, Vidinure, Voigt, Vosmaer, Wagner, Waldfchmidt, Walter; Wolf, Wunfch, von Wurmb, Zauschner, und fehr vieler andern ältern und neuern Zoologén, Syftematiker, Reifebefchreiber, Journaliften und Monographiften. In der Anführung felbft habe ich die willkührliche Crdnung beobachtet, dafs die neueften, bekannteften deutfchen Syfteme und andere Schriften voranftehen, diefen die Reifebefchreibungen, darauf die periodifchen, anatomifchen Schriften und Monographien, alsdann die ältern Syfteme und ausländifchen Schriften folgen. Ich behaupte nicht, dafs diefe Ordnung gerade die befte fey; -aber fie war für mich bey dér mühfamèn Befchäftigung die leichtefte; und es liegt ja auch in der That nichts daran, ob diefe oder jene Schrift vor - oder nachftehet. Bey Hausthieren habe ich auch am Ende einer jeden Gattung die vorzüglichften von folchen Schriften mit bemerkt, die die Zucht, Wartung, Pflege u. . w, diefer Thiere ganz befonders betreffen.

-3) Füge 
3) Füge ich aufser den trivialen Benennungen, auch die fyrtematifchen, und die verichiedenen Karaktere bey, womit andere Syftematiker das Thier bezeichnet haben.

4) Zeige ich an, ob das Thier abgebildet fey, oder nicht, ob die Abbildung gut oder fchlecht, oder mittelmäfsig fey: Wo ich gar keiner Abbildung gedenke, davon ift mir wenigttens noch keine bekannt geworden.

5) Gebe ich bey jeder Art, wo es nöthig ift, die vorzüglichfte Merkwürdigkeit an, und fuche fo viel als möglich, nach den neueften Quellen und richtigtten Beobachtungen die zoologifchen Irrthümer zu berichtigen. Wenn man alfo das, was $i c b$ von der Naturgefchichte des Thiers beygebracht habe, mit dem, was davon im Syftem felbft gefagt worden ift, zufammen nimmt, fo wird man eine, im Ganzen zwar immer kurze, aber doch fo viel davon bekannt, zienlich vollftändige Gefchichte deffelben 
haben. Ueber die Verbreitung der Thiere" habe ich der Kürze wegen nicht viel fagen können, und verweife deshalb auf Zimmermainn, und was vom Nutzen und Schaden der Thiere, auch von der Art, fie zu fangen, gefagt werden könnte, kann man zum Theil beym Gatterer finden; doch find des letztern Citata mit Behutfamkeit zư prüfen. .

6) Führe ich bey jedem Gefchlecht die noch unbefimmten Gattungen von Thieren, deren im Syftem nicht mit gedacht worden, und nicht gedacht werden konnte, mit Vergleichung der Quellen, an; auch bemerke ich allemal, wenn etwa diefe oder jene Gattung von einem andern Schrifteteller von Gewicht nur für eine Varietät oder eine im Syftem angenommene Varietät von einem andern für eine eigene Gattung gehalten wird. Hin und wieder habe ich auch felbit noch einige Varietäten hinzugefügt.

7) Bemerke ich bey jedem Gefchlecht oder Gattung; wo die XIII. Ausgabe von der 
der XIl. abweicht, was für Veränderungen das Syftem erlitten, um wie viel Gattungen jedes Geíchlecht vermehrt worden, u. f. w. Diefer Umfand wird denenjenigen zu Statten kommen, die an die fyrenatifchen Benennungen der XII. Ausgabe gewöhnt find, und nun ein Thier auffuchen wollen, das in der XIII. an einem ganz andern Orte fteht. Vergebens werden fie dann z. E. Mus Por. cellis, Citellus, Marmota, Quercinus, Longipes, Volans, etc. noch unter dem Gefchlecinte Mus fuchen. Sie finden aber alle diefe hier fehlenden Gattungen fehr leicht, wenn fie meine Bēyträge ain Schluss eines jeden Gefchlechts nachfehen wollen.

8) Verfehe ich jeden Band mit einem " umfändlichen Namen - Regiter, nach wel. chem jedes Thier fowohl nach feiner deutfchen als ausländifchen gemeinen oder fyfe. matifchen Benennung, für mehrere Syfteme fofort aufgefunden werden kann.

Ich denke das ganze Werk in drey Bänden $z u$ liefern, wovon der zweyte die Vö. 
gel, und der dritte die Amphibien und Fifche enthalten wird. Auf die Infekten und Würmer kann ich mich nicht einlaffen, weil wir eines Theils in Anfehung der Entomologie fchon die fchönen Beyträge von Goeze haben, andern Theils aber auch es mir an den hiezu nothwendig erforderlichen vielen und kottbaren Quellen fehlt, die ich hier in der Nähe nicht haben, und aus meinem Ver mögen nicht alle anfchaffen kann.

Dies wäre es ohngefähr, was ich über den Plan und die Einrichtung meiner Arbeit zu fagen hätte. Es wird nun dárauf ankommen, ob ich fie zweckmäfșig ausgeführt habe.

Freylich find mir nicht felten erhebliche Schwierigkeiten vorgekommen, of habe ich, befonders bey Reifebefchreibungen, um einer einzigen Stelle willen, funfzig andere nachfchlagen und vergleichen müfen, um kein Synonym mit dem andern zu verwechfeln, oder keinem unvollntändig und man- 
gelhaft befchriebenen Thiere eine unrechte Stelle anzuweifen. Oft ift mirs dann woht gelungen, hinter die Wahrheit zu kommen, oft bin ich aber auch felbet in der Ungewifsheit geblieben, und dann habe ich, um den Lefer nicht irre zu führen, die Stelle fragweife (?) hingefetzt, 'auch da, wo ich Be. denklichkeiten gefunden habe, in der Note dạrauf aufmerkfam gemacht. Aber eskann auch leicht feyn, dafs ich, aller angewandten Mühe ohnerachter, dennoch zuweilen $\mathrm{da}$ des rechten Wegs verfehlte, wo ich am ficherften zu gehen glaubte. Ja es find mir auch wohl Quellen entgangen, auf die ich bey meiner Arbeit noch hätte Rückficht nehmen foller. IVIänner in der Wiffenfchafr; die felbft in folchen Fächern gearbeitet haben, und denen dis mit einem folchen Un. ternehmen verbundene Schwierigkeiten nicht unbekannt find, werden folches der Billigkeit nach gewifs entfchuldigen, und auf eine, der Würde eines Gelehrten nicht unanftändige Art, die Mängel anzeigen, die fie entdecken möchten; ihreBelehrung foll von 
mir nicht ungenutzt bleiben. Wer aber Luft hat, mich dieferhalb mit;Bitterkeit zu tadeln, der thue es zu feiner eignen Schande, und verfuche, ob er felbft im Stande ift, hier etwas vollkommenes zu liefern. Leichter ifts, bey einer folchen Arbeit etwas zu tadeln, als es beffer zu machen.

Für mich ift es Belohnung genug, wenn das unpartheyifche Publicum mir nur die Gerechtigkeit wiederfahren läfst; dafs ich durch meine mühfelige Arbeit den Freunden 'der Naturgefchichte das Studium der Zoologie, und den Gebrauch des Linneifchen Syftems einiger Maafen erleichtert habe.

Bey der 15. und 16. Gattung des Affengefchlechts p. 31. des Syftems habe ich vergeffen mit anzuführen, dafs Schreber die Synonymen diefer beyden Gattungen zufammen genommen hat; und bey der 20. p. 37 . dafs Büffon und Pennant diefe mit der 29. für einerley halten, welches ich alfo hierdurch noch bemerken will. Von allen von jetzt an 
noch herauskommenden, oder mir ferner bekannt werdenden Schriften, werde ich bey dem folgenden Bande den Nachtrag zu diefem liefern, auch wohl nach mehrern Jahren, wenn ich durch keine erhebliche Hindernife abgehalten werde, noch einen Supplement-Band, zu dem Ganzen, hinzufügen, um dem Werke, als einem eigentlichen zoologifchen Repertorio, die möglichfte Vollifändigkeit zu geben.

Quedlinburg, vor der Oftermeffe 1792.

\section{J. A. Donndorff.}




\title{
ERSTE KLASSE.
}

\section{A M M A L I A. (Sângende Thiere.)}

\author{
ERSTE ORDNUNG.
}

PRIMATES. (INenfchenähnliche Thiere.)

I. GESCHLECHT. Homo. Der Menfch.

I. S A PIENs. Der Men $\int c h$.

Miiller, Linn. Naturfyftem I. p. 6r. der verniinftige Tagmenfch.

Zimmermann, geogr. Gefch. des Menfchen und der allgemein verbreiteten vierfüfs. Thiere, I. p. 3 T.

Schreber, Säugthiere, I. p. 5 .

Lefke, Naturgefchichte, I. p. IIO.

Blumenbach, Handbuch der Naturgefch. 4te Aufl.

p. 5 I.

Bechftein, gemeinnützige Naturgefch. Deutfchl.

I. p., I28.

Gatterer, vom Nutzen und Schaden der Thiere,

I. p. $x$.

Bilffon, allgemeine Naturgefch. V. p. I. Naturgefch. des Menfchen.

Klïgel, Encyklopädie, I. p. 283. die-Lehre vom Menfchen.

Wriin $c h$, kofmolog. Unterhaltungen, IIIter Band. 
Kant, Abhandl. von den verfchiedenen Racen der Menfchen: - in dem Philolophen für die Welt, II. p. I23.

Springer, natürliche Gefchichte des Menfchengefchlechts; a. d. Franz. des Pichon. Lemgo I768.

Mnofcati, von dem körperlichen Unterfchiede zwifchen der Struktur des Menfchen und der Thiere.

Sander, ökonom. Naturgefch. I. p. " 4 .

Plattner, Briefe tiber die Thiere und Menfchen. Lpz. 1775.

Bonnet, Betracht. uiber die Natur, überf, von Titius; 4te Aufl. Lpz. 1783. I. p. I46.

Handbuch der Naturgefchichte, aus dem Franz.

Nürnb. 1773. I. p. 29.

Neuer Schaupl. der Natur, V. p. 630.

Onomat. hift. natural. IV. p. 265.

Herder, Ideen zur Philolophie der Gefchichte der Menfuhheit, III Bde.

Ifelin, über die Gefch: der Menfchheit, II Bde. Adelung, Verfuch einer Gefchichte der Kultur des menfchl. Gefchlechts. Lpz. 1782.

Sömmering, vom Bau des menfchlichen Körpers. Frcf. a. M. I79r.

Groffe, Magazin für die Naturgefch des Mehn. fchen. I. III B. Zittau, I79I.

Houfen, Verfuch einer Gefchichte des menfchl. Gefchlechts. Halle, I77I. f.

Vierthaler, philof. Gefch. der Menfchen und Völǩer. I - IV B. Salzb. I784. ff.

Hallor, elementa phyfiologiae. (deut) ch: Anfangsgründe der Phyfiologie; überfetzt von Halle. Berl. 1765 - 75. 8 Bde.)

Bhumenbach, de generis humani varietate nativa: 
Bhumenbach, über den Bildungstrieb. Götting. 1789. 8.

Bhumenbach, über Menfchenracen und Schweine: racen; in Voigts Magazin für das Neuefte a. d. Phyl. u. N. G. VI. I. p. I.

Hacquet, Beytrag zu Blumenbachs Beobacht. über Menfchenracen etc. daf. IV. p. 28.

Beddome 2 diff. de hominum varietatibus et eorum cauflis. 1777.

Duglas, defcript. comparata mufculorum hominis et quadruped. I729.

Walter, obfervat. anatom. Berl. 1785. fol. INayer, anatom. Kupfertafeln, nebft dazu gehö. rigen Erklärungen. Berl. und Lpz. I783. ff. gr. 4.

Verfuch von den Urfachen der verfchiedenen Far. ben der Menfchen, in verfchiedenen Weltge. genden; im Hamb. Magazin I. p. 235. u. 378.

Hume, von der Menge der Menfchen bey alten Nationen; daf. X. p. 45r.

Von der Anzahl der Menchen in alten und neuern Zeiten; daf. XXIII. p. It5. u. 339.

Betrachtungen über den Menfchen; daf. XXV. p. 228.

Siifsmilch, göttliche Ordnung in den Veränderungen des menfchl. Gefchlechts; neuefte Aufl.

Von verfchiedenen Menfchenracen; Samml, zur Phyf. und N. G. I. p. 347 .

Eintheilung des Menfchengefchlechts in fünf Spielarten. Blimenbach Beytr. zur Naturgefch.

I. p. 79 .

Blafii anatomia animalium. Amf. 168r. 4 Sauri phyfique du corps humain, ou phyfiologie moderne. Paris, 12. Vol. I. II. I778.

Albini de natura hominis lib. Leid, x775. 8. A 2 Funk, 
Homo.

Finnk, de homine naturali. Erf. 1707 .

Weidner, de natura hominis. Roft. I7I4.

Erxleben, Naturgefchichte von Gmelin. 4te Auf, I791. §. 135 .

Donndorf, Natur und Kunf, II Band p. Iff.

a. Americanus. Der Amerikaner.

Miiller, Naturfyf. I. p. 89. 96.

Lefke, Naturgefch. I. p. II3. n. 6 .

Bhmenbach, Handb. d. N. G. p. 55 .

Bechftein, Naturgefch. Deutfchl. I. p. I3r. n. 6.

Kiïgel, Encyklop. I. p. 333:

Biiffon, allgemeine Naturgefchichte VI. p. 158. und p. 193 .

Paw, philofoph. Unterfuch. über die Amerika. ner. Berlin, 1769. T. I. II.

Organifation der Amerikaner; in Herders Ideen fi. p. $5^{2}$.

Befchreib. der Landeseinwohner von Amerika etc. im Hamburg. Magaz. XXIV. p. 46I.

Erxleben, Mämmalia, p. 2.n. 5 .

B. Europa Eus. Der Eltropizeti.

Miïler, Naturfyf. I. p. 89.

Lefke, Naturgefch. I. p. II2: n. 4.

Bihnmenbach, Handb. der N. G. p. 54. n. I.

Bechftein, Naturgefch. Deutfchl. I. p. I3r. n. 4. Klïgsl; Encyklopädie, I. p. 328.

Sömmering, über die körperliche Verfchiedenheit des Negers vom Europäer. Frcf. 1785 .

Camper, kleine Schriften, I. p. I6. (Unterfchied $z$ wifchen dem Mohren und Europäer:) Erxleben, Mammália, p. 2. $\delta$. 
\%. Asia ticus. Der Afiate.

Miiller, Naturfyft. I. p. 89.

Lefke, Naturgefch. p. II2. n. 2. 3.

Blumenbach, Handb. der N. G. p. 55. n. 2.

Bechftein, Naturgefch. Deutichl. I. p. 130. n.2. 3.

Biiffon, allgem. Naturgefch. VI: p. 89.

Organifation der Völker um den afiatifchen Rü-

cken der Erde; in Herders Ideen II. p. $1_{5}$ :

Erxleben, Mammalia, p. 2. $\gamma$.

8. AFER, Der' Afrikaner.

Miiller, Naturfyft. I. p. 89.

Zimmerinanı, geogr. Lool. I. p. 77.

Schreber, Säugthiere, I. p. 9. U. I5.

Lefke, Naturgefch. I. p. II2. n. 5 .

Blunienbach, Handb. d. N. G. p. 55. n. 3.

Bechféein, Naturgefch. Deutfchl.I. p. I31. n. 5 .

Demianet, hift, fur les Negres; im II Th. Seiner hiftoire de l'Afrique françoife.

Barrere, diff fur la caufe phyfique de la couleur des Negres, Paris, 1741.

Recherches fur la couleur des Negres. - Acad. d. Sc. de Berlin Tom. IX.

Camper, kleine Schriften, I. p. 24. Ueber den Urfprung der Farbe der Schwarzen.

Albin. diff de fede et caufa coloris Aethiopum et ceter. hominum. Lugd. Bat. I73I.

Haller, element. phyfiolog. Tom. V. p. 19. oder Lib. XII. Sect. I. \$. I2.

Bäk, Unterf, von der fchwarzen Haut der $\mathrm{Ne}$ ger; in den Schwed. Abh.X. p. II.

Sömmering, in der fub $\beta$ angef. Schrift. Adanfon, Reife nach Senegall, p. 55 . Le Cat, fur la couleur de la peau. 
Verfuch einer Frklärung der Urfach der Farbe bey den Schwarzen üherhaupt, und bey den weifsen und buntheckigen Negern infonderheit; in Hamb.'Magaz. XIX. p. 376.

Browne, error, popul. VI. c. Ir. I2.

Neue Anmerk. üher alle Theile der Naturlehre, II. p. 275 .

Waldfchmidt, diff. de colore Aethiopun. Marb. 1683.4 .

Hannemann, anatome Aethiopis foeminac; in Bartholini act. med. et philor. Haffn. Vol. IV. A. 1677 . obl. 17 .

Pechlin, de habitu et colore Aethiopum, qui vulgo Nigritae. Kiel, 1677.8.

Meckel, recherches anatom. fur la diverfité de couleur dans la fubttance medullaire du cerveau des Negres; in den Mem. de l'Acad. de Sc. à Berl. T. IX. oder An. i759. p. 79. Conment. Lipf. de rebus in fcient. nat. et med. geft. Tom. V: P. 2. p. 200.

Organifation der afrikan. Völker: - in Herders. Ideen, II. p. 34 .

Biiffon, allgem. Naturgefch. VI. p. 103. 7.4.

Ueber die Negern insbefondere; Blumenbach, Beytr. zur N. G. I. p. 84 .

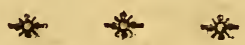

Beytrag zur Gefchichte von gefleckten IMenfchen. Schreber, Säugthiere, I. p. 15. - Naturforfcher, XVI. p. 169. XX. p. I23. XXIII. p. 213.

Durch die Kunft entftellte Menfchen. Biiffon, allgem. Naturgefch. VI. p: 25\%: 
Befchreibung cines fonderbaren Menfchen von Diequemare, in Lichtenbergs Magazin für d. Neuefte a. d. Phyf, u. N. G. II. II. p. 89.

Beyfpiele eintger in der. Wildnifs anfgervachfenen Menfchen.

Schreber, Säugthicre, I. p. 3r. - Büffon, allgem. N. G. VI. p. 263, - Beckmann, phyfik. ökon. Bibl. IX. p. 159.

Von riefenartigen Menfchen.

Mïller, Naturfyn. I. p. 1о2. - Zimmermunut; geogr. Zool. I. p. 63. - Schreber, Säugthiere, I. p.28. - Lefke, N. G. I. p. III. - Blumenrbach, Handb. der N. G. p. 56. - Biiffon, allgemeine Naturgefch. VI, p. 238 . Allgem. Magazin IV, p. 238 - Stralfundifches $\mathrm{Ma}$ gazin, I. p. 27: - Kliigel, EncykJ. I. p. 335. Schwed. Abhandl. 1765. p. 319. - Brydone, Reife um die Welt. I764. f. Vorrede, - Falkner, Befchreib. von Patagonien, p. 40.

Von zwergartigen Men fchẹn.

Mïller, Naturfyn. I. p. Ior. - Zinmmermann? geogr. Zool. 1. p. 73. - Schreber, Säugthiere, 1. p. 27. - Blumenbach, Handbuch d. N. G. p. 56. - Biiffon, allgem. N. G. VI. p.202. Changenx, von Riefen und $\mathrm{Z}$ wergen, und von der mittlern Gröfse des menfchl. Körpers; in Rozier obf. XII. p. I67. - Kofmolog. Unterhalt III. p. 94.

Beyfpiele von fehr alt gewordenen IMeñ chen.

Bunzlauer Unterhalt. I. p. 348. 382. II. p. I87. Anmerk. über alle Theile der Naturl. I. p. $315^{\circ}$ A 4 327. 
327. - Magazin für den Landmann, I. p. 73 . not. c. - Schriften der Drontheim. Gefellifch. I. p. 250. - Weber Nachrichten von Perfonen. welche ein ungewöhnlich hohes Alter errẹicht haben. Hannoṿer, 8.

Beyfpiele vor Menfcheirfreferey, waid Urfprung, diefer Gewohnheit.

Dappers Afrika, p. 538. 572. - Phyfikalifch. ökonom. Ausz, III, p. 235. - Hamb. Magazin VI. p. 53. 63. - Hamb. Journ. II. p. 103. Olearii Reifa, p. 80. - Allgem. Hift d.Reif, T. XII. p. 25. 27. T. XIV. p. 236. T. XV. p. I39. 282. T. XVI. p. 252. 293. 298. 648: Brand, chinef. Reife, Berl. I712. p. 66. - Gefchichte der Seereifen, überf. von Forfter $178 \%$ p. 474. - Tagebuch von Cooks neuefter Reife um die Welt. I. Lpz. 1776. - Forfter, Reife un dis Welt, ate Edit, in 8. p. 153. - Forfter, Bemerkungen auf feiner Reife um die Welt, p. 288. Hawkesworth, Samml. der neueften engl. Reifebefchr. Edit. in 4 III, 2 Buch, 9 Kap. - Ol. dendurp, Gefch. d. Miffion etc. auf den caraibifchen Infeln. Barby 1777. I. p, 25. - Tues Reife nach Indien und Perfien a, d. Engl. von Dohn, Lpz. 1774. I. p. II4 - Ellis Reife nach Hudfons Meerbufen. Götting. 1750: p. 190. 220: - Gooze, Natur, Menfehenlęben und Vorfehung, I. p. I42. - Nachricht, von merkwïrd. Verbrech. in Deutfchl: I. p. zo. - Iaco, bi, differt. de anthropophago Bercano, Ien. I77 r. m. e. K. - Dapper, Amerika, p. 283. Miller, in den philor, Tranfactions LXVIII. I. p. 168 , 
-Von den Kakertaken.

BhumenUach, Handbuch d. Naturgefch, p. 56. Bhumenuach, Beyträge zur Naturgefch. I. p. 119.

Vollfändiges Verzeichnifs der Nanien von menfchli. chen Sibarten durch Vermifchung der Spanier mit den Indiern.

Tivi/s, Reife durch Portugall und Spanien, I772. und 73. I. p. 326. - Beckmann, pliyfik ökon. Bibl: VIII. p. 517

2. GESCHLECHT. SIMI A. Affen. (I)

Miiller, Naturfyf. I. p. II8, Gen. II:

Zimmermann, geogr. Zool. II. p. I65. Gen. XIV: Schreber, Säugthiere. I. p. 45. Gen. II. Lefke, Naturgefch. I. p. II . Gen. H. Borowfky, Thierreich, I. p. 37 . Gen. I. Bhumenbach, Handbach d. N. G. p. 57. Gen. II. III. IV.

Funke, Naturgefch. u. Technologie, I. p. I7o. Biiffon, Naturgefchichte der vierfüls. Th. XVII. p. I23.

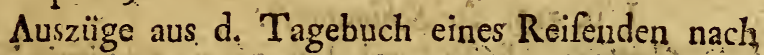
Afien: Lpz 1784. p $27 \mathrm{I}$.

Berlin. Samml. I. p. 378 .

Anatonifche Befchreib: vieler Affen, in Perraulí, Charras, und Dodaert, Abhandl. zur Natur. gefch. L.pz. 1757: I. p. 325. Tab. XLIV. und XLV:

\section{A 5 \\ Ervile}

(1) Unter den Gefchlecht Simia frod fowohl die eigentichert Affe", als die Baviane, und Meerkatzen, Sapajus und Sagoins begriffen, woraus Erxlebez u. a. befondere Gefchlechtes gemacht haben. 
Erxleben, Mammal. p. 6. 15.22. 44.55 . Gen. II. III. IV. V. VI.

Hermani, Tab. affinit. animal. Argent. I783. 4. p. 50 .

Lichtenftein, de Simiarum, quotquot veteribus innotuerunt, formis, earumque nominibus. Hamb. r79I:

\section{* Ungefchwänzte; die Affen der Altens.}

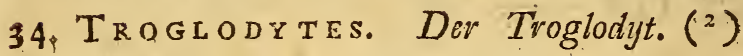

Miiller, Naturfyf. I. Tab. VI. Fig. r. unrichtig. Zinimermann, geograph. Zoolog: II. p. I7o. deas Troglodyt, n. 69.

Schreber, Säugthiere, I. p. 54. p. 62. der Chime paruzee.

Lefke, Naturgefch. p. Ir4. n. x. der. Troglodyt. Blumembach, Handb. d. Nat. Gefch. p. 57. der Schimpane, Pongo, Tocco, Barris, africaniche Waldmenfch.

Limné, auscerlefene Abhandl. I. p. 63. n. 2. der Satyv, Tab. 2. fig. 3 .

Goeze, Natur, Menfchenleben und Vorfehung, 1. p. 519. der Schimpanfe.

Gatterer, brev. Zool. I. p. I4. n. I. Simia Troglodytes; der Chimpanje,

Lichtenftein, de Sim. vett. p. 16. 72. Simia Trog. lodytes.

(2) Man mufs dicfen Troglodyten nicht mit Linne's Homo Troglodytcs. S. N. Edit. XII. p. 33. n.. 2. der ein Gemifch aus der Gefchichte der Kakerlaken, cet. und des Urangutangs war, verwechfeln. In der XII Euit. ift diefer S. Troglodytes als cine ₹rat. von S. SAtgrus aufgeführet; p. 34. I. $\beta$. 
I. SA TYR U S. Der Orang-Outang: (3)

Zimmermann, geogr. Zool. I. p. II7. II. p. 67 und 170 .

Schreber, Säugthiere, I. p. 54. Orang-Outang, Tab. II. A.

Lefke, Naturgefchichte, p. II5. n. 2. der Orang. utcang.

Blumenbach, Handb. d. N. G. p. 58. S. Satyrus, Capite minore, gracilior, hirfuta: pilorum humeri et ulnae contraria directione, pollice manuum anteriorum mutico, ungue deftituto; der oftindifche Waldmenfch; eigentliche Orangutang.

Halle, viẹrf. p. 549. der Waldniensch.

Gatterer, vom Nutzen und Schaden der Thiere,

I. p. 9. n. 3. der Orangitang.

Biiffon, vierf. XVH. p. 170. die Oraing - outangs, oder der Pongo unid der Tocko.

Borowfky, Thierreich, I. p. 4o. n. I. Orang: Outang, Waldmenjch, Tab. I. A.

Limné, auserlefene Abhandl. I. p. 62 . n. r. dẹv Pygniär. Tab.2. fig. 4. p. 66. der Troglodyte.

Goeze.

(3) Camper beweif aus 'der Zergliederung des Stimmwerkzeugs des Orangs und verfchiedener Affenarten, dafs fie nicht fathig find, Töne wie der Menfch zu bilden; denn die Luft, indem fie die Spalte der Stimmritze vorbeygeht, fallt unmittelbar in die Lufteácke, oder den Sack des Halfes, wie in den Affen, und mufs alro von da ohne einige Kraft oder Melodie, in die Kehle und den Mund diefer. Thiere zurückkehren. - Diefs. Thier wird mit dem vorhergehenden oft unter dern gemeinfchaftichen. Namen: Waldmen/chs, Pysmääe etc. verivęchfelt; und ift nach Camper (am a. O.) werter der Pongo noch der Iocko: Yon dem vorhergehenden unterfcheider er fich durch einen weit Schlankern fchmächtigern Wuchs, kleinern Kopf, ganz andere Gefichtsbildung, und einen dicht beharter leib. 
Goese, Natur, Menfchenleben u. Vorf. I. p. 520. der eigentliche Orangutang.

Martini, Naturlex. I. p. 55I. aer Bufchnnenfch; Erdmenath m. Büff. Fig.

Ausziïge a. d. Tagebuche eines Reifenden nach Afien, p. 256. n. 26.

Boddaent, im Naturforfcher, XXII. p. 2 ff. yon Wunnb, in Lichtenbergs Magazin für das Neuelte a. d. Phyf. u. N. G. I. IV. p. I. Voigts, Magazin für das Neuefte etc. V. II. p.I. (das Weibchen)

Camper, Naturgefch, des Orang - Outang etc. ins 1)eutfche iiberfetzt von Herbell. Düfeldorf, I79r. gr: 4 . 11 . K.

Camper, den Philof. Transact. LXIX. I. I779. p. $I_{39}$.

Camper kleine Schriften, II. II. p. 49. Vom Sprach Anatomie von Camper, in Lichtenbergs Maga. zin etc. II. I. p. I8z.

Sammlungen zur Phyf. u. Naturgefch. II. p.449. Pomnct; Botracht, uiber die Natur. II. p. $52 \%$ der Orang - Uiang.

Erxleben, Mammalia, p. 6. n. I. S. (Satyrus) ferruginea, lacertorum pilis reverfis, natibus tectis.

Gatterer brev. Zool. I. p. 14. n. 2. Simia (Saty. rus) capite minore; gracilior; hirfuta, pilo. rum humeri et uluae contraria directione; (ut in homine) der Orang - utang; Waldmenfich.

Vosmcier, defcription d'une efpece de Singe aufli rare que fingulier, 'nommé Orang - Outang. Amf. 1778 .

Hermmann, Tab.affinit, animal.p.52. Oran Utan. 
Lichtenftein, de Sim. vett. p. 30. 73. Simia Sa. tyrus.

35. La R. Der Gibbon.

Mitler, Naturfyft Suppl. p. 4. Tab. I. fig. I. der Langarm.

Ziminermann, geogr. Zool. II. p. 174. n. 71. der Gibbon; der laingarnige Affe.

Bïffon, vierf. XVII. p. 259. der Gibbon, m. 2 figg. Lefke; Naturgefch. p. II5. 11. 3. der langarmige Affe.

Boroweky, Thierreich, I. p. 42. n. 2. S. Longimana; der langarmige Affe, Gibbon, Golock.

Bhumenbach, Handb. d. N.G. p. 59. n. 3. Simia (Longimana) brachiis longifimis, talos attin: gentibus. Der Gibbon oder Golock.

IIIartini, Naturlex. I. p. 565. der indianifche lang. armige Affe ; der Schleuderaffe, fig. Büff.

Erxleben, Mainmalia, p. 9. n. 2. Simia (Longimana) brachiis longitudine corporis.

Philof. Transact. Vol. LIX. p. 418. (Abbild. und Befchreit. des Golock.)

Lichtenftein, de Sim. vett. p. 32. 73. Simia Lar. Gatterer, brev. Zool. I. p. I4. n. 3. Simia (Lon. gimana) brachiis longitudine corporis, talos attingentibus; der Langarm, Gibbon, Golock

2. Syivanus. Der gemeine Affe. ( $\left.{ }^{4}\right)$ Miiller, Naturfyftem, I. p. 120. n.2: der Wildtenfel.

\section{Zimuner-}

(4) Diefs ift der Affe, den -gèwöhnlich die Barenführer bey fich haben. - Seine Nahrung find. Früchte, Blätter, Brodt; auch Lyer und Infekted. Fleifch aber frifst er gar nicht. Alt gefan- 
Zinmmermann, geogr. Zoolog. II. p. I75. n. 72. der gemeine Affe; der Pithecke.

Schreber, Säugthiere, I. p. 68. der gemeine Affe, Tab. IV:

¿eske, Naturgefchichte, p. II5. 11. 4. der gemeine Affe.

Borowfky, Thierreich, I. p. 42. n. 3. Affe dev Alten; gemeiner Affe.

Blumerbach, Handb. d. N. G. p. 59. n. 4. Simia (Sylvanus) brachiis corpore brevioribus, natibus calvis. capite fubrotundo. Der gemeine tiiskifche Affe.

Bechftein, Naturgefch. Deutfchl. I. p. 132. der geineine Affe.

Meyer, Vorltellung allerh. Thiere, I. Tab. I3. (mittelmälsig) ein Aff.

Gatterer, vom Nutzen und Schaden der Thiere,

I. p. Ió. n. 4 der gemeine Affe.

Biiffon, vierf. XVII. p. 245 . der Pithekos, oder der gemeine Affe, m. e. Fig.

Martini, Naturlex. I. p. 546. Fig. Schreb. Affe der Altein.

Gesner, Thierb. p. ז. Simia, ein Aff, mit einer fchlechten Figur.

Erxleben, Mammalia, p. 75. n. 3. Simia (Sylvanus) brachiis corpore brevioribus, natibus calvis, capite fubrotundo.

Linné, Syft. Nat. VI. p.3.n. 4. Simia ecaudata, rufo nigricans.

Linné, Syft. Nat. X. I. p. 25. n. 2. Simia (Sylvanus.) ecaudata, clunibus tuberofo - callofis.

\section{Linné}

gen it er fehr unbändig. Er vermehre fich auch in der Gefangenfchaft, und das Weibchen geht in den zehnten Morath trachtig, und hat eine periodifche Reinigung. 
Linné, Syก. Nat. Edit. XII. I. p. 34. n. 2. Simia (Sylvanus) ecaudata, natibus calvis, capite fubrotundo.

Gatterer, brev. Zool. I. p. 15.n. 4. Sinnia (Sylvanus) brachiis corpore brevioribus, natibus calvis, capite fubrotundo; der gemeine tïrkiche Affe.

Ludolf, hif. Aethiop. Eib. I. cap. 10. $\$ .56 .57$. Raj, Synopf: Quadr: p. 149. Simia fimpliciter dicta, cauda carens.

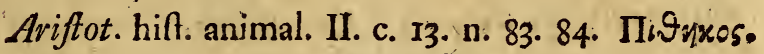
Aetian. animal. V. c. 26. M.

Plin. hift. natur. VIII: c. 54 XI. c. 37 : c. 44 : Simia.

Lichtenftein, de Sim. vett. p. 39. 74. Simia Sylvanus,

3. I n u u s. Der Hundskopf. (s)

Miiller, Naturfyf: I. p. I2I. n. 3. der Bufchgott; Pan.

Zimmermani, geogr. Zoolog. II. p. 176. n. 73: der Hundskopf:

Bhumenbach, Handb. der N. G. p. 59. n. 5. Simia (Innuus) capite oblongo, natibus calvis.

Gatterer, vom Nutzen u. Schaden der Thiere, I. p. 10. n. 5. der Bufchgott.

Biiffon, vierfüfs. XVII. p. 282. der Magot, mit 2 Figg.

Martin, Naturlexicon, I. p. 549. der Bufchgott, fig. Büff.

Erxleben, Mammalia, p. i3. n. 4. Simia (Innuus) capite oblongo, natibus calvis,

(s) Ein bürsartiges Thier, das eine ungemeine Starke befitzt, ma etwa die Gröfse von viar fufs erseicht. 
Linné, S. N. Edit. VI. p.3. n. 3. Simia ecaudata, clunibus tuberofis.

Ionfton, quadrup. p.' 144. Cynocephahis.

Gatterer, brev. Znol. I. p. I5. 11. 5. Simia (Innuus) capite oblongo, natibus calvis.

Lichtenftein, de Sim. vett. p. 43. 74. Simia In. nuus.

* * Thit kurzerz Schwänzen. PAv I A NE.

4. Nemestrin a. Der Schweinfchwanzaffe. $\left({ }^{6}\right)$ Miiller, Naturfyft. I. p. I2I. n. 4. der Waldgott. Zimmermann, geogr. Zool. II. p. 180. n. 79. der Schwein chwanzaffe.

Schreber, Saugthiere, I. p. 79. n. 7. Tab. IX. Simia Nementina, der Schreinfchwanzaffe. Borowfky, Thierreich, I. p. 44. n. 6. Waldgott; Schweing chrernzaffe.

Erxteben, Mammalia, p. 20. n. 4. Papio (Nemeflrina) fubbarbatus, grifeus, iridibus hrunneis, natibus calvis, cauda nuda contortuplicata. Büffon, hiftor. natur. XIV. p. I76. Tab. XIX. Le Mainnon.

Pennant, Syn. quadr. p. ro5. n. \r.

Gatterer, brev. Zool. I. p. 17, n. 4. Papio (Nemeftrina) fubbarbatus, grifeus, iridibus brunneis, natibus calvis, cauda nuda contortuplicata. Lichtenftein, de Sim. vett. p. 54. Simia NemeArina.

5. A PE.

(6) Das vollw üchfige Thier ift 2 bis $=\frac{\pi}{2}$ Parifer Fufs hoch, wenn es aufrecht fitzt; der Schwanz vier bis fünf Zull lang. Es ift gegen die Kalte empfindlich, und fchlaft fitzend. Das W zibchen hat eine Reinigung. 
3. A P E I A. Der kleine Pavian. (7)

Miiller, Naturfyf. I. p. 122. n. ร. der Kisrzfchwanz.

Zimmermann, geogr. Zool. II. p. 181. n. 80. der kleine Pavian.

Erxleben, Mammalia, p. 20. n. 5. Papio (Apedia) palmarum pollice approximato, unguibus oblongis: pollicum rotundatis, natibus tectis.

Linné, S. N. Edit. X. I. p. 25. n.4. Simia (Ape: dia) femicaudata, palmarum pollice approximato, unguibus oblongis, pollicum omnium rotundatis.

Gatterer, brev. Zool. I. p. 17.n. 5. Papio (Apedia) palmarum pollice approximato, unguibus oblongis, pollicum rotundatis; natibus tectis. Lichtenftein, de Sim. vett. p. 55. Simia Apedia.

6. SPні X. Der braume. Pavian. ( $\left.{ }^{8}\right)$

Miiller, Naturfy?. I. p. 122. n. 6. der Bavian. Zimmermann, geogr. Zool. II. p. 177.n. 75. der braune Pavian.

Boroweky, Thierreich, I. p. 44. n*5. der braune Pavian.

Halle, vierf. Th. p. 559. der Pavian.

Gatterer,

(7) Zimmermann legt ihm die Gtöfse cinet Katze bey.

(8) Die Nahrung diefes Thiers find blofs Früchite, Blatter, Wurzeln und Getraide. Das Weibchen tragt auf einmal nur Ein lunges. In kalten Ländern heckt es gar nicht, Büjfon, Scbreber, Zimmermann und andere Zoologen unterfcheiden zwey Varietäten, den grofsen und kleinen Pavian, welcher Unterfchied aber im Syftem p. 29. nicht mit bemerkt in. 
Gatterer, vom Nutzen u. Schaden der. Thiere,

I. p. 11. n. 7. der gemeine Pavian.

Klein, Quadr. difp. p.89. Cebus, Papio, Baboon, Hyaena Gesneri.

Klein, Claflif. d. vierI. Th. p. 272. n. 13. Meerkatze, Papion genannt.

Klein, natïrl. Ordnung d. vierf. Th. p. 95: n. 15. Bavian, Papio, Hundskopf mit ausgeftreck. ter Aumpfer Schnauze, wie ein Kettenhund.

Gesiner, Thierb. p. 357. fig. p. 358. Eine Art des Vielfrasses, Babuin.

Erxleben, Mammalia, p. 15. n. r. Papio (Sphinx) facie nigra, unguibus acuminatis, natibus calvis purpureis.

Limné, Syft. Nat. Edit. VI. p. 3. n. 5. Simia femicaudata, ore vibriffato, unguibus acutis.

Linné, Syft. Nat. Edit. X. I. p. 25. 'n. 3. Simia (Sphinx) femicaudata, ore vibriffato, unguibus acuminatis.

Kolbe, Vorgeb. p. 138. Tab. 5. fig. I. (fchlecht) Bavian.

Gatterer, brev. Zoolog. I. p. I6. n. I. Papio (Sphinx) facie nigra, unguibus acuminatis, natibus calvis purpureis.

Lichtenftein, de Sim. vett. p. 55. 76. Simia Sphinx.

Leon. Afric. defcript. Afr. p. 757. Babuini.

36. Mormon. Der Choras. (9)

Iniiller, Naturfyf. Suppl. p. 6. der Choras, Tab. I. fig. 2 .

Zimmer.

(9) Moguay bedeutet eine Marke, oder einen verkleideten Po. panz, Kinder zu exfchrecken; und wegen feines Gefichts, das, wie 
Zimmermanin, geogr. Zool. II. p. 178. n. 77. der Choras. Mormon.

Schreber, Säugth. I. p. 75, n. 6. der, Choras. Borowfky, 'Thierreich, I. p. 43.n. 4. der Choras." Tab. I. C.

Blumenbach, Handb. d. N. G. p. 60. n. I. Papio (Mormon) nafo miniato, ad latera coerule, fcente. Der Choras.

Berlin. Sammlung. I.,p. 387. Simia Mormon, fig. Alftröm.

Erxleben, Mammalia, p. 18. n. 3. Papio (Mormon) facie cyanea, pilis verticis faftigiatis, natibus calvis purpureis.

Schroed. Abhandl. XXVIII. p. 144. Tab. 3.

Gatterer, brev. Zool. I. p. I7. n. 3. Papio (Mormon) nafo miniato, ad latera coerulefcente, pilis verticis faftigiatis, natibus calvis purpureis. Lichtenftein, de Sim. vett. p. 54. 76. Simia Mormon.

7. M A I M O N. Der Mainon. $\left({ }^{10}\right)$

Mililler, Naturfyn. I. p. I23. n.7. der Teufel. B 2

Zimmer.

wie mit einer Mafke verftellt ausfieht, hat diefer Affe diefen Namen erhalten. Ex frifst faftige Frächte, das Fleifch der Citronen, Nüffe, die er aufknackt und mit der Schale zugleich hinterkailet, Hafer; infonderheit aber Eyer. Fleifch frifst er nur gekochtt, Wein und Prantewein verträgt er in Menge. Seine Höhe betragt nach Zimmermaxn etwas, über zwey Fufs. Das Weibchen hat feine Reinigung. Die Geilheit des Thiers if entfetzlich. Die Reihlichkeit aber liebt es fehr. Seine Stärke it beträchtlich, Sein Laut gleicht dem Grunzen einer alten Sau. Blumenbacb beftimmt feine Gröfse gegen fürf Fufs.

(10) Darbenton, Pennant, Scbreber i. a. geben die Gröfse des Maimon ohngefahr auf zwey Fufs an. Blumsubach aber verfichert, 
Zimmermann, geogr. "Zoolog. II. p. I78: n. 76. der Mainuon.

Bhumenbach, Handb. d. N. G. p. 60. n. 2. Papio (Mandril) facie violacea glabra, profunde fulcata.

Holle, vierf. p. 553. der Mamonnet.

Erxleben, Mammalia, p. 17. 11. 2. Papio (Maimon) facie violacea, unguibus acuminatis, natibus calvis purpureis.

Barttolin. acta Haffnienf. 167r. p. 67. fig. x. Cercopithecus Mamomet dicłus.

Gauterer, brev. Zool. I. p. 17. n. 2. Papio (Maimon) facie violacea glabra, profunde fulcata, unguibus acuminatis, natibus calvis purpureis; Mandril.:

Lichtenftein, de Sim. vett. p. 54. 76. Simia Maimon。

48. P O R C A R I A. Dev"Affe mit der Schiveinsfchnouze. (I)

Zimnermann, geogr. Zoolog. II. p. 197. (d.) der Affe nit der Schweins schnauze.

Pennant, Synopf. Tab. XIII. fig. s. Simia Porcaria?

Camper, Naturgefch. des Orang-Utang, p. II4. Choiropithecos.

Lichtenftein, de Sim. vett. p. 57.76. Simia porcaria.

$* *$ Mit

chert, dais es auch welche gibe, die fünf Fufs hoch wären, Handb. d. N. G. 3 Auf. p. 68.

(1) Arifloteles ift der einzige, der diefes Affen Erwähnung thut. Zimnermann fetzt ihn unter dic zweifelhaften Arten, nnd Cam7. per (Nat. Verh. I. p. 13.) bezweifelt gar feine Wirklichkeit. 
* * Mit langen Schwänzen. MEE R к AT Z EN.

* Mit Backentafchen, und kahlen Gefäfs chweieler.

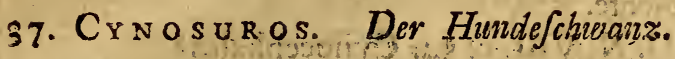

8. HA M A DR Y A S. Der graule Pavian. (2)

Miiller, Naturfyftem, I. p. 124. n. 8. di Waldmymphe.

Zimmermanir, geogr. Zoolog II. p. I\$2. n. 8r. der graule Pavian. (Affe.)

Borowfky, Thierreich, I. p. 45.11. 8. S. Hama: dryas; dev grais Paviano Hundskopf.

Gatterer, vom Nutzen und Schaden der Thiere, I. p. 12. n. 9 . die Waldriymphe.

Gesner, Thierb. p. 13 der Hundskopf, nit einer fchlechten Zeichnung.

Niebuhr, Befchreib, von Arabien, p. 167. Reife, ITh. p. 189.

Erxleben, Mammalia, p. 22. n. 1. Cercopithe-: $\operatorname{cus}^{\mathrm{T}}$ (Hamâdryas $)$ cinereus, auribus comofis, unguibus acutiufeulis, natibus calvis.

Linive, Syf. Nat: Edit. VI. p. 3. 1. 7 . Simia caudata imberbis, auribus comiofis.

Liiné, S. N. Edit. X. I. p. 27. n. Ir. Sinia (Haunadryas) caudata imberbis, auribus comofis.

Kolbe, Vorgeb. d, g. Hoffn. p. I38. der Bavian. sinu Die Figuren aber Tab. 5. fig. I. Tab. 2. fig. I. Aellen den gemeinen Affen vor; jene nach Ionf. Tab. 59. fig. It

B 3

Ionftore;

(2) Ein bürsartiges, geiles, aber doch gelehriges Thier, das oft fünf Fufs hoch ift. Sein Gebifs ift fürchterlich. - Seine Nahrung befteht vorzüglich in Früchten. In Arabien thut ex den Koffeebaumen, und auf dem Vorgebirge der guten Hoffnung den Gärten grofsen Sehaden. Fleifch frifst er nur gekocht. 
Ionfton, Quadruped, Tab. 59. fig: 1. (fchlecht) Cynocephalus 2. Pertrand.

Alpiz. hiff, nat. Aegypt. I. p. 242. Simia Cyno. cephalus ad vtramque aurem magna coma de. pendente.

Plin. H. N. VHI. c. 54. Cynocephalus?

Gatterer, brev. Zool: I. p. I8. H. I. Cercopithe. cus (Hamadryas) cinereus, auribus comofis, unguibus acutiufculis, natibus calvis.

9. VE TER." Der weifse Bartaffe. (3)

Miller, Naturfyf. I. p. I24. n. 9. der Alivater.

Zimmermann, geogr. Zool. II. p. I83. der weifst Bartaffe.

Schreber, Säugthiere, I. p. S6. n, 10. B. der weif a - Se Bartaffe. Weifs mit fikwarzen Barte.

Gatterer, vom Nutzen und Schaden der Thiere, slia: I. p.' I3. Mo ro der Altvaten.

Klein, Quadr, difp. p. 89. Cebus Elaurandus. Zeylanenfum.

Slein, Claflif, d. vierf. Th, p. 271. n. 12., Meepe katze Elaurąndus der Seilanenfer.

- Erxteben, Mammalia, p. 24. n. 2. Cercopithe. cus (Veter) barbatus albus, barba nigra.

- Gatterer, brev. Zool. I, p. 18. n, 2. Cercopithe cus (Veter) barbatus albus, barba nigra. Loa wando.

Lichtenfecin, de Sima vetti p. 64 77 . Simia

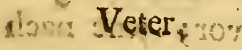

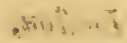
- . दे. क्. Zime

(3) Erxleben hat aus diefen beyden Varietäten zwey befondere Gattungen gemacht. Zimmemann und Scbreber führen fie zwar als Spjelnrien nuf, letzterer aber aweifelt noch mit erfterm, ob. fie nicht vielleicht, wie Knorr und Büfon anmehmen, gar mit cinander zu der folgenden Gattung gehürem. 
B. Zinmmermann, geogr. Zoolog. p. 185. a.

Schreber, Säugthiere, I. p. 86. n. 10. A. der vecifSe Bartaffe; ganz weifs.

Klein, Quadr. difp. loc. cit.

Klein, natürl. Ordn. d. vierfüfs. Th. l. є. Ganz weifse Meerkatze.

Erxleben, Manmalia, p. 24.n. 3. Cercopithecus (Senex) barbatus totus albus.

Gatserex, brev. Zool. I. p. I8. n. 3. Cercopithecus (Senex) barbatus totus albus.

Lichtenftein, de Sim. vett. p.65.77. Simia lactea barbata.

10. S Y L.ENus. Der fihwarze Bartaffe. (4)

Zimmermann, geogr. Zoolog. II. p. I84. n. 83 . der fihwarze Bartaffe.

Schreber, Säugthiere, I. p. 88. n. Ir. B. der fchwarze Bartaffe; ganz fchwarz.

Erxleben, Mammalia, p. 26. n. 5. Cercopithecus (Silenus) barbatus totus niger.

Linné, S. N. Edit. VI. p. 3. n. 13. Simia caudata caefaric prolixa faciem cingente.

Gatterer, brev. Zool. I. p. I8. n. 5. Cercopithecus (Silenus) barbatus totus niger.

Eichtenftein, de Sim. vett. p. 66.77. Simia Silenus.

B. Miiller; Naturfyft. I. p. I24. n. 10. der Weifsbart.

Zimmermann, geogr. Zoolog. II. p. 184. n. 83. b. Schwarz mit weifsem Barte.

Boroweky, Thierreich, I. p. 46. n. 9. Schwarzer Bartaffe.

$$
\text { B. } 4
$$

Gatterer,

(4) Auch diefe beyden Varietäten finden fich noch beym Erxlebein als zwey verfchiedene Gattungen. 
Gatterer, vom Nutzen und Schaden der Thiere, I. p. I4. 11. 13. das, Bartmännchens.

Knox, ceylan. Reifebefchr. p. 52. Wanderow mit einer Fig.

Klein, Quradr. difip. p. 89. Cercopithecus niger, barba incana promiffa.

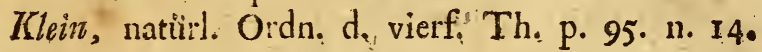
Wanduru aus Ceyloni.

Erxleben, Mammalia, p. 25. n. 4. Cercopithea cus (Vetulus) barbatus niger, barba alba.

Bosmann, Guinea, P. 302 . Bartminnerchen?

Heydt, Schauplatz von Africa und Ofind. p. 18\% Bartmünuchen?

Gatterer, brev. Zool. 1. p. 18. n. A. Cercopithecus (Vetulus) barbatus niger, barba alba.

Lichtenftein, de Sim, vett. p. 78. $\beta$.

Ix, FAunus. Der Malbrac. ( 5 )

Miller, Naturfyft. I. p. 125. n. Ir. der Löneene Schwanz, Wialdgeift.

Zimmernuann, geogr. Zoolog. II. p. I85, 11. 85. der Malbruc.

Borowesky, Thierreich, I, p. 45. n. 7. Simia Fau. nus; der Lönenfichvanz, Waldgeift, Tab. I. B. Halle, vierf. p. 554. der grosse brandfarbige Affe aus Guinea.

Klein, Quadr: difp. p. 89. Cercopithecus barba. tus primus Clufii.

Kein, Claflif. d. vierfüfs. Th. p. 270. n. z. bär. tige Meerkatze.

Klein,

(5) Nach Erxleben, Schreberi und Zimmermann gehört diefer Affe noch unter die zweifęlhaften Gattungen; und es if noch nicht genau betinnmt, ob der Faurus des Lihné mit dem Malbrse des Eivffon von einer Art fcy. 
Klein, natürl. Ordn. d. vierf. Th. p. 94. n. 8 .

Erxleben, Mammalia, p. 26. n. 6. Cercopithe. cus (Faunus) barbatus, cauda apice floccofa. Limne, S, N. Edit. VI. p. 3. n. 15. Simia cauda. ta barbata, cauda floccofa.

Linné, S. N. Edit, X. I. p. 26. n. 6. Simia (Faus. nus) caudata barbata, cauda apice floccofa. Ionfon, Quadruped. Tab. 74. Cercopithecus bar: batus Clufii,

Raj, Synopf. quadruped. p. 159. Cercopithecus barbatus I, Clufii.

Nieremberg, hif. nat. p. 177, Cercopithecus bara batus Clufii m. e. Fig.

Gatterer, brev. Zool. I. p. 18. n. 6. Cercopithe. cus. (Faunus) barbatus, cauda apice floccofa.

Lichtenftein, de Sim, vetto $p_{q}$ 68. 78, Simia Faunus,

\section{CY. omozers. Die Merkatza. ( $\left.{ }^{6}\right)$}

Miiller, Naturfyft. I. p. 127. n, i5, der Hzundsa beifer, angolifche Affe, Macaquo.

Zimmermann, geogr. Zoolog, II. p. r86. n. 86 , dia Meerkatze, Makack.

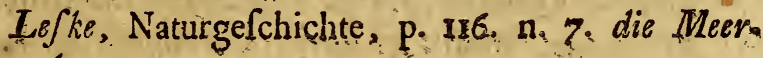
katze.

$$
3
$$

Blumerra

(6) Die warmetn Gegenden von Afrika wimmeln von diefen pofirlichen. Thipren. Durch ihren Schaden, den fie befonders den Reifsfeldern thun, nüthigen ffie die Neger, beftändig Wache bey ihren Aeckern zu halten, Diefe ftellen ihnen daher fehr nach, und das um defto mehr, weil fie das Fleifch mit Reifs gekocbt, oder grẳuchert effen. Man fängt fie in Schlingẹn, und fchiefst fie mit Pfeilen, oder Feuergewehr. Wenn einer angefchoffen if, fo kommen ihm die tubrigen zu Hülfe, und fuchen den Pfeil aus der Wunde zu ziehen, oder bẹifen wenigtens das Holz ab. Die Grölse des Tniełs ift etwa $x \frac{x}{2}$ Fufs, 
Blumenbach, Handb. d. N. G. p. 60. n. 6. Simia (Cynomolgus) cauda longa, arcuata, labio leporino. Der Macacso; die (insgemein $\int o$. gencinnte) Meerkatze.

Hialle, vierf. p. 556. der gröfsere aingolifche Affe. Gatterer, vom Nutzen und Schaden der Thiere,

I. p. 13. 11. 12. die Mcerkatze.

Klein, Quadr. difp.p. 89. Cebus angolenfis maior Macaquo Marcgr.

Klein, Clainf. der vierfüfs. Thiere, p. 269. n. 6. gröfsere angolenzffche Meerkatze.

Klein, natïrl. Ordn, der vierf. Th. p. 94. n. 6. MFacaquo aus Aingola.

Gesner, quadruped. p. 967. Cercopithecus: mit einer mittelmärsigen Figur.

Erxleben, Mammalia, p. 28. n. 7. Cercopithecus (Cynomolgus) imberbis, naribus bifidis. elatis, cauda arcuata, natibus calvis.

Linné, S. N. Edit. VI. p. 3. n. I0. Simia cauda. ta imberbis, naribus elatis bifidis.

Eimé, S. Nat. Edit." X. I. p. 28. n. I6. Simia (Cynomolgus) caudata imberbis, naribus bifidis elatis, clunibus tuberofis.

Ionfton, Quadrup. p. 143. Macaquo Congenfium. Marcgrav. Brafil, p. 227. Cercopithecus ango. lenfis maior, Congenfibus Macaquo dictus.

Gatterer, brev. Zool, I. p. 19. n. 7. Cercopithea cus (Cynomolgus) imberbis, naribus bifidis elatis, cauda arcuata, natibus calvis.

Lichtenftein, de Sim. vett. p. 78. Simia Cynomolgus.

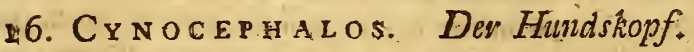
Miiller, Naturfynt. I. p. I28. 11. 16. der Hinds. kopf: 
Khin, Quadrup difp. p. 89. Cebus tertius Guineenfis.

Erxleberz, Manmalia, p. 30.n.8. Cercopithecus (Cynocephalus) imberbis flavefcens, ore producto, cauda recta, natibus calvis.

Icnfton, Quadrup. Tab. 59. die untere Fig. zur Rechten. Cercopithecus.

Marcgrav. Brafil. p. 228. Guineenfis alius.

Gatterer, brev. Zool. I, p. 19. n. S. Cercopithe. cu (Cynocephalos) imberbis flavefcens, ore producto, cauda recta, natibus calvis

17. Dia na. Die Diane. $\left({ }^{7}\right)$

Müller, Naturfyftem, I, p, 17, Diane, Tab. VI. fig. 2 .

Zimmermann, geogr. Zool. II. p. 18\%. n. $8 \%$ der Exquima; die Diane.

Boroweky. Thierreich, I, p, 47. n. 12. Diane; bürtige Meerkatze,

Halle, vierf. p. 600. bürtige Meerkatze; Diane. Klein, Quadruped. difp, p. 89, Cebus barbatusa Exquima Guineenfis.

Klein, Claffif. der vierf. Th, p. 270. n. 7a bärtí ge Meerkrotze.

\section{Klein,}

(7) Dicfer Affe frilst alterley Gewächfe, vorzüglich gern Nüfle, Mandeln und Rofinen, fonf aber auch Mühren, Rüben, Kohlwurzeln, Erbfen, Grütze, Kohl, Eyer u. dgl, Fleifch aber gar nicht. Die Wärme liebt er fehr; aber die brenpende Sonne if ihm zuwider. In kalten Năchten giebt er feine Fmpfindlichkeit durch Preifen zu erkennen. Er ift fehr reinlich. Alles, was: er freften will, beriecht er erft; ehe ex es koftet. Linné merise von ihm das Befondere an, dafs fich das aufserfte Ende feines Schwanzes jeden Monath cinmal öfnet, und einige Tago lang Blut fchwitzer, welches er ablecket ${ }_{2}$ und der Schwanz heil wiecer zu feiner Zeit. Schwed, Abh. XVI B. p. zrs. 
Kilein, natïrl. Ordn. d. vierfüfs. Th. p. 94. n. \%. Exquima aus Guinea, Erxleben, Mammalia, p. 30. n. 9. Cercopithecus (Diana) barbatus, fronte barbaque faltigiata.

Linné, S. N. Edit. VI. p. 3. n. I6. Simia caudata, harbata; harba cana, cauda fimplici.

Linne, S. N. Edit. X. I. p. 26: n. 8. Simia (Diania) cauda barbata, fronte barbaque faftigiata. Fonfton, Q tus Guineenfis.

Marcgrav. Brafil. p. 227. Cercopithecus barba- tus Guineenfis, p. 228. eine fehlechte Zeichnülig. Gatterer , bręv. Zoo!. I. p. 19. n. 9. Cercopithecus (Diana) barbatus, fronte barbaque faltigiata.

Lichtenftein, de Sim. vett. p. 78. Simia Diana,

78. S а в а с а. Der griine Affe. $\left({ }^{8}\right)$

Miiller, Naturfyft. I. p. I29. n. 18. der Mohr.

Zinmmermann, geogr. Zool. II. p. 188. n. 89. der grïne Affe.

Adanfon, Reife nach Senegall; (überf von Mar. tini ) p. IIr. $265 .(9)$

Forfter.

(8) Diefe Affen halten fich auf den Bäumen in grofson Haufen beyfammen, heobachten aber eine. folche Stille, dafs, da fie zwifchen dem Laube nicht wohl zu erkennen find, es fchwer baltep wïrde fie zu enrdecken, wenn fie fich nicht mit den $\mathrm{Z}$ weigen verristhen, die fie oft alsbrechen und berunterwerfen. Sic geben fogar nicht den geringiten Laut, wenn fie gefchoffen werden, defto mehr Grimaffen aber machen fie gegen ihren Feind, als ob fie ihn angreifen. wollten.

(9) In der dafelbft unien befindlichen Note o. o. ift vom Ueberfetzer aus Irrthum Simia Cynocepbalus, für Sabaea gefetzt. 
Forfter, Reife um die Welt, (Edit. in 8.) 1. p.44. St. Tago oder griine Affen.

Erxleben, Mammalia, p. 33. n. Ir. Cercopithecus (Sabaea) imberbis flavicans, facié atra, nảtibus calvis.

Gatterer, brev. Znol. I. p. 19. n. Ir. Cercopithecus (Sabaea) imberbis flavicans, facie atra, cauda cinerea, natibus calvis.

19. Ce P ù s. Der blanmä̀ilige Affe.

Miiller, Naturfyftem, I. p. Izo. n. 19. das Blawmonul.

Zimmermann, geogr. Zool. II. p. 189. n. 92. der blannüunlige Affe; der Mouftac.

Klein, Quadr. difp. p. 89. Cebus barbatùs alius Guineenfis.

Erxleben, Mammalia, p. 37. 11. 16. Cercopithecus (Cephus) buccis baccatis, vertice flavefcente, pedibus nigris ${ }_{\text {, }}$ cauda apice ferru. ginea.

Linné, S. N. Edit. VI. p. 3. n. I2. Simia caudata, genubus auribusque barbatis.

Linné, S. N. Edit. X. I. p. 2\%. n. 9. Simia (Ce. phus) caudata, buccis barbatis, vertice flave. fcente, pedibus nigris, cauda apice ferruginea.

Margrav. Brafil. p. 228. Alius Guineenfis.

Peninant, Synopf. quadruped. p. I14. n. 78. Munache.

Gatterer, brèv. Zoolog. I. p. 20.n. 16. Cercopithecus (Cephus) buccis barbatis, vertice flavefcente, pedibus nigris, cauda apice ferruginea. Muftak.

Iichtenftein. de Sim. vett. po 49. Simia Cephus. 
38. A Етнгор.s. Der weifsäugige Affe. ( $\left.{ }^{10}\right)$

Zimmermann, geogr. Zool. II. p. 191. n. 95. der re eissäugige Affe. Mangabey.

Haffelquift, R. nach Palaeft. p. 270. 190. Simia Aethiops.

Erixleben, Mammalia, p. 38. n. I7. Cercopithecus (Aethiops) interbis, vertice pilis arre. ctis lunulaque frontis albis.

Linné, Syft. Nat. Edit. XII. I. p. 39. Simia (Ce. phus) $\beta$.

Linné, Mur. Adolph. Fried. II. p. 4. Simia (Acthiops) caudata imberbis, vertice pilis arrectis lunulaque frontis albis.

Gatterer, brev. Zool. I. p. 20. n. $1 \%$. Cercopithecus (Aethiops) imberbis, vertice pilis arrectis lunulaque frontis albis.

ai. AYGuLA. Der. Tjükko. (')

Mitller, Naturfyf. I. p. I3I. n.2I. Eulaffe.

Zimmermann, geogr. Zool. II. p. I92. n. 96. det Tiökko.

Lefke, Naturgefch. p. I5 I. n. 9. der Tjähko.

Osbeck, Reife nach Clina, p. זзo.

Erxleb̆en, Mammalia, p. 39. n. 18. Cercopis thecus (Aygula) fubimberbis, grifeus, eminentia pilofa verticis reverfa longitudinali.

Limié,

(10) Ein gutartiges und gelebriges Thier, das etwa die Grớfse einer Katze hat. Den Schwanz trägt es gewöhnlich, in der Höhe, vorwärts nach dem Kopfe zu. Das Weibchen hat eine Reinigung.

(I) Tjükko ift der javanifche Name des Thiers. Wenn diefe Aften fchrafen, ftecken fie die Küpfe zufammen, fchreyen aber in der Nacht unaufhörtich. Grüne Sachen und Früchte find ihre liebte Nahrung. Nüffe beiffen fie felbft aut, und verzehren die Kernen. 
Linné, Syก. Nat. Edit. X. I. p. 27. n. Io. Simia (Aygula) caudata fubbarbata grifea, eminentia pilofa verticis longitudinali.

Pennant, Syn. quadr. p. II6. n. 8r. Egret ape. Gatterer, brev. Zool. I. p. 2I. n. I8. Cercopithecus (Aygula) fubimberbis grifeus, eminentia pilofa verticis reverfa longitudinali; die $A i$ grette.

23. Nictitans. Der veifsmånlige Affe. $\left.{ }^{2}\right)$

Miiller, Naturfyftem, 1. p. 132. n. 23. der nickende Affe.

Zimimermann, 'geogr. Zoolog. II. p. '190. n.' 93. der weissmäulige Affe.

Schreber, Säugthiere, I. p. 103. n. 20. der weifs. möulige Affe.

Erxleben, Mammalia, p. 35. n. 13. Cercopithecus (Nictitans) imberbis niger, punctis pallidis adfperfus, nafo albo, pollice palmarum. . breviffimo, natibus tectis.

Gatterer, brev. Zool. I. p. 20. n. I3. Cercopithecus (Nictitans) imberbis niger, punctis pallidis adfperfus, nafo albo, pollice palmarum breviffimo, natibus tectis.

Pennant, Syn. quadr. p. I20. n. 87 . the winking Monkey.

39. Sinica. Dev Hutaffe.

Miiller, Naturfyft. Suppl. p. 9. n. 23. C. der Hutaffe, Tab. II. fig. 3 .

Zimnnermann, geogr. Zoolog. II. p. 193. n. 98. der Hutaffe, die Chinefer Miitze.

Kinox

(2) Von dem befândigen Nicken mit alem Kopfe lat ex den.Namen erhalten. 
Knox, ceylan. Reifebefchreib. Lpz. I689. 4. p. 52. Rillow? M. e. fchl. Fig.

Evxleber, Mammalia, p. 4r. n. 20. Cercopithecus (Sinicus) imberbis, capillitio hotizontali, plano.

Gatterer, brev. Zool. I. p. 2r. n. 2a. Cercopithecus (Sinicus) imberbis, capillitio horizontali plano.

40. Nemaeus. Der Duk.(3)

Miiller, Naturfyft. Suppl. p. 10. n1. 23. d. der Duk, Tab. II. fig. 4 .

Zimmermann, geogr. Zoolog. II. p. I94. n. 100. $\operatorname{der}$ Duk; der Sifak.

Gatterer, vom Nutzen und Schaden der Thiere, I. p. 24. n. 14. $\operatorname{der} D u k$.

Erxleben, Mammalia, p.42.n.22. Cercopithecus (Nemaeus) mento buccisque barbatis, natibus pilofis, cauda alba.

Gatterer, brew. Zool. I. p. 21. n. 22. Cercopithe. cus (Nemaeus) mento buccisque barbatis, natibus pilofis, cauda alba.

41. Mon A. Die Mone. (4)

Miiller, Naturfyft. Suppl. p. 7. n. 17. a. die Monze. Tab. I. fig. 3. Büff.

Zimosiser-

(3) Det Duk hat keine Geftsfchwielen, fondern einen heharten Hintern, und macht alfo mit dem weifsmäuligen Affsn (S. Nicttans) eine Ausnahme in diefer Familie: Zimmermanse bàlt ihn für den Sifak des Flacour (Hift. de Madagafcar. p. 53.) welches Erxleben bezweifelt. Duk ift feia Name in Cochinchina.

(4) Auffer den gewöhnlichen Speifen der Affen frifst diefs Thier auch geisochtes Eleifch, Ameifen, Spinnen, und andere Infekten. 
Zimimermann, geogr. Zoolog. II. p. 187. n. 88 die Mone.

Gatterer, vom Nutzen und Schaden der Thieve, I. p. 13. n. Ir. die Mone.

Erxleben, Mammalia, p. 32. n. Io. Cercopithecus (Mona) barbatus, fupra rufofufcus, cauda grifea, natibus macula utrinque alba, pedibus nigris.

Alpinus, hift. Aegypt. IV. p. 245. Tab. 20. fig. 3. Monichus?

Gatterer, brev. Zool. I. p. I9. n. I0. Cercopithecus (Mona) barbatus, fupra rufo - fufcus, cauda grifea, natibus macula utrinque alba, pedibus nigris.

Leon. Afric. delcript. Afr. p. 757. INonae.

42. RvBR A. Der rothe Affe.(5)

Miiller, Naturfyn. Suppl. p. 7. n. 17. b. der rothe Affe, Tab. I. Fig. 4. Büff.

Zimimermann, geogr. Zoolog. II. p. I86. der ro. the Affe, der Patas.

Erxleben, Mammalia, p. 34. n. I2. Cercopithe. cus (Patas) barbatus, fupra niger, fubtus canus.

'Alpir. Aegypt. I. p. 244. Tab. XX. fig. 4. Jchlecht. Simia Callitrichus.

Aelian. de nat. anim. XVII. c. 8. Ǩ $\eta \pi \circ$ s?

Gatte-

(5) Die Neger am Senegall nennen diefe Affen Patas, und halten fie für wilde Menfchen, die nicht reden wollen, um nicht zur Arbeit gezwungen zu werden. Herr Brïe hat fie bey einer Reife auf Senegall zuerf bemerkt. Gegen ihre Vetfolger ver: theidigen'fie fich mit Steinen, und mit ihrem eigenen Uarath. den fie in die Hànde fallen laffen, und damit werfen. 
Gatterer, brev. Zool. p. 19. n. 10. Cercopithecus (Patas) barbatus, fupra ruber, fubtus canus, linea transverfa nigra fupra oculos inter aures.

\section{Tal a porn. Der chwarzanage Affe. $\left({ }^{6}\right)$}

Miiller, Naturfyft. Suppl. p.8. n. I8. a.der fchwarz. nafige Affe.

Zinnmermann, geogr.' Zoolog. II. p. I89. n. 91. der fchwarznafige Affe, der Talapoin.

Erxleben, Manmal. p. 36. n. 15. Cercopithecus (Talapoin) buccis barbatis, pedibus nigris, cauda fupra olivacea, fubtus cinerea.

Pennant, Synopf. quadrup. p. 115. n. 79. the Talapoin Monkey.

Gatterer, brev. Zool. I. p. no. n. 15. Cercopithecus (Talapoin) buccis barbatis, pedibus nigris, cauda fupra olivacea, fubtus cinerea.

\section{Peta u r is ta. Der veieisnafige Affe. (7)}

Miiller, Naturfyf. Supplem. p. 8. n. 23. 8. die Weifsnafe. Tab. H. fig. I. Allam.

\section{Zimmer.}

(6) Das Vaterland diefes Affen if nicht genau bekannt. Biiffors und Daubenton und mit ihin Erxleben fetzen es nach Indien. Letzterer ift fogar zweifelhaft, ob nicht diefe Gattung mit Simia Ceplous einerley feyn müge.

(7) Diefer Affe geht mehrentheils auf vier Füfsen, aufier wenn er etwas neues fichet, das er recht betrachten will, da er fich auf zwey Füfsen näbert. Ruhend hält er gemeiniglich den Kopf mit einem feiner Füfse, als ob er in Gedanken fâfse. Giebt man ihm etwas weiches zu freffen, fo rollt er es mit den Handen hin und her, wie cinen Teig, ehe er davon frifst. Bey dem Freffen lafst er fich nicht gern ftüren. Bey dem Saufen nimmt er den Bart fehr in Acht, dafs er nicht nafs wird, und trocknet ihn an irgend etwas snit fleifs ab, wenn es ja nafs geworden ware. 
Zinmermann, geogr. Zool. II. p. I9I. n. 94. der voifsnafige Affe.

Erxleben, Mammal. p. 35. n. 14. Cercopithe. cus (Petaurifa) barbatus, facie nigra, labiis macula triangulari alba, buccis auriculisque nudis.

Gatterer, brev. Zool. I. p. 20. n. 14. Cercopithe. cus (Petaurifa) barbatus, facie nigra, labiis macula triangulari alba, buccis auriculisque nudis.

45. Maura. Der Mohraffe:

Müller, Naturfyft. Suppl. p. 9. n. 23. b. der Mohr. affe, Tab. II. fig. I. Schre b.

Zimunermann, geogr. Zool. II. p. 193. n. 97. der IMohraffe.

Klein, Quadr. difp. p. 88. Cebus Simiolus Ceylonicus.

Klein, Claffif. d. vierf. Th. p. 268. n. 3. Meerkatze, kleiner ceylonifcher Affe.

Klein, natürl. Ordn. d. vierf. Thiere. p. 93. n. 3.

Ceylonifche Meerkatze.

Erxleben, Mammal. p. 4I. n. 19. Cercopithecus (Maura) totus niger.

Gatterer, brev. Zool. I. p. 21. n. 19. Cercopithecus (Maura) totus niger.

Lichtenftein, de Sim. vett. p. 70. 80. Simis Maura.

46. Rotoway. Der Palatinaffe. ( $\left.{ }^{8}\right)$

Miiller, Naturfyft. Supplem. p. 10. n. ^3. e. der palntinaffe, Tab. II. fig. 5. Allam.

$$
\mathrm{C} 2
$$

Zimmer-

(8) Er hat feinen Nanen von dem arn Kinne in eisen zweyfpalti* gen Bark verlangerten weifsen, lurzen Harftreifen, der ihm, 
Zinnmermann, gengr. Zool. II. p. 194. 17. 99. der Palatinaffe, der Roloway.

Erxleben, Mammal. p. 42. n. 21. Cercopithecus (Roloway) nigro-fufcus, barba alba bifida.

Gatterer, brev. Zool. I. p. 21. n. 21. Cercopithecus (Roloway) nigro - fufcus, harba alba bifida.

* Ohne Backentafchen und Gefäfschroielen.

* Mit langen Wickelfcherünzen; greifenden Schwönzen. (Sapajus, Büff. Cebus, Erxleb.)

12. Beеzzeвul. Der fchwarze Briillaffe.

IIiller, Naturfylt. I. p. I25. n. 12. der Fliegenfänger.

Zinmmermann, gengr. Zoolog. II. p. 200. n. I02. der fchwarze Briullaffe; der Aquiqui; Guariba.

Schreber, Säugthiere, I. p. II2. n. 28. der fchwarze Brillaffe.

Halle, vierf. p. $5 \%$. die fchwarze glatte Meerkatze. Klein, Quadr. difp. p. 88. Cebus Guariba Marc. grav.

Klein, Claflif. d. vierf. Th. p. 268. 11. 4. Meerkatae, Guaviba.

Klein, natürl. Ordn. d. vierfüls. Th. p. 94. n. 4. Guariba Marcgr.

Bankroft, Naturgefchichte von Guiana, p. 79. Gueveba.

Ei:xleben, Mammalia, p. 44. n. I. Cebus (Beelzebul) barbatus niger, caudae extremo pedibus. que brunneis.

Linné,

wenn das Thier fitzt, beynahe das Anfehen giebt, als wenn er vine Palatine um batte. 
Limné, Syft. Nat, VI. p. 3. n. I4. Simia caudata barbata, cauda prehenfili.

Ionfton, Quadrup. p. I42. Cercopithecus Guariba apud Brafilienfes dictus.

Dampier, Voy. III, p. 30z. Singes.

Pennant, Synopf. quadruped. p. I22. n. 9 r. the I'reacher Monkéy.

Laet, nov, orb. p. 553. Aquiqui,

Gatterer, brev. Zool. I. p. 22. n. I. Cebus (Beel. zebul) barbatus niger, caudae extremo pedibusque brunneis. Uarin.

13. Seniculus, Der rothe Britlaffe. $\left({ }^{9}\right)$

Miiller, Naturfyn. 1. p. I26. n. I3. der Greis. Zimmermann, geogr, Zoolog. II. p. 201. n. 103. der vothe Brillaffe; der Arabata.

Schreber, Säugthiere, I. p. II3. 11. 29. der rothe Briillaffe.

Gatterer, vom Nutzen u. Schaden der Thiere, I. p. 24. n. 16. $\operatorname{der}$ Greis.

Camper, Naturgefchichte des Orang-Utang, etc. p. 147. der Heuläffe.

Bankroft, N. G. von Guiana, p. 80. eine andere Meerkatze.
$\mathrm{C}_{3}$
Erxile

(9) Das Geficht diefes Affen kommt der Menfchengeftalt am nächften. Er fieht aus wie ein alter Mann mit einem Barte. Er hat, wie der vorige, einen eigenen Knochen im Halfe, wodurch reine Stimme fehr verfärkt wird; daher der Schreberfche Name: Brüllaffe, Am Tage fchlüft er, des Nachts" aber ilt er munter. "Die Gefangenfchaft vertragt er'nicht.' Die Wilden in America, und die dorigen Einwohner elTen ihn häufig. Das Fleifch if weifs, nicht fehr fett, und kümmt am Gefchmack dem Hammelfeifche bey. De Köpfe werden in Suppen gethan. Gefengt hat das Thier eine Aehnlichkeit mit einem kleinen Kinde, welches weinen will. 
Erxleben, Mammal. p: 46. n. 2, Cebus (Seniculus) barbatus rufus.

Pennant, Synopf. quadruped. p. I23. n. qr. $\alpha_{a}$ the royal Monkey,

Taet, nov. orb. p. 553. Rex Simiarum,

Demarchais, Voy. III. p. 3 II. 358.

Gatterer, brev. Zoolog. I. p. 22. 11. 2. Cebus (Se. niculus) barbatus rufus. Aluate.

14. PA Iscus. Der. Quota. (10)

Wiiller, Naturfyft. I. p. 136. n. I4. dier klcine Pañ oder Wraldgott.

Zinmermanu, geogr. Zoolog. II. p. 201. n. I04. der Quota.

Lefke, Naturgefchichte, p. II7. n. 9. dev vierfizygerige Affe.

Bhmenbach, Handb. d. N. G. p. 6I. n. I. Cercom. pithecus (Panifcus) ater, palmis tetradactylis, absque pollice; der Coaita, Beelzebub.

Bhumenbach, Samml. merkw. Reifegefchichten, I. 1.243. der Quotto.

Bankroft, N. G. von Guiana, p. 78. Quata.

Goeze,

(ro) Hecrdenweife in den Waldern von ganz Südamerica, wo fie oft dirch îhre Kïhnheit die läger erfchrecken. Der Schwanz leiftet diefen Thieren eben fo, und noch beffer als den übrigen Sapajoss die Dienfte einer fünften Hand. Sie halten fich damit an, wenn fie fich fchwingen oder falten wollen, indem fie ihn mit der Spitze um cinen Baumzweig, oder andern feften Körper herumwickeln. Ihre Nahrung befteht in Baumfrüchten. Sie freflen aber auch Fifche, Ungeziefer und Gewürme. Um. die Zeit, wenn die Früchte reif find, werden fie fehr fett, und man ifst fie dann bisweilen gebraten oder gekocht. Die Mănnchen fund fehr geil. Die IVeibchen haben eine regelmäsige Reinigung, und werfen auf ein Mal ein bis zwey lunge. Iung gefangen laffen fie fich zahm machen, legen aber ihre Falfchlieit und Tücke feiten ganz ab. 
Goeze, Natur, Menfchenleben und Vorfehung,

I. p. 531. der Cocita, Beelsebub.

Dobrizhoffer, Gefch. der Abiponer, I. p.388. das Thier Quati.

Fermin, Befchr. von Surinam, II. p. 115. Qilata. Erxleben, Mamalal. p. 46. n. 3. Cebus (Panifcus) imberbis, ater, palmis tetradactylis. Linné, Syn. Nat. Edit. X. I. p. 26. 11. 7. Simia (Panifcus) caudata barbata, cauda prehenfili, paimis fubtetradactylis.

Gatterer, brev.Zool. I. p. 22. n. 3. Cebus (Fanifcus) imberbis ater, palmis tetradactylis absque pollice. Waldtenfel, Coaita.

pennant, Syn. quadr. p. 124. n. 92. the fourfingered Moukey,

20. TREPIDA. Der Bufchfchwanzaffe.

Miiller, Naturfyf. I. p. 130. n. 20. der Zitteraffe. Zimmermann, geogr. Zoolog. II. p. 204. n. Ios. der Buechschuanzaffe.

Schreber, Säugthiere, It p. II8. p. 3I. Tab. XVII. der Bufchfchevanzaffe.

Erxleben., Mammal. p. 50. n. 6. Cebus (Trepidus) imberbis, capillitio arrerto, manibus pedibusque caeruleis, cauda villofa.

Gatterer, brev. Zool. 1. p. 23.n. 6. Cebus (Trepidus). imberbis, capillitio arrecto, manibus pedibusque caeruleis, cauda villofa.

pemnant, Syn. quadr. p. 126. n. 93. the Capucin Monkey.

28. Fatuezus. Der Hormaffe.

Miiller, Naturfynt. I. p. I35. n. 28. der Feldgott. 
Zimmermánn, geogr. Zoolog. II. p. 204. n. I09. der Hornaffe.

Schreber, Säugthiere, I. p. II8, n, 32. der Hornaffe.

Erxleben, Mammal. p. 5x.'n. 7 . Cebus (Fatuele lus) imberbiś, capitis fafciculis pilorum duobus erectis.

Penmant, Syn, quadr, p. 122. n. 96. the horned Monkey.

Gatterer, brev. Zool. I. p. 23. n. 7. Cebus (Im. berbis) capitis fafciculis pilorum duobus erectis,

39. APELLA. 'DeV Saju。

Mïller, Naturfyn. I. p. I36. n. 29. der Kahlbart。 Zimmermamn, geogr. Zoolog. II. p. 203. n. IoZ. der Saju.

Erxleben, Mammal, p. 50. 10.5. Cebus (Apella) imberbis, corpore fufco, pedibus nigris.

Limne, Syft, N. Edit, X. I. p. 28. n. 17. Simia (Apella) caudata imberbis, cauda fubprehenfili, corpore fufco, pedibus nigris.

Linné, Muf. Ad. Fried. I. p. I. Simia (Apella) imberbis, canda prehenfili, pedibus nigris, corpore fufco.

Gatterer, brev. Zool. I.p. 23. n. 5. Cebus (Apel= la) imberbis, corpore fufco, pedibus nigris.

30. Ca PUCINA, Der. Winelaffe.

Miiller, Naturfyft. I. p. 136. n. 30. der Capuziner. Zimmermann, geogr. Zoolog. II. p. 203. n. 106. der Winrelaffe; der Sai.

Lefke, Naturgefch. I. p. 108. n. 10. der Winfelaffe. Borowsky. Thierreich, I. p. 48. n. I4. Wine Selaffe.

Halle, 
Halle, vierf. p. 557. der ledergetbe IMuskusaffe? Fermin, Befchreib, von Surinam, II, p. II5, der branune Sapaju.

Dobrizhoffer, Gefch. der Abiponer, I. p. 384. Caui

Laet, nov, orb. p. 5.53. Cay?

Erxleben, Mammalia, p. 48. n. 4. Cebus (Ca. pucinus ) imberbis, fufcús, cauda hirfuta, pia leo artubusque nigris.

Linné, Syft. Nat Edit. X. I. p. 29: 1. 19. Simia (Capucina) caudata imberbis, cauda longa hir-futa, facie flavefcente.

Ionfton, Quadruped, p. I44. Caa apud Touvoupinamboutios?

Rai, Synopf: Quadrup. p. 155. Cay Brafilianis 'Touvoupinamboutis dictus?

Gatterer, brev. Zoolog, I. p. 22. n, 4. Cebus (Capucinus) imberbis, fufcus, cauda hirfuta, pileo artubusque nigris, Sai, Saju,

3r. Sciurea. Das. Todtenköpfichen.

Miiller, Naturfyftem, I. p. 136. 10. 31. der. Eich. hovinaffe.

Zimmermainn, geogr. Zoolog. II. p. 205. n. IIO. der Saitaca Saimivi; das. Todïenköpfchen.

Lefke, Naturgefch. p. iIs. n. II. das Todtenkköpfchen,

Fermin, Befchreib. von Surinam, II p, IIG. der gelbe Sapajou.

Erxleben, Mammalia, p. 5r, n. 8. Cebus (Sciureus) imberbis flavo-fufcus, ore caerulefcente, pedilus ferrugineis.

Limné, S. Nat, Edit, X. I. p. 29. n. 20. Simia (Sciurea) caudata imberbis, occipite prominua lo, unguibus quatuor plantarum oblongis. 
Gatterer, brev. Zool. I. p. 23. n. 8. Cebus (Sciu: reus). imberbis flavo -fufcus, ore caerulefeente, pedibus ferrugineis. Sajnirit.

3.2. Mor т A. Das Monkje. (Seb.) ( $)$

Mï̈ller, Naturfyft. I. p. 137. n. 32. der Fodtenkopf; Münch.

Halle, vierf. p. 558. der Todtenkopf:

Klein, Quadr. difp. p.88. Cebus, caput mortuum, vulgo.

Klein, Clallif. d. vierf: Th. p. 267. Mleerkatze, der Todtenkopf genamnt.

Klein, natürl. Ordn. der vierf. Th. p. 2,3. n. 2. der Todtenlepf.

Frermin, Surinam, II. p. II6. die Meerkatze mit dom Todtenkopf.

Linné, S. Nat. Edit. X. I. p. 29. n. 18. Simia (Morta), caudata imberbis, ore fufco, corpore fpadiceo, cauda nuda fubfquanofa.

33. Sч R I, н та, Der Magu. ( $\left.{ }^{2}\right)$

Miiller, Naturfyft. I. p. 137. n. 33. der Zwerg. Zimmermann, geogr. Zoolog. II. p. 199. 1. der. Magiv.

Schreber, Säugthiere, I. p. 124, 11. 36. der IMagit, Tab. XXXI. ein blofser Umrifs.

Linné, S. N. Edit. VI. p. 3. n. 6. Simia caudata, ore ciliisque vibrizatis.

Iinné;

(1.) Anch Erxleben, Mammalia, , p. 53. und Sçbreber, Säugthierc, p. 124. nehmen diefe Gatung als ein lunges der vorhergehenden an.

(2) Erxleben, Mammal: p. 53. rechnet diefe Gattung unter die Species abforias. 
Iinne', S. N. Ed: X. I. p. 29. n. I. Simia (Syrich-

ta) caudata imberbis, ore ciliisque vibriffatis.

Gatterer, brev. Zool. I. p. 23. n, , 10. Cebus: (Sy:richta) imberbis, ore ciliisque vibriffatis.

Rennant, Syn. quadr. p. 121. n. 90. the Philippine Monkey.

* Mit langess fihlaffen Schwoinzen.

(Sagoins, Büff. Callithrix, Erxl.).

2. Р гт несіа $D g r$ Sakia

Miller, Naturfyft. I. p. 132. n. 22. der Po/einm. reiser.

Zimmermann, geogr. Zoolog. II. p. 206. n. II2. der Saki.

Berlin. Samml. IV. p. 72. n., I. der Affe mit dem. Fuchsfchwanze; der Saki.

Erxleban, Mammalia, p. 55: n. I. Callithrix (Pithecia) Lubimberbis, vellere nigro, apice. albo, cauda nigra villofifima.

Ionfton, Quadrup. p. I43. Cagui Brafitienfibuss, Tab. LX. fig. Marcgr.

IIfarcgrav: Brafil. p. 227. Cagui Brafilienfibus; $m$. e. mittelnäfs. Figur.

Raj, Syn. quadruped. p. 154. Cagui maior Brafie lienfibus.

Gatterer, brev. Zoolog. I. p. 24. n. I. Callithrix (Pithecia) fubimberbis, vellere nigro, apice albo, cauda nigra villofifima.

24. I A C C Hu s: Der Sagoin. (3):

Miiller, Naturfyf. I. p. 13\%. 1n. 24. Bifamaffe.

Zinymere

(3) Von braner Farbe, und fo khein, dals er, in einer Cocusinusfchale Raum hat. "Scll befonders gean Fifche freffen. 
Zinumermanin, geogr, Zoolog. II. p. 207. n, II3. der Sagoin.

Schreber, Säugthiere, I, p. I26, n. 38. der Sagoin, , Tab. XXXIII.

Lefke, Naturgelch, p. II8. n. I2. der Sagoin. Boroweky, Thierreich, I. p. 48. n. I3. Sagoin, Bijamaffe.

Blumenbach, Handb. d. N. G. p. 62, n. 2. Cerco. pithecus (Jacchus) iuba pilofa alba ad genas ante aures, ca'nda villofa annulata. Der Uiftiti. Halle, vierf. p. 56I. das kleinfte Sagouinchen. Gatterer, vom Nutzen und Schaden der Thiere, I. p. 15.11 .17 . der Bifamaffe.

Klein, Quadr, difps p. 87. Cebus Sangouin die ctus Tab. 5. mit einer mittelmäls. Figur.

Klein, Claflif. der vierf, Th, p. 265. n. I. MLer. kat:se, Sagovin genannt.

Klein, natürl, Ordn, d. vierfüfs. Th. p. 93. n. I. Sagouiv.

Gesner, Thierb. p. 22. Galeopithecus Sagoin: Eine Art Meerkatzen; mit einer fehr fohlech. ten Figur.

Berlin. Samnul. IV. p. 78. dex Sagoin, Onif̧iti geranut.

Erxleben, Mammalia, p. 56. n. 2. Callithrix (Iacchus) auribus villofis patulis, cauda hirfu. tiffima, cinerea nigro annulata.

Linné, Syft. Nat, Edit. VI. p. 3. n. 8. Simia caudata imberbis, unguibus pollicum fubrotundis. Linné, Syft. Nat. Edit. X. I. p. 27. n. 12. Simia (Iacchus) caudata, auribus villofis patulis, cauda hirfutifima longiffima, unguibus fubulatis, pollicum rotundatis.

Zudolf; hif. Aeth. Lib. I. cap. ro. \$. 58. mit einer Figur. 
Edward, Befchreib. des Sanglins, oder kleinern

Cagui. Hamb. 1773. I Bogen 4.

$R a j$, Synopf. Quadrup. p. 160. Cercopithecus Sa• gouin Clụf.

Philos. Transat. Vol, XLVII. p. I46. Tab. 7. eine fchlechte Figur.

Rozier, Journal de Phyfique, 1787. Decemb.

p. 453. I779. Aout p. 153 .

Laet, nov orbis, p. 553. Sagovin:

Seligmann, Vögel, Tom. VII. Tab. XVI.

Nieremb. hiftor. nat. p. I77. Sagouin; mit einer erbärm! Zeichn.

Gatterer, brev. Zoolog. I. p. 24. n. 2. Callithrix

(Iacchus) auribus viliofis patulis, cauda hirfu. tifima, cinerea nigro annulata, iuba pilofa al. ba ad genas ante aures. Affennachtigall; $B i$. Samaffe; Sanguinzchen; Uiftiti.

B. Klein, Quadrup. difp. p. 88. Cebus Morchum redolens.

Klein, Claffif. d. vierf. Th. p. 269. n. 5. Meerkatze, welche wie Bifan viecht.

Klein, natürl. Ordnung d. vierf. Th. p. 94. n. 5 . Muskus. Meerkatze.

$R a j$, Synopf. quadr. p. I55. Caitaia.

Barrere, Fr. equinox. p. 151. Cercopithecus minor, dilute olivaceus, capite parvo.

25. OEDIPPUS. Der Pinche. (4)

Milller, Naturfyftem, I. p' 133. n. 25. der kleine Lö̋enaffe.

Zimmer.

(4) Zimmermann legt ihm die Gröfse von neun Zollen, und Scbreber die Gröfse von fechs Zollen bey. Limné fagt 'Schwed. Abh. am a. O.) es fey ras kleinfte Thier in feiner Art, und nicht gröfser als eine Ratte. 
Zinmmermann, geogr. Zoolog. p. 20\%. n. II4. der Pinche; das Löwenaiffchen.

Schreber, Säugthiere, I. p. 128. Tab. XXXIV. der Pinche.

Halle, vierf. p. 560. der Löwenaffe.

Klein, Quadrup. difp. p. 89. Cebus imberbis 2. Clufii.

Klein, Claffif. der vierfüls. Thiere, p. 27r. n. ro: Zwote Gattung unboirtiger Mlerkataen beym Chufuis.

Ktrin, natürl. Ordn. d. vierfüls. Th. p. 95.n. I2, Ohnbart; die zweyte des Chufus.

Klein, Quadrup. difp. p. 90. Cebus Liocephalus, L.eoniceps; Lüwenkatzz.

Klein, Claffif. d. vierfüfs. Th. p. 273.n. 14. Lö wen-Meerkatze.

Klein, natür. Ordnung d. vierf. Th. p. 96. n. x5. Löwen - Meerkatze.

Hamburg. Magaz. VI. p. 258. Pinche, Tamarin. Schwed. Abhandl. XXX. p. 157. Simia Oedippus. Erxleben, Mammal. p. 59.n.3. Callithrix (Oedip. pus) imberbis, capillo dependente, cauda rubra. Linmé. S. N. Edit. VI. p. 3. n. II. Simia caudkta imberbis, collo pectoreque iubatis.

Linné, S. N. Edit. X. I. p. 28. n. 13. Simia (Oedippus) caudata imberbis, capillo dependente. Condanine, Voy. p. 165. Pinche. Ionfton, Quadrup. Tab. LX. diefelbe Figur. Marcgr. Braf. p. 227. mit einer fchlechten Figur. Raj, Synop!. Quadr. p. 160. Cercopithecus non barbatus 2 Clufii.

Chuf. exot. p. 37I. Cercopithecus non barbatus II. Gatterer, brev. Zoolog. I. p. 24. n. 3. Callithrix (Oedippus) imberbis, capillo dependente, cauda rubrz. 
26. Rosal IA. Der Marikina. (s)

Miiller, Naturfyff, I. p. 134. n. 26. der Rofenaffe. Zimmermann, geogr. Zoolog. II. p. 208. n. II5. der Marikina.

Berlin-Samml. IV. p. 83. der kleinköpfige Löwen. affe. Marikina.

Erxleven, Mammalia, p. 60. n. 4. Callithrix (Rofalia) capite pilofo, faciei circumferentia pedibusque rubris.

Gafterer, brev. Zool. I. p. 24. n. 4. Callithrix (Rofalia) capite pilofo, faciei circumferentia pedibusque rubris.

47. Argentita. Der Miko. ( $\left.{ }^{6}\right)$

Miiller, Naturfy f. Suppl. p. ir. n. 26. a. der Miko; Tab. II. fig. 6. Büff.

Zimmermann, geogr. Zool. II. p.209. n. II6. der IMico.

Berlin. Samml. IV. p. 90. die kleine Meerkatze von Parn; der kleine langgefthwinzte Affe von Para.

Erxleben, Mammalia, p. 6t. n. 5. Callithrix (Argentata) imberbis, exalbida, facie rubra, cauda fufcefcente.

Condamine; Voyage fur la riviere des Amazones, p. 165. (deutfclie Ausg. p. 257.) Pinches àMay. nas, Tamarins.

Gatterer, brev. Zool. I. p. 25. n. 5. Callithrix (Argentata) imberbis, exalbida, facie rubra, cauda fufcefcente.

Reifên

55) Die Lange des Thierchens betrige neun Zoll; der Schwax ift drey Zoll langer.

(6) Dex Sshwanz ift faft noch einmal fo lang als das Thiet. 
Reifen der Miffionar. von Murr p. 206. Pinche oder Chichico; Sagovin, oder Tamarin. Ulloa, Voy. hift de l'A Amer. I. 50. Micos.

27. Midas. Der Tamari,

Miiller, Naturfy f. I. p. I35. n. 27. der Langohr, Tab. VI. fig. 4. Edwe.

Zimmermann, geogr. Zoolog. II. p. 209. n. II\%. der Tamari.

Sihreber, Säugthiere, I. p. I32. n. 42. der Tas. mari, Tab. XXXVII.

Lefke, Naturgefch. p. IIg. n. II3. der Tamarin. Fermin, Surinam II. p. II7. der fchwarze Sagoin mit gelben Fiifsein.

Berlin. Samml. IV, p. 75. n. Ir. der kleine Midas. affe; der fchwarze Affe.

Erxleben, Mammal. p. 62. n. 6. Callithrix (Mi. das) imberbis, labio fuperiore fiffo, auribus quadratis nudis, pedibus croceis.

Linne”, S. N. Edit. X. I. p. 28. n. I5. Simia (Mi* das) caudata imberbis, labio fuperiore filfo, auribus quadratis nudis.

Binet, Voy. à Cayenne, p.34r. Tamarin. Gatterer, brev. Zool. I. p. 25. n. 6. Callithrix (Midas) imberbis, labio fuperiore fiflo, auribus quadratis nudis, pedibus croceis.

A) Verönderungen gegen die XIIte Edition, und Ver. mehrung der Gattungen ailefes Gefchlechts,

Edit. XII.

Edit. XIII.

p. 34. n. r. ß. Satyrus In- p. 26. n. 34. Troglody* dicus. tes.

p. 39. n. r9. R. Cephus Si- p. 33. n1. 38. Aethiops. mia Aethiops. 
Diefs Gefchlecht if mit funfzehn Gattungen vermehrt, und S. Troglodytes, Lar, Mormon, Porcaria, Cynofuros, Aethiops, Sinica, Ne. maens, Mona, Rubra, Talapoin, Petauri. $\mathrm{fa}$, Maura, Roloway und Argentata hinzu gekommen.

\section{B) Unbeftimmtere Thiere.}

1. Der Affe mit den grofsen Hundszähnen. Simia (Madarogaftes) ecaudata, caninis fuperioribus exfertis, pectore abdomineque calvis, ver. tice cirrato.

Zimmermann., geogr. Zoolog. II. p. I76. n. 74 .

2. Der fchwarze Pavian. Papio (Äethiops) facie corporeque nigris (welcher mit Simia Aethiops im Syft. p. 33.n. 38. nicht zu verwechfeln in.)

Zimmermann, geogr. Zool. II. p. 180. n. 78 .

Turpin, Hilt. de Siam. I. p. 308 ?

Allgem. Reifen, XVIII. p. 425 ?

3. Der Affe mit flïgelähnlichem Barte. Cercopithe. cus (Kephalopterus) niger, barba alba triangulari, cauda longiore.

Zimmermann, geogr. Zoolog. II. p.'185. n. 84. Pennant, Synopl. quadrup. p. 109. Tab. XIV. fig. 2.

4. Der braungelbe Affe. Cercopithecus (Mulatta) fufcoluteus, caninis inferioribus magnis.

Zimmermann, geogr. Zoolog. II. p. 195. n. 10t. Pennant, Synopf. quadruped. p. 120. Tab. XIII. fig. 2.

Erxleben, Mammalia, p. 43. * 
5. Der grofse ungefchröinzte Affe von Benin. Zimmernlann, geogr. Zool. II. p. 196. a.

6. Der Boggo von Guinea.

Zimmernann, geogr. Zoolog. II. p. I96. b:

7. Dev Snitten von Giinea.

Zimnnernann, geogr. Zool, II. p. 197. c.

Bo/smann, Guinea, p. zor.

Barbot, in Allg. Reif. IV. p. 26r. 262.

8. Der Tretretretre von Madagafcar.

Zimmermann, geogr. Zool. II. p. I97. f.

Flacourt, Hift. de la grande Isle de Madagafear,

p. $15 \mathrm{I}$.

Dapper, afrik. Infeln, p. 32.

9. Der Affe mit.den Ziegezbarte.

Zimimernann, geogr. Zoolog. II. p. 198. g.

Pennant, Synopf quadr. p. 120. n. 88.

Erxleben, Mammalia, p. 43. **.

10. Der Affe mit geringeltem Schwcnze.

Zimmnernnann, geogr. Zoolog. II. p. 198. $\mathrm{h}$.

Pennant, Syn. quadr. p. I21. n. 89.

Erxteben, Mammalia, p. 44. $* * \cdots$ 。

11. Dev grofse Affe von Mralacca.

Zimmernann, geogr. Zool. II. p. 198. i.

Memoir. du Comte de Frobin. p. 219

12. Der vierfingerige Peruquenaffe. Cebus (Poly. komos) denfe comatus, fufcus, palmis tetradactylis, cauda alba.

Zinmmermagizi, geogr, Zool. II. p.202. n. 105. 
33. Der fchwarze Affe. Cebus (Lugubris) imberbis niger, facie ferruginea.

Zimmermann, geogr. Zool. II. p. 205. n. Irr. Erxleber, Mammalia, p. 53. n. 9.

14. Der Affe mit gelb i. Schworz gefprenkeltem Rücken.

Zimnermann, geogr. Zool. II. p. 206. a.

Penncint, Syn. quadr. p. 129. n. 97.

Erxileben, Mammalia, p. 54.*.

15. Ein niener Pavian.

Zinumermnam, geogr. Zool. II. p. 197. e:

Penuant, Syn. quadr. addit. Tab. XIII. fig. 2 :

Erxleben, Mammalia, p. 21. *.

16. WV anvenuven, eine dem Gibbon ähnliche Affenart. Lichtenberg, Magazin $\mathrm{f}_{\mathrm{i}}$ d. Neuefte a. d. Phyf: etc. II. I. p. I.

Camper, Naturgefch. des Orang-Utang, p. I2O. Wotneon.

Lichtenftein, de Sim. vett. p. 74. Simia Nanodes.

17. Der Kahau; aus der Infel Borneo.

Lichtezberg, Magaz. f. d. Neuefte etc. II. IV. p. I.

18. Simia Lugubris.

Erxleberi, Mammalia, p. 53. n. 9. Simia (Lu. gubris) imberbis niger, facie ferruginea:

Gattever, brev. Zool. I. p. 23. n:9.

19. Simia Lynx.

Lichtenftein, de Sim. vett. p. 75. Simia (Lynx) femicaudata, fubbarbata, facie fubrufa, fronte prominula, atra, natibus calvis fubrufis, fede feminae quafi prolapfu uteri protuberante. 
3. GESCHLECHT. LeMur. Der Maki; GeSpenfterthier; Fanlthieraffe.

Miiller, Naturfyft. II. p. I46. Gen. III.

Zimmermain, geogr. Zool. II. p. 210. Gen. XVI. Schreber, Säugth. I. p. I33. Gen. III.

Lefke, Naturgefch. p. II9. Gen. III. Borowesky, Thierreich, I. p. 49. Gen. II. Bhumenbach, Handb. d. N. G. p. 69. Gen. V.

Bïffon, vierf. XIV. p. I54.

Bat $\int \mathrm{ch}$, Thiere, I. p. 167. Gen. XX.

Erxleben, Mammalia, p. 63. Gen. VII.

Granmann, intr. in hiftor, nat: mammal. p. 23. Gen. VII.

Gatterer, brev: Zool. I. p. 25. Gen. VII.

1. TARdigradus. Der Loris.

Miiller, Naturfyft. I. p. I47. n. I. der Langfamfchleicher, Tab. VII. fig. I. Seb.

Zimmermant, gengr. Zool. II. p. 2 II. n. rI8. Lemur (I.ori) ecaudatus, corpore attenuato, artubus longioribus; der Lori. - p. 2I2. n.119. Lemur (Tardigradus) ecaudatus Linn. XII. p. 44. der Coucang; der träge Maki von Zeiv lan. ( 7 )

Lef'ke, Naturgefch. p.' I20. n. I. der Loris. Borowfky, Thierreich, I. p. 50. n. 2. Langfchlei. cher, Loris.

Blumenbach, Handbuch d. N. G. p. 62. n. I. Lemur (Tardigradus) ecaudatus; der Loris, Cucang.

Halle, vierf:p.551. der langgeftreckte röthliche Affe. IMeyer,

(7) Zimmerminn macht einen Unterfchied zwiichen dem Loris und dem Moki; der abor im System nicht aus einander ge= fetzt ift. 
Meyer, Thiere, III. Tab. III. Figur. Seb. der Foule mit dem Hundskopf beyderley GeSihlechts.

Klein, Quadr. difp. p. 86. Simia ceylonica, fu: periori labio leporino.

Klein, Claflif, d. vierf. Th. p. 259. n. 2. ceilonifcher Affe mit der obern Hafenlef $\approx e$.

Klein, natü!. Ordn. d. vierf. Th.'. p. 9r. n. 2. ceylonifcher Affe, mit einer Hafen charte.

Klein, Quadr." difp. p. 86. Simia roftro canino, capite efato.

Klein, Claflif. d. vierf. Th. p. 26r. n. 3. Affe mit der Hunds schnause mit erhöhetem Kopfe.

Klein, natürl. Ordn. d, vierfüfs. Th. p. 92. n. 3. Hunds chnlanze mit erhabenem Kopfe.

Martini, Naturlexicon, I. p. 563. der ceyloni/clie Affe.

Biiffon, Naturgefchichte der vierfüls: Th. XIV. p. 192. der Loris.

Erxleben- Mammalia, p. 63. n. I. Lemur (Tardigradus) ecaudatus.

Ausz. a. d. Tagebuch eines Reifenden nach Afien; p. 265. 11. 27. der Thevang oder Schleicher.

Linné, Syft. Nat, Edit. II. p. 42. Simia mammis quaternis, capite ad aures crinito.

Linné, S. N. Edit. VI. p. 3. n. 2. 'Simia ecaudata, unguibus indicis fubulatis.

Linné, S. N. Edit. X. I. p. 29. n. I. Lemur (Tardigradus) ecaudatus,

Linné, Mur. Adolph. Fried, I.p.3. Lemur (Tardigradus) ecaudatus.

Linné, Amoenit. acad. I. p. 558. Simia acauda, digitorum indicum ungue fubulato.

Graumann, intr. in hiftor, nat. manmm. p. 24. I. der Langfchleicher; ceyloni/che Affe.

Gatterer, 
Gatterer, brev. Zoolog. I. p. 25. n. I. Lemur (Tardigradus) ecaudatus; der Loris.

Mufo Baruth. p. I2. dẹr kleine oftindifche Affe, ohne Schucunz, mit den Hundskopf, Menfchenhï̈nden, and langen Spitzigen Nïgeln, an dem ziveyten Zehen des Fufses. Tab. IX. fig. I. das Weibchen.

Muf. Baruth: p. 19. der allerkleinfte oftindichè Affe, ohne Schwanz; mit dem Hundiskopf, MenSihenhïnden, und langen fpitaigen Nögein am ziveyten Zehen des Fisses, fo man noch nicht befinrieben, oder abgebildet findet. Tab. IX. Fig. 2. das Männchèn.

Vosmaer, défcription d'une Efpece de Parefleux. Amn. 1770. mit der beften Zeichnung:

9. IN DR I. Dev:Indri. $\left({ }^{8}\right)$

Sonnerat, Reifen nach Oftindien und China, aus d. Franz. überf. 4. I7.83: II. p. III. der Indxin. Tab. 88 .

5. Pотто. Der Potto.

Miiller, Naturfyft. Supplem. p. 12. n. I. der Potto.

Zimniermann, geogr. Zoolog. II. p." 218. a. der Potto.

Schreber, Säugthiere, I. p. 137. n. 2. der Potto. Bofsmann, Befchreibung von Guineà, p. 296. fig. 4 .

(8) Somnexat fagr: as fey die gröfste Gattung der Maki. 'Sein Aage if weifs tund fehr lebhaft. Indri heifst in der Sprache der Madagafien: Maldmench. 


\section{Mongoz. Der Mongus. (-9)}

a. Ganz grau oder braum.

B. Braun mit weifser $\mathrm{Nafe}$ und Händen.

$\gamma$. Braun, mit einem fchwarzen Flecke un die Augen.

8. Braun, mit einem fchwarzen Flecke um die $\mathrm{Au}$ gen, und hellgrauen Händen.

E. Dunkelbraun, mit fchwarzem Geficht und Händen.

3. Grau, mit fchwarzem Geficht und gelben Händen. Miller, Naturfyltem, I. p. 147. das Ringange. Müller, Naturfynt. Suppl. p. 12. n. 2. der Mongus. Zimmermann, geogr. Zoolog. Il. p. 214. n. 120. der Mongus.

Lefke, Naturgefch. p. 120. n. 2. der Mongus. Burowfky, Thierreich, I. p. 50 . n. 3. Mongus, Ringange.

Bhumenbach, Handb: der Naturgefch. p. 62. n. 2. Lemur (Mongoz) facie nigra, corpore ct cau. da grifeis; der Mongus.

D 4

Bech.

(9) Dẹr Mongus halt lich meift in ftarken Haufen auf den. Eäumen auf. Seine Nahrung find Obttrüchte, Salat, Rofinen, füfse Sachen, Brodt, Milch. Er nimmt die Speife theils mit dem Munde, theils mit der einen Vorderhand. Flïfsige Dinge leckt er wie die Hunde. $\mathrm{Er}$ if leicht zahm zu machen, und dann fehr fromm. Seine Zunge ift fo weich, wie die Zunge eines Hundes, An der Kette liegend macht er ein beftandiges Gerauch, weil er immer in Bewegung feyn will. . Eingefperrt oder allein nage er an feinem Schwanze, der daher faft niemals an der Spitze ganz if: Er kann mehrerey Ellen hoch furingen. Sein Gang ift immer vierfüfsig, und etwas fchleppend. Sein gewölinlicher Laut gieicht dem Grunzen eines jungen Schweines. Fir fchlaft oft, aber fehr leife, am liebften an einem finfern Orte, und halt fich fehr reinlich. Kalse ift ihm empindlich, daher er in Europa felten den Winter ansdauert.' Das Thier hat übrigens die Gröfse einer Katze, if aber hoçsbeinigter. 
Bechftein, Naturgefch. Deutfchl, I. p, I32. dev Mongus.

Bïffon, vierf. XIV. p. I86. m. 3 Figg.

Erxleberr, Mammalia, p. 66. n. 2. Lemur (Mongoz) caudatus, grifeus, cauda unicolore, pilofa.

Flacoust, IIif. de Madagafcar, p. I53: mit einer fehr fchlechten Figur.'

Couche, hift, de Madagafcar. p. 127. Singes bruns de couleur des cafiors.

3. Macaco. Der Vari. $\left({ }^{10}\right)$

$\alpha$. Schwarz,

$\beta$. Braun.

\%. Weifs,

$\delta$. Schwarz und weifs.

Miiller, Naturfyn, I, p. I47. n, 3, der Barte - kragen.

Zimmermann, geogr, Zoolog, II. p. 215, n. I2I, der Vari.

Biiffon, vierf, XIV, p. I90. der Vari; mit 2 Figg. Erxleben, Mammal, p. 67. n. 3. Lemur (Maca. co) caudatus niger, collari barbato, cauda pilora. Gratuman, intr, in hif, nat. mamm, p. 24. III, der Barikragen; Vavi.

Gatterev, brev. Zool. I. p. 26. n. 3. Lemur (Macaco) caudatus níger, collari barbato, cauda pilofa; der Vari; Bartkragen.

4. CAT.

(10) Unterfcheidet fich von dem Mongus durch einen Bufch langer Haare, die er an jedem Ohre hat, ift auch wolligter, und übertrift ihn an Gröfse. Iung gefangen läfst er fich zahm machen. Seine Speifen find Früchte, Butterbrodt, Kuchen. Seine Luftrühre bildet durch eine Erweiterung, bevor fie die Lunge be= rühret, eine Art von Trommel, wodurç eben die Stimme des Thiers das Füurchtęllịhe exthălt, 
4. Сатта. Der Mokoko. (')

Hhïller; Naturfyft, I, p. 148, n, 4. der Eichhorna affe, Táb. VII, fig. I. Edw.

Zimmermann, geogr. Zool, II, p. 216, n, 122. der Mokoko.

Lefke, Naturgefch. p. r21, n. 3. dér Mokoko.

Borows Sky, Thierreich, I. p. 49. n. I. Eichhorn.

affe, Maukauko.

Holle, vierf. p. 560, der Fichsaffe.

Gatierer, vom Nutzen und Schaden der Thiere,

I. p. I6. n. 19. Mokoko.

Naturforfch, XV. p. I3\%). Lemur Catta,

Klein, Quadr. difp, p. 90, Cebus capite vulpino;

Füchsèl-Mï̈nnchen.

Klein, Claflif, d, vierf, Th, p, 273, n, 15, Fiich Selmännchen.

Klein, natürl, Ordn, d, vierfüls. $T h_{i}$ p, 96. n, is. Fïichjelmännchen.

Büffon, vierf, XIV, p. 172, m. e.Fig, der Mokoko, Ives, Reife nach Indien, I, p, 29. Mocock.

Erxlebén, Mammal, p. 68. n, 4. Lemur (Catta) caudatus, cauda albo nigroque annulata, pia lofa.

Limné, S, N. Edit, X, I, p. zo, 1, 2, Lemur (Cato

ta) cauda annulata,

Scopoli, Ann, hif, V, p, zo, Lemur Catta,

Toreen, Reife nach Surate, p: 440. Lemur Catta, Seligmann, Vögel, VI, Tab. 92. Edww. der $M a_{a}$ kauko.

Flacourt, hif, de Madagafcar, p. 153.

Graunanu, intr, in hif, nat, mamm, $\mathrm{z}_{24} \mathrm{IV}$, Mankauko; Eichhornaffe.

D. 5

Gattes

(1) Der Gü̈fse nach gleicht das Thier einer mittelmälsigen Katzę Seine Linge if fechszehn Zoll. 
Gatterer, brev. Zool. I. p. 26. n. 4. Lemur (Catra) caudatus, cauda albo nigroque annulata, pilofa. Der Mokoko. .

7. MuR I nus. Die Ratze von Madagafcar. (Zimm.) Zimmermanin, geogr. Zoolog. II. p. 219. b. Biiffon, Suppl. Ir, p. I49. 'Tab, 20. Rat de Madagafcar.

\&, BICOLOR. Dev aweyfarbige Maki。

10. L A N I ER. Der wolligte Maki.

Sonnerat, R. nach OAtind. II. p. II2. Tab. 89: der wolligte Maki.

5: Korans. Der fliegends Maki.

Mitller, Naturfyftem, I. p. I49. n, 5: die file: gende Katze, Tab. VII. fig. 3. Seb.

Zimmernnann, geogr. Źoolog: II.: p. 3I6. n. I23. der fliegende MTaki.

Lefke, Naturgefch. p. I2I. n. 4. der fiegende IMaki. Hoille, vierf. p. 453. die fliegende. Katze.

Meyer, Thiere, III. die flegende ITatze von Ter. nate, Tab. 3\%. das Männchen.

Gatterer, vom Nutzen und Schaden der Thiere,

I. p. I6: n. 20. die fiegende Katze.

Eixleben, Mammalia, p. 71. n. 7. Lemur: (Vo. lans) caudatus, membrana ambiente volitans. Linné, Syft. Nat, Edit. X. I. p: 30. n. 3. Lemur (Volans) caudatus, membrana ambiente vo. litans.

Pennant, Synopf. quadruped. p. 139. 11. Io9. Ǩircher, China illuftrata, p. 84 . Vefpertilio, qui ob corporis molem Cattus yolans dicitur. Mit einer 
einer Figur. (Wird von den Einwolhnern ge: geffen, p. 85.)

Gaitever, brev. Zoolog. I. p. 26. n. 7. Lemur. (Volans) caudatus, membrana ambiente voli. tans. - Die. fliegende Katze.

A) Verönderungen gegen die XIIte Edition, unat Ver: mehrung der Gattuingen diefes. Gefchlechts,

Diefs Gefchlecht if mit finf. Gattungen vermehrt, und 1. Indri, Potto, Murinus, Bicolor und Laniger hinzugekommen.

B). Unbeftimmtere Thier:e.

1. Der kteinfte Maki.

Zimmennain, geogr. Zoolog. II. p. 219. $c_{9}$

Schreber, Säugthiere, I. p. I $\mathbf{4} \mathbf{I}_{\text {, }}$

Biiffon, vierf. XIV. p. I6?.

4. GESCHLECHT. VESPETILIO. DiQ Fiedermcaus.

Miiller, Naturfyn. II. p. 150: Supplemp. p. 14. Gen. IV.

Zimmermann, geogr. Zool. II. p. 408. Gen: XLI. Schreber, Süugthiere, I. p. I47. Gen. IV. Lefke, Naturgefch. p. F56. Gen. XXI. Borow fly, Thierreich, I. p. 5I. Gen. IU. Bhumenbach, Handb. der N. G. p. 67. Gen. XI. Funke, Naturgefch. u. Technologie, I. p. 182. Auszug a, d. 'Tageb. eines Reifenden nach Afien, p. 53.

Erxleben, Mammalia, p. 130. 141. Gen, XV. XVI. 
Graumann, intr, in hiff, nat. mamm, p. 33.34 Gen. XV. XVI.

Gatterer, brev, Zool, I. P. 37.39. Gen, XV.XVI. Pennant, Quadr. p. 359. Gen, Xl.III.

Briffon, Quadr, P. 153. 158. Gen, XXX, XXXXII. Hermann, 'Tab. affinit, animal, p. II3.

Miiller, diff, de Vefpertilionibus, Witteb. 1675.4. Pallas, f picileg. Zool, III. p. 3.

pallas, Naturgefch. merk v. Th. III, p. 3 .

\section{* Mit $\frac{4}{4}$ Vorderzähnenen. $\left({ }^{2}\right)$}

Pteropus Erril. Brif:

\section{VAmpY Rus. Der Vampyr: (3)}

a. Neun Zoll lang, klaftert mit den Fittigen drey Fufs, in fchwarz, auf dem Kopfe dunkel fuchsroth, von da fich ein dergleichen Streif an jeder Seite des Rückens über die Schultern und Lenden hinzichet; an dem Vorderarme und um den After herum heller; die Flughaut fchwärzlich, Schreb.

Boroweky, Thierreich, I. p. 52. der fliegende Hunnd, Bhutfauger; la Rouffette Büff.

Binmen-

(2) Die obere diefer beyden Zahlen deutet, wie bey Schreber und Zimmermann, auf die Vordẹtaăhne in der obern, die untere auf die in der untern Kinnlade.

(3) Eẹy den Thierarten, welche unter dẹ Namẹn des fliẹgendin Hundes, der fliegenden Katze oder Ratze befchrieben werden, herrfcht auf mehr als eine Weife grofse Unbeftimmtheir; und die Rouffette und Rougete find von den wenighten Scluriftellern unterfchiẹden, Ich will, fo gut ich kann'z, die zu jeder Varietat gehürige Schriften aus einander fetzen, und dann diejenigen Stellẹn hinzufügen, die vielleicht von der ganzen Gațtung handeln. Auch in Anfechung dẹ Gröfse ift ẹne grufse Verfchieden- 
Bhimenbach, Handb. d. N. G. p. 68. Vefpertilio (canis volans) ecaudatus, nafo fimplici, membrana inter femora divifa; der fliegende Hund, (Linnés Vampyras; Büffons RonifJette.)

Halle, vierf. p. 452. Tab. 28. der fliegende Hund. Meyer, "Thiere; III. Tab. 35. der fliegende Hund - von Terriate, beyderley Gefchlechts; fig. Seb. Neuer Schauplatz der Natur, III. p. II8. der fiegeride Hund von Ternate.

Frifch, Tábellen, p. 6. n. 4. die Tonga oder RoufJettè Büff.

Gesner, hift. Av. p. 772. Vefpertilio Borfippae. Klein, Quadr. difp. p. 6I. Vefpertilio Cynocephalus, Ternatanus.

Klein, Clafilf. der vierf. Th. p. i87. n. 8. Fle. dermans mit dem Hundskopf.

Klein, natürl.' Ordn. d. vierfüfs. Th. p. 6\%.' n. 6 . Hindskopf aus Ternato.

Biiffor,

heit in den Nachrichten, wiè die ängèführten Schriftèn beweifen. Diefe Thiere fliegen in fo grofsen Haufen, dafs fie die Luft verdunkeln. Sie nahren fich von den Früchten der Eäume; befonders follen fie den Saft 'der Palmbäume gèn fátifen, und fich darin fo beraufchen; dafs fie bisweilen wie todt zu Bo= den fallen. Sie haben einen widrigen Geruch; und beiffen fcharf; wenn man fie reitzt; fonft aber find fie harmlos. Sì thattern mit vieler t.eichtigkeit über dem $\vec{W}$ affer, und halten fich gern am Waffer auf, um durch Baden des Ungeziefers los zu werden. Forfter hat, auch einen fchwimmend gefunden. Nach feinem Bericht verferrigen die Einwoliner in Neukaledonien aus ihrem Haar Stricke und Quafte, womit fie ihre Kèlen auszieren, und verweben fie zu dem Ende mit Eàden, die aus dern Halm einer Art Cypergrafes gemacht werden. Forfer fand einen fehr "grofsen Keulenbaum; an welchem ïber 500 in verf́chiedenen Stellüngen, bald an deñ Vorder-bald an den Hinrerfüsen hiengen. Nähèrn Bebbachtungen zu Folge faugt ditofos Thier kein Blut, daher es' auch ganz unrishtig Vampyrt geaanat wird. $S$, die folgende Gattung: 
Biiffor, vierf. VII. p. 65. die Rotefette - An. hang p. 85 . und 89 .

Allg. Hift. d. Natur, V. 2. p. 33. Tab. 14. dib Rouffette.

Berlin. Samml. II. p. 423. die Ronffette. Erxleben, Mammalia, p. I3r. La Rouffette Bïf。 fonii; Chien volant Daubentonii.

B. Fünf Żoll lang, klaftert zwey Fufs, if fchwärzo lich grau, oder braun, auf dem Halfe röthlich. Die Schnautze fcheint an diefer Sorte etwas fpitziger zu feyn, als an der vorherge: henden. Sichreb.

Neuer Schauplatz der Natur, III. p. Ir\&. der.file. gende Hund rinit rothen Halfe.

Frifch, 'Tabellen, p. 6. 5. 17. 5. die Tani, oder Rougette Biiff:

Berlin. Samml. II. p. 423. die Rougette.

Biiffoin, vierf. VII. p. 65. die Rougette.

Allgem. Hif. der Natur, V. 2. p. 33. Tab. 17. die Rougette.

Erxlében, Mammalia, p. 133. ß. La Rougette Biiffonii, la Roufette Daubentonii.

Gatterer, brew Zoolog. I. P. 38. bo die Ru* gette.

$\gamma$. Nicht viel kleiner als die erfere, ohngefähr neun. tehalb Żoll lang. Strolifarbig. Schreb.

Biiffon, vierf. VII. p. 67.

Eirxtebin, Mammal. p. izj. $\gamma$. The lefier Ternate Pcrinainti.

Gatterer, brev. Zool. I. p. 38. e. die kleine Fledermains vors Ternate. 
Milller, Naturfyftern, I. p. I52. n. I. der fliegende Hund, Tab. VUI. fig. 1. Seb.

Zimmiermann, geogr. Zool. II. p. 62. die grössit Fledermaus, oder der Vampyr, p. 40̉8. n. 353. der Blutfouger.

Gatterer, vom Nutzen und Schaden der Thiere, I. p. 28. I'teropus Vampyrus, dèr fliegendè Hund?

Göttingifche Sammlung. merkwürd. Reifen, II: p. 307.

Forfter, Bemerk. auf feiner Reife un die Welt, p. 165. Vampyi, Bhitfanger.

Ives, Reife nach Indien, I. p. 3o. Fliegende. Fiichse.

Hawkesworth, Gefchichte der Entdeck. im Süd. meer, III. p. 156.227 .

Osbeck, Reife nach Chinà, p. ì37.

Seligmann, Vögel, VI. Tab. 7j. Grojse Fleder. mans:von Madagaskar.

Pallas, Naturgefch. merkiv. Thiere, III. p. 8. der Blutfanger.

Ersitebeni, Matimalia, p. I30. n. - I. Pteropus (Vampyrus) ecaudatus, nafo fimplici, mem. brana inter femora divifa.

Linnué, Syft. Nat: Edit. II. p. 45. Vefpertilio cauda nulla:

Limné, Syft: Nat. Edit. VI. p. 7. n. I. Vefpertilio cauda nulla.

Lininé, Syft. Nat. Edit. X. I. p. 3i. n. t. Vefpertilio (Vainpyrus) ecaudatus, nafo fimplici, membrana inter femora divifa.

Turpin, Hift. de Siam. p. 310.

Allgemeine Reifen, II. p. 398 . III. p. 336. VI. p. 95. XI. p. 404. etc. 
Gatterer, brev. Zoolog. I. p. 38. n. I. Pteropus (Vampyrus) ecaudatus, nafo fimplici, membrana inter femora divifa. Blutfauger; fliegende Hund.

\section{Spectrum. Die Trichternaje. (4)}

Miiller, Naturfyf. I. p. I53. n. 2. der Flatterer, Tab. VIII. fig. 2. Seb.

Zimmermann, geogr. Zoolog. II. p. 409 . n. 355. die Trichternafe.

Lefke, Naturgefchichte, p. I57. n. 2. die Fledermans mit der Trichternafe.

Bhumenbach, Handbuch d. Naturgefch. p. 67. n. I. Vefpertilio (Spectrum) ecaudatus, nafo infun. dibiliformi lanceolato. Der Vampyr.

Bhumèzbach, Samml. merk $\mathbb{z}_{2}$ Reifegefchichten I. p. $177 ; 233.256$.

Halle, vierf. p. 453. der gröfste fliegende Hund mit Ohren.

Meyer, Thiere, III. Tab. 36 . die gröfste fliegende Hiindin aus Neir panien.

Gatterer, vom Nutzen u. Schaden der Thiere, 1. p. 30. n. 4I. der Vimpyr.

Neuer

(4) Der eigentliche Bilutfauger , der wabre Vampyr. Berkel fagt in feiper Reife nach Berbice: (Blumenbatb, Reifegefch. am a. O.) man has fich am meiften vor ihnen zu fürchten, wenn man fich mnit blofsen Füfsen in die Hangmatte fshlafen legt; weil alsdann die Zehen, aus rènèn fie das Blut zu faugèn wiffen, fehr leicht žlu Schaden kommen. Ert thun tie ceinén Bifs, und dann fliegen fie weg, um zu fehen, ob der, den fie gebiffen haben, auch aufivacht. Gefchieht das nicht, fo fetzen tie fich auf den Zeh, und faugen fich dick voll Blut. Iunge zahme Tauben fand man oft auf diefe Art in Menge getödtet. In Surinam würde man Schweine in unend!icher Menge haben, wenn die Fledermâufe, die ihnen die Saugwarzen abbeiffen, es niche hinderter. Caraiben in Amerika halten fie für büfe Geifter. 
Neuer Schąuplatz der Natur, III.' p. IIg. der Vampyr.

Berlin. Samml. I. p. 53. Surinamifche Fledermärse. Berlin. Samml. II. p. 423. der Vampyr.

Frifch, Tabellen, p. 6. 5. n. 7. Sanguifuga; der Bhutfauger, oder Vampyr, in America.

Klein, Quadr. difp. p. 62. Vefpertilio Cynocephalus maximus auritus ex nova Hifpania.

Klein, Claffif. d. vierf. Th. p. I88. n. 7. grö/ste

Fledermaus mit dem Hundskopf und Ohren.

Klein, natürl. Ordn. d. vierf. Thiere. p. 65. n. 8. gröfsefter Hundskopf aus Neufpanien.

Pallas, Naturgefch. merkwiüt. Thiere, III. p. 8. Vefpertilio fpectrum.

Charlevoix, Gefch. von Paraguay. Nürnb. I768. 8. p. I23. gewifle fehr grofse Fledermäufe.

Schlözer, Erdbefchreibung von Amerika, p. 750. Fledermäuse.

Reifen der Miffionar. von Murr. p. 540. Flatterer, Trichternase.

Labat, Reifen nach Weftindien, I. p. 399.

Fermin, Befchreib. von Surinam, II. p. I20?

Nachrichtén von dem Lande Guiana, aus d. Ital. des Abts Gilii, p. 169?.

Allgem. Hift. der Natur, IX. p. 48. XV. p. I3. V.2. p. 33 .

Erxleben, Mainmal. p. 133. n. 2. Pterppus (Spectrum) ecaudatus, nafo infundibuliformi lan. ceolato.

Linné, Syff. N. Edit. X. I. p. ${ }_{3}^{\text {I. }}$ n. 2. Vefpertilio (Spectrum) ecaudatus, nafo infundibuliformi lanceolato.

Lery, Voyage fait en Terre de Brefil. p. 157 fq. Morcyrav. Brafil. p. 213. Andira aca Brafilien. fibus. 
Pallas, Spicil. Zool. Fafc. III. p. II. Vefpertilio Spectrum.

Pennzant, Syil. quadr. p. 362. n. 275 .

Grauman, intr. in hiffor. nat. mamm. p. 33. II. die Trichternafe.

Gatterer, brev. Zoolog. I. p. 38. n. 2. Pteropus (Speçrum) ecaudatus, nafo infundibuliformi lanceolato; der Vampyr, Flatterer.

3. Perspicillatus. Die Schanfelnafe. (5)

IViitler, Naturfyn. I. p. 154. n. 3. die Brillnafe, Tab. VIII. fig. 3. Seb.

Zimmermann, geogr. Zoolog. II. p. 409. n. 356. dis Schanfelnafe.

Halle, vierf. p. 58r. die gemeine amerikanifche Fle. dermaus.

Klein, Quadr. difp. p. 6r. Vefpertilio americanus vulgaris, longioribus auriculis, et e nafo erecta criflulat.

Klein, Clafif. d. vierf. Th. p. 186, n. 3. gemeine amerikanifche Fledernans, mit langen Ohrlap-pen, and einem anf der Nafe in die Höhe ftekenden Kamm.

Klein, natïrl. Ordn. der vierf. Th. p. 65. gemeine annerikanifche Fledermans.

pallas, Naturgefch. merkw. Thiere, III. p: 9 . Vefpertilio Perfpicillatus.

Erxleben, Mammal. p. 137. n. 5. Pteropus (Perfpicillatus) fubecaudatus rufus, nafo foliato plano acuminato.

Linné, S. N. Edit. II. p. 45. Vefpertilio caudatus, nafo foliato acuminato.

Linné, S. N. Ed. VI. p. 7. n1. 4. Vefpertilio caudatus, nafo foliato acuminato.

Linnzé

(5) Eànge des Kürpess drittehalb Zoll. 
Linné, S. Nat. Edit. X. İ. p. 31, n.3. Vefper. tilio (Perfpicillatus) ecaudatus, nafo foliato plano acuminato.

Graumann, intr. in hift. nat. mamm. p. 34. $\gamma$. Brillnafe; Schanfelnafe.

Gatterer, brev. Zoolog. I. p. 39. n. 5. Pteropus (Perfpicillatus) fubecaudatus, rufus, nafo fo. liato plano acuminato. Brillnaje, Schanfelnase.

4. Spasma. Die Herzinafe. $\left({ }^{6}\right)$

Milller, Naturfyf. I. p. I54. n. 4. die fliegende Ratze. Tab. VIII. fig. 4. Seb.

Zimmerwiann, geogr. Zoolog. II. p. 408. n. 354 . die Herzmaje.

Halle, vierfüfs. p. 55 ז. die fliegende Ratze, Tab. 27. B.

Meyer, Thiere, III. Tab. 39. die fliegende Ratz von Ternate; fig. Seb.

Klein, Quadruped. difp. p. 6t. Vefpertilio Rattus Ternatanus.

Klein, Claffif. d. vierfüls. Th. p. ז86. Ratzenar. tige Fledermanis.

Klein, natürl. Ordn. d. vierfüls. Th. p. 65. n. 5. Ternatanifche Fledersatze.

pallas, Naturgefch. merkwürd. Thiere, III. p. 9. Vefpertilio Spafma.

Biiffon, vierf. XIV. p. 237. die Her:znafe. Erxleben, Mammal. p. 135. n. 3. Pteropus (Spafma) ecaudatus, nafo foliato obcordato. Linné, Syñ. N. Edit. II. p. 45. Vefpertilio cau. datus, nafo foliato obverfe cordato.

Limné, S. Nat. Edit. VI. p. 7. n. 5. Vefpertilio caudatus, nafo foliato obverfe cordato.

$$
\text { E } 2 \text { Linn', }
$$

(5) J.ànge vier, det ausgebreiteten Fittige zwölf $\mathrm{ZOll}_{\text {, }}$ 
Limné, Syf. N. Edit. X. I. p. 32. n. 4. Vefpertilio (Spalma) caudatus, nalo foliato obcordato.

Granumann, intr. in hif. nat. mamm. p. 33. III. fliegende Ratze; Hersinafe.

Gatterer, brev. Zoolog. 1. p. 38. n. 3. Pteropus (Spafma) ecaudatus, nafo foliato obcordato; flie gridle Ratze; Herznase.

7. Hastatus. Die Kleellattrage. (7)

Miiller, Naturfyf. Suppl. p. I4. 11. 7. die Kleeblatt. nase.

Zimimermann, geogr. Zoolog. Il. p. 409. n. 357 . die Kleeblattnaje.

'Buffon, vierf. XIV. p. 209.

Erxlebeir, Mammalia, p. 136. n. 4. Pteropus. (Haftatus) ecaudatus nigricans, nafo foliato haftato.

Pallas, Spicil. Zool. III. p. 7. Vefpertilio haftatus. Pallas, Naturgefch. merk wïrd. Thiere, III. p. 9. Vefpertilio haftatus.

Gatterer, brev. Zoolog. I. p. 39. n. 4. Pteropus (Haftatus) ecaudatus nigricans, nafo foliato haftato; das Lanzenblatt; die Kleeblattnafe.

8. Soricinus. Die Sperrnafe. $\left({ }^{8}\right)$

Miisller, Naturfyft. Suppl. p. 14. n. 8. die Sperrnafe. Zimmermann, geogr. Zoolog. II. p. 410. n. 358 . die Sperrna $e_{\text {e. }}$

Gatterer, vom Nutzen u. Schaden der Thiere, I. p. 30. n. 42. die Spervinase.

Biiffon,

(3) Wird von einigen mit der Schaufabafe für einerley gchalten.

Länge des Kürpers viertehalb Zoll.

(s) Lange des liürpers nicht viel über zwey Zoll. 
Buffon, hifor. natur. XIII. p. 227. Une autre chauve - fouris.

Erxleben, Manmmal. p. I38, n. 6. Pteropus (Soricinus) fubecandatus cạnus, nafo foliato ovali acuminato.

Pallas, Mifcellan. p. 5r. Tab. 5. Vefjpertilio So. ricinus.

Pallas, Naturgefch. merkwürd. Thiere, III. p. 26. Tab. 3. die Spitzmausähnnliche Fledermans. (Zergliederung.)

Mèm. de l'Acad. r759. p. 388. Feuille Daubenton.

Graumann, intr. in hiff. nat. mamm. p. 34. VI. die Sperrnase.

Gatterer, brev. Zoolog. I. p. 39. n. 6. Pteropus (Soricinus) fubecaudatus canus, nafo foliato ovali acuminato; die Sperrnaje.

9. LÉór inus. Die Fledermans mit der Hajeñ. Scharte, (9)

Iitiiller, Naturfyntem, Supplem. p. I5. die Hafenfcharte.

Zimnuermann, geogr. Zoolog. II. p. 4 10. n. 359. die Fledermans mit der Hafen/fcharte.

Bechftein, Naturgefch. Deutfchl. I. p. r4r. n. 26. das Nachtthier; die kat:enartige Fledlermaus; das amerikanifche Nachtthier.

Halle, vierf. p. 45 o. die fliegende Katミe. Meyer, Thiere, III. Tab. 44. die einer Katze ühn liche amerikanifche Fledermans; das Männchen; fig. Seb.

$$
\text { E } 3 \text {. }
$$

Klein,

(9). Fant fo grofs als cine Ratte; die Flügelweite betragt 2 Fufs 3 Zoll; die Länge der Schwanzhaut = Zoll 7 Linien, und des Schwanzes 14 Linien. 
Klein, Quadrup, difp, p. 6r, Vefpertilio americanus, capite globofo, ore leporino.

Klein, Claffif, der vierfüfs. Thiere, p, I85. n. 2. amerikanif:che Fledermans.

Klein, natïrl. Ordnung d. vierf. Th. p, 65. n. 2. amerikanifche Flederratze, mit dickem runden, Kopfe, und einer Hiafenfcharte:

Biiffon, vierf. XIV, p. 239, die Hafenfledermans. Erxleben, Mammalia, p. 139. n, 7. Pteropus. (Leporinus) caudatus, lábio fuperiore fillo. Linné, S, N. Edit, II. p, 45. Vefpertilia caudatus, labia fuperiore bifido.

Linné, Mur, Ad, Fried, I, p. 7. Vefpertilio (Lea porinus) caudatus, labio fuperiore bifido, Pallas, Spicil, Z,oolog, III, p. 8, Vefpertilia Leporinus,

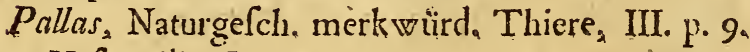
Vefpertilio Leporinus.

Gramonn, intr, in hif, nat. mamm, p. 34. VII. die Hasenfcharte.

Gatterer, brev, Zoolog. I. p. 39, n, 7. Pteropus (Leporinus) caudatus, Jabio luperiori fiflo: die Hasenscharte.

* * Mit $\frac{4}{6}$ Vorderzähnen.

5. A UR I Tus. Die langöhrige Fledermans. (10). Miiller, Naturfyfem, I. p. I54. n. 5. Langohr, Tab, VIII, fig. 5. Houtt.

Zinnmer.

(10) Hin und wieder in Deurfchland, am liebften in altem Gemäuer und Steinfelfen. Im Winter findet man fie haufenwẹife in Hüh-

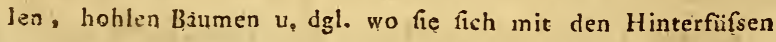
aufhangen, und die Flitgel um den Leib fchlagen. Sie bringen zvey Iungea Ihre Långe berragi nur zwsy Zoll. - Im Syntem 
Zimmermann, geogr. Zoolog. II. p. 4Ir. n. 360. die langöhrige Fledermáus.

Schreber, Säugthiere, I. p. 163. die langöhrige Fledermicus, Tab. L.

Lefke, Naturgefch. p." I58. n. 3. die langöhrige Fiedermanis.

Borowsky, Thierreich, I. p. 53. n. 2. langöhrige Flédermaurs.

Bhumenbach, Handb. d. N. G. p. 68. n. 3. Vefpertilio (Auritus) caudatus, auriculis maximis.

Bechftein, Nąturgefch. Deutfchl. I. p. 156. die langoührige Fledermans.

Neuer Schauplatż der Natur, III. p. Ir7.

Klein, Quadrup. difp. p. 61. Vefpertilio minor vulgaris,

Klein, Claffif. d. vierf. Th. p. 185. n. x. kleinere Fledermans, die gemeine.

Klein, natiirl. Ordnung d. vierf. Th. p. 64. n. I. Fi f cher, Naturgefch. von Livland, p. jo. n. I. Langohi, ohrigte Fledermaus.

Bock, Naturgefch. von Preuffen, IV. p. 5. n. I. der. Langohr.

Goeze, europäifche Fauna, I. p. 53. die langöhrige Fledermans:

Buiffon, vierf. V. p. 78. das Langohr; Grofsohr; die grofsöhrige Fledermans; mi. e. Fig.

Erxleben, Mammalia, p. 24r. n. r. Vefpertilio (Auritus) nafo oreque fimplici, auriculis capite maioribus.

Linné, S. N. Edit. X. I. p. 32. n. 6. Vefpertilio (Auritus) caudatus, nafo oreque fimplici, auriculis duplicatis, capite maioribus.

$$
\text { E } 4
$$

Gran-

fteht arriculis duplicatis; welches jedoch Blumenóacb in, a. niche als richtig annehmen. 
Gratmann, intr. in hif. nat. mamm. p. 34. I. die langöhrige Fledermans.

Gatterer, brev. Zoolog. I. p. 40. n. I. Vefpertilio (Auritus) nafo oreque fimplici, auriculis capite maioribus; das Langohr.

Belon, hiff. des oifeaux, p. 146. La.Sourichauve, fig. p. I47. mittelm.

Aldrovand. Ornithol. I. p. 57I. Vefpertilio, fig. p. 575. fchlecht.

Memoir. de l'acad, des Sc. I759. p. 379. L'Oreillar Daubenton, Tab. I. fig. 2.

Mïller, Dan. prodrom, p. 4. n. 22. Vefpertilio (Auritus) caudatus, nafo oreque fimpliẹi, atsriculis duplicatis, capite maioribus. IMuS. Wormiail. p. 308. Vefpertilio.

6. Murinus. Die gemeine Fledernans. ( ${ }^{\mathrm{x}}$ ) Miiller, Naturfyft. I. p. 154. 11. 6. Mar:eohr, Tab. VIII. fig. 6. Houtt.

Zimnnernann, geogr. Zoolog. II. p. 412. n. 36 I. $^{-}$ die gemeine Fledermaiss.

Schrbber, Säugthiere, I. p. 165. 11. 9. die gemeine Fledermanus. Tab. LI.

Lefke, Naturgefch. p. 158. n. 4. die gemeine Fledermaus.

Boroujky, Thierreich, I. p. 53. n. 3. Fledermans, Speckmants.

Bhumenbach, Handb. der Naturgefch. p. 68. n. 4. Vefpertilio (Murinus) caudatus, auriculis capite

(1) Die Lange des Kürpers betrage drittehalb Zoll; mit den Fittigen klaftert das Thier einen Fufs, und etwas druber. Ihr Bifamithnlicher Geruch im Sommer rührt vielleicht von dem Genufs des gro sen Bifamrogels (Spbinx Convolvuli L.) her, wovon fic lich büufy nahren. Winteraufenthalt und Vermebrung hat. he mit der vorhergahenden gemein. 
pite minoribus;-die gemeine Fledermans; Speckmaus.

Bechftein., Naturgefch. Deutfchl. I. p. I64. n. 2. gemeine Fledermans; p. 165.: A. das grofse Mane Seokr, der Nachtfchatten; p. 169. B. das kleine Manefeohy; die (eigentliche) gemeine Fle. dermaus. $\left({ }^{2}\right)$

Halle, vierf. p. 449. die gemeine Fledesmans. Meyer, Thiere, I. p. 4. Tab. 3. eine Fledermans; m. e. mittelm. Fig.

Sander, ökonom. Naturgefch. I. p. 263. n. Is. die Fledermans.

Neuer Schauplatz der Natur, III. p. II6. Beckmann, Naturhift. p. 24. n. 6. die Flederniaus. Beyträge zur Beförd. d. Naturkunde I. p. 55. n. 2. Flederonculs.

Fifcher, Naturgefch. von Livland, p. 50. n. I. gemeine Fledermaus, Maujeohr.

Klein, Quadrup. difp. p. 6r. Velpertilio maior vulgaris.

Klein, Claffif. der vierf. Th. p. 185. n. I. gröffere Fledermanus; die gemeine.

Ktein, natürl. Ordn. d. vierfüfs. Th. p. 64. n. r. Bock, Naturgefch. von Preuffen, IV. p. 5. n. 2. der Marifeohr.

Goeze, europäifche Fauna, I. p.' 36 . die gemieine Fledermanus.

Pallas, Reife-Auszïge, p.37o. die grofsenz Fledermänele.

Handb. d. deut fchen Thiergefch. Giefs. I790. p.37. Fledermaus, Speckmans.

Cetti, Naturgefch. von Sardinien, I. p. 248.

$$
\text { E } 5
$$

Biiffor,

(2) Ich habe diefen Unterfehied fonft nirgends als hiex und bey Göze gefunder. 
Biiffon, vierfüfs. V. p. 53. die Fledernnans; p. 76 . die eigentliche oder gemeine Fledermanus.

Errleben, Mammalia, p. 143. n. 2. Vefpertilio (Murinus) nafo oreque fimplici, auriculis longitudine capitis.

Linné, S. N. Edit. II. p. 45. Vefpertilio caudlatus, nafo oreque fimplici.

Linné, S. N. Edit. VI. p. 7. 1. 2. Vefpertilio candatus, nafo oreque fimplici.

Linné, S. N. Edit. X. I. p. 32. n. 7. Vefpertilio (Murinus) caudatus, nafo oreque fimplici, auriculis capite minoribus,

Linné, Fn. Suec. I. p. 7. n. I8. Vefpertilio caudatus, nafo oreque fimplici.

Graumainn, int: in hif. nat mamm. p. 34. II, Fledermaus, speckmans.

Gatterev; brev. Zoolog, I. p. 40. n. 2. Vefpertilio (Murinus) nafo oreque fimplici, auriculis longitudine capitis; gemeine Fledermans; Monjeohr.

Ionfton, av, p. 49. Vefpertilio, Tab. 20. die mittl. Fig:

Pennant, britt. Thiergefch, p. 50. Tab. XI. a. Pennant, arct. Zool. II, p, r8o. n. ro4. die gemeine Fledermans.

Miiller, Dán. prodrom. p. 4. n. 21. Vefpertilio (Murinus) caudatus, nafo oreque fimplici, alle riculis capite minoribus.

Daubenton, Mem, de l'Acad, de Par. 1759: p. 378. la chauve-fouris. Tab. I. fig. I.

Krom. Aufr. p. 3I4. Vefpertilio caudatus, ore nafoque fimplici,

Raj, Synopf. Quadrup. p, 243. Vefpertilio. plin. H. Nat. X. c. 61. XI. c. 37. 39. Vefpertilio. 
10. No ct u a, Die Spechmans. (3)

Miiller, Naturfyft. Suppl,p. I5. n, ro, Vefperti. lia Lardarius; die Speckmaus.

Zimmernann, geogr, Zool. II, p. 4I2, 11. 362, die Speckmauls.

Beihftein, Naturgefch, Deutfchl, I, p. I74. n. 3a' die (grofse) Speckmaus.

Neuer Schauplatz der Natur, III, p. II7.

Büffon, vierf. V. p. 8I, die nüchtliche Fledermans: Specknauss.

Goeze, europäifche Fauna, I. p. 60, der Nïchtling, Erxleben; Mammalia, p. 146. n. 3. Vefpertilio (Noctula) nafo oregue fimplici, auriculis capis te brevioribus, corpore cano.

Pennant, Synopf quadrup, p: $369, n, 287$, the Nóclule.

Daubenton, Mem, de l'Acad, de Par. 1759. p.380. La noctule Tab. 2. fig. I:

Graumann, intr, in hitt, nat, mamm, p. 35. III, die Specknaus,

Gatterer, brev, Zoolog, I, p. 41, n. 3. Vefpers tilio (Noctula) nafo oreque fimplici, auricua lis capite brevioribus, corpore cano; dio Speckniaus.

I1. SER OTINus, Die bla Je Ftedermans. ( $\left.{ }^{4}\right)$

Miiller, Naturfyf, Suppl, pa I6, n. II, die blafJe Fledermans.

Zimmermann, geogr, Zoolog. II, p. 4I3, n, 364, die bla fle Fledermuaus.

Bech.

(3) Grofse Fledermaus: Fledermaus miv dim Mausekopf: - An Grüfse gleicht fie der vorhergehenden.

(4) Lạnge des Körpers zwey und dreyviertel Zoll; der Schwąnz beỵabe fo lang als der Leib. 


\section{Mammalia Primates. Vefpertilio.}

Beckftein, Naturgefch. Deutfchl. I.' p. I80. n. 5; die blaffe Fiedermaus, Tab. II. Fig. I.

Never 3chaupl. d. Natur, III. p. Ir7.

Bïffor, vierf. V. p. 82. der Spätling; die blafje

Fledermans; m. e. Fig.

Cetti, Sardinien, I. p. 250 . die Serotine?

Goone, europäifche Fauna, I. p. 65. der Spät:

ling, oder die blafje Fledernuaus.

Erxleben, Mammalia, p. I47. n. 4. Vefpertilio

(Serotinus) nafo oreque fimplici, auriculis capite minoritius, corpore ferrugineo.

Pennant, Synopl, quadruped. p. 370 . n. 288. the Serotine liat.

Daubenton, Mem. de l'Academie de Par. I759: p. 380. La Serotine, Tab. II. fig. 2.

Graumicinu, intr. in hif. nat. mamm. p. 35. IV: die blaffe Fledermans.

Gatterer, brev. Zool. I. p.4I. n. 4. Vefpertilio (Serotinus) nafo oreque fimplici, auriculis capite minoribus, corpore ferrugineo; der Spü̈tling.

13. Pipistrellus. Die Zwergfledernans. ( $\left.{ }^{5}\right)$ Müller, Naturfy f. Suppl.p. I6. n. I2. die Zwerg. fledermants.

Zimmernanin, geogr. Zoolog. II. p. 4I3. n. 365 . Zwergftedermans.

Bech.tein, Naturgefch. Deutfchl. I. p. '177. die Zivergftedermans.

Gatterer, vom Nutzen u. Schaden der Thiere,

I. p. 32. n. 45 . die Zivergfledernanus.

Goeze, europailche Fauna, I. p. 65. die Zwerg. fledermans.

Neuer

(5) Die kleinfte, unter allen. Der Kürper it kaum anderthalb Zoll - lang; die Fligelweite nach Schreber acht Zoll. 
Never Schaupl. d.Natur, III. p. II7. Pipiffrelle. Biiffon, vierf. V. p. 83. Zwergftedernucus, mit einer Figur.

Cetti, Sardinien, I. p. 250. cine finfte Art kleiner Zwergfledemäule?

Erxleben, Mammalia, p. I48. n. 5. Vefperti. lio (Pipiftrellus) nafo oreque fimplici, auriculis. Iongitudine capitis, corpore fubtus nigricante.

Pallas Reife, I. p. 4r. eine Art kleiner Fledermïure.

Daubenton, Mem. de l'Acad. de Par. 1759. p.381. la Pipiftrelle, Tab. I. fig. 3.

Pennant, Synopf. quadruped. p. 370. n. 289. the Pipiftrelle Bat.

Graumann, intr. in hif. nat. mammal. p. $35 . \mathrm{V}$. die Zwergfledermaus.

Gatterer, brev. Zool. I. p. 4I. n. 5. Vefpertilio (Pipiltrellus) nalo oreque fimplici, auriculis longitudine capitis, corpore fubtus nigricante; Zwergfledermanis.

13. B A R B ASTELL US. - Das Kurzmant. $\left({ }^{6}\right)$

Miuller, Naturfyft. Suppl. p. 17. n. 13. das Kurzmanl. U. Gaktutule $193 \%$

Zimmermann, geogr. Zoólog. II. p. 414. n. 366 . das Kurzinaul.

Neuer Schaupl. der Natur, III. p. Irg. Bartaftelle. Biiffon, vierf. V. p. 85. das Kúu'zmaul.

Erxleben, Mammalia, p. 148. n. 6." Vefpertilio (Barbaftellus) buccis barbatis, auriculis longioribus frontem tegentibus.

Danben-

(6) Eine der feltenften. - Flügelweite nach Dasbenton zehn und einen halten Zoll. 
Daubenfon, Mem. de l'Acad. de Paris, I759. p.38r. la Barbaftelle, Tab. 2. fig. 3.

Pennant, Syn. quadr. p. 370. n. ngo. the Barbafelle Bat.

Graumain, intr. in hift. nat. mamm. p. 35 . VI. das Kurzmaut.

Gatterer, brev. Zoolog. I. p. 42. n. 6. Vefpertilio (Barbaftellus) buccis barbatis, auriculis frontem tegentibus; das Kurzmaul.

74. Hispidus. Die Bartfedermaus. (7)

Miiller, Naturfyft. Suppl. p. 17. n. I4. die Bart.

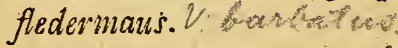

Zimmermanit, gcogr. Zool. II. p. 414. n. 367. die Bartfledermaus.

Biiffon, vierf. XIV. p. 227. die Bartfledermatus. Erxleben, Mammal. p. r49. n. 7. Vefpertilio (Hifpidus) auriculis longitudine capitis, fronte fulcata longius pilora.

Gramianin, intr. in hift. nat. manm. p. 35 . VII. die Bartfledermans.

Gatterer, brev. Zoolog. I. p. 42. n. 7. Vefpertilio (Hifpidus) auriculis longitudine capitis, fronte fulcata longius pilofa. Die Bartfledermaus.

$$
\text { * * * Mit } \frac{4}{8} \text { Vorderzähnen. }
$$

15. Prстus. Die Fledernans mit bunteni Fittigen. $\left({ }^{8}\right)$

IMiiller, Naturfy't. Suppl. p. I7. n.15. der Buntfliigel.

Zininer-

(7) Nicht viel grüfser als die Zwergfledermatss. An Kinne ift cine Art von Bart. Adanfon hat fie entdeckt.

(8) Lange des Körpers zwey bis dritthalb Zoll. 
Zimmermann, géogr. Zoolog. II. p. 415. n. 368. der Buntfligel.

Halle, vierf. p. 452. die afiatifiche. Fledermaus, Tab. 27. A.

Biiffon, vierf. XIV, p. 230. die buntgefliigelte Fledermans, mit einer Fig.

Erxleben, Mammalia, p. 150. n. 8. Vefpertilio

(Pictus) auriculis longitudine capitis; mem. brana volitante nigro friata.

Pallas, Spicil. Zoolog. III. p. 7. Vefpertilio pictus.

Pallas, Naturgefch. merkw. Thiere, III. p. 8. die gemahlte Fledermans.

Graumain, intr. in hift. nat, mamm. p. 35 . VIII. der Buntfliigel.

Gatterer, brev. Zoolog. I. p. 42̄. n. 8. Vefpertitilio (Pictus) auriculis longitudine capitis, membrana volitante nigro ftriata; der Bunt. fliigèl.

**** Mit $\frac{2}{6}$ Vorderzïhnen.

16. NIGRITA. Die fpitzöhrige Fledermaits.

Miiller, Naturfyft. Suppl. p. I8. n. I6. Vefpertilio Marmotte; das Spitzohr.

Zimmermanin, gengr. Zool. II. p. 415. in. 369 . die Spitzöhrige Fledermanis.

Biiffon, vierf. XIV. p. 215. das fliegende Matr. melthier, m. e. Fig.

Erxleben, Mammal. p. 151. n. 9. Vefpertilio (Nigrita) auriculis brevioribus, caudae apice extra membranam prominente, valvulis aurium acuminatis.

Graninann, intr. in hifor. nat. mamm. p. 36. IX. das Spitzohr. 
so Mamnialia Primates. Vefpertilio.

Gatterer, brev. Zool. I. p. 42: n. 9. Vefpertilio (Nigrita) auriculis brevioribus, caudae apice extra membranam prominente, valvulis aurium acuminatis; das Spitzohr.

\section{*****:Mit $\frac{2}{4}$ Vorderzähnen.}

77. Mocossus. Die hundsmäulige Fledernarus.

๙. Die gröfsere. (9)

B. Die kleinere. ( ${ }^{(0)}$ )

Miiller, Naturfyft. Suppl. p. 18. 11. 17. das Hinds. mant.

Zimmermann, geogr. Zoolog. II. p. 416. n. 370. die hundsmizunlige Fledermans.

Leeke, Naturgefchichte, p. 158. n. 5. die luunds. mäulige Fledermans.

Bïffon, vierf. XIV. p. 223. die hundismäulige Fledermants.

Bïffon, vierf. XIV. p. 225. eine andere Fledermais. Erxleben, Mammal. p. I51. n. ro. Vefpertilio - (Molofius) auriculis brevioribus, valvulis rotundatis, labiis pendulis.

Pallas, Spicil. Zool. III. p. 8. Vefpertilio MoIoflus Tab. IV. fig. I. (die Hirnfchale.)

Pallas, Naturgefch. merkw. Thiere III. p. g. den Moloffe, Tab. IV. fig. x.

Graumann, intr. in hiftor. nat. matrm. p. 36. X. das Hunzdsmaul.

Gatterer, brev. Lool. I. p. 42. n. ro. Vefpertilio (Molofius) auriculis brevioribus, valvulis rotundatis, labiis pendulis; das Hundsmaul.

(0) Linge des Kürpers zwey Zoll.

(10) Linge des jürpers cin und dregviertel Zoll. 
******* Mit 능 Vorderzähnen.

18. Cернадотеs. Die grofsköpfige Fledermans. Miiller, Naturfyft: Supplem. p. I9. n. is. der Grofskopf.

Zimmermani, geogr. Zool. II. p. 416. n. 37 i. die grofsköpfige Fledermaus.

Biiffon, vierf. XIV; p. 233. die grossköpfige $F$ Fledermaus; m. e. Fig.

Pallas, Naturgefch. merkw. Thiere, III. p. Ir. die Fledermaus mit dein Hundskopf. (Zergliederung.)

Erxleben, Mammalia, p. 152. n. ir. Vefpertilio (Cephalotes) labio fuperiore firfo, naribus cochleatis, valuulis auricularum nullis.

Pennant, Syn. quadr. p. 368. n. 285. the Moluc. ca Bat.

Graumann, intr. in hift. nat. mamm. p. $36 . \mathrm{XI}$. der Grofskopf.

Gatterer, brev. Zoolog. I. p. 43. n. Ii. Vefpertilio (Cephalotes) labio fuperiore fifo, naribus cochleatis, valvulis auricularum nullis; $\mathrm{der}$ Grojskopf.

******** Mit $\frac{0}{4}$ Vorderzähneit.

19. Lér TuR U-S. Die Beutelfedermans. (')

Mïller, Naturfyft. Suppl. p. 19. n. 19. Vefperti. lio Marfupialis, der Beiteiltrigger.

Zimmermann, geogr. Zoolog. II. p. 4I\%: n. 372 . die Bèntelfledermouss.

Biiffon, vierf. XIV: p. 242, die Bentelfledermans, m. e. Fig.

Erreles

(1) Lange des Küzpers anderchalb Zoll: 
Erxieben, Mammalia, p. 153. n. 12. Vefpertilio (Lepturus) membrana ad cubitum faccata. Graumann, intr. in hif. nat. mamm. p. 36 . XII. der Beuteltvïger.

Gattever, brev. Zoolog. I. p. 43. n. 12. Vefpertilio (Lepturus) membrana ad cubitum faccata; der Beutelträger.

20. Ferrum equinium. Die Hufeifemafe. ( $\left.{ }^{2}\right)$ a. Die grö/sere.

B. Die kleinere.

Miiller, Naturfyn. Suppl. p. 20. n.20. Vefpertilio equinus; die Hufeifennafe.

Zimmermann, geogr. Zool. II. p. 417. n. 373. die Hufeifennase.

Lefke, Naturgefch. p. 158. n. 6. die Fledermans mit der Huffrifennafe.

Bechfiein, Naturgefch. Deutfchl. I. p. I81. n. 6. die Fledermans mit der Hufeifennase, Tab. II. fig. 2. A. die gro/se Hufeifenna/e; p. I86. B. die kleine Hufeifennafe.

Neuer Schauplatz d. Natur, III. p. II8.

Biiffon, vierf. V. p. 87. das Huffeisen, die HufeiSennaje.

Goeze, europäifche Fauna, I. p. 66. das Hufeifen. Erxleben, Mammalia, p. 154. n. I3. Vefpertilio (Ferrum equinum) nafo foliato, ferri equini aemulo.

Daubenton, Mem. de l'Acad. de Paris I759.p. 382. Fer à cheval Tab. 2. fig. 4 .

Pennant, Syn. quadr. p. 369. n. 286 .

Gran.

(2) Wundernafe; Maske. Iaufig in den Mauern und Kellern alter Schlüfer. a. Von der Grifse der yembizen Iledirmasis. fo. Voa der Statur des Kurzmanls. 
Graumann, intr. in hif. nat. mamm. p. 36. XIII. die Hufeifennase.

Gatterer, brev. Zoolog. I. p. 43. n. I3. Vefper. tilio (Ferrum equinum) nafo foliato ferri equini aemulo; die Hufeisennaje.

\section{******** Ohne Vorderzähne.}

21. NOVEв ORACENSIS. Die nordamerikanifiche Fledermaus. ( 3 )

Miiller, Naturfyftem, Supplem. p. 20. n. 2I. Vefpertilio Borealis; der Neujorker.

Zimmermann, geogr. Zoolog. II. p. 418. n. 374. die nordamerikanifche Fledermaus.

Schreber, Säugthiere, I. p. I76. n. 2I. die nordamerikanifche Fledermaus.

Schöpf, Reife durch Nordamerika, I. p. 330. die nordamerikani/che Fledermaus.

Biiffon, vierf. XIV. p. 246. die nordamerikanifche Fledermans.

Erxleben, Mammalia, p. 155. n. I4. Vefpertilio (Noveboracenfis) apice nafi fubbifido, macula alba ad bafin membranae volitantis.

Pennant, arct. Zool. I. p. I78. n. IOI. die Fledermaus von. Neuyork.

Graumann, intr. in hift. nat. mamm. p. 36. XIV. der Nenjorker.

Gatterer, brev. Zool. I. p. 43. n. I4. Vefpertilio (Noveboracenfis) apice nafi fubbifido, macula alla ad bafin membranae volitantis; der Neujorker.

(3) Lànge des Körpers drittehalb Zoll, des Schwanzes ein und vierfünftel Zoll; Flügelweite zeha und sin halb Zoll, 
**********Von anbekannter Anzahl und

Ordiung der Zähne.

22. LASIOPTERUS. Die Fledernans mit beharten Fittigen. (Zimm.)

Zimmermann, geogr. Zool. II. p. 412. n. 363 .

Biiffon, vierf. XIV. p. 244. die Fledermans mit behaarten Fittigen; m. e. Fig.

33. L A S I U U s. Die rauchfcheänzige Fledermans. (4)

Biiffor, vierf. XIV. p. 245 . dic rauchfohedänzige Flédermiaus, m. e. Fig.

A) Veränuerungen gegen die XIIte Edition, "uatd Vermehring der Gattungen diefes Gefchlechts.

Das 26 te Gefchlecht der XIIten Edit. p. 88. Notililo ift ganz aufgehoben, und die einzige Species davon: Americanus, ift jetzt Viefjertilio Lepo. vinius, p. 47. n. 9 .

Diefs Gefchlecht if mit febzrkn Gattungen vermehrt, und V. Haftatus, Soricinus, Leporimus, Noetula, Serotinus, Pipiftrellus, Barbaftellus, Hispidus, Piotius, Nigrita, Molosfis, Cephalotes, Lepturus, Ferrum equinum, Noveboracenfis, Lafiopterus und Lafiurus hinzugekommen.

B) Unu

(4) Ift, nach Zimmermann am a. O. p. 418, nor, a. mit Vesp. Noveboracenfis einrerley, 
Mammalia, Prinates. Vefpertilio. 85

3) Unbefinmtere Thiere.

5. Der grofse Blutfauger von Siddamerika.

Bankroft, Naturgefchichte von Guiana, p. 87.

Gumilla, Hift, de l'Orenoque, III. p. 100.

Zimmermann, geogr. Zoolog. II. p. 419. a.

2. Der kleins Bhutfanger von Sïdamerika,

Gumilla, am angef, Ort.

Zimmermann, geogr. Zoolog, II, p. 419. b.

3. Die langharige Fledermaus.

Pennant, arct. Zcolog. I. p. I79. n. 102. (Vefp. Lafiopterus?)

4. Die grojse Flederminus.

Pennant, arct, Zoolog. I. p, 179. n. 103. (Vefp. Noctula?) 
ZWETTE ORDNUNG.

\section{B R U T A. (Thiere ohne Vorderzähne.)}

7. GESCHLECHT. BRA DY US. Das Fanlthier.

Mïller, Naturfyft. I. p. I77. Gen. VII.

Zimmermann, geogr. Zool.II. p.397. Gen.XXVII.

Schreber, Säugth. II. p. I96. Gen. V.

Lefke, Naturgefch. p. I22. Gen. IV.

Borowsky, Thierreich, I. p. 54 .

Finnke, Naturgefch. I. p. 175 .

Beckmann, Naturhiforie, p. Ig.

Biiffon, vierf. XIII. p. 234.

Erxleber, Mammalia, p. 84. Gen. IX.

Gatterer, brev. Zool. I. p. 29. Gen. IX.

Hermani, Tab. affinit, animal. p. 64.

3. TRIDAC, TYIUS. Der Ai. (5)

Miiller, Naturfyft. I. p. I7\%. n. I. das dreyfin. gerige Fiulthier.

Zimmermann, geogr. Zool. II. p. 398. der Ai; das Faulthier mit drey Klauen.

Schreber,

(5) Der Grölse nach gleicht der Ai einem Fuchfe von mittleter Statur. Er braucht eine Zeit von acht bis neun Minuten um einen Fufs nach dem andern fortzufetzen, und eben fo viel um dazwifchen auszuruhen; dabey fchleppt er den Bauch auf der Erde. Einen erkletterten Baum verläfst er nicht eher bis er ihn ganz abgefreffen hat. Den Hunger kann ex über einen. Monat aushalten. Ein Weg von einem Baume zum andern if für ihn eine weite Reife. Bey aller Tragheir aber ift er doch liftig genug, feinen Feinden, zumal den kleinen amerikanifchen Tigern, etc. auf allerhand Weife zu entgehen, und im Nothfalle ftark genug, fich gegen fie zu wehren. Hat eir áufsert zäbes Lehen, 
Schreber, Säugthiere, I. p. 197. n, I, der $A i$, Tab. LXIV.

Le $\int k e$, Naturgefch. p. т22. n. т. $\operatorname{der} A i$.

Boroweky, Thierreich, I. p. 54. n. r. dreyfingeriges Faulthier, Ai. Tab. IV.

Blumenbach, Handb. der Naturgefch. p. 63. n. I. Ignavus (Tridactylus) pedibus tridactylis, cauda brevi; der $A i$.

Bechftein, Naturgefch. Deutfchl. I. p. 134. der Ai. Halle, vierf. p. 39I. das fchmächtige, weifsgraue amerikanifche Foulthier, 'Tab. 15.

Meyer, Thiere, III. Tab. I. der amerikanifche Fanile.

Gatterer, vom Nutzen und Schaden der Thiere, I. p. 18. n. 25 .

Neuer Schauplatz der Natur, I. p. I62.

Onomat. hiff. nat. I. p. 212. $A i$.

Klein, Quadr. difp. p. 43. Ignavus Americanus rifum fletu mifcens.

Klein, Claflif. d. vierf. Th. p. 129. Americanifiher Faule.

Klein, natürl. Ordn. d. vierfüls. Th. p. 47. n. r. Fauler, dem es lärst, als lachte und weinte er zugleich.

Klein, Quadruped. difp. p. 43. Ignavus Marc. gravii.

Klein, Claffif. d. vierf. Th. p. 129. dev Faule des Marcgraf.

Klein, natürl. Ordn. d. vierf. Th. p. 47. n.'2. Fanler des Marcgravs, von Gröfse wie une $\mathrm{ev}^{\circ}$ Fuchs.

Klein, Quadr. difp. p, 44. Ignavus Americanus fomniculofus.

Klein, Clafif. d. vierf. Th. p. I30. Amerikanifcher Fiule; der Schlaffiichtige. 
Klein, natürl. Ordn. d. vierfüfs. Th. p. 48. n. 3. Fanler, der Schläfrige in Amerika.

Gesner, Thierbuch, P. I78. Hout, eine befon. dere Art Affen; Arctopithecus.

Biiffon, vierf. XIII. p, 234. der Ai.

Schlozer, Erdbefchreib. von America, p. 682. das Faulthier; Sloth.

Fermin, Surinam, II. p, 94. das Fanlthier. Bankroft, N. G. von Guiana, p. 88. das Faul. thier.

Reifen der Miffionar, von Muurr. p. 203. Perico. ligero.

Hartfink, Befchreib. von Guiana, I. p, Irr. $A i$ oder Haji.

Dobrizhoffer, Gefch, der Abiponer, I. p. 388. cier Ay.

Erxileben, Mammalia, p. 84 n. r. Bradypus (Tridactylus) pedibus tridactylis, cauda brevi. Linné, Syf. Nat. Edit. II. p. 42. Bradypus ma. nibus tridartylis, cauda brevi.

Iinné, Syft. Nat. Edit. VI. p. 3, n..I. Bradypus manibus tridactylis, cauda brevi.

Limné, Amoenit. acad. I. p. 495. Bradypus ma. nibus tridactylis, cauda brevi.

Limné, S. Nat. Edit. X. I. p. 34. n. I. Bradypus ('Tridactylus) manibus tridactylis, cauda brevi.

Grenimann, intr. in hifor nat. mamm, p. 27. I. das. Faulihier.

Gatterer, brev. Zoolog, I. p. 29, n, I. Bradypus (Tridactylus) pedibus tridactylis, cauda brevi : das amerikanifche Faulthier.

Ionfton, Quadruped, "p I45: Ignavus Tab. LXII. 74. fig. Cluf.

Marcgrav. Brafil, pe 221, Aifive Ignavus, mit einer mittelm, Figur. 
$R a j$, Synopf. quadrup. p. 245. Ai five Ignavus Marcgr.

Barrere, France equinox. p. 154. Ignavus Marcgrav. Ouaikaré Parefieux.

Laet, novus orb. p. 554. Hay, mit einer fehr fchlechten Figur.

Iacabaei Mufeum reg. p. Io. Ignavus feu pigritia. Laurent. Muf. reg, quadrup. :. 9I. Ignavus feu pigritia.

Olearins, Gottorf. Kunfkammer, p. 6. Ignavus Tab. VII. fig. 2. Cluf. mittelm.

Knorr, delic. natur. (edit. Müll.) II. Amerikani. fches Faulthier, Tab. K. fig. I. der Kopf; fig. 2. die Fürse; fig. 3. ein Iunges.

Dampier, Voy. III. p. 305. le Sloth ou le Pareffeux.

Seba, Thef. I. p. 53: Tab. 33. fig. 2, di feu Tar. digradus gracilis americanus. Abbild. gut

Binet, Voy, de Cayenné, p. 341 .

Maffé, hift. des Indes, p. $7 \mathbf{I}$.

Thevet, Sing, de la Fr. antarct. p. 99.

2. DIDAстуLUS. Der Unaru. $\left({ }^{6}\right)$

Miillér, Naturfyn. 1. p. 179. das zweyfingerige Fanlthier, Tab. IX. fig. 2. Seb.

Zimmermann, geogr. Zoolog, II. p. 398. n. 338. der Unaus.

$$
\text { F } 5 \text { Lefke }
$$

(6) Nicht ganz. fo langfam als der vorige. Zum Ruhen oder Schla= fen hangt er fich umgekehrt auf; er f́chlägt die vier Fǘsse dicht an einander, um einen Baumaft, und lâfst den gekrïmmten Leib frey herunter hàngen. Sein Schlaf dauert defta linger, je kâlter es ift. " Er frifst wenig, und fâft gern Milch mit Whaffè verdünnt, Sẹn Laut if ein einzelner kurzer kläglicher Ton, den er felten hüren läfst. - Unau ift die Benennung bey dẹt Anwohnern des Maragnonflufes; Potto, in Guinea, 
Lefke, Naturgefch. p. I23. n. 2. der Ulzan. Borowjky, Thierreich, I. p. 55. n. 2. das zweyfungerige Faulthier; Unau.

Halle, vierf. p. 390. der verkleidete Faulthieraffe mit den Hundskopfe.

Meyer, Thiere, III. Tab. 2. der afiatifche Faule. Neuer Schauplatz der Natur, IX. pa 20I. der Unan.

Onomatol. hiftor. natur. II. p. 29r. das zeylonifche Fanlthier.

Biiffon, vierf. XIII. p. 344. der Unau.

Klein, Quadrup. difp. p. 44. Ignavus orientalis minimus.

Klein, Clafifi. d. vierf. Th. p. 130. kleinfter orientalifcher Fanle.

Klein, natürl. Ordn. d. vierf. Thiere. p. 48. n. 4 . Fauler aus Oftindicn.

RoSsmann, R. nach Guinea, p. 296. Potto.

Erxleben, Mammalia, p. 88. n. 2. Bradypus (Didactylus) palmis didactylis, cauda nulla.

Limné, Syh. Nat. Edit. II. p. 42. Bradypus manibus didactylis, cauda nulla.

Linné, S. N. Edit. VI. p. 3. n. 2. Bradypus manibus didactylis, cauda nulla.

Limne', Syf. Nat. Edit. X. I. p. 35. n. 2. Brady. pus (Didactylus) manibus didactylis, cauda nulla.

Graumann, intr. in hift. nat. mamm. p. 27. II. der Unau.

Pennant, Synopf. quadruped. p. 321. n. 25r. the twotoed Sloth.

Gatterer, brev. Zoolog. I. p. 29. n. 2. Bradypus (Didactylus) palmis didactylis, cauda nulla; der Unan. 
8. GESCHLECHT. MYR MECOPHAGA: Ameifenfreffer; Ameisenbür.

Miiller, Naturfyft. I. p. 108. Gen. VIII. Zimmermann, geogr.Zool. II. p. 405. Gen. XL: Schreber, Säugthiere, II. p. 202. Gen. VI. Le $\int k e$, Naturgefch. p. I23. Gen. IV. Borowfky, Thierreich, I. p. 56. Gen. V. Blumenbach, Handbuch der Naturgefch. p. 64 Gen. VI.

Fisike, Naturgefch. I. p. I76.

Biiffon, vierf. VII. p. 201 .

Batfch, Thiere, I. p. I63. Gen. XVI. Esxleben, Mammalia, p. 90. Gen. X.

Graumann, intr. in hiftor. nat. mammal. p. $2 \%$. Gen. X:

Gatterer, brev. Zoolog. I. p. 30. Gen. X. Hermam, tab. affin. animal. p. 64. 66.

T. Did a c т г a. Der kleine Ameifenfreffer. (7) Miiller, Naturfyfem, I. p. I80. n. I. der zweyfingerige, oder kleine Ameifenbär, Táb. ro. fig. I. Seb.

Zimmermann, geogr. Zoolog. II. p. 405. n. 349. der kleine (zweyzehige) Ameifenfreffer.

Schreber, Säugthiere, I. p. 206. n. 3. der kleine Ameifenfreffer, Tab. 66.

Lefke, Naturgefeh. p. 124. n. 2. der kleine Ameisenfreffer.

Borowfky, Thierreich, I. p. 57. n. 2. der kleine Ameisenfresser.

Blumenbach, Handb. der Naturgefch. p. 64. n. r. Myrmecophaga (Didactyla) palmis didactylis, ungue

(7) Lunge des Körpers acht Zoll; der faft eben fo lange Schwanz haarig und greifend. 
ungue exterióre maximo, plantis tetradactylis, cauda prehenfili; der kileine Tamandua.

Halle, vierf. p. 383. der woifse amerikanifche AmeiSenfielder.

Illeyer, Thiere, III. Tab. 1o. dev weifse amerikanifche Ameifenfrel]er, fig. Seb.

Neuer Schauplatz d, Natur, I. p. 258. der Kleine Aneifenfireffer.

Onomatol, hifl, natur. V. p. 4, Myrmecophaga didactyla.

Klein, Quadrup. difp. p. 46. Tamandua alba altera, fou Coati.

Kiein, Claftif. der vierfüfs. Th, p. I338. weifser. Aneifenfehlucker.

Klein, natül. Ordn. d. vierfürs, Th. p. 5o, n. 3 . weifser Ameifenfrefser.

Martini, Naturlex, II. p. 274. der hieine zwozehi. ge Ameifenfrefjer, m. Schrcb. Fig.

Biiffon, vierfüfs, VII. p. 236. der"kleine Ameifenfreffer.

Fermin, Befchr. von Surinam, II. p. 87. die drit. te Art von Ameisenbären.

Molina, Naturgefch. v. Chili, p. 240.

Depper, Amerika, p. 308. ein kleiner Bär ohnè Mund mit longer Schnantze.

Erxleber, Mammalia, p. 90. n. I. Myrmecophaga (Didactyla) palnis didactylis, plantis tetradaclylis, cauda villofa.

Linné, Syf. N. Edit. II. p. 42. Myrmecophaga manibus monodacty lis, plantis tetradactylis. Linné, Syn. Nat. Edit. VI. p. 8. n. 2. Myrmeco. haga manibus didactylis, plantis tetradactylis. Limé, Syft. Nat. Edit. X. I. p. 35. n. I. Myrmecophaga (Didactyla) manibus didactylis, plantis tetradactylis. 
Graumann, intr. in hiftor. nat, mamm. p. $27 \%$ I. der kleine Ameifenfrêfer.

Gatterer, brev. Zoolog. I. p. 30. n. I. Myrmecophaga (Didactyla) palmis didactylis, ungue exteriore maximo, plantis tetradactylis, cauda prehenfili; der kleine Tamandia.

Gronov. Zooph. I. p. I. n. I. Myrmecophaga ro. fro brevi, pedibus anticis didactylis, pofticis tetradactylis.

Barrere, Fr. equinox. p. 63. Tamandua minos flavefcens.

Pennant, Synopf. quadruped. p. 333. n. 262.

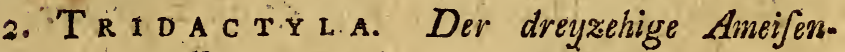
freffer. (Zimm.)

Mailler, Naturfy f. I. p. 18r. n. 2. der dreufingerigi, oder grosse Aneifenbizi. Tab. 10. fig. 2.

Zimmermann, geogr. Zool. II. p. 405. der drey. zehige Ameifenfreffer.

Halle, vierf. p. 393. der grosse Ameifenbür.

Meyer, Thiere, III. Tab. 9. dev Ameifenfreffer, fig. Sel).

Meyer, Thiere, III. Tab. I2. der brafilianifche AineifenfrefJer.

Never Schaupl. d. Natur, I. p/ 257.

Onomatol. hift. nat. V. p. 406.

Erxleben, Maminal. p. 92. n.2. Myrmecopha. ga (Tridactyla) palmis tridactylis, plantis pen. tadactylis, cauda villofa.

Linné, S. N. Edit. II. p. 42. Myrmecophaga ina. nibus tridactylis, plantis pentadactylis.

Linné, S.'N. Edit. VI. p. \&. n. 1. Myrmecophaga manibus tridactylis, plantis pentadactylis.

Graumanu, intr, in hift wat mamm. p. 28. II. der grofse Anuifonlior. 


\section{4
Mammalia Bruta.
Myrmecophaga.

Gatterer, brev. Zool. I. p. 30. n. 2. Myrmecophaga (Tridactyla) palmis tridactylis, plantis pentadactylis, cauda villofa; der grofse AmeiSenbiü.

3. I и в т а: Der grn/se Ameijenfreffer. ( ${ }^{8}$ )

Miiller, Naturfyht. I. p. 182. n. 3. der langharige Ameifenbär. (9)

Zimmermann, geogr. Zoolog. II. p. 406. n. 350. der grofse Ameifenfreffer.

Lefke, Naturgefch. p. 124. n. I. der grofse Amei: senfreffer.

Boroweky, Thierreich, I. p. 57. n. I. grofser Amei enfrelfer.

Bechftein, Naturgefch. Deutfchl. I. p. I35. der gro.jse Ameifenfredser.

Halle, vierf. p. 395. der grofse Amei cnbär.

Mheyer, Thiere, III. Tab. 13. der grö/ste Ameijenfreffer.

Gatterer, vom Nutzen u. Schaden der Thiere, I. p. 19. n. 28. der Tamanois.

Onomat. hift. natur. V. p. 404. der langhaarige Ameifenbür mit vier Zehen.

Klein, Quadrup. difp. p. 45. Tamandua - guacu, Tab. 5. eine ganz gute Fig.

Ktein, Claffif. d. vierfüfs. Th. p. 132. Tamandua-guacu, Tab. 5 .

Klein, natürl. Ordn. d. vierfüfs. Th. p. 49. n. x. Ameifenjöger, der gröfste.

Martini,

(8) Lânge des Thiers ohne den Schwanz beynahe vier Fufs. Die Zunge if gegen dritchalb Fufs lang. Diefe Ateckt das Thier, wi feine Verwandten in dic Ameifenbaue, und zieht auf die Weife damit feine Nahrung, die Ameifen, zu fich.

(9) Aber die Fig. 2. Tab. 10. gehürt nicht zu diefer, fondern zu der vorhergehenden Gattung. 
Martini, Naturlex. II. p. 27I. der grofse Annei. Senfreffer.

Bïffon, vierf. VII. p. 227. der grofse Ameijenfreffer.

Fermin, Surinam, II. p. 86 . die erfe Art von Ameisenbüren.

Hartfink, Befchr, von Guiana, I, p, 108. $W a$ riroe.

Reifen der Miffionar. von Murr. p. 2rr. der Ameifenbär. O/fo hormignero.

Frifch, vierf. Th. p. 5. n. r. der grö/ste AneiSenbiir.

Erxleben, Mammal. p. 93. n. 3. Myrmecophaga (Iubata) palmis tetradactylis, plantis pentadactylis, cauda iubata.

Linné, S. Nat. Edit. X. I. p. 35. n. 2. Myrmecophaga (Tridactyla) palmis tridactylis, plantis pentadactylis.

Graumann, intr. in hif. nat. mamm. p. 28. III. langhaarige Ameisenbär.

Gatterer, brev. Zool. I. p. 30. 11. 3. Myrmecophaga. (Iubata) palmis tetradactylis, plantis pentadactylis, cauda iubata; der Tamanoir.

Knorr, delic. natur; II. Tab. K. 9. der grofse ' dreyzehige Ameifenbär.

Barrere, Fr. equinox. p. 162. Tamandua maior, cauda paniculata: Ouariri; Grand mangeur de fourmis.

Ionfton, Quadruped. p. I36. Tamandua Guacu, Tab. 62. fig. Marcgr.

Marcgr. Brafil. p. 225. Tamandua Guacu Brafilienfibus; m. e. zieml. Figur.

Laet, nov. orb. p. 55r. Tamandua.

Nievemberg, hilt. nat. p. 190. Myrmecophagus, five Tamendoa, 
96 Mammalia Bruta. Myrmecophaga.

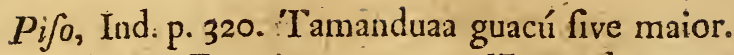
Charleton, Exercitat. p. I7. Tamandua guacu Brafilienfis.

$R a j$, Synopf. quadruped. p. 24I. Tamanduaguacu.

Laurent. muf. reg. Tab. 7 . fig. 7 T. Tanianduaguacu, vulgo Myreneter.

pallas, Mifcell. Zoól. p. 65. Myrmecophaga fciurea.

4. TE TR A D A T T L A. Der mittleré Ameifenfreffer. ( $\left.{ }^{10}\right)$

Miiller, Naturfynt. I. p. I83. der vierzehige AmeiSenbiir.

Zimmernuann, geogr. Zoolog. II. p. 407. n. 35 r. der mittlere (vierzehige) Ameifenfreffer.

Borowfky, Thierreich, I. p. 57. n. 3. mittlere Ameifenbär.

Halle, vierfüfs. p. 394. der mittlere falbe AmeiSenbär.

Meyer, Thiere, III. Tab. ir. der kleine amerikanifche Ameifenfreffer; fig. Seb.

Onomatol. hilt. nat. I. p. 505 . der vierzehige Amei. senbär mit kurzen Haaren.

Klein, Quadruped. difp. p. 46. Tamandua. I, Brafil.

Klein, Claflif, d. vierf. Th. p. 136 .

Klein, natïl. Ordn. der vierf. Th. p. 49: n: 2. Tamandua $\mathbf{1}$, aus Brafilien.

Biiffon, vierf: VII: p. 2 Io:

Frifch, Naturf, der vierf. Th: p. 5. 2. der mittlere Anueisenfreffer.

Martini,

(10) Die Lange des Thiers betragt über anderthalb Fufs. Schla* fond hält es den Kopf zwif̌hen dea Vorderfüfsen verborgen. 
Martini, Naturlex. II. p. 276. der mittlere vierzehige Ameifenfrefjer.

Schlözer, Erdbefchreibung von Amerika, p. 595. Ameifenbirio.

Fermin, Surinam, II. p. 87. die zwote Art von Ameifenbüren.

Erxleben, Mammalia, p. 95: n. 4. Myrmeco. phaga ('Tetradactyla) palmis tetradactylis, plantis pentadactylis, cauda calva.

Limné, S: N. Edit. VI. p. 8. n. 3. Myrmecophaga, manibus tetradactylis, plantis pentadactylis.

Linné, S. N. Edit. X. p. 35. n. I. Myrmecopha. ga (Tetradactyla) palmis tetradactylis, plantis pentadactylis.

Graumann, intr. in hif. nat. mamm. p. 28 . IV. vierzehige Am.

Gatterer, brev. Zoolog. I. p. 3I. n. 4. Myrmecophaga (Tetradactyla) palmis tetradactylis, plantis pentadactylis, cauda calva.

Barrere, Fr. equinox. p. 162. Tamandua minor, cinerea; Mangeur de fourmis.

Gronov. Zoophyl. I. p. I. n. 2. Myrmecophaga, roftro longifimo, pedibus anticis itetradactydis; plantis peritadactylis, cauda calva.

Ionfton, Quadrup. p. i37. Tamandua-I. Tab. 62. fig. Marcgr.

Marcgr. Brafil p. 225. Tamandua - I Brafilienfibus, p. 226. eine gute Fig.

Charleton, Exercit. p. I7. Tamandua-I.

Seba, Thef. II. p. 48. Tamandua formicis vefcens americana minor, Tab. 47. fig. 2. mittelm. Raj, Syn. Quadr. p. 242. Tamandua-I Brafilienfibus.

Pallas, Mifcell. Zoolog. p. 64. Myrmecophaga myofura. 


\section{Monmalia Brita. Myrmecophaga.}

5. C A $\mathrm{P}$ E N $\mathrm{I}$ s. Der afrikanifche Ameifenfreffer. (Zimm.)

Zimmermann, geogr. Zoolog. II. p. 407 n. 350 .

I. p. 305 .

Schreber, Säugthiere, I. p. 207. eine vierte Art Aneifenfrelfer.

Biiffon, vierfüfs. VII. p. 241. vom afrikanifchen AmieifenfrefJer.

Erxleber, Mammalia, p. 97.98.

Kolbe, Vorgeb. d. g. Hoffu. p. 165. Ausg. in Fol.

p. 337. Ausg. in Quart, Erdfchwein.

Comment. de rebus in feient. nat. geftis XV.p. 243.

Graumann, intr. in hift. nat. mamm. p. 28.

Pallas, am a. O. Míyrmecophaga afra.

Verändernngen gegen die XIIte Edition, und Vermehrung der Gattungen diefes Gefchlechts.

Diefs Gefchlecht if mit einer Gattung vermehrt, und M. Capenzifs hinzugekommen.

9. GESCHLECHT. M A Is. Das Schuppenthier. (Ceilonifche Armadill, Müll. Formuofonifche Tenfelchen, Blumenb.) Schuppeneideche.

Miiller, NaturfyAem, I. p. 185. Gen. IX.

Zimnnerniann, geogr.Zool.II. p. 403. Gen. XXXIX. Schreber, Säugthiere, II. p. 208. Gen. VII.

Leeke, Naturgefch. p. 125. Gen. VII.

Borowfky, Thierreich, I. p. 58. Gen. VI.

Blumenbach, Handb. d. N. G. p. 65. Gen. VIII.

Funke, Naturgefch. I, p. 177.

Biiffon, vierf. VII. p. 245 .

Errole. 
Erxleben, Mammalia, p. 98. Gen. XI.

Graumann, intr. in hiftor. natur. mamm. p; 28 : Gen. XI.

Gatterer, brev. Zoolog. I. p. 3I. Gen. XI.

Pennant, Syn. Quadr. p. 328. Gen. XXXVIII.

Hermann, tab. affinit. animal. p. 65.

Briffon, Quadr. p. 18. Gen. II. Pholidotus.

I. PEN T A D A C T Y L A. Das kurzgefchwänzte Schup. penthier. $\left({ }^{8}\right)$

Miiller, Naturfyf. I. p. I86. n. I. das fiunffingerige Schuppenthier; der javanifche Teufel.

Zimmermann, geogr. Zool. II. p. 403. n. 346. das kurzgefchwönzte Schuppenthier.

Leske, Naturgefchichte, p. 125. n. I. das kurzgefchwönzte Schuppenthier, fig. 19.

Borowfky, Thierreich, 1. p. 59. n. r. fiunffingeriges Schuppenthier, formofifches. Teufelchen, Tab. 6.

Halle, vierf. p. 396. 397. das formoffche Teufel. chen, Tab. I8.

Gatterer, vom Nutzen und Schaden der Thiere,

I. p. 19. n. 2. der Pangolin.

Neuer Schauplatz der Natur, VII. p. 778. der

Pangolin, Panggölin, seylonifcher Armadill,

Tenfel von Fava, chinefifcher Teufel.

Onomatol. hiftor. nat. V. p. 59. das formofif che Teufelchen.

Schwed. Abhandl. XI. p. 274. IManis, eizz oftina difches. Thier.

Klein, Quadr. difp. p. 47. Tatu muftellinus. Klein, Claflif. der vierfüfs. Thiere, p. I40."Wiea Jelihnnliches Panzerthier; ge/chuppter Armadill.

$\mathrm{G} 2, \ldots$ Klein,

(8) Lange des Thiers, bis asht Fufs. Dis Farbe der Schuppen if tütblich. 
Klein, natürl. Ordnung d. vierf. Th. p. 5r. n. r. wie.elelihnlicher Armadill; gepanserter Iltio, oder Tanuandua; tajovanifcher Tenfel der Chineser. Biiffon, vierf. VII. p. 245. das karragefchwoïnzite Schuppenthier.

Perrenilt, Charras und Dodart, Abhandl. aus der Naturgefch. II. p. 265. A natomifche Befchreibung einer Schuppeneideche. Tab. $77 \cdot 78$.

Erxlebein, Manumal. p: 98. n. r. Manis (Brächyura) cauda fere longitudine corporis.

Limné, S. N. Edit. VI. p. 8. n. I. Manis manibus pentadactylis, palmis pentadactylis.

Linné, S. N. Ed. X. I. p. 36.n. I. Manis (Pentadactyla) pedibus pentadactylis.

Linné, Muf. Adolph. Fried. II. p. 7. Manišs (Pentadactyla) pedibus pentadactylis.

Groumenn, intr. in hift. iat. mamm. p. 28. I. Manis brachyura, das, kuvzgefchwönzte Schuppenthier; der javanifche Teufel.

Gatterer, brev. Zool. I. p.3I. in. I. Manis Brachyura; der javanifche Teufel, Pangolin.

Gronov: Zooph. I. p. 2. n. 3. Pholidotus, pedibus anticis et pofticis pentadactylis, fquamis fub. rotundis.

Bont: hifor. nat. Ind: or. p. 60 . Lacertus indicus fquamofus; m. e. zieml. guten Figum.

Tacobaci Muf. reg. p. 9. . Lacerta fquaniofa ingens. Tab. 9. fig. 3 .

Lanrent. Muf. reg. Tab. 6. fig. 82. Lacerta, fquamofa ingens.

Men. de l'Acad. des Sc. de Paris, Tom. 3. P. 3. p. 85. Lezard écaillé Tab. I7.

Berichte der königl. dänifchen Miffionarien in Oftindien, 104 C. p. $20 \%$ m. e. doppelten Abbild. Alungu.

Philof. 
Philof. Transact. Vol. 6o. p. 36.

Muf. Barith, p. 4, das mit Schuppen gepanzerte

Thier, Manis, oder Armodillus genannt; Tab. 2. ein Iunges.

Des Marchais Voyage en Guinée, I. p. 179. Quogelo,

2. T E T R A A C T Y A. Das langgefchiounzte: Sihuppenthier ( $\left.{ }^{9}\right)$

Wiuller, Naturfyftem, I, p, I87, das vienfingerige Schuppenthier.

Ziminermann, geogr. Zoolog. II. p. 404. n. 347. das langge Schwänizte Schuppenthier.

Lefke, Naturgefchichte, p. I26. n, 2, das langgefchwinnte Schuppenthier.

Borowfky, Thierreich, I. p. 59. n, 2, das vierfingerige Schuppenthier.

Bhimenbach, Handb, d. N. G. p. 65. n. I. Manis (Macroura) cauda longiore; der Phatagin. Beihftein, IVaturgefch. Deutchl. I. p. 135. das. langgefchreönate Schuppenthier.

Nener Schauplatz der Natur, III. p. 779. der Phatagin.

Onomatol. hift. nat. V.p. 6 r.

Biiffon, vierf. VII. p. 245. das langgefchwcinzte Schuppenthier.

Erxleben, Mammalia, p. ror. n. 2. Manis (Macroura) cauda corpore longiore.

Graumiann, intr. in hift. nat. mamm. p. 29. n. 2.' Manis macroura; das langgefche ïnate Schupa penthier.

Gatterer, brev. Zool. I. p.3r. Manis (Macroura) cauda corpore longiore; der Phatagin.

$$
3
$$

Olear.

(9) Länga des Körpexs funfzehn. Zoll, des Schwanzes gegen vierzig. 
Olear. Gottorf. Kunftkammer, p. 7. Lacertus peregrinus fquamofus, Tab. 7. fig. I. mittelm. Mem. de l'Acad. des Sc. de Paris To. 3. P. 3. F.89. Lezard de Clufins.

Raj, Synopf. Quadr.' p. 274. Lacertus peregrinus fquamofus.

10. GESCHLECH'T. Dasypus. Das Gürtelthier, Armadill, Panzerthier, Tatu. Miiller, Naturfyft. I. p. I88. Gen. X. Zimnnermann, geograph. Zoologie, II. p. 399. Gen. XXXVIII.

Schreber, Säugth. II. p. 213. Gen. VIII. Lefke, Naturgefch. p. I26. Gen. VIII. Borow Sky, Thierreich, I. p. 60. Gen. VII. Blumenbach, Handb. d. N. G. p. 65. Gen. IX. Finnke, Naturgelch. I. p. 177.

Biiffon, vierf. VII. p. 257.

Erxleben, Marnmalia, p. I02. Gen. XII.

Graumann, intr. in hiftor. natur. mamm. p. 29. Gen. XII.

Gatterer, brev. Zool. I. p. 32. Gen. XII.

I. UN ICINc Tus. Das Gü̈rtelthier mit zwälf Gür. teln. (10)

Miiller, Naturfyn. Suppl. p. 23. n. 7. der zwölfgitrtelige Armadill.

Zimmermann, geogr. Zoolog. II. p. 402. 11. 344. das Giirtelthier mit awölf Gürteln.

Meyjer, Thiere, III. der afrikanifche Armadill, Tab. 27. das Männlein, Tab. 28, das Weib. lein; fig. Seb. -

Neuer

(10) Soll Jas gröfste Gürtelthier foyn. Länge des Rülekens etwas über eiren Fufs, an der Seite gemeffen, zwey Zoll mehr, Die zwülf Gürtel des Panzers haben viereckigte Schuppen. 
Neuer Schauplatz der Natur, I. p. 385 . n. 5. der Kaba/fiu, oder der Tatu mit zwölf Reifen.

Biiffon, vierf, VIl. p; зо9. das Giirtelthier mit awölf Gïrteln, oder der KabaJu.

Kkein, Quadrup. difp. p. 49. 'Tatu caninus, capite pedibusque anomalis.

Klein, Claffif. d. vierf. Th, p. 145. Panzerthier

mit dein. Hundskopf.

Klein, natïrl, Ordnung d, vierf, Th. p. 52. n. 5 .

Hutudstatu.

Martini, Naturlex. III. p. 232. Armadill mit zwölf

Gïrteln, der Kabafiu, af rikanifcher Armadill.

Mannichfaltigkeiten, III. p. 642.

Erxleben, Mammal, p. rIr, n. 6. Dafypus (Duodecimcinctus) cingulis duodecim, pedibus pentadactylis.

Linne, Syft. N. Edit. II. p. 45. Erinaceus loricatus, tegunine tripartito.

Linné, Syft. Nat, Edit. VI. p. 6. n. 7. Dafypus tegmine tripartito.

Grauniann, intr. in hif, nat, mamm, p. 3o. VI.

Dafypus Duodecimcinctus; Armadill mit zwölf Gürteln.

Gatterer, brev,Zool. I. p. 33. n. 6. Dafypus (Duo: decimcinctus) cingulis duodecim, pedibus pentadactylis; der Kaba/Ju.

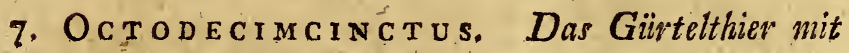
achtzehn Gürteln, ( $\left.{ }^{(}\right)$

Miiller, Naturfyf. I. p. I89. n, I. der eingürtlige Armadill.

$$
\text { G } 4 \text { Miller, }
$$

(1) Länge des Thiers zehn Zoll, des Schivanzes fieben. Srbreber hält es von dem Gürtelthier mit vier Gürteln $(n, 4$.$) nicht für$ verfchieden. 
Miller, Naturfyft. Suppl. p. 24. n. \&, der acht: zehngiurtelige Armuadill.

Zimmermanis, geogr. Zoolog. II. p. 402. n. 345. das Giirtelthier mit achtzchn Giurteln.

Schreber, Säugthiere, I, p, 227.n. 8. das Giirtel. thier mit achtsehn Gïrteln.

Halle, vierfüfs. p. 396. der gepanzerte Aneb. senbär.

Neuer Schauplatz der Natur, I. p, 386. n. 6. der Cirquinçon oder Cirquinchum, oder der Tatu mit achtzeln Giurtehn.

Biiffors, vierf. VII. p. 320. das Giirtelthier mit achtzehn Gïrteln oder der Cirquinachum.

Martini, Naturlexicon, III, p. 234, Armadill mit achtzehn Giurteln, Cirquincon, Tatur. wiefel.

Mannichfaltiģkeiten, III. p. 360.

Erxleben, Mammalia, p. I13. n. 7. Dafypus (Octodecimcinctus) fcuto poftico nullo, cingulis octodecim.

Iinné, S. N. Edit. VI. p. 6. n. I. "Dafypus cin. gulo fimplici,

Limne, S. N. Edit. X. I. p. 50. n, I. Dafypus (Unicinclus) tegmine tripartito, pedilus penta. dactylis.

Graumann, intr. in hif. nat. mamm. p. 30 . VII, der eingïrtelige Armadill, das Gürtelthier mit acht:alun Ringen.

Gatterer, brev. Zoolog. I. p. 33. n. 7. Dafypus (Ortodecimcinftus) fcuto poftico nullo, cingu= lis octodecim; das Tatruiefel.

Pennant, Syn. quadruped. p. 327. n. 257. the eighteęn - banded Armadillo. 
2. TRICINC TUS. Das Gürtelthier anit drey Gür. teln. $\left({ }^{2}\right)$

Miiller, Naturfyft. I. p. 190. n. 2. der dreygiirteli. ge Armadill.

-Zimmermann, geogr. Zoolog. II. p. 399. n.' 339.' das Gürtelthier mit drey Giirteln.

Lefke, Naturgefch. p. I27. n. I. das Gïrtelthier mit drey Giirtelin, Fig. 20.

Borowsky, Thierreich, I, p. 62. n. 2. der drey. giirtelige Armadill.

Bechftein, Naturgefch. Deutfchl. I. p. I36. das Giurtelthier mit drey Gürteln.

Halle, vierf. p. 399. der Jchönfte orientalifche Armodillo, Tab. 17.

Meyer, Thiere, III. Tab. 25. der oricntalifche Armadill, fig. Seb.

Gatterer, vom Nutzen und Schaden der Thiere, I. p. 20. n. 32. der Apar.

Neuer Schaupl. d. Natur, "I. p. 384. n. r. der Apar,

Tatuapara, oder Taiu mit drey Reifen.

Ktein, Quadrup. difp. p. 48. Tatu porcinus. Klein, Claflif. der vierfịifs. Th. p. 144. orienta lifches Schilduerkel.

Klein, natïrl. Ordn. d, vierfüfs. Th. p. 52. n. 4. fchönftes orientalifches Schildverkel.

Martini, Naturlex. III, p. 224. Armadill mit drey Ringer, Apar, Aparathier.

Biiffon, /vierfüls. VII. p. 265. das Giirtelthier mit drey Givrteln, oder der Apar, m. e. Fig.

Gesner, Thierbuch, p. 23I. nach Cluf. und Mą̣cgr.

$$
\text { G } 5 \text { Dapper, }
$$

(2) Yeder der drey Gürtel ift aus drey und zwanzig länglich viereckigen Stiicken zufammengefetzt, Länge des Kürpers, den Schwanz mitgerechnet, ein Furs. 
Dapper, Amerika, P. 445.

Erxleben, Mammal. p. I02. n. I. Dafypus (Tricinctus) cingulis tribus, pedibus pentadacty. lis, cauda brevi.

Linné, Sylt. N. Edit. II, p. 45. Erinaceus loricatus, cingulis tribus.

Limné, Syft. Nat. Edit, VI. p. 6.' n. 2. Dafypus cingulis tribus.

Linné, Syft, Nat. Edit. X. I. p. 51. n. 2. Dafypus (Tricinctus) cingulis tribus, pedibus pentadactylis,

Graumann, intr. in hiff. nat. mamm. p. 29. I. das Gintelthier mit drey Ringen.

Gatterer, brev. Zoolog. I. p, 32. 11. I. Dafypus (Tricinctus) cingulis tribus, pedibus pentada. ctylis, cauda brevi; der Apar.

Nieremberg, hiftor, nat. p. I5I. Armadillo genus alterum Clufii. Abbild. nach Cluf.

Ionfton, Quadr, p. 174. Tatus. Tab.80. eine fehr - fchlechte Figur.

Ionfton, Quadrup. Tab, 63. Tatu, Apara; nach Marcgr.

Pifo, Ind, p. 100. Tatu-apara; nach IMarcgr. $R a j$, Synopf. quadrup. p. 234. Tatu apara. Pennant, Synopf, quadr, p. 323. 11. 25 T.

3. Qua dricinctus. Das Gürtelthier mit vier Giirteliz. (3)

IMiïler, Naturfyf,- I. p. 190, n. 3. dev viergïrte. lige Avmadill.

Zinimermann, geogr. Zoolog. II. p. 400. n. 340. das Gürtelthiev mit vier Gürteln.

Schreber,

(3) Nach Erxlebsn eine unbeftimmte Gattung; nach Scbreber u. a, eine l'arięatat. Grö́se siner Katze. 
Schreber, Säugthiere, I. p. 217. 1. 2. das Gürtelthier mit vier Giirteln.

Martini, Naturlex. III. p. 225. Armadill mit vier Ringen, Schildkrötentigel, Schildkrötenf chweinn.

Bïlfon, vierf. VII. p. 27 I.

Erxleben, Mammal.p. 104. n. 2. Dafypus (Qua.. dricinctus) cingulis quatuor.

Linné, Syft. N. Edit. VI. p. 6. n. 3. Dafypus cingulis quatuor.

Linné, S. Nat. Edit. X. I. p. 51. n. 3. Dafypus (Quadricinctus) cingulis guatuor.

Graumann, intr. in hilt. nat. (mamm. p. 29. II. das Criirtelthier mit vier Ringen.

Gatterer, brev. Zoolog. I. p. 32. n. 2. Dafypus (Quadricinctus) cingulis quatuor; der Schild. krötenigel; das Schildkvötenfchwein.

4. SExcrnctus. Das Gürtelthier mit dem Halsfchilde. (4)

Miuller, Naturfyf. I. p. Igr. n. 4. der fechsgïrte. lige Armadill.

Zimmerinann, geogr. Zoolog. II. p. 400. n. 34r. das Givtelthier mit dem Hals fchilde.

Borowfky, Thierreich, I, p. 6I. n, I. der fechs. giirtelige Armadill.

Neuer Schaupl. d, Natur, I. p. 384. n. 2. der Ena cubert, der Tatz nit Jechs Reifen, Tatupeb, Tatupeba.

Martini, Naturlex. III. p, 226. Armadill mit Jechs Reifen; der Encubert, fig. Schreb.

Gesner, Thierbuch, p. $23^{\circ}$. die untere Fig. nach Bellon.

Bïffon,

(4) Lange des Thiers, ohne den Schwanz, exwas uber einen Schuh; die Breite etwa acht Zoll; "as Gewicht ohngàtahr zwölf Pfund. 
Bïffon, vierf. VIT. p. 273. das Gürtelthier. mit Jechs Gürteln oder der Enculiert, m. e. Fig.

Mannichfaltigkeiten, III. p. 634 .

Erxleben, Mammal. p. 105. 1. 3. Dafypus (Sexcinctus) cingulis fenis, pedibus pentadactylis.

Limné, Syft. Nat. Edit. VI. p. 6. 11. 5. Dafypus cingulis fex.

Linné, Syf., Nat. Edit. X. I. p. 5I. n. 4. Dafypus (Sexcinctus) cingulis fenis, pedibus pentacia. ctylis.

Graunuann, intr. in hift. nat. mamm. p. 29. n. 3. Das Gïrtelthier mit dem Halsschilde; der Jechs. gïrtelige Armadill.

Gatterer, brev. Zool. I. p. 32, n. 3. Dafypus (Sexcincius) cingulis fenis, pedibus pentadactylis; der Eacubert,

Bellon. Obfervat. p. 204. Tatu, m.e.mittelm. Fig. Nieremberg, hif, nat. p. I58. Armadillo Clufii; die mittl. Fig. nach Bellon.

Ionfton, Quadrup, 'Tab. 62. Tatu f. Armadillo. Fig. Bellon.

Chmleton, exercitat. p. I8. Tatu.

Pennant, Syn. quadr. p. 324. n, 253. the fixban. ded Armadillo,

5i Sertemcinctus. Das Gürtelthies mit Jechs geflammen Gïrtein. (s)

Miiller, Naturfy?t. I. p. 19I. n. 5. der fiebengïrtelige Armadill.

Borowefky, Thierreich, I. p. 62. n. 2. der fiebengiirtelige Armadill.

Gatteier, vóm Nutzen u. Schaden der Thiere, I. p. 21. n. 33. Dafypus feptemcinctus, die Tatluette?

Martini,

(5) Wira von Erreleben für den Dafypus Sexcinctus gehalten. 
Martini, Natudlex. HI. p. 22S. Avmadill mit Jechs geflamnuten, ingleichen mit fieben oder acht Gürteln; Tatuette.

Biiffon, vierf. VII. p. 283. das Giirtelthier snit acht Gürteln, oder der Tatuette; p. 289. das Gürtelthiev mit fechs geflammien Giivteln.

Schlözer, Erdbefchreib. von America, p. 595. Knorr, delic. nat. II. Tab. K. 3. fig. 2. Schildverkel oder Armadill aus Amerika, mit feben beneglichen Reifen.

Erxleben, Mammalia, p. 10\%. n. 4. Dafypus (Septemincinctus) cingulis feptenis, palmis tetradactylis, plantis pentadactylis.

Limné, S. N. Edit. VII. p. 6. n. 6. Dafypus cingulis feptem.

Limné, Syff. Nat. Edit. X. I. p. 5r. ni. 5. Dafypus (Septemcinctus) cingulis feptenis, palmis tetradactylis, plantis pentadactylis.

Graumann, intr. in hift. nat. mamm. p. 29 n. 4. der fiebengiirtelige Arnadill.

Gatterer, brev. Zoolog. I. p. 33. n. 4. Dafypus (Septemcinctus) cingulis feprenis, palnis tetradactylis, plantis pentadactylis; die Tatuette.

8. O c т o c I N c T u s. Das Gürteithier mit acht Gürteln. ( $\left.{ }^{6}\right)$

IViuller, Naturfyn. Suppl. p. 23. der achtgiirtelige Armadill.

Zimmer.

(6) Da nach Schreber der Unterfchicd diefer Gatung von der vorhergehenden und folgenden faft nur anf der mindern Anzahl dér Gürtel beruher, und folglich fo gering in, dafs man Grund hat zu ziveifeln, ob fie, wirklich von einander verfchieden, oder blofse Spielarten einer Gattung find, die Syionymen aber beym Erxlebon ganz unter einander geworfen find, fo hat es mir auch 
Zimmermann, geogr. Zool. II. p. 40r. 11. 342. das Gïrtelthier mit acht Gïrteln.

Neuer Schauplatz der Natur, I. p. 385. 11. 3. der Tatuette, oder Tatu, mit acht Reifen.

Biiffon, vierf. VII. p. 290. das Gïrtelthier mit acht Giirtelin.

Pennant, Synopf. quadr. p. 325. n. 254. Eightbanded Armadillo.

6. Novemcinctus. Das Gürtelthier suit neun Giirteln. (?)

Miiller, Naturfyf. I. p.irg2. n. 6. der neungiurtelige Armadill, Tab. 16. fig. 2. Seb.

Zimimermann, geogr. Zoolog. II. p. 40r. n. 343 . dàs Giirtelthier mit neun Giorteln.

Borowfky, Thierreich, I. p. 63. n. 4. der meum giirtelige Armadill.

Blumenbach, Handbuch d. Naturgefch. p. 65. n. r. Tatu (Novemcinctus) zonis dorfalibus IX. palmis tetradactylis, plantis pentadactylis; der Cafchicame.

Halle, vierf. p. 398. das amerikanifche Schildver. kslchen.'

Meyer, Thiere, I. Tab. 59. der Armadill. Ineyer, Thiere, III. Tab. 26. der anerikanifche Armadill.

Gattever, vom Nutzen und Schaden der Thie:e, I. p. 21. 11. 24. Dafypus novemcinctus; der Kachikame.

Neuer

bey Vergleichung der Quellen felbft, Mühe gekoftet, folche aus einander zu finden, und ich zweifle felbf, ob, wenn es wirlsich verfchiedeno Gattungen feyn follten, die Synonymen gehürig vertheilt find.

(7) If to kirre, dafs die Kinder damit fpielen. Ihre Baue werden auf allen von der See etwas entfernten Sandhügeln häufig ano getroffen。 


\section{Mammalia Bruta. Dafypus.}

Neuer Schauplatz der Natur, I. p. 385. n. 5. dev

Cachicame, oder Tatu mit neun Reifen; der Atuco.

Naturforfcher, XIV. p. 4I. Sanders Befchreibung eines in Paris befindlichen Dafypus Novemcinctus.

Klein, Quadr. difp. p. 48. Tatu porcinus;-Ta. tu finpliciter; Porcellus cataphractus; Armadillo communiter.

Klein, Claflif. d. vierf. Th. p. r43. Schildverkel; Verken.

Klein, natïl. Ordn. der vierf. Th. po 5r. n. 3. Schildverkel; Verken.

Martini, Naturlex. III. p. 230. Armadill mit neun Ringen, dev Kachikame.

Biiffon, vierfüfs. VII. p. 292. das Giirtelthier nit neur Giirteln, oder der Cachicamo; m. e. Fig. Bankroft, N. G. von Guiana, p. 86. der Tattyu, oder Armadillo von Guiana.

Hartfink, Befchreib. von Guiana, I. p. 106. das Schildfchreinchen, auch Tatou, und Armadille, und bey den Indianern Iefi genanut.

Dobrizhöfer, Gefch. der Abiponer, I. p. 390. Armadillo oder Tatiu.

Mannichfaltigkeiten, III. p. 64I.

Frxleben, Mammal. p. 109. n. 5. Dafypus (No. vemcinctus) cingulis novem, palınis tetradacty. lis, plantis pentadactylis.

Linné, S. N. Edit. II. p. 45. Erinaceus loricatus, cingulis novem, manibus' tridactylis.

Linné, S. N. Edit. VI، p. 6. n. \%. Dafypus cingulis novem.

Lininé, Syft. Nat. Edit. X. I. p. 5r. n. 6. Dafypus

(Novemcinctus) cingulis novem, palmis to tradactylis, plantis pentadactylis. 
Granmcun, intr. in hift nat. mamm. p.30. n. 5 .

Gatterea, brev. Zoolog. I. p. 33. n. 5. Dalypus (Novemcinctus) cingulis novem, palmis tetradactylis, plantis pentadactylis; der Kachikame.

Muf. Baruth. p. 23. ein gepanzertes amerikanifches Thier, Tatu, ocier Tatou genannt, mit einem Schueinskiopf, und fechs (ein Druckfehler, Atatt 9.) Panzergelenken auf dem Riicken.

Barrere, France equinox. p. 163. Tatus maior, molchum redolens; Taton - Kaba/fu.

Raj, Synopf. quadruped. p. 233. Tatuete Bra. filienfibus.

Penzant, Syn. quadr. p. 325. n. 255. Nine-banded Armadillo.

Philof. Transact. Vol. 54. p. 57. Tab. 7.

Gronov. Zoophyl. I. p. 2. n. 5. Cataphractus fcutis duobus, cingulis novem.

Iacobaei Mufeum reg. p. 8. Armadillo, feu Tatou Brafilienfum: Tab. 9. Fig. 4 .

Lanrent. Muf. reg. Tab. 6. fig. 79. Abbild. fchlechto

A) Verönderungen gegein dié XITte Edition, mua Vermehrung der Guttungen diefes Gefchlechts.

Edit. XII.

p. 53. n. ז. B. Dafypus cingulo fimplici.

\section{Edit. XIII.}

p. 55. n. 7. Dalypus Octo. decincinctus.

Diefs Gefchlecht ift mit ¿wey Gattungen verment,

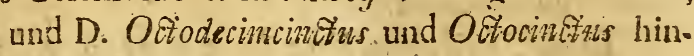
zugekommen.

B) Uin - 


\section{Mammalia Bruta. Rhinoceros.}

\section{B) Unbeftimmtere Thiere.}

\section{x. Das hundsköpfige Panzerthier.}

Halle, vierf. p. 4 or.

§. Die Gürtelthiere find weder beiflig, noch fonft böfe. Da ihr Harnifch, ob fie fich gleich vermitteln der beweglichen Gürtel zufammen rollen können, ihnen keinen vollkommenen Schutz gegen ihre Feinde giebt, fo befitzen fie dagegen eine fo grofse Fertigkeit zu graben, dafs fie fich in wenig. Minuten unter die Erde arbeiten, und dann halten fie fich mit den farken Klauen fo feft an, dafs es unmöglich ift fie beym Schwanze heraus zu ziehen. Aus dem Bau giefst man fie mit Waffer heraus, oder zwingt fie durch Rauch herauszugehen, und aus der Kugelform werden fie durch Hïlfe des Feuers gebracht, bey deffen Empfindung fie fich ausfrecken. Ihr Fleifch if in der Iugend wohlfchmeckend, be. kömmt aber im Alter einen Bifamgeruch. Aus der Haut macht man kleine Koffer.

36. GESCHLECHT. RHINOCEROOS. Das Nafehorn.

Miiller, Naturfyft. I. p. 469. Gen. XXXVI. Zimmermann, geogr. Zoolog. II. p. 59. p. 145: Gen. XI.

Schreber, Säugthiere, II. p. 228. Gen. IX. Lefke, Naturgefch. p. I27. Gen. IX. Borowfky, Thierreich, I. p. 63. Gen. VIII.' Bhumenbach, Handbuch der Naturgefch, p. 123. Gen. XXXIX. Bïffon, vierf. IX. p. 5 . Erxleben, Mammalia, p. 198. Gen. XXII. 


\section{Mommalia Bruta. Rhinocerose}

Graumann, intr. in hiftor.natur. maninial. p. 4r. Gen. XXII.

Gatterer, brev. Zoolog. I. p. 54. Gen. XXII. Pennant, Syn. quadr. p. 75. Gen, XI.

Hermann, tab. affin. animal. p. 97.

Briffon, quadrup. p. 78. Gen. XVI.

1. Un ICORNIs. Das (einhornige) Nafehorn.

Miiller, Naturfyfem, I. p. 469. n. I. das einhornige Nafehorn.

Zimmermann, geogr. Zoolog. II. p. 145. n. 34. das einhörnigte Nafehorn.

Borowjky, Thierreich, I. p. 63. das Nafehorn.

Blumenbach, Handb. der Naturgefch. p. I23. n. I. Rhinoceros (Unicornis) cornu unico, dentibus primoribus utrinque binis; das afiatifche Nafehorn.

Bechftein, Naturgefch. Deutfchl. I. p. 145. das Naseliom.

Neuer Schauplatz d. Natur, V. p. 62.

Onomatol. hiff. natur. VI. p. 824 .

Gesner, Thierbuch, p. 304. Rhinocer; fig. p.305.

Naturforicher, XIII. p. I. Sonder, vom Rhinoce. ros in Verfailles.

Neue gefellichaftl. Erzähl. II. p. 4I.

Berliv. Samml. VIII. p. 160.

Shaw, Reife, p. 370. Rhinnceros oder Rim?

Parfon, Hift. des Nafehorns. Nürnb. 1747.

Fravenzimmer Almanach, $1787 \cdot$ p. 248. m. e. illumin. Figur.

Evxleben, Mammalia, p. I98. n. I. Rhinoceros inicornis.

Limné, Sylt. Nat. Edit. II. p. 48. Elephas nafo cornigero. 
Mammalia Bruta. Rhinoceros.

Linné, S. Nat. Edit. VI. p. Ir. n. I. Rhinoceros cornu unico conico.

Linne', Syf. Nat. Edit. X. I. p. 56. n. I. Rhinoceros (Unicornis) cornu unico.

Graumann, intr. in hift. nat. mamm. p. 4r. n.22.

I. das Najehorn.

Gatterer, brev. Zool. I. p. 54. n. 1. Rhinoceros (Unicornis) ungulis tribus; das Nafehorn.

Ionfton, quadr, Tab. 38. Rhinoceros, Hornnase, Rhinocer.

Kram. Auftr. p. 3I7. Rhinoceros cornu unico co. nico.

Gronov. Zoophyl. I. p. I4. n: 13. Rhinoceros cornu unico conico.

Raj, Syn. quadr. p. I22. Rhinoceros.

Pennant, Synopf. quadruped. p. 75 . n. 58. the one - corned Rhinocéros.

Pallas, nov. comment. petropol. XIII. p. 447' Tab. 9. 10. XVII. p. 585. 'Tab. 15. fig. x. 2. 3. 16 :

Turpin, hift. de Siam. p. 291.

Borri hift. Cochin - chinae, p. 797.

Bergen, Oratio de Rhinocerote. Frcf. ad V. I746. 4 .

Aldrovand. de quadr. bifulc. p. 878. Rhinoceros.

Fig. p. 884.

Muf. Wormian. p. 336. Rhinoceros.

Bont. hiftor. nat. ind:- orient. p. 50 . Abada five

Rhinoceros. Fig. p. 51. Jchlecht.

Grew, muf. reg. Societ. p. 29. Rhinoceros.

Charleton, exercitat. p. 12. Rhinoceros.

Brifjon, quadruped. p. (mihi) 78. n. I. Rhino. ceros.

Chardin, Voy. II. p. 60. Rhinoceres. Tab. 40. Abbild. mittelm. 
I16 Mammatia Bruta. Rhinoceros.

Neuhoif, Gefandfch. nach China, p. 348. Rhinoceros, Fig. mittelm. (Fol. Amf. 1669.)

- Plin. hiftor. nat. VIII. c. 20. XVIII. c. I. Rhinoceros.

Aelian. de nat. anim. XVII. c. $45 .{ }^{\text {'P }}$ tvoregus. Oppiari. cyneget. II. 551. 'P $\mathrm{P}$ voxeģws.

2. BI cor.nis. Das doppelhörnigte Nafehorn.

Miillev, Naturfyft. I. p. 472 . n. 2. zweyhörniges Nofehorn.

Zimmermann, geogr. Zool. II. p. 148. n.65. das doppelhörnigte Na/ehorn.

Blumenbach, Handb. der Naturgefch. p. 123. n. 2. Rhinoceros (Bicornis) cornibus duobus, inciforibus nullis; das af irikanifche Nafehorn.

Neuer Schauplatz der Natur, VI. p. 62.

Onomat. hift. nat. VII. p. 824 .

Klein, Quadrup. difp. p. 26. Rhinoceros Tab. 2. das Horn.

Klein, Claffif. d. vierfüfs. Th. p. 85. Nafehorn. Klein, natül. Ordn. d.vierf. Th. p. 30. Nafehorn. Biiffon, vierf. IX. p. 40. das afrikanifche doppelhörnigie Nafehorn.

Sparrmann, Reife n. d. Vorgeb. d. g. H. p. 416. das aweyhornige Nafehorn.

Kolbe, Vorgeb. p. I59. Nafehorn oder Rhinoceros. Tab. IV. Fig. 2. Abbild. Sehr fchlecht.

Eberhard, Thiergefchichte, p. 273. das Nafehorn mit zwey Hörnern anf a'er Nafe, Tab. II. fig. 2.

Dapper, Afrika, p. 20. Rhinozeros, oder Rhinokeros.

Lichtenberg, Magaz. f. d. Neuefte etc. II. x.p. I85. Schwed. Abhandl. XL. p. 29r. m. e. Fig. Erxleben, Manmalia, p. 252. Rhinoceros (Bicornis) cornibus duobùs. 
Linné, S. Nat. Edit. VI. p. II. n. 3. Rhinoceros cornibus duobus cuneiformibus.

Linné, Syft. Nat. Edit. X. I. p. 56. n. 2. Rhinoceros (Bicornis) cornibus duobus cuneiformibus.

Limne', Muf. Ad. Fried. I. p. Ir. Rhinoceros bicornis.

Gatterer, brev. Zool. I. p. 54. n. 2. Rhinoceros (Bicornis) cornubus duobus; das zweyhörnige Naferiorn.

Ludolf, hift. Aethiop. Lib. I. c. 10. $\$ .78$. Nouvelle defcription du Cap. de b. E. Amf: I778. p. 37.

pennant, Synopf. quadruped. p. 75. n. 58. 4. the two-horned Rhinoceros.

Hiob. XXXVIII. 9. P Palm XXII. 22.

Verïnderungen gegen die XIIte Edition, and Ver: mehruing der Gätitungen diefes Gefchlechts.
Edit. XII.
Edit. XIII.

p. 104. Rhinoceros bicor- p. 57. n. 2. Rhinoceros nis $\beta$.

bicornis.

In der XII. Edit. fand das Rhinoceros unter den Belluis.

Diefs Gefchlecht if mit einer Gattung vermehrt, welche vorher als eine Varietät betrachtet wurde.

§. §. Das Nafehorn wird zwölf Fufs lang, und zwifchen fechs und fieben Fufs hoch. Das Horn if faferichter dichter Subftanz, und hat eine Länge von anderthalb bis zwey Fufs. Die Vorderzähne fcheinen bey der erften Gattung zu variiren, denn bey einigen fand man gar keìne, bey andern hingegen in jeder Kinnlade zwey. Die zweyte Gattung, 
die von d $\mathrm{d}$ eriftern völlig verfchieden ift, ift bis $z u$ Sparrmanns Entdeckung faft verkannt. Die Begattung des zweyhörnigen Nafehorns von hinten leug. net Sparrmann ganz, und zieht fie auch bey dem einhörnigen in $\mathrm{Z}$ weifel. Die Haut auf dem Rücken ift wenigitens anderthalb Zoll dick. Es werden Sp tzierföcke und Spiefsruthen davon gemacht, und das Horn verarbeitet man in Indien zu allerley Kun?. werken. Einige Völker effen auch das Fleifch.

5. GESCHLECHT. Elephas. Der Elephant.

I. M A x I m us. Der Elephant. (Faft in allen Sprachẹn.)

Niiller, Naturfyftem, I. p. 158. der Elephant. Tab. 29. Fig. 5 .

Zimmermann, geogr. Zool. II. p. 56. p. 155. 11.68. der' Elephant.

Schreber, Säugthiere, I. p. 24I. der Elefant. Tab. 78 .

Le $\int k e$, Naturgefch. p. 127. der Elefant.

Borowsky, Thierreich, I. p. 68. der Elephant.

Bhimenbach, Handb. d. Naturgefch. p. 120: der Elephant.

Bechftein, Naturgefch. Deutfchl. I. p. I39. 11. 5. der Elephant.

Funke, Naturgefch. I. p. I40. der Elephant. Halle, vierf. p. 374. der Elephant.

Meyer, Thiere, I. Tab. 69. der Elephant. Ab. bild. gut.

Gatterer, vom Nutzen u. Schaden der Thiere, p. 67. n. 60. der Elephant.

Neuer Schauplatz der Natur, I. p. 570. der Elephant. 
Onomat. hift. natur. I. p. 741. der Elephant. Klein, Quadrup. difp. p. 36. Elephas. Klein, Claflf. d. vierf. Th. p. rog. Elephant. Klein, natürl. Ordn, d. vierfüls. Thiere, p. 40.' Elephant.

Gesner, Thierbuch, p. 178. Helfant, Fig. p. 179. 180. und fchlechit p. 185 .

Biiffon, vierf. VIII. p. II4. der Elephant, mit 2 Fig. Männchen und Weibchen.

Biiffon, allgemeińe Naturgefchichte V. p. $5 \%$. VI. 148. 304. VII. 171.

Sparrmann, Reife nach dem Vorgeb. d. g. Hoffin.

p. 283. der Elephant.

$V$ aillant, Reife in das Innere von Afrika, I, p. I84. IJert, Reife nach Guinea, p. 64. 165.

Ives, Reife nach Indien, I. p. 103.

Adanfon, Reife nach Senegall, p. 73. IIO.

Du Halde, Befchreib. von China, III. p. 5 Ir. Auszïge a. d. Tagebuche eines Reif, nach Afien; - p. IIO.

Wolf, Reile nach Zeilan, p. 105.

Kolbe, Vorgeb. p. I48. Elephant, Tab. 4. Fig. 2. fehlecht.

Bofsmann, Reife nach Guinea, p. 284. Elephant. Röner, Nachrichten von Guinea, p. 280. Haid, of indifcher Schauplatz, p. 212. 281. Elephanten. Tab. 94.

Knox, ceylan. Reifebefchr. p. 4r. Elephanten. Thevet, Befchreib. der Morgenländer, 1554. p.70. Philipps Reife nach dem Orient, p. 36it. Perranlt, Charras und Dodart, Abh. a.d. N. G. II. p. $=275$. anatomifche Befchreibung eines Elephanten, Tab. 79 bis 84 .

Samml. zur Phyf. und Naturgefch. I. p. 340. Bonnet, Betracht. über die Natur, II. p. 5 I8. 
Campers kleinere Schriften, I. p. 50. die Zerglie: derung eines jungen Elephanten.

Zimmermann, Abbild. eines ungebornen Elephanten', nebft verfchiedenen bisher ungedruckten Nachrichten, die Naturgefch. des Elephanten betreffend. Erlangen I783. gr. 4 .

Bunzlauer Unterhaltungen, II. p. 26 .

Lichtenberg, Magazin für das Neuefte etc. I. I. p. 187. (ceylonifcher ungeborner, gefleckter.)

Naturgefch. des Elephanten; Wien I776. 8.

Sprengel, Tafchenbuch für r782. Zufätze p. XXIX. Nenhoff, Gefandfchaft etc. p. 349:: Elephant. Figur gut.

Abhandl. d. Gefellfch. in Batavia, I. p. 34I. weifse Elephant.

Choify, Tagebuch der Reife nach Siam." Par. 1687. p. 242.

Beyträge zur Länder und Völkerkunde, I. p. 69. Erxleben, Mammal. p. 203. Elephas Maximus. Linné, S. N. Edit.' II. p. 48. Elephas nafo cylindraceo elongato.

Limné, S. Nat. Edit. VI. p. Ir. n. I. Elephas. Linne', Muf. Adolph. Fried. I. p. Ir. Elephas indicus.

Linné, S. N. Edit. X. I. p. 33. n. I. Elephas (Maximus.)

Limné, Muf. Adolph. Fried. II. p. 6. Elephas (Maximus).

Gratimann, intr. in hiftor. nat. mamm. p. 4r. I. 、 Elephant.

Gatterer, brev. Zoolog. I. p. 55. n. I. Elephas (Maximus) pedibus pentadactylis; der Elephant. Schwenkfeld, theriotroph. Silef. p. 87. Elephas. Boullaye, Voyage, p. 250. Elephans. Marmol Afrique, I. p. 58. L' Elephant. 
Charleton, exercitat. p. 5. Elephas.

Ludolf, hift. Aethiop. I. c. Io. n. Is fq. Elephantus, fig. p. 147.

Leon. Africani defcriptio Africae, Lugd. Bat. 1632. p. 744 .

Nieremberg, hift. nat. p. 191. Elephas.

Gronov. Zoophyl. I. p. 2. n. 6. Elephas.

Pennant, Syn. quadrup. p. 85. n. 62. the great

Elephant.

Hermann; Tab. affinit. animal. p. 94.

Philof. Transact. num. 326. p. 51. m. Figg.

Ariftoteles, hift. animal. I. c. 9. n. 93-- II. c. 2.
n. 3. 4. c. 3. n. 6 . c. 4.11 .8 . c. 5. n. 24 .

c. 8. n. 38 . c. 9. n 46.5 I. c. II. n. 79.80.

c. I8. n. 133. c. 19. n. 155 . III. c. 1. n. 7 .

c. 9. n. II8. IV. c. 9. n. 293 . V. c. 2. n. 24.

c. 13. n. 140. VI. c. 27. n. 359 . VIII. c. 12.

n. II6. II7. IX.c. 72. n. 490-495. (nach der Ausg. von Iul. Caef. Scal. Toulouf. I6I9. Fol.) $\mathrm{E} \lambda \varepsilon \varphi$ ass.

Plinizus, hif. nat. VIII. c. I-r4. Elephas, Elephantus, Luca Ros.

Smellie, Philof. der Naturgefch. (von Zimmer. mann) Berlin I79I. I. p. 85.

Hartenfels; Elephantographia curiofa f. Elephanti defcriptio. Erf. I7IO. c. fig.

Phil. Okeimii Elephas. Lipf. 1652. 4.

Normanni Elephas breviter delineatus. Upfal. 1693. 8.

Neandri et Zobereri diff. de Elephante. Ien. I571. 8 .

Praetorii hiftoria Elephanti. Hamb. 1607. 8. Gilii defcript. nova Elephanti. Hamb. 16r4. 8. Hornii Elephas. Norimb. 1629. 8.

Gefchichte des Elephanten. Berlin 1777.8. 
Burchardi diff. de Elephante. Altorf. 1696. 4. Pallas, Reife Ausz. II. p. I85. Elephantenknochen am Tura. p. 199. mit Seekörpern begleitet. III. p. 253. am nördlichen Ienifey. p. 3r4. bey Krasnojarsk. II. p. 5. Elephanten Schenkelknochen in dem Ufer der Belaja.

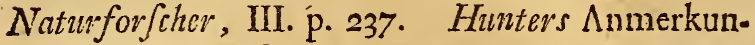
gen über die fogenannten Elephantenknochen, welche am Ohihofrom in Amerika gefundeh wurden; vergl. mit Zimmemamn, geogr. Zool. II. p. 157 .

Naturjorfcher, XX.p. Igs. Elephantenknochen im Mansifeldifchen gefunden.

Stralfundifches Magazin, I. p. I. von dén in Nordamerika entdeckten Elephantenähnlichen Gerippen.

Gmelin, Reife durch Rufsland, I. p. 34. von Ele. phantenknochen in Sibirien.

Walch, Naturgefch. der Verfteinerungen, II Th. II Ablchn. p. I64. ob die Elephanten ehemals in den nördl. Ländern einheimifch gewefen.

Hamb. Magazin, I, p. 453. von einem in der Erde gelegenen aufferordentlich grofsen Elephantenzahne.

Schriften der Berl. Gef. nat. Fr. VII. p. 374. befondere Merkwürdigkeit an einem ElephantenBackzahn.

Novi Comment. Göttingenf. I. 2. p. 96. (die Kunftgefchichte des Elfenbeins, und vornehmlich deffen Bearbeitung bey den Alten betreffend.)

Neue gefellfchaftl. Erzähl. I. p. II. von einem Elephantenzahne, in welchem eine kleine metallene Kugel eingelchloften gewefen. 
5. S. Unter den Elephanten giebt es fowohl in Anfehung der Farbe als der Gröfse beträchtliche Verfchiedenheiten. Die kleinften halten ausgewachfen über neun Fufs Höhe, und zwölf Ellen Länge. Oft erreichen fie eine Höhe von funfzehn bis achtzehn Fufs. Der Rüffel ift das Hauptorgan der Elephanten, der ihm zum Athemholen; zum äufserf feinen Geruch, zum Brïllen, zum Wafferfchöpfen, fein Futter damit zu faffen, und es, ins Maul zu fecken, zum Gefechte, und zu allerley künflichen. Verrichtungen ftatt der Hände dient. Er kann ihn drey Ellen ausfrecken, und bis auf eine Fille einziehen. Er kann damit Knoten aufknüpfen, Schnal. len auflöfen $u . \mathrm{dgl} . \mathrm{m}$. Das neugeworfene Iunge foll die Gröfse eines wilden Schweines haben. Die beyden grofsen Eckzähne. werden fieben bis ache Fufs lang, und man hat einzeln folche Zähne gefehen, die auf 160 Pfund wogen. Die kleinften Zähne eines ausgewachfenen Elephanten betragen zwifchen 60 und 70 Pfund. Das Alter des Elephanten erfreckt fich wahrfcheinlich über 200 Iahre. Die Laf, dic ein Elephant tragen Kann, wird auf 1500 bis 2000 Pfund gefchätzt; und was er mit slem Rüffel aufhebt, 200 Pfund. In einem Tage kann' er zehn und mehrere Meilen zurücklegen. , Seit den Zeiten des trojanifchen Kriegs hat man das Elfenbein zu allerley Kunftwerken angewandt. Das Fleifch des Thiers foll fchmackhaft feyn, und dem Rindfleifche gleichen. Sein getrockneter Miff wird auf Zeilan fatt Kohlen gebrannt, und auch von den Töpfern unter den Thon gemengt. - Auffer den gewöhnlichen grauen Elephanten hat man auch dunklere und zuweilen weifse. Die gefleckten oder fcheckigen find die felteniten. 


\section{I24 Mammalia Bruta. Trichecus.}

6. GESCHLECHT. TR I C E C US. Das Wallrofs.

Miiller, Naturfyft. I. p. I7I. Gen., VI.

Zimmermann, geogr. Zool. I. p. 299. II. p. 424.

Gen. XLIII.

Schreber, Säugth. II. p. 259. Gen. XI.

Lefke, Naturgefch. p. I28. Gen. XI.

Borowsky, Thierreich, I. p. 79. Gen. X.

Blumenbach, Handb. d. N. G. p. 128. Gen. XLIV.

Funke, Naturgefch. I. p. I6r.

Erxieben, Mainmalia, p. 593. Gen. XLVII.

Graimann, intr. in hifor. natur. mamm. p. 87 .

- Gen. XLVII.

Gatterer, brev. Zool. I. p. I59. Gen. XLVII.

Pennant, Syn. quadr. p. 335. Gen. XL.

BrifJon, quadr. p. 30. Gen. VI. Odobenus.

Hermainn, Tab. affinit. anim. p. I27.

1. Rosmarus. Das Wallross. ( ${ }^{\mathrm{I}}$ )

Miiller; Naturfyft. I. p. I7I. n. I. das Wallrofs, Tab. 29. fig. 2. Abbild. Jchlecht.

Ziwmernann, geogr. Zoolog. II. p. 424. n. 385 . das Wallrofs.

Leeke, Naturgefch. p. 129. das gemeine Wallross. Boroweky, Thierreich, I. p. 79. das Wallross. Tab. X.

Blumen-

(1) Diefe Thiere nähren fich von Seetang und Schaalthieren, dic fie mit ihren grofsen Hauzahnen loskratzen. Sie leben in Gefelffchaft und vertheidigen einander fehr beherzt. Das rothe Fleifch wird im Kochen weifs, wird aber nicht geachtet. Man fingt fie blofs um der Zähne willen, und zwar nur gelegentlich. lung find fie fchwarz, nach und nach werden fie blaffer, und im Alter weifs. Die Grönländer fchärften ihre.Harpunen mit den Zähnen. Die lungen haben keine Zähne. Das Zeugeglied if cin ellenlanger Knochen, und wird zu Mefferfchalen und andern Dingen verarbeitet. 
Blumenbach, Handb. d. N. G. p. 129. n. I. Trichecus (Rosmarus) dentibus laniariis fuperio. ribus exfertis longioribus; das Wallvo $\int s$.

Bechftein, Naturgefch. Deutfchl. I. p. 134. das Wallrofs.

Finnke, Naturgefch. I. p. 16r. das Wallross. Ebert, Naturlehre, I. p. 383. das Wallrofs. Halle, vierf. p. 583. das Wallross.

Gatterer, vom, Nutzen und Schaden der Thiere,

I. p. 407. n. 229. Wallrofs.

Neuer Schauplatz der Natur, IX. p. 225. Valrus, Wallrofs, Rofsmaul, Roftunger.

Onomatol. hift. nat. VII. p. 546. Trichecus Rofmarus, V. p. 662. Odobenus. Wallrofs, Walrus, Meerochs, Meerpferd.

Klein, Quadr. difp. p. 92. Rofmarus.

Klein, Claflif. d. vierf. Th. p. 278 . das Wallrofs; Robbe, mit hercaushiongenden Hunds. zähnen.

Klein, natürl. Ordn. d. vierfüfs. Th. p. 99. III. Wallross.

Schneider, Abhandl. a. d. Zoologie, p. 254. das Wallross, Morfe, Seekuh, Seepferd, IMeer. ochse.

Schlozer, Erdbefchreib. von America, p. 36. See. kuh, Morfe.

Phipps Reife nach dem Nordpol, p. 95. das Wallrofs.

Cranz, Hift von Grönland, p. I65. das Wallrofs. Egede, Befchr. von Grönland, p. ro6. Tab. 6. Meerpferd, Wallrofs.

Anderfon, Nachr. von Island, p. 230. 258. das Wallvo/s.

Steller, Befchreibung von Kamtfchatka, p. זos. Wallrộs. 
Kralchemminikow, Befchr. von Kamtfchatka, p. I47. Seepferd.

Forfter, Gefch. der Schiffarth im Norden, p. 88. Wallrofe.

Villeneuve, Hamburg. Magazin, XXIV. p. 598. Seepfera,

Olaffen, Reife durch Island, I. p. I\&9. Roftun. ger , oder Rofsmer.

Leem, Nachr. von den Lappen, p. II6. Wall. rolfe, Seepferde.

Pontoppidan, Norwegen, II. p. 299. Valrus, Rosmul, Rojtunger, Hav-Heft.

Pennant, arct. Zoologie, II. p. I4I. 1. 90. das Wallross.

I. G. Gmelin, Reife durch Sibirien, III. p. I65. Morfch.

Erxleben, Mammalia, p. 593.' n. I. Trichecus (Rostnarus) dentibus laniariis fuperioribus exfertis longioribus.

Limné, S. N. Edit. II. p. 44. Hhoca dentibus canimis exfertis.

Linné, S. N. Ed. VI. p. 6. n. 2. Phoca dentibus is caninis exfertis.

Limmé, S. N. Edit. X. I. p. 38. n. 3. Phoca (Rosmarus) dentibus lániariis fuperioribus exfertis. Linné, S. Nat. Edit. XII. I. p. 49. n. I. Triche. cus (Rosmarus) dentibus laniaris fuperioribus exfertis.

Granmann, intr. in hiltor. nat. mamm. p. 87. I. Wallross.

Gatterer, brev. Zoolog. I. p. 159. n. 1. Trichecus (Rosmatus) dentibus laniariis fuperioribus exfertis longioribus; das Wallro $\int_{s}$.

Ellis, Reife nach Hudfons Meerbufen, Tab. 7. ein Seepferd. Abbild. fchlecht 
Sibbald, Scotia illuftrat. II. p. to. Walrus feu Mors.

Mitller, Samml. ruf.Gefch. III. p.563. Wallrofs. Olaus Magn. de gent. Septentrional. p. 757. Rosmarus feu Morfus norvagicus; m. e. fchlechten Figur.

Gesner, Fifchbuch, p. 127. Rulfor oder Roftinger; mit Ol. Fig.

Miillev, Zool. dan. prodrom. p. r. n. r. Trichecus (Rosmarus) dentibus laniariis fuperioribus exfertis.

Fabric. Faun. Groenland. p. 4. n. 2. Trichecus (Rosmains) dentibus laniariis fuperioribus exfertis.

Pennant, Syn. quadruped. p. $335 . \mathrm{n}, 263$. the arctic Walrus.

Laet, nov. orb. p. 38. Animal, quod nofrates Walrus, Angli a Ruffis mutuato nomine Morsh vulgo nominant, m. e. guten Fig.

Charleton, exercitat. pifc. p. 49. Walrus. Denys, defcript. de l'Amerique Septent. I. p. 66. Charlevoix, nouv. Fr. III. p. I47. Vache Marine,

3. Dugong. Der Digung. ( $\left.{ }^{2}\right)$

Miiller, Naturfyft. Suppl. p. 2I. Trichecus Dugong; der Duging.

Zimmermann, geogr. Zoolog. II. p. 425. n. 386. der Dugung.

Sithreber, Säugth. II. p. 26\% n. \&. der Dugung. Gatterer, vom Nutzen u. Schaden der Thiere,

I. p. 4I4. n. 23r. das indianif che Wallio/s. Schneider, Abhandl. a. d. Zoologie. p. 256. der Dugon.

Barchoo

(2) Lange des Thiers über fechs Ellen. 
Barchewitz, oftind. Reifebefchreib. p. 381. der Dugong.

Leguat, Voyages, I. p. 36. m. e. fchlechten Fig. Erxleben, Mammalia, p. 599. n. 3. Trichecus (Dugung) dentibus laniariis fuperioribus exfertis brevioribus.

Graumann, intr. in hiffor. nat. mamm. p. 87. II. Seckuh.

Gaitterer, brev. Zool. I. p. 160. n. 2. Trichecus (Manatus) dentibus laniariis inclufis; die Seekuh.

2. Manatus. Der Manati. (3)

a. Der kleinere Manati (diffeits Amerika).

Zimmermann, geogr. Zoolog. II. p. 426. n. 388. der Kleinere Manati.

Schreber, Säugthiere, II. p. 269. der Manati.

B. Der Manati von Kamtfchatka (jenfeits Amerika). Zimmermann, geogr. Zoolog. II. p. 426 . n. 387. Manati Gigas, der Manati von Kamtfchatka. Schreber, Säugthiere, II. p. 274 .

Steller, nov. Comment. Petropol. II. p. 294. Pennant, arct. Zool. II. p. I7I. der Wallffich. fihwanz.

Siellers

(3) Zimmermann hat zueft die beyden Arten des Manati getrennt, und er macht aus ihnen ein eigenes vom Wallrofs verfchiedenes Gefchlecht. Die Nahrung des Manati befteht in Seegewiehfen. Das Fleifch ift blafsroth, und fehr wohlichmeckend. Es wird gebraten, eingefalzen und geräuchert gegeffen. Das Fett if weifs, und zwey bis drey Finger dick. Infonderheic wird der Schwanz eines jungen Thiers als ein Leckerbifen betrachtet. Die Lapides Manati, oder Seskubfteine, find nicht, wie Miillar. glaubt, hornartige Ballen aus dem Gehirn diefer Thiere, fondetn Knochen aus dem Kopfe des Pottiffches. S. Becknamn phyí. skon. Bibl. XIIl. p. $4 j \sigma$. Camper kleinere Schriften, UI. 1. p. 19 . Stabs, diffo de lapide Manati. Hal. 1699. 40 
Stellers Befchreib. des Manati, oder der fogenannten Seekuh, in Hamburg. Magazin, XI. p. 132 .

\section{$* \quad *$}

Miiller, Naturfy fem, I. p. 176. n'. 2. die Sbekuh, Tab. 29. fig. 3. Cluf.

Lefke, Naturgefch. p. 129. n. 2. der Manati.

Borowfky, Thierreich, I. p. 83. Seekuh, Manati. Bhumenbach, Handbuch der Naturgefch. p. I29.

Trichecus (Manatus) dentibus laniariis inclufis; die Seekuh.

Funke, Naturgefch. I. p. 162. die Seeknh.

Gatterer, vom Nutzen und Schaden der Thiere;

I. p. 4II. n. 230. die Seekuh, der IVteerochs, Manati.

Neuer Schauplatz der Natur, V. p. 17. Lamantin.

p. 341. Manatus, Matrulfa, Meerkih.

Onomatol. hiftor. nat. VII. p. 546. Trichecus Manatus. V. p. 32. Manatus, Manati, Seekuh, Seeochs.

Klein, Quadrup. difp. p. 94. Manatus, Seekuh, Seeochs.

Klein, Claffif. d. vierf. Th. p. 282. Seekuh, Seeochs. Klein, natürl. Ordn. der vierfüfs. Th. p. Ior. V. Seekinh, Seeochs.

Schneider, Abl. a. d. Zoologie, p. 257. der Ma. "nati oder die Seekuh.

Steller, Befchreib. von Kamtfchatka, p. 97. die Seekinh.

Adanfon, Reifẹ nach Senegall, p. 213. Sekuh. Bainkroft, N. G., von Guiana, p. I12. der. Manati, oder die Meerkuh.

Steller, Befchreib. von fonderbaren Meerthieren, p. 48. Meerkuh. 
Krafcheninnikow, Befchr. von Kamtfchatka, p. 53. 163. 165. 166. Seekilhe oder Manati.

Dapper, Amerika, p. I79. der Fifch IManati, m. e. fchl. Fig.

Le Gertil, Reifen in den indifchen Meeren, II. p. 48. die Seekuh.

Hamburg. Magazin, IV. p. 206. die Manatee oder Seekuh.

Hamb. Magaz. VI. p. 251. Seekuh oder Fifchochfe. Miïler, Sammlung ruif. Gefch. III. p. 251. die Seekuh.

Biifching, wöchentl. Nachr. II Iahrg. p. 69.

Gilii Nachr. von dem Lande, Guiana, p. 55. Manuasi, Seekuh.

Erxlebei., Mammalia, p. 596. n. 2. Trichecus (Manatus) dentibus laniariis inclufis.

Linné, Syft. Nat. Edit. II. p. 59. Trichecus. Manatus.

Limne', Syft. Nat. Edit. VI. p. 39. n. I. Trichecus Manatus.

Linné, Syf. Nat. Edit. X. I. p. 34, n. I. Trichecus (Manatus).

Linné, S. Nat. Edit. XII. I. p. 49. n. 2. Trichecus (Manatus) dentibus laniariis inclufis.

Grammann, intr. in hiftor. nat. mamm. p. 87. II. Seekuh.

Gatterer, brev. Zool. I. p. 160. n. 2. 'Trichecus (Manatus) dentibus Janiariis inclufis; dis Seekuh.

Milller, prodrom. Zool. dan. p. I. n. 2. Trichecus (Manatus) dentibus laniariis inclufis.

Fabric. fauna groenland.' p. 6. n. 3. Trichecus (Manatus) dentibus laniariis inclufis. Ionfton, pifc. p. 223. Marati Indorum. Charleton, exercitat. pifc. p. 49. Manati. 


\section{Mammalia Bruta. Trichecus,}

Laet, nov. orb. p. 6. Manati. Fig. Chuf.

Aldrovand. pifc. p. 728. Manati Indorum. p. 729.

Fig. Chis.

Pennownt, Syn. quadr. p. 352. n. 273, the Manati. Philof. Transad. LXV. p. 249.

De la Caille, voy. au Cap d. b. E. p. 229. le La mantin.

Condamine, voy* amer. p. I54. . Lamantin ou poifion boeuf.

Binet, voy. en Cayenne, p. 346.

Nouv. voy. aux Iles de l'Amer. Par. I722. p. Ioo. Dompier, voy.I. p. 46. Manates, ou vaches marines. Oexmelin, hift. des avant. II. p. 215.

A) Verändernengen gégen die XIIte Edition, und Ver. mehrung der Gattungen diefes Gefchlechts.

Diefs Gefchlecht ift mit einer Gattung vermehrt, und T. Dugung hinzugekommen.

B) Unbeftinmtere Thiere.

1. Der Seeaffe.

Zimmermann, geogr. Zool. II. p. 427: a:

Pennenst, Synopf. quadr. p. 356.

Erxleben, Manimalia, p. 600.

Pennant, arct. Zoologie, II. p. 155 .

Schreber, Säugthiere, I. p. 306.

2. Die Sirene.

Zimmermann, geogr. Zool. II. p. 427. b:

Erxleben, Mammalia, p. 600 . 
$D R T T T E$ ORDNUNG.

F E R A E. $\quad\left(R a u b t h i e r e_{0}\right)(4)$

II. GESCH LECH T. PносA. Der Robbe. (Seehund, Sällhund, Meerkalb.)

Miiller, Naturfyftem, I. p. 194. Gen. XI.

Zinnmermann, geogr. Zool. II. p. 419. Gen. XLI. Schreber, Säugthicre, III. p. 285: Gen. XII. Leeke, Naturgefch. p. I30. Gen. XII. Borow fky, Therreich, I. 2. p. 2. Gen. XI. Blumenbach, Handb. d. N. G. p. I26. Gen. XLIII. Pennant, arct. Zool. p. 147.

Bilffon, visrf. XVI. p. 77 .

Olaffen, Reife durch Island, I. p. 28r.

Erxleben, Mammalia, p. 579. Gen. XLVI.

Granmannu, intr. in hiftor. natur. mamm. p. 85. Gen. XLVI.

Gatterer, brev. Zoolog. I. p. I55. Gen. XLVI. p ennant, Syn. Quadr. p. 330. Gen. XLI. Brifon, Quadr. p. I62. Gen. XXXIII. Hermann, tab. affinit. animal. p. I23.

1. Ursina. Der Sebü̈r. (5)

Miiller, Naturfyf. I. p. 195. n. I. der Seebär.

Zimmer.

(4) Die Thicte diefer Ordnung find zwar nicht alle reiffend, und dem Menffchen oder grǘfsern Thieren furchtbar, fie ernähren fich aber doch faft alle von andern Thieren, und bemächtigen fich derfelben durch Gewalr oder Lift, daher fie Raubtbiere oder reifende Thiere genannt werder.

(5) Länge des Mănnchens neun Fufs, das Weibchen bleibe kleiner. Diefe Tiniere künnen in einer Stunde faft zwey deutfche Mei'en zurücklegen. Im Sommer finden fie fich heerdenweife 
Zimmermann, geogr. Zoolog. II. p. 419. n. 375. die Bürenrobbe, der Seebär, I. ṕ. 25 I. Le $\int k e$, Naturgefeliichte, I. p. I3I. n. I. der Seebär. Borowfky, Thierreich, I. 2. p. 2. der Sebbär. Blumenbach, Handb. der N. G. p. I28. n. 2. Phoca (Urfina) auriculata, collo laevi; der Seebär. Funke, Naturgefch. I. p. Ior. der Seebiir:. Halle, vierf. p. 585 . der Seebür.

Gatterer, vom Nutzen und Schaden der Thiere,

I. p. 384. n. 220. der Seebïr.

Beckmann, Naturhift. p.34. n. 3. der Seeboir.

Onomatol. hiffor. natur. VII. p. 663. Urfus marinus; der Seebörr.

Klein, natürl. Ordn. der vierf. Th. p. I03. der Seebär.

Pennant, arct. Zool. II. p. I6r. der Seebiir. Biiffon, vierf. XVI. F. 233. der Seebïr oder Bärenrobbe.

Steller, Befchreib, voin Kamt fchatka, p. 97. der Seebär.

Steller,

auf den In eln des kamtrchatkifchen Infelmeers, von da fie fich im Herbft wieder entferner. Ihr Winteraufenthalt if noch unbekannt; vermuthlich bringen fic denfelben auf den benachbarten, etwas füdlichern, Infeln des ftillen Meeres zu. Thre Stimme gleicht in der Ruhe dem Blöcken einer Kuh, beym Streit dem Brummen eines Bärs, nach einem Siege dem Girron einer Heime, und wenn fie den Kürzern gezogen, dem Zifchen einer Katze oder Meerotter. Die Fetthant if am Leibe an vier Zoll dick. Das Fett und Fleifch der alten Thiere erregt Ecke1 und Erbrechen; der Weibchen ihres hingegen felumeclit faft wie Lammfleifch. Die Iungen haben, gebraten, faft den Gefchmack der Spanferkel. Die Felle werden zum Befchlagen der Koffer gebraucht: die Kamtfchadalon befohlen damit ihre hölzernen Schneefchuhe, fo, dafs die Háare hinterwärts ftehen; die fibiri. fchen Bauern faffen ihre Pelzröcke damit eir ; die Fielle der aus der Mutter gefchnittenen lungen, werden unter die Kłeider gefuttert und theuer bezahlt. 
Steller, Befchr. von fonderb. Meerthieren, p. roz. der Mleerbär.

Steller, Befchr. des Seethiers, das Dampier zum erften Male gefehen, und unter dem Namen des Seebärs befchrieben hat; im Hamb. Magazin XI. p. 264. m. e, Fig.

Kra/cheninnikow, Befchr. von Kamtfchatka, p. 154. Seebär.

Forfter, Bemerk. auf feiner Reife um die Welt, p. 16\% der Seebuir.

Miiller, Samml. ruf. Gefch. III. p. 249. Seekat́zer.

Erxleben, Mammalia, p. 579. n. I. Phoca (Urfina) capite auriculato.

Linné, Syn. Nat. Edit. X. I. p.37. n. I. Phoca (Urfina) capite auriculato.

Graumann, intr. in hiftor. nat. mamm. p. 86. I. der Seebür.

Gatterer, brev. Zool. I. p. I55..n. I. Phoca (Urfina) capite auriculato; der Seebär.

Fabric. Faun. Groentand. p. 6. n. 4. Phoca (Urfina) capite auriculato.

'Pennant, Syn. quadruped. p. 344 . n. 27 r. the urfine Seal.

2. LEONINA. Der glatte Seelörve. $\left({ }^{6}\right)$

Miiller, Naturfyft. I. p. I97. 11. 2. der Selöwe.

Zinnmer.

(ต) Länge oft gegen zwanzig Fufs, doch find die Weibchen kleiner, und auch nicht mit dem Kamme verfeher, den die Mànnchen haben. Die Nahrung diefer 'Thiere befteht in Gras, Fifchen und andern Thieren. Ihr Rachen ift fo grofs, dafs eine Kugel von einem Fufs. im Durchfchnitt bequem hineingeht. Ihre Stimme ift, wie des vorigen, nach verfchiedenen Umftänden verfehieden. Sie find fo fett, dafs die Haut hin und her fehwan- 
Zimmermann, geogr. Zool. II. p. 420. n. 376. der glatte Seelöwe, I. p. 289.

Lefke, Naturgefch. p. I32. n. 2. der glatte Seclözoe. Borowfky, Thierreich, I. 2. p. 5. der glatte Seelöwe.

Gatterer, vom Nutzen u. Schaden der Thiere,

I. p. 387. n. 221. der Seclöwe.

Büffon, vierf. XVI. p. 125. der grofse Robbe mit runzelichter Schnouze, m. a Figg.

Schlözer, Erdbefchreibung von Amerika, p. 952. der Seelöwe.

Byron, Reife un die relt, p. I77. der Seelöwe. IMolina, Naturgefch. von Chili, p. 248. Phoca Elephantina, der Lamé.

Hawkesworth, Gefch. d. Entdeck. in der Südfee, II. p. 60 . und 382 .

Forfter, Bemerk. auf feiner Reife um die Welt, p. 167. Seélörve.

Erxleben, Mammal. p. 5ơn. n. 2. Phoca (Leonina) capite antice criftato, corpore fufco.

Limné. Syft. Nat. Edit. … I. p. 37. n. 2. Phoca (Leonina) capite antice criftato.

Iinné, Synt. Nat. Edit. XII. x. p. 55. n. 2. Phoca (Leonina) capite antice criftato.

Graumann, intr. in hifor. nat. mamm. p. 86. II. der glatte Seelözee.

Gatterer, brev. Zooleg. I. p. 155. 13. 2. Phoca (Leonina) capite antice criftato, corpore fufco; der Seelöreve.

Pennant, Syn. quadrup. p. 348. n. 272. the leonine Seal.

$$
14 \text { Danizier. }
$$

ket, wenn fie fich bewegen. Der Thran wird am Feuer, oder an der Sonne ausgelaffen, und ift frifch efsbar. Das Fleifch ift grob. Die Häute können zu Ueberzügen der Reifefäcke und Keffer, nad gejerbt zu Schuhmacherarbeit gebraucht werden. 
Dampier, Voy. I. p. Ir8. Le Lion marin.

Dampier, Voy. II. p. 268. Le Manate de Sainte Helene.

Parfon, philof. Transact. XILVII. p. 12r. Phoca maior, dentibus caninis tectis, palmis anterioribus digitatis, ungülatis, ponterioribus latis bifidis.

Rogger, Voy. autour du monde, I. p. $20 \% .223$.

4. Iu вата. Der zottige Selörve. (7)

Miiller, Naturfyn. Suppl. p. 25. n. 2. a. der Seelöre?

Zimmermann, geogr. Zoolog. II. p. 420. n. 377. der zottige Seelöive.

Lefke, Naturgefch. p. I32. n. 3. der zottige Seelöwe.

Borowjky, Thierreich, I.2. p. 6. n. 3. der zottige Seelöree.

Blumenibach, Handb. d. Naturgefch. p. I28. n. 3. Phoca (Leonina) capite auriculato, collo iuba. to; der Seelöwe.

Funke, Naturgefch. I. p. I6r. der Seslöwe.

Halle,

(y) Unterfcheidet fich von dem vorhergehenden befonders durch das grolse mähnenahnliche Nackenbaar des Mannes. Sie leben in der Polygamie. Die Weibchen werfen ein Iunges aut dem feften Lande; die Zeit ihres Trächtiggehens dauert wahrfcheinlich tuber neun Monat. Die Männchen ftreiten, wie die Seebüre, um den Platz und um die Weiber. Dem Menfchen weichén fie, wenn fiekönnen, fchon von ferne aus, werden fie aber in die Enge getrieben, fo vertheidigen fie fich mit äuferfter Wuth und grofsem Gebrülle. Ihre Nahrung find Fifche, Seeottern, Seehuide und andere Seethiere, Die Kamtíchadalen effen das Fleifch und das Fett diefer Thiere; aus der Haut verfertigen fie Schuhe und Stiefeln, auch Sohlen and Rienen; die Gedärme liefern den Einwohnern der Infeln hinter KamtÍchatka Oberkleider; andere Wilde nähen mit den Sehnen. 
Halle, vierf. p. 593. der Seelöwe.

Gatterer, vom Nutzen u. Schaden der Thiere,

I. p. 388. der zottige Selöwe.

Biiffon, vierf. XVI. p. 269. der Seelöwe, m.e. Fig. Onomat. hif. nat. IV. p. 785. Leo marinus; der Seelörve.

Peinant, arct. Zool. II. p. 167. n. 99. der zottige Seelöwe.

Steller, Befchr.v. Kamtfchatka, p. 97. der Seelöwe. Krafcheninnikow, Befchr. von Kamifchatka, p. I49. Seelöwe.

Forfter, Bemerk. auf feiner Reife un die Welt, p. 168. der Robbe mit der Mïhne.

Hamburg. Magaz. XI. p. 45I. der Seelöree (ruff: Siwutfcha).

Biffching, wöchentl. Nachrichten, 2 Iahrg. p. 63. Miiller, Samml. ruff. Gefch. III. p. 250. die See. low:n.

Erxileben, Mammalia, p. 582. n. 3. Phoca (Iubata) capite fubauriculato, collo (maris) iubato. Graumann, intr. in hift. nat. mamm. p. 86. III. der zottige Selöwe.

Gatterer, brev. Zoolog. I. p. 5. n. 3. Phoca

(Iubata) capite fubauriculato, collo (maris) jubato; der zottige. Seebür.

Miiller, prodr. Zool. dan. p. VIII. Phoca (Leo. nina) capite antice criftato.

Hift. des navigations aux terres auftrales, T. I. p. 22I.

3. Vitudin A. Der gemeine Seehund. (9)

Muiller, Naturfyftem, I. p. 198. n. 3. der Robbe oder Seehund.

I 5

Miiller,

(9) Lànge gegen fechs Fufs, If für die finnifchen Infulaner, fo wie für die Kamtfchadalen, befonders aber für die Grönlander 
Iiniller, Naturfyftem, I. p. 20I. eine andere Art eines Seehundes, Tab. Ir. fig. 6. Houtt.

Zimmermann, geogr. Zool. II. p. 42I. n. 378. die gemeine Robbe.

Lejke, Naturgefch. p. I32. n. 4. der gemeine Seehund.

Borowsky, Thierreich, I. 2. p. 7. n. 4. gemeiner Seehund, Robbe, Seckalb.

Blumenturch, Handb. der Naturgefch. p. I27. n. r. Phoca (Vitulina) capite laevi, auriculis nullis, corpore grifeo. Seehund, Robbe, Seekalb. Bechftein, Naturgefch. Deutfchl. I. p. 137. der Sechund; p. 402. der genueine Seehund ;- p. 408 . das Meerkalb, Seekalb, 'der Robbe, gemeine Robbe, Roppe, Saalhurizd.

Funke, Naturgefch. I. p. r59. der Sefinund. Hcille, vief. p. 579. das Seekailb.

Gatterer, vom Nutzen und Schader der Thiere, I. p. 39I. n. 223. das MLeerkalb; der Seehund; Robbe.

\section{Bock,}

und Efquimaux cin âufserft wichtiges Gefchöpf: die beyden letzten Völser zumal tuhrer. fich vor feinen Fleifch, kleiden fich in fein Fcll, beziehen ihre Sommerhüten und Fishbote damit etc. Der Thran davon dient ihnen zur Unterhaltung ihrer Lampen; mit den Sehnen nahen fie; aus den Gedärmen machen fie ihre Feufter und Hemden, aus dem Magen die Schlauche, worin fie den Thran aufbewahren. Der Speck wird zúm Schmälzen der Speifen gebratzcht, wuch wié Schweinefett gegeffen, befonders aber Thran daraus ausgelaffen.: Ein reche fetter Scehund griebt fuafzig bis fechigig Pfund. Die Milch, die fehr fett und ghranigt ift, giebt gersinchert eine Fetrigkeit, die man in Island in den Lampen brennt. In Furopa werden bekanntlich die Seehundsfelle am meiften zu Kofierbeíchlägen verbraucht. Vormals wurde das Fleifch in Norwegen und England felbit auf den Tafeln der Vornehmen gefpeifet. Die Häute der grofsen find fo grofs als Pferdeháure, 
Bock, Naturgefch. von Preuffen, IV. p. 7. n. 4. der Robbe, oder Seehund.

Biiffon, vierfüls. XVI. p. I84. der gemeine Robbe, m. e. Fig.

Bïffon, vierf. XVI. p. I83. der Robbe KaJigiak? Onomatol. hift. nat. V. p. 449. Phoca vitulina, das Seekalb, Roppe, oder Seehund.

Klein, Quadrup. difp. p. 93. Phoca; Robbe. Klein, Claflif. d. vierf. Th. p. 279. Robbe. Klein, natürl. Ordn. d. vierfüls. Thiere, p. 100. n. IV. Robbe.

Pennant, arct. Zool. I. p. I47. n..91. der gemeins Seehund.

Fermin, Befchreib. von Surinam, II. p. 107. dev Seehund.

Anderfon, Nachr. von Island, p. 260. der Seekund. Miarteizs Spitzberg. Reifebefchr.p. 75. Seehuna', den suan anch Rubbe oder Salhund nennt. Tab. P. fig. a. fchlecht.

Fifcher, Naturgefch. von Livland, p. 5o. Seehund, Sälhund, Robbe.

Steller, Befchr. von Kamtfchatlia p. 108. die dritte Sorte.

Olaffen, Reife durch Island, p.31. etc, p. 282/294. Tab. 32. Land - Selur.

Gcorgi, rufi. Reife, I. p. I56. 158 .

Ellis, Reife nach HudCons. Ázerbufen, p. 139. Sechund.

Cranz, Hift. von Grönland. Lpz. I779. p. I50.

11. I. Kaflgiak?

Pontoppidan, Norwegen, II. p. 236. Robbe oder Säilhund.

Fabric. Reife.nach Norwegen, p. 292. Seehunde. Hawkesworth, Gefch. der Entdeck. in d. Sïdfee, II. p. 278. I. p. 249. 
Pervault, Charras und Dodart, Abandl. aus der Naturgefcb. I. p. 219. anatomifche Befchreibung eines Seekalbes, Tab. 28. 29.

Phipps Reife nach dem Nordpol, p. 96. der ge= meine Seehand.

Uno von Troil, Reife nach Island, p. 98. Seehinnd.

Schwed. Alhandl. XXXIII. p. 5r. Ertner. Knutberg, vom Seehundfange; Schwed. Abhandl. XVII. p. I27.

Knorr, delic. natur. II. Tab. H. 8. Seehund. Miiller, Samml. ruff. Gefch. III. p. 559. Sechunde. Snuellie; Philof. der Naturgefch. I. p. 84. der Seehund.

Fabric. Faun. Grönland. p. 9. n. 6. Phoca (Vitulina) capite laevi, cervice retufufcula, nafo mediocri, corpore fubcylindrico, vibriflis undulatis medulla concolori.

Hartmann, diff. de Phoca, feu vitulo marino. Regiomont. $16 \$ 3.4$.

Schellhamner, tr. de Phocae maris anatome. Kilon. 1699.4 .

Evxleben, Mammalia, p. 583. n. 4. Phoca (Vitulina) capite laevi inauriculato, corpore fufco.

Linné, Syft. N. Edit. II. p. 44. Phoca dentibus caninis inclufis.

Linne', Syft. Nat. Edit. VI. p. G. n. I. Phoca dentibus caninis tectis.

Limué, Syft. Nat. Edit. X. I. p. 38. n. 4. Phoca (Vitulina) dentibus laniariis tectis.

Linné, Syf. Nat. Edit. XII. p. 56. n. 3. Phoca (Vitulina) capite laevi inauriculato.

Linné, Reif. d. Gothland, p. 270. Sechunde.

Limné, Reif. d. Weftgothland; p. I9I. Seehunde. 
Limné, Faun. Suec. Edit. I. p. 4. n. Ir. Phoca deutibus caninis tectis.

Linné, Fn. Suec. Edit. II. p. 2. n. 4. Phoca (Vi: tulina) dentibus caninis tectis.

Linné, Muf. Adolph. Fried. I. p. 5. Phoca (communis) dentibus caninis tectis.

Graumann, intr. in hift. nat. mamm. p. 86. IV. Robbe, Seehund, Seenalb.

Gattever, brev. Zoolog. I. p. I56. n. 4. Phoca (Vitulina) capite laevi inauriculato, corpore fufco; der Seehuind.

Olans MIagn. de gent. Septentr. p. 70I. Vitulus marinus.

IMnf. Wormian. p. 289. Phoca.

Gronov: Zoophyl. I. p. 7. n. 28. Phoca, pedibus anticis digitatis, unguiculatis, pofferioribus latis, unguibus teretibus.

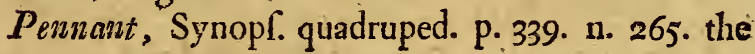
common Seal.

Parfon, phil. Transact. XLVII. p. I20. 'Tab. 6. Phoca'minor, dentibus caninis tectis, palmis anterioribus digitatis, ungulatis, pofterioribus latis ordinariis, ungulis teretibus donatis.

Charleton, exercitat. pifc. p. 48 . Phoca.

Charlevoix, hift. de la nouv. Fr. III. p. I43. le Loup marin.

B. Вот л I A. Der grane Seehund.

Schreber, Säugth. I. p. 309. der graue Seehund. Borowsky, Thierreich, I. 2. p. 9. der grane See. hund.

Bechftein; Naturgefch. DeutChl. I. p. 408. der grouve Seehunid.

Knneif, in den Schwed. Abh. XIX. p. T7r. das graue Seekalb.

Erxle 


\section{Mammalia Ferat. Phoca.}

Erxleben, Mammal. p.587. c. der graue Seehund. Granmann, intr. in hift. nat. mamm. p. 86. a. der graue Seehund.

Gatierer, 'brev. Zoolog. I. p. 157. a. der grause Seehund.

y. Sibirica. Der fibirifche Seehund.

Schreber, Säugthiere, I. p. 3IO. der fibirifche Seehund.

Borowfky, Thierreich, I. 2. p. 9. der fibirifche Seehund.

Steller, Befchreib. von Kamtfchatka, p. 108. dis vierte Sorte Seehunde.

Erxleben, Mammal. p. 588. $\beta$.

Graumann, intr. in hift. nat. mamm. p. 86. b. der fibirifche Seehuind.

Gatzerer, brev. Zool. I. p. I57. b. der fibirifche Seehund.

8. Cas P I c a, Der cajpifche Seehund.

Schreber, Säugth. I. p. 3Io. der cafpifche Seehund. Borowsky, Thierreich, I. 2. p. 9. der cafpifche Seehund.

S. G. Gmelin, Reife d. Sibirien, III. p. 246. der Seehund.

Pallas Reife, I. p. 430.

Erxlebes, Mammal. p. 588. $\gamma$.

Graumann, intr. in hifor. nat. manm. p. 86. e. der cafjifiche Sechund.

Gatterer, brev. Zool. I. p. 157. c. des cafpifche Seehwnd.

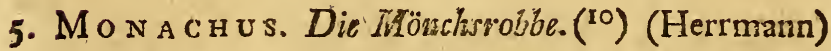
Zimmernann, geogr. Zoolog. p. 42I. n. 379. die Möachsrobbe.

Gesner,

(80) Lange des Kürpers acht Fufp. 
Gesner, aquat. p. 827. Vitulus maris mediterranei. Rondel.

Biiffon, vierfüfs. XVI. p. I97. der MÖnchsrobbe. m. e. Fig.

6. Groend a d d 1 3. Der Schwarajeitige Seehund. Sattler. ( $\left.{ }^{x}\right)$

Zimmermann, gengr. Zoolog. II. p. 422. n. 380. die fchwarzaseitige Robbe.

Schreber, Säugthiere, I. p. 3IO. 11. 5. der fchwarzSeitige Seehund.

Borowjky. Thierreich, I. 2. p.9. n.5. der JchwarzSeitige Seehund.

Gatterer, vom Nutzen und Schaden der Thiere,

I. p. 404. n. 224. der grönländifche Seehund, Sattler.

Bïffon, vierf. XVI. p. I63. der Robbe mit dem Mond; m. 2 Figg.

Pennant, arçt. Zool. II. p. I58. n. 96. der mit hal: ben Monden bezeichnete Seehrund.

Olaffen, Reile durch Island, I. p. 283. II. p. 42. Vade - Säl, Hav - Säl.

Steller, Befchreibung von Kamtfchatka, p. ro7. die andere Sorte.

Cranz, Grönland. p. 150. n. 2. Attarfoak. Egede, Grönland; p. Ioz. Tab. 6. SuartSiide.

Miiller, prodrom. Zool. dan. p. 8. Phoca groenlandica.

Bodd.

(1) Neun Fufs lang. Wandert jährlich von SüG - Island nach Norden, durch die Davisftrafse. Die Seehundjaiger auf Neyland sennen diefs Thier die Leyer oder das Herz, und feinefeichnung an den Seiten, den Sättel. Der Thran aus den Speck diefes Seehundes wird für den vorzüglichften gehalten. 
Bodd. El. p. 170. Phoca (Semilunaris) capite acutiore, corpore craffo grifeo, maculis lunaribus atris in lateribus.

Leppechin, act. Acad. Petropolitan. P. I. p. 259. Tab. 6. 7. Phoca oceanica, Krylatca Rufis.

Graumann, intr. in hift. nat. mamm. p. 86. V. der Schwaraseitige Seehuna.

Gatterer, brev. Zoolog. I. p. 157. n. 5. Phoca (Groenlandica) capite laevi inauriculato, corpore grifeo, lateribus luna nigra; der grönlïndifche Seehund.

Ellis, Reife nach Hudfons - Meerbufen, Tab. 7. Seehund mit einer fchwarzen Seite.

Fabric. Fauna groenland. 11. 7. Phoca (groenlandica) capite laevi, nafo longo, corpore de. preffo conico, vibriflis undulatis, medulla rubra apice fubcurvis.

7. His P I d A. Der rauhe Seehund. (2)

Miiller, Naturfyft. Suppl. p. 26. n. 5. der vauhe Seehunand.

Zimnermmnn, geogr. Zoolog. II. p. 422. n. 38I. die ranhe Robbe.

Pennant, arct. Zoo!. II. p. 155. n. 93, der yall he Sechund. .

Gatterer, vom Nutzen und Schaden der Thiere, I. p. 405. n. 225. der rauhe Sechund.

Cranz,

(2) Nach Schreber, Erxleben und Zimmèrmann von cier Grölse des vorhergehenden; nach Pennant nicht iber vier FuIs lang. Geht nie ins hohe Meer, fondern halt fich beftandig auf dem feften Zife in den abgelegenen Bayen auf. Lebt einfam, feiten parwife. Frifst kleine Fifche, Krabben u. dol. Haur, Sehnen, una Speck werden wie von andern Seehunden gebraucht. Das Fleifç ift roth, und vorziglich das der männlichen to ftinkend, tafs es feluht den Grönlandern Lickel verurfacht. 
Cranz, Grönland. p. İ́r. n. 3. Neitfek. Biiffon, vierf. XVI. p. I74. dev Robbe Neitfoak, m. e. Fig.

Rodd. El. p. 17í. Phoca (Hifpida) capite laevi inauriculato, corpore pallido - fufco, pilis furrectis hifpido.

Grammann, intr. in hift. nat. mamen; p. 86. VI. der rauhe Seehund.

Gattever, brev. Zoolog. I. p. I58. n. 6. - Phoca (Hifpida) capite laevi inauriculato, corpore pallide fufco, pilis furrectis hifpidis; der rau. he Seehund.

Faun. Grönland: n. 8. Phocà (Fcetida) capite laevi, nafo brevi, corpore fere elliptico, vibriffis undulatis, medulla lucida.

Perinant, Syn. quadrup: p. 34I. n. 267. the rough Seal.

8. Cris tata. Die Klappmitize. (3)

Zimmermann, geogr. Zoolog. II. p. 423. n. 382. die Klappmiitze.

Schreber, Säugthiere, I. p. 312: n. 7. die Klapp. miitze.

Gatterer, vom Nutzen und Schaden der Thiere, I. p. 406. ń. 226. die Klappmiitze.

Biiffon, vierf. XVI. p. I56. der Robbe mit der Kappe, m. e. Fig.

\section{Pointop.}

(3) Nach Zimmermann gegen zwölf, nach Pennanz acht Fufs Länge.

- Hat den Namen von dem auf der Stirne befindlichen, wie eine Blafe geftalteten Fell, welches das Thier bey Stürmen wie eine Mütze über die Angen zieht. Die Seehundjâger nennen ihn den gekappten Seebund. Erxleben zweifelt noch, ob nicht vielleicht diefs Thier mit Phoca Leonina einerley fey. Pomm aber verfichert das Gegentheil. 
Pontoppid. Naturgefch. von Norwegen, II. p. 237 . Klappmiitzen.

Cranz, Grönland. p. I41. n. 4. Neitferfoak. Egede, Grönland. p. 108. Tab. 6. die Klappmiitze. Ellis Reife nach Hudfons Meerbufen. Tab. 7 . - Seehuna mit einer Hanbe.

Pennant, arct.Zool. II. p. 157. n.95. die Klappmiitze. Bodd. El. p. I7o. Phoca (Cucullata) fronte cute denfa pilofa tecta, corpore pilis nigris, fub quibus lana nivea.

Granmuann, intr. in hiff. nat. mamm. p. 87. VII. P. Criflata.

Gatterer, brev. Zool. I. p. 158. n. 7. Phoca (Criftata) capite antice criftato, corpore grifeo; dic Klappmiitz:e.

Fabric. Fauna groenl. n. 5. Phoca (Leonina) ca. pite antice criftato.

9. Ba в ата. Der grofse Seehund. (4)

Zimmermann, geogr. Zoolog. II. p. 423. n. 382. die grofse Robbe.

Schreber, Säugthiere, I. p. 313. n. 8. der grosse Sechund.

Borowfky, Thierreich, I. 2. p. Io. n. 6. der grofse Seehund.

Gatterer, vom Nutzen und Schaden der Thiere,

I. p. 406. 11. 227. dev grofse Seehund.

Pennant, arct. Zoolog. p. 154. n. 92. der grosse Seehund.

Biiffon,

(4) Nach-Sibreber und Zimmermann, zehen, nach Pennant und Erxleben zwölf Fufs lang. Aus der Haut diefes Thiers, die fehr dick ift, fchneiden die Grönlànder Riemen zum Seehundsfange. Erxleben zweifelt, ob diefe Gattung wohl das Weibchen der vorhergehender feyr mö̈ge. 
Biiffon, vierfüls. XVI. p. I5r. Utfuk; p. I8I. der, Robbe Laktak aus Kamt fihatka.

Steller, Befchreib. von Kamtfchatka, p. Io7.

Steller, Befchreib. fonderbarer Meerthiere, p. 103. Lachtak.

Cranz, Grönland. p. 152. n. 5. Ut fuk.

Miiller, Samml. ruff. Gefch. III. p. 250. Seehunde von der gröfsern Art, die in Kamtfchatka Lachtak genannt werden.

Erxleben, Mammal. p. 590. n. 8. Phoca (Bar: bata) capite laevi inauriculato, corpore nigricante.

Graumanin, intr. in hif. nat. mamm.p. 87. VIII der grofse Seehund.

Gatterer, brev. Zoolog. I. p. 158. n. 8. Phoca (Barbata) capite laevi inauriculato, corpore nigricante; der grosse Seehund.

Parfons philof. Transact. XLVII. p. I21. Phoca maior, dentibus caninis tectis, palmis anterioribus digitatis, ungulatis, pofterioribus latis or. dinariis, corpore longiore.

Fabric. Faun. groenland. n. 9. Phoca (Barbata) pedibus anticis manus hominis referentibus, pollice breviore, vibriffis longis albis integris, apice curvis.

10. Pusit c a. Der kleine geöhrte Seehuind.(5)

Milller, Naturfyf. Suppl. p. 26. n. 4. der kleine Sechund; I. 'Tab. Ir. fig. 5. Houtt.

Zimmermann, geogr. Zool. II. p. 423. n. 384. die kleine geöhrte Robbe.

Borowjky, Thierreicil, I. 2. p. Io. n. 7. der kleine geöhrte Seehund.

Gatte:

(s) Lănge des Thiers etwas tuber zwey Fufs. 
Gatterer, vom Nutzen und Schaden der Thiere, p. 406. n. 228. der kiline Seehund.

Biiffon, vierf. XVI. p. 264. kleine geöhrte Robbe, m. 2 Figg.

IMolina, Naturgefch. von Chili, p. 244. Phoca lupina, der Urigne?

Erxleben, Mammal. p. 59r. n. 9. Phoca (Pufilla) capite laevi fubauriculato, corpore fufco. Granmann, intr. in hif. nat. mamm. p. 87. IX. der kleine Seehund.

Gatterer, brev. Zoolog. I. p. 158. n. 9. Phoca (Pufilla) capite laevi fubauriculato, corpore fufco; der kleine Seehund.

Gesner, aquat. p. 827 . Phoca feu vitulus maris mediteranei Rondel. m. Rond. Fig.

$p_{\text {ennnant, Syn. quadr. p. 343.11. } 270 .}$

Ariftot. hift. anim. I. c. 2. no 12. c. 23. n. 210.

II. c. 4. 11. I4. I9. c. II. n. 68. c. I8. n. 130.

I34. c. 20 . n. 158 . III. c. 16. n. 237 . V. c. 2.

11. 25. VI. c. II. n. 126-135. Ф

Plin. hiff. nat, VIII. c. 3i. IX.c. I2. I3. XI:

c. 37 . c. 40. Vitulus marinus.

- Plin.h. n. IX.c. 7. XI. c. 37. Phoca。

A) Vevïnderungen gegen die XIIte Edition, und Vermehrung der Gattungen diefes Gefchlechts.

Diefs Gefchlecht ift mit fisben Gattungen ver mehrt, und P. Tubata, Monachus, Groenlandica, Hi. Spida, Crifata, Barbata, und Pufilla dazugekonmen.

13) Unbefimintere Thiere.

1. Grammelelur.

Schreber, Säugth. I. p. 3I 4 .

Olaffen, Reife durch Island, I. p. 283. 


\section{Mammalia Ferae. Canis.}

2. Der hasenhaarige Seehund.

Zimmermann, geogr, Zoolog. III. p. 277. n. 3. Phoca leporina.

Pennant, arct. Zool. II. p. I58. n. 94.

Biiffon, vierf. XVI, p. 230. der haafenhaarige Seehund.

Bodd. El. p. r72. Phoca (leporina) capite et corpore albido, pilis mollioribus tecto.

Leppechin, art. Acad, l'etrop. I. p. 264. Tab. 8.9. Pennant, hift, guadr, 11, 382 . Leporine Seal.

3. Dev böndirte Seehund. Phoca fafciata. Pallas.

Zimmermann, geogr. Zoolog. III. p. 277. n. 2.

Phoca fafciata,

Pennant, arct. Zool. II. p. 160, n. 97 .

Büffon, vierf, XVI, p. 232. der bandirte Seehund; in. e, Fig.

Pennant, hilt, quadr, n. 380 , fig. p. 513. Rubbon Seal,

Voll den Gattungen der Robben in der Offee fiehe Oedmann in den neuen Schwed. Abhandlungen $V$. p; $8 \mathbf{I}$

12. GESCHLECHT, CAN İ, Der Hund. Miiller, Naturfyf, I. p. 206. Gen, XII. Zimmermann, geogr, Zool, II, p. 233. Genl. XVIII. Schreber, Säugthiere, III. p, 317. Gen. XIII. Lefke, Naturgefch. p. I32. Gen. XIII. Borowfky, Thierreich, I. 2. p.-II. Gen. XII. Blumenbach, Handbrich der Naturgefch. p. 89.

Gen. XXVI.

Bechftein, Naturgefch. Deutfchl. I. p. 189.

Funke, Naturgefch, u. Techn. I. p. 70 .

$$
\text { K } 3 \text {. }
$$

Erxle- 
Erxleben, Mammalia, p. 531. Gen. XLV.

Beckmann, Naturhift, p. 27.

Pemant, arct. Zool. II. p. 39.

Biiffon, vierf. II. p. 80. XV. p. 7. etc.

- Graumam, intr, in hif, nat, mamm.p.77. G. XLV.

Gatterer, brev. Zool. I. p. 142. Gen. XLV.

Pennant, quadr. p. I4I. Gen. XVII.

Briffon, quadr. p. 169. Gen. XXXV.

Hermann, Tab. affinit. anim. p. 67.

1. Familiakis. Der Hund. (')

Miiller, Naturfyf. I. p. 20\%. n. I. Suppl. p. $2 \%$. der gefellige Hund.

Zimmermann, geogr. Zool. I. p. I30." II. p. 235. n. I36. der Hund.

Schreber, Säugthiere, I. p. 3I8. n. r. der Hund. Lefke; Naturgefch. p. 133. der Hund.

Borowsky, Thierreich, I. 2. p. II. n. I. der eigentliche Hund.

Bhumenbach, Handb. d. N. G. p. 89. Canis (Familiaris) cauda recurvata, fubinde digito fnurio ad pedes pofticos; der Hund.

'Bechftein, Naturgefch. Deutfchl. I. p. 190. der (eigentliche) Hund.

Funke, Naturgefch. I. p. 70. der Hund. Halle, vierf, p. 47o. der Hunnd.

Gatterer, vom Nutzen und Schaden der Thiere, I. p. 345. n. 205. der Hund.

Neuer

(1) Hurd, weibl. Hindin; in gemeinen Ieben die Betze, Oberf. Petze, bey den lägern die Fähe, Wülfin, Deve, Niederfächf. Teve, Tiffe, Tieve, Thüle, Zippe, Luppe, Hannüv. Tache, alt nberd. Zaucbe, Zaucke, Zache; oberd. Bräcke, Mätze, Meze, Lufcb, Zaupe; Würtemb. Zatze; Schlef. Lutflé; Livl. Taufch. Iunge Hunde werden fowohl bey den lägern, als in Niederf. Wolfi, junge Wolfe, genannt. 
Neuer Schaupl. der Natur, IV. p. r42. der Hund. Onomat. hift. nat. II. p. 537. der Hind. Sander, ökon. Naturgefcli. I. p. 244. der Hund. Handb. d. Naturgef ch. a. d. Franz. Nürnb. 1.773 . I. I. p. 62. der Fiund.

Kriunitz, ökon. Encyklopädie, XXVI, p. 325. Kliigel, Encyklop. I. p. 223.

Handbuch der deutfchen Thiergefchichte, p. 52. der Hund.

Goeze, europäifche Fauna, I. p. 79. Houshund. Smith, über die Natur und Beftimmung der Thiere etc. p. 215 .

Auszug a.d.'Tagebuch e. Reifenden nach Afien, p. 57.11 .15$.

Gesner, Thierbuch, p. 211. Canis, ein Hunzt, m. e. Fig.

Klein, Quadrup. difp. p. 68. Canes proprie dicti. Klein, Claffif. d. vierfüfs. Th. p. 204. Hund. Klein, natüil. Ordn. d.vierf, Th. p. 72, Hisnd. Büffon, vierf. II. p. 88. der Hund. XV. p. 84. Cetti, Naturgefch. von Sardinien, I. p. 54. der fardinifche Hund.

Pontoppid. Norwegen, II. p. I7. Hunde.

Caver; Reif. durch d, Innere von Nordamerika, p. 363. der Hund.

Köupfer; Befchreib. von Iapan in du Haldo China IV. p. I37. Hunde.

Chandler, Reifen in klein Afien, p.7o. Hunde. Molina, Naturgefch. von Chili, p. 284. Hundde. Du Halde, China, II. p. I64. Hzande. Bo/smann, Reife nach Guinea, p. 282. Ellis, Reife nach Hudfons-Meerbufen, "p. 169. I. G. Gnelin, Reife d. Sibirien, I. p. 380.

Georgi, Rufsland, p. 77. 279.303.324.340. S. G. Gmelin, Reife d. Rufsland, III. p. 329 . 
Bock, Naturgefch, von Preuffen, IV. p. 12. n. 5.

I. Liplus, von wunderbarer Natur und Eigenfchaften der Hunde, a, d, Latein. Hanover I614. 4 :

Lindenkranz, diff. de Cynographia. UpГ. 1753.4. c. f. (Sub praef. Car. Limnaei.)

Bohemi, Bericht von den Hunden etc. herausgegeben von $C r u f u s$. Lpz. I677. 4 .

Paullini, Cunographia curiofa. Norimb, 1675.4. Erxleben, Mammalia, p. j3r. n, I, Canis (Fa, miliaris) cauda (finiftrorfum) recurvata.

Linné, S. N. Edit. II. p. 44. Canis cauda recurva. Limné, S. Nat. Edit. VI, p. 5. n, I. Canis cauda (finiflroifum) recurva.

Linné, Sylt. Nat. Edit. X. I. p. 38. n. I. Canis (Familiaris) cauda finifrorfum recurva.

Linne, Faun. Suec. I, p. 5. n. 12. Canis cauda recurva.

Linné, Muf. Ad. Fried. I. p. 6, Canis (Do. mefticus ) cauda finifrorfum recurva.

Kramer, Aufr. p. 312. Canis cauda recurva.

Gronov. Zoophy1. I. p. \&. n, 29. Canis cauda finiftrorfum recurva.

ITiller, prodr. Zool. dan. p. 2. n. 4. Canis (Fa: miliaris) cauda recurvata.

Pennant, Synopf. quadruped.' p. I4I. n. IIo. Arifroteles, hift anim, I. c. 6. n. 4 I. II. c. 5 . n. 23. c. II. n. 72. III. c. 7. 11. 95. V.c. II. 11. $125-I 27$. c, I3. I1. I38. VI. c. 20. n. 264 bis 291 . Kúwy.

Plin. "h. n. VIII. c. $40-42$. X. c. 63. Canis. Tavo, de re ruft. Lib. 2, c. 9, de canibus.

a. Domest icus. Der Schïferhand. Miiller, Naturfy,t. I, p. 207. n. a. der Haushund. 
Ziminermann, geogr. Zool. II. p. 235, n. a. der Schäf erhund.

Schreber, Säugthiere, I, p. 319. n, I, der Schäferhund,

Borove $\} k y$, Thierreich, I, 2. p. r4. n, a, der Schäferhind.

Bhimzenzach, Handb. d. Naturgefch, p. 90. f. der Houshand, Schïferhund.

Bechlitein, Naturgefch. Deutfchl. I. p. 195. n. c. der gemeine Schäferhund, Bauernhund, Haushunud, Hof fund.

Neuer Schauplatz d, Natur, I. p. I43. n. I, dev Haushund, oder Bauerhund.

Halle, vierf. p. 479. der Schifferhund.

Bock, Naturge[ch, von Preuffen, IV, p. 15. dey Hanushund.

Deutfche Zeitung, I785, p. 158 .

Handbuch der deutfchen Thiergefchichte, p, 5 ? cier Schäferhund.

Büffon, vierf. II. p. I64, n, 4. der Schäferhund, Hirtenhund, Phylax. Tab. 23. Fig. I.

Erxleben,-Mammalia, p. 537: n, o.

Graumann, intr. in hift, nat, mamm; p. 77, n. as Hainshund.

Pennant, Syn, quadruped, p, 144. n, r. the Shepherd's Dog,

B. Pomeranus, Der Spit:.

Zimumermann, geogr. Zoolog, II. p. 235. n. b. der Spitz, Pommer.

Schreber, Säugthiere, I, p. 319. 13. 2. der Spitz. Borowfky, Thierreich, I. 2. p, I4, n. b, det -Wolfshund, spitz, Pammer.

Blumenbach, Handb, der Naturgefch, p. 9r, der Spitz, ode" Pommer. 
Bechftein, Naturgefch. Deutichl. I. p. 195. 11. d. der Wolfshund.

Halle, vierf. p: 480 . der Wolfshund.

Kriinitz, ökon. Encyklop. XXVI. p. 348. n. 5. der Wolfshund.

Buiffon, vierf. II. p. 165. n. 5. der Wolfshund, Tau. 24.

Erxleben, Mammalia, p. 538. n. $\beta$.

Graumnann, intr. in hif. nat. mamm. p. 77 n. b. Wolfshand, Spitz.

Pennant, Syn. quadr. p. I44. n. I. \%. the Pomerian Dog.

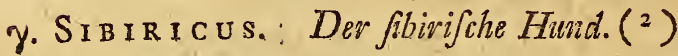

Zinimermann, geogr. Zool. II. p. 235. n. c. der fibirifche Hund.

Schreber, Säugthiere, I. p. 219. n. 3. der fibirifche Hinnd.

Borow jky, Thierreich, I.2. p. I4. n. c. der fibirifche Hund.

Blumeribach, Handb. der N. G. Ed. 3. p. I00. diso fibivifche Hund.

Halle, vierf. p. 480. der fibirifche Hundd.

Onomatol. hift. nat. II. p. 544. n. 7. der fibirifche Hund.

Pennant, arct. Zool, II. p. 4.r. Hunde.

Biiffon, vierf. II. p. I66. n. 6. der filiviche Hund. Tab. 25. fig. I. XV.p. 85 .

Krïnitz,

(2) Nahrt fich in Kamtchatsa von lauter Fifchen. Vom Frühjahr bis in den fpaten Herbft bekümmert man fich dafelbft um diefe Thiere gar nicht. Sie lavfen allenthalben frey herum, und lauern den gaizen Tag an den Flüfen auf Fifche, die fie gefchickt zu fangen wiffen, und von denen fe nur die Küpfe verzehren. Sie werden vor den Schlitten gefpannt, und machen überhaupt allein die ganze Klaffe von eigenen kamtfchatkifchen zahmen Thieren aus. 
Kriinitz, ökon. Encyklop. XXVI. p. 348. der fibi-

rifche Hund. p. 363 . der kamt chatkifche Hnnd.

Steller, Befchr. von Kamtfchatka, p. I32. Kosnc. Krafcheninnikoze, Befchreib. des Landes Kamt-

fchatka, p. I28. I76. 237.

Pallas Reife, Ausz. III. p. 16. Hurbde.

Erxleben, Mammalia, p. 538. n. $\gamma$.

Grounann, intr. in hift. nat. mamm. p. 77. n. d. der fulirifche Hund.

Pennant, Syn. quadrup. p. I44. n. I. $\beta$. the Sibirian Dog.

8. Is a a d Icus. Der isländifche Hund.

Zimmermann, geogr. Zoolog. II. p. 836 . n. e. der isländifche Hund.

Schreber, Säugthiere, I. p. 320. n. 4. der islöndifche Hitnd.

Borowl'ky, Thierreich, I. 2. p. 14. n. 4. Canis Getulus $\mathrm{f}$. Islandicus, der islëndifche Hund.

Blumienbach, Handbuch der Naturgefch. p. 9r. dor isländifche Hund.

Halle, vierf. p. 480. der islëndifche Hund.

Kriinitz, ökon. Encykl. XXVI. p. 348. der islïnzdifche Hund.

Biiffon, vierf. II. p. I67. n. 7. der islïndifche Hund. Tab. 24. fig. 2.

Erxleben, Mammalia, p. 538. n. $\delta$.

Graumann, intr. in hift. nat. mamm, p. 77.n. c. der islïndifche Hund.

2. A Quaticus. Der. Budel. (3)

Miiller, Naturfyf, I. p. 208, n, e. der Budel.

Zimmer.

(3) Caraccioli hat aus der Wolle eines fehr haarigten Pudels eis Paar Strümpfe machen laffen, die fehr gut, ausgefallen feyn follen. 
Zimmermann, geogr, Zoolog. II. p. 236. n. f. der Budel.

Schreber, Säugthiere, I. p. 320. n. 5. der Budel. Boronefky, Thierreich, I. 2.p. I4. n.e. der pudet. Blumenbach, Handb. d, N, G. p. 90, n. e. der Budel.

Bechftein, Naturgefch. Deutfchl. I. p. 203. 11. 4. dier grofse Budel, IVafjerhund, Barbet, ungarifcher Wafferhund.

Halle, vierf. p. 485. der Pudel.

Neuer Schauplatz der Natur, IV. p. I43. 13. 5. der Pudel.

Onomatol, hiftor, nat, II, p. 544, der grofse WafSerhund.

Bock, Naturgefch. von Preuffen, IV. p. 18. dier Pudel.

Biiffon, vierf. II, p, I74. n. II, der grosse Pudel. Tab. 3o. fig. I.

Krainitz, ökon. Encykl. XXVI. p. 350. n. II. der grosse Pudel, oder Budel, Pudelhind, Waf= Jerhind.

Erxlebon, Mammal. p. 539. n. $\varepsilon$,

Linné, Amoenit. acad. IV. p. 46. n. 5. Canis aquaticus,

Graumann, intr, in hif. nat, mamm. p. 77. n.e. Pudel, Budel.

İonfton, quadrup. Tab. 7o. Canes, Hünde; die unt. Fig. zur Rechten.

2.Mr-

len. Nạch feinẹ Mẹinung künnten einige Hünde jăhrlich zwey bis drey Pfund geben. S. deffen praktifche Landökonomie der Alten, Augsh. 1760. p. 57.

Merkwürdige Beyfpiele von künftlicher Befruchtung einer folchen Hündin liefet man in Spallanzani Verfuchen über die Erzeugung der Thiere und Pflanzen, p. 249. 3n4. 343. Lichienberg, Magazin für das Neuefte etc. 1. 4. p. Iys. 


\section{Mammalia Ferae. Canis,}

3. Minor. Der Zwergbudel.

Zimmermann, geogr. Zoolog. II. p. 236. n. g. der Zierergbudel.

Schreber, Säugthiere, I. p. 321. n. 6. der Zwerg. budel.

Boroweky, Thierreich, I. 2.. p. I4. n. f. der Zwergbuidel.

Bechftein, Naturgefch. Deutfchl. I. p. 204. n. a. der kleine Budel, Zwergbudel.

Halle, vierf: p. 488. der kleine Padel.

Kriinitz, ökon. Encykl. XXVI. p. 355. n. 18. der, kleine Budel.

Biuffon, vierf. II. p. 183. n. I7. der kleine Budei.

Tab. 3I. fig. 2.

Erxlebern, Mammalia, p. 540. n. $\tau$.

Graumani, intr. in hift. nat. mamm، p. 78. n. f. kleine Budel.

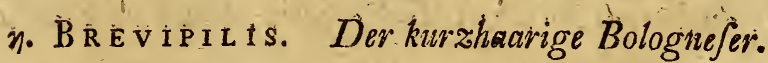
Zinmermain, geogr. Zool. II. p. 236. n. h. der kurahaarige Bolognefer.

Schreber, Säugthiere, I. p. 321. n. 7. der kurzhaarige Bolognefer.

Borow $\int k y$, Thierreich, I. 2. p. I4. n. 9. $\operatorname{der} B \dot{B}$. lognefer, englifche Wachtelhund.

Bechftein, Naturgefch. Deutfchl. 1. p. 204. n. c. der kuvzhaurige Bolognefer; p. 205. n. d. die $\dot{p}_{\text {yrame, }}$ der englifche Wachtelhund.

Halle, vierf. p. 485. der englifche Wachtelhuind. Biiffon, vierf. II. p. 176. n. I3. der eniglifche Wacts. telhund; Tab. 32. fig. I. Die Pyranise fig. 2. Erxleben, Mammalia, p. 540. n. 3 .

Graumann, intr. in hift. nat. mamm. p. 78. n. g. englifche Wachtelhurid.

Pemant, Syn. quadr, p، 45 , n. III. $\beta_{1}$ Pyrame. ?.Exं 
9. Ex т R a r us. Der Bolognefer Hund. Miiller, Naturfyft. I. p. 209. n. k. der Jpanifche Hund.

Zimmermain, geogr. Zoolog. II. p. 237. n. i. der (langhaarigte) Bolognefer; der Jpanifche Wachtelhund.

Schreber, Säugthiere, I. p. 322. n. 8. der Bolognefer Hund.

Borowfky; Thierreich, I. 2. p. 14. n. h. der Bolognefer; der fpanifche Wachtelhund.

Bech/tein, Naturgefch. Deutfchl. I. p. 204. 11. 5. der Spanifche Wachtelluind, der langbehaarte Bolognefer, Seidenzund, Seicienbutel.

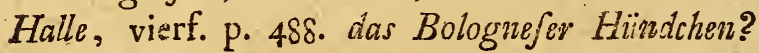
Tab. 57.

Neuer Schaupl. der Natur, IV. p. I44. n. IO. der fpanifche Hund.

Onomat. hift. nat. II. p. 540. Canis hifpanicus, canis extrarius.

Kriinitz, ökon. Encykl. XXVI. p.35r. der Spanifihe Wachtel-oder Hühnerhusbd, fpanifche klo. ne Budel.

Büiffon, vierfüls. II. p. I75. n. I2. der Jpanifche Wachtelhund. Tab. 3r. fig. I.

Erxleben, Mammal. p. 541. n. $\eta$.

Linné, Amoen. acad. IV. p. 47. n. 9. Canis extrarius. f. hifpanicus.

Gramann, intr. in hift. nat. mamm. p. 78. n. h. Bologneferhund.

6. Mel i taeus. Der angorifche Hund. Miiller, Naturfyft. I. p. 208. n. f. Bolognefer Hevnd.

Zimmermann, geogr. Zoolog. II. p. 237. n. k. des. angoriche Hund.

Schreber, 
Schreber, Säugthiere, I. p. 322. n. 9. der angorifche Hund.

Borowfky, Thierreich, I.2. p. I4: n. I. der ango. rifche Hunud.

Bechftein, Naturgefch. Deutfchl. I. p. 205. n. e. der angorifche Hund, Bologne erhiindchen, Mal. thejerhiindchen, Spanifches Hiindchen, Schooss. hiindichen.

Blumenbach, Handb. der Naturgefch. p. 9r. n. 9. das Bologneferhiindchen.

Neuer Schauplatz der Natur, IV. p. 143. n. 6. der Bologne erhund.

Onomatol. hift. nat. II. p. 540. Canis melitaens; Schoofshiindchen.

Bock, Naturgefch. von Preuffen, IV. p. ro. der Bolognefer.

Kriinitz, ökon. Encykl. XXVI.p. 355. n. 19. der bolognefer, malthefer, oder spanifche Hund, Schoosshund, Iungfernhund; p. 361. der angorifche Hinnt.

Biiffon, vierfüfs. II. p. I84. n. 18. das Bolognefer Hïndchen, Táb. 33. fig. I.

Erxleben, Mammal. p. 541. n. $\vartheta$.

Linnie, amoenit. acad. IV. p. 46. n. 6. Canis melitaeus.

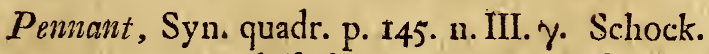
Ignat. Giorgi, diff. de canibus melitenfibus. Venet. 1730.4 .

Graunzann, intr. in hift. nat. mamm. p. 78. n. i. der angorifche Huind.

\%. LEONINUS. Der Lörernhund.

Zimnermann, geogr. Zool. II. p. 237. n. I. das Lörvenhiindchen.

Schreber, Säugth. I. p.322. n. I0. der Zözeenhund. Borowejky. 
Borowjky, Thierreich, I. 2. p. 14. n. k. das Löivenhiinząchen.

Bechftein, Naturgefch. Deutfchl. I. p. 205. n. f. das eigentliche Löwenhiinddchen.

Halle, vierf. p. 488. das Lörenhiündchen.

Kriniitz, ökon. Encykl. XXVI. p. 356 . der Lö. ioenhinit.

Biiffon, vierfüfs. Iİ. p. 185. n. 19. das Löwenhïndtheit, Tab. 33. fig. 2 .

Erxlebein, Mammal, p. 542. n. 6.

Graumann, intr. in hif. nat. mamm. p. 78.n.k. Löwenhuñ.

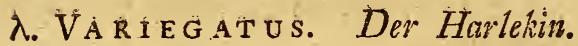

Zimmermann, geogr. Zool.' II. p. 238. n. m. der kleine dänifche Hund, cer Harlekin.

Schreberr, Säugthicre, I. p. 323. n. Ir. der Harlekint.

Borowsky, 'Thierreich, I. 2. p. 15. n. 1. der HarLekin, kleine dänilche Hund.

Bechftein, Naturgefch. Deutchl. I. p. 206. n. a. der kleinie dänifche Huvid.

Onomatol. hift. nat. II. p. 542. der Harlekini. Krïnits; ökon. Encykl. XXVI. p.'352. n. I5. der kleine dïnifche Hinnd.

Halle, vierf. p. 486. der kleine dönifche Hund. Biuffón, vierf. II. p. I77. n. 14. der kleine dönifche Huivd, 'Tab. 34. fig. I.

Erxleben, Mammalia, p. 542. n. k.

Granumaini, intr. in hiftor. nat. mamm. p. 79. Harleḳin.

$\mu_{0}$ H В В IDUs: Der Baftardmops.

Zimmermann, geogr. Zoolog. II. p. 238. n. n. Aer Baftardmops, Roquet.

Schreber, 
Schreber, Säugthiere, I. p. 323. n. I2. der $B a$. ftardmops.

Borowsky, Thierreich, I. 2. p. 55. n. m. Bafardmops; Roquet.

Bechftein, Naturgefch. Deutchl. I. p. 206. Roquet. Halle, vierf. p. 48\%. der Roquet.

Biiffon, vierfïls. II. p. IS8. n. 22. der Roquef, Tab. 34. fig. 2.

Erxlebein, Mammalia, p. 543. n. $\lambda$,

Graumann, intr. in hift. nat. mamm. p. 79, n. m. Baftardmops.

Pennant, Syuopf. quadrup; p. r47.n. V. $\gamma$. Ba. flard-Pug.

ข. FRICATOR. Der Mops.

Miiller, Naturfyf. I. p. 209. n. g. Mops, Steindocke.

Zimmermann, geogr. Zoolog. II. p. 238. n. o. der Mops.

Schreber, Säugthiere, I. p. 323. n. I3. der Mops. Borowfky, Thierreich, I. 2. p. 15. n. n. der Mops. Blumienbach', Handb. d. Naturgefch. p. 89. n. a. der Mops.

Bechflein, Naturgefch. Deutchl. I. p. J98. n. e. der Mops, Steindogge.

Halle, vierf. p. 488 . der Illops.

Neuer Schauplatz der Natur, IV. p. I43. n. 7. der Mops.

Onomatol. hill. nat. II. p. 540. der Mrops.

Bock, Naturgefchichté von Preuffen, IV. p. ig. der Mops.

Kriïntz, ökon. Encykt. XXVI. p. 356. der Mops, Mopshund, Moppel, Möppel, Steindocke. Biiffon, vierf. II. p. 186. n. 20. der Mops. Tab. 36. fig. 2. 
Erxleben, Mammal. p. 543. n. $\mu$.

Linné, Amoen. acad. IV. p. 46. n. 7. Canis fricator.

Graumann, introd. in hifor. nat. mamm. p. -79. n. 11. Mops.

a) Baftard vom Mops und Baftardmops.

Halle, vierf. p. 490. der Achtziger oder der Riilfellche Hund.

Biiffon, vierf. II. p. I88. n. 23. der artoiffche Hund.

Erxleben, Mammal. p. 544 .

b) Baflard vom Mops und Bolognefer Hund.

Halle, vierf. p. 490. dev alikantifche Hund. Büffon, vierf. II. p. I89. n. 24. alikantifche Hunde.

Erxleben, Mammalia, p. 544.

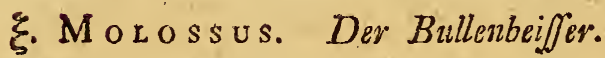

Miiller, Naturfyftem, I. p. 208. n. d. der Bullenzbeiffer, Wachthunut.

Zimunnermann, geogr. Zoolog. II. p. I38. n. p. der Bullenbeiffer.

Schreber, Säugthiere, 1. p. I24. n. I4. der Bullen. beifer.

Boroweky, Thierreich, I. 2. p. 15. n. o. der Bulienbeij]er.

Bhuménbach, Handb. d. Naturgefch. pq 9o. n. b. dei- Bärenbeifjer, Bullenbeiffer.

Bech.tein, Naturgefch. Deutfchl. I. p. 196. n.2. der Bullenbeifjer, Bürenhund, Bürenbeiffer, Wachthand.

Halle, vierf. p. 487. der Bullenbeiffer.

Neuer Schauplatz d. Natur, IV. p. I43. der Bullenbeiffer, oder Wachthund. 
Onomatol. hift. nat. II. p. 539. Canis maftinus; der Buillenbei/fer.

Bock, Naturgefch. von Preuffen, IV. p. x7. Bul. lenbeiffer.

Bïffon, vierf. II. p. 180. n. 16. der Bullenbeiffer, Tab. 36. fig. r.

Kriinitz, ökon. Encykl. XXVI. p. 354. n. r7. der Bullenbeiffer, Bärenhủad, Bärenbeiffer, Wachthund (von eirgigen: Schweifshund.)

Erxleben, Mammalia, p. 544. n. v.

Linné, amoenit. academ. IV. p. 46. n. 4. Canis Maftivus, feu Moloffus.

Graumiann, intr. in hift. rat, mamm. p. 79. n. o. Bullenbiffer.

- Anglicus. Die englifihe Dogge.

Zimmermann, geogr. Zoolog. II. p. 238. n. q; die englifche Dogge.

Schreber, Säugthiere, I. p. 324. n. 15. die englifiche Dogge.

Borowjky, Thierreich, I. 2. p. 15, n. p. dis énglifche Dogge.

Bechftein, Naturgefch. Deutfchl. I. p. 197. n. a. der englifche Hund, Dog, Kammerhund.

Halle, vierf. p. 488. die englifche Dogge, Tab. 32. Onomatol. hiftor. nat. II. p. 54i. Canis moloffus anglicus; die englifche Dogge.

Kriinitz, ökon. Encykl. XXVI. p. 357. n. 22. die - grofse, oder englifche Docke, der FleifcherMetzger-oder Schlächterhunid.

Bïffon, vierf. II, p. I86. n. 21. grofse Doggen, Tab. 37.

Erxleben, Mammal. p. 545. n. $\xi$.

Graumann, intr. in hift. nat. mamm. p. 80. n. q: englifche Dogge.

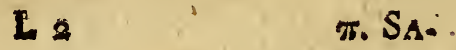




\section{itis Manmalia Ferce. Canis.}

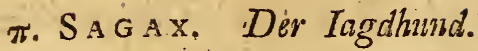

Miiller, Naturfyftem, I. p. 207 n. b. der Iägd. huind.

Zimmermann, geogr. Zoolog. II. p. 239. n. r. der Iogdhusd.

Schreber, Säugthiere, I. p. 224. 11. I6. der Iagdhund:

Borowfly, Thierreich, I. 2. p. 15. n. q. der Iagdhund, dent fiche Iagdhund.

Blumenbach, Handb. der Naturgefch. p. 90. n. c. der Iagdhund.

Bechftein, Naturgefch. Deutfchl. I. p. 198. n. 3. der Iagdhund.

Halle, vierf. p. 483. der Ingdhund.

Neuer Schauplatz der Natur, IV. p. 143. 13, 2. der Iagdhund (Spürhund).

Ononatol. hiff. nat. II. p. 539. der Togdhind. Schriften der berlin. Gef. naturf. Fr. VI. p. 429. Bock, Naturgefch, von Preuffen, IV. p. 16. dev" Iagdhund.

Erxleben, Mammalia, p. 546. n. 0.

Linné, Amoenit. acad. IV. p. 46. 1. 2. Canis Sagax.

Grammonn, intr. in hifor. nat. mamm. p. so. n. I. Iagdhund.

$\rho_{1}^{\prime}$ GaLlicus. Der Parforcehund.

Zimmermann, geogr. Zoolog. II. p. 239. n. s. der Parforcehund.

Schreber, Säugthiere, I. p. 325. n. I7. der Parforcehund.

Boronelky, Thierreich, I. 2.p. 15. n. r. der ?arforcehand.

Berhftein, Naturgefch. DeutCchl. I. p. 202. n.e. der Parforce-oder Lomflume. 


\section{Manumatia Ferae.}

Büffon, vierfüls. II: p. - I68. n. \& dev Iagdlund. Tab. 36.

Erxleben, Mammal. p. 546. n, $\pi$ :

Granmicnn, intr. in hif. nat. mamm.p. 80. n. s. Parforcehind.

o. Scior Icus. Der Schweifshind.

Zimmermann, géogr. Zool. II. p. 239. n. t. der Schweifshinnd.

Schreber, Säugthiere, I.p.325. n. ir. der Schueiifshiind.

Borowesky, Thierreich, I. 2. p. I5. n. s. der Schweis shund.

Bechftein, Naturgefch. Deutfchl. I. p. 20I. n. b. der Schweifshund:

Handbuch der deutichen Thiergefchichte, p. 55 . der Schweisshund,

Flemming, deutfcher Iäger, I. p. I74. fig. 2.

Erxleben, Mammalia, p. 547. n. $\varrho$.

Graumann, intr. in hift. nat. mamm. p. 80. n. to Schwei shuszd.

Aldrovand de quadrup. digit. p. 553 . Canis Sco. ticus Sagax; m. e. mittelm. Fig.

7. Venaticus. Der Leithund.

Zimmermann, geogr, Zoolog. IT. p. 239. n. u. der Leithund.

Schreber, Säugthiere, I. p. 225. n. 19. der Leiť hind.

Borousky, Thierreich, I. 2. p. I5. n.t. der Leithund.

Bechftein, Naturgefch. Deutchl. I. p. 199. n. a., der Leithnud, Spiishunnd.

Handbuch der deutfchen Thiergefchichte, p. 54 . der Leithuisd.

$$
\text { L } 3 \text { Heppe, }
$$


Heppe, praktifche Abhandlung vom Leithund. Auusb. 174I. 8.

Erxleben, Mammalia, p. 54\% n. $\sigma$ :

Graumann, intr. in hift. nat. mamm. p. 80. n. us Leithund.

บ. Av I cui arius. Der Hiihnerhund.

Miiller, Naturfyft. I. p. 209. n. I. der Hiihnerhund oder Wachtelhund.

Zimamermainn, geogr. Zoolog. II. p. 219. 11. v. der Hiihnernund.

Schreber, Säugthiere, I. p. 326. n. 20. der Hühnerkund.

Boronefky, Thierreich, I. 2. p. 15. n. u. der Hibihnerhund.

Blumenbach, Handb, d. N. G. p. 90. der Hiih. querknund.

Bechftein, Naturgefch. Deutfchl. I. p. 206. n. 6. der eigentliche Hiihnerhund, gemeiner Hühner. hund, Wachtelhunzd.

Halle, vierf. p. 485. der fpanifche Wachtelhund. Halle, vierf. p. 484. der bengalifche Tigerhund. Neuer Schaupl. d. Natur, IV. p. I44. n. 9. der Hihnevinund oder Wichtelsand.

Onomat. hift. vat. II. p. 540. der Hühnerhund. Bock, Naturgefch. von Preuffen, IV. p. 2I. der Hiihneren-oder Wachtelhund.

Kriinitz, ökon. Encykl. XXVI. p. 349. der bengalifche Spïr-oder Tigerhund.

Biiffon, vierf. II. p. I71. der Spürhund. Tab. 27. Biiffon, vierf. II, p. I7I. der Tigerhund, oder bengaliifche Spiörhund.

Erxleben, Mammalia, p. 547. n. $\tau$.

Linné, Amoenit, acad, IV.p. 47. n. 10. Canis avicúlarius. 
Graumann, intr. in hif, nat, mamm. p. 80. n. v. Fiihnerhund.

Q. A Quatilis. Der Wafferhind.

Zimmermann, geogr, Zoolog. II. p. 240. 1i. w. der Waferhund.

Schreber, Säugth. I. p.326, n.2x. der WafJerhund. Bechftein, Naturgefch. Deutfchl. I. p. 202. n. d. der Whafferhund.

Onomat. hif, nat. II. p. 539. der zottige Wafjerhussd.

Fermin, Befchreib. von Surinam, II. p. 106. dev W. afferhund.

Flemning, deutfcher Iäger, I. p. I8I. Tab. ZZ. fig. 4 .

Erxleben, Mammal. p. 548. n. v.

Granmann, intr, in hiftor. nat. mammal. p. 8I.' IV afferhund.

\section{x. Cursorius. Der Caurshund.}

Zimmermann, geogr. Zoolog. II. p, 240. n. y. der Curshund.

Schreber, Säugth. I. p. 327. n. 23. der Curshund. Bechftein, Naturgefch. Deutfchl. I. p. 208: n. e. der Curshund, Piirfchhund.

Flemming, deutfcher Iäger, I. p. I7r. Tab. TT. fig. $x^{\circ}$

Erxleben, Mammalia, p. 549. n. $\chi$.

Graumann, introd. in hifor, nat. mamm. p. $8 \mathrm{r}$. n. aa. Curshund.

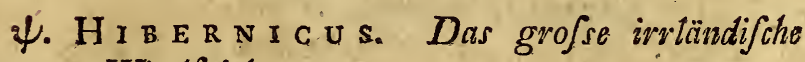
Wind fpiel.

Zimmermann, geogr. Zool. II. p. 240. n z. das grofse irrländische Windspiel.

$$
\text { L } 4
$$

Borou- 
Borowfky, Thierreich, I. 2. p. 16. n. w. das grof. Se irrländifche Windspiel.

Halle, vierf. p. 482. der Irrlïndifche.

Erxleberz, Mammal. p. 549. n. $\psi$.

Grammann, intr. in hif. nat. mamm. p. 81. n. bb. irrlëndifche Windhund.

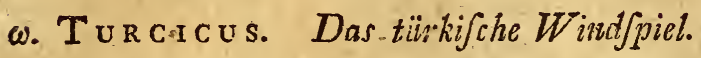

Zimmermanm, geogr. Zoolog. II. p. 24I. n. bb. das tiwrkif che Windspiel.

Schreber, Säugthiere, I. p.326. n. 25. das tiirkifche W ind fpiel.

Borow $\int k y$, Thierreich, I. 2. p. 16. n. x. das tïrkifche Windfpiel.

Erxleben, Mammalia, p. 549. n. w.

Graumann, intr. in hif. nat. mamm.!p. 8r. n. cce tiirkifche IVindfpiel.

«e. Grajus. Der gemeive Windhund.

Miller, Naturfyfem, I. p. 207. n.' c. der Wind. hund.

Zimmermann, geogr. Zool. II. p. 24r. n. cc. der gemeine $W$ indhund.

Schreber, Säugthiere, I. p. 327. n. 26. der gemeine. Windinund.

Borowlky, Thierreich, I. 2. p. 16. n. y. der gemeins Windhund.

Blimentbach, Handbuch der Naturgefch. p. 9I. n. i. das Wiudfpiel.:

Bechftein, Naturgefch. Deutfchl. I. p. 207. n. s. der gennive Windhund, Wind.

Halle, vierf. p. 481. der Windhund. Tab. 3.

Newer Schauplatz der Natur, IV. p. 143. n. 3. der IVináhund.

$\because$ Ononatol hift nat. II. p. 539. dev Windhund. Bock, 


\section{Mammalia Ferce. Canis.}

Bock, Naturgefch. von Preufen, IV. p. I6. dev W. indhund.

Kriinitz, ökon. Encyklop. XXVI. p. 346. der gemeine Windhind.

Biffon, vierf. H. p. 162. dev Windhund, Tab. 23. fig. 2. cias Windlpiel.

Erxleber, Mammalia, p. 550. n. ca.

Linne, Amoen. acad. IV.p. 46. n. 3. Canis graius. Graumann, intr. in hift. nat. mamm. p. 8I. n. dd. Windhund.

B€. HIR SU Tus. Der zottige Windhund.

Zimmemans, geogr. Zoolog. II. p. 24I. n. dd. dev zottige Windhund.

Scheber, Säugthiere, I. p. 328. 11. 27. der zottige Windthund.

Bechftein, Naturgefch. Deutfchl. I. p. 208. n. b. der zottige Windhind.

Evxleben, Mammalia, p. 5 z. n. $\beta \beta$.

Pennamt, Synopf. quadruped. p.I46. n. IV. $\beta .2$. Oriental Gre-hound.

Graumann, intr. in hift. nat. mamm. p. 82. n. ec. zottige Windhund.

\section{$\gamma \gamma$. I t a I I U s. Das kleine Windfpiel.}

Ziminermann, geogr. Zool. II. p. 24r. n. ee. das kleine Windspiel.

Schreber, Säugthiere, I. F. 328. n. 28. das kleine Wind/piel.

Bechftein, Naturgefch. Deutfhl. I. p. 208.,n. a. das Wind?piel, englifche Windspiel, dier kleine Windhund:

Erxteben, Mammal. p.55r. n. $\gamma \gamma$.

Graumunn, intr. in hift. nat. mamm. p. 82. n. ff. Wind/piel.

$$
L_{5} \quad \delta \delta . A E
$$


88. A E g y т I Us. Der tïrkifche nackte Hund. Miiller, Naturfynt. I. p. 210. n, I. der tiirkifche Hund.

Ziminermann, geogr. Zoolog, II. p. 24r. 11. ff. der türkifche nackte Hund.

Schreber, Säugthiere, I. p. 329. n. 29. der tiir. kifche nackte Hund.

Boroweky, Thierreich, I. 2. p. I6. n. z. der tiir. kilche nackite $H_{\text {nund. }}$

Blumenbach, Handb. der N. G. p. 9r. n. k. der ïgyptifche Hund, p. I8. Anm. 2.

Bechftein, Naturgefch. Deutfchl. I. p. 208. n. d. der tiirkifche nackte Hund.

Halle, vierf. p. 487. der titwisifhe Hund.

Neuer Schauplatz der Natur, IV. p. I44. n. Ir. der tiirkifche oder ägyptifch Hund.

Onomatol. hift. nat. II. p. 549. der nackte Hund. Bock, Naturgefch. von Preuflen, IV, p. 2I. dev tiirkifche odler Cyperhuizd.

Bïffon, vierfuifs. II. p. 178. n. 15. der tiirkifche, oder barbarifche Hund. Tab. 35. fig. I.

Krïnitz; ökon. Encykl. XXVI. p. 353. der ägy• ptifche, barbarifche oder tïrkifche Hund.

Erxleben, Mammal. p. 55r. r. $\delta \delta$.

Linné, Amoenit. acad. IV. p. 47. n. II, Canis aegyptius.

Graumann, intr. in hifor. natur. mamm. p. 82.

11. gg. der tïrkifche Hund.

b) Baftard vom türkifchen Hunde, und vom Harlekin.

Biiffon, vierf. II. p. 179. der tiirkifche Blendling. Tab. 35. fig. 2. 


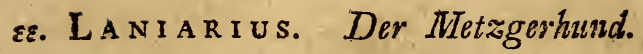

Zimmermann, geogr. Zoolog. II. p. 242. n.' hh. der Metzgerhund, der Haus - oder Banevihund. Schreber, Säugthiere, I. p. 329. n. 30. der Metz. gerhund.

Boronefky, Thierreich, I. 2. p. 16. n. aa. Canis villaticus; der Bauernhund, Haushund.

Bechftein, Naturgefch. Deutfchl. I. p. 197. n. b. der Metzger - ader Fleifcherhund.

Krïnitz, ökon. Encyklop. XXVI. p. 343. der Bauerhund, Banerreckel, Hanshund oder Hofhund; wenn er an die Kette gelegt wird, Kettenhund, niederf. Köther, Packan.

Biiffon, vierf, II, p. 159. n. I. der Bawernhund. Tab. 2r.

Erxieben, Mammal. p. 552. n. $\varepsilon \varepsilon$.

Linné, Amoenit. acad, IV, p. 46. n. x. Canis domefticus, $f$, villaticus?

Grammann, introd. in hifor. nat. mamm. p. 82 . n. hh. Bauerhund, Metagerhund.

32. APRIN Us. Der Sartfinder.

Zimmermann, geogr. Zoolog. II. p. 242. n. ii, der Sanfinder.

Schreber, Säugth. I. p. 329. n. 3r. der Saufinder. Bechftein, Naturgefch. Deutchl. L. p. I97. n. c. der Saufinoter. Saubeller.

Handb.d. deut. Thiergefchichte, p. 55. Salufinder.

Erxleben, Mammal. p. 553. n. 55.

Grannamn, introd, in hif, nat. mamm, p. 89. n. ii. Saufinder.

$\eta \%$ S utl zus. Der Sawiiden.

Zimmérmann, geogr. Zool. II. p. 242.n. kk. der Saurï̈den.

Schreber, 
Schreber, Säugth. I. p. 330. n. 32. der Sawvïdens. Bechftein, Naturgefch. Deutfchl. I. p. 197. n. d. der Scurriulen.

Erxleben, Mammal. p. 553. 11. $\zeta \zeta$.

Granmani, intr. in hift. nat. mamm. p. 82.n.kk. Sauriidîen.

99. Vertagus. Der Dachshund.

Miiller , Naturfyft. I. p. 209. n. h. der Dachs. hiund.

Zinmermann, geogr. Zoolog.II. p. 242. n. Il. der Dachshund.

Schreber, Säugthiere, I. 330. n.-33. der Dachshunid.

Borowfky, Thierreich, I. 2. p. I6. n. bb. der Dachshund.

Blumenbach, Handbuch der Naturgefch. p. pr. 11. h. der Dachshind.

Bechftein, Naturgefch. Deutchl. I. p. 209. der Dachshund, Dachshriecher, Dachsfoliliefer, Dachswiirger, Dachrffucier.

Halle, vierf. p. 485. dev Dachishund.

Neuer Schaupl. der Natur, II. p. 285. Dachshand. IV. p. I44. I45. Dachshund.

Onomat. hif, nat. II. p. 440. Canis muftelinus, Canis vertagus, Dachshuına.

Kriinitz, ökon. Encyklop. XXVI. p. 350. Dachshund, Fuchshund; VIII. p. 635 ff.

Bock, Naturgefch. von Proúfen, IV. p. 20. der Dachshund.

Büffon, vietfüls. II. p. I'72. die Dachshunde. Erxleben, Manmal. p. 553. 1. ทи.

L.inné, 'Amoenit. acad. IV. p. 47. n. 8. Canis V́ertagus. 
a) Mit geraden Beinen.

Bechftein, Naturgefch. Deutfchl, I. p. 210. n.b. der geradefihenklichte Dachshund.

Biiffon, vierfüfs. II. Tab. 29. fig. 2. der Dacks mit geraden Beinen.

Erxleben, Mammalia, p. 554. n. S.9.

Graumann, intr. in hifor. nat. niamm. p. 83 . n. $\mathrm{mm}$.

b) Mit krummen Beinen.

Bechftein, Naturgefch. Deutfchl. p. 210. n. a. der krummbeinige Dachshuma.

Biiffon, vierf. II. Tab. 39. fig. I. der krummbeinigte Dachshund.

c) Der zottige Dachshund.

Zimmermann, geogr. Zool. II. p. 243. n. mm. der zottige Dachshund.

Schreber, Säugthiere, I. p. 330. n. 34. der zottige Dachshurd.

Bechftein, Naturgefch. Deutfchl.I. p.210. n.c. der zottige Dachshund.

Biiffon, vierf. II. p. 199.'n. 25. Hunde von Burgos?

Erxleben, Mammal. p. 554. n. "6.

Granmann, introd. in hiflor. nat. mamm. p. 83. n. nn.

3. AMER I A ANus. Der Alho.

Zimninermann, geogr. Zoolog. II. p. 252 . n. c. der Alko.

Schieber, Säugth. I. p. 342. der Alko.

Borow fky, 'Thierreich, I. 2. p. I6. n.. cc. dev Alko. Neuer Schaupl. der Natur, I. p. 178. der Alka.

Klein- 
Klein, Quadrup. difp. p. 68. Canis mexicanis,' monftrofo corporis habitu, capite parvo, collo breviflimo et obefo, dorfo gibbofo, cauda brevi. Klein, Claflif. der vierfüfs. 'Th. p. 205. mexikaniicher Hund.

Klein, natür!. Ordnung d. vierf. Th. p. 72. n. 2. mexikanifcher Hund.

Halle, vierf. p. 490. dev mexikanifche Hund. Biiffon, vierf. II. p. 190. n. 27. der mexikanifche Hinnd.

Goeze, Natur, Menfchenleben und Vorfehung,

V. p. 331. der fogenannte wilde Hund?

Acofa, hint. nat. des Indes, p. 46.

Exxleben, Mammal. p. 554. unten.

a) Der IVichacanens.

Zimmermann, geogr. Zool. II. p. 253. n. d. der Michuacanens.

Gesner, Thierbuch, p. 225. Ytzcumbepotzotli. Ein indianifcher Hund. Canis mexicana; fig. p. 226. Hernand.

b) Techichi.

Zimmernann, geogr. Zoolog. II. p. 253. n. e. der Techichi:

Molina, Naturgerch. von Chili, p. 239. das Techichi.

vix. TER a a n ovae. Dei Hind von Nertfuszdland.

Blumenbach, Handb. der Naturgefch. p. 90. ni. c. der Nenfundländer.

$\rightarrow \quad \rightarrow$

Ausführliche Gefchichte der Hunde, von ihrer Natur, verfchiedenen Arten, Erziehung, Abrichtung, 


\section{Mammalia Ferae. Canis.}

richtung, Krankheiten, und mannichfaltigen pliarmacevtifchen Gebrauch. 1.pz. I78I. s.

Th. Bartholini variae in canibus obfervationes; in deffen hiftor. anatom. rarior. Cent. I. II. Amft. 1654. 8. Cent. 11. Hift. 82. p. 287-290.

D. überf. von mancherley Anmerkungen in den Hunden, in deffen ungewöhnlichen anatomifchen Gefchichten. I und 2 Hundert. Francf.

a. M. $1657 \cdot 8 \cdot$ p. $385 \cdot 388$.

Ger. Blafii, obfervata anatomica in cane; in defSen Obfervatis anat. pract. in homine brutisque variis. L. B. ef Amnt. 1694. 8. P. 33-35, und in M. B. Valentini amphith. zootom. Fref. a. M. I720. f. p. I83.

Io. Caji, de canibus britannicis liber unus. Lond: 1570. f. ib. 1724.4 .

Iames Duglas, myographiae comparatae fpecimen. Lond. 1707. I2. und L. Bat. I728. und 1738.8. von Schreiber.

Von der Natur und Wartung der Hunde f. Toh. Bennh. von Fifcher liefl. Land wirth Chaftsbuch. Halle I753. 8. p. 250.

Von den/verfchiedenen Arten der Hunde, einige curieufe Hiftorien von ihren Tugenden, und durch vernünftige Anführung erlangten $\mathrm{Ge}$ fchicklichkeit, und einige juriftifche Ergötzlichkeiten von diefen Thieren, f. Ioh. Chrift. Fritfch feitfame Gefchichtem. I, Lpz. I730. 4. p. 308.

Garengeot, Myotomie humaine et canine, à Par. I728. I2. 1750. I2. 2 Voll.

Io. Don. Horffii progr. ad anatomen canis gravio dae. Giefr. 1639. 4.

Car. Limnei diff. de Cyrographia. Upf. 1753. 4. fteht auch in defJ. Amoen. acad. Vol. IV.p.43. D. überí. 
D. überf. der Hund, befchrieben von Herrn Carl von Linné; in Scirebers neuer Camerallch. 4 Th. Halle 1766. gr. 8. p. ror.

De cane ciusque hiftoria naturali. S. Io. Tac. Manget $i$ bibl. pharmac. med. T. I. Genev. I730. f. p. 465 .

Marc. Anvel. Severini oblerv. anat. in cane foemina aqua fuffocata, in deffen Zoot. Democrit. p. 358 . und in $M$. B. Valentini amphith. zoo. tom. Fref. ad M. 1720. f. p. I39.

Io. Iahn, de cauibus notabiles quaedam obf. in defen Specul. phyf. math. hiltor. 'Tom. II. Norimb. I696. f. p. 303.

Naturgefchichte der Hunde, nach ihren verfchiedenen Arten, Erziehung und Abrichtung, nebf Anweifung, wie die Krankheiten der Hunde zu erkennen und zu heilen find. Augsb. 8. 1790.

Berger, gründliche Anweif. einen Hühnerhund abzurichten. Weimar 1787 .

\section{* *}

Frit $\int c h$, von den Urfachen der vielerley Biláun. gezz und Grölsen der Hunde; im Naturforfcher VII. p. 52.

Verfchiedene an Hünadimnen angeftellte leobachtungen. - Biiffon, allgem. Naturge Ch. III. p. 303. IV. p. 40.

Wilde Hisade. - Samml. zur Phyf. u. Naturgefchichte, II. p. 61о. - Sparrmann, Reile n. d. Vorgeb. d. g. Hoffn. p. I52. - Krïnitz, ökon. Encykl. XXVI. p.366. - Allgemeine, Reif. V. p. 89.

Begattet fich mit dem Wolf. - Zimmermenn, geogr. Zoolog. I. p. 15\%. - Baftarde vom 


\section{Mammalia Ferae. Canis.}

Hunde und einer Wölfin; Naturforfcher XV. p. 25. - Nord. Beytr. I. p. 154. - Pinnant, arct. Zool. II. p. 43.

Ift kein Originalthier. - Zimmermann, geogr. Zool. III. p. 190. I. p. 142. 143. 148. Hamb. Magazin, XI. p. 590. -

Tolle Hunde. - Kemme, de Hydrophobia eius. que fpecifico, meloe maiali et profcarabaeo. Hal. 1781. - Selle, neue Beytr. zur Natur. und Arzneywiffenfchaft, Berl. I Th. - Walther, Naturgefch. Deutfchl. p. 152. - Baldinger, Magaz. für Aerzte, IX B. - Goeze, Allerley, neue Aufl. III. p. 236. - Magazin für die gerichtl. Arzneykunde, II B. - Bunzlauer Unterhalt. III. p. 272. 300. II. p. II6. Kriinitz, ökon. Encyklopäd. XXVI. p. 488. Halle, Magie, I. p. 396. - Journal für Deutfchland, 1786. p. 217. - Scherf, Ar. chiv der medicinifchen Policey. _- Allgem. Magazin, I. p. 299. - Deutfche Zeit. 1784. p. 65.67.68.134. - Allgem. deut fche Bibl. LXIII. p. 99. LXI. p. 109. - Schwedifche Abhandl. XVI. p. 79. - Beckmann, phyr. ökon. Bibl. XIII. p. 133. - Kiihn, Beobach. tungen über den Tollwurm der Hunde, im Naturforfcher XVI. p. 89 ff. - MedicinalUnterricht zur Vorbeugung der Tollheit der Hunde, und Verhütung der gefährlichen Fol. gen derfelben. 4. Duisb. 1790. - Bechftein u. André, Spatziergänge, I. p. I28. $-U n$. zer, im Arzt, II. p. 427. 429. III. p. 280. 344. 358 . 387 IV. p. 579.

Soll fich mit Schafen belaufen. - Abildgaard, Unterricht von Pferden, Kühen, Schafen cet. Lpz. 577x. I. p. 240. (verdiente nähere Beflätigung.) 
Kusirt Lähnungen und Flechten. - Lichtenberg, Magazin für das Neuefte ctc. II. 4. p. 195. 96. vergl. mit Krünitz am angef. 0. p. $45^{\circ}$.

Mhit Papageyköpfen. - Hiftoire et mem. de l'acad. roy. des Sc. infcript. et bell. lettres de Touloufe. Tom. I. 1782. - Götting. gel. Zeit. d. 28 Febr. 1786. - Lichtenberg, Magazin für das Neuefte etc. II. I. p. 189.

Redende Hunde, - Neue Anmerk. über alle Theile der Naturlehre, III. p. 534. - Mercure de France, Janvier, 1728. - Sonderbare Erzähl. aus der Naturgefch. I. p. 34. - Bonnet, Betrachtung. über die Natur, 4 Aufl. II. p. 465. 1. 7. - Biiffon, allgemeine Naturgefch. V. p. 20. n. ro. - Halle, Magie, II. p. 408 .

Mit verflïnmelten Schwönzen, werfen auch häufg Solche Iunge. - Blumenbach über den Bil. duigstrieb. - Götting. gel. Anz. 1786. St. 6.

'-Wird gemäftet und gegeffen. - Forfter, Reife um die Welt. Edit. in 8. II. p. 289. - Schwed: Abhandl. XIV. p. 133. - Berlin. Samml. IV. p. 548. - Biiffon, allgem. Naturgefch. VI. p. 130. - Högfröm, Lappm. p. 150. Allgem. Reif. XVII. p. 324. XIX. p. 290. Grmelin, in den götting. Reif. V. p. I26. f. Trampler; Wallfifchfang. Lpz. 1771.8.p.63.Nerhoff, China, p. 348. - Sparmunn, Reife nach dem Vorgeb. d. g. H. p. 87. Forfter, Bemerk. auf feiner Reife, p. 177 .

Mit den Fellen Kleider gefuttert und Miitzen eingefafst. Forfter. Bemerk. auf feiner Reife um die Welt, p. 167. - Hawkesworth, Seereifen, III. p. 45. 46. - Steller, Kamifchatka 
p. 137. - Linné, Amoęnit academ. IV.

p. 62.

Iunge, mit Bandwürmern, können nicht bellen. -

Goeze, Naturgefch. der Eingeweidewürmer,

p. 27.

Sollen in Aegypten und Syrien nicht toll werden. Volney, Voy. en Syrie I. p. 217. Beckmann, Bibl. XV. p. 52.

Unterfchied des Bellens und Heilens. - Samml. zur Phyf. u. Naturgefch. I. p. 346.

Beyfpiel von langer Enthaltung von aller Nahrung: Voigt, Magazin für das Nevelte etc. IV. 3. p. 177 .

Wird von einem Aale vergiftet. - Allgem. Ma. gazin, I. p. 83 .

Eine Hiindin nährt zièy junge Kater. - Dafelbft p. 35 I.

Stammracen der Hunde. - Bhumenbach, Beyträ: ge zur Naturgefch.

Vorn Abrichten der Triiffelhunde. - Schlef. ökon.? Nachr. I. p. 24. ff.

Durch Frefen des Hundegrafes von Vergiftung ei. ner Kröte geheilet. - Schriften der berlin. Gefellfch. naturf: Freunde VI. p. 447 .

Defen Staminväter der Wolf, der Futchs, uind der Schakal. - Zinnmernann, geogr. Zool. I. p. 138 ff. - Pallas, obf. fur la formation des montagnes. Petersb. 1777. p. 15. die No. te. - Pallas, Naturgefch. merkiv. Thiere, XI. p. 3.

Kïnftliche Befruchtung einer Hiindin. - Spallan. zani, Verf. über die Erzeugung der Thiere II. p. $249 \cdot 304 \cdot 343$. 
2. Lupus. Der Wolf. (1)

Müller, Naturfyftem, I. p. 219. n. 2. der Wolf. Tab. 3I. fig. I. Suppl. p. 28.

Zimmermann, geogr. Zool. I. p. I48. II. p. 234. n. $135 . \operatorname{der} W o l f$.

Lefke, Naturgefch. p. 133. n. 2. der Wolf.

Borowsky, Thierreich, I. 2. p. I8. n. 3. der Wolf.

Bhumenbach, Handb. d. N. G. p. 92. n. 2. Canis

(Lupus) cauda incurvata; der Wolf.

Bechftein, Naturgefch. Deutfchl. I. p. 223. dor Wolf. Tab. 3.

Funke; Naturgefch. I. p. 99. der Wolf.

Ebert, Naturlehre, I. p. 368. der. Wolf.

Halle, vierf. p. 496. der gemeine Wolf.

Meyer,

(1) Lange des Kürpers ohagefahr viertehalb Fufs, Höhe dritcehals Fufs, der Schwanz anderthalb Fufs lang. So gierig der Wolf ift, fo fallt er doch nicht eher Menfchen an, bis ihn der aufferfte Hunger dazu zwingt. In Ermanglung des Fleifches frifs er auch Moos, Baumknofpen, u. dgl. Durft kann er weniger als Hunger ertragen, Er in ein gefahrlicher Feind des Rothwildes, und in den nordifchen Ländern der Elenne und Rennthicre; doch hat man Beyfpiele, lafs Wülfe yon Rennthieren durchftofsen find. Fr grabt auch Leichen aus, und eine folche náchtiche Erfcheinung auf Kirchhüfen mag vielleicht Anlafs zu der alten Sage von Withrwölfex gegeben haben. In Luandern, wo er fich zugleich mit dem Baren findet, herrfcht diefer doch mehr im Sommer, der Wolf mehr im Winter. Schafe mit Wolfskoth beftrichen, follen vor feinem Angriff ficher leyn. Sein Balg giebr gutes Pelzwerk. Der Zahne bedienen fich, in Sticle gefafst, Mahler, Goldfchmiede, Vergolder u. a. m. zur Clattung und Polirung ihcer Arbeiten. Das Fleifch vom Wolfe verachten, fogar gebraten, die Hunde, doch effen es Kalınucken, Tungufen, und die armften Lappländer. In England foll 3680. dér letzte Wolf gefchoffen feyn. In Perfien richtet man Wülfe zum Tanze $2 \mathrm{~b}$; und die Arnerikaner bedienten fich ehemals nichts als gezahmter Wölfe zur lagd. 
Meyer, Thiere, I. p. 27. Tab. 35. der Wolf. Ab. bild. gut.

Beckmann, Naturhif, p, 27, n. i2. der Wolf.

Gatterer, vom Nutzen und Schaden der Thiere,

I. p. 361. n. 206. der Wolf.

Neuer Schauplatz der Natur, X. p. r. des Wolf.

Onomat. hift, nat. IV. p. 87. der Wolf.

Sander, ökon. Naturgefch. I. p. 250 . der Wolf.

Handbuch der Naturgefchichte, I. 2. p. I34. der Wolf.

Kraft, Ausrott. graufamer Thiere, II. p. 547. der Wolf.

Handbuch der deutfchen Thiergefchichte p. 58. der Wolf.

Gesner, Thierb. p.348 ein Wolf. Abbild. fehr fchlecht.

Pennant, arct. Zool. II. p. 39, n, i2. der Wolf. Klein, Quadrup, difp. p. 70, Lupus vulgaris. Klein, Claffif. d. vierfüfs. Th. p. 209. gemei. ner Wolf.

Klein, natürl, Ordn, d, vierf. Th, p. 74. n.I. ge. meiner Wolf.

Fifcher, Naturgefch. von Livland, p. 50. n. 4 . der Wolf.

Fifcher, Zufätze zu Livl. p. 39.

Pallas Reife durch Rufsl. I. p. 234. 281. II. p. 660. III. p. ro. I7.

Hupel, topogr, Nachr. von Liefland und Ehftland, iI. p. 428. Wölfe.

Bock, Naturgefch, von Preuffen, IV. p. 28.n. 6. der Wolf.

Gooze, europäi Che Fauna, I. p. 135. dev Wolf. Schriften der hall. naturforfch. Gefell Ch. I. p. 86. Pallas Reife, Auszäge, I. p. 198. II. p. 15. III. p. 373 . 
Höft, Nachricht. von Marokos und Fes, p. 291. ier Wolf:

Carver, Reifen in d. Innere von Amerika, p. 362. der W.olf.

Cuhn, Samml, von Reifen d, d. Innere von Afri$\mathrm{ka}, \mathrm{p} .396$.

Taube, Befchreib. von Slavonien, p. 19. der IVolf:

Pontoppidan, Naturgefchichte von Norwegen, II. p. 34.

Steller, Befchr. von Kamt fchatka, p. II7. Wölfe, Kuorchu.

Dapper, China, p. 138. Wolf. Adanfon, Reife nach Senegall, p. 174 .

Leen. . Nachr, von den Lappen in Finnmarken, p. 73 .

$\boldsymbol{R}$ imer, Nachr. von der Küfte Guinea, p. 296.

Kalm, Reife nach d. nördlichen Amerika, II. p. 387.423 .

Georgi, Rufsland, I. p. 208. Wölfe.

Gmelin, d. ä. Reife durch Sibirien, I. p. 329.40r. II. p. 203. 470 . 126.

De la Caille, Reife n. d. Vorgeb. d. g. H. III, p. 247 .

Biiffon, allgemeine Naturgefchichte VII. p. I36. I38. 146.

Ludolf, hift. Aethiop. I. c. Io. \$. 49.

Neuhoff, Gefandfch. p. 348. Wölfe.

Miiller, Gedanken vom Iagen und befonders von der Wolfsjagd, Ien, $\mathbf{1 7 4 5}$.

Schreber, neues fchwedifches Magazin. I. p. 279. Wolfsjagd.

Danziger wöchentl, Anzeigen und Nachr, I76I. St. 28 .

Fabric. Reife nach Norwegen, p. 2 I\$. 
Leppechin, Tageb. der ruff. Reife; I. p. 20. 2 I. Linné, Reif. durch Schweden, I. p. 90.

Gmelin, Reife, in den Götting. Sammlungen V. p. 126. 127 .

Krafcheninnikow, Befchreib. von Kamtfchatka, p. 125 .

Büfforn, vierf. IV. p. 59. der Wolf. XV. p. 92. Bhimenbach, Beytr. zur Naturgefch. I. p. 29. Voigt, Magazin für das Neuefte etc. V. 2. p. 186. Pallas, nord. Beytr. I. p. 154 .

Miiller, Samml. ruff. Gefch, III. p. 546, 6,08. Wilfe.

Döbel, lägerpraktik, fol. p. 34. Wolf.

Ludreig, Kaufmannslexicon, V.'p. 961. Wolf.

Schwed. Abhandl. XV. p. 245. XXXV. p. 77. V. p. 188 .

Hamb. Magazin, VI. p. 59r. XIV. p. 6r4.

Geogr. hift. und phyf, Mancherley, II. p. 99 bis II4.

Leipz. Wochenblatt, IV. p. 97 - 103. p. 105-107. Reich der Natur und Sitten, III. p. 254.

Iherlin. Samml. V. p. 258.

Rübner, diff. de Lupo. Argent. 1686. 4.

Paullini, Lycographia. Frcf. 1694. 4.

Iahn, diff. de Lupo. Witt. I673. 4.

Fean de Clamorgan, la chaffe du Loup etc.

à Luneville, 1579. 4.

Erxleben, Mammal. p. 556. n. 2. Canis (Lu. pus) cauda incurvata.

Linne, Sylt. N. Edit. II. p. 44. Canis cauda rerta, corpore breviore.

Linné, S. Nat. Edit. VI. p-5. n. 2. Canis cauda incurvata.

Linné, S. N. Edit. X. I. p. 39. n. 2. Canis (Lupus) cauda incurvata. 
Linne', Faun. Suea I. p. 5. n., 13. Canis cauda incurvata.

Linné, Faun. Suec. II. p. 3. n. 6. Canis (Lupus) cauda incurva.

Linné, Muf. Adolph. Fried. I. p. 6. Canis (Lupus) cauda incurva.

Graumann, introd. in hif, nat, mamm. p. 83. II. der Wolf.

Gatterer, brev. Zool. I, p. 148, n, 2. Canis (Lu. pus) cauda incurvata; der Wolf.

Iiiller, prodrom. Zoolog. dan. p. 2. n. 5. Canis (Lupus) cauda incurvata.

Kramer, auftr. p. 313. Canis cauda incurvata. Rzaczynski, hift. nat. Polon. p. 219. Auct. hif. nat. Polon. p. 213. Lupus.

Sehefferi Lapponia, p. 338. Lupi.

Charleton, exercitat, p. 15. Lupus vulgaris. Olaus IMagn. de gent. feptentrional. p. 6Ir. Lupus.

Schwenkfold, theriotroph. Silef. p. 106. Lupus, Wagner, hif. nat. Helvet. p. I78. Lupus. Valentin, Amphitheatr. zootom. p. I40. (Ana. tom.)

Chardin, Voyage. Lond. 1686. p. 291.

Fifcher, mifcellan, phyf. med. 1729. p. II 8 .

Ariftot. hiftor, anim. V. c. 2. n. 2I. VI. c. 35. n. 419-42I. VIII. c. 9. n. 10I. $\Lambda$ uxos.

Plin. hiftor. nat. VII. c. 58. X. c. 63. 73. XI. c. 37. 43. 49. 50. Lupus,

Aclian. de nat. animal. III. c. 6. IV. c. 14. is. V. c. 19. VII. c. $20 . \quad$ X. c. 26. $\Lambda$ uxos.

(Weiffe Wïlfo. - S. Fifcher, Zimmermans, Carver, a. a. 0 .) 
8. Mexicanus. Der mexikanifche Wolf. (')

Miiller, Naturfyf. I. p. 228. n. 8. der mexikani. Sche Fuchs.

Zimmermann, geogr. Zool, I. p, I49. II. p. 234 . n. a. der mexikanifche Wolf.

Schreber, Säugth. III. p. 352, n. 3. der mexikani. fche Wolf.

Halle, vierf. p. 500 . der mexikanifche Wolf.

Halle, vierfüls. p. 49r. der nackte amerikanijche Hund.

Gesner, Thierb. p. 553. ein indianifcher Wolf. Biiffon, vierf. II. p. Igr. der nackte amerikank. fche Hund.

Erxleben, Mammal. p. 559. n. 3. Canis (Mexicanus) cauda deflexa laevi, corpore cinereo, fafciis fulcis maculisque fulvis variegato.

Graumann, intr. in hift. nat, mamm. p. 84. III. der mexicanifche Wolf.

Gatterer, brev. Zoolog. I. p. 149. n. 3. Canis (Mexicanus) cauda deflexa laevi, corpore cine. reo, fafciis fufcis maculisque fulvis / variega. to; der mexikanifcho Wolf.

Nieremberg, hift. nat. p. 180. Lupus indicus.

Gronov. Zoophyl. I. p. 8. n. 30. Canis cinereus, maculis fulvis variegatus, taeniis fubnigris a dorfo ad latera deorfum hinc inde deductis. Pennant, Synopf. quadruped, p. 151. the Mexi. can Wolf. (Mit dem vorhergehenden ver. bunden.)

\section{5}

9. Thous.

(2) Kommt an Givise ue. rhergehenden bey, if auch eben fo räuberifch; wagt fich an Stiere, zuweilen auch an Menfehen, - Fernandez und mit ihm Zimmermann, fehn ihn blofs als eine Spielart des gemeinen Wolfes an. 


\section{Mammalia Ferae. Canis.}

9. Tноч s. Der Jurinamifche Fatchs. (3)

Miiller, Naturfylt. I. p. 228. n. 9. der Jurina. mische Fuchs.

Zimmermann, geogr. Zoolog. II. p. 25r. n. 147. der furinamifiche Fuchs.

Schreber, Säugthiere, III. p. 37r. n. I4. der $\int u$ rinamifche Fuchs.

Büffon, vierfüfs. XV. p. 8. n. 'T. de!" Jurinamische Fuchs.

Exxleben, Mammal. p. 574. 11. I4. Canis'(Thous) cauda deflexa laevi, corpore fubgrifeo, fubtus albo.

Pennant, Synopf. quadr. p. I60. n. II\%. the Suriinam Dog.

Granmann, intr. in hift. nat. mamm. p. 85. XIV. dev furinami che Fuchs.

Gatterer, brev. Zoolog. I. p. 152. n. 14. Canis (Thous) cauda deflexa laevi, corpore fubgrifeo, fubtus albido; der furinamifche Fuchs.

3. Hyana. Die Hyäne. (4)

Miiller, Natưffyft. I. p. 222. 11. 3. die Hycinne. (Tab. 3̧. fig. I. gehört zur folgenden.)

Zinumer.

(3) Ein noch nicht hinlänglich bekanntes Thier.

(4) Diefe Hyäne in von Alten und Neuern oft mit dem Vielfrafs, dem Schakal, der Zibethkatze und dem Bavian verwechfelt worden. Ihre Kennzeichen find: 1) die Gröfse eines ziemlichen Hirtenhundes ; 2) der wolfsännliche Kopf; 3) aufrecht ftehende fteife, ganz nackte Ohren; 4) ein dicker, bemähnter Hals; 5) fchwarze Querftreifen am Leibe und Beinen; 6) ein von fteifen Haaren 'aufrecht ftehendet Rückenkamm; 7) eler Schwanz mit einem geringelten Haarbufch; 8) zwifchen dem Schwanze und After eine Querfpalte und Mofchusbeutel; 9) die Stimme kreifchend und katzenartig; 10) die Farbe weifsgraulich. - 
Zimmermann, geogr. Zool. II. p. 27. die Hyäne.

p. 56. n. 148. die geftreifte Hyöne.

Lefke, Naturgefch. p. I34. n. 5. die Hyöne.

Borow ky, Thierreich, I. 2. p. 23. n. II. die Hyä: ne, Grabthier, Abendwolf.

Blumenbach, Handb. der Naturgefch. p. 94. n. 8.

Canis (Hyaena) villofus, nigricans, facie nigra, iuba cervicis dorfique; das Grabthier, der Abendwoolf.

Funke, Naturgefch. I. p. 154. die Hyäne. Ebert, Naturlehre, I. p. 330. die Hyäne.

Halle, vierf. p. 500, der Meevwolf.

Gatterer, vom Nutzen und Schaden der Thiere,

I. p. 383. n. 218. die Hyäne.

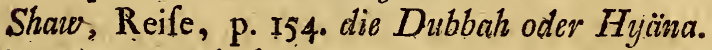

Neuer Schaupl, der Natur, IV. p. I8s. die Hyän6.

Onomat. hiff. nat. IV. p. 392 . Hyaena.

Niebuhr, Befchreib. von Arabien, p. 166. die Hyäne.

Cuhn, Samml, von Reif, in d. Innere von Afrika, p. 365 .

Goeze, Natur, Menfchenl. u. Vorf. I. p. 169. n. I. die kleine zottige, grane, hinten über den Rii. cken und an den Hiiften fchworarageftreifte Hyäne nit einem ftumpffpitzien IWolfsmant, und einem Schweinsborftigen Riickenkamm; die Wolfs. hyäne. p. 170. die zottige geftreifte Hyäne. Berlin. Samml. II. p. I86. die Hyäne.

Schreiben

Von feinem Wühlen in der Erde, und Ausgraben der Leichen hat das Thier den Namen Grabtbier erhalten. Es nährt fich auch von Wurzeln der Gewächfe, und den juagen Schöfslingeh der Palmbäume; und kạnn lange ohne 'Speeife dauern, Das Volk um Algier bildet fich ęin, dafs das Gebirn zu Zaubereyen dienlich fey, daher nach den Gefetzen der Kopf in Gegenwart ẹiniger Zeugen vérgraben werden mus. 
Schreiben eines Naturforfchers in Languedoc an einen feiner Freunde, worinn das Raubthier, die Hyäne, phyfikalifch befchrieben; und in Kupfer abgebildet ift; a. d, Franz. Francf, $u$, Lpz. I765. gr. 8.

Biiffon, vierf, VI. p. 320 . die Hyäne, m.e.Fig, Bat $\int c h$, 'Thiere, I. p. 189. die Hyäne. RiifJel, natürl. Hift. von Aleppo; in Hamburg. Magazin, XIX. p. 617. die Hyärze.

Erxleben, Mammalia, p. 575. n. 15. Canis (Hyaena) cauda recta, pilis cervicis erectis, auriculis nudis, pedibus tetradactylis.

Limné, S. Nat. Edit, VI, p. 5, n. 4. Canis pilis cervicis erectis longioribus.

Linve', Syft, Nat, Edit. X, I. p, 40. 11. 3, Canis (Hyaena) cauda recta anuulata, pilis cervicis erectis, auriculis nudis.

Limné, Syft, Nat. Edit, XII. I. p. 58. n. 3. Canis (Hyaena) cauda recta annulata, pilis cervicis erectis, auriculis nudis, palmis tetradactylis, Io. Vesling, obfervat. anatom. c. 6.

Nova act. Societat. Upfal. Vol. I. 7773 . p. 77 .

Ariftot. lib. de mirabilibus aufcultationibus; ex. plic. a Io. Beckmann. Gotting. I786. 4. 1) 323. Happel, relat. curiof. I. p. I86. das Wanderthier zu Fontainebleau?

Graumann, intr. in hif, nat, mamm. p. 85. XIV. Hyäne, Vielfrass.

Gatterer, brev. Zoolog. I. p. I53. n. 15, Canis (Hyaena) cauda recta, auriculis nudis, pedibus tetradactylis, corpore villofo, nigricante, fa. cie nigra, iuba cervicis dorfique. Hyïne, Abendwolf, Grabthier.

Severin, zool. Hungar. p. 6I. u. I. Hyaena, Lupus marinus. 
Nieremberg, hif. nat. p. 8x. animal necrophagum, five iefef, feu hyaena.

Kramer, Auftr. p. 373. Canis pilis cervicis ere. ctis longioribus.

Aviftot. hift. anim. VI. c. 32. n. $401-406$ VIII. c. 9. n. 102. 'T $T_{\text {Gover, }} \Gamma \lambda$ avos.

Plin: h.n.VIII.c.30. XI.c. 37.XXVIII.c.8.Hyaena. Aeliant, hiftor. animal. I. "c. 25. III. c. 7. VI. c. I4. VII. c. $22 .{ }^{\prime} T$ atve. Sirach, Kap. 13. v. 22. Hyäna. Leon. Afric. defcr. Afr. p. 756. Dabuh.

10. CR Oc UT A. Die gefleckte Hyäne. (s)

Zimmermann, geogr. Zool. II. p. 256. n. 149. die gefleckte Hyäne.

Schreber, Säugth. III. p.374. Spotted Hyaena Penn. Boroweky, 'Thierreich, I. 2. p. 25, n. 12. der Tis. gerwolf.

Onomatol, hif, nat. III. p. 474. Crocuta. Sparrmann, Reife nach dem Vorgeb. d. g. $H$. p. 36. 153. der Tigerwolf, die Hyäne.

Vaillant, Reife in d. Innere v. Afrika, t. p. 205. 206. 284. f. die Hyäne.

Forfter, Reife um die Welt, Ed. in 8. I. p. 87. fieckichte Hyöinen.

Gatterer, vom Nutzen und Schaden der Thiere, I. p. 384. n. 219. die gefleckte Hyäne.

Zauf chner, Beftimmung der Hundsart Krokute. Prag, 1788.4. m. e. Fig.

Goerze,

(5) Ihre Kennzeichen find: 2) Gröfse eines tarken Hundes; 2) der Kopf flach und grofs; 3). fehr kurze fpitze Ohren; 4) Gefiche und Obertheil des Kopfs fehwate ; st der Kürper rüthlich braun, mit runden (chwarzen Flecken; 6) der Schwanz kurz und haarigt; 7) das Gebifs fürchterlich; 8) die Stinme kakkernt, wie das menfehliche Lachen. 
Goeze, Natur, Manfchenl. und Vorf. I. p. 169. I73. I75. die gröfsere gefleckte tigerartige $H y \ddot{a}$ ne, mit länglichterm Kopfe und gröfserm Manl. Biiffon, vierf. VI. p. 339\% die geflechte Hyäne. Graumann, intr. in hiftor. nat. mamm. p. 85 . XVI. Tigernolf.

Gatterer, brev. Zoolog. I. p. I54. n. I6. Canis (Crocuta) cauda recta, corpore nigro maculato, pedibus tetradactylis; die gefleckte Hyäne.

7. A U RE U S. Der Schakall. $\left({ }^{6}\right)$

Miilter, Naturfyn. I. p. 227.n. 7. der Goldwolf. Tab. 30. fig. I.

Zimmermann, geogr. Zool. II. p. 33. der Schakkal und die Adive. p. 244. n. I37. der Sihakall. I. p. 141. der Iackhal.

Lefke, Naturgefch. p. I34. n. 4. der Schakai. Borowfky, Thierreich, I. 2. p. 16. n. 2. der. Goldhoolf, Schakall, Iakal. Tab. I2. A.

Blumenbach, Handb. d. Naturgefch. p. 93. n. 7 . Canis (Aureus) corpore fulvo, pedibus lone gioribus, caudae apice nigro; der Schnellwolf, Schakal, Thos.

Funke; Naturgefch. I. p. 99. der Schakal oder Goldwolf.

Ebert,

(6) Von dem Schakall als dem Stammvater des Hundes, f. bey Can is Familiaris. Shaw, Haffelquift, Michaelis, Biifching und Schreber halten die Schakalle für Simfons Fiabbjè, wogegen aber Faber manches erinnert hat. S. Scbreber, Saugthiere, III. p. 369. n. 1. Faber, Archäologie dér Hebräer, p. 140. Hart. mart, Beobacht. über den Orient, 11. p. 270. Oft ift diels Thier mit der Hyäne vermsngr. Nach Pallas and Gmelin vermifche er fich, befonders in Indien, zuweilen mit dem Hunde. Zimmermanis führe den wahrfcheinlichen Grund an, warum Buffon die Adive vom Schakall hat trennen wollen. 


\section{Mammalia Ferae. Canis.}

Ebert, Naturlehre, I. p. 368. der Goldwolf, Adive, Adil, Iackal.

Halle, vierf. p. 500. der Zwerg-oder Goldwolf. Gatterer, vom Nutzen und Schaden der Thiere, I. p. 38I. n. 216. Schakall.

Neuer Schauplatz der Natur, IV. p. 202. der Ia. ckal, oder Iakhal, Goldwolf.

Onomatol. hift. nat. II. p. 802. Chacal, das Chacalthier.

Klein, Claflif. d. vierf. Th. p. 209. goldgelber Wolf:

Klein, natürl. Ordn. d. vierf. Th. p. 74. n. 2. Goldemwolf.

Georgi, Rufsiand. I. p. 208. Schakale.

Biiffon, vierf. XV.p.7. der Schakal und die Adive, mi. 2 Figg.

Niebuhr, Befchr. von Arabien, p. I66. Iakkal, Tjihakkal.

Wolf, Reife nach Zeilan, p. r2r. Schakal. Hajfelquift, Reife nach l'aläftina, p. 2\%r. Canis Chical Turcarum.

Olear. Reife, p. 413. Schakal, eine Art Fiiche. Baldaeus, Zeilan und Malabar, p. 422. Iackhälle. S. G. Gmelin, Reile durch Rufsland, III. p. 80. Dieterich, Gefch. des Simfons. I. St. Götting. I878. 8. m. e. Fig.

Shaw, Reife, p. I55. der Dib oder Tackall. Auszug a. d. Tagebuch e. Reifenden nach Afien;

p. 56. n. I4. die Schakkale.

Erxleben, Mammalia, p. 571. n. 12. Canis (Au. reus) cauda recta, corpore pallide fufco.

Linné, S. N. Edit. VI. p. 5. n. 3. Canis lupus aureus dictus.

Limné, S. Nat. Edit. X. I. p. 40. n. 7. Canis (Aureus) Lupus aureus dictus.

Linné, 
Limne, Syft. Nat. Edit. XII. I. p. 57. n. 7. Canis (Aureus) Lupus aureus dictus.

Graumann, intr. in hift nat. mamm. p. 85. XII. Schakal.

Gatterer, brev. Zoolog. I. p. 152. n. 12. Canis (Aureus) cauda recta, apice nigro, corpore pallide fulvo, pedibus longioribus; Schnellwolf, Schackal, Goldwolf.

Oedmann, vermifçhte Samml. a. d. Naturkunde. Rof. 1787. Canis aureus', Schualim.

Pallas, Spicil. Zoolog. XI. p. 3. n." 1. Lupus aureus.

Pallas, Naturgefch. merkw. Thiere, XI. p. 3. n. $x_{\text {. Schakall. }}$

Huhn, Merkwürdigkeiten aus Bengalen; in den neuefters Mannichfaltigkeiten, II Lahrg. p. 779. IV. p. 786. Goldhund.

Berichte d. kön. dän. Mifr. in Oftindien, ${ }_{43}$ Cont. p. 840 . f. 48 Contin. p. 1641. - 21 Contin. p. 717 . - 22 Cont. p. $165 .-29$ Cont. p. 432. 56 Cont. p. 1306.

pallas, nord. Beytr. I. p. I53. Forscails canis ferus oder Kelbmitrre.

Charleton, Exercit. p. 15. Lupus aureus.

Dampier, Voyages, Amft. I7Ir. I. p. 255.

Boullaye, voy. et obfervat. p. 254. le Schakal. Gesner, quadr. p. 766. Thoes.

Ariftot. hift. anim. II. c. 19. n. 150. VI. c. 25. n. 426-428. IX. c. 70. n. 473-475. @ws. Plin. h. n. VIII. c. 34. X. c. 74. Thoes. Aelian. hift anim. I. c. 7. @ws.

I1. Mesomezas. Der capifche Schakall.

Zimmermann, geogr. Zool. II. p. 244. nl. I38. der Tenlie; der capifche Schakall. 
Borowfky, Thierreich, I.2. p. 23. n. 10. der capifche Schakal.

Gatterer, vom Nutzen und Schaden der Thiere, I. p. 383. n. 217. Kapifche Schakal.

$V$ cillant, Reife in d. Innere von Afrika, I. p.284. Iackals oder Schakals?

Biiffon, vierfüfs. XV: p. 40. der Tenlie oder ca. pifche Schakall. m. e. Fig.

Graumann, intr. in hift. nat. mamm. p. 85. XIIT. der capifche Schakall.

Gatterer; brev. Zoolog. 1. p. 153. n. I3. Canis (Mefomelas) cauda recta, corpore ferrugineo, fafcia dorfali nigra; der kap Jihe Schakal.

12. LY C A o N. Dev Jihwarze Fuchs. (7)

Miiller, Naturfyften, Suppl. p. 28. n. Ir. der Steilohr.

Zimmermann, geogr. Zoolog. II. p. 245. n1. 140: der fcheor:se. Fuchs.

Borow)ky, Thierreich, I. 2. p. 19. n.4. der fchwar. ze Fuchs.

Halle, vierf. p. 504. der fchoarze Fuchs.

Gatterer, vom Nutzen und Schaden der Thiere,

I. p. 369. n. 208. der jchivarze Fuchs.

Pallas Reife durch Rufsland, III. p. 136 .

Pennant, arct. Zool. II. p. 47. n. I5. der finiar. ze Fuchs.

Pontopp. Naturgefch. von Norwegen, II. p. 42. Steller,

(7) Zimmeimann hat in Petersburg fünf lebendige fchwarze Füchfe gefehen, die unfern Füchien an Geftalt fehr nahe kamen; und der Kaiferin auf jooo Rubel koften follten. Pallas fagt: die Chinefer bezahiten die Felle von $x$ bis zu roo Rubel. Nach Scbres ber wird ein Balg zuweilen mit 400 Rubel bezaht. Boddaers nimmt ihn für eine Spielart an; Elonch.p. 970 
Steller, Befchr. v. Kantfchatka, p. 124. Sihwarze Fïchse.

Strahleniberg, Afien, p. 355. der finwarze Fuchs. Kurze Nachricht der neu entdeckten Infeln zivifchen Afien und Amerika, 1776. p. 58. II3.

Gmelin, Reife durch Sibirien, I. p. 40I. Mitiller, Samml. ruff. Gefch. III. p. 535. Schwarze Fïchle.

Biiffon, vierfüls. VI. p. 370 . der fchwarze Wolf; p. 372. Anh. zuin fchwarzen Wolfe.

Charlevoix, hift. et defcri de la nouv. France, III. p. 133. Renards noirs.

Graumanz, intr. in hift. nat. mamm. p. 84. IV. fchworzer Fuchs.

Gatterer, brev. Zool. I. p. I50. n. 4. Canis (Ly. caon) cauda recta, corpore toto nigro; der fchuoware Fuchs.

4. VU L PES. Der Birkfuchs. $\left({ }^{8}\right)$

Miiller, Naturfyftem, I. p. 224. 11. 4. der Fuchs. Tab. 3r. fig. 2.

Zimmer.

8) Länge des Thiers enwas tiber zwey Fufs. Der Fuchs verbirge fich in unterirrdifche Gruben, oder bauet felbft welche. Gemeiniglich nimmt er fie dem Dachs ab, und erweitert fie, verfieht fie auch wohl mit Fluchtröhren. Anffer dem Fleifche frifst er auch Früfche, Kröten, Eidexen, Fifche, Krebfe und andere lufekten, imgleichen faftige Erd-und Baumfrüchte, auch Honig, und Wachs. Sein Geruch ift fo feil, dafs er fowohl feine Beute als feinen Feind anf 2 bis ;0o Schritte weit entdecken kann. Seine Stimme ift cin kurzes Bellen. Im Schlaf legt er fich wie der Hund in die Runde. Die Rauzzeit ift im Februar. Das Weibchen geht neur Wochen trächtig, und wirft 5 bis 7 lunge. Die Iungen werien blind geboren, und bleiben in diefem Zuftande 14 Tage. Das Fleifch des Fuchfes wird von einigen gegetien. Seine Fährte ift einer Hundsfährte nicht unahnlich. 
Zimmermann, geogr. Zool. I. p. 205 . II. p. 245. n. I39. der Fuchs.

Lefke, Naturgefch. p. 134.'n. 3. der Fuchs.

Borowsky, Thierreich, I. 2. p. 20. n. 5. der Birkfuchs.

Bhumenbach, Handb. der Naturgefch. p. 92. n. 4. Canis (Vulpes) cauda recta apice albo; der Fuchs, Birkfurhs.

Bechftein, Naturgefch. Deutfchl. I. p. .234. der Fuchs.

Funke, Naturgefch. I. p. 103. der Fuchs, der Birk-oder Rothfuchs.

Ebert, Naturlehre, I. p. 369. der Fuchs.

Halle, vierf. p. 501. der gemeine Fuchs, Bir. kenfiichse.

Meyer, Thiere, p. 27. Tab. 36. der Fuchs.

Gattever, vom Nutzen und Schaden der Thiere,

I. p. 370. n. 209. der Fuchs; Birkfuchs.

Beckmann, Naturhif. p. 27. der Fuchs.

Sander, ökon. Naturgefch. I. p. 249. der Fuchs.: Walther, ökonom. Naturgefch. Deutfchl. p. 130; der Fuchs.

Handbuch der Naturgefch. I. 2. p. 145. der Fitchs. Pennant, arct. Zool. II. p. 46. n. I4. der europäifiche Fuchs.

Neuer Schaupl. der Natur, III. p. 219. der Fuchs. Onomatol. hiftor. nat. VII. p. 835. Vulpes, der Fiuchs.

Naturforfcher, VII. p. 6r. 75. XV. p. 153. §. 2. Gesner, Thierb. p. I2I. der Fuchs. Abbild. gut. $\mathrm{N}_{2}$ Klein,

ahnlich. Er erreicht ein Alter von $1_{3}$ bis 14 Iahren. Gänfe follen vor ibm ficher feyn, wenn man ihnen den Kopf mir Theer befchmiert. In feinem Magen hat man mehrmals sicht nur, ẹine Ratte init ihren lungen, fondern auch das ganze Neft gefunden. Der Nutzen feines Balgs ift bekannt. 
Klein, Quadrup. difp. p. 71. Vulpes vulgaris. Klein, Clafif. d. vierf. Th. p. 212. Fuchs. Klein, natül. Ordnung d. vierf. Th. p. 75. n. I. gemeiner Fuchls.

Kraft, Ausiott, graufamer Thiere, II. p. 424. Fiiche.

Bock, Naturgefch. von Preufen, IV. p. 34. n. 7 . der Fuchs.

Goez:, europäifche Fauna, I. p. I62. der Fuchs. Büffon, vierf. IV. p. 97. der Fuchs.

Fifcher; Naturgefeh. v. Livl. p. 5r. der Fuchs. Fifcher, Zufätze zur Naturgefch. von Livl. p. 40. Carver, lksifen d. d. Innere von Nordamerika, p. 363. der Fuchs.

Cuhn, Samml. von Reif. in d. Inmere von Afrika, p. 365 .

Taube, Befchreib. von Slavonien, p. 20. Fï̈chfe. Olear. Reife nach Moskau u. Perfien, p. 86. Gmelin, in den götting. Reifen, V. p. I26. I27. H gfröm, Befchreib. von Lappland, p. 150-162. Leppechin, Tageb. der ruir. Reife, I p. 2I.

Pontoppidan, Naturgefch. von Norwezen, II. p. 42.

Steller, Befchreibung von Kamtfchatka, p. I23. Fiich $\ell$.

Adanfon, Reife nach-Senegall, p. 258. Fïche. Leem, Nachr, von den Lappen in Finnmarken, p. ror. Fitihe.

Georgi, Rulsland, 1. p. 208. gemeine Fiichle. Haff:slquift, Reife n. Palaeltina, p. I9I. 27I. Vulpes Auctorum.

Molina, Naturgefch. von Chili, p. 24r. Fïlche. Viciaure, Gefch. von Ghile, p. 89. Fuchs. Krafcheninnihow, Befchreib. vom Kamtfchatka, p. $\mathrm{II}_{5}$. 


\section{Manmatia Forae.}

Canis.

Kämpfer, Reife nach Iapan, in din Halúe China IV. p. 139. Fiich $e$.

Rytfchkow, orenb. Topogr. in Biifchings Magazin VII. P. 44 .

Olafjen, Reife durch Island. I. p. 3I. II6. u. p. 280. braune: Fiiches.

IMiller, Samml. rufr. Gefch. III. p. 53I. 608. Fiichese.

Cetti, Naturgefch. von Sardinien, I. p. 194. der Fichs.

Köhler, Samml. never Reifen, I. p: 325.

Schwed. Abhandl. IX. p. 192. XXIII. p. 278 .

XXIX: p. 300.

Bonnet, Betrachtung. über die Natur, II. p. 516 . der Fuichs.

Döbel, Iägerprakt. I. p. 38. der Fuchs.

Luciovici, Kaufmannslex. II. p. I89r. Fuchs.

Berlin. Samml. X. p. I48. Fiiichfs.

Gengr. hin. und phyl. Mancherley, II. p. I28.

Jherlin. Magazin, I p. 187-197.

Mannichfaltigkeiten, I. p. $243-245$.

Natürl. Gefch. von der-Louifiane; im Hamburg.

Magazin, XIV.p. 615 .

Deutfcher Merkur. 7785 . Ian. p. 39.

Onomat. foreftal. I. p. 955 .

Berichte der königl. dän. Mif. 25 Cont. p. 165.

43 Cont. p. 840 ?

Erxleben, Mammalia, p. 56r. n. 5. Canis (Vulpes) cauda recta apice albo.

Linné, Syft. N. Edit. II. p. 44. Canis cauda re. "Cla longitudine corporis.

Linné, Sylt. Nat. Edit. VI. p. 5. n. 6. Canis cauda recta extremitate alba.

Linné, Syft. Nat. Edit. X. I. p. 4o. n. 4. Canis

(Vulpes) cauda recta, apice albo. 
Linné, Faun. Suec. I. p. 5. n. I4. Canis cauda erecta.

Linné, Faun. Suec. II. p. 3. n. 7. Canis (Vulpes) cauda recta extremitate alba.

Linné, Muf. Adolph. Fried.I. p. 6. Canis (Vulpes) cauda recta, extremitate alba.

Graumann, introd. in hift, nat, mamm. P. 84. V. Fuchs, Birkfuchs.

Gatterer, brev: Zool. I. p. I50. n. 5. Canis (Vulpes) cauda recta, apice albo; der Fuchs. Miiller, prodrom. Zoolog: dan. p. 2. 11. 6. Canis (Vulpes) cauda recla, apice albo. Kramer, aufr. p. 313. Canis cauda erecta. Schefferi Lapponia, p. 314. Vulpeculae. Charlețon, exercitat. p. I5. Vulpes.

Olaus Magn: de gent. feptentrional, p. 634 . Vulpes.

Schwenkfeld, theriotroph. Silef. p. I33. Vulpes, Wagner, hift. nat. Helvet. p. I87. Vulpes.

Raaczynski, hift, nat, Polon. p. 3I. Auct. p. 324 . Vulpes.

Sibbald, Scot. illuftr. II. 2. p. 9: Vulpes. Forfk. faun, orient. p. III. Canis Vulpes.

Pennant, britt. zool. p. 28. the Fox.

Pennant, Synopf: quadruped. p. 152. n. II2. the Fox.

Forfter, philof: Transact. LVII. p. 342. Canis Vulpes:

'Ariftot. hiftor, anim, I. c. 6. n. 46. VI. c. 34.

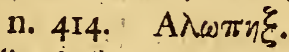

Plin. hilt. nat. VII. c. 58 . VIII. c. 28. X. c. 63. XI. c. 49. 50. XXIII. c. 8. Vulpes. Aelian. hift. animal. IV. c. 39. VI. c. 24. XIII. c. II. $\mathrm{A} \lambda \omega \pi \eta \xi$. 
5. A ц о в х. "Der Brandfuchs. (Köhler", Kohlbrenner.) ( $\left.{ }^{9}\right)$

Miiller, Naturfyft. I. p. 226. n. 5. der Feldfuchs. Zimmermann, geogr. Zool.t I. p. 207. II. p. 205. n. I39. $b$. der Brandfuchs.

Boroweky, Thierreich, I. 2. p. 2I. n. 6. der Brandfuchs.

Bhumenbach; Handb. der Naturgefch. p. 93. n. 5 .

Canis (Alopex) cauda recta, apice nigro. Bechfiein, Naturgefch. Deutfchl. I. p. 249. n. a. Brandfuchs, Rothfuchs, Feldfuchs. Funke, Naturgefch. I. p. 103. Brandfuichs. Halle, vierf. p. 5०4. der Brandfuchs.

Gatterer, vom Nutzen u. Schaden der Thiere, I. p. 377. n. 210. der Brandfuch.s.

Handbuch der deut chen Thiergefchichte, p. 59. die Brandfich/e.

Pennant, arct. Zool. II. p. 48. n. 17. der Brandfuchs?

Biiffon, vierf. IV. p. II3. Kohlenfiuchfe. p. II7• in. c. Brand - oder Braunfuichfe.

Molina, Naturgefch. von Chili, p. 24r. Chilla oder Brandfiuchhs.

Dïbel, Iägerprakt. I. p. 38. Brandfiich/e,

Onomatol. foreftal. I. p. 956 .

Beyträge zur Beförd. der Naturkunde, I. p. II3.

Canis Alopex. Fuchs.

Erxleben, Mammal, p. 565. n. 6. Canis (Alopex) cauda recta, apice nigro.

Linné,

(9) Diefen und die folgenden 13.14 .15 .16 . und 11, halten viele für Varietaiten vom gemeinen Fucbs. Der Brandfuchs unterfcheidet fich auch von ihm faft blofs durch die etwas kleinere Statur, dunklere Farbe und fchwarze Schwanzfpitze. Er hält fich mehr in gebirgigten Gegenden auf. Scin Balg wird weniger geachter. 
Linné, S. N. Edit, VI. p. 5. n. 5. Canis cauda re. cta, extremitate nigra.

Linné, Synt, Nat, Edit. X. J. p. 40. n. 5. Canis (Alopex) cauda recta, apice nigro.

Granmiann, intr. in hift. nat. mamm. p. 84. VI. Köhler, Kohlenbrenner, Brandfuchs.

Gatterer, brev. Zoolog. I. p. I5I. 11, 6. Canis (Alopex) cauda recta, apice nigro.

Severin, zool. Hungar, p. 93. a, der Brandfuchs,

33. Corsac, Dor Korfak. (10)

IMiiller, Naturfynem, Supplem. p. 28. n. ro. der Wolf shund.

Zimmerncann, geogr. Zoolog, II. p. 248. n. 143 . der Korfak.

Borowfly, Thierreich, I. 2. p, 2I. n.7. der - Korfak, Wolfstund.

Gatterer, vom Nutzen u. Schaden der Thiere, I. p. 377. 11. 212. der Wolfshund.

Pennant, arct. Zool, II. p. 49. 1, 18, der Corfak. Never Schaupl. d. Natur, X. p', r3. Wolfshund. Bïfon, vierf. XV.p.6r. cler Korfak. Fig. a. und b. Pallas, Reife durch Rufsland, I, p. 199. 234. Korfak.

Pallas, Reife, Ausz, I, p, 198. III. p. 373. Korfakin.

Georgi, Rufsland, I. p, 268. Sieppenfiiche.

Rytsh.

(ro) Kleiner als dep Fuchs, Der dicke Schwanz ift faft fo lang als der Leib, Er bellet, und wahnt unter der Erde. Der Balg ift ein wichtiger landelsartikel der Landex an laik und Irtis, wo man ihn in fo groiser Menge fängt, dafs oft jährlich 50,000 Bäla ge an die Ruffen verhandelt werden. Die Kirgifen brauchen fie im Handel und Wandel, beym Kauf und $T$ aufch faft wie Geld, und beftimmen den Prois ihreq Waaten nach der Anzahl der dafür zu gebenden Bàlge. 
Rytfchkow; orenb. Topogr,; in Biilchings Mag, VII. p. 43. Korfak.

Erxleben, Mammalia, p. 566. n. 8. Canis (Cor-, fae) cauda recta fulva, bafi apiceque nigra.

Pennant, Syn. quadr, p. 154. n. I12. E. the Corfac, Graumann, intr. in hift, nat. mamm. p. 84. VIII. Wolfshund.

Gatterer, brev, Zoolog. I. p. r51. n. 8. Canis (Corfac) cauda recta fulva, bafi apiceque nigra.

14. K A R A G A n.' Der Karagan. (Schnwarzohr.) (')

Zimmermann, geogr: Zoolog. II. p. 248. n. II2. der Karagan.

Schreber, Säugthiere, III. p. 359. n. 7\% der Karagan.

Biiffon, vierf. XV. p. 8I, n, 2, der Karagan, Step. penfuchs.

Ifert, Reife nach Guinea, p. 21r. der Bufchhund? Erxleben, Mammalia, p. 566. 11. 17. 11.7. 'Canis (Karagan) cauda recta, corpore grifeo.

Granmann, intr, in hiff. nat. naamm, p. 84. VIr. Steppenfuchs.

Gatterer, brev. Zool. I. p. I5r. n. 7. Canis (Ka. ragan) cauda recta, corpore grifeo.

15. Cinereo-argenteus. Der Griesfuchs. ( $\left.{ }^{2}\right)$ Miuller, Naturfyftem, Suppl. p. 29, n. I2. der Silberhunet.

Zimmermann, geogr. Zool. II, p. 250 . n. 145 . der Griesfuchs.

Bhumenbach, Handb. d. N. G. p. 22, Silberfuchs. N 5 Gatte.

(1) Sein Pelewerk ift ein Handelsartikel.

(2) Schreber zweifelt, ob er wohl picht mit dem folgenden einerley 1ey, und Blumen'sach bạlt ihn für eine Spielart von C. Lycaon, 
Gatterer, vom Nutzen und Schaden der Thiere, I. P. 378. n. 213: der Griesfuchs.

Pennant, arct. Znol. II. p. 50. n. 20. der Silber. fuchs, Griesfuchs.

Büffon, vierfüfs. XV. p. 59.' der Griesfuchs; m. e. Fig.

Batfch, Thiere, I. p. I9r. Silberfüchfe.

Granmann, intr. in hift nat. mamm. p. 84. IX. Silberlaund.

Gatterer, brev. Zoolog. I. p. 15I. n. 9. Canis (Cinereo-argènteus) cauda recta, corpore cinereo, collo lateribus fulvo; Silberinand, Gries. fuchs:

16. VIRG I N I A N US. Der virginifiche Fuchs. (3)

Zimmermann, geogr. Zool. II. p. 250. n. I46. der virginifche Fuchs.

Borowjky, "Thierreich, I. 2. p. 22. n. 8. der virgi." nifche Fuchs.

- Halle, vierf. p. 505. der amerikanifche Silberfuchs. Pennant, arct. Zoolog. II. p. 49. n. I9. der grane Fuchs.

Klein, Claffif. der vierfüfs. Thiere, p. 2I4. as/ch. graner amerikanifcher Fuchs.

Klein, natürl. Ordn. d, vierf. Th. p. 76. n. 2. grauer amerikanifcher Fuchs.

Biiffon, vierf. XV. p. 57. der virginifche Fuchs; in. e. Fịg.

Fermin, Befchreib. von Surinam, II. p. 91. die erfte Art Fï̈hfe in Surinam. Quaff?

Pennant,

(y) Penrant fagt: Er lebt von Vögeln und Hülinern, fallt aber nie Limmer an. Sein Fell dient zu Fütterung der Kleider, und die Haare gebrauchen die Hutmacher. Fr wird fehr fett. Aus den hohlen Băumen vertreibt man ihn vermittelf dès Rauchs. 
Pennant, Synopf. quadruped, p. 157. n, ri4. the grey Fox;

Grausuann, intr, in hifor, nat, mamm. p. 84. X. virginif che Fuchs.

Gatterer, brev. Zoolog. I. p. 152. n. Io. Canis (Virginianus) cauda recta, corpore ex cinereo albicans.

\section{La g o pus. Der. Stbinfuchs. (4)}

Miiller, Naturfyft. I. p. 226. n. 6. der Hafenfufs, Zimmermann, geogr. Zool. II. p. 249. n. I44. der Ifatis.

Borowfky, Thierreich, I. 2. p. 22. n. 9. der Steinfuchs; blaue, weifse Fuchs.

Bhimenbach, Handb, d. Naturgefch. p. 93. n. 6 . Canis (Lagopus) cauda recta, apice concolo. re, palmis plantisque villofiflimis; Polarfuchs, Steinfuchs, Ifatis.

Halle,

(4) Länge des Körpers gegen 2 Fufs. Der Schwanz ît einen Fufs lang, und dick behart. Die Speife diefer Thiere ift vorzügJich der Lemming; hienäch allerley Geflügel, infonderheit Waffervögel, die im Norden häufig niften, und deren Eyer; im Winter Hafen und Sçnneehühneer. Sie fieffen auch Fifche, und fogar Aas. Die Zeit ihrer Begattung fällt in den Aprịl. Das Weibchen wirft 7 bis 8 lunge, in Höhlen und Felfenritzen. Die Grönländer effen das Fleifch, und zerfpalten die Sehnen zu Zwirn. Steller fagt: fie ftinken noch weit häfslicher als die Ruthfüchfe. Nach Martenis gehen fie nicht ins Waffer. Pennant aber behauptet, fie fchwömmen fehr gut, und oftmals von einer Infel zur andern. Man fängt fie entweder in einer Art von Wolfsgruben, oder in Schlingen von Fifchbein, pder auch in Fallen. Die bläulichen Felle werden höher als die weifsen gefchätzt. Auffer den beyden im Syftem bemertkten V'arietä. ten findet man auch Spielarren, weifs mit fchwarzen Ohren und Füfsen; auch weifs mit fchwarzen Küpfen. (Leem, Martens, Pbipps) Der Balg des Ifatis gehört zu den Handelsartikeln des ötlichen Sibiriens. 
Halle, vierf, p. 504. der blaue Fuchs; der weifse Fuchis.

Gatterer, vom Nutzen und Schaden der Thiere, I. p. 379. n. 215."Steinfuchs, Eisfuchs. Pemuant, arct. Zool. II. P. 43. n. 13. der. Ifatis; aretifiche Fuchs.

Bïffon, vierf. XV.p. 42. der Ifatis; Tab. a. der blane Ifatis; Tab. b. der weifse Ifatis.

Steller, Befchreibung von Kamtfchatka, p. 126. $P_{e f} \int c i$, oder weifse Steinfuich $\int e$.

Steller, nov. Comment, Petrop. II. p. 32 r.

Cranz, Hilt. von Grönland, p. IoG. die Füch $/$ e. Phipps Reife nach dem Nordpol, p. 96. Polar. fiuchs.

Leppechin, Tagebuch der ruff. Reife, III. p. 218. Steinfuctus.

Leem, Nachr. von den Lappen, p, ro2. wei/se Fiichse.

Olaffen, Reife d. Island, I. p. 2r. weifse Fiichfe. Pontoppidan, Naturhif, von Lorwegen, II. p. 42. weifse Fiichfe.

IMartens Spitzberg. Reifebefchreib. p. 72. Fuchs. 'Tab, O. fig. b. fchlecht.

I. G. Gmelin, Reife durch Sibirien, III. p. 2 I4. weifse und blane Fï̌ch fe-

Strahlenberg, Afien, p. 355. weifse Fiic se. Miiller, Samml, ruff. Gefch. III. p. 539. Stein. fiich $\int e$.

Tremarec, Reife nach der Nordfee, p. 7o. Fïchfe. IMolina, Naturgefch, von Chili, p. 241. Canis L.agopus. Steinfuchs.

Cook, dritte Reife, II. p. 290.

Stährlin, kuize Nachrichten von den neu entdeckten Infoln, p. 113 .

Pallas, nord.Beytr.II, p.274. Stein: od. Eisfinclife. 
Krafcheninzinikow, Befchr. von Kamtfchátka, p. rrg. Steinfiichse.

Berlin. Samml, VII. p. 15r. Filichee.

Linné, Syft. Nat. Ed. VI. p. 5. n. 7. Canis cau. da recta unicolore.

Limné, Syft. Nat. Edit. X. I. p. 40. n. 6. Canis (Lagopus) cauda recia, apice concolore.

Linné, fn. Suec. II. p. 4. n. 8. Canis (Lagopus) cauda recta, apice concolore.

Eggede, Befchr. von Grönland. Berl. r763. p. 86. veifse, graue, oder blizuliche Fiichese.

Graumans, intr. in hift nat. mamm. p. 85. XI. Steinfuchs.

Gatterer, brev. Zoolog. I. p. I52. n. Ir. Canis (l agopus) cauda recta, palmis plantisque vil. lofifimis. Steinfachs.

Severin, zool. Hungar, p. 93. b. Lagopus. Piller, Elem. hift. nat. I. p. I6. Vulpes colo. ris albi.

Miiller, zool. dan. prodr. p. 2. n. 7. Canis (Lago. pus) cauda recta, apice concolore. Scheffer, Lappon. p. 340. Vulpeculae cinereae. Aldrovand. de quadr. digit. p. 222. Vulpes alba. Ionfton, quadrup. $\rho$. I33. Vulpes alba.

Fabric. Faun. groenland. p. 19. n. II, Canis (La* gopus) cauda recta, apice concolore. Forfter, phil. Transact. 1.XIl. p. 370. Arctic Fox. Pennaint, Synopf. quadruped. p. 155. n. II3. the arctic Fox. Tab. 17. fig. 3 .

\$S. VUL PES C R UCIGERA. Der Krenifuchs.

Miiller, Naturfyft. I. p. 225. der Creizafuciss.

Zimmermann, geogr. Zoolog. II. p. 250. Kreuz: fuchs.

Sthreber, Säugth. III. p. 364. Kr Euzfiichse. 
Borowfky, Thierreich, I. 2. p. 22. Kreuzfuchs. Blumenbach, Handbuch der Naturgefch. p. 93. Kreutzfuchs.

Bechftein, Naturgefch. Deutfchl. I. p. 250. n. b. Kreuzfuchs.

Ebert, Naturlehre, I. p. 363. Kreuz:fuchsw

Halle, vierf. p. 504. der Kreuzfuchs.

pennant, ardt. Zool. II. p. 48. n. 36. der Kreuz. fuchs.

Neuer Schauplatz der Natur, III. p. 219. der Kreuzfuchis.

Gesner: Thierbuch, p. I26. ein Krenzfuchs; m. e. mittelm: Abbild.

Bïffor, vierf. XV.p. 50. Krenz:afuchs. Klein, Quadr. difp. p. 7r. Vulpes fuper dorfum crucigerae:

Klein, Claffif. d. vierf. Th. p. 213.

Klein, natürl. Ordn. d. vierf. Thiere, p. 75. n. I. Leem, Nachricht. von den Lappen in Finnmark. p. IOI.

Miiller, Samml. ruff. Gefch. III. p. 534. Krenzfiich $\int e$.

Scheffer, Lappon. p. 314. Vulpeculae cruce notatae.

Errleben, Mammal. p. 570. Vulpes crucigera. Aldrovand. de animal. digitat. p. 221. Vulpes crucigera.

Ionfton, quadrup. p. I3ذे. Vulpes crucigera.

Schwenkfeld, theriotroph. Silef. p. I35. Vulpee crucigera.

Penmant, Syn. quadr. p. I52. n. II2. $\beta$. The Crols Fox.

Graumnm, introd. in hifor. nat. mamm. p. 85 . Kreuzfuchs. 
QS. Canis cula a eus. Der Culpeu.

Vidaure, Gefch. des Königr. Chile, p. 89. der Culpeu.

17. CERDa. Der Zerda.

Zimmermann, gengr. Zoolog. II. p. 247. n. I41. Canis (Zerda) pygmaeus, auriculis maximis, cauda attenuata, apice nigra; der Zercia.

Bhlumenbach, Handb. der Naturgefch. p. 85. n. 5 . Viverra (Aurita) auriculis ampliffunis; das Grofsoin.

Sparrmann, Reife nach dem Vorgeb. d. g. H. p. 45. Zerda.

Schwed. Abhandl. XXXIX. p. 248. ein kleines Sel-

tenes Tilier aus Afrika, cias zum Fuchsgefchlechte gehört. Tab. 6.

Lichteuberg, Magazin für das Neuefte etc. I. I. p. 92. 11. 22.

Biiffon, Suppl. quadrup. ed. in r2. VIII. p. 243. pl. 2o. Animal anonyme. $B$ :iffon, vierf. XV. p. 75. der Zerda. Bruce, Reifen nách den Quellen des Nils; im Anhang Tab. 22.

A) Verïnderungen gegen die XITte Edition, und $V e \eta$ * mehrung der Gattungen diefes. Gefchlechts.

Die erfte Gattung: Canis Familiaris ift mit zwey und zwanzig Varietäten; das ganze Gejchlecht aber mit fieben Gattungen vermehrt. Die Hyaena ift von der Crocuta getrennt; und C. Mefomelas, Lycaon, Corfac, Karagan, Cineveo-argenteus, Virginianus und Certa find dazu gekommen. Von den

Varietä- 
Varietäten der Hunde hat die Xlite Edit. nur

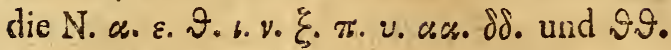

\section{B) Unbeftinmtere Thiere.}

1. Der grönländifche Hund. (Var. C. fam. Zimmermann, geogr. Zoolog. II. p. 236. n. d. Schreber, Säugth. III. p. 344. Egede, Befchr: von Grönl. Berl. 1763. p. 87. Cranz, Hift. von Grönl. Lpz. 1779. p. Io9.

2. Der. grofse dänifche Hunia. (Var. C. fam.) Zimmermann, geogr. Zoolog. II. p. 24I. n. x. Schreber, Säugth. III. p. 326. n. 22. der dïnifche Blenaling.

Halle, vierf. p. 482. dev grofse Dïnifche. Biiffon, hift. nat. V.p. 240. Tab.26. Grand Danois. Biiffon, vierfüfs. II. p. IGI. n. 2. der gro/se dïnijihe Hund. Tab. 22.

Erxleben, Mammal. p. 548. n. $\varnothing$.

Penncme, Synopf. quadruped. p. 146. In. IV. $\gamma$. danifh Dog.

Plin. hint. nat. VIII. c. 40 ?

3. Der MRebbia.

Zimmermann, geogr. Zoolog. II. p. 25t. n. a. Kolbe, Belchr. des Vorgeb. d. g. H. p. I52.

4. Inipaimpes, oder wilde Hurale von Sofala. Zimnermana, geogr. Zool. II. p. 252. n. bs Purchas, Pilgrim. II. p. 1545.

5. Der Coyotl.

Zimmermann, geogr. Zoolog. 11. p. 254. n. f. Fernand. hif. anim. nov. Hifp. p. 4. Coyotl, feu Vulpes indica. 
6. Der Koupara. Der Crabedago.

Zimmermann, geogr. Zool. II. p. 254. n. g. Berrere, France equinox. p. I49. Canis ferus maior cancrofus vulgo diclus: Konpara.

Onomat. hif. nat. IV. p. 597. Koupara.

7. Der Wolfsfuchs von den malouinifchen Infelu,

Zimmermann, geogr. Zool. II. p. 254. n. h. Bougainville, Voy. aut. du Monde, Bar. I77r.'

p. 65. Loup - renard.

8. Der Wolf von Neuholland.

Zimmermann, geogr. Zoolog. II. p. 255 . n. i.

Dampier, Voy. aux terres auftrales. Rouen I723: IV. p. $13 \%$.

13. GESCHLECH T. FEI Is. Die Katze. Miiller, Naturfyft. I. p. 229. Gen. XIII. Zimmermann, gengr. Zcol. II. p. 258. Gen. XX. Schreber, Säugthiere, III. p. 375. Gen. XIV. Lefke, Naturgefch, p. I35. Gen. XIV.

Borowsky, Thierreich, I. 2. p. 25. Gen. XIII. Blumenbach, Handb. d. N. G. p. 94. Gen. XXVIr. Bechftein, Naturgefch. Deutfchl. I. p. $25 \mathrm{r}$. Batfch, Thiere, I. p. r8o. Gen. XXI. Erxleben, Mammalia, p. 50o. Gen. XLIV. Pennant, arct. Zool. II. p. $5^{\circ}$.

Grammanin, introd. in hiftor. nat. mamm. p. 73.

Gen. XLIV.

Gatterer, brev. Znolog. I. p. 135. Gen: XLIV. Pennant, Syn. Quadr. p. 164. Gen. XIX. Briffou, Quadr. p. 19r. Gen. XXXIX. Hermani, tab, affinit. animal. p. 70. 


\section{LEO. Der Löwe. ( $\left.{ }^{1}\right)$}

Miiller, Naturfynt. I. p. 230. n. I. der Löwe. Tab. 29 fig. 4.

Zimmermann, geogr. Zoolog. II. p. 5t. p. 258. n. I50. der Löwe.

Lefke, Naturgefch. p. I35. n. I. der Lowe. Borowe ky, Thierreich, I. 2. p. 25. n. I. der Löwe. Bhmenbach, Handb. d. Naturgefch. p. 95. n. I. Felis (Leo) cauda elongata, floccofa, corpore fulvo; der Löwe.

Funke,

(1) Die Grürse des Thiers int verfchieden. Die geringfte und gröfste Länge deffelben mag etwa von 7 bis $y$ Furs ausmachen. Der Hauptitz der Löwen ift Afrika; und die Löwen in dem Innern von Afrika find gegen die ibrigen ihres Gofohlachts die gröfsten und ftärkften. Amerika hat nie Lösven gehabt. Den pordlichnten Grad des jetzigen Ausdaurens des Löwen kann man etwa auf den 34 Grad Breite ferzè. Das Weibchen unterf́cheider fich autserlich vom Männchen nicht nur durch die mindere Grüfse des Kürpers überhaupt; fondern auch dutch die beträchtlich klẹinere Mahne. Der Lüwe verträgt unfer Klima recht gut, lafst fich ausnehmend zahm machen, und felbft zum Zug, und zur lagd anderer Thiere abrichten. Er nährt fich biofs von feiner eigenen Beute, fchont klciner kraftlofer Gefchüpfe, entfetzt und fcheut fich aber vor dem Bärẹn. Das Weibchen wirft 3 bis 4 Iungre. In Neapolis hat cinmal eine Lüwin von einem mit ihr in der Gefangenfchaft befindlichen Lüwen fünf Iunge auf einmal zur Welt gebracht. Merkwürdig ift es, da?s die Nerven, und auch die Blutgefafse bey liefem Thier nur klein find, und gar nicht feiner Gröfse und Stăke proportionirt zu feyn fcheinen. Von der Gefchwindigkeit, womit das Geblüt bey ihm umläuft, leiten einige Anatomiker feine Stritke, Munterkeit und Unerfchrockenheit her. Adanfor fahe am Senegall nicht felten Löwen und Wölfe in Gefellfchaft gehen, und fagt : man hört hier faft alle Abend den Wolf neben dem brïllenden Lö. wen heulen. Die Urfách diefer fonderbaren Erfcheinung fetze er darin,' weil das Wolfsfleifch den Lüwen nicht zum Genufs deffelben reizen foll. Das Fleifch des Lüwen wird am Gefchmack mit dem Kalbfleifch verglichen. 
Fuake, Naturgefch. I. p. r49. 'der Löwe. Ebert, Naturlehre, I. p.375. der Löree. Halle, vierf. p. 534. der Lö́oe, die Lörvim. Gatterer, vom Nutzen und Schaden der Thiere, I. p. 322. n. I87. der Löwe. Beckmann, Naturhiftorie, p.30. n. 22. der Löwe. Handbuch der Naturgefchichte, I. 2. p. 207: der Lobive.

Neuẹr Schauplatz der Natur, V. p. I88. der Löree. Onomat. hift. nat. IV. p. 775: der Löre.

Gesner, Thierbuch, p. 246. ein Löu. p. 247. eine fchl. Fig.

Klein, Quădr. difp. p. 81. Leo.

Klein, Claflif. d. vierf. Th. pi 245. Löwe. Klein, natür!.Ordn. d. vierf. Th. p. 86. n. E. Löwe. Biiffon, vierf. V. p. 216. der. Löre, mit 2 Figg. Männchen und Weibchen.

Batfch, Thiere, I. p. 186. der Löwe. Cuhn, Samml, von Reifen in d. Innere von Afri. $\mathrm{ka}, \mathrm{p} .35^{2}$.

Shaw, Reife, p. 152. dev Lörèe. Adanfon, Reife nach Senegall, p. 174. der Löwe. Höft, Nachricht. von Marokos und Fes, p. 289.

Löwen; Sba.

Sparrmanit, Reife nach dem Vorgeb. d. g. H. p. 362. Jöwent.

Le Vraillant, Reife in das Innere von Afrika, I.

p. 268. 27 1. 277. Löwen.

Wolf, Reife nach Zeilan, p. 122. der Lörwe. * Knox, Reife nach Ceylon, p. 4r. Lötvent.* Reifen eines Officiers nach den Infeln Frankreich

u. Bourbon, p. 295 .

Kolbe, Vorgeb. der g. H. p. 154. der Lörese. Tab.5. fig. 3. Männchen und Weibchen unittelm. Edit. in 4. p. 306 . Tab. 3 I. 
Hafjelquift, Reife nach Paläftina, p. 562.

Condlawine Reifen; in den Götting. Samml. von

Reifen, II. p. 30 r.

Ridinger, Thiere, 32. 33. Lörve, Löwin.

Ridinger, jagdbare Thiere. Tab. I. Löwe.

Ridinger, Entwvurf einiger Thiere, II. Tab. Ig

bis 30 .

Ridinger, Löwe, Tab. x-8.

Dapper, Afrika, p. I8. der Leue.

Reife ins Südmeer, p. I7o. und 247. Löwe.

Perrault, Charras und Dodart, Abhandl. zur

Naturgefch. I. p. 3. anatomifche Befchreibung von vier Löwwen Tab. I. der Löve. Tab. 2. die Löwin; p. 23. anatomifche Befchr. dreyer Löwinnen. Tab. 3. Zerglied. des Männchen. Tab. 4. des Weibchen.

Neue Anmerk, über alle Theile der Naturlehre, III. p. 544.

Berlin. Samml. IV. p. 482 . V. p. 258.

Leipziger Wochenbl. VI. p. 46.

Teutfcher Merkur, I785. I Vierteljahr p. 38.

Goeze, Natur, Menfchenl. und Vorf. I. p. 55. Merklein, Thierreich, p. то5. Löre.

Oekonom. Zoologie, p. I6. n. I4.

Erxleben, Mammal. p. 500. n. I. Felis (Leo) cauda elongata, corpore heluolo.

Linné, Syft. Nat. Edit. X. I. p. 41. n. I. Felis (Leo) cauda elongata, corpore heluolo.

Grawnanin, introd. in hiftor. nat. mamm. p. 73 . Löre.

Gatterer, brev. Zoolog. I. p. 135. 11. I. Felis (Leo) cauda elongata, corpore fulvo.

Beckmaann, de hift. nat. vett. p. 33 .

Beyer, Schola Leonis, darin gehandelt wird von der Natur und Art des Löwens. Lpz. \&. 
Sperling, diff. de Leone etc. Witteb. I641. 4. Wolfart, diff. de Leone. Caffel izrr. 4. Kramer, Auftr. p. 3ro. Felis cauda elongata floc. cofa, thorace iubato.

Charleton, Exercitat. p. r4. Leo. Schwenkfeld, theriotroph. Silef. p. Ior. Leo. Muf. Wormian. p. ₹17. Leo. Bont. hift. nat. Ind. orient. p. 55 . Leones. Marmol. Afrique, I. p. 54. le Lion. Knox, relat. d'un voy. de Ceilan. Amft. 16.93. p. 89.

Leo African. defcr. Afric. I2mo p. 754.

Nouvelle defcript. du Cap de b. E. Amft. I778. II Part. p. 24.

Mem. de l'Acad. d. Sc. de Paris T. II. P.I. p. 3. Tab. I. 3. Lion.

Penanat, Syn. quadr. p. 164. n. r20. the Lion. Ariftot. hiftor, anim. II. c. I. 11.2. c. 5. n. 23. c. 7. n. 29 . c. 8. n. 4 r. c. 9. n. 50 . III. c. 7 . n. 102. c. 15. 11. 233. VI. c. 3I. n. 393-400. VIII. c. 9. n. 104. IX. c. 69 . n. $460-472$. $\Lambda \varepsilon(s), \Lambda \varepsilon \alpha$ เve.

Plin. hift. nat. VII. c. I. VIII. c. 15. 16. X.c. 45 . 63. XI. c. $37.39 .40 .43 .45 \cdot$ 53. Leo; Leaena.

Aelian. hift. anim. III. c. 1. 27. IV. c. 3. 34. 36. V. c. 39. VII. c. 23. IX. c. I. 30. XII. c. 7. XVII.c. 26. $\Lambda \varepsilon \omega \nu$.

Plutarch. de curiofit. p. 520. F. Tom. 2. edit.

Francf. 1620. $\Lambda \varepsilon \omega v$.

Phtarch. de folert.anim. p.966. C. Tom. II. $\Lambda \varepsilon \omega v$. Scaliger, exercit. edit. Francof. 1592. 208. p. 667. Martial, Spectac. Lib. Epigr. 18. Atbertus, de animal. Lib. 22. tract. 2. c. 2. fol. 221. b. edit. Venet. 1495 .

$\mathrm{O}_{3} \quad \mathrm{Carda}$ - 
Cardanas, de fubtilit. L. 10. p. 320 . edit. Bafil. 1560 .

Buch der Richter c. XIV.,v. I4: der Freffer.

2. TIGRIS, $\operatorname{Der}$ (königliche, wahre of̣indifche) Tiger. $\left({ }^{2}\right)$

Miiller, Naturfyn. I. p. 235. n, 2. der Tieger. Tab, 30. fig. 4.

Zinnmerniann, geogr, Zool. II. p. 259. n. 15r. der Tiger.

Le $k e$, Naturgefch. p. 136. n. 2. der Tiger. Borowfky, Thierreich, I. 2. p. 35. n. 10. der Tiger, Tab. 13. B. das Tigerthier.

Blumenbach, Handb. der Naturgefch. p. 95.n. 2. Felis (Tigris) cauda elongata, capite, corpore et cruribus nigro, variegato; das Tigerthier.

Funke,

(2) Die Grörse diefes Thiers ift oftmals ganz auferordentlich, - Man hat Tiger von funfzehn Fufs Länge gefehen, den Schwanz mitgerechnet. Seine liebfte Nahrung ift das Blut feiner Beute. Das Fleifch davon pflegt er nicht ganz aufzufreffen. Einen Bïffel, der dreymal fo grofs ift, als er felbft, fafst er mit den Krallen in den. Nacken, reifst ihn nieder, faugt ihm das Blut aus', und trăgt ihn mit gröfster Leichtigkeit davon, Fr würet gegenffeilien eignen. Gatten. Sein Gernch ift widrig. Zu feinef Beute foll er den Indier ftets dem Ausländer vorziehen. Das Weibchen witft im Frïhjahre drey bis vier Iunge, und foll zu diefer Zeit noç graufamer als das Mïnnchen feyn. Von dem Edelmuxh des Löwen finder fich bey dem Tiger gar keine Spur, Man fängt ihn in Gruben, und erlegt ihn mit Schiefsgewehr, Sein Fleifch wird gegeffen; von den Häuten werden Pferdedecken, u. dgl. gemacht. Die Araber rerkaufen ein Fell von einen alte: $T$ Iger uubereiter für is bis 20 Mark, und bęreitet für 40 bis so Mark. Die Benennung: königliches: Tiger, haben die Portugiefen aufgebracht, Nach Dio Caffus waren diejenigon! Tiger, welche die Indier dem "Auguft zum Gefchenke fchickten, die erften, welche die Rümer und Griechen gelehen hatten. 
Funhe, Naturgefchichte. I. p. I52. dev Tiger. Ebcrt, Naturlehre, I. p. 374. der Tiger. Halle, vierf. p. 530. der ceylonifche Tiger.

Gatterer, vom Nutzen und Schaden der Thiere, I. p. 326. n. I88. der Tiger.

Bat fch, Thiere, I. p. I84. der Tiger.

Beckmann, Naturhiftorie p. 29. n. 20. der Tiger. Handbuch der Naturgefch. I. 2. p. 212. der Tiger.

Neuer Schauplatz der Natur, IX. p. 68. der Tieger.

Onomatol. hif, nat. VII, p. 526. der Tiger, das Tigerthier.

Naturforfcher, VII. p. 42. Fin Tiger.

Gesner, Thierbuch p. 340. Tigerthier, m. e. mittelm, Fig.

Klein, Quadrup, difp. p. 80. Tigris afiatica. Klein, Claflif. der vierfüls. Th. p. 243. afiatijcher Tger.

Klein, natürl. Ordn. d, vierf, Th, p. 86. n. i. afiatifcher Tiger.

Biiffon, vierf. VI, p. I66. der Tiger, m. e. Fig. Auszug a. d. Tagebucli e. Reifenden nach Afien,

p. 63. der Tyger.

$H \ddot{f} f$, Nachr. von Marokos und Fes, p. 29r. Ti: ger, Enémer.

Kircher, China illufrata, fol. Amf. I667. p. 83. veră Tigridis forma.' Abbild. Schön.* Wolf, Reife nach Zeilan, p. I22. der Tiger. Ives, Reife nach Indien und Perfien, I. p. II2. der königliche Tiger.

D Halde, Befchreib, von China. IV. p. 32. Lao hou oder Tiger. p. 224. die Tigerjagd.

Dopper, Mefopotamien, p. İr.

$P_{i}$ o, de Ind, utriusque re naturali, p. 103.

Dänifche Miflionsberichte, 29 Cont. p. 432.

$\mathrm{O} A$

Deppet, 
Dapper, Afien, p. 5. Tiger.

Kinox, ceylan. Reifebefchr. p. 4I. Tiger.

Baldaens, Zeilan und Malabar. p. 42I.

Ridinger, Entwurf einiger Thiere, Tab. 35. Ti. ger mit länglichten Streifen.

Becknann, de hilt. nat. vett. p. 38 .

Saar, offindifcher Kriegsdienft. Nürnb. 1672. p. $18.7 \%$.

Perrault, Charras und Dodart, Abhandl. zur Naturgefch. II. p. I67. anatomifche Befchreib. eines 'Tigers und dreyer Tigerinnen, Tab. 61. der Tiger, das Tigerthier. Tab. 62. die Zergliederung.

Turpin, hiftoire de Siam, I. p. 296.

Neuhoff, Gefandfch. n. China, p. 350 . fig. p. 35 I. Tigerthier.

Dapper, Befchreib. von China, p. 138. Tiger. Auswahl kleiner Reifebefchreib. Lpz. I784. III. p. 683 .

Goeze, Natur, Meafchenl. u. Vorf. I. p. 57.

Jerlin Samml.VIII. I62.

Erxleben, Mammalia, p. 503. n. 2. Felis (Tigris) cauda elongata, corpore maculis omnibus virgatis.

Limné, Syft. Natur. Edit. II., p. 43. 'I'jgris macalis oblongis.

Linné, S. Nat. Edit. VI. p. 4. 11. 2. Felis cauda elongata, maculis virgatis.

Linné, Syf. Nat. Edit. X. I. p. "4r. n. 2. Felis (Tigris) cauda elongata, corporis maculis omnibus virgatis.

Graumann, intr. in hift. nat. mamm. p. 73. Tieger. Gatterer, brev. Zoolog. I. p. 136. n. 2. Felis (Tigris) cauda elongata, corpore, capite et cruribus maculis omnibus virgatis; der Tiger.

Kramer. 
Kramer, Auftr. p. 3ir. Felis cauda" elongata, maculis virgatis.

Charleton, exercitat. p. 14. Tigris.

Schwenkfeld, theriotroph. p. 130. Tigris.

Raj, Synopf. quadr. p. 165. Tigris.

Pennant, Synopf. quadruped. p. 167. n. I21. the Tiger.

Boullaye, Voy. et obfervat. p. 246. Tigre.

Plinius, h. n. VIII. c. 4. 17.28. Tigris.

Oppian. cyneget. III. 98. 340. Tryers?

Solin. Polyhiff. V. c. 20. Tigres.

Seneca, Hippolyt. Act. I. v. 345. Tigris virgata:

Albertus, de animal. L. 23. P. 2. c. I.

$V_{0} \int s$, obfervat. ad Pomp. Mel. L. 3. c. 5. S. I6.

Dio Cafius, Hift. rom. L. 54. p. 527. Tiyers.

Sueton. in Auguft. c. 43. \$. II. Tigris.

3. PARDUS. Der Panther. (3)

Miiller, Naturfyft. I. p. 237. n. 3. der Leopard. Tab. 30. fig. 5 .

Zimmermann, geogr. Zool. II. p. 26r. n. I52. der Panther.

\section{5 \\ Lefke,}

(3) In den Synonymen diefer, und dér folgenden Gattungen if grofse Verwirrung. Panther ift der eigentliche Name diefes Thiers; die Benennung Leopard kommt ihm gar nicht zu: Vielleicbt if diele Verwechslung daher entfanden, dafs man fich fonft einbildete, der Loopard fey vom Lüwenl und Panther entAtanden. Beyde Thiere haben dem erften Anfehn nach viel Achnliches mit einander, weichen aber doch merklich vori einander ab. Die Lange des Panthers ift, ohne den zwey bis dritthalb. Fufs langen Schwanz, fünf bis fechs Fufs. Die Grundfarbe ift bräunlich gelb. Das Fell des Fanthers ift bey weitem nicht fo fchön, als des Leoparden feins. Die Flecke find grörfer, weniger regulär, hin und wieder zufammengefloffen, bald in Hufeifenform, bald geringelt u. f. w. 
Lefke, Naturgefch. p. 136. n. 3. der Panther. Borowfky, Thierreich, I.2: p.29. n.2.der Panther. Blumenioach, Handb. d. Naturgefch, p. 96. n. 4 . Felis (Pardus) cauda fubelongata, maculis maioribus irregularibus, paffim confluentibus, et annulatis; das Pantherthier, der Parder. Funke, Naturgefch. I. p. 153. der Parder. Ebert, Naturlehre, I. p. 374. der Parde:. Halle, vierf. p. 528. a'er Parder.

Gattever, vom Nutzen und Schaden der Thiere,

I. p.329. n. 189. das Panterthier, der Parder. Batfch, Thiere, I. p. Is4. dev Panther. Beckmann, Naturgefch. p. 29. n. 21. der Parder. Newer Schauplatz der Natur, VI. p. 345. das grofSe Panterthier.

Onomat. hift. nat. V. p. 683. das Panterthier. Klein, Quadrup, difp. p. 78. Pardus, Pardalion. Klein, Claflif. der vierfüls. Thiere, p. 237. Panther, Panterthier, Pardel.

Klein, natiirl. Ordn. d, vierf. Th. p. 83. n. Is Parder, Panterthier, Leopard.

Biiffon, vierf. VI: p. 193. 216. 'der Panther.

Gesner, Thierbuch, p. 253. Leopard, oder Lep. pard, m. e. fchl. Fig.

Kolbe, Vorgeb. d. g. H. p. I7. Tygerthiere. Cuhn, Samml. von Reif. in d. Innere von Afrika, p. 357 .

Shaw, Reife, p. 152. der Panther.

Goeze, Natur, Menfchenleben und Vorfehong,

I. p. 62. Panterthier, Panther.

Erxleben, Mammalia, p. 505. n. 3. Felis (Pardus) cauda fubelongata, corpore fufco, maculis annularibus nigris, centro notatis.

Linné, Syft. N. Edit. II. p, 43. Tigris maculis orbiculatis. 
Iinate, S. Nat. Edit. VI. p. 4. n. 3. Felis cauda elongata, maculis fuperioribus orbiculatis, inferioribus virgatis:

Linné, S. N. Edit. X. I. p. 41. n. 3. Felis (Par. dus) cauda elongata, corpore maculis fuperioribus orbiculatis, inferioribus virgatis.

Graumann, introd. in hiftor, nat. mamm. p. 74. Panther, Parder, Leopard.

Gatterer, brev. Zool. 1. p. I36. 11. 3. Felis (Pardus) cauda fubelongata, corpore fufco, maculis maioribus irregularibus, paflim confluentibus et anuulatis; das Panterthier, der Parder.

Severin, zool. Hungar. p. 97. 11. 5. Telis Par. dus; der Parder.

Beckmann, de hift. nat. vett. p. 37.

Kramer, Aufr. p. 3 Ir. Felis cauda elongata, ma. culis fuperioribus orbiculatis, inferioribus vir. gatis.

Charleton, exercitat. p. 14. Pardus. Krzorr, delic. II. Tab. K. fig. 4. Pardus. Aldrovand de quadrup. digit. p. 64, Pardus. Alpin. hif. Aegypt. I. p. 237. Pardus, Panthera; Tab. 15. fig. I2.

Ionfton, quadrup. p. ir6. Pardus.

Pennant, Synopf. quadruped. p. "T70. n. 122. the Panther.

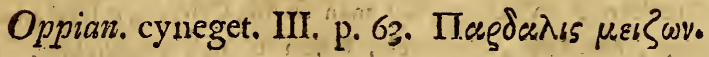

9. U N C I A. Di\& Unze. (4)

Miiller, Naturfyftem, Suppl, p, 29. n. 8. der kleine Panther.

Zinunuer.

(4) If weit kleiner als der Panther und Leopard; der Schwanz aber, der drey Fafs und drüber lang ift, verhältnifsmäfsig länger, 
Zimmermann, geogr. Zoolog. II. p. 40. p. 263. n. I53. die Unze.

Lefke, Naturgefch. p. 137. 11. 4." die Unze.

Borowjky, Thierreich, I. 2. p. 30. n. 3. Felis Panthera; der kleine Panther; die Unze.

Bhumenbach, Handb. der Naturgefch. p. 96. n. 5. Felis (Panthera) cauda elongata, corpore albido, maculis irregularibus nigris; das kleine Panterthier.

Meyer, Thiere, III. Tab. 23. der junge ceilanifiche Tiger.

Gatterer, vom Nutzen und Schaden der Thiere, I. p. 330. n. 190. der kleine Panther; die Unze. Never Schaupl. der Natur, VI. P. 346. der kleine Panther.

Onomatol. hiffor. nat. V. p. 686. die Onke. Gesner, Thierbuch, p. 256. das kleine Pantherthier; ein Unz; ein kleiner Leopard; ein Hunds - oder Wolfspard; m. e. fchl. Fig. Bïffon, vierf. VI. p. 193. 237. die Unze.

Share,

als bey beyden. Der ziemlich lang behaarte Kürper hat auf weifslichtem Grunde unregelmäfsige Flecken. Unter den groffen reiffenden Thieren des Katzengefchlechts hat die Unze ohnfireitig die gröfste Biegfam'seit des Naturells, bey der grö́fsten Erffreckung. Vorlängft hat man fie im Orient zur lagd kleirer Thiere, der Rehe, Gazellen, Haren u. dgl. abgerichtet. Der läger führt fie hinter fich auf dem Pferde. Wónn er das Wild eingeholt hat, lafst er fie auf felbiges los, welches fie fangt, und fich nachher wieder gutwillig greifen, und aufs Pferd nehmen lafst. Auch is Italien und Indien hat man fie in den mittlern Zeiten dazu gebraucht. Die Kürfchner nennen die Haut vom Panterthier Paxterfelle, die von der Unze nennen fie afrikanifche Tigerfelle, die vom Leopard heifsen fie ondlich fehr uneigentlich Tigerfelle. Die Háute, womit zuweilen die Kutfchpferde der Vornehmen gefchmückt find, find gewöhnlich Pantherbiute'. Die wahren Tigerhătite find viel zu rar. 
Showe, Reife, p. 153. der Faadh, oder Chamus. Olear. perf. Reife, p. 23I.

Rytfchkow, orenb. Topogr. in Biifchings Magazin VII. p. 39.

Cuhn, Samml. von Reif. in d. Innere von Afrika, I. p. 361. die Unże.

Le Vaillant, Reife in das Innere von Afrika I. p. 47. 52. der Panther. (Am Cap: Tiger; bey den Hottentotten: Garou, Gama, d. i. gefleckter Löwve.)

Perrault, Charras und Dodart, Abh, zur Naturgefch. II. p. I85. anatom. Befchreib. eines Panltherthiers. Tab.63. das Panterthier. Tab. 64. die Zergliederung.

Dänifche Miffionsberichte, XXXI Cont. p. 773.

Goeze, Natur, Menfchenl. u. Vorfeh. I. p. 63. die eigentliche Unze.

Erxleben, 1.c. p. 508. Felis Panthera.

Graumann, introd. in hifor. nat. mannm. p. 34 : der Kileine' Panther.

Gatterer, brev. Zoolog. I. p. 137. n. 4. Felis (Panthera) cauda elongata, corpore albido maculis irregularibus nigris.

Severin, zool. Hungar. p. 98. n. 6. Felis Panthera, clas Pantherthier.

Thevenot, relat. de la Chine. 1696. p. T9.

Sihwenkffeld, theriotroph. Silef. p. I02. Leo. pardus.

Pennant, Synopf. quadrup. p. I75. n. 126. the Once.

Plin. hift. nat. VIII. c. 15. c. 16. c. 17. X. c. 63. XI. c. 40. c. 49. c. 50. Panthera.

Oppian cyneget. MI. 95. Magdas $\tau \varepsilon \rho \alpha_{\text {. }}$ 
1ค. L E O P A R D US. Der Leopard. (5)

IViiller, Naturfyft. Suppl. p. 29. in. 9. der gutneifche Leopard.

Zimnuermann, geogr. Zool. II. p. 263. n. 154. der Leopard.

Lefke, Naturgefch.p.137. n. 5. der Leopard. Borowfky, Thierreich, I. 2. p. 31. n. 4· des Leopard.

Blumenbach, Handbuch der Naturgefch. p. 96. n. 3. Felis (Leopardus) cauda fubelongata, maculis numerofis, minoribus, obtufe angula. tis; der Leojard.

Funke, Naturgefch. I. p. 153. der Leopard.

Gatterer, vom Nutzen u. Schaden $/$ der Thicre, I. p. 33I. n. I91. der Leopard.

Neuer Schauplatz der Natur, VI. p. 346. der Leopard.

Onomat. hift. nat. V. p. 684. 687. der Leopard. Bïffon, vierf. VI. p. 193. 231. der Leopard. Kolbe, Vorgeb. d. g. H. p. 156. Leoparden oder Panterthiere.

Goeze, Natur, Menfchenl. u. Vorfel.. I. p. 64. Leopardez.

Berlin.

(5) Das Fell des Leoparden hat, einen goldgelben Grund, mit kleinen fchwarzen Flecken, die aber dichter und regelmärșiger als beym Pantherthier, und meift drey bis vier nahe beyfammen ftehen. Bruft und Bauch haben ein etwas längeres Haar, als der äbrige Körper. An dem dritthalb Fufs lnngén Schwanz find die Flecken langlicht und grofs, Die Neger fangen ihn ia Fallen, und graben Lücher in die Erde, welch" mit wenigen geflochtenen Hürden überlegt, und mit einer Lorikfpeife verfehen find, fo dafs die Leoparden, wenn fie fich anf die Hürde wagen, in die Grube fallen. Dq̣ Leopard in gröfser als die Unze, aber kleiner als der Panther. Er ift leichtitr zu zahmen als der Tiger. Sein Fleifch foll an Weifse dein Kalbileifch sleichon:? 
Berlin. Samml. VII. p. 418. (deutfche) Leo. parden.

Hamburg. Magazin, XVI. p. 183. (unrichtige Befchreibung.)

Batfch, Thiere, I. p. 185. der Leopard.

Pennant, Syn. quadr. p. I72. n. 123. the Leopard.

Graumunin, intr. in hifor. nat. mamm. p. 74. V. der guineifche Leopard.

Gatterer, brev. Zoolog. I. p. I37. n. 5. Felis (Leopardus) cauda fubelongata, corpore fufco, maculis numerotis minoribus, obtule angulatis; der Leopard.

Severin, zool.,Hungar. p. 99. n. 7. Felis Leopardus; der Lueopard.

Leon. Afric. defeript. Afr. p. 755. Leopardus.

4, O N C A. Des Taguar. ( $\left.{ }^{6}\right)$

Mitller, Naturfyn. I. p. 238. n. 4. der Panther: Tab. 30. fig. 6.

Zimmermann, geogr. Zoolog.j II. p. 7. 1. p. 268. n. 162. der Iaguar.

Borore.

(6) Kleiner als die Unze. Långe des Kürners ohngefahr dritthalb Fufs; des Schwanzes einen, und etwas druber; doch finden fichtowohl in Anfehung feiner Gröfse, als der Flecken felnes Pelzes vorzügliche Verfchiedenheiten. Die Bartbaare find an diefer Gattung befonders lang. Die Unze năhrt fich von allen Arten der Thiere; getic des Nachts in die Stâdte und Dörfer, um Hühner, Huncie, und andere kleine Thiere zu bolen; bey welcher Gelegenheit Ge zuweilen Kinder mitnimmt, und wenn diefs einmal gefchehen it, fo wird fie alsdann felbn erwachfenet Perfonen gefährlich. Bey allem dem aber it diefs Thier weit furchtfamer und feiger als die vorigen, fo dafs es tich, wenn es fart iff, fchon von einem nur märsig groifsen Hunde jagen lafst. Man fàngt es in Fallen und Schlingen'; die Mulatteri wiffen es im Zweykampfe zu tödten, nachoem. fis ihm beym Angriff dis Pfoten abgehauen haben. 
Borow Jky, Thierreich, I. 2. p. 33. n. 7. der Iaguar, amerikanifche Tyger, Tygerkat:ee.

Blumenbach, Handb. der Naturgefch. p. 97. n. 6. Felis (Onca) cauda fubelongata, corpore fufco-lutefcente, maculis angulatis, ocellatis, medio flavis; Halle, vierf. p. 531. der amerikanifche Tiger.

Gatterer, vom Nutzen und Schaden der Thiere,

1. p. 333. n. 195. der Iaguar.

Neuer Schaupl. der Natur, IV. p. 212. der Iaguar. Onomat. hift. nat. IV. p. 450 . Iaguara.

Kleinz, Quadr. difp. p. 80. Tigris americana Iaguara Brafil.

Klein, Claflif. d. vierf. Th. p. 244. amerikanifcher Tiger.

Klein, natürl. Ordn. d. vierf. Thiere, p. 36. n. 3 . amerikanifcher Tiger.

Geswer, Thierbuch, p. 342. Tlatlavhqui Ocelotl, feu Tigris americana; ein mexikanifeh Tiger. thier.

Biiffon, vierf. VI. p. 240. der. Iaguar; m.e. Fig. Carver, Reifen d. d. Innere von Nordamerika, p. 360. der Tiger.

Bunkroft, Naturgefch, von Guiana, p. \$2. der Tiger von Guiana.

Hartfink, Befchr. von Guiana, p. 104. Iawari, Inguar.

Dobrizhoffer, Gefch. der Abiponer, I. p. 32I. der Tieger; Onza and Mbaracaya. p. 33I. Yaguareté, Nihiranak, Apanigehak, Lapriratraye.

Falkner, Befchr. von Patagonien, p. 74. Tiger. Fermin, Befchreib. von Surinam, II. p. 85. die fogencanten Tiger.

Nachr. v. d. Lande Guiana, p. 176. Tiger. 
Reifen der Miffionar. in Amerika, von Murr, p. 542: Tiger.

Berkel, Reife nach Berbice; in Blumenbachs Rei. fegefchichten, I. p. I64. Tiger.

Dapper, Befchr. von Amerika, p. 288. Tiger? Samml.- zur Phyf. und Naturgefch. II. p. 606.

Tiger.

Berlin. Samml. IV. p. 151. der guianifche Tiger. Erxleben, Mammalia, p. 513. 11. 9. Felis (Onca) cauda fubelongata, corpore fufco - lutefcente, ocellis nigris, rotundato angulatis, medio flavis.

Limné, Syf. Nat. Edit. X. I. p. 42. n. 4. Felis (Onca) cauda elongata, corpore flavefcente, maculis nigris, rotundato angulatis, medio flavis.

Graumann, intr. in hift. nat. mamm. p. 74. IX. der amerikanifche Tiger.

Gatterer, brev. Zoolog. I. p. I38. n. 9. Felis (Onca) cauda fubelongata, corpore fufco-lute. fcente, maculis angulatis, ocellatis, medio fla. vis; der Iaguar.

Pennant, Syn. quadr. p. 176. n. 127. the Brafilian Panther.

5. Pardatis: Der Ozlot. (7)

Milller, Naturfyftem, I. p. 239. n. 5. die wilde Katze. 'Tab. 3०. fig. 7 .

\section{Zimmer.}

(7) Thut dem jungen Rindviehe, und dem Wilde viel Schaden, dem er auf den Bäumen auflatert; und vorzüglich das Blut daw von geniefst. Das Marnchen ift fchöner als das Weibchen, und fehr herrifch gégen diefes. Die Gröfse des Thiers fiade ich hỉ and wieder ganz verfchisden angegeben. 
Zinmernann, geogr. Zoolog. II. p. 269. n. 164. der $O=10 t$.

Borowfky, Thierseich, I. 2. p. 34. n. 9. der Ozlot.

Gatterer, vom Nutzen u. Schaden der Thiere, I. p. 335 . n. 196. $\operatorname{der} O \approx e l o t_{\text {s }}$

Onomat. hift. nat. II. p. 702. Catus Pardus; die Berghatze, die amerikanifche Pantherkatze; die amerikanifche wilde Kat:e.

Penzunzt, arct. Zool. II. p. 53. n. 24. die Pardel. katze; die geftreifte Bergkatze?

Klein, Claffif. d. vierf. Th. p. 238. Parder mit kurzem Schwanz; Kat 2 maparder.

Klein, natürl. Ordn。 der vierfüls. Th. p. \$3. n. 2. Katzenparter.

Büffon, vierf. XIV. p. 262. cier Osilot.

Cerver, Reifen durch Nordamerika, p. 363. die Bergkatse.

Bankr oft, Naturgefch. von Guiana, p. 83, der Lachs von Guiana.

Erxleben, Mammalia, p. 515. n. ro. Felis (Pardalis) cauda elongata, corpore fufco maculis ocellaribus elongatis.

Linné, Syn. Nat. Edit. VI. p. 4. n. 4. Felis cauda elongata, maculis fuperioribus virgatis, inferioribus punctatis.

Linné, Syit. Nat. Edit, X. I. p. 42. n. 5. Fe. lis (Pardalis) cauda elongata, corpore niaculis fuperioribus virgatis; inferioribus orbicu= latis.

Graumann, intr. in hift. nat. mamm. p. 74. X. die villa'e Katze.

Dampier, Voyage, III. p. 306. le Chat Tigre. Pennant, Synopfo quadrup. p. 185. 11. 134. the Mountain? 
Gatterer, brev. Zool. I. p. I38. n. ro. Felis (Pardalis) cauda elongata, corpore fufco, maculis ocellaribus elongatis; der $O z e l o t$.

11. I U в A T A. Der Gepard: $\left(^{8}\right)$

Miiller, Naturfyfen, Supplem. p. 30. n. I3. der Wolfstieger.

Zinnermann, geogr. Zoolog. II: p. 264. 11. 154. der Gepard.

Borowfky, Thierreich, I. 2. p. 32. n. 5. der Gb: pard, Wolfstieger.

Helle, vierf. p. 533. der Tiegerwolf.

Gatterer, vom Nutzen und Schaden der Thiere;

I. p. 332. n. 192. der Gepard.

Biiffon, vierf: XIV. p: 283. der Gepard.

Graumann, introd. in hift. nat. mamm. p. $74 . \mathrm{VI}$. der Wolfstieger:

Tavernier, Voy. I. p. 348. une certaine bête, ap: pellée Once:

(8) Länge dès Kürpers viertchạlb Fufs; des Schwanzes etwas über anderthalb Fufs. Weña das gezahmte Thier zu einer Antilopen oder Gazellenjagd gebraucht werden foll, fo führt man es auf einem kleinen Wagen an einer Kette, und mit verbunde-? nen Augen zu einer Heerde Gazellen, und lafst es los. Wenn es fich in Freyheit fieliet, drückt es fich an die Erde und fught fich ünvermerkt an eine Gazelle zu fchleichen; und fobald eq feinen Vortheil abgefehen hat, thut es mit einer uriglaublichen Gefchwindigkeit 5 bis $\sigma$ weite Sprünge auf diefelbe, reifst fie nieder, und geniefst nebit den Blute ein en Theil des Eingeweides: Verfehlt is aker die Gazelle, fo weifs es ihr nicht weiter nachzufetzen, und würde auch unvermögend feyn, fó lange zu laufen, als es die Gazelle aushẩle. Der Führer năhert fich ihm, alsảann behutfarn, giebt itim Fleifch, fchmeichelt ihm, bedeckt ihm wâhrend der Zeit die Augen vom 'neuen; legt ihm die Kette wieder an, und bringt es auf den Wagen zuriick: 
Gatterer, brev. Zool. I. p. 136. n. 6. Felis (Iubata) cauda mediocri, corpore fulvo, maculis nigris, collo iubato. Tiegerwolf.

12. Discoror. Der Schwarze Tiger.

Zimnnermann, geogr. Zool. II. p. 267. 11. 161. der Schwarse Tiger.

Halle, vierf. p. 531. der gröfste Tiger mit fchwarzen Flecken, anf lichtgelbem Grunde.

Neuer Schauplatz der Natur, IV. p. 2I3. Iagiarette.

Onomat. hift. nat. IV. p. 459. Iaguarete. Klein, Quadrup. difp. p. 81. Tigris Inguarete. Kbin, Claflif. d. vierf. 'Th. p. 245. Ingwarete. Klein, natürl. Ordn. d. vierfüfs. Thiere, p. 86. n. 3. Iaguarete.

Biiffon, vierf. VI.' p. 258. Inguarete, oder der fohwarze Tiger.

Berkel, Reife nach Berbice; in Blumenbachs Reifegefchichten, I. p. 238. der fchwarze Tieger. Hartfink, Befchreib. von Guiana, I. p. 105. der Iaguarete.

9. Concolor. Der Kuguar. (9) (amerikanifche Löwe.)

Miiller, Naturfyftem, Suppl. p. 3०. 11. 12. die brafilianifche Katze.

Zimmer.

(9) Da diefs Thier der Breite nach eine Strecke von melor als 95 Graden einnimmt, und aufserft verfchiedene Klimate ercrägt, fo mufs es hin und wieder, wie alle verbreitete Thiere, ausarten. Der Kuguar von Nordamerika ift fchwacher und nuthlofer, als der, welcher in den heifsern Gegenden wobnt. Bey den Irokefen ift er grau, bey andern röthlich gefärbt, etc. Sei- 
Zimmermann, geogr, Zoolog. II. p. 70. der Cuguar, oder der rothbraune Tieger. p. I63. 269. der Kuguar.

Borowe/ky, Thierreich, I. 2. p.32. n.6. der Kuguar, rother Tyger.

Blumenbach, Handb. d. N. G. p. 97. n. 7. Felis (Concolor) cauda mediocri, corpore immaculato fufco; der amerikanifche Löwe, Puma, Cuguar.

Halle, vierf, p. 533. der grofse amerikanifche Tiger. Neuer Schauplatz der Natur, II. p. 26I. Cigguacuanua.

Onomat. hift. nat, III. p. 499. Cuguacuarara. Klein, Quadruped. difp. p. 8I. Tigris Cuguacuarana Marcgr.

Klein, Claflif, d. vierf. Th, p. 245. Cuguacuarana.

Klein, natürl. Ordnung d, vierf. Th. p. 86. n, 4. Cuguacuarana.

Pennant, arch. Zool. II. p: 5o. n, 2I. der Pukn oder Cuguar.

$$
\mathrm{P}_{3} \quad \text { Biiffons }
$$

תe Länge if gewöhnlich viertehalb, des Schwanzes zwey Furs. Die Spizze des Schwanzes ift fchwarz. Sein Aufenthalt ift gewöhnlich in den Wäldern, wo er auf den Bäumen die Thicre belauert, In Nordamerika neant man ihn Passber, und er ift in dortigen Waldern allen Thieren fehr fchädlich. Za Zeiten gnurt er, wie dic Katzen, im Wohlbehagen, da er ein andermal ftark heult. Er greift auch reiffende Thiere, als Wölfe, an. Wenn er fich an feiner Beute gefättigt hat, fo vergräbt er die Ueberbleibfel unter den Laube, geniefst aber, nichts weiter davon, fobald er merkt, dafs cin anderes Thier fie berühret hat, Sein Fleifch wird gegeffen, und foll wie Kalbfleifch fchmecken. Aus feinem Felle macnen fich die Indianer Winterkleider, auch bereiten fie es zu Schuhen und Handfehuben. Die Begattung foll am Ende des Winters gefchehen, das Weibchen drey Monate trächtig gehen, und zwey Innge bringen. 
Büffon, vierf. VI. p. 26r. der Kuguar.

Goeze, Natur, Menfchenleben und Vorfehungs IV. p. 549. der amerikani iche Löwe.

Gettever, vom Nutzen und Schaden der Thiere, I. p. 332. n. 193. der Kuguar, grosse amerika. nifche Tiger.

Hartfink, Befchr, von Guiana, I, p. 105. Cagua cuarane.

Hartfink, Befchr. von Guiana, I. p. 108. Puma. Dobrishoffer, Gefch, der Abiponer, I. p. 332: der Löree.

Charlevoix, Gefch, von Paraguay; p. 225. die Lörent.

Schlözer, Erdbefchreibung von Amerika, p. 278: der amerikanifche Tiger.

Molina, Naturgefch, von Chili, p, 262. 11. 8. Felis Puma, der Pagi, ider Initzili; (in Mea xico) der Puma, (in Peru) p. 263 . der chiles fische Löwe.

Schöpf. Reif. durch die nordamerikan. Staaten, $\mathrm{I}$. p. 42I. 252. der rothe Tiger. II. p. 155. der. hieflge Tiger. p. 167. Cuguar.

Pilauve, Gefch. von Chile, p. 84. der Pagi. Berkel, Reife nach Berbice; in Bhinzenbachs Reie fegefch. I. p, 240. rothe Tiger.

Begert, Nachr.von Californien, p. 63. Leopard? Erxlebenr Mammal.p. 5II. n. 7: Felis (Concolor) cauda mediocri, corpore immaculato fulvo.

Graumanz, intr. in hift, nat, mamm. p. 74. VII, brafiliani/che Katze.

Charlevoix, hif: de la nouv. France, I. p. 44.

Gumilla, hif, nat. de l'Orenoque, II. p. 3. I.p. I72: Gatterer, brev.Zool. I. p. 138. n.7. Felis (Concolor) cauda mediocri, corpore immaculato, fulvo; der Kuguar. 
13. TI G In A. Die Miaragua.

Miiller, Naturfyft. Suppl. p. 29. 11. 10. der Margay. Zimmsermann, geogr, Zjolog. II, p. 27a, n. I65. die Maragua; die anterikanifche Tigerkatze. Borowjky, Thierreich, I.2. p. I7. n. Ir. die Tygerkatze, Maragua.

Halle, vierf. p. 522. dic Tigerkatze.

Halle, vierf. p. 523. die anerikanifche Katze.

Meyer, 'Thiere, III. Tab. 22. die wilde amerikanijihe Tigerkatze.

Gatterer, vom Nutzen und Schaden der Thiere, I.p. 336: n. 197. der Maragaa, Maragey.

Dapper, Amerika, p, 445. die vilden Katzen Margaja.

Onomat. hift, nat. II, p. 706. Catus Tigris fylveftris, die wilde Tigerkatze.

Klein, Quadrup. difp. p. 75. Alius Tepe Maxt. laton dictus, tygrinus ex Hifpaniola.

Klein; Claflif, d, vierf. Th, p. 228. Tepe MIaxtlaton.

Klein, natürl. Ordn, d, vierfüfs. Th, p. 80. n. 80.

Tepe Maxtlaton.

Biiffon, vierf. XIV. p. 274. d'er Margay. m.e. Fig; Bankiroft, Naturgefch. von Guiana, p. 83. Ti. gerkatze von Guiana.

Reifen der Miff. in Amerika, von Miv»r, p. 543. - Maracaja, der brafilicanifche. Fuchs.

Gilii Nachrichten von dem Lande Guiana, p. I80.' Tigerkatze.

Berkel, Reife nach Berbice; in Bhunenbachs Reifegefchichten, I. p. I65. Katzentieger.

Fermin, Befchr. von Surinam, II. j.8j. Felis fera tigrina; Tigerkatze.

Graumann, intr. in hiftor, nat. mamm. p.75. XI. dis Tigerkatze. 
Seba, thefaur, I. p. 77. Felis fylveftris tigrinus ex Hifpaniola. 'Tepe Maxtlaton Tab. 48. fig. 2.

Gatterer, brev, Zool. I. p. 138. n. Ir. Felis (Tigrina) cauda elongata, corpore fulvo, nigro friato, maculatoque, fubtus albido; der Margay.

6. Cатus. Die Kqtze.

Miiller, Naturfyft. I. p. 240. n. 6. die Hauskatze.

Zimnermann, geogr. Zool. I. p. 193. die Katze. Schreber, Säugthiere, III. p. 397. die Katze, Lefke, Naturgefch. p. 137. n. 6. die Katze. Borowfly, Thierreich, I. 2. p. 38, n. 12. die Katze.

Bhinienbach, Handbuch der Naturgefch, p. 98. n. 9. Felis (Catus) cauda elongata, friis dor. falibus, longitudinalibus, lateralibus fpiralibus; die Katze.

Bechftein, Naturgefch. Deutfchl. I. p. 25I. die Katze.

Flunke, Naturgefch. I. p. 76. die Katze.

Beckmann, Naturhiftorie, p. 28. n. 18. die Katze.

Handbuch der Naturgefch, I. 2. p. 79. die Katze.

Verfuch einer Katzengefch. Frankf. r772. 8.

Pennant, arct, Zool, II, p. 54. n. 25. Hauskatze. Haller, Elem. Phyfiolog. T. VIII. P. II. p. 93.

Naturforfcher XII. p. 100,

Gmelin, d. ä. Reife nach Sibirien, I. p. 315 .

Büffon, vierf. II. p. 260. die Katze.

Verfuch einer Katzengefchichte. Frcf, a. Lpz. I772. 8.

Carpzovii Kattologia; d. i. kurze Katzenhiforie, darin insgemein von den Katzen, auch infon. derheit von einer ungewöhnlichen Katzenge. burt, fo zu Leipzig 1713 . gefchehen, gehandelt wird. Lpz. x716.8. 
Erxleben, Mammal. p. 518. n. 12. Felis (Catus) cauda elongata, fufco annulata, corpore fafciis nigricantibus; dorfalibus longitudinalibus tribus, lateralibus fpiralibus.

Linné, Syf. Nat. Edit. II, p. 43. Felis cauda elongata, auribus aequalibus.

Linne, S, N. Edit. VI. p. 4. n, 6. Felis cauda elongata, auribus aequalibus.

Linné, Synt. Nat. Edit. X. I. p. 42. n. 6. Felis (Catus) cauda elongata, corpore fafciis nigricantibus; dorfalibus longitudinalibus tribus, la teralibus fpiralibus.

Kramer, Auftr, p. 3II. Felis cauda elongata, au. ribus aequalibus.

Charleton, exercitat. p. 20. Felis.

Granmonn, intr, in hif, nat, mainm. p. 75. XIr, Felis Catus.

Gattever, brev. Zoolog. I. p. 139. n. 12. Felis (Catus) cauda elongata, fufco annulata ; corpore falciis nigricantibus, friis dorfalibus longitudinalibus tribus, lateralibus fpiralibus; dis Katze.

Miiller, prodr, zool. dan. p. 2. n. 8. Felis (Ca. tus) cauda elongata, fufco annulata, corpore fafciis nigricantibus; dorfalibus longitudinali. bus tribus, lateralibus fpiralibus.

Pennant, Syn, quadr. p. I83. n. I33. the common Cat. Ariftot. biftor, anim. V. c. 2. n. 22. VI. c. 35 . n. 422. 423. Ainovejos.

Plin. hilt, nat. X. c. 73. XI. c. 37. Feles,

e. FER Us. Die wilde Katze. $\left({ }^{10}\right)$

Mïller, Naturfyn. I: Tab. 3r. fig. 5.

$$
\text { P } 5
$$

Zimmera

(10) If grơlser als, die zahme, und unterfcheidet fich überdiefs von derfelben aufserlich befonders durch die graue Farbe 
Zimmermańn, geogr. Zool. I. p. 193. II. p. 266. n. I60. die vilde Katze. Lefke, Naturgefch. p. I37. n.6. die reilde Katze. Borowfky, Thierreich, I. 2. p. 38. n. 12. a; Catus ferus fylventris, die vilde Katze. Bhumenbach, Handbuch der Naturgefch, p. 98. wilde Katze.

Bechftein, Naturgefch. Deutfchl. I. p. 262. b. die wilde Katz2.

Funke, Naturgefch. I. p. 76. die wilde Katze.

Halle, vierf. p. 521. Catus fylveftris; die reilde Katze.

Neuer Schauplatz der Natur, IV. p. 425. wilde Katzen.

Onomatol. hif. nat. II. p. 704 . Catus fylventis; eine wilde Katze; eine wilde IValdkatze.

Handbuch der deutfchen Thiergefchichte. Gief, 1790. p. 60. die vilde' Katze.

Gatterer, vom Nutzen u. Schaden der Thiere,

I. p. 336. n. 193. die vilde Katze.

Goeze, europäifche Fauna, I. p. 252. die wilde Katze.

MLurr, Iapan und China; im Naturforfcher, VII. p. 48. Felis fylveftris.

Gesner, Thierbuch, p. 246. Catus fylveftris; ein Baumreuter.

ITlein, Claflif. der vierfüls. Th, p. 227. n. 2. quilde Katze:

Kleinz

Ichwatzen Lefzen und Fufsfohlen. Der Kopf iff auch etwas weniger platt, und das Haar über den ganzen Leib länger und feiner. Sie nahrt fich nicht nur von Hamftern, Maulwürfen, Mäufen und Federwildpret, fondern ftellt fogar Hafen und felbft jungen Rehen nach; lauert auch im Schilfe am Ufer dem Wafterseflügel und Fifcheı aur; heckt in hahlen Baumen oder Felfen,' auch in Fuchs-oder alten Dachșauen. 
Klein, natürl. Ordn. d. vierf. Th. p. 80. n. 2. wile der Kater; Waldkater.

Kraft, Ausrottung graufamer Thiere, II. p. 440; wilde Katze.

Biiffon, vierf. II. p. 230. wilde Katze. Tab. 39.

Fontoppidan, Hif. von Norwegen, II. Ed. in 8,

p. I8. wilde Katzen.

Sparrmann, Vorgęb. d, g. H. p. I44. wilde Katze. Cetti Naturgefch. von Sardinien, I. p. 202. die wahre wilde Katze.

Ryt fchkow, orenburg. Topogr, I, p. 232. wilde Katzen.

Olaffen, Reife durch Island p. 30. Urdar-Kettir; wilde Katzen.

Miller, Samml. run. Gefch. III. p. 608. wilde Kntzon.

Bofsmann, Reife nach Guinea; p. 282.

Lichtenberg, Magazin für das Neuefte etc. III. 2 , p. 162.

Dübel, Iägerpraktik, I. p. 4r. vilde Katzen. Erxleben, Mammalia, p. 518. n. 12. c. Catus ferus.

Grammann, intr. in hift. nat. mamm. $p_{9} 75$. XII. $a_{9}$ wilde Katze.

Beytr, zur Beförder. der Națurk. I. p. Ir4. wilde Katze.

Schwenzfeld, theriotroph, Silef, p. so. Catus fil. veftris.

Wagner, hift. nat. Helvet. p. I75. Felis feu Cat tus filveftris.

Frorfter, Philof. Tranșact. LVII, p. 342. Felis Catus.

Sperling, not, ad Teftamentum Abfalonis: p. I2?: 
B. Domesticus, Die Hauskatze. (')

Miiller, Naturfyn. I. Tab. 3I. fig. 6.

Zimmermann, geogr. Zool. II. p. $26 \%$ n. $\%$. die gevöhnliche Hauskatze.

Le $\int k e$, Naturgefch. p. I37. n. 6. die zahme Kaize. Borowfky, Thierreich, 1. 2. p. 38. n. b. die Hanskatze.

Bhumenbach, Handb. der Naturgefch, p. 98. die Hauskatze.

Bechftein, Naturgefch. Deutfchl. I. p. 254. a. dio zahme Katze.

Funke, Naturgefchichte, I. p. 76. die zahme Katze.

Halle, vierf, p. 519. die Hauskatze.

Mleyer, Thiere,'I, Tab. 15. Eine Katz. Fig. mittelm.

Gatterer, vorn Nutzen und Schaden der Thiere, I. p. 398. n. 199. die Hanuskatze.

Handbuch der deutfchen Thiergefchichte, p. 6\%. die zalme Katze.

Sander, ökonom. Naturgefeh. I. p. 24 I.

Walther, ökon. Naturgefch: Deutfchl. p. I43.

Neuer Schauplatz der Natur, IV, p. 424. Katze.

Onomat. hift. nat. II. p. 698. Catus domefticus;

eine Katze; eine Hauskatzb.

Naturforfcher, XII. p. I07. zahme Katzen.

Gesner, Thierbuch, p. 239. Felis vulgo Catus: eine Katz; p. 240, eine gute Fig,

Klein,

(1) Eine blofse von der wilden Katze durch Kultur entfandene $\Lambda$ usartung. Sie begattet fich nicht felten mit der wilden, und verwildert auch fehr leicht wieder, wenn fie zufällig in Wildheir geräth. Merkwürdig ift die angffliche unüberwindliche Antipathie vieler Menfchen gegen die Katzen, Dafs diefe Thiere keine Flühe haben foltion, widerfprich der Erfahrung, die ein jeder loich felbft anftellẹn kann. 
Klein, Quadr. difp. p. 75. 'Catus domefticus; Fe. lis domeftica.

Klein, Clafif. der vierfüfs. Thiere, p. 226. n. I. Kater, Katze.

Klein, natürl. Ordn. d. vierf. Th. p. 8o. n。 $I_{0}$ zahme Katze.

Biiffon, vierf. II. p. 233. die Haliskatze. Tab. 38.

Pontoppidan, Norwegen, II. Edit. in 8. p. I8. zahme Katzen.

Krïnitz, ökon. Encykl. XXXVI. p. 20r. Hanls. oder zahme Katze.

Bock, Naturgefch. von Preuffen, IV. p. 37. n. 9. die Hauskatze.

Goeze, Naturgefch. der Hauskatzen; in der Mo. natsfchrift für allerley Lefer, I. p. 47I. Europäifche Fauna, I. p. 197. die zahme Katze. Beyträge zur Beförd. der Naturkunde, t. p.215. c. die Houskratze.

Berlin. Magazin, I. p. 228.586.

Merklein, Thierreich, p. Ioo ff. Katze.

Bunzlauer Unterhaltung. I. p. 292.

Anınerkungen über alle Theile der Naturl. I.

p. 54. 154 .

Schwed. Ablyandl. VII. p. 221.

Carpzov. Katzenhiflorie. Leipzig 1716. 8.

Verfuch einer Katzengefchichte. Frof. u. Lpz.

I772. 8 .

Schulae, de felis amore erga hominem; in den Mife. Nat. Cur. A. 1675. et 1676. obf. 162.

Hanemanin, obf. de antipathia cum felibus; in den Mifc. Nat. Cur. Dec. II. A. II. obf. 50.

Wochenblatt für den geneinen Mann, II.

p. 47 .

Lanzoni obferv. de fele rabida; in Mangetibibl. chirurg. III. p. 578.

Erxle- 
Erxleben, Mammalia, p. 520. n. ß. Catus do. mefticus.

Granincmin, intr. in hif. nat. mamm. p. 75 . XII.b. clie Hauskatze.

Ephemerid. Nat. curiof. Dec. I. an. 3. obf. 198. Schwernffeld, theriotroph. Silef. p. 79. Catus domefticus.

Sibbald. Scot. illuftr. II. p. 13. Felis.

Rzacz. hiftor. nat. Polon. p. 244. Feles feu Cati domeftici.

Pennant, britt. Zool. p. 2r. the domeftic Cat.

y. $\Lambda$ N G O E NSIS. Die angorifche Katze. $\left({ }^{2}\right)$

Zimmermanin, geogr. Zool. I. p. 197. II. p. 26\%. n. $\delta$. die angorifche Katze.

Borow ky, Thierreich, I. 2. p. 38.n. c. die ango. rifthe Katze.

Blumenbach, Handb. d. Naturgefch. p. 98. die angorifche Katze.

Neuer, Schauplatz der Natur, IV. p. 425. die ans: gorifche Katze.

Biiffan, vierf. II. p. $23 \%$. die angorifche Hairska: tzee. Tab. 40. Fig. 2.

Evxleben, Mammalia, p. 52 i. n. $\gamma$. Catus An: gorenfis: domefticus, pilis longioribus argen: teis fericeis, in collo longifimis.

Groumann, intr. in hif. nat. mamm. p. 75. XII. n. c. Catus Angorenfis.

Gatte:

(2) Zeichnet fich durch ihr fehr langes feidenartiges glänzendes Haar; das gemeiniglich fchneeweifs iff, oder etwas ins Geibliche fallt, deutlich aus. Sic kommt aus Angora in Syrien, und wird.häufig in Perfien, in Europa aber felten gehalten: In Deutfchland vermehrt fie fich vermuthlich deswegen nicht ftark; veil fie die Kälte nicht gut vertragen kasa. 
Gatterer, brev. Zoolog. I. p. 140: c. Angorenfis; die angorifche Katze.

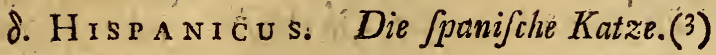

Zimmermann, geogr: Zoolog. I. p. 197. II. p. 267 . n. $\gamma$. die fpanifche Katre..

Borowf'ky, Thierreich, I. 2. p. 38. ti. d. die fpariifehe Katze.

Bluminbach, Handb. der Naturgefch. p. 98. die Spanifche oder Schildpattfärbige Katze.

Neuer Schauplatz der Natur, IV. p. 425. die fpanifiche Katze.

Biiffon, vierf. II. p. 234. die fpanifche Haiskatze. Tab. 39. fig. 2.

Erxleben, Mammalia, p. 522. n. $\delta$. Catus Hio fpanicus, nigro, albo aurantioque varius.

Graumiann, introd. in hiftor. nat. mamm. p. 75 . XII. n. d. Catus Hifpanicus.

Gaiterer, brev. Zoolog. I. p. 140. d. Hifpanis cus; die Spanische Katze.

8. CA ER UL EUs. Die Karthenerkatze. (4)

Zimmermann, gengr. Zoolog. I. p. 197. II. p.267\% n. B. die Kartheiserkatze.

Borow:

(3) Die röthliche, lebbafte und tiefe Fatbe, it das vornetimfte; und vielleicht das einzige Merlsmal, welches diele Katzen von andern unterfcheider. Die rüthliche. if wenigftens die Hauptfarbe; oft mit weifsen und fchwarzen Flecken. Auch das Haar ift etwas weicher.

(4) Von der blateit Farbe findet fich eigentlich bey diefer katze gar" keine Spur. Thr Haar ift auf dem gröfsten Theil feiner länge afchgrau, an der Spitze hingegen unter dem Ende fchwärzlich braủin. Die fehr bufchigten Haare liegen diclit über eirander. Die Mifchung von grau und braun tnterfolieidet fick blors in der Natié. 
Borowjky, Thierreich, I. 2. p. 38. n. $\varepsilon$ die Kartheuerkatze.

Blmmenbach, Handbuch der Naturgefch. p. 98. die blaulich giaure Cartheufer - oder Cyper. katise.

Neuer Schaupl. der Natur, IV. p. 425. die Cartheuferkatze.

Onomatol. hiffor. nat. II. p. 705. Catus fylveAris Cyaneus; die blane wilde Katze.

Buiffon, vierf. II. p. 236. die Karthenferkatze, oder a)chfarbige Hauskatze, 'Tab. 40. fig. I.

Beytr. zur Beförd. der Naturkunde, I. p. II5. n. d. die Karthenerviatze. Blane Katze.

Erxleben, Mammalia, p. 522. 11. ع. Catus Caeruleus.

Granmann, intr. in hiftor. nat. mamm. p. 75. XII. n. e. dic Karthene erkatze.

Gatterer, brev. Zoolog. I. p. I40. e. Caeruleus; die Karthenerkatie.

S. R U в ER. Die rothe Katze.

Onomat. hilf. nat. II. p. 706 . Catus fylveftris ru= fus; die rothe wilde Katse.

Erxteben, Mammal. p. 530.*** Stria pulchra fplendide rubra lata in dorfo a capite incipiens. Venter cinereus, five albicans.

Graumann, intr. in hif. nat. mamm. p. 76. XV. 11. 3. rothe Katzers.

14. Cayensis. Die Capkatze.

Zimnernann, geogr. Zoolog. II. p. 27 r. t1. a. die Capkatze.

Kolbe, Vorgeb. d. g. Hoffn. p. I54. Tygerbuschkatæen? 
-Sparrmann, Reife nach dem Vorgeb. d. g. H.

p. 534. Tigerbufchkatze. (s)

Buiffon, vierf. VI. p. 318. die Tigerkatze von Cap. Erxleben, Mammalia, p. 529. * (Species obfcura.) Longitudo fere trium pedum. Pili breves, folendentes, ferruginei. Facies lineis nigris deorfum verfis. Dorfum ab occipite ad caudam ufque ftriis oblongis nigris. Latera maculis parvis nigris numerofiflinis. Venter albus. Cauda élongata fufca, nigro maculata.

Granmann, introd. in hiftor, nat. mamm. p. 76. $\mathrm{XV}$. n. i.

\section{Manut. Der Manul.}

Zimmermarn, geogr. Zool. II. p. 265. n. 157. der (Karakal, ein Schreib - oder Druckfehler, für) Manul.

Schreber, Säugth. İI. p. 406. der Mannul. Biiffon, vierf. VI. p. 315. der Mannil.

Pallas Reife, Auszug, III. Anh. p. 2. n. 2. Fèlis Manul.

Pallas, nord. Beytr. III. p. I42. Stein-oder wilde Katzen.

16. Servat. Der Serval.

Mibiller, Naturfyft.Suppl. p. 30. n. ̈̈4. der Serval.

Zimmer-

(5) Diếs mufs ich wenigftens:aus. Sparrmanns eigener Erklärung fchliefsen, denn et giebt diefs Thier am angef. $O$. für eben daffelbe aus; welches Forfier in den phil. Transact. befchrieben hat; fürbt auch eben die Stelle an, die im Syftem felbft mit bemerkt it. Glèichwohl aber fagt, er p. $14 \%$. wo er deffelben Thiers fchon Erwähnung that; er kß̋nne nicht anders fchliefsen, als dafs es mit Büffons Serval einerley fey. Ich getraue mit nicht diefe Zweydeutigkeit $z u$ heben. 
Zimmermann, geogr. Zool. U. p. 265. n. 955. der Serval.

Borowelky, Thierreich, I. 2. p. 41. n. 13. der Serveal; Katrenpardel.

Halle, vierf. p. 529. der Ǩatzenparder.

Gatierer, vom Nutzen und Schaden der Thiere,

I. p. 340. n. 200. der Serval, Katzenpander. Never Schaupl. der Natur, VIII. p. 219. Serval, Maraputé, Tiegerkatze, Pferdekatze.

Sparrmann, Reife n. d. Vorgeb. d. g. H. p. 146.

Tigerkatze; Tigerwaldkat:e?

Bïffon, vierf. XIV.p. 256. der Serval.

Perranlt, Charras und Dodart, Abhandl. zur

Naturgefch. I. p. 125." anatomifche Befchreib. einer Pardelkatze; Tab. XIV. Tiegerkatze. Tab. XV. die Zerg!iederung.

Graumanu, introd. in hiftor. nat. mamm. p. 75 . XiII. Serval.

Gatterer, brev. Zoolog. I. p. I40. n. 13. Felis (Serval) cauda fubabbreviata, corpore cinereo, nigro, oblcure ftriato et maculato; Katzenparder.

7. Cir A u s, Der Kirmyschak.

Zimmermann, geogr. Zoolog. II. p. 266. n. 159. der Kyvmischak.

Bïffon, vierf. VI. p. зI6. der Kirmyfchak.

Borowejiky, Thierreich, I. 2. p. 43. der Kirmy. Schak.

18. C A r a c a L. Der Karcikal. (6)

Iiiiller, Naturf. Suppl. p.30. 11. 15. Ner Caracal.

Zinnmer.

(6) Kümmt an Grüfse dem Luchfe bey; năhrt fich von allerley

Thiercn, und fattigt fich bisweilen von den Ueberbleibfeln des 
Zimmermann, geogr. Zoolog. II. p. 39. der Ca. racal.

Borowfky; Thierceich, I. 2. p. 4I, n. I4. der Karakal.

Halle, vierf. p. 527. Luchskatze nit gelben Haa. ren, ohne Flecken.

Gatterer, vom Nutzen und Schaden der Thiere, I. p. 34I. n. 20I. der Karakal; perfianifche Luchs.

Neder Schaupl. 'd. Natur, II. p. 43. Caracal. Klein, Claffif. d. vierf. Th. p. 235. Luchs mit dem Kälberfchwanze.

Klein, natürl. Ordn. d. vierf. Thiere, p. 82. n. 3 . Kalbluchs.

Büffon, vierfüfs. VI. p. 299. der Karakal; m. e. Figur.

Sparrmann, Vorgeb. d. g. Hoffn. p. 145. die raus he Katze; Pennant's, perfifche Katze; Biif: fons Karakal.

Hiöft, Nachr. von Marokos und Fes, p. 294. wilde Katzen ; Gat el Kalä.

Shaw, Reife, p. 155. Gat el Khallah; Siyah ghush; oder Karrah Ku-lak.

Erxleben, Mammal. p. 524. r. I4. Felis (Caracal) cauda fubabbreviata, auriculis apice barbatis, corpore immaculato.

Limné, Syft. Natur. Edit. II. p. 43. Felis cauda elongata, auribus penicilliformibus.

Linné, S. Nat. Edit. VI. p. 4. n. 5. Felis cauda elongata, auribus penicilliformibus.

$Q_{2}$

Grait.

Löwen, welches zu der Erzahlung Anlafs gegeben hat, 'er fey cin Spion des Lüwen, und jage ihm Thiere zu, wovon hernach letzterer ihm etwas zur Belohnung übrig laffe. in Oftindien wird er zur lagd, aúf Hâen, Kaninchen, und grofse Vügel ab. gerichtet. 
Groumann, intr. in hift. nat. mamm. p. 76. XIV. Caracal.

Gatterer, brev. Zoolog. I. p. I40. n. I4. Felis (Caracal) cauda fubabbreviata, auriculis apice barbatis, corpore pallide ex rufo fufco, immaculato; der perfianifche Luchs.

Thevenot, Voy. au Levant, II. p. II4. les Karacoulacs.

Voyages d'Orient, du Pere Philippe. Lyon 1699. L. 2. p. 76 .

IVusladini Saadi (vel Scheikh Saadi) Gulifan, feu Rofarium politicum, feu amoenum fortis humanae theatrum, de perfico in latinum verfum, a Georgio Gentio. Amf. 165I. fol. p. 81.

19. RuFA. Der Rothluclis. (7)

Zinmermann, geogr. Zoolog. I. p. 286. der Rothluchs.

Bechftein, Naturgefch. Deutfchl. I. p. 27r. der Rothluchs.

Gatterer, vom Nutzen und Schaden der Thiere, I. p. 345. n. 204. Rothluchs.

Pennant, arct. Zoolog. II. p. 53. n. 23. der Rothluchs.

Biiffon, vierf. VI. p. 3I7. der Rothluchs. Giildenftidt, Nov. Comment. Acad. Petrop. XX. p. 499. Felis rufa.

Erxieben, Mammal. p. 529. oben.

\%.LYNX.

(7) Wird von Zimmermanz uad Fraleben fiir eine Vatietat vom Luch/b gehalren. 
7. LYNx. Der Luchs. $\left.{ }^{8}\right)$

a. Der gewöhnliche rothbraune Luchs; der Wolfs. luchs.

Müller, Naturfyftem, I, p. 24I. n, 7. der Luchs. Tab. 30. fig. 3 .

Zimmermann; geogr. Zool. I. p. 284. der Luchs. Lefke, Naturgefch. p. 138. n. 7. der Luchs. Borowfky, Thierreich, I. 2. p. 42. der Luchs. Bhumerbbach, Handb. der Naturgef.h.' p. 97. n. 8. Felis (Lynx) cauda abbreviata, apice atro, auriculis apice barbatis, corpore maculato, plantis palmisque ampliffimis; der Luchs. Bechftein, Naturgefch. Deutfchl. I. p. 267. n. 2. der Luchs. p. 277. gemeiner Louchs, Hivecho. luchs, Hirfchwolf. 'Tab. 4.

Funke, Naturgefch. I. p. I02. der Luchs. Ebert, Naturlehre, I. p. 273. der Luchs, Hirfch: wolf.

Halle, vierf. p. 524. der Luchs,

$$
\text { Q } 3 \text {. }
$$

Gatte:

(8) Der Luchs kơmmt an Gröfse dem Puchfe ziemlich gleich; Seine Länge betrágt dritthalb Fufs Er ift das einzige getiegerte, oder gefleckte Thier, das in der kalten Zone lebt, und ein, dem kleinen fowohl als grofsen Wilde fehr fchädliches Raubthier. Hat er ein Thier erreicht, fo wirft er fich auf felbiges und greift mit den Krallen fo tief ein, dafs er nicht herunter 2u bringen it. Dann würgt er es ${ }_{2}$ faugt ihm das Blut aus den Droffeladern, verzehrt einen Theil des Fleifches, und zwar zuerft die Keulen und Lenden; das übrige verfcharrt er bis zum. folgenden Tage. Fr verliert fich zwar immer mehr aus den bewohbten Gegenden, doch hat man noch vor einigen Iahren welche auf dem thüringer Walde gefçhofen. In den oberfchlefifchen Wäldern, und in der hernftädtfchen Haide kiommt er auch noch vor. Sein Fleifch wird von einigen in Liefland gegeffen. Der Balg giebt ein koftbares Pelzwerk: Das Stück wird mit so bis is Thalern bezahlt. Doch haben die Haare bey aller Weiche eine Sprödigkeit, dafs fie bald abbrechen. 
Gatterer, vom Nutzen und Schaden der Thiere, I. p. 34.1. n. 202. der Luchs.

Meyer, Thiere, III. Tab. 3I. der Luchs. Abbild. gut.

Beckmannl, Naturhiforie, p.29. n. 19. der Luchs. Pennanat, arct, Zool. II. p. 52. n. 22, der Luchs. Never Schauplatz der Natur, V. p. 236. Luchs, Hirfohiuchs.

Onomat. hhif. nat. IV. p. 9r2. Lynx; Lupus Cer, varius; Luchs.

Gesner, Thierbuch, p. 354. ein Luchs, Lutz, oder Lux; ein Thierwolf. Mit fchlechten Figuren.

Klein, Quadrup. difp. p. 77. Lynx.

Klein, Claffif. der vierfüfs. Th, p. 235. afrika. mifcher Luchs.

Klein, natürl. Ordn, d. vierf, Th, p. 8s. n. I. Hirfchluchs.

Bock, Naturgefchichte von Preuffen, IV. p. 38.

n. 10. der Luichs.

Goeze, europäifche Fauna, I. p. 252. der Luchs. Biiffon, vierf. VI. p. 278. der Luchs; m. e. Fig. Handb. d. deut. Thiergefchichte, p. 60. der Luchs, Hirfcherolf.

Fifcher, Naturge $\int \mathrm{ch}$, von Livland, p, 52. n. 6. Luchs.

Kraft, Ausrottung fchädl. Thiere, II. p. 565. der Luchs.

Taube, Befchr. von Slavonien, p. 2I. Luchs. Hiipel, topogr. Nachr. von Liefland und Ehfland, II. p. 428. Lauchs.

Pontoppidan, Hift. von Norwegen, II. p. 40. der Luchs.

Rytfchkow, orenb. Topogr. I. p. 237. der Luchs. Döbel, Iägerpraktik, I. p. 33. Lux. 
Ryt fchkow, orenb. Topogr. in Biifchings Magazin VII. p. 46. Lacks.

Pallas, Reife, Ausz. I. p. 96. III. p. 285. Luich/e. Strihrlin, Kabardey; in Bïfchings Magazin, VI. " P. 467. Luchs.

Perreult, Charras und Dodart, Abhandl. zur Naturgefch. I. p, I25. anatomifche Befchreibung eines Luchjes. Tab. 18. der Luchs. Tab. 19. die Zergliederung.

Miiller, Samml. ruff. Gefch.III. p. 5\$8. 607. Lauchfe. Berlin. Samml. von Reifebefchr. V. p. 230. Mannichfaltigkeiten, I. p. 607 . II. p. 435.

Onomatol. foreftal. II. p. 759.

Ridinger, Entwurf einiger Thiere, Tab. 65.66. Luchs.

Ridinger, jagdbare Thicre. Tab. Io. Luchs. Erxlehen, Mammal. p. 525. n. 15. Felis (Lynx) cauda abbreviata apice ața, auriculis apice barbatis, corpore maculato.

Linné, Syft, N. Edit. II. p. 43. Felis cauda truncata, colore rufefcente maculato.

Linné, S. Nat, Edit. VI. p. 4. n. 7. Felis cauda truncata, corpore rufefcente maculato.

Linne, Syft, Nat. Edit. X. I. p. 43. n. 7. Felis (Lynx) cauda abbreviata, apice atra, auriculis apice barbatis.

Graumann, introd, in hift. nat. mamm, p. 76. XV. Luchs,

Gatterer, brev, Zoolog. I. p. 141. n, 15. Felis (Lynx) cauda abbreviata, auriculis apice barbatis, corpore maculato, plantis palmisque ampliffimis; der Luchs.

Müller, Zoolog. dan. prodrom. p. 2. n. 9. Felis

(Lynx) cauda abbreviata, apice atra, auriculis apice barbatis. 
Giildenftüdt, Nov, Comment, Acad. Petrop. XX, p. 485.

Schlef. ökon. Nachrichten, Iahrg. I781.

Kramer, Auft, p. 3II. Felis cauda truncata, cor. pore rufefcente maculato.

Charleton, Exercitat. p. I4. Lynx,

Olaus Magn. de gent. Septentrion. p. 610. Lynx, Schwenkfeld, theriotroph. Silef. p. ros. Lynx.

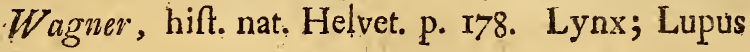
cervarius.

Rzaczynnsk. hift. nat. Polon. p. 222. Lynx, Hernandez, thefaur, p. 526. Lupus cervarius f. Lynx,

'Ariftotel. hiftor. anim, c. 7, n. 31. c. 9. n. 50 , $\Lambda$ ขy $\xi$.

Plin. h. n. VIII. c. 22. Cervarii lupi. Plin. h. n. XI. c. 46. XXVIII. c. 8. Lynx. Aelian. anim. XIV، c. 6. $\Lambda \nu \gamma \xi$. Oppian, cyneget, III. 84. $\Lambda$ ư $\xi$.

B. Die Luchskatze.

Zimmermann, geogr, Zool. I. p. 285. der weifses deutlicher gefleckte Luchs; die Luchskatze. Schreber, Säugthiere, III. p. 410. die Luchskatze. Borowflyy, Thierreich, I. 2. p. 42. die Luchskatze, Bechftein, Naturgefch, Deutfchl, I, p, 27.7. Luchs. katzen. ( $\left.{ }^{9}\right)$

Halle,

(9) Hier fcheint mir eine Verwechfelung der Synenymen zu feyn. Lucbskatze ift die eigentliche Benennung diefer Varietăt, und der Name Katzenlucbs ift ebenfalls gewöhnlich. Die Benennung Kalbiucbs aber, woraus auch. viell eicht Lucbskalb enttanden feyn mag, gehök gar nịchṭ hieher, fondern für den Karakal, Siehe Klein, natürl. Ordn. etc. p. 82. n, 3. Eben diefe Verwechfefung finde ich auch in der Onomat. hift. nat. ain a. O. desgleio chen in Fifchers livl, pa $52, n .7, \$$, auch Klein , Claftif, dẹn vierf. Th. $\mathrm{P}: 348$ 
- Mammatia Eerae. Felis.

Halle, vierf. p. 527. die Luchskatze mit fohwar. zen Flecken anf wei/sem Grunde.

Neuer Schaupl, der Natur, V. p. 226. Katzers. luichs.

Onomatol. hiffor. nat.' IV, p, gr6. Livchkatzo mit fchwarzen Flecken anf weifsem Grunde. Klein, Claflif, der vierfüfs. Th. p, 235. weifser Luchs mit fchwarzen Flecken, und abgekiirstem Schuranz. Kattlo.

Kiein, natürl. Ordnung d, vierf. Th, p. 82, n. 2, Fatzenluchs.

Biffon, vierf. VI. p.'288. n. 5 .

Pontoppidan, Hif, von Norwegen, II. p. ,44. Kat:entuchs.

Erxleben, Mammal. p. 528. Varietas maior al. bicans', maculis nigris.

Linné, Syft, Nat. Edit. II. p. 43. Felis cauda truncata, colore albo maculato,

Linné, S. N. Edit, VI, p. 4, n. 8. Felis cauda truncata, corpore albo maculato.

Millex, Samml. ruff. Gefch. III. p. 60\%. Irbis.

Graumani, intr, in hift. nat. mamm. p. $76 . \mathrm{XV}$, Katzenluchs.

Gatterer, brev. Zoolog, I. p. I4tr.

A) Verönderungen gegen die XITie Edition, und Ver. mehrung der Gattungen viie es Geffchlechts.

Die Sechfte Gattung: Felis Catus ift mit vier Varietäten vermehrt, die Hauskatze von der wilden getrennt, und die $\int p a n i \int c h e-k a r t h e t=$ Ser - und rothe Katze hinzu gekommen. Bey der fiebenten Gattung, Felis Lynux ift die Luchskatze wieder von dem Wolfhuchs getrennt. 
Das ganze Gefchlecht aher if mit zwölf Gatt!ngen vermehrt, und F. Uiuca, Leopardus, Iubata, Difcolor, Concolor, Tigrina, Capenfis, IVIanul, Serval, Chaus, Caracat, und Rufa, dazu gekommen.

\section{B) Unbeftinumtere Thiere.}

I, Die chinefifche weifse Katze mit fchlappen Ohrer. Zimmermann, geogr. Zoolog. I. p. I97. 'II. p. $267 . \mathrm{n}, \varepsilon$. Neuhof, Gefandfch. nach China, p. 347.

2. Der Kleine Leopard des Pennant.

Zimmermann, geogr. Zool. II. p. 272. n. b, Pennant, Synopf. quadrup. ps 173. n. 144, the leffer Leopard.

Erxleben, Mammal, p. 530.***

3. Die kurzgefineänzte Hanskatae von Iapan. Ziminermann, geogr. Zool. II. p. 272. n. $\mathrm{e}_{8}$ Kämpf. Iapan, I. p. I38.

4. Die wilden gefprenkelten Katzen von Guinea. Zimmermann, geogr. Zool. II. p. 272. n. $d_{\text {}}$ Bofsmann, Reife nach Guinea, p. 298.

5. a) Der Schenfar. b) Der Aekfch. c) Der Uabr. Thiere des Katzengefchlechts in Arabien.

Zimmermann, geogr. Zoolog. II. p. 272. n. e, Forskial, defcript. animal. Havniae, I775. Fn. orient. p. V. n. 30. 3r. 34.

6. Die wilde Katze von Neufpanien.

Zimmermann, geogr. Zool. II. p.272. n. f.

Büffon, 
Biiffon, Suppl, III. p. 227. Tab. 43. Chat fauvage de la nouvelle Efpagne.

Biiffon, vierf. XIV. p. 288. Katze von Neufpa. nien, oder Katzenparder.

Du Prati, Louif. II. p. 92. le Pichou?

7. Le Gaques ou Leopard.

Zimmermann, geogr, Zool. II, p. 273.

Biiffon, Supplem. III, p. 220. Tab. 38.

8. Die wilden Katzen von Californien.

Zimmermann geogr, Zoolog. II. p. 273. n. $h_{\text {. }}$ Begert, Gefch. von Californien, I. p. 55 .

9. Der grofse Tieger von Patagonien. Zimmermann, geogr, Żool. II. p. 273.

10. Der Luchs aus Canzada.

Zimmermannt, geogr. Zool. I. p. 286。

Biiffor, hift. nat. Supplem. III. p. 229.

Samml. zur Phyfik und Naturgefch. I. p. 32\%.

14. GESCHLECH T, VIVERRA. Das Stinkthier.

Miiller, Naturfyf, I. p. 243, das Frett. Gen. XIV. Zimmermann, geogr, Zool. II. p.282. Gen. XXIII, Schreber, Säugthiere, III. p. 4I7. Gen, XIV. Lefke, Naturgefch. p. I38. Gen, XV. Borowsky, Thierreich, I. 2. p. 43. Kaninchen. wiefel. Gen. XIV.

Blumenbach, Handb. d. N. G. p. 84. Gen. XXIII. Bat $\int c h$, Thiere, I. p. 200. Gen. XXV. Erxleben,-Mammalia, p. 480. Gen, XLIII. Granmann, introd, in hiftor. nat. mamm. p. $7 \mathrm{r}_{\text {, }}$ Gen. XLIII. 
Gatterer, brev, Zoolog. I. p. I30, Gen. XLIII, Hermann, tab. affinit. animal. p. 73 .

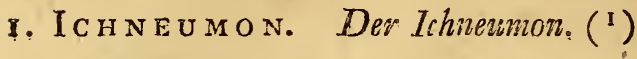

IViiller, Naturfyftem, I. p. 244, n. x. Pharaoratze.

Zimmermann, geogr. Zoolog. II. p. 44. p. 286.

n. I78. der Ichnenunon.

Lefke, Naturgelch. p. 139. n. 2. der Ifhntumon. Borowfky, Thierreich, I. 2. p. 45. n. 2. die Pharaoratze, Ichneumon.

Blumenbach, Handbuch der Naturgefch, p. 85. n. 4. Viverra (Ichneumon) caudae bafi incraflata, fenfim attenuata, pollicibus remotiu. fculis; die Pharaonsmans, der Minngo.

Funke, Naturgefch. I. p. Inr. der Ichneumnon, Pharaoratte, Mungo.

Ebert;

(I) Diefe Gattung gränzt fo nahe an die gleich darauf folgrende, dass Linné, Eräleben, Bilffon, Daubenton, u. a: m. beyde Thiere nur für Varietäten unter einander anfehen. Scbreber, Briffon, Edwards und Gmelin aber, hạlten dẹn Icbneumon für verfchicden von der Mangufte. Ueberein kommen beyde: r) in der Geftalt und Verhältnifs der Theile; 2) in der Lebensart; 3) in den Sitten; 4) in dem von ihnen bewohnten Klima. Die Manguffe weicht aber von dem Içhneumon $a b:$ I) durch das (pitz zulaufende Ende des Schwanzes, da fich der Schwanz des Ichneumons mir einer Quafte endigt; 2) durch die mindere Gröfse; die Mangufte ift bis zum Schwanzende 15 bis 17 Zoll, aler Ichneumon hingegen mehr als 21 Zoll; ; 3 ) auch durch die Farbe. Der Schwanz des Ichneumuns mifse 8 Zoll. Da Aegypten fo überhäuft mit Mäufen ift, dafs aus den Spalten des ausgetrockneten Erdreiçhs unzählige von allerley Gațungen herauslaufen, wenn man ftark darauf tritt, auch dị Häufe davon wimmeln, des Ichneumons Nahrung aber vorzüglich in Mäufen und alleriey Amphibien befteht, fo wird er durch die Verminderung der Anzahl fchädlichen Thiere feinem Vaterlarsde überaus nützlich, 
Ebert, Naturlehre, I. p. 366. Pharaonsmanis, Pharaonsratze.

Batfch, Thiere, I. p. 203. der eigenitliche Ichneumon.

Halle, vierf. p. 460. die Pharaomans, Ichnenmon.

Gatterer, vom Nutzen und Schaden' der 'Thiere,

I. p. 3ro. n. I72. Viverra Ichneumon; Pharaonsratie.

Neuer Schauplatz derNatur, VI. p. 574. Pharao. ratze.

Onomat. hiftor. nat. IV. p. 49I. Ichneumon, die Pharaonsmans, egyptifches spiurwiefelchen.

Klein, Quadrup. difp. p. 64. Muftela aegyptiaca. Klein, Claflif, der vierf. Th. p. 194. ägyptifche Wiefel, Ichueumon, ägyptifche Ihaus, ägypti. Sche Otter.

Klein, natürl. Ordn. d. vierf. Thiére, p. 68. n. 8. egyptifche Wiefel, Spürwiefel, Pharaonismans.

Gesner, 'Thierbuch, p. 273. indianifche Mians; m. Bellon.' Fig.

Shawe, Reife, p. 157. Ichneumon oder Tezerdéa. Kolbe, Vorgeb. d. g. Hoffn. p. I59. der Maus. hund?

Sparrmann, Vorgeb. d. g. H. p. 44. Pharaosra. tre. (Maishund.)

Lichtenberg und Voigt, Magazin für das Neuefle etc. IV. 2. p. 64. Miungo oder Pharaons. manus.

Berlin. Samml. IV. p. 370. der egyptifche Icha nerumon.

Bunzlauer Unterhaltungen, I. p. 300 . Ichneumont, egyptifches Spiirwiefel.

Erxleben, Mammal. p. 480. n. I. Viverra (Ich. neumon) cauda e bafi incraffata fenfím atte. muata, pollicibus remotiufculis.

Graus. 
Graumanu, intr. in hift nat. mamm. p. 71. Pharcoratze.

Severin, tentam. zoolog. p. 84. r. Ichneumon.

Gatterer, brev. Zoolog. I. p. 130. n. r. Viverra (Ichneumon) cauda e bafi incraflata, fenfim attenuata, pollicibus brevioribus.

7. Mun Go. Die MIangiffe. (2)

Miiller, Naturfylt. I. p. 245. 1. c. der Sichlan. gentödier.

Zimmermann, geogr. Zoolog. II. p. 44. p. 286. 11. I79. die Mangufte.

Biiffoin, vierf. XIV. p. II2. die IMangnfte. Batfch, Thiere, I. p. 204. die Iilangufte. Auszug a: d. Tagebuch e. Reifenden nach Afien, p. 59. Ichneumon.

Pallas, nord. Beyträge, IV. p. 95. Ichneunon. (ruff. die bagdadifche Maws; perf. Kusma.) Buchodz, hift. univerf. du regne végetal, T. I: dec. 8. pl. 8. f. 2.3. Moncus, Mangoufe.

8. Cafra.

(2) Fin nunteres betiendes Thier, das oft auf den Hinterfüfsen fitze, und fehr gefchwind laufen und klettern kann, Den Ratzen fchleicht und gräbr lie in ihre Löcher nach; das Geflügel aber foll fie auf diefe Art berücken, dafs fie fich fo lange als tode hinftreckt, bis fich diefe in Menge unh fie verfammelt haben, die fie fodann mit leichte: Mühe erhafchet: Den gefangenen Thieren beifst fie die Kehlè oder dèn Kopf entzwey, und faugt das Blut aus; ehe fie das Fleifch anrühret, Was fie nicht auf einmal bezwingen kann, verfcharre fie zu einer àndern Mahleeit. Brodt frifst fie nicht, aber doch Kirfthen, Plauz. men, und andere faftige und fifse Früchte. Sie trinkt viel. Unter dem Freffen"brummt fie wie eine Katze. Wenn mạn fie böfe macht, ftrătubt fie die Haare. Àm Tage plégt fie viel źu fchlafen, und des Nachts wachfam zu feyn. Zum Schlaf legt fie fich in einen Kreis zufammen, dafs man weder Kopf noch Eeine fiehet. Ihren Unrath trïgt fie an eizen befondern Ort.' 
Mammalia Ferae. Viver
8. C A F RA. Dev capifche Ichinemmon.

Zimmermann, geogr. Zoolog. II. p. 286. n. 180. Viverra Ichneumon Capenfis, Pallas: das ca. pifche Ichrueumon.

27. Ze N I . Der Zenik.

Sonnerat, Reife n. Oftind. u. China, II. p. II4، der Zenik der Hottentotten. Tab. 92.

Das ganze Thier in röthlich grau, und hat zehen fchwarze Streifen quer über den Leib.

9. Tет R D A т т a. Der vierzehige Riiffel trïger. (3)

Zimmermann, geogr. Zoolog. II. p. 287. n. I8I。 der Surikatt; der vierzehige Riifseltrüger.

Lefke, Naturgefch. p. I40. 11. 3. das vierzehige Stinkthier.

Borow $k$ ky, Thierreich, I. 2. p. 4G. n. 3. der vier. - zehige Rilfjeltrïger.

Gatterer, vom Nutzen und Schaden der Thiere, I. p. 3II. 11. 175. die Surikate.

Batfch, Thiere, I. p. 204. der Suricatte.

Biiffon, vierf. XIII. p. 273. der Surikate; mit einer Figur.

Erxleben, Mammal. p. 488: n. 4. Viverra (Suri: catta) pedibus tetradactylis.

Pallas, mifcellan. zool.p. 60.

Graumann, intr. in hift. nat. mamm. p. 72. IV. die Ratelmans; (welche Benennung aber dem Thier eigentlich nicht zukömmt). Pennant, Syn. quadr. p. I2s. n. 163.

Gatteiver,

(3) Diefs Thier pflegt feinsn eigenen Harn zu faufen, und lesist gerne Speichel. 
Gattever, brev. Zoolog. I. p. 13I. n. 4. Viverra (Suricatta) pedibus tetradactylis.

3. NasuA. Der rothe Riiffeltrï̈ger.

Mïller, Naturfy f. I. p. 247. n. 2. das Nafenfrett. Tab. 15. fig. 2.

Zimmermann, geogr. Zoolog. II. p. 290. N. I85. der rothe Riilfeltriiges; der Coati.

Lefke, Naturgefch. p. I40. n. 4. der voine Riifjeltrïger.

Borowsky, Thierreich, I. 2. p. 47 . 11. 4. das Nofenfrett; Koatimondi.

Bechfteint, Naturgefch. Deutfchl. I. p. I38. das Nasenfiett.

Halle, vierf. p. 505. das Koatimonciti.

Ebert, Naturlehre, I. p. 370 . der Coati; der bra filianifche Fuchs.

Neuer Schauplatz der Natur, II. p. I69. Coatimondi. VI. p. 62. Nafenfrett.

Onomat. hilt. nat. III. p. 3. Coati mondi mit geo ringelten Schuanz: VII. p. 783. der Nafua. Klein, Quadr. difp. p. 72. Coati Mondi Marcgr. Klein, Claffif. der vierfüfs. Thiere, p. 216. Klein, natürl. Ordn. d. vierf. Th. p. 76. 11. I. brafilianifcher Halbfuchs.

Bïffon, vierf. V. p. 194. das Nafenfrett. Koati. Mit 2 Figg.

Bankroft, Naturgelch. von Guiana, p. 84. dev Dachs in Guiana.

Perrault, Charvas und Dodart, Abhandl. zur Na. turgefch. 1. p. 28\%. anatomifche liefchreibung von vier Coatien. Tab. 38. Coati-mondi. Tab. 39. die Zergliederung.

Erxleben, Mammal. p. 485. 11. 2. Viverra (Nafua) rufa, cauda albido annulata. 
Linné, Sy凡t. Nat. Ed. XII. I. p. 64."n. 2. Viverra (Nafua) rufa, cauda albido annulata.

Graumann, introd. in hift. nat. mamm. p. 7r. II. Naspafrètt.

Valeatin. amphith. zool. p. 123.

Gatterer, brev. Zool. I. p. I30. n. 2. Viverra (Nafua) rufa, cauda albido annulata; das $\mathrm{Na}$. senfrett.

3. Narica. Der braune Riiffelträger. (4)

Miiller, Naturfyf. I. p. 250. n. 3. der Frettbär. Zimmermann, geogr. Zoolog. II. p. 291. n. I86. der braune Riiffelträger; der Frettbär.

Lefke, Naturgefch. p. I40. n. 5. der braune Riil. Seltrüger.

Boroweky, Thierreich, I. 2. p. 47. n. 5. der Freitbïr.

Ebert, Naturlehre, I. p. 37r. Coati mondi. Neuer Schaupl. der Natur, III. p. 195. Frettbär. Onomatol. hiff. nat. III. p. 3. Coati mondi. VII, p. 779. der Narika.

Gatterer, vom Nutzen u. Schaden der Thiere, I. p. 3Ir. n. I74. der Frettbär.

Bïffon,

(4) Diefs Thier ift mit dem vorhergelienden fo nahe verwandt, dafs beyde von Pennant und Büffon nur als Spielarten angefehen werden. : Der vornehmfte Unterfchied befteht in folgendem: 1) Die Narica ift etwas gröfser als die Nafua. 2) Die Farbe des Kürpers ift nicht fuclisrcth, und am Schwanz ift kein weifser Ring, auch ift der Schwanz an der untern Seite nicht flach. 3) Unter dem Auge if bey der-Narica keine Warze zu finden, die bey der Nafua deutlich iff. 4) Die. weifsen Streifen vom Auge herunter nach der Nafe, oder hinter jedem Mundwinkel, zeigen fich nicht bey der Nafua. Die Länge des Thiers beträgt gegen zwey Fufs. Nach Europa kommt es weit feltener, als das rothe. 
Biiffon, vierf. V.p. 202. der Erettbär, oder $\mathrm{Na}$ vika. Lin.

Limné, in den Schwed. Abhandl. XXX. p. I52. das Thier Narica. Tab. 4.

Erxleben, Mammalia, p. 486. n. 3. Viverra (Narica) fubfuíca, cauda concolore.

Berlin. Samml. III. p. 199. ein Seltnes Thier, $\mathrm{Na}$ rica genannt; m. Linn. Fig.

Graumanu, intr. in hift. nat. niamm. p. 7r. III. Frettbiö.

Pennant, Synopf. quadrup. p. 23c. n. 164 .

Gatterer, brev. Zool. I. p. I3r. n. 3. Viverra (Na. rica) fubfufca, cauda concolore; der fchue ïrzliche Konti.

10. Vulpecula. Die Coase. (\$)

Milller, Naturfy f. Suppl. p.32. 11. 7. das Braunfrett.

Zimmermann, geogr. Zoolog. II. p. 29I. n. I87. die Coase; der Yzquiepatl.

Borowlky, Thierreich, 1. 2. p. 48. das Braunfrett; die Coafe.

Halle, vierfüfs. p. 509. das Izquiepotl; Stinkthier.

Meyer,

(5) Diefe und die folgenden Arten geben einen fo peftilenzialifchen, faft erfickenden Dainpf oder Geftank zu ihrer Verchei. digung ron fich, dafs felbft die auf fie jugdmachenden Hunde, ofrmals zu ihrer Erholıng die Nafé gegen die Erde halten müffen, daher fie vom Büfion Mouffittes, oder Strinker genannt werden. Der Geftank rühret von einer, in zwey Drüfen, die an beyden Seiten des Schwanzes liegen, befindlichen Feuchtigkeit her, die fie, wenn fie geängftigt werden, von 'fich fprïtzen. Die Confe halt fich in Felfenklüften auf, wo fie auch inre lungen wirfe Vom Federvieh foll fie hauprfachlich dur den Kopf frefien. 
Meyer, Thiere, III. Tab. II. der mexicanifche Ameifenfreffer.

Gatterer, vom Nutzen und Schaden der Thiere, I. p. 313, n. I77. der Izquiepattel.

Neuer Schauplatz der Natur, II. p. I67 Coafe.

Onomat. hift. nat. VII. p. 785 . $Y_{\text {zquiepatl; (das }}$ Thier if aber hier mit - dem folgenden verwechfelt.)

Gesner, Thierbuch, p. 126. Tzquiepatl, ein indianifcker Fuchs; m. e. fchl. Fig.

Klein, Quadrup. difp. p. 72. Coati Hernondefii. Klein, Claffif. d. vierf. Th. p. 217. Yrquiepatl. Klein, natürl. Ordn. d. vierfüfs. Th. p. 77. $\Upsilon_{z}$. quiepatl.

Bïffon, vierfüfs. XV. p. 201. 204. Yzquiepatl; Coafe. p. 222. Coafe, m. e. Fig.

Erxleben, Mamesalia, p. 490 . n. 6. Viverra (Vülpecula) ceflanea, fubtus flavefcens.

Grmumann, intr. in hift. nat. mamin. p. 72. VI. das Braunfrett.

Seba, thef. I. p. 68. Ichneumon de Yzquiepatl, feu vulpecula americana, quae colore Maizium torrefactum aemulatur. Tab. 42. fig. I.

Gatterer, brev. Zool. I. p. I3I. n. 6. Viverra (Vulpecula) caftanea, fubtus flavefcens.

11. Qu As JE. Das Quasje.( $\left.{ }^{6}\right)$

Zimmermann, geogr. Zoolog. II. p. 293. n. I88. das Quasje.

$\mathrm{R} 2$

Schreber,

(6) Seba erhielt ein folches Thier lebendig aus Surinam. Es grub fich mit der Schnauze und den Vorderpfoten gern in die Erde; in einem Loche, das es fich gemacht hatte, fchlief es am Tage, und war die ganze Nacht hindurch in Bewegung. Die kalte Hexbft- 
Schreber, Säugth. III. p. 44I. n. 14. das Quasje. Biiffon, vierf. XV. p. 20I. n. 2.

4. Pu torius., Der Skunk. (7)

Miiller, Naturfyftem, I. p. 25r. n. 4. Supplem. p. 3I. das Stinkthier.

Zimmermain, geogr. Zoolog. II. p. 293. n. I89. der Skwnk.

Lefke, Naturgefch. p. I4̧0. n. 6. das Stinkthier, der Shumz.

Boro:vfly, Thierreich, I. 2. p. 48. n. 6. das Stinkthier.

Blunnen-

Herbftwitterung tödtete es. Biiffor und Linné nehmen diefs Thier fitr nicht verfchieden von der vorhergehenden Art an, aber Scbreber itt anderer Meynung.

(7) Pennant kann sen Gefrank diefer Thiere nicht abfcheulich genug befchreiben. Perfonen, die das Unglück haben davon in in. cirt zu verden, verfagt man die Rechte der Gaffreylieit, und. verfchliefst die Thür vor ihnen. Der Prof. Kalm lief Gefahr von dem Geftank eines folchen Thiers, welches in das Haus, worin er fich befand, verfolgt wurde, erftickt $z u$ werden. Eine Dienftmagd, weiche eiris diefer Thiere in einer Speifekammer antraf, und erfchlug, ward von dem Dunfte fo feht angegrifen, daifs fie mehrere Tage hindurch krank blieb, und die Speifen músten weggeworfen werden. Reifende finden fich mitten in den Waldern oft genüthigt, die Nafen zuzuhalten, um den Wirkungen diefes Geftanks vorzubeugen. Selbft die Thieve erfahren die traurigen Wirkungen diefer Ausdüntungen. Das Hornvieh fängt ängftlich an 2 a blöken, und nur gut abgerichtete Hunde greifen die Sränker an. Sie behalten augh viele Tage nach dem Kampfeinen unausftehlichen Geftank. Des fcheufslichen Geftanks aber ohneyachret, wird das Fleifch diefer Thiere dennoch gegeffen; nur müifen gleich nach dem Tode die Dräfen ausgefchnitsen, und das Fell abgezogen wercien. Aus dem Felle machen die Indianer Tabackbeutel. Das Thier heckt an der Erde und in hohlen Baumen. 
Bhumenbach, Handb. d. Naturgefch. p. 84. n. 3.

Viverra (P'útorius) lineis quinque dorfalibus parallelis albis; das Stinkthier, Conepatl. Funze, Naturgéfch. I. p. IIo. das Stinkthier. Halle, vierfüfs. p. 460. der amerikanifche Livreiiltis.

Gatterer, vom Nutzen und Schaden der Thiere, I. p. 312. n. 176. das Stinkthier.

pennant, arct. Zool. II. p. 85. n. 39. das geftreifte Wiefel.

Neuer Schauplatz der Natur, II. p. 204. Conepatl.

Onomat. hift. nat. VII. p. 785. das Stinkthier. Klein, Quadr. difp. p. 64. Muftela americana foetida.

Klein, Claffif, d. vierf. Th. p. 193. amerikanifche finkende Wiefel.

Klein, natürl. Ordn. der vierfüfs, Th. p. 68. n. 5 . geftreifte Stankwiefel.

Schlüzer, Erdbefchreib. von America, p. 75. der Skunk; Pole-cat; das ftinkende Thier.

Hamb. Magazin XIV. p. I68. das finkende Tiier. Sparrmann, Reife n. dem Vorgeb. d: g. Hoffn. p. 45. das Stinkthier.

Kalm, Reife nach d. nördlichen Amerika, II. p. 412, ein gewiffes vierfiifsiges Thier.

Batfch, Thiere, I. p. 203. das eigentliche Stinkthier.

Bïffon, vierf. XV. p. 209. der Conepatl. Erxleben, Mammalia, p. 488. n. 5. Viverra (Putorius) nigricans, lineis quinque dorfalibus parallelis albis,

Linné, Syft. Nat. Edit. X. I. p. 44. n. 3. Viverra (Putorius) fufca, lineis quatuor albidis dorfalibus parallelis. 
Limné, Syก. Nat. Edit. XII. I. p. 64. n. 4. V i. verra (Putorius) fufca, lineis quatuor dorfalibus parallelis albidis.

Granmann, intr. in hifor. nat. mamm. p. 72. V. das Stinkthier.

Gatierer, brev. Zoolog. I. p. I3r. n. 5. Viverra (l'utorius) nigricans, lineis quinque dorfalibus parallelis albis; das Stinkthier.

Severin, zoolng. Hungar. p. 85. n. 5. Viverra Putorius; das Stinkthier.

12. Conepatr. Das Conepatl. ${ }^{(8)}$

Zimnermann, geogr. Zoolog. II. p. 294. n. 190. der Conepatl.

Schreber, Säugthiere, III. p. 443. n. 16. das Conepatl.

Lefke, Naturgefch. p. I4I. Conepatl.

13. Mephitis. Der Chinche. (9)

Initiller, Naturfyftem, Supplem. p.32. n. 8. Viverra Chinche; dir Cinunk.

Zinmuernann, geogr. Zoolog. II. p. 294. n. 19 r. der Chinathe.

Lefke, Naturgefch. p. I4r. der Chinche. Borows k'ky, Thierreich, I. 2. p. 49. n. 8. der Chinche, Chunk.

Gatte-

(8) Biffon zieht diefe Art mit der vorhergehenden zufammen. Scbreher aber fondert beyde von einander $a b$, weil der Conepatl anflatt fünf nur zwey Streifen über dem Rtïcken hat, die auch iiber den Schwanz gehen.

(9) Pennant fagt: Diefe Art bewohut das fefte Land von Amerika, von der Hudionsbay bis nach Pert hinab. In Peru heifst fie Cbinche. Man trift fie auch in Mexiko an, wo fie Conepatl genannt wird; odér der kleine Fuchs der Kinder. - Sonft wird der Conepatl von den Zoologen von dem Chinche getrénnt. 
Gatterer, vom Nutzen und Schaden der Thiere; I. p. 21 I3. n. I78. der Chinche.

Penuant, arct. Zool. II. p. 68. n. 40. der Chinche. Neuer Schauplatz der Natur, II. p. II4. Chinche. Onomat. hift. nat. VII. p. 786. Chinche. Biffon, vierf. XV. p. 2ir. p. 223. der Chinche. Mclina, Naturgefch. von Chili, p. 255. Viverra Chingha, der Chinghe.

Vicaure, Befchreib. von Chile, p. 90. das Thier Ciine.

Byron, Reife um die Welt, p..9I. Chieche. Berlin. Sámml. IV. p. 298. der Chinchi, oder das Stinkthier. (Unter grofser Verwechfelung der Synonymen.)

Erxleben, Mammal. p. 49r. n. 7. Viverra (Mephitis) dorfo albo linea longitudinali nigra.

Groumain, intr.' in hift. nat. mamm. p. 72 . VII. Viverra Mephitis.

Gatterer, brev. Zoolog. I. p. I3I. n. 7. Viverra (Mephitis) dorfo albo, linea longitudinali nigra.

Severin, zool. Hungar, p. 85. Viverra Mephitis.

14. Zorilla. Die Zorilla.

Miiller, Naturfyfem, Suppl. p. 32. n. 9. Viverra Mapurita; der Mapurit.

Zimmermann, geogr. Zool. II. p. 295. der Zorill. Le $\int k$, Naturgefch. p. I4I. die Zorilla.

Neuer Schauplatz der Natur, X. p. 270. der Zo- rill im fiidlichen Amerika.

Gatterer, vom Nutzen und Schaden der Thiere,

I. p. 314. n. 179. der Mapurit.

Bïffon, vierf. XV. p. 225. die Zorille; m. e.Fig. MIurr, Reifen der Miffionar. in Amerika, p. 215.

Zorillo.

$$
\text { R } 4 \text { Begert, }
$$


Begert, Nachricht. von Californien, p. 64 .

Dobrizhoffer, Gefch. der Abiponer, I. p. 343. der Zorinno.

Charlevoix, Gefch. von Paraguay, p. 227. der Zorillo.

Bat fch, Thiere, I. p. 203. die Zorille.

Fernand. hift. nov. Hifpan. p. 6.

Garcilafjo, hift. de Peru, p. 33r.

Erxleben, Mammalia, p. 492. n. 8, Viverra

(Zorilla) albo nigroque variegata.

Grcumann, intr. in hift, nat. mamm. p. 72. VIII. Viverra Zorilla.

Gatterer, brev. Zoolog. I. p. I32.n. 8. Viverra (Zorilla) albo nigroque variegata.

Pennant, Syn. quadr. p. 533. n. I68. the Zorilla. Severin, zool. Hungar, p. 85. Viverra Zorilla.

15. M Á P R I T o. Der Mapririto. $\left({ }^{10}\right)$

Zimmermann, geogr. Zool. II. p. 295. n. 192. dier Mapurito.

Schreber, Säugth. III. p. 445. n. 9. der Mapurito. Lefke, Naturgefch. p. r4I. der Mapurito.

Neuer Schauplatz der Natur, X. p. 270. der Zo= rill in Neufpanien; (die Befchreibung mit dem vorhergehenden verwechfelt.)

Biuffon, vierf. XV. p. 227. der IMapurito. Schwed. Abhandl. XXXII.p. 68. (v. Syft.) Linné, Mantifia, II. p. 522. Viverra Putorius.

16. Viтtт та. Der Grifon.

Zimmermann, geogr, Zool. II. p. 296. n. 194. der Grifon.

Lefke,

(10) Erxleben hält diefs Thier fütr einerley mit dem Cbikche, welas ches auch Zimmermann niche unwahrfcheinlich findet. 
Lefke, Naturgefch. p. 141. der Griforb.

Borowefky; Thierreich, I. 2. p. 49. n. 9. der Grifon.

Biiffon, vierf. XV. p. 238. der Grifon, m. e. Fig. Erxleben, Mammal. p. 499. Viverra vittata.

Graumann, intr. in hif. nat. mamm. p. 73. XIV. V. Vittata.

7. Z EYLANICA. Der Boshond.

Zimmermann, geogr. Zool. II. p. 288. n. I83. der Bofshond.

Schreber hält den vilden Hund des Vosmaer für diefs Thier, weil $V$ osmater diefem Thiere vorn und hinten fünf Zehen zufchreibt, deren der Schakal hinten nur viere hat. S. Schreb. a. a. O. p.452. Syft. Linn. p. 72. ad 7 .

18. C'A PEN S Is. Das Stinkbinkfen.

Zimimermann, geogr. Zool. II. p. 289. n. I84. der Stinkbieksen.

Graumann, intr. in hift. nat. mamm. p. 72. IX. V. Capenfis.

Kolbe, Vorgeb. Edit, in 4. p. 347. dev Stinkdachs. Severin, zool. Hungar. p. 85. Viverra Capenfis-

19. Civetra. Die Civetie. (I)

Miiller, Naturfyf. Supplem, p. 30. n. Ir. Felis Mofchata; Biefamlatse?

Zimmermann, geogr. Zool. II. p. 47. die Zibet. katze. p. 2\$2. 11. 173. die Civette.

Gesner, Thierbuch, p. 244. Zibethkatz; mie einer fchl. Fig.

$$
\mathrm{R}_{5} \quad \text { Biiffon }_{2}
$$

(1) S. die forgende Anmerkung. 
Bïlfon, vierf. VI. p. 34I. die Civette; m. e. Fig. Never Schauplatz der Natur, X. p. 208. die Zi. bethkatze.

Ifert, Reile nach Guinea, p. 192. Zibethkatse. Bofsmann, Reife nach Guinea, p. 298. Zibeth katzen.

Projart, Gefch. von Loango. Lpz. I778.

Santos, in Purchas Pilgr. II. p. I545.

Flacourt, hift. de Madagafcar. p. I54. Falanouc? Marmol, Afrique, I. p. 57.

Mem. pour fervir à l'hift. des animaux, I. p. 157. Tab. 23. Civette.

Pennant, Syn. quadr. p. 234. n. 170. the Civet. Leon. African. defcr. Afr. p. 756 . Felis excre: mentum emittens.

5. Zів тетн. Das Zibeth. (')

\section{Miiller, Naturfyf. I. p. 253. n. 5. das Zibeththier; Tab. I3. fig. I.}

\section{Zimmer-}

(2) Erxleben, Lefke, Blumenbach, und uberhaupt die allermehreften Naturhiftoriker nehmen die Civette und das Zibetb nur als Spielarten von einander an; auch findet man bey vielen, wo fie wirklich getrennt zu feyn fcheinen, doch die Synonymen üfters verwechfelt. Buiffon hat die wichtigften Unterfchiede beyder Thiere befonders ans einander gefetzt. Beyde find an Farbe und Sitten einander ähnlich; aber Kopf, Schnauze und Ohren find beym Zibet langer, als bey der Civette; auch fehlt ihm das mähnenabnliche Nackenhaar. Der Schwanz if viel län̨er, dabey dick und fchwarz geringelt. Das Schild oder der fchwarzo Fleck am Halfe ift mit mehrern weifsen Linien durchfchnitten. - Beyde Thiere find räuberifch und haben einerley Nahrung. Sie faufen wenig. Ihre Augen funkeln in der Nacht. Sic bringen beyde dic bekannte öligte, fchmierige, ftarkriechende Feuchtigkeit, den Zibet, in einem eigenen, neben den Zeugungstheilen liegenden Beutel, durch hiezu be- 
Zimmermann, geogr. Zoolog. II. p. 47. das Zibeththier; p. 283. n. 174. das Zibet. Leske, Naturgefch. p. 139. n. I. die Zibethkatze. Boroweky, Thierreich, I. 2. p. 44. n. I. das Zibetthier; Tab. $\mathbf{4}$.

Bhimenbach, Handb. der Naturgefch. p. 84. n. r. Viverra (Zibetha) cauda annulata, dorfo cinereo nigroque undatim friato; die Zibethkatze.

Funke, Naturgefch. I. p. IIo. die Zibethkatze. Ebert, Naturlehre, I. p. 371. das Zibeththier, die Zibethkatze.

Halle, vierf. p. 510. die Zibethhatze, Zibeththier. Meyer, 'Thiere, III. 'Tab. 32. die Zibethkat:e. Gesner, Thierbuch, p. 245. eine andere Art Zi. bethkatzen.

Beckmann, Naturhift. p. 28. n. 16. das Zibetthier, oder die Zibetkatze.

Gatterer, vom Nutzen und Schaden der Thiere, I. p. 3I4. n. 180. die Zibethkatze.

Neuer

ftimmte Glandeln hervor. Diefe Materie hat die Confiftenz des Honigs oder der Butter. Sie fammelt fich in folcher Menge, dafs die Thiere; fie oft von fich laffen, wenn man fie ihnen nicht nimmt, welches wöchentlich uhngefähr zwey bis dreymal, mittelf eines Löffels gefchielzet, nachdem das Thier vorher in ein enges Behältnifs, worin es fich nicht umwenden und beiffen kann, gefperret, und an den Hinterbeiner' herausgezogen worden. Die Farbe des Zibets ift erft weifslicht, hernach gelblicht, bräunlicht, oder f́chwärzlicht, welcher Unterfchied duch niche allein vom Alter, fondern oft asch von der Verfalfchung herrïhret. In Holland, und befonders in Amfterdam wird die Zibetkatze von mehrern um des Gewinftes willen geheget, doch mufs fie dann im Winter warm gehalten werden. Der reinfte Zibet kommt auch aus Holland. Der von dem Zibetthier foll, nicht fo gut feyn, als der von der Zibetkatze. Ueberhatup wurde diefe Materie ehedem mehr als jetzt zum Parfumiren, und in der Arzeney gebraucht. 
Neuer Schaupl. der Natur, X. p. 208. das Zibeththier.

Onomat. hift. nat. VII. p. 787. das Zibetthier. Klein, Quadrup. difp. p. 73. Coati Civetta vulgo. Klein, Claflif. der vierf. Th. p. 218. Zibethkatze. Klein, natürl. Ordn, d. vierf. Thiere, p. 77. n. 5. Zibethkatse.

Biiffon, vierf. VI. p. 34I. das Zibeth; m. e. Fig. Perrault, Charras und Dodart, Abhandl. zur Na. turgefch. I. p. I83. anatomifche Befchreibung von fünf Zibethkatzen. Tab. 24. die $\dot{Z}$ ibeth katze. Tab. 25. die Zergliederung.

Pomet, Drogues, II. p. I7. Civette.

Walbaum, Hiftorie von Grofs-Iava. Lpz. 1754 .

p. IIO,

Tumpin, hift. de Siam, I. p. 300.

Ruyjch, theatr. an. I. p. I57.

Berlin. Samml. II. p. 188. 194. Zibethkatze.

Erxleben, Mammal. p. 493. n. Io. Viverra (Zibetha) cauda annulata, dorfo cinereo nigroque undatim friato.

Limné, Syn. Nat, Edit, II, p. 45. Meles unguibus uniformibus.

Linné, Syn. Nat. Edit, VI. p. 6. n. 2. Meles unguibus uniformibus, cinerea.

Limné, Syft. Nat. Edit. X. I, p. 44. n. 4. Viver. ra (Zibetha) cauda annulata, dorfo cinereo nigroque undatim friato.

Graumann, intr, in hif. nat. mamm. p. 72. X. Zibethkatze, Zibeththier.

Pennant, Synopf, quadruped, p. 235. $\beta$. Civet Weefel,

Gatterer, brev̀, Zoolog. I. p. I32, n, I0. Viver. ra Zibetha; das Zibetthier. 


\section{$+\quad$ - *}

Hernandez, Mex. p. 5338. Zibethicum animal americanum; m. e. mittelmäfs. Fig.

Della Valle, fameux voyages, I. p. 275. Chats mufquez.

Rauwolf, Reife in die Morgen]änder; Frcf. 1582.

II. p. I33. Thierlein, fo den Zibeth tragen.

Ionfton, quadrup. p. I57. Animal Zibethi. 1.

Ionfton, quadrup. 'Tab. 72. Felis Zibethi. Zibet Katz.

Charleton, exercitat. p. 20. Animal Zibethi.

Pyrart, voyages, III. p. I4.

Forskail, faun. orient. p. III. Viverra Zibetha.

20. HERMAPH ODI TA. Das Znitterftinkthier. Zimmermann, geogr. Zool. II. p. 285. 11. I77. das Zwitterfinkthier.

6. Geneтta. Die Genette. (3)

Miiller, Naturfyfem, I. p. 256. n. 6. die Ge. nettkatze.

Zimmermann, geogr. Zoolog. II. p. 283. n. I75. die Genette.

Borow.

(3) Das Thier, welches Sparrmann (Schwed. Abhandl. am a. O.) befchreibt, roch ftark nach Mofchus oder Bifam, wenn es in einem warmen Zimmer gehalten wurde, in káltern ${ }^{-}$Zimmern verminderte fich aber der Geruch betrachtlich, Das Thier liebte felr die Warme, und hüllte fich oft in eine Menge Spreu, welche zu feiner Bequemlichkeit hingelegt war. Des Nachts gieng es auf feine Nahrung aus. "Es hatte grofse Luft Hühner zu fangen. Zur Nahrung nahm es mit rohen Fleifchfücken vorict, anch verfchmähete es andere Fleifch - Fifch - Melnlund Milchfpeifen nicht. Aepfel liebte es vozzüglich, und nahm auch gern Zuclier. Es murrete und zifchte beynahe wie eine Katze, wenn es gereizt wurde. 
Borowfky, Thierreich, I. 2. p. 50. n. 10. die Genettkatze.

Bhumenbach, Handbuch der Naturgefch. p. 84. n.2. Viverra (Genetta) cauda annulata, corpore fulvo - nigricante, maculato; die Genettkatze.

Ebert, Naturlehre, I. p. 37r. die Genette, Genzettkatze, Spanifche Katze.

Halle, vierf. p. 509. die Genettkatze.

Gatterer, vom Nutzen und Schaden der Thiere, I. p. 315. n. I8r. die Genettkatze.

Neuer Schauplatz der Natur, III. p. 322. G Gnette.

Onomat. hift. nat. VII. p. 778. die Genettkatze. Gesner, Thięrbuch, p. 243. Genetha; eine Geneth-Katz; mit Abbild. des Felles.

Klein, Quadr. difp. p. 73. Coati Genetta.

Klein, Clanfif. der vierfüls. Thiere, p. 277. Genithkatze.

Klein, natürl. Ordn. d. vierf. Th. p. 77. n. 4. Genethkatze.

Bïffon, vierf. VI. p. 663. die Genitte; m. e. Fig. Sparrmann, zool. Bemerk. über die Viverra Genetta; in den neuen Schwed. Abhandl. VII. p. 66.

Sparrmann, Reife nach dem Vorgeb. d. g. H. p. 44. Genettkatze?

Erxleben, Mammal. p. 496. n. II. Viverra (Genetta) cauda annulata, corpore fulvo - nigricante maculato.

Limné, Syf. Nat. Edit. VI. p. 5. n. 8. Muftela cauda annulis nigris albidisque cincta.

Linné, Syft. Nat. Edit. X. I. p. 45. n. 5. Muftela (Genetta) cauda annulata, corpore fulvo. nigricante maculato. 
Graumann, intr. in hif. nat. mamm. p. 72. XII. Genottkatie.

Gatterer, brev: Zonlog. I. p. I33. n. Ir. Viverra (Genetta) cauda annulata, corpore fulvonigricante maculato; die Genetthatze. Aldrovand. de quadrúp. digit. p. 337. Genetta. Aldrovand digit. p. 339. Ginetta Bellonii, Felis fpecies; m. Bell. Fig.

Ionfton, quadr. p. 157. Genetta.

Ionjzon, quadr. 'Tab. 72. Genetta, Geneth-Katz; m. Bell. Fig.

Charleton, exercitat. p. 20. Genetta:

Raj. Syn, quadr. p. 20I. Genetta vel Ginetta.

Pennant, Synopf. quadruped. p. 236. n. 271. the Genet.

\section{2r. Foss a. Die Fo/fane. (4)}

Miiller, Naturfyft. Suppl: p. 32. n. 10. das melirte Frett.

Zinmermann, geogr. Zool. II. p. 50.p. 284. n. 160.' die offane.

Borowfky, Thierreich, I. 2. p. 5I. n. Ir. die Bie. famkatze; das melirte Frett.

Neuer Schaupl. der Natur, III. p. 322. die Genette von MTrdagafcar.

Biifon, vierf. XIV. p. 140. die Fo/fane, m. e. Fig. Gatterer, vom Nutzen und Schaden der Thiere, I. p. 316. n. I82. die Folfane.

Kolbe, Vorgeb. d. g. Hoffn. p. 154. Edit, in 4. p. 348. Biefamkatze?

Dapper,

(4) In dem Gefitigel gefährlich, frifst aber auch gern Früchte, befonders Baranafien, und lafst fich fchwer zahm machen. Die Benennung Biefamkatze wird ihr wohl mit Unrecht gegeben. 
Dapper, afrikan. Infeln, p. 32. Foffe.

Granuiann, intr. in hift. nat. mamm. p. 73. XII. die Biefamkatze:

Gatterer, brev. Zool. I. p. 133. 11. I2. Viverra (Foffa) cauda annulata, corpore cinereo nigro maculato; die Folfone.

Fincourt, hift. de Madagafcar. p. 152. Foffa.

22. Tigrina. Die Bifamkatze. (s)

Zimmermain, geogr. Zoolog. II. p. 285. n. a. Biefamkatze vom Vorgeb. d. g. H.

Biiffon, vierf. XIV. p. I45. die Bifankatze des Vosmaer; m. e. Fig.

Erxleben, Mammalia, p. 499. Viverra tigrina. Graumann, intr. in hift. nat. mamm. p. 73. XIII. Viverra tigrina.

23. Ca udivolvuca. Der Wickelschwanz. $\left({ }^{6}\right)$ Miiller, Naturfyft. Suppl. p. I3. n. 4. der Wickel. Schoonz. Tab. 2. fig. 7. Penn.

Zimunermannu, geogr. Zool. II. p. 297. n. 195. der Wickelschwanz.

Biiffon, vierfüs. XV. p. 182. der Kinkajou oder Potto?

pallas, Spicil. zool. XIV. p. 26. e. Meles caudi. volvula.

Erxle-

(5) It diefs vielleicht Kolbens Biefamkatze; falls diefe Gattung von der vorhergehenden wirklich verfchieden feyn follte? Die Gattungen grenzen fo nahe an einander, und die ältern Befchreibungen find oft fo unbeftimmt, dafs es fchwer bält, etwas mit völliger Gewilsheit davon zu behaupten.

(6) Ein artiges Thierchen, das gern auf den Minterbcinen fitzt, und von Brodt, Früchten, Fleifchwerk, Milch u. dgl. lebt. Es fchlaft bey Tagre, und erzürnt fich, wenn mán es in Schlafe ftört. Scinen Schwanz wickelt es im Schlafe um den Hals. 
Erxleben, Mammal. p. 7o. 1. 5. Lemur (Flavus) flayus, cauda prehenfili.

Graumann, introd. in hift nat. mamm. p. 24. V. der' Wickelschwowz.

Gatterer, brev. Zoolog. I. p. 26.' n. 5. Lemur (Flavus) flavus, cauda prehenfili.

Q4. Mezrivora. Der Ratel. (7)

Zimmermann, geogr. Zoolog. II. p. 287. 1. 182. der Honigfucher; der Ratel.

Bhumenbach, Handbuch der Naturgefch. p. 86. 11. 2. Meles (Mellivorus) dorfo cinereo, fafcia laterali nigra, abdomine nigto; dev Honig. dachs; Rattel.

Funke, Naturgefch. I. p. io6. der Honigdachs.

Gatterer, vơm Nutzen und Schaden der Thiere,

I. p. 321. n. I86. der Honigfucher.

Sparrmann, Befchreibung der Viverra Ratel: in den fchwed. Abhandl. XXXIX. p. I34.

Sparrmann, Reife n. d. Vorgeb. d. g. H. p. 480 . Ratel, Rateifrett.

Goeze, Natur, Menfchenleben und Vorfehung;

I. p. 556. der afrikanifche Honigdachs.

Gattever, brev: Zoolog. I. p: I34. n. 16. Viverra (Mellivora) dorfo cinereo, fafcia laterali nigra, abdomine nigro, unguibus longis; fubtus cavis fofloriis; der Honigfucher.

25. FA S I A TA. Die indifche woilde Katze mit fchwar: zen Streifen.

Sonnerat, Reife n. Oftind: u. China, II. p. Iiz.

İch

(7) Sparmann halt diefs Thier für de la Caille's Blairean puant, und Schrebers Viverra capenfis. Vid. Sylt. p. 89. n. 18. 
Ich habe das Original nicht zur Hand. In der vor mir liegenden Ueberfetzung heifst es: fie in vom Kopfe bis zum Ende des Schwanzes zwey Fufs lang; die Länge des Schwanzes beträgt neun Zoll..

26. Malaccensis. Die Zibethkatze von Malack $a$.

Sonnerat, Reife nach Oftindien und China, II. p. II3.

Das Thier in fo wild, dafs es gegen den Schufs läuft", wenn es nur verwundet ift.

A) Veröndernngen gegen die XITte Edition, und Vermeinung der Gattungen diefes Gefchlechts. Edit. XII.

Edit. XIII.

p. 63. n. I. B. Muftela pag. 84. n. 7. Viverra Glauca. Mungo.

Auffer dem, dafs die Mhangufte vom Ichneumon getremnt worden, ift das Gefchlecht noch mit ein and zwonzig Gattungen vermehrt, und V. Zemick, Tetradactyla, Vulpecula, Quasje, Co. mepatl, Mephitis, Zorilla, Mapurito, Vittata, Zeylanica, Capsinjis, Civetta, Flermaphrodita, Folfa, Tigriana, Candivolvula, Mellivora, Fa- Sciata, und Malaccers is hiuzugekommen. 'Auch if das Zibetthier von der Zibetkatze abgefondert.

.B) Unbeftimntere Thiere.

1. Der Sumxis der Chinejen.

Zimumernann, geogr. Loo!. I.p. 197. II. p.299.n.a. Boym, 
Boym, Flora Sinenf. tab. penult.

Muurr, Beytr. zur Naturgefch. von Iapan und Si. na; im Naturforfcher VII. p. 48. Song - Schu.

2. Der Nems.

Zimmermanin, geogr. Zool.II. p. 299.n. b. Viverra Bïffon, Suppl. III. pl.27. Le iNems. YIchneu* Biiffon, vierf. XIV. p. 152. Nems. J J mon?

3. Die Katze mit Spitzzulanfendem Kopfe ans $N_{\text {New- }}$ Spanien.

Zimmuermanni, geogr. Zool. II. p. 300 . n. e. Seba, thef. I. p. 76. Tab. 48. fig. I.

Erxleben, Mammalia, p. 499 .

4. Die bräunliche Viverre mit einem voeifsen Stricto an jeder Seite.:

Zimmermann, geogr. Zoolog. II. p. 300. n. d. Brown, Iamaic. p. 486. n. I. Im Syftem if diefe Stelle p. 89. bey n. r8. vermuthungsweife an. geführt.

5. Der Squafch des Dampier.

Zimmermann, geogr. Zool. II. p. 30o. n. è.

6. Der Chinche des Fenille.

Zimmermann, geogr. Zool. II. p. 30 t. n. $f_{s}$

Fenillé, Voyag. I. p. $272 . \quad$ If im Syftem p. 88. bey $\mathbf{n}$ : 16. vermuthungsweife angeführt.

7. Der Maikel; Yagitane.

Zinumermann, geogr. Zool. II. p. 3or. n. g: Falkner, Befchreib. von Patagonien, p. $1580^{-}-$ Im Syftein vermuthungsweife p. 88 . bey n. I6. 


\section{Mammalia Ferae. MuRela:}

15. GESCHLECHT. MUStela. Das Wiefel. Der Marder.

Miiller, Naturfÿf. I. p. 258. Gen. XV. Zimmermann, geogr. Zool. II. p. 3oI. Gen. XXIV. Schreber, Säugthiere, III. p. 455.47I. Gen. XVI. XVII.

Lefke, Naturgefch. p. I4I. I43. Gen. XVI. XVII. Borowesky, Thierreich, I. 2. p. 5I. Gen. XV. Bluntenbach, Handb. d. Naturgefch. p. 8I. p. I25. Gen. XXII. XXXXII. Bechftein, Naturgefch. Deutfchl. I. p. 278. Erxleben, Mammalia, p. 445. 452. Gen. XLI. XLII.

Graumann, intr. in hift. nat. manm. p. 68.69 . Gen. XLI. XLII.

But fch, Thiere, I. p. 20I. Gen. XXVI. XXVII. Gaiterer, brev. Zool. I. p. I2I. I23. Gen. XLI. XL.II.

Pennant, Syn. quadr. p. 21I. 238. Gen. XXIII. XXIV.

Briffon, quadr. p. I75. 20I. Gen. XXXVI.'XL. Hermann, tab. affin. animal. p. 74 .

* Mit verwachenen Fiijsen. OT TER

I. Lutris. Der Meerotter. ( $\left.{ }^{(}\right)$

Miiller, Naturfyftem, I. p. 259. n. I. der Meer. otter.

Zimnmera

(1) Sehr viele Zoologen, auch die mehreften von denen, die ich angeführt habe, verwechfeln den brafilianifchen und kamtfchatkifchen Otter, und halsen fie für einerley Thier. Erxlebin war es noch nicht entfchicden. Schreber bezweifilt es, und Zimmernann hat lie föllig von einander getrennt, weil der brafilianifche Otter nicht im Merwaffer lebt, weil er nur cinem mito telmafsi- 
Zimmermann, geogr. Zoolog. II. p. 3I3. n. 2 II. der Meerctter.

\section{$S_{3}$ \\ Lefle,}

telmäfsigen Hunde an Gröfse gleich kommt, da hingegen jener drey Fufs lang ift; weil er ferner eine gelbe Kehle hat, und end́lich weil er in einem fehr heifsen Klima zu Haufe gelıört. Der Laut diefes an den Flüfren des füdlichen Amerika gemeinen Thiers gleicht deni von einem jungen Hunde. Tr lebt von Fifchen und Krebfen, liefert ein brauchbares Pelzwerk, und efsbares Fleifch.

Die Miecrottern werden zwifchen dem 50 und 56 Grade der Breite an den Küften des Meers, welches Afien von Amerika trennet, am haufighten aber auf dẹn Infeln in demfelóen gefunden. Im Winter liegen fie an dem Merresufer, oder auf dem Eife, welches fie bey anhaltendem Oftwinde hẩufig aụs den Infeln nach der Weftküfte von KKamtfchatka bringt. Im Sommer fteigen fie in die Flüfe und Landfeen hinauf. In warmen Tagen fuchen fie den Schatten tiefer Tisaler. Oetrter, wo fich Robben, Meerlöwen, und Meeroären aufhalten, vermeiden fie forgfaltig. Thre Nahrung befteht in allerley Fifchen, Seekrebfen, Mufcheln, Schnecken, Blackfifchen, im Nothfall auch Fleifch und Tạng. Ihre Speife fuchen fio zur Zeit der Ebbe, wenn das Meer niedrig ift; auf. Sie laufen gefchwind, fchwimmen in vẻrfchiedenen Lagen, tauchen unter, künnen aber nur kurze Zeit unter dem Waffer bleiben. Zum Schlaf begeben fie fich aus dem Meere aufs felte Land, ind legen fich krumm wie die Hunḑe. Ihr Geruch und Gehör ift fturker als ihr Gefịcht. "Sié find furchtfam, aber fchlau den Nąchftellungen zu entgehen. Sie leben in der Monogamie; Das Weibchen foll acht bis neun Monat trachtig gehen, wirft auf dem Lande, aber hüchlt felten mehr als Ein lunges, welches mit offenen Augen, und ailen Zahnen zur Welt kommt. Die Mutter liebt ihr lunges fehr zärtlich, und lịfst es, wenn'fie auch verfolgt wird, nie aus dem Munde. Die Begattung gefchiehet zu allen lahrszeiten, ein Weibchen aber begattet fich in demfelben labre, da es tràchrịg gewefen, nicht wieder. Diefen Tnieren wird wegen ihres koftbaren fchwarzen und filbergrauen Balgs fehr nachgeftellt. Die beften Bälge fallen im Marz, April und May. Die meiftẹn gẹẹn nach China, wo der Hof zu Peking, und die 
Lefke, Naturgefch,-p. I42. n. 2. der Meerotter: Borowelky, Thierreich, I. 2. p. 63. n. 1. der Sebotter, Meerotter.

Bhunnenbach, Handb. der Naturgefch. p. 126. n. 2. Lutra (Marina) plantis pilofis, cauda corpore quadruplo breviore.

Funke, Naturgefch. I. p. 97. Meerotter.

Halle, vierf. p. 567 . Seeotter.

Pennant, ard. Zoolog. II. p. 92. n. 44. dev Meevotter.

- Gatterer, vom Nutzen u. Schaden der Thiere, I. p. 273. n. I57. der Seebirber, die Meerotter. Neuer Schauplatz der Natur, IX. p. 780. Mieer otter oder Seeotter.

Batfch, Thiere, I. p. 280. Meerotter. Steller, Befchr. von Kamtfchatka, p. 97. der Sega biber, oder vielmehr der Seeotter.

Steller, Befchr, fonderb. Meerth. Halle 1756. p. 6r. Krajcheninnitiow, Befchr. von Kamt fchatka, p. I6r. Seebiber.

Hamb. Magazin, XI. p. 460. Meerotter; m. e. guten Figur.

Onomatol: hiftor. nat. IV. p. 903. Seeotter. Beckmann, Naturhiftorie p.32. n. a. Seeotter. Pallas, Reife d. Rufsland, III. p. 127. 137. Pallas, nord. Beytr. II p. 279. Seebiber. Miiller, Samml. ruff. Gefch. III, p. 529. Kamt. fchatkifche Biber.

Limué,

Vornehmften im Reich, davon Verbrämungen an den Kleidern rragen, Ein Balg wird, nachdem er fchön ift, mit 90 bis 140 Rubel, fo wie die zu Mützengebrämen und Handfchuhen gebräuchlichen Schwänze mit 2 bis 7 Rubel bezahlt. In Kamtfchatka trägt man zum Staat Gebrame davon an Kleidern von weifsen Rennthierhăuturen, Das Fleifch ift unf̣chmackhaft und q⿳亠乛冖h 


\section{Mammatia Ferae. Mufela.}

Iinné, Syft. Natur. Edit. II. p. 44. Lutra pol. lice digitis breviore.

Linné, S. Nat. Edit. VI. p.5. n. 2. Lutra pol. lice digitis breviore.

Linné, Syft. Nat. Edit. X. I. p. 45. n. I. Muftela (Lutris) plantis palmatis pilofis, cauda corpore quadruplo breviore.

Granumann, intr. in hiftor. nat. mamn. p. 68.1. Meerotter.

Gatterer, brev, Zoolog. I. p. I2r. n. I. Lutra (Marina) plantis pilofis, cauda corpore quadruplo breviore. Secotter, Meerotter, Seebieber.

Pennant, Syn. quadr. p. 241. n. 175. Sea-Otter.

B. Der Guachi; die Saricovienne.

Zimmermann, geogr. Zoolog. II. p. 3I个. n. 214. der Guachi, die Saricovienne, Iiya.

Klein, Claflif, der vierfüls, Th. p. 275. n. 2. brafilianifche Otter.

Klein, natürl. Ordn. d, vierf, Th. p, 98. n. 2. brafilianifche Otter.

Biiffon, vierf. IV. p. I45. der Iiya, oder Caregueibeja.

Bïffon, vierf. XVI. p. 3. die, Saricovienne; mit einer Figur,

Ionfton, quadr. p. 150. Carygueibein, alias Tiya. Charleton, exercitat. p. ig. Saricovienne et $\mathrm{Ca}$ rygueibein.

Des Marchais, voy. en Guinée, III. p. 290. Lou. tre, que les Indiens appellent Carigueibeiu.

Gunilla, hift. de l' Orenoque, III. p. 293. n. 240. Guachi.

Fermin, Hollande equinox. p. Io.

$$
\mathrm{S}_{4} \quad 2 . \mathrm{Lu} \text {. }
$$




\section{0

2. Zutra. Die Fifihotter. ( $\left.{ }^{2}\right)$

Iniiller, Naturfyft. I, p. 262. n. 2. Fhilsotter. Tab. I4. fig. I. Houtt.

Zimmermann, geogr. Zool. I. p. 276. der. Fhussn otter. II. p. 313. der Fischotter.

Lefke, Naturgefch. p. I42. n. I. Lutra' vulgaris; Fifchotter.

Borowlky, Thierreich, I. 2. p. 65. n. 2. Lue tra vulgaris; Fifinotter.

Blumenbach, Handb. d. N. G. p. I25. n. I. Lu. tra (Vulgaris) plantis nudis, cauda corpore di. midio breviore; die Fifchotter.

(2) Länge des Kërpers über zwey Fufs, des Schwanzes anderthalb. Das Thier hat in Anfehung des Vaterlandes, Aufenthalts, und überhaupt in feiner Lebensart manches mit dem Biber gemein, obwohl beyde in ihrem Kürperbau, zumal in Gebifs, verfchieden gebildet find. Der Fifchotter beifst mehr Fifche todt, als er verzehren kann. Die kleinen frifste ér ganz, von den gröfsern aber lafst er Kopf und Rückgrad liegen. Er fchwimmt fehr gefchickt, kann fich auch unter dem Waffer von einem Oțte zum andern begeben, aber doch nicht lange in der Tiefe bleiben. Vom Waffer entfernt er fich nie weit. Einen gefangenen Fifch trägt er jederzeit anfs Land. Am liebften raubt er in der Nacht. Am Tağe bärt er fich mehrentheils in feinem Bau auf, oder fetzt fich auf alte, im Waffer befindiiche Stöcke und Steine in die Sonne, aber gegen den Wind; und begicbt fich bey dem gevingften Verdachte in Sicherhẹit. ITetern Nachichicen zu Folge foll der Balg des Thiêrs weit electrifcher, als der vọn der wilden Katze feyn. Man will fogar bemerkt haben, daís wenn es ins Waffer geht, und durch daffelbe bey Nachtzeit fehwimmt, er folches an feinem Kürper leuclitend mache, und dadurch leicht entdęckt werde. Man fangt die Fifchottern am beften mit dem Tellereiren, welches an Orten, wo fie ibren Auswurf gelaffen, aufgeftellt, und fo lang gelafen wịd, dafs fie damit das Waffer erreichen künnen, wo fie erfticken; fonft beiffen fie fich das Bein ab, woran fie gefangen worden, müffẹ aber doch umkom- 


\section{Mamimatia Férae. Mufela.}

Bechftein, Naturgefch. Deutfchl. I. p. 320. der Fifchotter. P. 329. der gemeine Fifchotter, Flussotter, Landotter, Otter, Fi/chdieb, Fifchottermarder.

Funke, Naturgefch. I. p. 97. Fifchotter:

Ebert, Naturlehre, I. p. 381. Fifchotier.

Halle, vierf. p. 564 . cise Flus sotter. p. 599. die Fifchotter.

Beikmann, Naturhiftorie, p.32. n. 6. die Fhusso otter.

Neuer Schaupl. d, Natur, IX. p. 7.8I. Flurfsotter. Onomat. hift. nat. IV. p. 399. Flulsotter.

Handbuch der Naturgelch. I. 2. p. ד.52. Fifchotter: Handb.d. deut. Thiergefchichte, p. So. Fifchotter. Pennant, arct. Zool. II. p. 89. n. 42. die gemeine Flussotter.

Walther, ökonom. Naturgefch. Deutfchl. p. 128. Fif chotter.

Gatterer, vom Nutzen und Schaden der Thiere,

I. p. 276. n. 158. Flufsottex, Fifchotter.

Gesner, Thierbuch, p. 302. der Otter; Figur

p. 303. fchlecht.

Klein, Quadrup. difp. p, 9r. Lutra.

Klein, Claffif. d. vierf. 'Th. p. 275. Otter.

Klein, natürl. Ordn. d. vierf. Thiere, p. 98. n. т. gemeine europizifche Otter.

Biiffon, vierf. IV. p. I37. XVI. p. 38. Fifchotter. Bock, Naturgefchichte von Preuflen, IV. p. 40.

n. II. Fis chotter:

Goeze, europäilche Fauna, 1. p. 326. F/lı $\int$-oder Fifchotter.

Batfch, Tliere, I. p. 208. der gemeine Fifchotter. S 5 Kraft,

umkłommen, weil fie nicht meht fchwimmen künnen. Dás Fleifch wird gegefien, fohmeckt aber fifchartig. Die virginjfclién Bälge find die feinten. 
Kraft, Ausrottung graufam. Thiere, I. p. 203. Fifchotter.

Perrault, Charras und Dodart, $\Lambda$ bhandl. zur Naturgefch. I. p. 175. anatomifche Befchreib. einer Fifchotter. Tab. 22, die Fifchotter. Tab. 23. die Zergliederung.

Pallas, Reife durch Rufsland, I. p. I99. 429. II. p. 569. III. p. 87. 288. 37\%.

Carver, Reife d. Nordamerika, p. 38r. Fifchotter.

Schöpf, Reife durch Nordamerika, I. p. 422. Ottern.

Pontoppidan, Norwegen, II. p. 54. Fifchotter. Steller, Befchr, von Kamt́chatka, p. I28. Fifchotter.

S. G. Gmelin, Reife, III. p. 285 und 273.

Leem, Nachr. v. d. Lappen, p. III. Fifchotter. Fif cher, Naturgefch. von Livland, p, 52, n. 8. Fifchotter.

Nenhoif, Gefandtfchaft rach China, P. 393.

Fermin, Befchr. v. Surinam, II. p. 82. Fifchotter. Beytr. zur Béförder. der Naturk. I. p. II6. Fijch. otter.

Lichtenberg und Voigt, Magazin für das Neue. fte etc. IV. 4. p. 157. Fifchotter.

Loto, Schwed. Abhandl. XIV. p. 147. Fifch. otter?.

Beckmamn, Befchreib, der Mark Brandenburg, I. p. 586.

Ridinger, Entwarf einiger Thiere, Tab. 82. 83. Fifchotìter.

Ridinger, jagdbare Thiere. Tab. I6. Fifchotter. Döbel, Iägerprakt. I. p. 40. Fijchotter. Schörfeld, Landwirthfchaft, p. 619. 674. IMerklein, Thierseich, p. I52, Otter.

Onomat. 
Onomat. foreftal. I. p. 804. Canis fluviatilis. Fi: chotter.

Heppe, wohlredepder Iäger, p. I23.

Oekonom. Zoologie, p. 21. n. 23. die Flnfsotter. Icblonfky, allgem. Lex. p. 337. Fifchotter.

Miiller, Samml. Fuff. Gefch. III. p. 531. Otter, Flusfsotter, oder Fifchotter.

Leipz. geogr, hiftor. und phyf. Mancherley, I. p. II5 - II9.

Linné, Syf, Nat. Edit, II. p. 44. Lutra digitis aequalibus.

Limné, S, Nat. Edit, VI. p. 5. n. I. Lutra digitis aequalibus.

Linné, Syft, Nat. Edit. X. I. p, 45. n. 2. Muftela (Lutra) plantis palmatis nudis, cauda corpore dimidio breviore.

Graunann, intr, in hiftor. nat. mamm, p. 69.II. Lutra Vulgaris; die Fifchotter.

Gatterer, brev. Zool. I. p. I2r. n. 2. Lutra (Vulgaris) plantis nudis, cauda corpore dimidio breviore. Flufsoiter, Fifchotter.

Muiller, Zoolog. dan. prodrom. p. 2. n. ro. Muftela (Lutra) plantis palmatis nudis, cauda corpore quadiuplo breviore.

Denys, defcription de l'Amerique feptentr. II. p. $28 \mathrm{I}$.

Turpin, hif, du roy. de Siam, I. p. 3I6. Kramer, Ault. p. 312. Lutra digitis aequalibus. Charleton, exercitat. p. I8. Lutra. Schwenkfeld, theriotroph. Silef. p. IO7. Lutra. Wagner, hift. nat. Helvet. p. I78. Lutra, Enydris" Rzacz. hiftor. nat. Polon. p. 22I. Auct. p. 3is.

\section{Lutra.}

Severin, zool. Hungar. p. Ios. n. 2. Lutra vulga. ris; Fifchotter. 
Sibbald, Scot. illunt. II. p. Io. Lutra.

Gronov. Zoophyl, I. p. 8. n. 3I. Lutra digitis omnibus aequalibus.

Bellon, aquaiil p. 3I. Lutra. p. 32. eino mitrel. märsige Figur.

Schoneveld, ichthyolog. p. 46. Lutra.

Pennant, britt. Zool. p. 32. Otter; mit einer guten Abbildung.

Forfter, philof. transact. LVII. p. 342. Mufta Lutra.

Ayricola, de animal. fubterran. p: is. Lutra,

Ariftot. hit. anim. I. c. 2. n. 8. VIII. c. 9. n. I05. Evudess.

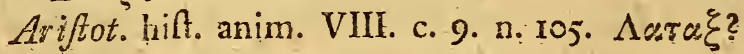

3. Lutreola. Der Nör. (3)

Miiller, Naturfyft. I. p. 264. n. 3. die Sumpfotter. Zimunersncnu, geogr. Zoolog. I. p. 278. der kleine Sumpfotter. II. p.3I4. n. 2riz, der Nörz, sumpf: otter.

Borowfky, Thierreich, I. 2, p. 66. n. 3. Lutra minor: der Sumpfoiter, Nörz.

Bechfintin, Naturgefch. Deutchl. I. p. 330. n. 2. der Nörz. p. 333. kleiner Fif chotter, Swmpfotter, kleiner Sumupfotter, Nerz, Krę̧sutter, Sumpfotternarder, Steinhunzd.

Gatte.

(3) Länge des Köppers etwas tiber einen Fufs, des Schwanzes. wenig tiber fechs Zoll. Seinen Aufenthalt hat er wie der Fifichotter, an den Ufern cer Bache und anderer Gewaffer, befonders in waldigen Gegenden, wo das Walfer im Winter nicht ganz ausfrieret. Eri friss auch Krebfe und Wafferkäfer, und kommt übrigens in der Lebensart mit den Fifchotter ziemlich iberein. Der Balg wird in dem Vaterlande des Thiers getragen, aber felten zu uns gebracht, if auch von etwas geringerm Werthe, als der Fifchotterbalg. 
Gatterer, voin Nutzen und Schaden der Thiere, I. p. 281. n. 159. Steinhund, Nerz, Simup: otter.

Penunent, arct. Zool. II. p. 90. n. 43. der Sumpf: otter, der Nörz.

Neuer Schaupl. d. Natur, IX.p. 782 . Sumpfotter.

Onomat. hif. nat. V. p. 386. Sumpfotter.

Gesner, Thierbuch, p. 347. Nörz-Wiefelein, Noertz, Nertz, oder Nörtz.

Büfon, vierf. XVI. p. 99. der Sumpfotter oder Noerz; m. e. Fig.

Goeze, europäifche Fauna, I. p. 341. eine kleinere Race des Fifchotters.

Bat/ch, Thicre, I. p. 207. der Nörz.

Pallas, Reife durch Rufsland, 1. p. 96. eine kleine Art von Ottern.

Carver, Reife durch Nordamerika, p. 382. die Sumpfotter; Mink.

Fifcher, Zunü̈tze zur Naturgefch. von Livland; p. 48. Suzuipfotter.

Rytfchkow, orenb. Topogr. I. p. 237. Norka. Ryt fchkow, orenb. Topogt. in Buifchings Magazin VII. p. 46.

Oekon. Zool.p. 22. n. 24. die Sumpfotter; Nörzo Schöpf, Reife durch Nordamerika; I. p. 422 . Minks.

Pallas, Reife, Ausz. II. p. is. Wafferwiefel.

Graumann, intr. in hift. nat. mamm. p. 69. III. Lutra Minor; die Sumipfotter.

Gatterer, brev. Zoolog. I. p. I22. 11. 3. Iutra (Minor) plantis hirfutis, digitis aequalibus, ore albo. Simpfotter.

Rzacz. hiftor. nat. Polon. p. 2I8. Latax; germ. Nörtz; nobis Nurek.

Furfter, phil. transact. LXW. p. 37 I. Leffer Otter. 
Severini, zoolog. Hungar. p. IoS. n. 3. Lutra Minor, feu Paluatris. Schuppotter.

5. Vison. Das Vifon. (4)

Zimmermamn, geogr. Zoolog. II. p. 315. n. 213. das Vifon.

Pennant, arct. Zool. II. p. 8r. n. 36. der Vifon. Neuer Schauplatz der Natur, IX. p. 259. Vifon. Wifon (mit dem Pekan verwechfelt).

Biiffon, vierf. XV. p. 246. der Vifon; in. e. Fig:

* Mit gefpalienen Fiifsen. W. ESEL.

4. Barbara. Die Tayre. (5)

Miiller, Naturfyft. I. p. 265. 13. 4. der fchiearzt Wiefel.

Zimmernamn, geogr. Zoolog. II. p. 3ro. n1. 209. die Tayra.

Schrebsi, Säugthiere, III. p. 493. n. 8. die Tayra. Boronefky, Thierreich, 1. 2. p. 55. n. 2. dev Schroma IV iefel.

Erxleben, Mammal. p. 452. n. I. Muftela (Bar. bara) atra, collo fubtus macula alba, triloba.

Linné,

(f) Linge des Thiers gegen fechszehn Zoll, des Schwanzes fieben. Hühner, Ginfe, und Ënten beifst es mehrentheils tcht, faugt ihnen das Blut aus, und läfst das Fleifch licgen. Eg kann anch zahm gemacht werden. Pennant und Erxleben vermengen das Thier mit dem Pekan, Biffon findet es dem Iltis ähnlich, und will es als eine Spielart davon anfehen. Schreber zweifelt, ob es nicht vielleicht nit dem Nürz nur eine einzige Art ausmache, weil der Untetfhicd zwifchen beyden faft nur anf dem weifsen Munde beruhe. II der artifcken Zoologie if der pekan vom Vijon getrennt.

(5) Reibe fich, nach Barrere, gern an den Biumen, und ber Echmiert tie mit ciner Art von Bifam. 
Linné, S. N.Ed.X.I.p. 46.n. 4. Muftela (Barbara) pedibus fiflis, atra, collo fubtus macula alba, triloba.

Graumann, intr. in hilt. nat. mamm. p. 69. I. der fchwarze Wiefel.

Gatterer, brev. Zool.I. p. I23. n. I. Muftela (Barbara) atra, collo fubtus macula alba, triloba; das fihwarze Wiefel.

Severin, zool. Hung. p. 83. n. s. Muftela Barbara.

\section{Galera. Dev Vanfire.}

Zimmermann, geogr. Zoólog. II. p. 307. n. 203. der Vinfire.

Schreber, Säugthiere, III. p. 493. n. 9. Tab. 135 . Borowlky, Thierreich; I. 2. p. 55. 11. 3. der, Vanfire.

Neuer Schauplatz d: Natur, IX. p. 229. Vanfire. Biiffon, vierf. XIV. p. I47.der Vonfive; m.e. Fig. Handbuch der Naturgefch. I. 2. p. 166. der Tayra. Graumann, introd. in hif. nat. mamm. p. 69. II. Muftela Galcra.

Gatterer, brev. Zoolog. I. p. I23.'n. 2. Muftela (Galera) tota fufca; guineifiche Wiefel.

Severin, zool. Hungar. p. 83. n. 8. M. Galera.

13. Canadensis. Der Pekar.

Zimmermann, geogr. Zoolog. II. p. 309. n. $20 \%$. déer Pekan.

Borowelky, Thierreich, I. 2. p. 55. n. 4. dev Pekan.

Gatterer, vom Nutzen und Schaden der Thiere, I. p. 283. n. 160. der Pekan.

Pennant, arct. Zool. II. p. So. n. 35. der Pekan. Neuer Schauplatz der Natur, VI. p. 405. Pekan. Biiffon, vienf. XV.p. 246. der Pekan; m.e. Fig. 
Charlevoix, defcript. de la nouv. Fr. III. p. 164. les Pekans.

Graumanz, intr. in hif. nat. mamm. p. 69. III. M. Canadenfis。

Gatterer, brev. Zoolog. I. p. İ3. n. 3. Muftela (Canadenfis) corpore fulvo nigricańté, pectore macula alba; Pekan, Wifon.

Severin, zool. Hung. p. 83. n. 8. M. Canadenfis.

\section{F O INA.- Der Steimmarder. ( $\left.{ }^{6}\right)$}

Zimmermann, geogr. Zool. I. p. 267. der HansStein-oder Buchnarder. II. p. 302. n. I96. der Steinmarder, Hausmarder.

Leeke, Naturgefch. p. 144. der Steinmarder.

Borose:

(6) Lange des Körpers etwa io Zoll, des Schwanzes gegen 9. Er uniterfcheidet fich von dem Baummarder 1) durch die.etwas geringere Gröfse; 2) den etwas langern Kopf und kürzere Beine; 3) durch die weifse Kehle; und 4) durch den fchlechtern, weinger feinen Peiz. Zimmermaniz hált mir Linné diefe und die. folgende Gattung nur für.Varietäteh. Nach Gesner und $R a j$ begàtten fich beyde mit einander Der Steinmarder hat feinen Aufenthalt in Klippen, Steinbaufen, aten Gemânuer, Scheuren, Stäler; und felbet Wohshäufern, wo er Gelegenheit fich zu verbergen findet. Im Winter, wenn es ihm an Nahrung manselt, nagt $\mathrm{cr}$, um zu den Hühnerfällen und Taubeghhẩufern zu gelangen, oft die Strohdächer und folbft Bretter durch. Er etwhirgt aber meht als ex verzehrt. Sein Auswurf hat einen blSamartigen Geruch. Zum Schlaf legt er fich, wie die Hunde, kugelrund zufammen. Ër fcheint den ganzen Sommer hindurch zu ranzels, denn man findet vom Frühling ah, bis in den Herbft; Iunge. Das Werfen gefchiehet unter dem Heu oder Stroh, unter Reisholze, im Genüuer oder Steinklippen, auf einem Lager von $\mathrm{Hell}$, Stroh, oder Moos, das fie fich zufammén tragen. Der Balg ift zu allerley Kleidungsftïcken branchbar. Am beften fangt man das Toier in dem Schwarenhalfe. Es hált aber noch fehwerer einen Marder, als einen Fuchs-zu fangen. 
Boraw Jky; Thierreich, I. 2. p. 57., n. 6. der Steinmarder.

Blumenbach, Handb. der Naturgefch. p. 82. n.2.

Muftela (Foina) corpore fulvo nigricante, gula alba; der Hansmarder, Steinmarder.

Bechftein, Naturgefch. Deutfchl. I. p. 279. der

Steinmarder; p. 287. Hans - and Dachmarder, (von einigen fälfchlich Feld-und Buchmarder.)

Funke, Naturgefch. I. p. I09. Steinmarder, Haus. marder.

Halle, vierfüls. p. 459. der Steinmarder.

Ebert, Naturl. I. p. 366. der Stein-oder Hausmarder.

Meyer, Thiere, II. p. 2. Tab. 4. der Steinmarder, oder Buchmarder.

Gatterer, vom Nutzen und Schaden der Thiere,

I. p. 286. n. 162. Steinmarder, Hausmarder.

Beckmann, Naturhiftorie, p.25. n. 7. der Marder. Neuer Schaupl. der Natur, V.p. 376. Haius - oder

Steinmarder, von einigen Buchmarder.

Onomatol. hift. nat. V. p. 388. der Steinmarder,

Hausmarder, Buchmarder.

Handb, der Naturgefch. I. 2. p. I60. Hausmarder. Handbuch der deutfchen Thiergefchichte, p. 49.

Hausmarder, Steinmarder.

Sander, ökonom. Naturgefch. I. p. 259. der Marder; p. 260. der Hansmarder.

Gesner, Thierbuch, p. 346. Tachmarder, Haus. marder, Steinmarder, Buchmarder.

Klein, Quadr. difp. p. 64. Martes faxorum non fagorum, feu domefticus; Steinnarder.

Klein, Claffif. der vierf. Th. p. I93. Steinmarter oder Hausmarter.

Klein, natül. Ordn. d. vierf. Thiere, p. 68. n. 5.' Steinmarter.

$$
\text { T Bock, }
$$


Bock, Naturgefch. von Preuffen, IV. p. 42. der Steinmarder.

Goeжe, europäifche Fauna, I. p. 263. Haus. marder.

Biiffon, vierfüfs. IV. p. I46. der Hausmarder; Tab. 6I. a.

Gmelin, Reife durch Rufsland, III. p. 370. der Marter?

Fifcher, Naturgefch. von Livl. p.53. n. 10. Steinmarder.

Beyträge zur Beförd. der Naturkunde, I. p. II7. Steinmarder mit weiei ser Kehle.

Schönfeld, Landwirthfchaft, p. 676. 66I.

Döbel, Iägerprakt. I. p. 42. Steinmavaier.

pennant, britt. Thiergefchichte, Tab. 6. Hans'marder.

Loniceri, Kräuterbuch. Ulm, 1703. fol. p. 622. Buch-Bach - Haus - Steinmarder.

IMerklein, Thierreich, p. 217. 11. 2. Dach-oder Tachmarder, Buch-oder Steinmarder.

Grannann, intr. in bifor. nat. mamm. p. 70. V. Hausmarder, Steinmarcier.

Gatterir, brev. Zoolog. I. p. 125. n. 5. Muftela (Foina) corpore fulvo-nigricante, gula alba; der Steinmarder.

Severin, zool. Hungar. p. 83. n. 7. Muftela Foina, faxatilis.

Agricola, de animal. fubterran. p. 38. Martes in Saxis.

Schwenkfeld, theriotroph. Silef. p. IIO. Martes faxatilis.

Rzacr. hif. nat. Polon. p. 222. Martes. Pyrard, voyages, I. p. 87. Fouines. 
6. Martes. Der Bánmmarder. (7)

Miiller, Naturfyft. I. p. 267. n. 6. der. Marder. Zimmermann, geogr. Zool. I. p. 267. der braus ne Feld-oder Tannenmarder; II. p. 303.n. 197. der Baummarder, Feldmarder.

Lefke, Naturgefch. p. I44. n. 2. der Banminarder:' Borow Jky, Thierreich, I. 2. p. 56. n. 5. der. Bausmmarder.

Bhumenbach, Handb. d. Naturgefch. p. 8r. n. I. Muftela (Martes) corpore fulvo-nigricante, gula flava; der Baum-Edel-Tamen-WildFeldnarder.

Bechftein, Naturgefch. Deutfchl. I. p. 287. der Banmmarder; p. 293. Edelmarder, GoldWald - Buch - Büchen - Bufch - Fichten - KieferTannen - Birken - Espen - Vieh - Licht - und Feldmarder.

Funke, Naturgefch. I. p. 109. der Baummarder. Ebert, Naturlehre, I. p. 366. der Baummarder. Halle, vierf. p. 457. Banmmarder.

Meyer, Thiere, II. Tab. 5. der Feldntarder oder Baummarder.

\section{$T_{2} \quad$ Gatte}

(7) Lange des Körpers gegen 19 Zoll, des Schwanzes 10. Diefe Thiere entfernen fich von den bewohnten Gegenden fo viel als möglich, in dicke, und vornämlich -aus $\mathrm{Nadeltiolz} \mathrm{beftehende}$ Wallder. Ihr Auswurf riecht biefamartig. Ihr Pelz gehürt unter die fchönften Rauchwerke. Bey den lagden der kanadifchen Wiiden, und dem dortigẹn Pelzhandel machen fie einen Hauptartikel aus. Sie finden fich da, tnd in dem brittifchen Amerika in folcher Menge, dafs man lahre weifs, wo die Engländer und Franzofen allein aus diefen Gegenden über foooo Marderfelle zum Handel brachten. Das Fleifch wird in Frankreich gegeffen. Auch der Feldmarder läfst fich zăhmen. Cetti fagt: er habe einen gefehen, Gen man fo wcit gebraclit hatte, dafs er unter Küchlein fchlief. In Sardinien macht man zuweilen : Prefence damit. 
Gatterer, vom Nutzen und Schaden der Thiere, I. p. 283. n. I6r. Feldnarder, Baumnarder. Sander, ökonom. Naturgefch. I. p. 260. der Feldoder Banmmarcerer.

Handb. der Naturgefch. I. 2. p. 160. Feldmarder.

Handb. d. deutfch. Thiergefchichte, p. I48. Baummarder, Edelmarder, Wildmarder, Tannenmarder, Feldnarder.

Pennant, arct. Zool. II. p. 79. n. 34. Baummar. der, Feldinarder.

Neuer Schaupl. d. Natur, V. p. 376. Feld-oder Waldnucrder.

Onomat. hiff. nat. V. p. 390 . Baummarder, Feldmarder, Wald-oder Tannenmarder.

'André und Bechftein, Spatziergänge, III. p. 42. der Banmarder.

Gesner, Thierbuch, p. 345. ein Marder, m. e. fchlechten Figur. p. 346. ein wilder IMarder, Feldmarder, Wildinurder, Baumnarder, Tannenmavder, Viehmarder.

Klein, Quadruped. difp. p. 64. Muftela Martes abietum; Baummarder.

Klein, Claffif. d. vierf. Th. p. 192. Bauminarter. Klein, natürl. Ordn. d. vierf. Thiere, p.68. n. 6. Baunmmarter.

Biiffon, vierfüfs. IV. p. 156. der Feldnarder: Tab. 6r. b.

Bat fch, Thiere, I. p. 206. der Baummarder.

Bock, Naturgefchichte von Preuffen, IV. p. 42: der Banmmarder.

Goeze, curop. Fauna, I. p. 279. Bannnnarder. Leffeps, Reife durch Kamtichatka und Sibirien, p. I60. Marder:

Fifcher, Naturgefch. von Livland, p. 53, n. to. Banmunarder.

Carver, 
Carver, Reif. d.Nordamerika, p. 373. der Marder. Cetti, Naturgefch. von Sardinien, I. p. 203. der Feldmarder.

Miiller, Samml. ruff. Gefch. III. p. 515. Marder. Pallas, Reife durch Rufsland; II. p. 570. 214. 52. 143.20 .270$.

Ryt fchkow, orenb. Topogr, in Bïfchings Magaz. VII. p. 44 .

Hog/tröm, Befchr. von Lappland, p. 22. Pontoppidan, Norw. II. p. 46. \$. I2. der Marder. Leem, von den Lappen in Finnmarken; p. Ic6. Steinmarder, Birkenmarder, Kiefermarder. (Benennung und Bëfchreibung verwechfelt.) Pennant, britt. Thiergefch. p. 34. Batummarder. Merklein, Thierreich, p. 218. Baum - Feld-oder Wildmarder.

Döbel, Iägerprakt. I. p. 4I. 42. Bannmmarder. Heppe, wohlredender läger, p. Ir4. Schönfeld, verbeff. Landwirthfchaft, p. $616 \mathrm{ff}$. Beytr. zur Beförd. der Naturk. I. p. II7. Baummarder mit gelber Kehle.

André und Bechftein, Spatziergänge, II. p. 400. Baummarder.

Ludovici, Kaufmannslexicon, III. p. I8I\%.

Onomat. foreftal. II. p. 776 .

Linné, Syft. Nat. Edit. II. p. 44. Martes.

Linné, S. Nat. Edit. VI. p. 5. n. 2. Muftela ful. vo-nigricans, gula pallida.

Linné, Sylt. Nat. Edit, X. I. p. 46. n. 5. Muftela (Martes) plantis fiffis, corpore fulvo-nigricante, gula pallida.

Linné, Fn. Suec. I. p. 3. n. 7. Muftela fulvo-nigricans, gula pallida.

Limné, Fn.S.II. p.6.n. I5. Muftela (Martes) pedibus fiflis, corpore fulvo- nigricante, gula pallida. 
Gaumann, intr, in hift. nat, mamm. p. 69. IV. Feldmarder, Wildmarder, Baummarder.

Gatterer, brev. Zoolog. I. p, I24. n. 4. Muitela (Martes) corpore fulvo-nigricante, gula flava; Feldnarder.

Severin, zool. Hungar. p. 82. 1n. 3, Mufela Mar. tes; der Marcler.

Miiller, Zoolog. dan. prodrom. p. 3. n. x2. Muftela (Martes) pedibus fiflis, corpore fulvo. nigricante, gula pallida.

Kramer, Auftr.p. 3Ir. Muftela fulvo-nigricans, gula pallida.

Scheffer, Lapponia, p. 34r. Mardures,

Chäleton, exercitat. p. 20. Martes.

Schwenkfeld, theriotroph. Silef. p. IIO. Martes arborea.

Agricola, de anim. fubterr. p: 38 , Martes in arboribus.

Wigner, hift. nat. Helvet. p. 8I. Martes.

Rzacs. hint nat. Pol.p. 222. Martes. Auct. p.314. Sibbald, fcot. illufr. P. II. T.II p. Ir. Martes.

Charlevoix, hift. de la nouv. France, III. p. I34. les Martres.

Forfter, phil. Transact. LXII.p. 372. Pine Martin. Ionfton, quadr. p. 156. Martes fylveftris.

Q. Zibetrina, Der Zobel. $\left({ }^{8}\right)$

Miiller, Naturfyftem, I. p. 272. der Zobel. Zimmermann, geogr. Zool. I. p. 287. der Zobel. II. p. 303. n. 196. der Zabele

Lejke,

(8) Länge des Thiers gegen ry Zoll. Ber Zobel hat feinen Auf enthalt in einfamen, wüften, dick bewaldeten, auch feifigen Gegenden, in Höhlen unter der Erde, unţcr den Baumwurzeln, auch 
Le he, -Naturgefch. p. $34 \%$ n. 53 der Zobel. Borowfky, Thierreich, I. 2., p. 52. n. I. des Zobet. it

Bhumenbach, Handb. der Naturgefch. p. 82. n. 4. Muftela (Zibellina) corpore fulvo- nigricante, facie et gula cinereis; der, Zobel.

Funke, Naturgefch. I p. Tos. der Zobel.

Ebert, Naturlehre, I. p. 366. der Zobel. Tab.22. Halle, vierfüls. p. 459. der Zobel.

Gattever, vom Nutzen u. Schaden der Thiere;

I. p.29r. n. I66. der.Zobel.

Beckmnann, Naturhiftorie, p.26. n. Io. der Zobel. Pennant, arct. Zool. II. p. 82. n. 37. der Zobel. Pennont, arct.Zool.II. p.85. n.38. das itifcherwiefel. A. $\quad \mathrm{T} 4$ Neuer

auch in hohlen Bäumen. Wenn er fich fatt gefreffen hat, fchläft ex forfeft, dafs man ihn aufnehmen, ftofsen und techen kann, ohne dafs er erwacht. Zur Zeit der Begattung fallen unter den Mànchen eben lo blutige Aufritte, wie unter den Katern vor. In Sibirien fąngt man diefe Thiere in Schlagbäumen mittelf einer Azung; die aus einem Stǘck Fleifch oder Fifch befteht, auch in Netzen, die man vor den Oeffnungen ihrer Baye, oder um die hohlen Bäume anftellt, tind treibe fie mit Rauch, oder durch Umháựng' der Bäume heraus; Sie werden anch mit vorn breiten Pfeilen gefchoffen. Der Zobelfang wird in Gefellfchaften, dic zuweilen to Manu ftark find, vorgenommen. Die feinften Bàlge fallen um lakuzk. Die beften werden vom November bis Febriar gefangen. Die Sommer - und Herbftzobel find kurzhaarig. Die Mãnnchen find grölser und dickhariger.als die Weibchen. Die feialten Zobel werden nach ausgef chnittenen Bäuchen (die Schwänze behalten fie) paarweife zifanumen genăhet, fchlechte hingegen ganz gelaffen, und ihnen nur etwa die Schwànze abgefchnitten. Beyde Sorten wérden zimmerweife verkautr. Ein Zimmer hălt 49 Stück. Der Preifs ift fehr verfchieden. Es giebt Zobel, die auf der Stelle das Stück 25 Kopeken, oder ein Viertel und fo fort bis funfzig und mehr Rubel gelten. Die Schwanze werden das Hundert zu is bis 40 Rubel verkauft. 
Neuer Schauplatz der Natur, X. p. 26r. Zobel.

Onomatol. hiftor. nat. V. p. 392. Zobel.

Gesner, Thierbuch, p. 347. Muftela Zobela; Zobelivieselein, Zobel.

Klein, Quadruped. difp. p. 64. Muftela Zibel. lina.

Klein, Claffif. der vierf. Th. p. 193. Zobel, ruffische, fiberifche Wiefel; Scytifcher Marter; Sar. matifche Maus; fcytifche Maus.

Klein, natürl. Ordn. d. vierf. Th. p. 68. n. 7. Zobel; von etlichen auch: ruffische, fibirifche aind Scytifche Marter.

Biiffon, vierf. XV.p. 255. der Zobel; m.e. Fig. Batfch, Thiere, I. p. 205. der Zobel.

Steller, Befchr. von Kamtfchatka, p. Irg. Zobel.

I. G. Gmelin, Reife durch Sibirien, I. p. 39r. Zobel.

Strahlenberg, Europa und Afien, p. 430. Zo. beln oder Sobeln.

Krächeniinnikow, Befchr. von Kamtfchatka , p. II\%. II8. I32-I39. Zobel.

Leffeps Reife d. Kamtfchatka u. Sibirien, p. 23. Zobel.

Miiller, Samml. ruff. Gefch. III. p.495. der Zobel. Schlözer, Erdbefchr. v. Nordamerica, p. 75. dev Marder oder Zobel.

Leppechin, Tagebuch der ruff. Reife, III. p. I8. Befchreib. des Zobelfangs.

Pallas, Reife d. Rufsl. II. p. 214. 227. Tab. 7. III. p. 570 .

Erxleben, Mammal. p. 467. n. 9. Muftela (Zibellina) corpore obfcure fulvo, fronte exal. bida, gutture cinereo.

Erxleben, Mammalia, p. 470 . n. Io. Muftela (Pennanti) corpore nigro, lateribus fufcis. 


\section{Mammialia Ferae. Muftela. 297}

Linné, Syft. Nat, Edit. II. p. 44. Mufta Zi. bellina.

Linné, Syft. Nat. Edit. VI. p. 5. n. 7. Muftela Zibellina.

Linné, Syft. Nat. Edit: X. I. p. 46. n. 8. Muftela (Zibellina) plantis fiflis, corpore obfcure fulvo, fronte exalbida, gutture cinereo.

Graumann, intr, in hif. nat. mamm. p. 7o. IX. Zobel. X. Muft. Pennanti.

Gatterer, brev.Zool.I. p. I26. n.9. Muftela (Zibellina) corpore obfcure fulvo, fronte exalbida, gutture cinereo; der Zobel.

Gatterer, brev. Zoolog. I. p. I27. n.'1o. Muftela (Pennanti) corpore nigro, lateribus fufcis; das Fifclierzuiefel.

Severin, zoolog. Hungar. p. 82. n, 5. Muftela $\mathrm{Zi}^{-}$ bellina, Sobella; der Zobel.

Scheffer, Lapponia, p. 343. Sabellinae. Deutfiche Ausg. p. 387 .

Charleton, exercitat, p. 20. Muftela Zibellina; Ariftoteli Satherius; Nipho Cebalus; Alciato Mus Sarmaticus et Scythicus.

Agricola, de animal. fubterr. p. 39. Zobela. Rcaczyn fki, auct. hift. nat. Polon. p. 317. Mu. ftela Sobella.

Raj, Syń. quadr. p. 20I. Muftela Zibellina.

7. PUTORIUS. Der Iltis. (9)

Miiller, Naturfyft. I. p. 269. n. 7. der Iltis.

Zimmermanı, geogr. Zoolog. II. p. 304. n. 199: der Iltis.

$$
\mathrm{T}_{5} \quad L_{\mathrm{e}} \int k e_{\text {, }}
$$

(9) Lánge des Körpers etwa fiebzehn Zoll, des Schwanzes nur fechs. Er beifst alles Geflügel, was" er habhaft werden kann, todit, 
Lefke, Naturgefch. p. 145. n. 4. der Ittis. Boronefky, Thierreich, I. 2. p. 59. n. 8. dev - Iltis.

Bhimenbach, Handb, der Naturgefch. p. 82. n. 5. Muftela (Putorius) flavo - nigricans, ore et auricularum apicibus albis; der Iltis, Ilk, Stärzkirratz.

Bechflein, Naturgefch. Deutfchl. I. p. 294. der Iitis. p. 302. Eltis, Ilk, Ulk, Elfke, Elb. thier, Ellenkatze, Stinkthier, Stänker, Stänkerratze, finkende Wiefel, Tenfelskind, Haus. wink, Unke, Illing, Buntfing, Nölling, Iltis. marder; in Thüringen Ratze.

Finnke, Naturge(ch, I. p. IIo. Iltis; Stinkthier. Ebert, Naturlehre, I. P. 365. Iltis, Stinkthier; finkendes Wiefel.

Halle, vierf. p. 456 . der Iltis; Stinkthier. IMeyer, Thiere, II. Tab. 6. Iltiv. Abbild gut: Gatterer, vom Nutzen und Schaden der Thiere, I. p. 287. n. 164. der Iltis, Ilk.

Goeze, europäifche Fauna, I. p. 285. der Iltis. Batfch, Thiere, I. p. 207. der Iltis.

Georgi,

codt, und trägt es weg, oder frifse ihm das Gehirn aus. In die Scheunen und Stalle grabt er Lücher, macht auch wohl Rühren unter der Eràe hin. Wenn er angegriffen wird, "ftellt er fich mit einem Katzenbuckel und funkelnden Augen zur Wehre, zifcht, grunzt, und giebt den ihm eigenen ibblen Ges ruch ftárker als gewöhnlich. Das Weibchen wirft feine Iungen an fillen, einfamen Orten, in Gebäuden, in hohlen Baum wurzeln und Felsklüften. Gegen den Herbft verlaffen die Iungen die Mutrer. Man fangt diefe Thiere auf eben die Art, wie die Steinmarder. Der Ealg wird von den Kürfchnern durch Sehwärzen der längern Haare verfchünert, und verarbeitet, verliert aber den widrigen Geruch des Thiers nicht, und if daher węniger im Gebrauche, als er feiner Güte naç feyr könnte. 
Georgi, Rufsland, I. p. 208. Iltiffe.

Becknann, Naturhiforie, p.25. n. 8. der Iltis, oder das Stinkthier.

Pennant, arct. Zool, II. p. 89. der Iltis.

Neuer Schauplatz der Natur, VIII. p. 585. Stinkthier.

Onomat, hit. nat. VI. p. 7ro. Iltis; Stinkthier.

Gesner, Thierbuch, p. 346. ein Iltis, mit einer fchl. Fig.

Klein, Quadruped. difp. p. 63. Muftela foetida: Putorius. Iltis; Tenffelskind.

Klein, Claflif. der vierfüls. Th, p. I92. Iltis; Teufelskind.

Klein, natürl. Ordnung d. vierf. Th. p. 67. n. 4. finkende Wiefel; Iltis, Teufelshind.

Bock, Naturgefch. von Preuffen, IV. p. 44. n. I3. der.Iltis.

Büffon, vierf, IV: p. I69. der Iltis, Tab. 63. Sander, ökon. Naturgefch. I. p. 258. der Iltis. Handbuch der deutchen Thisrgelchichte; p. 49. Iltis, Ilt, Stönokerratz, ftinkendes Wiefel. Handbuch der Naturgefch. I. 2. p. 16r. der Iltis. Kraft, Ausrottung fchädl. Thiere, II. p. 542. dev Iltis.

Beytr. zur Beförd. d. Naturk. I. p. Irs. Iltis, Stäna kermart.

Pallas, Reife, I. p. 129. weifsliche Iltiffe.

Fifcher, Naturgefch, von Livland, p.54. n. IF. Iltis, Teufelskind.

Hupel, Befchr. von Livland, II. p. 435. Iltis. Schreber, (d. ä.) Cameralfchriften, XII. p. 495. Halle, Magie, I. p. 335. Iltisfang.

Berlin. Samml. II. p. 400. Iltisfang; IV. p:302. europäifches Stinkthier, oder Iltis. Berlin, Magazin, III. p, 429. Iltisfang. 
Ridinger, Entwurf einiger Thiere, Tab. 87. Iltnis. Ridinger, jagdbare Thiere. Tab. 2o. Iltnis. Merklein, Thierreich, p. 2I8, n. 3. Iltis.

Döbel, Iägerprakt. I. p. 42. Iltis, Illing, EllKatze, Stänkervatz, Elbthier.

Heppe, wohlredender Iäger, p. I25. Iltis. Neue Mannichfaltigk. I. p. 817-823. Fig. p. 824. Pennant, britt. Thiergefch. p. 32. Tab. V. der Tlits, Teufelskind, ftinkende Wiefel.

Schönfeld, verbefferte Landwirthflhaft, p. 660. André u. Bechftein, Spatziergänge, I. p. 95. Iltis. Erxleberi, Mammalia, p. 463. n. 7. Muftela (Putorius) flavo-nigricans, ore auricularum. que apicibus albis.

Linné, Syft. Natur. Edit. II. p. 44. Putorius. Linne', Syft. Nat.'Edit. X. I. p. 46. n. 6. Muftela (Putorius) plantis fiíis, corpore flavonigricante, ore auriculisque albis.

Graumzann, introd, in hifor. nat, mamm. p. 70 . VIII. Iltis.

Gatterer, brev, Zoolog. I. p. I25. n. 7. Muftela (Putorius) flavo - nigricans, ore auricularum. que apicibus albis; der Iltis.

Severin, zool, Hung. p. 8r. $\mathrm{n}_{2}$ 2. Muftela Putorius, - vulgo Viverra; der Iltis.

IMilller, zool. dan. prodrom. p. 3. n. r3. Muftela (Putorius) pedibus fiffis, corpore flavo - nigricante, ore auriculisque albis.

Kramer, Aufr, p, 312. Muftela flavefcente-nigricans, ore albo, collari flavefcente.

Charleton, exercitat. p. 20. Putorius.

Schwenkfeld, theriotroph. Silef. p. I20. Putorius, Agricola, de anim. fubterr. p. 37. Silveftris.mufela เærıs?

Wagner, hif. nat. Helvet. p. I82. Putorius, Viverra. 
Rzacz. hif. nat. Pol. p.236. Auct. p.329. Putorius. Sibbald, Scot. illuftr. P. II. T. II. p. Ir. Putorius. Forfter, phil. transact. LVII. p. 342. M.Putorius. Brown, effay fur les erreurs populaires, I. p. 252. cap. 4 .

8. F u Ro. Das, Frett. $\left({ }^{10}\right)$

Miiller, Naturfyft. I. p. 27t. n. 8. das Kaninchemviefel.

Zinmermann, geogr. Źoolog. II. p. 305. 11. 200. das Frett.

Lefke, Naturgefch. p. 146. n. 5. das Fret.

Borowfky, Thierreich, I. 2. p. 60. n. 9. das Kaninchemeiefel, Frett.

Bhumenbach, Handb. der Naturgefch. p. 83. n. 5 . Muftela (Furo) corpore pallide flavo; das Frettel.

Bechftein, Naturgefch. Deutfchl. I. p. 303. das Frett; p. 305. Furett, Frettele, Frettchen, wilde Wiefel, weifse Wiefel, Kaninchenwiefel, Kaninchenjöger, Frettmarder.

Funke, Naturgefch. I. p. Ir3. Frettchen:

Ebert, Naturlehre, I. p. 364. das Frett; Kanizchemeiefel.

Halle, vierfüfs. p. 456. das Frett, oder der Ka. ninchenjäger; Kaninchenzwiefel.

Meyer,

(10) Länge des Körpers etwa vierzehn Zoll; des Schwanzes fünf. Gehört eigentlich in der Barbarey zu Haufe. Von da hat mans nach Spanien gebracht, um die Kaninchen zu vertilgen, und nun hat fichs fchon weiter in Europa verbreitet. Die Kaninchen haben eine unglaubliche Furcht vor ihm. Es kriecht ihnen in ihre Höhlen nach, iagt fie heraus, oder tödtet fie auch wohl darin, und faugt ihnen das Blut aus. Es begattet fich auch mit lltifen, und bringt fodann braunhärige Baftarde. Sein Geruch ift wiarig, wie beym lltis. 
Meyer, Thiere, II. p. 7. Tab. 22. das Frettel. Gatterer, vom Nutzen und Schaden der Thiere,

I. p. 290. n. I64. das Frett, Frettel.

Goeze, europäilche Fauna, I. p. 298. das Frett. Batjik, Thiere, I. p. 207. das Frett.

Neuer Schaupl. d. Natur, III. p. I95. Frett. (In den Stellen, auf welche hier hingeviefen wird, fteht nichts.)

Onomat. hift nat. V. p. 38r. Wildwiefel, Frettwiefel, Kaninchenusiefel.

Handbuch d. Naturgefch. I. 2. p. 163. das wilde Wiefel. Viverra, Furo, oder Furunculus.

Gesner, Thierbuch, p. 344.'Frett, Frettel, oder Furettel. Fig. mittelm.

Klein, Quadr.difp. p. 63. Muftela, Viverra dicta: Ǩlein, Clafiff, d. vierfüfs. Th. p. rgr. Frettviejel.

Klein, natürl. Ordn. der vierfüfs. Th. p. $67 \cdot$ n. 3 . Fretwiesel.

Biiffon, vierfüfs. IV. p. I78. das wilde Wiefel. Tab. 64. p. r8o. wilde Iltiswiefel. Tab. 65. das gelbe Wiefel.

Beytr. zur Beförder. der Naturkunde, I. p. Irs. n. 9: Fret.

Verfuch einer Anweifung zur Anlegung etc. der Wildbahnen etc. Berlin und Stettin, 1779. gr. 4. p. 323. Naturgefchichte des Frettchen. Mlierklein, Thiere, p. 2I7. 1. 3. Frett, Furet, Frettele.

Döbel, Ïgerpraktik, II. p. I24. Fretgen. Limné, S. N. Edit. II. p. 44. Viverra. Limné, Sŷt. Nat. Edit. VI. p. 5. n. 4. Muftela fylveftris, Viverra dicta.

Limné, Syft. Nat. Edit. X. I. p. 46. n. 7. Muftela (Furo) plantis fiflis, oculis rubicundis. 
Graumann, intr. in hif. nat. mamm. p. 70. VIII. Frettel.

Gattever, brev. Zool. I. p. I26. n. 8. Mufela (Furo) corpore pallide flavo; KaninchenwieSel, Frettel.

Severiar, zool. Hungar. p. 82. n. 4. Muftela Furo feu Sylveftris.

Charleton, exercit. p. 20. Viverra.

Agricola, de animal. fubterr. p. 40. Viverra." Rzacz. hift. nat. polon. p. 235. Muftela filveftris. Sibbald, fcot. illuftr. P. II. T. 2. p. Ir. Viverra. Plin. hift. nat. VIII. c. 55 . Viverra XXIX. c. 4. Muftela fylveftris.

Ariftot. hift. anim. IX. c. 9. n. Ios. Ixtıs.

15. S A R M A T I CA. Der Tiegeriltis. (")

Miiller, Naturfyft. Suppl. p. 33. n. I3. der Sar. matier.

Zimmermann, geogr. Zoolog. II. p. 305. n. 20 . der Tigeriltis.

Borowfky. Thierreich, I. 2. p. 58. n. 7. cier Tigeriltis.

Gatterer, vom Nutzen und Schaden der Thiere, I. p. $287 . \mathrm{n}$. I63. Tiegeriltis.

Gesner, Thierbuch, p. 348. Wormlein - Wie. Selein.

Pallas,

(1) Sène lagdzeit ift die Nacht. Am Tage rubet er in den Bauen anderer 'Thiere, auch gräbt er felbft welche. Ir läuft hüpfend mit gekrümmtem Leibe, und gerade hinats geflrecktem Schwanze, wie alle eigentliche threlel. Das Thier ift bösartig. Sein Geruch ift fehr fein. Die Länge des Kürpers beträgt etwa gegen vierzehn Zoll, des Schwañzes gegen fieben. Das Pelzwerk wird in dern Vaterlande des Thiers nịcht geachitet; kümmt aber bisweilen nach Deutfchland, und ift dann ziemlich im Prsife. 
Pallas, Reife, I. p. I75. Tiger-Iltis.

Pallas, Reife, Ausz. I. Anh. p. I. Muftela Sarmatica, III. p. 477. Tiger-Iltis.

Graumann, intr. in hift. nat. mamm. p. 70. VI. M. Sarmatica.

Gatterer, brev. Zool. I. p. I25. n. 6. Muftela (Sarmatica) fufco luteoque varia.

16. Síirica. Der Kulon. ()

Miiller, Naturfyft. Suppl. p. 34. n. I4. die fibirifche Wiejel.

Zimmernann, geogr. Zoolog. II. p. 306. 12, 202. der Koulon.

Schreber, Säugth. III. p. 495. 11. ro. der Kulon. Bororelky, Thierreich, I. 2. p. 60. n. Io. fibirificher Wiefel, Kulon.

Gatterer, vom Nutzen und Schaden der Thiere, I. p. 300 . n. 167 . Kulon.

Pallas, Reife, II. p. 570. eine noch nicht befchrie. bene Wiefelart.

Pallas, Reife, Ausz. II. Anh. p. I. n. I. Muftela Sibirica. Tataris Kulon.

Graunnainz, intr. in hif. nat. mamm. p. 70. XI. fibirifches Wiefel.

Gatterer, brev. Zoolog. I. p. I27. n. II. Muftela (Sibirica) fulva, palmis plantisque hirfutiffimis.

IO. ER-

(2) Lange dés Kürpers etwa zwölf Zoll; des Schwanzes fechs. Der tatarifche Name foll einen Vielfrafs bedeuten, weil man bemerkt haben will, dafs diefs kleine Thier alle Arten von Thieren, dis in Schlingen und Fallen gefangen find, wenn der läger zu fpät kommt, auffreffen, und auf einmal eine ftarke Mahlzeitithun foll. Das Pelzwerk wird in Rufsland nicht geachtet, geht aber häufig nach China. 
10. ERm I nea. Die grofse Wiefel. (3)

a. AESTIVA; die Sommerfarbe. Wiefel; grolse
Wiefel.

B. HY BERNA; die Winterfarbe. Hermelin.

\section{Mïller, Naturfyftem, I. p. 276. b. der Herme: limwiefer.}

\section{Zimmer-}

(3) Die Benennung Hermelin kommt ihm nur zur Zeit des Winters zu, wenn der Balg ganz weifs ift. Die Farbe verändert fich im Herbf und Frühlinge mit den Haaren. Die Wiefeln warmer Länder, iḿ füdlichen Turopa, in Perfien, und weiter hinunter in Afien, zwifchen den Wendekreifen, verfärben ihr Haar gar nicht. In Sibirien und Norwegen macht diefs Pelzwerk einen beträhtichen Handelsartikel aus. Im erfern Lande koften 100 Felle auf der Stelle zwey bis drey Pfund Sterling. Man fänt die Thiere in Schlagbäumen, Fallen und Schlingen, oder fchiefst fie mit ftumpfen Pfeilen. Die aufferordentliche Kalre einiger Winter hat auch felbft diefe, dauerbaften Thiere gezwungen, auszuwandern, wie man in den Iahren 1730. und 374. gefehen hat. In Rufsland find die Hermeline in den dünnen Gehülzen fo häufig, dafs man wohl eher in kurzer Zeit zwanzig taufend Stück zufammengebracht hat, die nach Fetersburg verlangt worden. Leppechin, der diefe und die folgende Gattung in eifernen Käfigen gehalten hat, fagt: das Thier lâfst fich niemals zahm machen, wenn mans auch noch fo lange fuittert. Es ift fo gefräfsig, dafs es in einem Tage weit mehr verfchlinget als fein ganzer Kürper beträgt. Sein Futter reifst es zu kleimen Stïcken aus den Händen, und wonn es gereitze wird, fällt es den, der es reitzt, mit Ungeftïm an, zifcht wie ein Sperling und hat funkelnde Augen. Sperrt man es in eine Scheune bey die Märfe, fo bleibr keine am Leben, und wenn" ihrer taufend da wären. Eben fo verhâlt fichs auch mit den Wiefeln. (M. vulgaris) Daher tödten die Banern die Hermeline und Wiefeln, die fich bey den Getraidefchobern aufhalten, niemals; fondern fchonen fie forgfaltig. Die rotbbraune Farba im Sommer, und die weifse im Winter, kommr diefen Thieren in beyden Iahrszeiten zu ihrer Erhaltung zu ftatten, weil des Sommers die weifce Farbe fie unter dem Grünen, und des 
Zimmermann, geogr. Zool. I. p. 243. das Her. melin. p. 245. das Hermelin oder Wiefel mit fihwarzer Schwanzfpitze. II. p. 308. n. 205. das gröfsere WViefel; das Hermelin.

Lefke, Naturgefchichte, p. I46. n, 6. das grofse iviefel.

Borowfky, Thierreich, I. 2. p. 6I. n. I2. der Hermuelin.

Blumentach, Handb. d. N. G. p. 83. n. 6. Mufela (Erminea) caudae apice atro; das grofse Wiefel, Hermelin.

Bechfeein, Naturgefch. Deutfchl. I. p. 305. das (grofse.) Wiefel.

Fumke, Naturgeich. I. p. II3. das grofse Wiefel, Hermelin.

Ebert, Naturlehre, I. p. 365. der Hermeliz.

Halle, vierf. p. 455. das Hermelin.

Gatterer, vom Nutzen und Schaden der Thiere, I. p. $5^{\circ} 4$. n. 169. das Hermelin.

Beckntann, Naturhiforie, p. 25, n.9. das Wiefel; das Hermelin.

Pennant, arc: Zool. II. p. 78. n. 33. das gröfsere Wiejel, der Hermelin.

Neuer Schauplatz der Natur, III. p. 806. Hermelint.

Onomat. hif. nat. V. p. 379. Muntela candida f. Ermineùn; das Hermelin. p. 381. KönigsweieSel; Muftela regalis.

Gesner,

Winters dic rothbraune auf dem Schnee; den Iigern leicht in die Hande liefern künnte. Verfchiedenheit der Nahrungsmittel, innere Schwachung, gehemmte Ausdünfung, gehäufte innere

- Feuchtigkeit; und deren Einfuís auf Haar und Gefieder, find vielleicht Mliturfachen des Weifswerdens vieler Thiere in kalten Ländern zur Winterszcit. Siche \&. G. Cmelin Jeife d. Rursl. 1. p. 39 ff. 
Gesner, Quadrup. p. 852. Armelini.

Handbuch der Naturgefch, I. 2. p. 168. das Her. melin. Hermellanus.

Klein, Quadrup. difp. p. 63. Muftela Armellina. Klein, Claffif. d. vierfüfs. Th. p. 190. Hermelin; das weifse Wiefelein, welches nur am Schwanz fchwarz ift.

Klein, natürl. Ordn. d. vierfüfs. Th. p. 67. n. 2: Hermelin; Königsweiefel.

Büffon, vierf. IV. p. I96. das Hermelin. Tab. 67. Bock, Naturgefch. von Preuffen, IV. p. 46. der Hernelin; p. 48. b. das Hermelinueiefel.

Goeze, europäifche Fauna, I. p. 304. das gro/ss Wiefel.

Batfch, Thiere, I. p. 206. das. Hermelin.

Fif cher, Naturgefch. von Livland, p. 55. 11. 13 . Hermelinchen.

Pontoppidan, Norwegen, II. p. 48. das Her melin.

Steller, Befchr. von Kamtichatka, p. 125. Hermelins.

Leppechin, Tagebuch der ruff. Reife, I. p. I75. $^{2}$ Hermeline.

Schlözer, Erdbefchr. v. Nordamerica, p. 75. der. Hermelin.

Pallas, Reife d. Rufsl. I. p. 129. Hermeline. Miiller, Samml. ruff. Gefch. IIL p. 516. Hermelinen. Georgi, Rufsl. I. p. zose Hermeline.

Pallas, nov. fp. quadr. e glirium ord. p. 188.

Erminea.

Köhler, neue Reifebefchr. I. p. 325. Hermelin.

Samml. der neueften Reifebefchr. I. p. I91. 192. V. p. 220.

Pennant, britt. Thiergefch. p. 25. Tab. 7. b. das Hormelin. 
Mannich faltigkeit. I. p. 172. $188.355 .435 . \mathrm{m}$. Fig. Erxlebsn, Mammalia, p. 474. n. I3. Muftela (Erminea) auricularum marginibus albis, caudae apice nigro.

Linné, Syfl. Nat. Edit. VI. p. 5. n. 6. Munela candida $\int$. Ermineum.

Granmonn, intr, in hift. nat. mamm. p. 7r. XIII. Hermelin.

Gatterer, brev.Zool.I. p. 128. n. I3. Muftela (Erminea) auricularum marginibus albis, caudae apice nigro; dis Hermelin.

Severin, zoolog. Hungar. p. 83. n. 6. MuRtela Erminea; Mus Ponticus; das Hermelin.

Scheffer, Lapponia, p. 343. Hermelini.

Chanleton, exercitat. p. 20. Hermellanus.

Schuenkfeld, theriotroph, Silef. p. II8. Muftela candida.

Agricola, de anim. fubterr.p. 33. Mus Ponticus, quem hodie vocant Hermelam.

Wagmes, hift. nat. Helvet. p. 180. Muftela alpina candida.

Rzacz. hift. nat. Pol. p. 235. Auct. p. 328. Muftela alba; Turnebio Hermelinus.

Forfter, philof. transact. LXII. p. 37r. Stoat and Ermine.

Ionflon, Quadrup. p. I53. Hermellani.

I. Vulgaris. Die kleine Wiefel. $\left({ }^{+}\right)$

\%. A $E$ S T I va; clie Scminerforbe.

Miiller, Naturfyftem, I. p. 274. n. Io. der Hermelin; n. a. genieine kleine Wiefel.

Zimmere

(4) An Geftalt, Farbe, ind Eigenichaften kommt dies Thier den vorbergehenden bey, aber nichi an Grüfse und Wildheit. Es 
Zimmermann, geogr. Zool. I. p. 243. das Wiefel. p. 244. das Wiefel ohne fchucurae Schwanzsfipitze.

Boronefky, Thierreich, 1. 2." p. 61. nI. II. das gemeine Wiefel.

verwandelt anch feine Farbe in kalten Ländern, nicht aber in Deurfchland, und andern wärmern Lảndern, gegen den Winter in weifs. Linné verwechfelt es mit dem Hermielin; und die meiften Naturhiftoriker vermengen in ihren Synonymen und Befchreibungen beyde Gattungen mit einainder. Boddaest fiẹhe diefe kleine. Wiefel für eine blofse Varietat von der grofsen Wiefel an. Auch Zimmermann hält aus Grïnden, die der Prüfung wïrdig find, beyde für einerley. Erxileben hat die Muftele nivalis-als eine eigene Gattung von der affiva getrennt; aufsert aber doch felbft die Vermuthung, dafs beyde wohl nur Varietîten einler Gattung feyn müchten. - In den Scbived. Abisandl. werden folgende Verfchiedenheiten zwifchen der Nivilis und Vulgaris Nxxl: angegeben: 1) Vulgaris lebe in gelindern Erdftrichen, fcheuct die kaltern, welche Nivalis wählt. 2) Vulgaris if das ganze lahr hindurch in Häufern und bewohnten Oertern, andert felten thre Farbe, und vermuthlich nie nach der Jahrszeit: die wenigen weifseh diefer Art, die man dann und wann gefunden hat, beftäk en das wenigftens nichr, vielleicht rührt die Farbe bey ihnen von Krankheir oder andern Urfachen her, bleibt auch beftândig, wie bey weifsen Mâufen, Aelftern u. dgf. Nivalis ift noch nì̀ im Sommer weîfs, im Winter grau gefehen worden. 3) Vulgaris giebt allemal cinen witrigen Geftank; Nivalis hat faft keinen Gerùch, wenigftens nie fo widrig, als der Iltis, welches doch von Vulgaris allgemein bezengt wird. 4) Der Schwanz bey Vulgaris ift zwar in Vergleichung mit dem Körper kürzer als bey àndern Muffelis, aber doch nicht fo kurz, dafs er nach der Zoologen Sprachgebrauch abbreviata heifsen könnte, welches bey Nivalis ftatt findet; aufferdem 5) bey Vulgaris allemal am Ende gelbbraun, da Nivalis ihn bey allen Abwechfelungen von einerley Farbe mit dem Körper hat; die fchwarzen Haare, die man bey einem I und dem andern antrift, machen keinen merktichen Unterfchied der Farbe. 
Bhmenbach, Handbuch d. Naturgefch. p. 83. n. 7 . Mufta (Vulgaris) corpore ex rufo fufco, fub. tus albo; das gemeine Wiefel.

Bechftein., Naturgefch. Deutfchl. I. p. 3I5. das Heemuänntiven. p. 319. das kleine Wiefel, Hous - und Speicherneiefel.

Funke, Naturgefchichte, I. p. II4. das gemeinze Wiefel.

Ebert, Naturlehre, 1. p. 364. das gemeine Wiefel. Halle, vierf. p. 454. das gemeine Speicherwiefel. Meyer, Thiere, II. Tab. 24. das rothbraune Wiefel.

Gatterer, vom Nutzen und Schaden der Thiere, - I. p. 301. n. I6́s. áas Wiefel.

Bock, Naturgefchichte von Preufen, IV. p. 46. das lichtbraune oder röthliche gemeine. Wiefel. Ridinger, Entwurf einiger 'Thiere, Tab. 89. Wiefel.

Gesizer, Thierbuch, p. 342. ein Wiefelein oder Wiefel; etliche nennen es Hermelein, andere Hëernulein ; m. e. fchlechten Figur.

Thaft, Ausrottung fchädl. Thiere, I. p. 425. das Wiefelein.

Santer, ökon. Naturgefch. I. p. 258. das Wiefel. Handbuch der deutfchen Thiergefchichte, p. 50. das gemeine Wiefol.

Klein, Quadruped. difp. p. 62. Muftela vulgaris. Klein, Claflif. d. vierf, Th. p. 189. Haus jpeichsr. woiefel.

Klein, natioil, Ordn. d. vierf. Thiere, p. 66. n. x. gemeine Wiefel; Han'sspeicherwiefel.

Biiffon, vierf. IV. p. I86. das gemeine Wiefel. Tab. 66.

Fifcher, Naturgefch. von Livland, p. 54, 11. I2. gemeines oder Hauswiefel.

Beytr. 
Beytr. zur Beförder, der Naturk. I. p. I20. n.'a. brann Wiefel; gemein roth Wiefel; Haus. reiefel. .

B. NIVAL is; die 'Winterfarbe,

Miiller, Naturfyft. I, p. 276 . n. b. das Schnee - viefel.

Zimmernamn, geogr. Zoolog. II. p. 308. n1. 206. das kleine Wiefel.

Meyer, Thiere, II. Tab. 23 das weifse Wiefel. Pensant, arct. Zool. II, p. 77 , n. 32. das gemeine Wiefel.

Neuer Schauplatz d. Natur, IX. p. 780. Muftela nivalis; das Schneeroiefel.

Onomat. hif. nat. V. p. 39r. Muftela nivalis; der Schneewiefel.

Goeze, europ. Fauna, I. p. 316. das kleine ge: meine Wiefel.

Leppechin, Tageb. d. ruff. Reife, I. p. 175. Wiefel. Neue fichwed. Abhandl, VI. p. 209. Schneeviefel. Tab. 8.

Beyträge zur Beförd. der Naturkunde, I. p. I2I. weifs Wiefel, Hermelin.

Erxleben, Mammal.p. 476. n. r4. Muftela (Nivalis) corpore albo, caudae apice vix pilis ullis nigris.

Linné, Faun. Suec. II. p. 7. n. 18. Muftela (Nivalis) caudae apice vix pilis ullis nigris.

Aiiller, zool. dan. prodrom. p. 3. 11. 15. Muftela (Nivalis) pedibus fiflis, corpore albo, caudae apicè vix pilis ullis nigris.

Graumann, intr. in hift. nat, mamm. p. 7r. XIV. - das Schneeviefel.

Müller, Samml. ruff. Gefch. III. p. 517. ein beSonderes Gejchlecht von Hermelinen. 
Gatterer, brev. Zoolog. I. p. I29. n. I4. Mufela Nivalis; Schneeneiefel.

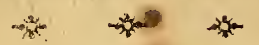

Pallas, Reife; I. p. «29. die grmeine Wiefel.

Pallas, nov. fpec. quadrup. e glir. ord. p. 8.9. Muftela.

Titius, Wittenberg. Wochenblatt, I773. p. II. Wiefel.

Döbel, Iägerprakt. I. p. 43 .

Onomat. foreftal. III. p. 392.

Shaw, Reife, p. 157. Wiefel oder Fert el Haile? Batfch, Thiere, I. p. 207. das gemeine Wiefel. Linnzé, Sylt. Nat. Ed. II. p. 44. Muftela vulgaris. Charleton, exercitat. p. 20. Muftela. Schwenhfeld, theriotroph. Silef. p. Ir6. Muftela. Agricola, de animal. fubterr. p. 36. Muftela do. meftica, Vifela.

Rzacs. hift. nat. Polon. p. 235. Muftela.

Rzacz. hift. nat. Polon. p. 235. Muftela alba vul. garis

Sibbald', Scot. illuftr. P. II. T. 2. p. Ir. Muftela vulgaris.

Plin. hir. nat. XXIX. c. 4. Muftela, quae in do. mibus noftris oberrat.

Severin, zool. Hung. p. 81. n. r. Muftela vulgaris.

17. Qu1Qux. Der Quiqui.

Von der Spitze der obern Lippe bis zum Anfange des Schwanzes in gerader Linie gementen, beträgt die Länge des Thiers $1_{3}$ Zoll. Dis platto Nafe ill auf der Mitte mit einem weifsen Flecken gezeichnet. Da das Thier wild und wiitend if? fo nennen die Eingebornen in Chili alle diejenigen Quiqui, die fich vom Zorne himreiffen laffen.

A) $V e r$ - 
A) Verändernngen gegen die XITte Edition, wnd Vermehrung der Gattungen diefes Gefchlechts.

Edit. XII.

Edit, XIII.

p.67. n. 5. Muftela Gulo. ' p. 104. n. 8. Urfus Gulo.

Der brafalianifche Otter ift als eine Varietät vom kamtfchatkifchen; der $V$ anfire als eine befondere Gattung von der Tayra; der Steinnarder auf eben die Art vom Bannmarder; - das Hermelin von der kieinen Wiefel getrennt; und von letzterer beyde Varietäten aus cinander gefetzt, und berichtiget. Uebrigens ift das $G e$ jehlecht nit fechs Gattungen vermehrt, und $M$. Vifon, Galera, Canadenjis', Foina, Samatica, Sibivica, und Quiqui hinzugekommen. Der hinzugekomimenen neuen Gattungen find eigentlich fieben; aber das Gefchlecht an fich if doch nur um fechfe färker geworden, weil die fiunfte des XII Edit, daraus weggelafen if.

\section{B) Unbeftimntere Thieve.}

$*$

1. Die grofse Otter von Giujana.

Zimmermann, geogr. Zool. II. p. 3I8. a.

Biiffon, Supplem. III. p. I59.

2. Die gelbliche Otter von Gujana.

Zimmermann, geogr. Zoolog. II. p. 3Is. b, Biitfon, Supplem. III. p. 158 .

3. Otterïhaliche Thiere von Neuland?

Zimnnermann, geogr. Zoolog. II. p. 318. c.

$$
\text { V } 5
$$


4. Die Ottern von Siam.

Zimmernnama, geogr. Zool. II. p. 318. d.

Turpin, hiftor, civ, et nat, du Roy. de Siam, I. p. 316.

5. Die graue Otier in der Gegend von Batavia. Lichtenberg und Voigt, Magazin für das Neue. fte etc. III. 2. p. Io.

$$
\text { ** }
$$

6. Die javcnijche Wiefel.

Zimnnermann, geogr. Zool. I. p. 246 . II. p. 308. 1. 204. das jovanifche Wiefel; der KogerAgar:

Halle, vierf. p. 460. die javanifche Wiefel.

Briffon, quadr. p. I78. n. 5. (ic.i habe die hollürad. Edit. in 8. von 1762 . zin Honid) Munela (Viverra Iavanica) fupra rufa, infra dilute flava, caudae apice nigricante. $L_{B}$ Furet de Java.

Seba, Thef. I. p. 77. Tab. 48. fig. 4. Muftela Ia. vanica.

Klein, Quradr, difp. p. 64. Muftela iavanica, Ko. ger - Agan dicta.

Klein, ClaAff. der vierfürs. Thiere, p. 194. IEvawifches Wiefelein.

Klein, natïl. Ordn, d. vierf. Th. p. 69. n. 9. Iavanifche Wiefel; Koger - Agan.

Biiffon, vierfüls. IV. p. 194. kleines javanilfches Wiefel.

Erxleben, Mammal. p. 454 .

Graumans, intr. in hift. nat. mamm. p. 69. ad II. Muftela Iavania.

Limué, Synt. Nat, Edit. XIII. p. 98. in not. 


\section{Mammalía Ferae. Mufela.}

7. Der Kokeboe.

Zimmermann, geogr. Zool. II. p. 3Ir. a.

Bofsmann. Reife nach Guinea; p. 299. Koekeboé. Schreber, Säugthiere, III. p. 495, oben. Kokobo.

8. Der Infire.

Zimnermann, geogr. Zool. II. p. 3rr. b.

Pigafetta, Befchr. von Kongo, in Purch. Pilgr. II. p. 1002 .

9. Der Tamiki.

Zimmermann, geogr. Zool. II. p. 3r. c. Kömpf. Iapan. Lond. 1727, fol. Vol. I. p. I26.

o. Der. Itutz.

Zimmermann, geogr. Zool. II. p. 3rr. d.

1. Der Tirr.

Zimmermann, geogr. Zoolog. II. p. 3̈r. ó

12. Der Quoll.

Zimmernann, geogr. Zoolog. II. p. 3r2. f.

13. 'Der kleine Marder ans Gujana.

Zinmermann, geogr. Zool. I. p. 3i2. g.

Biiffon, Suppl. III. p. 162. Pl. 24. ed. Paris, La pe. tite Fouine de Guyane.

1. Das verkehrte Hermelin.

Zimmermann, geogr. Zool, I. p. 312, h. Halle, vierf. Th. p. 462.

Erxleben, Mammal. p. 476.

5. Die Boccamele.

Cetti, Naturgefch.v. Sardinien, I. p. 2rr. Tab. 5 . Zimmievinann, geogr. Zool. II. p. 43I. Beckmant, phyf. ökon. Bibl. X. p. 540. Linné, Syn. Nat, Edit. XIII. p. 98. in not. 


\section{Mrammaliz Ferae. Urfus.}

16. GESCHLECHT. URsus. Der Bär.

Miiller, Naturfyf. I. p, 277. Gen. XVI.

Zisnmermann, gecor. Zcol. II. p.274. Gen. XXI.

Schreber, Säugthicre, III. p. 50I. Gen. XVIII.

Leeke, Naturgef ch. p. 147. Gen.. XVII.

Borowsky, Thierreich, I. 2. p. 66. Gen. XVI.

Blumeinbarh, Handb. der Naturgefchichte, p. 87 .

Gen. XXV.

Bechftein, Naturgefch. Deutfchl. I. p. 138.332.

Funke, Naturgefch. I. p. I55.

Peinant, arct. Zool. II. p. 55 .

Thatfch, Thiere, I. p. I95. Gen. XXIV.

Erxleben, Manmalia, p. Iś. Gen. XVII.

Graumann, introd. in hifter, nat mamm. p. 37. Gen. XVII.

Gaitever, brev. Zool. I. p. 44. Gen. XVII.

Peninant, Syropf. guadr. p. I90. 20I. Gen. XX. XXI.

Briffon, quadruped. p. I\$3. I87. Gen. XXXVII. XXXVIII.

Hermaniz, tab. affin. animal. p. 68 .

1. A к то оs. Der Landbär. (5)

Q. Niger. Der fchwarze Bär.

Zimmermann, geogr. Zool. H. p. 274. n. I66. b. der Schwarze.

Schreber,

(5) Ein phlegnatifches, brummigtes, aber im Grunde gurmüthiges Gefchüpf, das, wovon man wenigftens in Polen und Preuffen Beyfpiele hat, zuzeilen eine Grüfse von fünf bis fechs lillen erreicht. Seinen Raub fchlägt ef mit der Tatze daniecer, und faugt alshenn zuerft das Blut aus. Was er nicht verzehren kann, verbirgt er. Auch die braunen Bären geniefsen V'egetabilien, und die Schwarzen ebenfalls Fifche und Fleifchwerk, wiewohl 
Schreber, Säugth. III. p. 505. der fchwarze Bör. Lefke, Naturgefch. p. 148. der fchwarze Btir. Borowe ky, Thierreich, I. 2. p. 7o. der fchwar. ze Bür.

Steller, Befchr. von Kamtfchatka', p. 113. Schwarze Bären?

Erxleben, Mammal. p. I59. o. Niger, minor? Grinumann, istrod: in hifor. natur. mammal. p. 37. I. a.

Gatterer, brev. Zoolog. I. p. 45. a. Niger, maior? der Ameifenbär.

wiewohl letzteres feltener. Der Ameifen bemächtigt fich der Bär auf die Art, dafs er feine Zunge an den von ihm zerftürten Ameifenhaufen bringt, die Thierchen darauf kriechen lafst, und fie, wenn ihrer genug beyfammen find, verfchlingt. Er fàuft faft wie der Hund. Er fchivimmt gut', hält es aber nicht lange aus. Fin Schlag auf den Kopf tödtet ihn leicht. Sein laut if ein Brummen und Schnauben, im Zorn mit Zähneknirfchen vergefellfchaftet. Im Herbit wird er fehr fett. Den Winter bringt er zwar nicht fchlafend oder erftarrt, aber doch in einer ununterbrochenen Ruhe und olme Nahrung zu, und dennoch kann die Mutter dabè ihre lungen faugen. Nach Weihnachten ohngefálie häuten fich die Fufsfohlea des Bärs, und dann kann er kaum wenige Scbritte gehen, ohne die noch zarte Haut daran zu verletzen. Wenn er aus dem Lager geht, geniefst er zuerf Ameifen, oder die Wurzel der Calla, wovon fich der Leib öffnet; hernach das junge hervorfproffende Eipenlaub. Im zweyten labre verwachfen die meiften Baren die weifsen Ringe, die fie bis dahin um den Hals hatten; einige behalten fie immer, und die heifsen Ringelbären. Sie wachfen bis übar das 20 lahr. Ihr Alter aber ift unbekannt. Dafs fie unförmlich zur Welt kommen follten, if eine Sage, die keinen Grund hat. Das Fleifch wird von einigen Völkern gegeffen, foll aber einen füfslichen Gefchmack haben. Aus den Tatzen aber wird felbft auf den $T$ afeln der europatichen Grofsen ein Leckerbiffeñ gemacht. 
B. Fuscus. Dev branne Bär.

Zimmermann, geogr. Zool. II. p. 274. n, I66. a. cinc braune.

Schreber, Säugth. III. p. 504. 505. der bratuze Bïr. Lefke, Naturgefch. p. 147. der branne Bïr.

Boroweky! Thierreich, I. 2. p. 62. der broune Bür.

Erxleben, Mammalia, p. 159. 3. Fufcus, aut ferrugineo - fufcus.

Gramnanis, introd. in hift. nat. mamm. p.37.I.b. Gatterer, brev. Zool. I. p. 45. b. der Honigbär.

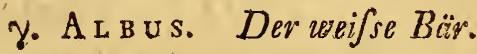

Zimmermann, geogr. Zoolog. I. p. 210. véifse Landbairen.

Klein, Quadrup. difp. p. 82. Minimus, Argentinus.

Klein , Claffif. der vierfüfs. Th. p. 249. Silberbiir.

Klein, natürl. Ordn. d. vierf. Th. p. 88. n. I. Silberbär.

Pennant, arct. Zoolog. II. p. 64. n. 28. c. ganz weifse Landbären; p. 65. d. grauliche Bäre, Silberbäre.

Bïfon, vierf. V. p. 94. weifse Landbiore.

Erxleben, Mammalia, p. 159. $\gamma$. Albus, vel niger, admixtis pilis albis, vulgo Germanis: der Silberbär.

Grammann, intr. in hift. nat. mamm. p. 37. I. c. der Silberbïr.

Rzacz. auct. hift. nat. Pol. p.322. Mininus argentinus. Pilis nigris et argentei coloris mixit.

Relation de la grande Tartarie. Amft. I\%37. in I2mo p. 8 . 
Gatterer, brev. Zoolog. I. p. 45. e. der Zeifel. bär, Silberbärs.

8. VAR I fGAT us, Der weifscheckigte Bior:

Zimmermann, geogr. Zoolog. I.- p. 210. Weifs; Scheckigte Bären.

Schreber, Säugthiere, III. p. 504. Schwarz unbt veifs. checkige Büren.

Pallas, Reife durch Rufs!l. II. p. 69r.

Pennant, arct. Zool. II. p. 64. b. mit Weiss go. fleckée Büre.

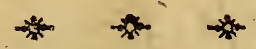

Mïller, NaturfyA. I. p. 273. n. I. der Landbiatr. Zimnmermann, geogr.Zool. I. p. 209. der Bär. Lefke, Naturgefch. p. I47. n. I. der Landbör. Borowylky, Thierreich, I. 2; p. 69. n. 2. der - gemeine Bär, Landbär.

Bhimenbach, Handb. der Naturgefch. p. 87. n. r. Urfis : (Arctos) fulco nigricans, collo brevi; - der Bär.

Bechftein, Naturgefch. Deutfchl. I. p. 333. n. I. der Landbör. p. 344. der Bör, gemeine Bür, Ringelbär, (wenn er.die jugendlichen weiffen Kinge behält) Zeidelbïr (wenn er noch klein iff.) Der grofse fchwarze und fanftere Bär heifst auch Grafebrïr, Ancifenbür; und der kleine rothe Pferdebïr und Honigbirr.

Funke, Naturgefchi. I. p. 155. der Bär. p. I56. der Landbär.

Ebert, Naturlehre, I. p. 377. der Bïr. Halle, vierfüfs. p. $54 \mathrm{I}$. der gemeine Bär. Meyer, Thiere, I. Tab. 26. der Bür. Abbildung gut. 
Gatterer, vom Nutzen und Schaden der Thiere, I. p. 32. n. 46. der Riar.

Krituitz, ökon. Encykl.III. p. 432. Bär.

Becímamn, Naturhiftorie, p. 30. n. 23. der Bär.

Pennant, arct. Zoolog. II. p. 64. n. 28. der branne Bär.

Neuer Schauplatz der Natur, I. p. 47 . Bär. Onomat. liift. nat. VII. p. 655' der' gennsine Bür. Klein, Claflif. d. vicrfüfs. Th. p. 249. einlündiffher polnifcher Bär.

Klein, natürl. Ordn. der vierfüfs. Th. p. 88. n. I. poinifcher Biiv:

Gesner, Thierbuch, p. 25. Bär. Fig. mittelm. Biffon, vierf. V. p. gr. der Biv; m. e. Fig. Bntfch, Thiere, I. p. 195. der Landbiry. Kraft, Ausrottong fclädl. Thiere, II. p. 338. Bür; p. 340. Steinbür, Schlachbör, Hautptbär, Fifchbär, Bienenbär, Inmenbär, Ameisbür, Obftbör.

Fifcher, Naturgefch. von Livland, p.55. 11. I4. Gemeiner Bïr.

Taube, Befchrẹibung von Slavonien, I. p. I6. der Bir.

Pontoppidan, Norwegen, II. p. 24. der Bür. Woif, Reife nach Zeilän, p. I22. der Bär.

Shatw, Reife, p. 157. Bären oder Dabh?

Bock, Naturgefch. von Preuffen, IV. p.49. 1. 15. der gemcine Bür.

Goeze, europäifche Fauna, I. p. 345. der Lancibär. Höpfner; Magazin für die Naturkunde Helvetiens, II. St. 9.

Knox, ceylan. Reifebefchreib. Lpz. 1689. 4. p. 4I. Büren.

Schöpf, Reife durch die nordamerikan. Staaten, II. p. 254. ein Bär? 
Leppechin, Tagebuch der ruff. Reife, I. p. I9. Büreni: (Bärenfalle.) II. p. 42. Bärenfalle und Selbftgefchofs, Tab. I.

Georgi, Rufs!. I. p. 21. 57. 83. Bären.

Gmelin, (d. ̈̈.) Reife, I. p. 309. II. p. 20\%' p. 470. Bär.

Palias, Reife durch Rufsland, I. p. I43. I98: II. p. 209. 207. 470.569 . III. p. I9. Selbftfchüffe mit $\mathrm{Pfeilen.}$

De Kerguelen Tremarec, Reife nach der Nordfee, p. 70. Bären.

Dı Halde, Befchreib. von China, I. p. 34. das Thier Ginn hinng, oder Bïrmen/ch.

Baldaens, Befchreib. von Malabar und Koromandel etc. Amft. 1672. fol. p. 42r. Büren.

S. G. Gmelin, Reife durch Rufsland III. p. 2.93. Bär.

Carver, Reif d.Nordamerika, p. 361. der Bür? Schlözer, Erdbefchr. von Nordamerika, p. 74.' Bären. p. 234. Bitren?

Neuhoff; Gefandfch. nach Chira, p. 348. Bären. Condamine, Voyages, p. 163. Ours?

Fifcher, mifcellan. phyf. med. I729. p. II8.

Condamine, Reifen; in Gött. Reifen, II. p. 302 ? Cook, Tagebuch neueft. Reife um die Welt. Lpz:

1776. p. 269.

Leffeps, Reife d. Kamtfchatka u. Sibirien, p. 46. Bärent.

Krafchenimnikow, Befchreib: von Kamtfchatka, p. I21. Riureir.

Leem, von deen Lappen in Finnmarken, p. 98. Bïreñ.

Olear. Reife nach Mofkau und Perfien, p. is8. (von e. befoffenen Bären.)

Köhler', Samml. neuer Reifen, I.p. p.32r. 
Dapper, Befchr. von China, p. I38.

Perrault, Charras und Dodart, Abhandl. zur Na. turgefch. I. p. 95. anatomifche Befchreibung zweyer Bären. Tab. X. der Bär. Tab. XI. die Zergliederung.

Neues fchwed. -Magazin (von Schreber) I. p. 297. Bören - und Woifsjagd.

Börner, Stadt - und Landivirthfchaft, II. p. IgI.

Schwed. Abhandlung. XXXIV. p. 349. Bären.

XXXV. p. 7.7. Bävenfang.

Merklein, Thiere, p. 5. Bör, Obfbür, Schlachtbär, Steinbär, Eauiptbïr etc.

Kriinitz, ökon. Encykl. III. p. 418. Bür.

Döbel, Iägerpraktik, I. p. 32. II. p. I25. III. p. 98. I4I. 160. Bär.

Heppe, wohlredender Iäger, p. $53^{\circ}$

Ludovici, Kaufmannslexicon, I. p. .1145.

Mannichfaltigkeiten, I. p. 643 . II. p. 435。 III. p. 172.

Nachricht von den Thieren des Königreichs

Siam; in den Bertin. Samml. VIII. p. $255^{\circ}$ Bären.

'Kurtis', Nachr. von dem Lande Labrador; in den Berlin. Sennt. IX. p. 373. Bären.

Naturgefchichte von Georgien und Carolina; im Hamb. Magasin, VI. p. 589. Bär e:

Naturgefch. von Iapan; in den Berlin. Samml. X. p. 147. Büren.

Roberg, diff. de Urfo. Upf. 1702. 4.

Riolinger, Entwurf einiger Thiere, Tab.39-44. Bär.

Ridinger, jagdbare Thiere. 'Tab. 3. Bär. Schreber, (d. ä.) Cameralfchriften, V. p. 281. Linné, Syft. Nat. Edit. II. p. 43. Urfus cauda abrupta. 
Linné, Syft. Nat. Edit. VI. p. 4. n. I. Urfus cauda abrupta.

Linné, Synt. Nat. Edit. X. I. p. 47. n. I. Urfus (Arctos) cauda abrupta.

Linné, S. N. Edit. XII. I. p. 69. n. I. Urfus (Arctos) cauda abrupta.

Linné, Fn. Suec. I. p. r. n. 2. Urfus cauda abrupta.

Linné, Fn. Suec. II. p. 7. 11. I9. Urfus (Arctos) cauda abrupta.

Graumann, introd. in hif. nat. mamm. p. 37. r. Bür, Bärin.

Mitller, Zoolog. dan. prodrom. p. 3. n. I6. Urfus (Arctos) cauda abrupta.

Kramer, Auftr. p. 3ro. Urfus cauda abrupta.

Charleton, exercitat. p. I4. Urfus.

Schwenkfeld, theriotroph. Silef. p. I3I. Urfus.

Wagner, hift. nat. Hẹlvet. p. 187. Urfus.

Rzaczinf ki, hift. nat. Pol. p. 225. Urfus.

Muf. Wormian. p. 318. Urfus.

Agricola, de anim. fubterr. p. 43. Urfus.

Forfter, philof. transact. LVII. p. 342. Urfus Arctos.

Oeuvres de Regnard, I. p. 168.

Le Gentil, voy. autour du monde. Amf. I728. III. p. 62.

Alpin. hift. nat. Aegypt. p. 233.

Ariftot. hilt anim. II. c. 5. n. 23. V. c. 2. n. r8. VI. c. 30. n. 384 - 392 . VIII. c. 9. n. I03' c. 22. n. I81. 182. IX. c. 7. n1. 90. 91. A grtos. Plin. hiftor, nat. VIII. c. 35.36 .58$. X. c. 63 : 72. 73. XI. c. $37 \cdot 40.45 \cdot 49.50$. Urfus. Aelian. hiftor. anim. II. c. 19. V. c. 49 . VI. c. 3. 9. Apxros.

Oppian. cyneget. III. 139. Aģxтos. 
5. Maritimus. Dev Eisbïr. (6)

M:ller, Naturfyftem, I. p. 279. weifse Bären.

Zimmermann, geogr. Zool. I. p. 210. der weifse Bär, der Polarländer. II. p. 275. n. I67. der Eisbär.

Le $k e$, Naturgefch. p. I48. n. 2. der Eisbär. Borow $k \mathrm{ky}$, Thierreich, I. 2. p. 73. n. 3. der Eisbör.

Blumerzbach, Handb. der Naturgefch. p. 88. n. 2. Urfus (Maritimus) albus, collo et roftro elongatis; der veifse Bär, Polcrbür.

Fuinhe, Naturgefch. I. p. 156. der Eisbör oder Potarbär.

Halle, vierf. p. 546. der nordliche weifse Bär.

Gatterer, vom Nutzen und Schaden der Thiere, I. p. 45. n. 47. Eisbür, Polarbär. Pennant, arct. Zool. II. p. 55. 12. 26. der Eisbïr.

Neuer

(6) Långe des Thiers oftmals über żwülf Fufs. Diefer Polarbär darf ja nicbt mit der weifsen Spielart des gemeinen Bären verwechfelt werden. Er hat eine ganz andere Stimine, fchlankere Glieder -und weifses, Jangzortiges, weiches. Haar. Ir grábt Leichen aus, und geht Menfchen an, ohne fich an überlegene Zahl oder Gewehr zu kehren. Er fchwimmt fertig, und kann es lange aushalten, auch auf kurze Zeit untertaichen. Tief ins Land geht er niemals. Fifche zieht er dem Fleifchévor, befonders wenn fie gefroren find. Er foll fogar feines gleichén freffen. Das Weibchen wirft feine lungen in Wohnungen oder Lägern, innerhalb grofser Eismafien, die bey ihrem wùnderbaren Zufammenthürmen hiezn bequeme lï̈hlen bilden, und liebt fie auferordentlich. Diefe Thiere werden fo fett, dafs man oft von einem einzigen hundert Pfund Fett beliömmt. Das. Fett wird ausgelaffen, und als Thran verbraucht. Das Eleifch if fehr weifs, aber grob, und wird von einigen gegeffen. Den Winter follen fiefich in tiefen, in Schnee gemachten Höhlen verbergen. Mit Schlägen auf aien Kopf find fie fchwer zu tüdten. 
Neuer Schauplatz der Natur, II. p. 479. Seebär, . Eisbär.

Onomat. hift. nat. VII. p. 660. dev weifse Bär. in Grönland.

Klein, Claflif. der vierfüfs. Th. p. 250. weifser Spitzbergifcher Bär.-

Klein, natürl. Ordn. d. vierf. Th. p. 88. n. 2. weifser Bär.

Bïffon, vierfüfs. V.p. I27. der weifse oder der Eisbirn; m. e. Fig.

phipps Reife nach dem Nordpol, p. 96. der weifJe Bär.

Pontoppidan, Hiff. von Norwegen, II. p. 25. der, weifse Bär.

Egede, Be[chreib. von Grönland, p. 83. Büre. Ellis Reife nach Hudfons-Meerbufen, p. 39. der weilse Biir.

Cranz, Hiftorie von Grönland, p. I07. weifse Bärent.

Pallas, Reife, Ausz. III. Anh. p. I. Urfus ma:rinus.

Fabricii faun. Groenland. p. 22. 11. I3. Urfus Maritimùs.

Hons und Ifrael de Bry, wahrhafte Contrafeyhung der See - Mappen, Infeln, Völkẹ etc. Frcf. am M. 1599. fol. Tab. $36 \cdot 57$. befonders Tab. 39. 40. 43. 46. 48. 53 .

Trampler, Befchreib. des grönländ. Wallfifchfangs; p. 63.

Allgemeines Magazin, II. p. 314: veifse Bären. Schriften der berlin. Gefellfch. naturforfch. Fr.'

VI. p. 20I. Eisbär.

Miiller, Samml. ruff. Gefch. III. p. 550 .

Berlin. Sammlung. IX. p. 562. n. 4. der weifse

Bär.

$$
\mathrm{X}_{3} \text { Goease, }
$$


Gooze, Natur, Menfchenl. u. Vorf. I. p. 558. der. weifse See - oder Polarbär.

Bat fch, Thiere, I. p. 197. der Eisbär.

Linné, Syft. Nat. Edit. X. I. p. 47 . Urfus (Ma. ritimus) albus maior, arcticus. (Mit dem vorhergehenden verbunden.)

Linné, Syft. Nat. Edit. XII. I. p. 7o. Urfus (Maritimus) albus maior areticus. (Mit dems vorhergehenden verbunden.)

Graumann, intr. in hift.' nat, mamm. p. 37. II. der weifse Bär.

Gatterer, brev. Zool. I. p. 45. n. 2. Urfus (Maritimus) albus, roftro colloque elongatis; der Eisbür, weifse Bür, Polarbär.

Miiller, prodrom. Zool. dan. p. 3. n. 16. b. Urfus albus.

Voy. de Compagn. des Ind. orient. Rouen I725. I. p. 124 .

6. AMERICANUs. Der Schwarze amerikanifche Bär. (7)

Schreber, Säugthiere, III. p. 512. Bäre der neuen Welt.

Pennant,

(7) Die Naturhiftoriker find noch nicht einig, ob diefor amerikanifche Bar wirklich der Gattung nach von dem gemeinen Land. bär verfchieden fey oder nicht. Ponnant hat eine eigene Gattung daraus gemacht; Pallas ift gleichfalls geneigt, ihn dafitr anzunehmen; Scbreber hält es noch fïr unausgemacht; und Zimmermann hält ihn für eine blofse Varietät von den vorher. gehenden. Die Sache wäre alfo noch einer genauern Unterfuchung würdig. Macht er wirklich eine eigene Gattung aus, fo müchten vielleicht die Citata, die ich bey, der erftern Gattung aus Schöpf, Carver, Scblözer und Condamine angefithre habe, auch hieher gehösen. Pennant rechnet fogar Stellers fobwarzen 


\section{Mammalia Frrae. Urfus.}

Pennant, arct. Zool, II. p. 60, n. 27, der fchwarze amerikanifche Bär.

Dapper, Amerika, p. 144. pechfchwarze Bären? Du Pratz, hilt, de la Louifiane, II. p. 77 . Ours. Natürl, Gefch, von der Louifiane; im Hamburg: Magazin, XIV. p. 6I2. der Bär.

Kalm Reife nach dem nördl. Amerika, II. p. 265. $\operatorname{der} B \ddot{z} r$.

Schöpf, Reife durch Nordamerika, II. p. '154 Büren?

2. MELes. Der: Dachs. $\left({ }^{8}\right)$

Miiller, Naturfyft. I. p. 28I. n. 2. der Daths. Zimmermann, geogr. Zool. I. p. 293. II. p. 280.' n. I7. Ier Dachs.

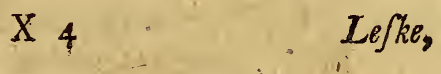

Bär mit dahin. Aus den Befchreibungen habe ich wenigftens nichts Entfchẹidendes nehrnen künnen. Das Fleifch wird in Nordamerika frifch und gerạuchert gegeffen; und das Fett gebrauchen die Wilden wie eine Salbe.

(8) Der Dachs bauet unter der Erde einen tiefen Keffel, zu welchem verfchiedene Röhren oder Gange führen. Er frifst wenig, und trägt zwar etwas, aber nur fehr wenig in feinen Bap. Die Ruhe macht ilnm aber dennuch überaus fett. Am fetteften ift er im Herbfte; dann trägt er Laub in feine Höhle, macht fich davon ein Bette, und ruht den ganzen Winter darauf, ohne auszugehen. Ununterbrochen fehlaffer er aber nicht. Sein Lauf ift nicht íchnell, und ẹn Menfeh, der Rark geht, kạnn ihn einholen, noch viel leichter die Hunde. Wenn er von diefen angefallen wird, fo legt er fich auf den Rücken, und vertheidigt fich nachdrücklich mit Gebifs und Klauen. Das Fleifch des Dachfes ift efsbar, und fein Fett in Apotheken, eîngeführt. Von dem Balge wird wenig Gebrauch gemacht, Merkwürdig ift an diefem Thięre, dafs die Rander der Pfannen, in welchen fich die Küpfe der untern Kinnlade bewegen, fo um diefe herum anfchliefsen, dafs die Kinnlade ohnmöglich vorwärs', fon- 
Lefke, Naturgefch. p. 149. n. 3. der Dachs. Borowe'ky, Thierreich, I. 2. p. 75. n. 4. der Dachs.

Blumenabach, Fiandb. der Naturgefch. p. \$6. n. 3. Meles (Trxus) cauda concolore abdomine nigro; der Dachs.

Bechftein, Naturgefch. Deutfchl. I. p. 349. der Dachs. p. 367. niederfächf. Grüruing, oder Grefing; Dáchsbär.

Funke, Naturgefch. I. p. 106. der Daihs. Ebert, Naturlehre, I. p. 372. der Dachs. Halle, vierf. p. 515. der Dachs.

Meyer, Thiere, II. Tab. 3r. der Dachs. Ab4, bild. gut.

Gatterer, vom Nutzen und Schaden der Thiere, I. p. 317. n. I83. der Dachs.

Bock, Naturgefchichte von Preuffen, IV. p. 56. n. I6. der Dachs.

Goeze, europäifche Fauna, I. p. 393. der Dachs, Batfch,- Thiere, I. p. 198. der gemeine Dachs. Georgi, Rufsl. Io' p. 208. Dachfe. Beckmanin, Naturhiftorie, p. 28. n. 17. der Dachs, Neuer Schauplatz der Natur, II. p. 283. Dachs. Onomatol. hiftor. natur. V. p. I39. Meles; der Dachs.

Gesner, Thierbuch, p. 63. Dachs; m. e. mittelm. Fig.

Klein, Quadrup. difp. p. 73. Coati cauda brevi; Taxus; Meles.

Klein, Claffif, d. vierf. Th. p. 220. Dachs, Halb. fuchs mit kurzon Schwanz.

Klein,

dern nur auf und nieder, und nach beyden Seiten bewegt werden kann, Eịn Bau, der grofse Stärke im Gebifs, aber langfames Kauen verurfacht, Die Eintheilung der Dachfe in Hundeund Scbweinedacb/e, if unwicintig. 
Klein, natürl. Ordn. d. vierf. Thiere, p. 77. n. 6 , Dachs.

Bïffon, vierf. IV. p. 123. der Dachs; Tachs. Kraft, Ausrottung graufam. Thiere, II. p. 406. Dachs.

Sander, ökon, Naturgefch. I. p. 260. der Dachs. Walther, ökonom. Naturgefch, Deutfchl. p. I2r. Dachs.

Handbuch der Naturgefch. I. 2. p, 148. der Dachs; Meles, Melis, Taxus,

Handbuch der deut fchen Thiergefchichte, $p, 5$. der Dachs.

Kriunitz, ökon. Encykl. VIIl. p. 623. Dachs.

Fif cher, Naturgefch. von Livland, p. 55, n. 15. Dachs.

Taube, Befchr. von Slavonien u. Syrmien, p. 2I. der Dachs.

Pontoppidan, Hift. von Norwegen, II. p. 54. der Dachs.

Bankroft, Naturgefch. von Guiana, p. 84. der Dachs.

Leem, Nachr, von den Lappen, p. 124, in not. Urfus Meles.

Gmelin, (d. j.) Reife, III. p. 432. Dachs. Pallas, Reife d. Rufsl. I. p. I29. III. p. Iro. Rytfchkow, orenb. Topogr. T. p. 226. Barfuk, Dache.

Kalm, Reife nach Amerika, II. p. 332. Dachs. Ridinger, Entwurf eiviger Thiere, V. Tab. 78.79. Tachs.

Döbel, Iägerprakt. I.p. 37. Dachs.

Geogr. hift. u. phyf. Mancherley, I. p. 7r.

Neue Mannichfaltigkeiten, I. p. 255-26I.

Beytr. zur Beförder. der Naturkunde, I. p. I23,

n. I, Tachs.

X 5 Loni= 
Loniceri, Kräıter -u. Thierbuch, p. 616. c, 48 . Onomat. foreftal. I. p. 44r.

Amdré und Bechftein, Spatziergänge, I. p. I37. 190. Dachs.

Erxleben, Mammalia, p. x6r. n. 3. Urfus (Meles) cauda concolare, corpore fupra cinereo, fubtus nigro, fafcia longitudinali per oculos auresque nigra.

Limné, Syft. Natur. Edit. II. p. 45. Meles unguibus anticis longiffimis.

Linné, Syn. Nat. Édit. X. I. p. 48. n. 3. Urfus (Meles) cauda concolore, corpore fupra cinereo, fubtus nigro, fafcia longitudinali per oculos auresque nigra.

Linné, Fn. Suec. I. p. 6. n. 15. Meles unguibus anticis longiflimis.

Graumann, introd. in hift. nat. mamm. p. 37. III. Dachs, Tachs.

Gatterer, brev. Zoolog. I. p. I33. n. I3. Viverra (Meles) cauda concolore, corpore fupra cinereo, fubtus nigro, fafcia longitudinali per oculos auresque nigra.

Severin, zool. Hungar. p. Ior. n. 3. Urfus Mea les, Taxus,

Miuller, zool. dan. prodr. p. 3. n. 17. Urfus (Me. les) cauda concolore, corpore fupra cinereo, fubtus nigro, fafcia longitudinali per oculos auresque nigra.

Kramer, Auftr. p. 313: Meles unguibus anticis longiffimis.

Charleton, exercieat. p. I8. Taxus. Schreenkfeld, theriotroph. Silef. p. 122. Taxus. Wagner, hift nat. Helvet. p. I86. Taxus. Melis. Rzacz, hiftor, natur. Polon. p. 233. Auct. p. 327 . Meles. 
Rzacz. Auct. hift. nat. Polon. p. 315. Melis, Sibbald, Scot, illuftr. P. II. T. 2. p. II. Taxus Suillus.

Dufouilloux, venerie, p. 72. 73. Teffons cher nins et porchins.

Pennant, britt. Zool. p. 30. the Badger, Plin. hif, nat. VIII.' c. 38. Melis,

B. А с в а. Der weifse Drichs,

Zimniermann, geogr. Zoolog. I. pa" 294. der ganz weifse Dachs! II. p. 281. n. 172. der weifse nordanerikanifche Dachs.

Schrever, Säugthiere, III, p. 52I. der vebi/se Dachs.

Biiffon, vierf. IV. p. I35. ein weifser Dachs.

Erxleben, Mammalia, p. 165. Varietas alba nove eboracenfis.

\%. M a c u ata. Der gefleckte Dachs.

Zinimermann, geogr. Zoolog. II. p. 28r. der Dachs mit gelbröthlichen wnd. kaftanienbrausnen. Flecken.

Schréber, Säugthiere, III. p. 520. ein weifsev Dachs mit gelbröthlichen, und dunkelkaftanienbraunen Flecken.

7. LA B R d OR I us, Dev labradorifche Dachs. (9) Pennant, arct. Zool. II. p. 74. n. 30. der nord. amerikanifche Dachs.

Bïffon:

(9) Bey diefer Gattung findet fich in Anfeluung der Synonymen manche Verwechfelung. In der aretifchen Zoologie ift das Citatum aus Briffon, 'welches nach unferm Syftem zu dér weifsen Varietàt des gemeinen Dachfes gehüren foll, wit angeführt. 
Biiffon, vierf. XV: p. 193. der nordamevikanifche Dachs.

Batfch, Thiere, I. p. 198. der labradorifche Dachs. Boddciert, Elench. anim. p. 80. Meles, americanus, linea alba nigraque a nafo trans frontem ad dorlum, abdomine cinereo.

Erxleber, Mammal. p. I64. unten.

3. Lỏ tor. Der Schupp. $\left({ }^{10}\right)$

Miiller, Naturfyftem, I. p. 284 , n. 3. der Coati. Tab. 15, fig. I.

Zimmer.

In Biiffons vierf. XV. $\mathrm{P}$. ros. ift auf Zimmermanns geogr. Zonlogie hingewiefen, und gleichwohl hat Zimmermann dafelbft ebenfalls das Brifonfche Citatum. Ift alfo vielleicht, wie auch Erxleben p. 164. vermuthet, diefer labradorifche Dachs nur eine Varistät vom gemeinen? Die Benennung Carcajou kommt ihm wenigfters nicht zu.

(10) Länge des. Thiers beynahe zwey Fufs, des Schwanzes ein Fufs. Seine Nahrung beftehe auch in Mays, wenn die Achren noch weich find, Zuckerrohr, allerley Baumfrüchten, befonders Aepfeln, Kaftanien, wilden Weintrauben, Milch, Măufen, Maulwürfen, Spinnen, Käfern, Regenwüusern u. dgl. m. und er geht feiner Nahrung in der Nacht nach. Am Tage kommt er nur bey trübem Wetter zum Vorfchein. Bey fchlin.mer Witterung, Schnee und Sturmwetter liegt er wochenlang in feinem Schlupfwinkel. In ohngefahr drittehalb Iahren erreicht er feine völlige Gröfse. Sein Fleifch ift efsbar; aus den Bälgen werden Müffe gemacht, und die Schwänze um den Hals getragen. In Nordamerika werden diefe Thiere häufig in den Häufern gehalten, und mit Brodt, Fleifchwerk, Knochen, Brey, Suppen u. dgl. unterhalien. Vögeln, die fie ertappen können, beiffen fie den Kopf entzwey, faugen das Blut rein aus, und laffen das übrige liegen. Sie faufen wenig, und ziehen das Waffer in den wagrecht hinein gehaltenen Mund, wie die Eichhörner. Jhres Auswurfs entledigen fie fich an einem entfernten Orte. Am 22 May 1790 , warẹn hier in Quedlinburg 
Zimmermann, geogr. Zool. II. p. 278. n. 170. der Raccoon; der Schup.

Lejke, Naturgefchichte, p. 149. n. 4 der Schup. Borow fky, Thierreich, I. 2. p. 77. n. 6. der schupp, Koiti.

Blunnenbach, Handb. d. N. G. p. 85. n. 4. Meles (Lotor) cauda annulata, fafcia per oculos transverfali nigra; der Rackun, Coati.

Halle, vierfüfs. p. 596. der ameri,anifche Bär. p. 506. das Koati.

Meyer, Thiere, III. Tab. 18. die wilde amerikanifche Katze.

Gatterer, vom Nutzen und Schaden der Thiere, I. p. 318. n. 184. der Raton, Sjupp.

Pennant, arct. Zoolog. II. p. 72. n. 29. der Ra. coon, der Schupp.

Neuer Schaupl. der Natur, II. p. 168. der Coati.

Onomat. hif. nat. III. p. i. der Coati, das Coatithier.

Handbuch der Naturgefch. I. 2. p. 15I. der Raton. Klein, Quadrup. difp.p. 72. Coati hrafilienfium. Klein, Claflif. d. vierfüls. Th. p. 214. brafilianiScher Halbfuchs.

Klein, natürl. Ordn. d. vierfüfs. Th. p. 76. n. I. brafilianifcher Halbfuchs.

Biiffon, vierf. V. p. I82. der WVafchbior, Abjpiiler, Raton; m. e. Fig.

Batfch, 'Thiere, I. p: 199. der Scloupp.

Carver, Reife durch Nordamerika, p. 372. der Coati.

Schlözer, Erdbefchreib. von America, p. $235 . d e r$ Racoon. (Amerikanifche Dachs.),

Siȟöpf,

Männclien und Weibchen zu fehen, dic fo zahim waren, dafs die Kinder mit ihnen fpielten, aber wild wurden, fobald fie einen Hund in der Nihe erblickten. 
Schöpf, Reife durch die nordamerikan. Staaten, II. p. 456. cier Racoon.

Kalm, Reile nach Amerika, II. p. 246. der Bär mit dem langen Schwanse; Rakkoon.

Kaln, Reife, II. p. 35I. der Raccoon, den wiv Sjupp nenuen.

Kalm, Reife, III. p. 3I. der Bïr, dzr Raccoon oder Efpan gencannt wird.

Goeze, Natur, Menfchenl. u. Vorfeh. V. p. ror. der kleine amerikanifche $W$ a fichbür.

Schwed. Abhandl. IX. p. 3oo. ein amerikanifches Thier; Tab. 9.

Erxleben, Mammal. p. I65. n. 4. Urfus (Lotor) cauda annulata, fafcia per oculos transverfali nigra.

Linné, Syft. Natur. Edit. II. p. 43. Urfus cauda elongata.

Limné, Syft. Nat. Ed. VI. p. 4. n. 2. Urfus cauda elongata.

Linné, Syft. Nat. Edit. X. I. p. 48. n. 4. Urfus (Lotor) cauda annulata, fafcia per oculos trans. verfali.

Graumann, intr. in hiftor. nat. manm. p. 37. IV. der amerikanifche Bür; der Coati.

Gatterer, brev. Zool. I. p. 134. n. 14. Viverra (Lotor) cauda annulata, fafcia per oculos transverfali nigra; das Coati, dev Raton, Raccon, Sjupp.

Charleton, exercitat. p. I5. Vulpes americana, Mapach dicta.

Marcgrav. hilt. nat. Brafil. p. 228. Coati. Ab. bild. mittelm.

Thevet, cosmogr. univerf. II. fol. 937. a. Coaty. Laet , nov. orb. - defcript. Ind. occident. p. 553. Cuati. 


\section{Luscus. Die Wolverene. (")}

IMïller, Naturfyn. I. p. 285. n. 4. der Woifsbïr.

Zimmermani, geogr. Zoolog. II. p. 276. n. 169. cier Quickhatch; die Wolverene.

Schreber, Säugth. III. p. 530. die Wolverene.

Boroweky, Thierreich, I. 2. p. 78. n. 7. der Wolfsbärs, die Wolveréne.

Halle, vierf. p. 5I8. der gröfste amerikanifche Hallifuchs.

Gatterer, vom Nutzen und Schaden der Thiere, I. p. 320. n. 185 . die Wolverene.

Onomatol. hift. nat. VII. p. 662. der Wolfsbür. Klein, Quadruped. difp. p. 74. Coati Urfulo affi. nis, americanus.

Klein,

(1) In Anfehung diefes Thiers find die Meynunizen der Naturhiftóriker in mehr als einer Hinficht getheilt, Büffon und Pennant halten es mit dem folgenden für einerley. Erxtebex hat zwey verfchiedene Gattungen aus beyden, gemacht. Zimmermann nahm anfanglich mit Linné und Exxtebin die Wolverene für verfchieden von dem Vielfrafs an; ich finde aber, dâfs er in der Folge feine Meynang geàndert, und beyde Thiere nur für eine Art angenommen hat, and Schreber läfst die Sáche ganz unentfchieden. Ohne einem oder dem anders diefer berühmten Mănner zu nahe zu treten, glaube ich doch, dafs es keinem Zweifel mehr unterworfen fey, dafs nicht die Wolverene ein von dem Vielfrafs ganz verfchiedenes Thier feyn folite. Ich habe zwar den letztern nicht gefehen; aber die Wolverene habe ich am it May 1788. hier lebendig gefehen. Wegen ibrer Wildheit hatte man ihr dic Augen ausgeftochen, und einen Ring in die Nafe gelegt, den fie fchon einige Mal ausgeriflen hatte. Die Abbildung, welche Znufchner davon geliefert bat, ift fehs treffend, und wenn man diefe mit Scbrebers Zeichnungen vom Vielfrafs vergleicht, wird man ohnmöglich beyde Thiere für einerley halren künnen. Darin hat pennan* recht, dafs er dem Thiere vorn und hinten fünf Zehen beylegt. Ob Cranzens und Egedens Am arok die Wolverene ader der Vielfrafs feyn mag? 
Klein, Claflif. der vierfüfs. Th. p. 78. n. 9. anierikanifcher Haibfuchs, aus dem Gefchlecht der Wilfe.

Klein, natürl. Ordnung d. vierf. Th. p. 78. n. 9. gröfster Halbfuchs, Quilik-hatch, Wolverene.' Biiffon, vierf. XV. p. I77. der Quickhatch oder Wolverene; m. e. Fig.

Anfon, Reife, I. p. 58. Quichhatch.

Zaufchner, Beftimmung der Bärenart Saribur. Prag 1778. 4. p. 8. Urfus (Saribur) cauda abbreviata, villofa, auriculis mediocribus, erectis, ovato-acuatis, pilofis, pedibus pertadaAylis. Tab. 2.

Penncint, arct. Zoolog. II. p. 69. n. 28. der Viel. frafs oder die Wolverene.

Cranz, Hilt. von Grönland, p. Ios. Amarok?

Egede, Befchr. von Grönland, p. 84. Amarok?

Cook, dritte Reife, III. p. 39r. Wolverene oder

Vielfrafs.

Catesby, Carolin. Append.p. XXX. Urfulo affinis americana; Quickhatch.

Erxieben, Mammal.p.I67.n: 5. Urfus (Lufcus) cauda elongata, corpore ferrugineo; rofro fufco, fronte plagaque laterali corporis longitudinali pallida.

Linné, Syft. Natur. Edit. X. I. p. 47. n. 2. Urfus (Lufcus̄) cauda elongata, corpore ferrugineo, roftro fufco, fronte fafciaque laterali longi. tudinali albida.

Graumann, intr. in hiffor. nat. mamm. p. 38. V. dev Tholfsuirr.

Gatierer: brev. Zoolog. I. p. 134. n. 15. Viverra (Lufca) cauda elongata, corpore ferrugineo, roftro fufco, fronte plagaque laterali corporis longitudinali pallida; der Wolfsbour. 
Fabric. Fn. groenl. p. 24. n. 14. Urfus (Lufcus) cauda elongata, corpore ferrugineo, roftro fufco, fronte plagaque laterali corporis pallidiore?

8. Guco. Der Vielfrass. (2)

Miiller, Naturfyft. I. p. 265. n. 8. der Vielfrafs; Tab. 14. fig. 4. Houtt.

Zimmer:

(2) It fo ftark, dafs er felbnt Rennthiere tiberwaltigon kann, denen er aber, da fie ihm zu fchnell find, um fie im Laufe yzu fangen, im Sommer auf den Bäumen auflauert, im Winter aber fie im Schlafe befchleicht, oder wenn fie das Moos unter dem tiefen Schnee hervorfuchen, und den Kopf unter dem Schnee haben, da er ihnen denn auf den Rücken fpringt und fie tüdtet. Vügel fpürt er von weitrem aus, und befchleicht fie oft. Die Schneehühner (Tetrao Lagopus) weifs er im Winter unter dem Schnee zu fangen. W Was er nicht verzehren kann, begräbt ex, oder fchleppt es in cine Felfenkluft. Iung gefangen und zahm gemacht, läfst er fich mit allerley rohem Fleifchwerk, Fifchen, Knochen, auch gekochten, nur nicht gern mir vegetabilifchen Speifen unterhalten. Das Waffer leckt er wie ein Hund. Sein Auswurf ift mehrentheils dünne und übelriechend. An fich hat der Vielfrals aber keinen tublen Geruch, und halt fich reinlich. Er fchlafr fant mehr bey Tage als in der Nacht, und legt fich dazu kugelrund nieder. Er itt immer in Bewegung, klettert, kratzt, grabt, walzt fich, und läuft mit gewohnten Leuten, wie ein Hund. Er geht auch ins Waffer. Bey zunehmendem Alter gewinit die Liebe zur Freyheit die Oberhand, wenn man ihn nicht an eine Kette legt. Húger mache ihs unbandig. Schlägt man ihn mit dem Stocke, fo knurrt er wie ein Hund, hauet mir den Pfoten zu wie eine Katze, und fafst den Stock zwifchen die Vorderbeine. Hiande fallt er an, ob fie ihn gleich an Grö́se đubertreffen. Im Kampfe mit ihnen bedient er fich des Gebifes und der Klauen zugleich, wird er aber in die Enge getrieben, fo giebt er einen Stra! von Unrath von fich, deffen 'übler Geruch fie verícheuchr. Eben diefs thut er auch, wenn man ihn allzubüfe macht, - Klein 
Zinnnermann, geogr. Zool. I. p.280. II. p.276. n. I68. ader Vielfirass.

Lefke, Naturgefch. p. 150. n.5. der Vielfrafs. Borow fky, Thierreich, I. 2. p. 67. n. I. der Vielfirafs, Rofomak:

Bhnasenbach, Handb. d. Naturgefch. p. 85. n. I. Meles (Gulo) corpore rufo - fufco, medio "dorfi nigro; der. Vielfrafs, Rofonlak.

Beihf in, Naturgefch. Deutchl. I. p. 344. n. 2 . der Vielfrass. p. 349. Rofonak und Wolverene. (Vielfrassmarder.)

Funke, Naturgefch. I. p, r55. der Vielfrass.

Ebert, Naturlehre, I. p. 378. dev Vielfra/s.

Halle, vierf. p. 548. der Vielfra/s.

Gatterer, vom Nutzen und Schaden der Thiere,

I. p. 307 . n. 170. der Vielfrafs.

Beckmann, Naturhiltorie, p. 31. n. 24. der Vielfrafs.

Neuer Schauplatź der Natur, IX. p. 264. der Vielfirass.

Onomat. hift. nat. V. p. 383. Muftela Gulo; der Vielfr $\sim a f s$.

Gesner, Thierbuch," p. 380. ein andrer Vielfrafs. Klein, Quadiuped. difp. p. ab. 5. Gulo; Vielfrals. (Verwechint "Thier mit der Hyäne.

Klein, Clatfif. d. vierfüls. Thus: Tielfrajs.

Klein, natürl. Ordn. der vierfüls. ih. rev Vielfrafs.

Bïffon, vierfüfs. XV. p. 120. der Vielfi. in. 2 Figg.

Fif cher,

und Briffort haben den Vielfrafs mit der Hyone verwechfelt. Dafs ihn mehrere mit der Wolverene für einerley halten, habe ich in der vorhergehenden Note bemerkt. 
Fifcher, Naturgefch. von Livland, p. 53. n. 9. wilder Vielfrass.

Pontoppidan, Hilt. von Norwegen, II. p. 44; Vielfrafs, Ierv, Gievv; nord. Ierven oder Erven; in Drontheim Kola.

Steller, Befchr, yon Kamtfchatka, p. I18. Rofo: macken, oder Vielfrä/se, Timuch genannt.

I. G. Gmelin, Reife durch Sibirien, III. p. 492. Vielfrafs.

Leppechin, Tageb. der ruffifch. Reife; III. p. 58: Muftela Gulo; $V$ ielfrass.

Pallas, Reife d. Rufsl. II. p. 209. 377. 571. 67 r III. p. 10. 87. Rofomak.

Krafcheninnikow, Befchreib, von Kamtfchatka, p. 120. Vielfrals.

Rytfchkow, orenb. Topogr. I. p. 237. Rofomach, Vielfrafs.

$R_{y t}$ fchk. orenb. Topogr. in Biifchings Magazin VII. p. 46 .

Miiller, Samml. ruff. Gefch. III. p. 549.'Viel. frafse.

Siheffer, Lappland, p. 383. Vielfrafs.

Pallas, Reife, Auszing, III. p. 285. V ielfrass.

Genberg, Bericht von einem Tärf oder $V$ ielfra/s, der jung gefangen, und bis ins andere Iahr gefuttert ward; in den Schwed. Abh. XXXV.

p. 20 r.

Lindreall, Befchreib. des Thiers lärf oder Viel. frafs; in den Schwed. Alh. XXXV. p. 208. Tab. 7. 8 .

Leem, von dẹ Lappen in Finnmarken, p. I06. der Vielfrass.

Högftröm, Schwed. Lappland, p. 22.

Berch, Schwed. Magazin, I. p. 242.

Berlin. Samml. II. p. 193. dev Vielfres/s. 
Neue gefellfchaftl. Erzähl. III. p. 27 . Vielfrafs. Goeze, europäilche Fauna, I. p.371. der Vielfrafs. Batfch, Thiere, I. p. 197. der Vielfrafs.

Erxleben, Mammalia, p. 477. n. 15. Muftela (Gulo) corpore rufn-fufco, medio dorfi nigro.

Linné, Syft. Nat. Ed.'II. p. 44. Gulo.

Linné, Syft. Nat. Edit. VI. p. 5. n. I. Mufela rufo - fufca, medio dorfi nigro.

Linné, Syft. Nat. Edit. X. I. p. 45. n. 3. Muftela (Gulo) plantis fiffis, corpore rufo-fufco, medio dorfi nigro.

Linnié, Fn. Suec. I. p. 2. n. 6. Muftela rufo-fufca, medio dorfi nigro.

Grannzann, intr. in hift. nat. mamm. p. 7r. XV. Muftela Gulo. Vielfrafs.

Gatterer, brev. Zoolog. I. p. 129. n. 15. Muftela (Gulo) corpore rufo-fufco, medio dorfi nigro; der Vielfrafs.

Severin, zool. Hung. p. Ior. Urfus Gulo.

Muïller, dan. zool. prodrom. p. 3. n. Ir. Muftela (Gulo) pedibus fiffis, corpore rufo-fufco, medio dorfi nigro.

Fabric. faun. grönland. p. 2r. n. I2. Muftela (Guio) pedibus fiffis, corpore rufo-fufco, medio dorfi nigro.

Kramer, Auffr. p. 3ir. Muftela rufo - fufca, medio dorfi nigro.

Charleton, exercitat. p. 15. Gulo. Olaus Magn. de gent. feptentr. p. 605. Gullo. Megabeni hiforia Gulonis. Vien. Aufr. 1688. Severin, tentam. zoolog. p. ror. Urfus Gulo. Herrmiann, affinit. animal. p. 69. Urfus Gulo. Relation de la grande Tartarie. Amit. I737. pag. 8 . 
Veränderungen gegen die XIIte Edition, und Ver: mehruing der Gattungen diefes Gefihlechts.

Bey der erfern Gattung find vier, bey der zvieyten zwoy Varietäten aus einander gefetzt. Aus dem Gefchlecht der Muftelen ift die fünfte, Gulo hieher verfetzt, aufferdem $U$. Maritimus, America. sus, Labradorius hinzugekommen, alfo das Ge. fchlecht überhaupt mit vier Gattungen vermehrt.

17. GESCHLECHT. DI DEL'PH IS. Das Bentelthier; Philander.

IMiiller, Naturfyf. I. p. 286. Gen. XVII. Zimmerminnn, geogr. Zool. II. p. 220. Gen. XVII: Schreber, Säugthiere, III. p. 532. Gen. XIX. Lefke, Naturgefch. p. I50. Gen. XVIII. Boro:e $k y$, Thierreich, I. 2. p. 79. Gen. XVII. Bhumenbach, Handb, dẹr Naturgefchichte, p. 77 . Gen. XVIII.

Funke, Naturgefch. I. p. 180,

Martini, Naturlex. VII. p. 214.

Batfch, Thiere, I. p. 219. Gen. XXXII.

Erxleben, Mammalia, p. 73. Gen. VIII.

Graumanı, introd, in hiftor. nat. mamm. p. 25. Gen. VIII.

Penncme, quadr. p. 204. Gen. XXII.

Briffon, quadrup. p. 207. Gen. XLII. Philander.

1. Marsu Pialis. Das Marfupial.(3)

Miiller, Naturfyftem, I. p. 288, n. I. die Beus. telratze.

\section{$\mathbf{Y}_{3}$}

Zimmer-

(3) Biffon, Erxleben, Borous $/ k y$, und mehrere Naturhiftoriker von Gewicht, halten diefs Thiez mit dem Opoljum für einer- 
Zimmevimann, geogr. Zoolog. II. p. 221. n. I25. der Iiarfupial.

Lefke, Naturgefch. p. I5i. n. I. die Rentelratte. Borowsky, Thierreich, I. 2. p. 79. n. I. dev gröfste Philander; dis Bewalratze.,

Bhimenbach, Handbuch d. Naturgefch. p. 77. n. 2. Didelphis (Marfupialis) mammis intra fac. cum abdominalem, die Beutelvatze; der Opoffum; Philander?

Funke, Naturgefchichte, I. p. I81. Marfupial oder Beuteliatte.

Halle, vierf. p. 44т. der giöfste Philander?

Meyer, Thiere, III, tab, 8. die grösste orientali. Jche Boutelratze?

Gatterer, vom Nutzen und Schaden der Thiere, I. p. I6. n, 21. Didelphis Marfupialis; die Beutelratze.

Neuer Schaupl, d. Natur, VI. p. 579. Bentelratze. Onomat. hift. nat. V. p. 708. die Benteliatze; der gröfsie oftindifche Philander:

Klein, Quadr; difp. p. 59. Mus marfupialis ma. ximus,

Klcin, Claffif, d. vierf. Th, p. I\$o. gröfste oriens talifche Beutelratze.

Kleitss

ley; einige vermengen es auch mit dem Faras; daher die grofse Verwechfelung der Synonymen uild Befchreibungen, welche die genauere Auseinanderfetzung der Gattungen ungemein erfchwert, Hiezu kommt die von einer und eben derfelben Gattung hin und wieder ganz verfchieden angegebene Reftimmung der Grörse, einzelner 'Theile fowohl, als des Ganzen; ferner, des Vaterlandes, und mancher Nebenumftande. Ich habe daher die Synonymen fo gut geoordnet, als ich, den Umfiänden nach, gekonnt habe. Bey Schreber und Zimmermann findet man die unterfcheidenden Merkmale der Gattungen näher beftimmit und aus cinander gefetzt. Das Morfựigl bewohnt Südamerika, und siche Oftindien. 
Klein, natürl.' Ordn, d. vierfüfs. Thiere, p. 62. gröfsefte Bentelratze aus Oftindien. Biiffon, vierf, VIII. p. 97. das Marfupial. Batfch, Thiere, p. 221 das Marfupial. IIItrtini Naturlex, VII. p. 238. Marjupial. Goeze, Natur, Menfchenleben und Vorfehung, I. p. 554. die Belutelratte.

Ecrmin. Befchr. v. Surinam, II. p. 100. der Philander; $\Lambda$ wari; Mus Marfupialis; Didelphis Marfupialis; die Beutelratze.

Dapper, Amerika, p. 58. Abbild. u. Befchreib. abfurd.

Beckmann, phyf. ökon. Bibl. XIV. p. 28. Didelphis Marfupialis? (Aus Iacquin, den ich nicht zur Hand habe.)

Lichtenberg und Voigt, Magazin für das Neue. fte etc. VI. 4. p. 107. Didelphis Marfupialis. Beutelratte. (Die Iacquinfche Erzählung.)

Erxleben, Mamnalia, p. 73. n. r. Didelphis (Marfupialis) mammis intra funiculum umbilicalem. (Mit dem Opoffum verbunden.)

Linné, Syft, Nat. Ed. II. p. 44. Didelphis. Linné, S. Nat. Edit, VI. p. Io. n. r. Jidelphis mammis intra abdomen.

Limne', Syft. Natur, Edit, X..I. p. 54. n. r. Didelphis (Marfupialis) mammis 8. intra abdomen.

Linné, amoen, acad, I. p. 559. Didelphis mam. mis intra abdomen,

Gratmans, intr, in hift. nat, mamm. p. 25 . I. Beutelratze; Waldratzi; (mit dem Opolo fum verbundeì.)

Gatterer, brev, Zoolog. I. p. 27. n. I. Didelphis (Marfupialis) mammis intra faccum ab, dominalem.

$$
\mathbf{Y}_{4} \quad 2, \mathrm{PHI}
$$


3. Philander. Der Faras. (4)

Milller, Naturfyftem, I, p. 291. n. 2. der Philander. Tab. I8. fig. I.

Zimmermann, geogr. Zoolog. II. p. 224. n. 127. der Faras.

Borowfky, Thierreich, I. 2. p. 80. n.'2. der Faras, der Philander.

Mleyer, 'Thiere, III, Tab. 6. die Bofchratte ; fig. Seb.

Gatterer, vom Nutzen und Schaden der Thiere, I. p. 17 . 11. 22.

Neuer Schaupl. der Natur, VI. p. 580 . Philander. Onomatol. hiff. nat. VI. p. 447. der Philander. Klein, Quadr. difp. p. 59. Mus Tlaquatzin, feu Tai-Ibi Brafil.

Klein, Claffif. der vierfüls. Thiere, p. $x 78$. n. 15 . Bofchratte.

Klein, , natiirl. Ordn. d. vierf. Th. p. 62. n1. I2. Busctiratte.

Biilfon, vierf. VIII. p. 98. n. 3. der Baras. Batfch, Thiere, I. p. 222. der Faras. Martini, Naturlex. VII. p. 222. Faras. Lichtenberg und Doigt, Magazin für das Neuefle etc. V. 2. p. I3. Philander?

Erxle.

(4) Wenn unter dem Pbilander im Voigtfchen Magazin wirklich diefe Gattung zu verftehen ift, welches fich hin und wieder aus der Befchreibung und der angegebenen Anzahl der Zitzen fchliefsen läst, fo pafst doch die Gröfse durchaus nicht. Diefe wird hier auf neunzehn.Zoll gefetzt; nach Sclureber, Zimmermann und Erxleben aber beträgt die Grüfse des Faras nur neun.Zoll. - $\mathrm{Ob}$ der Faras oder. Ravale des Gumilla hieher gehöre, fcheint ebenfalls noch nicht ganz ausgemacht zu feyn; wenigftens if Zimmermainn der Meynung, dafs die Befchreibung des Gumilla eben fo wohl auf den. Opoffum gedeutet werden müge. Die Namen Tlaquatzin und Tai-ibi kommen eigentlich nicht diefem Thiere, fondern dem Opoffum zu. 
Erxeleben, Mammal. p. 78. n. 2. Didelphis (Philander) auriculis pendulis, mammis qua. ternis.

Iimné, Syf. Nat. Edit. X. I. p. 54. n. 10. Didel. phis (Philander) cauda bafi pilofa, auriculis pendulis, mammis quaternis.

Graumann, intr. in hift, nat. mamm. p. 25. II. D. Philander.

Gatterer, brev.Zool.I. p.27.n.2. Didelphis (Phi- lander) auriculis pendulis, mammis quaternis:

3. Opossum. Der OpolJum (5)

Miiller, Naturfyf. I. p. 292. n. I. der Walct ratze.

\section{Y 5 Zimmer.}

(5) Halt fich unter dem Zubbe der Bäume verfeckt, und fucht allerley Vögel zu ertappen, denen er das Blut ausfaugt, ohne allemal das Fleifch zu freffen. Das Thier hat ein hartes Leben, wie eine Kasze. Sein Laut in ein Grunzen, das man niche, weit hört. Das trächtige Weibchen macht fein Neft vón dürrem Grafe in dichtes Geftraich an der Wurzel eines Baums. Die lungen, werden blind gehoren, und von der Mutrer einige Wochen, bis fie fehend werden, und Haare bekommen, in der grofsen Tafche am Bauche, die durch befondere Mufkeln und dünne Knochen gefchloffen, und geüffnet werden kana, und in deren Boden die Zitzen liegen, aufbewahrt, und da von der Muttermilch genahret. Nach Verlauf diefer Zeit läfst fie fie heraus an die Sozne, nimmt fie aber bey dem geringften Geraufch oder Verdacht einer Gefahr wieder darin auf, und uffret den Beutel nicht, wenn man fie auch lebendig uber das Feuer hängt. Zahme Weibchen aber lafen fich felbigeu aufmachen. Das Thier giebt einen unangenehmen Geruch von fich, welches einer fchmierigen Feuchtigkeit zuzufchreiben if, die in zwey Drïfen am After, und in dem Beutel des Weibchens abgefondert wird. Doch wird das Fleifch von einigen gegeffen. Das rauhe und fchmuzig anzufehende Haar fpinnen die Wildinnen in Louifiana, und weben daraus Strumpfbänder und Gürtel. 
Zimmerinann, geogr. Zool. II. p. 222. n. 126. der Opoffum.

Lefke, Naturgefch. p, $x_{52}$, n. 2. der Opo/fum.

Borowfky, Thierreich, I. 2. p. 80. Opo/fum. (Mit dem Marfupial verbunden.)

Bechftein, Naturgefch. Deutlchl. I. p. I39. der Opofjim.

Ebert, Naturlehre, I. p. 35r. das Beutelthier, Sonft auch Beutclratze, Sarige und Philander gew nannt. Tab. I7.

Halle, vierf. p. 436 . die amerikanifche Beutelratte. Tab. 25 .

IMeyer, Thiere, III. Tab. 4. die Beutelratze; das Männtein; fig. Seb.

Becknann, Naturhiforie, p. 24. n. 4. die Beutel. ratze.

Pennant, aret. Zool, II. p. 75. n. 3r. der Opo/fums. Neuer Schaupl. d, Natur, VI. p. 580. Opoffum; die Waldrataie.

Onomat. hift. nat. II. p. 647: das Beutelthier.

Gesner, Thierbuch, p. 2I. Sinivulpa, Fichs. aff. Abbild. äufserft fchlecht.

Klein, Quadrup. difp. p. 59. Mus marfupialis; Sylveftris; Brafilienfis.

Klein, Claffif. der vierfüfs. Th. p. 176. Bentel. ratze; Philander.

Klein, natürl. Ordn, d. vierf, Th. p. 62. n." II. Bentelratze.

Biiffon, vierfits. VIII. p: 24. der Sarige, oder Opofium; m. 2 Figg.

Batfih, Thiere, I. p. 222. das Opoffium. Ilartini, Naturlexicon, VII. p. 239. Opolfum; in. 2 Figg.

Handbuch d. Naturgefch. I. 2. p. 3II. der Sarige sder. Opoßjum.

Samm. 
Sàmml. zur Phyf. und Naturgefch. II. p. 609. der Sarigue oder Opoffum; in Guiana Pian, oder Piunzt.

Schlözer, Erdbefchreibung von Amerika. p. 236. das Opolfum. (Beutelthier.)?

Schöpf, Reife durch die nordamerikan. Staaten, I. p. 15. Opolfim oder Beutelthier.

Liclitenberg und Voigt . Magázin für das Neuefte etc. V: 2. p: 29 . Genauere Umflände von der merkw ürdigen Fortpflanzungsweife der weiblichen Beutelvatíe.

Goeze, Natur, Menfchenl, u, Vorfeh, V. p. 334. die Beuteliatze.

$D_{\mathcal{E}}$ Chaftellux, voy. dans l'Amerique feptentrionale. Paris 1786: II. p. 333.

Limné, Syft. Nat. Edit. X. I. p. 53. n. 3. Didel. phis (Opoffum) cauda femipilofa, fupercilio. rum regione pallidiore, mammis binis.

Zimné, Syf., Natur. Edit. XII. I. p. 75. n. 3. Didelphis (Opoflum) cauda femipilofa, fuperci. liorum regione pallidiore, mammis binis.

Iinné, Muf, Adolph. Fr. I. p: to. Didelphis (Opoffam) mammis intra abdomen.

Nieremberg, hiftor, natur, p. 156. Tlaquatzin; m. e. Fig.

Ionfton, quadrup. Tab. 73. Tlaquatzin; m. Nie. remberg. Fig.

Ionfton, quadrup. p. 135. Tab. 63. Carigueia et Taibi; m. Marcgr. Fig.

„2.: Ionifton, Quadruped, Tab. 58. Semivulpa; mie Gesn. Figur,

Valentin, amphith. zoot. I. p. 130. Tab. 26. 2\%. das Weibchen; p. 136. Tab.28. das Männchen. Rochefort, hif. des Antilles, p. 337 Opo:Jum. Abbild. fehlecht. 
Du Tertre, hif. gen. des Antilles; 11. p. gor. lo Manitou de la Grenade.

Feuillée, Journal des obf. III. p. 206, le Manicou: Lawesor, Carolin. p. 120. Polfum; m. e. Fig. $R a j$, Syn. quadr, p. 185. Taii-ibi Brafilienfibus. Du Pratæ, hiftor. de la Louifiane, II. p. 94. Io Rat de bois; m. e. Fig,

Dumont, mém. de la Louifiane, p. 84. Laet, nov. orb, p. 55r. Carigue.

Charlevoix, hiff. de la nouv. France, III. p. 134? le Rat de bois,

B. Molucca. Der molukkifche Opolsum. ( $\left.{ }^{6}\right)$

Zimmermann, geogr. Zool. II. p. 223. a.

Schreber, Säugthiere, III. p. 539. eine ähnliche Art, deren Schnauze ein klein wenig lönger, und die Forbe etwas rother ift.

Heidt, Schauplatz von Afrika und Oftindien; p. 186.

Pallas, mifcellan.' p. 62. Didelphis Opoffum; varietas orientalis.

Barchewitz, oftind. Reife, p. 532. 368 ?

Seba, thef. I. p. 61. Tab. 38. fig. I. Philander orientalis, cum catulis fuis in facco ventris hae. rentibus, femina?

6. Cay o p ol In. Der Cayopollin. (7) Miiller, Naturfyft. Suppl, p. 35. n. 6. der Cayo: pollin.

Zimmer.

(6) Zimmermanr glaube nicht, dafs das Thior anf Ceilan zu Haure fey, und es ift ihm wahrfcheinlich, dafs die angeführten Stel- len auf den Filonder des de Brun, oder auf den KuJkus gehen.

(7) Seba, und die ihm gefolgt find, goben irrig Afrika für das $V$ aterland diefes $T$ :iers an. Der Beutel fehlt diefer, und den folgcsaden vier Gattungen. 


\section{Mammatia Ferae. Didelphis}

Zimmermann, geogr. Zoolog. II. p. 224. n. 128. der Kayopollin.

Borowfky, Thierreich, I. 2. p. 81. n. 3. der Kayopollin.

Halle, vierfüls. p. 453. die afrikanifche Maus, Kayopollin.

Neuer Schauplatz der Natur, II. p. 84. Cayopollin.

Klein, Quadrup. difp. p. 58. Mus africanus Kajo. polin dictus.

Klein, Claffif. d. vierfüfs. Th. p. I72. die afrikanifche Maus Kajopolin.

Klein, natïrl. Ordn. d. vierfüfs. Th. p. 61. n. 4: die afrikanifche Maus Kajopolin.

$B$ iffori, vierf. VIII. p. 83. der Cayopollin.

Samml. zur Phyf. und Naturgefch. II. p. 609. der Cayopollin.

Handb. der Naturgefch. I. p. 312. der Cajopollin. Martini Naturlexicon, VIII. p. 220. Cayopollin. Bat $\hat{c}$ h, Thiere. I. p. 222. der Kayopollin.

Erxlebpn, Mammal. p. 82.

Graumann, intr. in bift. nat. mamm. p. 26. V. b: die afrikanifche Maus.

Limné, Syft. Nat. Ed. VI. p. ro. n. 2. Didelphis mammis extra abdomen.

Charlezon, exercitat. p. 25. Mus indicus dictus Cayopollin.

Ionfton, Quadr. p. 170. Tab. 67. Coyopollin.

4. Murina. Die Marmofe. (8)

Iniiller, Naturfyftem, I. p. 293. die Bufchratte: Tab. 18. fig. 3. Seb.

Zimmer.

(8) An der Schreberifcben Figur fcheinen neun Eutex zu feyn. Dex Schwanz ift greifend, tattenaholich und lang. 


\section{Mammalia Ferak. Didelphis.}

Zimmermann, geogr. Zool. II. p. 225. n. 128. die Marmofe.

Borow kky, Thierreich, I. 2. p. 81. n. 4. die Marmo $\int e$, die Burfhratzie.

Halle, vierf. p. 432. die langfiifsige amerikanifche Waldmans. Tab. 24. $\mathrm{k}$.

Gatterer, vom Nutzen u. Schaden der Thiere, I. p. 17. n. 24. die Bufichratte.

Nequer Schaupl. der Natur, V.p. 38\%. MLarmoSe; Bufchratze; wille Ratze.

Onomat. hift. nat. III. p. 620. das mansä̈hnliche Beutelthier.

Klein, Quadrup. difp. p. 58. Mus Scalopes. Klein, Claffif. d. vierf. Th. p. I7t. n. 7. amerikamifche IV aldmans.

Klein, natürl. Ordni d. vierf. Thiere, p. 6t. n. 4. IV aldinaus aus Annerika.

Biiffon, vierfüfs. VIIl. p. 73. dis Marmofe; mit 2 Figg.

Handb. der Naturgefch. I. p. 312. die Mharmofe.

Martini, Naturlex. VII. p. 235. Marmofe.

Batfch, Thiere, I. p. 222. die Marnofes

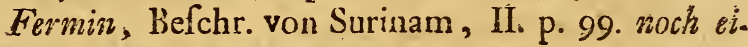
ne andere wilde Ratze.

Evxlebrin, Mammal. p. 80. 13. 5. Didelphis (Murina) roftro acutiufculo, ungue pollicum palmarum acuto, cauda femipilofa.

Linné, Sy凡. Nat. Ed. X.I. p. $55 \cdot$ n. 4. Didelphis (Murina) cauda femipilofa, mammis fenis.

Gransmann, intr. in hift. nat. matum. p. 25. V. die Buzchratze.

Pennant, Synopf quadrup. p. $20 \%$. n. 145. Mu. rine opoffum.

Gatterer, brev. Zool. I. F. 28. n. 5. Didelphis Murina. Bufchratte, Marnofe. 
5. DORSIGERA. Die Bufchratte. (')

Milller, Naturfyftem, I. p. 293. n. 5. die Schwanz. ratte. Tab. 18. fig. 4 . Seb.

Zimmermanin, geogr. Zool. II. p. 226. n. 129. der Aeneas, die Bufchratte.

Lefke, Naturgefch. p. 152。 n. 3. die Bufchratte. Borow Jky, Thierreich, I. 2. p. 82. n. 5. dis Schwanzratze; der furinamifihe Aeneas.

Blimenbach, Handb. der Naturgefch. p. 77. n. Is - Didelphis (Dorfigera) cauda bafi pilofa, dorfo fulco, abdomine albido; der Jurinamifche Aeneas.

Funke, Naturgefch. I. p. I8I. die Bufchratte, oder der Jurinamifche Aeneas.

Halle, vierf. p. 433. der Surinamifche Aeneas. Tab. 34 A.

Neuer Schauplatz der Natur, I. p. II3. Aeneas. VIII. p. s. Schwanzratze.

Onomatol. hiftor. natur. III. p. 619. das furina. mifche, feine Inngen ouf dem Riicken tragende Beutelthier.

Kiein, Quadr. difp. p. 58. Mus fylveftris americanus, femina; catulos fuos fupra dorfum gerens.

Klein, Claffif. der vierf. Th. p. rs2. n. 9. ameri. kanifche Waldmans.

Klein, natürl. Ordn. der vierf. Th. p. 6r. n. 6. indianifche IV aldratze.

Biiffon, vierfüfs. VIII. p. Ior. die Bufchrotte; m. e. Fig.

Martini,

(9) Von dem Inftinke, ihre Iungen bey anctrohender Gefalts auf dem Rücken fortzutragen, hat ilir Halle zuerft durch, eine Umwendung den Namen Aeneas (der feinen Vater Anchifes auf dem Rücken aus der brennenden Stadic Troja getragen haben (oll) gegsben. 
Martini, Naturlex. VillI. p. 218. Bufchratte.

Fermin, Befchr. von Surinam, II. p. Ior. die Waldmaus.

Dapper, Amerika, p. 303. ein namenlo/es Thier. Erxleben, Mammal. p. 83. n. 6. Didelphis (Dorfigera) cauda bafi pilofa, corpore longiore, digitis manuum muticis.

Limne, Syft. Nat. Edit. X. I. p. 55. n. 5. Didelphis (Dorfigera) cauda bafi pilofa, corpore longiore, digitis manuum muticis.

Graimann, intr. in hif. nat. mamm. p. 26. VI. die Schwanzratze, die Waldmaus.

Gatterer, brev. Zool. I. p. 28. n. 6. Didelphis (Dorfigera) cauda bafi pilofa, corpore longio$\mathrm{re}$, digitis manuum muticis, dorfo fufco, ab. domine albido; die Schwonzarattę, der Aeneas.

7. Cancrivora. Der Krabbenfrefjer. $\left({ }^{10}\right)$

Zimmermann, geogr. Zoolog. II. p. 226. n. 130. Didelphis (Karkinophaga) caninis fuperioribus exfertis, vellere hirto, cauda fquamofa; der Krabbenfreffer.

Schreber, Säugth. III. p. 547. n. 7. der Krabbenfrefter.

Borow Jky, Thierreich, I. 2. p. 82. 11. 6. der Krabbenfrefer.

Biiffon, vierf. VIII. p. 103. n. 7. der Krabbenfrefer; m. e. Fig.

Martini Naturlexicon, VII. p. 229. der Krabben- freffer.

Batfch, Thiere, I. p. 222. der Krabbenfreffer.

8. BRA.

(10) Länge des Schwanzes funfzehn Zoll. Das Fleifch des Thiers effen die Wilden. 
8. B R A C н Y.U R A. Das kurzafchwänzigte Beutelthier:

Zimmermann, geogr. Zoolog. II. p. 227, n. I3r. das kurzschwönzigte Bentelthier.

Borowl'ky, Thierreich, I. 2. p. 83. n. 7. das kurzgefchwönste Bentelthier.

MIartini Naturlexicon, VII. p. 227. das kurzfchwönzige Buitelthier.

Halle, vierf. p. 435. die amerikanifche Waldmans. Biiffon, vierf. VIII. p. 107. das knirz/chwönzige Bentelthier; m. e. Fig.

Erxleben, Mammal. p. 80. n. 4. Didelphis (Bre: vicaudata) cauda abbreviata craffa.

Graumann, intr. in hif. nat. mamm. p. 26. VII. Didelphis Brachyura.

Gatterer, brev. Zool. I. p. 28. in. 4. Didelphis (Brevicaudata) cauda abbreviata craffa.

9. OrIEnTalis. Der Kufkus. (")

Miiller, Naturfyf. Suppl. p. 35. Didelphis indica; der Amboinefer.

Zimmuermann, geogr. Zoolog. II. p. 228. n. I32, $\operatorname{dev} K u \int k u s$.

Borowe ky, Thierreich, I.2.p. 83, n. 8. der Kufkus. Halle, vierf. p. 434. die gröfste amerikanif che dick. köpfige Waldmans.

Biiffon, vierfüls. XIII. p. 288. der Phalanger; m. 2 Figg.

Martini,

(1) Eine änliche Art hat Banks auf Neufüdwallis oder an der Onkiufte' von Neuholland gefunden. S. Hawkeswortib Gefch. der engl. Seereifen, III. p. 183. Sçbreber, Säugth. III. p. S I Zimmermann, geogr. Zool. Il. p. 229. 2. 
Martini, Naturlex. VII. p. 23r. Kuekus, Phalanger, Coefcoes.

Argenfola, Befchreibung der Molukken. Lpz. I710. p. 167.

Klein, Quadruped. difj. p. 58. Mus americanus maior, agrentis, capite grandi.

Klein, Claflif. der vierfüls. Th. p. I74. n. Io. anterikuni/che gröfseve Waldmans, nuit einem grofsen గ̆opf.

Klein, natürl. Ordn. d. vierf. Th. p. 6I. n. 7. Felduraus mit grofsem Kopfe.

Graumanin, intr. in hift. nat. mamm. p. 25. III. Phalanger.

Gatterer, brev. Zoolog. I. p. 28. n. 3. Didelphis (Orientalis) digitis duobus intermediis plantarum coadunatis.

\section{BRUNII. Der Filander.}

Zimmermann, geogr. Zoolog. II. p. 230. n. 131. Didelphis (Brunii) roltro elongato, conico, pedibus pofticis longioribus, cauda mediocri; der Filander.

Biiffon, vierf. VIII. p. rog. der Filander; m. e. Fig. Martini, Naturlex. VII. p. 228. Filander. Bat Sch, Thiere, I. p. 223. der Filander.

Graumann, intr. in hift. nat. mamm. p. 26. VIII. Didelphis Brunii.

11. Gigantea. Das Kenguruh.( $\left.{ }^{2}\right)$

Miiller, Naturfyft. Suppl. p. 62. n. 40. Mus Canguru; das Känguruh.

Zimmer-

(2) Diefes durch Cooks erite Reife nach der Südíe bekannt gewordene Thier bat in der Bildung des Kopfs viel vom Wind- 


\section{Mammalia Ferae. Didelphis.}

Zimmermann, geogr. Zoolog. II. p. 231. n. I34. Didelphis (Kenguru) gigantea, abilomine incraftato, pedibus anticis breviffimis, pofticis longioribus, cauda attenuata, longitudine corporis; der Kenguru.

Boroweky, Thierreich, I. 2. p. 84. n. 9. das Könguruh.

Blumenbach, Handb. der Naturgefch. p. 78. 11. I9. Iaculus (Giganteus) cauda attenuata; der Kön. guruh.

Biiffon, vierf. VIII. p. IIr. n. Ir. der Kengurnh. Martini, Naturlex. VII. p. 286. Känguruh, Kengurreh.

Nachricht von den neueften Entdeck. der Engländer in der Südfee. Berlin 1772. p. 220. 221.

Gefchichte der Seereifen nach dem Südmeere. Frcf. und Lpz. 1775. 8. III. p. 76. Kängurnh; m. e. Fig.

Krïnitz, ökon. Encykl. XXXIV. p. 123. Känge. ruh, Känguruh.

Gatterer, vom Nutzen und Schaden der Thiere,

I. p. 254. n. 144. der Känguhruh.

Bat fch, Thiere, 1. p. 223. der Kenguruh.

Camper, Naturgefch. des Orang - Utang, p. Is. I Canguroo.

Merrem, zool. Abhandl. p. 34. n. r. Künguiru. Erxleben, Mammal. p. 409. n. 2. Iaculus (Gigantens) cauda attenuata, longitudine corporis. Graumann, intr. in hif. nat. mámm. p. 64. II. Iaculus Giganteus; das Kïnguruh. $\mathrm{Z}_{2}$

Gatte.

fpiel, if aber tiber 80 Pfund fchwer, und hat ein fehr fchmackhaftes Fleifch. Er geht nicht auf vier Füfsen, fondern hüpft mit grofsen Sätzen blofs auf den Hinterfüfsen fchnell fort. Der Schwanz ift beynahe von der Lange des Kürpers. Die Hinterfuffe haben, nach Camper, nicht drey, fondern vier Finger. 


\section{Mammalia Ferae. Didelphis:}

Gatterer, brev. Zoolog. I. p. II2. n. 3. Iaculus (Giganteus) cauda attenuata, longitudine cor. poris.

12. Macrotarsus. Der Táfier. (3)

Zimmermann, geogr. Zoolog. II. p. 2I\%. 13. I24: der Tarfier, der Podje.

Borowfky, Thierreich, I. 2. p. 84. n. Io. der Turfier.

Neuer Schauplatz d. Natur, VIII. p. 793. Far er. Biilfon, vierfüfs. VIII. p. II3. XIII. p. 28I. der Tarfer; m. e. Fig.

Fabricins Briefe aus London, p. 94.

Erxleben, Mammal. p. 71. n. 6. Lemur (Tar. fier) cauda gracili nuda, apice fubfloccofa, tibiis pofticis nudis.

Granmann, introd. in hiff. nat. mamm. p. 26. X. Didelphis Macrotarfos. p. 24.VI. Lemur Tarfier. pallas, nov. fp. quadr. e glirium ord. p. 275 . not. a. Lemur fpectrum.

Gatterer, brev. Zool. I. p. 26. n. 6. Lemur Tar. fier. Der Tarfer.

Verïnderungen gegen die XIIte Edition, and Vermehruing der Gattungen diefes Gefchlechts.

Der molukkifche Opoffitm in als eine Varietät vom Opo/fum getrennt; überdem ift das Gefchlecht mit

(3) Der Buffonf̣che Name Tavfier ift von den langen Fufswurzeln (Tarfus), die allein fo lang als der übrige Fufs find, hergenommen. Vom Vacerlande des Thiers meldet Pallas (am angef. O.) folgendes: Patriam, quat buffonium latuit, moneo efe exttremas infulas Oceani indici, praesertin Amboynam, inale non nifi parce in Belgarum Mufaed venit, "et wbi nomine Macafarienfibus ufitato, $P$ odje notum efi. 
mit fiebesc Gattungen vermehrt, und D. Cayo. pollin, Cancrivora, Brachynura, Orientalis, Bru. mii, Gigantea und Macrotarfus hinzugekonmen.

18. GESCHLECHT. TXLPA. Der Maúlvourf, Moll.

Miiller, Naturfyfen, I. p. 295: Gen. XVIII. Zimmermanni, geograph. Zoolog. II. p. 388. Gen. XXXIV.

Schreber, Säugthiere; III. p. 557. Gen. XX. Lefke, Naturgefch. p. 153. Gen. XIX. Borow Jky, Thierreich, I. 2. p. 84. Gen. XVIII. Bhumenbach, Handb. der Naturgefchichte, p. 76 . Gen. XVII.

Bechftein; Naturgefch. Deutfchl. I. p. 376. Onomatol. hiff. nat. VII. p. 4 I8. Biiffon, vierf. V. p. 4I. Erxleben, Mammalia, p. II4. Gen. XIII. Granmonn, intr. in hiftor. nat. mamm. p. 30 .' Gen. XIII.

Batfch, Thiere, I. p. 209. Gen. XXVIII. Gatterer, brev. Zool. I. p. 33. Gen. XIII. Pennant, Syn. Quadr. p. 3ir. Gen. XXXIV. Briffon, quadr. p. 203. Gen. XLI. Hermann, Tab.' affinit. anim. p. 78 .

1. E u R O PA E A." Der gemeine IManherurf. (4) Miüller, Naturfyftem, I. p. 295. n. I. der euro: paìiche Manheurf.

\section{$\mathrm{Z}_{3}$ \\ Zimuner-}

(4) Mollmaus, Moltwurf, Scherrmaus', Erdratie. Das Thier hat einen fehr feinen Geruch, wociurch es eben im Stande ift, feine Nahrung in der Erde zu entdecken. Bey bevorftehendem Regen-und Thauwetter if der Maulwurf mehr. an der Oberfläche der 
Zimmermann, geogr. Zoolog. II. p. 388. n. 324. der gemeine Manherurf.

Lefke, Naturgefchichte, p. 153. n. Ir der gemeine IMTulwourf.

Borowesy. Thierreich, I. 2. p. 86. n. 2. der gemeine Manlwurf.

Blumenbach, Handb. d. N. G. p. 76. n. I. Talpa (Europaea) cauda breviore, auriculis plane nullir; der Mauhourf; äie Schermaus.

Bechftein, Naturgefch. Deutfchl. I. p. 377. n. I. der gemeine Mauhenrf. p. 388. Moll, Scharrmains, fchwarzer, fchwarzifahler, europäifcher Minulivurf.

Funke, Naturgefch. I p. I25. der Mauhwirrf. Ebert, Naturlehre, I. p. 352, der Manhwnrf. Halle, vierfüfs. p. 445. der gemeine Manlwourf. Tab. 26. A.

Meyer, Thiere, Tab. I. fig. 2. ein Manheinurf. Abbild. gut.

Gatterer, vom Nutzen und Schaden der Thiere, I. p. 2I. n. 35. der Manlwurf.

Becknann; Naturliftorie, p. 24. n. 5. der Manilwourf.

Pemant, arct. Zoolog. II. p. I40. n. 88. der eusropäif che Maullourf.

Never Schauplatz der Natur, V. p. 423. Maulwurf.

Onomat. hift. nat. VII. p. 420. der éuropiii che oder gemeine Mandwurf; die Scharrmans.

Sander,

der Erde zu merken, weil die Regenwürmer dann in die Höhe gehen. In trocknem Wetter, da fich diefe tiefer hinunter ziehen, gehen ihnen die Maulwürfe nach, und werfen alfo feltner auf. Die erfte Nahrung der lungen foll, nächft der Muttermilch, in der Wurzel der Zeitlose (Colchicum autumnale Linn.) bertehen. 


\section{Manmalia Ferae: Talpa.}

Sander, ökon. Naturgefch.I. p. 26r. der Mauheurf. Handb. der Naturgefch. I. 2. p. 190. der IILailuenurf. Handbuch der deutfchen Thiergefchichte, p. 43. der Maulevurf.

Gesner, Thierbuch, p. 257. Talpa; ein IMaulwuirf, Schär, oder Schärmaus; in. e. fchl. Fig. Klein, quadr. difp. p. 60. Talpa noftras. Klein, Claffif. der vierfüfs. Th. p. 82. n. I. einlïndilcher Mantwurf.

Klein, natïl. Ordnung d. vierf. Th. p. 63. n. I. fchevarzer europaiifcher Maulweurf. Kraft, Ausrottung graufam. Thiere, II. p. 443. der Manherurf.

Büffon, vierf. V. p. 33. der Maulleurf; m. e. Fig. Fif cher, Naturgefch. von Livland, p. 56. n. 16. gemeiner Maulwourf.

Kolbe, Vorgeb. d. g. Hoffn. Edit. in 4. p. 34r. Miaulieiirfe.

Pontoppidan, Norwegen, II. p.55.der Mauhwurf; nordifch Vond.

Shane, Reife, p. 157. der Maulweurf.

Bock, Naturgefch, von Preuffen, IV. p. 58. n. I7. der europäifche Mantieurf.

Goeze, europäifche Fauna, I. p. 433. der gemeine europäif che Maulwurs.

Bat fch, Thiere, I. p. 2 Io. der gemeine Manheurf. Blumenbach, Beytr. zur Naturgefchichte, I. p. 43. (das Gerippe.)

Bonniet, Betracht. uiber die Natur, II. p. 104. n. 4 . Merklein, Thiere, p. II8. Mantherinf.

Derham, Phýfikotheol. p, 155. 340. 350. 409. 521.525.

Beyträge zur Beförd. der Naturkunde, I. p. I23. Mauheurf

Stuttgard.allgem. Magazin 1767.p. 790.378 .

$$
\text { Z } 4 \text { Gefell. }
$$


Gefellfchaftl. Erzählungen, II. p. 150-160: Bernhard, Abhandl. vom Wiefenbaue, p. 348 .

Magazin für den Landmann, II. p. I74. Halle, Magie, III. p. 308. 321. 585.

Goeze, Allerley, II. p. 243. neue Aufl. I. p. 398:

Voigt, phyfikal. Zeitvertr. p. 22I.

Ludovici, Kaufmannslexicon, III. p. I223.

Hantiurg. Magazin, II. p. 38. III. p. 304. 305:

XXIV.p. 217 .

Berlin. Samml. IV. p. 47 I. 473.548. V.p.383.59I. Schwed. Abhandl. IV. p. 214. XXIII. p. 3I. 32 I.

Schwed. ökon. Wochenbl. p. I95. not.

Koburgifches Magazin, I77r. p. 92.

Stuttgard. Realzeit. 66. p. 39\%.

Thüring. neue Beytr. I. p. 453.

Reichard, Land-und Gartenfchatz, VI. p. I73:

IIitls prakt. Feldwirthfchaft; III. p. 406.

Lemmery, Materiallexicon, p. IIO3.

Springer, Abh. vom deutfchen Weinbau. Lemg:

I769. p. 227.

Eckhard, Experimental Oekonomie. Ien. I777.

p. I2r.

Zinken, allgem. ökon. Lex. p. I817.

André u. Bechfteinu, Spatziergängge, I. p. 169:207.

Moyen fûr et facile de detruire les Taupes, dans

les prairies, et dans les jardins. à Paris 1770 .

8. avec figg.

Comment. Lipf: XIX. p. I44.

Zimué, Syft. Nat. Edit. II. p. 45. Talpa caudata.

'Limne', Syif. Nat. Edit. VI. p. 7. n. I. Talpa caudata.

Linné, Syf. Nat. Edit. X. I. p. 52. n. I. Talpa

(Europaea) caudata, pedibus pentadactylis.

Limné, Fn. Suec. I. p. 7. n. 17. Talpa caudata.

Graunann, 
Grammani, introd. in hifor. nat. mamm. p. 30. Maulevurf.

Milller, Zoolog. dan prodrom. p. 3. n. Is. Talpa (Europaea) caudata, pedibus pentadactylis.

Kramer, Aufr. p. 3r4. Talpa caudata.

Cinarletor, exercitat p. 25. Talpa.

Sinwenkfeld, theriotroph. Silef. p. I29. Talpa. Agricola, de anim. fubterr. p. 70. Talpa.

Rzacz. hiftor natur. Polon. p. 236. Talpa.

Sibbiald, Scot. illuftr. P. II. T. II. p. II. 'Talpa.

Gronov. Zoophyl. I. p. 9. n. 32. Talpa caudata, pedibus péntádacylis.

Pennant, britt. Zoolo.p. 52. the Mole.

Pennant, britt. Thiergefch. p. 47. IManhenrf.

$V$ alent. amphith. zootom. p. I83.

Ari Apollinio, hieroglyphica: Parif I55I. p. 60.

Arifot. hift anim. I. c. 2. n. 15. IV. c.8. n. 240.

A $\sigma \pi \omega \lambda a \xi$.

plin. h. n.XI. c. 37. XXX. c: 3.c. 69. Talpa.

Aelian. de nat. anim! XVII c. ro. A $\sigma \pi \omega \lambda e_{0} \xi$.

Oppian. cyneget. II. 6I2. A $\sigma \pi \sigma_{0} \lambda \alpha_{0} \xi$.

B. VA I E G ATA. Der weifsgeffeckte Manheurf.

Milller, Naturfyat. Suppl. p. 36. n1. 5. Talpa frifius; der gefleckte Manhwirrf.

Zimmermann, geogr. Zool. II. p. 389. n. .. dev. wei/sgefleckte Maulwurf.

Sihreber, Säugthiere, III. p. 559. B. der weifsAckkigte Mauherurf.

Borow Jky, 'Thierreich, I. 2. p. 87. a. der marmorfarbene oft friefische Maulwerurf.

Bechftein, Naturgefch. Deutfhl. I. p. 388. a. der gefleckte, oder. marmorfarbene oftriefiche IManlwourf. 
Halle, vierf. p. 448. der marmorfarbene offriefische Maulwurf.

Kiein, Quadruped. difp. p. 60. Talpa maculata, Ooft. Frifia.

Klein, Claflif. der vierf. Th. p. 183. n. 4. fleckig. ten Maniwerurf.

Klein, natü̈rl. Ordn. der vierf. Th. p. 64. n. 4. gefleckter, offfriesländifcher Manhwurf.

Biiffon, vierfüls. V. p. 42: der fchwarze mit weifsen Flecken aus. Oftfriesland.

Berlin. Samml. VI. p. 319. der fleckigte Maulweurf; m. Edw. Fig.

De la Faille, Naturgefch. des Maulvurfs, p. 25. der gefleckte oder bunte Miuthenurf.

Erxleben; Mammal, p. II7. c. Talpa albo-ma. culata.

Graumann, introd. in hiftor. nat. mamm. p. 36 . Talpa E, albo-inaculata ooffrifia.

Gatîter, brev. Zoolog. I. p.34. a. der fleckigte Manhwurf.

\%. А в в А. Der weifse Mamlwurf.

Zimmermann, geogr. Zool. II. p. 389. B. der weifse IMaudieurf.

Schreber, Säugthiere, III. p. 559. der weifse Mauhwourf.

Boroweky, Thierreich, I. 2. p. 88. b. der weifse Maulwourf.

Bechftein, Naturgefch. Deutfchl. I. p. 387. n. I. der queifse Mauhourf.

Biiffon, vierfüfs. V. p. 4I. der ganz weifse IMaulvenrf.

Klein, Quadrup. difp. p. 60. Talpa alba noftras. Berlin. Sammlung. VI. p. 320. der ganz weifse Mauleurf. 
De la Faille, Naturgefch. des Maulwurfs, p. I8. Talpa albica; der neifse Mauhenurf. Erxleben, Mainmal. p. 117. ß. Talpa tota alba. Linné, Fn. Suec. II. p. 9. n. 23. Talpa alba noftras,

Wagner, hift nat. Helvet. p. 185. Talpa alba. Rzacz. Auct. hift. nat. Polon. p. 329. Talpa alba. Graumann, intr. in hift. nat. mamm. p. 30 . b. 'Talpa, E. tota alba.

Gatterer, brev. Zool. I. p. 34. b. tota alba.

8. Flava. Der gelbo Maulwourf.

Zimmermanin, geogr. Zool. II. p.389. $\gamma$. der gelbe Maniheurf.

Schreber, Säugthiere, III. p. 559. . der gelbe Mautheurf.

Borow $k y$, Thierreich, 1. 2. p. 88. c. der virginifche Mauhourf.

Erxleben, Mammalia, p. 128. $\gamma$. Talpa E. fla. vefcens.

Graumans, intr. in hift. nat. mamm. p. ${ }_{3}$ I. d. Talpa E. flavefcens: Virginiana.

Gatterer, brev. Zoolog. I. p. 34. c. flavefcens; der vinginifche Mauhourf.

8. Cinerea. Der graue Maulwurf.

Zimmermann, geogr. Zoolog. II. p. 390. $\delta$. der graue Mantwourf.

Schreber, Säugth. III. p. 159. E. der graue Maulienrf.

Borotefky, Thierreich, I. 2. p. 8. n. d. Eifliaca cinerea, der grané Maulwunrf.

Hiipsch, am a. O. eine bisher nubekannt gewefene Art von Maulwiirfen, der graue bifelifche Mandiourf.

Bechftein, 
Bechftein, Naturgefch. Deutfchl. I. p. 388. n. 3. der grane Marihourf.

Biüfon, vierf. V. p. 43. der grane Manhenrf.

De la Faille, Naturgefch. des Maulwurfs, p. 95."

Evxleben, Mammalia, p. II8. $\delta$. Talpa E. fupra fplendens grifea, fubtus flavefcens.

Granmann, intr. in hiftor. nat. mamm. p. 3 r. d. T. fupra fplendens grifea, fubtus flavefcens.

Gattever, brev. Zoolog. I. p. 34. d. der eifelifche. Manlwirf.

3. L O N G I C A U D A A: - Der langgefchwönzte Maulterurf.

Zinumermann, geogr. Zool. II. p. 290. n. 325. der langgefche änzte Maulewrurf.

Schrever, Säugthiere, III. p. 561 . n. 20. der lang: gefchwoinste Maulwurf.

Boronefky, Thierreich, I. 2. p. 88. n. 3. der lang-. gefchwïnzite Manheurf.

Pennant, arct. Zool. II. p. I38. n. 85. der langgeL. Schröanzte Mantwurf.

Granunann, introd. in hift. nat. mamm. p. 3r. II. Talpa longicaudata.

Gatterer, brev. Zoolog. I. p. 34. n. 2. Talpa (Longicaudata) cauda mediocri, pedibus pentadactylis, polticis fquamofis.

4. Ruвra. Dev rothe Manherurf.

Miiller, Naturfyft. Suppl. p. 35. n. I. Talpa Virginea; der Purpurmanlwourf.

Miiller, Naturfyftem, Suppl. p. 36. Talpa americana; der rothe Maulwourf.

Zimmernanu, geogr. Zoolog. II. p. 290. n. 326. der rothe Maulwurrf:

Schreber, 
Schreber, Säugthiere, III. p. 56I. n. 3. der rothe Maulwurf.

Borowfky, Thierreich, I. 2. p. 89. n. 4. der. rothe Manlwurf, Purpuirnanlwurf.

Halle, vierfüls. p, 447. der amerikanifche rothe. IIIantourf.

Neuer Schaupl. der Natur, V. p. 424. röthlichs Manlevitrf:

Onomat. hif. nat. 'VII. p. 418. Talpa americana rufa. p. nig. dev rothe amerikanifche Moulwurf. Klein, Quadrup. difp. p. 60. Talpa rubra americana.

Klein, Claffif. d. vierf. Th. p. 383. n. 2. rother. amerikanifcher Maulwenrf.

Klein, natïrl. Ordn. d. vierf. Thiere, p. 63. n. 2:' rother Mandwenrf.

Biiffon, vierfüfs. V. p. 4I. der rothe amerikanzfihe Maulwerurf.

De la Faille, Naturgefch. des Maulwurfs, p. 26: Talpa americana rubra.

Graumann, intr. in hif. nat. mamm. p. 3I. III. der rothe Matihurf, der Purpurinautwenrf.

Gatterer, brev. Zoolog. I. p. 35. n. 3. Talpa Rubra.

3. As i at I ca. Dev Goldmanhwurf. (')

Miiller, Naturfyn. I. p. 299. der afiatifche Maulथurf. Tab. I7. fig. 2.

\section{Zimmer-}

(5) Seba, der diefs Thier zuerft bekannt gemacht hat, hat in Anfehung des Vaterlandes deffelben, wofür er Sibirien angiebt, die Zoologen lange fehr irre geführt, daher fich auch diefer Irrthum noch in der xil Edition unfres Syftems erhalten hat. Sparrmann technet es zu den Spitzmäufen, und giebt feine Länge auf fïnf bis fechs Zoll an. 
Zimmermann, geogr. Zoolog. II. p. 39r. n. 327. der Goldmaulworrf.

Borowsky, Thierreich, I. 2. p. 85 . n. I. der afatifiche Maulwurf; Goldmaulwurf.

Lefke, Naturgefch. P. 154. n. 2. der Goldmaulvourf, Talpa aurea.

Halle, vierf. p. 447. der bunte fibirifche Maulwurf.

Neuer Schaupl. der Natur, V. p. 424. 'fibirifche Maulwürfe.

Onomat. hiltor. nat. VII. p. 419. der afiatifche Manlwurf; der bunte fibirifche Maulwurf. Klein, Quadrup. difp. p.60. 'Talpa fibiticus ver. ficolor.

Klein, Claffif. der vierfüfs. Thiere, p. I83. n. 2. bunter fibirifcher Manlwurrf:

Klein, natürl. Ordn. d. vierf. Th. p. 53. n. 3. bunter fibivifcher Mlaulwin.

Biiffon', vierf. V. p. 40. der Gbirifche Mauhourf. Sparrmann, Reile n. d. Vorgeb. d. g. H. p. 497. Sorex aureus; die angefchuïn:te Gold jpitzmans.' De la Faille, Naturgelich. des Maulwurfs, p. 28. der Manlwurur von Sibirien.

Erxleben, Mammal. p. 120. 1. 4. Talpa (Afiatica) ecaudata, palmis tridactylis.

Limné, Syft. Natur. Edit. II. p. 45. Talpa cau. da nulla.

Linné, Syft. Nat. Ed. VI. p. 7. n. 2. Talpa ecau. data.

Linné, Syf. Nat. Edit. X. I. p. 53. n. 2. 'Talpa (A fratica) ecaudata, palmis tridactylis.

Graumanu, intr. in hift. nat. mamm. p. 3i. IV. der afiatifche Maulwurf.

Gatterer, brev. Zool. I. p. 35. n. 4. Talpa (Afiatica) ecaudata, palmis tridactylis. 
A) Veränderungen gegen die XIlic Edition, und Ver. mehrung der Gattungen diefes Gefihlechts.

Bey der erften Gattung find vier Varietäten diftinguirt. Bey der letztern Nr. 2. in das Vaterland berichtiget; das ganze Gefchlecht aber if mit zivey Gattungen vermehrt, und T. Longicaudata und Rubra dazu gekommen.

\section{B) Unbeftimmtere Thiere.}

1. Der Tucan.

Zimmermani, geogr. Zool. II. p. 39r. a.

Schreber, Säugth. III. p. 562. b. c.

Fernant. anim. p. 7. Tucan, feu Talparum in. dicarum quoddam genus.

2. Der gro/se.capifche Mantheurf:

Zimmermann, geogr. Zoolog. II. p. 39r. b.

3. GESCHLECHT. SOREX. Die Spitzmaus. Miiller, Naturfyft. I. p. 300 . Gen. XIX. Zimmermani, geogr. Zool. II. p. 38I. Gen.XXXIII. Schreber, Sąugthiere, III. p. 565. Gen. XXI. Lejke, Naturgefch. p. 154. Gen. XX. Boroweky, Thierreich, I. 2. p. 89. Gen. XIX. Blumenbach, Handb. der Naturgefchichte, p. 75. Gen. XVI.

Bechftein, Naturgefch. Deutfchl. I. p. 388. Neuer Schauplatz der Natur, VIII. p. 38I. Onomat. hif. nat. VII. p. 202. Bat fch, Thiere, I. p. 209. Gen. XXIX. Erxleben, Mammalia, p. I2r. , Gen. XIV. Graumann, introd. in hiftor. nat. mamm. p. 31 . Gen. XIV. 
Boddaert, Elèchus, p. I26. Gen. XXIV.

Gatterer, brev. Zool. I. p. 35. Gen. XIV.

Pennant, Synopf. quadr. p. 307 . Gen. XXXIII.

Briffon, quadrup. p. I26. Gen. XXVII.

Hermann, tab. affin. animal. p. 79.

i. C, R Is tatus. Die Kamminae.

Miiller, Naturfyft.I. p. 300 . n. I. die Hacinaje. Zimmermann, geogr. Zool. II. p. 386. n. 322. die Kaminnafe.

Schreber, Säugth. III. p. 566.'n. 2. die Kammnafe. Lefke, Naturgefch. p. 155. n. I. die Spitzmans mit der Kammnnase.

Borowfky. Thierreich, I. 2. p. 9I. n. 3. die gekammine Spitzmonis.

Pennant, arct. Zool. II. p. 138. n. 86. die Kammwase (unter den Maulwüirfen).

Neuer Schaupl. d. Natur, VIII. p. 382. Haarnafe.

Onomat. hiftor. nat. VII. p. 206. die Spitzmaus mit der Igelnase.

Batch, Thiere, I. p. 211. die Kaminnafe.

De la Faille, Naturgefch. des Maulwurfs, p. 30. Talpa (Canadenfis) cauda nodofa longiffima, nafo multiplicibus mufculis, tanquam fpinis coronato, der Maailwurf von Cancada. Tab. I.

Erxleben, Mammal. p. I2I. n. I. Sorex (Crifta. tus) naribus carunculatis, cauda breviore.

Limné, Syft. Natur. Edit. X. I. p. 53. n. 2. Sorex (Criftatus) naribus carunculatis, cauda corpore breviore.

Linné, S. N. Edit. XII. I. p. 73. n. I. Sorex (Criftatus) naribus carunculatis, cauda corpore breviore.

Graunann, intr. in hifor. nat. mamm. p. 3I. I. die Haarnafe. 
Gatterer, brev. Zool. I. p. 35. n. I. Sorex (Crifta. tus) naribus carunculatis, cauda breviore.

2. Minutus. Die kleine ungefchwoinate fibirifche Spitzmaus.

Miiller, Naturfyft.I. p.30r. n: 2. die Zwergmans. Zimmernann, geogr. Zool. II. p. 285. n. 318. die kleinfte ungefchwönate fibirifche Spitzmons.

Borowfky, Thierreich, I. 2. p. 92. n. 4. die Zwergmaus, ungefchwänzte fibir. Spit:mmaus. Neuer Schauplatz der Natur, VIII. po" 382. Zwergmans.

Onomat. hift. nat. VII. p. 206, die kleinfte Mants. Evxleben, Mammalia, p. I22. n. 2. Sorex (Minutus) roftro longifimo, cauda nulla.

Graumann, intr. in hift. nat. mammal. p. 3r. II. die Znergmans.

Gatterer, brev. Zool. I. p. 36. n. 2: Sorex (Minutus) roftró longifimo, cauda nulla.

3. A QUA T I U s. Der Weifsfchwans.

Miiller, Naturfyft. I. p. 302. n. 3. die WafSermaius.

Zimmermann, geogr. Zool. II. p. 387. n. 323' der. IV eifs shwanz.

Borow fky, Thierreich', I. 2. p. 9I. 11. 2. dit Waferspitzinans.

Pennant, arct. Zool. II. p. I39. n. 87. der Wifs. Schwanz.

Halle, vierf. p. 447.dervirginifche Oder gehören , Mauneurf? diefe SynonyBriffon, quadr. p. 205. Talpa (Vir-, men vielleicht giniana) caudata nigricans, ex zu pag. IIo. faturate purpureo mixta, pedibus $\mathrm{Nr}$. $i$. $\delta$. des anticis et pofticis pentadactylis? Syftems?

Onomat, 
Onomatol. hift. nat. VII. p. 203. die amerikani. Sihe Spitzmaus.

Evxleben, Mammal. p. I23. n. 4. Sorex (Aquaticus) plantis palinatis, palmis caudaque breviore albis.

Linné, Sy凡. Nat. Edit. X. I. p: 53. n. 3. Sorex (Aquaticus) plantis palmatis, palmis caudaque corpore breviore albis.

Granmann, intr. in hift. nat. mamm. p. 32. IV. Waffermatus.

Gatterer, brev. zool. I. p. 35. n. 4. Sorex (Âqua. ticus) plantis palmatis, paimis caudaque breviore albis.

\section{Moschatu.s Der Wiichurchol. (')}

$\rightarrow$

\section{Milller, Naturfylem, I. p. 332. n. 2. die Biber- ratzz.}

Zimmer:-

(1) Im Winter hat das Thier keine andere Luft, als die unterirrdifche in feiner Hühle; hingegen fieht man es, fobald das Eis vergangen, fleifsig auf dié Oberiache des Wallers kommen, und an der Sonne fpielen. Das Athemholen kann es zwar länger als sndere Säugrhiere, entbehren, mufs es aber lange unter dem Waffer verweilen, fo erftickt es. Man findet daher die Biefamratzen, die fich in den Fifchreufen und Stellnetzen gefangen haben, gemeiniglich todt. Im Trocknen kann es daher gut aushalten, ohnerachter der gemeine Mann in Rufsland-das Gegentheil glabbt. Man hürt das Thier oft mit den Lippen, wie eine Ente im Wafter fchnattern, wobey es den Rüfel in dén Mund nimmt. Wenn es aber gereitze wird, fo läfst es eine fchwache quitternàe Stimme von fich hüren, und beifst gefährlich. Die Eingeweide haben, auch wenn fie frifch find, einen Arengen Schwefelgeruch. Das in den Bläschen des Schwanzkolbens enthaltene zahe Wefen hat einen fo farken Geruch, dafs ex den Geruch des fogenannten Bibergeils an Stärke noch iibertreffen foll. Der belz. ift zwar glinzend, wird aber des un- 
Zimmermann, geogr. Zoolog. II. p. 283. n. 315. der Wiichnol, die Biefamratte.

Lefke, Naturgefch. p. I55. n. 2. die Bifamratte. Borow $\int k y$, Thierreich, I. 2. p. 89. n. I. die 'Bi. Samratte; wohlviechende Wafferratte. Tab. I9. Fqunk, Naturgefchichte, I. p. 96. die Biberratze; Bifanratze; Bifambiber.

Halle, vierf. p. 426. die wohlriechende WafJer: ratte.

Gatterer, vom Nutzen und Schaden der Thiere, I. p. 27. n. 39. die Biefam - oder Bieberratze. Neuer Schaupl, d. Natur, II. p. 312. der Desman; die Bifonvatze von Mofkau.

Onomat. hif. nat. II. p. 675. die Mofchusratte. Klein, Quadrup. difp. p. 57. Glis mofchiferus; odore Mofchi.

Klein, Claffif. d. vierf. 'Th. p. I68. n. 6. Muskus: Siebenfchlïfer; Walfer - ausländifche Maus. Klein, natürl. Ordn. der vierf:Th. p. 60. n. 6. IVuskusratze; Waffernummelthier.

Biiffon, vierfüls. VI. p. 376. der Desman; die Zibethpitzmous; der Wychuchol; die rulfische Bifanuratie; m. e. Fig.

Batfch, Thiere,-I. p. 2i2. die Bifamratte.

A a 2

Pallas,

leidlichen Geruchis wegen, den er beybehâlt, nur in fchmalen Streifen, eines Fingers breit, zur Verbrämung der Schlafpelze, die man in den Stuben trägt, gebraucht. Den Schwanz thut man in die Kleiderfchränke, um die Motten von Kleidern, befonders von Zobelbälgen abzuhalten; aber der Geruch zieht fich-in - die Kleider. Das Haar/würde zu Hutfilzen eben fo dienlich feyn als das Biberhaar. Die riechende Materie brennt wie Oel, init einem Sprenzelm. An dem Wäls und dem Hecht hat der Wüchuchol grofse Verfolger, welche ihn häufig freffen. Letzterer bekoinmt davon einen fo ftarken Zibethgeruch, dafs er nicht zum effen taugt. Uebrigens ift das Thier fehr menfchenfcheu, und verbirge fich, fobald es jemand gewahe wird, 
Pallas, Reife durch Rufsl. I. p. 130. 156. Sorex Mofchatus; Bifamratte.

Pallas, Reife, Ausz. I. p. 107. Bifamrattern.

Schlözer, Erdbefchreibung von Amerika. p. 75. die Biefamratze.

Rytfchkow, orenb. Topogr. I. po 229. Wychochol, Wafer - oder Bifanuratze.

IMiller, Samml. ruff. Gefch. VII. p. 4I. die BifamRatze an der Wolga.

Goøze, Natur, Menfchenl. u. Vorfeh. IV. p. 362. Bifonratze.

Bock, Naturgefchichte von Preufien, IV. p. 73 . die Bieberratze.

Merrem, zoolog. Abhandl. p. Ig. n. 2. Biefamb bieber.

Linné, Syft. Nat. Ed. II. p. 47. Caftor cauda lineari plana.

Linnz', Syft. Nat. Edit. VI. p. זo. n. 2. Caffor cauda longa lanceolata plana.

Linné, Syft. Nat. Edit. X. I. p. 59. n. 2. Caflor (Mofchatus) cauda longa lanceolata plana.

Limmé, Fn. Suec. I. p. 9. 11. 24. Caftor cauda longa lanceolata plana.

'Linne', weflgoth. Reif. p. I6r. die Biefamratte. Caftor mofchatus.

Graunuann, intr. in hift. nat. mamm. p. 32. IX. die Bifannratte, die Biberratze.

Gatíterer, brev. zool. I. p.37. n. 9. Sorex (Mofcha' tus) pedibus palmatis, cauda compreffo-lanceolata.

Charleton, exercitat. p. 25. Sorex Mofcoviticus.

Mufeum Wormianum, p. 334. Mus aquaticus Clufii. 
7. FODIENS. Die Wa Werspitzmauls. ( $\left.{ }^{2}\right)$

Minller, Naturfyft. Suppl. p. 36. n. 6. Sorex aquaticus, die Wa/ferfpitzmans.

Zimmermann, geogr. Zool. II. p. 15. dic Was-, Serfpit $\approx m a u s$.

Zimmermann, geogr. Zoolog. II. p. 283. n. 3r4: Sorex (Daubentonii) Erxl. - Sorex Fodiens Pallas - Sorex (Carinatus) atrocinereus, fubtus albidus, inciforibus brunneis, macula retro oculos alba, cauda attenuata fubtus pilofocarinata Herrmann.

Boroneky, Thierreich, I. 2. p. 93. n. 6. Sorex Daubéntonii Erxleb. Die grabende Spitzmaus, der Gröber.

Blumenbach, Handb. der Naturgefch. p. 76. n. 2.' Sorex (Daubentonii) habitn talpae, digitis ciliatis; die Wafferfpitzmans.

Bechflein, Naturgefch. Deutfchl. I. p. 394. n. 2: die Wafferfpitzmans. p. 399. kleine Waffermans; kleiner Manhenurf; Gröber. Tab. 9.

Gatterer, vom Nutzen u. Schaden der Thiere, I. p. 26. n. 37. Wafjerfpitzmanis. A a 3

Neuer

(2) Merret hat fie zuerf s67o. namentlich angezcigt; fis if aber, nacher in Vergeffenheit gerathen, und von Daubenton, der fie in Bourgogne fand, 1756. als ein neu entdecktes Thicr befchrieben worden; daher die Erxlebenfche Benennung. Die Nahrung des Thiers befteht vorzüglich in Regenwütmern, Infektenlarven, kleinen Wafferfchnecken ' $u$. dyl. Seine Füfse haben zwar keine Schwimmhaut; jeder Zahe ift aber zu beyden Seiten mit kurzen Härchen befetzt, die die Füfse zum kudern unge. mein gefchickt machen. Die Oeffnung des Gehörgangs kann es durch eine Klappe zufchliefsen, fo lange es unter Waffer ift. Es kommt wenig zum Vorfchein, läfst fich am meiften früh Morgens erblicken, - ift aber wegen feiner Behendigkeit fchwer zu fangen. Die Begattung gefchichet zu Anfang des Maies. Das Weibchen trägt 3 Wochen. Die lungen werden blind geboren. 
Neuer Schauplatz der Natur, IX. p. 582. Waffer. Spitzmaus.

Handb. der Naturgefch. I. 2. p. I\$g. die WaJerSpitzmans.

Handb. d. deutfch. Thiergefchichte, p. 43. Waf. Serspitzmans.

Biiffon, vierfüfs. IV. p. 267, 268. die Wasferfpitzmans; m. e. Fig.

Batfch, Thiere, I. p. 213. die Wa/Serfpitzmans; der Gräber.

Geeze, Natur, Menfchenl. u. Vorf. I. p. 553. die Wasferfpitzmaus.

Goeze, europäifche Fauna, I. p. 477. die WafSerfpitzmaus.

Oedmann, in den neuen fchived. Abhandl. IX. p. 299. Sorex Fodiens, Wa/Jerfpitamaus.

Pallas, Reife durch Rufsland, I, p. IIs. Sorex fo. diens. II. p. 664.

Daubenton, Mem. de l' Acad. de Paris, I756. p. 21I. Tab. 5. fig. 2. Mufaraigne d' eau.

Graumuann, intr. in hift. nat, mamm. p. $32 . \mathrm{V}$. die Waffermaus.

Gatterer, brev. zool. I. p. 36. n. 5. Sorex (Daubentonii) cauda mediocri fubnuda, corpore nigricante, fubtus cinereo, digitis ciliatis.

4. Murinus. Die javanifche Spitzmans.

TMiiller, Naturfyftem, I. p. 302. n. 4. der Maib, Sekopf.

Zimnnermann, geogr. Zool. II. p. 384. n. 316. die javanifche Spitzmais.

Schreber; Säugthiere, III. p.576. n.8. die javanifche Spitzmans.

Borowfky, Thierreich, I. 2.p. 93.11. 7. die javanijche Spitzmans.

Onomat. 
Onomat. hift. nat. VII. p. 207. die javaniffche Spitzmans.

Pennant, Syn, quàdr. p. 309.-n. 238. the murine Shrew.

Erxleben, Mamnália, p. I24. n. 6. Sorex (Murinus) cauda mediocri, corpore fufco, pedibus caudaque cineress,

Graumann, intr. in hif. nat. mamm. p. 32. VI. Sorex murinus.

Gattever, brev, zool. I. p. 36. n. 6. Sorex (Murinus) cauda mediocri, corpore fufco, pedibus caudaque cinereis; der Manekopf.

5. A r a eus. Die gemeine Spit:manis.(3)

Miiller, Naturfyft. I. p.302. n. 5. die Spitsmaus." Tab. I\%. fig. 3 .

Zinmersnann, geogr. Zoolog. II. p. I4. p. 382. 11. 3 Iо. die gemeine Spitzmans.

Lejke, Naturge\{chichte, p. 155. n. 3. die genieine Spitzmaus.

\section{A a 4}

Borow-

(3) Diefe Thiere zeichnen fich befonders durch ihre Gefchicklichkeit im Graben, Gefchwindigkeit im Laufen, und dureh ihre luftigkeit aus. Sie halten fich im Freyen in Gefellfchaft zufammen, in Gebänden aber wohnen lie einzeln in Winkeln, wo es feucht ift. Ihre Nanrung befteht in Mehl, Fleifch, Brod, Regenwürmern, Infekten etc. Sie benagen auch die Wurzeln der Weinföcke, Eichen, Buchen, Wacholder und Oḅtbäume. Wahrfcheinlich gehen fie auch im Felde den jungen Vögeln, die auf der Erde ausgebrüret find, nach; da fie, nach fichern Erfahrungen, die Stubenvögel, z. F. Rothkehlchen, todtbeiffen. Sie begatten fich mehrmals im Iahre, Das Weibchen geht an drey Wochen trächtig, und wirft feine lungen in einer Kluft in dem Mifte oder im Grafe, unverfteckt, auf cinem von allerhand Genifte, Stroh und Grashalmen verfertigten tunden Nefte, Dafs diefe Maus giftig fey, oder den Pferden? in den Leib- krieche, u. f. w. find ungegründete Sagen. Zuwcilen, aber felten, finden fich weilfse Spitzmüufe. 
Borolefky, Thierreich, I. 2. p. 93. 11. 8. die ge. meine Spit:mans.

Bhumenbach, Handb. d. Naturgefch. p. 75. $\mathrm{n}_{4} \mathrm{I}$. Sorè (Araneus) cauda mediocri, abdomine albido; die Spitzmans.

Bechftein, Naturgefch. Deutfchl. I. p. 389. n. I, die gemeine Spitzmans. p. 393. Bifammaus, Miiger, Zismans, Angelmans, Rentmaus."

Funke, Naturgefch. I. p. I22. die Spitzmans,

Halle, vierf. p. 435. die Spitzmans.

Gatterer, vom Nutzen und Schaden der Thiere,

I. p. 26. n. 38. Spit «mans.

Penunant, arct. Zool. II. p. I37. n. \$̣4. die gemeine Spitzmaus.

Neuer Schauplatz der Natur, VII. p. 38I. Spitzmaus.

Onomat. hiftor. nat. VII. p. 204. die gemeine Spitzinans.

Handbuch d. Naturgefch. I. 2. p. 189. die Spitz. macus. Mus Araneus.'

Handbuch der deutfchen Thiergefchichte, p. 43. Spitzinaus.

Gesner, Thierbuch, p. 27i. Miitzer, Spitzmans. Abbild. fchlecht.

Klein, Quadruped. difp. p. 58. Mus Araneus, roltro productiore.

Klein, Claflif. d. vierfüls. Thiere, p. I74. n. Ir. Spitzmans.

Klein, natïrl. Ordn. d. vierf. Thiere, p. 6I. 1. 8. Spitzmaus.

Biiffon, vierfüfs. IV, p, 263. die Spitzmans; m. e. Fig:

Kraft, Ausrottung graufam. Thicre, II. p. 492. Spitzmans.

Batfich. Thiere, I. p. 2Iว. die gemeine Spitzmanus. Gooze, 
Goeze, europäifche Fauna', I. p. 465. die ge. meine Spitzmaus.

Fifcher, Zufätze zur Naturgef́ch. von Livland, p. 40. n. 484. Spitimiaus.

Beyträge zur Beförd. der Naturkunde, I. p. I24. n. I3. die Spitzmans.

Ant'vé und Bechftein, Spatziergänge, III. p. 120. die gemeine Spitzmants.

Iablonjky, allg. Lex. p..IIr. Spitzmaus. Sorex. Pallas, Reife durch Rufs!. II, p, 664. Erxleben, Mammal. p. I25. n. 7. Sorex (Arạneus) cauda mediocri, corpore fubtus albido. Linne', Syft. Nat. Edit. II. p. 48. Sorex. Limné, S. Nat. Edit. VI. p. Io. n. I. Sorex. ILininé, Syft. Nat. Edit. X. I. p. 53. n. 1., Sorex (Araneus) cauda corpore longiore, Linné, Fn. Suec, I. p. II. n. 33. Sorex. Linné, Muf. Adolph. Fr. I. p. Io. Sorex vulgaris.

Graunumn , intr, in hift. nat, mamm. p. 32. VII. Spitzimaus.

Gatterer, brev. Zool. I. p. 36. n. 7. Sorex (Araneus) cauda mediocri, corpore fubtus albido.

Severin, zoolog. Hungar. p. 7r. n. I. Sorex Araneus; feu Mus Araneus.

Miiller, zool. dan prodrom. p. 4. n. 19. Sorex (Araneus) cauda mediocri, corpore fubtus albido.

Heucher, diff. Araneus homini perniciofus et fa: lutaris. Witt. r7or.

Kramier, Auftr. p. 317. Sorex.

Chárleton, exercitat. p. 25. Mus araneus.

Coler, oeconom. rural. et domeft. Mogunt. 1665 . fol. p. 150 .

Schwenkfeld, ther. Silef. p. IIA. Mus arancus. 
Agricola, de asim. fubterr. p. 35. Mus araneus. Pemnant, britt. zool. p. 54. the Shrew Moufe; m. e. Fig.

Pennant, britt. Thiergefch. p. 49. Tab. 9. e.

Daubenton, Mem. de l'acad. de Yaris 1756 . p. 21 r. Tab. 5. fig. 2. Moufaraigne.

Forfter, phil. transact. LXII. p. 380 . foetid Shrew. Plint. hifor. natur. VII. c. 58 . XXIX. c. 4. Mus aràneus.

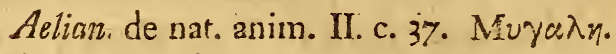

Columella, de re ruf. VI. c. 17. Mus araneus, quem Graeci $\mu$ rya $\lambda$ zv appellant.

8. SUR INAMEN IS. Die furinamifche Spitzmaus.

Zimmnermann, geogr. Zoolog. II. p. 386. n. 320.' die furinamifche Spitz:naus.

Schreber, Säugthiere, III. p. 575. n. 6. die Jurinamifche Spit:mans.

Borowe $k y$, Thierreich, I.2. p. 94. n. Io. die Jus rinami fche Spitzmanu.

9. Pusilius, Die perfifche Spitzmens.

Zimmersnamn, geogr. Zool. II. p. 385 . n. 317. die perfifclie Spitzmaus.

Schreber, Säugth. III. p. -576. n. 7. die per/fische Spitzmaus.

Boroufiny, Thierreich, I, 2. p. 92. n. 5. die per. fische Spitzmanus.

Severin, zool. Hung. p. 7. n. 3. Sorex Pufillus,

10. BR ASILIENSIs. Die brafilifche Spitzmunes. Mïller, Naturfyn. Suppl. p. 36. n. 7. Sorex americanus; die úrafilionifche Spitzmans.

Zimmernuann, geogr. Zool. II. p.385. n. 321. die brafilifche Spitzmans,

Schreber, 
Schreber, Säugthiere, III. p. 577. n. 7. die brafilifche Spitzinicus.

Borowfky, Thierreich, I. 2. p. 94. n. 9. die brafilianijche Spitsmans.

Neuer Schaupl. der Natur, VIII. p.382. die brafilianifche Spitzmaus.

Onómat. hiftor. nat. VII. p. 205. die braflianifihe Spit:malis.

Brifjon, quadr. F. I27. n.. 3. Mufaraneus (Brafilienfis) fufcus, tribus taeniis in dorfo nigris.

Graumanin, intr. in hift. nat, mamm, p. 32 . VIII. die braflicanif che Spitzmanis.

Gatterer, brev. Zool. I. p. 37. n. 8. Sorex (Brafilienfis) fufcus, dorfo friis tribus nigris,

Severin, zool. Hungar. p. 7I. n. 4. Sorex Brafilienfis.

11. ExIL is. Die kieinfte gefchnocinate fibirifche Spitzmaus. (4)

Zimmermann, geogr. Zoolog. II. p. 385, n. 319. die kleinfte gefchwönzte fibirifche Spitzmaus. Schreber, Säugthiere, III. p. 577. n. Io. die kleinAte gefchwöninate fibirifche Spitzmaus.

Bhumenbach, Handb. der Naturgefch. p. 76. n. 3. Sorex (Exilis) minimus, cauda craflifima tereti.

Pallas., Reife, II. p. 664, eine Art unglaublicts kleiner Spitzmäufe.

Erxleben, Mammalia, p. 130. * *

Grmumann, introd, in liffor, nat. mamm. p. 32 .

IX. 2.

A) $V e r$.

(t) Linge des Kơrpers noch nicht trey, des Schwanzes $1 \frac{1}{3}$ Zoll. 
A) Verïnderungen gegen die XIIte, Edition, nund Ver. mehrung der Gattungen diefes Gefchlechts.

Aus dem 23. Gefchlecht der XII Edition ift die zweyte Gattung Caftor. Mofchatus p. 79. hieher verfetzt; init diefer das Gefichlecht um fechs Gattungen vermehrt, und $S$. Fodiens, Surinamenfis, Pufillis, Brafilienfis, und Exilis hinzugekominen.

\section{B) Uribeftimntere Thiere.}

1. Dev Weifs $\approx a h n$.

Zimnmernamn, geogr. Zoolog. II. p. 382. n. 3 Ir. Sorex (Leucodon) atro-cinereus, fubtus albidus, inciforibus candidis, cauda tereti pilofiore; der Weifszahn.

Bechftein, Naturgefch. Deutfchl. I. p. 399. 11. 3. der: WWeifszahal.

2. Die Spitzmaus mit vier Seitigem Schwanze.

Zimmermann, geogr. Zool. II. p. 383. n. 3I2. Sorex (Tetragonurus) atro-cinereus, fubtus pallidior, cauda fubquadrata, dentibus inciforibus brunneis.

Bechftein, Naturgefchichte Deutfchl. I. p. 399.

n. 4. die Spitzmans mit ciem vierseitigen Schewanze.

3. Die Spitzmans mit verkehrtem Schwanze.

Zimnmermann, geogr. Zoolog. II. p. 383. 4. 313.

- Sorex (Confrictus) atro-cinereus, unicolor, caudae bafi confricta.

Bephftein, Naturgefch. Deutchl. I. p. 400. 11. 5. die Spitzonaus mit verkehrtent Schwanse. 
4. Spitzmäufe, welche einen Mofchusgeruch haben. Zimmermann, geogr. Zool. II. p. 387.a. Bofsmann, Reife nach Guinea, p. 298.

5. Die gröjste Spitzimaus vom Cap.

Zimmermann, geogr. Zoolog. II. p. 388. b. Petiver. Gazophylac. nat. et art. Tab. 23. fig. 9.

Mus araneus Capenfis maximus.

Erxleben, Mammal. p. 130. *

6. Die Spitzmaus von 'der Hudfonsbay.

Zimmermann, geogr. Zool. II. p. 388. c. Forfter, philof. transact. LXII. p. 38I. Shrew. Erxleben, Mammal. p. 1зо. **:

20. GESCHLECHT. ER IN ACEu s. Der Igel. Miiller, Naturfyft. I. p. 304. Gen. XX. Zinmermann, geograph. Zoolog. II. pag. 392. Gen. X̃XV.

Schreber; Säugthiere, III. p. 579. Gen. XXI. Lefke, Naturgefch. p. 159. Gen. XXII. Boroneky, Thierreich, I. 2. p. 95. Gen. XX. Bechftein, Naturgefch. Deutfchl. I. p. 367. Neuer Schauplatz der Natur, VIII. p. 434. Onomat. hift. nat. III. p. 8I3. Bat $\int h$, Thiere, I. p. 209. Gen. XXX. Erxleben, Mammalia, p. I69. Gen. XVIII. Grammann, introd. in hiftor. nat. mamm. p. 38 . Gen. XVIIII.

Gatterer, brev. Zoolog. I. p. 46. Gen. XVIII. Penniant, Syn. quadr. p. 3I6. Gen. XXXV. Briffoin, quadr. p. I28. Gen. XXVIII. Herrmann, tab. affinit. animal. p. $2 \Omega_{3}$ 
I. Eu O OAEUs. Der gemeine Iyel. (1)

Ihitller, Naturfyft. I. p. 304. der gemeine Igel. Tab. 17. fig. I.

Zimmermann, geogr. Zoolog. II. p. 21. p. 392. 11. 328. der genieine Igel.

Le $\int k e$, Naturgefch. p. I59. der gemeine Igel.

Borowfky, Thierreich, I. 2. p. 96. n. 2. der ge= meine Igel.

Blumenbach, Handb. d. N. G. p. 66. n. r. Hyftrix (Erinaceus) auriculis rotundatis, naribus crifatis; der Igel.

Bechfiein, Naturgefch. Deutfchl. I. p. 368. n. I. der genneine Igel.

Finnke,

(1) Man unterfcheidet insgemein zwey Varietaten bey diefer Gattung, Hurdsigel und Schweinigel, deren Verfchiedenheit befónders in der Bildung der Schnauze beftehen foll, aber wohl tmehr in der Einbildung beftchen mag. Der Igel ift ein unfchuldiges.furchtfames Thier, das Niemancien beleidigt, und auch nicht einmal beifst, wenn es beleidigt wird. Zur äufserften $\checkmark$ ertheidigung benetzt er fich mit feinem übelriechenden Urin: Wenn er fich einmal zurammengerolle hat, fo läst er fich eher zerreiffen, als mit Gewalt zur Aufwickelung bringen., Doch kann man ihn durch Begiefsen imit Waffer, und Eintauchen in daffelbe, wodurch ihm das Athemholen benommen wird, dazu bewegen. Der Fuchs bedient fich dazu feines ftinkenden Harns. Durch die V'erzehrung mancherley Ungeziefers. tvird der Igel ein überaus nüzzliches Thier, und man verfolgt: ilin nur, wegen des vielleicht unbeträchtichen Schadens, den er an dem Federviehe thut. Man will auch bemerkt, haben, dafs er die Hühnernefter plïndert, und die Eyer verzehrt. Das Weibchen geht fieben Wochen trachtig, fingt die lungen, die anfangs weifs, und axf ihrer Haut nur mit Spuren von Stacheln verfehen find, vier Wochen; und träst ihnen Schnecken, Regenwürmer, Weintrauben, und abgefallenes Obft zu ihrer Nahrung zu. Merkwürdig it es, dafs der lgel fogar fpanifche Fliegen frifst, und deren tiber hundert auf einmal ohne Schaden verzebren kann. Im Herbfte wird er fehr fett. Sein Fleifch foll aber nicht efsbar feỹ. 
Funnke, Naturgefch. I. p. 178. der Iget. Ebert, Naturlehre, I. p. 355. der Igel. Tab. 17. Halle, vierfüfs. p. 463. der gemeine Schweinigel. Theyer, Thiere, I. Tab. 95. 96. der Igel. Abbild. git.

Gattcrer, voin Nutzen und Schaden der Thiere, I. p. 47. n. 48. der Igel.

Beckmann, Naturhiftorie, p. 26. n. 12. der Igel oder Schweinigel.

Pennant, arct. Zool. II. p. 149. 13. 89. der gemeine Igel.

Sander, ökon. Naturgefch. I. p. 260. der Igel. Handb. der Naturgefch. I. 2. p. I83. der Igel.

Handbuch der deutfchen Thiergefchichte; p. 37. der Igel.

Newer Schauplatz.d. Natur, VIII. p. 434. der Igel. Onomat, hift. nat. III. p. 8r4. der enropiäiche Igel. Gesnev, Thierbuch, p. 227. Herinaceus; ein Igel. Abbild. gut.

Klein, quadrup. difp. p. 66. Acanthion vulgaris noltras.

Klein, Claflif. der vierfüfs. Thiere, p. 199. Igel, Schweinigel.

Klein, natürl. Ordnung d. vierf. Th. p. 70. n. r. geneinev Igel, Schweinigel.

Büiffon, vierf. V. p. I6. dier Tgel; m. e. Fig. Kraft, Ausrottusg graufan. Thiere, - II. p. 434. dier Igel.

Batfch, Thiere, I. p. 214. dev geneine Igel.

Goez:, europäifche Fauna, 1. p. 4 18. der europaiifche Igel.

Bock, Naturgefch, von Preuffen, IV. p. 62. n. Ig. der europiiiliche Igel, Scheeinigal.

Fifcher, Naturgefch. von Livland, p. 56. n. I7. Schweinigel. 
Pontoppidan, Norwegen, II. p. 55. der Igel. Cetti, Naturgefchichte von Sardinien, I. p. 233. der Tgel.

Pervault, Charras und Dodart, Abhandl. zut Naturgefch. I. p. 305, anatomifche Befchreib. von vier Igeln. Tab. 4.2. der Igel. Tab. 43. die Zergliederung. p. 319. Hunzie - u. Schweinigel, Dornfthwein, Heckenfcherein.

Krinitz, ökon. Encykl. XXIX: p. 416. Igel. Klïgel; Encyklop. I. p. 238. der gemeinê Igel. Hamburg. Magazin, XI. p. 306. Zergliederung der Igel.

Beyträge zur Beförd, der Naturkunde, I. p. 125. n. 14. Igel, Schueeinigel.

Becknann, phyf. ökon. Bibl. X. p. 437. der Igel. Schwed. Abhandl. XVI. p. 129. 1 gel.

Hepse, wohlredender läger, p. 184. Tgel.

Börneq', Stadt - u. Landwirthfchaft, II. p. I92. Igel. Merklein, Thierreich, p. 91. Igel.

Andve' und Bechftein, Spatziergänge, I. p. 266. der gemeine Igel.

Erxleben, Mammal. p 169. n. I. Erinaceus (Europaeus) auriculis rotundatis, naribus criftatis.

Limé, Syft. Natur. Edit. II. p. 45: Erinaceus fpinofus auriculatus.

Jinné, Syft. Nat. Ed. VI. p. 6. n. I. Erinaceus auriculatus.

Limné, Syft. Nat.' Edit. X. I. p. 52. n. I. Erina ceus (Europaeuś).

Linné, Fn. Suec. I. p. 6. n. 16. Erinaceus fpino: fus auriculatus.

Linné, Muf. Adolph. Fried. I. p. 6. Erinaceus (Vulgaris) auriculatus.

Linn?é, Reife d: Gothland, p. 282. Erinaceus europaeus; Igel. 
Graumanin, intr. in hilt. nat. mainm. p. 38. I. Igel, Zaunigel, Schweinigel, Sauigel.

Gatterer, brev. Zool. I. p. 46. n. I. Erinaceus (Europaeus) auriculis rotundatis, naribus criAtatis; der Igel; 'Zaninigel.

Miiller, prodrom: Zool, dan. p. 4. n. 2o. Erina: ceus (Europaeus) auriculis rotundatis, nari: bus criftatis:

Kramer, Auftr. p. 313. Erinaceus auriculatus:

Charleton, exercitat. p; 19. Erinaceus:

Schwenkfeld, theriotroph. Silef. p. 96. Herinaceus:

Wagner, hift. nat: Helvet. p: r75. Erinaceus terreftris. Echinius:

Rzacz. hiftor natur. Polon. p. 233. auct. p. 326 .

Herinaceus:

Agricola, de annim. fubterr. p. Io. Erinaceus:

Mufeum Worm. p. 334. Echinus terreftris.

Sibbald, Scot. illuftr. P. II. T. II. p. Ir. Erinaceus: Forsk. faun. orient: p. III. Erinaceus.

Pallas, nov. comment. Petrop. XIV. p. 578 .

Pennanit, britt. Zool. p. 5 I. the Hedge-hog or Urchin.

Peninant, britt. Thiergefch. p. 46. Tab. Io.

Flacourt, hift. de Madagafcar. p. 152. Heriffoin.

Sora.

Forfter; philof. transact. LVII. p. 342 . Erinaceus europaeùs.

Plin. hift: nat. VIII. c. 37 . c. 58. X. c. 63. Herinaceus:"

Ariftot. hif. anim. İ. c. 7. n. 62 . III. c. I. n. 6 . V.c. 2. n. 19. IX. c. 9. n. ro7. Exเvos.

Aeliani. de nat. anim. III. c. Iọ. IV. c. I7. VI.

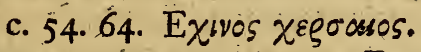

Oppian. cyneget. II. 598. Exivos.

B b

2. IN- 
a. IN A U R Is. Der ungeöhrte Igel. $\left({ }^{2}\right)$

Miiller, Naturfyf. I. p. 307. n. 2. der Kahlohr.

Zimmermann, geogr. Zool. II. p. 393. 11. 330. der ungëhrte Tgel.

Boloweky, Thierreich, I. 2. p. 97. n. 3. der weilse amerikanifche Igel.

Halle, vierf. p. 464. Tab. 3I. der, weifse anerikaniifche Igel.

Neuer Schauplatz der Natur, VIII. p. 435. der Kahlohr.

Onomat. hif. nat. III. p. \&14. Erinaceus amerio. canus; der amerikanifche Igel.

Klein, Quadrup. difp. p. 66. Acanthion echinatus; Erinaceus americanus albus.

Klein, Claffif. d. vierf. Th. p. 199. amerikanifcher Schiseinige!.

Klein, natürl. Ordn. d. vierf. Thiere, p. 70. n. 2. furinamifriser Igel.

Biiffon, vierf. XIII. p. I30: dor ungeöhrte Igel. Bankroft, Naturgefch. von Guiana, p. 86. der amerikanifche Igel.

Fermin, Befchr. von Surinam, II. p. I03. Erinaceus furinamenfis; der Igel.

Adanfon, Reife nach Senegal. p. 247. der Igel?

Erxleben, Mammalia, p. 173. n. 3. Erinaceus (Inauris) auriculis nullis.

Linné, Syft. Nat. E.dit. II. p. 45. Erinaceus fpinofus, vix auriculatus.

Linne', Syft. Nat. Edit. VI. p. 6. n. 2. Erinaceus fubauriculatus.

Graumann, intr. in hif. nat. mamm. p. 38. III. der Kahlohr.

(2) Länge des Kürpers acht Zoll. Sibreber, halt ilın mit dem Tendirac für einerley; p. 584 . 
Raj. Syn. quadr. p. 232. Echinus indicus albus. Pennant, Syn. quadr. p.318. n. 249. the Guiana Hedge-hog.

Gatterer, brev. Zool. I. p. 44. n. 3. Erinaceus (Inauris) auriculis nullis.

3. M A a c cens is. Der malackifche Igel.

Miiller, Naturfyft. I. p. 308. n. 3. der malaccifchi Igel. Tab. 19. fig. 2. Seb.

Zimmermann, geogr. Zool..II. p. 394. a. Erinaceus (Malacceinfis).

Borowfky, Thierieich, I. 2. p. 98. n. 4. der malackische Igel.

Bhumeinbach, Handb. der Naturgefch. p. 66. n. 2: Hyftrix (Malaccenfis) auriculis pendulis. Funke, Naturgefchichte, I. p. 178 . der Igel von Malakka.

Halle, vierf. p. 468. Tab. 30. das Stachelfchwein. Gatterer, vom Nutzen und Schaden der Thiere; I. p. 49. n. 49. der malackifche Igel.

Neuer Schaupl. d. Natur, VIII. p. 435. der malak. kifche Igel.

Onomat. hift, nat. III. p. 817 . der Igel von Ma: lacca. IV. p. 446. das Stachelfchuein voin Malacca.

\section{$\mathrm{Bb}_{2}$}

Frifch,

(3) Der in der Gallenblafe diefes Thiers zuweilen erzengte Stein, der unter dem Namen Lapis porcinus, oder Scibweinftin bekannt ift, wurde ehemals für ein herrliches Atzeneymitiel gehalten, und ein Loth davon mit einigen hundert Thalern bezahlt. Fr fîeht fchwärzlich aus, ift fehr bitter, und theilt Gefchnack und Farbe dem Waffer mit, worin er liegt." In Europa ift er fchon längft nicht mehr im Gebrauch. Scbreber if geneigt diefs Thier, mit Hyftrix Criffata für einerley zu halten. S. Th. IV. p. 602. wovon aber Erxleben das Gegentheil behauptet. S. Schulz de bile medicina. Guetting. 1775. p. S. 
Frifch, Naturfyft. der vierf. Th. p. Io. n. 3. das -malekkiche Stachelfchwein.

Biiffon, vierf. XIII. p. I32. der malackifche Igel; in.e. Fig.

Batfch, Thiere, I. p: 215 . der malakkijche Igel. Kiein, Quadruped. difp. p. 66. Acanthion aculeis longiffimis.

Kleine, Claflif. der vierfüfs. Th. p. 200. Stachel. fthwein, Stachelthier mit den längften Stacheln. Klein, natül, Ordn. d. vierf. Th. p. 70., n. 2. Stacheifchwein mit den längften Federn.

Erxleber, Mammalia, p. 173. n. 4. Erinaceus (Malaccenfis) auriculis pendulis.

Limné, S. N. Edit. Il. p. 46 Hyfrix pedibus pentadactylis, cauda exferta.

Limné, Syft. Nat. Edit. VI. p. 9. n. 4. Hyfrix pedibus pentadactylis, cauda truncata.

Grawnans, intr. in hiftor. nat. mamm. p. 38. IV. der malactifche Igel.

Gatterer, brev. Zoolog. I. po 47. n. 4. Erinaceus (Malaccenfiss) auriculis pendulis.

\section{A U R I T U S. Der lengöhrigte Igel. (4)}

Zimmermann, geogr. Zool. II. p. 393 . n. 329. der langährigte Igel.

Lefke, Naturgefchichte, p. 160. 1. 2. der langöhrige Igel.

Borowfky, Thierreich, I. 2. p. 95. n. t. der langöhrige Igel. Tab. 20.

Biiffon, vierfüls. XIII. p. I35. der langöhrigte Igel.

Gatte.

(4) Lange' des Körpers gegen fieben Zoll. In Aftrachan halt man ihn baufig um der Maufe willen, und ernährt ihn. vorzüglich mit Milch. 
Gatterer, vom Nutzen und Schaden der Thiere,

I. p: 50. n. 50. der giofsöhrigte Igel.

Georgi, Reife, I. p. I60. Io $c h$, auf ruffilch.

S. G. Gmelin, Reife durch Rufsland, II. p. I74 dev geöhrte Igel.

Bechinann, plyf. ökon. Bibl. V. p. 337. Stacheligei mit langen $O_{\text {irren. }}$

Bat $\int c h$, Thiere, I. p. 214. der langöhrige Igel.

5. Setosus. Der Tendrac. (\$)

Zimimermann, geogr. Zoolog. II. p. 393. n. 33r. der Tendrac.

Borovefky, Thierreich, I. 2. p. 98. n. 5. Erinaceus Tendrac; der Tendrak.

Neuer Schauplatz d. Natur, VIII. p.783. Teirdrak. Dapper, afrikan. Inf. p. 32. Tendrak.

Frifch, Naturfy h. p. 5. n. 5. Erinaceus africanus; der afrikanische Igel oder Tendrak; der kleinAte Igel.

Biiffon, vierf. XIII. p. I24. der Tendrac; m. e. Fig. it. p. 128.

Sonnerat, Reife nach Oftindien und China, II.

p. Ir4. der kleine Tandrek von Madagafcar.

Flacourt, hift. de Madagafcar. p. I5I. Tendrac. Erxleben, Mammalia, p. 175. * (Species ob: fcura.)

\section{$\mathrm{Bb}_{3}$}

Grau:

(5) Diefe Thiere haben einen langfamen Gang; fie grunzen, wie die Schweine, und haben daher von den Europäern die Namen : Erdfchwein und Stadbelfchwein erhalten. Sie graben fich in die Erde, und verfchlafen drey Monate des lahrs. Bey Tage verbergen fie fich, und kommen ert nach Untergang der.Sonne hervor, ihre Nahrung zu fuchen, die aus Früchten und Kräutern befteht. Ihr Körper ift eigentlich ein blofser Fettklumpen. Die Bewohnér von Madagafcár verfpeifen fie, aber eben.nicht als befondere Leckerbiffen. 
Graumann, introd. in hif. nat. mamm. p. 38. V. Erinaceus (Setofus) auriculis brevibus, fpinis brevibus, cauda breviflima.

\section{Ecauda tus. Der Taniec. $\left({ }^{6}\right)$}

Zimmermann, geogr. Zoolog. II. p. 394. 11. 332. der Tourec.

Borow Jky, Thierreich, I. 2. p. 98. ad n. 5. der Tunrek.

Neuer Schauplatz der Natur, VIII. p.783. Tanvek. Biiffon, vierfüs. XIII. p. 124. 128. der Tanrec; m. e. Fig.

Gatterer, vom Nutzen und Schaden der Thiere, I. p. 50. n. 51. der oftindifche Igel.

Frifsh, Naturfyft. d. vierf. Th. p. 5. n. 4. Erina. ceus indicus. Indifche Igel, oder der Tamvek. Erxleben; Mammal. p. I75.**. (Spec. obfcur.) Cauche, Kelation de Madagafcar, p. I27. Certaines beftes en $l^{\circ}$ isle Saincte Marie.

Pennant, Synopl, quadr, p. 317. n. 248. the afratic Hedge - hog. (mit dem Tendrak verbunden.) Graumann, intr, in hif, nat. mamm, p. 39. VI. Erinaceus (Ecaudatus) corpore minus f pinofo, auriculis apparentibus.

Gatterer, brev. Zool. I. p. 47. n. 5. Erinaceus (Tanrec) auriculis brevibus, roftro longo.

A) Veränderungen gegen die XIIte Edition, und Ver. mehrung der Gattungen diefes Gefchlechts.

Diefs Gefchlecht if mit drey Gattungen vermelirt, und E. Auritus, Setojus und Ecaudatus hinzu. gekommen.

B) Un?

(6) Pennant, Erxleben, und Borowf ky halten diefe Gattung mit der vorhergehenden für einerley. 


\section{3) Unbeftimmtere Thiere.}

\section{Der fibirifche Igel.(7)}

Erxleben, Mammal. p. I72. Erinaceus (Sibiricus) auriculis planis, nafo fimplici.

Seba, thef. I. p. 79. Tab. 49. fig. 4. Erinaceus

(Sibiricus) fuperne confpicuus, capite ad pe. des contracto.

Seba, thef. I. p. 79. Tab. 49. fig. 5. Erinaceus

(Sibiricus) mas fupinus.

Briffon, quadrup. p.'129. n. 2. Erinaceus (Sibiricus) auriculis planis.

Halle, vierf. p. 465. der fibirifche Igel.

Klein, Qnadrup. difp. p. 66. Acanthion echinatus.

Echinus fibiricus.

Klein, Claffif. der vierfüfs. Th. p. 200. fibirifcher Schrveinigel.

Klein, natürl. Ordn. d. vierf. Th. p. 70. n. 3.' fibirifcher Igel.

Onomat. hift. nat. III. p. 817 . der fibirifche Igel.

Graumann, intr. in hift, nat. mamm. p. 38. II. fiberifche Igel.

Severin, tentam. zool. p. 80. n. 2.

(7) Erxleben hält ihn für eine Varietät vom gemeinen Iget. In der Biifforfchen Naturgefch. der vierf. Thiere aber XIII. p. 35. find die mehreften diefer Synonymen bey, dem langöbrigen Igel mit angeführt. 


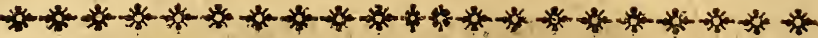

VIERTE ORDNUNG.

GLIRES. (Rattenartige, nagende. Thiere.)

2I. GESCHLECHT, HY S T I X. Das Stachelthier.

Milller, Naturfyftem, I. p. 3Ir. Gen. XXI:

Zimnermann, geograph. Zoolog. II. p. 395.

Gen. XXXVI.

Sichreber, Säugthiere, IV: p. 599. Gen. XXIII.

Lefke, Naturgefch. p. I6r. Gen. XXIII.

Borowe $k y$, Thierreich, I: 3. p. 4. Gen. XXI.

Blumenbach, Handb. d. Naturgefch. p. 68. Gen, X.

Bechftein, Naturgefch. Deutfchl. I. p. I40.

Onomatol, hiff, nat. V. p. 442,

Biiffon, vierf. XIII. p. 88 ff.

Bat $\int c h$, Thiere, I. p. 242. Gen. XLI.

Merrem, vermifchte Abliandl. aus der Thierge:

fchichte p. 5. 17.

Erxleben, Mammalia, p. 340. Gen. XXXIII.

Graumann, intr. in hiftor. nat, mamm. p. 56 .

Gen. XXXIII.

Gatterer, brev. Zool. I. p. 98. Gen. XXXIII. Boddaert, Elenchus, p. 127. Gen. XXV.

Pennant, Syn. Quadr. p. 260. Gen. XXVIII, Briffon, quadr. p. 85. Gen. XX.

Hermuann, tab. affin. animal. p. 78.

1. CR rs tata, Das Stachelfchwein. (')

Miiller, Naturfyftem, I. p. 312. das afrikanifche Stachelfchwein.

Zimmer:-

(1) Mit dem Schweine hat diefs Thịer, das Grunzen ausgenommen, garlkeine Aehnlichkeit, und pievon ift vielleicht der 
Zimmiermann, geogr, Zoolog. II. p. 22. p. 3959

n. 333. das Stachelfchieein.

Lejke, Naturgefchichte, p. I6I. n. I. das Stachelfchwein.

Borowesy, Thierreich, I. 3. p. 5. n. 2. das gehaubte Stachelfchwein.

Blumenbach, Handb. der Naturgefch, p. 66. n. 3. Hyftrix (Criftata) capite criftato, cauda ab. breviata; das Stachel/chwein.

Bechftein, Naturgefch. Deutfchl. I. p. r40. das Stachelf chwein.

Funke, Naturge[ch. I. p. 179. das Stachelfchwein. Ebert, Naturlehre, I: p. 356. das Stachelfchwein. Tab. 18 :

Halle, vierfüls. p. 465. das Stachelfchweinn mit dem Bufche am Kopfe.

Gatterer, vom Nutzen und Schaden der Thiere, I. p. 213. n. IIr. Stachelschwein.

$$
\text { B b } 5
$$

Bẹck:

Name hergenommen, wiewohl Sparrmann vermuthet, dafs fein Fleifch, welches dem Schweinefieifche fẹr ähñlich fey, dięfe Benennung veranlafst habe. Iung gefangen lärst es fich zwar zăhmen, behält aber doch immer eine geviffe Furchtfamkeit. Die Stacheln find oft anderthalb Fufs lang. Zuweilen, befonders im Herbf, fallen fie $\mathrm{hhm}_{\text {aus; }}$ dafs er fie aber gegen feine Verfolger von fich fchiefle, darüber if noch kein glaubwürdiges Zeugnifs vorhanden, ob es gleich Wolf mit vialliger Gewifsheit behauptet. Schöpf und le Vaillant befchreiben indeffen die Verwundungen, welche an Menfchen oder Thieren, durch diefe Stacheln, wenn das Thiẹ lich damit vertheidigt, verurfacht yerden, fehr gefăhrlich. Am Cap fiehet man die Stachęlfchweine als Leckerbiffen auf den vornehınften Tafeln, aber das Fleifch mufs gerauchert werden. In Rom wird es auf dem Markte verkaufte. Die Zähne gebraucht das Thier ñicht als Waffen, auffer um fich in Freyhęix zu fetzen,. wenn es in hü!zerne Behälțiffe eingefperrt wird, die es bald zernaget. Von dem Schrweinẹteine hat. Sparrmann am Cap nichts gehörț 
Beckmann', Naturhiftorie, p. 22. n. 6. das Stachelfcinvein. (.Begreift mehrere Gattungen unter fich.)

Neuer Schaupl. der Natur, VIII. p. 435. das Stachelfchroein.

Onomat. hift. nat. IV. p. 443. das Meer/chwein, Dornfchwein, Stachelfchwein.

Klein, Quadruped. difp. p. 66. Acanthion criAatus.

Klein, Claffif. d. vierf. Th. p. 201. Stachelthier mit einem Kamm.

Klein, natürl. Ordn. der vierf. Th. p. 7r. n. 5. gekröntes Stachel/chwein.

Gesner, Thierbuch, p. 67. Dornfcheein. Fig. mittelm.

Biuffon, vierf. XIII. p. 88. das Stachelfchwein.

Bat fch, 'Thiere, I. p. 243. das gehassbte Stachelfolwein.

Kraft, Ausrottung graufam. Thiere, II. p. 416. Dorn - oder Stacheljchwein.

Kolbe, Vorgeb. d. g. Hoffn. p. 66. Tab. 6. fig. 6. Edit. in 4. p. 337. das Stachelfchwein.

Merrem, zool. Abhandl. p. 18. n. 3. Haubenfta: chelfchwein.

Sparrmann, Reife n. d. Vorgeb. d. g. H. p. I46. das afrikanifche Stachelfchwein; Yzer-verken. (Eifeufch wein.)

Le Vaillant, Reife nach Afrika, I. p. 226. das Stachelfchwein.

Wolf, Reife nach Zeilan, p. I22. das grofse Stacheifcherein.

Balciaeus, Malabar und Zeylon, p. 422. StachelSchweine.

Brydone, Reife d. Sicil. u. Maltha. I. p. 12. I3. Haffelquift, Reife n. Paläftina, p. 555. Hyftrix. 
Bofsmann, Reife nach Guinea, p. 295 . Shaw, Reife, p. 156. Stachelfchweine. Schöpf, Reife durch die nordamerikan. Staaten, I. p. 18. Stachelfchweine.

Frich, Naturfyft d. vierf. Th. p. 1o. n. 2. Hyftrix africana maior; das Quinica der Afrikaner?

Berlin. Samml. VIII. p. 263. Stache!fchweine.

Merklein, Thierreich, p. 92. der Meerigel oder das Stachelfchwein.

Oekon. Zoologie, p. 31. n. 37. das gemeine Sta. chelschwein.

Perrault, Charras und Dodart, Abhandl. zur Nat turgefch. I. p. 305. anatomifche Befchreibung von acht Stachelfchweinen. Tab. 42. das Stachelfcheein. Tab. 43. die Zergliederung.

Erxleben, Mammal. p. 340. n. T. Hyftrix (Criftata) palmis tetradactylis, plantis pentadacty. lis, capite criftato, cauda abbreviata,

Linne', Syft. Natur. Edit. II. p. 46. Hyftrix manibus tetradactylis, plantis tridactylis, capite criflato.

Linné, Syft. Nat. Edit. VI. p. 9. n. I. HyArix manibus tetradactylis, plantis pentadactylis, capite criflato.

Limné, Syft. Nat. Edit. X. I. p. 57 . n. x. Hyfrix (Crillata) palmis tetradactylis, plantis pentada. ctylis, capite criftato, cauda abbreviata.

Gratmann, intr. in hift. nat. mamm. p. 56. I. Stachelf chwoin.

Gatterer, brev. zool. I. p. 98. n. I. Hyftrix (Criflata) palmis tetradactylis, plantis pentadactylis, capite crifato, cauda abbreviata; das ge meine Stachelfchwein.

Severin, zool. Hungar. p. 8I. Hyftix.

Kramer, 
Kramer, Auftr. p. 314. Hyftrix manibus tetrada: ctylis, plantis pentadactylis, capite criftato.

Charleton, exercitat. F. I9. Hyftrix. Mufeum Worm. p. 335 . Hyftrix.

Tavernier, voyages, 1.p. 344. Porc-epys. Mem. pour fervir à l'hift. des animaux, II. p. 33. Tab. 4r. Porc. épic.

Agricola, de animal. fubterr. p. 486.

Ludolf, hift. Aethiop. I. c. Io. n. 74. Hyftrix,

Dampier, voyage. Amf. I7II. III. p. I39. Ariftot. hif. anim. I. c. 7. n. 62. VIII. c. 22.

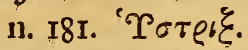

Plin. hift, nat, VIII. c. 35 . Hyftrices.

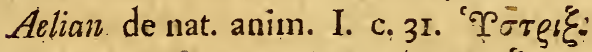

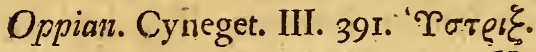

Scaliger, exercitat, 198. p. 64I. Hyftrix,

Clandian. epigramm. 45. Hyhrix.

2. Prehensilis. Der Cuondu.

Miiller, Naturfyft. I. p. 314: das gefchwcinzte Stachelfchwein.

Zimmernnann, geogr. Zoolog. II, p. 396. n. 336. der Cuandu,

Le $\int k e$, Naturgefch. p. I62. n. 2. der Kuandh. Borow $k y$, Thierreich, I. 3. p. 6. n. 3. der Kuare: du; das gefchwö̈nzte Stachelfchwein.

Gatterer, vom Nutzen und Schaden der Thiere,

I. p. 2 I4. n. ¥12. der Kuandu.

Neuer Schaupl: d. Natur, II. p. I74. Coendu, Onomat. hif. nat. IV. p. 450 , das Stachelfchweinz mit dem verlängerten halb-nackten Schwanze. Gesner. Thierbuch, p. 70. indianifches Dorn. fchwein ; mit Marcgr. und Hernand. Figg. Büffon, vierfuîls: XIII. p. I04. der Cucnudui; m. e. Fig. 
Frifch, Naturfynt. d. vierf. Th. p. ro. n. $6 . \mathrm{Hy}$ Arrix Cuandu; das eiferne Ferken dér Holländer. Fermin, Befchri von Surinam, II. p. 81. Hyftrix hougus caudatus, brevioribus aculeis; das Stachelf shwein.

Mìlina, Naturgefch. von Chili . p. 259. das chilefifche Stachellchwein.

Batfch, Thiêre, I. p. I43. der Kuandui. Merrem, zool. Abhandl. p. 17. 11. I. Cuandal. Erxleben, Mammal. p. 342. n. 2. Hyfirix (Prehenfilis) pedibus tetradactylis, cauda elongata, preheivifili, fubnuda.

Limné, Syft. Nat. Edit. VI. p. 9. n. 2. Hyfrix, pedibus tetradactylis, cauda exferta, prehenfili, feminuda.

Linné, Syft. Nat. Edit. X. I. p. 57 . n. 2. Hyfrix (Prehenfilis) pedibus tetradactylis, cauda elongata, prehêfili, feminuda,

Graumann, intr, in hiftor. nat. mamm. p. 56. II. das gè fchwoinzte Stachel]chwein.

Gatterer, brev. zool. It p. 98. n. 2. Hyfrix (Prehenfilis) pedibus tridactylis, cauda elongata,

prehenfili, feminuda.
Des Marchais, voyage en Guinée, III. p. 288 . Cuaindu, Chat epineux.

Miffion du P. $d$ 'Abbeville au Maragnon. Paris, 16r4. feuil. 249. Coendu.

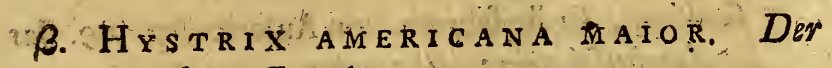
gröfsere. Cutandu.

Zimimermann, geogr. Zool. II. p. $397 \%$

Schreber, Säugthiere, IV. p. 605 .

Biiffon, vierfüfs. XIII. p. ro8.

Bonit. hilf. nat. ind, orient. p. 54. Hyftrix? mit Pifon. Fig. 
y. Hystrix novae hispaniae. Dey Hoitzlacuatzin.

Biiffon, vierf. XIII. p. 107. 109.

Schreber, Säugthiere, IV. p. 605.

3. Dorsata. $\operatorname{Der} \operatorname{Ur} \operatorname{Tont}_{4}\left({ }^{2}\right)$

Miiller, Naturfyftem, I. p. 315. n. 3. der Sta: chelvïcken.

Ziminermann, geogr. Zool. II. p. 396. in. 335. der Urfon:

Borowfky, Thierreich, I. 3. p. 4. 11. I. das ver. larvte Stachelfihwein.

Halle, vierf. p. 406. das verlarvte Stachelfcheein von der Hudfonsbuicht.

Gatterer, vom Nutzen u. Schaden der Thiere; p. 215 . n. 113. Urjon.

Onomat. hif. nat. IV. p. 445. das Meerfchwein von Cenadi.

Klein, Claflif. d. vierfüfs. Th. p. 152. Afterhaaje von der Kiifte Hudfon.

Klein, natürl. Ordn. d. vierfüfs. Th. p. 54 . n. 8. Afterhaje aus der Hundonsbay.

Biiffon, vierf. XIII. p. I15. der Ur fon; m. e. Fig.

Carver, Reifen durch Nordamerika, p. 371. das amerikanifche Stachelfchivein.

Pennonut, arct. Zool. II. p. rog. n. 5 o. das cancdifche Stachelfchwein.

Schlözer, Erdbefchreib. von America, p. 109. das Stachelfchiecin; Porcupine.

Ellis,

(2) Lánge des Kürpers zwey Schuh, des Schwanzes, acht Zoll. Die Wilden efien das Fleifch, und gebrauchen die Stacheln ftatt der Nadeln. Der Geftalt nach gleicht das Thier einem Biber. Im Winter frifst es die Fichtenborke, daher alsdana reine Knochen grïn gefarbt find. 
Ellis Reife nach Hudfons-Meerbufen, p.39. das Stachel] chroein.

Batfch, Thiere, I. p. 243. das verlarvte Stachel: fonwein.

IMerrem, zool. Ábhandl. p. 18. n. 4. verlarvtes Stachelfchwein.

Becknann, phyf. ökon. Bibl. V. p. 204. Hyfrix dorfata.

Erxleben, Matnmal. p. 345. n. 3. Hyfrix (Dor-

- fata) palini tetradactylis, plantis pentadacty. lis, cauda mediocri, dorfo folo fpinofo.

Linné, Syft. Nat. Edit. X. I. p. 57. 11. 3. Hyftrix - (Dorfata) palmis tetradaefylis, plantis pentadactvlis, cauda elongata, dorfo folo fpinofo.

Linné, Syft Natur. Edit. XII. I. p. 76.n 3. Hy. ftrix (Dorfeta) palmis tetradactylis, plantis pen. tadactylis, cauda mediocri, dorfo folo fpinofo.

Grawnonn , intr. in hift nat. mamm. p. 56. III. der Stacheiniicken.

Gatterer, brev. Zoolog. I. p. 99. n. 3. Hyftix (Dorfata) palmis tetradactyli, plantis pentada. ćylis, cauda mediocri, dorfo folo fpinofo.

Penucnt, Syn. quadr. p. 266. n. 196. the Canada Porcupine.

Forfter, philof. transad. LXII. p. 374. Canada Porcupine.

4: M a c r o u R. Das lang/chwänzige Stachelthier. Iniiller, Naturfyft. I. p. 316. Langfchwanz.

Zimmermonn, geogr. Zool. II. p. 395. 1.334. das lang chioünzige Stachelthier.

Boroufky, Thierreich, 1. 3. p. 6. n. 4. das lang. Schwänzige Stachelthier.

Halle, vierf. p. 496. das langfchwänzige StachelScinwein. 
Gatterer, vom Nutzen und Schaden der Thiere, p. 216. n. II4. Lang chwanz.

Onomat. hift. nat. IV: p. 448. das langfchecïn- zige Stachelfchweini.

Klein, Quadruped difp: p: 6\%. Acanthion cauda praelonga, acutis pilis horrida, in exitu quafi paniculata.

Kilein, Claflif. d. vierfüfs. Thiere, p. 203. Stachelthier mit einem Sehr laingen Schwanze.

Ǩlein, natïrl. Ordn. d. vierf. Thiere, p. 7r. n. 6. Sonderliches Stachelfchwein.

Biiffori, vierfüls. XIII. p. I2I. das laingfchwänzige Stachelthier; m. e. Fig.

Batfch, Thiere, I. p. 242. das langfchwoinzige Stacheifchwein.

Merrem, zoolog: Abhandl. p. í. n. 2. Lang. Schionio.

Erxleben, Mammal. p. 346. n. 4. Hyfrix (Ma: croura) pedibus pentadactylis, cauda longiffima; aculeis clavatis.

Lisiné, Syft. Nat. Edit. II. p. 46. Hyftrix pedibus pentadactylis, cauda exferta.

Linnié, Syff: Nat. Edit. VI. p. 9. n. 3. Hyftrix pedibus pentadactylis, cauda exferta.

Linné, Syft. Nat. Ed. X. I. p. 57. in: 4. Hyfrix (Macroura) pedibus pentadactylis; cauda elongata, aculeis clavatis.

Grausstann, intr. in hift, nat. mamm. p. 57 IV. das lang/chwönzige Stachelfchwein.

Gatterer; brev. zool. I. p. 99. n. 4. Hyfrix (Macroura) pedibus pentadactylis, cauda longifli: tha, aculeis clavatis.

Penizant, Syn. quadr. p. 263. n. 194. the long. tailed Porcupine. 


\section{Mammalia Gilires. Cavia.}

* (EINGESCHOBENES GESCHLECHT.)

Cav r A. Die Savia, Szavia, Kavia, After hase, Halbkaninchen; Halbhaje.

Zimmermann, geograph. Zoolog. II. pag. 223. Gen. XXVII.

Schreber, Säugthiere, IV. p. 608. Gen. XXIV. Le $\int k e$, Naturgefch. p. 163. Gen. XXIV.

Boroweky, Thierreich, I. 3. p. I9.

Bhumenbach, Handb. der Naturgefchichte, p. 8I.

Gen. XXI.

Bechftein, Naturgefch. Deutfchl. I. p. 140. 410.

Ebert, Naturlehre; I. p. 333.

Frifch, Naturfyft. p. 9.

Batfch, Thiere, I. p. 23 r.

Erxleben, Mammalia, p. 348. Gen, XXXIV.

Granmann, introd. in hiftor. nat. mamm. p. $5 \%$.

Gen. XXXIV.

Gatterer, brev. Zoolog. I. p. 99. Gen. XXXIV.

Severini, zoolog. Hungar, p. 62.

Klein, Quadr. difp. p. 49.

Pennant, Syn. quadr. p. 243. Gen. XXXIV.

Herrmann, tab. affinit. animal. p. 53.85.

1. Pa a. Der Paca. (')

Miuller, Naturfyft. I. p. 341. n. t. die brafilianifche Kanizichenmans.

Zimmer.

(1) Êin gutartiges Thier, das feinen Gefchäften des Nachts nachgeht, und am Tage nicht zum Vorfchein kommt, auffer wenn ihn feine Nothdurft heraustreibt; denn in feinem Bau teidet es nicht die geringfte Unreinlichkeit. Wenn er wieder hineingeht, verftopft er die Zugănge mit Blättern, und kleinen Zweigen. Wird ef verfolgt, fo flieht er ins Waffer, tancht unter, und Ateckt den Kopf nur ron Zeit zu Zeit horaus, um Athem zu 
Zimmermann, geogr. Zool. II. p. 324. n. 220. der Paka.

Lefke, Naturgefch. p. 164. n. 4. der Paka.

Borow $k y$, Thierreich, I. 3. p. 23. n. 6. brafilianifche Kaninchenmaus; Paka.

Blumenbach, Handb. d. Naturgefch. p. 89. n. 2. Cavia (Paca) caudata, corpore fulvo, falciis fateralibus punctatis flavis. (Edit. 3.)

Ebert, Naturlehre, I. p. 335. der Paca. Halle, vierfüfs. p. 404: die grofse brafilianifche Mous.

Gatterer, vom Nutzen u. Schaden der Thiere, I. p. 218. n. II8. der Paca.

Neuer Schauplatz der Natur, VI. p. 269. Paca, Pag, Pague; Mins Paca Linn.

Onomat. hift. nat. II. p. 715. die gro/se brafilianifche Ratte; cias braflifche Coatiasthier; das grofse brafilifche Kaningen.

Gesner, Thierbuch, p. 177. Paca; die dritte Art aus Brafilien. Mit Marcgr. Fig.

Klein,

holen. Seine Nahrung befteht in Brod, Rüben, Sellery, Zwiebeln, Knoblauch, und andern Wurżeln, Kohl, fogar Baumrinden; befonders liebt er Früchte und Süfsigkeiten. Er frifst auch Körner. Das. Thier wird fehr fett. Sein Fleifch, aus welchem eine grofse Delicateffe gemacht wird, ift durchaus mürbe, und fo weifs wie Kalbfleifch. Man brühet fie in heifsem Waffer, fchabt die Haare $a b$, und kocht fie in der Haut, es fey denn dafs man fie braten wollte. Der Paka fáuft wie ein Hund; öfters fitzt er auf den Hinterbeinen, und wifcht fich den Kopf und Bart mit den Vorderfuifsen, die er jedesmal leckt. Seinen Zorn giebt er durch eine Art von Zähnklappen und Grunzen zu erkennen. Im Nothfall vertheidigt er fich hartnäckig gegen die Hunde. Sein Urin ift dick und übelriechend; fein Unrath langlicher, als der vom Hafen und lianinchen. - Blumsnbacb hat in der vierten Atiflage, feines Handbuchs der Naturgefchichte diefs Thier weggelaffen, und dagegen den Aguti angefithrt. 
Klein, Quadr. difp. p. 50. Cavia Paca.

Klein, Claffif. der vierfüfs. Thiere, p. 149. n. 3. Afterhaafe; grofse brafilifche Manis.

Klein, natürl. Ordn. d. vierf. Th. p. 53. n. 3. Afterhaase Paca.

Biiffon, vierfüfs. VIII. p. 7. der Paca; mit einer Fig.

Bat fch, Thiere, I. p. 23r. der Paka.

Merrem, zool. Abhandl, p. 25. n. r. gefleckter. Kleinange.

Frifch, Naturfyft. p, 9. n. 2. das Pacoene.

Fermin, Befchreibung voin Surinam, II. p. ros. der Paka.

Berkel, Reife nach Berbice; in Blimenbachs Reifegefch. I. p 160. Hafen.

pallas Naturgefch, merkwürd. Th. II. p. 2r. Ca. via Paca.

Hartfink, Befchreib. von Guiana, I. p. IIr. Pag, oder Pague.

Graumann, intr. in hif. nat. mamm. p. 58. VH. die brafilianifche Kaninchemmaus.

Gatterer, brev. Zool. I. p. Ior. n. 7. Cavia (Pa: ca) caudata, pedibus pentadactylis, corpore fufco, fafciis lateralibus punctatis flavis. Pallas, fpicil. zool. II. p. 18. Cavia Paca. Laet, nov. orbis, p. 55t. Pacae, Pag, aut Pague, p. 6r8. Pac.

$D^{\prime}$ Abbeville, miffion au Maragnon, p. $25 \mathrm{I}$. Lery, hift. d'un voyage au Brefil, p. I57. MMaffée, hift des Indes. Paris 1665. p. 70. Herrera, defcript. des Indes occident. . Paris 1622. p. 252.

Binet, voyage à Cayenne; en 1652 . Paris 1662. p. 340 . 
2. Aсисну. Der Akoufchy. (2)

Zinmermann, geogr. Żoolog. II. p. 326. 11. 222. der Akoufchy.

Ehert, Naturl. I. p. 334. Aguchi, oder Akuchi. Handb. der Naturgefch. I. 2. p. 152. Akouchi. Biiffon, vierfüls. V. p. 210. Aguchi.

Gatterer, vom Nutzen und Schaden der Thiere, $\therefore$ I. p. 218. n. 120. der Akiz $c h i$.

Samml. zur Phyf. und Naturgefch. II. p. 608. der Aconchi.

IMerrem, zool. Ábhandl. p. 24. n. 5. 3. Akuschi. Graunicmi, introd. in hiftor. nat. mamm. p. 57 . Cavia Acouchy.

Des. Marchais, voyage en Guinée, III. p. 287. Agouchi.

\section{A GU I. Der Aguti.(3)}

Miiller, Naturfyf. I. p. 33\%. n. 3. das Ferkel. kaninchèr.

\section{Zimmer.}

(2) Giebt eben den Iaut wie das Meetfchweinchen, aber felten. Ins Waffer geht er nicht; fein Fleifch fchmeckt nicht fo gut, als das vom Aguti:

(3) Wenn man diefe Thiere zahm gemacht hat, fo gewöhnen fie fich wegzulaufen und wieder zu kommen, und freffen alles was man ihnen giebt, Brod, Körner, Früchte, Wurzelwerk, Sallat, Kohl, und andere Blatter, aber kein Fleifch. Das Ueberiüfsige vergraben fie. Getränke nehmen fie wenig, und zwa: faugend in fich. Sie fchlafen wenig, mit offeren Augen, fitzend oder liegend. Wenn fie büfe werden, frauben fie die Haare, von der Mitte des Rückens bis hinten, empor, und ftampfen mit den Hinterfüisen ftark gegen die Frde. 1hr Laut, den man aber felten hört, gleicht dem Grunzen eines Ferkels, und bisweilen, befonders wenn man ihnen zu frellen giebt, fclonurren fie wie eine Katze, wenn fie fpinut. Linné hat diefe und mehrere Bemerkungen an emem-zahmen Aguti gemacht. 
Zimmermamn, geogr. Zool. II. p. 325. n. 221 . der Aguti.

Lefke, Naturgefch. p. 164 n, 3. der Aguti.

Borow 5 ky, Thierreich, I. 3. p. 19. 11. I, das Ferkelkaninchen.

Bhumenbach, Handb. der Naturgefch. p. 8r. n. 2. Cavia (Aguti) (Piculi) caudata, corpore ex rufo fufco, abdomine flavefcente; das . Ferkel. kaninchen:

Ebert, Naturlehre, I. p. 333. der Aguti, oder Acuti; das brafilianifche Kaninchen.

Halle, vierfüls. p. $4 \circ 3$. das braune brafilianifche Ferkelkaninchen, mit kurzem Scheanze.

Gatterer, vom Nutzen und Schaden der Thiere,

I. p. 217. Il. II7. das Ferkelkaninchen.

Neuer Schauplatz der Natur, I. p. 148. Agriti.

Dnomat. hift. nat. II. p. 710. das gemeine amerikanifche Kaninchen, das brafilifohe Meerschweinchen, das indianif che Agutithier.

Handb. der Naturgefch. I. 2. p. I51. Aguti.

Frifch, Naturfy h, p. 9. n. 3. Aguti.

Batfch, Thiere, I: p. 232. das Ferkelkaninchen.

Camper, Naturgefch: des Orang- Utang etc. P. 53. Aguti.

Gesner, Thierbuch, p. 177. Aguti, vel Acuti; cine Art-Küniglein, aus Bráflien.

Klein, Quadr. difp. p. 5o. Cavia Aguti, vel Acuti. Klein, Claflif, der vierfüfs. Thiere, p. I48. n. 2. Afterhaase, Cavia, Aguti oder Akuti.

Klein, natürl. Ordn. d. vierf. Th. p. 53. n. 2. Afterhaaje auls Brafilien.

Biiffon, vierfüfs. V. p. 206. das Ferkelkaninachen. Aguti; in. e. Fig.

Martini, Naturlexicon, I. p. 653. Agnti; kaninchenartige IMaus. 
Kriinitz, ökon. Encyklop. I. p. 389. Aguti.

Dapper, Amerika, p. 225. Aguti.

Labat, Reifen, I. p. 394. Aguti.

Schwed. Abhandl. XXX, p. 27. ein brafilifches Thier; Aguti.

Pallas, fpicil. zool. II. p. r8. Aguti.

Pallas, Naturgefch. merkw. Th. II. p. 21. Agou. ti, oder Acuti.

Samml. zur Phyf. und Naturgefchichte, II. p, 608. Aguti.

Baion, Mem, pour fervir à l' hiftoire de Cayenne. Paris, 1778. II. p. 195. Aguti. (Befchreib. der innern Theilc.)

Bankroft, Naturgefch. von Guiana, p. 84. Puc. carara?

Hartfink, Befchreibung von Guiana, I. p. 2 ro. der Agouti.

Mherrem, zool. Abhandl. p. 24. n. 5. «. Hajen. ferkel, Aguti.

Laet, novus orbis, p. 55r. Acuti five Agouti. Rochefort, hilt. des Antilles, p. 139. L' Agouty. De Bry, hif. de l'Amerique, III. p. 156.

Graumann, intr. in hif. nat. mamm. p. 57. IV. Ferkelkaninchen.

Gatterer, brev. Zool. I. p. roo. n. 4. Cavia (Agu-

ti) caudata, corpore ex rufo fufco, âbdomine flavefcente.

B. Leporina. Der Haje von Iava.

Milller, Naturfyft. I. p. 338. n. 3. die Ha enmanus. Zimumermann, geogr. Zool. II. p. 326. der Hafe von Tava.

Borowj"ky, Thierreich, I. 3. p. 23. 11. 5. Cavia Leporina; der javanifche Halbhase; Hafen-. mans. Halle, 
Halle, vierf. p. 405.' der javanifche Halbhase. Onomat. hif. nat. II. p. 715. "das Kaninchene vors. Iava; der javifche Haas.

Klein, Quad uped. difp. p. 50. Cavia Iavenfis."

Lepus Iavenfis Catesb.

Klein, Claflif. d. vierf. Thiere, p. 150. javaniSihor Afterhaase.

Klein, natürl. Ordn. d. vierf. Thiere, p. 54. n. 7: Afterhajo aus Iava.

Frifch, Naturfyft. d. vierf. Th. p. 9. n. 7. $A$ fterhafe aus Iava.

Meriem, zool. Abliandl, p. 25. $\gamma$. das javanifche Ferkel.

Linnt, Syf. Nat. Edit. X: 1. p. 59. n. 2. Mus (Leporinus) cauda abbreviata, palmis tetrada: ctylis, plantis pentadactylis.

Graumanin, intr, in hiftor. nat, mamm. p. 57. VI. Haferimaus.

Gatterer, brev. zool. I. p. roo. n. 6. Cavia (Lepo: rina) caudata, corpore fupra rufo, fubtus albo.

\%. A M ER ICA N A. Das Jivinamifche Kanincheno' Halle, vierf. p. 405, das furinamifche Kaninchen. Onomat. hift. nat. II. p. 717. das amerikanifche Kaningen; das-Jurinamifche Cavia - Thier. Frifch, Naturfyf. der vierf. Th. p. 9. n. 5. Cavia; furinamifche Afterkaninchen.

Klein, Quadruped. difp. p. 5o. Cavia Surinamenfis.

Klein, Claflif. der vierfüls. Th. p. r49. Jurina: mifcher Afterhaase.

Klein, natürl. Ordn. d. vierf. Th. p. 53. n. 5. Afterhafe aus Surinam. Cuniculus americanus Seb. 


\section{8

4. Aperea. Der Aperea. $\left({ }^{4}\right)$

Miiller, Naturfyftem, Suppl. p. 40. 11. 24. Mus Aperea; die brafilianifche Maus.

Zimmermiann, geogr. Zoolog. II. p. 327. n1. 223. der Aperea.

Schreber, Şäugth. IV. p. 6Iб. n. 4. der Aperea. Boronesky, Thierreich, I. 3. p. 21. n. 2. der Ape= rea; das brafilianifche Koninchen.

Ebert, Naturlehre, I. p. 334. der Aperea, Cori. Halle, vierfüfs. p. 404. die braflianifche Bufchvatte.

Gatterer, vom: Nutzen und Schaden der Thiere,

I. p. 217 . n. I16. die brafilianifche Maiis.

8il Batfch, Thiere, I. p. 232. der Aperiea. Merierin, zool. Abhandl. p. 24. n. 4. Aperea, Neuer Schaupl. d. Natur, I. p. 343. Aperea.

Onomatol. hiftor. nat. II. p. 7II. das brafilifche Apereathier; das brafilijò Kaninichen.

Klein, quadrup. difp. p. 50. Cavia Aperea.

Klein, Claflif. der vierfüfs. Thiere, p. I49. n. 4. Afterhafe Aperea.

$\therefore$ Klein, natürl. Ordnung d. vierf. Th. p. 53. n. 4 . Afterhase Aperea.

Miartini Naturlexicon, III. p. 4, Aperea, die braflianifche Bufchratte, das brafilianifche Kanimschen; m. e. Fig.

Gesner, Thierbuch, p. 177: Aperea, Cuniculi fpecies; ein Feldratte; mit Marcgr. Fig.

Berkel, Reife nach Berbice; in Blumenb. Reife. gefchichten, I, p. 160. Kanincheir.

Charle-

(4) Das Fleifch diefer. Thiere if wollfchmeckender als Kaninchenfleifch, aber nicht fo fchmackhaft, als das vom Aguti, denn es ift trockner; es wird aber hảufig gegeffen, und deswegen das Thier mit Hunden gefaugen. 
Charlevoix, Gefch. von Paraguay, p. 24. Kanivn. then, welche die Spanier Apercos nennen.

Severin, zool. Hungar. p. 63. C. Aperea, die brafilianische Mans.

Bankroft, Naturgelch. von Guiana, p. 84. Pul carara?

Granmani, intr in hif, nat mamm. p. 57 . I. die brafilianifche Bufchratte.

Gatterer, brev. Zoolog. I. p. 99. n. r. Cavia (Aperea) ecaudata, corpore ex cinereo rufo,

5. Совауа. Das Mberschwein. (s)

Miiller, Naturfyftem, I. p. 334. n. r. das IMeer. fchweinchen.

\% i C C 5 Zimmer

(5) Das Meerfchwein fcheint erft zu der Zeit, da die vercinigten $\therefore$ Niederlañde Brafilien in Befitz bekamen, nach Europa gekommen zu feyn, und feitdem hat man es auch hâufg zum Vergnïgen gezogen. Diefe Thiere freffen wie die Eichhörner, auf den Hinterfüfsen fitzend. "Sie fafen mit ausgeftreckter Zunge, wie die Hunde; lieben die Milch fehr, find aber auch ohne Saufen fortzubringen, wenn fie nur feuchte Speifen bekommen. Eine Speife werden fie leicht überdrüfsig, und erkranken, wenn man ihnen nicht trockne und fenchte Nahrungsmittel wechfelsweife giebt. Nach der Mahzeit ruhen fie fitzend. Sia laufen am liebrten an den Wanden hin, und das Mannchen treibt feine Gattin yor fich her. Die Furse wafchen fie öfters mit der Zunge. Vor dem Schlafe freffen fie gerne etwas, und wafchen fich. Im Schlafe fchliefsen fie die Augen nie ganz, auch if ihr. Schlaf ktirz, Männchen und Weibchen fchlafen nicht mit einander, fondern eins bowacht das andere. Das Weibchen geht drey. Wochen trachtig, und bringt gemeiniglich zwey bis drey, felten mahr als vier lunge. Die lungen werden fehend und haarigt geboren; zwölf Stunden nach der Geburt kisnnen fie fchon laufen, und fangen bald an zu freffen. Den Hunger geben diefe Thiere durch Grunzen, ihre Zilfriedenheit/ durch ein gewiffes Murmeln, und den Schmerz mit einem durchdringenden Gefchrey zu erkennen. Dás Fleifch ift efsbar; aber unf́chmackhaft. Die Ratten follen vor ihnen weichen. 
Zimmermann, geogr. Zool. I. p. 298. das Mheer: Schweinuchen. II. p. 327. in. 224. der Cobaya, das Meerfohweinchenz.

Lejke, Naturgefchichte, p. I63. n. I. das Mieer. fchweingen.

Borow Sky, Thierreich, I. 3. p. 21. n. 3. das Meerfchweinchen.

Blumenbach, Handb. der Naturgefch. p. 8I. n. r. Cavia (Porcellus) ecaudata, corpore variegato; das Meerschweinchen.

Bechftein, Naturgefch, Deutfchl. I. p. 4rr. n. r. das Meerfchweinchene. p. 4is. Meerferkel, Meersäulein, Afterhase, Afterkaninchen, $F_{e r}$. kelmaus, Cobaya.

Funke, Naturgefchichte, I. p. 88. Meerschweinchen, Halbkaninchen.

Ebert, Naturlehre, I. p. 333.'das Meer Cchweinchen. Halle, vierf, p. 402. das Meer fchweinchen; Tab.9. Meyer, Thiere, I, tab. 82. das Meerfchweinchen. Gatterer, vom Nutzẹı und Schaden der Thière,

I. p. 216. n. I15. das Meer sihweinchen.

Beckmann, Naturhiftorie, p. 20. n. I. das Meerfchweinchen.

Neuer Schauplatz der Natur, V. p. 547. Meer: Schweinchen.

Onomat. hift. nat. II. p. 7i4. das indianifche Ka: ninchen, das indianifche Schweinchent.

Handb. der Naturgefch. I. 2. p. I82. das MMeerschweinchers.

Frifch, Naturfyn. p. 9. n. I. das Meerfchweinchen: Gesner, Thierbuch, p. I76. indianifche Künig. lein. Abbild. mittelın.

Klein, Quadrup. difp. p. 49. Cavia Cobaya Brafil. quibusdam mus Pharaonis; Tatu pilofus; Por. cellus; Mus indicus. 
Klein, Claffif. der vierfüls. Th. p. 147. brafilifcher Afterhafe.

Klein, natürl. Ordn. d. vierf. Th. p. 53. n. x. Pharaonis Maus; haarigter Tatu; indianifche Maus; amerikanifches und guineifches $K a$. ninchen.

Büffon, vierfüfs. V. p. 7. das Meerfchweinchen; m. e. Fig.

Bat fch, Thiere, I. p. 232. das Meerfchreinchen. Murr, Reifen einiger Miffionar, p. 198. Meessficherinlein, Cary.

Pallas, Naturgefch. merkw. Thiere, II. p. 21. das Meer Chweinchen.

Bock, Naturgefch. von Preuffen, IV. p.75. n. 23 . das Meer/chweinchen.

Linmé, auserlefene Abhandl. I. p. I35. Meer. - Schwein.

Beyträge zur Beförd. der Naturkunde, I. p. I26. n. 17. Mus Porcellus; Cuniculus indicus; MeerSchweinchen.

Dicquemarre, über einige im Uterus eines Meer. fchweins gefundene Steine; in Lichtenbergs und. Voigts Magazin für das Neuefte etc. IV. 2. p. 73 .

André und Bechftein, Spatziergänge, I. p. 38\%. das Meerfchweinchen.

Wittenberg. Wochenblatt, 68. p. 428. Meer. fchoveinchen.

Neue Mannichfaltigkeiten, III. p. 17. m. e. Fig. Iablon אky, allgem. Lex. p. 656. Meer - Färklein. Erxleben; Mammalia, p. 349. n. 2, Cavia (Por. cellus) ecaudata, corpore variegato,

Limné, Syft. Nat. Edit. VI, p. ro. n, r. Mus cauda abrupta, palmis tetradactylis, plantis pen. tadactylis. 
Limne', Syf. Nat. Edit. X. I. p. 59. n. r. Mus (Porcellus) cauda nulla, palmis tetradactylis, plantis peritadactỵlis.

Grammamn, intr. in hifor, nat. mamm. p. 57. Ir. IMeerfchweinchen.

Gatterer, brev. Zoolog, I. p. 99. n, 2. Cavia (Porcellus) ecaudata, corpore variegato:

Severin, zool. Hungar. p. 63. C. Porcellus; das Meerscinueins:

Kramer, Auftr. p. 3r5. Lepus cauda nulla.

$R z a c z$ auct. hift. nat. Pol. p: 333. Porcellus indicus.

Ciarleton, exercitat. P: 24. Porcellus indicus five Guinenfis.

6. CAPYBAR A. Der Capybara. (6)

IMiiller, - Naturyft. I, p. 467. n. 4. das Stumpf: fchwein.

Iniiller, Naturfyfh. Suppl. p. 40. n. 25. Mus Cabiai; die amerikanifche Mans.

Zimmermann, geogr. Zool. II. p. 323. n. 219. der Capibara, das Wafferfchwein.

Lefke, Naturgefchichte, p. 164. n. 5. der Ca. pybara.

Borowfky, Thierreich, I. 4. p. 70. n. 2. das amerikanif che Wafferfchwein.

Halle, vierf. p. 368. das amerikan. Wafferfocheein. Neuer:

(o) Frifst mịt Hülf̣e der Vorderfüisse auf den Hinterfüfșen fitzend, und geht des Nachts feiner Nahrung parweife nach: Das Thier kann lange unter dem Wafer ausdauern, hat eine Art von Efelsfimme, und wird zuweilen bis hundert Pfund fchwer. Sein Fleifch hat einen thranigten Gefchmack, den es aber verliert, wenn das Thier nit vegetabilifcher Koft ernahrt wird. Wegen des fonderbaren Baues feiner Füfse ift diefs Thier eines der zweifelhafteften in der Naturgefchichte; Merrem, am angeführten Ort. 
Neuer Sihauplatz d. Natur, II. p. 4. Cabiak.

Onomat. hift. nat. II. p. 38i. Cabiai; das grofse Meer-oder Wafferfchwein.

Onomat. hift. nat, VII. p. 377. Sus Hydrochaerus. Handbuch d. Naturgefch. I. p. 292. dér Cabiai. Biiffon, vierf. XIII. p. 79. der Cabiai, m. e. Fig. Bat fch, Thiere, I. p. 23I. das WafJerfchavein. Merrem, zool. Abh. p. 22. n. 1. WafJer-Ferkel. Berkel, Reife nach Surinam; in Blumenb. Reife. gefch. I. p. s37. Tapir Capybara; eine Art Schreeine.

MLurr, Reifen einiger Miflonar. p. 2n. Capiva ¿ra, Ungumayo.

Eerminin, Befchr. von Surinam, II p. 8o. Porcus - fluviatilis feu Sus maximus paluftris; der Kabiai. Pallas, Naturgefch. merkwürd. Thiere, II. p. 2I. Capybara.

Hartfink, Befchreib. von Guiana, 1. p. Io7. der WafJerhase, Caibiai, Tybuaite.

Dobrizhoffer, Gefch. der Abiponer, I. p. 406. das Wafserfchwein, Capiiguara, Atopehenra.

Charlevoix, Gefch. von Paraguay, p. 22\%: der Capivara.

Evxleben, Mammalia, p. 193. n. 2. Hydrochaerus (Capybara) dentibus primoribus utrinque duobus.

Graumann, intr. in hif. nat. mamm. p. 40 . II. das Sumpffchwein.

Gatterer, brev. Zoolog: I. p. 52. n. 2. Hydro. chaerus (Capybara) dentibus primoribus utrinque duobus, cauda nulla; das Sumpffchrein. Des Marchais, voyage en Guinée, III. p. 298. Cochon d' eau?

Severin, zool. Hungar. p. 53. n.2. Hydrochaerus Capybara. Sus paluftris americanus. 


\section{Mammalia Glires. Cavia.}

A) Verïnderungen gegen die XIIte Edition, und Vermehrung der Gattungen diefes Gefchlechts.

Das ganze Gefchlecht if hier neu. "Die erfte, dritte, fiinfte und Jechfte Gattung find aus andern Gefchlechtern hieher verfetzt: Die zweyte und vierte Gattung $C$. Acouchy und Aperea aber find neu. Die Benennung Cavia hat zuernt. Klein, und nach diefem Pallas gebraucht.

\section{B) Unbeftimmtere Thiere.}

x. Die Cavie von der Hudfonsbay.

Zimmermann, geogr. Zoolog. II. p. 33r. a.

Ellis Reife nach Hudfons Meerbufen, p. II8. I84. das Kaninchen der Hudfonsbay?

2. Der Hafe von Patagonien.

Zimmerniann, geogr. Zool. II. p. 328. n. 225: Cavia (Patagonum) cinereo-fulca, abdomine albo, cauda nuda breviflima, lumbis macula alba notatis.

3. Der Cori.

Zimmermann, geogr. Zoolog. p. 23r. b.

Hift. des voy. par du Perrier de Mont Fraizier:

Paris 1707. p. 43 .

Charlevoix, hif. de St. Domingue, I. p. 35 .

23. GESCHLECHT. C'ASTor. Der Biber. Miiller, Naturfyftem, I. p. 326. Gen. XXIII. Zinmermanin, geograph. Zoolog. II. p. 320. Gen. XXVI.

Schreber, Säugthiere, IV. p. 622. Gen. XXV. Lefke, Naturgefch. p. 165. Gen. XXV. 
Borow Sky, Thierreich, I. 3. p. 13. Gen. XXIII. Bhumenbach, Handb. der Naturgefchichte, p. 124. Gen. XLI.

Bechftein, Naturgefch. Deutfchl. I. p. I44.

Frifch, Naturfyft. d. vierf. Th. p. Io.

Bat $\int c h$, Thiere, I. p. 242. Gen. XLII.

Erxleben, Msmmalia, p. 440. Gen. XE.

Graumann, intr. in hiftor. nat. mamm. p. 68. Gen. XL.

Gatterer, brev. Zool. I. p. I20. Gen. XL. Pennant, Syn. Quadr. p. 255. Gen. XXV. Briffon, quadr. p. 90. Gen. XXI. Boddaert, Elenchus, p. I66. Gen. XLI. Hermann, tab. affin. animal. p. 81.

1. Fí: Der Biber. (')

Miiller, Naturfyftem, I. p. 326. der Caftor. Tab. 3०. fig. 2.

\section{Zimwer.}

(1) Wird wegen feine: feinen Haare für die Handlung, und für die Arzneykunft wegen des fogenannten Bibergeils wichtig, das fich bey beyden Gefchlechtern in befondern Behältern unter dem Schwanze findet. Am berühmteften find dicfe Thiere furch die bewundernswürdigen Kunftriebe, mit welchen fie, befonders da, wo fie fich, wie in Innern von Canada, noch in grofser Menge beylammen finden, ihre berühmten Gebäude aufführen; da fie z. E. fich za mehrern Hunderten am Ufèr des Fluffes oder Sees verfammeln, Büue fällen, fie zu Pfälen behauen, fie an Ort und Stelle flöfsen; Kanale und Flofsteiche zur Erleichterung des Transports graben; im Fall das Waffer zu feichte ift, vorher grofse, faft unverwüfliche Damme aufführen, und dann etft ihre eigentlichen Wohnungen dahinter bauen, die nach der verfchiedenen Anzahl der Familien, die fie beziehen follen, auch von verfchiedener Gröfse, von viez bis zehen Fufs im Durchfchnitt, meiftens drey Stockwerk hoch und oben gewölot find. Das untere, im Waffer befindliche 
416 Mammalia Glires. Caftor.

Zimmermann, geogr. Zool. I. p. 272. II. p. 32 I. n. 217 . der Biber.

Lefke, Naturgefcli. p. i65. der Biber. Borow ky, Thierreich, I.3. p. 15. n.2. der Kaftor, der Biber.

Bhumenbach, Handb. der Naturgefch. p. I24: n. I. Caftor (Fiber) cauda depreffa, ovata, fquamofa. Bechftein, Naturgefch. Deutfchl. I. p. 414. n. r. der genieine Biber. p. 425. Caftor, Biber, Erd-oder Lanabiber.

Funke, Naturgefchichte, 1. p. 9r. Biber:

Ebert, Naturlehre, I. p. 379. der Biber. Tab. 18. Halle, vierf. p. 573 . Bibẹr.

Gatterer, vom Nutzen und Schaden der Thiere,

I. p. 264. Biber.

Beckmann, Naturhiforie, p. 33. n. 2. der Biber, oder Kaftor.

Pennant, arct. Zool. II.' p. 98. n. 48. der Biber... Neuer Schaupl. der Natur, I. p. 7or. Biber:

Onomat. hift. nat. I. p. 186. Biber.

Handbuch der Naturgefch. I. 2. p. 202. der Biber. Handbuch der deutfchen Thiergefchichte, p. 78. der Biber.

Klein, Cuadruped. difp. p. 9i. Caftor, Biber. Klein, Claffif. d. vierf. Th. p. 276. Biber. Klein, natürl. Ordn. d. vierf. Th. p. 95. Biber. Frifch, Naturfyn. d. vierf. Th. p. Io. der Biber. Mnartini, Naturlex. VII. p. 327. Biber, Bieber:

Gesner,

Stockwerk, dienť zum Màgazin für den Wintervorratl. An der Hudfonsbay find die Riber fo häufig, dafs man oft in kurzer Zeit über so,000 tödter. Die Hitze fcheint ihnen zuwidet zu feyn, denn kein Reifender erwähnt des Bibers in der heiffen Zone. Dielungen werden itn dritten lahre vollwüchlig. Das Alter des Bibers foll fich auf is bis 20 lahre erftrechea. Ein erwachfen gefangener Biber wird nie zahm. 
Gesner, Thierbuch, p. 39. Biber. Abbildung inittelmäfsig.

Biiffon, vierf. V. p. 139. der Biber; m. e. Fig. Kraft, Ausrottung graufam. Thiere, II. p. 386.

Biber.

Bock, Naturgefchichte von Preuffen, IV. p. 69.

n. 22. der Bieber, Caftor.

Bat fch, 'Thiere, I. p. 244. der gemeine Biber. Merrem, zool. Abhandl. p. 6. Biber., p. 18. n. r. gemeiner Bieber.

Kriinitz, ökon. Encykl. IV. p.387. Biber, Bieber. Kligel, Encyklopäd. I. p. 226. der Biber. Fifcher, Naturgefch. von Livl. p. 59. Biber.

Carver, Reif. d. Nordamerika, p. 376. der Bieber. Dobrizhoffer, Gefch. der Abiponer, I. p. 409. der Bieber.

Taube, Befchr. von Slavonien u. Syrmien, p. 20. Biber oder Caftor.

Pontoppidan, Norwegen, II. p. 51. der Biber.

Dapper, Amerika, p. 146. Biber.

Hermanin, Reife durch Oefterreich etc. I. p. 549. Biber.

Leem, von den Lappen in Finnmarken, p. To8. Kaftor oder Biber.

Allgem. Gefch. der Länder und Völker von Amerika, II. p. 495 . Biber.

Schlözer, Erdbefchr. von Amerika, p. 25. Biber. Taube, Naturk. des Herzogth. Zelle, II. p. I42. Biber.

Paillas, Reife, I. p. 198. 283. III. p. I7. Biber. Köhler, neue Reifen, I. p. 314. Biver: Kalm, Amerika, III. p. 28. 606. Bieber. Mïller, Samml. ruff. Gefch. III. p. 528. Riber. Gmelin, (des ält.) Reife, III. p. 487. Biber: Ellis Reife nach Hudfons Meerbufen, p, 166. Bieber. D d Bo/fi, 
Bo/fii Reife nach Weftindien, I. p. 182. Bieber. Gottwald, phyf. anatom. Bemerk. über den Biber, a. d Latein. überf. Nürnb. I782. 4. m. F. 'Perrault, Charras und Dodart, Abhandl. zur Naturgefch. I. p. 157. anatomifche Befchreib. eines Bibers, Tab. 20. der Biber. Tab. 2I. die Zergliederung.

Gisler, Schwed. Abhandl. XVIII. p. 196. Biber. Holften, Sch wed. Abhandl. XXX. p. 292. Biber. Pallas, nov. Sp. quadr. e glir. ord. p. 85. Tab.25. fig. 2. glans e genitali Cafloris. Kulmus, Anat. des Bibers; in Kanold Anmerk: von Natur - und Kunftgefch. I. Suppl. p. 107. Hellwing, Relation von dem Biber; dal. p. 96. Bonnet, Betracht. über die Natur, II. p. 315.318. die Bieber.

Sulıer, über die Schönh. der Natur, p. 12r. Biber. Merklein, Thierreich, p. Io. Bieber.

Börner, Stadt-u. Landwirthfchaft, II. p. 198. n.8. " Schönfeld, Landwirthfchaft, p. 68I.

Döbel, Iägerpraktik, I. p. 36. II. p. I37. 151. III. p. 98.

Heppe, wohlredender läger, p. 67. Allgem. ökon. Forfmagazin. Lpz. 1767. p. 297. Heifen, Abhandl. vom Biber oder Caftor; im 35-37 St. der gel. Beytr. zu den Braunfchw: Anz. 1763.

Anmerk. uiber alle Theile der Naturl. I. p. 400. Inblonfky, allgem. Lex. p. 136. Bieber.

Lemmery, Materiallexicon, p. 255.

Geogr. hift. phyl. u. moral. Mancherley, I. p. rog. Magazin für den Landmann, I. p. 38. 70. 87. I43. 167. 183.

Schlef. ökon. Nachrichten, Iahrg. 1785. Angenelime u. nuitzl. Lekt. Starg. I772. p. 203. 
Wochenbl. für Kinder, IX. p. 89.

Donndorf; Natur u. Kunft, I. p. 205.

Hamb. Magazin, IV. p. 359. Biberfang:

Hamb. Magazin, I. p. 460 . anatom. Beınerk. von den Behältniffen des Bibergeils.

Greifswald. Beytr. zum Nutzen und Vergnügen, 1757. 4tes St. p. 14i-144. Nachricht von den canadifchen Bibern.

Allgem. Hift. der Reifen, XVII. p. 67. 79. 89. 224. Biber.

I. C. Frommann, diff. de Caftore f. Fibro. Co. burg. 1686.

Erxleben, Mammal. p. 440. n. r. Caftor (Fiber) cauda depreffa ovata.

Linné, Syft. Nat. Edit. II. p. 47. Caftor cauda ovata plana.

Linné, Syft. Nat. Edit. VI. p. Io. n. I. Cafor cauda ovata plana.

Linné, Syf. Nat. Edit. X. I. p. 58. n. t. Caftor (Fiber) cauda ovata plana.

Linné, Syft. Nat. Edit. XII. I. p. 78. n. r. Caftor (Fiber) cauda ovata plana.

Linné, Fn. Suec. I. p. 9. n. 23. Caftor cauda ova ta plana.

Graumann, intr. in hiftor: nat: mamm. p: 68. $\mathrm{I}$; Biber, Bieber.

Gatterer, brev. Zoolog. I. p. I20. n. t. Caftor (Fiber) cauda depreffa ovata, pedibus polticis palmatis; der Bieber:

Mitller, zóol, dan. prodr. p. 4. n. 25. Caftor (Fi. ber) cauda ovata plana.

Kramer, Auftr. pi 3.15. Caftor cauda ovata plana: Scheffer, Lapponia p. 339. Caftores:

Charleton, exercitat. p. 18. Caftor. Olans Magiz. de gent. feptentr. p. 604. Caftor.' Dd 2 Schwenks 
Schwenkfeld, theriotroph. Silef. p. 94. Fiber. Schonevelde, ichthyologia. Hamb. I624. 4. p. 34. Fiber five Cafor.

Agricola, de anim. fubterr, p. 20. Fiber. Wagner, hift. nat. Helvet. p. I72. Caffor, Fiber. Mufeum Wormian. p. 320. Caftor.

Rzacz. hiffor. nat. Polon. p. 215. Auct. p. 306. Caftor, Fiber.

Forfter, philor. transact. LXII. pे. 375. Beaver. Valentin, amphith. zoot. I. p. I69. Caft. anatome. IVIangeti, bibl. pharmac. med. Genev- 5703. I. p. 489. (Marii Caftrologia.)

Caftor Maş, Gedani diffectus a. 1684. menf. April. ab E. G. H.(eife) in den Act. erud. Lipf.a. I684. M. Aug. p. 360 . Ebendeff: Caftoris femellae difiectio. daf. p. 362 .

Anatomica Caftoris defcriptio; in den Comment. ad illuftr. hift. animalium naturalem, iuflu Ludovici XIV. Parif. I671. m. Fig.

Defcription anatomique de l'ours, du Caftor, de la Gazelle etc. Paris 1669. 4.

Les Voyages du Bàron de Hontan. II. p. 155.

Bacqueville de la Poterie, hift. de l' Amerique feptentrionale à Rouen I722. I. p. I33.

Dierville, relat. d' un voy. en Acadie. à Rouen 1708. p. 126.

Charlevoix, hifl. de la nouv. France, II. p. 98. Sagard Theodat, voyage au pays des Hurons. à Paris 1632. p. 319.

Oeuvres de Regnard. Par. 1750. I. p. I72.

Hiftoire de la Virginie, p. 406.

Haller, element. phyfiolog. VII. p. I47.

Plin. hiffor. nat. VIII. c. 3 o. Fiber. XXXII. c. 3.

Fibri, quos Caftores vocant.

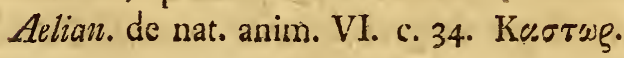


B. Castor al bus. Der weifse Biber.

Zimmermann, geogi. Zoolog. I. p. 275. ganz weifse Biber.

Schreber, Säugth. IV. p. 624. weifse Biber. Klein, Quadruped. difp. p. 92. Caftor albus.

Klein, Claflif. d. vierfüfs. Th. p. 277. weifsev Biber.

Onomat. hif. nat. II. p. 674. Caftor albus; der weifse Biber.

Bïffon, vierfüfs. V. p. 162. ganz weifse Biber. Rzaczynfki, hift. nat. Polon. p. 216.

Diefem könnte noch beygefügt werden:

y. Castor colore stramineo. Der frohgelbe Biber.

Zinmmermann, gangr. Z.ool. I. p. 275. der gelboder Atrohfarbigte Biber.

Bïffon, vierf. V. p. I63. Arohgelbe Biber.

Charlevoix, hilt de la nouv. France, II. p. 940

2. HuाD в в IUS. Der Guillino.

Molina fagt: das Thier fey fo wild, und fo muthig, dafs es die Fifche felbft vor den Augen der Fifcher aus den Reufen hole; das Weibchen gehe, wie er glaube, nicht länger als fünf Monat trächtig; das Haar nehme leicht jede Farbe an, : und man mache Hüte daraus; die den ächten Kaforhüten nichts siachgäben. Die Benennung $\mathrm{Ca}$ for Huidobrius habe er ihm zum Andenken eines feiner Freunde, mit Namen Huidobrio gegeben. 
Verciuderungen gegen die XIIte Edition, und Vere mehrung der Gattungen diefes Gefchlechts, Edit. XII. Edit. XIII.

p. 79. n. 2. CaftorMofchatus. pag. II3. n. 6. Sorex Mo: fchatus.

p.79.n.3.Caftor Zibethicus. p. 125.11.2. Mus Zibethicuse

Die zweyte Gattung Huidobrius if neu hin. zugekommen.

34. GESCHLECHT. Mus. Die Mans.

IViiller, Naturfyft. I. p. 334: Gen, XXIV. Zinmuermann, geograph. Zoolog. II. pag. 359. Gen. XXXII.

Schreber, Säugthiere, IV, p. 635. Gen. XXVI. Lefke, Naturgefch. p. I66. Gen. XXVI. Borowe fky, Thierreich, I. 3. p. 24. Gen. XXIV. Blumenbach, Handb, der Naturgefchichte, p. 73. Gen. XV.

Bechftein, Naturgefch. Deutfchl. I. p. 426. Funke, Naturgefch. I. p. I2I. Bat/ch, Thiere, I. p. 224. Gen. XXXIII. Ebert, Naturlehre, I. p. 336. Onomat. hift. nat. V. p. 327 . Erxleben, Mammalia, p. 38r. Gen. XXXVII? Pennanut, arct. Zoolog. II. p. 128.

Graumann, introd. in hiftor. nat. mamm. p. 60. Gen. XXXVII.

Boddart, Elenchus, p. 105. Gen. XX. Gatterer, brev. Zool. I, p. ro6. Gen. XXXVII, Pennant, Syn. quadr. p. 299. Gen. XXXII. Briffor, quadr. p. Ir8. Gen. XXVI. Hervmann, tab. affinit. animal. p. 88 . 
* Flachfchwänzige.

\section{J. Coypus. Der Coypu.}

Von der Grölse eines Fifchotters, Das Thier läfst fich zahm machen, und frifst alsdenn alles, was man ihm giebt. Seine Stimme int ein fcharfes Gefchrey, die es aber nicht anders hören ]äfst, als wenn es beleidigt.vird. Das Weibchen führt feine Iungen immer mit ficle, wenn es Nahrung fucht.

\section{Zí тетісus. Der Ondathra. (i)}

Miiller, Naturfylt.I. p. 333.n. 3. die Zibethratze: Dd 4

Zinumer.

(') Die Häufer, welche diefe Thiere 'ạuen, find rund, wie ein Backofen, etwa zwey Fufs weit, aus Binfen und Brde etwa drey Zoll dick verfertigt, und mit einem wohl dreymal fo dichten Flechtwerke von Binfen überzogen. Der Eingang ift über der Wafferfläche, Inwendig find verfchiedene Rühren, theils zur Flucht, theils zur Entledigung des Unraths, theils um zu den Wurzeln, die ihnen zur Nahrụng dienen, zu gelangen. In Landern, die wärmer als Kanada find, bauen fie keine Häufer, fondern bervohnen blufs die Röhren, welche Ge in den Ufern haben. - In jedem Haure wohnen_den Winter hindurch mehe rere beyfammen. Sie bewohnen aber folches nur ein lahr, und bauen im Herbft allemal ein neues. Der Bifamgeruch des Thiers ift poch an dem getrockneten Balge zu fpüren, daher itt auch das Fleifch nicht efsbar. Die Kürfchner verarbeiten die Felle, und die Hutmacher das Haar, zuweilen wie Biber. haár. Man ftellt ihnen daher Fallen; an welchen Aepfel die Lockfpeife find, oder tüdtet fie in ihren Rühren durch Schwefeldampf, Diefe Thiere erleichtern den Perlenfuchern manchmal ihre Mühe, weil fie grofse Liebhaber des Bewohners diefer Mufcheln find. Sie halren ihre Mahlzeiten gern an einem ftilJen Ort des Fluffes, auf den über das Wafer hervorragenden Steinen oder Sand. Die Perlen, wenn fie welche finden, fpucken fie aus, und diefen Umftand benutzen die Pẹlenfucher, 
Zimmermann, geogr, Zoolog, II, p, 32r, n, 2 I8. Uer Ondathra.

Le $\int k e$, Naturgefch. p. I66. n. I. die Zibetmaus. Borowfky, Thierreich, I, 3, p. I3, n. I. Caa for Zibethicus; die Biefanratte; der Biefanbiber; ; der Ondatra. 'Tab. 23. A.

Pennañt, arct. Zool, II. p. Ioz. n. 49. der One datra.

Gatterer, vom Nutzen und Schaden der Thiere, I. p. 271. n. 156. der Ondathra; die Zibeth. rat:ze.

Neuer Schaupl. d. Natur, X. p. 2Ir, Zilethratze. Onomat. hift. nat. II. p. 676 . die condidifche Mo. Schusratte.

Handbuch der Naturgeích, I, 2, p. 224, der Ona diatra.

Buiffon, vierf. VI, p. 376, der Ondatra; die $Z i_{4}$ bethratte; kanadifche Bifamratte.

Batfch, Thiere, I. p. 245. der Ondathra.

Carver, Reifen durch Nordamerika, p. 374. die Biberratze, Muskratze, Musquafh,

Schöpf, Reife durch die nordatnerikan. Staaten,

I. p. 207. p. 33r. Biefamiratzen, Mufkrat.

Natürl. u. bürgerl. Gefch. von Californien, I. p. 35 . Bonnet, Betracht, über die Natur, II. p. 325. Bi= Santratze.

Kalm, Amerika, III. p. 25. die Biefamratzen. Erxleben, Mammal, p. 444.n.2. Caftor (Zibethicus) cauda longa compreffo - lanceplata, pedi, bus fifilis.

Graumann, introd, in hif, nat, mamm. p. 68. II, die Zibetratze,

Gatte.

und fincien, wenn fie dẹn Sand an folchen Orten fleifsig durchfuchen, viele, und rein ausgemachte Perlen, ohne Mühe, lung gefangen lâfsţ fich der Ondathra auch zahm machen. 
Gatterer, brev, Zool, I. p. I2I, 11. 2, Caftor (Zibethicus) cauda longa compreffo - lanceolata, pedibus fifis; die Bifamratze; Zibetratze.

Charlevoix, hif. de la nouv. France, III. p, 107, Rat Mufqueé.

Forfter, philor, transact. LXII. p. 375. Mufk - Beaver.

Hermann, tab, affin, animal, p, $\delta \mathrm{I}$, Caftor Zibe, thicus,

* Rattenfchu änzige.

3. PILORIDEs, Der Piloris.

Zimmermann, geogr, Zool. II. p. 361. n, 269, der Piloris; der ceilanifche Piloris.

Zimmernann, Zoolog. geogr. (edit. lat.) 'p. 325 . Mus amphibius javenfis.

Schreber, Säugthiere, IV, p. 642, 13, 2. der $P_{i}$ loris.

Merrem, zool, Abhandl, p. 29. n. I. Mo chiusmans,

B. Der amerikanifiche Piloris.

Zimmermann, geogr. Zool. II. p. 360 . der ameri= kanifche Piloris,

Zimmermann, Zoolog. geogr. (edit. lat.) p. 509. Cafton Piloris,

Schreber, Säugthiere, IV, p. 643. der amerikanifche Piloris.

Biiffon, vierf. VI. p. 378, die antillifche Biefann. ratte,

Du Tertve, hift, gen, des Antilles, Paris, I676, II. p. 302 .

Erxleben, Mammal, p. 357. * (Spec, obfcur.) Graumann, intr, in hif, nat, mamm, p. 58 . 


\section{Cа п ас O. Der Karako. (2)}

Zinmerinann, geogr. Zool. II. p. 36r. n. 270. des. Karako.

Pennant, arct. Zoolog. II. p. I29. n. 69. der $K a$, rako; die grofse Maus der Mongolen.

Merrem, zool. Abhandl. p.30. in. 2. grofse, Maus.

6. DeCumanus. Die Wanderratte. (3)

Zimmermann, geogr. Zool. II. p. 12. p. 36r. dis Wanderratte; der Siìmuloto

Borone.

(2) Hat grofse Aehnlichkeit mit dem Siirmulot, unterfcheidet fich aber docch von derfelben durch den kleinern Kopf, bläfere Obervorderzähne, ftärkere Hinterfülse, kleinere und weichere Daumenzehe, Verbindung fammtlicher Zehen unter einander, Kürze des Schwanzes, weicheres Haar $u_{\bullet}$ dgl. $m_{q}$,

(3) Diefe Thiere richten in den Häufern, wo fie fich einquartirt haben, grofse Verwüftungen an, werfen in den Kellern Berge von Erde auf, brechen durch gemauerre Wände, zerfreffen Teinwand, Kleider $\mathrm{u}$. dyl. kommen aber nie in die obern Zinmer, und auf dic Kornbüden, fondern bleiben immer nahe an der Krde. Es ift fchwer diefe Ratten, die fchädlichftep unter allen, los zu werden, da fie fchwer in die Fallen und an das Gift gehen, auch die Katzen fich nicht gern daran machen, Sie find ạuch, befonders die Munnchęn, fo kühn, dafs fie fich zur Wehre fellen', wenn man fie verfolgt, gehen aber sloch auf Zuckerwurzeln und Zwiebeln. In den lahren 1778 , und 1779. waren diefe Thiere hier, in Quedlinburg, fo häufig in den Häufern, dufs fie entfetzlichen Schaden thaten, befonders in den Häufern, 'die arn Waffer liegen. Die erften waren vorn Harze mit einem Fuder Wafen herunter gekommen. In Blankenburg hatten fie faft alle Häufer untergratien, und befonders in den Papiermithlen grofsen Schaden gethan. Hicr bey zins hatte mạn die Bemerkung gemacht, dafs fie nach abgefchälten Zuckerwurzeln, gebratenen Zwiebeln und Speck am erften giengen. In lulius 1781 . waren fie zum Theil noch in manchen Hüufern dẹr Stadt feht häufig and holten des Nạhts 
Borowfky, Thierreich; I. 3. p. 24. n. 1. Mus Norwegicus; die grofse Waldratte.

Bechftein, Naturgefch. Deutfchl. I. p. 436. n. 2. dic Wanderratte. "p. 44I. die gro/se Ratte, grofse Waldratte, wilde Ratte, Springratte, hïpfende Ratte, Sïmuillot, Erdratte, grosse Wajesratte, Felạratte. (Fälfchlich norwegifiche Maus.)

Halle, vierf. p. 424. die granefchwärsliche norwegi. Sche Ratte.

Gatterer, vom Nutzen und Schaden der Thiere, I. p. 235, 11. 13. der Siirmiilot, die, Wander. ratte.

Pennant, arct. Zool. II. p. I29. n. 68, den Siir: miilot, die Wanderratte.

Handhuch der deutfchen Thiergefchichte; p. 43. die fuchsbraune grofse Erdratte.

Khlein, Quadrup. difp. p. 56. Glis Norwagicus.

Klein, Claflif, der vierfüfs, Th. p. 166. n. 2. norwegifcher Siebenfchlïfer.

Klein, natüul. Ordn. d. vierf. Th. p. 59. n. 2, norwegifcher Siebenfchläfer.

Biiffon, vierfüls. IV. p. 290. die gro/se Waldratte, Siirmiilot,

Bat $\int c h$,

viel junges Federvieh. An einigen Orten anf dem Harge haben fie des Nachts den Kindern die Haut von den Fingerir abgefreffen. Merkwürdig ift es, dafs diefe Thiere unţer'allen Säuğthieren varzüglich von Eingeweidewürmern frey find, Brifon hat van diefem Thicr die erfte Nachricht gegeben. Un Paris ift es erft uns 1 ahr 17.53 , in England gegen $173^{\circ}$, in A trachan feit 1727 , bekannt. Die Natuiforfcher, die ihm den Namen zorwegifche Maus gegeben haben, find wahrfcheinlich durch den Seba dazu verleiret worden, deffen Stelle auch beym Errlebes mit angeführt itt. Aber Seba meint entweder ein anderes

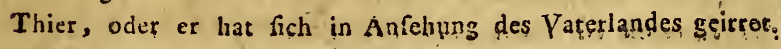
p.sllas glir. p. 96 . 
Batfch, Thiere, 1. p. 225. die grofse Waldratte. ILerrèm, zool. Abhandl. p. 30. 11. 3. Wandermazs.

Pallas, Reife durch Rufsl. I, p. 304. Goeze, Naturgefchichte der Eingeweidewürmer, p. 223 dic branne Erdratte.

Löree, phyf. Zeit. I784. p. 184. Windertatze. André und Bechftein, Spatziergänge, I. p. 74. die Wanderratte.

Becknann, phyf. ökon, Bibl. I. p. т86. norwegiSche Maus,

Graumann, intr, in hifor, nat. mamm. p. 61. Mus Norwegicus; die grofse 1 aldratte.

Gatterer, brev. zool. 1. p. Io6. n. I. Mus (Norvegicus) cauda elongata, palmis tetradactylis, cum unguiculo pollicari, corpore rufo; der - Sïrmulot, die grofse Waldratte.

Severin, zool. Hungar. p. 73. a. Mus Surmulote tus, feu Rattus migratorius.

12. R aт т̦ us. Die Housratte. (4)

Ilïller, Naturfyft.I. p. 347 . n. 12. Hansratze.

Zimners:

(4) Wenige andere Thiere find fo aufserft gefräisig als die Racten. Sis freffen fogar Scorpione, und zichen dem Menfchen und feinen Victualien überall nach, fogar den Bergleuten in die tiefften Schachte. Sie verlafien die ankommenden Schiffe, wenn fie ausgeladen werden, fchwimmen ans Land, und beziehen fie wieder, fnbald fie vom neven befrachtet werden. Sie benagen fogar fchlafende Menfchen, haben aber auch oft in Hungersnoth, zumal auf Schiffen, vielen zur E.rhaltung als Nahrungsmittel dienen miiffen. Die Mürter vertheidigen ihre Iungen mit eigener Lebensgefahr, felbft gegen gröfsere Katzen, dagegen werden auch alte kraftlofe Ratten von den jüngern beforgt und,gefütrert. Solche bejahrte Ratten verwickeln fich zuweilen zu fechs, acht und mehrern mit den Schwänzen in einane 
Zinmermann, geogr. Zool. I. p. 23\%. die Ratze. II. p. 362. n. 272. die Hausratte. Lejke, Naturgefchichte, p. 167. n. 3. die Ratte. Borvw fky, Thierreich, I. 3. p. 25. die Ratte. Bhmenbach, Handb. der Naturgefch. p. 75 . n. 5. Mus (Rattus) cauda elongata, palinis tetrada. ctylis, cum unguiculo pollicari; die Ratte. Bechfteinz, Naturgefch. Deutfchl. I. p. 428. n. $\mathrm{x}_{\text {. }}$ die Ratze. p. 435. Ratten, Hausratten, Rattenunciule, grofse Hausmäule, Huusratzen. Funke, Naturgefchichte, I. p. Ir8. Ratten.. Halle, vierf. p. 427 die grofse Hausmaus. (Ratte.) Meyer, Thiere, II. tab. 83. die Ratte.

\section{Gatte-}

einander, und diefs find die fogenannten Ratienkörtige. Urfprünglich ift die Ratte wohl im mittlern Europa zu Haufe. Die alten Griechen und Römer gedenken des Thiers nie; und in die neue Welt if es erft feit ihrer Entdeckung von Europa aus ütiergebracht worden. Naç Südamerika follèn die Ratten zuerft is 44. gekommen feyñ. Eben fo haben fie unfere Srhiffe in verfchiedene Infeln des Südmeers gefchlèppt. Auf den Marquefen - Eilanden. den Societät's - und freundfchaftlichen İnfeln, den neuen Hebriden, und fogar auf Neu- Fingland liat fie ForAer angetroffen. In den Societäts - Infeln, vorzüglich auf Otaheiti, find fie in unglaublicher Menge vorhandén, nähren fich von den Ueberbleibfeln der Mahlzeiten, welche in den Wohnungen der Einwohner liegen bleiben, und von den Blattern und Schoten des Korallbaums (Erytbrina Corallodendron orientale Linn.) von Pifang und andern Früchten, und in Ermanglung diefer von allerley Exxcrementèn. In Guiana halten fie fich vorzüglich im Zuckerrohr auf: weswegen diè Einwohner, nachdem fie das Rohr ausgefchnitten und wèggefchaft haben, die Atehen gebliebenen Blatter davon 'rings umher anzünden, woaurch vieletaufend verbrennen müfen. Auf Barbadós find die Ratten fo häufig', dafs die 'Einwohner der Kirchf́piele S. Iofeph und S. Andrew, durch den Schaden, den fie am Zuckerrohr thun, jahrlich einen Verluft von zwey bis drey taufend Pfund leiden. Von den lakuten und einigen andern Vülkern werden die Ratten gegetran. 
Gatterer, vom Nutzen u. Schaden der Thiere, I. p. 236. n. 132. die Ratte.

Beckmann, Naturhiftorie, p.27. n. 5. die Ratze. Pennant, arct. Zool. II. p. I28. n. 67 . die Haus. ratte.

Neuer Schauplatz der Natur, VII. p. 36. Ratte. Onomat. hift. nat. V. p. 346. die gro/se Hausmaus, Hausratze, Ratte.

Handbuch der Naturgefch. I. 2. p. 172. die Ratze. Handbuch der deutfchen Thiergefchichte, p. 43. die Ratte.

Sander, ökon. Naturgefch. I. p. 257. die Ratte. Frifch, Naturfyft. p. 7. 13. 1. Rattus domefticus; Haus - Rat $\approx$.

Klein, Quadr. difp. p. 57. Mus, Rattus; Mus criftinarius.

K!ein, Claffif. der vierfüfs. Thiere, p. I69. n. t. Maus, Ratze.

Klein, natürl. Ordn. d. vierfüls. Th. p. 68. n. I. Ratze, Hausrat:e.

Gesner, 'Thierbuch, p. 263. die Ratte. Abbil. dung gut.

Buiffon, vierfüls. IV. p. 22I. die Ratte.

Bock, Naturgefch. von Preuffen, IV. p. 78. n. 27. die Housratze.

Batfch, Thiere, I. p. 225. die gewöhnliche Ratte. Merrem, zool. Abliandl. p. 3o. n. 4. Ratze. Kraft, Ausrottung graufam. Thiere, II. p. 463. Ratten oder Ratzen.

Fifcher, Naturgefch. von Livland, p. 60. n. 21。 Ratte.

Molina, Naturgefch: von Chili, p. 242. grofse Hausratzen.

Cetti, Naturgefchichte von Sardinien, I. p. 344. die Ratte. 
Wolf, Reife nach Zeilan, p. I2s. Ratzen. Knox, ceyl. Reifebefchr. p. 63. Ratten.

Schlözer, Erdbefchreib. von America, p. 48t, Ratzen.

Pontoppidan, Norwegen, iI. p. 56. Ratten. Bankroft, Naturgefch. von Guiana, p. 85. Ratteri. Olaffen, Reife d. Island, II. p. 46. Ratzen.

Forfter, Bemerk. auf feiner Reife um die Welt, p. 164. die gemeine Ratte.

Kalm, Amerika, III. p. 14. Ratzen.

Pauv, Unterf. üb. d. Amerik. I. p. 230. Ratten, Ococha.

Kolbe, Vorgeb. d. g. Hoffn. p. 163. Edit. in 4. p. 34r. Ratten.

Kümpfer, Befchr. von Iapan; in du Halde China IV. p. 138. Ratzen.

Gefch. fchädl. Infekten; überf. von Goeze p. 15. Ratten.

Goeze, Natur, Menfchenleben und Vorfehung,

I. p. 527. (vom Rattenkönige.)

Beyträge zur Beförd. der Naturkunde, I. p. 120. n. 21. Ratze, Ratte.

Berlin. Sammlung. IV. p. 478. Ratzem.

Berlin. Samml. V. p. 384. Rattent.

Hamb. Magazin, III. p. 304. X. p. 557. XIX. p. 456. Ratten. (Fürforge anderer für eine blinde.)

Magazin für den Landmann, I. p. 516. 514, II. p. 9. Ratzen.

Merklein, Thierreich, I. p, i22. Ratze.

André und Bechftein, Spatzietgänge, I. p. 33. die Ratze.

Wittenberg. Wochenblatt, 1774. p. 46. u. 69. Stuttgard. phyf. ökon. Ausz. III. p. 236. Schwed. Abhandl. XXIII. 'p. 80. 
Jablonfky, allgem. Lex. p. 874. Ratte, Ratze. Select. phyf. oeconom. I. p. 483.

Eraleben, Mammal. p. 382. 11. 2. Mus (Rattus) cauda elongata, palmis tetradactylis; cum unguiculo polícari, corpore grifeo.

Linné, Sylt. Nat. Edit. II. p. 47. Mus domefticus maior.

Linné, Syî. Nat. Edit. VI. p. ro. n. 6. Mus cauda longa fubnuda, corpore fúfco - cinerafcente.

Linné, Sylt. Natur. Edit. X. İ. p. 61. in. 9. Mus

(Rattus) cauda elongata fubnuda, palnis tetradactylis, cum unguiculo pollicari, plantis pentadactylis.

Granmann, intr. in hift. nat. mamm. p. Gr. II. die Ratte, Ratie.

Gaiterer, brev. Zool. İ. p. Io6. n. r. Mus (Rattus) cauda elongata, palmis tetradactylis, cum unguiculo pollicari, corpore grifeo.

Severin; zool. Hungar. p. 73. n. I. Mus Rattus. Kramer, AuAr. p. 316. Mus cauda longa fubnuda, corpore fufco-cinerafcente.

Charleton, exercitat. p. 25. Sorex domefticuss. Sihwerikfeld, theriotroph. Silef. p. II5. Mus domenticus maior.

Sibbald, Scot. illuftr. II. 2. p. I2. Mus domeftieus maior, feu Sorex.

Fubric. fil. groenl. p. 29. n. 3. Mus Rattus.

Rochefort, hif. d'Antilles. Rotterd. 1659. p. 26r. les Rats.

Forfter, phil. Transact. LVII. p. 343. Mus Rattus. Duverney, oeuvr. anatom. II. p. 384 .

Valentin. Muf. mufeor. II. p. I5I.

Schellhamner, Mifc. Acad. Nat. Curiof. Dec. II. an. IX. obf. 147. p. 254. 
Geoffroy, mater. med. VIII. p. I89. Avicenna, L. II, Tract, 2. c. 498.

B. Weifse Ratten.

Schreber, Säugthiere, IV. p. 649. ganz wєifse Ratten.

Naturforfcher, I. p. 63. n. 15. ganz weifse Ratten. Fifcher, Naturgefch. von Livland, p. 60. 11. 21. ein ganzer Whurf. weifser Ratten mit rothen fourigen Angen.

Wolf, Reif. nach Zeilan, p. I28. ganz veifse Ratten.

Beckmann, phyf. ökon. Bibl. V. p. I02. vergl. mit II. p. 588.

33. Muscurus. Die Hansmans. (s)

IMüller, Naturfyft. I. p. 349. n. I3. die Hausmaus: Zimmermann, geogr. Zool.I. p. 237. die MIans. II. p. 363. n. 274. die Hansmans. Le $k k e$, Naturgefch. p. 167 n. 4. die Housmanls. Borowfky, Thierreich, I. 3., p. 27. n. 5. die Hansmuans.

Bhumenbach, Handb. der Naturgefch. p. 74. n. 4. Mus (Mufculus) cauda elongata, palmis tetradactylis,

(5) Ift bekannter Maafsen der Ratte wie in der Bildung, fo in der Lebensart ähnlich, doch dafs fie fich mehr häustich bält, und nicht fo wie jene umherfchweift; auch hat fie nicht das boshàfte Naturell der Ratre. So furchtfan diefe Thiere find, fo werden fie doch ziemlich dreuft, wenn fie merken, dafs man fie nicht verfolgt, und laffen fich fo gar aus der Hand futtern: Man hat-bemerkt, dars fie den Seidenwürmern kachrehen. Auch follen fie eine Art von Wuth, wie die Hunde bekommen. Nicht an allen Orten, wo Ratten find,' finden fich auch Mafue; wie z. E. letztere in Sardinien fehlen, obgleich enfere angetroffen werden.

Ee 
dactylis, pollice palmarum mutico; die Hous mants.

Bechftein, Naturgefch. Deutfchl. I. p. 44I. n. 3.

die Hansmans. p. 447. die gemeine Manis.

Funke, Naturgefch. I. p. I2\%. die Hansmaus.

Halle, vierfüfs. p. 43r. die kleine Hausmaus.

Meyer, Thiere, I. Tab. I. eine Maus.

Gatterer, vom Nutzen und Schaden der Thiere,

I. p. 245. n. I35. die Maus.

Pennant, arct. Zoolog. II. p. I30. n. 7r. die Hanismans.

Neuer Schauplatz der Natur, V. p. 427. Maus, Hansmans.

Onomat. hif. nat. V. p. 337. die kieine Hanusmens. Hanib. der Naturgefch. I. 2. p. I78. IVTaus.

Handbuch der deutfch. Thiergefchichte, p. 42 . Hausmans.

Sander, ökon. Naturgefch. I. p. 252. die Hansmans. Frifch, Naturfy h. p. 7. n. I. Mus domefticus vulgaris; gemeine Hansmons.

Klein, Quadr. difp. p. 57. Mus minor. Mufcu. lus vulgaris, domefticus.

Klein, Clafff. d. vierf. Th. p. 169. n. 2. Manks.

Klein, natürl. Ordn. d. vierf. Thiere, p.60. n. 2. Mous.

Gesnev, Thierbuch, p. 260. eine Mans. Abbild. fchlecht.

Biiffon, vierfiifs. IV. p. 238. die Miaus. Tab. 70. Bock, Naturgefch. von Preuffen, IV. p. 85. n. 28. die Hausmans.

Bat fch, Thiere, I. p. 236 . die Hausmans. Merrem, zoolog. Abhandl. p. 3г. n. 6. Hausmatus.

Kraft, Ausrott. graufam. Thiere, II. p. 459. Atüdtifche Mäule, Haus - MIc̈ule. 
Fifcher, Naturgefch. von Livland, p. 60. 11. 22: gemeine Maus.

Molina, Naturgefch. von Chili, p. 242. die klei. ne Hausmans.

Pontoppidan, Norwegen, II. p. 56. Mïn é.

Olaffen, Reif. d. Island, II. p. 46. Mäuse. Kömpfer, Befchr. von Iapan; in du Halde China IV: p. I38. Mänee.

Steller, Kamtfchatka, p. I29. ganz kleine und zahme Mänse?

Gmelin, (des ält.) Reife; I. p. 315. II. p. "37r. Neuhof; Gefandichaft nach China; p. 348 .

Beytr. zur Beförd. d. Naturkunde, 'I. p. 2 I0. n. 20. Maus, Hansmans.

Goeze, Natur, Menfchenl. und Vorfeh. I. p. I. Naturgefch. der Hansmans:

Schwed. Abhandl. I. p. 174. Mäufe. VUI. p. 273. Mïuraje:

Afti, vom Gift der Thiere. p. 6 .

Berlin. Samml: IV. p: 470. Hansmäuse.

IMerklein, Thierreich, p. I22. Maus.

Andvé u. Becluftein, Spatziergänge, II. p. 285.294: Hausniaus.'

Erxleben, Mammalia, p. 39r. n. 5. Mus (Mufculus) cauda elongata, palmis tetradacty. lis absque unguiculo pollicari, corpore grifeo:

Linne', Syn. Nat. Edit. II. p. '47. Mus domenti: cus vulgaris.

Linné, Syn. Nat. Edit. VI. p. Io. n. 8. Mus cauda nudiufcula, corpore cinereo-fufco, abdo. mine fubalbefeente.

Lisné, Syft: Nat. Edit. X. I. p. 62. n. ro. Mus (Mufculus) cauda elongata fubnuda, palmis te. tradactylis, plantis pentadactylis.

Fe 2

Grasen. 


\section{Mammatia Glires.:- Mus.}

Graumann, intr. in hiftor. nat. mamm. p. 6r. $\forall$. Mans, Hausmaus.

Gatterer, brev. Zoolog. I. p. I08. n. 5. Mus (Mufculus) cauda elongata, palmis tetradactylis absque unguiculo pollicari, corpore grifeo; die Mans.

Stanve, differt: de muribus, eorumque damnis. Ien. 1676. in 4.

Miuller, zool. dan prodrom. p. 5. n. 28: Mus (Mufculus) cauda elongata fubnuda, palmis tetradactylis, plantis pentadactylis, pollice mutico.

Kramer, Aufr. p. 316. Mus cauda nudiufcula, corpore cinereo-fufco, abdomine fubalbefcente.

Schwenkfeld, theriotroph. Silef. p. Ir3. Mus do. metticus minor.

Sibbald, Scot. illuftr. II. 2. p. 12. Mus domefticus minor.

Forfkial, fn. oriental. p. 4. Mus domeficus. Fabric. fn. groenl. p. 29. n. 4. Mus mufculus.

Pennant, britt. zool. p. 302. n. 229. the common Moufe.

Pennant, britt. Thiergefch. p. 45. fp. VII.

Forfter, philofoph. "Transact. LVII. p. 343. Mus Mufculus.

Ariftot. hil. anim. I. c. 2. 11. 15. Mvs.

Plin. hift. nat. VIII. c. 56. X. c. 65. c. 73. Mus.

B. Weifse Mänle. ( $\left.{ }^{6}\right)$

Zimmermana, geogr. Zoolog. I. p. 242. veifsc Miüulese.

Schreber,

(6) Ohnftreitig gehüren einige der von mir angeführten Stellen wohl mit zu der nachft folgenden Gattung; aber die Befchrei- 
Schreber, Säugthiere, IV. p. 658. voeifse Müufe.

Naturforfcher, I, p. 64. -1. I7. ganz wijse Haus: mäus $s$.

Klein, Quadr. difp.p. 5\%. Toti albi.

Klein, natürl. Ordn der vierf. Th. p. 60. n. 2. D. Tchnéveiveifse.

Klein, Naturfeltenheiten des Königreichs Ungarn, p. 57. weifse Mäuse.

Fifcher, Livland, p. 60. n. 22. weifse Maus.

Olaffen, Island, I. p. II7. weifse Mïufe?

Schwed. Abhandl: XXIII. p.3I7. Bergius. Befchreibung einer ganz weifsen Maus.

Kanold, Samml. von Natur - und Medicingefch. XV. Verf. p. 285. und Supplem. IV, p. 88. 9I. weifse IMïuje.

Phyfikal. Belun. III. p. 437. weifse Mans.

Schivenhfeld, theriotroph. Silef. p. II4. Mus albus; Rzacz. Aud: hiftor. nat. Polon. p. 328 . Mus al. bus domefticus.

Erxleben, Mammalia, p. 394. Varietas alba.

37. Sil varicus. Die grofse Feldmans. (7).

Ziitller, Naturfyf. I. p. 352. n. 17. die Wald: mians.

Ee 3

Zisnmers:

bungen find zu unbeftimmt angegeben, als dars ich es mit $\mathrm{Zu}$ verficht hätte wagen können, fie gehörig aus einander zu fetzen; und im Syftem felbet finde ich ihrer nicht gedacht.

(7) Diefe Mäufe hecken des láhrs mehrmal, und bringen auf jeden Wurf zehn bis zwölf lunge. lhre Vermehrung if daher betrachtlich, and wenn ihre Arzahl, wie in manchen Iahren gefchiehet, ftark anwächif, fo verheeren fie Garten und Holzungen. Dem ausgefäeten Holzfamen, befonders den Eicheln gohen fie-ftark nach, und die jungen Stamme der Obft-uud wilken Bäume benagen fie, dass fie verdorren, Biiffộ hat mit 
Zininermconn, geogi. Zoolog. II. p:15. die Waldmans. p. 362. n. 273. dise grosse Felamans; die Waldments.

Borowfing, Thierreich, I. 3. p. 27. n. 4. die Wald-Mious.

Bhunenbath, Handb. der Naturgefch. p. 74. n. 2a Mus (Silvaticus) cauda mediocri, pectore flavefcente, abdomine albido; die Waldmaus; grofse Feldmauts.

Bechftein, Naturgefch. Deutfchl. I. p. 447. n. 4. die grofse Feldmaus. p. 453. Waldratte, Walle mans, gelbbraune Feldnaus, Heermaius, Feli. manus, branne Mans, (fälfchlich die kleine Hios Selinans) Nieliticuls.

Funke, Naturgefchichte, I. p. I22. die Feldnans. Halle, vierf: p. 431. 11.4. die Feldmaus? IMeyer, Thiere, I. tab. I. die Feldmans?

Pennant, arct Zool. II. p. .30. 11. .72. die grofSe Fildmuns.

Gatterer, vom Nutzen und Schaden derThiere, I. p. 242.n, I34. Miilot; grofse Feldmans. Neuer Schaupl. d; Natur; IX. p. 459. Walcanculs. Onomat. hift. nat. $V$. p. 350 . die Waldmans. Handboch der deutfchen "Thiergefchichte, p. 42 a Hanmms, Banmatze, Waldmaus, grofse Feldinans.

Sander, ökonom. Naturgefch. 1 p, 254 die Felda gucuts?

\section{… Biiffon,}

aufgeftellten platten Steinen, und einer gehratenen Nuifs zer Zockfpeife, in Zeit von drey Wochen über taufend wegfangen laffen, Ihre Löcher nind eine halbe bis eine ganze Elle tief unter der Erde, und beflehen öfters aus zwey Kammern, in deren einer der Vorrath if, und in der andern die Maus eiszeln wohnt. Die Zugänge find eine fenkrechte und fchräge Röhre, vor dẹiẹi Oefrnung keine aụfgeworfene Erde zu bemerlien iłt. 
Bïfforn, vierf, IV, p. 243. die grofse Feldmaus; m. e. Fig.

Batfch, Thiere, I. p. 226. die Waldmans. Merrem, zool. Abhandl. p. 31. n. 5. Feldmanis. Fifcher, Naturgefch. von Livland, p. 60. n. 24. Waldmaus.

Leem, Nachr. von den Lappen in Finnmärken, p.121.122.

Andirk and Bectaftein y Spatziergänge, I. p. 284. die grofse Feldmaus.

Reichard, Land -und Gartenfchatz, IV. 4.217.

Duroy, Baumzucht, II. p. 256.

Allgemeine Haushalt. und Landwinthfch. IV. p. 313. Feddminge?

Erxleben, Mammal. p. 38s. n. 4. Mus (Sylvaticus) cauda mediacri, corpare cano pilis nigris, pectore flavefcente, abdonine albo.

Linîué, Syft. Nat. Edit. III. p. 47. Mus domefticus medius.

Linne, Sylt. Nat. Edit. VI. p. เo. n. 7. Mus cauda longa, corpore nigro-flavefcente, abdomine álbo.

Linné, Sylt. Natur. Edit. X. I. p. 62. n. I2. Mus (Silvaticus) cauda Ionga, palmis tetradactylis, plantis pentadactylis, corpore grifeo, pilis nigris, abdomine albo.

Limé, Fn. Suec. I. p. Ir: n. 30 . Müs cauda longa, corpore nigro flavefcente, abdomine albo.

Graumani, intr, in hift, nat, mamm. p. 6I. IV. Feldmaus.

Gatterer, brev. Zool. I. p. I08. n. 4. Mus (Silvaticus) cauda mediocri, corpore cano pilis nigris, pectore flavefcente, abdomine albido; die Waldmaus; die grosse Feldmains. E e 4

Severing, 
Severin, zool. Hungar. p. 75. n. 4. Mus fylvati. cus; die $W$ aldniaus.

Miller, zool. dan. prodrom. p. 5. n. 29. Mus (Silvaticus) cauda mediocri, palmis tetradactylis, plantis pentadactylis, corpore grifeo pilis nigris, abdomine albido.

Kramer, Auft, p. 316. Mus cauda longa, corpo: - re nigro - flavefcente, abdomine albo.

Schicenkfeld, theriotroph. Silef. p. Ii6. Mus Sil: vaticus.

Sibbald, Scot. illufr. II. 2. p. r2. Mus Silvaticus. Aldrovand. de quadrup. digit. p. 436. Mus agreitis maior.

Pennont, britt. Zoolog. p. 40. Long-tailed. Field-moufe.

Pennaint, britt. Thiergefch. von Murr. p. 44. Tab. Ir. d.

Forfter, philof transact. LXII. p. 380. Field. Moufe.

B. Harvest-rat. Pemi. $\left({ }^{S}\right)$

Pallas, glir. p. 95. Rattus mefforius.'

Erxleben, Manmal. p. 399.

Graumann, intr. in hiftor. nat. mammal. p. 62 ad VIII.

7. AGR ARIU's. Die Brandmans. (?)

Miẗler, Naturfyn. Suppli p.4I. n. 2\%. die Acker: matss.

Zimmer.

(8) Erxleben bält diefe mit der Brandmaus fïr einerley.

(9) Der Schwanz ift etwas über halb fo lang als der Leib, dïnner aber etwas dichter behaart, als an der Hausmaus, hat ohngefáhr neunzig Ringe, und eine der Lânge nach getheilte, oben Ichwärzliche, unten weifse Farbe. Um die lahre $176_{3}$ oder 64 . 
Zimmermiann, geogr. Zool. II. p. 363. n. 275. die Brandmaus.

Gatterer, vom Nutzen und Schaden der Thiere, I. p. 249. n. 137. die Ackermans, Brandmaus.

Bechftein, Naturgelch. Deutfchl. I. p. 453. n. 5. die Brandmans. p. 456. Ackermans, Streif mans, Erbsmaus. Tab. 10. fig. 2.

Pallas, Reife durch Rufsl. II. p. 651.

Pallas, Reife, Ausz. I, Anh. p. 2. Mus Agram rius.

André und Bechftein, Spatziergänge, I, p. 253. die Brandmans.

Merrem; zool. Abhandl. p. 32. 1. 7. Brandmanus.

Beckmann, phyf. ökonom. Bibl, XII. p. 3Ir. Mus Schwenkfeldii.

Er xleben, Mammal, p. 398. n. 8. Mus (Agrarius) cauda mediocri, corpore fupra luteo, fubtus canefcenti albo , linea dorfali nigra.

Groumann, intr. in hift nat mamm. p. 62. VIII. Ackermanes,

Gatterer, brev.Zool. I. p. IIo. n. 8. Mus (Agrá rius) cauda mediocri, corpore fupra luteo, fubtus canefcenti albo, linea dorfali nigra; die Ackermanus.

Severin, zoolog. Hungar, p. 73. n. 7. Mus Agra. rius; die Ackermanis.

\section{Ee 5}

8. $\mathrm{Mr}$

hat diefe Mäufeart die Gegenden um Kafan und Arfk fo überfehwemmt, dafs nicht nur die ganze Flur, fondern auch die Häufer, in welche fie fonft nicht gern gehẹt, davon voll geworden; wo fie für Hunger das Brod von den Tifchen, und den Leuteh faft ans den Händen holten, bis det Wiater dẹt Plage ein Ende machte, 
8. Mrnu Tus; Die Zwergmans. (10)

Miiller, Naturfy@. Suppl. p. 41. n. 28. die Zwergmaus.

Ziminermann, geogr. Zoolog. II. p. 363. n. 276. die Znegrgmaus.

Borowejh, Therreich, I.3. p.29. n.g. die Zwerg. maus.

Gatterex, vom Nutzen und Schaden der Thiere,

I. p. 251. n. 138. die Zwergmanis.

Neuer Schauplatz der Natur, X. p. 3Ir. Zwerg• matas.

Pallas, Reife, Ausz. I. Anh. p. 2. Mus minutus. Merrent, zool. Abh. p.33. n. ro. Zwerg-Maus. Erxleben, Mammalia, p. 404. n. Ir. Mus (Mi. nutus) cauda elongata, corpore grifeo. lute fcente, fubtus fordide albo.

Grantinunz, intr. in hif. nat mamm. p. 63. XL Ziovergmans:

Gatterer, brev.Zoolog. I. p. III. n. Ir. Mus (Minutus) cauda elongata, - corpore grifeo-lutefcente, fubtus fordide albo; die-Zivergmans.

Severin, zhol. Hungar. p. 73. n. 6. Mus minutus; die Zuergmaus.

70. SorICINus. Die Rüfelmans. (')

Zimmernanu, geogr Zool. II. p. 364. n. 277. die Rilfelmauls.

Bech:

(10) Der Schwanz hat fünf Viertel der Länge des Leibes, und . nd: 30 zarte Ringe, ift etwas hạarigter, als an der Brand̦maus, oben braun, unten grau. Lange des Leibes zwey und ein viertel Zoll; die Schwere gegen anderthalb bis zwey Quentchen.

(1). Ift zuerft bey der grofsen Ueberfchwemmung 24 Ende des $\because$ Oetobers:1778. auf den Aufenwerken der. Stadr Strafsburg gefanger: 
Bechfeein, Naturgefch. Deutfchl. I, p. 456 . n. 6. die Rilfelminus. 'Tab. ro.fig. 3 .

Merrem, zaol. Abhandl, p. 33.n. 13. Rilfelmans.

I4. VAG Us Die Strifmans. (2)

Miiller, Naturfyl. Suppl. p. 46. Mus fubtilis;

if an der Zürtling.

Zinuniermant, geogr. Zoolog. II. p. 364. n. 278: die Streifmauss.

- Wistirerrem, zool. Abhandl p. 33. n. Ir. Schlafnaus. Borowfky, Thierreich, I. 3. p. 30. n. 10. Mus fubtilis; der: Züztting.

Pallas, Reife, Ausz. II. Anh. p. 6. Mus fubtilis. Erxlgben, Mammalia, p. 402, n. I2. Mus (fub. tilis) cauda elongata, corpore cinereo - canefcente, fáfciola dorfali nigra.

Granmann, intr in hift naf. mamm. p. 63. XII. der Zärtling.

Gatterer, brev, Loolog. I. p. Irr. n. I2. Mus (fubtilis) cauda clongata, corpore cincreo-ca. nécénte, fáciola dorfali nigra, dert Zürtling.

15. Be t Uil r nu s. wie Birknans. (3)

Ziminernann, geogr. Zoolog. II. p. 365. n. 279. die Birknatis.

Af

Pallas,

(2) Länge des Körpers zwey Zoll, eine bis fieben Linien; des . St... Schwanzes zwey Zoll ; fieben bis eilf und eir. Viertel Linien; das, Gewicht der kleinften zwey Quentchen, Der Schwanz hat ohngefähr 170 fchuppige Ringe. Mittelft der ausgefpreitzten langen Zehęn und des greifenden Schwanzes neigt das-Thien mit grolser Leichtigkeit an den Pfanzenftängeln hinauf, um fich der Gefíme zu bemächtigen. Es erfarrt bey geringer Kälo to f felbft in kaltẹn Frühlịingsnàchten.

(3) Länge des Körpers zwey Zoll viertehalb Linien; des Schwana zes drey Zoll zwey und ein Viertel Linien; Gewicht ein, und cina 
Pallas, Reife, II. p. 408, eine Art kleiner grauer Schlafmicuis $\int$.

Pallas, Reife, Ausz. II. p. 306. eine Art klleiner. grauer Schlafmän fe.

Pallas, Reife, Ausz. II. 'Anh. p. 6. n. II. Mửs * fubtilis $\beta$.

Erxlebcn, Mammalia, p. 402. Varietas (Muris fubtilis) colore fulvefcenti-luteo, fafcia Ppinali fimili, cauda longiore.

Merrem, zool: Abhandl. p. 33. n. I2. Birkmaus.

18. Pu mi r o. Die Miais von Sitzicamiá.

Neue fchwed. Abhandl: V. p. 239. eine necue Maubs aus dewi fidlichen Theile von Afrika.

Götting. gel. Anz. I786. St. 24.

19. StRIATUS. Die Perlinalis.

IMiiller, Naturfyf. I, p, 352, n, 19, dic oftindifche geftreifte Ratze.

Zinmermann, geogr. Zoolog. II. p. 366 . n. 280 . die Pirlmanus.

Schreber, Säugth. IV. p.665.n. I4. die Perhnaus. Borowf.ky; Thierreich, I. 3. p. =2. n. 8. dia oftindiche geftreifte Ratte.

Halle, vierf. P. 432. dic rothe orientalifche Mauns.

Onomat:

ein Drittheil, hüchftens drey Quentchen. Das Thier wird leicht zahm, und lâfst fich gern in die Hạnd nehmen. Bey einer geringen Kalte erfarrt es, wehrt fich mit den Fufsen etwas, wenn es gereitzt wird, und giebt eirien 'fchwachen', fpitzmatsartigen Laut von fich; bey farkerer Kăke fchlaft es gar ein. Dẹn Winter hindurch verkriecht es fich in Baumhöhlen, wo es wie eine Kugel zufammen gewitkelt liegt, bis es durch die Warme wieder belebt wird, Nach Pallas ift es noch nicht ausgemacht, ob diefs eine eigene Gattung, oder nur eine Varietat von der vorhergehenden fey. 
Onomat. hif. nat. V.p. $35^{\circ}$. die geftreifte oftindifiche Mous.

Klein, quadrup. difp. p. 57. Mus orientalis.

Klein, Claffif. der vierfüfs. Thiere, p. I7I. n. 6. orientalifche Maus.

Klein, natürl. Ordwung d. vierf. Th. p. 6r. n. 3. feuerrothe orientalifche Maus.

Frifch, Naturfyft. p. 7. n. 5. Mus orientalis ruber; rot': Mans mit granen Streifên.

Merrem, zool. Abh. p. 32. 11. 9. geftreifte Maus.

Erxleben, Mammal. p. 400. n. Io. Mus (Striatus) cauda elongata, corpore grifeo ftriis punctatis albis.

Linné, Syft. Nat. Edit. X. I. p. 62. n. I3. Mus (Striatus) cauda elongata, fubnuda, palmis tetradadylis, plantis pentadactylis, corpore friis. punciatis.

Graumann, introd. in hift. nat. mamm. p. 63. X. die rothe geftreifte Maus.

Gatterer, brev. Zool. I. p. IrI. n. Io. Mus (Striatus) cauda elongata, corpore grifeo ftriis punctatis albis; die oftindifche yefireifte Ratze.

20. B A R B A R U S. Die geftrichelíe Maus.

Miiller, Naturfyftem, Suppl. p. 47. 1. 39. die wilde Mans.

Zimmermann, geogr. Zoolog. II. p. 366. n. 28 r. die geftrichelte Maus. -

Schreber, Säugth. IV. p. 666. n. 15. die geftrichelts Maus.

Merrem, zool. Abhandl. p. 32. n. 9. dreyfingrige Maus.

Erxleben, Mammalia, p. 399. ๒. 9. Mus (Barbarus) cauda elongata; corpore fufco, friis decem pallidis. 
Grawmana, intr. in hift, nat. mamm. p. 62. IX. die vilde Maus.

Catterer, brev. Zool. I. p. IIr. n. 9. Mus (Barbarus) cauda elongata, corpore fufco, ftriis de. cem pallidis; die wilde Hilaus.

$$
\text { * * Haarschrönzige. }
$$

21. SaxAtilis. Die Klippmatis. (4)

Zimmermann, geogr. Zoolog. II. p. 267. n. 282. die Klippnanis.

Merrem, zoolog. Abhandl. p. 29. n. Ir. Klipp. Zeift.

22. CYANus. Der Guanqué.

Moline fagt: er wohnt in einer zehn Fufs langen horizontalen Röhre, die den andern vierzehn Löchern oder Kammern, wovon fieben in jeder Reihe liegen, und ohngefähr einen Fufs lang find, zum Vorfaal dient. Beym Eintritt der regnigten Iahrszeit fängt es an, von dem Vorrath zu leben, der in den hinterften Kammern liegt, weil diefer zuerft hineingelegt wurde; die Schale der verzehten Knollen trägt das Thier imrier heraus. Die Familie befteht aus Männchen und Weibchen, und fechs Iungen, die zu Ende des Herbftes zur Welt kommen; die andern fechs Iungen, die im Frühling gebohren wurden, find um diefe Zeit fchon von den Alten getrennt.

Ir. A AI.

(4) Bauet fich zwifchen den Felfenklippen, in deren rnit Erde angefullten Spalten ein mit weichem Iicu ausgepolnertes Neft, zu welchem eine "fchrige, und eine oder mehrere faft fenkrechte, anderthalb bis zwey. Ellen lange ungerade Rithron fuliren. 
1. A м H в г Us. Die Waffermans. (5)

Miilles", Naturfyftem, I. p. 346. n. ir. die WafSerratze.

Zimmernann, geogr. Zool. I. p. 295. die Wa Jerratze. II. p. 367 . n. 293. die W afferratte. Lefke, Naturgefch. p. 167. 11. 2. die Wafermans. Borowsky, Thierreich, I. 3. p. 26. n. 3. die Wafa Serratte. Tab. 24 .

Bechftein, Naturgefch. Deutfchl. I. p. 458, n. I. die Wafferratte, der Erdwolf.

Ebert, Naturl. I. p. 347. die IV afermanus.

Halle, vierf. p. 430 . die WaJlerratte.

Meyer, Thiere, II. Tab. 84. die Wafferratte.

Gatterer, vom Nutzen und Schaden der Thiere,

I. p. 24r. n. I33. die Wafjerratze.

Perinant, arct. Zool. II. p. I29. n. 70. die WafSerratte.

Neuer Schaupl. d. Natur, IX. p. 57i. Waffermaus: Onomat. hift. nat. V. p. 229. die Walderratte, Waffermaus.

Klein, Quadr. difp. p. 57. Mus aquatilis.

Klein, Claffif. der vierfüfs. Thiere, p. 169. die Wádferratze.

Klein, natïrl. Ordn. d. vierfüfs. Th. p. 60. n. I. Wafferratze.

Biiffon, vierfüfs. IV. p. 25I. die WaJermaus, WafJerratte. Tab. 72 .

Kraft,

(5) Fangt fich oft zufalligct Weife in Fifchreufen, und komme darin um, weil fie kam eine halbe Minute unter dem Waffer awshalten känn. In Frakkreich machen die Bauern eine Faftenfpeife daraus. In einigen Gegenden Schwedens braucht man gegen die Wafferfuche eine Waftermaus mit Haar und allem zu Pulver geftof́sen, cingenommen. Von den Wanderungen diefer Thiere hat pallas in den nordifchen Eeyträgen, (am a. O.) merkwürdige Nachrichten mitgetbeilt. 
Kraft, Ausrottung graufam. Thiere, II. p. 473. die Waffermaus.

Handb. der Naturgefch. I. 2. p. 18r. Mus aquaticus; die Wafermans.

Handb. d. deutfchen Thiergefch. p. 42. Mus aqua. ticus; Waffermans.

Bock, Naturgefchichte von Preuffen, IV. p. 77. Wh:Jervatze.

Batfch, 'Thiere, I. p. 245. die Waferratte.

Merrem, zool. Abh. p. 27. n. 4. Waffer-Zeift.

Fifcher, Naturgefch. von Livland, p. 6r. 13. 27. Waferratte.

Pontoppidan, Norwegen, II. p. 56. WafJerratten. Leenz, Nachr. von den Lappen, p. I24. Mus amphibius?

Pallas, Reife durch Rufsland, III. p., I9.

Pallas, nord. Beytr. I. p. 335. grofse Waffer. ' mäuse.

Schwed. Abhandl. XXIX.p. 306 . Waffermaus. Reichard, Land - und Gartenfchatz, VI. p. 222. Becknamin, phyf. ökon. Bibl. I. p. 187. Wa/ferratte.

Limné, Syff. Nat. Edit. II, p. 47. Callor cauda lineari tereti.

Linnzé, Syft. Nat. Edit. VI. p. Io. n. 3. Caftor cauda lineari tereti.

Linné, Syft. Natur. Edit. X. I. p. 61. n. 8. Mus (Amphibius) cauda elongata pilora, plantis palmatis.

Limné, Fn. Suec. I. p. 10. n. 25. Caftor cauda lineari tereti.

Graumann, intr. in hifor. nat. mainm. p. 6r. III. Wafferratze.

Gatterer, brev. Zool. I. p. 107. n. 3. Mus (Amphibios) cauda mediocti, palmis tetradactylis, 
eum unguiculo pollicari, corpore nigricante, abdomine ferrugineo; die Waferratze.

Severin, zool. Hungar. p. 75. n. 5. Mus Amphibius, Aquaticus; tie WalJerratte. Miiller, zool. dan. prodr. p. 5. n. 3. Mus (Am: phibius) cauda elongata pilofa, plantis pal. matis.

Charleton, exercitat. p. 25. Mus aquaticus. Schwenkfeld, theriotr. Silef. p. 115. Mus aquaticus. Sibbald, Scot: illuftr. II. 2. p. 12. Mus aquaticus. Agriciola, de anim. fubterr. p. 58. Mus aquatilis. Bellon. de aquatil. p. 35. Mus aquaticus? Abbild. p. 36. Schlecht.

Ionfton, quadr. p. I69. Mus aquaticus.

Aldrovand. de quadr. digit. p. 447. Mus aquatilis,

B. Terrestris. Die Erdmails. (6)

Miiller, Naturfyft.I. p. 345. n. Ió. die Erdmaus. Borow $k$ y. Thierreich, I. 3. p. 28. 11. 7. die kleia ne Feldmans. (mit Mus arvalis verwechfelt.) Neuer Schaupl. der Natur, II. p. 80z. Feldmans. Onomat. hift. nat. V. p. 35 I. Feldmaius. Bechftein, Naturgefch. Deutfchl. I. p. 468. Feldmans, Feldratze, Reutmans, Evdfchiiffel, Scharrmans; Scheermaus.

Klein, Quadrup. difp. p. 57. Mus agreftis, capite grandi.

Klein, Claflif. d. vierf. Th. p. t7o. die Feldmans. Klein,

(6) Es ift fchwer, bey diefor; der fechszelinten; fiebzebnsen, und acit und zwanzigften Gattung die Synonymen gehürig zu ordnen: Auch im Syftern find nut wenige bemerkt. Ersleben hall die Erdmaus und die gefellige Maus für Varietaten von der Feldmaus. Zimmermann hall Linne's und Erxlebens Misis Terroftrio fü einerley: 
Klein, naturl. Ordn d. vierf. Th. p. 61. n.

Feldmais mit grofsem Kopfe.

Bock, Naturgefch. von Preuften, IV. p. 76, n. 25 . Feldmaus?

IMolini, Naturgefch. von Chili, p. 242. Feld. maus?

Fabric. Reife wach Norwegen, p. 217. Mus Terreftris.

Beytr. zur Beförd. der Naturkunde, I. p.İ7. n. I9. Erdmous. (mit Mus arvalis verwechfelt.)

Limné; Syrt. Nat tedit. II. p. 47. Mus agreftis, capite grandi, brachyurus.:

Linné, S. Nat. Edit. VI. p. Io. n. 5. Mus cauda longa pilofa, auribus fubrotundis, vellere brevioribus.

'Limé, Syf. Nat. Edit. X. I. p. G̈. Mus (Terreftris) cauda mediocri fubpilora, palmis fubtetradactylis, plantis pentadactylis, auriculis vellere brevioribus.

Linné, Fn. Suec I. p. IO. n. 29. Mus cauda longa pilofa, auribus fubrotundis, vellere brevioribus.

Kramer, Aufr. p. 3i6. Mus cauda longa pilofa, auribus fubrotundis, vellere brevioribus.

Miiller, zool. dan. prodr. p. 4. n. 27. Mus (Terreftris) cauda mediocri fubpilofa, palmis fub. tetradactylis, plantis pentadactylis, auriculis vellere brevioribus.

Pallas, nov. fpicil. quadr. e glir. ord.p. 80.

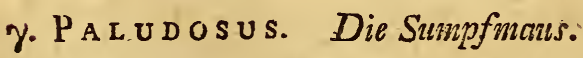

Milller, Naturfyft: Suppl. p. 39. n: 22. die Sumpfmazes.

Boroweky, Thierreich, I. 3. p. 28. n. 6. die Sulinpfinaus. 
Biiffon, vierfïls. IV. p. 252. die Sumpfratte. Batfch, Thiere, I: p. 226. die Sumpfinaus. Merrem, zool. Abhandl p. 28. $\gamma$. die Sumupfmans.

Erxleben, Maminal. p. 394. n. 6. Mus (Paludofus ) cauda elongata; auriculis vellere brevio. ribus, corpore nigro.

Graumann, intr. in hiltor, nat: mamm: p. $62 . \mathrm{VI}$. die Sumpfmans.

Gatterer, brev. Zool. I. p. ròg. n. б. Mus (Pa ludofus) cauda elongata, auriculis vellere bre: vioribus, corpore nigrb.

- NIGER. Die fchivarze Waflermanis.

Schreber, Säugth. IV: p. 670. einè ganz Jchwarz⿺ Spielart.

Pallas, glir: p: 8I. Varietas tota atra:

ع. M A c U L A T u s. Die gefleckte Wafjermaiis. Schreb̉er, Säugthiere, IV. p. 6700: eine andere, die fich durch einien grossen weifseri Flech von unregelniässiger Geftalt; mitten auf dem Rïcken iiber den Schultern; und einen kleinnen weifsen Strich anf der Brinft auszéichnet.

Pallás, glir. p: 8i. alia varietas; quae macula alba irregulari in medio dorfo fupra fcapulas, fimul que litura faepe parva alba ferni infignitur.

23. A L I A R I Us. Die Knoblauchsmanis. (7)

Zimmerinann; geogr. Zool. II. p. 368 . n. 284. die Knoblauchmans.

\section{Ff 2}

Gatte-

(7) Die Ruffen und Sibirialen haben von diefer Maus den Vortheil, dafs fie die von ihr zum Wintervorrath in ihre Löcher, eingetragene $Z$ wiebeln ausgraben; und fich derfelben zu threm Gebrauch bedienen. 
Gatterer, vom Nutzen u. Schaden der Thiere,

I. p. 25 r. n. 139. die Knoblauchsmaus.

Merrem, zool. Abhandl. p. 29. n. 10. KnoblaichZeift.

24. RuTilus. Die rothe Mans. $\left(^{8}\right)$

Zimmermonn, geogr. Zoolog. II. p. 7. die rothe

Maus. p.368. n.285. die rothe fibirifche Maus. Gatterer, vom Nutzen und Schaden der Thiere,

I. p. 253. n. 142. fibirifche rothe Maus.

penvant, arct. Zool. II. p, I34. n. 79. die rothe fibirifche Maus.

Steller, Kamtfchatka, p. I29. Tfchetanauftchu;

die dritte Sorte, die rothe Maus.

Merrem, zool. Abhandl. p. 29. n. 8. rother Zeift.

ß. Min or. Cauda proportione tenuiore et longiore. Pallas glir. p. 247.

Habitat circa mediam Volgam, praefertion in Cafanienfibus et Simbirskienfibus agris, frequens hyeme circa pagos et granaria.

Pondus in plurimis $4 \frac{x}{2}$ val 5 drachmas aequat. Longitudo corporis $3 \frac{x}{3}$ pollicum; capitis $I \frac{x}{6}$, caudae $I \frac{\pi}{2}-1 \frac{2}{3}$ pollicis.

25. GREGALIs. Die Zwiebelmans. (9)

Merrem, zool. Abh. p. 29. n. 9. Zwiebel-Zeift.

Gatte-

(8) Fängt fich öfters in den fitr Hermeline und dinliche Thiere mit Fleifch aufgeftellten Fallen. Sie wird von den lakuten an der Lena gegeffen. Selbtt foll fie keine Provifion eintragen, aber im Wincer dic Vortathskammern der Wurzelnaus beAtehlen.

(9) Gewicht der Weibchen I Unze 2 Quentchen, der Männchen 6 Quentchen. Ueber. das Neft machen fie von der ausgewühl- 
Gatterer, vom Nutzen und Schaden der Thiere,

I. p. 25 I. n. 140. die Zwiebelmans.

26. OEc o-i omus. Die Wurzelmans. $\left({ }^{10}\right)$

Zimmermann, geogr. Zoolog. II. p. I6. die öko: nomifche Maus. p. 369. n. 287. die Wurzelmans; die ökonomifche Mans.

Bhumenbach, Handb. der Naturgefch. p. 73. n. r. Mus (Oeconomus) cauda fubfesquiunciali, auriculis nudis, vellere molli latentibus, palmis fubtetradactylis, corpore fufco; die $W u w z c l$. mans.

Gatterer, vom Nutzen und Schaden der Thiere, I. p. 252. n. I41. die Wurzelmaus.

Pennant, arct. Zool. II. p. 133. n. 78. die Wur. zehnaus.

$$
\mathrm{Ff}_{3} \quad \because p_{\text {allas, }}
$$

ten Firde einen gewölbten Haufén, um den Regen abzuleiten. In jedem Nefte in allemal eine gąnze aus einem Paar, und den lungen defelben lahres beftehende Familie anzutreffen.

(10) Lạnge des Mạnnchens olıne den Schwanz 3 Zoll, 3 bis $6 \mathrm{Li}$ nien; des Schwanzes eilftehalb Linie: des Weibchens vier Zoll drittehalb Linie; des Schwanzes eilftehalb Linie; gröfserer Weibchen 4 Zoll 5 Lin., des Schwanzes I Zoll 2 Lin. Gewicht des Männchens obngefähr I Loch; des Weibchens I Unze I-2 Quentchen, Der Vorrathsliammern bey einem Nefte find oft drey, vier, und mehre:e, und jede enthält oft 8-10 Pfund gereinigter Wurzeln. Der Abzug diefer Thiere, der in grofsen Haufen in gerader Linie gegen Nordweften gefchiehet, kann fo zahlreich feyn, dafs er zwey Stunden in einem fortwähret. Sie gehen um den grofsen penfhinifchen Meerbufen herum, und dann füdlich bis ohṇgefähr gegen den 57 Grad der Breite herunter, wo fie um die Mitie des Iulius anzukommen pflegen. Im October kommen fie auf eben die Art wieder in Kamtfchatka an. Fine folche Auswanderuing wird für ein Zeichen küinftiger naffen und ftürmifchen Witterung; dic Rückkehr hingegen für die Vurbedeutung cines zum Wild-und Fịfchfange glücklichen lahrs angefehen. 
Pallas, Reife, Ausz. III. p. 159. Anh. p. 3. n. 4 Mus aeconomus; Buraeto - Mongolis Uru? - gundfchi-Cholgona. Iacutis Kutujach. Steller, Kamtfchatka, p. 129. Taegult fchitfch. Krafchenimnikare., Befchr. von Kamt lchatka, p. 126. die erfte Sorte Teguktfchitfch genannt.

Merrem, zool. Abh. p. 28. n. 7. Wurzeb.Zeift. Georgi, Rufsland, II. p. 320. Mïuse.

Pallas, nord. Beytr. I. p. 335. die fibivifche IMagazinmaus.

Olaffen, Reife d. Island, I. p. 97. Waldmö̈ufe? p. II7. Mön $\int e$ ?

27. L A N I E R. - Die Chinchilla. Molin,

76. Arvar ys. Die kleine Feldmaus: (1)

Miiller, Naturfyft. I. p.345. n. 16. die Feldmans. Zimmernann, geogr. Zool. I. p. 296. II. P. 3770. 11. 289. die Feldmanus.

Bechftein, Naturgefch. DeutChl. I. p. 469. n. 2. die kileine Feldnuans. p. 474. Heerdenmabs, kleine StoSsmans, kleine Reutmans, Kampagnol, Erdfahren, Ackermans.

Bhumenbach, Handb. der Naturgefch. p. 74. n. 3 . Mus (Terrefter) çauda mẹdiocri, dorfo ferrugineo,

(4) Die Irohlen, welche diefe Thiere machen, haben eine, oder. gewöhnlicher zwey fchrảge und verfchiedentlich gebogene, kaum daumensdicke. Röhren, anderthalb bis zwey Fufs tief angelegt. Die Höhle hat die Gröfse einer Fauft, und ift mit weichen Grasfchnitchen ausgepolftert. Diefe Maus vermehrt fich zuweilen fo nark, dafs fie dem Getraide auf dem Felde nachtheilig wird. Ins Waffer geht fie nie. In Ânfehung der zu diefer Gattung gehörigen Synonymen wiederhole ich dasjenige, was ich bereiss in der Annerkung bey Mus Amphib. Terriftris angeführe habe. S. Schreber. IV. p. 682. 
gineo, abdomine cinereo; die Feldnnaus, Stofs: mans.

Halle, vierf. p. 431. die Scharrmans.

Gatterer, vom Nutzen und Schaden der Thiere,

I. p. $24 \%$. n. 136. die Feldmaus, kleine Feldmaus, der Kampagnol.

Merrem, zool. Abhandl. p.28.' 11. 5. Erd-Zeift. Pennant, arct. Zool. II. p. x32. n.76. die Feldmans. Neuer Schaupl, der Natur, II. F. 670. Eramans. Onomat. hift. nat. V. p. 334 die Erdmaus.

Gesner, Thierbuch, p. 264. Mus fubterraneus; Nül-oder Wiihlmats; in. e. fchl. Fig. p. 265 . Feldmaus, Evdmuns, Schnorrmanus, Scharrmaus, Stofsmaus, Lücknaus, Ackermaus.

Handb. der Naturgefch. I. 2. p. i80. Camupagnol. Handb. d, deutfchen Thiergefch. p. 42. Mus Terreltris; die Felamaus, Stossmaus, kleine Feld. maus.

Naturforfcher, XII. p. 88. Erdmcuus mit kurzen Ohren and Schwanze.

Biiffon, vierf, IV. p. 256. die kteine Feldmans, der. Kampagnol. Tab. 73

Kraft, Austottung graufam. Thiere, II. p. 475.' Niill-Maus, Sihnorr-oder Schör-Maus, Stockmans, Ackermans.

Beytr. zu Beförd. d Nakurkunde, I. p. 210."n. 23 . Feldmans, Ackermans.

Wolf, über die Fèldmäufe, \&. Hamb. I786. Zorn, Gedanken über die Müufeplage, 1742. und $1773^{\circ}$

Wittenberg. Wochenbl. 73. p. 261. 269. u. 305. Hïpfch, ökonom: Vorfchläge die fchädlichen Ackermäule zu vertilgen. Köln 1768.8 .

Berlin. Samml. IV. p. 470. Garten-zund Faldmaàife. VI. p. 144. Felduniüufe.

$$
\text { Ef } 4 \text {. Einné, }
$$


Linné, Syf. Natur. Edit. VI. p. ro. n. 4. Mus cauda bevi, corpore nigro-fufco, abdomine cinerafcente.

- Linnęé, Fn. Suec, I. p. 10. n. 27. Mus cauda hre. vi, corpore nigro - fufco, abdomine cinera. fcente.

Graumann, intr, in hilt, nat, mamm. p. 62. VII. kleine Feldnuaus.

Gatterer, brev. Zool. I. p. rio. n. \%. Mus (Ter= reftris) cauda mediocri, auriculis vellere bre. vioribus, (das find fie aber nicht) corpore fupra ferrugineo, fubtus cinereo; die stossmazis, Feldinaus.

Kramer, Auft, p. 316. Mus cauda brevi, corpore nigro - fufco, abdomine cinerafcente.

Schwenkfeld, theriotr. Silef. p, ni6. Mus agreftis, Aldrovand. de quadrup. digit. p. 436. Mus agre. ftis minor.

Pennant, britt. Zoolog. p. 50. the fhort tailed Fieldmoufe.

Pemant, britt. Thiergefch. p. 45. Tab. XI. e. Forfter, philof, transact. LXII. p. 3\$0. Shorts tailed Moufe.

28. Socratis. Die Tulpermails. $\left({ }^{2}\right)$

IMiiller; Naturfyft. Suppl. p. 45, n. 36. dis gefellio ge Matus.

Zimmermamn, geogr. Zool. II. p. 370. n. 290. di Tulpenmunus.

Pallas, Reife, Ausz, II. Anh. p.5. n, Io. Mus focialis, Pallas.

(2) Erxieben und die, die ihm gefolgt find, rechnen, wie ich fchon bemerkt habe, die gefellige Maus zu der Erdmaus, und machen dagegen aus den beyden, im Syftem angeführten Synonymen des Gmelin, die eigentich mit bieber gehören, zwey befondere Gattungen, $S_{9}$ Pallas glir. p. 219. not, b. 


\section{Mammalia Glives. Mus.}

Pallas, Reife, Ausz. III. p. I60. eine andere lichtgraue and kleinere Art von Müufen.

Merrem, zool. Abhandl. p. 28 .n. 6. Tulpen-Zeift. Erxleben, Mammalia, p. 403. n. I3: Mus (Aftra. chanenfis) cauda mediocri, corpore fupra flavo, fubtus cincreo.

Erxleben, Mainmal. p. 403. n. 14. Mus (Microuros) cauda abbreviata, corpore fupra cinereocanefcente, fubtus fordide albo.

Graumann, intr, in hift, nat. mainm. p. 63. XIII, Mus Aftrachanenfis; XIV. Mus Microuros.

Gaitterer, brev. Zoolog. I. p. III. n. I3. Mus (Afrachanenfis) cauda mediocri, corpore fupra flavo, fubtus cinereo.

Gaiterer, brev. Zool. I. p, Irr. 11. 14. Mus (Mi. crouros) cauda abbreviata, corpore fupra cine, reo-canefcente, fubtus fordide albo.

29. L A G UR US. Die Schreertelmaus. (3)

Miiller, Naturfyft. Supplem, p. 45. n. 35. den Rauchfchwanz:

Zimmermann, geogr.' Zool. II, p. 37r. n. 29 r. die Schevertelmans.

Pennant, arct. Zool, II. p. I32, n. 77. die Schrpere telinaus.

Pallas, Reife, Ausz. II. p. 5. n. 9. Mus Lagurus. Merrem, zool. Abhandl. p. 27. 11. 2. Z ug -Zeift. Erxleben, Mammalia, p. 375. 11. 12. Glis (Lae gurus) auriculis vellere brevioribus, corpore fupra cinereo, fubtus albo, fria dorfali nigra, Ff 5

Grals:

(3) Die Nefter diefer Thiere baben zwey Röhren, die eine fenkrecht, etwa einer Qirerhand tief, die andere fchräge, und etwa eine Spanne lang; beyde laufen zuweilen vor dem Eingange in àas Neft zufammeñ. 
Graumann, intr. in hif. nat. mamm. p. 60. XII, der Rauch chwernz.

Gatterer; brev. Zool. I. p. 104. n. II. Glis (Lagurus) auriculis vellere brevioribus, corpore fu. pra cinereo, fubtus albo, ftria dorfali nigra; dev Rauchfcherauzz.

\section{Torquatus. Die Uralmaus.}

Zinmermann, geogr. Zoolog. II. p. 372. ne 292. die Uralmans.

pennant, arct. Zool. II. p. 336. n. 82. die Maus. mit der Halsbincte; die Uralnaus. Merrem, zool. Abhandl. p. 27. n. 3. Kragen-Zeift.

\section{Lem u us. Der Lemming. (4)}

Müller, Naturfyften. I. p. 339. n. 5. dęr Lemming. Tab. 20. fig. r. Worm.

Zimnner:a

(4) Theilt fich in zwey Racen, die norwegifche und die rufifiche. (Peftrufsbka.) Länge der criftern $5 \frac{\mathrm{T}}{4}$ Zoll, des Schwanzes $7 \frac{\mathrm{T}}{3} \mathrm{Li}$ pien; der letzern $3 \frac{5}{6}$ Zoll, des Schwanzes $5 \frac{2}{3}$ Linien; beydes ohnic die an der Spirze befindlichen Haare. Die Wanderungen. diefer Thiere find fehr merkwïurdig, befonders die Umftände, init welchen eine folche Wanderung gefchiehet. Das ganze Volk verfammelt fich in Herhft damu in Haufen, und jeder zieht in gerader Richtung, in langen Colonnen, die eine bis zwey Spannen breit, oft mẹthere Ellen von einander in Abftande und einander payallel find, vom Gẹirgẹ hinabu. Untẹrwegs freffen fie alles Grïne auf der Erde weg, fo dafs die öden Spuren ihres Zugs faft das Anfehen eines geptiligten Feldes haben, denn es cmigriren zuweilen gañze Legionen wie Zugheufchrecken von einer Gegend in die andẹre. Auf ihrem Zuge laffen fie fich weder durch Berg, noch Thal, noch Feuer, noch Waffer vor der geraden Linie abbringen, th und finden fogar im Waffer; die Richtung ibres Weges wierier, wenn man fie davon s.bgebracht hat. Eines im Wege ftehenden Felfenwant, die io 
Zimmermann, geogr. Zool. II. p.5. p. 372. n. 293. der Lemining.

Borowfky, Thierreich, I. 3. p. 30. n. I. Glis Lemmus; der Lenming, die norwegifche IVIus. Tab. 25.

Bhumenbach, Handb. der Naturgefch, p. 72. n. 4. Marmota (Lemmus) capite acuto, corpcre nigro fulvoque irregulariter maculato; der. Eemming. Funke, Naturgefcbichte, I. p. 123. der Lemming. Halle, vierf. p. 436. die norwegifche IMaus, Lem. ming. p. 603. die norevegifche Bergmans: Lemming.

Gatterer, vom Nutzen' und Schaden der Thiere, I. $p_{2}$. 132. n. 126. der Lemming.

Pernant

fteil ift, dafs fie nicht hinan klettern küňnen, müfmen fie zwat ăusweichen; fie marchiren aper blofs ụm felbige herum, und fetzen fodann itren $W$ eg in der numlichen Linie weiter fort. Dabey gehen fie weder Menfchen noch Vieh aus dem Wege, fetzen fich, wens man ihnen in den Weg tritt, auf die Hinterfüfse, wehren fich und beiffen in den, ihnen vorgehaltenen Stock fo feft, dafs man fic damit auflheben kann. Der Weg der Haufen, die fich gegen Weften wenden, endigt $f_{\text {ich }}$ in Meere; des gegen Often ziehenden aber im bottnifchen Meerbufen, worin manche umkommen. Diejenigen, welche die Reife aushalten, und nicht durch Eisfüchfe, Wiefel und Raubvougel, . diẹ ihṇen in Mạnge nachziehen, oder durch andere Schicksale aufgerieben werden, kommen dann im künftigen Sommer, aber in fo geringer Anzahl zurïck, dafs man kaum éinen gegen hundert rechnen kann. Die Züge. der Peftrufchlea géhen vom nürdlichen Ural gegen Weften bis ins ruffifche Lappland, und gegen Often bịs an den lenifei. Die unerwartete uni unbemerkte Ankunft diefer Thiere an dem Orte, wo fie fich niederlaffen wollen, fo wohl, als auch der Fall, dafs, dann und wann welche, die von den Raubvügeln in die Luft gehoben worden, fich los gearbeitet haben, und herunter getallen find u. dgl. hat in yorigen Zeiten zu der Șage Anlaş zegeben, dâfs es Lemminge vom Himmel regnete. 
Pennant, arct, Zoolog. II. p, 135. n. 80. der Lemming.

Neuer Schauplatz d. Nạtur, V. p. 90. Lemming, Lasmmil.

Onomat. hift. nat. IV. p. 764. noorwegifche Maus. Klein, Quadr. difp. p. 58. Mus Lemingus, Nor. vagicus,

Klein, Claffif. d. vierfüls. Th. p. 175. norwegiSche Mans.

Klein, natür!. Ordn. d. vierf. Th, p. 6т̣. n. ra: norwegifche Maus, Lemming.

Gesner, 'Thierbuch, P. 275. Leming, norwegifihe Maus, Leminger, Lemender, Lommet. p. 275, Abbild. p. 277. das Skelet.

Biiffon, vierfüls, XV. p. 293. der Lemming: Bat fch, 'Thicre, I. p. 229. der Lemuning. Merrem, zool, Abhandl. p. 26. n. I. Lemming. Leem, Nachr, von den Lappen, p. I2r. Berg mäne

Olear. Gottorf. Kunfkammer, p. 20, Tab. 12. fig. 6. ein Sonderlich rauch Thierlein. Abbildung gut.

Bonnet, Betracht. über die Natur, II. p, 254. dic Ratzen, welche in Heerden herumziehen.

Goeze, Allerley; neue Aufl. I. p. 298. eine Avt von Bergmänjen, Lemninge.

Goeze, Natur, Menfchenl, und Vorf. I. p. 526.

V. p. 433. die nomeegifchen Lemminge.

Kliigel, Encyklopäd. I. p. 231. Lemmuing. Liane', Abhandl. über die Thiere, von denen in Norwegen gefagt wird, dafs fie aus den Wol. ken kommen; in den Schwed. Abhandl. II. p. 75. 76. Schwedifche Rergmaus, Zobelmaus, Rothmaus, nordifche Lönuing, Lömmer, Lombmel lappländifcise Lansmik. 


\section{Mammalia Glives. Mus $46 x$}

Högfrön, Anmerkung tiber die Thiere, die in Norwegen aus den Wolken kommen follen; in den Schwed. Abhandl. XI. p. Ig.

Derhan, Phyficotheol. p. Io. Lemming.

Erxleben, Mammal. p.37i. n. 8. Mus (Lemmus) corpore fulvo nigroque vario.

Zinné, Syff. Nat. Edit. II. p. 47. Mus norvegi॰ cus, vulgo leemming.'

Linné, Syft. Nat. Edit. VI. p. io. n. 2. Mus cauda abrupta, corpore fulvo nigroque vario.

Linné, Syft. Natur. Edit. X. I. p. 59. n. 3. Mus (Lemmus) cauda abbreviata, pedibus pentadactylis.

Limné, Fn. Suec. I. p. Io. n. 26. Mus cauda brevi, corpore fulvo nigroque variegato."

Graumann, intr. in hift. nat. mamm. p. 59 . VIII. Glis Lemmus; norwegifche Bergmaits.

Gatterer, brev. Zool. I. p. I03. n. 8. Glis (Lemmus) capite acuto, corpore nigro fulvoque ir regulariter maculato; der Lemming, die norwegifche Maus.

Milller, zool. dan, prodrom, p. 4. n. 26. Mus (Lemmus) cauda abbreviata, pedibus pentadaCtylis corpore fulvo nigroque vario.

Mitller, zool. danica, II. p. 9. Mus Lemmus. 1'ab. 43 .

Olans Magiz. de gent. feptentr. p. 726. Murium multitudo de nubibus demifia.

Scheffer, Lappon. p. 344. Beftiae minutae Lem'mus dictae.

Scheffer, Lappland, p. 388. Lemming.

Charleton, exercitat. p. 25. Mus Norvegicus.

Iacobaei, Mufeum reg. p. 6. Mus norvagicus, Leming vel Lemend dictus.

Hermanzm, tab. affin. anim. p. gr. Mus Lemmus, 
Valentini. Amphith. zoot. Tab. 38. fig. Worm: Valentin. Muf. mufeor. (deutfche Ausgabe) it. Tab. 27. fig. 3. Worm:

Blafii anatom. animal. Tab. 34. fig. 5. 6.

31. Hudsonius. Die labrádorifche Maus.

Zimmermann, geogr. Zool. II. p. 373: n: 294: dic labradorifche Minus.

Pennant, arct. Zool. II. p. 13̈7. n. 75. die Labra. dor-Maius.

Forfter, philofoph. Transact. LXII. p. 379.

Penncint, hift. quadr. n. 3rg. Hudfons = Rat:

32. Ma U I INUS. Die grofse Waldmaus. Molin. Ift 1764. zuerft gefunden.

**** Hamjermäufe, mit Backeatajcheq.

3.3. A CREDU A, Die Iaikmars. (’)

Miiller, Naturfyf. Suppl. p: 43. n. 3I. Mus Mi: gratoritus , die Zngmiaus:

Zimmernann, geogr. Zoolog. II. p. 376. n. 301. die laiknaus.

Borow $k y$; Thierreich, I. 3. p. 38. in. 6. Glis migratorius; die Zugmaus.

Gatterer, vom Nutzen und Schaden der Thiere; I. p. 233. n. I27. die Znigmans.

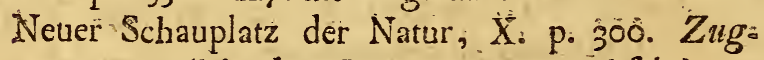
matus: (Mit dem Lemming verwechlelt:) Batfch, Thiere ; I. p. 230. Zugmänife. Merreni, žool. Abh. p. 44. n. 9. Zng-Ziefel. Pallas, Reife, Ausz. II. Anh. p. 3. n. 5. Mus migratorius:

Ëxles:

(5) Hat den deutfchen Namen von dem İaik, im Orenburgifchez, an welchern fie zuerft if entdeckf worden. 
Erxlebrin, Mamnialia, p. 373. n. 9. Glis (Migratorius) corpore fupra cinereo, fubtus candido.

Graumtann, intr: in hiff. nat. mamm. p. 60. IX. Glis migratorius; die Zugmans.

Gatterer, brev. Zoolog. I. p. 104. n. 9. Glis ( $\mathrm{Mi}$ gratorius) corporefupra cinereo, fubtus candido; die Zugmaus.

Severin, zool. Hungar. p. 73. b. Mus migratorius.

9. Cri c т Us. Der Hamfter. ( $\left.{ }^{6}\right)$

Miiller, Naturfyh. I. p. 345. n. 9. der Hamfter. Zimmermanin, geogr. Zool. II. p. 10. 376. n. 300. disior Hamifer.

(6) Ein beifliges böshaftes Thier, das auffer dem Zorn kaum eine andere Leidenfchaft zu kennen fcheint. Bey einer fehr unbeträchtlichen Leibesgröfse ftellt er fich doch dem Menfchẹn zur Wehr, und Hunde, die des Hamfterfangs ungewohnt find, ziehen leicht gegen ihn den Kürzern. Frifse autfer den Vegetabilien aùch Käfèr, kleine Vügel, denen er žuerft die Flïigel ent: zwey heifst, und Mäule. Man hat fogar gefuricén, dafs Hams Aer, einander felbft auffreffen. Des Saufens künnen fie lange enthehren. Bisweilen farten fie ihren eigenen Harn. Um das Keimen dés Getraides in ihren Kammern zu verbüten; beiffen fie düin Korne vorher die Keime ab. Eine Hühie tält manchmal auf fechszig Pfund. Wenn ter Hamter ruhet, liegt er wie eine Kugel zufammen geballt, mit unter die Bruft gezogenem Kopfe. Greift man ihr an; indem er mit vollen Backen-nach Haus kehree, fo itt ér wehrlos, und es ift keine Gefahr bey ilum zu beforgen. Er fucht die Backen zuerft auszuleeren; und dann fpringt er mit klappernden Zähnen, fchnellem $A$ shmen, fchnarchendem Aechzen, und aufgeblafenen Backentafchen auf feinen Feind los; dem er, falls er weiche, nachhüpft. Sein Bifs ift gefahrlich, weil er fich leicht verbeifst. Mehrere zufammengefperre Hamfter reiben fich unter einander fo auf; dafs zuletzt nur einer übrig bleibt. Das Alrer eines Hamfters rechnet 
Lefke, Naturgefch. p. 169. 11. I. Cricetus vulgaris; der gemeine Hamfter.

Borowjky, Thierreich, I. 3. p. 35. n. 4. Giis Cricetus; $\operatorname{der} \operatorname{Ham} / \mathrm{tez}$. .

Bhumenbach, Handb. der Naturgefch. p. 72. n. 3. Marmota (Cricetus) abdomine nigio; der Hamiter, Kornferkel.

Bechftein, Naturgefch. Deutfchl. I. p. 475. n. r. der gemeine Hamfter. p. 489. Hamftemans, grofse Feldmans, frrafsburgifches Murmelthier, Kornhamfter, Kornferke!, Grentfch, Grutfchel, (Erdwolf) Krietfch.

Funke, Naturgefchichte, I. p. II6. des Hamfter.

Ebert, Naturl. I. p. 346. Hamfter, grofse Feldmalus.

Halle, vierf. p. 424. der Hamfter. Tab. 23. Gatterer, vom Nutzen und Schaden der Thiere, I. p. 223. der Hamfter.

Beckmann, Naturhiforie, p. 2I. n. 4. der Hamfler. Neuer Schaupl. d. Natur, III. p. 639. Hamfter. Onomat. hift. nat. III. p. 462. Hamfter, Kornt farle.

Handbuch der deutfchen Thiergefchichte; p. 39. Marmota Cricetus; der Hamfter, Komferkel. Sander, ökon. Naturgefch. I. p. 256. der Hamifter. Frif ch, Națurfyft. p. 9. n. 3. Marmota Cricetus der Hamfler.

Klein, Claflif. der vierfifs. Thiere, p. 166. n. 3 . Anfter.

Klein,

rechnet than auf fieben bis acht tahre. Die lungen wercen nackt und blind, aber mit allen. Zähnen gebohren. In manchen Iahren, und in manchen Gegenden vermehrt fich diefe Thierart ganz auferordentlich. Im Gothaifchen hat man fchon in einem Iahre über 80,000 gefangen, und binnen drey lahrea $1461_{3} 9$ Hamíterfelle geliefert. 
Klein, natürl. Ordn. d. vierf. Thiere, p. 59.n. 3. Hamfter.

Gesner, Thierbuch, p. 267. Hamfter, Komle fïrklein; m. e. fchl. Fig.

Biiffon, vierf. XIV. p. 5. der Hamfter; m. e. Fig. Bat $\int c h$, Thiere, I. p. 229. der Hamfter.

Merrem, zool. Abhandl. p. 43. n. 8. Hamfter. Kraft, Ausrott. graul. Thiere, II. p. 486. Hamıfter: Fif cher, Naturgefch. von Livland, p. 60. n. 21. Hamfter.

Kriinitz, ökon. Encykl. XXI: p. 354. Hamfter. Goeze, Allerley, neue Aufl. I. p. 84, Hamjter. Beytr. zur Beförd. der Naturkunde, I. p. 127. n. I8. Hamfter.

Denfo, monathl. Beytr. zur Naturk. Berl. I752。 III. p. I93. Hamfter.

Mannichfaltigkeiten, I. p. 624. III. po 177 . Hanifter.

Alijcher, Obf. voin den Hamftern in Schlefien; in den Brefsl. Samml. Verf. 17. Sept. 1721. Cl. 4. Art. 6. 21 Verf. Sept. 1722. Cl. 4. Art.6. Büchiner, ausführl. Befchreib, des Hasnfters, bey Gelegenheit der Menge derfelben im İari im 2. Suppl. der Brefsl.Samml. Budiff. 1728.4 . Art. 7. p. 92. und $\mathrm{im}_{1}$ 2. Th. der vermifchten ökon. Samnl. Lpz. 175i. 8. p. í42.

Strelitzer, neue Beytr. II Iahrg. I. Q. 1769. p. I.6. Hamfter.

Berlin. Samml. I. p. 37r, Hamfiter.

Döbel, Iägerpraktik, I. p. 43. Hamfter.

Iablonfky, allgemein. Lex. p. 428. Hamfter.

Syftem. Lehrbuch über die drey Reiche der Na.

tur, 1. p. 167. n. 9. Hamfter.

Reichard, Land - und Gartenfchatz, VI. p. 203. Homfter. 
Andvé und Bechfein, Spatziergänge, Io p. 5 s. Hanifter.

Oekonom. Zoologie, p. 35. n. 42. der Hamfter. Ludovici Kaufmannslexicon, III. p.II\%.

Bergen, diff. de animalibus hieme fopitis. Frcf. ad V. 1752 .

Erxleben, Mammalia, p. 363. n. 4. Glis (Cricetus) corpore fubtus nigro, lateribus rufefcentibus, maculis utrinque tribus albis.

Linné, Syft. Nat. Edit. II. p. 47. Mus Cricetus Gesneri.

Linné, Syft. Nat. Edit. VI. p. Io. n. Io. Mus cauda elongata, corpore cinereo, rutilo nigroque longitudinaliter vario.

Linnié, Syf́. Nat. Edit. X. I. p. 60. n. 6. Mus (Cricetus) cauda fubabbreviata, auriculis rotundatis, corpore fubtus nigro, lateribus ru. fefcentibus.

Granniann, intr. in hift. nat. mamm. p. 59. IV. Hameter:

Gatterer, brev. Zoolog. I. p. I02. n. 4. Glis (Cricetus) corpore fubtus nigro, lateribus rufefcentibus, maculis utrinque tribus albis; $d e e^{*}$ Hamfter.

Severin, zoolog. Hungar. p. 66. Glis Cricetus; der. Hamfter.

Hildebrand, obf. de fyncope aut fopore cricetorum, per plures continuante menfes fine putredinis labe, et vitae fufpendio; in den Nov. act. phyf. med. Acad. N. C. Norimb. I767. obf. 33. p. 135 .

Hildebrand, Wahrnehmung von den Hamfern etc. im nenen Homburg. Magazin St. 25. p. 87.

Valentin. amphitheatr. zootom. p. I54. 
Kramer, Auftr. p. 317. Mus cauda elongata, corpore cinereo, rutilo nigroque longitudinali ter vario.

Rzacz. hiftor. nat. Polon. p. 332. auct. p. 326. Cricetus.

Hiinerwolf, obf. de elephantiafi ex ufu criceto. rum; in den Mifc. Nat. Cur. Dec. II. A. VIII. obf. I6.

B. CRICETUS TOTUS NIGER. Der fchwar. ze Hamfter.

Zimmermann, geogr. Zoolog. II. p. Ir. eine ganz Schwarze Spielart.

Biiffon, vierf. XIV. p. I6. gans fchwarse. Pallas, Reile, Ausz. I. p. 86. fchearar Hamfter. Naturforfcher, II. p. 9. ganz fchwarze Hamfter. S. G. Gmelin, Reife d. Rufsl. I. p. 33. Táb. 6. Georgi, rurt. Reile, II: p. 82 I.

Merrem, zoolog. Abhandl. p. 44. der ficwarze Hamfter.

Allgem. Gefch. der neuéten Entdeck. I. p. 40. und p. 24.7 . Erxleben, mammalia, p. $365 . ?$ pedibus, ore, et
Varietas nigra,

Gatterer, brev. zool. I. p. roz Sau ricularum Varietas nigra, margine albis.

34. A R E A R I US. Die fibirifehe Sandmaus. (7)

Miiller, Naturfyf. Suppl, p. 44. n. 33. die Sand. maus.

\section{$\mathrm{Gg} 2$}

Zinimer.

(7) If von Pallas, im Iunius 1772. entdeckt. Das Thier mache ziemlich grofse Baue in die Sandhägel. Mànnchen und Weibchen fcheinen befonders zu wohnen. In einem Nefte hat man das Weibchen mit fünf lungen gefunden, Dia lungen wollten nicht zahm, werden, 


\section{Mammalia Glires. Mus.}

Zimmermann, geogr. Zoolog. II. p. 377. n. 302. die fibirifche Sandmians.

Boroweky, Thierreich, I. 3: p. 38. n. 7. Glis Arènarius; die Sandmanus.

Pallas, Reife, Ausz. II. Anh. p. 4. n. 7. Mus arenarius.

Pallas, Reife, II. p. $485 \cdot 493$.

Merrem, zool. Abhandl. p. 43. n. 7. Sandziefel. Erxleben, Mammalia, p. 375. 11. Ir. Glis (Are. narius) corpore fupra cano, fubtus pedibusque caudaque albis.

Graumann; intr. in hift. nat. mamm. p. 60. XI. die Sandmaus.

Gatterer, brev. Zool. I. p. I04. n. Ir. Glis (Are. narius) corpore fupra cano, fubtus pedibusque caudaque albis; Sandoncaus.

\section{Phateus. Die Reismails.}

Zinumernann, geogr. Zoolog. II. p. 377 . 11. 303 . die Reismanus.

Merrein, zoolog. Abhandl. p. 44, n. I0. Schlaf: lojer Ziefel.

36. Song A U Ús. Die. Fleckmanis. $\left({ }^{8}\right)$

Miiller, Naturfyftem, Suppl. p. 43. n. 32. dit Hamftermans.

Zinumes:

(8) Von Palias im Iun. $37 \%$. entdeckt. Ein Weibchen ward in einem Nefte, ztu welchem eine etliche Spannen lange Röhre führe, mit fieben noch blitden Iungen gefunden. Die Jungen wurden zahm, frafsen unter andern verfchiedene Schotengewàchfe, bewegren fich langfam, im Graben aber, womit fie fich in einem Kaften mit Sande befchäffigten, selchwind, waren am Tage wach, und fchliefen die Nacht hindurch zufam. mengerollt, gaben, wenn, fie etwas geplagt wurden, eine piepende, 
Zimmermann, geogr, Zoolog. II. p. 376. n. 304 . die Fleckenmanis.

Borowfky, Thierreich, I. 3. p. 38. 11.8. Glis oeco: nomicus; die Hamftermanus.

Gatterer,-vom Nutzen und Schaden der Thiere,

I. p. 233. n. I28. die Hamftermans.

Merrem, zoolog. Abhandl. p. 44 in. Ir. Stunupffchwanz.

Pallas, Reife, Ausz. II. Anh. p. 3. n. 6. Mus fongarus. Tab. B. fig: I.

Erxleben, Mammal, p. 376. n. 13., Glis (Oeconomicus) corpore cinereo, lateribus variegatis, ftria dorfali nigra.

Graunuann, intr. in hift, nat, mamm, p. 60. XIII.' Hamftermaus.

Gatterer, brev. Zoolog. I. p. 104. n. 13. Glis (Oeconomicus) corpore grifeo, lateribus varie: gatis, ftria dorfali nigra; die'Hanftermans.

37. Fur unculus. Die Obmans.

Mïller, Naturfyft. Supplem, p. 44a n, 34, die Obymóns.

Zimmermann, geogr, Zool, II, p. 378. n. 305. die Obmaus:

Pallas, Reife, Ausz. II. Anh. p.4. n. \&. Mius baraberzis.

Merrem, zool. Abhandl, p. 43. n. 6. Spitzkopf:

Erxleben, Mammalia, p. 374. n. 10. Glis (Barae benfis) corpore grifeo-lutefecente, fubtus albido, fria dorfali nigra.

Graumann, intr. in hif. nat. mamm, p. эо. $X_{0}$ Glis Barabenfis; die Obymaus.

\section{$\mathrm{Gg} 3$}

Gatte-

pende Fledermausfinime von fich, und exfickick nach zwey, Monaten im Fett. 
Gatterer, brev. Zool. I. p. 10.4. n. 10. Glis (Barabenfis) corpore grifeo-lutefcente, fubtus albido, Aria dorfali nigra; die Obymans.

$$
\text { *****Evdnàuse. }
$$

38. TALPINUS. Die Mamharfsmans. (9)

Zimmermann, geogr. Zoolog. II. p. 378. n. 306. die Mauhenrfsmauts.

Lejke, Naturgefchichte, p. I68. n. 2. Spalax minor; der kleinere Slepez.

Borowfky, Thierreich, I. 3. p. 39. n. 2. Spalax minor; der Eldgrä̈br, die Reitmans.

Gatterere, voin Nutzen und Schaden der Thiere,

I. p. 234. n. r3o. die Reitmons.

IMerrem, zool. Abhandl. p. 26. 11. 5. Grabender Kleinainge.

Graumann, intr. in hiftor. nat. manm. p. 60. I. Spalax minor.

Gatterer, brev. Zcol. I. p. I05. n. 2. Spalax (Minor) roftro abbreviato; die Manlwurfsmaus. -

39. Ca pensis. Der Blesmoll.

Zimmermann, geogr, Zoolog. II. p. 378. n. $30 \%$. der Sandmill.

Sparr.

(9) Liebr fchwarzes Frdreich, fonderlich die Weiden um die Dörfer. Die Robren, die fie unter oder in dem Rafen wählt, find mehrere Klaftern lang, und überall $z u$; hin und wieder wirft fie daraus kleine, kaum fpannenbreite Erdhanfen auf. Abends und Morgens ift fie damit befchäffigt, ihre Röhren zu reinigen und za verlängern, wobey fie zuweilen mit dem Kopfe heraus kömmt, und frifçhe Luft fchöpft. Im Winter ift ihr Aufenthalt in dichtern Gebiufchen, oder unter Heuhaufen, wa fie in grösserer Tiefe als die Röhren ein Neft, und daneben eine 
Sparmann, Vorgeb, d. g. Hoffn, p. 496. Blä/smanhunif.

Merven, zool. Abh. p, 26, n. 4. Sand - Kleinange.

40. Már i tr mus. Der Sandmoll.

Sparrmann, Vorgeb. d. g. Hoffu. p. 496. Sandmaulwarf.

Gatterer, vom Nutżen und Schaden der Thiere; I. p. 234. n. 129. Glis capenfis; der kapfche Mautwurf?

41. As PAlax. Die Scharrmanus. $\left({ }^{10}\right)$

Zimmiermann, geogr. Zool. II. p. 379. n. 308. die Scharrmaus.

Pallas, Reife, Ausz. III. Anh. p. 2. Mus Afpalax. Schwed. Abhandl. XXXV. P. I26. Semlianaga Medwedka ruff.

Beckmann, phy!. ökon. Bibl.'II. p. 593. dev Myaa - Spalax.

Merrem, zool. Abh. p. 26. n. 3. Unterivdifcher Kleinaugé.

42. T ч рй us. Die Blindmazus. (II)

Zimmermann, geogr. Zoolog. II, p. 380. n. 309: die Blindmans.

$$
\mathrm{Gg} 4
$$

Vorrathskammer anlegt, und jenes mit weichem Heu, diefe mit Vorrath anfüllet. Ihr Laut, dẹn fie abẹ felțen họ̣̈̂ lüst, gleicht dem Pipen junger Mäufe.

(10) Pie Röhren, die das Thier mit der Oberflàche der Erde, oder dem Rafen parallel gräbt, find oft einige hundert Klafter lang. Hin und wieder wirft gros Erdhaufen auf. Es ift ichwer zu fangen.

(II) Pallas hat gar keine Augen an dem Thier gefunden, auch nichț nachdem das Fell abgezogen worden, docha rudinentu pou- 
Lefke, Naturgefch. p. I68. n. I. Spalax maior; der gröfsere Slepez.

Borowfky, Thierreich, I. 3; p. 39. n. I. Spalax maior; der Slepez.

Binmuenbach, Handb. der Naturgefch. p. 73. 11. 5. Marmota (Typhlus) ecaudatá, palmis pentadactylis, inciforibus fupra infraque latis, pal. pebrarum apsrturis auriculisque nullis; die Blindmuaus, Slepez.

Funke, Naturgefch. I. p. I23. die Blinámaus. Merrem, zoolog. Abhandl. p. 36. n. 2. blinder Kleinange.

Becknuann, phyf. ökonom. Bibl. V. p. 335. Spalax microphthalmus. XI. p. 428. Mus typhlus.

Graumann, intr. in hiftor. nat. mamm. p. 60. I. Spalax maior.

Gatterer, brev. Zool, I. p. I05. 11, I. Spalax (Ma, ior) roftro elongato attenuato.

A) Veränderungen gegen die XIIte Edition, and Vor mehrung der Gattungen diefes Gefchlechts.

Edit. XIT.

Edit. XIII.

p. 79. n. I. Mus Porcellus. p.122. 1.5. Cavia Cobaya,

p. 80, n, 2. Mus Aguti. p. I21. n. 3. Cavia Aguti,

p. 80. n, 3. Mus Leporinus. p. r21. n.3. B. Cavia Aguti, leporina.

p. 80. n, 4. Mus Citellus, p. I44. n. 6. Arefomys $\mathrm{Ci}_{2}$ tellus.

Edit.

burusk, die mit Hâten und Murkeln bedeckt wạren, fand $\mathrm{er}_{2}$ und diefe nicht gröfser als Mohnfamen; fo wie auch Leppecbin angemerkt hat. Ich bemerke hiebey noch, dafs das Thier in der Ueberfetzung der Leppechinfchen Reife (das Original kann

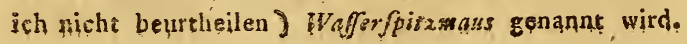


Edit. XII.

Edit. XIII.

p. 81. n. 6. Mus Paca. p. 120. n. I. Cavia Paca.

p. 81. n. 7. Mus Marmota. p. 141. n. I. Arctomys Mar. mota.

p. 81. n. 8. Mus Monax. p. 142. n.2. Arctomys Mo. nax.

p. 82. n. ro. Mus Terre- p.132.n. I1. $\beta$. Mus Amphiflis. bius Terreflris.

p. 83. n. 14. Mus Avellana- p. 156. n. 4. Myoxus Mus. rius. cardinus.

p. 84. n. 15. Mus Querci- p. 156. n. 3. Myoxus Ninius. tela.

p.84. n. 16. Mus Gregarius, p. 134. n. 16. Mus Arvalis. p. 84. n. 19. Mus Longipes. p. 159. n. 4. Dipus Meridianus.

p. 85. n. 20. Mus Iaculus. : p. 157. n. r. Dipus Iaculus, p. 85.n. 21. Mus Volans. p. 153. n. 26. Sciurus Vo. lucella.

Nach diefem Verzeichnifs find alfo zivey bisher verfchiedene Gattungen, nunmehro als Va. rietäten von andern angenommen; zwölf aus diefem, in andre, neue Gefchlechter verfetzt, und fiinf und dreyssig neue Gattungen, worunter jedoch eine aus dem 23 ten Gefchlecht hieher verfetzt worden, hinzugekommen.' Ueberdem if das ganze Gefchlecht in fünf befondere Abtheilungen nach den Schwänzen beftimmter geordnet, und bey der IIten Gattung 5 , bey der 9ten aber 2Varietäten aus einander gefetzt.

\section{B) Unbeftimntere Thiere,}

ก. Glis Tjcherkaficųs.

Erxleben, Mammal. p. 366. n. 5. Glis (Tfcher kafficus) corporę caftaneo. 
Miiller, Samml. rufr. Gefch. VIİ. p. 124. Thier: chen, die die Einwohner zu den Feldnöus Jen rechinen.

Pennant, Syn. "quadr. p. 278. n. 205, the Circaflian Marmot.

Grannann, intr. in hift. nat. mamm. p. 59. V. Glis Tfcherkaficus.

Gatterer, brev, zool. I. p. 102. n. 5. Glis (Tf̧cherkaficus) corpore caftaneo; das tfchivkafjiche IIturnelthieg:

2. Glis Zonuni.

Gatterer, vom Nutzen und Schaden der Thiere,

I. p. 232. n. 125. das podolif che Miturmetithier.

Hermann, tab. affin. animal. p. 91. Mus Zemni.

Evxleben, Mammal. p. 370. n. 7. Glis (Zemni) corpore profunde grifeo.

Rzaizynifki, auct. hift. nat. Polon. p. 325. Cu. niculus fubterraneus.

Biiffon, hift. nat. Ed. in 4. XV. p. r42. Le Zemni. Pennanit, Syn. quadr. p. 277, n. 204. the Podo. lian Marmot.

Graumann, intr, in hift. nat. mamm, p. 59. VII. Glis Zemni.

Gatterer, brev. Zoolog. I. p. ro3. n. 7. Glis (Zemni) corpore profunde grifeo; das padolifche Mivimelthier.

Schreber, Säugth. IV. p. 7rg. not. a.

Vielleicht Mus Typhlus? Ich finde das Citatum von Rzaczyn $k i$ bey Zimmermann p. 380 .

n. 309 .

3. Die Sandmants.

Zimmermann, geogr. Zoolog. II. p. 370. n. 288. Mus Glareolus; die Sandnuars.

Sckreber, Säugth, IV.p. 679. 680. Tab. I90. B. 


\section{IIanmalia Glires. - Mus.}

4. Gmelins Mans.

Pennant, ard. Zool. II. p. I36. n. 8I. Lena Rat. Gmelins Maus.

Pallas, nov. fp. quadr. e glir ord. p. 195. Dubia fpecies, Lemmo faltem et $M$. torquato proxima.

5. Die Maus Tfchelagat/chitfch.

Pannant, arct. Zool. II. p. I37. n. 83.

Steller, Befchreib. von Kamtlchatka, p. I29.

6. Der Chirofo.

Zimnermann, geogr. Zoolog. II. p. 380. a. Voyages du Sieur de la Boullaye le Gouz. p. 16.

7. Eine andere Art indifcher Ratzen.

Zimmérmann, geogr. Zool. II. p. 381. b. Boullaye le Gouz, am angef. $O$.

8. Die Bontees.

Zimmermann, geogr. Zoolog. II. p. 38r.c. Bofsmann, Reife nach Guinea, p. 297 .

9. Mus Norkegicus.

Seba, thefaur. II. 64. Tab. 63. fig. 5 .

30. Der Chologona der Mongolen, oder der $T \int_{c}$ hin geroh der TunguJen, oder deve Longker der Lappen.

Zimmermann, geogr. Zoolog. II. p. 381. e. Pallas, nov. fp. glir. p. 72 . not. a. Mefjerfchmidt, Xen. Ifidis Sibiricae, MSt: 


\section{Mnmmalia Glives. Arctomys.}

11. Glis montannas brachyurinos anterius pofteriusquo pentadactylus.

Zimmernarn, geogr. Zoolog. II. p. 38I. f, Pallas, nov. fp. glir. p. 72 . not. a. Mus montanus vel riparius; e cinereo rufus, brachyournus, anterius 4,5 dactylus pofterius.

* (EINGESCHOBENES GESCHLECHT.) AŔ с т о ч

Zimumermann, geogr.Zool. II. p. 373. C. Schreber, Säugthiere, IV, p. 72J. Gen. XXVII. Bechftein, Naturgefch. Deutfchl. I. p. 489. Pennant, arct. Zool. II. p. Irr. Pallas, nov, fp. quadr. e glir. ord, p. 74.

1. Mar mota. Das Alpenmurmelinier. ( $\left.{ }^{1}\right)$

Miiller, Naturfyftem. I. p. 341. n. 7. das Marmelthier.

Zimnuemann, geogr. Zool. I. p. 29r. das Mhurmel. thier." II p.373. n. 395, das Alpenanumelthier, Leske

(1) Zahm läst fich das. Thier am beften mit Milch erhalten. Im Herbft find fie fehr fett, fo, dafs das Gewicht des Fettes oft das Gewicht des Fleifches und der Knochen überfeigt. Das Fleifch wird frifch und geräuchert gegeffen. Zum Ràuchern werden fie, wie die gefchlachteten Schweine, mic heifsem Waffer gebrithet und effchabet; andere baigen fie aus. Das. frifche Fleifch und Fett in Waffer gekocht, macht eine milchweifse Brihe. Merkwürdig ift, dafs man das Thier auf der alléc blanche in Savoyen theiis auf ifolitten Klippen findet, die wie Infeln auf diefem Eismeer hervorragen, ${ }_{2}$ etliche Stunden weit von aliem unbesiften Erdreich entfernt, und im ganzen lahr etwa fechs Wochen lang vom Schnee befreyet find; fo dafs es fcheint, die dafigen Murmelthiere durchfchlafen wenigftens, zehen Manat vom lahr, und bringen nur einen aulserft klẹinen Theil ihrer Exiftenz wachend zu. 
Lefke, Naturgefchichte, p. r7ó. n. 3. Cricetus Marmota; das. Murmelthier.

Borowfky, Thierreich, I. 3. p.32. n. 2. Glis Marmota; dis Murmelthier.

Blumenbach, Handb. der Naturgefch. p. 7I. n. I. Marmota (Alpina) corpore fupra fufco, fubtus flavefcente; dos Murmelthier.

Bechftein, Naturgefch. Deutfchl. I. p. 490 . n. r. das Mhurnelthier. p. 500. Murmelmans; Murmentle, Miffbelierle, Alpenratze, Bergratze. Tab. XI. fig. I.

Funke, Naturgefch. I. p. I79. das Murmelthier. Ebert, Naturlehre, I. p. 335. das IIurmelthier. Halle, vierf. p. 42r. das Murmelthier. Meyer, Thiere, I. Tab. 81. das Murmelthier. Gatterer, vom Nutzen und Schaden der Thiere, I. p. 218. n. I21. das Murmetthier.

Beckmann, Naturhiftorie, p. 2r. n. 3. das IMurmelthier.

Onomat. hiff. nat. V. p. 114. Marmotta Alpina. Bürmants; dis Murnielthier.

Handbuch d. Naturgefch. I. 2. p. 196. Mus, Al. pinus; das Murmelthier.

Fi i ch, Naturfynt. p. 9. n. T. Mus Alpinus; das rechte Minurnelthier, Alpenmcus.

Klein, Clalfif. der vierfïfs. Th. p. I66. n. 4. Murmelthier.

Klein, natürl. Ordn. der vierf. Th. p. 59. n. 4. Miurmelthier.

Gesner, Thierbuch, p. 267. Mnumelthier, Murs mentle, Miltbellevle. Fig. p. 268. mitteim. Biiffon, vierfïfs. IV. p. 296. äas Mnumelihier. Tab. 8c.

Kraft, Ausrottung graufam. Th. I1. p. 502. das Murmalthier. 
Altmann, helvet. Eisgeb. p. 199. Bergdachs. Merklein, Thierreich, p. 132. Mitunuelthier.

Bonnet, Betrachto über die Natur, II. p. 448.das Murmelthier.

Klïgel, Encyklopädie, I. F. 231. das Alpenmur. melthier.

Kloin, Naturfeltenkeiten des Königr. Ungarn, p. 57. Murmelthier; Murzerchen.

Merrem, zool. Abhandl. p. 4I. n. I. Murmelthier.

Georgi, Rufsland, I. p. 208. Marmoten.

Perrault, Charras und Dodart, Abhandl. zur

Naturgefch. II. p. 205. anatomifche Befchreib. eines Murmelthiers. Tab. 67. das Murmelthier; Tab. 68. die Zergliederung.

Lichtemberg und Voigt, Magazin für das Neue. fle etc. IV. 2. p. 17. das Murmelthier.

Hambúrg. Magazin XXVI. p. 419. das Muurmelthier.

Am Stein, Naturgefchichte des Murmelthiers; im Sammler, IV. p. $21 \%$.

Schwed. Abhandl. II. p. 8I. Murmelthier.

Bechncinn, phyf. ökon. Bibl. XIII. p. 26. Mure melthier.

Harder, anatome Muris Alpini; in den Mifcell. Nat, Cur. IV. 2. ob!. I22.

Klein, hifh. natur. $f$. de vita, genere moribusque Minvis Alpini; in den philof. Transact. XLV. Nr. 486. (Iteht auch in Deffen hift. av. prodron. Lub. $175^{\circ}$. p. 230.

Klein, Vorhereit. zu einer vollftändigen Vögel.

hiftorie. Lpz. I760. p. 414. Murmelthier.

Schenichzer, anatome Muris Alpini; in Büchner mifcellan. phyf. med. mathem. A. 172S. Erf. I732. 4. p. 804 . 
Velfch. anatome Muris Alpimi; in den Mifcellan. nat. curiof. 1760 . obf. 160.

Abhandl. d. Röm. k. Akad. d. Naturf. Nürnberg 1755. 4. I. p. 291.

Iablonfky, Lexicon, p. 707. Inummelthier. Ludoviti, Kaufmannslexicon, III. p. 2269.

Neue Anmerk. über alle Theile der Naturlehre, III. p. 538 .

Maninichfaltigkeiten, I. p. 6I7.

Limné, Syft. Nat. Edit. II. p. $47-$ Mus alpinus Plinii.

Linné, Syn. Natur. Edit. VI. p. I0. n. Ir. Mus cauda elongata nuda, corpore rufo.

Linné, Syf. Nat. Edit. X. 1. p. 60. n. 4. Mus

(Marmota) cauda'abbreviata fubnuda, auriculis rotundatis, buccis gibbis.

Graumann, intr. in hift. nat. mamm. p. 58. I. Glis Marmota; das Murmelthier.

Gatterer, brev. Zool. I. p. Ior. n. r. Glis (Marmota) corpore fupra fufco - cincreo, fubtus cinereo-flavefcente; das IVurmelthier.

Severin, zoolog. Hungar. p. 64. Glis Marmota; das Murmelthier.

Kramer, Auftr. p. 317. Mus cauda elongata nuda, corpore rufo.

Charleton, exercitat. p. 19. Mus alpinus, Marmota.

Agricola, de animal. fubterran. p. 28. Mures alpini.

Wagner, hif. nat. Helvet. po 179. Mus alpinus, Mus montanus, Marmota.

Raacz. hiff. nat. P'ol. p. 233. auct. p. 327. Mus alpinus.

Plin. hiftor. nat. X. c. 65. Mures alpini. 
2. Monax. Der Monax. (2)

Miiller, Naturfyft. I. p. 343. n. 8. das amerikanifche Murmelthier.

Zimmermann, geogr. Zool. II. p. 375. n. 299. das virginifchs Murmelthier.

Borowjky, Thierreich, I. 3. p. 35. n. 3. Glis Mo. nax; das amerikanifche Murmelthier.

Halle, vierf. p. 426. das amerikanilche Murmelthier.

Pennant, arct. Zool. II. p. Itr. n. 52. das virginifche Murmelthier.

Onomat. hiftor. nat. V. p. 336. Mus Monax; das amerikanifche Miurmelthier.

Gatterer, vom Nutzen und Schaden der Thiere, I. p. 222. das amerikanifche Murmelthier.

Klein, Quadrup., difp. p. 50. Cavia Bahamenfis. p. 56. Glis Marmota, Americanus.

Klein, Claffif. der vierfüls. Th. p. 150. Baha. menfifcher Afterhaale. p. 167. 11. 5. amerika. nifches Marmelthier.

Klein, natürl. Ordn. d. vierf. Th. p. 54. n. 6 . Afterhare aus lava. p. 59. n. 5. cmerikani. fihes Mummelthier.

Biiffon, vierfüls. XIV. p. 53. der Monax; mit eirer Fig.

Merrem, zool. Abhandl. p. 42. n. 4. graner Ziefel. Carver, Reif. d. Nordamerika, p. 372. der Hams fter. (Woodchuck.)

Schöpf, Reife durch die nordamerikan. Staaten, I.p. 422. Grounadhog, Woocichuch. II. p. 456 .das anerikanifche Minurmelthier, Rabbet, Woodjack. Selignucin, Vögel, IV. Tab. Ioz:

Linne $_{3}$

(2) Das Fleifch wird gegeffen, und in Anfchung des Gefchmacks mit dem jungen Schweinefleifche verglichen. 
Linné, Syft. Natur. Edit. X. I. p. 60. n. 5. Mus (Monax) cauda mediocri pilofa, corpore grifeo, auriculis fubrotundis, palmis tetradacty. lis, plantis pentadactylis.

Groumam, intr. in hiftor. nat. marnm. p. 58. II. Glis Monax, das amerikanifche Murmelthier. Gatterer, brev. Zool. I. p. Io2. n. 2. Glis (Monax) corpore fufco, lateribus ventreque palli. dioribus; das ainerikanifche Murmelthier.

Briffon, quadr. p. (mihi) n15. n. 4. Glis (Mar. mota Bahamenfis) fufcus.

Catesb. Carol. II. p. 79. The Bahama Coney. app. p. XXVIII. The Modax.

3. Вовас. Der Bobac. (3)

Mitller, Naturfyft. Suppl. p, 40. n. 26. Mus Bo. bac; die poinifche Maus.

Zimmermann, geogr. Zoolog. II. p. 374. n. 296. der Bobak, das wiffiche Murmelthier.

Pennant, arct. Zool.II. p. I15.n. 56. der Bobak. Fvifch, Naturfyft. p. 7. n. 2. Marmota polonica; der Bobok.

Mherrem, zoolog. Abhandl. p. 4I. i1. 2. polnifcher Ziefel.

Biiffon, vierf. XIV. p. 35. der Bobak; m.e.Fig. Pallas, Reife, I. p. 96. die Murmelthiers.

Pallas, Reife, I. p. 129. die "rulfichen Murmel. thiere.

Pallas,

(3) Mattufcbles's Marmotte war achezehn Pfund fchwer, da fie den 4ten October in ihren hau gieng, und wog fieben Pfund weniger, als fie den irten Mărz zum erften Male wieder hervorkam. Erxleben und Zimmermann halten den Bobac rom Alpinn murmelthier nicht für wefentlich rerfohieden. 
Pallas, Reife, Ausz. I. p; 86. die ruffifchen Muss' ineithieve.

Leppechin, ruf. Reife, I, p. 195. rudi fiche Mura melthiere.

I. G. Greelin, Reife durch Sibirien, II. p. 445 . eigentiiche Mismelthiere.

S. G. Gimelin, Reifel aurch Rufsland, J. p. 30. Mnurnelthier:.

Pallas, nord. Beytr. Il. p. 343. n. 3. Bobak, Bai. bak. (fchwarze und weifse Varietäten.)

IMefferfichnidt, Reil. in Taurien; in Pallas nord. Beytr. III. p. I28. eine befondere Art Murmel. thiere, Turbagan.

Steller, Befchr. von Kantichatka, p. I26. Tarpagarizen oder Murmelthiere.

- Mattufchka, in den Schriften der berl. Gefellfch. naturf. Fr. VI. p. 400. IX. p. 88. Mirmotta Bambuc.

4. EM PETRA. Das kanadifche Murmelthier.

Zinumermann, gengr. Zool. II. p. 375. n. 298 . das canadifche Murnueithier.

Pennant, arct. Zoolog. II. p. IIt. 11. 5I. das cas. madifche Mummelthier.

Merrene, zoolog. Abhandl. p. 43. n. 5. bunter Ziefel.

Forfter, Beytr. zur Länderkunde, III. p. 192. das canadif che Murmeithier.

Dв la Hontan, voyage, I. p. 95. p. 233. Siffleur. Graumann, intr. in hiftor. nat. mamm. p. 58. III. Glis Canadenfis.

Gatterer, brev. zool. I. p. 102. n. 3. Glis (Canadenfis) corpore grifeo, ventre cruribusque aurantiis; das quebeck/che Murmelthier. 


\section{PRUINOSA. Das berbifte Murnelthier.}

Zimmermann, geogr. Zool:. III. p. 274. n. I. des graue bereifta Murmelthier.

Schreber, Säugthiere, IV. p. 745: n. 5. das bereifte Minurmelithier.

Pennant, arct. Zool. II. p. It2. n. 53. das bereifte, das weifslichgraue Murmelthier.

6. Ciтexlus. Der Ziefel. (*) (Der gewäfferte Ziefel.)

Miiller, Naturfyffem, I. p. 339. n. 4. die pol: nijche Manes.

Miiller, Naturf. Suppl. p. 39. n.2. die Ziefelratze. Zinmermann, geogr. Zoolog. II. p. 8. die Ziejelmans oder der Souslic. p. 374. n. 297. des. Ziefel, der 'Söuslic.

Hh 2

Lefke,

(4) Die Höhlen diefer Thiere liegen einige Spannen tief unter der Erde; der Weibchen ihre aber an anderthalb Klafter tief Sie haben ohagefähr einen Schuh im Durchmeffer, find gewölbt, langlich tund, und mit trocknem Grafe ausgefuttert. Im September, wo fie am fetteften find, ziehen fie fich in diefe Höhlen zurïck, verfchliefsen ihren bisherigen Gang,-graben den künfuigen bis an den Rafen; und überlaffen fich dann dem Winterfchlafe. Sobald aber der Schnee gefchmolzen ift, durchbohren fie ihren reuen Gang vollends, und kommen abgezehrt aus ihren Winterquartieren hervor. Man fangt fie in Schlingea oder Fallen; fe wercen auch atisgegraben, oder durch in die Hühlen gegoffenes Waffer aus felbigen gelockt. Die Felle find fehr wohlfeil, dalser werden nur die vorziiglich fchün gefleckten, die in Sibirien am Lenaftrom gefundên werden, als Kaufmannsivaare an die Chinefer verhandele, die ihre Bälge felbat theuter, als die der grauen Eichhörner bezahlen. Die Korjaken thd Kamtfehadalen bedienen fich ihrer fehon lange wegen ihrer Schönheit und Leichtigkeit zu Sommerkleidern. Taufend Balge koften bey ihnen nur 8 bis ro Rubel. Kleinern Thieren ftreifen die ungarifchen Bauern das ganze Fell ab, und brauehen es zum Geldibuted. 
Lefke, Naturgefch. p. 169. n.2. Cricetus Citellus; die Ziefelmaus.

Borowsky, Thierreich, I. 3. p. 27. n. 5. Glio Citellus; die Ziefelratte.

Bhimenbach, Handb. d. Naturgefch. p. 7r. n. 2. Marmota (Citellus) corpore longiore, capite parvo, pedibus brevibus pentadactylis; das Erdzeifelchen, Suslik.

Bechftein, Naturgefch. Deutfchl. I. p. 500. n. 2. die Ziefelmons; p. 505. der Ziefel, Zeifel, Zi. Sel, Erdzeifel, Erdzêifelchen, die Ziefrlratte. polnifche Maus, Bilgmaus, der Suslic (Kritfch) orientalifche Hamfter.

Halls, vierf. p. 424. die langgeftrockte fchmächtige Zieselratie.

Gatterer, vom Nutzen u. Schaden der Thiere, I. p. 231. n. 124. dir Bilgmans, das Suslik. Merrem, zool. Abhandl. p. 41. n. 3. Suslik. Pennant, arct. Zoolog. II. p. I13. n. 55. der Ziefel.

Neuer Schaupl. d. Natur, X. p. 217. Ziefelratze.

Onomat. hiftor, nat. V. p. 333 . Mus Citellus; die lange fchmöchtige Ziefelratte.

Handbuch der deutfchen Thiergefchichte; $p .39$. Marmotta Citellus; Ziefelratte, Erdseifelchein, Suslik.

Klein, Quadruped. difp. p. 56. Mus Noricus vel Citellus Gesneri.

Klein, Claffif, der vierf. Th. p. 165. die gro/se Haselmons des Gesners.

Gesner, Thierbuch, p. 266: Bilchmanss, Bilchmans, Zyfel, Zyfelmans, Zysmans, grofse Hafelmaus.

Bïffon, vierfüls. XIV.p. 37. das fibirifche Thier, das die Rusfen levrafchka nestnen. 
Krroft, Aústottung graufam. Thiere, II. p. 483. Ziefel-Maus, Bilch-Maus, Zifs-oder grosse Hajelmans.

Batfch, Thiere, I. p. 229. die Ziefelratte.

Klügel, Encyklopädie, I. p. 232. die Ziefelnuans: Leppechin, Tageb. d. ruff. Reife, I. p. 231. die Ziefelmaus? p. 242. Ziefalmiciuse.

I. G. Gmelin, Reife durch" Sibirien, I. p. 443 . Ievrafchka.

Pallas, Reile, I. p. 50. 129. I48. 207. 22i. 319. II, p. II2. 164. III. p. 687. Ziefelmanus.

Pallas,- Reife, Ausz. I. p 86, Ziefelmans. III. p. 383. 477.'Ziefelmiöuse.

Pallas, nord. Beytr. II. p. 316. Ziefelmiäufe, oder kleine Murmelthiere. (Ievrafchki,) III. p. 282. Zeisel.

Steller, Befchreib. von Kamtfchatka, p. 126. Tebrafihken; ;uff. Pifchtfchuiga, (wegen der letzten Benennung f. Pallas glir. p. I2r. not, e.) Krafcheninnikow, Befchr. von Kaintfcliatka, p. IIg. Murmelthiere von dev kleinern Art; Bergratzen. Georgi, Reife d, Rufsl. I. p. 208. Ziefelmänufe. Bock, Naturgefchichte von Preuffen, IV. p. 76. die polnifche Maus.

Beckmann, phyf. ökon. Bibl. II. p.586. III, p.r73. V: p. 338 . Ziefelmunts.

Linné, Syft, Nato. Edit. II. p. 47. Mus noricus vel Citellus.

Linné, Sy凡. Nat. Edit. VI. p. Fo, n. 3. Mus cauda brevi, capite inauri.

Graumann, intr. in hift. nat, mamm. p. 59. VI. Glis Citellus; die Ziefelmanus.

Gatterer, brev. Zool. I. p. 103. n. 6. Glis (Citel, lus) auriculis nullis, capite parvo; das Erd. zeifelgeı, die Ziefelnuans. 
Le Bruyn, voy, en Mofc. II. p. 402. Tfitsjan. Severin, zool. Hungar. p. 6G. Glis Citellus; dic Ziefelratze.

B. LEUCOPICTUS. Der geperite oder gefleckte Ziefel.

Magnitudine mediocri, pondere unciarum circiter decem, cauda brevi, nigricante et teretiufcula, colore in dorfo grifeo-fufcefcente, punctis albis pulchre guttato Pallas glir. p. I24. Tab. VI, B.

Zimmernann, geogr. Zoolog. II. p. ro. getieger. to oder' gek opfte Ziefel:

Schreber, Säugthiere, IV. p. 748. dev geperlte Ziefel. 'Tab. 2 Ir. B.

Bechftein, Naturgefch. Deutichl. I. p. 502. ge. perlte oder getiegerte Zig.jelmanus.

\%. Flavescens. Der gelbliche Ziefel.

Pallas, glir. p. I27. Citellus favefcens.

Zimmermann, geogr. Zoolog. II. p. Io. der gelb, lichte Ziefel.

Schreber, Säugthiere, IV. p. 748. der gelbliche Zirelel.

Bechftein, Naturgefch. Deutfchl. I. p. 502. die gelbliche Ziefelinauss.

7. GuNDI. Der Gundi.

Zimmermann, geogr. Zoolog. II..p. 43r. Mus Gundi.

Schreber, Säughthiere, IV: p. 754. n. 7. der Gundi, 
Vercünderungen gegen die XIIte Edition, und Ver-mehrung der Gattungen diefes Gefchlechts.

Das ganze Gefchlecht if hier neu... Die erfte, aiveyte und fechlfe Gatung find aus dem vorber. gehenden Gefchlecht hieher verfetzt; die dritte, vierte, fiunfte und ficbente aber neu hinzuge. kommen,

25. GESCHLECHT. SCIUR Us. Das Eichhorn.

Mïller, Naturfyीem, I. p. 355. Gen. XXV.

Zinimermann, geograph. Zoolog. II. p. 339. Gen. XIX.

Schreber, Säugthiere, IV. p. 755. Gen. XXVIII. Lefke, Naturgefcl. p. I7r. Gen. XXX.

Borowsky, Thierreich, I. 3. p. 43. Gen. XXVII.' Bhumenbach, Handb. der Naturgefchichte, p. 69 . Gen. XII.

Bechftein, Naturgefch. Deutfchl. I. p; 5ุig.

Pennant, arct. Zool. II. p. IIs,

Batfch, Thiere, I. p. 239.

Frifch, Naturfy f. p. 8...

Onomat. hif, nat. VII, p. Ir.

Erxleben, Mammalia, p. 4II. Gen. XXXIX.

Graumann, intr, in hiftor, nat, mamm, p. 64, Gen. XXXIX.

Gaiterer, brev. Zool. I. p. II3. Gen, XXXIX. Boddaext, Elench. animal. Gen, XXI.

Pennant, hif. Quadr. p. 406. Geir. XXX, Briffon, quadr. p. 104. Gen. XXIV. Hermann, tab. afinit. anim. p. 88 . 


\section{* L a ufende Eichhörner.}

I. VULGARIs. Das gemeine Eichhorn.(')

Miiller, Naturfyft. I' p. 355. n. I. das gemeine Eichhorx.

Zimmer.

(1) Springfufs, Eicblatzl, Eicbbermelix, Eckereben, Eicbkatzerl. Länge des Thiers obne Schwanz acht Zoll, fechs Linien; die Schwere neun Unzen ein Quentchen. Die Nefter diefer Thiere find rund, und oben mit einer kleinen Oefinung verfehen, dis wiederum von einem konifchen Deckel gegen die Witterung geíchütze wird. Sie beziehen auch :wohl verlafiene Nefter wilder Tauben und anḑerer Vögel. Oft bewohnen iurer zwey bis drey ein Neft gemeinf́chaftliçh. Auffer ihrer gewöhnlichen Nahrung, die in Nüffen, Bucheckern, Ficheln, Saamen von Nadelholz, u. dgl. beftehr, fammeln fie auch allerley Erdfehwämme, (Fungi) ftecken folche befonders in der Nachbarfchaft ihres Neftes in die Eaumritzen zwifchen die Aefte, oder an die Spitzen abgetrochener Aeitchen, um fie für den Winrer zu dürren. In Lappland weifs man fie daher auch mit einer Art der Blätterfchwamme in die Fallen zu locken. In Lappland, Norwegen und Sibirien hat man öfters wahrgenommen, dafs die Eichhürner, vermuthlich aus Mángel der Nahrung, Wanderun. gen angeftellt haben, und haufenweife von einem Orte zun andern gezogen find. In Rufslind find fie fo' häufig, dafs mancher liger jeden Winter gegen taufend Felle liefert, und

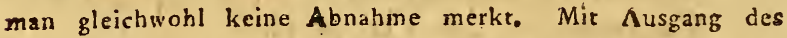
Winters maufen fie fich, und nehmen dann gemeiniglich ihre rothe Farbe wieder an, die im erften Iahre am fchünften ift, und im Herbft und Winter zuweilen in fchwarz und grat, doch mit etwas roth untermifcht übergeht. Diefe Farbenveränderung gefchiehet nur allmăhlich und gradweife, 'ift aber niche allgemein; und erfteckt fich im Ganzen in Sibirien und den angränzenden Ländern ohngefăhr bis an den foten, in Rufsland bis an den sote?, und im nördlichen Europa bis etwa nur den Goten Grad N. Br. herab: viele bleiben aber unverändert. Der Balg dẹs europäifchen Eichhorns ift von geringem Werthe; die Winterbälge der ruffích - fibirifchen aber, die unter dem Namen des Granzwerks bekannt find, find ein fchätzbares Peiz- 
Zimmexnann, geogr. Zool. I. p. 230. das Eichhorri. II. p. 239. 11. 237. das gemeine Eichhorn. Lejke, Naturgefchichte, p. I72. n. 2. das gemeine Eichhorn.

Borowfky, Thierreich, I. 3. p. 44. n. 2. das Eichhörnchen; das geneine Eichhorn.

Blumembach, Handb. der Naturgefch. p. 69. n. 2. Sciurus (Vulgaris) auriculis apice barbatis, cauda dorfi concolore; das Eichhörnchen.

Bechftein, Naturgefch. Deutfchl. I. p. 520 . n. I. das gemeine Eichhorn.

Funke, Naturgefch. I. p. Ir4. das Eichhörncheir. Ebert, Naturl. I. p. 345. das Eichhorn. Halle, vierf. p. 4I5. das Eichhörnchen. Meyer, Thiere, I. Tab. 97. das Eichhorn.

Gatterer, vom Nutzen und Schaden der Thiêre, I. p.254. n. 145. das Eichhörnchen.

Beckmann, Naturhift. p. 30. n. 2. das Eichhorn.

Pennant, aret. Zool. II. p. I21. n. 63. das gemeine Eichiborn.

Neuer Schaupl. d. Natur, II. p. 502. Eichhorn. Onomat, hiftor, natur. VII. p.'23. das gemeine Eichhorn:-

Handb. der Naturgefch. I. 2. p. 169. Sciurus; das Eichhorn.

Handb. d. deutfchen Thiergefch. p. 39. gemeines Eichhörnchen.

Sander, ökon. Naturgefch. I. p. 263. das Eich. horn.

Walther, ökonom. Naturgef̣ch. Deut [chl. p. I23, Eichhors.

werk, das defto koftbarer ift, je fchöner und dunkler grau es ausfiehet. Die Haare aus dem Schwanze werden zu Pinfeln dgl, m. gebrauchte Das Fleifch kann auch gegeffen werdon, 
Merzen, zool. Abhandl. p. 36. 1, r. gemeines Eichhörnchen.

Gesner, Thierbuch, p. 22. Aychhorn, m. e. mittelm. Fig.

Frifch, Naturfyf. p. 8. n. 1. Sciurus Ivulgaris; gemein Eichorn; n. 2. Sciurus nobilis; das Graurerk.

Klein, Clamf. der vierfüfs. Thiere, p. I58. n. $\mathbf{I}_{\mathbf{1}}$ gemeines rothes Eichhorn.

Klein, natürl: Ordn. d, vierf: Th. p. 56. n. I. gemeines rothes Eichhorn.

Biiffon, vierfürs. IV. p. 208. das Eichhörnchen. Tab. 68 .

Batfch,"Thiere, I. p. 239. das gemeine Eichhorn. $F_{1}$ fcier, Naturgefch, von Livland, p.6r..11.2I, gemeines Eichhorn.

Limué, Reife d. Gothl. p. 222. die Eichhörner.

Pontoppidar, Norwegen, II. p. 47. das Eichhörncinen. (Egerne, Ikhorn.)

Pontoppidenn, Dännemark, p. 162. Eichhörnzhen. Lecm, Nachr, von den Lappen, p. r18. Eichhörner.

Carver, Reile durch Nordamerika, p. 375. das rothe Eichhärnchen?

Pallas, Reife durch Rufsland, II. p. 642. 660: Eichhorn.

Georgi, ruff. Reife, I. p. IG2. Eichhorm:

Lägus, in den Schwed. Abhandl. XXXV. die Eichhörner.

Gooze, Allerley, neue-Auf. I. p. I28. Eichnorn.

(Die Fehwammen und das Grauwerk.)

Khlïgel, Encyklopädie, I. ‥ 233. das gemeine Eichhorn.

Beytr. zur Beförd. der Naturkunde, I. p, 212. n, 24. Eichhorn, Eichhürnichen. 
Beckmamn, plyf. ökon. Bibl. VIII. p. 49. Eich. förner.

Berlin. Samml. II. p. 59r. Eichaörnchen, das ge. meine Eichhörnchen.

Schwed. ökonom. Wochenblatt; 1765. p. 283.

Eichhörner.

Merklein, Thierreich, p. 24. Eichhorn.

Iablonfky, allgem. Lexicon, p. 287. Eichtionn.

Tänzer, Iagdgeheimniffe, p. 50. Eichhorn.

Leipz. Wochenbl. f. Kinder, II, p. 9 ff:

Lomiceri, Ktäuter-und Thierbuch, p. 6 I6.

Andre' und Bechfein, Spatziergänge, II. p. 225 .

p. 242. das gemeins Eichhorn.

Oekonom. Zoologie, p. 36. n. 44. das Eich.

hörmgen.

Krinitz, ökon. Encyklop. X. p. 307. Eichhorn.

Dübel, Jägerprakt. I. p. 32. Eichhörnlein.

IIIofer, Grundfätze der Forftökonomie, II. p. 6r4. Eichhörner.

Heppe, wohlred. Iäger, p. 420. Eichhorn.

Beckmann?, fchädl. Befchäfftigungen der Eichhörner in den Waldungen; im Forfmagazin V. p. 42.

Limne', Syft. Nat. Edit. II. p. 46. Sciurus folis palmis faliens.

Linné, Syft. Nat. Edit. X: I. p. 63. n. I. Sciurus (Vulgaris) ąuriculis apice barbatis, palmis tetradactylis, plantis pentadactylis.

Linné, Fn. Suec, I. p. 8. n. 2it. Sciurus palmis fo. lis faliens.

Graumann, intr. in hiftor. nat. mamm. p. 64. I, Eichhorn, Eichhörnchen.

Gatterer, brev. Zool. I. p. II3. n. I. Sciurus (Vul. garis) auriculis apice barbatis, cauda dorfi con. colori ; das Eichthärnchon. 
Miller, zoolog. dan. prodi, p. 5. n. 32. Sciurus (Vulgaris) auriculis ápice barbatis, palmis tetradactylis, plantis pentadactylis.

Kramer, Auftr. p. 3r4. Sciurus palmis folis faliens. Charleton, exercitat. p. 24. Sciurus.

Rzaczynifki, hift. nat. Polon. p. 224. auct. p. 320. Sciurus.

Gronov zoophyl. I. p. 4. n. 17. Sciurus auriculis barbatis, palmis tetradactylis, plantis penta. dactylis.

Sibbalci, Scot. illuftr. II. 2. p. Ir. Sciurus.

Pennant, britt. Zoolog. I. p. 44. the Squirrel.

Pennant, britt. Thiergefch. p. 39. Tab. 8. a.

Paullini, obf. de ictero ex morfu fciuri; in den Mifc. Nat. Cur. Dec. II. A. IX. obf. x8s.

Forfter, philof. transact. LXII. p. 378. Common Squirrel.

Plin. hiftor, nat. VIII. c. 38. c. 43 . Sciurus.

Oppian. cynegét. II. 586. Exbougos.

B. VARIUS. Das wieifsgrane Eichhorn.

Onomat. hiffor. nat. VII. p. 2I. Sciurus varius; das Granuerk, oder Fech, Veh.

Gesner, Thierbuch, p. 24. Mus Ponticus five Venetus; das Veeh.

Miiller, Samml. ruff. Gefch. III. p. 517. Eichhörner oder Graunererk.

Berlin. Samml. II. p. 603 das weifsgraue Eicho hürnchent.

Krïnitz, ökonom. Encyklop. X. p. 314. das weifsgraue Eichhörnchen.

Graumann, intr, in hift. nat, mamm, p. 64. I. a. Gatterer, brev. Zool. I. p. II4. a. Sciurus hieme caerulefcenti-cinereus, aeflate ruber, abdomine albo; das weifsgraue Eichhörnchen.

Merrem, 
Merrem, zool. Abhandl. p. 37. B. Granwerk. Charleton, exercitat. p. 24. Sciurus, five Mus Ponticus.

Scheffer. Lappon. p. 342. Sciuri.

Rzacz. auct. hift. nat. Polon. p. 318. Mus ponti. cus feu venetus:

y. NIGER. Das (gemeine) Jchwarze Eichhorv.

Zimmermann, geogr. Zoolog. I. p. 233. Jchwar. ze Eichhörner.

I. G. Gmelin, Reifed. Sibirien. II Vorrede.

Graumann, intr. in hiftor. nat. mamm. p. 65. b. Sciurus niger.

Gatterer, brev. Zool. I. p. II5. b. Sciurus niger; das fchwarze Eichhörnchen.

Merrem, zool. Abh. p. 37. $\gamma$. Schwarzes.

8. Al вus. Das weifse Eichhorn. ( $\left.{ }^{2}\right)$

Zimmermann, geogr. Zool. I. p. 232. weifse Eich. hörner.

Schreber, Säugth. IV. P. 762. ganz wieifse Eichhörner.

Bechftein, Naturgefch. Deutfchl. I. p. 530. n. 6. fchneeweifse mit rofenrothen Augen.

Kriinitz, ökonoin. Encykl. X. p. 3I3. n. 2. das weisse fabirifche Eichhörnchen.

Onomat. hift. nat. VII. p. I2. das weifse fibirifiche Eichhorn.

IKLerrem, zool. Abh. p. 37. \$. weifses mit rothen Angen.

\section{Linné,}

(2) Eines rothen Eichhorns mit einem fchneeweifsen Schwanze, weifsen Fitifsen, und dergleichen breiten Bauchgtirtel, der faft his an den Rücken reichet, findet man gedacht im NaturforScber, p. 64. n. 19. Erxleben, Mammal. p. 416. d. Merrem, zool. Abhandl. ?. 37. 2. Linué, Syt. Nat. I. p. 86. Edit. XII. 
Linne', weetgoth. Reif. p. 8. das weifse Eichhorn. Berlin. Samml II. p. 600. das weifse Jeltene fibi. rifche Eichhörnchen.

Graunann, intr. in hif. nat. mamm. p. 65. c.

Gatterer, brev. Zool. I. p. II5. c. Sciurus totus albus, oculis rubris; das weifse fibirifche Eich. hörnchen.

Pallas, glir. p. 376. Sciurus fibiricus albus.

3. Niger. Das fihwarae Eichhorn.

Miiller, Naturfyf. I. p. 356. n. 2. das Schrearzé. Eichhorn.

Zimmermann, geogr. Zoolog. II. p. 347. 11. 253 . das amerikanifche fchworse Eichhorn.

Borowfky, Thierreich, 1. 3, p. 45. n. 2. das Schworze Eichhorn.

Halle, vierfifis. p. 417. das fchrearze indianifche

Eichhörnuchen.

Gatterer, vom Nutzen und Schaden der Thiere,

I. p. 258 . n. I46. das Jihwarze amerikanifche

Eichhörnchen.

pennant, arct. Zool. II. p. II9. n. 59. das Schwar. ze Eicihorn.

Onomat. hif. nat. VII. p. I8. das fchecarze Eich. horn.

Frifch, Naturfyfem, p. 8. n. 4. Sciurus america. nus niger; das fchwarze amerikanifche.

Klein, Claflif. d. vierf. Tbiere, p: I59. n. 2. fchivarzes Eichhorn.

Klein, natüt. Ordn. d. vierf. Thiere, p. 56.n. 2. Schwarzes Eichinorn.

Carver, Reife durch Nordamerika, p. 375. das fchwarse Eichorn.

Kriinitz, ökon. Encykl.X.p.3I2.n. I. das fchwav. ze Eichhörncheir. 
Büffon, vierf. VII. p. 165. das fchwar a amerikinifche Eichhorn; m. e. Fig.

IMerrem, zoolog. Abhandl. p. 38. 13. 5. Schevarzes Eichhörnchen.

Berlin. Samml. II. p. 598. das Jchudtze Eich. hörnchen.

Linné, Syी. Nat. Edit. X. I. p. 64. n. 2. Sciurus (Niger) niger.

Graumann, intr. in hift. nat mamm. p. 65. II. das fchwarze Eichhorn.

Gatterer, brev. zool. I. p. Ir5. n. 2. Sciurus (Ni. ger) niger, auriculis imberbibus; das fchwar. ze oder mexikanifche Eichhörnchen.

Ionfton, quadruped. p. 163. Prior Quautechallot Species.

8. Vucpinus. Das Fuchseichhorn. (3)

Zimmermann, geogr. Zool. II. p.347.ad n. 253 . das Katziglu-Eichhorn.

Pennant, ard. Zool. II. p. I18. ß. das Katzen. eichhorn.

Schöpf, Reife durch die nordamerikan. Staatèn, II. p. 8. Eichhörner.

3. CINEREUS. Das virginif che graue Eichiorn. (4) Miiller, Naturfyft. I. p. 356 . n. 3. das afchgrane Eichhorn.

\section{Zimmer.}

(3) Lange des Kiorpers 12 Zoll; des Schwanzes 12 Zoll. Pennant hảl es für eine Varietät von der folgendèn Gattung.

(4) Anderthalb Zoll linger als das gemeine Eichhorn; die Nahrung ohngefähr eben diefelbe. Von dem Mays verzehren fie nur den inperfen fufselten Kern. Sie beiffen auch gern die Eichankatzchen (Amenna) $2 b$, doch ohne fie $z u$ frefen. Zum 
Zimmermann, geogr. Zool. II. p. 345. n. 249. das amerikanif che graue Eichhorn.

Borow $5 k y$, Thierreich, I. 3. p. 46. n. 4. das afchgraue Eichhorn.

Pennant, arct. Zool. II. p. 116. n. 58. das graue amerikanifche Eichhorn.

Gatterer, voin Nutzen und Schaden der Thiere. I. p. 258. n.'147. das afchgraue virginifche Eich. hörnchen.

Onomat. hif. nat. VII. p. 54: das gro/se grause virginifche Eichhom.

Kriinitz, ökonom. Encyklop. X. p. 3I3. n. 3. das gro/se graue canadifche oder virginianifche Eichhorn.

Klein, Claffif. der vierfüís. Thiere, p. 159. n. 3. gröfseres fifchfarbiges virginianifches Eich. horn.

Klein, natürl. Ordn. d. vierf. Th. p. 56. n. 3 . virginianifches Eichhorn.

Frifcin,

Wintervorrath tragen fie die beften Niffe und Eicleln? zufammen, und vergraben fie; tragen auch einen Theil in ihre Hühlen. Im Winter halten fie fich bey fchicchtem Wetter mehrere Tage inne, und nahren fich von dem eingetragenen Vorrath. Bey gelinderm Wetter kommen fic hervor, und fcharren einea Theil der vergrabenen Nahrung aus, um ihn theils $z u$ freffen, theils in die Nefter zu tragen. Die Schweine fowohl als auch manche Landes Einwohner fpüren ihre Vorrathskammern aus, und berauben 'fie. $\mathrm{Zu}$ manchen Zeiten, wenn entweder ein kalter Winter bevorfteht, oder fie Mangel an Nabrung haben, ftellen fie Wanderungen an, gehen im Herbft aus den höhern in die niedrigern Gegenden, und kommen in folgenden Frühling wieder/zurück. lung gefangen werden fie leicht zahm. Ihr Balg wird als Pelzwerk in Amerika nicht fonderlich geachtet, aber doch im Nothfall gebraucht; und gewühnlich zt Riemer verfchnitten. Das Fleifch wird rọn einigen als Leckerbiffen gegeffen. 
Frifah, Naturfyft. p. 8. n. 5. Sciurus virginicus; das kurzohrige, graue.

Biiffon, vierf. VII. p. I48. das afchgranse Eich. hörnchen; m. e. Fig.

Schöpf, Reife durch Nordamerika, I. P. 42I. graue Eichhörner.

Merrem, zool. Abhandl. p. 38. n. 6. grazes Eich. $h$ rnchen.

Kalm, Reif. nach Amerika, II. p. 352. 450. die grauen Eichhörner.

Berlin. Samml. II. p. 600. das grofse graue vir: ginianische Eichhorn.

Limné, Syft. Nat. Edit. X. I. p. 64. n. 3. Sciu. rus (Cinereus) virginianus cinereus maior.

Grampann, introd. in hift. nat. mamm. p. 65.III. das graue Eichhorn..

Gatterer, brev. Zoolog. I. p. II5. n. 3. Sciurus (Cinereus) ventre albo, auriculis imberbi. bus; das grane Eichhörnchen.

Pallas, glir. p. 370 . Sciurus americanus cinereus.

12. Hudson I Us. Das labradorifche Eichhorri.(5)

Zimmermann, geogr. Zool. I. p. 232. das Eich. horn cier Hudfonsbay. p. 344. n. 248. das ka. bradorifche Eichhorn.

Pennnnt, aact. Zool. II. p. II5. n. 57. das labra. dorifche Eichhorn.

Forfter, Beytr. zur Völker - und Länderkunde, III. p. 192. 11. 13. das eifenfarbige Eichiorn.

Biiffors,

(5) Länge des Körpers 8 , des Schwanzes 4 Zoll, bis an die Spitze der längften Haare aber etwas ïber 5 Zoll. (Nach einem ausgeftopften Balge.) Lebt von Tannenzapfen, uńd bleibt den gar: zen Winter hindurch in feinem Nefte. 


\section{Mammalia Glires. Sciurus.}

- Biiffon, vierfüls. VII. p. I7r. das Hitidfons. Eichihosn.

Carver, Reif. d. Nordamerika, p. 375. das rothe Eichhorn?

Erxieuent, Mammalia, p. 416.. $\varepsilon_{0}$ Sciurus hudfo. nicus.

Graumian, intr. in hiftor. nat. inamm. p. 65. e. Sciurus hudfonicus:

Gatterer, brev. Zool. I. p. rt5. e. Sciurus hud. fonicus.

Penmant, hift. quadr. p. 412. n. 274. t. 43. fig. I.

13. CA R OL INENSIS. Das carolinifche Eichhom. $\left({ }^{(5)}\right.$ Schreber, Sïugthicre, IV. p. 779. n. 6. das caro- , linische Eichhorn.

Pennant, arct. Zool. II. p. II6. a. das Eichhorn aus Carolina.

Pennont, hif. quadr. p. 412. n. 274. Carolina Squirrel.

34. PERsicus. Das perfifche Eichhorn.

Zimmermann, gengr. Zool. I. p. 233. II. p. 34t. n. 240 . das perfifiche Eichhorn.

Schreber, Siugth. IV. p. 780. n. 7. Tal. 235. B. das perfische Eichhorn.

Biffon, vierf. VII. p. 173. das perfifche Eichhorn. Erxleben, Mammal. p. 417. $\eta$. Sciurus perficus. Graumann, intr. in hiftor. nat. mamm. p. 65. g. Sciurus perficus.

Gatterer, brev. Zoolog. I. p. II5. g. Sciurus perficus.

15. A NOMALUS. Das georgifche Eibhhorn. I6. $\mathrm{Br}$.

(6) Pemant hält es. fütr eine Varietit ven den voshergehendea. 


\section{Mannmalia Glires. Sciurus.}

16. Bicol or. Das jävanifchle Eichhorn.

Zinumermann, geogr. Zoolog. II. p. 342. n. 243. das javanifche Eichorn.

Palias, glir: p. 377. Sciurus javenfis.

17. ERYTHRAEUS. Das rothbïuchige Eichhom.

Zimmermann; geogr. Zoolog. II. p. 342.n.242。 das rothbiuuchige Eichhorn.

Schreber, Säugth. IV. p. 782. n. 10. das rothbiäz. chige Eichinon.

Bïffon, vierfüls. VIİ. p. 172. das rothbauchige Eichtiorin.

Merrem, zoolog. Abhandl. po 38. n. 4. rothes Ëiçһ̆örnuchen.

18. M A C R OU R Ús. Das langfchwänzige Eichhorn. Zimnmermann, gengr. Zoolog. II: p. 340. n. 238. das langJchwönzzige Eichiorn.

Borowe ky, Thierreich, I. 3. p. 46. 11. 5. das gelb. baiuchige indianifthe Eichhörnchen.

Halle, vierf: p. 4I7 das gelbbauchige indianifche Eichlörrachen.

Gatierer, vom Nutzen und Schaden der Thiere, I. p: 260. 11. 148. das ceylonif che Eichhörnchen. Bi:ffon ${ }_{3}$ vierf. VII. p: 169. das langfchwö̈rzige Eichhorn; m. e. Fig.

Merrém, zool. Abhandl. p. 37. n. 2. lang fchwörz ziges Eichhörnchein.

Berlin: Samml. II. p. 612. das gelbbäuchige inidià nifche Eichhörnchen.

Forffer, zool. ind. fel. p. tr. tab.1. Sciur. macrourus. Murr, Beytr. zur Naturgefch. von Oftincien; im Naturforfcher, 1. p. 264. n. x. das langge: Schwö̈nzite Eichhörnchen. 
Graumann, intr. in hift. nat. mamm. p. 66. IV. das gelbblane Eichhörnchen.

Gatterer, brev. Zoolog. I. p. Ir6. n. 4. Sciurus (Macrourus) cauda corpore duplo longiore, grifea, corpore fubtus rubro; das ceyloniSche Eichhörnchen.

19. M A X I M U S. - Das malabarifche Eichhorn.(7)

Sonnerat, Reife nach Oftindien und China, II. p. 109. Tab. 87. das grosse Eichhörnchen von. der Kiifte Malabar.

20. AвESSIN ICUs. Das abe Jinifche Eichhorn.

Zimmermann, geogr. Zoolog. II. p.341. n. 239. das Eichhorn von Alby finien?

Schreber, Säugthiere. IV. p. 785. n. 13. das abeffinifche Eichhorn.

Biiffon, vierfüls. VII. p. I7. das Eichhomn von Aby Jinien.

21. IN DICUs. Das Eichhorn von Bombay.

Zimmermann, geogr. Zoolog. II. p. 34r. n. 24r. das purpurfarbine, das malabarifche Eichhorn.

Schreber, Säugthiere, IV. p. 786. n. I4. das Eichhorn von Bombay.

Graumann, intr. in hiftor. nat. mamm. p. 66. V. Sciurus indicus.

Gatte-

(7) Auf der Küfte Malabar wird es die grofse Walchatte' (grand zat de bois) genannt. Vorziiglich häufig bält es fich auf den Kardamomenbergen, einem Theil des Geburges Gate auf. Der bufchige Schwanz ift länger als der Leib, und eben fo dicke, wenn es ihn fräubt. Wenn das Thier im Walde laxuft, hălt es ihn beyaahe fenkrecht. Wem die Kokosnüfe reif find, beifst os fie auf den Batumen auf, um blofs den Saft daron zu trinken. Zähm wird es leicht. 
Gatterer, brev. Zool. I. p. I16. n. 5. Sciurus (Indicus) cauda longitudine corporis, apice aurantio; das oftindifche Eichhörnchen.

Merrem, zoolog. Abhandl. p. 37. n. 3. indianifches Eichhörnchen.

4. F L a v us. Das blonde Eichhorn.

Mitller, Naturfýtem. I. p. 357. n. 4. das gelbe Eichhorn.

Ziminermann; geogr. Zool. II. p. 342. n. 244. das blonde Eichhorn.

Schreber, Säugth. IV. p. 786. n. 15. das blonde Eichhorn.

Botow fky, Thierreich, I. 3. p. 47. n. 7. das gelbe Eichhorn.

Ononiat. hiftor. natur. VII. p. I5. das gelbe Eich. horn: .

Merrem, zool. Abhandlo p, 36. n. 9. gelbe Ratze.

Berlin. Samml. II. p. 6Ir. das gelbe Eichłörnchen von Karthagena.

Linne', Syf. Nat. Edit. VI. p. 9. n. 3. Sciurus cauda teretiofcuta auribus fubrotundis nudiș.

Linné, Syft. Nat. Edit. X.I. p:64. n. 4. Sciuru (Flavus) auriculis fubrotundis, pedibus pentadactylis.

Graumann, intr in hift nat mamm. p. 66. VIII. das gelbe Eichhora woli

Gatterer, brev: Zonlog. I. p.rib. n. s. Sciurus (Flavus) auriculis fubrotundis, corpore luteo; das gelbe Eichbörnchen.

Du Pratz, hift de Ia Louif. II. p. 99. Les qua. triémes Ecureuils.

Pennant, hiftor. quadrup. p. 413. n.276. Fair Squirrel. 
5. P a m a R u m." Das Palmen - Eichhorn.

Miiller, Naturfyft. I, p. 357. n. 5. das Wiefel. eichhörnchiten.

Zimmermann, geogr. Zoolog. II. p.3,43. n. 245 . der Palmift.

Borowfky, Thierreich, I. 3. p. 47. n. 8. der Palmifl, das Wiefeleichhorn.

Onomat hittor. nat. VII. p. İ. das Wiefel-Eich. hörnlein.

Halle, vierfüfs, p. 418. Nierembergs Zwergeich. hörnciien.

Handb, der Naturgefch. I. 2. p. 170. der Palmift, oder die Palnnessratie.

Merrem, zoolog. Ahhandl. p. 38. 10. 7. Palmo. Eichbörnchen.

Frifch, Naturfyft.p. 8: n. I?, die Palm-Ratte.

Kriinitz, ökonom. EncyklopäjJ. X. p. 3r4. das Pain-Eichhönnchen, der Pahnift: die Palmenratze.

Biiffon,-vierfüls. VII. p. I74. das Palmeneichhörm. chenz; m. e, Fig.

Berlin. Samml. II. p. 603 der Patmift, die Pal. menratze, das Palmeneichiournchen.

Berlin. Samml. II p. 614: ein fogenanites Zwerg. eichhöruchen.

Exxleben, Mammalia, p: 423. n. 9. Sciurus (Pal. marum) fubgrifeus, Ariis tribus favicantibus, cauda albo nigroque lineata.

Granemanu, intr. in hin. nat. mamm, p. 66. IX. das WTiejelsichhörncherb:

Gatiever, brev. Zool, I, p. irz, n. 9. Sciurus (Pal. marum) fubgrifeus, friis tribus flavicantibus, cauda albo nigroque lineata; der Palmilt. 


\section{Mammalia Glives:}

Sciurus.

6. GeтUu U'S. Das Liberey- Eichhorm. ( $\left.{ }^{8}\right)$

Miiller, Naturfyf. I, p. 357. n. 6. das afrikani. fche Eichorin.

Zimnernnann, geogr. Zoolog. II. p. 343. n. 246. "das Eichhorn mit vier Strkifen, das LibereyEirhhorn.

Bornolky, Thierreich, I. 3. p. 48. n. 9. das afrikanifche Eichhorn.

Halle, vierf. p. 4 II . das afrikanifcine Liebereyeichhörnchen.

Halle, vierf. p. 42r. das amerikanifche geftreifts fliejende Eichhorn. (It sicht aus Amerika; Hiregt auch nicht.)

Onomat. hiftor. nat. VII. p. 15. das afrikanifche Eichhorn.

Frifch, Naturfyก. p. \&. n. 13. Gurbarifch geftreiftes Eichhorn.

Kriinitz, ökonom, Encyklop. X. p. 3r5. 11. 5: das Eichbürnchen von der Kïfke der Barbarey, odes der Barbaresque.

Gesner, Thierbuch, p. 24. afrikanifches. Aych. horn, Sciurus Getulus genannt. Abbild, fchl. Klein, Quadr. difp, p:54. Sciurus Getulus Caii apud Gesnerum.

Klein, Quadr. difp. p. 55. Sciurus ex cinereo rubicundus, latoribus friatis, anribus brevioribus. Klein, Claflif. der vierfüls. Thiere, p. I60. n.. 7 . Getulifches Eichloraz.

Klein, Claflif. der vierf. Th. p. 163. n. ro. Eich. horn, de.]en Farbe aus dein Afchgrauen in das Röthliche fültît.

Klein, natürl. Ordn. d. vierf. Th. p. 57. n. 8 . Eichhorn aus der Barbarey.

$$
\text { Ii } 4 .
$$

Klein,

(8) Ponnant halt es fits eine Spiehart des vorigent 
Klein, natürl. Ordn. d. vierf. Th. p. 58. n. Ir. grauröthliches Eichhorn.

Büffon, vierf. VII. p: 174. das Eichtorn aus der Barbarey; m. e. Fig.

Merrem, zool. Abhandl. p. 38. n. 8. barbarifches Eichhörnchen.

Seligmann, Vögel, VI. Tab. 93. das Eichhörn. chen aus der Barbarey.

Berlin. Samml. II. p. 605. das Eichhörnchen von der Kuifte der Barbarey.

Berlin. Samnl. II. p. 610. das afrikanijche Lievereyeichhörnchen.

Limne, Syft. Nat. Edit. II. p. 46. Sciurus genitalibus maximis.

Linné, Sylt. Nat. Edit. X. I. p. 64. n. 5. Sciu. rus (Getulus) fufcus, friis quatuor albis longitudinalibus.

Erxleben, Mammal. P. 525.11. 10. Sciurus (Getulus) fufcus, nriv quawer albidis longitudinalibus.

Graumann, intr. in hiffor. nat. mamm. p. 66. X. das Lievereyeichhörnchen.

Gatterer, brev. Zoolog. I. p. I17. n. ro. Sciurus (Getulus) fufcus, frris quatuor albidis longitudivalibus; das afrikanifche Eichhörnchen.

Charleton, exerc. p. 24. Sciurus, five Mus Getulus. Peninant, hift. quadr. p. 416. 11. 280. $\beta$. Barbary Squirrel.

7. S т r IAtus, Das Jchwarzgeftreifte Erd-Eich. horn. (9)

Miiller, Naturfyft. I. p. 358. 11. 7. das geftreifte Eichhorn. Tab. 21. fig. 2. Houtt.

Zinimer.

(9) Das afiațifche und amerikanifche geftreifte Eichhorn werden wegen ihrer grofsen Uebęrẹnftimmung von den Zoolugen nuc 
Zimmermann, geogr. Zool. I. p. 297. das geftreif. te Erdeichhorn. II. p. 345."n. 250. das geAreifte Erdeichhorn.

Boroweky, Thierreich, I. 3. p. 43. n. I. das geftreifte Eichhorn. Tab. 27 .

Gatterer, vom Nutzen und Schaden der Thiere, I. p. 260. n.' 150. das Erdeichiörnchen.

für ${ }_{1}$ eine einzige Gattung angefehen. Scbreber ift der erfte, der fit fyftematifch, doch auch nur als Varietaten yon einander getrennt hat; er halt aber, und zwar hatiptfichlich wegen der Entrernung, derjenigen Gegenden in Afien und Amerika," in welchen diefe Thière fich auflialten, die fpecififche Identität beyder fitir noch nicht fo ganz entichieden: - Die afiatifcben geftreiften Eichhürner find in Sibirien in Birken - und Nadelwäldern, befonders wio es Zederfichten giebt, haufig, balten fich. aber mehr an der Erde als auf den Bäumen aiff. Ihre Höhlen, die fie mehrentheils unter die Wurzeln der Bäume graben, find kaum eine Spanne tief unter der Erde. In diefen Höhlen halten fie fich den Winter hindurch auf, und leben von ihrem gefammeltan Vorrath, womit ie die Kammern im Herbft angefillt haben. In mancher Höhle hat man wohl 1.o bis 15 Pfund der auserlefenften Zirbelnüffe u. dgl. m. gefunden. Auf den Wejen fuchen fie die Körner und Samen aus dem Pferdemifte. Die zahmen fretren auch Fleifch, und find ihrer mehrere beyfammen, fo beiffen fie fich auch wohl felbit die Schwänze ab. Sie vermehren fich fehr ftark. Aus dem Pelzwerk macht man nicht viel, daher fangt man dic Thiere auch nicht fonderlich. In Sibirien fchont man fie vielmehr, weil die Zobel und Marder flark nach ihnen gehen. Am ftärkften if noch der Fang an der Lena, wo das Taufend Felle o bis 8 Rubel, koftet. Man fängt fie in Fallen, oder fchiefst fie mit ftumpfen Pfeilen. Die Hühlen der amerikanifcben liegen riefer, und der Zugang geht bis 4 Fufs fchrage hinunter. 'In ihter Lebensart kommen fie fehr mit den afiatifchen überein. In ihren Vorrnthskammern findet man wohl ganze Hutküpfe voll Mays, Eicheln, Zwergkaftanien, u. dgl. Zuweilen grahen fie fich durch die Erde in die Keller, wo Aepfel aufbewahrt werden, oder fallen in dic Schęunen, wo Maýs aufgefchüttet ift, Die Katzen ftellen ihnen fehre nach. 
Merrem, zool. Abhandl. p. 39. n. 9. geftreiftes Eichhörnchen.

Pemnant, arct. Zool. II. p. 125. n. 65. das gefureifte Eichhorn.

Onomat. hiftor. nat. VII. p. 20. das gefreifte Eichhorn.

Schlözes, Erdbefchr. von Amerika, p. 238. Eichhörncher?

Kriinitz, ökonom. Encykl. X.p. 315. a'ns EvdEichhorn, das geftreifte virginifche Lucihbörnchen; das kleine Eichthönchen von Carolina.

Berlin. Samml. II. p. 605. der Schweeizer, das fcheseizerifche Eichhörnçien, u. f. w.

Berlin. Samml. II. p. 608. das gelbe fibivifche Eich. hörnchen, mit fünf brannen Streifen.

Batfch, Thiere, I. p. 239. n. 2. das geftreifte Eich'rorn.

Erxleben, Mammal. p. 426. n. II. Sciurus (Stria. tus) flavus, friis quinque fufcis longitudinalibus. Linné, Syft. Nat. Edit. X. I. p. 65. n. 6. Sciurus (Striatus) flavus, friis quatuor fufcis longitudinalibus.

Graumann, intr, in hift. nat. mamm. p. 66. XI. das geftreifte. Fichhorn.

Gatterer, brev. Zoolog. I. p, rr7. n. Ir. Sciurus (Striatus) flavus, firis quinque fuf́cis longitudinalibus; der Schweizer, das geftreifte Eich hörnchen ; Erdeich̆örnnchern.

4. Asraticus, Das afiatiche.

Schreber, Säugthiere, IV. p. 792. das ajiatif che fincervigeftreifte Eichhorn.

Biffon, vierf. VII. p. 174. der Schweitzer. Wollas , Reife, II. p. 209. 665. die geftreiften Fichhö̀nuhęza 
Leppechin, ruff. Reife, III p. si. die geftreifterz Eichhörner. (Burunduk.) p. 47. das kleine gefrreifte Eichhorrz.

B. A M E ICA A US. Das amerikanifche.

Halle, vierfüls. p. 416. das geftreifte virginifche Eicihbömchen.

Klein, Cuadruped. difp. p. 53. Sciurus Ariatus. K! cin, Claffif. d. vierf. Thiere', p. 159. n. 4. Eichhorn mit Streifen.

Klein, natïrl. Ordn. d. vierf. Thiere, p. 57. n. 4 . geftreiftes Eichhorn.

Kalm', Amerika, II. p. 462. die Erdeichhirner. Carver, Reile durch Nordamerika, p. 375. das bunte Eichhorn.

Seligmınn, Vögel, Tab. 76. das carolinifche Eichhorn.

Pallas, glir. p. 38 I. Sciurus ansericanus Ariatus. Du Pratz, hift, de la Louif. II. p. 98. les Ecureuils Suiffes.

Denys, defcript. de l'Amerique feptentr. Paris 1632. Il. p. 33I-332.

Theodat. voy. du lays des Hurons. Paris 163r. p. 305.306 .

9. Aestuans. Das brafilifche Eichorom.

Miiller, Naturfyn. I. p. 359. n. 9. das furinamifine Eichtion.

Zimmermann, geogr. Zoolog. II. p. 347.11. 254 das braflianifche Eichhor?.

Schreber, Säugthiere, IV. p. 787. 13. I6. das brafilifche Eichhovi.

Borowsky, Thiercich, I. 3. p. 47 , n. 6. das. furinamifche Eichhorn.

Onomata 


\section{Manmalia Glives. Sciurus.}

Onomat. hiftor. nat. VII. p. I2. das furinamifche Eichhorn.

Berlin. Samml. II. p. 6ro. das gelb und braunbun. te Eichhörnchen aus Brafolien! mit wei/sen Streifent an den Seiten.

Berlin. Samml. II. p. 612. das Jurinamifche Eich. hörnchen.

Merrem, zoolog. Abhandl. p. 39. Surinamifches Eichhörnchen.

Erxleben, Mammal. p. 421. n. 7. Sciurus (Aeftuatis) grifeus, fibtus flavefcens.

Graumann, intr. in hift. nat. mamm. p. 66. VII. das furinamifche Eichhorn.

Gatterer, brev. zoolog. I. p. II6. n. 7. Sciurus (Aeftuans) fubtus flavefcens; das furinamische Eichhörnchen.

Penvant, hil. quadr. p. Ir4. n. 277. Brafilian Squirrel.

22. Dschinschicus. Das Eichhorn aus Dechinfthi.

Schreber, Säugthiere, IV. p. 788. n. 17. das Eichhorn aus Dfchinfchi.

Somerat, Reife nach Oftindien, II. p. IIo. das Eichhörutchen aus Gingi.

Pennant, hiftor. quadrup. p. 416. n. $\gamma$. Plantane Squirrel?

a3. Variegatus. Der Coquallin.

Zimnermann, geogr. Zoolog. II. p. 346. n. 252. das Coquallin.

Biiffon, vierfüfs. XIII, p. 296. der Cóquallin; m. e. Fig.

Gatterer, vom Nutzen und Schaden der Thiere,

I. p. 260.n. I49. der Coquallin. 
Mervem, zool. Abhandl. p. 39. n. 10. buntes Eichhörnchen.

Graumann, intr. in hif. nat. mamm. p. 66. VI. Sciurus variegatus.

Gattever, brev. Zoolog. I. p. 116. n. 6. Sciurus (Variegatus) corpore fupra nigro, albo et fufco variegato; der Coquallin.

Pennant, hiftor, quadrup. p. 413. n. 275. Varied Squirrel.

24. DEgus. Der Degu.

25. Mexicanus. Das Band-Eichhom.

Zimmerminn, geogr. Zoolog. II. p. 346. n. 25 x. das Bandeichhorn.

Schreber, Säugth. IV. p. 808. ni: 22. das BandEichiorn.

Halle, vierfüfs. p. 4I6. das fchönfte amerikanifche Lievereyeichhörnchen. Tab. 21.

Onomatol. hif. nat. VII. p. I8. das amerikaniSche Livreyeichhörnlein; das Eichhorn mit äftigem Schwanze aus Neu-Spanien.

Klein, Quadr. difpor. p. 53. Sciurus rariffimus, ex nova Hifpania, taeniis albis.

Klein, Claffif. der vierfüls. Thiere, p. 159. rarftes Eichhorn aus Nenfpanien, mit weifsen Streifen.

Klein, natürl. Ordn. d. vierfüfs. Th. p. 57. n. 5. Fichhorn, mit äftigem Schwanze," aus NeuJpanien.

Berlin. Samml. II. p. 609. das fchönfte amerikaniSche Lieverpyeichhörnchen; das weifsgeftreifte Eichhorn aus Nenfpaniers.

Merrem, 7.0ol. Abh, p. 39. 1. Ir. Liverey - Eich. hörnchen. 
Grauinann, intr: in hif. nat. mamm. p. 67. XII. das weifsge/treifte Eichhörnzchen.

Gatterer, brev. Zoolog. I. p. II7. n. 12. Sciurus (Mexicanus) cinereo-fufcus, friis quinque ad feptem albidis longitudinalibus; das mexicnmifche Eichörnchen.

Pennant, hilt. quadrup. p. 4I4. n. 278. Mexican Squirrel.

29. Madagascariensis, Der Aye-Aye. $\left({ }^{\circ}\right)$ Sonnerat, Reife n. Oftindien, II. p. 107. Tab. 86. $\operatorname{der}$ Aye-Aye.

* Flizegende Eichhörner.

86. Vólucetca. Das virginifche fliegerde Eichhorn. (')

Miiller, Naturf. I. p. 354. n. 21. der Flieger."

Zimmer.

(10) Sonnerat hat von diefen Thieren ein Par gehabt, die aber nur zwey Monate lebten, und mit gekochtem Reifs gefuttert wurden. Sie liebten die Warme, krochen immer zufatrmen, * um zu fchlafen, legten fich auf die Seite, den Kopf ${ }^{-}$zwifchen die Vorderfüfse, und konnten nur durch vieles Rütteln dahin gebracht werden, dafs fie fich bewegten. Der Name Aye-Aye, den er diefem Thier gegeben hat, ift ein Schrey, den die Einwohner von Madagafiar zur Bezeugung ihres Erfaunens von fich geben.

(1) Bey den fliegenden Eichhörnern hertcht in Anfehung der Synonymen grofse Verwirrung. Biiffor halt das virginifche fliegende Eichhorn mit dem ruffifchen für einerley. Erxiebes: zählet die zu Linnés Mus Volass, oder Pallas Sciurus V'olucella gehürigen Synonymen aus Brown, Raj, Fernandez, Catesby, Edward, Pesnant, du Pratz zu feinem Sciurus Volans p. 435. (Linn. Sc. volans,) bringt Linnés Mus Volans, und Klein's Sciurus' Petaurigha volans unter feinen Siciurus Petaurifa, p. 438. u. dgi. pallas 
Zimmermann, geogr. Zoolog. II. p. 348. n. 256.: das anerikanifche fliegende Eichhorn.

Penmant, arct. Lool. II. p. I19. 11.60. das fliegen. de Eichhorn.

Onomat. hiftor. nat. V. p. 352. Mus volans; der Flieger.

Frifch, Naturfyfem, p. 8. n. 16. Sciurus volans Americanus; der AJjaponik.

Biiffon, vierf. VII. p. Io7 der Polatufche; m. e. Fig. Merrem, zool. Abhandl. p. 40. n. 16. flattern. des Eichhörnuchen.

Kalm, Reife nach d. nördl. Amerika, II. p. 460. fliegende Eichhörnes.

Carver, Reife darch Nordamerika, p. 375. das fliegende Eichhörncheru.

Sihlözer, Erdbefchr. von Amerika, p. 238. das fiegende Eichhörnchen.

Laet, nov. orb. p. 82. Affapanik.

Brickell, North-Carol. p. 129. Hying Squirrel.

27. HuD.

Pallas hat aber die Irrthümer genau unterfucht, uad demnachit Scbreber die Synonymen zuerit richitig aus eivander gefetzt, nach welchem fie nunmehro in unfer Syftem whergetrajers find. - Das virginifche fliegende Eiclbborn kommt anderwärts nicht, als in feinem eigentlichen Vaterlande, dem gemálsigten und warmen nürdlichen Amerika, vor. Is kann vermittelft der ausgefpannten Flughaut, und der Sciwingungen des Schwanzes von éinem Baume zum andérn über dreyfsig Fufs weit fpringen. Es flieg: oder-fpringt aber allemal gerade; Wendungen im Fluge zu machen ift ihm unmöglich. Es bewegt auch feine Flughaut im Fluge nicht fo, wie die Vügel ihre Flïgel. In Laufe ift die Flughaut an den Körper angezogen; auch felộ im Schwimmen breitet es fie niche aus. In eisem Nefte werren oft fieben, zwölf und mehrere beyfammen gefunden, und darin bringen fie auch beym gemeinfchaftlichen Wintervorrathe den ganzen Winter hin. Das Thier feheint die Wäme zu lieben, es verkriecht fich in die Wolle, die man ihm giebt, und lafict fich auch gerne mir zu Betse nehmen. 
27. Huds on Ius. Das hudfonsfche fliegende Eich. horn.

Schreber, Säugthieı, IV. p. 812. n..24. das hudSonifche fliegende Eichhorn.

Pennant, arct. Zool. II. p. I21. n. 62. das gröfSere flirgende Eichhorn.

Forfter, Beytr. zur Völker-u. Länderkunde, III. p. 193. das gröfsere fliegende Eichhorn.

10. Vor A s s. Das europäifch - afiatifche fliegende Eichhorn. ( ${ }^{2}$ )

Müller, Naturfyftem, I. p. 359. n. 10. das ficegende Eichhorn. Tab. 2I. fig. 3. Houtt.

Zimmer.

(2) Lange des Körpers von der Nafe bis an deg Schwanz 6 Zoll 4 Linien. Schwere fechftehalb Unze. Mit dem Fluge diefer Thiere hat es ganz diefelbe Bewandnifs wie beym virginifchen Eichhorn. Sie finden fich vorzüglich in allen grüfsern und kleinern Birkenwaldern Sibiriens, und halten fich immer in dee Höhe auf. Da ihre Nahrung vorzüglich in den fogenannten Schäfchen befteht, die auf den Birken wachfen, und im Winter klein und braun, im Frühling blühend, im Sommer zum Theil mit Saamen gefunden werden, fo haben fie das ganze Iahr hindurch ihr Futter daran. Wo Fichtenwaldungen find, da freffen fie auch die Keimblumen und Fruchtknofpen diefer Bäume, und alsdenn riechen ihre Eingeweide fehr harzig, die fonft den Birkengeruch haben. Von diefer Nahrung nimmt fowohl der im Darmkanal enthaltene Speiferaft, als die dem Mäufekoth ahnliche Lofung eine gelbgrüne Farbe, und fo harzige Eigenfchaft an, dafs letztere trocken am Fetier mit einem Harzgeruch in heller Flamme brenne. Auf die Erde kommen fie nur, wenn fie fich ibres Unraths entledigen wollen, daher ift ihr Aufenthalt an der davon unter dem Baume befindlichen Spur leiche ausfindig zu machen. Inre Stinme ift im Schmerz pfeifend, im Zorn brummend. Die lungen kommen ganz kahl und blind zur Welt, bleiben wenigfens fechs Tage nackt, und, welches man noch bey keinen vierfüfsigen Thiere beob- 
Zimmermann; geogr. Zool. 1. p. 289. das fliegende Eichhorn. (Mit Nr. 26. verbunden.) II. p. 348. n. 255. das ruffische fliegende Eichhorn. Le $\int k e$, Naturgefch. p. I7I. n. I. das fliegende Eichhorn.

Borowsky, Thierreich, I. 3. p. 50. n. 13. das fliegende Eichhorn.

Blumenbach, Handb. d. Naturgefch. p. 69. n. I.' Sciurus (Volans) duplicatura cutis laterali a pedibus anterioribus ad pofteriores; das fliegende Eichhörnchen, der Polatilfché.

Funke, Naturgefch. I. p. Ir4. fliegenide Eichhörner. Ebert, Naturl. I. p. 345. fliegende Eichhörnchén. Halle, vierf. p. 4I8. das graue fliegende Eichhörm. chen, ans Russland.

IMeyer, Thiere, III. Tab. 14. das fliegende Eich: horn, das Männlein.

Gatterer, vom Nutzen und Schaden der Thiere, I. p. 264. n. 154. das filegende Eichhürnchen. Pennant, ard. Zool. II. p. I23. n. 64. das euro. päif che fliegende Eichhorn.

Onomat. hiftor. nat. VII. p. 22. das fliegende Eichhorn.

Handb. der Naturgefch. I. 2. p. I7r. Polatouché. Firifh, Naturfyn. p. \&. n. 15. Aliegend Eichhorn. Büffon, vierfüs. VII. p. I38. das rulfiche flie. gende Eichhorn.

Batfch,

achtet hat, wohl vierzehen Tage blind. Nach fechs Tagen bekommen fie auch "die Vorderzähne. Die Mütter fitzen am Tage über den Iungen, und hüllen fie in ibre Flughaut ein; des Nachits aber, wenn fie felbft auf Nahrung ausgehen, de-. cken fie fiz mit Moos zti. Die Bälge haben eine fehr dünne Haur, und zu weiches Haar, geben alfo ein f́chlechres Pelzwerk. 
Bat fch, 'Thiere, I. p. 241. das mufische fiegende Eichhorn.

MTerrem, zool. Abhandl. p. 40. n. I5. fliegendes Eichhörnzhen.

Klein, quadr.difp. p. 54. Sciurus petaurifta volans. Klein, Claffif. der vierfüls. Thiere, p. I60. n. 8.' fliegendes, oder durch die Luft fpringendes Eichhorn.

Klein, natürl. Ordn. d. vierf. Th. p. 57. n. 90 ftiegendes polnifches Eichhorn; Luft fpringer. Gesner, Thierbuch, p. 24. Mus Ponticus aut Scyticus volans; das fliegende Veeh. Fig. p. 25. mittelmälsig.

Fifcher, Naturgefch. von Livland, p. 62. fiegencies Eichhorn. Tab. I. Abbild. Cchl.

Pallas, Reife, II. p. 439. das fiegende Eichhorn. Pallas, Reife, Ausz. II. p 3II. fliegende Eichhörner.

Leppechin, Tageb. d. ruff. Reife, II. p. 3. fliegende Eichhörner.

Georgi, ruff. Reife, I. p. 163. fliegende Eich. hörner. :

Miiller, Samml. ruff. Gefch. III. p. 524. fliegende Eichhörner.

Berlin. Samml. III. p. 432. der Polatuche, oder das fliegende Eichhorn.

Duvernoi, Bemerk. von einem fliegenden vierfüfsigen 'Thiere in Rufsland; im Hamb. Magazin, II. p. I99.

Leem, Nachr. von den Lappen, p. Irg. * das fiegende Eichhorn.

Wagner, Muf. Baruth. p. 6. I4. Tab. 4. das enlropäifche fliegende Eichhorn.

Erxleben, Mammal. p.435. n. I7. Sciurus (Volans) hypochondriis prolixis volitans, cauda rotundata. Linné, 
Linné, Syft. Nat. Edit. II. p. 46. Sciurus hypo. chondriis prolixis volitan.

Linné, Syft. Nat. Edit. VI. p. 9. n. 2. Sciurus hy: pochondriis prolixis volitans.

Linné, Syfl. Nat. Edit. X. I. p. 64. n. 7. Sciurus (Volans) hypochonidriis prolixis volitans.

Limnié, Fn. Suec. I. p. 9. n. 22. Sciurus hypochon: driis prolixis volitans.

Graumainn, intr. in hift nat, mamm. p. 67. XVII: fliegendes Eichhorn.

Gatterer, brev. zool. I. p. IIg. n. 17. Sciurus (Vo.' lans) duplicatura cutis laterali a pedibus ante. rioribus ad pofteriores; das fiegende Eichhörnchen.

Miitller, zoolog. dan. prodr. p. 5. n. 33. Sciurus (Volans) hypochondriis prolixis volitans, cau. da rotundata.

Kramer, Auftr. p. 315. Sciurus hypochondriis pro. lixis volitans.

Pallas, glir. p. 350 . Sciurus volans.

Pennant, hift. quadrup. p. 420. n. 285. european flying Squirrel.

I I. S A G I t T A. Das javanifche fiegende Eichhorn.'

Miiller, Naturfyn. I.p.360.n. Ir.der Pfeilfchivanz. Zimmernanin, geogr. Zoolog. II. p. 349. n. 257. das jowanifche fliegende Eichhorn.

Schreber, Säugthiere, IV. p. 8I8. n. 26. das java. nifche fliegende Eichhorn.

Neuer Schauplatz der Natur, VI. p. 468. Pfeil. Schwanz, das oftindifche fliegende Eichhörnchen. Onomat. hiftor. nat. VII. p. I9. der Pfeilfchwanz, oder das javanifche fliegende Eichhorn.

Biiffon, vierf. VII. p. I35. das javaniffhe fliegende Eichhorn.

Kk 2

Merrem, 
Merrem, zool. Abhandl. p. 40. n. I4. Schuelles Eichliörnchen.

Erxleben, Mammal. p. 439. n. 19.'Sciurus (Sagitta) hypochondriis prolixis volitans, cauda plano - pinnata lanceolata.

Graumann, intr. in hift. nat. mamm. p. 68. XIX. der Pfeilfchwans.

Gatterer, brev. Zool. I. p. II9. n. I9. Sciurus (Sagitta) hypochondriis prolixis volitans, cauda plano-pinnata, lanccolata; der Pfeilfchrecinz.

Pallas, glir. p. 353. Sciurus Sagitta.

28. PETA URISTA. Der Taguan.(3)

Zimmermann, geogr. Zoolog. II. p. 349. n. 258. der Taguan.

Schreber, Säugth. IV. p. \$19. n. 27. das indianifche fliegende Eichhorn, oder der Tagnan.

Mervem, zool. Abhandl. p. 40. n. I3. Jegeindes Eichhörnchen.

Biifforn, vierf. VII. p. I24. der Taguan, oder das grofse fliegeinde Eichhörnchen.

Vosmaer, defcript. Amf. 1767. Ecureuil volant. Tab. I.

Pallas, glir. p. 149. 153. Sciurus Petaurifta. Biiffon, hift. nat. fuppl. III. p. 150. Tab. 21. a. b. Taguan, ou grand écureuil volant.

Mémoires geograph. phyf. et hiftoriques. Paris I767. II. p. I84. Taguan.

Pennant, fyn. quadr. p. 292. n. 270 . Tab.27. hift. quadr. p. 4I7. n. 28I. Tab. 44. Sailing Squirrel.

A) $V i r$.

(3) Lânge des Körpers 23 Zoll, des Schwanzes 21 Zoll Parifer; I Schuh 5 Zoll rheinifch Maafs; das grüfste von allen bisher bekannten Eichhörnern. Es ift fchüchtern und wild. Mit feinen fcharfen Zähnen kann es, in einer Nacht ein hölzernes Haus, das man ihm eingiebt, mit leichter Mühe zerftücken. 
A) Verïnderungen gegenu die XIIte Edition; und Vera mehrung der Gattungen diefes Gefchlechts.

Edit. XII.

Edit. XIII.

p. 87. n. 8. Sciurus Glis. $\quad$ f. I55. n. I. Myoxus Glis.

Die XIIte Edition hat nur eilf Gattungen, wovon die achte in ein neues Gefchlecht verfetzt worden. Die fechs und zwansigfte if aus dem vier und zwanzigften Gefchlechte, hieher aufgenommen, und auffer derfelben das Gefchlecht mit achtzehn Gattungen vermehrt, überdem bey der er.ften Gattung vier, und bey der fiebenten zilvey Varietäten aus cinander gefetzt.

B) Uibeftimmtere Thiere.

1. Das Kappen - Eichhorn.

Zimmermann, geogr. Zool. II. p. 350. a. das virginifche flieginde Eichhorn.

Schreber, Säugth, IV.p. 822. n. 28. das Kapperem Eichhorn.

Borowsky, Thierreich, I. 3.p. 5I.n. I4 das virginifche fliegende Eichhorn.

Holle, vierfüfs, p. 420. Tab. 22. das virginifche fiegende Eichhürnchen.

Meyer, Thiere, III. Tab. 15.das virginifche fliegende Eichhorn.

Klein, Quadrup. difpof. p. 53. Sciurus virginianus Petaurifta.

Klein, Claflif, der vierfüfs. Thiere, p. I62. n. 9. virginianifches Eiclhorn.

Klein, natürl. Ordn. d. vierfüls, Th. p. 58. n. Io. virginifcher Lafterpinger.

Seba, thefaur. I. p. 72. Tab. 44. fig. 3. Sciurus virginianus volans.

$\mathrm{Kk}_{3}$

Brifont, 
Briffon, quadr, p. 159. n. I4. Sciurus virginianus volans. Sc. cute a pite ad anum membranae in modum lateraliter extenfa volitans.

Pennant, arct. Zool. II. p. I20. n. 6I. das behaubte fliegende Eichhorn.

Erxleben, Mammal. p. 438. n. I8. Sciurus (Petaurifta) membrana a capite ad caudam per pedes expanfa volitans.

Linné, Syft. Nat. Edit. II. p.-46. Sciurus cute a capite ad caudam relaxata volans.

Groumann, intr. in hif, nat, mamm. p. 68. XVIII. Sciurus petaurifta.

Gatterer, brev. Zoolog. I. p. IIg. n. I8. Sciurus (Petaurifa) membrana a capite ad caudam per pedes expanfa volitans.

pennant, Synopf. quadrup. p. 294. n. 221. $\beta$. hiftor. quadrup. p. 419. n. 284. Hooded flying Squirrel.

Pallas, glir, p. 254. Animal paradoxon.

* (EINGESCHOBENES GESCHLECHT.) M ч oxus. Der Schläfer, Winterfchläfer, Schlafratte, Fettmans.

Zimmermann, geograph. Zoolog. II. p. 35 I. Gen. XXX.

Schreber, Säugthiere, IV. p. 824. Gen. XXIX. Binmenbach, Handb. der Naturgefchichte, p. $7 a_{4}$ Gen. XIII.

Bechftein, Naturgefch, Deutfchl. I: p. 505. Kliigel, Encyklopädie, I. p. 233. Bat $f \mathrm{ch}$, Thiere, I. p. 238. Pennant, hift, quadrup. p. 422. Boddaert, Elenchus, Gen. XXII, 


\section{Gris, Der Billich. (4)}

Miiller, Naturfyftem, I. p. 358. n. 8. das Maureeichhörnuchenz.

Zimmermann, geogr. Zool. II. p. 20. die Schlaf: ratte. p. 35I. n. 259. der Billich, die Schlafratte, der Siebenfchläfer.

Schreber, Säugthiere, IV. p. 825. n. 1. Myoxus (Glis) cauda longa villofifima, corporeque cano, fubtus albo, oculis annulo fufco cinctis; der Billich.

\section{Kk 4 Boroz.}

(4) Liebt trockne und befonders folche Gegenden, wo klüftige - Felsklippen nicht felten find. In hohen, Gebirgen aber findet man ihn nicht, noch weniger in Schneegebirgen. Ihr Futter nehmen dicfe Thiere auf den Hinterfüfsen fitzend, wie die Eichhürner. Von den faftreichen Speifen werden fie im Herbft fo fett, dafs man Beyfpiele hat, dafs das Fett bis fechs Linien dick gewefen ift. Ihr Laut if ein röochelndes Schnarchen, das mit dem Ein-und Ausathmen wechfelsweife fchwàcher upd ftärker wird, aber lange fortdauern kasin, ohne unterbrochen, zu werden. Wenn fie fçhläfrig 'find, laffen fie es nur ftofsweife hören. Sie leben parweife, und begatten fich in Frühjahr. Die Iungen konmen nackt zur Welt, und wachfen gefchwind, follen aber nicht über 6 lahr alt werden. $\mathrm{Zu}$ ihrem Winterfchlaf legen fie fich im Heŗbft auf eingetragenes weiches Moos kugelrụnd zufammę", unḍ erfarrèn bey einfạllender Kălte, Bey gelindem Wetter findet man fie nicht immer erftarrt, aber dnch fchliafrig. Dabey freffen fię aber doch etwas, und tragen alfo, um nicht darlien zu dürfen, Wintervorrăthe ein. In einer beftändig geheitzten Stube erftarren fre gar nicht. Aber in einem kühlen Zimmer erfarren fie wohl fchon manchmal im Auguft,fo, dafs fie in etlichen Tagen nichta zunı Vorfohein kommen; wie man fie denn auch bey Sommerszeit in Fiskel. lern zur Erftarrung bringen kann. In Italien werden fie noch tis jetzt gemäftet und gegreffen. Um fie zu fangen bereitet man ihnen beleckte Winterlager von Moos, mit einer Kứrnung von Buctreckern, in welche fịe fich in Herḅ̂t verkriechen, erftarren, und dann heraus genommen werden. An Wię̧eln, lltif fer, und Mardern haben fiẹ betrăchtliçhe Fẹinde. 
Borow $k y$, Thierreich, I.z. p. 48. n. Io. Sciurus Glis; der, Siebenfchläfer, die' Schlafratte, der preulfische graue Tagfchläfer.

Bhimenbach, Handb. der Naturgefch. p. 7o, n. I. Glis (Efculentus) grifeus, fubtus albidus, auriculis rotundatis, nudis; der Siebenschlä̈fer, Ratz, Billich, die Rellmaus.

Bechftein, Naturgefch. Deutfchl. I. p. 506. der Siebenfchliffer. p. 509. Rellmans, Micuseichtion, Billich, Schlafratte, Ratz, Schlafratz, Greuel, Grauwerk, Rafjelizaus. Tab. I2. fig. I. Funke, Naturgefch. I. p. I21. die Rollmous. Ebert, Naturlehre, I. p. 348. der Siebenf chläfer. Halle, vierf. p. 4I8. der prendfiche Tag chliafer. Gatterer, vom Nutzen und Schaden der Thiere, I. p. 26I. n. I5I. der Siebenfchläfer, die Rell. manus.

Neuer Schaupl. d. Natur, VII. p. 66r. Schlafratte. Onomat. hift. nat. VII. p. I6. Sciurus Glis; die

Hafelmaus, Greul, oder Rellmans, der Sięben-. Schläfer, das grane Schläfrige Eichhorn.

Handbuch d. Naturgefch. I. 2. p. 195. der Siebenlchlïfer. Glis.

Handbuch der deut chen Thiergefchichte, p. 38.

Glis efculentus; Siebenfchläfer, Schlafratze. Ratz, Billch, Rellnnaus.

Klïgel, Encyklop. I. p. 253. Schlafratte. Klein, Claflif. der vierf, Th. p. 163. Siebon fçhläfer. Klein, natürl. Ordn. der vierfüls. Th. p. 58. B. Siebenschläfer.

Gesner, Thierbuch, p. 272. Glis; ein Greuel, ein

Rell, ein Rell-oder Schrotmans, eine grofse Haselmans.

Büffon, vierf. IV. p. 27̧o. der. Siebenfchläfer. Tab. ?6. 
Kraft, Ausrott. graufam.'Thiere', II. p. 496. RellMaus.

Bat $\int c h$, Thiere, I. p.238. n. 2. der Sieben/chläfer. Merklein, Thierreich, p. Iз gr. grosse Hajelmans. Ratz. Glis.

Merrem, zool. Abhandl. p. 35. n. 8. Schlafratze. Taube, Slavonien u. Syrmien, I. p. 2I. Gebirg. miärese, oder Biliche.

Pallas, Reife, I. p. 154. die Schlafratte.

Pallas, Reife, Ausz. I. p. I06. die Schlafratte.

Perrault, Charras und Dodart, Abhandl: zur

Naturgefch. II. p. 205. anatomifche Befchreib. einer Bergratte. Tab. 67. die Bergratte. Tab. 68. die Zergliederung.

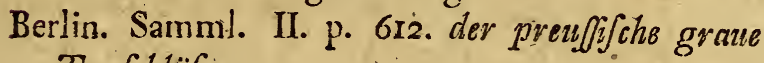
Tagfchläfer.

Iabionfky, allgemein. Lex. p. I44. Billich, Pillich. Tolvaffor, Ehre des Herzogthums Krain, I. p. 437. Pouh.

Erxleben, Mammal. p. 429. n. 13. Sciurus (Glis) canus, fubtus albidus.

Graumann, intr. in hift. nat. mamm. p. 67. XIII. der Siebenschläfer.

Gäterer, brev. Zoolog. I. p. II7. n. 13. Sciurus (Glis) canus, fubtus albidus; der Siebenj/chläfer. Rzaєz. auct. hift. nat. Pol. p. 329. Pouch.

Charleton, exercitat. p. 25. Glis.

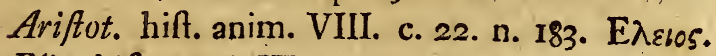

Plin. hît. nat. VIII. c. 58. XVI. c. 6. XXXVI. c. I. Glires.

Oppian. cynèget. II. p. 574: Muokos.

Matthiol. comment. in Diofcor, Ven. 1565. p.205.

Glires.

Varro, de re ruftica, III. c. 15. Glires. (Gliraria.)

Apjicius, de re culinaria, VIII. c. 9. Glis.

Kk's

2. DRYAS 
2. Dryas. Der Eichfchläfer. (s)

Schreber, Säugthiere, IV. p. 83r. n.2. Myoxus (Dryas) cauda longa villofiflima, corpore fulvo, fubtus albicante, fafcia oculari longitudinali atra; der Eich.Schliifer.

\section{Ni tel a. Der Gartenfchläfer. $\left(^{6}\right)$}

IMiiller, Naturfy f. I. p. 351. n. 15. die Eichelmans. Zinnmermann, geogr. Zool. II. p. 21. p. 35I. n.260. die grosse Hajelmans.

Schreber, Säugthiere, IV. p. 833. 11. 3. Myoxus (Nitela) cauda longa fubfloccofa, corpore rufefcente, fubtus albo, fafcia oculari auricularique nigris confluentibus; der Gartenfchläfer.

Borowesky, Thierreich, I. 3. p. 5 o. n. 12. Sciurus Quercinus; die gro/se Hafelmans, Eichelmans. Bechftein, Naturgefch. Deutfchl.' I. p. 509. die grofse Haselmaus. p. эr6. Schlafratte, Eichelmans, Eichenmans, weifse Ratte, Holzmans. Tab. I2. fig. 2.

Funke, Naturgefch. I. p. I2I. die grofse Hajelmanis.

Ebert, Naturl. I. p. 348. die grofse Hajelmans. Halle,

(5) In in einer Gegend in Rufsland, an der untern Wolga, gefunden, und auch in Georgien entdeckt worden. Lange des Kürpers 4 Zoll, des Schwanzes, mit Inbegriff der tiber die Spitze hinaus gehenden Haare, 2 Zoll 6 Linien. (Nach einem aưsgeftopften Balge.)

(6). Trägt im Herbft Nüfle und andere Saamen ein; erftarrt aber wenn es kalt wird, in welchem Zuftande man oft mehrere beyfammen antrift. In ibrem Magen will man zur Sommerszeit häufig Stücken ven Rofskäfern gefunden haben. Auch follen fie den jungen Vögeln und Vogeleyern nachgehen. Das Weibe chen geht viertehalb Wochen trächtig, Die Iungen wachfen

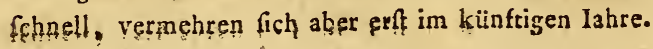


Halle, vierf. p. 422. die Schlafratte?

Gatterer, vom Nutzen und Schaden der Thiere,

I. p. 262. n. 152. die grofse Haselnnaus.

IMerrem, zool. Abhandl. p. 35. n. 6. Eichelratze.

Pennant, arct. Zool. II. p. I27. n1. 66. die grosse HaJelmans.

Neuer Schauplatz d. Natur, VII. p. 662. die grofse Hajelmaus.

Onomat. hiftor. nat. V. p. 344. Mus quercinus; die Schlafratte, die grofse Hafelmans, die Eichelmaus.

Handbuch der deutfchen Thiergefchichte; p. 38. Glis quercinus; die grofse Haselmans.

Klein, Quadrup. difp. p. 56. Mus avellanarum maior Raji.

Klein, Claffif. der vierfüfs. Th. p. 165. die HaSelmaus des Rajus.

Biiffon, vierfüfs. p. 281. die grofso Hajelmaus. Tab. 77 .

Kraft, Ausrottung graufam, Thiere, II. p. 478. Ha/el-Maus.

Batfch, Thiere, I. p. 239. die grofse Hafelmaus. Kriinitz, ökon. Encykl. XXII, p. 197. die gro/se Hafelmaus. (Die Synonymen verwechfelt.)

Cetti, Naturgefch, von Sardinien, I. p. 239. die grofse Hufelnaus.

Pallas, Reife, Ausz. III. p. 477. Hafelmaus. Linne', Syft. Nat, Edit. II. p. 47. Mus avella. narum maior.

Gratumann, intr. in hif. nat. mamm. p. $6 \% \mathrm{XV}$.

Glis quercinus; die grofse Hafelmaus.

Gatterer, brev. Zool. I. p. II8. n, 15. Sciurus (Quercinus) canus, fubtus albidus, macula nigra fub oculis; die Eichelmaus, grofse Hasel. smans,

Charletong 
Charleton, excrcitat. p. 25. Mus avellanarius. Racacz. audt. hint. nat. Polon. p. 315. Mus avella. natum.

Mem, de l'Acad. de Paris. III. P. 3. p. 40. Loir.

Penncint, hiflor. quadr. p. 424. n. 228. Garden Dormoufe.

4. Muscardinus. Der Haselfchlüfer. (7)

Miiller, Naturfyft. I. p.350.n. I4 die Hafelmans. Zimmernann, geogr. Zoolog. II. p. 353. 11. 262. die kleine Hafelmaus.

Schreber, Säugthicre, IV. p. 835. n. 4. Myoxus (Mufcardinus) cauda longa , fubfloccofa, corpore fulvo, gula alba, pollicibus plantaribus muticis; der Hafelfchläfer.

Borowfky, Thierreich, I. 3. p. 49. Sciurus Avellanarius; die Haselmaus.

Biumenbach, Handb. der Naturgefch. p. 70. n. 2. Glis (Avellanarius) rufus, pollice plantarum mutico, auriculis rotundatis; die kleine $\mathrm{Ha}$ Selmaus.

Bechftein, Naturgefch. Deutfchl. I. p. 516. n. 3. die Kleine Hafelmans. p. 519. Hajelmans, Schlaf. ratte, Nuissbiffer, Waldmaus, rothe Waldmanus, auch der Siebenfchläfer. Tab. I2. fig.3. Funke,

(7) Ein niedliches, munteres; dabey unfchuldiges und furchtfames Thierchen, das Niemand beleidigt, und fich nicht einmal welurt, wenn es beleidigt wird, und viel von dem Betragen des Eichhürnchens hat. Es wohnt in Vorhülzern, wo es Hafelftauden giebt, einfam, einzeln oder paarweife; fcheint aber überall nur in geringer Menge zu feyn. Sein Neft ift inwendig etwa 6 Zoll weit, hat oben eine Oeffnung zum Eingange, und if mit weichem Moos ausgefuttert. Es fcheint leichter zu erftarren, als die übrigen Schläfer, und ift im Winter faft fo fetr als der Billich. 


\section{Maminatia Glires. Myoxus. 525}

Funke, Naturgefchichte, I. p. I22. die kleine Ha. felmans.

Ebert, Naturlehre, I. p.349. die kleine Hafélmants, kleine Schlafratze.

Halle, vierf. p. 443. kleine Hafelmans.

Neuer Schauplatz der Natur, VII. p. 663. die klei. ne Hafelmonas.

Onomat. hift. nat. V. p. 331. Mus avellanarius; die kleine Haselmans.

Handb. d. deutfchen Thiergefch. p. 39. die kleine Haselmaus.

Gatterer, vom Nutzen u. Schaden der Thiere,

I. $p_{1}$ 263. n. I53. die kleine Hajelmans.

Batfch, Thiere, I. p. 239. die Hafelmans.

Merrem, zool. Abhandl. p. 35. 11. 7. Ha elratze. Frifch, Naturfyft. p. 8. n. 3. Mus avellanarum minor; kleine. Hafelnsaus.

Gesner, Thierbuch, p. 265. Hrafelmans? Biiffon, vierfüls. p. 285. die kleine Haselmans. Tab. 78.

Kriinitz, ökonom. Encyklop. XXII. p. 198. dis kleine Haselmaus.

Fifcher, Naturgefch. von Livland, p. 60. n. 23. Haselmans.

Bock, Naturgefch. von Preuffen, IV. p. 85. die Hafelmans.

Schriften der berlin. Gefellfch. naturforfch. Fr. III. p. 222. ein Siebenfichläfer.

Perlin. Samml. VI. p. 318. die kleine Huselmans; in. Edw. Fig.

Limné, Syft. Nat. Edit. II. p. 47. Mus avellana. rum minor.

Linné, Syrt. Nat. Edit. VI. p. 10. n. 9. Mus cauda pilofa elongata, corpore rufo, gुula al. bicante. 
Linné, Syft. Natur. Edit. X. I. p. 62. n. II. Mus (Avellanarius) cauda elongata pilofa, corpore rufo, gula albicante.

Linné, Fn. Suec. I. p. Ir. n. 32. Mus cauda longa pilofa, corpore rufo, gula albicante.

Grommann, intr. in hif. nat. mamm. p. 67. XVI.

Sciurus avellanarius; die kleine Hafemanus.

Gatterer, brev. Zoolog. I. p. Ir8. n. 16. Sciurus (Avellanarius) corpore rufo, gula albicante, pollicibus plantarum muticis; die kleine $H a$ Selmans.

Kramer, Auftr. p. 316. Mus cauda pilofa elongata, corpore rufo, gula albicante.

pemant, britt. zool. I. p. 95. Leffer Dormoufe; m. e. Fig.

Pennant, britt. Thiergefch. p. 40. Tab. VIII. b. die kleine Haselmaus.

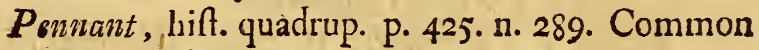
Dormoufe.

A) Verönderningen gegen die XIIte Edition, und Vermehrung der Gattungen diefes Gefchlechts.

Das ganze Gefchlecht ift neu. Die erfte, dritte und vierte Gattung find aus andern Gefchlechtern hieher verfetzt; die zweyte Gattung aber neu hinzugekommen.

13) Unbefinintere Thiere.

1) Die Jurinamifche Goldratte.

Zimmermann, geogr. Zoolog. II. p. 352. n. 26r. Miyoxus (Chryfurus) corpore fufco-purpureo, linea frontali mediaque cauda aurei coloris. 


\section{Mammatia Glives. Dipus.}

Allamand. Biiffon Suppl. IV. ed. d'Holl. p. I64. P1. 67. Le Lerot à queue dorée.

2. Die ungeöhrte Schlafratte. Zinmernain, geogr. Zoolog. III." p. 275.

26. (EINGESCHOBENES GESCHLECHT.) D I P u s. Der Springer, Springhafe, Erdhase. Zimmermenn, geograph. Zoolog. II. p. 354. Gen. XXXI.

Schreber, Säugthiere, IV. p. 840. Gen. XXX. Lefke, Naturgefch. p. 170. Gen, XXIX. Borow $k y$, Thierreich, I. 3. p. 40. Gen. XXVI: Blumenbach, Handib. d. N. G. p. 78. Gen. XIX. Bat $c h$, Thiere, I. p. 237 . Biiffor, vierf. XIV. p. 65. Merrem, zoolog. Abhandl." p. 33." Erxleben, Mammalia, p. 404. Gen. XXXVIII: Graumann, intr. in hiftor. nat. mamm. p. 63 . Gen. XXXVIII.

Gatterer, brev. Zool.'I. p. riz. Gen. XXXVIII. Pennant, hill. quadr. p. 427. Gen. XXXII. Hermainn, tab. affinit. anim. p. II7.

7. I A c u. u s. Der Alakdaga. $\left({ }^{(}\right)$

Zimmermann, geogr. Zool. II. p. I7. der Erdoder Springtice e. " p. 255. n. 263. der Spring. hase, Her Alakdaga.

Schreber,

(8) Nach Rallas giebt es von diefer. Gattung drey befondete Spielarten, eine gröfsere, wie ein Lichhorn; eine mittlere, wie cine Hausratze; und eine kleinere, von der Gröfse einer Feldmaus. Diefe Thiere machen ihre Baue in jedem fenen Boden, am liebAten an höhern Oertern. Die Báue beftehen aus Röhren, die 
Schreber, Säugth. IV. p. 842. n. I. der Alakdaga. Blumenbach, Handb. der Naturgefch. p. 79. die fitivifche Alactacha.

Merrem, zool. Abhandl. p. 34. n. I. fibirifcher Springer.

Biiffon, vierfüls. XIV. p. 67. Alogdaga. Strahlenberg, nord. und öftl. Theil von Europa und Afien, p. 36r. der fliegende Haase.

S. G. Gmelin, Reife d. Rufsl. I. p. 26. der Erdhaafe. 'Tab. 2.

Niebuhr, Befchreib: von Arabien, p. 167. dic. Iarboa?

Miiller,

fchief und gekrimmt mehrere Ellen weit in der Erde bis zum geräumigen und mit weichen und faubern lräutern ausgefutter* ten Nefte fortlaufen. Das Neft pflegt einen Fufs tief, oder auch tiefer, zu liegen, Nebenkammern zu haben, und diene einem, bis direy Paaren zum Aufenthalt. Die Röhre verräth fich' durch den Ausgang, vor welchem die herausgeworfene Erde liegt, pflegt aher am Tage inwendig immer mit Erde verftopft zu feyn, wenn das Thier zu Haufe it, fo wic der offne Zugang eine verlaffene Wohnung andeutat. Ob fie gleich von dẹ Kälte erftarren, fo künnẹn fie doch die Tageshitze nicht vertragen, und kommen nur nach Sonnenuntergange hervor. Die grüfsern künnen fo fchnell fpringen, dafs fie auch fchwerlich vor, einem Pferde eingeholet werden künnen. Ihre Nahrung nehmen fie mit den Vorderfüfsen auf, und verzehren fie in aufrechter Steilung. Da fie faftige Pflanzen freffen, fo bedürfen fie keines Getränks. Auch rohes Fleifch freffen fie, und die Eingeweide der Vügel zieten fie felbft den Kräutera vor. Werden ihrer mehrere zufammen eingefpert, fo greifen fie fich einander felbft an, und an den Getödteten findet man die Augen, und durch die Augenhöhlen das Gehirn ausgefreffen. Für den Winter tragen fie nichts ein, weil fie ihn ganz und gar ohne alle Nahrung verfchlafen. Sie fcheinen dén Sommer hindurch mehrmals zu werfen, und die Iungen werden blind gebohren. Getrocknete und gepuilverte Thiere diefes Art halten die Kirgifen. und Barabinzen für eine bey fchweren Geburten und Steinfchmerzen nütrliche Arzeney. 
Miller, Samml. ruff. Gefch. VII. p.I24. Zits -jam Olear. mufcow. und perf. Reif. p. 740. Seltzanle Art Fetdmüuese, Terbuab.

Paillas, nord. Beytr. I. p. 155. Mus Iaculus. Stralfund. Magazin, I. p. 189. Erd-oder Spring. hafe.

Leppechin, ruffifche Reife, I. p. 258. Erd-oder Springhaafen.

Beckmann, phyf. ökon. Bibl. p. 551. Sprinyratze. Krünitz, ökon. Encyklop. XXXIV. p. I57. das hleins Kaninchen, mit Jehr langem Sichenze. Shaw, Reife, p. 30I. das Daman Ifrael.

Pallas, glir. p. 87. n. 27. Mus (Iaculus) cauda longiffima, apice pennata, nigro alba, pedibus pofticis maximis, pentadactylis. Monogr. p. 275.

Aldrovand. de quadrup. digit. p. 395. Cuniculus feu lepus indicus Vtias dictus.

Alpin. hiftor. nat. Aegypt. p. 232. Agnus filio. rum Ifrael.

I. G. Gmelin, nov. comment. Petrop. V. p. $35 x$. Tab. II. fig. I. Cuniculus pumilio faliens, cauda longifima. Abbild. Schlecht.

Briffon, epit. p. 103. n. 9. Cuniculus cauda longiflima.

Muf. Petropol. I. p. 344. n. I23.

Biiffon, hift. nat. XIII. p. I4r. Alagtaga.

Pennont, Syn. quadr. p. 205. n. 222. hift. quadr. p. 429. n. 292. Tab. 45. Sibirian Ierboa.

2. Sagit t a. Die Ierboa. (?)

Miuller, Naturfyft. I. p.352. n. 20. der Springer.* Miiller.

(9) Unterfcheidet fich unter andern von dem vorhergehenden durch den weniger dicken Kopf, die kleinere Nafe, etwas 
Miiller, Naturf. Suppl.p. 46. n. 38. Mus Sagitta; die' Pfeilmaus.

Zimmermann, geogr. Zoolog. II. p. 19. die klei" nere Terbua, oder die fpringende Mans. p. 355. 1. 264. die klainere Ievboa; die Springende Hiaus.

Schreber, Säugthiere, IV. p. 849. n. 2. die Ierboa.

Le $\int k e$, Naturgefch. p. I 70 n. I. Iaculus orientalis; dev afiatifche Erchifofe. *-

Boroujky, Thierreich, I. 3. p. 40. v. I. Iaculus orientalis; die egyptifche Bergratte, der Springer. Tab. 26.*

Binmenbach, Handb. der Naturgefch. p. 78, it. 2. Iaculus (Ierboa) cauda floccofa, plantis tridaAylis; der. Springhase, Erdhase, die sweybeimischte Bergmaus.

Finnke, Naturgefchichte, I. p. I23. der Springer, Erdinaase.*

Ebert, Naturlehre, I. p. 35I. der Gerbo, oder Ierbuah.

Halle, vierf. p. 595. egiptifche Bergratte.

Gatterer, vom Nutzen und Schaden der Thiere,

I. p. 253. n. 143. der Erdhaf?, Tevboa.*

Merrem, zoolog. Abhandl. p. 34. 15. 2. ägypti. joher Springer.

Onomat.

kürzern Füfse, die drey Zehen an den Hinterfïfsen u. dgl. Die Schwere beträst drey Unzen fiebentehalb Drachmen. Diefe, Thiere halten fich am liebrten in weichem Sande auf, den jene nicht lieben. Ihre Wohnungen find den vorizen ahnlich; auch in der Nahrung und Lebensart kommen fie mit dem Alaldaga uberein, nur dafs lie etwas beiffiger find, und wenn fie beunruhiget werden, einen andern, nämlich kläglichen und fchwachen Ton hüren laffen, da hingegen der Alakdaga, wenn ex gereitzt'wird, eine Stimme wie eine jurge Katze, und im Zorn ein befonderes. Grunzen von fich giebt. 
Onomat. hif. nat. IV. p. 457. die. Ratte mit ver. längertem Schwanz.

Gesner, Quadrup. p. 837. Mures aegyptii alii. Biiffon, vierf. XIV. p. 66. der Gerbo; oder dit eigentlich $\int 0$ gentannte Gerboife.

Batfch, Thiere, I. p. 237. die Terboa's.

Haffelquift, Reife nach Paläftina, p. 277. Mus

(Iaculus) pedibus pofticis longiffimis, cauda extremo villofa.

Hafelquift, act. Upfal. 1750. p. I7. Mus (Aegy ptius) pedibus pofticis longiffimis, cauda corpore longiore, extremo villofa.

HafJelquift, in den Schwed. Abhandl. XIV. p. 129. die ägyptifche Bergratte. Tab. 4. fig. I. Niebuhr, Befchr. v. Arabien, p. 167. die Iarbod? Shaw, Reife, p. 156. Lerboa, oder Terboc. Forfkàl, fn. orient. p. IV. n. ro. b. Mus Iaculus; Djarbua.

Biiffon, hiff. nat. XIII. p. I41. Gerbo ou Gerboife: Bruyn, voy. au Levant, p. 406 . Gerbo. Pallas, Reife, Ausz. II. p. 7. n. I2. Mus Sagitta. Pallas, glir. p. 306. Mus Sagitta.

Falk, Beytr. zur topograph. Kennitnifs des ruff. Reichs, III. p. 3 io.

Berlin. Samml. IV. p. 542. die egyptifche Berg* ratte.

Somnini de Minioncourt, Beobachtungen über den Springhafen; in Voigts Magazin für das Neué. ne aus der Phyf. u. f. w. VI. 3. p. 70.

Sonnini de Manoncourt, journ. de l'hyfique $\mathbf{1} 78 \%$.

Nov. Nr. 2.

Voy. du P. Lucas. Paris 1712. II. p. 73. Erxleben, Mammal. p. 404. n. r. Iaculus (Orien. talis) palmis tetradactylis cum unguiculo pole licari, plantis tridactylis. * 
Linné, Syft. Natar. Edit. IX. n. 4. Lepus cauda elongata.

Linné, Syft. Nat. Edit. X. I. p. 63. n. I5. Mus (Iaculus) cauda elongata hoccofa, palm is pentadactylis, plantis tridactylis, femotibus longiffimis, brachiis brevifimis.

Iinné, Muf. Adolph. Fr. II. p. 9. Mus (Iaculus) cauda elongata, floccofa, palmis fubpen. tadactylis, plantis tridactylis, femoribus longiffmis, brachiis breviflimis.

Linné, Syก. Nat. Edit. XII. I. p. 85. n. 20. Mus (Iaculus) cauda elongata floccofa, palmis fubpentadactylis, femoribus longiffimis, brachiis brevillimis. *

Graumann, intr. in hifor. nat. mamm. p. 63. I. Iaculus Orientalis; der Evdhase, der Springhale, die Bergratte. *

Gatterev, brev. Zoolog. I. p. II2. 11. I. Iaculus (Orientalis) palmis tetradactylis, cum unguicu* lo pollicari, plantis tridactylis; der Erdhase, die ïgyptifche Bergratte. *

Edw. glean. I. Tab. 219.' Gerbua.

Pennant, Syn. quadrup. p. 295. 11. 222. Tab. 25. fig. 3. hift quadr. p. $427 \cdot$ n. 291. Aegyptian Ierboa.

Plizr. hift. nat. X. c. 65. Aegyptii mures.

3. CAFER. Der kapifche Springer. $\left({ }^{10}\right)$

Zimmermann, geogr. Zoolog. II. p. 256. n. 265. der copifche Springer.

Schreber,

(10.) Länge des Körpers 16 , des Schwanzes i 7 Zoll engl. Maafses. Wohnt in der Erde, nährt fich von Gras und Getraide, fchläft am Tage, und geht in der Nacht feiner Nahrung nach. Er bedient fich der Vóderfüfse nur zum Freffen und zum Putzen. 
Schreber, Säugthiere, IV. p. 854. n. 3. der kapifche Springer.

Pallas, glir, p. 87. n. 29. Mus (Cafer) cauda lon- giflima, apice floccofa nigra, pedibus polticis longiffimis tetradactylis.

Biiffon, vierf. IV. p. 78. der Jpringende Hafe. p. 98. der grofse Gerbo, od. Springhafe ani Kap. Sparrmann, Reife n. dem Vorgeb, do g. Hoffn. p. 495. der capfche Gerbua.

Schiwed. Abhandl. XL. p. I९̧. Irз. Yerbúa caperifis.

Biiffon, Suppl. VI. p. 260. Tab. 15. Grande Gerboile ou lièvre fauteur.

Allomant, hilf. nat. du Gnou, du grand Gerbo, et de l'Hippopotame. Amfterd. I776. p. II7. Tab. 15. (Gchört noch mit zum XV Th. der Amfterd. Ausg. des Büff. Werks.)

Merrem, zoolog. Abhandl. p. 34. n. 3. capifcher Springer.

Pennant, hilf. quadr. p. 433. n. 299. Cape Gerbo.

4. ME R I D I A U S. Der dïnnfchwänzige Springer. ( $\left.{ }^{1}\right)$ Miiller, Naturfy f. I. p. 352. n. 19. der Langfuss. Suppl. p. 42. 11. 30. der Langfis s.

Zinmermann, geogr. Zoolog. II. p. 357. n. 266. Dipus longipes; die langbeinigte Maus.

\section{3 Schreber,}

Auf den Hinterfufsen geht er, und macht kleine Schritte, erfchreckt aber grofse Sprünge von $20=30 \mathrm{Fu}$ s. Spin Lut it cine Art Grunzen oder Meckern. In der kalten lahrszeit erftarrt er. Das Weibchen bringt 3.4 Junge. Er wird fehr zahm. Sein Fleifch wird am Kap von einigen gegeffen.

(1) Linge des Körpers 4 Zoll 4 Lin. des Schwanzes 4 Zoll 9 Lin. Schwere ohngefähr 2 Unzen. Der langen Hinterfüfse ohnerachtet hat man diefe Thiere loch nicht wie die vozigen hüpfen, fondern wie die Mạufe laufen gefehen. 
Schreber, Säugthiete, IV. p. 856. n. 4. Dipus lon: gipes; der diinngefchwönste Springer.

Borowfky, Thierreich, I. 3. p. 42. n. 3. Iaculus Torridarum; der Langfuiss.

Pallas, glir. p. 88. n. 30. Mus (Longipes) cauda longa vefita rùfa, corpore fulvefcente, fubtus albo, pedibus pofticis maiufculis pentadactylis. Monogr. p. 3r4.

Pallas, Reife, Ausz. II. Anl. p. 2. n: 4. Mus Meridianus.

Onomat. hifor. nat. V. p. 336 . Mus longipes; $\operatorname{der}$ Langfus s.

Iimné, Syit. Natur, Edit. X. I. p. 62. n. I4. Mus (Longipes) cauda longa veftita, palmis tetradactylis, plantis pentadactylis, femoribus lon. gifimis.

Miiller, Samnıl, ruf. Gefch. VII. p. 124.Zits.jam? (Tfitsjan.)

Shaw, Reife, p. 156. der Iird?

Merrein, zool. Abhandl. p. 34. n. 2. Langfufs.

Granman, intr, in hift. nat. mamm. p. 64. III. Iaculus Torridarum.

Gatterer, brev, Zool. I. p. Inz. n. 3. Iaculus (Tor ridarum) palmis tetradactylis, plantis pentadaCtylis; der Langfu/s.

Seba, thef, II. Tab, 29. fig. 2. Serpentum pabulum. Peinzcnt, hill. quadr. p. 433.11.294. Torrid Ierboa.

5. Tamanicinus. Der ringelfcheönzige Springer: Mritller, Naturfyft. Suppl. p. 42. n. 29. Mus ta. marifinus; die Tamariskemnaws.

Zimmer-

(2) Schwere des T'hiers ohngefahr 4 Unzen. Von feinen Sitten in nichts weiter bekannt, als dafs es atich des Nachts umher fchweift. Seine Begattungszeit fchcint der Fruhhling zu feyn; tim diefe Zeis in auchi das \$ă am lichünten. 
Zimmermann, geoĝr. Zoolog. II. p, 357. n. 267. die cafpifiche Maus.

Schreber, Säugth. IV. p. 859. n. 5. der ringelfchuvänzige Springer.

Pallas, glir. p. 88. n. 31. Mus (Tamaricinus) cauda longiffima, veftita, fufco-fubannulata, corpore grifeo cinerafcente, fubtus albo. Mo: nogr. p. 323.

Pallas, Reife, Ausz. II, Anh. p. 2. n. 3. Mus taimarifcinus.

Merrem, zool, Abhandl, p. 35. n. 3. Tamaris. kent. Ratae.

Erxleben, Mammal, p. 431. n. I4 Sciurus (Tamarifcinus) grifeo-lutefcens, fubtus albus, cauda fufco obfolete variegata.

Graumonn, intr. in hift, nat. mamm. p. 6\%. XIV; die Tamariskbmmans.

Gatterer, brev. Zoolog. I. p. II 8 . n. I4. Sciurus (Tamarifainus) grifeo-lutefcens, fubtus albus, cauda fufco obfolete variegata; die $T a$. mariskezmaus.

Pennant, hiftor. quadr, p. 43\%, n. 296. Tama. risk Rat,

6. Hud on I s. Der labradorifche Springer.(3)

Zimmermann, geogr. Zoológ. II. p. 358. n. 268. die langbeinigte Mans der Hudfonsbay.

Schreber, Säugthiere, IV. p. 86I. 11. 6. der labradorifice Springer.

$$
\mathrm{II}_{4}, \mathrm{P}_{\text {ennant }}
$$

(3) Lânge des Körpers $3 \frac{\mathrm{T}}{4}$ Zoll, des Schwanzes $4 \frac{3}{4}$ Zoll englif́chen Maafses. Bewohnt die Hudfonsbay, und die Küfte von Labrador. Pennant vereinigt das Thier mit dem Mus longipes Palla wovon er den Mus lengipes Linis. (Dipus meridianus) trenne, und diefen unter die Springer, jenen unter die Mäufe rechnet. 
Pennant, arct. Zool. II. p. I31. n. 74. die langbeinigte Mans der Hudjonsbay.

Pennant, hiftor. quadrup. p. 435. n. 295. Labrador Rat.

Verïnderungen Igegen die XIIte Edition, and Vere mehrung der Gattungen diefes Gefchlechts.

Das 26 Gefchlecht der XIIten Edit. Noctilio in ganz aufgehchen, und die einzige Gattung davon: Ameritanus in jetzt Vefpertilio Leporinus, p. 47.n. 9. Das Gefchleche Dipzis if neu; die erfte und vierte Gattung find aus dem vier und zwonzigftein Gefchlecht hieher verfetzt; die zwey. te, dritte und finfte find neu hinzugekommen; und die jechlite habe ich, nach Schreber, noch beygefügt,

22. GESCHLECH T. Lepus. Der Haje. Milller, Naturfyftem, I. p. 3I8. Gen. XXII. Zimmermann, geograph. Zoolog. II. p. 332. Gen. XXVIII.

Sihreber, Sängthiere, IV. p. 862. Gen, XXXI. Lefke, Naturgefch. p. I72. Gen. XXXI. Boroiefky, Thierreich, I. 3. p. 7. Gen. XXII. Blumenbach, Handb. d. N. G. p: 79. Gen. XX. Bechftein, Naturgefch. Deutfchl. I. p. $53 \mathrm{~F}$. Pennant, arct. Zool. II. p. 95. Onomat. hiftor. nat. IV. p. 791.

Erxleben, Mammalia, p. 325. Gen. XXXII. Grannnonn, intr. in hifor. nat. mamm. p. 54 . Gen. XXXII.

Gatterer, brev, Zool, I. p. 93. Gen, XXXII. 
Boddaert, elench. anim. Gen. XVII.

Pennant, hilf quadr. p. 363. Gen. XXVI.

Briffon, quadr. p. 94. Gen. XXII.

Hermann, tab. affinit. anim. p. 85. Irr.

* Ge cchwänzte.

5. Viscaccia. Die Vifcachos.

Zimmermann, geogr. Zoolog. II. p. 339. c. die $V_{i} \int$ cachos:

Vidaure, Gefch. des Königreichs'Chile, p. 90. Vifcacha.

Dobrizhoffer, Gefch, der Abiponer, I. p. 348. der Bifcacha.

Eqxxleben, Mammalia, p. 339. * (Spec. obfcur.)

Garcilafjo, hift. d. Incas, p. 33 r.

Linfchotens, Befchreib. der neuen Welt durch Artus. Frcf. bey de Bry, IXter Th, von Ame. rika", fol. p. I78.

Cieza, Peru, p. 236. Vifcalchos.

I. Timid us. Der gemeine Haje. (4)

Mïller, Naturfyn. I. p. 3I8. n. x. der Feldhafe. L. 5

Zimmer.

(4) Hafe, Rammler, (das Männchen) Setzbafe, Häfin, (das Weibchen). Von der Befchaffenheit feines gewöhnlichen Aufenthalts bekümmt er den Namen: Berghafe, Sumpflase, Feldbaje, Holzbafe, Sandbafe, Brucbbafe, Grundbafe, Steinbafe u, a. m. Man hürt von diefem Thiere keinen Lant, nur fchnaubt es, wenn es in Angft if, und fchreyet blofs dann, wenn man es quall, und zwar ziemlich ftark; Das Weibchen bringt das erfte Mal - 2 hernach auch wohl 5- 6 lunge. Die lungen werden mis offenen Augen gebohren, und 20 Tage gefaugt. Das Alter der Hafen erftreckt fich auf 7-8 lahre. lung gefangen laffen fie fich zähmell, werden aber doch wieder fcheu, wenn fie nicht immer um Menfchen find. Durch ausgefiete Peterfilien kann 
Zinmermann, geogr. Zool. I. p. 214. der Haje. II. p. 335. n. 23I. der gemueine Hirfe.

Schreber, Säugthicre, IV. p. 865 . n. I. der geo meine Hase.

Lefke, Naturgefch. p. 173. n. I. der gemeine Hase. Borowfky, Thierreich, I. 3. p. 7. n. I. der Feldhase.

Bhmenbach, Handb. der Naturgefch. p. 79. n. I. Lepus (Timidus) auriculis apice nigris, corpore et pedibus pofticis longioribus; der $\mathrm{Ha}$ e. Bechfein, Naturgeích. Deutfchl. I. p. 53I. der ge* meine Hafe.

Funke, Naturgefch. I. p. 85. der Hoafe. Ebert, Natullehre, I. p. 34,4. die Hafen. Halle, vierfuils. p. 408. der Haje. Meyer, Thicre, II. Tab. 32. der Has. Abbild. gut, Gatterer, vom Nutzen und Schaden der Thiere,

I. p. 203. n. 105. der Hivge.

Merrem, zool. Abhandl. p. rg. n. r. Felähafe. Beckmann, Naturhiforie, p. 23. n. 2. der Hcafe. Neuer Schaupl. d. Natur, III. p. 667. Hafe.

Onomat. hift. nat. IV. p. 792 . der gemaine Hafe. Handbuch d. Naturgefch. I. 2. p. II2. der Haas. Handbuch der dentfchen 'Thiergefchichte, p. 44. der Hame.

Sancier, öknn. Naturgefch. I. p. 25I. der Hafe. Walther, ökonom, Naturgefch. Deutfchi. p. 133. der Hase.

Frif ch, Naturfyft. p. 9. n. I. Lepus vulgaris vel campeftris; der rechte Hafe, oder Feldhafe.

Klein, Quadr، difp. p. 5r. Lepus vulgaris cinereus.

Klein,

man fie berbeylocken. Der Hafe ift unter den Fufsfohlen, und fogar zum Theil im Munde behaart. Pallas führt cin Beyfpiel yon einem zahmen Hafen an, der fich mit einer Hündin begatret hat, $S$ : Naturgeich, merkw. Thiere, XI. p. 37\% 
Klein, Claflif. d. vierf. Th. p. I55. gemeiner Haafe. Klein, natürl. Ordn. der vierf. Th. p. 54. n. I. Feldhase.

Gesner, Thierbuch, p. 167. Has. Abbild. mittelmälsig.

Bïffon, vierf. p. 147. der Hafe. Tab. 50.

Batfch, Thiere, I. p. 234. n. 2. der gemeine Feldhoafe.

Bock, Naturgefch. von Preufen, IV. p.64. der Feldhase.

Molina, Naturgefch. von Chili, p. 241. der Hafe. Dobrizhoffer, Gefch. der Abiponer, I. p. 349. der Haaje?

Hartfink, Befchr. von Guiana, I. p. rog. tie Hafen? Höft, Nachricht. von Marokos und Fes, p. 294. Hafen, Erneb?

Adanson, Senegal, p. 37.277. Hajen?

Baldauus, Zeylan, p. 42r. Hare.

Wolf, Reif. nach Zeilon, p. I2r. Has $6 u$. Buswell, Befchr. von Corfica, p. 44. Hajen. Knox, ceylan. Reifebefchreib. p. 4I. Hafen.

Cetti, Sardinien, I. p. I89. der Hafe. Miiller, Samml.ruff. Gefch. III. p. 525. Harfen. Shaw, Reife, p. 157. der Hafe.

Neuhof, Gefandfchaft nach Clina, p. 348. Haferz. Bonnet, Betrachtung. über die Natur, II. p. 5I4, $\operatorname{der} H_{a} \int_{b}$.

Beytr. zur Beförd. d. Naturkunde, I.p. 125. Hafe. Anveifung zur Anleg. der Wildbahnen, p. I80. der Hase.

Krïnitz, ökon. Encykl. XXII. p. 100. der Hafe. Le Verrier de la Conterie, normännifcher Täger, Münft. 1780. p. 64. Haje.

Döbel, Iägerpraktik, I. p. 29. Hase. Mlerklein, Thierreich, p. 65. Hanfa.

Schweds 
Schwed. Abhandl. XXXV. p. 92. (Ein junger aus dem Leibe gefchnitten, und aufgefuttert.)

Sch wed. Abliandl. XXXVIII. p. 48. (Durch die Schafe mit Blattern angefteckt.) Hif. phyf. u. moral. Mancherley, II. p.345. Hafe. Heppe, wohlredender Iäger. p. 158. Ha e.

Oekon. Znologie, p. 32. n. 38. der gemeine Haaje. Iablon kky, allgem. Lex. p. 433. Haje, Haase.

Judovici, Kaufmannslexicon, III. p. 302.

Lemmery, Materiallexicon, p. 63I.

Goth. genieinnuitz. Wochenbl. 1779. p. 60.

Donndorf, Natur und Kunf, I. p. 536. Haje.

Limné, Syn. Natur. Edit. II. p. 46. Lepus cauda abrupta, pupillis atris.

Linne', Sy?. Nat. Edit. VI. p. 9. n. 2. Lepus cauda abrupta, pupillis atris.

Linné, Syft. Nat. Edit. X. I. p. 57. 11. I. Lepus (Timidus) cauda abbreviata, aúribus apice nigris.

Limné, Synt. Nat. Edit. XII. p. 77. n. I. 'Lepus (Timidus) cauda abbreviata, auriculis apice nigris.

Limné, Fn. Suec. I. p. 8. n. I9. Lepus cauda abrupta, pupillis atris.

Limné, Muf. Ad. Fried. I. p. 9. Lepus (vulgaris) cauda abrupta, pupillis atris.

Grawmann, intr. in hiftor. nat. mamm. p. 54. I. Hule; Rommler, Setzhaje.

Gatterer, brev. Zool. I. p. 93. n. I. Lepus (Timidus) cauda abbreviata, pedibus polticis longitudine corporis dimidii, auriculis apice nigris; der Hafe.

Ro:mmel, difcurf. phyf, med. ad Weichmannum de foetibus leporinis, extra uterum repertis, aliisque tam de Leporibus etc. Ulm I699. 4 . 
M:ller, zool. dan. prodr. p. 4. n..23. Lepus (Timidus) cauda abbreviata, auriculis apice nigris.

Kramer, Aufr. p. 315. L.epus cauda abrupta, pupillis atris.

Charleton, exercitat. p. 23. Lepus.

Schienkfellt, theriotr. Silef. p. 103. Lepus.

Rzaczynfki, hift. nat. Polon. p. 219. aucq. p. 3ir.

Lepus.

Sibbald, Scct. illuftr. II. 2. p. Ir. Lepus.

Forfkal, fn. orient. p. IV. n. 20. Lepus Ârnäb.

Muleum Wormian. p. 32 I. Lepus.

Pallas, glir. p. 30. Lepus (Europaeus) apice aurium, capite longiorum, caudaque fupra atra.

Hagedorn, de pellis leporinae in vigiliis vfu; in den Mifc. Nat. Cur. Dec. II. an. 5. obf. 94.

Ovelgün, de pinguedinis leporinae, maris et feminae, ac oculorum leporinorum efficacia; in den Act. med. phyf. Acad. nat. cur. X. obf. 48 .

Lentilii, obf. de ufi axungiae Leporinae ad oculorum pannum; in Bartholin. act. med. et philof. Hafnienf. V. a. 1677-1679. obf. 124. und' in Manget. bibl. chirurg. III. Genev. I72I. p. 293.

Forfter, philof. transact. LVII. p. 343. Lepus timidus.

Pennant, britt. zool. p. 41. Hare.

Pennant, britt. Thiergefch. von Murr, p. 36.

$p_{\text {ennant }}$, hiftor. quadrup. p. 368. n. 24I. Common Hare.

Plin. hiftor. natur. VIII. c. 55. 56.58. X. c. 63. XI. c. 37-39. Lepus.

Aelimn. de nat. animal. II. c. 12. VI. c. 47. XIII. c. $12-15 . \Lambda$ a $\gamma \omega$ s.

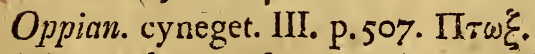
$V$ arro, de re ruh. Lib. III. c. I2. Lepus. 
B. Cornutus. Der gehürnte Hafe.

Schreber, Säugthiere, IV. p. 875. mit Geweihen verfehene Hafen.

Bhumenbach, Handb. der Naturgefch. p. 80.

Ebcrt, Naturlehre, I. p.344. die gehörnten Hasen. Onomat. hift. nat. IV. p. 797. gehörnte Hafen. Klein, Claflif. der vierfüfs. Th. p. I56. gehürnte Hajen.

Klein, natül. Ordn. d. vierf. Th. p. 55. n. 4. gehörnter Haje.

Meyer, Thiere, II. tab. 33. zwey gehörnte HaSen. Gesner, Thierbuch, p. 173. ein frembde Art der Hafen, mit Hönnew; m. e. Fig.

Biuffon, vierfüs. IV. p. r78. gehürnte Hasen. Tab. 51.

Heppe, wohlred. Täger, p. 158 .

Anmerk. über alle Theile der Naturl. III. p. 540. Hafen, welche Hörner tragen.

Erxleben, Mammal. p. 330. $\gamma$. Lepus cornutus.

Gatterer, brev. Zoolog. I. p.95. c. der gehörnte Haje.

Hermam, tab. affin. anim. p. II2. Lepus cornutus. Iacobaei, muf. reg. 'Tab. ̧. fig. 6. 7. 8. Cornua leporina.

Muf. Wormian. p. 32 I. Lepores cornuti. Ionfton, quadr. Tab. 65. Lepus cornutus. Mufeum Beslerianum, p. 38. Tab. Io.

Mifc. Acad. Natur. Curiof. dec. II. an. 6. 1687. p. 368. obf. 183. de lepore cornuto fig. 73 . Pallios, glir. p. 14. Lepus offeis cranii excrefcentiis difformiter cornutus.

Panllini, Lagographia, Sect. I. c. 3. S. I. Welch. hecatoft. I. p. 3r. Tab. 2. fig. I. 2. 3. Turre, element. phyl. Tom. V. p. 44 . 


\section{VAR I a B I Is. Der veründerliche Hafe. (")}

Zinimermann, geogr. Zool. II. p. 335. n. 232. der veriuderliche oder der nordliche Hafe.

Blumenbach, Handb. der Naturgefch. p. 79. eine befondere weifse Spietart.

Pennant, arct. Zoolog. II. p. 95. n. 45. der veränderliche oder nordifche Haaje.

Bechftein, Naturgefch. Deutfchi. I. p. 544. 2 ei $\int_{s e}$ Hâjen.

Klein,

(5) Die Naturhiftoriker find nicht einig, ob diefe.und die vorhergehende Gattung blofse Varietäten, oder wirklich verfchiedene Arten ausmachen. Erxleben und Biiffon fehen beyde Thiere als blofse Varietäten an. Pennant und Pallas halten fio für verfchiedene Arten. Zimmermanin ift Büffons und Erxlebens Meinụng zugethan, und hält den gemeinen Hafen, den beftän diğ weifsen grönländifchen, und den. Alpenhafen des Pennant blofs für drey Racen von einer Art, weil fie in Anfehung des ganzen Aeufsern, der Lebansart, des Gefchmacks des Fleifches, u. dgl. m. bis auf geringe Abweichungen mit einander iibereinkummen, ohne die grofse Verbreitung des Thiers, und die daher rührende Verfchiedenheit der Wirkung des Klima's auf daffelbe in Erwägung zu ziehen, es auch bekannt fey, dafs es felbft unter den európäifchen Hafen oftmals wei/se, ja gañz fcbwarze gebe. (Von erftern findet fich unter andern auch ein Beyfpiel im Naturfarfch. I. p. 64. n. 20.) In Grönland bleiben diefe Hafen beftändig weifs. In Lappland find fie 10 Monate des lahrs tiber weirs, und nur in den zwey heifseften Monaten des Sommers nehmen fie ihre falbe Farbe wieder an. Neben der Tungunska has man Heerden von $5-600$ fchneeweifser Hafen angetroffen. Sie follen dort grofse Wanderungen anftellen. Alle Frühjahr ziehen fie in noch gröfsern Heerden gegen Süden, und wenn die Tungunska, wieder überfroren if, wandern fie zurück gegen Norden. In Grönland, wo fie fich ron Gräfern, von dem dafelbft wachfenden weifsen Moofe nahren, und gewöhnilich fehr fett werden, ifst man das Fleifch gekocht, das im Magen befindliche Futter aber roh. Thr Unrath giebr'den Grönländern Daclite für die Lampen, und der weiche warme Pelz Kleidung für ihre Kinder. 
Klein, Claflif. der vierfürs. Thiere, p. 155. Sttin-, Sandhaae.

Klein, natürl. Ordnung d. vierf. Th. p. 55. n. 2. weilser Steinhase, Sandhase, Berginafe.

Büffon, vierfüfs. IlI. p. I66. die Jchweiserifchen Berghasen.

Merrem, zool. Abhandl. p. 20. n. 2. Berghajè. Fifcher, Naturgefch. von Livłand, p. 57. gemeiner' Hafe.

Ietze, phyfico-theol. Betracht. über die weifsen Hafen in Liefland. Lïbeck 1749.8.

Leem, Nachr. von den Lappen, p. 97. Hasen. Altmann, helvẹt. Eisgebürge, p. 210. die Berghafen.

Pontoppidan, Norwegen, II. p. r9. Hafen.

Steller, Kamtfchatka, p. 126. Haafent.

Krafcheninnikow, Kaintfchatka; p. I19. Hawene. Lefeps; Kamtichatk. u. Sibirien, p. 54. Hajen.

Penznant, Reife durch Schottland, I. p. 7I. eine befondere Art von Hasen.

Pennant, Reife durch Schottland, I. p. I24. weifse Hajen.

Egede, Naturgefchichte von Grönland, p. 86. Hajen.

Cranis, Hift. von Grörl. p. ro4. Hajen.

Strahlenberg, nord. und öft. Th. von Europa und Afien, p. 16I. Hafe.

Ryt fchk. orenl. Topogr. I. p. 230. Hafen.

Anmerk. über alle Theile der Naturlehre, III. p. 529. $\$ .5$.

Fabric. fn. groenland. p. 25. n. 15. Lepus Timidus. Lepus groenlandicus, tam aeftate, quam hieme albus, exceptis apicibus fummis auricularum nigris.

Scheffer, Lappon. p. 346. Lepores. 
Pallas, glir. p. 30. n. 2. Lepus (Variabilis) apice aúrium atro, cauda concolore alba.

Erxleben, Mamnalia, p. 328 n. o. Lepus alpinus, minor, auriculis brevioribus apice nigris, cruribus gracilioribus.

Linné, Syft. Nat. Edit. XII. p. 77. n. 1. ß. Lepus caudatus, plane candidus.

Graumann, iner. in hift nat. mamm. p. 54. I. a: derifchweitzerifche Berghase.

Gatterer, brev. zoolog. I. p. 94. a. L. alpinus; der fchweitzerifche Berghafe.

Melanges d'Hift. et de la Litter. par Vigneut Marville. Tom. I. p. 8.

Penuant, britt. zoolog. p. 40. T2b. 47. Alpine Hare.

Plin. hiff. nat. VIII. c. 55. Lepores candidi.

$V$ arro, de re rult. III. c. I2. toti candidi.

B. Niger. Der fchuarze Hafe.

Zimmermann, geogr. Zoolog. I. p. 215. ganz Schwarze Hafein.

Bhunnenbach, Handb. der Naturgefchichte, p. 79.' Schivarze Hasen.

Onomat. hif. nat. IV. p. 797. vollkommen fchwar. zer Hafe.

Klein, Claffif. der vierf. Thiere, p. 156. Schwarzer Hafe.

Klein, natürl. Ordn. d. vierf. Th. p. 55. n. 3. Schwarzer Hafe.

Bïffon, vierfüfs. III. p. 167. fchwarzev Hafe. Merrem, zogl. Abhandl. p. 19. B. der fchwarze Feldhaje.

Pallas, glir. p. 13. Lepus niger.

Muf. Wormian. p. 32I. Lepus plane niger. Erxleben, Mammal. p. 323. ,. Lepus niger. 


\section{6 । Mammalia Glires. Lepus.}

Graumann, intr. in hift. nat. mamm. p. 54. I. b: Schwarser $\mathrm{Ha} / e_{0}$.

Gatterer, brév. Zool. I. p. 94. b. Lepus niger; der fchwarze Haje.

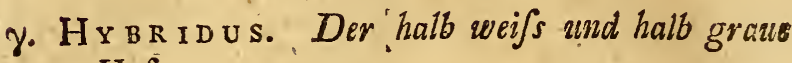
Hase.

Pallas, glir. p. 15. Varietas hybrida femigrifea.

7. A MER I C ANus. Der nordannerikanifche Hafe. $\left({ }^{6}\right)$

Zinimermann, geogr. Zoolog. II. p. 336. n. 223. der HaJe von der HudJonzsbay.

Schreber, Säugth. IV. p. 88I. n. 3. Lepus (Nanus) auribus extrorfum nigro - marginatis, cauda fupra nigricante; der Wabus, oder amerikanifine Hase.

Merrem, zool. Abhandl. p. 20. n. 5. Hudfonio fcher Hafe.

Pennant, arct. Zoolog. II. p. 96. n. 46. der Hase von der Hudfonsbay.

Kalm, Amerika, III. p. 349. die Hafcn.

Forfter, von den Thieren der Hudfonsbay; in

Sprengels Beytr. zur Völker - und Länderkunde, III. p. 189. der amerikanifche HaJe.

Ellis,

(6) Unterfcheidet fich von dem Europäifchen 1) durch die unverânderliche kleine Statur und Gewicht, da feine gröfste Länge nie über anderthalb Fufs, und das $G$ ewicht ohngefăhr zwey und ein Viertel höchftens drey Pfund beträgt; 2) durch die Farbe: 3) durch die Ohren, die a) wo nicht kürzer, doch nie lange: als der Kopf, b) deren Spitze ftumpfer, und nur mit einer fchmalen Einfaftung verfehen, und die c) hinten ganz nackend find. Man fangt diefe Thiere in dräthernen Schlingen. In der Hudfonsbay find zwifchen den lahren 1708. bis 1714. in einem lahre 25000 Stück gefangen. In dem kultivirten Amerika find fie den Kohl - und Rübenpflanzungen fehr nachtheilig. 
Ellis Reife nach Hudfons Meerbufen, p. 181. 184.' Hafen.

Pallas, glir. p. 15. Species quam Barringtonus a Lep. variabili diftinctam fabilivit.

Graumann, intr. in hiftor. nat. mamm. p. $55 . \mathrm{I}_{\text {q }}$ Lepus Americanus.

Gatterer, brev. Zool. I. p. 95. n. 2. Lepus (Ame: ricanus) cauda abbreviata, pedibus poftiois, corpore dimidio longioribus, auricularum caudaeque apicibus grifeis; der amerikanifche Hafe. Pennant, hiftor. quadr. p. 372. n. 243. American Hare.

\section{Tola I. Der Tolai.(7)}

Zimmermann, geogr. Zoolog. II. p. 237. n. 235 • der Tolai.

Schreber, Säugthiere, IV. p. 877. n. 2. der Tolai. Borowfky, Thierreich, I. 3. p. II. n. 5. Lepus Dauricus; das langgefchwänzte Kaninchen.

Gatterer, vom Nutzen und Schaden der Thiere, I. p. 213. n. 108. Tolai.

Klein, natürl. Ordn. der vierf. Th. p. 55. Cuniculus Dauricus caudatus.

Biiffon, vierfüfs. III. p. 219, das langge/chwiinate Kaninchen.

Kriinitz, ökon. Encyklop. XXXIV. p. 158. das langgefchwönste Kaninchen.

$\mathrm{Mm} 2$

Merrem,

(7) Halt fich' getn im Freyen, unter niedrigen Gebüfchen ron Robinien und Weiden auf, die ihm auch vorzüglich zur Nahrung dienen. Im Laufen macht er nicht fo, wie der veränderliche und gemeine Hafe, Umwege, fondern lauft gerade aus, und verbirgt fich, wenn er in Gefahr if, in Felfenklïften und Murmelthierhühlen. Selbft gräbt er nicht. Der Balg ift yon geringem Werthe, und nicht im Handel. Die Tanguten haben diefs Thier unter die Flecke des Mondes verfert. 
Merrem, zool. Abhandl. p. 20. n. 3. Tolai. Bat fch, Thiere, I. p. 235. der Tolai. Pallas, Reife durch Rufsland, III. p. 220. Tolai. Briffon, quadr. p. 97. n. 8. Lepus cauda in fupina parte nigra, in prono alha.

Pallas, glir. p. 30. Lepus (Tolai) auribus apice nigro-marginatis, cauda fupra atra. Meder fchmidt, Xen. Ifidis Sibir. et Hodegetic. MSt. - Catal. Muf. Petrop. I. p. 314. Cuniculus dauricus caudatus, Tolai Mongolis dictus.

Graumann, intr. in hift. nat. mamm. p. 55 . V. Lepus Dauricus; das langgefchwönzate $K a$ ninchen.

Gatterér, brev. Zool. I. p. 96. n. 5. Lepus (Dau. ricus) cauda elongata, gula nigra; das langSchröinzige Kaninchen.

Severin, zool. Hung. p. 69. Cuniculus Dauricus. Penncint, hiftor. quadrup. II. p. 374. n. 245. Baikal Hare.

9. Minimus. Der Cuy. Molin.

3. Cuniculus. Das Kaninchen. $\left.{ }^{8}\right)$

Miiller, Naturfyft. I. p. 322. n. 2. das Kaninchen. Zimmermann, geogr. Zool. I. p. 214. 'II. p. 337. n. 224. das Kaninchen.

Lefke,

(8) Ift urfprünglich in den wärmein Zonen der alten Welt zu Haufe, ift aber nun auch in nordifchen Gerenden einheimifeh geworden. Zum Hafen verhält es fich dergeftalt, dafs von zwey alten; zu gleicher Zeit und ohngefahr von gleichem Alter gebohrmen, das Kaninchen vier Pfund wog, ein Fufs drey Zull lang; vorn fünf Zoll und hinten neuntehalb Znll hoch war, und der Leib, wo er am dickften ift, im Unfange eilf Zoll ausmachte; der Hafe aber wog fieben Pfund, war ein Fufs eilftehalb Zoll lagg, vorn zehn Zoli, und hinten ein Fufs drey 
Le $\int k e$, Naturgefch. p. 174. n.2. das Kaninchen. Borowfky, Thierreich, I. 3. p. 9. das Kaninchen. Blumenbach, Handb. d. Naturgefch. p. 80. n. 2. Lepus (Cuniculus) auriculis nudatis, corpore et pedibus pofticis brevioribus; das Kaninchen. Bechftein, Naturgefch. Deutfchl. I. p. 544. das Kaninichen. p. 545. das wilde Kaninchen. Eunke, Naturgefch. I. p. 98. das Kaninchen. Ebert, Naturl. I. p. 344. Kaninichen. Halle, vierf. p. 413. das Kaninchen. Meyer, Thiere, I. Tab. 83. das Caninichen. Gatterer, vom Nutzen und Schaden der Thiere, I. p. 209. n. 106. das Kaninchen. Beckmamn, Naturhiforie, p. 23* n. 3. das Kaninchen.

Neuer Schaupl. d. Natur, IV. p.372. Kaninichen. Onomat. hiftor. nat. IlI. p. 503. das Koninichen. Handb. der Naturgefch. I. 2. p. IIg. das Kaninichen; Cuniculus oder Lepus Hifpanicus.

Handbuch der deutfchen Thiergefchichte, p. 47. das Kaninchen, Haajenkiihlein.

$\mathrm{Mm}_{3}$.

Sander,

Zoll hòch, und hatte der Umfang dés Lcibes, wo er am dickften it, zivölftehalb Zoll. Der Hafe if alío um ein Drittheil länger, und noch einmal fo hoch and fchwer als das Karinchen. Die Fruchtbarkeit diefer Thiere ift weit forker als bey dem Hafen. Sie künnén, wenn fie fünf oder fechs Monate alt find, fich vermehren, Man hat, wiewuhl felten, Beyfpiele, dafs ein Weibchen zwölf lunge gebracht hat. Rechnet man nur fieben. Würfe im Iahre, und auf jeden Wurf acht lunge, fo kann Ein $P$ aar Kaninchen fich in vier lahren auf $1,274,840$ vermehren. Auf der St. Peters In'el, ohnweit Sardinien, war 1736. die Menge der Kaninchen fo grofs, dafs fie zur Landọlage worden, und die anbauenden Menfchen vertrieben. Sie kommen auch in ganz, wüften Gegenden, z. E. auf Volcano, der funft fo üden liparifchon Infel, fort. Ihr Fleifch wird befonders in England gefpeif, wo man fie zu diefer Abficht in eigenen Ställen füttert. Sie leben in der Monogamie. 
Sander, ökonom. Naturgefch. I. p. 25I. das Caninichera.

Walther, ökonom. Naturgefch. Deutfchl. p. IIg. Caninchern.

Frifch, Naturfyn. p. 9. n. 2. Cuniculus vel!Le. pus fubterraneus; das rechte Kaninchen.

Klein, Qiadr. difp.p. 52. Lepufculus, Cuniculus, terram fodiens.

Klein, Claffif. der vierf. Thiere, p. 156. Kaninchert. Klein, natürl. Ordn. d. vierfüs. Th, p. 55. n. 5 . Kaninchen.

Gesner, Thierbuch, p. I74. Küniglein. Abbil. dung' 'gut.

Bïffon, vierfüfs. III. p. I84. das Kaninchen. Biuffon, vierf. III. p. 206. das wildé Kaninchen, Tab. 52.

Merrem, zool. Abhandl. p. 20. n. 4. Kaninchen. Bat $c h$, Thiere, I. p.235. das gemeine Kaninehen. Bock, Naturgefch. von Preuffen, IV. p. 67. 11. 2I. das Kaninchen.

Fifcher, Naturgefch, von Livland, p. 59. n. 19. Kaninuchen.

Dobrizhoffer, Gefch, der Abiponer, I. p. 349. Kaninchen, Conejos.

Cetti, Naturgefch, von Sardinien, I. p. I89. das Kaninchers.

Kolbe, Vorgeb. d. g. Hoffn. p. 153 . edit. in 4.

p. 346. das Caninchen.

Shaw, Reife, p. I57. dias Kantrithen.

Bonnet, Betrachtung. über die Natur, II. p. 440. das Kaningen.

Beytr. zur Beförd, ler Naiurkunde, I.p. I25. n. I6. Kaninichers.

Mellin, Anweifung zur Anleg. der Wildbahnen, p. 788. dat Kaninshen; m. e. Fig.

Kriunitz, 
Kriinitz, ökon. Encykl. XXXIV.p. 124. Kanin, Kaninchen, Kiiniglein, Kuniglhase, Kunele, Kïnlein, Hasenkiiihnlein; Küngelin, Künelle, Kïlle, Kïngele, Kenienken, Karnickel, Zwerghase. (Rammler, das Männchen; Lampreten, die Iungen.

Döbel, Iägerpraktik, I. p. 3 I. Kaninchen, Küllein, Cannickel.

Heppe, wohlredender Iäger, p. 84.

- Oekon. Zoologie, p.32. n.39. das Kaninchen. Iablonfky, allgem. Lex. p. 199. Caninchen, Kitlen, HaJenkillen, Hafen - königlein.

Beckmann, phyf. ök. Bibl. V. p. I94 Kaninchen. Ludovici, Kaufmannslexicon, III. p. 850.770. Lemmery, Materiallexicon, p. 378 .

Hamburg. Magazin, XXIV. p. 218, Caninchen. Hunvich, Anleit. zum Deich-und Schleufenbau.

Brem. I77r. II. 2. S. 31. Kaninchen.

Börner, Stadt -u. Landwirth fch. I, 2. p. 197.

Krünitz, gemeinnütz. Vorrath auserlef. Aufiatze.

Lpz. 1767. p. 65:

Hannöv, nützl. Samml. 5758. n. 22, .

Stuttgard. Ausz. II. 4. p. 490.

Taube, Gefch. der engländ. Handelfchaft. Lpz. I776. p. 203.

Allgem. Haushalt, und Landwiflenfch, I, p. Sos. Cuninchen.

Frankf. neue Ausz. I768. VIII. p. 57 I.

Erxlehen, Mammal. p. 331. n. 3. Lepus (Cuniculis) cauda abbreviata, pedibus pofticis corpore dimidio brevioribus.

Linné, Syft. Natur. Edit. II. p. 46. Lepus eauda abrupta, pupillis rubris.

Limmé, Syft. Nat. Edit. VI. p. 9. 11. 3. Lepus cauda abrupta, pupillis rubris, 
Limé, Syft. Nat, Edit. X. I. p. 58. n. 2. Lepus (Cuniculus) cauda abbreviata, auriculis nudatis. Lininé, Fn. Suec. I. p. 8. n. 20. Lepus cauda abrupta, pupillis rubris.

Graninann, intr, in hift. nat. mamm. p. 55. III. Kanisachen.

Gatterer, brev, Zool, I. p. 95. n. 3. Leppus (Cuniculus) cauda abbreviata, auriculis nudis, pedibus pofticis corpore dimidio brevioribus; das Kaninzchen.

Miiller, zool. dan. prodr. p. 4. n. 24. Lepus (Cu. niculus) cauda abbreviata, auriculis nudatis.

Kramer, Aufr. p. 315. Lepus cauda abrupta, pupillis rubris.

Charleton, exercitat. p. 23. Cuniculus.

Schroenkfeld, theriotr. Silef. p. 86. Cuniculus.

Rzacsynfki, hift. nat. Polon. p. 240. Cuniculi.

Sibbald, Scot. illuftr. II. 2. p. Ir. Cuniculus.

Leon. Afric. defcript. Afr. p. 757. Cuniculus.

Pennant, britt. zool. p. 43. Rabbet.

Pennant, britt. Thiergefch. von Murr, p. 38 . Aviftot. hiftor. animal. II. c. 9. n. 50. V. c. 2 a
11. I2. I3.
VI. c. 33. n. 407-412. $\triangle 4 \sigma v \pi 045$.

Varro, de re ruft. Lib. III. c. I2. Cuniculi.

3 Buch Mol. XI. v. 5. Caninchen.

C. Domesticus. Das zahme Kanincherr.

Bechftein, Naturgefch. Deutfchl. I. p. 550. das zahme Kaninchen. p. 554. Kaninchen, zahme Hiven, Kanickelchen, Karnützchen, Kiillen, Hafenkïhlein.

Biiffon, vierfüls. III. p. 207. das zahme oder das. Hanskaninchen. Tab. 53 .

Allgemeine Haushalt. u. Landwiffenfch. I. p. 8II. zahne Caninchen. 
Argenteus. Das reiche Kaninchen.

Zimmermann, geogr. Zoolog. I. p.218. das weiche (reiche) Kaninchen.

Borowsky, Thierreich, I. 3. p. Io. b. das reiche Kaninchen.

Biiffon, vierfüf: III. p. 208. das reiche Kanivchen. Tab. 54.

Kriinitz, ökon. Encykl. XXXIV. p. I56. das reiche Kaninichen.

Erxleben, Mammalia, p. 334. ß. C. argentéus. Varietas pilis ex argenteo grifeis, pedibus fulcis.

Graumann, intr. in hiftor. nat. mamm. p. 55: b. das reiche Kaninchen.

Gatterer, brev. Zoolog. I. p. 96. b. C. argen. teus; das reiche Kaninchen.

\%. ANG ORENS Is. Das angorifche Kaninchen.

Zimmermanin, geogr. Zoolog. I. p. 218. das angorifche Kaninushen.

Lefke, Naturgefch. p. 174. das angorifihe Kaningen.

Borowelky, Thierreich, I. 3. p.-ro. a. das angorifche Kaninchen. Tab. 22.

Blumenbach, Handb. der Naturgefch. p. so. die langhaarichten angorifchern Kaninchen.

Bechftein, Naturgefch. Deutfchl. I. p. 554. das angorifche Kaninchen. p. 557. Seidenhasen, englijche Hafen, Rupfinafeñ, Königshajen, Hafenkönige, ungarifche, mofkowitifche und Seidenkaninchen.

Büffon, vierf. III. p. 209. das angorifche Kaninchen. Tab. 55. Das angorifche Kaninchen in . der Manee. Tab. 56 ,

Krïnitz, ökon. Encyklop. XXXIV. p. 153. das. angorifche Kaninchen.

Funke. 
Funke, Naturgefchichte, I. p. 88. Seidenha/e.

Meyer, Anweifung zur angorifchen Kaninchenzucht. Dresd. 1789.

Erxleben, Mammal. p. 334. a. C. angorenfis. Varietas angorenfis pilis longioribus, undulatis, fericeis.

Granmani, intr. in hiftor. nat. mamm. p. 55. a. das angorifche Kaninchei.

Gatterer, brev. Zool. I. p.96. a. C. angorenfis; das angorifche Kaninchen.

8. Russicus. Das ruffeche Kaninchen. ( $(9)$

Zimmermann, geogr. Zool. I. p. 219. das rulfische Kaninchen.

Pallas, glir. p. 17. Cuniculus rufficus.

Erxleben, Mammal. p. 335 \% \% C. rufficus.

Graumann, intr. in hifor. nat. mamm. p. 55.c. C. ruflus.

Gatterer, brev. Zoolog. I. p. 96. c. C. ruflus.

3. C A pensis. Der Cap-HaJe.

Miuller, Naturfyft. I, p. 324. n. 3-dir -capfchs Hafe.

Zimmermann, geogr. Zoolog. II. p.'338. n. 236. $\operatorname{der~Cap~-~Ha\int e.~}$

Borovefky, Thierreich, I. 3. p II. der kapifche Hafe.

Oñomat. hif. nat. IV. p. 79r. der Haaje vom Vor. gebiirge der guten Hoffunng.

Büffon,

(9) Soll ẹin doppeltes Fell auf dem Riicken haben, worin es feinen Kopf zurück ziehen kann, und noch einen, diefem ähnlichen Sack unter dem Halfe, um die Fülse darin zu verbergen, In dem lafen Felle find kleine Oeffnungen; das Licht durchzn, laften; ubrigens ift der Leib grau, und der Kopf bratu. Pallas fagt: en kenne das Thier gar nicht. 
Bïffon, vierf. III. p. 215. der kapifche Hafe.

Sparrmann, Reife n. dem Vorgeb. d. g. Hoffn. p. 256. capfcher Hafe?

Kolbe, Vorgeb. d. g. H. p. 346. die dritte Art?

IMerrem, zoolog. Ábhandl. p. 20. n. 6. capifcher $\mathrm{Ha}$ e.

Erxleben, Mammal. p. 335. n. 4. Lepus (Capen. fis) cauda longitudine capitis, pedibus rubris. pallas, glir. p. 30. Lepus (Capenfis) auribus cauda rufa.

Linné, Syft. Natur. Edit. X. I. p. 58. n. 3. Lepus (Capenfis) cauda longitudine capitis, pedibus rubris.

Graumann, intr. in hiftor. nat. mamm. p. 55. IV. der cap fche Hase.

Gatterer, brev. Zoolog. I. p. 96. n. 4. Lepus (Ca. penfis) cauda longitudine capitis, pedibus ru॰ bris; der capifche Haje.

$$
\text { * * Ungeschwänzte. }
$$

4. Brasiliensis. Per Tapeti.

Miiller, Naturfyftem, I. p.325. n. 4. das unge. Schwönste Kanincheñ.

Zimmermann, geogr. Zoolog. II. p. 334. n. 230. der Tapeti.

Borow fky, Thierreich, I. 3. p. II. n. 4. der Tapeti. Neuer Schauplatz d, Natur, VIII. p. 788. Tapeti. Onomat. hif. nat. IV. p. 79r. der brafilianifche Haase.

Gesner, Thierbuch, p. 177. Cavia Cobaya; die zweyte Art ans Brafilien; m. Marcgr. Fig.

Biiffon, vierfüfs. III. p. 216. das ungefchwönzte brafilifche Kaninchen. Dev Tapeti der Braflianer.

Iriinuitz: 
Krïnitz, ökon. Encykl. XXXIV.p. 158. das ungefchwönzate brafolifche Kaninchen.

Gatterer, vom Nutzen u. Schaden der Thiere, I. p. 212.n. 107. der Tapeti.

Merrem, zool. Abhandl. p. 21. n. 7. Tapeti. Erxleben, Mammalia, p. 336. n. 6. Lepus (Bra-' filienfis) cauda nulla, auriculis elongatis, corpore ex rufo fufco.

Linné, Sy凡t. Nat. Ed. II. p. 46. Lepus cauda nulla. Linné, Syft. Natur. Edit. VI. p. 9. n. I. Lepus cauda nulla.

Linné, Syft. Nat. Edit. X. I. p. 58. n. 4. Lepus (Brafilienfis) cauda nulla.

Granmann, intr. in hift. nat. mamm. p. 55. VI. das ungefchwönzte Kaninchen.

Gatterer, brev. Zoolog. I. p. 97. n. 6. Lepus (Brafilienfis) cauda nulla, auriculis elongatis, corpore ex rufo fufco; das ungefchuoüinate Kaninchen.

Briffon, quadr. p. (mihi) 97. Lepus (Brafilianus) ecaudatus.

Ionfton, quadrup. Tab. 63. Cavia Cobaya, Cuniculi Brafilienfis fpecies. Fig. Marcgr.

Fernandez, hift. anim. Now. Hifp. p. 2. Citti, feu lepus?

Waffer, voyage, p. 224. Lapin? (von langen Ohren if hier nicht die Rede.)

Bankroft, Naturgefch. von Guiana, p. 84. indianifches Kanin, Puccarara?

10. Pusiluus. Der Ziererghafe. (10)

Miiller, Naturf.Suppl. p.37.n.5. der Zwerghaaf̧.
Zinntier-

(10) Ein artiges Thier, das einzeln in bufchigten und krăuterreio chen Gründen wohnt, fich ziernlich tiefe Löcher mit einem oder 
Zimmermann, geogr. Zoolog. II. p. 332. n. 227. der Zwerghaje.

Merrem, zool. Abh. p. 21.'n.3. kleiner Graber. Borowsky, Thierreich, I. 3. p. 12. n. 7. der Zwerghase.

Lefke, Naturgefch. p. 174. n. 3. der kleinfte Hafe. Pallas, Reife, Ausz. I. p. Io6. eine Art Zwerghafen.. III. p. I79. der allerkleinfte Erdhaafe.

Beckmann, phyf. ökon. Bibl. I. p. 630. Lepus pufillus.

Graumann, intr. in hif. nat. mamm. p. 56. VIII. Ziverghafe.

Gatterer, brev. Zool. I. p. 97. n. 8. Lepus (Pufillus) cauda nulla, auriculis brevibus rotundatis; der Zwerghase.

- oder mehrern Zugàngen gräbt, und den ganzen Tag darinn verfleckt hält. Seine, dem Wachtelton faft ahnliche Stimme kann man einige Werfte weit hören. Die lungen werden blind und kahl gebohren, find aber nach ihrer Art fchon ziemlich grofs, und nehmen gefchwind zu. Im Winter macht das Thier kleine Laufgraben unter dem Schnee auf dem Rafen, um feine Nahrung zu fuchen, die alsdens in dem Auswurf pflanzenfreffender Thiere, als der Pferde und Schafe befteht. Fben daher entfehen rach Pailas Vermuthung die Afcariden, die er bey diefem Thier faft fo grofs als bey Pferden gefunden hat. Im Sommer aber if das Laub vom Strauchklee und faftige Pfianzen fein liehftes Futter. Gleichwohl if fein Mift fehr trocken, und fiehet faft wie Sctirot-oder Pfefferkörner aus. Man hat auch daran das befte Zeichen, um die Hühle des Thiers zu finden, weil es denfelben an gewiffen Stellen, nicht weit von feiner Wohnung beyfamtnen abzulegen pflegt. Merkwürdig ift die Geftalt des Grimmdarms, und die Anzahl der Rippen, deren fiebzehn Paar fird, in deren Menge alfo der $Z$ werghafe alle bekannte Thiere ïhertrift, ausgenommen den Hyrax Capen/s, welcher zwey und awanzig Paar hat. Die Haare werden durch das geringtte Reiben elekrrifch. 
I. A l p x nus. Der Berg-oder Alpenihase.

Miiller, Naturf. Suppl. p.37. n. 6. der Berghaafe.

Zimmermann, geogr. Zoolog. II. p. 333. n. 228. der Berg-oder Alpenhase.

Borowfky, Thierreich, I. 3. p. I2. n. 6. dev Berghane.

Gatterer, vom Nutzen und Schaden der Thiere,

I. p. 213. n. mo. der fibirifche Berghase.

Merrem, zool. Abhandl. p. 21. 11. 2. Berggraber.

Pennant, arct. Zool. II. p. 98. n. 47. der ungeSchwönzte Hase, der Alpenhase, Berghase.

Pallas, am angef. O. Eine Art von Steinhasen. Pallas, Reife, Ausz. II. p. r. n. 2. Lepus alpinus. III. p. 179.285. kleine Klippenhasen, Kilbe.

Granmann, intr. in hift. nat. mamm. p. 56. VII. der Berghase.

Gatterer, brev. Zool. I. p. 97. 1. 8. Lepus (Al. pinus) cauda nulla, auriculis elongatis, corpore lutefcente; der Berghase.

2. Ogotona. Der Ogotona.

Zimmermann, geogr. Zoolog. II. p. 334. n. 229. der Ogotona.

Pallas, Reife, Ausz. III. p. I79. Ozotona.

Merrem, zool. Abhandl. p. 2I. n. I. Felfengraber.

A) Verainderungen gegen die XIIte Edition, und Vermehrung der Gattungen diefes Gefchlechts.

Die zu diefem Gefchlecht gehörigen Thiere find in zwey Unterabtheilungen, gefchroinzte und ungefchwänzate, gebracht. Von der evften Gattung ift die Varietät $\beta$. p. 161. 12. 6. als eine befon- 
befondere Gattung angenommen und davon drey, von der zweyten Gattung aber vier Spielarten nebit den Unterfpielarten, aus einander gefetzt. Uebrigens ift das Gefchlecht mit acht Gattungen ver. mehrt, und. L. Vifcaccia, Variabilis, America. nus, Tolai, Minimus, Pufillus, Alpinus, und Ogotona, hinzugekommen.

13) Unbeftimmtere Thiere.

x. Die Kaninchen von Neuhollaind.

Zimmermann, geogr. Zoolog. II. p. 338. a." Dampier, voyage, T, III. 'p. r38. ed. de Rouen.

2. Die Hafen und Kaninchen von Californien. Zimmermann, geogr. Zool. II. p. 338., b. Begert, Nachrichten von Californien, p. 62.

* (EINGESCHOBENES GESCHLECHT.) Hy в a x. Das Fettthier. Schrëber, Säugthiere, IV.

I. C A P e nsis. Der Klipdas. (1)

Miiller, Naturfyftem, Supplem. p. 48. n. I. die caapsche Savia. Tab. 3.fig. I.

Zimmer.

(1) Der äufsern Geftalt nach einem kleinen Bären nicht unähnlich, der nicht grölser als cin Kaninchen wäre. Ein ausgewachfenes Weibchen, welches der Graf von Borke befafs, wog zwey und ein halbes Pfund berliner Gewicht. Das Thier ift von Natur fehr wehrlos, aber doch beiffig und hefrig, wenn es gereitzt wird. Es fchlyft feft, und fteckt ith Schlafe die $\mathrm{Nafe}$ zwifchen die.Hinterkculen. Auffer allerley grünen Gräferı, Brodt, Obft, Karroffeln a: dgl. dio der Klipdas gern "frifst, nimmt 
Zimmermann, geogr. Zoolog. II. p. 329. n. 226 . der Klipdas; die cap fche Cavie.

Lefke, Naturgefchichte, p. 163. n. 2. die cap $\int$ che Szavie. Cavia Capenfis.

Borowfky, Thierreich, I. 3. p. 22. Cavia Capenfis; die capifche Kavia.

Frifch, Naturfyftem, p. 9. n. 8. Cavia capenfis; (PallaJens) Halbkaninichen.

Biiffon, vierfüfs. XIV. p. 59. das capfche Muvmelthier.

Gatterer, vom Nutzen und Schaden der Thiere,

I. p. 2I8. das cap che Halbkaninchen.

Batfch, Thiere, I. p. 233. der Klippdachs.

Merrem, zool. Abhandl. p. 24. n. 3. capifches Ferkel.

Pallas, Naturgefch. merkw. Thiere, II. p. I9. das Halbkaninchen voin Cap. Tab. 2. 3.

Eberhard, Thiergefchichte, p. 263. das Halbkaningen von Kap. Tab. I. fig. I.

Krïnitz,

nimmt es auch gern das. Moos, welches auf der Rinde der Eichen fitzt, zu Eeiner Nahrung. Er fauft fehr ivenig.' Seines Unraths entledigt er fich immer an demfelben Orte, und verfcharrt ihn, wie die Katzen. Un fich des. Ungeziefers zu entledigen, wälzt er fich oft in Sande herum. Wenn er lofen will, mufs er eine aufgerichtete Srellung annehmen, die Hinrerfuifse gegen eine Wand, oder fonft etwas ftammen, und die Vorderfüfșe auf eine bequeme Erhabenheit ftellen. Dabey fteckt er die Zunge aus, und leckt fich immer das Maul. Die Ausleerung feines Körpers fcheint alfo mit einiger Befchwerde für ihn beģleitet zu feyn. - Das Thier unterfcheidet lich von den Cavien 1) durch die vier untern Zähne, deren die Cavien nur zwey haben; 2) durch die fonderbare Bildung der Zehen uberhuapt, und befonders des Nagels am dritten Zeben des Hinterfufses; 3) durch feine Heimath, welclie in der alteŕ Welt ift; auch zum Theil 4) durch feine Lebensart. Daher es aus diefem Gefchlechte weggelaften, und ein eigenes daraus gemacht worden ift. 


\section{Mammalia Glires: Hyrax. $\quad 56 \mathrm{t}$}

Kriinitz, ökon. Encykl.XXXX. p. 604. Klipdas. Kolbe, Vorgeb. d. g. H. p. 145. Dachs.

Sparrmann, Reife nach d. Vorgeb. d. g. Hoffn.

p. 279. Dachs, Das.

Pallas, glir. p. 278. Cavia capenfis.

Graumann, intr. in hift. nat. mamm. p. 57. III.

Cavia Capenfis.

Gatterer, brev. zoolog. I. p. 100. n. 3. Cavia (Capenfis) ecaudata, dentibus primoribus infra quatuor.

For $k$ all, fn. orient. p. V. n. 34. Uabr? feli fimilis, fine cauda, herbiphagus, monticola. Caro incólis edulis.

Spriiche Salom. XXX. v. 26. Caninichen?

Hermann, tab. affin. anim. p. 83. Cavia capenfis.

2. SYRIAC U s. Das fyrifche Fettthier.

Schreber, Säugthiere, IV. p.....

Das Gefchlecht fowohl, als beyde Gattungen find neu. 


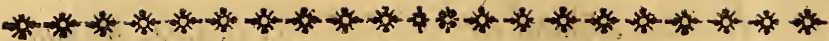
FÜNFTE ORDNUNG.

PECORA (Wiederkäuende Thiere.)

Thiere mit gefpaltenen Klauen.

27. GESCHLECHT. CAMELU S. Das Kameel.

Miiller, Naturfyfern, I. p. 365. Gen. XXVII. Zimmerniann, geogr. Zool. II. p. I39. Gen. IX. Lefke, Naturgefch. p. 175. Gen. XXXII. Borovelky, Thierreich, I. 3. p. 52. Gen. XXVIII. Blumienbach, Handbuch d. Naturgelchichte, p. 103.

Gen. XXIX.

Onomat. hiftor. nat. II. p. 464 .

Frifch, Naturfyn. p. 4 .

Batfch, Thiere, I. p. r21.

Erxleben, Mammalia, p. 218. Gen. XXV.

Graumanin, intr. in hifor. nat. mamm. p. 43. Gen. XXV.

Gatterer, brev. Zool. I. p. 6I. Gen. XXV.

Klein, Quadrup. difpof, p. 4r.

Sevérin, zoolog. Hungar. p. 56.

1. DROMEdarius. Der Dromedar.(')

Miiller, Naturfyft. I. p. 365. n. I. das Kamcel.

Zimmermann, geogr. Zoolog. I. p. 26. der Dro. medar. p. 139. n. 56. das einbuckeliclite Ka. meel; der Dromeciar.

Schreber,

(1) Das wichtigfte Hausthier für den ganzen Orient, das in einem fanften. Trabe zwölf Meilen in einem Tage zuruicklegen kann. Es kann 6 bis 8 Tage chne Geträak leben, fäuft aber dagegen 
Scíreber, Säugthiere, IV. Tab. 303. Camelus Dromedarius.

Lefke, Naturgefchichte, p. I76. n. 1. das gemeine Kameel.

Borowfky, Thierreich, I. 3. p. 56. n. 3. das ara. bifche Kameel; der Dromedar.

Bhumenbach, Handb: der Naturgefch. p. 103. 11. I. Camelus (Dromedarius) topho dorfi unico; das gemeine Kameel.

Bechftein, Naturgefch. Deutfchl. I. p. 142. das ge. meine Kameel.

Eunke, Naturgefch. I. p. I29. der Dromedar.

$\mathrm{Nn}_{2}$

Ebert.

ungeheuer viel auf einmal, da fich diefs Waffer lange Zeit in feinem Magen unverảndert erhälr." Dafs et nicht triuken follte, ohne das Waffer mit den Füfsen zu triben, ift Erdichtung. Bey der Begattung legt das Weibchen fich platt auf den Bauch nicder, und das Männchen bringt, wie $H \ddot{b} / t$ fagt, öfters eine ganze Nacht vergeblich darüber zu, wenn es der Kameeltreiber nicht befürdert. - Zur Brunftzeit wird es fo wütend, dafs es felbft feinen Herrn und Führer verkennt. Der weifse Schaun bedeckt den halben Kopf, die Zunge hängt lang aus dem Maul, und man hört befandig ein abfcheuliches Gefchrey. Der Höker wird nicht durch Krïmmungen des Rückgrads hetvorgebracht, fondern ift ein Gewächs einer drïfigten und fleifchigten Subftanz. Nach Olenrius begattet fich das einbucklichte Kameel mit dem zweybucklichten. Der Künig von Fes und Mar rokos bat einige büfe Kameele, die abgerichtec find, Menfchen umzubringen, und die einen folchen Unglïcklichen mit den Zähnen faffen, ihn hoch in die Luft werfen, wnid hernach mit den Knieen zexquetfchen. Aus dem Buckel von jungen $\mathrm{Ka}$ méelen witd eine Delikiteffe gemacht. Beyde, fowohl diefe, als die folgende Gattung; haben eine grofse Schwiele vorn an der Bruft, vier kleine an den Vorderfitisen, und zwey dergleichen an den Hinterfüfsen, die ihnen zum Auffemmen dienen, wenn fie müde find, und fich niederlegen, und die fchon bey den ungebornen Kameelen zu fehen find, mithin nicht erft is der Folge durch das Niederknieen entitehen. 
Ebert, Naturlehre, I. p. 322. das arabifche Kameel, der Dromedar.

Halle, vierfüfs. p. 386. der Dromedar- Tab. I4. Halle, vierf. p. 388. das kleine Pofkambel, mit kleinern Haarfattel.

Gatterer, vom Nutzen und Schaden der Thiere,

I. p. 99.n. 67. das Kameel; Dromedar.

Beckmann, Naturhifforie, p. I8. no.I. das Kameel. (Mit Inbegriff des folgenden.)

Neuer Schauplatz der Natur, IV. p. 354. Kameel. p. 355. das arabifche Kameel, Dromedar, Lanfkameel.

Onomat. hifor. nat. II. p. 464. Komeel mit einem Hecker auf dem Buckel. p. 473. der Droe medar.

Handbuch d. Naturgefch. I. 2. p. 246. Camelus: Dromas; der Dromedar.

Frifch, Naturfyft. p. 4. n. I. Camelus vetus; das rechte Kameel.

Klein, quadr. difp. p. 42. Camelus Imel, Arabicus. Klein, quadrup. difp. p. 42. Camelus minimus;

Dromedarius.

Klein, Claffif. d. vierf. Th. p. I24. Kameel, Imel. Klein, Claflif. der vierf. Thiere, p. 124. kleinfter Kameel, Dromedarins.

Klein, natürl. Ordn. d. vierf. Th. p. 45. n. 2. Kameel. n. 3. Kameel, Dromedar.

Biiffon, vierfüls. IX. p. 46. der Dromedar; mit einer Figur.

Batfch, Thiere, I. p. 122. der Dromedar.

Dapper, Afien, p. 349. das arabifche Kameel. p. 376. Kamehle.

Chandler, Reife n. Klein-Afien, p. 32. Kameele. Reife nach der Infel Frankr. u. Bourbon, p. 294. das Kameel. 
Shaw, Reife, p. I48. das Kameel. p. I49. Dro: mas, Maihari, Afhaari.

Georgi, Rufsl. I. p. I773. 206 . II. p. 407. 423.

447. (ein - und zweybucklichte) Kameele.

Adanfon, Reife nach Senegall, p. 53. Kameele.

Niebuhr, Befchr. v. Arabien, p. I6r. Dronedarien: Hijt, Marokos und Fes, p. 287. Kameele, Sghimél. (Nago, die Kaneelin.)

Pokoke, Befchreib. des Morgenlandes, I. p. 230: Kanueel.

Haffelquift, Reife nach Paläftina, p. 281. das. Konueel.

Olearii mufcow. u. perf. Reife, p. 570 . Camehle. I. G. Gmelin, Reife d. Sibirien, II. p. 127, Ka: meele. p. 552 . Kamcel.

Rytfchk. orenb. 'Topogr. in Bïfchings Magazin; VII. p. 40.

Ausz. aus dem 'Tageb. eines Reif. n. Afien, p. 134: der'Dromedar.

Agatharchides, de mare rubro, p. 58. in Hudfon: geogr. veter. fcriptor. graec. Oxon. 1698.

Perrault, Charras und Dodart, Abhandl. zur Naturgefch. I. p. 83. anatomifche Befchreibung zweyer Kameele. Tab. 7. der Dromedar. Tab. 9. die Zergliederung. (Die Abbildung if fehr fchlecht.)

Klïgel, Encyklopädie, I. p. 247. der Dromedar. Smellie, Philof. der Naturgefch. I. p. 80. der Dromedar.

Krinitz, ökono!n. Encyklop. IX. p. 640, Dromedar, Dromthier.

Kriintitz, ökonom. Encyklop. XXXIII. p. 2. das Kamel, oder Kamelthier. p. 3. der Dromedar, das Dromedarkamel, das Dromthier, Windkamel. p. 4. das arabifche Kame!. 
Iablonfky, allgemein. Lex. p. 194. Cameel. (Mit dem folgenden verbunden.)

Merklein, Thierreich, p. 95. Kamel.

Oekonom. Zool. p. 38. n. 45. der Dromedar.

Mannichfaltigkeiten, II. p. 586. III. p.342. Dramedar.

Neuefte Mannichfaltigkeiten, III. p. 59I. Dro. medar.

Erxleben, Mammal. p. 218. n. I. Camelus (Dro. medarius) topho dorfi unico.

Limne', Syft, Nat. Edit. II. p. 49. Camelus topho dorfi urico.

Linné, Syft. Nat. Edit. VI. p. I3. n. r. Camelus topho dorfi unico.

Limne', Syf, Nat. Edit. X. I. p. 65. n, I. Came. lus (Dromedarius) topho dorfi unico.

Graumzann, intr. in hiftor. nat. mamm. p. 43. I. Dromedar.

Gatterer, brev. Zoolog. I, p. 6I. n, I.. Camelus (Dromedarius) topho dorfi unico.

Kramer, Auftr. p. 318. Camelus topho dorfi unico.

Schwenkfeld, theriotroph. Silef. p. 73. Camelus Dromedarius.

Ludolf, hit. Aethiop. Lib. I. c. 10. \$. 13. Cameli. Aldrovand. quadr. bifulc. p. 889. Camelus.

Aldrovand. quadrup. bifulc. p. 908. Camelus Bactrianus; m.e. Schl. Fig.

Boullaye - le - Gouz, voyages, p. 225. les Cha. meaux.

Marmol Afrique, I. p. 48. le Chameau, Tavernier, voyages, I. p. J06. le Chameau. Chardin, voy. en Perfe, III. p. 37. Le Chameau, Brown; Iamaic. p. 488. Camelus topho dorfi unico, vertice glandulofo.

Cardan, 
Cardan. de rer, variet. L. 7. c. 33. L. Io. p. 30I. Cameli.

Ariftot. hift, animal. II. c. 4 , n. 17 . c. 6. n. 26. c. \$. n. 42 . c. 9. n. 50 . c. II. n. $62 . \quad$ V. c. 2. n, 23. є. 13. n. 132. VI, c. 26. n. 358 . VIII. c. II. n. I15-II7. IX. c: 73. n. 496. $497 . \mathrm{Ke}$ -

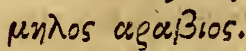

Plin. hif. nat. VIII. c, 18. XI. c. 4r. Camelus Arabiae.

2. BACTRIANUS, Das Trampelthier. ( ${ }^{2}$ )

Mïller, Naturfyn. I. p. 369 . n. 2. der Dromedar. Zimmermann, geogr. Zoolog. II. p. 26. das Kameel. p. 140. n. 57. das zweybuckelichto Kameel.

Schreber, Säugthiere, IV. Tab. 304. Camelus Bactrianus.

Lefke, Naturgefchichte, p. I76. n. 2. das Tianpelthier.

Boroweky, Thierreich, I. 3. p. 59. n. 4. das baktrianifche Kameel.

$\mathrm{Nn} 4$

Bhumen.

(2) Findet fich mehe im mittlern Afien bis gen China, zumal in ganzen Heerden in Beffarabịn, und wį̣d nicht fo häufig wie die vorige Gattung zum Laftragen, fondern feines fçhnellen Trabes und nätürlichen Sattels wegen, meht zum reiten, und bey den Tataren vorzüglich zum Zug gebrnucht. - In Aushalten oder Dauer in Arbeit übertrift das Kameel alle Quadrupeden. Beladen geht es gewöhnlich nicht über drey englifche Meilen in einer Stunde. So geht es aber 16 Stunden von 24 fort, und hält diefe Arbeit viele Wochen hindurct aus. Von dem Trampelthier fagt Höft, dafs' es in einem Tage vie:zig dänifche Meilen laufe, dafs es aber gelibte Reuter erfordere, die doch am Ende am Blutfurz fterben, ob fie gleich unter Wegs Nafe und Ohren mit Baúmwolle zugeftopft haben: Von vielen Schrifttellern und Rèifenden wird diefe Gattung Dromednv genannt, 
Blumenbach, Handb. der Naturgefch. p. 104. n. 2 .

Camelus (Bactrianus) tophis dorfi duobus.

Funke, Naturgefch. I. p. 129. Trampelthier.

Ebert, Naturlehre, I.ip. 322. das baltrianifche oder tiirkifche Kameel.

Halle, vierf. p. 384. das baktrianifche Kameel.

Gatterer, vom Nutzen und Schaden der Thiere,

I. p. I03. n. 68. das Trampelthier.

Neuer Schauplatz der Natur, IV, p, 355. das baEtrianifche oder tiirkifche Kameel.

Onomat. hift. nat. II, p. 470. ein eigentlich $K \mathrm{Ka}_{\text {- }}$ meel, oder Bactrian.

Handb. d. Naturgefch. I. 2. p. 246. das Kameel; Cameluss.

Frifch, Naturfyftem, p. 4. n. 2. Camelus Bao ctrianus.

KKiein, quadrup. difp. p. 4r. Camelus, Befchet; Backrianus fortiffimus.

Klein, Claflif. der vierf. Thiere, p. r23. Kameel.

Klein, natürl. Ordnung d. vierf. Th. p. 45 . n. r. Kamęe?

Geswer", Thierbuch, p. 23r. Kameelthier. Figur p. 232. mittelm.

Buiffon, vierfüfs. IX. p. 46. das Kameel.

Bat $f$ ch, Thiere, I. p. 123. das battrianifcho Kameel.

Niebuhr, Befchreib.' von Arabien, p. I6r. Ka. meele. p. I64. Kameele.

HHöf, Marokos und Fes, p. 289. Dromedaren; Meheri, Hafghün.

Ausz. a. d, Tageb. eines Reif, ñ. Afien, p. I3I. das eigentliche Kameel.

Pallas, Reife, I. p. 397. Kamele.

Pallas, Reife, Ausz. I, p. 339. Kamele. p. 257. Kamele. 
Pallas, Naturgefch. merkw. Thiere, XI. p. 5 . zweybucklichte Kameele.

Du Halde, Befchr, von China, II. p. 183. Cameele. Di Halde, Befchr. von China. III. p. 5r3. wilde und zahme Cameele.

Leibnitz, novifima Sinica, 1699. p. 166.

Olear. mufcow. und perf. Reife, p. 570. Dro: medarii.

Perrault, Charras' und Dodart, Abhandl. zur Naturgefch. I. Tab. 8. ein Kameel.

Falk, ruff. Topogr. II. p. 509. Kameele.

Reife nach der Inf. Frankr. u, Bourbon, p. 294: der Dromedar.

Smellie, Philof. der Naturgefchichte, I. p. 80. das Kameel.

Kriinitz, ökonom. Encyklopäd. XXXIII. p. 4. das bactirianifche oder tïrkifche Kamel.

Oekon. Zoologie, p. 38: n.46. das Kameel. Mannichfaltigkeiten, II. p. 584. III. p. 342 . Kancel.

Neuefte Mannichfaltigkeit. II. p. 15I. III. p. 590. Kameel.

Erxleben, Mammal, p. 221. n. 2. Camelus (Bactrianus) tophis dorfi duobus.

Linné, Syn. Natur. Edit. II. p. 49. Camelus to. phis dorfi duobus.

Linné, Syft. Nat. Edit, VI. p. I3. n, 2. Camea lus tophis dorfi duobus.

Linné, Syft. Nat. Edit. X. I. p. 65. n. 2. Camelus (Bactrianus) tophi dorfi duobus.

Graumann, intr. in hiftor. nat. mamm, p. 43. II. Kameel.

Gatterer, brev, Zoolog. I. p. 62. n. 2. Camelus (Bactrianus) tophis dorfi duobus; das Transo pelthier. Kamieel.

$\mathrm{Nn}$ ร.

Kramer, 
Ki:mier, Auft. p. 318. Camelus tophis dorfi duobus.

Charleton, exercitat, pag. I3. Camelus baçtrianus.

Leon. African. defeript. Africae, p. 745. Camelus.

Ariftot. hifor, anim. II. c. 3. n. 17. c. 6. n..26. Kosprinos Baxreicives.

plin. hift. nat. VIII. c. I8. Camelus Bactriae.

Aelian. de nat. anim. III. c. 47. IV. c. 55. VI.

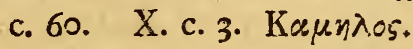

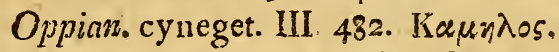

Iefaia, c. 60. vo 6. die Liüufer aus Midian und Epha?

In folgenden Stellen der heil. Schrift wird der Kameele ferner gedacht:

I R. Mof. c. 12. v. I6,

I 13. Mof. c. 24 . v. 35 .

I B. Mol. c. 30 v. 43 .

I B. Mof. c. 32. v. 15 .

2 B. Mof. c. 9. v. 3 .

I B. $\mathrm{Mof}_{9}$ c. 24. v. Ir.

I B. Mof. c. 3I. v. I7.

I B. Mof. c. $37 \cdot$ v. 25 .

3 B. Mof. c. II. v. 4 ;

5 B. Mof. c. I4. v. 7 .

I Sam. c. 15. v. 3 .

I Sam. c. 27. v. 7 .

v. 17.

I Chron. c. 5. v. 2f. c. I2. v. 40.
I Chron. c. 27. v. 30.

2 Chron. c. 14.v. 15. c. 9. v. $x$.

Iob. c. I. v. 3.

Iob. c. 42. v. 12.

Ierem. c. 49. v. 28.29.

I B. d. Kön, c. Io. v. I.

2 R. d. Kön. c. 8. v. 9.

Nehem. c.7. v. 69.

Ief. c. $30 . v .6$.

Ief. c. $2 I . v .7$.

c. 30. B. d. Richter, c. 6. v. 5 . c. 7 . v. 12. c. 8. v. 2 I. 26.

Zach. c. I4. v. I5. 


\section{G ц A м. Die Kameelziege. (3)}

Miiller, Naturfyft. I. p.37I. n. 2. das perivianifcke Schafkameel.

Zimmermann, geogr. Zoolog. II. p. 73. das Guamico.. p. 140. n. 58. das Guanico.

Schreber, Sïugth. IV. Tab. 306. Camelus Glama. Borowsky, Thierreich, I. 3. p. 58. n. I. der Lama, das pernanifche Schafkameel, die $K \boldsymbol{K}_{\text {. }}$ meelziege. Tab. 28.

Bhumenbach, Handb. der Naturgefch. p. Io5. n. 2. Camelus (Llacma) dorfo laevi, to pho pectorali; die Kameelziege, Guanaco. Funke, Naturgefch. I. p. זзо. die Kameelziege, Lama.

Ebert,

(3) Bey den Synonymen diefer und der folgenden Gattungen herrfcht grofse Verwechfelung, weil mehrere Naturhiftoriker manche derfelben mit andern für einerley halten. Ich habe die im Syftem nicht:mit befindlichen Stellen fo gut zu ordnen gefucht, als mirsinter diefen Umftänden, und bey don oft man. gelhaften Befchreibungen müglich gewefen ift. In der That gränzen diefe Gattungen fehr nahe an einander. Die Llacma hat in der Bildung viel von der Ziege. Sie war, nebft dem Pacos das einzige Gefchöpf, das die Amerikaner fchon vor Ankunft der Spanier als Hausthier hielten, und noch jetzt lieben fie es aufs zärtichfte, putzen es, und richten es heerden, weife, aber mit grofsen Feyerlichkeiten, zum laftragen u. f. w. ab. Das Thier wird unter andern auch vorziglich und in ganzen Caravanen zum Tranfport der Silberbarren aus den Berg werken von Fotofi gebraucht. Sein Schritt gleicht dem von einem Kameel, und feine Höhe dor von einem ein - bis zweyjäh. rigen Efel. Fis macht aber nur kurze Tagereifen von wenigen Meilen, und wenn es gewaltfam fortgetrieben, oder iberladen wird, legt fichs auf der Stelle, nieder, und ift durch kein Mittel wieder zum Aufitehen zu bringen', fondern mufs gefchlachtet werden, wehrt fich-auch durch einen ätzenden Unrath, dẹn es auf die fich ihm nâhernden Perfonen ausfpeyet, und dẹ cine Art von krätze auf der Haut verurfachen foll. 
Ebert, Naturlehre, I. p. 325. Lama, Gamne Glama.

Halle, vierf. p. 309. die Kameelziege.

Gatterer, vom Nutzen u. Schaden der Thiere,

I. p. 104. n. 69, das peruanifche Schafkameel.

Neuer Schauplatz d, Natur, V. p. 14. Lama, das peruanifche Schaf, Erdfchaf, Schafkameel. p. 15. Glama, Lhama.

Onomat. hiftor. nat. II. p. 477. das pernanif che Kamel; der pernvianische Hamnel, peruvia. nifches Schaf, Glama oder Lhama.

Handb. der Naturgefch. I. p. 298. der Lamia.

Frifch, Naturfyftem, p. 4. n. I. das Lama oder Glama.

Klein, Quadr. difp.p. 42. Camelus fpurius Peruanus, Glama dictus.

Klein, Clafif. der vierf. Thiere, p. 124. Afterkameel, Hivschkameel.

Klein, natürl. Ordn. d. vierfüls. Th. p. 46. n. 4. annigentliches Kaneel, Glana, Ljanna.

Bïffon, vierfüfs. I. p. 314. XIII. p. 179. 219. Lama.

Batfch, Thiere, I. p. 124. der Lama, Glama, Clacinu.

Molina, Naturgefchichte von Chili, p. 275. 28I. Gliama.

Vidaure, Chile, p. 87. der peruanifche Llama. Schlözer, Erdbefchr, von Amerika, p. 893. der. Lama.

Byron, Reife um die Welt. p. I89. Guanacos? Dapper, Amerika, p. 32. Glama.

Dobrizhoffer, Gefch. der Abiponer, I. p. 340. die peruanifchen Schaafe, Llamas.

Charlevoix, Gefchichte von Paraguay, p. 228. Llanzaez. 
Murr, Journal zur Kunfgefch. u. f. w. II. p. I74• Liama.

Kriinitz, ökonom. Encyklop. XXXIII. p. 26. das pernanifche Kaneel.

Khïgel, Encykl. I. p. 248. die Kameelaiege.

Beckmann, phyf. ökon. Bibl. II. p. 489. Glama.

Felice, Encycl. ou Diction. univerf. Yvercion.

1770. ff. II. p. 232. Glama, Vigogne.

Evxlebeñ, Mammal. p. 224. n. 3. Camelus (Lla.

ina ) dorfo laevi, topho pectorali...

Limmé, Syft. Nat. Edit. II. p. 49. Camelus dor. fo laevi, pectore gibbofo.

Linné, Syft. Nat. Edit. VI. p. I3. n. 3. Camelus dorfo laevi, pectore gibbofo.

Linné, Syft. Nat. Edit. X. I. p. 65. n. 3. Camelus (Glama) dorfo laevi, topho pectorali.

Linné, Syft. Nat. Edit. XII. I. p. 9т. n. 3. C2. melus (Glama) corpore laevi, topho pectorali.

Graumann, intr. in hift. nat. mamm. p. 43. II. das Schafkameel.

Gatterer, brev. Zoolog. I. p. 63. n. 3. Camelus (Llama) dorfo laevi, topho pectorali; das pernani/che Schafkameel, das Schafdromedar, die Kameelziege.

Severin, zoolog. Hungar. p. 58. n. 3. C. Lama; das Sichafkameel.

5. Huan acus. Der Guanaco. (5)

Miller, Naturfyftem, Suppl. p. 50. u. 5. Camelus guanicae; der Schafdromedar.

Schreber, Säugth. IV. Tab. 305. Camelus Huanacus; Mol.

Ebert,

(4) Wird von Biiffon, Erxleben, Zimmermann, u. a. mit der Glama für einerley gehalten. Nach Vidime und Falkner findet fici bey ihm der Bezoarlein. 


\section{Minmmalia Pecura. Camelus.}

Ebert, Naturl. I. p. 325. Hitanacus und Guanaco. Bïffon, vierfüfs. XIII. p. 320. das Guanaco. Frifch, Naturfyftem, p. 4. n. I. a. das Guancaci. Kriinitz,-ökou. Encyk!. XXXIII. p. 30. das wilde peruanifche Kameel, Gucnaco, Huanaco, oder Huanacu.

Byron, Reife um die Welt, p. 189. Guanacos. (Mit dem vorhergehenden verwechfelt.)

Vidaure, Chile, p. 87. der Huanaio oder Guzanaco.

Schlözer; Erdbefchr. von Amerika, p. 894. der Guanaco.

Dobrizhoffer, Gefch. der Abiponer, I. p. 338. - der Huenaick.

Charlevoix, Gefch. von Paraguay, p. 227. das Guanaco oder Hutunaco.

Falkner, Befchr. v. Patagonien, p. I12. Guanicoes. Gorz:e, Natur, Menfchenl. und Vorf. V. p. 279. der Guanaco.

6. Araucanus. Der Hueque, Rehueque. Mo. lin. (j)

Bïffon, vierf. XIII. p. 229. der Chilihueque. Vidauve, Chile, p. 87. der Chilihueque. Goeze, Natur, Menfchenl. und Vorf. V. p. 280. der Chilihueque.

7. VICUGNa. Die Vicugna. ( $\left.{ }^{6}\right)$

Zimmermann, geogr. Zool. U. p. 74. p. I40.11. 49. die Vicunna.

Sitreber,

(5) Ift nach Erxleben mit der Glasia einerley.

(6) It nach Linné, Erxleben, Büffon, Zimmermann, Beckinann u. a. mit der folgenden Gattung einerley, und beftehet nach diefen 
Schreber, Säugthiere, IV. Tab. 307 . Camehtis Vi. cugna. Molin.

Blumenbach, Handb. d. Naturgefch. p. I05. n. 4. Camelus (Vicunna) tophis nullis, pectore lanato; das Schafcameel.

Eunke, Naturgefch. I. p. 129. Schaafkameel, Vicuinina, oder Vigogne.

Ebert, Naturi. I. p. 326. Vicunna.

Gatterer, vom Nutzen und Schaden der Thiere,

I. p. 106. n. 70. dis Vigogne.

Biiffon, vierfüls. XIII. p. 203. Vigogne. p. 227.

Vicogna.

Frifch, Naturfyfem, p. 4. n. 2. b. Vicunja.

Kriinitz, ökon. Encykl. XXXIII. p. 32. Vicunna, Vicunha.

Kliigel, Encyklop. I. p. 248. das Schanfkameel. Vidaure, Chile, p. 88. das Thier Vicunne.

Dobrizhoffer, Gefch. der Abiponer, I. p. 340. die Vicunna.

Falkner, Patagonien, p. Ir2. Vicuırias.

Murr, Journ. zur Kunftgefch. II. p. 246. die $V i$ cunzuas.

Schlozer, Erdbefchr. von Amerika, p. 894. die Vicunna.

Goeze, Natur, Menfchenl. u. Vorf. V. p. 277. 28I. die Nicugna.'

Beckinann, phyf, ökon. Bibl. II. p. 489. Vigo. gne. VIII. p. 449. äie Vicunnas.

Oekonom. Zoologie, p. 39. 11. 47. das Vigogne. Kameel.

4.Paco.

der Unterfchied zwifchen beyden, fo wie zwifchen dem Lama und Guanaco nur auf dem gezăhmten oder wilden Zulande. Das wilde Lama heifst nach Büffon Huanato, oder Guaniso, fo wie dai wilde Paco, Vicunna oder Vigogne. 


\section{Mammalia Pecora. Camelus.}

4. Pac o. Der Paco. (7)

Müller, Naturfyft. I. p. 372. n. 4. das chilefifche Schafkameel.

Lefke, Naturgefch. p. 177. n. 3. das Schafkanel.

Borowsky, Thierreich, I. 3. p. 55. n. 2. das chilifiche Schafkameel, der Pako.

Bluquenbach, Handb. d. Naturgefch p. 105. Pacos. Ebert, Naturl. I. p. 326. der Paco. (Mit Vicunna verbunden.)

Halle, vierf. p. 389. das peruanifche Schaafkameel. (Mit Lama verw echfelt.)

Neuer Schaupl. d. Natur, VI. p.27I. Paco.

Onomat hiftor. nat. II. p. 479. das perivianische Schaf, der pernwianifche Wollenträger, der peruvianifche Pacos.

Handb. der Naturgefch. I. 2. p. 298. der Paco. Kleirs, Claffif. d. vierf. Thiere, p. I25. wolltragender Kameel, pernanifches Schaaf.

Klein, natürl. Ordn. d. vierf. Thiere, p. 46. n. 5.

Pacos. (Mit Vicunna verbunden.)

Biiffon, vierf. XIII. p. 179. p. 228. der Paco.

Batfch, Thiere, I. p. 124. der Pako.

Molina, Naturgefch. von Chili, p. 274.275. 278. der Paco oder Alpaca.

Dobrizhoffer, Gelch. der Abiponer, I. p. 340. die Pacos.

Erxleben, Mammalia, p. 226. n. 4. Camelus (Pacos) tophis nullis, corpore lanato.

Linné, Syft. Nat. Edit. II. p. 49. Camelus, gib. bis nullis.

Linné, SyA. Nat. Edit. VI. p. I3. 11. 4. Camelus gibbis nullis.

Linne', Syn. Nat. Edit. X. I. p. 66. n. 4. Camslus (Pacos) tophis nullis, corpore lanato.

Limbe,

(7) Man fehe die vorhergehende Anmerkung. 
Linné, Syft. Nat. Edit. XII. I. p. 94. n. 4. Ca. melus (Pacos) tophis nullis, corpore lanato.' Graumann, intr. in hif. nat. mamm. p. 43. IV. das chiliff flche Schafkameel.

Gatterer, brev. Zoolog. I. p. 63. n. 4. Camelus (Pacos) tophis nullis, corpore lanato; das chilififche Schafkameel, Vicogne.

Severin, zool. Hung. p. 58. n. 4. C. Pacos. Marcgr. Brafil. p. 244. Ovis chilenfis? Ionfton, quadr. Tab. 23. Vervex alius peregrinus?

Veränderungen gegen die XIIte Edition, und Vorn mehrung der Gattungen diefes Gefchlechts.

Das Gefchlecht if mit drry. Gattungen vermehrt, und C. Huanacus, Araucanus, und $\mathrm{Vi}$. cugna hinzugekommen.

28. GESCHEECHT. Moschus, Das Mo: fchusthier, Bifamthier.

Miiller, Naturfynem, I. p. 374. Gen. XXVIII. Zimmermann, geograph. Zoolog. II. p. 134: Gen. VIII.

Lefke, Naturgefch. p. 177. Gen. XXXIII. Borowfky, Thierreich. I. 3. p. 60. Gen. XXIX. Blumenbach, Handb. der Naturgelchichte, p. II6. Gen. XXXV.

Onomat. hiftor, nat. V. p. 25 I. Erxleben, Mammalia, p. 319. Gen. XXXI. Graumann, intr. in hiftor. nat. mamm. p. 53. Gen. XXXI.

Gátterer, brev. Zoolog. I. p. 9ז. Gen. XXXI. Brifjon. quadr. p. 65. Gen. XuI.

O. I. Mo: 


\section{Manmalia Pecora. Mofchus.}

1. Moschiferu's. Das Biefamthier. ( $\left.{ }^{8}\right)$

Miiller, Naturfyft. I. p. 375. n. I. das tartarifche Biefamthier.

Zimmermann, geogr. Zool. II. p. 135. n. 52. das Biefamthier, der Glax.

Lefke, Naturgefchichte, p. 177. n. I. das tatarifche Bifantinies.

Borowfky, Thierreich, I. 3. p. 60. n. 2. das tar. tarifche Birfamthier.

Bhumenbach, Handb. der Naturgefch. p. II6. 11. I. Mofchus (Mofchiferus) funiculo umbilicali; das Bifamthier.

Bechftein, Naturgefch. Deutfchl. I. p. 142. das tatarifche Bifamthier.

Funke, Naturgefch. I. p. 83. das Bifamhirfchchen, oder Bifamthier.

Ebert, Naturl. I. p. 3or. Bifamreh, Bifamhirfch, Bifansiege.

Halle, vierf. p. 317. der Muskusbock.

Gatterer, vom Nutzen'und Schaden der Thiere, I. p. 200. n. 102. Bifannthier, Mofchus - oder Mus kusthier.

Pennant,

(8) Die Hauptnahrung diefer Thiere befteht in Moofen, Flechten, Sandbeeren und Alpbalfam. Sie find befchwerlich zu jagen. Man fängt fie in Fallftricken, auch werden fie durch Bügen gefchoffen, die man ilınen fo in den Weg legt, dafs fie durchs Auftreten den Drücker des Bogens losdrücken. Die Tungufen Íchiefsen fie mit Bogen und Pfeilen. Den Mofchusbeurel haben

- nur die Mäunchen; beym Weibchen if Keine Spur davon. Der Bifam riecht zur Brunftzeit am ftarkften. Der tybetifche, deffen weit ftarkerer Geruch wahrfcheinlich von dem wărmern IKlima, und von den ftarkriechenden Pflanzen, mit welchen fich diefe Thiere dort nähren, herrühren mag, ift der vorzüglichfe. Das Weibchen foll im May, hüchntens in Iunius, ein oder awey lunge werfen. 
Pennant, arct. Zool. II. p. 37. n. 1. der Glax, das Bifamtnier.

Neuer Schauplatz d. Natur, I. p.774. Bifamthier.

Onomat. hift. nat. V. p. 256. der Mujkus -Bock, das Bifanthier.

Handb. der Naturgefch. 1. 2. p. 290. Bifamthier. Frifch, Naturfyot. p. 2. 12. 9. Tragus molchifer; Murkus - Tnier.

Klein, Claffif. der vierf. Th. p. 59. Muscusbock, IMus cusziege.

Klein, natütl. Ordn. d. vierf. Th. p. 20. n. 5. Mnufcusbock.

Martini, Naturlex. VII. p. 69r. Bifanthier, Bi. funthierlein, Biefan, Bifen, Bifemthier, Mo. fchus., Iluskusthier; Tibetifche, tatarifche, fibirifthe Bifamthier.

Biiffon, vierfüfs. XIII. p. 5. das Bifamthier; mit einer Figur.

Bat $\int \mathrm{ch}$, Thiere, I. p. I3r. das eigentliche Bifambthier.

Kiiigel, Encyklop. I. p. 246. das Bifamthier.

Kriinitz, ökon. Encyklop. V. p. 494. Bifam. thier.

IIIIller, Samml. ruft. Gefch. III. p. 56 r., Kabarga. Pallas, nord. Beytr. IV. p. 277. Muskus - oder Bifanthier.

Georgi, rufr. Reife, I. p. I63. Bifamthier,

S. G. Ginetir, Reifed. Rufsland, III. p. 326.529. Bifamthier.

I. G. Gmelin, Reife durch Sibirien, II. p. 239. Bifomthiere.

Du Halde, Befchr. von China, I. p. 35. das Thier Hiang - tchang - Te; die wohlriechende Gem/e.

Du Halde, Refchreib. von China, II. p. I83. der wohlirischends Rehbock.

$\mathrm{O}_{2}$

Neuhof. 
Nezhof, Gefandfchaft nach China, p. 35r. Biefemithierlein, $X e$ genanunt; m. e. guten Fig. Pallas, Reife durch Kufsland, III. p. I2. I4I. Mo/chusthiere.

Pallas, Reife, Ausz. III. p. 12. Mofchusthiere. Strahlenberg, nord. u. öfl. Europa und Afien, p. 335. Cabardyn.

Becknann, phyf. ökon. Bibl. V. p. I27. M0. fchusthier.

Rozier, Abhandl. über die Natur und Kunft,

I. p. 63. Bifanthier; Tab. 4.

Neue Anmerk. über alle Theile der Naturlehre,

II. p. 4iz. Minfius.

Aiberti, orat. de cognitione herbarum; de Mhofhis natura etc. Norimb. 1585..

Landsberg, diff. fcholaft. de Mofcho. 8. Schenke, diff. de Mofcho. Ien. I667.8. Merklein, Thierreich, p. I6. Bifan - Thier. Oekon. Zoologie, p. 40. в. 48. das Bifamthier. Erxleben, Mammal. p. 319. Mofchus (Mofchiferus) folliculo umbilicali.

Linzé, Sy凡. Nat. Edit. VI. p. I3. n. I. Mofchus. Linné, Sy.f. Nat. Edit. X. I. p. 66. n. I. Mofchus (Mofchiferus).

Granmann, intr. in hiffor. nat. mainm. p. 53. I. das tartarifche Bifomtinier.

Gatterer, brev. Zoolog. I. p. 91. n. I. Mofchus (Mofchiferus) folliculo umbilicali; das BiSam-oder Mafchusthier.

Severin, zoolog. Hungar. p. 50. n. x. Mofchus mofchiferus; das Biefemthier.

Della Valle, voy. II. p. 387. Cerains animaux, qui pcrtent le mufc.

Tavernier, voy: II. p. 3I6. Animal qui produit le mufc. 
Daubenton, Mem, de l'Academ. des Sc. 1727. II. p. 215 .

Valentin. Muf. mufeor, III. c. II, Amphith. zootom. p. 108.

2. I N D I U S. Das indifche Mofihusthier. (9)

Schreber, Säugthiere, V. p....

Dictionnaire des animaux, Paris I759. I. p.602. Chevrotin des Indes.

3. Pygmaeus. Das guineifche Tho/chisthierchen. $\left({ }^{10}\right)$

Müller, Naturfyl. I. p. 37\%.n. 3. das kleine guineiSche Reh.

Zinmermann, geogr, Zool. II. p. I38. n. 54. das guinueifche Mo/chusțilerchezl.

Schreber, Säugthiere, Tab. 244. Molchus Pygmaeus.

Borowe ky, Thierreich, I. 3. p. 62. n. 4. das guim neifeche Reh, das Zicerghirfchchen.

Bliménbach, Handb. d. Naturgefch. p. II6. n. 2. Mofchus (Pygmaeus) fupra fufco-rufus, fubtus albus; unguiculis fuccenturiatis nullis; das kleize guineifche Rehgert.
Óo 3
Halle,

(9) Briffon und Erxleben verwechfeln diefe Gattung mit der folgenden. Letzterer verfichert auch p. 323. dafs ihm, unter allen Sàugthieren, keine mehr Mühe gekoftet, als Antilope regic (Pygmaea), Cervus Capreolus americanus, Mofchus Meminna, Pysmaeus, und Americanus.

(10) Das kleinfte Thier diefer Ordnung, dẹffen Länge noch nicht einen Fufs beträgt. F.s hat den Wuchs eines Rehes, if aber fo zart, dafs feine ganzen Beine kaum einen kleinen Finger lang find, und ohngefahr die Dicke eines Pfeifenftiels haben. Seine Füfse werden in Gold eingefafst, und als Tabacksttopfẹr gebraucht. Goeze, Natur, Menfchenleben und Vorfehung, IV. p. sru. Das Thier mufs mit der Zweagantilope nicht verwechfelt werden. 
Halle, vierf. p. 320. das fchmichtige afrikanifche Hurfshyen, ohne Hörner.

Halle, vierf. p. 321 . das ungehörnte afrikanifche Hirfchgen, wit gekräuleltem in die Höhe gefoilagenem Hunds Schuanze.

Haile, vierf. p. 321. das afrikanifiche Hir.chgen mit rothem Haar.

Gatterer, vom Nutzen und Schaden der Thiere,

I. p. 202. n. 103. das guineifche Reh, der Zwerghirfch.

Oncmat, hifor. nat. V. p. 258. das kleine guineifche Reh.

Frijch, Naturfyftem, p. 2. n. 4. das guimeifche Rehzieglein.

Klein, Claffif. der vierf. Thiere, p. 7r. das af rikanifche Böckicin, kleines guineilches Reh.

Klein, Clatlif. der vitf. Th. p. zr. afrikanifches Böcklein, oder Rehböcklein, trelches Sehr diin ift; fchiüchitger afrikanifcher Hirfchbo:k.

Kilein, Claflif, der vierfüls. Th. p. 73. guineifches Böcklein, wit langen rothen Hnaren.

Kleizs, natürl. Ordn. der vierfüfs. Th. p. 24. guineifches Bücklein; kleinftes afrikanifches Geislein,

Biiffon, vierf. XII. p. 106. das oftindifche Zwerg. hivechchen; m. e. Fig.

Batfch, Thiere, -1. p. IzI. das Zwerghirfchchen. Camper, Naturgefch. des Orang-Utang, p. IOr. Mofchus Pygmacus.

Linné, Syft, Nat. Edit. II. p. 50. Capra pedibus digito humano anguftioribus.

Linné, SyA. Natur, Edit, VI. p. 14. n. 4. Capra pedibus digito humano anguftioribus.

Graunnann, ịıtr, in hift. nat. mamm. p. 54. III. das kleine gruizgifche Reh.

Gatte, 
Gattever, brev. Zoolog. I. p. 92. n. 3. Mofchus (Pygmaeus) fupra fufco - rufus, fubtus albus, ungulis fuccenturiatis nullis; das kleine guineifche Rehgen.

Severin, zoolog. Hungar, p. 60. n. 2. M. Pyg. maeus; das kleinie guineijche $R b h$.

4. MemIN Na. Der Meminina. (')

Zimmermann, geogr. Zoolog. II. p. .137. n. 53. der Meminna.

Borowsky, Thierreich, I. 3. p, 63, n. 3. $\operatorname{der}$ MB. minna.

Ebert, Naturlehre, I. p. 300. Mheninina. (Mit dem vorhergehenden verwechfelt.)

Biiffon, Suppl. quadrup. VIII. p. 164. Tab. 15.' Meminna, ou Chevrotain de Ceylon.

Biiffon, vierfüfs. XII. p. то6. ceylonifche Zwerg. hirch; m. e. Fig.

Frifch, Naturfyft, p. 2. n. 5. das ceylonifche Meminnza.

Bat $c$ ch, Thiere, I. p. 131. dor IMeminnu. Knox, ceylan, Reifebefchr. p. 4r. Meminna,

Graninann, intr. in hiftor. nat mamm, p. 54. II. M. Meminna.

Gatterer, brev. Zoolog. I. p. 92. n. 2. Mofchus (Meminna), fopra cinereo-olivaceus, fubtus albus , lateribus albo maculatis; das indianifhe Moschusthier.

5. I A v a N I Cus. Das javanifche Zuerrghirgckchen. Biiffon, vierf. XII. p. I06. e. javanifche Zwerg. hivgch.

O० 4

Lich:-

(1) Gewicht des Thiers fechftehalb Pfund. 
Lichtembergu. Voigt, Magaz. für das Neuefte aus der Phyf. u. Naturgefch. III. 2. p. I. Zwerghirfchgen von der Infel Iava; Kantil.

6. AMERICANus. Der Wirrebocerra. $\left({ }^{2}\right)$

Zimmermann, geogr. Zoolog. II. p. I38. 11. 55 . der Wirrebocerra.

Borowejky, Thierreich, I. 3. p. 60. n. I. das filrinamijche Hivrchgern. Tab. 29.

Halle, vierfüfs. p. 32r. das furinamifche röthlichs Hirfchgen, mit weifsen. Flecken und langen Ohren.

Gatterer, vom Nutzen u. Schaden der Thiere, I. p. 203. n. ro4. das brafiliznifche Mofchus. thier.

Klein, Claflif. der vierf. Thiere, p. 27. Surinamifches Böcklein.

Klein, natiirl. Ordnung der vierfüls. Th. p. 24. furrizamisches Böcklein.

Bankroft, Naturgefch. von Guiana, p. 73. der Wirrebocerra.

Hartfink, Befchreib. von Guiana, I. p. 109. $W_{i}$ ribiciri.

Fermin, Befchr. von Surinam, II. p. 89. die zwey. te Art von Hirrchen.

Groumann, intr. in hiftor. nat. maamm. p. 54.V. das furinamifche rötthliche Hirfcligen.

Gatterer, brev. Zoolog. I. p. 92. n. 4. Mofchus (Americanus) rufo-fufcus, ore nigro, gula alba; das furinamische Hirfchgen.

Severin, zool. Hungar. p. 50. M. Americanus.

Vevän.

(2) Aus dem Fleifche diefer Thiere wird eine Delikateffe gemache. 
Vercüdernugen gegen die XIIte Ediion, und Vermehrung der Gattungen diefes Gefchlechts.

Edit. XII.

p. 92. n. 2. Molchus Grim. mia.

Edit. XIII.

p. 191. n. 19. Antilope Grimmia.

Das Gefchlecht ift mit vier Gattungen" vermehrt, und M. Indicuis, Meminna, Iavaniciis und Ambricanus hiuzugekommen. Es enthält allo drey Gattungen mehr als die XIIte Edition.

29. GESCHLECHT. CERVUS. Der Hirfch.

Miller, Naturfyfem, I. p. 379.. Gen. XXIX.

Zimmermann, geograph. Zoolog. II. p. I27.

Gen. VII.

Lefke, Naturgefch. p. I78. Gen. XXXIV.

Borow $5 \mathrm{ky}$, Thierreich, I. 3. p. 63. Gen. XXX.

Blimentach, Handibuch d. Naturgefchichte, p. II3.

Gen. XXXIV.

Bechftein, Naturgefch. Deutfchl. I. p. 558.

Pennant, arct. Zool. II. p. 20.

Onomat. hiftor. nat. II. p. 790.

Frifch, Naturfyf. p. 3.

Erxleben, Mammalia, p. 294. Gen. XXX.

Graumann, intr. in hiffor. nat. mamm. p. 5 I. Gen. XXX:

Gatterer, brev. Zool. I. p. 85. Gen. XXX.

Boddaert, elench. anim. Gen. XXIX.

Brifforz, quadr. p. 58. Gén. XII.

Hermanm, tab, affin. animal, p. 103.

005

1. PY. 


\section{6 - Mamnialia Pecora. Cervus.}

1. Pỵgargus. Der Ahu.(3)

Miiller, Naturfyftem, Supplem. p. 5I. 11. 8. der uilde Hir.ch.

Zimmermann, geogr, Zoolog. I. p. 222. Pygargus Saiga.

Boronfky, Thierreich, I. 3. p. 77. n. 7. der wilde Hirfich.

Pennant, arct. Zool. II. p. 36. der Ahu, das un. gefchvöinzte Reh.

Pallas, Reife, Ausz. I. p. 65. Rehe.

Pallas, Reife, Ausz. I. Anh. p. I. n. I. Cervus Pygargus.

Erxleben, Mammal. p. 31\%. n. 8. Cervus (Pygargus) cornibus trifurcis, cauda nulla.

Gratimann, intr. in diff. nat. mamm. p. 53. VIII. der wilde Hirfch.

Gatterer, brev. Zuol. I. p. 91. n. 8. Cervus (Py. gargus) cornibus trifurcis, cauda nulla; der roilde Hirfch.

2. Atces. Das Elennthier.(4)

Müller, Naturfylt. I. p. 38I. n. 2. das Elend. thier.

\section{Zimmer-}

(3) Die Tataren und Tffhuwafchen fangen diefe Thiere zu Ausgàng des Wìnters in Schlingen und Fallen, oder jagen ihnen auf Schneefchuhen nach, womit fie fie leicht einholen, weil fie die, auf dem ticfen Schnee, erzeugte Rinde durchbrechen, und niche wohl darauf fortkommen können. Pennant und Zim. mermann halten den Ahu für eine Varietät von, Cervus Capreolus.

(4) Unterfuçhunģen haben gelehrt, dafs das amerikanifçhe MoofeDeer der Englander, oder das Orignal der Franzofen", mit dem Elenn ein und eben daffelbe Thier fey. Die Schwere diefes Thiẹs beiragt uber $1200 \mathrm{Pfund}$, und fein Geweihe wiegt 
Zimmermann, geogr. Zoolog. 'I. p. 263. das Elenn. II. p. 127. n.- 40. das Elend:' III. p. 157. n. 10. das Elendthier.

Le $\int k e$, Naturgefch. p. I78. n. 2. das Eleni. Borow $\int k y$, Thierreich, I.3. p.66. n. 2. das Elenn. Bhumenbach, Handb. der Naturgefch. p. I13. n. r. Cervus (Alces) cornibus planis ycaulibus, palmatis; das Elennthier.

Funke, Naturgefch. I. p. I⿳ิ77. das Elent.

Ebert,

auf $56 \mathrm{zu} 75$. Pfund.' Sie halten fich gews̈hnlich in den Waldern anf, und nahren fich von Baumrinile, den jungen Trieben und Schüfslingen der Bäume, auch von Laub, fettem Grafe, Krant und gtïnem Getraide. Die Begattungszeit fallt in den Auguft, Das Weihchen geht neun Monat trächtig, und wirfe in April gewöhnlich nur ein, zuweilen auch zivey lönge, welche der Mutter uiber ein lahr folgen. Den Somner hindurch halten fie fich in Familien zufammen. Bey tiefem Schnee verfamıneln lie fich in den Nadelhölzern in Haufen. The Gang. ift ein fchaukelnder Trott, wodurch fie fehr fchnell fortkommen. Nur die Männchen haben Geweihe, welche fie alle Iahr abwerfen. Das Fleifch ift füls und nahrhaft. Die Zungen fchmecken vortrefflich, und die $\mathrm{Nafe}$, die völlig wie Mark reyn foll, wird in ganz Canada für den gröfsten Leckerbiffén getialten. Das Fell wird gegerbe, und zu allerley Arbeiten verwendet. Aus deın fchaụfelförmigẹn Theil der Hörner machen die Wilden Lüffel, In Preuffen wird das Pfund Fleifch für 2- 3 Grofchen verkauft. Die Meynung, dafs das Thier der fallenden Sucht unterworfon fey, und dafs es fich davon kurire, indem es fich mit den hintern Klauen bis zum Bluten die Ohren kratze, braueht jetze keiner weitorn Widerlegung. Von diefer Sage if vermuthlich die Schreibart des Namens Elend liergekommen. Andere fchreiben Flen, und Elent, und leiten es von dem flavonifchen Worte: Ielen, der Hirf $c h$, her. Aber Elent heifst im alten Deutfchen Stärke, wie Alce im Griechifchen. In einẹ alten Bibclüberfetzung aus dem Xlliten Seculu finder fich der elenthafie Simpson, und der elenthafie Degens Gedeon, der wyje. Auch lonatbas, den Wygant, mafbte dieg

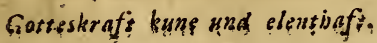


Ehert, Naturlehre, I. p. 306. das Elendthier. Halle, vierf. p. 338. das Elendthier, Elendkuh. 'Tab. 8.

Gatterer, vom Nutzen u. Schaden der Thiere, I. p. 173. Elendthier.

Beckmanni, Naturhiflorie, p. I4. n. Ir. das Elendthier.

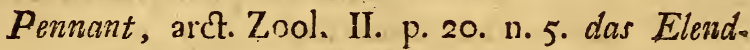
thier, Mus. Thier. Tab. \&.

Neuer Schaupl. d. Natur, II. f. 568. Elend.

Onomat: hifor. nat. I. p. 274. das Elendthier.

Handb. der Naturgefch. 1. 2. p. 270. das Elendthier.

Frifch, Naturfy R. p. 3. n. 3. das Elend-Thier. Klein, Quadr. difp. p. 24. Cervus Palmatus Alce; vera et legitima.

Klein, Claflif. der vierf. Thiere, p. 80. 8. Elent. Klein, 'natïrl. Ordn. d. vierfüls. Th. p. 37. n. 3. Elend.

Gesner, Thierbuch, p. 84. Elend; m. Figg.

1 Biiffon, vierf. X. p. 178. das Elennthier; m.e.Fig, Batfch, Thiere, I. p. 138. das Elemu. Bock, Naturgefchichte von Preuffen, IV. p. 94. das Elendthier:

Fifcher, Naturgefch. von Livl. p. 33. 11. 3. Elend. Pontoppidan, Norwegen, II. p. 20. Elendthiere. Schöpf, Reife durch Nordamcrika, II.' p. I6\%: das Orignat.

Carver, Reile durch Nordamerika, p. 365. das Eilendíthier.

Carver, Reile, p. 366. das Muesethier.

Schlüzer, Erdbefchr. von Amerika, p. 73. p. 235. das Elendthier.

Schlözer, Erdbefchr. von Amerika, p. 149. Mut. rethier: 
Pallas, Reife, I. p. 198. II. p. 258 . III. p. 12. 89. Elennthier.

Pallas, Reife, Ausz. I. p. 129. Elendthiere. II. p. 12. Elenn.

I. G. Gmelin; Reife durch Sibirien, I. p. 296. Elende. II. p. 165. Elende.

Kalm, Amerika, III. p. 582. Elendsthier.

Dapper, Amerika, p. 145 . Elande.

Miiller, Sainml. ruff. Gefch. III. p. 552 . Elende, Elendthievé.

Leppechin, Tageb. d. ruff. Reife, II. p. 4. Elevid. III. p. 57. Elende.

Hupel, Topogr. von Liefl. II. p. 439. Elend. Ausz. a. d. Tageb. eines Reif. n. Afien, p. so. das Elendthier.

Schlözer, Briefwechfel, I. p. 79. Elendthier. Krünitz, ökon. Encyklop. X. p. 705. Elendthier, Elend.

Dobrizhoffer, Gefch. der Abiponer, I. p. 334. das Elendthier, oder die grofse Beftie.

Perrault, Charras und Dodart, Abhandl. zur Naturgefch. I. p. 207. anatomifche Befchreib. eines Elendthiers. Tab. 26. das Elendthier. Schlecht. Tab. 29. die Zergliederung. Stralfund. Magazin, I. p. 382. das Elennthier. Oekon. Zoologie, p. 40. n. 49. das Elendthier. Allgem. Magazin, XII. p. 290. Elendthiere. Mannichfaltigkeiten, II. p. 413.429. Elennthier; m. e. Fig.

Beckmann, Befchr. d.Mark Brandenburg, I. p. 786. Beckmann, diff. de praecipuis Germaniae antiquae animalibus, p.39-49. Alces, Achlis, Tarandus. Brefsl. Samml. Aug. 1718. Cl. 4. Art. ir. u. Oct. 1780. Cl. 4. Art. 9. Elendthier. Sept. 1725. S1.4. Art. S. Elendthier. Weygand; 
Weygand, Bericht vom Elendthiere; in Kanold IV Suppl. Budiffin, 1729.4. p. 35. m. e. Fig. Tablonsky, allgem. Lex. p. 294. Elend, Elendthier. Döbel, Iägerpraktik, I. p. Ig. Elendhir $\int i$. Merklein, Thierreich, p. 37. Elendt. Erroleben, Mammal. p. 298. n. 2. Cervus (Alces) cornibus acaulibus, palmatis.

Linné, Syfh. Nat. Edit. II. p. 5o. Cervus cornibus acaulibus, palmatis.

Linné, Syft. Nat. Edit. VI. p. I3. n. 2. Cervus cornibus acaulibus, palmatis.

Linné, Syft. Natur. Edit. X. I. p. 92. n. 3. Cervus (Alces)- cornibus acaulibus, palmatis, caruncula gutturali.

'Linné, Fn. Suec. I. p. 13. n. 37. Cervus cornibus acaulibus palmatis.

Graumann, intr. in hiffor. nat. mamm. p. 5I. II. Elend, Elendthier.

Gatterer, brev.Zool. I. p. 85. n. 2. Cervus (Alces) cornibus acaulibus, palmatis; das Elendthier. Heinfii, diff. de Alce. Ien. 1697. 4. m. e. Fig. Baccii, tract. de magna beftia, Alce, Elenad. Stuttg. I598.

Goebelii, hiforia brevis de Alce. Venet. 1595. m. e. Fig.

Menabenii, tract. de magno animali, quod Alcen vocant, etc. Mediol. 1581. 4.

Dodonaei de Alce epiftola. Colon. 158r. (Bey dem vorhergehenden befindlich.)

Wigandi vera hiftoria de fuccino Pruffico, de Alce Boruflica. Ien. 1590. 8.

Hermann, de fceleto feu ollibus Alcis, Maslae detectis. Hirfchb. 1729. 1731. 4. m. e. Fig.

Valentin, amphith, zootom. p. 57. de Alce. (Die Leupoldifche Befchreibung. 
Mangeti, bibl. pharmac. med. I. Gen. 1703. f. P. 544. de Alce.

IMenzel. obf. de alces monfrofo partu et gratitudine in homines; in dent Ephem. N. C. Dec. II. A. 5. obf. 3 .

Severin, zoolog. Hungar. p. 43. C. Alces; das Elendthier, Iavor, Los.

Miiller, zool. dan. prodr. p. 5. n. 34. Cervus (Alces) cornibus acaulibus, palmatis, caruncula gutturali.

Rzacz. hift. nat. Polon. p. 212. auct. p. 304. Alce feu Alces.

Diere'ville, Acadie. Amf. 1710. p. 122. l'Ori-' gnal ou l'Elan.

Denys, defcr. de l'Amerique feptentr. I. p. 27. Hiftnire de la Virginie. Orleans I707. p. 213.

La Hontan, voyage, I. p. 86 .

Catesby, Carol. append. p. XXVII. Alce maxima americana nigra; the Moofe or Elk.

Pennant, hift. quadr. n. 43. Elk; Moofe-Deer.

3. Elaphus. Der Hiv ch. (\$)

Miiller, Naturfyft. I. p. 389. n. 3. der Hirfch.

Zimmermann, geogr. Zool. I. p. 220. II. p. I29. der Hirfch.

$$
\text { Lefke, }
$$

(s) Edler Hirfch, Rotbbirfch, Hirfcbbock (das Munnchen); Hirfcb. kuh, Hinde, Hindin, Tbier (das Weibchen); Hirfcbkalb (das männliche lunge); Thierkalb, Hindekalb, Wildkalb (das weibliche lunge); Scbmaltbier (ein Hirfchkalb tiber Reben Munate); Spiefshirfcb (im zweyten lahre); Gabelbirf $c h$ (im dritten lahre). Verândert nach Verfchiedenheit der Iahrszeit feinen Stand und Gröfse. Die Zahl der Enden des Geweihes richtet fich nichs genau nach dem Alter des Thiers. Im vierten lahre ift es fechsendigr, und nash dem ashten lahre ift die Anzabl der Enden 
Lefke, Naturgelch. p. 180. n. 3. der Hirfch. Borowsky, Thierreich, I. 3. p. 68. n. 3. der Hirfch. Bhomenbach, Handb. d. Naturgefch. p. 115. n. 4. Cervus (Elaphus) cornibus ramofis, totis teretibus recurvatis, apicibus multifidis; $\operatorname{der} H i r f c h$. Bechftein,

unbeftimmt. Die gröfsten Geweihe find von 64 Enden.. Selten fallen beyde Stangen zugleich ab, und oft behälr der Hirfch die andere Stange zwey bis drey Tage, nachdem er dis erfte abgeworfen hat. Die ftarken Hirfohe werfen gewöhnlich zuerft ab. Gemeiniglich ift das fpare Abwerfen eine Folge eines lan. gen und harten Winters. Man hat Boyfpiele, dafs zwey Hirfche beym Kampf in der Brunftzeit fich mit den Stangen dergeftalt verwickelt haben, dafs fie nicht wieder loskømmen konnten, und im Walde todt gefunden wurden. Die Brunftzeit dauert etwa vier Wocher. Ein Hirfch von 88 Monaten ift im Stande fich forrzuplanzen. Die jungen haben über den ganzen Leib weifse Flecken, die fie erft gegen den Winter verlieren. Aus der Anwendung des Ueberfuffes der Nahrung, bey dem Hirfch, läst fich der Grund angeben, warum die Thiere keine Gehürne haben, weil bey ihnen der Ueberflufs an Nahrungsfáften cben zu der Zeir eintritt, wenn fie tragend find, und cr alfo zum Unterhalt der Frucht, oder nachher zur Milch für das faugende Kalb gebraucht wircl. Der Hirfch hat ein fcharfes Geficht, ein leifes Gehür, und, wenn er unter dem Winde ift, einen weiten Gernch. Seine Stimme if, befonders zur Brunftzeit, fo ftark, dafs fie oft über eine halbe Meile weit gehört werden kan^. Seine Nahrung richtet fich nach der Verfchiedenheit der lahrszeit. Im Hcrbft befteht fie in Knofpen des Nadelbolzes, Heidekraut, Brombeeren u. .ow. In Winter in Baumrinde, Moos u. dgl. Im. Frühjahr in Kátzchen der Hafeln', Aefpen, Wciden, und den Knof́pen der aufbrechenden Büfche. Im Sommer hat er zwar Ueberflufs, doch zieht er Koggen und Hafer allen übrigen Getraidearten vor, ift auch begierig auf die Flachsknoten, die aber das Thier verfchmăhet. Nur zue Brunfteit trinkt er viel; im Winter und Frühjahre aber fehr wenig. Dafs er keine Cralle an der, Leber hat, ift bekannt; ob fie" fich aber in der Blume oder im Schwanze befindet, wie einige aus der inwendigen grünlichen Farbe, und dem bittern Gefchmack muthmafsen, if unentrchieden. 
Bechftein, Naturgefch. Deutfclil. I. p. 558. der, Hirsch.

Funke, Naturgefchichte, I. p. 80. der edle Hirfch.' Ebert, Naturlehre, I. p. 303. die eigentlichen Hir. Sche; edle Hirfche.

Halle, vierf. p. 326. der Hirfch.

IMeyer, Thiere, I. Tab. 22. der Hirfch.

Gatterer, vom Nutzen und Schaden der Thiere;

I. p. 174. Hirfch.

Beckmann, Naturhiforie, p. 13. n.9. der Hirfch.

Pennant, arct. Zool. II. p. 3̧o. n. 7. der Hirsch.

Neuer Schauplatz der Natur, IV. p. 29. Hirfch,

Onomat. hiftor. nat. II. p. 79 r. ein Hirfch.

Handbuch der Naturgefch. I. 2. p. 87. Cervus; der. Hir ch.

Handb. d. deutfchen Thiergefch. p. 73. der Hiv. ch, Edelhirfch, Brandhirfch.

Walther, ökonom. Naturgefch. Deutfchl. p. I38. Hirfch.

Frifch, Naturfyn. p. 3. n. x. Cervus vulgaris; 'der. europüiliche Hirfch.

Klein, quadrup. difp. p. 23. Cervus nobilis.

Klein, Claffif. d. vierf. Th. p. 76. der edle Hirfch: Klein, natürl. Ordn. d. vierf. Th. p. 26. n. x. edler Hirfch.

Gesner, Thierbuch, p. 189. Hirfch. Abbildung mittelmälsig.

Büffon, vierfüfs. III. p. 23. der Hirfch; Tab. 4r.' die Hirfchkuh; Tab. 43... das Hirfchkalb; Tab. 44 .

Batfch, Thiere, I. p. I36. der gewöhniliche Hirfch. Bock, Naturgefch. von Preuffen, IV. p. 125. n. 34. der edle Hirfch.

Doüriahoffer, Gefch. der Abiponer, I. p. 350: der Hirfch. 
Hartfank, Befchr. von Guiana, p. 109. Hivfire? Baldaeus, Zeylon, p. 422. Hirfchen Wolf, Reife nach Zeilai, ,p. r20. Hiwfhè.

Du Halde, Befchreib. von China, IV. $2 \times 3$. die Hirfche.

Pontoppidan, Norwegen, II. p. 18. die Hiv fhe.

I. G. Gimelin, Reife durch Sibirien, I. D. 2390 Hirfche. p 296 . Hirrche.

S. G. Gmelin, Reife d. Rúfsl. III. p. 432. Hinfolso Neuhoff, Gefandfch. n. China, p. 348 Hirfche. Dapper, Amerika, p. 624. Hirveche.

Keife nach den Infeln Frankreich und Bourbora, p. 293. Hirfiche.

-.... Pallas, Reife, III. p. 10. p. 449. Hirfche. Pallas, Reife, Ausz. IIII. p. I2. I3. Hirrfhe.

Bonnet, Betrachtung. über die Natur, II. p. $5^{14}$. der Hirfch.

Beytr: zur Beförd. d. Naturkunde, I. p. 2 212. Hir $\epsilon_{\text {tr. }}$ Burgsdorf, in den Schriften der berlin. Gefelifeh. naturf. Fr. VI. p. 4II. Rothiir/ch.

Miller, Samml. ruff. Gefch. III. p. 553. Hirfthe. Penuant, Reife d. Schottland, I. p. I24. Hirfche. Mellin, Anveifung zur Anleg. der Wildbahnen, p. I29. der Hirfch; m. e. Fig.

Krïnitz, ökon. Encykl. XXIII. p. 590. Hir. Fch. Le Verrier de la Conterie, normännifcher Iäger, p. 164. Hirfch.

Döbel, Iägerpraktik, p. I. Hir.ch, edler Hir.ch, Rothhirfch.

Merklein, Thierreich, p. 72. Hirfch. Heppe, wohlredender Iäger, p. r. Hirfch. Oekonom. Zool. p. 41. n. 5o. der Hirrch. Iablonfky, allgem. Lex. p. 455. Hirfch. 'André und Bechfein, Spatziergänge, II. p. II\%. der Hirfch.

Beckmanns. 
Beckmann, phyl. ökon. Bibl. XIV. p. 63. Hir fch. Linne', goth. Reife, p. 56.130 .148 . der Hi fich. Ludovici, Kaufmannslex. III. p. 417. Lemmery, Materiallexicon, p. 279.

Anmerk. über alle Theile der Naturl. III. p.597. Hannöv. Magaz. I77I. p. 209-222. p. 233240. Brefsl. Samml. Aug. 1726. p. 236. Febr. p. $2+8$. Erxleben, Mammalia, p. 3 or. n. 3. Cervus (Ela: phus) cornibus ramofis totis teretibus recurvatis.

Linné, Syft. Nat. Edit. II. p. 5o. Cervus cornibus ramofis teretibus incurvatis.

Linné, Syft. Nat. Edit. VI. p. 13. 1. 3. Cervus cornibus ramofis teretibus incuryatis.

Linné, Syft. Nat. Edit. X. I. p. 67. n. 3. Cervus (Elaphus) cornibus ramofis teretibus incurvatis. Linné, Fn. Suec. I. p. 13. n. 38. Cervus cornibus ramofis teretibus incurvatis.

Graumann, intr. in hift. nat. mamm. p. 5r. III. Hirjch, Hindin.

Gatterer, brev. Zoolog. I. p. 86. n. 2. Cervus (Elaphus) cornibus ramofis totis teretibus recurvatis; cier Hirfch.

Severin, zool. Hungar. p. 43. n. 2. C. Elaphus; Hirfch, Szarvus, Ieleus.

Vallevii, diff. de Cervis. Upf. s778. 8. Albini, diff. de Cervo. Frcf. ad Viadr. 1686.4: Von der Natur der Hirfchen. Augsp. 1612. 4. Gundelii, Mifcell. de Cervo. 1715. 4.

Langelotti, obf. de cerva cornuta; in den Mife.' N. C. A. 1678. 1679. A. 9. et Io. obf. 88.

Ledel. obf. de cerva cornuta; daf. Dec. II. A. 2. ohf. 98 .

Hoffinann, de cervae gravidae anatome; in denz Append. ad Eph. N. Cur. Cent. 9. et 10. p. 467.

$$
\mathrm{Pp} \text { ?. Hoff. }
$$


596 Manmalia Pecora. Cervus.

Hoffinann, de cervo, cultro anatomico fubiecto; in dem Append. ad Eph. Nat. Cur. Cent. 9. et 10. p. 448. (vid. Syft.)

Ludovici, obf. de foetus cervini conflitutione; in den Mifc. N. C. A. 1675. et 76. obf. 242.

Manget. bibl. pharm. med. I. p. 544. de cervino genere, eiusque hif:'naturali.

Volckanniner, defer.anatom cervae; in den Mifc.

N. C. D. II. A. 6. obf. 223. p. 459. and Valent. amph. zoot p. 74.

Stail, de corm cervi deciduo. Hal, I699. 4. Wepfer, de lachryma cervi; in den Mifc. N. C. Dec. II. A. 6. obf. II8.

Manget. bibl. pharm. med. I. p. 545. de lachry. ma cervi.

IVIlley, zool. dan. prodrom. p. 5. n. 35. Cervus (Elaphus) corinitus ramolis, totis teretibus recurvatis。

Kramer, Auftr. p. 3I9. Cervus cornibus ramofis teretibus incurvis.

Penticnt, britt.Zool. p. 15. the Stag or Red Deer. Aelinn. hiltor. anim. VI. c. II. I3. VIII. c. 39. XII. c. I8. Eגaşos.

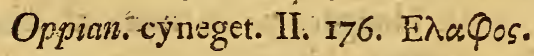

6. Hiprelaphus. Der Brandhirfch, Ro/shirech. Miiller, Naturfyfem," I. p. 394. der böhmifche Brandhirfch.

Zimmermani, gengr. Zool. I. p. 225. dev Tragelaphus und Hippelaphus der Alten, Brandhirfch, ardennifine Hirfch.

Bechflein, Naturgefch. Deutfchl. I. p. 584: Brandhivfche.

Ebert, Naturlehre, I. p. 304. der böhmifche, Jo genomite Brandhirfch.

Beytr. 
Beytr. zur Beförderung d. Naturkunde, I. p. 213. Brandhirfolu.

Biiffon, vierf. III, p. 26. A, der ardennifche Hirfch. Tab. 42. edler Hirfch mit 66 Enden.

Wald - Forft - und läger - Lexicon. Prag, I768.

p. 145.

Granincuna, intr. in hiflor. nat. mamme p. 52. a. Brandhirfch.

Gatterer, brev. Zool. I. p. 87. a. Hippelaphus; Maior, pilis in collo longioribus, quafi iubata; fenior: der Brandhirfch, Pférdehirfch.,

Severin, zool. Hungar. p. 44. des Brandisirfich.

Hieher gehört der Berg-oder Gebirghirfch.

y. Corsicanus. Dov korflkanifche Hirfch.

Mïller, Naturfyf. I. p. 394 . der kleine corficanio fiche Hirfin, Tab. 22. fig. 2. Hoútt.

Zimmermann, geogr. Zoolog. I. p.223. 225. der korfikanifche Hirgch.

Borowfky, Thierreich, I. 3. p. 72. b. der korjkanifche Hirch.

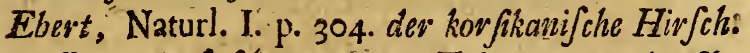
Biiffon, vierfüls.' III. p. 80. Tab. 45. der korfika. mifiche Hirfch.

Frifch, Naturfynt; p. 3. n. 3. Cervus minor; der corficanifche kleime.

Cetti, Naturgefch. von Sardinien, I. p. 126. der Hirfch.

Grawnann, intr. in hiftor. nat. mamm. p. 50. b. C. Corficanus;

Gatterer, 'brev. zoolog. I.' p. 87. b. Corficanus. Vulgàri dimidio minor, fufcus; der corgakianifche Hirfch. 
8. C An a d ins Is. Der kanadifche Hirfch.

Miiller, Naturfyft. I. p. 393, der Hirfch vor, Canada. Tab. 22. fig. I.

Zimmernamn, geogr. Zoolog. I. p.220. 225. der kanndif che Hirjch.

Borowjky, Thierreich, I. 3. p. 72. d. der canadenfifche Hirfch.

Eliert, Naturl. I. p. 304. der Hirfch von Canata. Klein, Quadrup. difpuf. p. 23. Canadentis. Klein, Claffif, der vierf. Thiere, p. 76. der Hirfch aus Canada.

Carver, Reife durch Nordamerika, p. 365. der Hir. Ch.

Perrault, Charras und Dodart, Abhandl. zur Naturgefch. II. p. I anatomifche Befchreibung eines Hirfches aus Canada. Tab. 46. canadifcher Hirfch. Tab. 47. die Zergliederung.

Gramman, intr. in hiftor. natur. mamm. p. 52. C. Canadenfis.

Gatterer, brev. zool. I.p.87. c. Canadenfis. Cornibus ampliffimis; der kanzadenfjeche Hir $\mathrm{fch}$.

\section{Tarandu's. Das Rennthier. ( $\left.{ }^{6}\right)$}

Miiller, Naturfyft. I. p. 394. n. 4. das Rennthier: Tab. 23. fig. Houtt.

\section{Zimmer.}

(0) Es giebt wenig Gefchöpfe in der Natur, die fo, wie das Rennthier, faft alle Bedürfniffe des Menfchen $z u$ befriedigen im Stande wären, daher es auch von den Lappländern, Koräten, Tungufen, Samojeden, u. dgl, auf alle Weife benutzt wird. Sie nähren fich von feinem Fleifch und Milch, kleiden fich! in fein Fell, und bezichen ihre Schlitten und Zelte damit; brauchen es zum Laftragen und zum Zug, verfertigen allerhand Gerätice aus feinen Hörnern, Nadeln aus feinen Knochen, $\mathrm{Fa}$. den aus feinen Sehnen, und Beutẹl. und Elafchen aus feine: 
Zimmermann, geogr. Zool. I. p. 259. 'II. p. I28.

n. 4 r. das Rennthier.

Lefke, Naturgefch. p. 179. n. 3. das Rennthier. Borowfky, Thierreich, I. 3. p. 73. n. 5. das Rennthier. Tab. 30. B.

Blumienbach, Handb. der Naturgefch. p. ri4. n. 3.

Cervus ('Tarandus) cornibus longis, fimplicibus, teretibus, fummitatibus fubpalmatis, iuba gulari pendula; das Renthier. Fanke, Naturgefch. I. p. I33. das Rennthier. Ebert, Naturl. I. p. 307. das Renntilier. Halle, vierf p. 343. das Remuthier. Gatterer, vom Nutzen und Schaden der Thiere, I. p. ,180.' n. 99. das Rennthier: Beckmann, Naturhiftorie, p. 14. n. 13. das Renizthier.

Pennant, arct.Zool. II. p. 25. n. 6. das Rennthier. Pp 4 Neuer

Harnblafe. Die Rennthierbutter ift unfchmackhaft, aber die Kä́fe defto vorzüglicher. Die Grönländer effen das Fleifch roh, gekocht, getrocknet, und mit Moos geräuchert, trinken das Blut mit Heidebeeren zubereitet, verzehren felbft dasjenige, was fie im Magen dies Thiers finden, kochen die Eingeweide, und geniefsen vorzüglich das Fett. Die Koraten haben oft Heerden von zwanzig taufend Stick, da fie fehr wohlfeil zu ernähren find, und von dürrem Laube, vorziiglich von Rennthier-Moos leben, das fie unter dem Schnee hervorfcharren. Renathiere, die in ihrer Freyheit leben, bringen nach Büffon ihr Alrer auf 28 bis $30 \mathrm{lahr}$, nach der bey allen vierfitisigen Thieren angenommenen Regel, dafs fie fiebenmal fo lange leben als fie lahre brauchen, um ihren vollkommenen Wachsthum zurückgelegt zu haben. Im vierten lahre aber hat das Rennthier feine ganze Gröfse und Stärke erreicht. Naçh der Erzählung der Koräten follen zwey vor einem Wagen oder Schlitren gefpannte Rennthiere in einem Tage 150 Werfte, oder $21 \frac{3}{7}$ deutfche Meilen zurücklegen. Das Thier fangt fich, da wir kein höheres Land kennen, mit Spitzbergen an, wo es mit dem weifsen $B a$ r und dem Ifatis die ganze Lifte der dortigen Quudrupeden ansmacht. 
Neuer Schaupl. d. Natur, VII. p. 90. Rennthier.

Onmat. hiftor. nat. VI. p. 796. Rangifer; das. Rennthier.

Handbuch der Naturgefch. I. 2. p. 270. Tarandus Rargifer; das Rennthier.

Frifch, Naturfy/t. p. -3. n. I. Rangifer Lapponicus; das Remnthier.

Klein, Claffif der vierf. Th. p. 76. Rennthier. Klein, natïl. Ordn. d. vierf. Th. p. 26, n. 2 , Rennthier.

Gesner, Thierbuch, p. 210. Ein Seltzanne Avt Hir ch?

Bïffon, vierf. X.p.I78.das Renntinier; m.2 Figga Batfch, Thiere, I. p. I38. dits Renin.

Miiller, Samml, ruff. Gefch. III. p. 553. Renzzthiere.

Phipps Reife n. d. Nordpol, p. 97. Rennthier. Leppechin, ruff. Reife, III. p. 57. Rennthiere. Steller, Kantichatka, p. Ir4. II9. 252. Rennthier. Cranz, Grönland, p. 104. Rennthiere. p. 106. Renntizieve.

Egede, Gröniand, p. 84. Rennthiere.

Martcns, Spitzberg. Reife, p. 74. Hirch den man Rehe nennt. Tab. O. fig. 3. fchlecht.

Krafcheninnikow, Befchr, von Kamtfchatka, p. 125. die Remnthieve.

Lefips, Kamtfchatka und Sibirien, p. 139. Remn. thiere. p. 180 . Rennthiere.

Pontoppidan, Norwegen, II. p. 21. Rennthier.

Leemi, Nachr. von den Lappen, p. 95. Rennthiere.

Pallas, Reife, III. p. 25: 46. 69.470. 597. Rennthisr.

Pallas, Reife, Ausz. III. p. 42. Rennthieve.

Pallas, Naturgefch. merkw. Thiere, XI. p. 33. Rennthieve. 
Högftöm, Lappland, p. 87. p. 107. Rennthiere. De Kerguelen Tremarec, Reife nach d. Nordfee, p. 188. das Rennthier.

Utio von Troil, Reife nach Island, p. 107. Renn. thiere.

Diereville, Akadien. Götting. Reif. III. p. I54. Rennthièr.

Camper, Naturgefch. des Orang-Utang, des afrikan, Nalehorns, und des Rennthiers; ins Deutfche überfetzt von Herbell. Düffeidorf, I79I. gr. 4. m. K. p. 66.

Georgi, Rufsland, II. p. 279. 280. 313.547.35\%. Rennthieve:

Comper, kleine Schriften, II. 2: p. 40. Rennthier. Lichtenberg, Magazin für das Neuefte etc. II. 3. p. 94. Rennthier.

Mellin, in den Schriften der Berlin. Gefellfch. IV. p. 128. Rennthier, Rennhirfch. Tab.5.6. 7.8. Linne, in den Schwed. Abhandl. I. p. I45. Revanthieve.

Tricuald, in den Schwed. Abhandl. I. p. 158. Rennthiere.

Hagftröm, in den Schwed. Abhandl. XII, p. 96. Rennthiere.

Gifsler, in den Schwed. Abhandl. XXI, p. 286. Rennthier,

Holften, in den Schwed. Abh. XXXVI. p. 129. Rennthier,

Schwed. Abh. II. p. 77. XXI. p. 226. XXXV, p. 75. Rennthiere.

Stralfund. Magazin, I. p. 394. Rennthier. Sanml. zur Phyf. und Naturgefehichte, I. p. 337. Rennthier.

Oekon. Zoologie, p. 42. n. 51. das Rsmnthier. Iablonfky, allgem. Lex. p. 912. Rennthier, Reiner.: 
Merklein, Thierreich, p. 74. Rennthier: Berlin. Samml. IX. p. 373. 465. Rennthier. Linné, Syn. Nat. Edit., II. p. 50. Cervus cornibus ramofis teretibus, fummitatibus palmatis.

Limné, Syft. Nat. Edit. VI. p. 13. n. 4. Cervus cornibus ramofis teretibus, fummitatibus palmatis.

Linné, Syft. Natur. Edit. X. I. p. 67. n. 4. Cervus (Tarandus) cornibus ramofis teretibus, fummitatibus palmatis.

Linné, Fn. Suec. I. p. 14. n. 39. Cervus cornibus - ramofis teretibus, fummitatibus palmatis.

Erxleben, Mammal. p. 305. n. 4. Cervus (Tarandus) cornibus ramofis recurvatis teretibus, fummitatibus palmatis.

Graumann, intr. in hiftor. nat. mamm. p. 52. IV. Rennthier.

Gatterer, brev. Zool. I. p. 8s. n. 4. Cervus (Tarandus) cornibus ramofis recurvatis teretibus, fummitatibus palmatis; das Rennthier.

Fabricii, faun. Groenland. p. 26. 11. 16. Cervus (Tarandus) cornibus ramofis recurvatis, teretibus, fummitatibus palmatis:

Severin, zoolog. Hungar. p. 45. n. 3. Cervus Tarandus.

Kramer; Auftr. p. ${ }^{19}$. Cervus cornibus ramofis terètibus, fummitatibus palnatis.

Rzaczynsk. hiftor. nat. Polon. auct, p. 3 I8. Ren. fchieron.

Maupertuis, oeuvres. Lyon, 1768. III. p. I97. Le Renne.

Nouveau Memoire fur la grande Rufie, II. p. I8I.

Pennant, hift, quadr. n. 43. Rein, 
๔. RangIfer. Das eigentliche Rennthier.

Hulden. multifariae utilitatis Rangifer. Ien. 1696.4. Menaber. tract. de Alce; it. hiftoria Cervi Rangiferi. Colon. 1581. 8.

Graan delineatio Rangiferi. Upf. x685. 4.

f. G R O E N L A N D I C U s. Der grönländifche Hir $\int c h$.

Miiller, Naturfyn. I. p. 394. der grönlëndifche Hivfch. Tab. 22. fig. 3.

Borowjky, Thierreich, I. 3. p. 72. c. Cervus Groenlandicus; 'der grönlïndifcise Hirfch. Klein, quadr. difp. p. 24. Cervus Groenlandicus. Klein, Claflif. der vierf. Thiere, p. 77. Edwards grönlc̈ndifcher Hirfch.

Klein, natürl. Ordn. d. vierfüls. Th. p. 26. der grönlïndifche Hirsch.

\%. Carib ou. Der Karibou.

Miuller, Naturfyft. I. p. 394. der burgundifche Hirfch.

Zimmermann, geogr. Zsolog. I. p. 260. 262. der Karibou.

Camper, Naturgefch. des Orang-Utang, p. 79. Caribou.

Ebert, Naturlehre, I. p. 308. Caribu.

Frifch, Naturfyfr. p. 3. 11. 2. Rangifer Americanus; Caribu.

Carver, Reife durch Nordamerika, p. 367. das Rennthier.

Ellis Reife nach Hudfonsmeerbufen, p. 88. eim Hirfch?

Stralfund. Magazin, I. p. 394. Macaribo, Cari, bou, Pohano. 
5. D Ам А. Dev Dambirfch. (7)

Miiller, Naturfyft. I. p. 399. n. 5. der Damhirfet. Tab. 22. fig. 4. Büff.

Zimmuermann, geogr. Zool. II. p. 24: der Damhivfch.: p. โ28. n. 42. der Dannutirfch.

Lefke, Naturgefchichte, p. I79. n. 4. der Damehivjch.

Borowsky, Thierreich, I. 3. p. 72. n. 4. der D.anuhirfch.

Bhumenbach, Handb, der Naturgefch. p. rı. n. 2. Cervus (Dama) cornibus fubramofis compreflis, fummitate palmata; der Damhirfch, Tannhivech.

Bechftein,..Naturgefch. Deutfchl. I. p. 584. n. 2. der Damhirfech. p. 589. Damminirfch, Däme lein, Dïmmhirgch, Tawnhirfch, wilder Reh. bock; (die guten und ftarken: Schauf plitiveche.) Funke, Naturgefch. I. p. 79. der Tan:hirfch. Ebert, Naturl. I. p. 307. Damhir.ch, Tanithier. Halle, vierf. p. 342. der Dainmhivech.

Meyer,

(7) Dambirfch, Dambock, Dämling (das Männchen)i Damgeifs, Dambirfcblub, Damtbier, 'Damwild (बas Weibchen); Dämlein, Tannkitızle, Tannenwilchalb (das Kalb); Damuildbrẹtt. Die Brunftreit diefer Thiere fangt etwa den tuten Ootober an, und dauert gew ishnlich nut is Tage. Um diefo Zeit ift das Fleifch, wegen des tiblex, fchon von weitem zu fpürenden Geruchs nicht efsbar. Lin Hirfch befchlagt viele Thiere, treibt fich aber fehr mit ihtien herum, ehe fie fich ergeben. Scin Gefchrey ift nicht fo ftark und anhaltend, als beym Edelhirfch, fondern in kurzen Sarzen, doch kann es bey der Nacht wohl eine Viertelmeile weit gehört werden. Die lungen find etft in vicr oder fünf Tagen im Stande der Mutter z̀u folgen, und pflegen im folgenden Iahrẹ zu brunften. Schmalthiere, die zum crften Male ferzen, laffen bisweilen ihre lungen venhungern, weil ihnen das Gefauge fchmerzet, funft erziehen fie lie mir vie!er Sorgfalt. 
MLeyjer, Thiese, II. 'Tab. 7r der Tonnhirfch mit kleinen Flecken. Tab. 72 das Thier von Tanuhirr cheni.

Gattever, vom Nutzen und Schaden der Thiere.

I. p. 196. der Damhirfch.

Beckmann, Naturhiflorie, p. İo n. i2. der Dam. hirfsh.

Pennaru, arct. Zool. II. p. 36. der Damhirfch. Neuer Schaupl. d. Natui, II. p. 289. Damhir cch.

Onomat. hifor. nat. III. p. $585^{\circ}$ der wilite Reh. bock, der Dawihirfch.

Handb. der Naturgefch. I. 2. p. 103. Damirhich.

Frifch, Naturfyn. p. 3. n.2. der rechie Damhirgck. Klein, Claffif, der vierfüls. Thiere, p. 82. Dam, Tannhivech.

Klein, watürl. Ordn. der vierf. Tï. p. 28. n. 4 .

Dammhivfch, . Tannenhirfch.

Biiffon, vierfüfs. III. p. no. der Damhirfch. Tab. 46. und 47.

Batfich, Thiere, I. p. 137. dev Damhivfch.

Bock, Naturgefch, von Preuffen, IV." p. I29. der

Dainhirfch, Tannenhirgch.

Cetti, Sardinien, I. p. 135. der Damhir chh. Haffelquift, Paläflina, d. 342. Cervus (Dama). Beytr. zur Beförder. der Naturkunde, I. p. 213. Danhirfih, Tannhirfich. Mellin, Anveif. zur Anleg. der. Wildbahnen, p. I5x. der Damhirfch; m. e. Fig.

Kriinitz, ökon. Encykl. VIII. p. 658. Damhir $c h$. Döbel, Iägerpraktik, p. 21. Tamn-oder. Donnzhirfih.

Heppe, wohlred. Iinger, p. 293. Damhivech. Inblonfky, allgem. Lex. p. 259. Dampn-Hivech, Dänmlein, Dann - Hirfich, Tann - Hivfch. Oekon. Zoologie, p. 43. n. 52. der Dawhirfch. 
Ludovici, Kaufmannslex. II. p. 804.

Geogr. hift. phyf. Mancherley, I. p. 235.

Limné, Syft. Nat. Edit. II. p. 50. Cervus corninibus ramofis compreflis, fummitatibus palmatis.

Limmé, Syf. Natur. Edit. VI. p. 13. n. 5. Cervus cornibus ramofis compreffis: fummitatibus palmatis.

Linné, Syft. Nat. Edit. X. I. p. 67. n. 5. Cervus (Dama) cornibus ramólis compreffis: fummitate palmata.

Linné, fn. Suec. I. p. 14. n. 4. Cervus cornibus ramofis compreflis, fummitate palmata.

Erxleben, Mammal. p. 309. n. 5. Ceryus (Da. ma) cornibus ramofis recurvatis compreflis; fummitate palmata.

Graumanin, intr. in hiftor. nat. mamm. p. $52 . \mathrm{V}$. Damhirfch, Tannhirfch.

Gatterer, brev. Zool. I. p. 89. n. 5. Cervus (Dama) cornibus ramofis recurvatis compreflis: fummitate palmata; der Damhirf6h.

Severin, zool. Hungar. p. 46. n. 4. C. Dama; der Damhirfch.

Miiller, zool. dan. prodrom. p. 6. n. 37. Cervus (Dama) cornibus ramofis recurvatis compreflis, fummitate palmata.

Kramer, Auttr. p. 319. Cervus cornibus ramofis compreflis: fummitatibus palmatis.

Rzaczynfki, hift. nat. Polon. p. 217. auct. p. 308. Dama.

Bellon. obfervat. p. 57. Platogni.

Pennant, britt. zool. p. 15. the Buck.

Pennant, britt. Thiergefch. (von Murr) p. I4. Varro, de re ruf. II. c. I. Caprae platycerotae. 
8. VIRGI NIANUs. Der virginifche Hirfch. $\left(^{(8)}\right.$

Zimmermann, geogr. Zool. II. p. 24. p. 129. n. 44. der virginifche Hirjch.

pennant, arct. Zool. II. p. 31. n. 8. der virginiSihe Hirfch.

Naturgefehichte von der Louifiane; im Hamburg. Magazin, XIV. p. 613. Rehböcke.'

Scḧ̈pf, Reife durch Nordamerika. II. p. 52. Rehe. Schïpf, Reile, II. p. 167. der virginifche Hir.ch, oder das Jo genannte Deer.

Boddaert, Elench. anim. I. p. ז36. Cervus (Virginianus) cornibus multirainis, antrorfum cur. vatis, corpore pallide fufco cinereo.

Erxleben, Mammal. p. 312. Americanus.

\section{Axis. Der Gangeshirsch.}

Zimmernann, geogr. Zoolog. II. p. I3O. n. 45 , der Axis, der Gangeshirfich.

Borow fky, Thierreich, I. 3. p. 76. n. 6. der Axis.

Biiffon, vierfüfs. IX. p. 335. der Axis; m. 2 Figg. Perrault, Charras u. Dodart, Abhandl. zur Na. turgefch. II. Tab. 46. Sardinifche Hirfchkuh? Bellon. obf. p. I20. Cervi genus Platiceroti fimile, olim Axis nuncupatum.

Gran:

(8) Diefe Thiere werden fo zahm, dafs fie von den Indianern gebraucht werden, die wilden Hirfche bis zum Schufs anzulocken. Ihre Häute machen einen beträchtlichen Handelsartikel aus. Rey einem öffentlichen Verkaufe von 1764 . waren von Neujork und Penlylvanien 25027 Haute eingebracht. Sie grafen in unzahlbaren. Rudeln mit Hirfehen und Büffeln. Die Wilden trock; nen das Fleifch, in kleine Stückchen zerfchnitten, bey mäfsigem Fener, oder reiben es zu einer Art Pulver. Als eine Delicateffe effen fie auch Hirfchkälber, die aus Mutterleibe gefchnitten, und in dem natürliclaen Beutel (Amnios) gekoche find. 
Grazmanin, intr. in hift. nat. mapnm. p. 52. VI. C. Axis.

Gatterer, brev. zoolog. I. p. 90. n. 5. Cervus (Axis) cornibus ramofis teretibus erectis, fummitate bifida, corpore albo maculato; $d e r$ Gangeshirfch.

B. Der einfarbige Gangeshirfch.

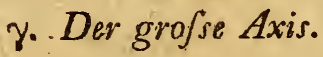

Zimmernamn, geogr. Zool. II. p. 130.

Erxleben, Mammalia, p. 313.

10. Porcinus. Der dickleibigte Hirfch.

Zimmernanis, geogr. Zoolog. II. p. I3I. n. 46. Cervus (Porcinus) cornibus reclinatis, trifurcis, corpore crafliore; der dickleibigte Hiv $/ c h$. Erxleben, Mammal. p. 318. * (Sp. obfcur.)

11. Mexicanus. Das mexikanifche Reh.

Zinmermann, geogr. Zoolog. II. p. I36. N1. 50. der Apara?

Zimmermann, geogr. Zool. II. p. 133. n. 5 I. der Cugicacti?

Zimnnermann, geogr. Zool. II. p. I34. e. die Mazamen.

Halle, vierf. p. 320. das Temamacama?

Halle, vierfüfs. p. 337. das brafilifche Rehe, Cagua- tuete?

Halle, vierfuils. p. 337. das brafilicnilche Cagua. cutpara?

Meyter, Thiere; III. Tab. 20. der Hir Cch Mecathchichiltic oder Tecamamana gezannt?

Pennant, arct. Zool. II. p. 35. n. 9. das mexiccunte Jche Reh. 


\section{Mammalia Pecora. Cervus. 609}

Klein, quadrup, difpof. p. 2I. Tragulus Temamacama?

Klein, Claffif. d. vierf. Thiere, p. 69. Tema. makamijches Böcklein?

Klein, natürl. Ordn. d. vierf. Thiere, p. 24. To mamacama?

Onomat. hiff. nat. II. p. 603. das brafilifche Reh? Biiffon, vierf. XII. p. I22. die Mazamien. Tab. 44. der Cariacu. Hirfck?

Bankroft, Naturgefch. von Guiana, p. 73. Bajert. Fermin, Befchreib. von Surinam, II. p. 89. die Waldhinzdin?

Linné, Syft. Nat. Edit. X. I."p. 67. n. 6. Cervus (Bezoarticus) cornibus ramofis teretibus erectis, rainis tribus.

Briffon, quadr. p. (mihi) 46. ni. 13. Gazella no. vae Hifpaniae. Hircus cornibus teretibus, cir. ca medium inflexis, ab origine ad flexuram fpiraliter canaliculatis, a feexura ad apicem laevibus?

Charlevoix, nouv. Fr. III. po 132. le Cheureuil?

Charlevoix, Gefchichte von Paraguay, p. 25: die Rehe?

Gronov. zoophyl. L p. 3. ni. 9. Cervus cornibus ramofis teretibus erectis, ramis tribus.

Ionfton, quadr. Tab. 33. Capreolus marinus?

6. CAPREOLUS. Das Rehe. (9)

IMiiller, Naturfyftem, I. p. 40\%. n. 6. das Reh. Tab. 22. fig. 5. Houtt.

Zimmer.

(9) Reb (die ganze Gatung); Rebbock (das Munnchen); Rebziege Rehgeifs, Geifs, Rieke, Rebebille (das Weibchen); Rebkützlein, Rébknlb, Rehekitzchen (die Iungen); Schmalrïeken, Spie/s. 
Zimmermann, geogr. Zoolog. I. p. 220. 227. II. p. I3I. n. 48. das $\cdot R e h$. Lefke, Naturgefch. p. I80. n. 6. das Reh. Bhumenbach, Handb. d. Naturgefch. p. II5. n. 5 . Cervus (Capreolus) cornibus ramofis, teretibus, . erectis, fummitate bifida; das Reh

Bechftein N. G. Deutchl. 1. p.589. n. 3. das.Reh. Funke, Naturgelch. I. p. 83. das Reh., P. 84. zuzJer gemeines Reh.

Ebert. Naturlehre, I. P. 305. das Rehe. Halle, vierfuils. p. 334. das Rehe. Meyer, Thiere, II. Tab. 73. der Rehqbock. Tab. 74. das Rehr.

Gatterer, vom Nutzen u. Schaden der Thiere, I. p. 197. n. IOI. der Rehbock, das Reh.

Becknamn,

böcke (nach der Brunft, nach Verfchiedenheit des Gefchlechts.) Im fechften Monat wächl den Reheböcken das erfte Gehörn, welches, weil diefe Thiere zu der Zeit, wenn es nuch weich ift, folches in niedrigen Strauchen und Gebüfchen oft anftorfen, und beugen, öfter als bey andern Gattungen diefes Gefchlechts ganz fonderbar und monftrös gebildet ift. Man hat auch einzelne Beyfpiele, von gehürnter Rieken. Beyde Gefchlechter haben keine Blume. Sie leben in der Monogamie. Männchen und Weibchen erziehen ihre lungen, deren gewöhnlich zwey find, cin Bock, und eine Rieke, gemeinfchaftich: und die Iungen verlaffen die Eltern erf, wenn fie ein lahr alt find. Die Brunftzeit dauert drey bis vier Wochev. Das Alter des Thiers erftrecke fich etwa auf funfzehn lahre. Neuern Beobachtungen des Grafen von Mellin zu Folge, geht das Kehwildpret sleun Monat tragbar. Die Nahiung ift rach der lahrszeit verfchieden, und beftehet in Winter in Bromranken, Genfter, der mąnnlichen' Blüre der Weiden und Hafeln, Rinde von Aefpen, Flieder, jungen Kiefern u. . w. im Frühjahr in Baumknofpen, jungen Blattern, auch grünen Saaten; im Sommar fuchen fie befonciers feine Krauter, Laub von Stauden, Brombeerblitter, Weinblicter, Rïfternlaub, Heidekraut u. $f$. $w$. 
Beckmann, Naturhiforie, p. 13. n. 10. das Reh. Pennant , arct. Zool. II. p. 35. n. 10. das Reh. Neuer Schaupl. d. Natur, VII. p. 79. Reh. Onomat. hiftor. nat. II. p. 603. ein Reh. Handb. der Naturgefch. I. 2. p. 107. das Rehe. Handbuch der deutfch. Thiergefchichte, P. 74' das Reh.

Walther, ökon. Naturgefch. Deutfchl. p. I63. Reh. Frifch, Naturfyft. p. 3. n. I. Capreolus vulgaris; gemeines Rehe.

Klein, Claflif. der vierf. Thiere, p. 79. Rehe. Klein, natürl. Ordnung der vierfüfs. Th. p. 28: n. 5. Relie.

Buiffon, vierf. III. p. I23. der Rehbock und das Reh. Tab. 48. 49 .

Batfch, Thiere, I. p. I36. das Reh. Bock, Naturgefchichte von Preuflen, IV. p. I30: n. 36. das Reh.

Hartfink, Befchr. von Guiana, I. p. Iog. Rehen? Dobrizhoffer, Gefch. der Abiponer, I. P. 35I. das Reh?

Wolf, Reife nach Zeilan, p. I20. Rek.

Baldaeus, Zeyl. p: 422. Rehieri.

Pontoppidan, , Norwegen, II. p. 19. Rehe.

Kolbe, Vorgeb. d. g. Hoffn. edit. in 4 p. 332. Rehbork?

I. G. Gmelin, Reife durch Sibirien, I. p. 296. II. p. 165. Rehe.

Neuliof, Gefandfehaft nach China, p. 348. Rehe. Cetti, Naturgefchichte von Sardinien, I. p. I34: das Reh.

Pallas, Reifed. Rufsi. III. p. 12.127.339.407. Rehe. Pallas, Reife, Ausz. III p. 12. Rehe.

Pennant, Reife durch Schottl. u. do hebrid. Inf. I. p. 124. Rehe. 
Reife nach den Inf. Frankr. u. Bourbon, p. 293.

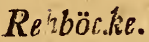

Beytr. zut Beförd. d. Naturk. I. p. 214. n. 27. Rehe. Mellin, Anweir. zur Anlegung der Wildhahnen, p. I65. das Reh.

Döbel, Iägerprakt. I. p. 26. Reh.

Flemming, deutfch. Iäger, I. p. ror. Reh.

Brcknamn, Befchreib. der Mark Brandenburg, I. p. 775 . Rehe.

Rochowe, Schr. der berlin. Gefellfch, IV. p. 385, (fchwarze) Rehe.

MIellin, Schr. der berlin. Gefellich. VIII. p. I95. Rehwilipret.

Liidovici Kaufmannslex. IV. p. In24. Reh. Merklein, Thierreich, p. 172. Rehe. Iabicnfky, allgem. Lex. p. 895. Rehe. Oekonom. Zoologie, p. 43. 11. 53. das Reh. André u. Beshftein, Spatziergänge, II. p. 56. 358. das Rer.

Becknann, phyf. ökon. Bibl. II. p. 371. das Rek. Linné, Syrt. Nat. Edit' II. p. 49. Cervus cormibus ramofis teretibus erectis.

Linné, Syft. Nat. Edit. VI. p. I3. n. 6. Cervus cornibus ramofis teretibus erectis.

Linné, Syft. Nat. Edit. X. I. p. 68. n. 7. Cervus (Capreolus) cornibus ramcfis teretibus erectis, fummitate bifida.

'Linne', fn. Suec. I. p. I4. n. I. Cervus cornibus ramofis teretibus erectis.

Limné, Muf. $\Lambda$ d. Fried. I. p. Ir. Cervus cornibus ramofis teretibus erectis.

Graumann, intr. in hilt. nat. mamm. p. 53. VI. Rehbock, Reh.

Gatterer, brev. Zoolog. I. p. 90. n. 6. Cervus (Capreolus) cornibus ramofis teretibus erectis, 
ctis, fummitate bifida, corpore rufo.fufco; das Reh.

Severin, zool. Hungar, p. 46. n. 5. C. Capreo. lus; das Reh.

$M$ Iller, zool. dan. prodr. p. 6. n. 38. Cervus (Capreolus) cornibus ramofis teretibus erectis, fummitate bifida.

Kramer, Auftr. p. 319. Cervus cornibus ramofis teretibus erectis.

Ludolf, hiftor. Aethiop. Lib. I. c. Io. $\$ \cdot 74$. Capreolus.

Pifo, Liftor, nat. et med. utriusque Ind. p. 97. Cuguacurte? (vielleicht ein Irrthum, für Cuguapara?) Mit einer Figur, welche unferm Rehe gleicht.

Trampler, grönländ. Wallfifchfang, p. 67. Re. hen? ?

Pennant, britt. Zoolog.'p. 18. the Roebuck; mit einer Figur.

12. Mun т jac. Der Muntjak,

Zinumermann, geogr. Zoolog. II. p. 131. n. 47. Cervus (Muntjac) cornibus trifurcis, uncinatis, corpore crafliore, facie fulcata; dev Muntjak.

7. Guineensis. Das guineifche Hirfchgent. Miiller, Naturfyftem, I. p. 403. das guinueiSche Reh.

Zimmermann, geogr, Zool. II. p. 134. d. das guivneifche Hiv fchger.

Erxleben, Mammalia, p. 318. ** (Sp. obfcur.) Linné, Syft. Natur. Edit. X. I. p. 68. n. I. Cervus (Guineenfis) grifeus, fubtus nigricans.

$$
\text { Qg } 3 \text { A) Vering. }
$$


A) Veränderungen gegen die XIIte Edition, und Ver. mehrung der Gattungen diefes Gefihlechts.

Edit. XII.

Edit. XIII.

p. 92. n. I. Cervus Camelo- p. I8I. n. I. Camelopar: parialis. dalis Giraffa.

Das Gefchlecht if mit Jechs Gattungen ver. mehrt, und C. Pygargus, Virginianuls, Axis, Porcinus, Mexicanus und Munntjac hinzugekom$m * n$. Ueberdem find bey der dritten Gattung vier, und bey der vierten dreiy Varietäten von ein. ander unterfchieden.

\section{B) Unbeftimmiere Thiere.}

I. Der Tonkong. Zimmermann, gengr. Zool. II. p. I33. 2.

2. Eine grofse Art Hirfche auf Zeilan. Zimmermann, geogr. Zoolog. I. p. I34. b. Pennant, fyno quadrup. p. 50 .

3. Der Kileine chineffjche Hir.fch aus der Provinz Simnuan.

Zimmernnan, gergr. Zoolog. II. p. 134.c. Naturforfcher, vil. p. 45 .

4. Der Scoadilu.

Zimmermann, gengr. Zool. II. p. 134. f. Lery, Schiffahrt in Brafilien durch de Bry. Frankfurt. 
Mamminatia Pecora. Camelopardalis. 6 r5

* (EINGESCHOBENES GESCHLECHT.)

CAMEL OPARDALIS. Der Kameelparder.

Zimmernann, geograph. Zoolog. II. p. I25: Gen. VI.

Bhumenbach, Handbuch d. Naturgefchichte, p. Irz: Gen. XXXIII:

Briffon, quadr. p. 37. Gen. VIII.

Frifch, Naturfyft. p. 2. Gen. X.

Hermann, tab. affin. animal. p. 105.

1. GIRAFFa. Die Giraffe.

Müller, Naturfyft. I. p. 379. n. I. das Kameelpardel.

Zimmermann, geogr. Zoolog. II. p. 125. n. 39: Cervus (Camelopardalis) cornibus fimpliciffimis, rectis, tuberculo frontali, iuba dorfali, femoribus anticis longioribus, tergore maculato; der Kanielparder.

Lefke, Naturgefch. p. I78. n. r. Cervus Camelo: pardalis; der Kampelparder.

Borowfky, Thierreich, I. 3. p. 64. n. I. Cervus

Camelopardalis; die Giraffe, Kameelparder;

Tab. 30.A.

Bhumeinbach, Handb. d. Naturgefch. p. Ir3. n. I. Giraffa (Camelopardalis) cornibus folidis fimpliciflimis, brevibus, rectis, tuberculo frontali, dorfo declivi; die Giraffe.

Funke, Naturgefch. I. p. I81. die Giraffe.

Ebert, Naturlehre, I. p. 302. Kamee!parder, Znursiaba, Sirapha, Girnaffe, Giraffe. Halle, vierf. p. 322. die Giraffe.

Beckmann, Naturhiftorie, p. 13. n. 8. der Kameelparder.

Neuer Schaupl. d. Natur, IV.p.360. Kameolparder.

$$
\text { Qq } 4 \text { Onomat. }
$$




\section{I Mammalia Pecora. Camelopardalis,}

Onomat. hift nat. II. p. 462. der Kameelparder. Handb. der Naturgefch. I. 2. p. 296. der Ka. meelpardel.

Naturforfcher, VI. p. 239. der Cameelpardel.

Frifch, Naturfy h, p. 2. n. Io. das Kameelpardel, Nabis.

Klein, Claffif. der vierf. Th. p. 73. Kameeipardel. Klein, natürl. Ordn, d. vierf. Thiere, p. 24. n. Io. Kameslpardet.

Büffon, vierf. XIII. p. r43. die Giraffe; m.e. Fig. Batfch, Thiere, I. p. 136. die Giraffe, oder der Kameelpardel.

Sparrmann, Reife nach d. Vorgẹb. d, g. Hoffu. p. 53I. der Kameelpardel.

Le V'aillant, Reif. in das Innere von Afrika, II. p. 33r. die Giraffe. Tab. 5. das Männchen. Tab. G. das Weibchen.

Forfter, Reife um die Welt. Edit. in 8. I. p.84. Giraffe.

Shaw, Keife, p. 360. 368. das Camelopardalis, oder Feraffa. (Zomer.)

Reife 11. d. Inf. Frankr. u, Bourbon, p. 293. eine Giraffe.

Dapper, Afrika, p, 22. der Kamehlpardel.

Berlin. Samml. I. p. 6rf. die Giraffe, oder der. Kanneel-Parder.

Iablonfky, allgem. Lex. p. 195. Camelopardel. p. I443. Zivafa, Girafa, Nabuna, Tirataka. Beckmann, phyf. ökon. Bibl, III. p. 424, X. p. 6. Camelparder; Giraffe.

Krïnitz, ökonom. Encyklopäd, XXXIII. p. 45. Kannel-Pardes, das indianifche Kanneel, dio Giraffe.

Kliiget, Eneyklopädie, I. p. 246. n. 35: der Ka. meelparder. 
Ṃammalia Pccora. Camelopardalis. 617

Merklein, Thierreich, p. 96. der Kamel-Pard, Nouvelle defcript. du Cap de b. E. p. 25. 27:36. Leo Afric, defeript, Afric, Lugd. Bat. 1632, p.745. Giraffa.

Erxleben, Mammal.p, 294. n. x. Cervus (Camelopardalis) cornibus fimplicifimis, pedibus anticis longiflimis.

Linné, Syft. Nat. Edit. II. p. 49. Cervus cornibus fimpliciffimis, pedibus anticis longiffimis.

Linné, Syf. Nat. Edit. VI. p. I3. n. I. Cervus cornibus fimpliciflimis, pedibus anticis lon. giffimis:

Linne', Syft, Nat. Edit. X. I. p. 66. n. 1. Cervus (Camelopardalis) cornibus fimpliciflimis, pe. dibus anticis longiffimis,

Graumann, intr. in hiftor. nat, mamm. p. 5r. I. das Kameel-Parder.

Gatterer, brev. Zoolog. I. p. 79. n. 4. Antilope (Canelopardalis) cornibus rectis teretibus totis annulatis, pedibus anticis longifimis, collo longiflimo, tuberculo frontali, iuba dorfali; die Giraffe, das Kambelparder.

Severin, zool. Hungar. p. 4r. IV. Camelopars dus, feu Camelopardalis; Giraffa.

Valentin. muf. mufeor. II. p. 143 .

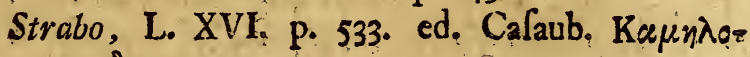

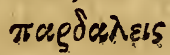

5. \$. Die deutfche Benennung: Kameelparder if daher entflanden, $\square$ eil das Thier an Grölse und Län. ge des Halles mit einem Kameel, wegen der gefleck. ten Haut aber mit dem Parder eine Aehnlichkeit hat, In Anfehung des Vordertheils ift die Giraffe das höchfte unterilallen vierfụ̂fsigen Thieren. "Dẹr gröfsa te Menfch reicht kaum bis an ihre Knie, und nach Q9 Ludolf 
Ludolf ftofst ein Reuter zu Pferde erft an feinen Bauch an. (Equitem erecto corpore equo infidentem ventrem illizs fubire poffe. A Е т н то Р, 1. с.) Die Hörner find fieben Zoll lang. Der Lauf der Giraffe befteht nicht, wie man font glaubte, in einem Hinken, fondern theils im ordentlichen Gehen, theils im Galoppe. So oft fie einen Vorderfufs aufhebt, neigt fie den fechs Fufs langen Hals, den fie fouft gerade trägt, hinterwärts. Demohnerachtet gehört ein fchrelles Pferd dazu, "das 'Thier einzuholen. Das Fleifch von den Iungen foll ziemlich fchmackhaft feyn. Die Hottentotten finden das Mark fehr wohl. fchmeckend; und aus der Haut machen fie Gefäfse, worin fie felbft Wafler aufbewahren können. Der lange Hals, der kurze Körper, der abhängige Rücken, die Hals "und Rückenmähne, das gefleckte Fell, u. dgl. geben dem Thiere ein auszeichnendes Anfehen, dafs es allerdings verdient, in einem eigenen Gefchlechte von den Hirfchgattungen abgefon. dert zu werden.

* (EINGESCHOBENES GESCHLECHT.) A v т il a e. Die Antilope; Gazelle.

Zimnnermann, geogr. Zool. II. p. 98. Gen. V. Lefke, Naturgefch. p. I8r. Gen. XXV. Borowesky, Thierreich, I. 4. p. 12. Gen. XXXII. Bhumenbach, Handb. der Naturgefchichte, p. 108. Gen. XXXI.

Bechftein, Naturgefch. Deutfchl. I. p. 599. Ebert, Naturlehre, I. p. 295.

Pallas, Naturgefch. merkw. Thiere, I. p. I. Biiffon, vierfüls. XIII. und XIV. B.

Batfch, Thiere, I. p. I32.

Erxleben, Mammalia, p. 268. Gen. XXIX. 
Graumann, intr. in hifor, nat. mamm. p. 47. Gen. XXIX.

Gatterer, brev. Zool. I. p. 77. Gen. XXIX.

Hermann, tab. affinit. anim. p. 106.

1. Le u o p a e A. Die blangraine Antilope.

Miüller, Naturfyit. Suppl. p. 52. n. I. Antilope capenfis; der blane Bock.

Zimmermann, geogr. Zool. II. p. 106. n. 17. die blangraue Antilope.

Rorow Jky, Thierreich, I. 4. p. 15. n. 3. die blaue Antilope.

Halle, vierfüls. p. 308. der himinelblaue afrikaniSche Bock.

Frifch, Naturfyff. p. 2. n. 6. Antilope caerulea; die lichtgrane Antilope.

Martini, Naturlex. II. p. 68r. die lichtgrane Antilope. Büffon, vierf. XII. p. 268. die blaue Ziege, oder blangraue Antilope; m. e.Fig.

Bat fch, Thiere, I. p. I33. die blangraue Antilope. Gatterer; vom Nutzen u. Schaden der Thiere, I. p. 166. n. 89. der.blaue Bock.

Kolbe, Vorgeb. d. g. Hoffn. Edit. in 4. p. 332. die blauen Ziegen.

Sparimann, Reife n. dem Vorgeb. d. g: Hoffn. p. 516. der blaue Bock.

Vaillant, Reife nach Afrika, p. 94. 95. der blaue Bock.

Forfter, Reife um die Welt, Edit. in 8. I. p. 86. die blaue Anteloje.

Pallas, Naturgẹch. merkw. Th. I. p. 5. n. I. die lichtgraue Antilope.

Erxleben, Mammal. p. 27I. n. 2. Antilope (Leucophaea) cornibus erectis, retrorfum flexis, corpore fupra caeruleo. 
Graumann, intr. in hiftor. naţ. manum, p. 48. II. der blane Bock.

Gatterer, brev. Zoolog. I. p. 78. n. 2. Antilope (Leucophaea) cornibus erecis, retrorfum llexis, corpore fupra caerulen; der blanic Bock.

2. LERWIA. Der Kob.

Zimimermann, geogr. Zool. II. p. I24. n. $38^{\circ}$ der Kob, oder Lervi.

Ebert, Naturl. I. p. 297. das Thier Kob.

Biiffon, vierfüfs. XI. p. I8r. der Kob. p. 264. der Kob.

Martini, Naturlex. II. p. 678. die kleine braune Kuh von Senegal.

Shaw, Reife, p. I5I. p. 358. der Fifhtall, Lerwi.

Graumann, intr. in hift. nat. mamm. p. 5 I. XXIII. die kleine branne Kuh von Senegal.

Gatterer, brev. Zonlog. I. p. 84. n. 25. Antilope (Kob) cornibus medio flexis, bafi annulatis, apicibus maxime approximatis; die kleine brans ne Kreh.

3. Rupic a r a. Die Gems. (')

Miiller, Naturfylt. I. p. 409. n. 4. die Gems.

Miiller;

(1) Die Genfe wag? fich nicht fo, wie der Stteinbock, auf die äufferften Felfenfpitzen, fondern häli fich mehr in den mittlern Berggegenden auf. lung eingefangen laffen fich diefe Thiere zahmen, fo, dafs fie mit den Hausziegen auf die Weide getrieben werden. Auch will man Reyfpiele haben, dafs zahme Gemfen fich mit den Ziegen gepaaz̧, und Baftards erzougt haben. Um die Brunftzeit kümpfen die Böcke fo lange um ein Weib, bis der ftarkere den Sieg davon trägt. Das Weibchen gebt 20-22 Wochen trächtig. Die Iungen hoit oft der Alpengeyer, Beyde Gefchlechter haben Hörner. Die Gemfenjagd ift 
Müller, Naturfyft. Suppl. p. 53. n. 2. Antilope europaea ; die Gemfe.

Zimmermann, geogr. Zool. II. p. Io5. n. I6. dio Genns.

Liefhe, Naturgefch. p. I8I. n. t. die Gemfe. Borows ky, Thierreich, I. 4. p. 13. n. 2. die Gem$f_{e}$, Felfengeis.

Blumenbach, Handb. der Naturgefch. p. rog. n. I. Antilope (Rupicapra) cornibus erectis uncinatis; die Gemfe.

Bechftein, Naturgefch. Deutfchl. I. p. 600. n. I.

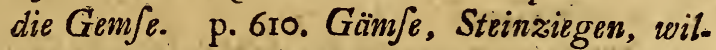
de'Feldgeis, Felfongeis, Steingeis, Felfenantilope. (Gemfenbock, Damhirzlein.) Tab. 13. b. fig. $\mathrm{f}$.

Funke, Naturgefchichte, I. p. 66. die Gemee. Ebert, Naturlehre, I. p. 294. die Gems. Halle, vierf. $\mu .314$. die Gemfe. Tab. 3. Iheyer, Thiere, I. Tab. 66. die Gems. Gatterer, vom Nutzen und Schaden der Thiere, I. p. 164. r. 88. die Gemse.

Beckmann, Naturhiftorie, p. I2. n. 5. die Gemfe. Neuer Schauplatz der Natur, III. p. 319. Genzs. Onomat. hiftor. nat. VI. p. 855. die Genife. Handbuch d. Naturgefch. I. 2. p. 277. die Gems. Handb. d. deut fchen Thiergefch. p. 67. die Gemse. Frifch, Naturfyf. p. 2. n. I. Rupicapra; die Gems. fe, das Waldthier, und das Grathier.

Klein,

höcht gefährlich. Wenn die Gemfe keinen andern Ausweg Leht, fpringt fie auf den Iager $z u$, und ftürzt ihn in den Abgrund. Selten ftirbt ein Gemfenjäger des natürlichen Todes, oder mit unverftïmmélten Gliedmafsen. Die von den unverdaulichen Fafern des Furters in dem Magen der Gemfon gebildeten Kugeln, denen inan fonft feltfame Heilkrafte andichtere, haben jerzic viel yon ihrem Werth verlusen. 
Klein, Claffif. d. vierf. Th. p. 56. Geinse.

Klein, natül. Ordn. d. vierf. Th. p. 19. n. 3.

Gemese, Grathiere und Waldthiere.

Martini, Naturlex. II. p. 656. die Gemse, FelSengeis.

Bïffon, viertürs. XI. p. 7. 109. die Gems.

Batfch, Thiere, I. p. 134 die ziegenförmige Gems. Aitmann, belvet. Eisgeb. p. 184. Gemeen.

De Liic, Reife nach den Ei geb. p. 58. Gemfe.

Pallas, Naturgefch. merkw. Thiere, I. p.6. n.2. die Gemele.

Höpfner; Magazin für d. Naturkunde Helvetiens, II. p. IIr. Gemfe.

Perranlt, Charvas und Dodart, Abhandl. zur Naturgefch. I. p. 335. anatomifche Befchreib. einer Gemfe. 'Tab. 30. die Gem/s. Tab. 3r. die Zergliederung.

Krïnitz, ökon. Encykl. VII. p. 29r. Gemes; wil$\therefore$ de Feld-oder Felfen-und Steingeifse, Steinsziege.,

Kliigel; Encyklop. I. p. 243. die Geme. Merklein, Thierreich, p. 60. Gembfs.

Iablonkyy, allgem. Lex. p. 377. Gems, Gemfe. Döbel, Iägerpraktik, I. p. 29. Geme e.

Forit. Filch-und Iagd-Lexicon, I. p. IO21. Gems u. f. w.

Oekonom. Zoologie, p. 45. n. 55. die Gemfe.

Neue Anmerk. über alle Th.der, Naturl. III. p. 53I.' Gensen.

Mannichfaltigkeiten, I. p. 579. Gemee.

Becknamn, phyl. ökon. Bibl. XV. p.380. Gemee.

Linnié, Syft. Nat. Edit. II. p. 50 . Capra cornibus furrectis', uncinatis.

Linné, Syft. Natur. Edit. VI. p. 14. n. 5. Capra cornibus erectis, uncinatis. 
Linné, Syff. Nat. Edit. X. I. p. 68. n. 3. Capra (Rupicapra) cornibus erectis, uncinatis.

Graunami, intr. in hiftor. nat. namm. p. 48. I. Gemene.

Gatterer, brev. Zoolog. I. p. 77. 1. I. Antilope (Rupicapra) cornibus erectis, corpore fupra. ferrugineo; die Gemse. Gratthiere und Waldthiere.

Severin, zool. Hungar. p. 40. c. Rupicapra, Ca. prea; die Gemfe.

Lebwald yon und zu Lebenwald, Damographia, oder Gemfenbefchreibung, von den edlen Gemfen, von der kraft - und tugendvollen Gemfenkugel. Salzb. 4. ohne Iahrzahl m. Fig. Kramer, Auntr. p. 320. Capra cornibus erectis uncinatis.

Muf. Wormian. p. бI. (Filae rupicaprae, Gemfenkiigel:)

Velfch. diff. 2. de Aegagropilis, e figg. 1668. 4. Harder, in Mif. Nat. Cur. Dec. II a. I. App. de Aegagropilis.

Schurig, Lithol. hiftorico-med. I744; p. 488. etc. Valentin. Muf mufeor. p. 3.3

4. Dam A. Der Nanguer.

Miiller, Naturfyn. Suppl. P.53.n. 3. die afrikaniSche Gempe.

Zimmermanu, geogr. Zoolog. II. p. II4. n. 27. der Nanguer.

Borow $\int k y$, Thierreich, I. 4. p. 25. n. 16. die Damnyirgch Antilope; die Dama der Alten. Ebert Naturl. I. p. 298. Nanger. Frifch, Nanrfyn. p.2.n. 3. Nanguer; die Dama der Alten.

Klein; Quadr. difp. p. I7. n. 16. Dama veterum. Büffon, 
Buiffon, vierf. XI. p. 191. XII. p. 145. Nangueur, oder Nanguer.

Batfch, Thiere, I. p. I34. die Dammhirfchantilope.

Martini, Naturlex. II. p. 645. Dam-oder Dam. hirfchantilope.

Pallas, Naturgefch. merkw. Th. I. p. 7. n. 3. die Dammhirfch Antilope.

Erxleben, Mammal. p. 280. n. II. Antilope (Da* ma) cornibus in frontem reduncis, corpore fupra fulvo, fubtus albo.

Gratumann, intr. in hift. nat. mamm. p. 49. XI. die afrikanishe Gemse, Dannantilope.

Gatterer, brev. zool. I. p. 8s. n. I3. Antilope (Dama) cornibus in frontem reduncis, corpore fupra 'fulvo; fubtus albo; die africanio sthe Gempes

5. RE D UNCA. Der Nagor.

Miiiler, Naturfyftem, Supplem: p. 53. n. 4. das Krumwihoriz.

Zimmermann, geogr. Zoolog. II. p. II4. 11. 28. der Nagor.

Frifch, Naturfyft. p. 2. n. 2. Nagor; die rothe Gazelle.

Martini, Naturlex. Iİ. p. 672. Antilope mit vor: wö̀rts gebogenen Haken.

Büffon, vierf. XII. p. 145. Nagor; m. e. Fig. Pallas, Naturgefch. merkw. Th.j. p. 8. n. 40 die Antilope mit zuriickgebogenen Haken.

Erxleben, Mammal. p. 281. n. 12. Antilope (Re. dunca) cornibus in frontem reduncis, corpore toto rufo.

Graumann, intr. in hif. nat. mamm. p. 49. XIII. das Krummhorn. 
Gatterer, brev. Zoolog. I. p. 8r. n. I4. Antilope (Redunca) cornibus in frontem reduncis, corpore toto rufo; das Krummhorn.

6. Tragocamezus. Der Biggel.

Miiller, Naturfyft. Suppl. p. 53. n. 5. der Ka. meelbock.

Zimmerniann, geogr. Zoolog. II. p. Ir3. n. 25. der Biggel. Kameelsbock.

Borowjky, Thierreich, I. 4. p. 18. n. 7. der. Kameelbock.

Halle, vierf. p. 319. der Bockhirfich.

Martini, Naturlex. II. p. 643. Bockskameel.

Bïffori, vierfüls. XII. p. 272. Bockskameel, oder Biggel; m. e. Fig.

Pallas, Naturgefch. merkw. Thiere, I. p. 8. n.5: die Antilope, das Bock-Cameel.

Bat fch, Thiere, I. p. 135. Kameelbock.

Graumann, intr. in hif. nat. mamm. p. 49. IX. Kameelbock.

Gatterer, brev. zoolog. I. p. 80. n. It. Antilope (Tragocamelus) cornibus in frontem reduncis, corpore fupra cinereo, fronte macula rhombea nigra; der Kameelbock, das Bockkameel.

Raj. Syn. quadr. p. 82. Tragelaphus Caii apud Gesnerum?

\section{Picta. Der Nyl-ghax.(2)}

Zimmermanin, geogr. Zool. II. p. Ir3. n. 26. der Nylgan, oder die weissfiifsige Antilope.

Bïfron;

(2) Das Wort $\mathrm{Ny} \dot{l}$-gau, welshes eigentich Neel-gau ausgefpro. chen wird, bedeutet eine blause $K u b$, oder eigentlich einen blaren Ochfon; weil das mannliche Thier fowohl in Anfehung Re " der 
Biiffon, vierf.XII. p. I86. dev Nilgaut; m. 2 Figg. Batjch, Thiere, I. p. I34. Nilgau.

Granmann, intr: in hiffor. nat. mamm. p. 49. X. Antilope Albipes.

Gaitever, brev. Zoolog. I. p. 8́. n. 12. Antilope (Altipes) cornibus in frontem reduncis, ma. culis albis fupra ungulas; der Nilgau.

8. SAÏGA. Die Śriga.

Miiller;, Naturfyft. I. p. 4I7. n. II. die tatarifche Ziege.

Miziller, Naturfyftem, Suppl. p. 53. n. 6. Antilope Scytica; der Tartar.

Zimmermann, geogr. Zool. II. p. I2r. n. 36. die Saiga, der Suhac.

Borowfky, Thierreich, I. 4. p. 2I. n. 2I. die quilde Steppenziege.

Ebert, Naturl. I. p. 298. Seiga, oder Seigak. Halle, vierfüls. p. 322. das Ziegeneinhorn. ', Neuer Schaupl. d. Natur, VIII. p. I83. Seigak.

Handb. der Naturgefch. I. 2. p. 279. das Thier Saiga.

Martini, Naturlex. II. p. 682. Jcytiche Antilope, der nngarifche Ziegenbock, unbärtige Steinbock, hochgehörnte kretifche Bock.

Gesnev, Thierbuch, p. 156. andere Art wilder Schaafe, ein Straulschanf.

Biiffon, vierf.XI. p. I23. das Thier Saige; m. I Fig. Batfch,"Thiere, I. p. I33. die Saiga, oder Steppenziege.

Gatte-

der Farbe als vieler Theile des Kürpers manche Aehnlichkeit damit har. Seine Hörner find fieben Zoll lang, und betragen an der Wurzel fechs Zoll in der Runde. Das Weibchen hat vier Zitzen, foll neun Monat trächtig gehen, und tringt gemeiniglich tis, felten zwey Iunge. 
Gatterer, vom Nutzen und Schaden der Thiere, I. p. 169. n. 96. die Steppenziege.

Georgi, Rufsland. I. p. 208. Saiga.

Rytfchk. orenb. 'Topogr. I. p. 231. wilde Ziegen; die Saigi genannt werden.

Pallas, Naturgefch. merkw. Thiere, I. p. 9. n.6. die fcytifche Antilope.

Pallas, Reife, I. p. 2rI. die wilde Steppenziege.

Pallas, Reife, Ausz. I. p. 144. die wilden Step. penziegen, oder Antilopen.

Leppechin, ruff. Reife, I. p. 307. die Saigaken, oder Jogenannten wilden Zieger.

Graumann, intr. in hilt. nat. mamm. p. 50. XIX.

A. Scytica; die wiilde Steppenziege.

Gatterer, brev. Zoolog. I. p. 83. n. 21. Antilope (Scytica) cornibus rectiufculis diaphanis, corpore albido; die tatarifche Ziege.

9. Gutтuros. Die Kropfgazelle.(3)

Zimmermann, geogr. Zool. II. p. 120, n. 35. die Kropf Gazelle.

Schreber, Săngthiere, V. Tab. 275. Antilope Gưtturofa Pall.

Gatterer, vom Nutzen und Schaden der Thiere, I. p. 168. n. 95. die chinefifche Gafelle.

Martini, Naturlex. II. p. 653. Caprea campeftris gutturofa.

\section{$\operatorname{Rr} 2$}

Pallas,

(3) Die Hörner find gegen zehn Zoll lang. Das Weibchen if kleiner als das Mannchen, und unbehörnt, hat auch nicht den Ktopf am Halfe. In China gehen diefe Thiere zu 1000 bis 2000 Stück in der Wildnifs zufammen. Beym Erxleben find die Synonymen von diefer, der zwölften Gattung, und dem Ahu (Cervus Pygargus) unter einander geworfen, auch der Tzeiran, Dfchairan der Perfer mit hieher gereahnet. Mammax. p. 288 . 


\section{Mammalia Pecora. Antilope.}

Pallas, Reife, Ausz. IIK. p. I63. die wafJer/chene. Ziége.

Du Halde, Befchreib.vơn China, IV. p. 137.gel. be Ziegen.

Olear. Reife, p. 449. eine Art Hirfche, Ahm gee nannt? Oder gehört diefes letztere viel. leicht Cervis Pygargus? oder zu Antilope Pygarga?

Erxleben, Mammalia, p. 288. n. 18. Antilope (Orientalis) cornibus bafi annulatis, medio flexis, corpore rufefcente.

Graumanin, intr. in hifor. nat. mamm. p. 50 . XVIII. Ahu.

Gatterer, brev. Zoolog. I. p. 82. n. 20. Antilope (Orientalis) cornibus bafi annulatis, medio flexis, corpore rufefcente; die chine fif che Gem/e.

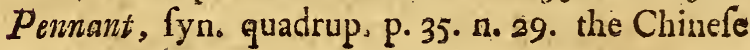
Antelope.

33. S U в G U т т บ R O A. Die kleinkropfigte $G$ a. zelle:

10. PygARGA. Der Pygarg. (4)

Miiller, Naturfynt. Suppl. p. 54. 13. \%. Antilopo Pygargus; der Weifsarjch.

\section{Zimmer.}

(4) Die Ausćinanderfetzung der' Synonymen bey diefer Gattung ilt, für mich wenigtens; mit vielen Schwierigkeiten verbunden gewefen. Im Syftem find bey der folgenden Gattung p. 187 . $n_{0} \mathrm{Ix}$. die fchwedifchen $A$ bhandlungen citirt. Ich vermuthe, diefs fey ein Schreibfchler, denri in dem angeführten Bande fteht thithts davon. Bey diefer Gattung n. 10. if Sparmann in eben diefen $A b$ handlungen von 3780 . angeführt, und nach diefem Citatd gehören auch die von mir aus deffen und Vaillant's Reifen beygebrachten Stellen hieher. Sparrmann aber giebt diefs Thier für den Difobon des Mufes, aus, für. welchen jedoch 
Mannmalia Pecora. - Antilope. 629

Zimmermann, geogr. Zool. II, p. IIg, n. 34. der. Pygarg.

Ebert, Naturlehre, I. p. 297. Koba?

Neuer Schaupl. d. Natur, IV. p. 644. Koba?

Mhartini, Naturlex. II. p. 678. die gro/se braune

Kuh von Senegal?

Kritmitz, ökon. Encyklop. XL. p. 2. Koba?

Biiffon, vierf. XI. p. 181. 264. Koba?

Biiffon, vierf. XII. p. 229. die Springende Gazelle;

oder der Springbock; m. e. Fig.

Batfch, Thiere, 1. p. I33. der Springbock.-

pallas, Naturgefch. merkw. Th. I. p. ro. n. 7 . die roilde Hivch Antilope.

Sparrmann, Reife nach d.Vorgeb.d.g. H. p.397.

Springbock. Tab. \&.

Vaillant, Reifen. Afrika, I. p. 235. Spring.Böcke.

Forfter, Reife un die Welt, Edit. in 8. I. p. 86. der Springbock.

Neue Schwed. Abh. I. p. 262. die Luftfpringer Gazelle, der Coloniften Springbock. Tab. 9.

Beckimansz, phyf:ökon. Bibl. III. p. 304. Antilope Pygargus.

Erxleben, Mammal. p. 287. n: 17. Antilope (Py: gargus) cornibus medio flexis, apicibus appro: ximatis, facie alba.

Grammann, intr. in hift. nat. mamm. p. 50 . XVII, $\operatorname{der} W e i f s a r f c h$.

Gatterer, brev. zoolog. 1. p. 82. n. 19. Antilope (Pygargus) cornibus medio flexis, apicibus ap. proximatis, facie alba; der Weifsarfch.

$\mathrm{Rr} 3$

Evxle=

nach dem Syftem die folgerde Gattung gehalten wird. Die Vermengung der Thiere Pygarg, Koha, und Tzeiran fuwohl, als die oft nicht übereinftimmenden Abbildungen, und verfchiedenen Angaben der Gröfsen, und anderer Umftände exfchweren die Sache noch mehr. 
Erxleben, Mammal. p. 293. n. 22. Antilope (Koba) cornibus medio flexis, macula genuum nigricante?

Graumann, intr. in hift, nat. mamm. p. 5I. XXII. die grofse braune Kuh von Senegal?

Gatterer, brev. zool. I. p. 84. n. 24. Antilope (Koba) cornibus medio flexis, macula genuum nigricante; die gro Sse bramie Kuh?

II. Dorcas. Die Gazalle.

Miiller, Naturfyft. I. p. 4I6. n. I0. die afrikanifche Ziege.

Miiller, Naturfyn. Suppl. p. 54. n. 8. die Gazelle.

Zimanermann, geogr. Zool. II. p. II7. n. 3I. die Gaะelle.

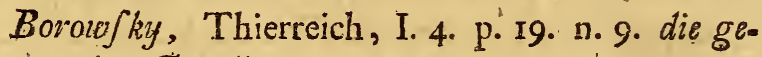
meine Gazelle.

Bhmenbach, Handb. der Naturgefih. p. Iog. n. 2. Antilope (Dorcas) cornibus teretibus annulatis, medio flexis, apicibus laevibus approximatis; die Gazelle.

Gatiever, vom Nutzen und Schaden der Thiere, I. p. 168. n. 94. die Gaselle.

Neuer Schauplatz der Natur, III. p. 289. die Gazelle.

Handbuch der Naturgefch. I. 2. p. 280. die $G a$ zellen. p. 281. die gemeine Gazelle.

Frifch, Naturfy@t. p. 2. n. I. Gazella vulgaris; die Dorcas.

Martini, Naturlex. II. p. 646. die gemeine Gazelle. Kliigel, Encyklopädie, I. p. 244. die Gazelle. Krinitz, ökon. Encykl. XVI. p. 502. Gazelle. Büffon, vierf. XI. p. 161. die gemeine Gazelle. Batfch, Thiere, I. p. I33. die gemeine Gazelle. 
Pallas, Naturgefch, merkw. Th. I. p. II. n. 8. die Antilope Dorcas.

Shane, Keife, p. 357. das Tzebi.

Erxleben, Mammal. p. 285. n. 15. Antilope (Dor-

cas) cornibus teretibus annulatis, medio flexis, apicibus approximatis.

Linné, Synt. Nat. Edit. II. p. 5r. Capra cornibus teretibus perfecte annulatis, arcuatis. Linué, Syfl. Nat. Edit. VI. p. r4. n. 9. Capra cornibus teretibus perfecte annulatis, arcuatis. Linné, Syft. Natur. Edit. X.I. p. 69. n. 9. Capra (Dorcas) cornibus teretibus perfecle annulatis, contortis.

Graumann, intr. in hift. nat. mamm. p. $50 . \mathrm{XV}$. die gemeine Gazelle, libyjche Ziege.

Gatterer, brev. Zoolog. I.p. 82. n. 17. Antilope (Dorcas) cornibus teretibus annulatis, medio Hexis, apicibus lacvibus approximatis; die gesneine Gazelle.

I B. der Chron. XII. v. 8. - 2 B. Samuel. II. v. Is. Rehe.

1a. Kevella, Der Kevel. (5)

Miiller, Naturfyftem, Supplem. p. 54. n. 9: das Platthorn.

Zimmermann, geogr, Zoolog, II. p. II7. ㅍ. 32. der Kevel.

Borowfky, Thierreich, I. 4. p. 20. n. Io. die Kewelle, der afrikanifche Stembcck.

$$
\text { R r. } 4
$$

Ebert,

(5) Nach Pallás gèhürt der Tzeiran; (B̈̈ffon, vierf. XI, p. 17\%. 25\%.) der bald mit der Kropfgazelle, bald mir dem Abu verwechfelt ift, hieher. Er gleicht dem Kevel völlig in Anfehung der Hörner, ift aber gröfser, und zu beyden Seiten des Riuckens haben beyde Gefchlechter einen hellen Streifen. Zimmermann, geogr. Zool. 11. p. 118 . 
Ebert, Naturl. I. p. 297. Kevel.

Neuer Schauplatz der Natur, IV. 'p. 468. Kevel.

Handbuch d. Naturgefch. I. 2. p. 28I. der Kevel.

Frifch, Naturfyft. p. 2. n. 2. Gacella media; das. Kevel.

Kriinitz, ökonom. Encyklopäd, XXXVII. p. 21 r. Kevel.

Martini, Naturlex. II. p. 649. afrikanijche Antilope, oder Kevella.

Biffon, vierf. XI. p. 166. der Kevel; m. e. Fig. cf. p. 255

Pallas, Naturgefch. merkw. Th. I. p. 12. n. 9. dio Antilope Kevelia.

Erxleben, Mammalia, p. 287. n. 16. Antilope (Kevella) cornibus planis annulatis, media flexis, apicibus approximatis.

Granmann, intr. in hift. nat. mamm, p. 50. XVI. das Plâthorn.

Gatterer, brev. Zoolog. I. p. 82. n. 18. Antilope (Kevella) cornibus planis annulatis, medio fle. xis, apicibus approximatis; das Plathorn.

\section{Corinna. Die Korinne. $\left({ }^{6}\right)$}

Zimmernann, geogr. Zool. II. p. II8. n. 38. die. Corinne.

Ebert, Naturlehre, I. p. 297. Korinna.

Handb. der Naturgefch. I. 2. p، 28r. das Korine.

Frifch, Naturfyftem, p. 2. n. 3. Gacella minor; das Korin.

Biiffon, vierf. XI. p. I7o. Corine; m. e. Fig.

\section{Pallas,}

(6) Kömmt mit den Kevel to fehr überein, dafs Pallas fie für das Weibchen des Kevels hält. Es giebt auch gefleckte Corinnen, daher Pennant die bunten Böeke, des Jolbo mit hieher rechnet, Kolse, Vorgeb. p. 142. 
Pallas, Naturgefch. merkw: Thiere, I. p. 12. Corinna.

Halle, vierfüls. p. 309. der gefcheckte afrikanifche Bock.

Erxleben, Mammal. p. 291. n. 20, Antilope (Corinna) cornibús medio flexis, faciei lateribus linea alba.

Graumann, intr. in hif, nat. mamm. p. 50. XX. bunter Bock.

Gatterer, brev. zool. I. p. 83.n. 22. Antilope (Corinna) cornibus medio flexis, facici lateribus linea alba; der gefleckte afrikanifche Bock.

Pemant, Synopr. quadrup. p. 37. n, 3I. Spotted Antilope.

13. BuBalis. Der Bubal.(7)

Miiller, Naturfynt. Suppl. p. 54. n. 10, die BlaSengazelle.

Ziminermann, geogr, Zool. II. p.'r22. n. 37. der Bubal, der Bubalus der Alten.

Schreber, Säugth. V, Tab. 277. Antilope Bubalis, Pallas.

Borowfky, Thierreich, I. 4. p. 24. n. I4. die Kuhantilope.

Halle, vierfüfs. p. 277. das Rindvieh ans der Barbarey. Tab. 2.

Frifch, Naturfynt. p, 2. r. I. Bubalis der Alten, Stierhirfch, grofses Thier.

Martini, Naturlex. II. p. 679. afrikanifche Kuhantilope.

\section{$\operatorname{Rr} 5$}

Biiffon,

(7) Hat eine Thränenbühle. Beyde Gefchlechter find gehürnt, Das Haar if fehr fein, etwa einen Zoll lang, übrigens wie bey dén Hirfchen oder Gazellen. Die äufserften Schwanzhaare etwa einen hal hen Fufs lang: Aus den Hornern verfertigen die Kole: niften auf dem Cap Iöffel. 


\section{Mammatia Pecora. Antilope.}

Biiffow, vierf. XII. p. Ir. der Bubal; m. e. Fig. Pallas, Naturgefch. merk würd. Thiere, I. p. 13. n. 10. die Antilope Buibalis. Sparrmanm, Reife nach d. Vorgeb. d. g. Hoffn.

p. 125. 24.8. 249.339.499. 583. Hir fohthiere. (Harte-Beeften.) Tab. II. Hirfchantilope.

Forfter, Reife um die Welt. Edit. in 8. I. p. 86. Antilope Bubalis.

Perrault, Charras u. Dodart, Abhandl. zur Naturgefch. I. p. 297. anatomifche Befchr. einer Kuh aus der Barbarey. Tab. 40. die Kuh aus der Barburey. Tab. 4r. die Zergliederung. Sparmann, in den Schwed. Abh. XLI. p. 129. Harteberft oder Dorcas.

Shaw, Reife, p. I51.358. Bekker el Wafh. Graumann, intr. in hift. nat. mamm. p. 5I, XXI. Blafengazalle, Kuhantilope.

Gatterer, brev. Zoolog. I. p. 83. n. 23. Antilope (Bubalis) cornibus medio flexis fpiralibus, faciei linea alba; die Bla engazelle, afrikanifche Kuhantilope.

I B. von den Königen, IV. v. 23. Weid-Rinder.

25. GNu. Der Gmu.(8)

Zimmermann, geogr, Zoolog. II. p. I02, n, 15: Antilope (Gnou) cornibus laevigatis, horizon. taliter procurrentibus, bafi fronti incumbentibus,

(8) Die 19 Zoll langen Hörner haben an der Wurzel 17 Zoll Peripherie. Das Haar if hirfchartig. Die Beine fchlank, wie am Hirfch. Das Thier hat eine doppelte Stimme, wovon die eine "dem Blücken deś Ochfen fich nähert; die andere dem Gefchrey des Hirfches gleicht, aber ungleich heftiger klingt. Fine befondere Gewohnheit des Thiers ift, dafs es fich zuweilen mit den Vorderbeinen auf die Knice niederlert, und dann nit 
bus, conniventibus, poftea recurvis, uncinatis, cervice gibbofa iubata, fronte, collo, palearibusque villofis, cauda equina; der Gnou. Schreber, Säugth. V. Tab. 285. Antilope Gnu, Z Zinmm.

Borowsky, Thierreich, I. 4. p. 22. n. I3. $d e v$ capfche Gnou. Tab. 32. D.

Bhumenbach, Handb. der Naturgefch. p. 109. n. 3. Antilope (Gnu) cornibus antrorfum directis, apicibus reflexis, mente barbato; iuba cervicali et pectorali ; das Gun - Tinier. Batfich. Thiere, I. p. 133́. das Gnous.

Sparrmann, Reife, p. 439. 44I. 476. das t' Gmu. Tab. 1o. das Gau-Tizier.

Vaillant, Reife nach Afrika, I. p. 244. das Gini. Forfter, Reife um die Welt, Edit. in 8. I. p. 85. Gmi.

Biiffon, vierf. XII. p. 206. der Gnov; m. 3 Figg. Beckmann, phyf. ökon. Bibl. X. p. 7. Gnou.

Gatterer, brev. zoolog. I. p. 80. n. 10. Antilope (Capenfis) cornibus in frontem reduncis, recurvis, uncinatis; cervice iubata et gibbofa; mento, fronte, collo et palearibus villofis; cauda undique fetofa, et pedum fêrme longitudine; die kapifche Gajelle.

Allamand, hilt. nat. du Gnou, du grand Gerbo, et de l'Hippopotame. Avec Fig. Amfterd. 1776. in 4.

Nouvelle defcription du Cap de b. E. Amft. 1778. 8. p. 53. Gnou.

Defcript. des Animaux, et des Plantes des Indes, tirée de la Topographie de Cosmas le Solitaire;

grofser Gefchwindigkeit fortrutfcht, wobey es zugleich mit. den Hörnern auf dem Boden fortgleitet. - Die Bubals follen in der nämlichen Pofitur mit einander kämpfen. 


\section{Mammalia Pecora. Antilope.}

re; in Thevenot Relat. de divers voy. Paris 1696. fol. T. II. p. 18. Le Taureau-cerf?

14. Or.x. Der Pafan.(9)

Miiller, Naturfynt. I. p. 412. n. 7. die Gafelle. - Tab. 24. fig. I.

Miiller, Naturfynt. Suppl. p. 55. 11. Ir. Antilope bezoartica; der Bezoarbock.

Ziminevmann, geogr. Zoolog. II. p. 107. n. I8. der Pafan.

Boronofky, Thierreich, I. 4. p. I2, n. I. Antilope recticornis; die Bezoar-Antilope.

Halle, vierf. p. 309. der wei/sgeftreifte, rundhörnige, afrikanifche Bock?

Neuer Schaupl. der Natur, III. p. 290. die andere Ant Gazellen.

Frifch, Naturfylt. p. 2. n. 3. Adaçes 'Pafan; der Bezoar-Bock.

Martini, Naturlex. II. p. 640. Bezoarasitilope, Bezoarziege.

Kliggé, Encykl, 1. p. 244. der Pafan. Ebert, Naturiehre, I. p. 297. die B6zoargazelle, der Pajan.

Kriinitz, ökon. Encyklop. XVI. p. 503. Pafan. Biifforr, vierf. XI. p. I86. die Bezoargazelle, der Pafan. p. 266. die Pafan -Gazelle; m.e.Fig. Pallas, Naturgeich. merkw. Thiere, 1. p. 15.1. I. die Bezoar - Antilope.

Sparrmann, Reife, p. 5I4. der Gemsbock. Forfter, Reife um die Welt, Edit, in 8. I. p. 86. Pafan.

(9) In dem Pfaitser (abonafo) diefes Thiẹs wird auch der orientalifche Bezoar, aber nicht fo läufig als bey der funfzehnten Gattung erzeugt. 


\section{Mammalia Pscora. Antilope. 6 az}

Linné, SyR. Nat. Edit. II. p. so Capra cornibus teretibus rectiflimis longiffimis, bafi annulatis.

Lininé, Syft. Nat. Edit. VI. p. I4. n. 8. Capra cornilius teretibus rectiflumis longiffimis, bafi annulatis.

Linne', Syft. Nat. Edit. X. I. p. 69. n. 7. Capra (Grzella) ccrnibus teretibus rectiflimis longiffi. mis, bafi annulatis.

Graumann, intr. in hift. nat. mainm. p. 48. III. A. Recticornis; der Bezoarbock.

Gatterer, brev.zool. I. p. 78. n. 3. Antilope (Re(ticornis) cornibus teretibus rectiflimis, bafi annulatis.

Gronov. zoophyl.I. p.3. n. 8. Capra cornibus teretibus rectiflimis longiflmis, bafi annulatis.

Forfk. fn. orient. p. IV. n. 15. a. Capra Gazella. Nouv. defcript. du Cap de b. E. II. p. 56. le Pafan. Abbild. gut.

26. Oreotagus. Der Klippjpringer. $\left({ }^{10}\right)$

Zimmermann, geogr, Zoolog. III. p. 269. 'der Klippenjpringer.

Büffon, vierfüls. XII. p. 248. der Klipp $p$ pringer; oder Sauteur de rochers; m. e. Fig.

Sparrmann, Reife, p. 43. 127. Felfenfpringer. p. 520. Klippenfpringer.

Batjch, Thiere, I. p. 135.der Klippspringer.

I⿳亠.冋. GA-

(10) Hat feinen Nanet ron feinem fehr fchnellen Laufen, und den weiten Sätzen, die er felbft an den feilften und fteinigtten Oertern machr, daher er fich auch nicht von den Hunden fangen läsr. Hürnér fünf Zoll lang. Grüfse einer Ziege. Der Kopf rundlich. Das Maul fehwarz. Thränenhühlen. Das Weibchen ohne Hürnery. 
15. Gazel a. Die Bezoargazelle. (')

Miiller, Naturfyf. I. p. 415. n. 9.'der Bezoarbock. Zimmermann, geogr. Zool. II. p. Iro. n. 21. die Bezoar Gazelle.

Lefke, Naturgefch. p. 182. n. 2. der Bezoarbock. Halle, vierf. p. 3r8. der Bezoarbock, Hirfchziege? Klein, quadr. difp. p. 19. Tragulus Bezaarticus; Bezoarbock?

Klein, Claffif. der vierfüfs. Thiere, p. 62. Bezoarbock?

Klein, natürl. Ordn. der vierf. Th. p. 21. n. 7 . Bezontock?

Gatterer, vom Nutzen und Schaden der Thiere, p. 167. n.91. Hirfchziege.

Martini, Naturlex. II. p. 676. Antilope mit bogenförnig gekrïnmten Hörnern.

Bitffon, vierf. XI. p. 184. Algazel.

Pallas, Naturgefch. merkw.Th. I. p. 15. Antilope mit bogenförmig gekrïmmten Hörnern.

Erxieben, Mammal. p. 274. n. 4. Antilope (Bezoartica) cornibus teretibus fubarcuatis, totis annulatis.

Graunann, intr. in hift. nat. mamm. p. 48. IV. der Bezoarbock, Hirfchziege.

Gatterer, brev. Zoolog. I. p. 79. n. 5. Antilope (Bezoartica) cornibus teretibus fubarcuatis, totis annulatis; die Hirfchziege, der Bezoarbock. Charleton, exercitat. p. II. Hircus bezoarticus? I6. LEU.

(1) Ich weifs nicht, ob ich alle Synonymen bey diefem Thier gehörig georduet habe.' Die meiften Zuologen verwechfeln es mir dem wilden Ziegrenbock, (Aegagrus) und werfen die Synonymen von diefen und mehreren Gattungen unter einander. Er:xleben fagt felbf, er wiffe nicht, was er aus dem Thiere machen foll. Aus diefer Ungewirsheit if denn auch manche nicht richtige Beftimmung der Character entfanden. 
Mammalia Pecora. Antilope.

.639

16. Le u C о Yx. Die weifse Gazelle. (2)

Zimmermann, geogr. Zoolog. II. p. 108. n. I9.

III. p. 269. die weifse Gazelle.

Büfon, vierf. XII. p. 271. die wifse Gazelle.

17. Oreas. Der Empophos. (3)

Miiller, Naturfyrt. Suppl. p. 55. n. 13. Antilope Oryx; der Cudu.

Zimmermann, geogr. Zoolog. II. p. Io9. n. 20. der Empophos.

Borow $5 k j$, Thierreich, I. 4. p. I5. n. 4. Antilope Oryx; die grofse wilde Ziegen-Anilípe.

IMartini;' Naturlex. II. p. 685. grofse vilde Zieg̀enantilope.

Biiffon, vierf. XII. p. 152. der Cudus oder Canna; m. e. Fig.

Batfch, Thiere, I. p. 133. das Canna. Pallas, Naturgefch, merkw. Th. I. p. r7. n. 14: Antilope Oryx; die wilde Ziegen Antilope.

Schwed. Abhandl. XLI. p. 134. Eland, oder das capsche Elend.

Kolbe,

(2) Hürner über drey Fufs lang, und bis an die Spitze geringelt,

(3) Wenn diefe Thiere gejagt werden, laufen fie, wenns möglich if, allezeit gegen den Wind, felbft dann, wenn ihnen der läger von diefer Seite entgegen eilt. Vielleicht können fie unter diefen Umftänden wegen ihrer Fettigkeit leichter Othem holen. Sie haben viel Fett; hefonders ums Herz, welches auch wie Ganfefchmalz auf dem Brodte gegeffen wird. Die Männchen findi allemal die fetteften, und die erften, die der ligger zu Pferde erreicht. Die Haur bey den Munnchen ift am Halfe fehr dicht und zähe, und wird nächlt der Büffelhaut für die tüchtigfte zu Ochfenriemen; Zugfeilen, Feldfebuhen u. dgl. gehalten. Die Rruft des Thiers wird unter die Leckerbiffen gerechaet. Beyde Gef́fhlechter haben Hürner. 
Kolbe, Vorgeb. d. g. Hoffn. Edit. in 4. p. 33t. das africanifche Elend - Thier.

Sparrmann, Reife, p. 410. 442. Elenne. p. 426: 438. 583. Elenthiere. p. 440. cap chíes Elennn, kappche Eland. p. 504 . capfches Elennthier. Tab. 12. Elenn-Antilope. 'Tab. 3. fig. 3. das Horn.

Le Vritlant, Reife, I. p. 264. Kudus. Evxleben, Mammal.p. 275.11.5. Antilope (Oryx) cornibus rectis, ultra medietatem bafi fpiraliter tortis, corpore grifeo.

Graumann, intr. in hift nat. mamm. p. 48. V. A. Oryx; Cudu, grofse reilde Ziegenantilope. Gatterer, brev. Zoolog. I. p. 79. n. 6. Antilope (Oryx) cornibus rectis ultra medietatem baff foiraliter tortis, corpore grifeo; die gro/se wil. de Ziegenantilope, das Kututhier.

Lobo, Abyffin. I. p. 292. Empophos.

18. S C R I P TA. Der Guib. (4)

Miiller, NaturfyRem, Suppl. p. 55. n. 13. dep Creutzbock.

Zimmernsani, gengr. Zool. II. p. IIr. n. 22. dev Guib.

Ebert, Naturl. I. p. 299. der Gib, oder Guib. Frifch, Naturfyh. p. 2. n. 4. Antilope Guib; dit Schriftantilope.

Martini, Naturlex. II. p. 643. die bunte Antilope. Biiffoiz, vierf. XII. p. 72. cier Gib; in. e. Fig. Pallas, Naturgefch. merkw. Th. I. p. 16. n. I3. die Schrift Antilope.

Sparrmann, Reife, p. 125. 255. der bunte Bock. p. 516. der bunte oder bemahlte Bock; die angefchirrte Antilope, die beriahlte Antilope.

Lie Vail.

(4) In Heerden zu 2000. Das Weibchen hat keine Hürner. 
Le Vaillant, Reife, I. p. 98. die bunten Böcke. Erxleben, Mammalia, p. 276. 11. 6. Antilope (Scripta) cornibus rectis fpiraliter tortis, corpo. re rufo-fufco, friis albis.

Graunainn, intr. in hift. nat. mamm. p. 48. VI. der Creutzbock.

Gatterer, brev. Zoolog. I. p. 79. n. 7. Antilope (Scripta) cornibus rectis, fpiraliter tortis, corpore rufo-fufco, Atriis albis; der Kreutz. bock, Gib.

19. GRIM M I D. Die grimmifche Antilope.(5)

Miiller, Naturfyftem, 1. p. 376. n. 12. Mofchue

Grimmia ; affikanifche MIOfchis.

Miiller, Naturfyft. Suppl. p. 55. n. 12. der Iung. fermbock.

Zimmermann, geogr. Zoolog. II. p. IIr. n. 23. die grimmifche Antilope, Ziege des Dr. Grimm. Borowejky, Thierreich, I. 4. p. I7. n. 6. die grimonifche Antilope.

Halle, vierf. p. 318. die amerikanifche Gazelle mit dem Haarbiifchel anf dem Kopfe.

Handb. der Naturgefch. 1. 2. p. 289. die grimmi. fiche Ziegé.

Naturforfcher, VII. p. 46. n. 3. die grimmifche is Ziege.

Frifch, Naturfyf, p. 2. n. I. Antilope Grimmii. Klein, Claflif, d. vierf. Th.p. 62. Scheonlskopf. Klein, natür. Ordn. d: vierf. Th. p. 21. n. 6. Grimins Waldbock.

\section{Martini,}

(s) Statt des Thränenfacks unter den Augen eine Höhle, worin fich eine farl riechende öligte Materie fammelt. 
Martini, Naturlex. II. p. 668. grimmijche Antilope; mit Pall. Fig.

Biiffor, vierf, XII. p. 79. die grimmifche Ziege; m. 2 Figg.

Bat fit, 'Thiere, I. p. '33. die grimmifche Antilope.' Palisis, Naturgefch. merkw. Th. I. p. I6. n. I2. - die grimuifche Antilope.

Sparrauchn, Reife, p. 520. Pallas Iurigfernboik. Grenmanin; intr. in hift. nat. mamm: p. 49. VII. der. Turigfernbock.

Gatterer, brev. zoolog. I. p. 80े. n. 8. Antilope (Grinmia) cornibus rectis, bafi fubannulatis, inter cornua fafciculo pilofo; der Iung ernbock:

\section{P Y $\mathrm{G}$ A EA. Die Zuergantilope. $\left({ }^{6}\right)$}

Zimmerminn, geogr. Zoolog. II. p. II 2 11. $246^{\circ}$ dis Zwery - Antilope, der Guevei.

Ebert, Naturl. I. p. 300. das guineifche Zwerg. hirgchchen, Guevei.

Halle, viçrfïls. p. 3aI. das bleirifte guilteifche Hirfchgen.

Handb. der Naturgefch. I. 2. p. 288. der Guèuei. Frifch, Naturfynt p. 2. n. 3. Guvezi Kagor.

Klein, Quadr. difp. p. 2t. Traguli Guineenfes? Klein, Claffif. der vierf. Thiere, p. 69: guineifeines Böckleizt, Hirfchrehtein?

Klein, natürl. Ordn. d. vierfüls. Th. p. 23. n. و. gnineifcines Bücklein?

Biiffon, vierfüls. XII. p. Irt. das andere Zwierg. hiv.chcher. p. II6. IIS. Gruevei.

Adinifon, R. nach Senegall, p. I7o. kleine guinei. fche Bö̈ckchen oder Hirjchrehe.

Sparr:

(6) Muis nit dem guineifcben ingibustbierchen (SyAt. Nit. p. 173 , n. 3.) nicht verwechfelt wetden. 
Mammalia Pecora. Antilope. 643

Sparrmann, Reife, p. 249. 256. Graometje, Err: vetje?

Schived. Abhand. XLI. p. 129. *

Erxleben, Mammal. p. 278, n. 8. Antilope (Re. gia) cornibus brevifimis rectiufculis, corpore rufo - fufco.

Graumann, intr. in hift. nat. mamm. p. 49. VIII, das kleinfte guineifche Hirfchgein.

Gatterer, brev. zoolog. I. p. 80. n. 9. Antilope (Regia) cornibus breviffimis rectiufculis, corpore rufo-fulco; der König der Hirgche.

Des Marchais, voy. en Guinée, I. p. 275. Bi. ches d' une petiteffe extraordinaire.

27. Súcuat ica. Die Waldantilope. (7)

Zimunermann, geogr. Zool. III. p. 268. n. 3. An. tilope fylvatica:

Büffon, vierfüls. XII. p. 258. der Bosbock oder Bir chbock; in. e. Fig.

Sparrmann, Reife, p. 249 . die Waldböcke. p. $5 \mathrm{I} \%$. der Bufch. oder Waldbock. Tab. 6. Waldantilope.

at. STRE Ésickos. Der Kudu.

Miiller, Naturfyll. I. p. 429. das af ricanifche Koutou, oder Kututhier; Tab. 26. fig. i. gut. fig. 2. das Horn.

Miller.

(7) Diefe Thiere Jeben in der Monogamie, tund haltea fich paarweife zufammen. Des Nachts thun fie den Weinbergen und Kohlgärten Schaden. Da fie fchwerfallig tatuen, fo gelinge es manchmal, fie mit Hunden zu fangen. Wenn fie keine andere Ausfucht fehen, ftèlien fie fich mit den jcharfen Hürnern zus Gegenwehr. Das Fleifch hat mit dem Fleifche des Guib eine Aehnlichkeit. Die Stinme foll einem ganz abgebrochenen kurzen, leifen, und ziemlich treifern Dellen des Huñdes glaichen. In Fallen fangen fic fich nich: leicht. 
Miiller, Naturfyft. Suppl. p. 55. n. 15. der Co3s. doma.

Zimmermann, geogr. Zool. II. p. Ir5. n. 29. der Kudiu.

Borowfky, Thierreich, I. 4. p. I6. 11. 5. das afri. canifche Kututhier. Tab. 32. 13.

Ebert, Naturl. I. p. 300 . das Kuduthier.

Neuer Schaupl. d. Natur, II. p. 203. Condoma.

Frifch, Naturfyft. p. 2. n. 5. Antilape Condoma; die Schlangenhörnige Antilope.

Biiffon, vierf. XII. p. 38. der Condoma oder Coes. does; m. 2 Figg.

Bat fch, Thiere, I. p. I32. das Condoma:

Martini, Naturlex. II. p. 677. Antilope mit gesundenen Hörnern.

palias, Narurgefch. merk w. Th. I. p. 19. n. 15. die Antilope mit gewandenen Hönern.

Sparrmann, Reife, P. 5Ir. 572.584 . Koedoe. (Kudu.)

Erxleben, Mammalia, p. 282. n. I3. Antilope (Strepticeros) cornibus fpiralibus, facie nigricante $\mathrm{V}$ albo notato.

Grammann, intr. in hif. nat. mamm. p. 49. XIII. Antilope mit gevenndenen Hörnevin.

Gatterer, brev. zoolog. I. p. 8I. 11. 15. Antilope (Strepficeros) cornibus fpiralibus, facie nigricante V. albo notato; der Condoma.

Nouv, defcript. du Cap de b. E. II. p. 42. Coesdoes. Abbild. gut.

Hersican, tab. affinit. animal. p. I07. Antilope Strepficeros.

22. Cervicapta. Die Antilope.

Miiller, Naturfyf. I. p. 414. 11. 8. Capra Cervica: pra; der Hirfchbock. Tab. 24. fig. 2.? 
Milller, Naturfyft. Suppl. p. 56. n. 16. Antilope Rupicapra; die bengalif he Antilope.

Zimmermann, geogr. Zoolog. II. p. Ir6. n. 30: die Antilope, die Hirfchziege.

Borow/ky, Thierreich, I. 4. p. 18. n. 8. der Be: zoarbock, dis Cervicapra.

Halle, vierfuifs. p. ${ }^{116}$. Tab. 4 die afrikanifche Gazelle.

Onómat. hift. nat. IV. p. 16, die Gazelle aus Afris $k a$, nit gedrehsten Runzeln an den Hörnern. Meyer, Thiere, III. Tab. 33. die afrikanifche Gazelle.

Frifch, Naturfynt. p. 2. n. 2. die Lidme Antilope! 11. 3. die grofse Hirfch Antilope.

Klein, Claflif, der vierf. Thiere, p. 58. gewnn? den Horn.

Klein, natürl. Ordn. der vierf. Th. p. 20. n. 4 : Gazelle.

Martini, Naturlex. II. p. 673. der Bezomb bock. Biiffon, vierfüls. XI. p. 194. 277. die Antilope; m. 2 Figg.

Batfch, Thiere, I. p. 132. die Bezoarantilope.

Pallas, Naturgefch, merkw. Th. I. p. 20. 2I: die Cervicapra. Tab. I.

Perrault, Charras und Dodart, Abhandl. zur Naturgefch. I. p. 109. anatomifche Befchreib. von fieben Gazellen. Tab. 12. dia Gazelle? Tab. I3. die Zergliederung.

Shaw, Reife, p. 152. 359. der Lidmi.

Graumann, intr. in hift, nat. matnm, p. 50. XIV. die bengalifche Antilope.

Gattever, brev, zoolog. I. p. 85. n. 76. Antilope.

(Cervicapra) cornibus fpiralibus annulatis (interdum vero laevibus) corpore fupra fufco, fubtus albo; der Hirfchbock, die Antilope.

$\mathrm{Ss}_{3}$ A) $\mathrm{Ver}$ : 
A) Vorcunderningen gegen die XIIte Edition, uind Vor. mehrung der Gattungen diefes Gefchlechts.

Das ganze Gefchlecht if neu. Die 3. 8. Ir. 74. 15. 19. und 22te Gattung find aus andern Gefchlechtern hieher verfetzt; die I. 2. 4. 5. 6. 7.92 10. 12. 13. 16. 17. 18. 20. 21, 23. 24. 25. 26. und 27 te Gattung aber find neu hinzugekonumen.

\section{3). Unbeftimntere Thiere.}

- j; Der Inpangueza.

2. Der Impalankile.

3. Die wilate Ziege Goulonga. Allgemeine Reife, V. p. 85 .

Zimmernann, geogr. Zoolog. II. p. I64. b. c. de

30. GESCHLECHT. CAPRA. Die Ziege. Miller, Naturfyftem, I. p. 404. Gen. XXX. Zimmermann, geograph. Zoolog, I. p. 159. $16 \%$. II. p. 95 . Gen. IV.

Lefke, Naturgefch, p. 182. Gen, XXXVI.

Boroweky, Thierreich, I. 4. p. 5. Gen. XXXI. Bhumeinach, Handbuch d, Naturgef Chichte, p. 106. Geti. XXX.

Becíftein, Naturgefch. Deutfchl. I. p. 610.

Onomat. hiftor: nat. II. p. 593.

Erxleben, Mammalia, p. 256. Gen. XXVIII.

Grammann, intr. in hiftor. nat, mamm. p. 46, Gen. XX'VIII.

Gatteier, brev. Zoolog. I. p. 73. Gen. XXVIII. Boddaert, Elenchus anim. Gen. XXXI. Pennant, hilt. quadrup. Gen. IV. Briffori, quadr. p. 38. Gen. IX. 


\section{Mranmalia Pecora. Capra.}

\section{AEGAGRUS. Der wilde Ziegenbock. (')}

Ziminermann, geogr. Zool. I. p. 177. Aegagrus,

II. p. 95. a. 13, der wilde Bock, die Bezonrziege.

Sthreber, Säugth. IV. Tab. 282. Capra Aegagrus. Borow $5 y$, 'Thierreich, I. 4: p. İo. b. die Bezourziege.

Bhimenbach, Handbuch der Naturgefcli. p. roy. Aegagnus.

Funke, Naturgefch. I. p. 65. Bezuarzieg8.

Gatterer, vom Nutzen und Schaden dęr Thiere,

I. p. 160. n. 84. ail bezoaraigge.

Biiffon, vierf. XI. p. 82. Aegagmus; m. Abbild. d. Höner. (unch Pallas.)

Pallas, Naturgeful. merkw. Thicre, XI. p. 45. eine eigene Gattung woider Bergziegen, die Bezoaraiege, Aegigrus.

pallas, nord. Beytrïge, IV.p. 387. Beromrbock, Bergzièze.

Beckmann, phyf. ökon. Bibl. VI. p. 266 . VIII. p. 133. Pajeng der Perfer:

Erxleben, Mammal,p. 260. Aegagrus.

Graumann, introd. in hiflor. nat. mamen. p. 46. Aegagrus.

Gatterer, bicev. Zool. I. p. 74. c. Aegagrus. Severin, zool. Hungar. p. 40. b. Aegagrus,

$$
\text { Ss } 4 \text {. }
$$

3. HIR.

(1) Lebt heerdenweife auf den erhabenten Felfen. In furchtram, belaufr fich im Herbfte, und wirft im Frühling Der von diefem Thiere kommende Bezoat ift nichts anders als eine zu Stein gewordene Velhtrtung gewiffer in dem Magen erzeugter Safte, die fich nach und 'nach fchichtenweife anhäuf́. Dér Preifs diefes Steins fteigt nach Verhältnifs , der Gröfse, wie bey den Diamanten. Ein Stein von einer Unze wird in Perfien ohngetahr mit hundert Kronen (die Krone zu einem halbẹ Livre gerechnet) bezahlt. Wiegt er aber vier und eipe Viętel Unze, fo wird er für 2000 Livres verkaufe. 
6. Hircus. Der zahme Ziegenback. (2)

Miller, Naturfyf. I. p. 404. n. F. der Bock.

Zimmermann, geogr. Zool. I. p. 159. die Ziege.

II. p. 97. c. der zahme Ziegenbock.

Schreber, Säugthiere, V. Tab. 283. Capra Hira cus vulgaris.

Lefhe, Naturgefch. p. 182. 11. 1. der Bock:

Borolesky, Thierreich, I. 4. p. 7. n. 2. der Zil= genbock.

Blumbenbach, Handb. d. Naturgefch, p. II\%. n. 5.

Capra (Hircus) mente barbato, cornibus arcuatis, carinatis; die Ziege.

Bechftein, Națurgefch. Deurfchl. I. p. 616. n. 2. die Ziege, der Bock.

Funke, Naturgefchichte, I. p. 6\%. Ziege.

Ebert, Naturlehre, I. p. 292. dis gemeiness Ziegen.

Halle, vierf. p. 302. der Ziegenbock, die Ziege. Meyer, Thiere, 1. Tab. 67. der Ziegenbock mit - vier Hörquera.

Meyer, Thiere, I. Tab. 68. die Ziege,

Gatte:

(2) Es ift fonderbar, dafs bey diefer Gattung ungleich hăufigex als bey andern Săugthieren Beyfpiele von Mănnchen geíunden worden, die Milch aus ihren Zitzen gegeben. Schon Ariftoseles befchreibt dergleichen melkbare Bücke von der Infel Lemnos. Mattbiolus hat dịe Abbildung eines folchen Bocks gegeben, und Blumexbach hat felbft einen dergleichen unterfucht und beTehrieben. Ein Beyfpiel davon findet mạn auch in Bocks Naturgefch. von Preuffen a. a. O. - Der Ziegenbuck zeugt mit dem Schafe, alier der Widder nicht mit der Ziege. - In neuern Zciten hat man Verfuche gemacht, aus den feinften Ziegenhaaren Strümpfe zu verfertigen, die den Kaftorftrümpfen gleich gewefen find. In Marokko macht man Zelte und Tauwerk davon. Aus Norwegen werden jährlich allein an rohen Bockfellen, 70 bis 80000 Stück ausgefülurt; die berciteten un gerechnet. 
Gatterer, vom Nutzen und Schaden der Thiere,

I. p. 152. n. 82. dev Ziegenbock und die Ziege. Beckmann, Naturhiftorie, p. II. n. 3. der Ziegenbock.

Neuer Schaupl. d. Natur, X. p. 2Ir. Ziege.

Onomat. hifor. nat. II. p. 598. Capra domentica; der einheimifche Bock.

Handb. der Naturgefch. I. 2. p. 46. die Ziege. Handbuch der deutfchen Thiergefchichte, p. 66, die Ziege.

Peninant, arct, Zoolog. II. p. 19. die zahnese Ziegen.

Sander, ökon, Naturgefch. I. p. 231. die Ziegen, Walther, ökonom. Naturgefch. Deutfchl. p. 172. Ziegt.

F. meiner Ziegenback.

Klein, Claffif, der vierf.' Th. p. 51. Ziegenbock. Klein, natürl. Ordn. der vierfüfs. Thiere, p. 18. Honusbock.

Bïffon, vierf. II, p. 3. die Ziege. Tab. 13. u. 14. Batfch. Thiere, I. p. 128. der geteöhnliche Ziegenbock.

Bock; Naturgefch. von Preuffen, IV. p. 132. 1. 37. der Ziegenbock, Hazusbock.

Cetti, Sardinien, I. p. rog. die Ziege.

Niebuhr, Arabien, p. 161. Ziegen.

Ḧ̈t, Marokos und Fes, p. 293. Ziegen.

Olaffen, Island, I. p. 29. Ziegen. II. p. 46. Ziegen.

Reife nach den Inf. Frankr. u, Bourbon, p. I87: Zieger.

Shawe, Reife, p. 150. die Ziege.

Pontoppidan, Norwegen, II! p. 14. Böcke und Zirgens. 
Kolbe, Vorgeb. d. g. H, p. 141. Edit. in 4. p. 3332 zainme Ziegen.

Adanron, Scnegall, p. 19. Ziegen.

Chandler, Klein-Afien, p. 184. Ziegens.

Sthweiggers, Reyfs. Befchr. aus Teufchl, nach

Conftantinopel. Nürnb: 1608. 4. $\Gamma_{2}: 263$.

pallas, Reife d. Rufsland, III. p. 142.

Oldendorp, Miflion auf den Karaiben. Barby 1777 . p. 82.

Pemant, Reife durch Schottland, I. P. I24. Ziegerv.

S. G. Gmelin, Reife d. Rufsland, III. p. 496. unnSer Hausbock.

Reytr. zut Beförder. der Naturkunde, I. p. 2I4. Ziegenbock, Zifge.

Morklein, Thierreich, p. 52. Gei/s.

Oekonom. Zoologie, p. 44. n. 55. die Ziege.

Zablonfing, allgemeines Lexicon, p. 1436. Ziege, Geifs.

Abhandl. der ökon, Gefellich, zu St. Petersburg,

II. p. 190. III. p. 67 . Ziegen.

Schwed. Abhandl. XXXVIII. p. 227. Ziegen.

Hamb. Magazin, III. p. 475. Ziegen.

Ziiskert, Speif. aus dem Thierreiche, p. zo ff.

Ludovici, Kaufmannilex. II. p. 638. HI, p. 8,

19. 445 .

Beckmann, phyl. ökon. Ribl. I. p. 185. IV. p. 37. 330. VII. p. 386. XIII. p. 410.

Becknnan, Landwirthfeh. p. 463. 464.

Bcrlin. Samml. V. p. 383. VI. p. 518. X. p. 145. Hiükel, Abhandl. von den Ziegen. Frcf, 1756 . Erxleber, Mammal. p. 256. n. x. Capra (Hircus) cornibus carinatis arcuatis.

Einné, SyR. Nat. Edit: II. p. 5o. Capra cornibus carinatis arcuatis. 
Limne, Syfl. Nat. Edit. VI. p. I4. n. I. Capra cornibus carinatis arcuatis.

Linné, Syft. Natur. Edit. X. I. p. 68. n. x. Capra (Hircus) cornibus carinatis arcuatis,

Limné, Fn. Suec. I. p. 15. n. 42. Capra cornibus carinatis arcuatis.

Graumann, intr. in hiffor. nat. mamm. p. 46.I, Bock, Ziege.

Gatterer, brev. Zool, I. p. 73. 11. 1, Capra (Hircus) cornibus carinatis, arcuatis; die Ziege, der Bock.

Severin, zoolog. Hungar. p. 38. Capra.

Fabric. fn. groenland. p: 32. 11. 6. Capra Hircus.

Kramer, Nuftr. p. 319. Capra cornibus catinatis, arcuatis.

Brown, Iamaic. p. 488. Capra cornibus carinatis, arcuatis?

Forffer, philor. transact. LVII. p. 344. Capra Hircus.

Pennant, britt: zool. p. I3: the Goat.

Varro, de re ruft. II. c. 3. caprae, hirci, et hoedi.

Cohtmella, de re ruft. Lib. VH. c. 6. caprinum pecus.

y. Angorensis. Die angorifche Ziege.(3)

Miiller, Națurfyftem, J. p. 406. die Ziegen vons Angora.

\section{Zimmer:}

(3) Die afiatifehe Türkey liefęr das Meifte von dem vortrefflichen Ziegenhaar, welches unter dem Namen der Kameelbarre zu ans gebracht wird. Es ift verboten, die Ziegen felbft aufferhalb Laudes zu fuhren. Im lahr 1723. hat man fie nach Schweden verpflanzt. Auch in B.ıyern hat man den Perfuch damit semacht. Erft die dritte Zeugung hatte vollkommen angorifche feidenhafte Haare. 
Zinnmermann, geogr. Zoolog. I. p. 167. die ant: gorifcite Ziege.

Schreber, Säugthiere, V.'Tab. 284. Capra Hircus angorenlis.

Lefke, Naturgefch. p. 182. der angorifche Bock.

Borow ${ }^{k}$ y, Thierreich, I. 4. p. 9. a. der angori. Sche Ziegenbock.

Blumenbach, Handb. der Naturgefch. p. Ios. dio angorifche Zirge, oder Kämmelthier.

Bechftein, Naturgefch. Deutfchl. I. p. 629. dis angorifche Ziege.

Funke, Naturgefch. I. p. 67. die angorifche oder risünmelziege.

Ebert, Naturl. I. p. 293. Kameelziegen.

Halle, vierf. p. $30 \%$. der angorifche Bock.

Gatterer, vom Nutzen und Schaden der Thiere,

I. p. 159. 11. 83. die angorifche Ziege, oder Kämnelsiege.

Beckmam, Naturhiftorie, p. Ir. die Kameelziege. Firifich, Naturfyft. p. I. n. 2. Caper Angolenfis; angolifcher Ziegenbock, mit widderartigenz Hörnern.

Büffon, vierf. II. p. 24 der angorifche Bock und Ziege: Tab. 15. und 16 .

Batfich, Thiere, I. p. 128. die angorifche Ziege. Bock, Naturgefch, von Preuffen, IV. p. 134. an gorifche Ziegen.

Bemerk, der churpfälz. Gefellfch. 177r. p. 280. Horneck, Bemerk, über die öflerreich. Staatsökon: (von Hermann) Berl. 1784. p. 85.

Schwed. Abhandl. V. p. $222 \mathrm{ff}$.

Beckntann, Landwirthfch. p. 464. 465 :

Beckmann, phyf. ökon. Bibl. IV. p. 37. VII. p. 529:

XIII. p. 523. XV. p. 340.

Ludovici, Kaufmannslex. III. p. .7. 
Iufti, ökon. Schrifien, II. p. 465 .

Hannöv. Samml. IV. p. 45 .

Stuttgard. phyf. ökon. Ausz. II. p. 495.

Schreber, Samml. verfchied. Schriften. III. p. 98. Erxleben, Mammiál. p. 259. Varietas Angorenfis. Graumain, intr. in hift. nat. mamm. p. 46. an" gorifche Ziege:

Gatterer, brev. Zoolog. I. p. 74. b. Varietas Angorenfis; angorifche oder Kämelziege.

2 B. Mof. Cap. 25. v. 4. Cap. 26. v. 7. Cap. 36. v. 14. Ziegenhaare?

8. M A mb I CA. Die Mamberziege. (4)

Miiller, Naturfynt. I. p. 408. n. 2. die fyrifche. Ziege.

Zimmernann, geogr. Zool. I. p. 168. die Mam. berziege.

Schreber, Säugthiere, V. Tab. 285. Capra Hircus mambrica.

Borowsky, Thierreich, I. 4. p. Io.n. 3 die Sy. rifine Ziege.

Halle, vierfüls. p. 302. das Jyrifthe Schaf mit lap. pigen Ohren.

Gatterer, vorn Nutzen und Schaden der Thiere, I. p. 163.n. 86. die Jyrifche Ziege.

Neuer Schauplatz der Natur, X. p. 212. die $\int y$. rifche Ziege.

Gesner, 'Thierbuch, p. 153. eine indianifche $G_{8 i} \int_{s}$; Figg. p. 154. inittelm.

Biiffon, vierf. XI. p. 35.39. die Mambrine oder levantifche Ziege.

Erxleben, Mammal. p. 264. n. 3. Capra (Mambrica) cornibus reclinatis, auriculis pendulis.

Linne,

(4) Hat ihren Namen rom Berge Mamber in Syrien; ob tie gląich durch gasz Klein-Afien, Indien und Aegypten geht. 
Linné, Syft. Nat. Edit. II. p. 51. Capra auribus pendulis longifimis.

Linazé; Sylt. Nat. Edit. VI. p. I4. n. Ir. Capra auribus pendulis longifimis.

Limé, Syft. Nat. Edit. X: I. p. 7c. n. rì. Capra (Mambrica) auribus pendulis longifimis.

Graumanin, intr. in his. nat. manm. p. 47. III. die Jyrifche Ziege.

Gatterer, brev. zool. I. p. 75. n. 3. Capra (Mam. brica) cornibus reclinatie, auriculis pendulis; die Syrifche Ziege, Mambrine.

Luillier, voy. Rotterdam. I726. p. 30.

E. DEPRESSA. Der afrikanifche Ziegenbock.

Miiller, Naturfyht. I. p. 4II. n. 5. der Zwergbock. Zimmernanin, geogr. Zool. I. p. 169. der afrikanis she Ziegenbock.

Schreber, Säugth. V. Tab. 287. Capła Hircus depreffa.

Borowejky. Thierreich, I. 4 p. II. n. 4 det Zwergbock.

Gattbrer, vom Nutżen u. Schaden der Thiere, I. p. I63. n. 87. der Zwergbock.

Beckmann, Naturtiff. p. I2. n. 7. die Zivergböcke? Biiffon, vierfürs. XI. p. 38. die kleine Ziege; init 2 Figg. der afrikanifche Ziegenbock, und die Zwergaiege.

Erxleben; Mammal. p. 266. n. 4. Capra (Deprefia) cornibus deprefis incurvis minimis, cranio incumbentibus.

Linné, Sylt. Nat. Edit. II. p. 50. Capra corni. bus depretlis incurvis minimis, cranio incum. bentibus.

I*inné, S. N.Ed: VI. p. I4. 1. 2. Capra comibus deprellis incurvis minimis, cranio incumbentibus. 
Linné, Syft. Nat. Edit. X' I p. 69. n. 4. Capra (Depreffa) cornibus depreflis incurvis minimis, cranio incumbentibus.

Linné, Syft. Natur. Edit. XII. p. 95. n. 5. Capra (Depreffa) cornibus depreflis incurvis minimis, cranio incumbentibus, gula barbata.

Graumnani, intr. in hin. nat, mamm. p. 47 IV. der Zwergbock.

Gatterer, brev. Zoolog. I. p. 76. n. 5. Capra (Depreffa) cornibus depreftis incurvis minimis, cranio incumbentibus; der Zwergbock, afrika. hiffhe Bock.

3. REversa. Die Ziege von Inida. (5)

Tizitler, Naturfyltem, I. p. 411. 1, 6. die amerio kanifche Geinse.

Zimmersnann, geogr. Zool. I. p. 168. n, 4. die - Ziege von Inidi, oder Whida.

Schreber, Säugthiere, V. Tab. $2 \$ 6$. A. B: Capra Hircus reverfa.

Borosesky, Thierreich, I. 4. Ir n. 5 . die amevikanifche Gemje, dèr Bock von Iuda.

Biiffon, vierfüls. XI. p. 38. die kleine Ziege mit geraden Hörnern, und umgebogenen Spitzen. p. II8. die Ziege von Iuda; m. 2 Figg.

Beckmanin, phỳ ökon. Bibl. III. p. 304. itie Ziege voin Iuda.

Erxleben, Mammal.p. 267; n. 5. Capra (Reverfa) comibus erectis, apice recurvis.

Limmé, Syft Nat. Edit. II. p. 5o. Capra cornibus "erectis, apice recurvis.

Limné, Synt. Nat. Edit. VI. p. 69. n. 5. Capra cornibus ereclis, apice recurvis.

Limié,

(5) Erxleben hat diefe, und die beyden vorhergehenden Varietatan, als befondere Gattungen aufgefihts. 
Limne, Syft. Nat. Edit. X. I. p. 69. n. 5. Capra (Reverfa) cornibus erectis, apice recurvis.

Graumann, intr. in hif. nat. mamm. p. 47. Vे. die amerikanifche Genite.

Gatterer, brev. zool. I. p. 76. n. 5. Capra (Reverfa) cornibus erectis, apice recurvis; der Bock and die Ziege von Inda, die amerikanische Gemss.

7. C APRICORNUS. Der Capricom. $\left({ }^{(}\right)$

Zimmermani, geogr. Zool. I. p. 177. II. p. 98. der Capricorn.

Biiffor, vierf. XI. p. 38. n. 2. der Capricorm.

Erxleben, Mammal. p. 264. Capricornus.

Graumian, intr. in hift. nat. mamm. p. 47. Ca. pricornus.

Gatterer, brev. zool. I. p. 75. Capricornus.

2. IBEX. Der Steinbock.( ()

Miiller, Naturfyft. I. p. 407 n. 2. der Steinbock. Zimmermanit, geogr. Zool. I. p. I74. II. p. 96. 11. 14. der Steinbock.

\section{Schreber}

(6) Nach B̈̈iffort ein Mittelding zwifchen dem Steinbock nnd dem "zahmen Ziegenbock; nach Penmat eine blolse Varierät deg Ziegenboeks.

(5) Wird grürser als eine Ziege, und wiegt im Alter wohl einige Centner, kann aber doch mit grofser Leichtigkeit jähe Felfenwande hinanfetzen, und über tiefe Abgründe von einer Klippe eur andern fpringen. Erft im dritten lahre follen diefe Thiere anfangeri Hüner aufzufetzen, und jedes derfelben dann jählich einen neuen Knoten erhalten; daher diefe Knoten; oder hükrigen Querkanten jedesmal in defto grüfserez Anzahl vorhanden find, je äleer das. Thier ift. Bey einem ganz ausgewachfenen Steinbock ift ihre Zahl gewöhnlich zwanzig. Sonft bäit 
Schreber, Säugth. V. Tab. 281. Capra Ibex. Lefke, Naturgefch. p. 183. n. 2. der Steinbock. Borowfky, Thierreich, I. 4. p. 5. n. I. der Steinbock. Tab. 3r.

Bhumenbach, Handb. der Naturgefch. p. I08. n. 4. Capra (Ibex) mento barbato, cornibus lunatis maxinis, fupra nocofis, in dorfum reclinatis; der Steinbock.

Bechftein, Naturgefch. Deutfchl, I. p. 6IO. dey Steinbock, Tab. 13.

Fanke, Naturgefch. I, p. 66, der Steinbock.

Ebert, Naturl. I. p. 293. der Steinbock.

Halle, vierf. p. 312. der Steinbock.

Gatterer, vom Nutzen u. Schaden der Thiere,

I. p. 161. n. 85. der Steinbock.

Beckmann, Naturhil. p. II. n. 4. der Steinbock. Pennant, arct. Zool. II. p. 19. n. 4 der Steinbock. Neuer Schaupl. d. Natur, VIII. p. 505. Steinbock. Onomat. hiffor. nat. IV. p. 48r. Steinbock. Handbuch der Naturgefch. I. 2. p. 277. dev Steinbock.

Handbuch d. deutfchen Thiergefch. p. 66. der Steinbock.

Frifch, Naturfyft. p. I. n. 1. Capricornus, Ibex; der europöif che Steinbock.

Klein, Claffif. der vierf. Th. p. 53. Steinbock.

Kllein,

fich die Länge dec. Hürner gemeiniglich zwichen zwey Furs fechs Zoll und drey Fufs, und das Gericht von beyden zwifchen lechzehn und zwanzig Pfunden. Das Fleifch wird zwar ivi der Schweiz gegeffen, ift aber zahe uad unverdaulich. Aus den Hörnern macht man Trinkgefchirre, und die Türken verfertigen Bogen daraus. "Aus der Haut wird Leder bereitet, Der Steinbock foll der Blindheit fehr unterworfen feyn. Seis 200 Iahren hat fich diefe Thierart febr. vermindert. 
Klein, nat. Ordn. d. vierf. Th. p. 18. n. 2. Steinbock. Gesner, Thierbuch, p. I48. Steinbock, Tbfchen, eder $T b \int c h . G e y \int s ;$ m. e. mittelm. Fig.

Biiffon, vierf. XI. p. 7. der Steinbock. p. 60. der fibirifche Steinbock; m. e. Fig.

Bat fch, Thiere, I. p. I28. der Steinbock.

Pallas, Naturgefch. merkw. Th. XI. p. 32. der fibirifche Steinbock.

Höpfner, Magazin für d. Naturkunde Helvetiens, II. p. 23. Steinbock.

Pallas, Reife, Ausz. III. p. 297. Steinzböcke.

Lichtenberg u. Voigt, Magazin für das Neuefte etc.

III. 2. p. 78. Steinbock.

Wolf, Reife nach Zeilan, p. 12r. Steinbock.

Beckmann, phyf. ök, Bibl. XV. p. 380 . Stcinbock. Tablonfky, allgem. Lex. p. rr29. Steinbock; lbex. Prinz Eugens Thiergarten. Augfp. I734. Tab. 3. Steinbock.

Linné, Syft. Nat. Edit: II. p. 50. Capra cornibus nodofis in dorfum reclinatis.

Linné, Syft. Natur. Edit. VI. p. I4. n. 6. Capra cornibus nodofis in dorfum reclinatis.

Linné, Syf. Natur. Edit.X. I. p. 68. n. 2. Capra (Ibex) cornibus nodofis in dorfum reclinatis.

Graumann, intr. in hift. nat. mamm. p. 47. II. Steinbock.

Gatterer, brev. Zoolog. I. p. 75. n. 2. Capra (Ibex) cornibus lunatis rotundatis, fupra nodofis, in dorfum reclinatis; der Steinbock. Severin, zool. Hungar. p. 39. Ibex; Steinbock. Kramer, Auftr. p. 32I. Capra cornibus nodofis, in dorfum reclinatis.

Forfk. fn. orient. p. IV. n. 15. b. Capra Ihex. Aelian. de nat. anim. XIV.c. 16. Alyas aygrob ev $\Lambda$ is ${ }_{\text {vin? }}$ 


\section{Mammalia Pecora. Capra.}

659

3. CA U A s r ca. Der kaukaffeche Steinbock. ( $\left.{ }^{8}\right)$

Schreber, Säugth. V. Tab. 281. B. Capra caucáfica Güildent.

B iffon, vierf. Xİ. p. Iö2. der kaukajifche Stein. bock; m. e. Fig.

Pallas, nord. Beytr. IV. p. 386. der kaikafif che Steinbock. Tab. 2.

Lichtemberg, Magazin für das Neuefte etc. II. 4 . p. 44. der kaikafifche Sieinbock.

Verïnderangen gëgen die XIIte Edition, und Veru mehrung der Gattungen diefes Gefchlechts.
Edit. XII.
Edit. XIII.

p. 95. n. 4. Capara Rupica- p. 182.n. 3. Antilope Rupi pra. capra.

p. 95. n. 7. Capra Gazella. p. 189.n. 14. Antilope Oryx. p. 96. n. 8. Capra Cervica- p. 192. n.22. Antilope Cerpra. vicapra.

p. 96. n. 9: Capra Bezoar- p. 190. n. 15. Antilope Ga: tica. zella.

p. 96. n. 10. Capra Dorcás. p. 187. n. ri. Antilope Dor= cas.

97. n. Ir. Capra Tata p. 185. n. 8. Antilope Saiga: rica.

p.97.n. 12. Capra Ammón. p. 200. n. 2. Ovis Ammon.

$\mathrm{Da}$ diefs Gefchlecht in der XIIten Edition 12 Gattungen hatte, in der XIIIten aber nur 3 hat, fo ift es hier um 9 Gattungen vermindert; $\mathrm{Tt} 2$ wovou

(8) Das Fleifch diefes Thiers wird unter dèn Bergïülkern und Georgianern für eine Leckerfpeife gehalten, und die Hürner dienen ihnen ftatt der Pokale. Iung Gefangene fterben, wie die Gemfen, bald in der Gefangenfchafe. 
wovon 7 in andere Gefchlechter verfetzt, dio erjte, dritte, vierte und fünfte aber fub $\beta, \delta, \varepsilon$, und $\zeta$, als Varietäten von der neuen Gattung Aegagrus angenommen find. Die dritte Gattung Capra Caucafica if gleichfalls neu hinzugekommen; und Biiffons Kaprikarn fub n als Spiel. art zur erften Gattung gerechnet.

31. GES CHLE CHT. Ovis. Das Schaf. Miller, Naturfyrism, I. p. 42r. Gen. XXXI. Zinmermann, geograph. Zoolog. I. p. 159 : II. p. 94. Gen. III. Lefke, Naturgefch. p. 183. Gen. XXXVII. Borow kyy, Thierreich, I. 4. p. 25. Gen. XXXIII. Bechftein, Naturgefch. Deutfchl. 1. p. 63I. Peniant, arct. Zool. II. p. 15. Neuer Schauplatz der Natur, VII. p. 566. Onomat. hift. nat. V. p. 826. Biiffon, vierfürs. I. p. 286 . IX. p. 249. Erxleben, Mammalia, p. 242. Gen. XXVII. Graumann, intr. in hiftor. nat. mamm. p. 45 . Gen. XXVII.

Gatterer, brev. Zoolog. I. p. 68. Gen. XXVII. Boddaert, elench. anim. Gen. XXXII. Pennant, hift. quadr. p. 38. Gen. III. Briffon, quadr. p. 48. Gen. X. Hermann; tab. affin. animal. p. Ioz: rog. IIo.

1. ARIES. Das gemeine Schaf. (1)

Miiller, Naturfyftem, I. p. 422.n. I. der Widder. Ziszmer.

(1) Widaler (das Männchen), Schaf (das Weibchen), Scböps, Hammel (verfchnitten), Lamm (ein Iunges), Stöbrlamm, Bocklamms 
Zimmermanin, geogr. Zool, I. p. 159 . II. p. 95. das Scilaaf.

Lefke, Naturgefch. p. x83. n. I. das gemaine Schaf. Borowsky, Thierreich, I. 4. p, 26. n. I. dev Widder.

Blumenbach, Handb. d. Naturgefch, p. 106, n. $\mathbf{x}$. Capra (Ovis) mento imberbi, cornibus compreffis lunatis; das Schaquf.

Bechftein, Naturgefch. DeutChl. I. p. 632. das gen meine Schaf:

Fumke, Natargefch. I. p. 24. das Sthaaf.

Ebert, Naturl, I. p. 288. Schaf.

Halle, vierf. p. 28r. Wiuder, Schaf. Tab.1r.

Meyer, Thiere, I.'Tab. 5o. der ungarifche Widder.

Meyer, Thiere, I. Tab. 5r. das Schaaf.

Gatterer, vom Nutzen und Schaden der Thiere,

I. p. 136. 11, 78, das Schaaf, der Widder.

Beckmann, Naturhiftorie, p. 10. n. 2. das Schaaf. Neưer Schauplatz der Natur, VII. p. 566. Scharff.

Onomat. hif. nat. V. p. 328. das gemeine Schaaf, der. Widder.

Handbucl der Naturgefchichte, I. 2. p.35, das Schaaf.

Handbuch der deutfch. Thiergefchichte, p. 63. das Schaaf.

\section{Tt 3}

Sonder,

Iamin (männl. Gefchl. im erften Iahr), Schaf-Kälber-KilberScbibben-Zibben-Lamm (weibl: Gefchl. im erften Jahr), gefibnitten Lamon (Hammel im erften lahi), zweyzäbriger Iährling (im zwcyten), vierzäbriger (im dritten), Jechszähniger (im vierten), vollmäuliger Hnmmel (im fünften lahre), Mutterlamm (Lamm im erften), Ithrling (im zweyten), Schilke (im dritten), vollwäulig (im vierten lahre). Da das Schaf fich, fehr leicht mit der Ziege begatret, und ïberhaupt in feiner ganzen Oekonomie und Körperbau fehe nahe mit ihr verwandt if, fo rechnen bewatrte Naturhiftoriker es mit derfelben zu cincm Gefchlechte. 
Sander, ökon, Naturgefch. I. p. 22r. dis Schafe. Walther, ökon, Naturgefch. Deutfchl. p. 165़. Schafe.

Frifch, Naturfynt. p. I. n, I. Ovis vulgaris; $\mathrm{g}_{*}$ mein Wollen - Schaf.

Klein, quadr. difp. p. 13. Aries, Widder; Ovis, Schaaf.

Klein, Claflif. d, vierf. Thiere, p. 44. Wido der, Schaaf.

Klein, natürl. Ordn, d. vierf, Thiere, p. 15. II. Widder, Schaaf.

Biiffon, vierfüfs. I. p. 286. der. Widder and das Schaf. Tab. I2.

Bat $/$ t? Thiere, I. p. 125. das Schaaf.

Bock, Naturgefchichte von Preuffen, IV, p. 138, das Schaf.

Fifcher, Naturgefch. von Livland, p. r\%. Sehafe. Fif ches, Zufätze zur Naturgefch. yon Livl. p. 36. Schafe.

Dobrizhoffer, Gefch, der Abiponer, I. P. 315. Schaafe.

Taube, Slavonien und Syrmien, I. p. 38. Schafe, Pennant, Reile durch Schottland, II. p. 272. Schanfe.

Cetti, Sardinien, I. p. 85. Schaf:

Pontoppidan, Norwegen, II. p. 13. Schafe.

Merklein, Thierreich, p. 188. Schaaf.

Oekonom, Zoologie, p. 46. n. 56. das gembine

Schaaf.

Beckmaznn, Landwirthfchaft, p. 44\%. Schaaf:

Beytr. zur Beförderung d. Națurkunde, I. p. 215: Schaf und Widder.

Jablonfky, allgem, Lex. p. 974. Schaaf. Erxlebier, Mammal. f. 242. n. I. Ovis (Aries) cornibus compreffis lunatis: 
Linné, SyfR. Nat: Edit, II. p. 5 I. Ovis cornibus compreffis lunatis.

Linné, Syft. Natur. Edit. VI. p, 15. n. I. Ovis cornibus compreflis lunatis.

Linné, Syft. Nat, Edit, X, I, p. Yo. n. I. Ovis (Aries) cornibus compreflis lumatis.

Linné, Fu. Suec, I, p. 15. n. 43. Ovis cornibus compreflis lunatis.

Graumann, intr, in hifor. nat. mamm. p. 45. I. Widder, Schafbock.

Gatterer, brev. zool. I. p. 68, n. T. Ovis (Aries) cornibus compreflis lunatis; der Widder, das Schaf.

Severin, zool. Hungar. p. 57. Ovis.

Fabrir. faun, groenl. p. 39. n. 5. Ovis Aries.

Milller, zool, dan, prodrom. p. 6. n. 40. Ovis

(Aries) cornibus compreflis lunatis.

Kramer, Auftr, p. 321 . Ovis cornibus compreffis . lunatis.

Schecenkfeld, theriotroph Silef. p. 55. Aries. Forfter, philoloph. Transact. LVIl. p. 344. Ovis Aries.

Pennant, britt. zool. p. ro. the Sheep.

Varro, de re ruft, L, II. c. 2, de ovibus, arietibus et agnis.

Cohumella, de re ruft. L. VII, c. 2. ovillum pecus.'

a. A N g I I A. Das engländifche Schaf. ( $\left.{ }^{2}\right)$

Miiller, Naturfyftem, I. p. 422. B. das englündiSche Schaf.

\section{Tt 4}

Zimmer-

(2) In England felbft gilt ein guter Widder $=$ bis 3 no Thaler, und noch dazu ift die Ausführung bey fchwerer Strafe verboten: Ein zweyiahriges Mutterfchaf liefert an 6 Pfund Wolle, ein Widder bis 8 , und ein Hammel bis 9 Pfund alle lahre. Gemeiniglich find beyde Gefchlechter gehơrnt. 
Zinmermama, geogr, Zool. I. p. 167. das horis. lnfe Schaf.

Borowjky, Thierreicl, ,I. 4. p. 28. a. das englifche Schaf.

Bechftein, Naturgefch. Deutfchl. I. p. 668. b. das engliscine Schaf.

Funke, Naturgefch. I. p. 26. die englifchen Schaafe. Neuer Schaupl. der Natur, VII. p. 566. das engli- Sche Schaaf.

Onomat. hift. nat. V. p. 858. das englifche Schaaf. Pallas, Naturgefch. merkw. Thiere, XI. p. 6I. * die gemeinen englifchen Schaafe.

Ellis, englifche Schafzucht; in Schrebers Samml. verfch. Schrifren, XI - XVI Th.

Erxleben, Mammal. p. 246. a. O. Anglica.

Graumann, introd, in hifor. nat.mamm. p. 45. a. O. Anglica.

Gatterer, brev, Zoolog, I. p. 7r. a. O. Anglica; das englifche Schaf.

Severin, zool, Hungar. p. 57. a. Anglica; pro: ceritatis magnae lanaque molli,

B. Rus т ic a. Das nordifche Schaf.(3)

Miiller, Naturfyf. I. p. 422. A. nordifches Schaaf. Borow fky, Thierreich, I. 4. p. 22. b. das nordifche Schaf.

Zimmermann, geogr, Zool, I. p. 163.

Neuer Schauplatz der Natur, Vil. p. 566, das nor. difine Schanf:

Onomat. hilt. nat. V. p. 837. das nordifche Schaaf. Biiffon, vierf. IX. p. 262. das nordifche Schaaf.

Pallas,

(3) Lảmmer, die aus der Vermirchang diefes Schafs imit dem Geifsbock entftehen, fehen der Mutter ganz ăhnlich, auffer dafs fie eine fehr harigre Wolle haben. 


\section{Mammatia Pecora. Ovis.}

Pallas, Reife d. RufsI. I. p. 78. Schaafe.

Pallas, Naturgefch. merkw. Th. XI. p. 60. dic zweyte Spielart, das milfichs Sckaaf. p. 63. die kurzfchwönzige Sorte.

Erxleben, Mammal. p. 246. B. Ruftica.

Graumann, intr. in hiffor. nat. mamm. p. 45. b. nordifches Schaaf.

Gatterer, brev. Zoolog. I. p. 70. b. O. Ruftica; das genseine Schiaf.

\%. His P AN I A. Das fpanifche Schaf:(4)

Müller, Naturfyft. I. p. 423. C. das Jpanifche Schaaf.

Borovefky, Thierreich, I. 4. p. 29. c. das fpantiSche Schaf.

Bechftein, Naturgefch. Deutfchl. I. p. 667. das Spanifche Schaf; Tab. 13. b. fig. 2.

Funke, Naturgefch. I. p. 26. die Spani/chen Schaafe. Ebert, Naturlehre, I. p. 289. die Spanifchen Scíafe.

Neuer Schaupl. der Natur, VII. p. 566. das Spamifche Sehaaf.

Onomat. hift. nat. V. p. 837. die Jpanifchen Schaafe. Lefke und Hindenburg, Magazin zur Naturkunde, III. p. 319. Spanifche Schafe.

Stumpf, Gefchichte der Schäfereyen in Spanien. Lpz. I785.

Limné, weftgoth. Reife, p.-58. die Spanifchen Schaaf 8 .

Linné, Verfuch einer Natur - u. f. w. HiA, von einigen Schwed. Provinzen, p. 90. die Jpanifthen Schaaf b. p. I72. die Spanifchen Schaafböcke.

$$
\text { T } 5 \text {. }
$$

Char.

(4) In Spanien werfen 6000 folcher Schafe Inach Abzug aller KO. ten ẹinen Gewingn von 36000 .Thalexn, unfres Geldes ab. 
Clarkes, Briefe von Spanien, p. 733.

Magazin für den Landmann, I. p. 377.

Mannichfaltigkeiten, II. p. 592.

Hanuöv. Magazin, XIIl. p. 24I.

Oekonom. Nachrichten, XIII. p. 69.

Erxleben, Mammal. p. 247. $\gamma$. O. Hifpanica.

Graumamn, intr. in hifor. nat. mamm. p. 45 . c. das Jpanifche Schaaf.

Gatterer, brev. zoolog. I. p. 7x. e. O. Hifpanica; das Spanifche Schaf.

Severin, zool. Hung. p. 38. b. O. Hifpanica.

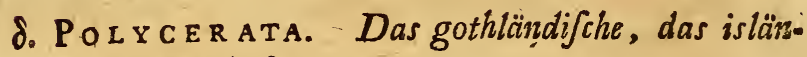
difche Schaf. (')

Miiller, Naturfyft. I. p. 423. D. das gothländifche Schaf.

Zimmermann, geogr. Zoolog. I. p. 163. das nor. difche Schaf.

Schreber, Säugthiere, V. Tab. 289. Ovis Aries brachyura islandica.

Borowfky, Thierreich, I. 4. p. 29. d. das isländiche Schaf.

Buiffon, vierfüls. IX. p. 253. Schafe in Island; $\mathrm{m}, 2$ Figg.

Neuer Schaupl. der Natur, VII. p. 567. das gothtimdifche Schaaf.

Olaffen,

(5) Sonderbar ift der Gebrauch, dấs man in Island die Bücke durch ein kleines durchlüchertes Brett,-welches in der Wolle feft genthet, und nicht eher als gegen Weynachten abgenommen wird, abhalt, die Schafe zu befpringen, damit fie nicht zu früh werfen, und bey einem eintretenden harten Frühjahr das Leben darüber einbiilsen mïgen. In den Iahren 1740 bis \$750. hat die Schafpeat ganz enorm in Island gewütet. Olaffen und Uno von Troil leugnen das Dafeyn der wilden Sçhafe, welches einige Fremde vorgegeben haben. 
Mammualia Pecora. Ovis. 667

Olaffen, Reife nach Island, J. p. 28. 102. 279. II. p. 45. I18. 198. Schaafe.

Uno von Troil, Reife nach Island, p. ror. die Schafe.

De Kerguelen Tremarec, Reife nach der Nordfee, p. 72. Schaafe.

Limne', goth. Keife, p. 248. Schaafe.

Beckmann, phyf. ökonom. Bibl. VI. p. 184. die - Schafe.

Handb. der Naturgefch. 1. 2. p. 260. die isländiSchen Schaafe.

Erxleben, Mammalia, p. 247. $\delta$. Ovis Polycerata.

Graumann, intr. in hifor. nat, mamm, p, 45. d. das gothländifche Schaaf.

Gatterer, brev, Zoolog. I. p. 71. d. O. Polyce. rata; das isläridifche oder gothlündifche Schaf.

Severin, zool, Hungar, p. 38. c. O. Polycerata,

\&. A F R I C A A. Das afrikanifche Schaf. $\left({ }^{6}\right)$

Mïller; Naturfyn. I. p. 423. E. das afrikanifche Schaaf.

Borowjky. Thierreich, I, 4. p. 30. c. das afrikianifche Schaf.

Neuer Schaupl. der Natur, VII. p. 567, das afri. kanifche und äthiopifche Schaf,

Erxleben, Mamınal. p. 248. \&. O. Africana.

Graumann, intr. in hiftor. nat. mamm. p. 45 . c. das afrikanifche Schaaf:

Gatterer, brev. Zoolog. I. p. 7r. e. O. Africana; das af rikanifche oder äthiopifche Schaf.

S: Gul-

(5) Kommt foṇt an Geftalt mit unferm Schafe überein, hat aber mehr boxtenartige Haare als Wolle. 
そ. Gurneensis. Der Adimain.(?)

Miiller, Naturfyntem, I. p. 428. n. 2. das guineifche Schaaf.

Zimmermain, geogr. Zoolog. I. p. 166. der Adimain.

Sthreber, Säugthiere, V. Tab. 294. A. B: C. Ovis Aries guineenfis.

Bororefky, Thierreich, I. 4. p. 32. n. 3. das guineifche Schaf.

Ebert, Naturlehre, I. p. 290. das guineifche, angolifche, oder Senegalifche Schaf, der Adimain oder Adimmain.

Halle, vierfüls. p. zor. das guinneifche Schaf.

Gairerer, vom Nutzen und Schaden der Thiere,

I. p. 191. "11. 80. das guisseifche oder ina'iani. Sche Schaof.

Neuer Schaupl. der Natur, VII. p. 568. das guineifche Schaaf.

Onomat. hiftor. nat. V. p. 843. die Schaafe aus Guinzea.

Onomat, hif, nat. I. p. 774. das angolifche Schaf. Frifch, Naturfyftem, p. I. n. I. Ovis Guineenfis; das Adimari.

Klein, Claflif. der vierf. Th. p. 48. guinefifcher Widder.

Klein, natürl. Ordn. d. vierf. Th. p. I7. n. 3. guineificher Widder.

Biiffon, vierfüls. I. p. 287. n. 4. das guinzifche Schaf; der guineifche Widder oder Verföhn. bock.

Biiffon,

(7) Ift in keiner Gegend mit Wolle bedeckt, fondern trāgt pald ein fprödes, bald ein weicheres Haar, nachdem das Klima, wn es lebt, heifs ift. Hat, wie das kirgififche Schaf, Dütten untep dem Halle. Erxleben hat eine eigene Gattung daraus gcmacht. 


\section{Mammalia $p_{\text {ecora. }}$ Ovis.}

Buiffon, vierfüls. IX. p. 260. 265. der Adimain; m. 3 Figg.

Adainfon, Keife nach Senegall, p. 53. die Hammel oder die Widder.

Shaw, Reife, p. 15o. das Schaf in der Sahara. Erxleben, Mammal. p. 253. n. 3. Ovis (Guineenfis) auribus pendulis, palearibus laxis pilofis, occipite prominente.

Limne, SyAt. Natur. Edit. II, p. sr. Ovis auribus pendulis, palearibus laxis, occipite prominente.

Linné, Synt Nat: Edit. VI. p. 15. n. 3. Ovis auribus pendulis, palearibus laxis, occipite pro. minente.

Linné, Syft. Nat. Edit. X. I. p. 7r. n. 2. Ovis (Guineenfis) auribus pendulis, palearibus laxis, occipite prominente.

Graumann, intr. in hift. nat. mamm. p. 46. III. das givineifche Schaaf.

Gatterer, brev. zool. I. p. 72. n. 3. Ovis (Gui. neenfis) auribus pendulss, palearibus laxis piLofis, occipite prominente; das guineifche oder indianifche Schaf.

Leon. African. defcript. Afr. p. 753. Adimain.

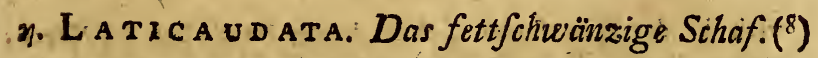
Miiller, Naturfyn. I. p. 423. F. das arabifche. Schaaf.

Zimnner.

(8) Das grüfsts und ungeftaltefte von allem Wollesvieh.' Wiegs oftmals, 200 Ptund. Har einen flark gebogenen Kopf, grofse hàngende Oliren und ojfters Dürten, oder fo genannte Haárglocken, einzeln oder doppelt am Halfe. Der Schwanz, der fich in zwey Theile theilt, unten faft ganz kahl ift, wird an to Pfund facher, und giebt 20 bis $z^{\circ}$ Pfuad Talg. Bey keinem Wollen- 
Zintmermann, geogr. Zool. 1. p. 163. das Sciraf mit dem dicken Schwanze.

Sihreber; Säugthiere, V. Tab. 292. Ovis Aries Reatopygos.

Borowfky, Thierreich, I. 4. p. zo. g. das arabi. Sche langgefchwänzte Schaf.

Bhumenbach, Handb. der Naturgefchichte, p. I06. die arabifchen Schaafe.

Funke, Naturgefchichte, I. p. 24. die'aribifeilent Sc haafe.

Ebert, Naturl. I. p. 290. das arabische Schaf.

Halle, vierf. p. zor. das arabifche breitf chroänzige Schaf.

Neuer Schauplatz der Natur, Vil. p. 567. das ardbische Schaaf.

Onomat. hiff. nat. V. p. 843 das arabifche Schaaf. Klein, Claffif. der vierf. Thiere; p. 46. orientalifcher brieitgefchwänater Widder.

Klein, natürl. Ordn. d. vierffüls. Th. p. I7. n. $\mathbf{r}$. breitgefchwoürzter Widder.

Biiffon, vierf. I. p. 287. n. 2. das arabijche breit. Schwönzzige Schaf.

Büffon, vierf. IX, p. 254. das Schaf aus Arabienti.

Ausz.

vieh in der Welt finden fich fo häufig Haarballen im Magế, als bey diefem. Cremeiniglich beftehen fie aus zarter bráunlicher Kancelivolle, die wie die feinfte Filzmaterie lagenweife durch die Bewegung des Magens zufammengeballt ift. Die Urfach hiezu giebr der falzige Schweifs, der auf dem Leibe der Kameele, die fich faft von lauter Salzpflanzen nahren, bey trocknem Wetter, fonderlich wenn fie durch Laufén erhitzt gewefen, überał ausbricht. Die Schafe ftellen fich um die liegenden Kameele, und lecken diefen Salzreif, wobey-fie viel Kameelhaare mit verfchlucken, woraus die Haarballen entftehen. Man findet he von der Grớse einer Nufs bis zum Hülinerey. - Die Widdet find durchgingig, und auch die Hammel grürstentheils gehürnt: 
Ausz. aus dem Tageb. eines Reif. n. A fien, p. 82 . das Schaaf.

Pallas, Naturgefch. merkw. Th. XI. p. 65. fettSchwänzige Schaafe. p. 83. der kirgififche Widder. Tab. 4.

Pallas, Reife, Ausz. I. p. 256. kalmiickifche Schaa.

fe. p. 34r. die kirgiffichen Schaafe.

Niebuhr, Lrabien, p. 16r. Schafe.

S. G. Gmelin;, Reife durch Rufsland; II. p. 156. kalmiickifche Schafe mit. Fettfchwönzen.

Pallas, nord. Beyträge, IV. p. 393. kalmiickifche Schafe mit polfterförmigem Fettcinwonze.

Reife nach der Infel Frankr. u. Bonibon, p.294. Scháa mit einem dicken Schwanzs.

Leon. Afric. defcript. Afr. p. 753. Aries.

Georgi, Rufsland, I. p. 174. 207. 218. breit. fcherinzaige Situafe.

Shane, Reife, p. 150. Schanfe mit einem breitert S.hwanze.

Beckinann, phyf ökon. Bibl. VII. p. 394. Schafe mit dem Fettfchwanze.

Neuhof, Gefandfchaft nachi China, p. 346. perfifche und tartarifiche Schafe?

Erxleben. Mammalia, p. 248.- 3. Ovis Laticaudata.

Granımann, intr. in hiftor. nat. mamm. p. 45 . f. das arabifche Schaaf.

Gatterer, brev. Loolog. I. p. 7r. f. O. Laticaudata;' das arabifche, abefinifche oder breitgeSchwänzte Schaf.

Severin, zoolog. Hungar. p. 38. d. O. Laticaudata.

2 B. Mof. e. 29. v. 22. - 3 R. Mof. c. 7. v. 3. c. 8. v. 2.25. c. 9. v. 19. Widider. 
9. B ч С а в I с A. Das bucharifche Schaf. (")

Zimmermann, geogr. Zool. I. p. 164. die bucharifchen Schafe.

Pallas, Naturgefch. merkw. Th. XI. p. 60. 8I. bucharifche Schaafe.

Beckmann, phyf. ökonom. Bibl. VIII. p. 135. die bucharifchen Schafe.

Georgi, Rufsland, I. p. I48. eine breitfchwänzige Art, mit längerm Schwanze, und feiner Wolle.

6. LONGICAUDATA. Das langgefohwainzte Scinaf: $\left({ }^{10}\right)$

Zimmermann, geogr. Zoolog. I. p. 165. das Schaf mit dem langen Schwanze.

Borowjky, Thierreich, I. p. 30.g. das arabifche langge/chwänzts Schaf.

Halle, vierf. p. 302. das arabifche langfchwain. zige Schaf.

Biiffon, vierf: I. p. 287. s. das arabifche 'bang. fchwänæige Schaf.

Biiffon,

(9) Trägt die koftbarfte feidenartige Wolle, die fich an den Spitzen zufammenrollt, und in kleine Locken legt. Die Häute der jungen Lümmer, oder auch der aus Mutterleibe gefchnittenen, gleichen dem gewalferten Mohr, und find, wenn lie fchwarz find, fehr theuer.

(10) Der Schwanz diefer Scháfe it, fonderlich bey den Widdern, fo lang, dafs er faft auf der Erde fchleppt, und mit Wolle bewachfen, weiche an der Spitze einen Quaft, gleich dem Liwenfchwanz bildet. In Podolien und in der Ukraine if die Wolle diefer Schafe gewaffert und kleinlockigt. Man nattet die Schafe dort, um die Güte derfelben zu befürdern, in Leinwand ein, und begiefst fie täglich einmal mit warmem Waffer, wodurch fich die Wolle kräufelt, und dicht zufammen legrt. 
Büffon, vierf. IX. p. 255. Schafe, die einnen langent und Schweren Schwanz haben.

Pallas, Naturgefch. merkw. Thiere, XI. p. 6I, das lang/chwinzige vder tfcherkaffiche Schaaf.

Pallas, Reife, Ausz. I. p 134. Schaafe.

Höft, Marokos und Fes, p. 293. Schaafe und Schaufböcke.

Dapper, afrikan. Inf. p. 52. Schaffe die lange und dicke Schwönze haben.

Erxleben, Mammal. p. 249. भ. Ovis Longicau. data.

Groumann, intr. in hiftor. nat. mamm. p. 45.g. das langfchw ünzige Schaaf.

Gatterer, brev. zool. 1. p. 7I. g. O. Longicaudata ; das arabifche langfchwionzige Schaf.

Severin, zoolog. Hungar. p. 38. d. O. Longicaudata.

\%. CA PENSIS. Das capfche Schaf. (1)

Zimmermann, geogr. Zool. I. p. 165.

Borowshy, Thierreich, I. 4. p. 31. h. das capifche Schaf.

Handb. der Naturgefch. I. 2. p. 26,3. die Schaafe auf dem Vorgeb. der guten Hoffrnung.

Biiffon, vierfuils. IX. p. 257. 0 .

Pallas, Naturgefch. merkw. Thiere, XI. p. 83. ** die kaapifchen Schaafe.

Kolbe Vorgeb. d. g. Hoffn. p. I2r. Schafe.

Kolbe, Vorgeb. d. g. Hoffn. Edit. in 4. p. 245. die Schaafe.

Erxleben, Mammal. p. 250. ๆ. O: Capenfis.

Grau.

(1) Die länglich viereckigten Fettfchwänze diefer Schafe find oft bis 20 Pfund fchwer. 
Grammann, intr. in hiftor. nat. mamns. p. 46. h. O. Capeufis.

Gatterer, brev. Zool. I. p. 7r. h. O. Capenfis; das capifche Schaf.

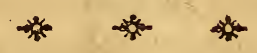

Grbjot, ForA. Handbuch. Nürnb. 1771. p. 330. Atmofphäre der Schafe.

Sammlung einiger befondern auf die Natur fich gründenden, und durch neuere Erfahrung be. fätigten Anmerkungen über die Schafzucht; in den Schriften der Lcipz. ökon. Societc̈t. I. Dresd. I77I. p. 57.

Schlefifche Landwirthfchaft. I. Breslau, 177r:

p. 200.

Carlier, 'Traité des bêtes à laine. Paris 1770.

Haffer, Unterricht von der Zucht und Wartung der beften Art Schafe. Göttingen, 1754. 8. Neue Aufl. ebend. 1764 .

Berliner Beytr. zur Landwirthfehaftswiff. 1. Berl.' 1774. p. 127. II. p. I. IV. p. 29.

S. G. Gmelin, Reife durch Rufsland, III. p. 486. oriental. Schafe.

Wiegand, Handb. zum Unterricht für die öfterreichifchen Schaafmeitter. Wien, 1775. 8.

Brigido, Unterricht, wie die Schafe zur vollkommenfen Art gebracht und erhalten werden köunen; in den Samml. der k. k. GefellSchaft des Ackerbaues u. . w. in Krain. Laybach, I773. 4. II. p. 77.

Stier, vom Drehen der Schafe; in den ökonom.

Nachr. der patriot. Gefellfch. in Schlefien 1775 .

p. III.

Unterricht von der vortheil hafteften Schaafkultur. Ulm 1776.8 . 


\section{Mammalia Pecora. Ovis.}

Taube, Slavonien und Syrmien, III. p. 57. cle, mentinifche Schafe.

Sanıml. einiger $A$ bhaindlungen von verfchiedenen Krankheiten der Pferde und Schaafe. Nürnb. 1779. 8. p. 49. (v. Hartmann.)

Lefke, vom Drehen der Schafe u. f. w. Leipzig, I780. 8.

Goeze, Naturgefch. der Eingeweidewürmer thier.

Körper. Blankenb. 1782. 4. p. 248-257. vom Blafenbandwurm im Hirnmark drehender Scha. fe. p. 369. vom Schafbandwurm.

Rozier, obfervations fur la phyfique etc. XIV.

1779. p. 57.

Hift. de l' acad. roy. des Sc. I772. p. 436.

Rozier, dictionnaire univerfel de l' agriculture etc.

Par. 178r. Tom, I. voc. Berger, Brebis.

Schubart, Erweifs, dafs alle Schäfereyen ohne

Hutung beftehen können. Lpz. 8 .

Liittwitz, wider die Stallfütterung der Schafe; in den fchlef. ökonom. Nachr. III. 1782. p. 175.

Wichmann, Katechismus der Schafzuchto Leipz. 1784. 8. m. K.

v. Bentekendorff, kleine ökonom. Schriften. Kü. frin 1784. 8. I. p. $x$.

v. Lamotte, Beytr. zur Cameralwiffenfchaft. Lpz. II. $1784^{\prime}$ p. 127.

Infruction fur le parcage des bêtes à laine. Publíe par ordre du Roi. (Ein Bogen in 4. gedruckt 1785. jedoch ohne Anzeige des Iahrs.)

Bibliotheque phylio-économique. Paris $1784 \mathrm{ff}$. an. 1786. p. 137.

Der Hausvater. V. Lpz. I786. p. 481.

Neue Abhandl. der kön. Grofsbr. u. f. w. Landwirthfchafts-Gefellfchaft zu Celle. II. Celle, I788. p. I. 
De Lamerville, obf: pratiques fur les bêtes à laine, dans la Province du Berry. Paris 1786.

Germershane en, das Ganze der Schafzucht u. f. w. I. II. 'Th. Lpz. 1789 ff. 8 .

Hïckel, Abhandl. vom Schafvieh. Starg. 1745.

Haltfer, Goldgrube eines Landes, oder von Verbefferung der Schafzucht. Koppenl. 1756 .

v. Tam, Horn - Schaf - Pferde - und FederviehArzneykunt. Wien und Prag; 1765. 8. P.39I bis 508 .

- Abilgaard, Unterricht von Pferden, Kühen, Schad fen und Schweinen. Koppenh. 177I. p. 216 bis 240.

Schreber, Samml. verfchied. Schriften, I. p. 43. von der Schafzucht in der Laufitz.

Boutrolle; gelchickter Viehhirte. Wittenb. I77x. II. Abth. p. 69-75.

La medecine des Bêtes à laine: Par. 1769. I2. v. Iufti, ökonom. Schriften, II. p. 43r.

Neue ökonom. Nachrichten, II. St. XVİ: p. 992. Krïnitz, auserlef. Auffätze u. f. w. Lpz. 1767. S. I. p. 78.

Ephem. Nat. Curiofor. V. An. 1675. et 1676.

Schäfer, von der Egelfchnecke in den Lebern der Schafe u. f. w. Regensb. I762. neue Auflage in $4 . \mathrm{m}$. $\mathrm{K}$.

Siegward, diff. de Scabie ovium. Tubing. I763. Beckmann, phyf. ökon. Bibl. I. p. 160. 367. 45 I. 536. II. p. 393. 439 . III. p. 8I. I2I. 170. 407. 57I. IV. p. 3. V. p. 20. 138. 159. 208. 43I. VI. p. 156. 181. 253. 264. 493. 497. VII. p. 393. 43I. 433. 46I. 528. 582 . VIII. p. IIg. I3I. 585. IX. p. $97.433 .484 . \quad X$. p. I2. 182. 360.565. XI. p: 66. 67. 329. $355.407 . \quad$ XII. p. 5.407. 440. XIII. p. I39. 374.382 .44 I. 458.596. XIV. 
$\mathrm{XIV}$. p. 196. 202. 326. 382. 386. 399. XV. p. 155. 246. 28I. 370.

Berlin. Samml. I. p. $372.375 . \quad$ III. p. 401.426.

IV. p. 69. VIII. p. 35. IX. p. 237.

Ellis, von der Käude an Schafen und Lämmern;

in Hamb. Magazin, V. p. II4.

Schlettwein, von Verbeff. der Schafwolle; im

Hamb. Magazin: XIX. p. 170.

Schwed Abhandl. III. p. 245. 246. V. p. 222.

232. VII. p. 105.220. IX. p. I75. XII. p.73.

XIV. p. 165. I66. XV. p. 245. XVI. p. 239.

XVIII. p, 445 . XLI. p. I46.

Hamburg. Magazin, VI. p. 439. X. p. 137.22I. XXII. p. 5 I8.

Chomel, ökonom. phyfik. Lex. Art. Schaaf.

Allgemeine Haushaltungs , und Landwiffenfchaft. I.pz. 1763, ff. I. p. 769. II. p. 384. III. p. 80. 170. IV. p. 259. (betrift die Schafucht in England.)

Mofer, Grundfätze der Forft-Oekonomie, I. p. 44I. 460.

Hammard, Reife nach der Ukraine, I. p. 194ff. Loffius, letzte und naturlichfe kehandlung der Schafe, diefelben vor allerley böfsartigen $\mathrm{Zu}$ fällen zu verwahren, und die Sch.̈fereyen in Aufnahme zu bringen. L.pz. 179r. \&.

Limme', gothländ. Reife, p. I04. I28. '242. 266. 291. 304. 353 .

Riemifch - Reuterifche ausführl. Praktik des veterinairifchen Trokarirens irrgehender Drehfchafe; oder ökonom. chirurg. 'Unterricht für Landwirthe u. Schäfer, das Dúmm-Drehen zu kuriren. Mit I Kupf. Dresd. u. Ljz. 1791.

Gafchitz, kurzer und gründlicher Unterricht zur beften Behandlung und Benutzung des Pferie- 
Rind - Schaf - Schweine - und Feúct viehes u. dgl, Lpz. I791. 8.

Strunz, Briefe über die Schafzucht in Böhmen und Oefterreich, Wien, I7 $\$ 89.8$.

2. AMMONi Der Argali.(2)

Miuller, Naturfyfem, I. p. 4I7. n. I2. die fibiri fche Ziege.

Zimmernamn, geogr. Zoolog. I. p. 169. der Argali oder Múuflon.

Zimmernann, geogr. Zool. II. p. 94. n. I2: der Argali, das wildo Schaf.

Schreber, Säugthiere, V. 'Tab.288. Ovis Ammon, Lefke, Naturgefch. p. 184. dev Muflon.

Borowsky, 'Thierreich, I. 4. p. 31. n. 2, das orientalifche Schaf:

Bhlumenbach, Handb. d. Naturgefch. p. I06. n. 2, Capra (Ammon) cornibus arcuatis circumfle. xis, fubtus planiufculis, palearibus laxis pilofis; das Mulfelthier, Argali.

Funke, Naturgelch, I.p.24. Mufflons od, Argalis. Ebert

(3) Wird vor Büfon, Pallas, Zimmeruann, Erxteben, u. a. m. für den Stammvater des Schafs (wie der Aegngrus für den Stammyater der Ziege) gehalten, dem or fich auch unter allen Thieren auf der bekannten Erde am meiften nähert. Die Hörser des mannlichen Thiers erwachfen zu einer ungeketuern Gröfse, und wiegen zufammen oft über dreyfsig Pfund; dennoch $w$ :rden fie beym Kampí nicht felten zerbrochen. Sa fcheu und wild das erwachfene Thier ift, fo leicht find die gofangenen Lammer zu zahmen. Sic werden theils mit Hunden, theils mit Feuergewehr und Pfeilen, theils in Gruben gefongen. Die Widder wiegen zuweilen bis 300 Pfund, die Weibchen aber felten iber 200 . Die Kamtfchadalen verarbeiten die His nex zu Trinkgefçhirren, Löffeln, Tabạckbüichfen u. f. w. Aus der Haut macht man in Sardinien, Corfica, und Cypera, corduan. 
Ebert, Naturl. I. p. 29r. Argali; Stepnie-Ba. ramni, Maiflon.

Gatterer, vom Nutzen und Schaden der Thiere, I. p. 150. n. 79. das wilde Schaaf, der Muflon.

Pennant, arct. Zoolog. II. p. 15. n. I. das voilds. Schaaf, der Argali.

Neuer Schauplatz der Natur, X. p. 213. fibirifthe Ziege.

Onomat. hiftor. nat. II. p. 602. der orientalifche Bock. V. p. 830 . Mufflon.

Handbuch der Naturgefchichte, I. 2. p. 258. der. Minflon.

Frifin, Naturfyf. p. I. n. I. der Muffon, cyprifine wilde Widder:

Kleirs, Clanflif. der vierf. Th. p. 66. Hir fchbock. Klein, natürl. Ordn. der vierf. Th. p. 23. 11. 8. Muffon.

Biiffon, vierf. II. p. 12. die orientalifche oder fabi. rifihg Ziege.

Buiffon, vierfüfs. IX. p. 249. 282. Muflon, Ar. gali; m. 2 Figg.

Bat $\int \mathrm{ch}$, Thiere, I. p. 127. der Mouflon.

Pallas, Reife, III. p. 23r. das vilde Steinfihaaf oder Argali.

Pallas, Reife,-Ausz. III. p. Iss. die veilden SteinSchaafe oder Argali.

Pallas, Naturgefch, merkw. Th. XI. p. 3.' der. Argali oder fibirifche wilde Schaaf:

$C_{b t t i}$, Naturgefchichte von Sardinien, I. p. 142. Muflon. Tab. 3 .

Boswell, Befchr. von Corfica, p. 42. Muffoli. Leffeps, Kamtfchatka und Sibirien, p. 48. Argalis oder wilde Widder.

Georgi, Rufsland, I. p. 208. vilde Schaafe, Arkal. 
Beckmann, phyf. ökon. Bibl. VIII. p. I3I. Muts. mon, Argalis.

Linné, Syft. Nat, Edit. X. I. p. 70. n. 12. Capra (Ammon) cornibus arcuatis, collo fubtus bar. bato, cauda nigra.

Graumann, intr. in hift. nat. maamm. p. 46. II. das orientalifche Schaaf, das wilde Schaaf.

Gatterer, brev, zool. I. p. 7I. 11. 2. Ovis (Ammon) cornibus arcuatis, femicircularibus, fub. tus planiufculis, palearibus laxis pilofis; der IMiiffoin, das orientalifche Sthaf.

Severin, zoolog. Hungar, p. 38. e. Argalis, feu Ammon.

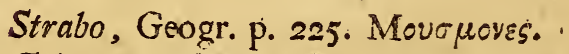

Cohmella, de re ruft. Lib. 7. cap. 2 .

4. Pupu. Der Pudu. Molin.

3. S TR R PSICER os. Das cretifche Schaf.(3)

Miiller, Naturfyft. 1. p. 429. 11. 3. das cretenffiche Schaaf.

Zimmermann, geogr. Zool. I. p. 165. das Sihaf. Strepfikeros.

Schreber, Säugthiere, V. Tab. 29I. A. B. Ovis Aries Strepficeros.

Borowfky, Thierreich, I. 4. p. 32. n. 4. das hochgehïrnte cretenafiche Schaf.

Ebert, Naturlehre, I. p. 290. das cretenffische Sthaf.

Halle, vierf. P. 302. das hochgehörnte kretifche Schaf.

Gatterer, vom Nutzen und Schaden der Thiere,

J. p. I5r. n. 81. das kretifche Schaaf.

Neuer 
Mammalia.Pecora. Ovis, $68 \mathrm{I}$

Necuer Schaupl. der Natur, VII. p. 568. das cre- tenfifche Schaaf.

Onomatol. hiftor. natur. V. p. 880 , das creten $f$ f che Schaaf.

Frifch, Naturfyftem, p. r. n. I. das SchraubhornSchaf, das cretifche, oder kandifche, Zickel. Schaf:

Klein, Claffif, der vierf. Th. p: 47. hochgehörn. ter cretenfficher Widder.

Klein, natürl. Ordn. d. vierf. Th. p. 17. n. 2. hochgehörnter Widder.

Biiffon, vierfüfs. I. p. 288. n. 5. dev hochgehörnte Widder.

Biiffon, vierf. IX. p. 258. 264. das. Sehaf Sirepficeros. p. i32r. der wallachifche Widder, das wallachifche Schaf; m. 2 Figg.

Erxleben, Mammal. p. 255. n. 4. Ovis (Strepfi. ceros) cornibus rectis carinatis flexuofo - fpiralibus,

Linné, Syft. Natur. Edit. II. p. 51. Ovis cornibus rectis fpiralibus.

Linné, Sylt. Nat, Edit, VI, p. 15. n. 2, Ovis cor: nibus erectis fpiralibus.

Limné, Syft: Nat. Edit. X. I. p. 7r, n. 3. Ovis (Strepficeros) cornibus rectis fpiralibus.

Graumann, intr, in hif, nat, mamm, p. 46. III. das cretenfifche Schaaf, Zackl.

Gatterer, brev, Zool. I, P. 72, n. 3. Ovis (Strepfceros) cornibus rectis carinatis flexuofo - fpiralibus; das kretenfliche Schaf:

Severin, zool. Hungar; p. 37. Ovis Strepficeros. Kramer, Auftr. p. 322. Ovis cornibus erectis fpi: ralibus. 
V̌rründernngen gegen die XIIte Edition, sud Ver. mehnung der Gattumgen diefes Gefchlechtș.

Die zweyte Gattung ift aus dem dreyfsigften Gefchlecht hieher verletzt, und die vierte ift neu. Die zweyte Gattung der XIIten Edit. p. 98. if in der XIII. p. I98. द. als eine Varietät von der er. ftern angenommen, und auffer derfelben noch die Spielarten $\vartheta, b$, und $x$, hinzugekommen.

32. GESCH L ECHT. Bos. Der Ochfe:

Miller, Naturfyftem, I. p. 43r. Gen. XXXII. Zimmermann, geograph. Zoolog. II. pag. 8. Gen. II.

Lefke, Naturgefch. p. I84. Gen. XXXVIII. Boronelky, Thierreich, I. 4. p. 33. Gen. XXXIV. Blumenbach, Handb. der Naturgefchichte, p. 109.

Gen. XXXII.

Bechfteini, Naturgefch. Deutfchl, I. p. 668.

Pennant, arct. Zool. II. p. 3.

Onomat. hif. nat. II. p. 278.

Bat $\int c h$, Thiere, I. I38.

Frifoh, Naturfyftem, p.. .

Erxleben, Mammalia, p. 228. Gen. XXVI.

Graumann, intr. in hiftor. nat. mamm. p. 43 .

Gen. XXVI.

Gatterer, brev. Zoolog. I. p. 64. Gen. XXVI.

Boddaert, elench. anim. Gen. XXXIII.

Pennant, hift. quadr. Gen. II.

Briffon, quadr. p. 5I. Gen. XI.

Hermanm, tab. affu. animal, p. Iog.

I. TAU: 
3. Taunús. Der Stier. (')

Miiller, Naturfyfem, I.p. 432. n. I. der Stier. Zimmermann, geograph. Zoolog. I. p. IjI. der Oches.

Bhimenbach, Handb. der Naturgefch. p. Iro. n. I; Bos (Taurus) cornibus teretibus extrorfumcurvatis, palearibus laxis; der Ochfe.

Lefke, Naturgefch. p. 184. n. I. der Stier. Erxleben, Mammal. p. 228. n. 1. Bos (Taurus) cornibus teretibus extrorfum curvatis, palearibus laxis.

Linné, Syft. Nat. Edit. II. p. 5I. Bos cornibus teretibus flexis.

Linné, Sy凡. Natur. Edit. VI. p. 15. n. I. Bos cornibus teretibus flexis.

Linné, Syft, Natur. Edit. X. I. p. - †r. n. I. Bos (Taurus) cornibus teretibus extrorfum curvatis, palearibus laxis.

Graimann, intr. in hiftor. nat. mamm. p. 43. İ. B. Taurus.

Gatterer, brev. zoolog. I. p. 64. n. 1. Bos ("Taurus ) cornibus teretibus, extrorfum curvatis, palearibus laxis; der Stier.

Severin, zool. Hungar. p. 33. Bos.

\section{C, FERUS.}

(1) In dem Magen" diefer Thiere finden fich zuweilen Ballen, die aber weder fteinartig, wie die Bezoare, noch van vegetabilifcher Subftanz wie die Gemfenkugeln , fundern blofs aus hìaren zufammen gebacken find, die fie fich abgelecks und eingefchluckt haben. Die diefer Gattung eigene Viehfeuche ift zwar fchon den Alten bekanne gewefen, hat abef doch eqf feit 178. da fie fiçh von Ungarn aus durch Italiẹ über ganz Euro. perbreitete, allgemeineł grafirt, 
«. F E R U S. Der witlde Stier.

a. URus. Der Auerochs, Urochs. $\left({ }^{2}\right)$

Miiller, Naturfyfem, I. p. 436. B. der wilde oder Aueroch $\int e, W$ aldoch $\int e$.

Zinimermann, geogr. Zoolog. II. p. 82. n. 5. der Auerochse.

Schreber, Säugthiere, V. Tab. 295. Bos Urus. Borowfky, Thierreich, I. 4. p. 39. der Auerorhs. Funke, Naturgefch. I. p. 34. Ur-oder Auevoch Se. Ebert, Naturlehre, I. p. 284. der Urochs, oder Auerochs.

Halle, vierfüfs, p. 280. der Auerochs.

Gatterer, vom Nutzen und Schaden der Thiere,

I. p. 107. n. 7I. der Aueroghis.

Beckmann, Naturhilt, p. Io. b. der Auevochs.

Neuer Schaupl. der Natur, I. p. 433. Auerochs.

Onomat. hift. nat. VII. p. 678. der Auerocins.

Frifch, Naturfynt. p. I. n. I. nordischer Auerochs. Klein, Claflif, der vierf. Thiere, p. 36. Urochs, Auerochs.

Klein, natürl. Ordn. der vierf. Th. p. 12. n. 3. Auerochs, Urochs, Bergochs, wilder Oihs.

Martini, Naturlex. III. p. 693. Auerochs.

Biiffon, vierf. IX. p. II2. 149, 223. der Auevochs. Batfch, Thiere, I. p. I4I. der Auerochs. Bock, Naturgefch. von Preuffen, IV. p. I9r. der wilde oder Auevochs.

\section{Hart-}

(2) Ur heifst im Altdeutfchen Wald; Urocbs oder Auerochs alfo Waldocbs; wie Auerbabn Waldhahn. Ehemals lebte der Auerochfe auch in Deutfchland, und foine weiten Hörner dienten unfern Vorfahren zu Trinkgefáfsen. Die Römer machten dünne polirte Tafeln daraus, die fie tatt des Glafes in Laternen zebrauchten. Aus der Haut wird ein fehr ftarkes Leder bereitet. - In Preuffen haben die Auerochfén anfgehört; und auch in Polen find fie faft ausgeftorben. 
Hartknoch, Preuff.Hift. I. c. I3. S. S. Aner-Ochs. Pallas, nord. Beytr. 1. p. 6. der preulfische und titthauifche Anierochs.

Döbel, Iägerpraktik. I. p. 20. Auer Ochfe.

Byron, Reife um die W elt, p. I86. wilde Ochfen? Hiineken, Befchr. der Natur und Eigenfchaften eines Auerochfen; a. d. Lat. überf. Berl. 1705.4. Mafcov. diff. I. II. de Uro. Regiom. I705. I718.4. Menzel. Scholium de Uro; in den Mifc. Natur.

Cur. Dec. II. A. 2. p. 22..

'Hellwing, Obferv. von denen, wie Mofchus rie. chenden Haaren des Auerochlen; in den Brefsl. Samml. VII Verf. Febr. 1719: p. I9o.

Kriunitz, ökon. Fncykl. II. p. 722. Auer. Ochs, Aur-Ochs, Ur-Ochs.

Klïgel, Encyklop. I. p. 241. Aurochs.

Merklein, Thierreich, p. 136. Anter-Ochen.

Iablonfky; allgem. Lex. p. 82. Auerochs.

Onomat. foreftal. I. p: 153. Aueruchs.

IM. D. Efá fur l'hifloire literaire de Pologne. Berlin, 1778. p. r3o.

Graumann, introd. in hiftor. nat. mamm. p. 43. Urochs, Auerochs.

Gatterer, brev. Zoolog. I. p. 64. a. Urus; ferus; der Auerochs.

Severin; zool. Hungar. p. 36. b. Bos ferus fyl. vefris; Urüs; der Auerochs.

Plin. hift. nat. XI c. 37 . Urus,

b. Bon as us. Der Bonafus. (3)

Miiller; Naturfyf. I. p. 437. h. 2. afrikanifcher wilder Ochs.

Zimmer.

- 32 Soll fich mit feinèn átzenden brenuenden Unrath oder Mifte vertheidigen, den er weit von fich wirft. Erxleber macht aus " dem 
Zimmermann, geogr. Zool. II. p. 93. a. der Ba. nafus der Alten.

Borowfky, Thierreich, I. 4. p. 40. 11. 3. der afvikanifche wilde Ochs.

Gatterer, vom Nutzen u. Schaden der Thiere, I. p. 128. n. 73. der afrikanifche wilde Ochs. Onomat. hif. nat. II. p. 262. der Bonalis. Klein, Quadr. difp. p. 13. Bonafus. Klein, Claffif, der vierf. Thiere, p. 39. Bonafus. Klein, natürl. Ordn. der vierfüls. Thiere, p. I4. Bonajus.

Gesner, Thierbuch, p. 297. Muniffier, odes Mäneftier" ; mit Abbild. des Kopfs.

Büffon, vierf. IX. p. 112. 128. 144. 145. der Bo. nafus.

Bat/ch, Thiere, I. p. I4I. der afrikanifiche wikde Ochs.

Erxleben, Mammal. p. 233. n. 2. Bos (Bonafus) cornibus deorfum inflexis, iuba longiflima.

Linné, Syf. Nat. Edit. II. p. 5r. Bos iuba longiffima, cornibus in fe flexis.

Linné, Syft. Nat. Edit. VI. p. 15. n. 2. Bos iuba longiffima, cornibus in fe flexis.

Limné, Syft. Natur. Edit. X. I. p. 7r. n. 2. Bos (Bonafus) cornibus in fe flexis, iuba longiffina.

Graumann, intr!' in hiftor. nat. mamm. p. 44. II. afrikanifcher wilder $O_{6} h$.

Gatterer, brev. Zoolog. I. p. 66. n. 2. Bos (Bonafus) cornibus deorfum inflexis, iuba longif. fima; der afrikanifche wilde Ochs. Severin, zool. Hungar. p. 35. Bonafus.

Ariftot.

dem Thier eine eigene Gattung. Biiffor hatt ihn fiir eine Varietät des Ochfen; und Zimmernamn ferzt ihn unter die unbeftimm. tern. Thiere. 
Ariflot. de mirabil. aufcultat. ed. Beckmann. c. t. Bonajits.

- Bison. Der europaiifche Bifon. $\left({ }^{4}\right)$

Miiller, Naturfyftem, I. p. 439. A. der gemeine Bifon.

Bhumenbach, Handb. d. Naturgefch. p. rIo. Biforz der alten Welt.

Zimmermann, geogr. Zool. II. p. 84. der Bifon. Ebert, Naturl. I. p. 285. der Bifon.

Neuer Schaupl: der Natur, p. 39. Höckerochs, Buckelochs.

Onomat. hif. nat. II. p. 198. der Vifent, oder BiSentochs, Bifenftier.

Bermouilli, Reifen durch Brandenburg, Preuffen,

Polen und Rufsland, VI. p. 40. Bifon.

Berlin. Samml. V. p. 36. Bifon oder Buckeloch/e;

m. e. Fig.

Titius, Wittenberg. Wochenblatt, 1772.

Erxleben, Mammal. p. 235. n. 3. Bos (Bifon) cornibus divaricatis, iuba longifima, dorfo gib. bofo. (Mit Bos Americanus p. 204. n. 2. verwechfelt.)

Linné, Syft. Nat. Edit. II. p. 51. Bos iuba longiflima, dorfo gibbofo.

Linne, Syf. Nat. Edit. V1. p. 15. n. 3. Bos iuba longiffima, derfo gibbofo.

Limé, Syft. Nat. Edit. X. I. p. 72. n. 3. Bos (Bifon) cornibus divaricatis, iuba longiffuna, dorfo gibbofo.

Graumann, intr. in hif. nat. mamm. p. 44. III. Bifon, Buckelochje...

Gatte.

(4) Wird yoo den mehreften Syftematikera mit dem amerikanifchen Bifon p. 204. n. 2. verwechfelt. 
Gatterer, brev.'Zoot. I. p. 67. n. 3. Bos (Bifon) cornibus divaricatis, iuba longiflima, dorfo gibbofo; der Buckelochs, vierfache Teufel. Severin, zool. Hungar. p. 36. B. Bifon.

3. Domesticus. Der zaime Stier.(") Miiller, Naturfylt. I. p. 432. A. zahme Rinder. Miiller, Naturfyf. Suppl. p. 57. n. I. der Maft. ochee.

Schreber, Säugthiere, V. Tab. 297. Bos Taurzs. Borow jky, Thierreich, I. 4. p. 35. 11. 2. der Ochs, der Stier.

Bechftein, Naturgefch. Deutfchl. I. p. 673. n. 2. der Ochs, Stier, die Kuh.

Finnke, Naturgefchichte, I. p. 35. unefer einheimi. fohes Rinduieh.

Ebert, Naturl. I. p. 283. der zahime Stier.

Halle, vierf. p. 260. der zahme Oihs. IMeyej, Thiere, Tab. 4r. der Ochs. Meyer, Thiere, Tab. 42. der Stier.

Gatterer, vom Nutzen und Schaden der Thiere,

I. p. I09. n. 72. der Stier, das Rindvieh. Beckmann, Naturhiftorie, p. 9. n. ì. der Och/e. Neuer Schauplatz der Natur, VI. p. I8I. Och e. Onomat. hift. nat. II. p. 278. ein zahmer Ochs, ein Rind, Rindvieh.

Handb.

(5) In gemeinen Leben heifst eigentlich Stier das erwachfene Männchen; Oclsese, Riricl, das verfchnittene Männchen; Kalb, ein lunges; Bolle odet Bulle, ein Stiet der über 4 lahr alt if: $K u h$, das Weibchen; Farfe, ein Kuhkalb im erften lahr. Man hat Beylpiele von ungeheurer Gröfse und Fettigkeit gemàfteter Ochfen. Im lahr 1775, wurde zu Nürnberg ein Ochí von 25 Centner und $40 \mathrm{Pfund}$ gefchlachter. Er hatte $34^{\circ}$ Pfund Talg, und die Haut wog $7 \circ$ Pfund. Im lahr 1692 . wurd e aber zu Lincolnfhire ein Ochfe gefchlachtet, der diefen am Gewichte noch weit tibertraf, und 35 Centner 77 Pfund wog. 
Handb. der Naturgefch. I. 2. p. r. der Ociss. p. 25. die Kuh.

Handbuch der deutfch. Thiergefchichte, p. 68. zahmer Ochs. p. 69. die Kuh.

Sander, ökonom. Naturgefchichte, I. p. 194. das Rindvieh.

Walther, ökon. Naturgefch. Deutfchl, p. 146. Kiihe: p. 156 . Ochs.

Frifih, Naturforfch. IX. p. 35. Stammochfen.

Frifch, Naturfyft, p. I. n. I. Bos vulgaris; ge- meiner Ochs.

Klein, Claflif. d. vierfüls. Thiere, p. 3i. der Housbol'e, und die zahme Kuh.

Klein, natürl. Ordn. d. vierf. Thiere, p. ri. n. I. Hausboll, zahmer Ochs.

Gesner, Thierbuch, p. 277. Ochfe, Kuh; mit Figuren.

Gesner, Thierbuch, p. 291. Stier; m. e. Fig. Gesiner, 'Thierbuch', p. 294. Kalb; in. e. Fig. Biiffon, vierfüls. I. p. 232. der OGhse. Tab. ro. dis Kun und das Kalb; 'Tab. Ir.

Bock, Naturgefch. von Preufen, IV. p. 168. zahmer Ochs, Rindvieh.

Batfch, Thiere, I. p. I40. der gewöhnliche Othfe. Pontoppidan, Norwegen, II. p. 8. Ochfen und Kiuhe.

Cetti, Sardinien, I. p. 62. Rindvieh.

Olaffen, Island, I. p. 27. 101. 188. 278. II. p. Ir7. 198. Hornvieh.

Uno von Troil, Reife nach Island, p. 99. Rindvieh.

De Kerguelen Tremares, Reife n. der Nordfee, p. 74. Ochsen und Kiihe.

v. Taube, Slavonien und Syrmien, I. p. 34. das. Rindvieh. 
I. G. Gmelin, Reife durch Sibirien, I. p. 221 . Kiihe. p. 429. Ochjen.

Schlözer, Briefwechfel, VI. Heft p. 343 .

Schlözer, Erdbefuhreib. von Amerika, p. 916. Rindvieh; 274.

Falkner, Patagonien, p. 52. Hornvieh. p. 54. Rindvieh.

Byron, Reife um die Welt, p. 186. zahmes Hornvieh.

Molina, Naturgefch. von Chili, p. 284. Och/en. p. 285. Hornvieh.

Fabric. faun. groenland. p. 29. n. 7. Bos Taurus. Niebuhr, Arabien, p. 165. Ochfen und Kïhe. Höft, Marokos und Fes, p. 293. Rindvieh. Beytr. zur Beförd. der Naturkunde, I. p. 217.30. Rindvieh.

Dobrizhoffer, Gefch. der Abiponer, I. p. 276. - Ochsen.

Hartfink, Befchr. von Guiana, I. p. Irs. Kithe. Boswell, Befchr. von Corfica, p. 4r. die Kïhte. Shaw, Reife, p. I5o. das Hornvieh.

Fermin, Surinam, H. p. 76. Ochfen und Kiihe. Hammard, Reife nach der Ukraine, I. p. I89. Ochfeir.

Pennant, Reife durch Schottland, II. p. 270. Kiihe. I. p. 346. das Hornvieh.

Kämpfer, Iapan; in dit Hälde China, IV. I. p. 137. Ochfen and Kühe.

Schöpf, Reife durch Nordamerika, II. p. I39. Hornvieh. p. 386. das Rindvieh.

Georgi, Rufsland, II. p. 447. das Rindvieh. Ives Reife nach Indien, I. p. 68. Ochlen. p. 103. Ochsen.

Prïtorius, Gefch. der Infel Minorka; p. I24. das Rindvieh. 
Leppechin, Tagebuch der ruff. Reife, I. p. 63. Hornvieh.

Mhetager, Schriften der berlin. Gefellfchaft, IV. p. 42r. Rindvieh.

Pallas, Reife d. Rufsl. I. p. 397.320. 321. 315.

Oeknnom. Zoologie, p. 48. n. 57. der Ochse. Merklein, Thierreich, p. 135. Ochje.

Iablonfky, allgem. Lex. p. 746. Ochs. Erxleben, Mammal. p. 230. B. B. domefticus; Manfuetus, cultus.

Limné, Fn. Suec. I. p. 15. n. 44. Bos cornibus teretibus flexis.

Linné, Fn. Suec. II. p. 15. n. 46. Bos (Taurus) cornibus teretibus extrorfum curvatis, palearibus laxis.

Graumiann, intr, in hiftor. nat. mamm. p. 44. b. Stier, Ochje, Rind.

Gatterer, brev. zoolog. I. p. 64. b. B. domefticus; der zahme Stier.

Kramer, Auftr. p. 322. Bos cornibus teretibus flexis.

Miiller, zoolog. dan. prodrom. p. 6. n. 4I. Bos (Taurus) cornibus teretibus, extrorfum curva. tis, palearibus laxis.

$\mathrm{V}_{\mathrm{arro}_{3}}$ de re ruAt. Lib. II. c. 5. boves et vaccae.

Columella, de re ruft. Lib. VI. c. 3 . Boves. c. 27. Tauri. c. 28. Vaccae.

a. Indtcus iraIOR. Der grojse indianifche Biiffel.

Zimmermanin, geogr. Zoolog. I. p. 154. der Ochfe in Madagafiar.

Zimmermann, geograph. Zoolog. II. p. 83. e. der Buckelochfe der heifsen Liinder der alteiz Welt. 
Bïffon, vierf. IX. p. 163. Ochfen, deren Hücker 40 bis so Pfund wiegent.

b. INDICUS MINOR. Der Zebu.( $\left(^{6}\right)$

Miiller, Naturfyft. I. p. 442. n.6. der Zwergbiiffel.

Zimmermann, geogr.Zool. II.p. 92.n. 10. der Zbbu.

Schreber, Säugthiere, V. Tab. 298. Bos indicus.

Borowejky, Thierreich, I. 4. p. 4I. n. 4. der Zwergbiiffel, kleine indianifche Biiffel. Tabula 34 . B.

Ebert, Naturlehre, I. p. 287. der Zebu. (Mis Nr. f. verwechfelt.)

Gatterer, vom Nutzen und Schaden der Thiere, I. p. 135. der Znergbiiffel, Zebu.

Neuer Schaupl. d, Natur, X. p. 3Ir. Zwergbïffel. Frifch, Naturfyftem, p. I. 12. 6. Bos minimus; das Zebu.

Biiffon, vierf. IX. p. 112. 139. 163, 184, I87. Zebu; m. e. Fig.

Batfch, Thiere, I. p. 140. der Zebu.

Krinitz, Encyklop. VII. p. 369. der indianifche oder chinefische Zwergbiiffel.

Ausz. aus dem ' ageb. eines Reif. n. Afien, p. 87. Zwerg - Bifonen.

Berlin. Samml. IV. p. 310. der kleine indianifche Biiffel; m. Edw. Fig.

Erxleben, Mammal. p. 240. n. 6. Bos (Indicus) cornibus aure brevioribus, dorfo gibbo, iuba nulla.

Linné, Syft. Nat. Edit. X. I. p. 72. n. 5. Bos (Indicus) cornibus aure brevioribus, dorfo gibbo, iuba nulla.

Graus-

(6) Diefe Varietat wird oft mit der fub Nrr. f. verwechfelt. Aber, wie leicht ift das nicht möglich! Erxleben hält fie fïr eine eigene Gattung. 
Graumann, intr. in hif. nat. mamm. p. 44. VI. Zwergbiiffel.

Gatterer, brev. zool. I. p. 68. n. 6. Bos (Indicus) cornibus aure brevioribus, dorfo gibbo, inba nulla; der Zebu, Zivergbiiffel.

Severin, zool. Hung. p. 36. c. Bos Indicus; Zebu. Pennant, Syn, quadrup. p. 6. Tab. I. fig. 2. the Indian Bull.

c. Bos abessinicus. Das aby. Jinifche Rindvieh.

Zimmermann, geogr. Zool. I. p. 157. die Schlachtochsen der Abyjinier.

Zimmermann, geogr. Zoolog. II. p. 94. f. dev abyffinifche Ochfe, mit welken oder fchlappent hangenden Hörnern.

Dapper, afrikan. Inf. p. 3r. Ochfen mit hangenden oder lofen, and nicht an dev Haut des Kopfs angewachenenon Hörnern.

Lobo, voy. hiftorique d'Abiflinie. Amf. 1728. 4. p. 7 o.

d. BOS MADAGASCARIENSIS NIVEUS. Der weifse madagafkarifche Stier.

e. Bos tinianensis niveus. Das Rindvieh von Tinian.

Zimmermann, geogr. Znol. II. p. 83. b. das Rindvieh ouf der Infel Tinian.

Anfor, Reife um die Welt, p. 285.

f. BOS AFRICANUS NIVEUS CELER. Der Lant.

Ebert, Naturlehre, I. p. 287. der Lant, Lampt oder Dant.

Dapper, Afrika, p. 24. das Thier Lant, oder Dant.

$$
\mathrm{X}_{3} \quad \text { Frifich, }
$$


Firich, Naturfyfem, p. r. n. 5, Bos humilis; das Dante oder Lampt.

Leon. Afrikan, defcript. Afr. p: 75r, Lant five Dant.

S. Bos eur OPAEUS. Das europäifche Rindo vieh.

Zimmermann, geogr. Zool. II. p. 83. a, der enropaii flche zahme Oches; u. f, w:

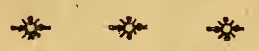

Scopoli, annus hifor. natur. 1769. JII. 2.

Bemerk. der phyf. ökon. Gefellifch. in Lautern, 1769. p. 220 .

Kocziann, Prüfung der Urfach der Hornviehfeua che. Wien 1769.8 .

Höknert, Beytr. zur Landwirthfchaft. Bremer, 5772. 8. P. 10.

Yowng, Reife durch England. Leipzig, iz72. I. p. 259.

v: Schünfeld, Landwirthfchaft, p. 300 .

Oeconomia forenfis, I. p. $4 \mathrm{II}$.

Abhandl. der ökonom. Gefellfchaft izu Bern, 1772. p. 51.

Gefch. der Einimpfung der Hornviehfeuche; aus d. Dänl. überf. von TTode. Koppenh. I775. gr. 8. Scopoli, Bemerk. a. d. Naturgefch. III. p. 45. von der Kindviehfeuche.

Beobacht, und Verfuche meiftentheils durehs Einimpfen an Rindvieh gemacht u. f. w. aus $d$. Holländ. Wefel 1777. 8.

Von der Vielzzucht. Brandenb. 1777. 4:

Beytr. zur Gefch. der Rindviehfeuche im Hannö. verfohen. Altenb. I776. 8.

Berlin. Beytr. zur Landwirth chaftsw. III. p. 3r3. 
Manmmalia Pecora. Bos. $\quad 695$

Bemerk. der churpfälz, ökonom. Gefellfch. vom Iahr I775. Lautern I779. p. 2 I2.

Beytr. zur Gefch. der Hornviehfeuche in einigen Kreifen der Altmark. Göttingen I777. 8.

Die gehobene Gefahr beym Eintritt der Rindviehfeuche. Berlin I779. $8 . \mathrm{m}$. e. K.

Oeffentl. Bekanntmachung der nunmehr fattfam geprobten, und in Mecklenburg allgemein gewordenen Inoculation der Kindviehfeuche. Hamb. 1779. 4.

Beikmann, Beytr. zur Oekonomie u. f. $\mathbf{}$. V. p. I. Der patriot. Gefellfch. in Schlefien neue ökonom. Nachr. III. p. r.

Riem, prakt. ökonon. Encyklop, III. p. I.

v. Lamotte, prakt. Beytr. zur Cameral iff. IV. p. 192.

Schriften der Leipz. ökon, Gefellfch. VII. p. I. Lehrbegriff der ökon. u. Cameral wiff. I. I. p. 146. Preuff. Sammler, St. 63: u. 64.

Kriinitz, Verzeichnifs der vornehmften Schriften von der Rindviehfeuche. Lpz. 1767.8 .

Berlin. 'Samml. I. p. 296. II. p. 314. 402. 627.' III. p. 297. 398. 616. IV. p. 262. 263. 586. V. p. 20I. 280. 38I. VI. p. I38. 497. VII. p. 162. 617. VIII. p. 282. 617. IX. p. 6. 167. X. p. I. 5.272.

Beckmann, phyf. ökon, Bibl. I. p. 317. 429. 537. 598. II. p. I7. III. p. 54. 120. 204. 578. IV. p. 34. 162. 383. 420.45 I. 523. V. p. 55. I4I. 258. 282. VI. p. 107. I20. 426. 440. 490. 54I. VIII. p. 20I. 211.213 .214 .289 .520 .6 II. IX. p. 139. 360. 484. X. p. r4I. 35I. 36r. 556. 562. 569. 579. 582. 588 . XII. p. 48. 69. 185. 371. XIII. p. 380. XIV. p. 43. 449. XV. p. 336.19 .593 .

$\mathrm{X} \times 4$ Schwed: 
Echwed. Ahhandl. II. p. 247. VII, p. 220 . VIII.

p. 291. X. p. 194. XVIII. p. I4I. XIX.

p. 278. XX. p. 47. XXVI. p. 52. XXXI.

p. 46. 84. 323. XXXIV. p. 278. XXXVII.

p. 154. XLI. p. 144 .

Hamburg: Magazin, I. p. 97. V. p. 102. VI.

p. 429.390. XXV. p. 3r.

Ziickert, Speif. aus dem Thierreiche, p. $40 \mathrm{ff}$. Abilgaard, Unterricht von Pferden, Kühen, u. dgl. p. 196.

Chomel, ökon, phyfik. Lex. Art. Kuh. Rindvieh. Allgemeine Haushalt. u. Landwiffenfch. I. p. 733. 738. 753.763. III. p. 333. IV. p. 226.

Fifcher, liefländ. Land wirth chaftsbuch, p. 160. Oekonom. phyl. Abhandl. I. p. 604. II. p. 695. Halle, Magie, II. p. 276 . III. p. 2IS.

Hacquet, Beobacht. u. Heilungsmethode einzel. ner Hornviehfeuchen, welche durch Gifte aus den drey Naturreichen verurfacht werden. $S_{0}$ Leipziger Magazin, I. p. 529.

2. Americanus. Der (amerikanifche) Bifon. (7) Muiller, Naturfyfem, I. p. 440. B. amerikanifche Bifon.

Zimener.

(7) Das gröfste Landthier der neuen Welt. Soll fich in fo ungaheurer Menge findẹn, dafs bey einer lagd oft 1500 bis 2000 getüdtet werden. Die Stiere werden fo fett, dafs man zu Zeiten von einem einzigen 150 Pfund Talg bekümmt. Der ftärkfte Mann ift nicht im Stande das Fell eines folchen Thiers von der Erde aufzuheben. Das Fleifch der Kuthe wird dem Fleifche der Stiere vorgezogen. Erxleben, und andere, haben diefe Gattung mit dem europäifchen Bifon zufammengezogen. "Gmelin felbs ift ungewifs, ob fie nicht eine Varietät vom Stier fey, da beyde fich mit einander begatten. Es ift uberhaupt fchwer, die 
Mammalia Pecora. Bos.

Zimmermann, geogr. Zool. II. p. 85. der ameri. kanifche Bifon.

Borow $5 k y$, Thierreich, I. 4. p. 42. n. 5. der Bul ckelochfe, Bifon, Wifent. (Mit Nr. I. a. c. p. 203. vervechfelt.)

Blumenbach, Handb. d. Naturgefch. p. IIr. a. 3 . Bos (Bifon) cornibus divaricatis, iuba longiffima, dorfo gibbofo; der Buckelochfe.

Schïpf, Reife durch Nordamerika, II. p. I67. der Bifon.

Halle, vierf. p. 281. der amerikanifche Anerochs. Gatterer, vom Nutzen und Schaden der Thiere, I. p. 128. n. 74. der Buckelochs, Bifon.

Pennant, arct. Zool. I. p. 3. der Bifon.

Carver, Reife durch Nordamerika, p. 364. der Bifon.

Hamb. Magazin, XIV. p. 6ro. der wilde Ochs. Biiffon, vierf. IX. p. 147. 221. 232. nordamerika. nifcher Bifam. Ochs.

Pallas, nord. Beyträge, I. p. 5. der nordamerikanifche Bifon.

Valentin. Muf. mufeor. deutfch. Ausg. II. p. I34.

3. Maschatus. Der Mufkusochse. $\left({ }^{(8)}\right.$

Zimmermann, geogr. Zool. II. p. 86. n. 6. Bos (Molchatus) cornibus magnis iuxta bafin prope. $\mathrm{X} \times 5$ modum

die Arten des Ochfengéfchlẹchts genău zu -bẹtimmen, da die Kennzeichen leicht in einander laufen.

(8) Lieht die felfigten öden Gegenden, und kömmt nur felten zu den waldigten Thälern des Iandes; läuft fchnell, und erklettert die Felfen mir vieler Gefchicklichkeit. 'Das' Haar fchleppt faft auf der Erde. Das Herz des Thiers fchmeckt befonders unausftehlich ftark nach Mofchus. Man finder Heerden von 20 bis 30 .Stück zurammen. Auch diefs Thier hat man gewơhnlich mit dem vorhergehenden verwechfelc. 
modum iunctis, iuba longiffina, mofchum redolens; der MLufkusochfe, der Churchills-Büffel.

Schreber, Säugthiere, V. Tab. 202. A. B. Bos mofchatus.

Pennant, arct. Zool. I. p. II. n. 2. der MofchusOchfe. Tab. 7 .

Buiffon, vierf. IX. p. I93. 228. der IMuskusochs; m. E. Fig.

Pallas, nord. Beytr. I. p. 340. amerikanificher Bifamoche.

Herrmann, Naturforfcher, XIX. p. 9I. Bifamb ochfe aus der Hudfonsbay. Tab. 5. der Kopf. Fabric. faun. groenland. p. 28. Bos grunniens? (S. Pennant, arct. Zool. I. p. 15.)

Nov. Comment. Petropolit. XVIII. p. 6or.

4. Gru NNIENs. Der tibetanifche Büffel.(?)

Müller, Naturfyf. I. p. 44I. n. 4. der afiatifche Brummochse.

Zimmermann, geogr. Zool. II. p. 88. n. 7. der Sarluk, der grunzende Ochje.

Schveber, Säugth. V. Tab. 299. 1. B. Bos grunniens.

Borowfky, Thierreich, I. 4. p. 43. n. 6. der afiatifche Brunochs.

Bhumenbach, Handb. der Naturgefch. p. I24. n. 4. Bos (Grunniens) cornibus teretibus, introrfum curvatis, vellere propendente, cauda undique iubata;

(9) Gewöhnlich kleiner als unfer Hornvieh. Die Chinefer wiffen das weifse Haár vor einigen diefer Büffel recht brennend roth zu fathen, und tragen faft durchgängig Quaften davon auf ihren geflochtenen Sommerhüten. Witfen ift der einzige, der einer Art Bezoar gedenkt, die im Magen diefer füffel von der Gröfse ẹines Gänfeeyes gefunden werden, und im Orienț fehr gefchätzt feyn foll. Schṿimmt gut. 
iubata; der Bïffel mit dem Pferdefchweif, Ziegenoch $\int e$.

Gatterer, vom Nutzen u. Schaden der Thiere,

I. p. 135. 11. 76. der Brummochs.

Biiffon, vierfüfs. IX. p. I89. die tatarifche Kuih; m. e. Fig.

Pallas, Reife, Ausz, III. p. I26. voilde tangutifche Kiihe.

Georgi, Rufsland, I. p. 208, kalnuiikifche Kïhe. Erxleben, Mammal. p. 237. n. 4. Bos (Grunniens) cornibus teretibus, introrfum cúrvatis, vellere propendente, cauda undique iubata.

Graumann, intr, in hif, nat. mamm. p. 44 . IV. der afutifche Brammochfe.

Gatterer, brev. Zool. I. p. 67: n. 4. Bos (Grunniens) cornibus teretibus, introrfum curvatis, vellere propendente, cauda undique iubata; der Brummochs.

Severin, zool. Hungar. p. 36. D. Bos Grunniens, Grew, mufeum regalis focietat. p. 26.

Fabric. faun. grconland. p. 28. Bos Grunniens? Witfent, Nord - en Oft-Tartarye. Edit, 2.I. p. 66.342 .258 .

5. Bubalus. Der B̈̈ffel. $\left({ }^{10}\right)$

Miiller, Naturfyft. I. p. 44I. n. 5. der Bïffel. Zimmermann, geogr. Zool. I. p. I55. II. p. 43. der Elephantenochse.

Zimmer-

(10) Zwey diefer Thiere find im Stande eine Laft zn ziehen, die fechs Pferde kaum zu bewegen im Stande feyn würden. Das fchwarze, dünnbehaarte Fell ift aufferordentlich fark, und vorzüglich zu Schlauchen tauglich. Ihr Fleifch ift zwar fchlechter, aber ihre Milch, und die daraus gemachten Käfe und But= tef, unglẹch fchmackhafter, als die vom gemeinen Hornvieh. 
Zinmeymann, geogr. Zoolog. II. p. 40. p. 90. n. 8. der Biiffel.

Schreber, Säugth. V. Tab. 300. A. B. Kos Rubialus. Lefie, Naturgefch. p. I85. n. 2. der Bïffel. Boroweky, Thierreich, I. 4. p. 33. der Biiffelochs, Biiffel. Tab. 34. A.

Blumenbach, Handb. der Naturgefch. p. IIr. 11. 3. Bos (Buffelus) cornibus refupinatis intortis, antice planis; der Biiffel.

Bechftein, Naturgefch. Deutfchl, I. p. 669. n. I. dev Biiffel.

Funke, Naturgefch. I. p. 34. der Bïffel.

Ebert, Naturl. I. p. 285. der Biiffel.

Halle, vierf. p. 278. der Biiffelochs.

Meyer, Thiere, II. Tab. 43. der Bïffel.

Gatterer, vom Nutzen und Schaden der Thiere,

I. p. I3r. n. 75. der Bïffel.

Beckmann, Naturhilt. p. ro. a. der Bïffelochs. Neuer Schaupl. d. Natur, I. p. 1030. Biiffel.

Onomat. hiftor. nat. I. p. 286.393. Biüffelochsen. p. 306. ingarifcher Biiffelochs.

Handbuch der Naturgefch. I. 2. p. 255. dev Biiffel.

Frifch, Naturfyf. p. I. n. I. Bubalus vulgaris; der gemeine Biiffel.

Klein, Claflif. der vierf. Thiere, p. 34. Bïffel. Klein, natürl. Ordn. d. vierf. Th. p. 12. n. 2. Bïffel.

Büffor,

Ein ausgewachfener Büffel wiegt 800 bis 1000 Pfund, und wird mit 60 bis 90 Thalern bezahlt. Die Haut koftet etliche und 20 Thaler. Die alten Rümer verfertigten Harnifcha daraus. In der brittifchen Sammlung if ein Horn, das $2 \mathrm{r}$ Pfund wiegt, und 4 Quarts Waffer fafst. Urfprïnglich ftammt das Thier wohl aus Tybet. Zwifchen den lahren 59r. und 6í. ift es von Indien in die Lombardey eingeführt. Zimmermann if nicht der Meynung, dafs der Elephantenochfe des Luiolf hieher gehüre. 
Büffon, vierf. IX. p. I12. 203. der Bïffel.

Bat/ch, Thiere, I. p. I4r. der Biiffel.

Niebuhr, Befchr. von Arabien, p. 165. Biiffel. ochsen, Büffel.

HaJfelquift, Reife nach Paläftina, p. 577. Biiffel.

Kolbe, Vorgeb.'d. g. Hoffn. edit. in 4. p. 327.

Bïffel-Ochsen.

Baldiutus, Zeyl. und Malabar, p. 421. 422. Biiffel.

Dapper, Afrika, p. 551. der Biiffel.

Shaw, Reifen, p. 359. der Biiffel oder Bufalo.

Ausz. a. d. Tageb. eines Reifenden nach Afren,

p. ro3. der Bïffel.

Pallas, Reife, Ausz, I. p. 317.369. Biiffelsschädel, Biiffelsköpfe.

Knox, ceylan. Reifebefchr. p. 4r. Biiffel. Ochfen. Pallas, nord. Beytr. I. p. 9. der Biiffel.

Hanokesworth, Gefch. der engl. Seereifen, III.

p. 355.362 .

Ellis Reife nach Hudfonsmeerbufen, p. 257.

S. G. Gmelin, Reife durch Rufsl. III. p.326. 433 .

Olear. mofcow. und perf. Reif. p. 278.

Berlin. Samml. VÍII. p. 166. Biiffel.

Kriinitz, ökon. Encyklopäd. VII. p. 365. Biiffel, Bïffelochs, Piiffel.

Kliigel, Encyklop. I. p. 24r. der Bïfet.

Hamburg. Magazin, VI. p. 59r. Bilffelochjen.

Börner, neue fchlef. ökon. Nachr. II. p. 23. n.41. lios Bubalis.

Merklein, Thierreich, p. 136. Biiffel, Piiffel.

Oekonom. Zool. p. 50. n. 52. der Biiffel.

Iablonfky, allgem. Lex. p. I83. Biiffel.

Beckmann, phyC. ökon. Bibl. III. p.340. der Büffel.

Onomat. foreftal. I. p. 4 10. Biiffel.

Erxleben, Manmal. p. 238. n. 5. Bos (Bubalis) corsibus refupinatis, intortis, antice planis. 
Linné, Syft. Nat. Edit. II. p. 51. Bos cornibus vaftis intortis.

Linné, Syit. Natur. Edit. VI. p. 15. n. 4. Bos cornibus valtis intortis refupinatis.

Linné, Syft. Natur. Edit. X. I. p. 72. n. 4. Bos (Bubalis) cornibus refupinatis, intortis, antice planis.

Graumann, intr. in hiftor. nat. mamm. p. 44. V. Biiffel.

Gatterer, brev. zoolog. I. p. 67 n. 5. Bos (Bubalis) cornibus refupinatis, intortis, antice planis; der Biiffel.

Severin, zoolog. Hungar. p. 36. E. Bos bubalis; der Büffel.

Kramer, Aufr. p. 322. Bos cornibus valtis intortis refupinatis.

Warnefrid, de geftis Longobardor. Lib. IV. c. 2.

Ludolf, hiftor. Aethiop. Lib. I. c. 10. n. 66. Bubalus.

Demanet, Afriq. franc. II. p. 128.

Turpin, hift. de Siam, I. p. 301.

6. CAFFER. Der afrikanifche Büffel.(')

Zimmermann, geogr. Zool. Il. p. 9ò. n. 9. Bos (Caffer) cornibus bafi craffiffumis, fubcompla. natis,

(x) Die Stärke diefes Thiers if fo grofs, dâs der Löwe, der dach dem gröfsten Capochfen mit einem Schlage den Rücken bricht, oftmals im Zweykampfe mit ihm untèrliegt. Man hat bey mehr als einem folcher Büifel auf der Nafe die Narben der einge. druckten Lüwenklauen gefunden. Falfchheit und Heimtïcke find Hauptzïge in feinem Karakter. Er fillt oft plörzlich ïber Menfchen und Thiers aus einem verborgenen Hinterhalte her, wirft fie nieder, zerqueticht fie mit Hürnern und Füfsen, und leckt felbat nash ihrem Tode ihnen das Fleifch 2 wiederholten 
natis, rugofis, fronti incumbentibus, medio attenuatis, teretibus, deorfum inclinatis, poftea furfum flexis, apice mucronatis; der af $i$ kanifche Biiffel.

Schveber, Säugthiere, V. Tab. 30r. Bos Caffer. Bïffon, vierf. IX. p. 196. der afrikanifche Biiffel; in. e. Fig.

Sparmann, Reife n. dem Vorgeb. d. g. Hoffin. p. 257. 379. 389. 393. 435. der Biiffel. 'Tab. 2. Le Vaillant, Keife nach $A$ frika, I. p. 158. die Biiffel.

Forfter, Reife um die Welt, Edit. in 8. I. p. 85. der wilde Biiffel.

B. Búbalus africanus. Der Dante.

Zimmermann, geogr. Zool. II. p. 92. n, Ir. der Dante.

Borowfky, Thierreich, I. 4. p. 42. der afrikaniSche Zwergochs.

Halle, vierfüfs. p. 278. der kleine afrikanifche Zwergochs.

Klein, Claffif. der vierf. Thiere, p. 35. ein kleiner afrikanifcher Ochs.

Klein, natürl. Ordn. der vierfüls. Thieré, p. I2. ein kleiner afrikanificher Ochs.

Frifch,

Malen ab, Durch das ftarkfte Bufchwerk rennt er mit eben der Leichtigkeit, als durch ein Kornfeld. So bald er warm gehetzt worden, wirft er fich in jedes Waffer ohne Unterfchied; daher glaube man auch, dafs man keinen Nutzen davon haben würde, wenn man ihn zihmen wollte, um ihn vor dem Wagen zu gebrauchen, weil er, fo bald er heifs und müde ware, fich ins nachfte Wafter legen, oder vielleicht andere Streiche ausiiben würde. Sparmann fiefs, weit vom Cap, gegen das Innere von Afrika, einmal auf eine Schaar, die aus to bis 80 Iungen und Alten beftand. Am Cap felbit ift das Thier unbekannt. 
Frifch, Naturfyftem, p. r. n. 2. der afrikanifche Biiffel.

Biiffon, vierf. IX.p. 128. der kleine Ochs des Belon. Erxleben, Mammalia, p. 24I. Marmol. Afrique, I, p. 52. le Dante.

A) Verïnderningen gegen die XIIte Edition, und Ver mehrung der Gattungen diefes Gefchlechts.

Edit. XII.

Edit. XIII.

p. 99. n. 2. Bos Bonafus.

p. 99. n. 3. Bos Bifon.

p. 202. n. I. a. b. Bos Tau. rus, ferus, Bonajus.

p. 203. n. c. Bos Taurus, ferus, Bifon.

p. 99. n. 3. B. Bifon Amea p. 204. 11. 2. Bos Americaricanus. nus.

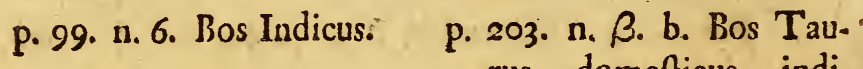
rus, domeflicus, indicus minor.

p. 99. n. 6. B. Bubalus Afri- p. 207. n. 6. B. Bubalus Africanus. canus.

Es find alfo drey Gattungen der XIIten Edit. hier als Varietäten, und eine Varietät aus derfelbrn hier als eine eigne Gattung angenommen; die Gattungen Nr. 2. und 6. B. Mo fchatus und Caffer neu hinzugekommen, dafs alfo die Anzahl der Gattungen eben diefelbe geblieben if.

\section{3) Unbeftimmtere Thier'.}

1. Der zeilde Ochfe vom Gebirge Nerever.

Zimmermann, geogr. Zool. 15. p. 93. b.

Thevenot, voyage, III. p. II . 


\section{Mammalia Pecora. Bos, $\quad 205$}

2. Die braunen wilden Othjen von Duguela.

Zimmermann, geogr. Zool. II. p. 93. c. Marmol, Afrique, III. p. 66 . und $x_{57}$ :

3. Der Gauvera anf Zeilan.

Zimmermanan, geogr. Zoolog. II. p. 93. d. Knox, hifor, Relat. of Ceilod. Lond. 168it. pag. 2t.

Knox, ceylan. Reifebefchreibung. LPZ 6689 .

p. 4r. Gawvera.

Allgem. Reifer, VIII. p. str.

4. Der Annoa auf Celebes.

Zimmermann, geogr. Zoolog. U1. p. 93. e.

Penniant, fyn quadrup. p. 6 . 


\section{SECHSTE ORDNUNG.}

\section{BELLUAE. (Thiere mit Pferdegebifs.)}

33. GESCHLECHT. ERUUs. Das Pferd.

Muiller, Naturfyftem, I. p. I45. Gen. XXXIII. Zimmermann, geogràph. Zoolog. II. pag. 79. Gen. I.

'Lefke, Naturgefch. p. I86. Gen. XXXIX. Borows ky, Thierreich, I. 4. p. 44. Gen. XXXV. Blumenbach, Handb. der Naturgefchichté, p: 99. Gen. XXVIII.

Bechltein, Naturgefch. Deutfchl. I. p. 709.

Onomat. hift. nat. III. p. 807 .

Batfch, Thiere, I. p. I45.

Frifch, Naturfyftem, p. I.

Erxleben, Mammalia, p. 20\%. Gen. XXIV.

Graumann, intr. in hiftor. nat. mamm. p. 42 . Gen. XXIV.

Gatterer, brev. Zoolog. I. p. 56. Gen. XXIV. Brifjon, quadr. p. 69. Gen. XIV. Hermann, tab. affin. animal. p. Ior.

* Mit getheiltem Hufe.

6. Bis uccus. Der Guenul, oder Huemuil.

Vidaure, Gefchichte des Königreichs Chile, p.88. das Thier Guemul.

Goeze, Natur, MenIchenl. u. Vorfeh. V. p. 280.' das Guemul. 
* Mit ungetheiltem Hufe.

1. Ca b a llu s. Das gemeine Pferd. ( $\left(^{2}\right)$

Miiller, Naturfyft. I. p. 446. n. I. der Hengft. Zimmermann, gèogr.Zool. I. p. 178. II. p. 79. n. I. das Pferd.

LeJke, Naturgefch. p. 186. n. I. das Pferd. Borowsky, Thierreich, I. 4. p. 46. n. 2. das Pferd.

Bhumbenbach, Handb. d. Naturgefch. p. 99. n. I. Equus (Caballus) cauida undique fetofa; das. Pferd.

Bechftein, Naturgefch. Deutfchl. II. p. 709. n. I. das Pferd.

\section{$\mathrm{Y}_{\mathrm{y}} 2 \quad$ Funke,}

(2) Pferd, Ro/s (die ganze Gattung), Hergft (das Mannchen), Stute, Stutte (das Weibchen), Wallacb (ein verfchnittener Hengft), Futlén, Foblen (ein Iunges). Der Nutzen diefes Thiers für die Menfchen ift hinlanglich bekannt. Die arabifchen Pferde zeichnen fich durch ihre Leichrigkeit und Dauerhaftigkeit, die perfifchen durch ihren ausnehmend fchönen Bau aus. Unter den etropaifchen find die panifohen, (befonders die aus Andalufien) die neapolitanifchen und englifchen, die vorzüglichften. Die Araber haben zwey Racen Pferde, wovon fie die eine Kadifcbi, d. i. Pferde von unbekaninter Abkunft, nennen, die in Arabìen nicht hơher als die gemeinen 'Pferde in Furopa gefchätzt werden; die andere Race heifst Köcblani oder Köbejle, d. i. Pferde, deren Abkunft man bereits von zwey taulend Iahren her aufgefchrieben hat. Diefe follen urfpränglich aus der Stutterey des Kijnigs Salomon abftanımen, und werden gemeiniglich um fehr hohe Preife verkauft Klein gedenkt eines Pferdes in det Galerie zu Dresdert, welches ifabellfarbig, deffen Mahne 9 Schuh, der Stirnzopf fechftehalb, und der Schwanz 25 Schub hielt. Die Rammhare des oldenburgifchen Pferdes, der Kravich genannt, waren achtehalb Schuh, und der Schwanz 17 Schuh 4 Zoll lang. In Arabien und Perfiem gefchiehet das Wallachen nicht, und die unnatürliche Mode des Anglifirens kounmt felbft in England wieder ab. Stirbe von bittern Mandela. 
Funke, Naturgefch. I. p. 44. das Pferd.

Ehert, Naturlehre, I, p. 278. das Pferd.

Halle, vierf. p. 227. das Pferd.

Meyer, Thiere, I. 'Tab. 24. das Pferd.

Gatterer, vom Nutzen und Schaden der Thiere,

I. p. 80. n. 6r. das Pferd.

Beikmann, Naturhiforie, p. 8. n. I. das Pferd.

Neuer Schauplatz der Natur, VII. p. 260. Ro $s$.

Onomat. hif. nat. III. p. sos. das Pferdt.

Handb. der Naturgefch. I. p. 247. dos Pferd.

Handbuch d. deutchen Thiergefch. p. 6I. das Pferd, Ro/s, der Gaull.

Sander, ökon. Naturgefch. I. p. 182. das Pferd. Walther, ökonom. Naturgefch. Deut fchl. p. 16r.

Pferd:

Frifch, Naturfyft. p. r. n. r. das Pferd.

Biiffon, vierfüls. I. p. I3. das Pferd. Tab. 1 . das barbarifiche Pferd. Tab. 2. das fpanifche Pferd. Tab. 3. das englifche Pferd. 'Tab. 4. das neapolitanifche Pferd. Tab. 5. das frieffine Pferd. Tab. 6. das Pferd.

Batfch, Thiere, I. p. I45. das gemeine Pferd. Bock, Naturgefchichte von Preuffen, IV. p. I99. der Hengft.

Molina, Naturgefch. von Chili, p. 284. Pferde. Fidaure, Gefch. von Chili, p. 84. Pferde. Hartfenk, Befchr. von Guiana, I. p. II3. Pferde. Dobrizhoffer, Gefch.d. Abiponer, I. p. 284. Pferde. Cetti, Sardinien, I. p. 27. das Pferd. Pontoppidan, Norwegen, II. p. 4. Pferde. Boswell, Befchr. von Corfica, p. 4r. die Pferde. Niebuhr, Arabien, p.'16r. Pferde. Höft, Marokos und Fes, p. 29r. Pferde. Mariti, Reifen durch Cypern, Syrien u. f. $w$. 2 2 İ. Pferde. 
Wolf, Reife nach Zeilan, p. IIs. Pferde.

Byron, Reife um die Welt, p. 13. die Pferde. Linné, goth. Reife, p. 136. die (öländijihnn) Pferde.

Pennant, Reife d. Schottland; II. p. 27 r. Pferde. Schöpf, Reife d. Nordamerika, II. p. 34. Pferde. Krafcheninnikoro, liefchr. von Kanitchatka, p. I3I. P'ferde.

Leffeps, Kamtfchatka und Sibirien, p. 5o. Pferde. Du Halde, China, IV. p. 33. tartarifche Pferde. Känıfer, Iapan; in du Halde China, IV. p. 343 . Pferde. I. p. 137. Pferde.

Taute, Slavonien und Syrmien, I. p. 40. I' ferde. Adanfon, Senegall, p. 238 . Pfer.de. Bankroft, Guiana, p. zi. das I'fert. Steller, Kantfchatka, p. I40. Pfertie. Kolbe, Vorgeb. d. g. Hoffn. Edit. in 4. p. 334. Pferde.

Ausz. a. d. Tageb. eines Reif. nach Afien, p. 179. das Pferd.

Baldaeus, Zeylon und Malabar, p. 42ะ. Pferde.

p. 398. Pferde.

Dapper, Afrika, p. 19. 20. barbarifche Pferde. Olaffen, Island, I. p. 27. 100. 188. 278. II. p.44. 117. 198. Pferde.

Uno von Troil, Reife n. Island, p. Ior. Pferde. De Kerguelen Trémarec, Reile nach d. Nordfee,

p. 7 I. Pferde.

Slaw, Reife, p. 147. das Pferd.

Fermin, Surinam, I. p. 89. 93. II. p. 83. Pferde.

Reife nach der Infel Frankr. u. Bourbon, g. 294.

Pferde.

Sparrmann, Reife, p. 74. ein Pferd. I. 21 Ir. die Pferde.

Falkwer, Patagonien, p. nza zahme Pferde.

$$
\mathrm{Y}_{\mathrm{y}} \mathrm{I} . \mathrm{G} \text {. }
$$


I. G. Gmplin, Keife durch Sibirien, I. p. 99. 168. 274. 333. 335. 441. Pferde.

Sehlözer, Erdbefchreib. von Amerika, p. 274. Pferde.

Tavernier, oriental. Reifebefchreib. Genf, I68I. fol. III. p. 66. 79. Pferde.

Georgi, Rufsland, I. p. I40. I48. 183. 235. II, p. 313.320.390. Pferde.

Paltas, Reife, I. p. 315 ff. 333 . III. p. 12\%. Pferde.

Pallas, Reife, Ausz. III. p. 19. Pferde.

Kleemann, Reife. L.pz.1773. 2 Auff. p. 85. Pferde. Anfon, Reife un die Weit, p. 64 Pferde.

Neuhof, Gefandfch nach China, p. 347. Pferde. Debes, natürl. u. polit, Gefch, der Infeln Feroë. Koppenh. I757. p. IIO.

Beytr. zur Beförderung d. Naturkunde, I, p. 218. Pferd, Rofs.

Merklein, Thierreich, p. 160 . Pferd.

Oekonom. Zool. p. 52. 11. 59. das Pferd.

Iabloufky, allgem. Lex. p. 80r. Pferd.

Erxleben;, Mammal.,p. 207. n. r. Equus (Caballus) cauda undique fetola.

Linné, Syft, Nat. Edit, II. p. 48. Equus cauda undique fetola.

Linne, Syf. Nat. Edit. VI, p. II. n. I. Equus cau. da undique fetofa,

Linné, Syft. Nat. Edit. X. I. p. 73. n. I. Equus (Caballus) cauda undique fetofa.

Limué, Fu. Suec. I. p. 43. n. 34. Equus cauda undique fetofa.

Linne', Fn. Suec. II. p. 15. n. 47. Equus (Cabal, lus) cauda undique fetofa.

Graumann, intr, in hiftor, nat, mamm. p. 42. I. Pferd, Ross. 
Gatterer, brev. zoolog. I. p. 56. n. I. Equus. (Caballus) cauda undique fetofa.

Severin, zool. Hungar. p, 28. n. x. Equus, Caballus, Sonipes; das Pferd.

IMïller, zool. dan. prodr. p. 6. n. 42. Equus (Ca: ballus) cauda undique fetofa.

Kramer, Auftr. p. 3I7. Equus cauda undique fe: tofa.

Outier, Journal d' un voyage au Nord, p. roo. Voyages della Valle. Rouen 1745. V. p. 284. Voyages du Sieur le Gouz de la Boullaye. Paris 1657 .

Bougainville, voy. aut. d. monde, p. I3 T. Ludolf, Aethiop. Lib. I. c. I0. n. 68. Equus.

Leon. Afric. defcript. Afr. p. 750 . Equus Barba. ricus.

Forfter, philor. Transact. LVII. p. 345. Equus Caballus.

$p_{\text {ennant, britt. zool. p. I. n. I. the Horfe. }}$

Varro, de re ruft. Lib. II. c. 7 . de equis et equabus.

Procop. de bello Gothor. Lib. IV: c. 24. I $\pi \pi$ or.

Cohumella, de re ruft. Lib.VI. c. 27. genus equinum.'

๙. FER U s. Das wilde Pferd.

Schreber, Säugthiere, V. Tab, 309. Eqquus Cabale lus ferus.

Borowfky, Thierreich, I. 4. p. 47 das wilde Pferd.

Ebert, Naturl. I. p. 279. wilde Pferde.

Cetti, Sardinien, I. p. 27. das wilde Pferd. Pallas, Reife, Ausz. I, P. I42. wilde Pferde. Falkner, Patagonien, p. 53. wilde Pferde.

Dobrizhoffer, Gefch, der Abiponer, I. p. 284. herumiersende Pforde.

$$
\mathrm{Yy}_{4} \text { Scílizer. }
$$


Schlözer, Erdhefchr, von Amerika, p. 276. die wilden Pferde,

$D u$ Halde, China, IV. p. 32. die wilden Pferde: Pallas, Naturgefch, merkw. Th. XI. p. 6. wilde Pferdie.

Pallas, Reife, Ausz. III. p. 346. die wilden Pfern de, oder Tarpanzm.

Newhof, Gefandfch. nach China, p. 347. wilde Pferde.

Schriften der berlin. Gefellfch. IX. p. 189. wilde Pferde.

Pritzelizus, Befchreib. des fo bekannten Sennergefuttes, in der Graffchaft Lippe, Lemgo 1770.8.

Becknann, phyf. ökonom. Bibi, II. p. 589. wilde pjeritis. VII. p. ri2.

Dapper, Afrika, p. 20 wilde Pferde. Linné, goth. Reife, p. 248. die vilden Pferde. Leon. Afric. defcr. Afr. p. 75 r. Equus fylvaticus,

B. Domesicus. Das zahme Pferd. Schreber, Säugthiere, V. Tab. 310. Equus Ca. ballus domefticus.

Klein, Claffif. der vierf. Thiere, p. 13. das Hauspferd, oder das zahme Pford.

Klein, natül, Ordn der vierf. Th. p. 4. n. I. Hofpferd , Haus - Bauerpferd.

Fabric faun. groenl. p, 22. n. g. Equus Caballus.

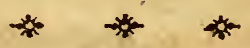

v. Sind, Unterricht in den erften Wiffenfehaften eines Stallmeifters, Göttingen, 1770. 4. Init Is Kupfer.

Abildgacird, Unterricht von Pferden, Kühen, Schaafen, u. Schweinen. Lpz. 777 1. 8 . 
Hurel, Abhandl. über den Wurm der Pferde; a. d. Franz, brefsl. I77r. 8.

Ridinger, Vorfell, der Pferde nach ihren Haupt. farben. I770. m. só K.

Bevenger, hiftory and Art of Horfemanship, Lond. 177r. gr. 4.

La Fole, Cours d'Hippiatrique, ou Traité coms. plet de la Médecine des Chevaux, orné de foixante et cinq planches. Par. 1772. Landchar. tenformiat.

v. Schönfeld, Landwirthlchaft, P. 349-496.

Der königl. grofsbritt. u. f. ww. Landwirth fch. Gef:

Nachrichten, von Verbeff. der Landwirthfch.

II B. 6 Samml. Zelle, 1772. p. 694.

Zückert, von einem bösartigen Entzündungsfieber der Pferde, welches im Aug. 1773. um Berlin gewvïthet hat; in den Befchrifft. der berlin. Gefellfch. I. p. 138.

Za Fo/fe, dictionnaire raifonné d'Hippiatrique,

Cavalerie, Manège, et Marechallerie. Paris, 1775. Tom, I:IV. 8.

Sihreber, Beyträge zur Beförd. der Haushaltungs. kunde u. $f_{\imath}$ w. Münf. 1776. 8. p.326.

Hartmann; Pferde-und Maulthierzucht. Stuttg, 1777. 8. 2te Aufl, 1786.

Prizelins, volifändige Pferdewiffenfchaft. LPz. 1777. gr. 4. m. .51 K.

v. Reizenftein, vollkommener Pferdekenner. Uf: fenh. I764.

Aufgedeckte Rofstäufcherkünfte, L.pz. 1780.

Berlin. Beytr. zur Landwirth fchafts wiffenfchaft, III. p. 205:

Drumpimond de Mielford, traité fur lá Cavalerie. Paris, 1776. fol.

Von Pferdekrankheiten u,f. w, Brandenb. I778.8. Y 5 Bewälırte 
Bewährte-Arzneymittel vor die Krankheiten der Pferde. Erfurt, 1777. 8.

Bounwinghaufen von Wallmerode, praktifche $\mathrm{Ab}$. handlung, von dem Unterfchiede zwifchen der Drufe und Strengel der Pferde. Stuttg. I776. 8 .

Bartlet, Pharmacopöe oder Apotheke eines Rofs. arztes u. $f_{\text {. }}$ v. aus d. Engl. überf. von Buchholz. Weimar $1778.8 . \mathrm{m}$. K.

Samml. einiger Abhandl. von verfchied. Krankheiten der Pferde und Schaafe u. f. w. Nürnb. I719. 8 .

Vitet, Unterricht in der Vieharzneykunft; a. d. Franz. überf. von Hennemann. VI. Lemgo, I785. 8 .

Der Hausvater, II. p. 55. 63. 478. V. p. 469. $B u f c h$, Archiv für Rofsärzte und $\mathrm{Pferdeliebha-}$ ber. Marb. 1788. \&.

Ploen, Anleitung zur äufsern Pferdekenntnifs. Berlin, I790. 8 .

Schreber, Samml. verfch. Schriften u. f. w. Halle, I759. IV. p. 24I. 294. 35I. ff.

Gefellfchaftl. Erzählungen. Hamburg, I753. IV. p. 49. II. p. I29.

Bracken, verheff. Rofsarzneykunft u. $f$. w. aus d. Engl. Altenb. r758. 8.

Zeiher, Lehrbegriff von den Krankheiten derPfer-

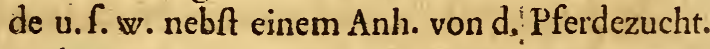
Berlin, I77r. 8.

Erxleben, prakt. Unterricht in der Vieharzneykunft. Gött. 1771. 8.

Zehntner, Unterricht von der Pferdezucht. Berl. I754. 8 .

Zehntner, gründl. Abhandl. der Kunf, Pferde zu erkennen. Berl. 1757.8. 
Garfoult, Unterricht für Liebhaber der Pferde und Reiter; a. d. Franz. von Kriinitz. Berlin, I770. 8. $\mathrm{m}, 7 \mathrm{~K}$.

v. Saunier, vollfändige Erkänntnifs von Pferden u. f. w. Lpz. 1767. fol.

Kölner, Verfuch über die Farben und Eigenfchaften deutfcher und ausländ. Pferde. Greiz, I791. 8.

Der deutfche Rofsarzt zu Heilung der Krankheiten der Pferde, für Verwalter, Schmiede und Bauern, Halle, r79r. 8.

Ridinger, Entwurf einiger Pferde, nach ihrem unterfchiedl. Aiter und Gebrauch, nach dem Leben gezeichnet. Augśb. I755. fol.

Marx. Fugger, von der Zucht der Kriegs , und Bürigerpferde, nach der altdeut Chen Ausgabe von 1578. überfetzt; mit Anmerk. und dem sten Theil vermehrt, von $I_{i} G$. Wollftein. gr. 8. Wien 1786.

Wilhelm, Herzogs von Neukaftel Reitbạhn. Nürn. berg, 1764. fol,

2. Berga, neue Reitkunf. 'Tüb. I755. \&.

v. Eifenberg, wohleingerichtete Reitfchule u. f. w. a. d. Franz. $\Lambda m f$. u. Lpz. I746. lang fol.

Die Zaumkunf, durch Hippophilum. Herborn. 4738. fol.

Trichter, von der Pferde-Anatomie. I7I6. 8. Die hefte und bewährtefte Erfind. wider das Kop. pen der Pferde. Anfp. 1764.8 .

Anonymi, wohlvorficht. Pferdearzt, Schwabach 1766.8.

Fuchfens aufrichtiger Rofsarzt. Lpz. 1733.8. Beckmann, Bibl. I. p. 184. 342. II. p. 107. 205. 361. 474. 578. III, $p_{q}$ 155. 257.260.496. IV. $\begin{array}{lll}\text { p. } 64.317 \cdot 452 . & \text { V. p. } 352.493 \cdot 542 \text { VI. }\end{array}$ 
p. 222.435 .490 . VII. p. 196. 326 . VIII. p. 582. IX. p. 53. 71. 205. 476. X. p. 82.228. 229. 235. 247.562. XIII. p. 317. 593. XIV. p. 230. $235.375 .399 . \quad X V \cdot$ p. 426 . XVI. p. 425 .

Schwed, Abhandl. II. p. 246. III. p. 294. IV. p. 312. VII. p. 229. 22I. VIII, p. 6I. X. p. 142. 144. XI. p. 81. XV. p. 292. XVIII. p. 70. XIX.p. 278. XX.p. 55. XXIV.p. 45. XXXIV. p. 278. XL. p. 25 ff.

Berlin. Samml. II. p.396. III. p. 206. IV. p. I29. 316. $372.55 \mathrm{I}, \quad$ V. p. $38 \mathrm{I} .386$. VI. p. 502. VII. p. 58. X. p. 273 .

Hammard, Reife nach der Ukraine, I, p. 190 ff.

- Guevinieve, vollt, Anveif. zur Reitkunft u.f. w. a. d. Franz, von Knöll; m. K. Marb. I79r. \&.

5. Henionus. Der Dfiggettai.

Zimmermann, geogr. Zool. I. p. 183. II. p. 80 . der Djeggettai.

Sehreber, Säugthiere, V. Tab. 3rr. Equus Hemionus.

Lefke, Naturgefch. p. 187. n. 2. dev DShikket äi. Borowfky, Thierreich, I. 4. p. 5I. 11. 3. der DSchikketäi, das Langohr.

Bhmenzach, Haindb. der Naturgefch. p. IIr. ** der Dfinggetäi:

Gatterer, voin Nutzen und Schaden der Thicre,

I. p. 98. n. 66. dev TJchicketäi.

Bat fch, "Thiere, I. p. 147. der" Dfiggetai.

Klïgel, Encyklopädie, I. p. 249. der Dfikkotäi. Pallas, Reife, Ausz. III. p. 174. DJhiggetai. Naturforfcher, VII. p. 54. Caigitay. Gmelin, Reife durch Sibirien, II p. ro7. Tfchigitai, 
Catal. Mufaei Petropol. I. r. p. 335. Mulus dauricus foecundus Ariftotelis, Cappadocicus Errefii.

"Erxleben, Mammalia, p. 211. Equus Hemionus, Mongolis Dfchikketaei dic\{us.

Gatterer, brev. zool. I. p. Gì. n. 4. Equus (Hemionus) auriculis magnis erectis, cauda extre. mitate fetofa, corpore luteo fufco; das Lang. ohr, der Halbefel.

Hermann, tab. affinit. animal. p. I02. Equus Hemionus.

2. As Inus. Der Efel.

Miiller, Naturfyfem, I. p. 453. n. 2. der Efel. Zimnnermann, geogr. Zool. I. p. 178. II. p. 80. n. 3. $\operatorname{der} E \int e l$.

Le $\int_{k e}$, Naturgefch. p. 187. n. 3. der Efel.

Borowfky, Thierreich, I. 4. p. 52. n. 4. der ESel.

Blimenbach, Handb. der Naturgefch. p. ror. n. 2. Equus (Afinus) cnuda extremitate fetofa, cruce dorfali nigra; der Efel.

Bechftein, Naturgefch. Deutfchl. I. p. 745. n. 2. $\operatorname{der} E \int e^{\prime}$.

Funke, Naturgef chichte, I. p. 54. der EJel. Ebert, Naturl. I. p. 280. der F.jel.

Beckmann, Naturhiftorie, p. 8.n. 3. der Efel. Neuer Schaupl. d. Natur, II. p. 709. Efel.

Onomat. hiflor. nat. II. p. x. der Efel.

Handb. der Naturgefch. I. p. 28r. der Egel.

Handbuch der deutfchen Thiergefchichte, p. 62. der Efel.

Sander, ökon. Naturgefch. I. p. 240. der Efel. Walther, ökon. Naturgefch. Deutfchl. p. I26. Efel. Sander, Naturforfch. XVI. p. 85. von einem wiitenden ESel. 
Frifch, Naturfyft. p. I. n. I. der Efel. Bïffon, vierfüls. I. p. 187. dier ESel. Bat $c h$, Thiere, I. p. 146.der Efel. Bock, Naturgefch. von Preufien, IV. p. 230. der Efel.

Molina, Chili, p. 284. Esel.

Vidaure, Gefch. von Chili, p. 85. Ejel.

Dobrizhoffer, Gefch. d, Abiponer, I. p. 307. Efel. Cetti, Naturgefchichte von Sardinien, I. p. 42. der EJel.

Boswell, Befchr, von Corfica, p. 4I. Efel.

Niebuhr, Arabien, p. I61. Ejel.

Hijt, Marokos und Fes, p. 293. Ejel.

Byron, Reife um die Welt, p. I⿳̧. E Eel.

Taube, Slavonien und Syrmien, I. p. 40. Efel. Adanfor , Senegall, p. I76. EJel.

Bankroft, Naturgefch. von Guiana, p. 7r. EJel.

Kolbe, Vorgeb. d. g. Hoffn. edit. in 4. p. 326. ceylanifiche Efel.

Ausz. aus dem Tageb. eines Reif. n. Afien, p. 163. der Efel.

Shaw, Reife, p. 148. der Efel.

Fernin, Surinam, II. p. 80. die Efel.

Reife nach d. Inf. Frankr.u. Bourbon, p. 294. EJel. Beytr. zur Beförderung d. Naturkunde, I. p. 218. n. 32. ESel.

Merklein, Thierreich, p. 40. Efel.

Oekonom. Zoologie, p. 52. n. 6o. der EJel. Tablonfky, allgem. Lex. p. 310. E/el.

Krimitz, ökon. Encykl. XI. p. 542. Esel, Hanisefel, Steinefel.

Kliigel, Encyklop. I. p. 249. der Efel. Erxleben, Mammal. p. 212. 12. 2. Equus (Afinus) cauda extremitate fetofa, cruce nigra fupra huineros. 
Graumann, intr. in hifor. nat. mamm. p. 42. II. E. Âfinus.'

Gatterer, brev. zoolog. I. p. 59. n. 2. Equus (Afinus) cauda extremitate fetofa, cruce nigra fupra humeros.

Severin, zoolog. Hungar. p. 3o. n. 2. Afinus; der Efel.

Linné, Syf. Natur. Edit. ЦI. p. 48. Equus cauda extremitate fetofa.

Linné, Syf. Nat. Ed. VI. p. II. n. 2. Equus cauda extremitate fetofa.

Limné, Syf. Nat. Edit. X. I. p. 73. n. 2. Equus (Afinus) caủda extremitate fetofa, cruce nigra fupra humeros.

Lettres edifiant. Collect. 10. p. 96 .

Ludolf, Aethiop. Lib. I. c. Io. p. 69. Afinus.

Gesner, de antiqua afinorum honeftate. Cons. ment. Götting. T. IJ.

Cafori, bibl. Efcurial. T. I. p. 208.

Varro, de re ruft. Lib. II. c. 6. Afini.

Cohumella, de re ruft. Lib. VII. c. r. Afellus.

a. FER Us. Der wilde Efel.

Zimmermann, geogr. Zool. I. p. 183. II. p. 80. der wilde Ejel.

Schreber, Säugthiere, V. Tab. 3I2. Equus Afinus Onager.

Lefke, Naturgefch. p. 187. der vilde Efel. (Onager) Borowsky, Thierreich, I. 4. p. 52. a. der wilde E/el, Waldefel.

Blumenbach, Handbuch der Naturgefch. p. IOI. der wilde Efel.

Funke, Naturgefch. I. p. 54. der wilde Efel, oder WTaldefel.

Halle, vierf. p. 252. der wilde EJel, Waldefel. 
Gatterer, vom Nutzen und Schaden der Thiere, p. 93. n. 62. der wilde EJel.

Frifch, Naturfynt. p. 1. n. 3. Onager, der Waldefel.

Klein, quadr. difp.p.7. Afinus fylveftris, Afinus ferus, Onager.

Klein, Claflif. der vierf. Th. p. 23. Walde/el.

Klein, natürl. Ordn. der vierf. Th. p. \&. n. 3 .

Waldefel, Feldefel.

Pallas, nord. Beytr. I. p. 152. Onager.

Pallas, Naturgefch. merkw. Thiere, XI. p. 6. wilde Efel.

Glas, Gefch. der kanarifch. Infeln, p. 237. Ejel, Dapper, Afrika, p. 22. wilde Efel.

Rytfchk orenb. Topogr. I. p. 233. Kulany.

Rubruquis, allgem. Gefch. der Reifen, VII Th.

I7 B. 2 Kap. I Abfchn. Kulan.

Ludolf, Aethiop. Lib. I. c. 10.73. Afinus fylve. Aris; i. e. Onager.

Leon. Afric. defcr. Afr. p. 752. Afiuus fylvaticus. Ranuolf, Reife. Augfp. 1583.4. p. 65.

Olear. Reife nach Perfien. Schlefsw. 1656. p. 526. Pietro della Valle, voyage. Amft. 1766. III. p-137. VI. p. 105. VIII. p. 49.

Graumann, intr. in hift. nat. mamm. p. 42. a. der wilde EJel.

Gatterer, brev. zool. I. p. 59. a. der wilde Efel. Severin, zool. Hungar. p. 31. Onagri, feu Afini fylveftres.

Varro, de re ruft. Lib. 2. cap. 6. Onagri. Coluquella, de re ruft. Lib. VI. c. 36. Onager:

B. Domesticus. Der zahme Efel.

Schreber, Säugthiere, V. Tab. 313. Equus Afinus domefticus.

Borowfky, 
Borowsky, Thierreich, I. 4. p. 53.b. der Efel. Bhimenbach, Handb. d. Naturgefch. p. roI. dev. zahine EJel.

Halle; vierf. p. $24 \%$. der Ejel.

Mheyeir, Thiere, II. Tab. 44. der ESel.

Gatterer, vom Nutzen u. Schaden der. Thiere;

I. p. 94. n. 63: der Efêl.

Klein; Claffif: der vierf. Th. p. I\%. der ESet.

Klein, natürl: Ordn. d: vierf. Thiere, p. 6. Efel.

Chandler, Reif, in Klein-Afien, p. 46. Efel.

Ryt fchk. orenb: Topogr. I. p. 23ö. Ifchaki oder - Ejel.

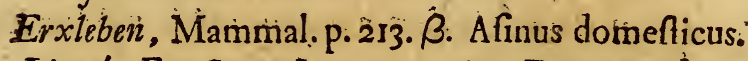
Linné, Fn: Suec. I. p. t2: n. 35. Equus cauda exa' trema fetola.

Grammani, intr: in hifor. nat: mamm: p. 42 : b. der Efel.

Gatterer, brev. Zool. I. p. 59: b. A. Domefticus; ' der zahme oder Müllerefel.

Kranier, Auftr: p: 318. Equus cauda extrema fe: tofa:

Brown, Lamaic: p. 48\%. Equus cauda extrema fetofa:

Pennant, britt. zool: p. 5. n. 3, the Afs.

y. Mutus: Das Maulthier:

Miiller, Naturfyfem, I. p. 455. A. das Maulthier, defen Vater der ESel und die IMutter ein P. ferd ift.

Zimmermann, geogr: Zoolog: İं: p.: 8o. a. das Manilthier:

Schreber, Säugthiere; V: Tab. 3it. Eqưus Mulus: Lefke, Naturgefch. p. i88: der Maiulefel. Boronefky, Thierreich; I. 4. p: 54. c. dis Mauls thier.

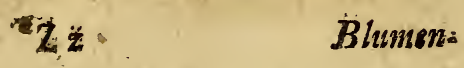


Blimenbach, Handb. der Naturgefch. p. I12. das gemeine Manlthier.

Bechftein, Naturgefch. Deutfchl. I. p. 752. n. I. das Mantthier.

Funke, Naturgefch. I. p. 57. ein Manlthier.

Ebert, Naturlehre, 1. p. 291. die Maulefel.

Halle, vierfüfs. p. 252, der Maulefel.

Gatterer, vom Nutzen und Schaden der Thiere,

I. p. 97. n. 64. das Maulthier.

Beckmann, Naturhifforie, p. 9. das Mazulthier.

Neuer Schauplatz der Natur, V. p. 422. Mandefel. (mit dem folgenden verbunden.)

Onomat. hift. nat. V. p. 280. Maul-Efel, Zwitter - Efel. (m. d. folg. verbunden.)

Handb. dar Naturgefch. I. p. 287. der Maulefel.

Handbuch der deutfch. Thiergefchichte, p. 62. Manithier.

von der Belır, oftind. Reife. Frcf, 1689. 4. p.Ir2. Maul. Efel.

Sander, ökonom. Naturgefch, I. p. 241. Manlefel. Naturforfch. VII. p. 55. Maulefel.

Frifch; Naturfyft. p. I. n. 2. Mulus foecundus; das natiirliche Maulthier.

Klein, Claflif. der vierf. Thiere, p. 19. Minulefel, Zivitterefel.

Klein, natürl. Ordn. d. vierf. Th. p. 7. n.2. Maul. efel, Zwitterefel.

Biiffon, vierf. I. p. 223. die grofsen Maulesel; Tab. 9. der Manilesel.

Bat.jeh, Thicre, I. p. I46. das Maulthier.

Dobrizioffer, Gefch. der Abiponer, I. p. 306. 309. Maulthiere.

Cetti, Naturgefch. von Sardinien, I. p. 53. Mauls efel.

Boswell, Befchr. von Corfica, p. 4̦. Maulthiere. Niebuhr. 
Niebuhr, Arabien, p. 16r. Manle.el.

Hïf, Marokos und Fes, p. 292. Maulthieve.

Hantfink, Befchr, von Guiana, p. tr.3. Manlefel.

Bankroft, Naturgelchichte von Guiana, p. 7 . MInuie fel.

Kolbe, Vorgeb. d. g. Hoffn. Edit. in 4. p. 326. Manl Efel.

Shaw, Reile, p. 148. Maulesel. p. 368. Maulesel. Fermin, Surinam, II. p. 83. Maulefel.

Du Halde, China, IV. p. 34. ITaulthier.

Beytr. zur Reförderung der Naturk. I. p. 219. der IMculejel.

Neve gefellfch. Erzähl. II. p. 162. 189. Manlthier. Bonnet, Betrachtung. tiber die Natur, I. p. 329 . Maule $e l$.

Beckmann, phyf. ökon. Bibl. IX. p. 54. Maulthiere. IV. p. 299 Maulefel.

Spallanzani, über die Erzeugung der Thiere und $\begin{array}{ll}\text { Phanzen, I. p. } 218 \text { ff. Maulesel. } & \text { p. 307. Manl- }\end{array}$ thier.

Iablonjky, allgem. Lex. p. 648. Maul, Maulesel, IMaulthier.

Erxleben, Mammal. p. 215. $\gamma$. Mulus: Hybridus ex Afino et Equa.

Graumcan?, intr. in hiflor. nat. mamm. p. $42 . c_{\text {. }}$ Maulthier, Manlesel.

Gatterer, brev. Zoolog. I. p. 60. c. Mulus, das Manilthier, der grofse Maulejêl.

Liudolf, hift Aethiop. Lib. I. c. 10. n. 69. Mulus. Panllini, Onographia, f. de Afino. Frcf: 1695.8. Severin, zoolog. Hungar. p. 31. n. 3. Mulus, Manile el.

Pennant, britt. zool. p. 6. the Mule.

Varro, de re ruft. Lib. II. cap. 8: Mulus.

Cohumella, de re ruft. Lib. VL . 36.37 . Mulus. 
8. Hinnus. Der Maulejel.

Miiller, Naturfyf. I. p. 455. B. das Maulthier, delfen Vater ein Pferd und die Mutter ein ESel ift.

Zimmernann, geogr. Zoolog. II. p. 80. b. der Miailesel:

Schreber, Säugthiere, V. Tab. 315. Equus Hinnus: Lefke, Naturgefch. p. I88. dois Maulthier.

Borowjky. Thierreich, I. 4. p. 54. d. der kleine Maule el.

Blumenbach, Handb. der Naturgefch. p. I02. der Manilefel.

Bethftein, Naturgefch. Deutfchl. I. p. 752. n. 2. der Maule el.

Funke, Naturgefch. I. p. 57. der Maulefel.

Beckmann, Naturhiftorie, p. 9. der Maulefel.

Biiffon, vierf. I. p. 223. die kleinen Manlefel.

Erxleben, Mammal. p. 216. $\delta$. Hinnus, Hybridus ex equo et afina.

Graumann, intr. in hiftor. nat. mamm. p. 42.8 . k̉leiner Maulesel.

Gatterer, brev. zoolog. I. p. 60. $\delta$. Hinnus; der. Maule $\int e l$, der kleine Maule $e l$.

Varro, de re ruft. Lib. II. cap. 8. Hinnus.

Columella, de re ruft. Lib. VI. cap. 37. Hinni.

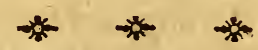

Von den Iumars, die von einigen angenommen, von den mehreften aber aus Gründen bezweifelt werden, handeln folgende Stellen:

Gesner, quadrup. p. 19. Peculiare quoddam mulorum genius; tunar.

Gesner, quadrup. p. 106. Muli vel inni genus, ex afina ct tauro: 
Gesner, quadrup. p. 799. Quartum muli genus. Aldrovand, Solidung. p. 358 . Iumar.

Wagner, Helvet. p. 188. Hippobus vel Hippo. taurus.

Shaw, Reife, p. I48. der Kumrah.

Miiller, Naturfyft. I. p. 455. Ochfenefel, oder Ochfenpferd.

Borow/ky, Thierreich, I. 4. P. 40. der Maulochs, Iunnar, Gemar.

Buiffon, vierf. I. p. 224. Manulochfen, Onotauri, Iumars, Gemars.

Zimmermann, geograph. Zoolog. I. p. I30. b. der Iumar.

Bhuszenbach, Handbuch der Natargefch. p. Ir2. Iumar.

Spallanzani, über die Erzeug. der Thiere u. f.w. I. p. 241. 349. Iumars.

Saniml. zur Phyf. und Naturgefchichte, I. p. 334. Inmars.

Goeze, Natur, Menfchen]. u. Vorfel. I p. 573. Inmara.

Beckmamn, phyf. ökon. Bibl. I. p. 3I2̀. Lumars. VII. p. $35^{\circ 0}$ XIV. p. 414.

Götting. Kalender, 1779. P. 55.74 . Allgem. deut fche Bibl. LXI. P. 322.

Bhumenbach, de gen. hum. variet. p. I3. Iumarus: Hamburg. Magaz. X. p. 444. Inmarts.

Pallas, Naturgefch. merkiw. Thiere, XI. p. 4r.

Von Efeln und Maulefeln, und deren einzelnen Theilen handeln noch folgende Schriften:

La Foffe, Cours d'Hippiatrique etc. Paris 1772. p. 165 ù. 167. (Vergleichung der Zeugungstheile in Pferden nud Maulefeln.)

$$
\mathrm{Zz}_{3}
$$

Mem. 
Mern. de l'academ, roy. des Sc. Aṇn. I769. Paris 1772. p. 47. (Beyfpiele, da Maulefelinnen von Hengften trächtig geworden.)

Berlin. Beytr. zur Landwirthfchaftswiffenfchaft, III. p. 303. (Vom Nutzen der Elel, vom Nutzen der Maulthierzucht.)

Selecta phyfico-oeconom. Stuttg. IZ49. Iq. I, p. 386.

Lemmery, Materiallex. p. II3. 749.

Hamb. Magazin, XXIII. p. 4 3. XXI. p. 30I.

Mannichfaltigkeiten, III. p. 8-II. (Chagrin.)

Krünitz, gemeinnïtz, Vorrath auserlef. Auffätze,

Lpz. $1767 \cdot$ p. 81 - 86.

Leipz. Intelligenzblatt v. Iahr $1775 \cdot$ p. 474 ;

Hamburg. Unterhalt. 1765. p. $343-345$.

Berlin. Samml, IV. p. $38 \% \cdot 396$.

Frankf. neue Auszïge, III. p. 224.

Becknuann, Grundf. der deutfchen Land wirthfch.

p. 43 I.

Allgemeine Haushalt. uud Landwif̣enfchaft, I. p. 723.

(Pfeifer) Lehrbegriff fämmtl, ökon. u. Cameral. wiffenfch. I. p. I44.

Unzer, Arzt, V. p. 33r. 373. neue Auflage III. p. 273.300.

Chomel, allgem. Lex. Art. Efel, Manlthier.

Hartmann, Pferde - u. Maulthierzucht. Stuttg. İ 86 . Pallas, nord. Beytr. I. p. 325 .

\section{Z I B R. Der Zebra. (3)}

ALiiller, Naturfynt. I. p. 456. der geftreifte Efel.

Zimmer.

(3) Vor mehretn fahren hat fich ein weiblicher Zebra in Lord Clives Menagerie zu London nach vielen vergeblichen Verfichen 
Zimmermann, geogr. Zool. II. p. So. n. 4. des" Zebra.

Sihreber, Säugthicre, V. Tab. 316. Equus Zebra. Lofke, Naturgefch. p. I8s. n. 3. das Zebrapferd. Borowe ky, Thierreich, I. 4. p. 45. 11. I. das afrikanifche Tygerpferd, das Zebrc. 'Tab. 35. Blumenbach, Handb. der Naturgefch. p. 102. 11. 3. Eyuus (Zebra) zonis fufcis et albidis, maxime regularibus.

Funke, Naturgefchichte, I. p. 54. das Zebra. Ebert, Naturl. I. p. 279. das Zebra oder Zecora, der geftreifte Efel oder Efelpferd. Tab. 12.

Halle, vierf. p. 245. das Zebra. Tab. I.

Gatterer, vom Nutzen und Schaden der Thiere,

I. p. 97. 11. 65. das Zebra.

Beckmann, Naturhiforie, p. s. n. 2: Zelra.

Neuer Schaupl. d. Natur, X. p. I6r. Zebra.

Otiomat. hiftor. nat. VII. p. 869. das Zebra.

Handb. der Naturgefch. I. 2. p. 266. das Zebra. Frifch, Naturfyft. p. I. n. 2. Cebra; der Zebra, afrikanische Atreifige witde Pferd.

Klein, Claffif. der vierf. Thiere, p. 15. unngezihn • tes, oder Jeiner Art wach willies Pferct.

Klein, natürl. Ordn. der vierf. Thiere, p. 5. 11. s. wildes Pferd.

Bïffon, vierf. X. p. 42. das Zebra; m. 2.Figg. Bat $\int c h$, Thiere. I. p. 147. das Zebra.

Goeze, Natur, Menfchenl. u. Vorfel.. V. p. 285. das Zebra.

\section{$\mathrm{Zz} .4$} Kolbe,

chen von einem, mannlichen Efel, den mán wie einen Zebra mit Streifen bemahlt hatte, befpringen laffen, und eine Art Maulthier zur "Welt gebracht, das in der Bildung völlig das Mittel zwifchen feinen beyden Eltern hielt, and von grauer Gruņdfarbe wie der Vater, aber fchwarz geftreift, wic die Mutter, war. 
Kolbe, Vorgeb, d. g. Haffn. Edit. in 4: p. 326. Wald-Efel. Tab. 33. Zecoa, oder af rikanifoher W'aldefel.

Sparrmann, Reife, p. 126. 210. Zebra.

Le. Vaillant, Reife, I. p. 99. Zebra's.

Bankroft, Guiana, p. ż. die Zebra.

Reife nach der Infel Frankr. u. Bourbon, p. $293 \%$

Zebra.

Dapper, Afrika, p. 55r. Azebro oder Zebra. Samml. zur Phyf: u. Naturgefchichte, I. p. 340: Zebra.

Nouvelle defcript: du Cap de b. E. Amn, 1778:,

p. 52. not.

Neue kurzgefafste Befchreib. des Vorgeb. d. g: H.

Lp7. 1779. p. 172 . not. 3\%.

The Zebra, or wild Afs. Von G. Stubbs: mit lebendigen Farben, I77.

Iablonfky, ailgem. Lex. p. 1323. der afrikanif 6 ho Waldefel.

Oekonom. Zool, p, 53. n. 6r. geftreifte Efel. Neuefte Mannichfaltigkeiten, II. p. I32." III: p. 267. Zebra.

Erxleben, Mammal. p. 216. n. 3. Equưs (Zebra) falciis fufcis transverfis.

Limné, Synt. Natur, Edit. II. p: 48. Equus lineis transverfis yerficolor.

Limmé, Syn. Nat, Edit, VI. p. II. n. 3. Equus lineis transverfis verficolor.

Limmé, Syf. Nat. Edit. X. I. p. 74. n. 3. Equus (Zebra) fafciis fufcis verficolor.

Graumanus, intr. in hift. nat. mamm. p. 42. III. der geftreifte E.jel, das Zebra.

Gatterer, brev, zool. I. p. 60. n. 3. Equus (Ze. bra) fafciis fufcis transverfis; der geftreifte Efel, das indianifche Pferd.

Severin, 
Soverin, zool. Hungar, p. 3r. n. 4. Zebra, Z cora.

Nieremberg. hift, nat. p. 168. Zebra,

4. QuAGgA. Der Quacha. (4)

Zinmermann, geogr .Zool. II. p. 81. ce der Que. cha. III. p. 267. der Qwagga.

Schreber, Säugth. V. Tab. 3I\%. Equus Quagga. Biiffon, vierf. X. p. 6\%. der Kwagga; m. e. Fig. Batfch, Thiere, I. p. 147. das Quagge. Sparrmann, Reife, p. 127. 210. 220. 339. 38\%. 443. 445.583. der Quagga.

Nouvelle defcript. du Cap de b. E. p: 36 .

A) Vevcindernngen gegen die XIIte Edition, und Vorn mehrung der Gattungen diefes Gefchlechts.

Bey der erften und zweyten Gattung find die Varietäten $\alpha$ und $\beta$ aus einạnder gefetzt; die vier te, fiinfte und fechfte Gattung find neu hinzuge: kommen.

\section{3) Unbeftimsmtere Thize.}

1. Der Mangarfahoc.

Zimmermann, geogr, Zoolog. II. p. 8r. a.

Flocoust hift. de Madagafcar. Par. 166r. p.154.

$$
\mathrm{Zz} 5 \text { 34. GE. }
$$

(4) Unterfcheidet fich vọn dem Zebra dutch die kürzern Ohren, und die ihm theils an den Vorderbeinen, theils an den Lenden fehlenden Streifen des Zebra. Edwards hielt der Aehnlichkeit wegen diefs Thier für das Weibchen vom Zebra. Es find aber zwey von einander ganz verfchiedene Gattungen, die fich auch in verfchiedenen, und oft weit von einandęr entlegenen Gegenden aufhalten. Beyde Gefchlechter des Quagga find fo, wie des Zebra, gleich gezeichnet, nur dafs die Farbe der Hengfe frifcher und auffallender ift. 
34. GESCHLECHT, HiPPOPOTAMUS. Das Fluefspferd.

r. А менгі геs. Der Hippopotamus.

Miiller, Naturfyft. I. p: 457. der. Behemoth́.

Zimmermann, geogr.Zool. II. p. I5I. 11. 66. das Nilpferd.

Schreber, Säugthiere, V. Tab. 3I8. Hipfopotamus amphibius.

Lefke, Naturgefch. p. Igo. das Fhisspferd.

Borovelky, Thicrreich, I. 4. p. 55. das Nilpferd. Tab. 36 .

Bhnmlenbach, Handbuch der Naturgefch. p. I24.

Hippopotamus Amphibius; das Nilpferd; (Edit. 3. p. 136. WafJerfchivein.)

Bechftein, Naturgefch. Deutfchl. I. p. 144. n. 36. dis. Fluifspferd.

Funzke, Naturgefch. I. p. 146. das Flu Sspferd, oder

Hippopotamus.

Ebert, Naturlehre, I. p. 3I - das Flusspferd, Nilpferd, WafJerochse.

Halle, vierf. p. 372 . das Nilpferd.

Gatterer, vom Nutzen und Schaden der Thiere,

I. p. 6I. 11. 38. das Nilpferd.

Beckmlann, Naturhif. p. I6. n. I. 'das Nilpferd.

Neue: Schaupl. der Natur, III. p. 164. Flufspferd. Onomat. liff. nat. IV. p. I8r. das Wallro $s$, das Nilpferd, der Flus s. Ochfe.

Handb, d, Naturgefih, I. 2. p. 268. das Fhusspferd. Frifch, Naturfyntem, p, 4. Equus Niloticus; das Nilpferá.

Klein, Claffif. der vierf. Thiere, p. то3. Wafeerochs, Finfspferd.

Klein, natïl. Ordn. cer vierf. Th. p. 38. Nilpferd, Seepferd, Wafserochs, Wafferkith, Behenoth. Biiffun, 
Biiffon, vierfüfs. X. p. 78. das Fhufspferd; mit 5 Figg,

Bat $\int c h$, Thiere, I. p. 147. das Nilpferd.

Sparrmann, Reile, p. 553.562-572. das Fhuss. pferd. Tab. I3. I4.

Le Vaillant, Reife, I. p. 286. 294. Hippopotamiis. Bankroft, Guiana, p. 76. Hippopotamuls.

Adanfon, Senegall, p. 106. Wallroffe, fonft auck Seepferde genannt.

Forfter, Reife um die Welt, Edit. in 8. 1. p. 84. das Fhifspferd, Seekuh.

Ifert, Reife nach Guinea, p. Ir7. See-Elephant.

Shaw, Reife, p. 306. das Fhufspferd. p. 367. der Behemoth,

Kolbe, Vorgeb. d. g. H. Edit. in 4. p. 328. See. $p f^{e}$ rd, oder Hippopotamus.

Bomnet, Betracht. uiber die Natur, I. p. 127. das Flu/spferd.

Battara, muf. Kircherianum. Rom. r773. gr. fol, I. Tab. 3. fig. 50, Abbild, fchlecht.

Lichtenberg und Voigt, Magazin für das NeueAte u. f. w. IV. I. p. 32. die Nilpferde.

Schwed. Ablandl. XL. P: 3 18. Tab. X. Abbildung eines lebendig gefangenen Iungen.

Bunzlauer Unterhalt. II. p: 300. Nilpferd, oder Fhuspferd.

Nouv. defcript. du Cap de b. E. p. $x$.

Kurze Naturgefch. des Vorgeb. d. g. H. p. 148 .

Seekuh.

Berlin. Samml. I. p. 5 I4 das Nilpferd; m. e. Fig. Iablon/ky, allgem. Lex. p. 659. Meerpferd.

Oekonom. Zool, p. 54. n. 62. das Nilpferd, der Fhussoch/e.

Hippopotamus. Orrelins Djurkiärninggen. Stockholm, 1777.8. 
Allamand, bif, du Gnou, du grand Gerbo, et de l'Hippopotame. Amft. 1776. Tab. 3.

IIIThaelis, deutfche Ueberfetz. des? alten Teftaments mit Aumerk. für Ungelehrte, I. p. 80 . u.r75. Goeze, Allerley, I. p. I90. neue Aufi. I. p. 120.

Allgem, deutfche Bibl. LXV. p. 438. Fhufspferd. Beckmann, phyf. ökonom. Bibl. VI. p. 8. IX. p. 329. XIII. p. 248. 473. Hippopotamus, Flusspferd.

Erxleben, Mammal, p. 195. n. x. Hippopotamus Amphibius,

Graumann, intr. in hift nat. mamm. p. 4I. Nilpferd, Seepford, Behemoth, Wafjerochs. Linné, Syft. Nat. Edit. II. p. 48. Hippopotamus. Linné, Syft. Natur. Edit. VI. p. II. n. I. Hippopotamus.

Gatterer, brev. zoolog. I. p. 53. 1. r. Hippopotamus (Amphibius) pedibus tridactylis; das Nilpferd, Seepferd, der Wafferochs. Der Behemoth?

Severin, zool. Hung. p. 52. Hippopotamus. Behemot Iobi; das Nilpferd.

Tachard, voyage de Siam. Lib. I. p. 106. Ifalm 68. v. 3r. das Thier im Rohr. Leon, Afric. defcr, Afr. p. 758. Hippopotamus.

§. §. Ein äufserf plumpes, mifsgeftaltes Thier, mit einem unförmlich grofsen Kopfe, ganz ungeheurem Rachen, dickem Leibe, kurzen Beinen etc. Die über zwey Schuh lange Zunge wird als ein vorzïgliches und fchmackhaftes Effen betrachtet. Die Weite des Rachens beträgt über zwey Fufs. Die Art, den Hippopotamus zu fangen, befeht, aufler dem 


\section{Mammalia Belluas. Tapir.}

dem Schieffen, darin, dafs man ihm da, wo er feinen Weg nach den Flïffen hat, Gruben gräbt. In Aegypten ftreut man ihm fo viele Erbfen und Bohnen hin, als er nur auffreflen kann, wovon ihm alsa denn der Magen auffchwillt, dafs er platzen und fterben mufs. Da er das Feuer fcheuet, fo wird folches an den Orten; wo er fich aufhäl, zur Vorficht auf den Schiffen unterhalten. Die fpätern rö: mifchen Kaifer haben of Nilpferde zur Schau nach Rom kommen laffen. M. Scausus war der erfte, der es dafelbht in einem dażu ausgegrabenen See zeigte: Auf den alten Kuntwerken fellt diefs Thier das.Symbol des Nils vor. Die alten Aegypter bil. -deten auch, dem Shav zufolge, durch daffelbe entweder die Unverfchämtheit, oder auch den Typhon, d. i. den Weft, ab, der die Sonne verzehrit und wegtrinkt.

* (EINGESCHOBENES GESCHLECHT.) TAPI R. Der Tapir.

Zimmermann, geogr. Zool. II. p. I53. Gent. XIII. Lefke, Naturgefch. p. 190. Gen. XLI.

Bhumenbach, Handbuch d. Naturgefchichte, p. 120. Gen. XXXVII.

Beinftein, - Naturgefch. Deutfchl. I. p. 145.

Briffon, quadr. p. 8r. Gen. XVIII.

Hermanin, tab. affin. animal. p. 96.

x. Americanus. Der Anta.

Miller, Naturfyftem, I. p. 460. Landhippopo. tamus.

Zimmermann, geogr. Zoolog. II. p. 154. n. 67. der Mainguri, Tapirete, Anta. p. 429. der Tapir. 
Schreber; Säugthiere, V. Tab. 319. Tapir amea ricanus.

Lefke, Naturgefch. p. 190. der Tapir.

Borowefky, Thierreich, I. 4. p. 98. 11. 1. Hydrochaerus Tapir; der Tapir, das Antathier. Tab. 28.

Bhimenbach, Handb. der Naturgefch. p. r20. n. I. Tapir (Suillus) dentibus primoribus utrnque 1o, roltro fpithamaeo retractili; der. Tapir, Anta.

Funnke, Naturgefch. I. p: I48. der Tapir.

Ebert, Naturl. I. p. 315. Gier Tapir, Anta, bra. filianifche Ejelskich, Berghuh, wilde Maulthier, Wafferfichein. 'Tab: 13:

Gatterer, vom Nutzen und Schaden der Thiere, I. p. 60. n. 56. das Wafferfchwein, der Tapir:

Neuer Schaupl. d. Natur, I. p. 330. Anta.

Onomat. hift. nat. V. p. 54. Manipouris, Tapivus. Handbuch der Naturgefchichte, I. 2. p. 245. der Tapir, oder Anta.

Martini, Naturlexicon, II. p. 6I6. Antathier; mit Büff. Fig.

Frifch, Naturfynt. p. 4. n. I. Tapir.

Klein, Claflif, der vierf. Th: p. ros: Tapiisete, Anta.

Klein, natürl. Ordn. der vierf. Th. p. 40. Tapiierete.

Biiffon, vierfüfs. X. p. 7. der Tapir oder Anta; m. e. Fig.

Bat fh, Thiere, I. p. I5r. dis Flufspferd oder der Tapiv.

Sthlözer, Erdbefchr. von Amerika, p. 815. Danta. Reifen der Miflonar: in Amerika, (von MTurr.) p. 210. Danta, oder gran Beftia, Lacha-Vacca, Waldkuh. 
Falkner, Patagonien, p. Ir2. der Anta.

Hort fink, Befchreib. von Guiana, I. p. Io7. der

Waldefel, Cama, Maipari.

Dapper, Amerika, p. 445. das. Thier Tapiire-ete.

Charlevoix, Gefch. von P'araguay, p. 26. p. 226. Anta oder Danta.

De la Condamine, Reife auf d. Amazonenfrome; im Hamb. Magaz. VI. p. 257. das Elendthies.

Graumnann, intr. in hift. nat. mamm. p. 40.n. I. das Wafferjchwein; Antathier.

Gatterer, brev. Zool. I. p. 52. n. I. Hydrochaerus (Tapir) dentibus primoribus utrinque de$\mathrm{ccm}$, roftro fpichameo retractili; iuba cervicalis, corpus fuillum; das WafJer/chivein, der Landhippopotamus.

Severnn, zool. Hungar. p. 53. n. I. Hydrochaerus Tapir; Sus aquaticus; das Wa Jer chwein.

5. 5. Das gröfste Landthier in Südamerika; von der Grölse eines mittelmäfsigen Ochfen. Der Kopf und die Schenkel find ohngefahr wie beym Schwein; der Rünel faft wie am Elephanten, nur weit kürzer, und ohne die hakenförmige Spitze. Setzt fich gewöhnlich auf die Hinterfüfse wie cin Hund. Der kurze Rüffel in doch ganz beweglich, und das Thier weifs fich deffelben mit vielem Ge. fchick zum Aufheben, Abreiffen und Anfaften zu. bedienen. Sein Gefchrey befleht in einem lebhaften und farken Gepfeife, welches die Iäger ziemlich genau nachahmen, um fie herbeyzulocken, und in der Nähe zu fchieffen. Zu Cayenne hat man einige diefer Thicre als Hausthiere aufgezogen. Die Iagd auf fie wird des Nachts angeftellet. Man erwartet fie in ihten Lagern, wohin fie fich haufenweile begeber: und wenn man fie ankommen ficht, 
geht man ihnen mit brennenden Fackeln entgegèn, twelche fie dermaafsen blenden; dafs fie über einander zur Erde fürzen. Sie werden auch in Schlingen gefangen. Aus der Haut, machen die Wilden Schilde.

35. GESCHLECHT. SUS. Das Schwein.

Miiller; Naturfýftem; I. p. 46r. Gen. XXXV:

Zimmiermiann, geograph. Zoolog. II. pag. I4İ。 Gen. X.

Lejke; Naturgefch. p. I88. Gen. XL.

Borowsky; Thieireich, I. 4. p. 58. Gen. XXXVIÍ. Bhumenbach, Handb. der Naturgefchichte, p: $11 \%$ \% Gen: XXXVI.

Bechftein, Naturgefch. Deutfchl. İ: p. 755 .

Neuer Schauplatz, der Natur, VIII. p. 16.

Onomat: hift. nat. VIII. p. 37 r.

Erxteben; Mammalia, p. 176. Gen. XIX.

Graimnan; intr: in hiftor. nat. mamm. p. 39 Gen: XIX:

Gatterer, brev. Zoolog. I: p. 48. Gen. XIX. Bat $\int c h$; Thiere, I. p. I48. Brifjon, quadr. p: 73. Gen. XV. Herwann; tab. affin: animal. p. 95. 97. Toto

1. Scí of A. Das gemeine Schruein.

a. FE R U S. Das wilde Schroein.( $\left.{ }^{1}\right)$

Miiller, Náturfyftem, I. p. 463. A. das wilde - Schweims

Zinumer.

(1) Wila Scbwein, Hauer, Kabler (das Munnchen), wilde Sar.

- Bache (das Weibchen), Frifchling (ein Iunges). Das wilde Schwein hat eine langere Schnauze, kürzere aufrechte Ohren; 
Zimmermann, geogr. Zool. I. p. 189. der wilde Eber. II. p. 141. n. 60. n. a. das wilde Si hwein. Schreber, Säugthiere, V. Tab. 320. Sus Scrofa Aper. Tab. 322. Sus Scrofa falciatus. Lefke, Naturgefchichte, p. 189. das gemeine Schwein.*

Borowfky, Thierreich, I. 4. p. 60. n. a. das wilde Schreeir.

Bhumenbach, Handb. d. Naturgefch. p. II7. n. t. Sus (Scrofa) dorfo fetofo, cauda pilofa; dias Schwein. *

Bechftein, Naturgefch. Deutfchl. I. p. 771. das wilde Schwein. (ritterliche Thier.)

Funke, Naturgefch. I. p. 58. das wilde Schwein; Schwarrwild.

Ebert, Naturlehre, I. p. 309. das Schwein. * Halle, vierfürs. p. 359. das seilde Sinuecin.

Gatteres.

grüfsere Fangzahge als unfer Hausfchwein, auch keinen Speck, und ift faft immer von fchwarzgrauer Farbe. Es wird durch reine Fänge furchtbar, womit es, fich, wie man in der Barbarey zuweilen bemerkt hat, felbft gegen Lüwen fattfam vertheidigt. Duch hat man auch Beyfpiele, dafs fich Frifchlinge haben kirte machen laffen; und wenn fie fchoun erivachfen, ihrem Herri gefolgt find u. $f$. w. Die Fárbe der lungen if hellcaftanienbraun, mit dunkelbraunen Streifen, die fich über die ganze länge des Kơrpers erftrecken. Nach einigen Monatèn verliaren fich' diefe Streifen; fie werden einfärbig dunkelbraun, und in den folgenden tahrer verfarben fie fich noch dunkler, bis fie endlich ganz fchwarz find. Den Namen des ritterlicber Thiers hat das wilde Schwein von feinem Muth und Tapterkeit erhal= ten, worin ihm keines tunfrer edlen oder efsbaren Thiere beykümmt. P. Servilius Rultus, der Vater des Rullus der unter dem Konfulat des Cicero das Ackerveitheilungsgefetz (Legem agrariam) gáb, war det erfte unter dea Rümern, der ein wilo des Schwein im Gaftmahl auffetzte. 
Gatterer, vom Nutzen und Schaden der Thiere, I. p. 50 . 11. 52. das Schweint. *

Beckmann, Naturhift. p. 15. n. 14. das Schreein.* Neuer Schaupl. d. Natur, VIII. p. I6. Schwein. * Onomat. hift. nat. VII. p.378. cias Hausfchreein, die Sau, das wilde Schwein. *

Handb. der Naturgefch. 1. 2. p. 52. das Schwein. * Handbuch d. deutfchen Thiergefch. p. 77. die wilden Sïue.

Sander, ökon. Naturgefch. I.p. 233. die Schucine. * Waither, ökonom. Naturgefch. Deutfchl. p. $16 \%$. Schrveine. *

Frifch, Náturfynt. p. 3. n. 5. Aper vulgaris; das zesilde Schevein.

Klein, Clanif: der vierf. Th. p. 83. Schwein. Klein, naturl. Ordin. d. vierf. Th. p. 28. V. Schwein. Bïfon, vierfüls. II. p. 35. das veilde Schueinb. Tab. 18. fig. I. der wilde Eber. Tab.19. fig.r. der Frifchling, oder das junge woilde Schwein. Bat $\int \mathrm{ch}$, Thiere, I. p. 149. das wilde Schwein. Bock, Naturgefch. von Preufien, IV. p. 232. das wilde Schreein.

Hartfink, Befchreib. von Guiana, p. I05. wilde Schreeine?

$H: A$, Marokos und Fes, p. 294. wilde Schweine. Fifcher, Zufätze zur Naturgefch. von Livl. p. 4r. n. 485 . wildes Schwein.

Pallas, Reife, II. p. 393. wilde Schweine. Pallas, Reife, Ausz. III. p. 373. wilde Schweine. Cetti, Naturgefchichte von Sardinien, I. p. 185. das wilde Schwein.

Bankroft, Guiana, p. 74. wilde Schweine. Bankroft, Guiana, n. 75. das Warreefchwein? Schlozer, Erdbelchr. von Amerika, p. 682. dev Warre?? 
Georgi, ruft. Reife, I. p. I64. wilde Schweine.

I. G. Gmelin, Reife durch Sibirien, I. p. I92: wilde Schweint.

Shaw, Reife, p. 157. das wilde Schwein.

Reife nach d. Inf. Frankr. u. Bourbon, p. 187. ent. lanfene Schweine.

Boswell, Befchr, von Corfica, P. 43. die vildenz Sihweine.

Baldaeus, Zeylon und Malabar, p. 422. wildé Schweine.

Wolf, Reife nach Zeilan, p. r20. Schwein.

Grangers, Reife durch Egypten, in den Göt. ting. Reifen, III. p. 449 .

Beytr. zur Beförderung d. Naturkunde, I, p. 2 Ig. weild Schwein.

Mellin, Anveif. zur Anleg. der Wildbahnen, p. 172. die Sauen; m. e. Fig.

Merklein, Thierreich, p. zoI. Schwein. *

Döbel, Tägerpraktik, I. p. 23. wilde Sauen.

André u. Bechftein, Spatziergänge, II. p. 375.38t.

das wilde Sihwein.

Bhmenbach, Beytr. zar Naturgefchichte, I. p. 36. iiber die Ausartung des Schuveinis.*

Beckmann, phyf. ökon. Bibl. III. p. 189.529.

Ryt fchkow, d. j. Reife durch Rufsl. Riga 1774. p. 324. Schweine *

Krox, ceylan. Reifebefchreib. p. 4I. Schiseine.

Pennant, Reife d. Schottland, I. p. I24. Schwarzwildpret.

Kliigel, Encykl. I. p. 239. das gemeine Schwein. * Oekonom. Zoologie, p. 55. n. 63. das gemeine Schwein. *

Iäblonfky, allgem. Lex. p. 1039. das wilde Schroein. Linné, Syft. Natur. Edit. II. p. 49. Sus dorfo antice fetolo, cauda pilofa. 
Limé, Syf. Nat. Edit. VI. p. I2. 11. I. Sus dcrfo antice fetofo, cauda pilofa. *

Linné, Syft. Natur. Edit. X. I. p. 49. n. I. Sus, (Scrofa) dorfo antice fetofo, cauda pilofa. *

'Limé, Fn. Suec. I. p. 12. n. 36. Sus dorfo antice fetofo, cauda pilofa. *

Graumann, intr. in hiftor. nat. mamm. p. 69. a. seild Schwein.

Gatterer, brev. 200l. I. p. 48. n. 1. a. Sus (Scrofa) Aper; nigro-canefcens, lana nulla inter fetas; Auriculae breviores, fubrotundae. Roftrum productius. Dentes laniarii longiores. Setae profundius radicatae. Lardum nullum. Iunior flavefcente et fufco longitudinaliter ftria. cus; das wilde Schwein.

Severin, zoolog. Hungar. p. 49. n. 2. Sus ferus, Aper; das wilde Schwein.

Wiiller, zoolog. dan. prodrom. p. 6. n. 43. Sus (Scrofa) dorfo antice fetofo, cauda pilofa. * Kramer, Auftr. p. 31\&. Sus dorfo antice fetofo, cauda pilofa. *

Gronov. zoophyl. I. p. 3. n. Ir. Sus caudatus, auriculis oblongis acutis, cauda pilofa. *

Forfter, phil. Trans. LVII. p. 342. Sus Scrofa. * Penzanat, fyn. quadr. p. 68: n. 54. the common Hog. *

Varro, de re ruf. Lib. 3. cap. 13. Aper.

C. Domestrus. Das zahme Schwein.

2. V U L A R I S. Das gemeine zahme Schwein. ( $\left.{ }^{2}\right)$ Miiller, Naturfyftem, I. p. 464. B. das zahme Schwein.

Miiller,

(2) Eiber, Kempe (das Minnchen), Bork (das gefchnittene), Sau - (das.Weibchen), Börgen (das verfchnitrene), Ferker, Ferkel ein 
Miiller, Naturfyn. Suppl, p.58. das Maft fchwein. Zimmmermann, geogr. Zoolog. II. p. 141. 11. 60. b. das zahme europaiifche Schwein.

Schreber, Säugthiere, V. Tab. 321. Sus Scrofa domefticus.

Borowjky, Thierreich, I. 4. p. 62. b. das zahme Schwein, Hous fohwein.

Bechftein, Naturgefch. Deutchl. I. p. 756. das, zahme Schwein.

A a 3

Funke,

(ein lunges), Sparnferkel (wenn es noch faugt), Abfeszferkel (wenn es von der Sau genommen wird). Wenig Thiere find fo allgemein faft tiber die ganze Erde verbreitet, und einige Vülker ausgenommen, welche aus Religionsprincipien, die fieh doch auf medicinifche Urfachen gründen, kein Schweinefleifch effen dürfen, wird es feir den alteften Zeiten, und fatt unter allen Himmelsírichen verfpeift, hat auch yor den übrigen den grofsen Vorzug, dafs es durchs Rauchern und Einfalien fich fo lange erhalten lifst. Wie weit man es mit der Maftung der Schweine bringen künne, beweifen folgende merkwürdige Beyfpiele: In Danzig wog ein Schwein in Iahr 1714 . fieben hundert und fechszehn Pfund. Ein Fleifcher hatte dafelbft 74 t. ein Speckfchwein gemältet, welches fünf hundert und fiebzig Pfund danziger Gewicht hielt. Nach dem Schlachten fand man auf dem Rückgrad I Schuh hoch Fleifch, wovon 9 Zoll Speclì waren. $\mathrm{Zu}$ Fürth, bey Nitrnberg, wurde im Iahr ${ }^{1748 . ~ e i r x ~}$ Schwein gefchlachtet, welches fieben hundert Pfund wog, und fieben Fufs lang war, In Ludwigsburg ein neunvierteljahriges, von ache hundert und vier und achtrig Pfund; es wąr 9 Schuh 4 Zoll lang, und 4 Schun 5 Zoll hoch. Die Hühe des Speciss auf dem Rücken betrug 7 , und an den Seiten 9 Zoll; das inwerdige Fett belief fich auf 87 Pfund. In Dresden hatten die Fleifcher 1729. ein Schwein, das 2 Ellen hoch, und eben fo lang war, und am Gewicht über fechftehalb Ceatner hatte: Der Hr. geh. Finanzrath von Brenkenboff hat einmal zwey fette Schweine, das Stück zu 7o Thaler naeh Berlin verkauft, wovoij jedes 1000 Pfund wog. - Unter allen Thieren mit gefpaltenen Klauen wirft das_Schwein die meillen lungen. - Die mit cinem * bezeichneten Stellen begteifen das wille und zahme Schwein zugleich unter fich. 
Funke, Naturgefch. I. p. 59. uñ $\mathrm{fer}$ zahmes Hons. fchwein.

Halle, vieirfüls. p. 346. Hantschwein.

Meyer, Thiere, I. Tab. 49. das Schwein.

Handbuch der deutfchen Thiergefchichte, p. 78 . das zahme Schwein.

Frifch, Naturfynt. p. 3. n. x. Porcus vulgaris domefticus; gemein zahn Schwein.

Biiffon, vierfüls. II. p. 35. das gemeine zahme Schwein. Tab. I7. der zahme Eber. Tab. 19. fig. 2. das Spanferkbl, Sogfchwein.

Batfch. Thiere, I. p. r49. das zahme Schwein. Bock, Naturgefchichte von Preuffen, IV, p. 234. das zahme Schwein.

Schäpf, Reife d. d. nordamerik. Stąten, II. p. Ióg. die Schweine.

Hariffnk, Naturgefch. von Guiana, I. p. II 3 . zah. me Schuevine.

Höft, Marokos und Fes, p. 294. zahme Schuceine. Boswell, Corfica, p. 43. die zahmen Schweine. Cetti, Sardinien, I. p. Irg. das Schrein.

Bankroft, Guiana, p. 74. Schweine, welche ans Europa find heriiber gebracht worden.

v. Taube, Slavonien und Syrmien, I. p. 36. die Schreeine.

Byron, Reife um die Welt, p. 13. Schweine. Pontoppidan, Norwegen, II. p. I6. Sthweine. Uno von Troil, Reife n Iland, p. I06. Sihweine. Olafjen, Island, I. p. 29. II. p. 46. Schweine.

Reife naçı der Infel Frankr, u, Bourbon, p. I88. die Sauen.

Mafch, Naturforfch. XV. p. 28. Baftarte von ei. nem wilden Eber, und einer zahmen Sau.

$B_{y j}$ ron, Unglücksfälle des Schiffs Wager, Nürnb. $1769 \cdot$ p. 152 . 
Iabionfky, allgen. Lex. p. 1038. Scliercin.

Erxleben, Mammal. p. 179. $\beta$. Sus: Donenticus Mitonfues.

Graumann, intr. in hiftor. nat. mamm. p. 39.b. Eber, Kempe, Sau.

Gatterer, brev. zool. I. p. 49. b. Sus: Domefticus. Auriculis longioribus, acutis, fubpendulis, colore faepe albicante, fed et luteo, nigro, rubro, cinereo, unicolor, difcolorve; das Hausfchuiein.

Severin, zool. Hungar. p. 47. n. I. Sus domefìcus.

Fabric, faum. groenland. p. 29. n. 8. Sus Scrofa:

Pennant, britt. zool. p. 19. the Hog.

Varro, de re ruft. Lib. 2. cap. 4. Sus.

Columella, de re ruft. Lib. VII. cap. 9. Suillum pecus.

b. Moxungulus. Das einhufige Scheein.

Zinsmermann, geogr. Zool. I. p. rgr. die einhufigten Schweinu vor Päonien.

Zimniermann, geogr. Zool. II. p. I4I. n. 60. e. das einhufigte Schwein.

Borowesky, Thierreich, I. 4. p. 65. Schreine mit ingefpaltenen Klauten.

Bhumenbach, Handb. d. Naturgefch. p. II8. Schweine mit ungefpaltenen Klauen.

Erxleben, Mammalia, p. I82. Mcnungula varietas domeftica.

Gatterer, brev. zool. I. p. 5 I. Moniungula varietas domeftica,

Beckmann, phyf. ökonom. Bibl. X. p. 54I.

Hanow, Seltenheiten der Natur, III. Tab. I. fig. I.

$$
\text { Aaa } 4 \text { c. Si- }
$$


c. Sinensis. Das clinefffche Schwein.

Müller, Naturfyftem, I. p. 463. b. das chineffifche oder famifche Schwein.

Zimmermann, geogr. Zoolog. I. p. 192. das fian mifche Schwein.

Zinumermann, geogr. Zoolog. II. p. I4I, n. 60. ca das framifche Scine ein.

Borow/ky, Thierreich, I. 4. p. 64. c. das chinzefi fche, fromifiche Schoein.

Beckmann, Naterhiftorie, p. 15. n. 14. b. das chin nefifche Schwrin.

Handb. der Naturge[ch, I. 2. p. 59. die chinefifchore Schereine.

Bïffon, vierf. II. p. 7\%. die chinzefifchen Schweine. Frifch, Naturfylt. p. 3. n. 4. chinej/3 ch Schwein. Käupfer, Iapan; in du Halde China, IV. I. p.137. Schueine, die von den Chinefern hieher gebracht worderr.

Sihölf, Reife durch Nordamerika, II. p. $38 \%$ ein chinzefifches Schwein.

Neuhoff, Gefandfch. nach China, p. 347. Schweine. Du Halde, China, II. p. 164. Schweine.

Forfter, Reife, Edit, in 8. I. p. 332. die tahiti. fchen Schweine.

Forfter, Reife, II. p. I2. die Schueine.

Forfter, Pemerk. auf feincr Reife um die Welt etç, p. I66. die Schweine.

Beckmann, phyf. ökon. Bibl. III. p. 407. die chiuefifchen Schweine.

Briffon, quadr. p. (mihi) 75. n, 2. Sus (Sinenfis) caudatus, ventre ad terram usque propendente, cauda pilofa.

Erxleben, Mammal. p. I8I. $\gamma$. Sinenfis.

Gatterer, brev, zoolog. I. p. 51. c. Sinenzis; das chinefische, javanifche, oder fiamifche Schwein.

Pennani, 
Pennant, fynopf, quadr, p. 70. n. 54. $\beta$. the Chinefe Hog.

\section{$-*$}

Hannöv, Magazin, 1770. p. 402.

Der Landwirth in und nach dem Kriege. Berlin,

I779. 8. P. 345 .

Goeze, neuefte Entdeckung, dafs die Finnen im Schweinefleifch keine Drüfenkrankheit, fondern wahre Blafenwürmer find. Halle 1785 .

Vitet, Vieharzneykunft; a. d. Franz. von Hewne. mann. VI. Lpz. 1785. p. 246.

Verbefferte Brau - und Brant weinurbar u. f, w. Lpz. 1787. p. 220.

Wittenberg. Wochenblatt, XIX. p. $25 \%$.

Oekonom. Nachrichten, Lpz. 7749. p. 131. 1754. p. 297.

Leipz. Saminl. I749. p. 868. - 1750. p. 193. 1753. p. 246.

Allgem. Haushalt. und Landwiffenfch. V, c. 20. 2I. 22.

Berlin. Samml, IV. p. 69. VII. p. 615. VIII, p. 254. 624. IX.p. 238. X. p. 145.

Hamb. Magazin, X. p. 136.604. XIII. p. 234. XXIII. p. 459. XXVI. p. 538.

Wöltner, ökonom. Bibl. I. p. 315 .

Schwed. Abhandl. III. p. 245. 297. IV. p. 745.

V.p. 20. VI. p. 275. IX. p. 203. 257. 259.

XII. p. I06. XV. p. r9. XXIII. p. 260. $\mathrm{XXV}$. p. 266.

Beckmann, phyf. ökoniom. Bibl, I. p. 324. 653 , III. p. 196. 216.570 . V. p. 20. $41 \% \cdot 581$. VII. p. 150. 157. 161. IX. p. I12. X. p. 330.593. XII. p. 99. 553. XIII. p. 154. XIV. p. I23. 238. $\mathrm{XV}, 275 \cdot 345$. 
Chomel, allgen. Lex. Art. Schrein.

Beckniann, Landwirthfch. p. 466 .

Hammard, Rẹife nach der Ukraine, I. p. 198 ff.

2. Porcus, Das guineifche Schweinz:(3)

Mïller, Naturfyft. I. p. 465. n1. 2. das guineifche Schwein.

Zimanermann, geogr. Zool. I. p. 192. das gui. neifche Schueein.

Zimmermann, geogr. Zool. II. p. 14I. n. 6o. d. das guiszeifhe Schioin.

Borosejthy, Thierreich, I. 4. p. 65 n. 3. das guineifche Schevein.

Halle, vierfüfs. p. 366 . das langöhrige gainueifche Schieéin.

Gatterer, vom-Nutzen u. Schaden der Thicre, p. 58. n. 53. das guineifche Schwein.

Ebort, Naturlehre, I. p. 310. das guineijche Sihwein.

Neuer Schaupl. d. Natur, VIII. p. 20 . das guineiSche Schwein.

Onomat. hiftor. nat. VII: p. 377. das guineifche Schwein.

Frifch, Naturfyftem, p. 3. n. 2. guineifch Scheein. Klein, Clafif. der vierf. Th. p. 84. Spitzohr, guineifcreses Schwein.

Klein, natïr!. Ordn. 'd. vierf. Thiere, p. 29. n. 3. guineifches Schrein.

Biiffon, vierf. II, p. 37. b. das guineife he Schwein. Batfch,

(3) Pesnant und Biiffors halten diele Gattung für eine Varietăt von der vorhergehenden. Das gleich folgende framifche Scbwein aber wird yon Erxleben und mehrern mit dem clinefychen für einerley gehalten. Ueberhaupt find die Synonymen und Befchreibungen bey diefen beyden Varietäten fo fehr verwechfelt, dafs es fchwer liält, fie gehörig von einander zu trenner. 
Batfch, Thiere, I, pag. I51. das guineische Schroein.

Goeze, Natur, Menfchenl. u, Vorfeh, V. p. 328 . Cochon de bois.

Erxleben, Mammal. p. 184. n. 2. Sus (Porcus) dorfo poftice fetofo, cauda longitudine pedum, umbilico cyftifero.

Linné, Syft. Nat. Edit. II.'p. 49. Sus dorfo pone fetolo, cauda nuda.

Linné, Syft. Nat. Edit, VI. p. 12. n. 2. Sus dorfo pone fetolo, cauda nuda.

Linné, Syfl. Natur. Edit. X. I. p. 5o. n. 2. Sus (Porcus) dorfo pontice fetofo, cauda longitudi. ne pedum, umbilico cyftifero.

Graubmann, intr. in hiftor. nat. mamm. p. 40 . II. das guimeifche Schueitu.

Gatterer, brev, zoolog. I. p. 51. n. 2. Sus (Porcus) dorfo poftice fetofo, cauda longitudine pedum, umbilico cyftifero; das guinneifche Sicheein,

Pennant, fyn. quadr. p. 69. the Guinea Hog.

B. Das fiamifche Schwein.

Schreber, Säugthiere, V. Tab. 324. Sus Scrofa Siamenfis.

Neuer Schaupl. der Natur, VIII. p, I8, das chinzefis che oder famifche Schwein?

Frifch, Naturfyft. p. 3. n. 8. Porcus Siamenfis; das famifche Schwein.

Biiffon, vierf. II. p. 77. 79. 83. Tab. 18. fig. 2. das famifche Schwein.

Turpin, über verfchiedene Thiere des Königreichs Siam; in den Berl. Samml. VIII. p. $255^{\circ}$ dic Walds hhweine? 


\section{Ta Jassu. Das Mofchusfchwein. (4)}

Miiller; Naturfyftem, I, p. 465. n. 3. das Murcus oder Bifanifchwein.

Zimmermann, geogr. Zoolog. II. p. 143. n. 63 .

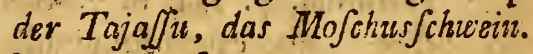

Lefke, Naturgefchichte, I. p. I89. n. 2. das BifamSchwein.

Borowfky, Thierreich, I. 4. p. 66. n. 4. das Muskus fchwein, Biefanfohwein.

Blumenbach, Haindb. der Naturgefch, p. IIs. n. 3. Sus (Tajafiu) cauda nulla, folliculo mofchifero ad coccygem; das Nabilfchwein, $B$ iSamechweiñ.

Ebert, Naturlehre, I. p. 310. das Bifam fhwein. Halle, vierf. p. 364. das kurzigefchwänate mexicanifche Muskus fchwein. 'Tah. 10.

Gatterer, vom Nutzen und Schaden der Thiere, I. p. 58. n. 54. das Bifanf chwein, der Pecari.

Neuer Schauplatz der Natur, VI. p. 40I. Pecari. Taja\iu, Cojcui, Caaigoara, Iavari, Coyanetl. Onomat. hift: nat. IV. p. 478. das kurzge/chwcinz. te mexicanifche Mufcus Schwein.

Handbuch der Naturgefchichte, I. 2. p. 58. das Pecari.

Frifch,

(4) Diefs Thier wird hüchftens fechzig Pfund fchwer. Die Warouwen ftellen in Guiana im April eine lagd gegen fie an. Wenn fie truppweife zichen, legt jedes feine Schnauze auf den Hintertheil des Schweins, welches voran geht, daher man, wenn man ihre Spur weifs, nicht auf das erfte, fondern auf das folgende fchiefst, welches man, wenn es.verwundet if, laufen lalst, und wenn es fich verblutet hat, mittelft der Spur des Bluts wiederfindet. Man fängt fie auch, wenn fie mit der Schnauze auf des andern Hintern, über einen Flufs fchwimmen, da man fie denn im Ueberfchwimmen mit einem Schlage auf die Schnauze tödtet. 
Frifch, Naturfynt. p. 3. n. I. das Bifamechweine. Klein, Claffsf. d. vierf. Th. p. 83. Mus cuss fchweiss. Klein, natürl. Ordn. der vierf. Th. p. 29. n. I. Muscusfchivein aus Mexico.

Biiffon, vierf. YII. p. 45. der Pecari oder Tajacu; m. e. Fig.

Batfch, Thiere, I. p. I5r. das Bifamfchwein. Hartfink, Befchreib. von Guiana, I. p. 105. die Nabel-Schwoine, Pafquiros, Aboujer.

Dobrizhoffer, Gefch. der Abiponer, I. p. 342. Tayacd, Ahergranraik, Iavali.

Dapper, Amerika, p. 445. TajalJou.

Bankroft, Naturgefch. von Guiana, p. 74. der Picary.

Schlözer, Erdbefchreib. von Amerika, p. 68r. der Pecaree.

Berkel, Reife nach Rio de Rerbice; in Bhumenibachs Reilegefch. I. p. 161. die wilden Schweine. Berkel, Reife nach Surinam; in Blument. Reifegefch. I. p. 237. die erfteri.

Reifen der Miffionar. von Murr. p. 199. Cahuscuma.

Charlevoix, Gefch. von Paraguay, p. 25. die witden Schweine.

Gozze, Natur, Menfchenl. u. Vorfeh. V. p. 328. das Patira.

Poynz, Naturgefch. der Infel Tabago; in Hamm burg. Magazin, IV: p. 203. der Pickery.

Kliigel, Encyklop. I. p. 239. das Bifamfihwein. Erxleben, Mammal. P. 185. n. 3. Sus (Tajaffu) dorfo cynfifero, cauda nulla.

Linne, Syn. Nat. Edit. II. p. 49. Sus dorfo cynifero, cauda nulla.

Linné, Syft. Natur. Edit. VI. p. 22. n. 3. Sus dorfo cyltifero, cauda nulla. 
Linné, Syft. Natur. Edit. X. I. p. 50. n. 3. Sus (Tajacu) dorfo cyftifcro, cauda nulla.

Graumann, intr. in hiftor. nat. mamm. p. 40. III. das Bijamfchwein.

Gatterer, brev. zoolog. I. p. 5r. n. 3. Sus (Ta. jaflu) cauda nulla, folliculo mofchifero ad coccygem; das Nabel-oder Bifamichusein, der Pecari.

Severin, zool. Hung. p. 5. n. 4. Sus americanus; Tajafju.

Gronov. zoophyl. I. p. 3. n. Io. Sus dorfo omnino fetofo, cauda nulla.

\section{A F RICANUS. Das afrikanifche Schwein.}

Miiller, Naturfyftem, I. p. 464. d. das capsche Sihuein?

Frifch, Naturfyft. p. 3. n. 7. Verres africanus; gro/ses wildes Schwein in Afrika.

Biiffon, vierf. II. p. 57. $\delta$. das cap fche Schwein? Pallas, Naturgefch. merkw. Th. II. p. 6. das wilde Schwein des afrikanifchen grünen Vor. gebiirges.

4. A ет н г о I C US. Das äthiopifche Schrein. (\$)

Miiller, Naturfyft. I. p. 464. c. das Schweint aus Acthiopien.

INiiller,

(5) Unterfcheidet fich von allen andern Arten der Schweine auch dadurch, dafs es vier befondere Auswüchfe oder Drïfen hat. Zwey davon fitzen eine Hand breit gerade unter jedem Auge, find breit und platt, und halten etwa 2 Zoll im Durchmeffer: die beyden andern find kugelrund, einen Zoll hoch, und befinden fich aut dor Schnauze in einem Abftande von drey Znll in gerader Linie hinter den Winkeln des Mauls. Die Bufchhottentotten haben yor dem Thiere eine folche Furcht, 
Thiller, Naturfyft. Suppl. p. 59. C. das äthiopifche Schwein. Tab. 3. fig. 2. Pall.

Zimnuermann, geogr. Zool. II. p. I4I.' n. 61. der Engalla, das äthiopifche Schwein.

Borowjky, Thierreich, I. 4. p.67. n. 5. das äthiopi/che Schroein.

Bhimenbach, Handb. der Naturgefch. p. II8. n. 2. Sus (Aethiopicus) facculis mollibus fub oculis; das Engalo.

Onomat. hiftor. nat. VII. p. 372. das aithiopifche Schwein.

Frifch, Naturfynem, p. 3. n. r. Aper aethiopicus; das Seltfame afrikanifthe wilde Sciwein.

Biiffon, vierf. II. p.36.76. das äthiopifche Schwein. Batfch, Thiere, I. p. IsI. das äthiopifche Schwein. Sparrinann, Reife, p. 350 . Waldfchereine, wilde Schweine. p. 353. die afrikanifchen WaldSchweine.

Pallas, Naturgefch. merkw. Thiere, II. p. 5.das äthiopifche wilde Schwein." Tab. I.

Acanfon, Senegall, p. Irr. ungeheure wilde Schweine?

Dapper, Afrika, p. 552. Emgalo.

Eberhard, Thiergefchichte. Halle 1768. '8. p. 269. das äthiopifche wilde Schwein. Tab. I. fig. $\mathbf{2}$.

Kliigel,

dals fie liebet einen Lüwen auf freyem Felde anzuggreifen wagen, als ein afrikanifches wildes Schwein, weil es wie ein Pfeil auf fie losfchöfie. Thre Wohnufgen haben diefe Thice unter der Erde. Sparrmann verfichert, er, wiffe es ziemlich zuverläfsig, dafs es einem Bauer in Kandebo gelungen fey, von afrikanifchen Waldfchweinen, die, man unit Schweinen von der gemeinen Art hat begatten laffen, Iunge zu betommen, die fich weiter fortgepflunat hatten. Das Fleifch foll dem gewöhnlichen Schweinefleifche feiı ahnlich fchmecken. Bey der Verfolgung hält das Thier den Schwanz gerade in die Höhe. 
Kliigel, Encyklopädie, I. p. 240. das itthiopifche Schwein, der Engalla.

Erxleben, Mammal. p. 187. n. 4. Sus (Aethiopicus) facculo molli fub oculis.

Graunzana, intr. in hifor. nat. mamm. p. 40. IV. das ätíiopifche Schwein.

Gatterer, brev. Zool. I. p. 5r. n. 4. Sus (Aethio. picus) facculo molli fub oculis; das äthiopifihe Schwein.

\section{B A в Y R Ussa. Der Hirfiheber. ( $(9)$}

Miiller, Naturfyft. I. p. 467. n. 5. der Hirfcheber. Zimmermann,- geogr. Zool. II. p. I43. n. 62. des Babiru $\int J a$, der Hiv Cheber.

Lefke, Naturgefch. p. 189. 11. 3. der Hirfcheber. Borowejky, Thierreich, I. 4. p. 58. n. I. der Hirfcheber, Babymefe. Tab. 37.

Blumenuach, Handb. der Naturgefch. p. IIg. n. 4. Sus (Babyruffa) dentibus laniariis fuperioribus maximis, arcuatis; der Schweizhirfch, Hirfch eber.

Ebert, Naturlehre, I. p. 3ir. Hirftheber oder Eberhirfch, gehörnies Schivein.

Halle, vierf. p. 366. das Rarbiroefa.

Gatterer, vom Nutzen und Schaden der Thiere,

I. p. 59. 11. 55. der Hirfcheber.

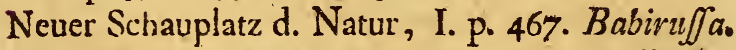

Onomat. hif. nat. II. p. II4. das Babyruffathier. Handb. der Naturgefchichte, I. 2. p. 292. der Babirulfe.

Frifch,

(6) Babà heifst auf Malaiifch das Scluwein, mgla der Mirfch. Der Nutzen der faft zirkelfürmigen grofsen Eckzähne des Oberkiefers if wohl noch niche beftimmt. Dafs das Thier fich damit, wie Betfcb meynt, an den Buimen aufhangen follte, wenn es Achend fchlaft, if nichs wahrfcheinlich. 
Frifch, Naturfyftem, p. 3. n. I. Babiruffa; der Schweinhirfch, oftindifches fonderbares Schwein. Klein, Claflif. der vierfüfs. Thiere, p. 84. Ba. birous.

Klein, natürl. Ordn. der vierf. Thiere, p. 29. n. 2 : Babiroufja, Babi-Roefa.

Martini, Naturlex. IV. p. 673. Babiröfathier.

Biiffon, vierfuils. II. p. 38. c. der Hirfckeber oder Eberhirsch.

Biiffon, vierf: XIII. p. 68. der Babirufa; m. e. Fig: Bat fch, Thiere, I. p. 150. der Hirrcheber. Adanifoin, Senegall, p. IIr. ungeheure voilde Schiveine?

ש. Bergen, Nachricht vom Kopfe des Babyrouffa; im Hamboirg: Màgazin XI: p. 188. m. K. Kliigel, Encyklop. I. p. 239. der Hirfcheber. Linne', Sỳft. Nat. Edit. II. po 49. Sus dentibus duobus, fronti inhatis:

Linné, Synt. Nat: Edit. Vİ. p. I2. n. I4. Sus dena tibus duobus; fronti innatis.

Linné, Syft. Nàtur: Edit. X. I. p. 50. n. 4: Sus (Babyruffa) dentibus duobus caninis; fronti innatis.

Graumani, intr: in hifor: nat: mamm p: $40 . \mathrm{V}$. der Hirfcheber:

Gatterer; brev: Zoolog. I. p. 52: 11: 5. Sus (Baby= ruffa) dentibus caninis verfus frontem reclinatis; der Hirfcheber, Sihwbinhirfch.

Severin, zoolog. Hungar: p. 49. n. 3. Sus l3aby. ruffa.

Charleton, exercitat. p. i4. Jabirouffa, feu por. cus indicus:

Gronovi. zoophyl. I. p. 3. n. t2. Sus caudatus, dentibus caninis fuperioribus ab origine furfúm verfis, arcuatis, cauda floccofa. 
Valentin. muf. mufeor. III. p. 268.452 : Bartholin. hift. nat. Cent. II. hif. 96.

Derïndernngen gegen die Xllte Edlition and Ver: mekrung der. Gdttungen diefes Gefchlechts.

Edit: XII. Edit. XIII.

P. 103. n. 4. Sus Hydro. p. I23. n. 6. Cavia Ca chaeris. pybara.

Aufer dem, dafs bey der erftern Gattung die Varietäten $\alpha$ und $\beta$ genauer beflimmt, upd von Letzterer wieder die Unterabtheilungen $a, b, c$ aus cinander gefetzt find, if das Geifchlesht nit zwey Gattungen vermehrt, und S. Africamus und Aethisopicis neu hinzugekommen.

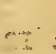


SIEBENTE ORDNUNG.

\section{E T E. (Säugende Seethiere.)}

37. GESCHLECHT. MONODON Der Ein horiffsh.

i. Mo Noceños. Der Narwal:(7)

Miiller, Naturfyn. I. p. 477 . der Norreal.

Lefke, Naturgefch. p. 193. der Narioal:

Borows.ky, Thierreich, II. I. p. 8. der Einhorn: fisch, Narwal. Tab. I.

Blumenbach, Handb. der Naturgefchi p. I30. n. I; Monodon (Narhwal) fiftulis refpiratoriis dua: bus, confluentibus; das See - Einhorn.

Bechftein, INaturgefcl. Deutfchl. I. p. I46. der, Narwal.

$\mathrm{Bbb} 2$

Funke,

(7) Nosrbual bedeutet eigentlich ein Wallfifchas, vom Norw: Nanr, eine Leiche, und Hual. Das. Thier if mit den eigendichèn Wallfifchè am riachften verwandt: Es hat nicht viel Speck, aber der Thran davon if düner, tind rithe fo ubeltiechend, als det vom Walifiche. Gewünlich halten fich die Narwals im Nordmeere auf, werden aber auch bisweilen weiter verfchlagen. So kam 1736 . im December ein Narwall mit einer hohen Fluth in die Elbe, und frandete bey Hamburg sach erfolgter Ebbe. In det Strafse Davis verfammeln fie fich hauferiweife um die Fisfelder mit Lüchern", dadurch fie Luft fchöpfen künnen. Im Schwimmen thun fie fich mit dem langen Zihhne kẹinen Schaden, fondern lëgen ihn einander glểichram auf den Riicken. Sie leben von Schollen und groisen Seequallen. (ACtiniił.) Die Grönländer halten fie für Vorboten der Wallfifche, und ruften fich bey ihrem Anblicke zus lagd. Man verarbciter die Zahne wie Elfenbein zu Kunftachen, und die Grönländer brauchter fie ehedem, in Ermanglung des Holzes, zu Sparren unter ihre Hütten. 


\section{Mammalia Cete. Monodon.}

Funike, Naturgefch. I. p. 169. der Narwall.

Ebert, Naturlehre, II. p. I73. der Narwhal, Eishornfifch, Seeeinhorn, Ziveyzahn.

Gatterer, rom Nutzen und Schaden der Thiere, I. p. 446. n. 244. das Einhorn, der Narwal.

Beckmann, Naturhift. p. 68. n. 2. der Narwall, oder das Einhorn.

Neuer Schaupl. der Natur, II. p. 5II. Einhorn.

Onomat. hiftor. nat. V. p. 237. der Einhornfifch, der Nahrival.

Handb, der Naturgefch. II. p. 249. IMeercinhorn, Narwhal.

Batfch, Thiere, I. p. 256. der Narwal.

Egede, Befchr. von Grönland. Berlin 1761. p.99: -... Sereinhorn. Tab. 5 .

Cranz, Hiftorie vón Grönland, Frcf. u. Lpz.

1779. p. 134. der Einhornfish, oder Narhval.

Eberhard, Thiergefch. p. I46. der Narhwall.

Steller, Befchreib. von fonderbaren Meerthieren,

p. 208. Meereinhoriz.

Schneider, Abhandl. zur Zoologie. Berlin 1784. p. 252. der Naarhual.

Pontoppidan, Norwegen, II. p. 259. Narhval, Narwal.

Olaffen, Island, II. p. 29o. Naa.Hualen, Nar-Hval. Zordrager, grönländ. Fifcherey, p. 33.

Berlin. wöchentl. Relat. der merkw. Sachen a. d.'

Naturreiche, 1754. p. 719. Seeeinhion.

Bartholini relat. de Grocnland. unicornu; in defSen Adt. med. et phyl. Haffn. II. A. 1673. p. 70.

Hampe, philol. Transact. 1738. N. 447. p. 149. Narhual.

Lange, von dem Unicornu, oder Meereinhorn, welches in Halle $1736.2 \mathrm{u}$ fehen gewefen; in den Hall. Anz. 1736. n. тg. 
Quellmals, obferv de unicoruu marino; in den Comment. lit. Norw. 1736. Hebd. XXII. n. 4. p. 17\%.

Reifel, obf. de unicornu marino duplici; in den Mifc. Nat. Cur; Ann. 7. u. 8. Dec. 3. obf. 208. Tulpii obferv. de unicornu marino; in deffen obf. zned. Edit. 5. Lugd. Bat. I7i6. Lib. 4. c. 59. p. 374 .

Allgemeines Magaz. der Wiffenfchaften, II.p. 322. der Narsoall.

Neue gefellfch. Erzähl. III. p. 33. von den zwey. ten Ziahne des Narivals.

Hamburg: Magazin, XVI. p. 178. Narhwall.

Goeze, nützl. Allerley, II. Aufl. I. p. IIg. Eixhorizfifch.

Bunzlauer Unterhalt. II. p. 14. Norwall.

Iablonfky, allgem. Lex. p. 1050. See-Einhorri. p. I32I. der Narhwal.

Oekonom. Zool. p. 57. n. 65. der Narwal.

Lnedovici, Kaufmannslex. II. p. II72.

Erxlebes, Mammalia, p. 626. 11. I. Monodon (Monoceros).

Linmé, Synt. Nat. Edit, II. p. 59. Monodon Monoceros. Unicornu.

Linné, Syft. Natur. Edit. VI. p. 39. n. I. Monodon Monaceros:

Linné, Syft. Nat. Edit, X. I. p. 75. n. r. Monodon (Manoceros).

Linné, Fn. Suec, I. p. 98. n. 263. Monodon.

Graimann, intr. in hifor. nat. namm. p. 90. I. den Narwal, das Einhorn.

Gatterer, brev, Zoolog. I. p. I66. n. r. Monodon (Monoceros); der Narwall, das Seecinhorn.

Larker, Monoceros pifcis hand monoceros ad veram formam nuperi ex mari Groenlan$\mathrm{Bb} \mathbf{b}_{3}$ dieo 


\section{Mammalic Cete Monodon.}

dico hofpitis depictus et defcriptus. Hafniae IyOz.

Fabric. faun. groenland. p. 29. n. I8. Monodon (Monoceros) dente cornuformi, fpirali, ra. rius duplici, recto, praelongo, exferto in maxilla fuperiore.

Sachs, Mononerologia, Rạceb. I676. 8. Hermann, tab. affinit, animal. p. $12 \%$.

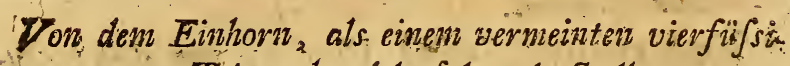
gen Thiere, handelun folgende Stellen:

Zimmernannz, geogr. Zoolog. II. p. I5\%. Schreber, Säugth. II. p. 237.

Strabo, geogr. Lib. I5. p. 1037. Edit. Almeloz. Plin. hiff. nat. Lib. 8. cap. 21.

Aelian. hiftor animal Lib. 6. c. 20. p. 888. Ed, Gronov.

Phile, de animal. proprietate; ed, Pauv. Traj, ad Rhen. I\%30. p. I6r.

Dapper, Amerika, p. 145 .

Gesner, hift. animal. p. 689.

Geszer, Thierbuch, p. 7r. mit vielen Figg.

Bochart, hierozoicon, I. p. 934 .

Bartholin, de unicornu. Amn. 1678. p. 218.

Garzias ab Horto, Aromat. hift. Lib.I.c. I4.

Lobo, voy. hiftor. d. Abyff. Amft. 1728. p. 69. und 230 .

Ottonis de Guerike, experiment. nov. Magdeb. de vac, Ipat. Amft, 1672. fol. p. 155 .

Leibnitz, Protogaea. Edit. Scheid. p. 64. Táb. 12. Ammanni cafus difcuff. et refponf. Facultat. med, Lipf. d. d. 2 Mai 1658, concernens probatio. nem unonocerotis; in defen Medic. critica, f. dẹciforia. Stad, 1677. 4. Caf. 67. p. 415. 
Behrens, diff. de Monocerote. Lipf. I672. 4\%

Bellon. obf. de cretenfi ariet. Strepficeros nomina: to: Difceptatio praeterea edocens, quid fit Unicornú; in defenz Obf. Lib. 2. c. I4. c. fig. Frenzel, diff de Unicorne. Wittemb. 1675. 4:' Kirchmaier, differt. de Monocerote feu unicornu.

Wittemb. $1660 / 4$.

Reijel. obf. de Unicornu; in dest Mifc. Nat. Cur.' 1671. obf. IIt.

Connel. Stalpart van der. Wiel, diff. de Unicornu; in deffen obfervat. rar. anat. chir. med. 1727.8 . p. 463. m. c. Fig.

Suden, Uuterfucli. ob man noch Einhörner habe? in defjerz gelehrten Critico. Leipzig, 1715.8. I. p. 873 .

Wedel. progr. de unicornu et ebore foffili. Ien. 1699. 4.

Merklein, Thierreich, p. 28. m. Figg. Ramusit, collect. Vened. 1563. p. 163. b. Valentin. muf. mufeor. Frcf. 1714. p. 483. Behreles, Hercynia curiofa, p. 35. 11. 2. p. 42 . Sander, kleine Schriften, von Götz. Lpz. I784. 8. I. p. Ior.

Sparmainn, Reife nach dem Vorgeb. d. g. Hoffu. p. 455.

Wallnann, von den Alterthümern der Stiftskirche zu Quedlinburg. 1776. p. 125 .

Goeze, über das vermeinte bey Quedlinburg, gefundene Einhor!. Quedl. I786. 8. Ladolf, hift. Aethiop. Lib. I. c. Io. n. 84 .

v. Murr, Befchreib. der Merkwürdigk. von Nürnberg. Nürub. I778. 8. 13. 63\%.

Defeript: du Cap de b. Efper - p. $4 \mathrm{r}$.

Camper, Schriften der berlin. Gefellechaft, IX. p. 219 . 
v. Murr, Naturforfcher, VII. p. 43 f:

Goeze, nützl. Allerley, I. p. Ir6.

Allgem. deutf he Bibl. LXI. P. 435:

Erxleberi, Mammal. p. 201.

B. SpuRIUs? Der Anarnak?

Fabric. faun. groenland. p. 3r, u, 19. Monodan

(Spurius) dentibus duobus minutis in maxilla

fuperiore, dorfo pinnato,

Schneider, zool. Abhandl. p. 253. Anarnak.

38. GESCHLECHT: BALAEN A: Der? Wallfifch.

Miiller, Naturfy:fem; I. p. 480. Gen. XXXVIII, Lefke, Naturgefch. p. 193. Gen. XLIV.

Borowsky, Thierreich, II. I. p. 10. Gen. II.

Blumenbach, Handb. der Naturgefchichte, p. $130^{\prime}$

Gen. XI.VI.

Onomat. hif. nat. II. p. II6.

Neuer Schauplatz der Natur, IX. p: 467.

Schneider, Abhandl. zur Aufkl. der Zool. p. I75:

Zordrager, alte und nẹue grönländifche Fifche.

rey; a. d. Holländ. Lpz. 1723.4.

Sibbaldi Balaenographia nova, five obfervationes,

de rarioribus quibusdam balaenis in Scotiae lite

tus nuper eiectis. Edinb. 1692. 4: Neue Aufl.

Lond. 1773. gr. 8.

Achurelii diff hiftorico-phyfica de Cetis; Aboae 1683. 8.

pol. Leyfer, diff. de Cetis. Lipf 1680. 4 .

Klein, hiforiae pifcium naturalis promovendae

- miffus fecundus de pifcibus per pulmones fpi-

rantibus: Gedan. 774 . gr. 4.

Erxleben, Mammalia, p. 60r. Gen. XLVIII.

Grats: 


\section{Mammalia Cete. Balaens. $\quad 26 \mathbf{z}$.}

Graumann, intr, in hiffor. nat. mamm, p. 88 . Gen. XIVIII.

Gatterer, brev. Zoolog. I. p. I6r. Gen. XLVIII. Hermam, tab, affin, animal. p. 128.

3. MYS т I CEт S. Der gemeine oder grönländ: Sche Wallffich. $\left({ }^{8}\right)$

Miiller, Naturfyft. I. P. 48̊r. n. r. der gröraländifche Wallfifth.

Lefke., Naturgefchichte, p. 193. n, r. der gemeine Wallffich.

Borowjky, Thierreich, II. I. p. 10. n. I. des grönländifche Wallffich. Tab.2. A. B.

Bilunzen.

(8) Wallffcb - eigentlich. Hualfffch, vọm Norweg. und Island. Huals; altdeutfeh: Guallfffch; das gröfste aller bekannten Thie ze, das über 100,000 Pfund am Gewicht halt; ift auch in fuidlichen Gegenden, im atlantifchen Ocean, auch um China herum, und im ftillen Meere, wo es die alten Peruaner an.o. bezezen, zu Haufe. Das Weibchẹn ift grö́fser als dạs Minnnchen. Das neugeborne Thier ift zwanzig Furs lang. Man hat Beyppiele von Wallfifcben, die 130 Tonnen Thran, und die Zunge allein 20 Tonnen gegeben hat. Ein grofser Fifch giebt a 000 Pfund Fifchbein. Die Begattung gefchiehet nux allę zwey Iahre. Die Wallfifche halten filch in Gefellichaft von 100 zu. farmen, und thun grofse Reifen. Tede Viertelftunde kommens fie gewöhnliçh einmal empor, um Waffer zu blafen, und frifche Lứ zu fchüpfen. Am Bnde des lahres geh der eigentliche Wallffich weftwarts, im Frïhjahr aber oftwärts. Wenn er bläft fo braufet es fo, dafs man ihn auf eine Meile weit hören kann, befonders wenn er verwundet ift, und vor Sçhmerzen wütet. Sẹn Auswurf fueht wie ein, feuchtes Zinnobexpulver aus. Sein daumendickes Fell ift oft mit Seegewżchfen, Korallen, und Murcheln befetze, An den Sigeffschen und Speckhauern. (Squalus Priftis und Delpbinus Orca) hat er beträehtliche Feinde. Sic fallen iha truppenweife an, jagen ihn aut den Strand, reiffen itum grolso Stücken aus dem Ieibe, und peinigen ihn 20 Tods. 
Blumeerbach, Handb. der Naturgefch. p. I30. n. I. Balaena (Myfticetus) dorfo impenni; der Wallffch.

Bechftein, Naturgefch. Deutfchl. I. p. 147. der gemeine Wallfisch.

Finnke, Naturgefch, I. p. s62. der Wallffich.

Ebert, Naturlehre, II. p. 167. dex grönländiJche Wallffich. Tab. 29. fig. I.

Gatterer, vom Nutzen und Schaden der Thiere,

I. p. 415. der grönländifche gemeine Wall. fifch.

Beckmann , Naturhifforie, p. 69: a. der grönlündi: fche Wallfisch.

Neuer Schaupl. der Natur, IX. p. 470. n. I. der eigentlich fo genannte grönlëndifche Wallffich.

Onomat. hift. nat. II. p. Irg. der Wallfich, gemeine Wallffich, grönlärzdifche Wallfifch.

Handb. d. Naturgefchichte, III. p. 240. der grörs. ländifche Wallffich.

Batfch, Thiers, I. p. 2 ģ7 der gemeine grönländifche Wallffich.

Schneider, Abhandl. zur Zoologie, p. r94. n. r. der eigentliche oder gemeine grönländifche Wallifich:

Cranz, Grönl. Frcf. I779. p. 122. dev eigentlick Sogernannte grönländifche $W$ allffich,

Egede, Grönland. Berlin I763. p. 90. Bartffich. Anderforn, Island, P. 212. der rechte grönländiSche Wallffich,

Trampler, Befchreib. des grönländ. Wallfifchfangs. Lpz. r77r. S. p. 27. der Wallffich.

Pontopipidan, Norwegen, II. p. 223. der Wallfifch, Hualffsh: Qual.

Phipps Reife nach dem Nordpol, p. 97. der gemeine Walffiftr. 
EVlis Reife nach Hudfonsmeerbufen, p. I4r. 349. die Wallfiche.

Olaffen, Island, I. p. 287. Wallffich.

Uno von Troil, Rẹife nach Island, p. 97. die Wallfifche.

$D_{6}$ Kerguelen Tremarec, Reife nach d. Nordfee, p. 82. Wallfifche.

Molina, Naturgefch. von Chili, p. 203. der grof: Se Wallfich.

Vidaure, Chili, p. die Wallfifctue.

Steller, Kamtfchatka, p. 98. 104. 309. Wallfiche. Le Gentil, Reifen in den indifchen Meeren, II. p. 50. Wallfifiche?

Fabric. neue Samınl. der Schriften der dän. Gef. I. n. 24 .

Faber, Comment. ad Recchi hiftor. Mexican, p. 568.

Camper, kleine Schriften, II. r. p. r.

Berlin. Samml. IX. p. 564. n. 9. der gemeine Wallfisch.

Iablonfky, allgem. Lex. p. 1326. der rechte grö̈z. ländische Wallfish.

Eberhard, Thiergefchichte, p. 141. der grönlïhn difche Wallffich.

Kliigel, Encykl. I. p. 209. der gemeine Wallffch. Utlloa, phyf. und hiftor. Nachricht von Amerika,

I. p. $15 \%$

Hanoy, Seltenheiten der Natur, I. p. 465. Horrebow, Nachricht von Island. Koppenh. 1753.

5. 54. p. 185 .

Neuhaff, Gefandich. nach China, $\mu$. 354. Abbild. der Wallfifche, bey Homanns Erben, in

Landkartenformat Fig. I. 2.

Oekon. Zoologie, p. 58. n. 66. dev Wallffych. Ludovici, Kaufmannslex. II. p. 158I. V. p. 6II.

Goeze. 
Goeas, nützl. Allerley, ste Aufl. III. p. 468.

Donndorf, Antipandora, I. p. 37x.

Doxisdorff, Natur und Kunf, I. p. 222.

Bunzlauer Unterhalt. I. p. 176.205.

Erxleben, Mammal. p. 601, n. r. Balaena (My.

fticetus) naribus flexuofis in medio capite, dorfo impinni.

Zimmé, Syf. Nat. Edit. II. p. 59. Balaena groenJandica.

Linné, SyR. Nat. Edit. VI. P. 39. n. I. Balae. na fiftula in medio capite, dorfo caudam verfus acuminato.

Iinne', Syft. Nat. Édit. X. I, P, 75. n. r. Balaena (Myfticetus) nạribus flexuofis in medio capite, dorfo impinni.

Linné, Fn. Suec. I. p. 98: n. 264: Balaena fiftula in medio capite, dorfa caudan verfus acuminato.

Einné, muf, Ad. Fr. I. p. 5r. Balaena (Groenlan. dica) frfula duplici in fronte, maxilla inferio. re multo latiore.

Graumann, intr. in hifor. nat. mamm. p. 88. I. grönlöndifcher: Wallfifch.

Gatterer, brev. Zoolog. I. p. I6r. n. r. Balaena (Myfticetus) fiftulis refpiratoriis duabus, diftinctis in medio capitis, dorfo impinni; der grönläradifche gemeine WVallfifch.

Fabric. faun. groenl. p. 32. n. 20. Balaena (Myfticetus) naribus flexuofis in medio capite, dorfo impinni,

Miiiller, zool. dan. prodrom. p. 6. n. 45. Balaena (Myfticetus) naribus flexuofis in medio capite, dorfo impinni.

Ruysch. Thefaur. anatom. II. Tab. I. fig: 9. 


\section{B. Is I A N D I C A. Der Eiswallfifch. (9)}

Miiller, Naturfyftem, I. p. 494. der Nordkaper. Borowejky, Thierreich, II. r. p. I8. Balaena Glacialis; der Nordkaper; der Eiswallfich.

Neuer Schauplatz der Natur, IX. p. 48r. n. 3. Balaena glacialis; Eisfifch. n. 6. Balaena bo" realis; Nordkaper, p.482. Eiswallfifch, p. 493. Bat $f c h$, Thiere, I. p. 259. der Eiswalfich, oder Nordkaper.

Iabionjky, allgem. Lex. p. 1328. der Nordkaper. Pontoppidan, Norwegen, II. p. 226. Silde-Qual. Crainz, Gröuland, P. 132, n. 2. der Nord-Caper. Egede, Grönland, p. 95. Nordcaper. Eberhard, Thiergefch. p. 14i. der Nordcaper. Chemnitz, Schriften der berl. Gefellfch. V.p. 463. Nordcajer.

Erxleben, Mammalia, p. 604:

IViuller, zool dan prodrom. p. 7. n. 49. Balaena (Glacialis).

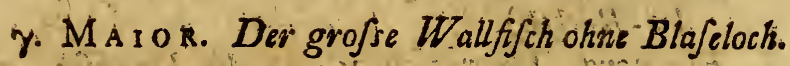

Erxleben, Maminalia, p. 602. pofit. 5. (fictam putat Artedi)

2. Ph Y S A L US. Der Fimnfich.(10)

Milller, Naturfyn. I. p. 49r. n. 2i der Finnfifch.

Borow kny,

(9) Die Benennung. Nordkapsr wird auter diefem auch dem Balaes na Miffulus, und dem Dielpbinus Orea gegeben. Man mufs fich daher vor Verwechfelungen hüten. Uelierhaugr finden fich in Anfehung der Synonymen und Befchreibungen ber diefem Ges fchlecht haufige Verwechfelungen und unerklärbare Zweifel.

(vo) If wegen feiner fchnellen Wendung gefalrilicher zu t'jdien, als der Wallfifch. Wena die Finnfifche kommen, fieht man keinen Wallfifch mehṛ. 
Borotefky, Thierreich, II. I. p. 19. n. 2. des Finnfich. Tab. 2. C.

Bhmenbach, Handb. der Naturgefch. p. I3I. n. 2. Balaena (Phyfalus) pinna dorfali; der Finz. fisith.

Ebert, Naturlehre, II. p. I7r. der Fimafich.

Gatterer, vom Nutzen und Schaden der Thiere,

1. p. 434. 1. 233 . der Finnfisch.

Beckmann. Naturbift. p. 70 . b. der Finanfich.

Neuer Schaupl. d. Natur, III. P. 55. Finnfifch.

Onomat hift, nat. II. p. I23. der Spritzesall, oder

Finnfijoti der Schweden.

Batfin, Thiere, I. p. 259 der Finnfich.

Schneider, Abhandl. zur Zoologie, p. 203. der Finnfich.

Phipps Reife nach dem Nordpol, p. 97. der Firngifch.

Cranz, Gröntand, p. 13. n. 3. der Finnfifch. Egede, Grönland, p. 89. Fin. Fifch.

Olaffen, Island, I, p. 288. b. Finufiuatkr (Bitk: kel-Rückerat.

Eberhand, Thiergelch. p. I42. der Finnfich. Iablonjky, alfgem.Lex. p. xว328. der Finnfifch. Berliu. Samml. IX. p. 565. 11. 7. der Fininffch. Abbild. der Wallfifche; bey Homanns Erben, Fig. 5. 6.

Pontoppidan, Norwegen, II. p. 232. Rörqual. Forfter, Bemerk. -auf feiner Reile un die Welt, p. 169. der Finnfisoh.

Eyxleben, Mammal. p. 605. n. 2. Balaena (Pty. falus) fiftula duplici in medio capite, dorfo ex. tremo pinna adipora.

Limué, S.yft. Nat. Edit. VI. p. 39. n. 2. Balaena finula in medio capite, tubero pinniformi in extremo dorfo. 
Lininé, Syft. Nat. Edit. X. I. p. 75. n. 2. Halaena (Phyfálus) naribus in medio capite, dorfo extrenio pinna ddipofa.

Linvé, fun. Suec. 1. p. 99. n. 265 . Balaena fifula in medio capite, tubero pinniformi in extremo dorfo.

Grinmannn, intr. in hiftor. nat. mannm. p. 88. If. Finnfifch.

Gatterer, brev.zool. I. p. 161. n. 2. Balaena (Phy. falus) fiftula duplici in medio capite, dorfo extremo pinna adipofa; der Finnfic $c h$

Miiller, prodr. zoolog. dan. p. 7. n. 46. Balaena (Phyfalus) fiftula duplici in medio capite; dorfo extremo pinna adipofa.

Fabric. faun. groenland. p. 35. n. 21. Balaena (Phyfalus) fifula duplici in thedio capite, dorfo extremo pinna adipofa.

\section{Boops. Der Intpiterfifch. (1)}

Miiller, Naturfyft. I. p. 492 . der Schnabelffich. Borowlky, Thierreich, II. I. p. 21 . n. 3. der Gus bartes, Gibbar, Schriabelffsch.

Gatte.

(1) Bey diefer Gattung herrfoht in Ánfehung der Synonymen grofse Verwirung. Die verdorbene Benenning Iupiterffscb fcheint aus dem Namen Gibbar, den die franzüfifchen und biskayifchen Wallfifchfanger gebrauchten, entrtanden zu feyn. Mitler nennt ihn ganz unfchicklich Scbnabelffich, da er doch keinen Schnabel, fondern eing gerade ftumṕfe Schnauze hat. Fabric. halt ihn für den Butzkopf des IEgede; worunter doch der Delpbinus Orca zu verftehen ift; u. f. w. - Er ift só bis 54 Fufs lang. Die Bauchfalten kann é zufähmenzichen und erweitern. Der dicke Speck giebt nicht fo viel Thrán, als von den ubrigen. Das Fleifch ift roth. Im Winter lebt er in der See, im Sommer und Herbft befuche er die Küften und Buchten. $D_{\text {as }}$ Weibchen wirft in Fruhjikr ein Iunges, welches der.Mutw 
Gattever, vom Nutzen und Schaden der Thiere,

I. p. 435. n. 234. der Iupiterfifch, Schnabliffch. Neuer Schaupl.d. Natur, VII. p.717. Schnabelffich.

Onomatol. hiftor. nat. II. p. I23. der dreyflofigte Wallfich his mit doppelter, Riiffelröhre.

Batfch, Thiere, I. p. 260. der Tibbar.

Schneider, Abhandl. zur Zool. p. 206. der ochfento äugigte Wallfich.

Olaffen, Island, II. p. 289. b. Hrafin-Reydur, oder Hrefiza.

Cranz, Grönland, p. i33. n. 4. der Iupitexffich; (beffer Gubartas oder Gibbar.)

Molina, Naturgefch. von Chili, p. 203. der Iiups$\tan r f$ s ch:

Tablonfky, allgem. Lex. p. 1328. der Iupiterfifch. Eberhard, Thiergefch. p. 142. der Finufich. Forfter, Bemerk: auf feiner Reife um die Welt, p. 169. der langgefchnauzte Wallfisch.

Ifrxleben, Mammal. p. 608. n. 3.Balaena (Boops) fiftula duplici in roffro, dorfo extremo protubeb rantia cornea.

Linvie, Syft. Natur. Edit. X. I. p. 76. n. 3. Balaena (Boops) fiftula duplici in roftro, dorfo ex: tremo protuberantia cornea.

Graile-

ter folgt bis fie zuth zweytenmal wirft. Er lebt vom Tobiasfifch, Lachs, und dem nördlichen Nautilus. Wenn è freffen will, fperrt et, wie der Wallfifch, den Rachen vreit auf, und verfchluckt die Beute fammt dem Waffer. Air blafst nicht fo nark als die übrigen, indem er gerade fortgeht, und fich hatufig untertaucht... Er fteigt fchief herab, und hält dabey den Schwanz tuber dem Waffer; kommt auch in eben der Richtung in die Höhe. Bey Atillerr Meere ruhet er oft auf dem Waffer als wena er fchliefe, und diefs in die bequennte Zeit zum Fanbe. Ex if furchefam; und hat feinen ärghten. Feind an dem kleinargigert Cacbelot. Das IVeibchen befchitut feln lunges mit don Schwanzichlngen. 
Graumann, intr. in hif. nat. mamin. p. 89. III. der Schnabelfifch.

Gatterer, brev. zoolog. I. p. 162. n. 3. Balaena (Boops) fiftula duplici in roftro, dorfo extre. mo protuberantia cornea; der Schnabelffich.

Miiller, zool. dan. prodr. p. VIII. Balaena (Boops) fiftula duplici in roltro, dorfo extremo protuberantia cornea.

Fabric. fn. groenl. p. 36, n. 22. Balaena (Boops) fiftula duplici dorfo extremo protuberantia pinnaeformi, capite recto obtufo, ventre fulcato. Keporkak.

Hermenn, tab. affinit. animal. p. I29. Balaena Boops:

5. Gi в в оs A. Der Knoten. und Pflockffch.

c. Der Knotenfifch.

Mïller, Naturfyf. I. p. 493. b. der Knotenfffich. Neuer Schauplatz d. Natur, IX. p. 474. n. 6. der Knotenfifch. p. 493. b. der Knotenfifch.

Gatterer, vom Nutzen und Schaden der Thiere,

I. p. 435 . n. 256. der Knotenfish.

Eberhard, Thiergefch. p. 142. der Knotenfifch. Schneider, Abhandl. zur Zool. p. 219. der Kno. tin - oder Knobbelfifch.

Kranz, Grönland, p. 133. n. 6. der KnotenFilch.

Iablonyky, allgem. Lex. p. I329. der Knotenfifch oder Knobbelfich

Graumanin, intr. in hiftor. nat. mamm. p. 89. V. der Knotenfifch.

Gatterer, brev. Zool. I. p. 162. n. 5. Balaena (Gib. bofa) gibbis dorfalibus fex; der Knotenffich. 


\section{Der Pflockifich.}

Miiller, Naturfyfem, I. p. 493. a. der Pflockff $6 h$. Neuet Schauplatz der Natur, VI. p. 553. Pflockfifch.

Newer Schaupl. der Natur, IX. p. 474. n. 5. der Pflockfich., p. 493. der Pflockjifch.

Bat fih, Thiere, I. p. 260. der. Pfockfifch.

Eberhard, Thiergefch. p. 142. der Pflockffsch oder amerikanifche Wallfisch.

Schneider, Abhandl. zur Zoologie, p. 208. der Pflockffich.

Boroweky, Thierreich, II. I. p. 21. der Pflockfich. Cranz, Grönland, p. 133. 11. 8. der Pflock-Fifch. Pontoppidan, Norwegen, II. p. 232. Tuequal, Pflockfisch?

Iablonfky, allgem. Lex. p. 1329. der Pflockfifch. Erxleben, Mammalia, p. 607.

4. Musculus. Der Nordkaper. (2)

Mhiller, Naturfyft. I. p. 492. n. 4. Breitmaul. Borowfky, Thierreich, II. I. p. 22. n. 4. der Knotenfich, das Breitmaul. Tab. 2. D.

Gatte-

(2) Der Kopई des Thiers, welches Sibbald unterfucht hat, betrug in der Lánge dreyzehn Fufs dritthalb Zoll; der offene Rachen ftellte ein gleichfeitiges Dreyeck vor. Es ftanden vierzehn Menfchen darin zufammen, und ein kleines Fahrzeug fuhr mit der Fluth gerade hincin. Die. Zunge war funfzehn Fufs achthalb Zoll lang, und an dem dickften Ende ehenfalls funfzehn Fufs breit. Das Zeugeglied war fünf Fufs lang, und am Grunde vier Fufs im Umfange dick. Die Haut war einen halben Zoll dick. Man erhielc kaum dreyfsig Tonnen Thran davon, fonft nichts vom Werth. Die Fifcher hatten bemerkt, dafs diefs Thier immer den Heringen nachgieng, und aus feintr Gegenwart fchlofen fie auf einen guten Fang. - Man bat diefe Gattung oft mit dem Eiswalfffch verwechfolt. 
Gatterer, vom Nutzen und Schaden der Thiere, I. p. 435. n. 235 . das Breitmaul.

Neuer Schauplatz der Natur, IX. p. 493. n. 4 . Breitmaul.

Onomat. hiftor. nat. II. p. 153. der dreyflo/fichte

Wallfich mit einffacher Röhre.

Bat fch, Thiere, I. p. 260. das Breitmanul.

Eberhard, Thiergefchichte, p. 143. der Wallfich mit rundem Unterkiefer.

Schneider, Abhandl. zur Zoologie, p. 210. Nordkaper.

Olaffen, Island, I. p. 288. Steipe Reydur.

Goeze, Allerley; neue Auflage, II. p. 470. der Nordkaper.

Erxleben, Mammal. p. 609. n. 4. Balaena (Mufculus) fiftula duplici in fronte, maxilia inferiore multo latiore.

Linné, Syft. Nat. Edit. VI. p. 39. n. 3. Balaena fiftula duplici in fronte, maxilla inferiore mul. to latiore.

Linné, Syf. Natur. Edit. X. I. p. 76. n. 4. Balaena (Mufculus) fifula duplici in fronte, ma. xilla inferiore multo latiore.

Granmann, intr. in hiftor. nat, mamm. p. 89. IV. Breitmaul, Knotenffych.

Gatterer, brev. zoolog. I. p. 162. n. 4. Balaena (Mufculus) fiftula duplici in fronte, maxilla inferiore multo latiore; Breitmaul.

Miiller, zool. dan. prodrom. p. 7. n. 47. Balaena (Mufculus) fiftula duplici in fronte, maxilla inferiore multo latiore.

Fabric. fn. groenland. p. 39. n. 23. Balaena (Mufculus) fiftula duplici in fronte, maxilla infe. riore multo latiore.

Ccc 2 6. Ro. 
6. Ros TRATA. Der Schnabelffech.(3)

Borow $\int k y$. Thierreich, II. I. p. 23. n. 6. der kleinfte Wallfich.

Bat fch, Thiere, I. p. 260. dev kleinfte Wallfich. Gatterer, vom Nutzen ưnd Schaden der Thiere, I. p. 436. der'kieinfte WVallfifch.

Scliweider, Abhandl. zur Zoologie, p. 213. dẹ Sihurabelfich.

Olaffen, Island, I. p. 289. c. Adarneffa.

Pontopipidan, Norwegen, II. p. 233. Nebbe. Hual.

Steller, Kantfchatka, p. ro5. Morskox Woik, Plebuin, Tfchefchchot?

Erxleben, Mammalia, p. 6rr. * (Sp. cblcur.)

Hermann, tab. affinit. anim. p. 129. Balaena roftrata.

Veriünderungen gegen die XIIte Edition, nund Ver. mehwing der Gattungen diejes Gefchlethts.

Diefs Gefchlecht in mit zwey Gattungen vermehrt, und B. Gibbofa und Roftrata hinzugekommen; auch bey der"erftern Gattung der fo. genannte Eiswallffich als eine Varietät vom gemeinen grönlïndijchein Wallffich getrennt.

39. GE.

(3) Auch diefem Thiere hat man mit Unrecht die Benennungen Boops; Butzkopf, Iupiterfffcb u. a. m. gegeben, und es mit denen, welchen diefe Namen eigentlich zukommen; verwechfelt; wie Chemnitz in den Befcib. der berlin. Gefellfch. a. a. $O$. ebenfalls gethan hat. 
39. GESCHEECHT. PHYSETER. Der Kachelot.

Miiller, Naturfyftem, I. p. 497. Gen. XXXIX. Lefke, Naturgefchichte, p. 194. XLV. Borowsky, Thierreich, II. 1. p. 24: Gen. III. Blumenbach, Handb, der Naturgefchichte, p. I32. Gen. XLVII.

Neuer Schaupl. der Natur; IX. p. 493.

Oromat. hiftor. nat. V. p. $48 \mathrm{I}$. Schneider, Abhandl. zur Zool. p. 219.

Erxleben, Mammalia, p. 6rr. Gen. XLIX.

Gramann, intr. in hiftor. nat. mamm. p. 39. Gen. XLIX.

Gatterer, brev. Zoolog. I. p. 163. Gen. XLIX. Hermaziz, tab. affin. anim. p. 127. I28.

1. C A T O DON. Derkleine Kachelot. $\left({ }^{4}\right)$

Miiller, Naturfyf. I. p. 495. n. I. der Weifsfifch. Borowsky, Thierreich, II. I. p. 24. n. I. dev Weifsfifch:

Neuer Schauplatz der Natur, IX. p. 493. n." r. der Weifsfifch. - VI. p. 698. erfter Potfijch oder Kachelot.

Onomat. hifor. nat. VI. p. 479 . der kleine $K a$ chelot oder Milfich.

Batfch, Thiere, I. p. 262. der Weifsfifch.

\section{C c 3}

Gatte-

(4) Wird unrechtmäfsiger Weife mit dem Namen Weifsfffch belegt, der dem Delpbinus Leucas zukommt, und mit diefem haufig verwechfelt. Fabricius hat den Catodon und Turfo mit einander vereinigt, (Fiun. grocnland. p. 44) deutet dẹn Turfio auf feinén eignen Catodoin, und zieht Xinne's Catodon zim Weifsfifche. (daf. p. 50.) Er lifst alfo den Catodon des Linné als einen Cachelott eingehen, und macht einen Delphir. daratus, worin ihm Schroider bespflichtet. Zool. Abhandl.p. 2320. 
Gatterer, vom Nutzen u. Schaden der Thiere; I. p. 436. n. 238. der Wittfich, Weifsfiscin.

Beckmann, Naturhiforie, p. 68. b. der Wittfisch. Schneider, Abhandl. zur Zool. p. 233, Catodon. Erxleben, Mammal. p. 6ri. n. I. l'hyfeter (Cato. don) dorfo impinni, fifula in roftro.

Limné, Syft. Nat. Edit. VI. p. 39. n. 1. Catodon fiftula in roftro.

Limon?, Syft. Natur. Edit. X. I. p. 76. n. I. Phyfeter (Catodon) dorfo impenni, fiftula in roftro. Graumann, introd. in hift, nat. mamm. p. 89. I. der Weissficen.

1 Gatterer, brev. Zoolog. I. p. 163. n. 1. Phyfeter (Catocion) dorfo impinni, fiftulis doabus coale. fcentibus in roftro; der Weissfich, Wittfifch, Miiller, zool. dan. prodr. p. 7. n. 5r. Phyfeter (Catodon) dorfo impinni, fiftula in roftro.

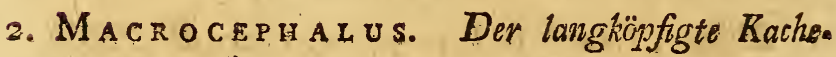
lot. (io)

Miiller, Naturfyftem, I. p. 498. n. 2. der Pottffch. Lefle, Naturgefch, p. I94. n. I. der Potffch.

Bechfein,

(5) Die wenigften Syfematiker haben die im Syftem bemerkten drey Varietäten aus einander gefetzt, upd Erxleben rechnete die beyden letztern noch zu den unbekannten Gattungen. Es if auch wirklich nicht leicht, die einer' jeden diefer Varietaten zugehürigen Sỵnonymen vüllig richtig zu ordnen, da ,man in den Befchreibungen mehrentheils alle drey mit einander verwechfelt findet, Blumenbafbs und Fabrizens Macracephalus, künmen z. E, nach allen Umftanden keine andere, als die crffe Varietăt a feyn; gleichwohl legt Fabriz feinem Macrocephal fehr kleine Augen bey, welche nach unfrem Syftem ein Unterfcheidungszeichen der dritten Varietat $\gamma$ feyn follen. Die obere langere Kinntade, und die darin befindlichen ausgehühlten Be-

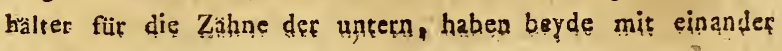
gemein. 
Bechftein, Naturgefch. Deutichl. I. p. 147. der Potfich.

Gatterer, vom Nutzen und Schaden der Thiere,

I. p. 437. n. 239. der Pottfich, Kafchelott. Neuer Schaupl. d. Natur, IX. p. 486. 月. I3. p. 487.

n, I4. p. 493. n. 2. Cachelot, Potfich. VI.

p. 698. zweyter Potfifch oder Kachelot.

Onomatol. hiff. nat. II. p. 686. der Potwallfifch.

VI, p. 48r. Phyfeter Macrocephalus.

Bat $\int c h$, Thiere, I. p. 26r. der Pottfich

Schneider, Abhandl. zur Zool. p. 220. der lang: köpfigte Cachelott.

Goese , Allerley; neue Aufl. III. p. 474 der Pottfich.

Handbuch der Naturgefchichte, II. p. 25I. der Potfich.

Le Vraillant, Reife in das Innere von Afrika, I. p. 25. der Kachelot.

Pontoppidan, Norwegen, II. p. 232. Troldqual. (Trollfifch.)

Erxleben, Mammalia, p. 612. 17. 12. Phyfeter (Macrocephalus) dorfo impinni, fifula in cervice.

Limné, Syn. Nat: Edit. II. p, 59. Catodon Ceto Clufii.

Linné, Syf. Nat, Edit, VI. p. 39. n. 2. Catodon fiftula in cervice.

Linné, Sy凡. Nat. Edit. X. I. p. 76. n. 2. Phyfeter (Macrocephalus) dorfo impenni, fitula in cervice.

Linné, Fn. Suec, I. p. 98. n. 262. Catodon fifula in cervice.

Limmé, Fn. Suec. II. pe 18. n. 53. Catodon (Macrocephalus) dorfo impenni, fintula in cervice. 


\section{Mammalia Cete. Phyfeter.}

Graumann, intr. in hiffor. nat, mamm. p. 89. II. der Pottfifch.

Gaitever, brev. Zoolog: I. p. 163. n. 2. Phyleter (Macrocephafus) dorfo impinini, fifula in cervice.

Miller, zool. dan. prodrom. p. 7. n, 52. Phyfeter (Macroccphalus) dorfo impinni, fiftula in cervice.

Hartenfiein, de magno pifce, qui Ionam vatem deglutivit. Witteberg, ryo5. 4.

Pfeifer, diff. pifcem Ionae deglutitorem fuiffe Balaenam. Lub. 1692.4.

Pechlin, de pifce Ionae deglutitore, non fuiffe Balaenam. Lub. 1694. 8.

Baring, de Ceto Ionae. Brem. 1689.

Bransmann, diff. de Ceto Ionae, qua eum verum füffe Cetum oftenditur. Ien. 1687.8 .

Roede, diff. de pifce qui Ionam deglutivit'; cuius.

$\therefore$ nam fpeciei fuerit. Hafin. 1744. 4.

Engelbrecht, diff. duae de pifce, Ionae deglutito. re. Lipf. 1702. 8.

a. Der Pottffich.

Boroveffy, Thierreich, II. 1. p. 25 . der. Pottfffch, Kafíchelot.

Blumeinbach, Handb. der Naturgefch. p. I32. n. I. Phyfeter (Macrocephalus) dorfo impinni, dentibus inflexis, apice acutiufculo; der Cachelot, Pottfffh. p. 670. (Ámber.)

Futuke, Naturgefch. I. p. 168. der Pottfjchi. Ebert, Naturiehre, II. p. I74. der Potfifeh. Beckinmin, Naturhiff. p. 68. a. der Potfijch. Cranz, Grönlànd, p. r36. n. 9. der Cafchelot oder Pottffico.

Egede, Grönland, p. 97. Cachelot od. Pottffifh. 


\section{Mammalia Cete. Phyfeter.}

Zordrager, grönland. Fifcherey, p. 328. 39r, Potfifch:

Camper, Schriften der berlin. Gefellfch. MI."p. 396. Cachelot.

Robertfon, phil. Transact. LX. p. 32r, Phyfeter Catodon.

Lichtenberg Magazin für das Neuefte etc. II. 4. p. 204 , Potfifich.

Schwedianer, Samml, zur Phyf. und Naturgefch. III. p. 333. über den Urfprung des Amibers, (a. d. philor. Tr. LXXIII. p. 226.) p. 336. der Cachelot.

Reich der Natur und Sitten. Halle 1758. \&- III. p. 12. Potfifch.

Schueider, Abhandl. zur Zool. p.235. (vom Wallrath und Amber.)

Beckmann, phyf. ökon. Bibl, III. p. 428. Phyfeter Catodon,

Abhildung der Wallfifche; bey Homanns Erben, Fig. 4 .

Ferber, neue Beyträge zur Mineralgefchichte $I$. Mietau I778. p. 366. (Bereitung des Wallraths.) Iablonfky, allgein. Lex p. 834. Pottfjch, Poth. wallfifich.

Eberhard, Thiergefch. p. I44 der Cachelot. Oekonom. Zool. p. 59. n. 67. der Cachelot. Halle, Mágie, IV. p. 573. (was det graue Ambra eigentlich fey.)

Bunzlauer Unterhalt. I. p. 238. der Pottffeh oder Wallrath and Ambrafifch.

Bartholin. Act. Hafnienf. Vol. II: p. 67.

Fabric. faun. groenl. p. 41. n: 25. Phyfeter (Macrocephalus) dorfo impinni, dentibus inflexis, apice acutiufculo. Kigulitik. 
B. Der weifslichte Kachelot.

Eberhard, Thiergefch. p. 144. der Weifsfifch.

Egede, Grönland. p. 98. Weifsfich.

Ebert, Naturlehre, II. p. 176. der Weifsfich.

Iabionfky, allgem. Lex. p. 1407, Witt-Fifch;

Weissfisch?

Erxleben, Mammalia, p. 616. *

Graumann, intr. in hift, nat, mamm. p. 89.a.

\section{y. Der Kachelot von Neuengland.}

Borowfky, Thierreich, II. I. p. 32. der netle englündifche Kafchelot,

Eberhard, Thiergefch. p. 145, der Cachelot von Neuengland.

Neuer Schaupl. der Natur, IX, p; 488. Dudleji Balaena.

Schneider, Abhandl. zur Zoologie, p. 237.

Erxieben, Mammalia, p. 617. **

Granumann, intr. in hif, nat. mamm. p. 89. b.'

3. Mr C OPs: Der kleinäugige Kachelot.

Miiller, Naturfyftem, I. p. 50r. n. 3. das Kleine: auge.

Gatterer, vom Nutzen und Schaden der Thiere,

I. p. 44,o. n. 240 . das Kleinange.

Pemant, arct. Zoolog. II. p. 158. Phyfeter microps.

Neuer Schauplatz der Natur, VI. p. yor. dritter Pottfifch oder Kachelot. IX. p. 493. n. 3. Kleinauge.

Onomat. hiftor. nat. VI. p. 48r. der kleinaugichte Kachelot.

Bat/ch, Thiere, I. p. 262. der kleinaingige $K a$. fiselot?

Goeze, 
Goeze. Allerley, neue Aufl. III. p. 478. das Kleinange.

Schneider, Abhandl. zur Zool. p. 225 Kleinauge. Erxleben, Marnmal. p. 6I4. n. 3. Phyfeter (Microps) dorfo pinna longa, maxilla fuperiore. longiore.

Linné, Syft. Nat. Edit,"VI. p. 39. n. r. Phyfeter maxilla fuperiore, longiore, fpina longa in dorfo.

Linné, Syft. Nat. Edit. X. I. p. 76. n. 3. Phyfeter (Microps) dorfo fina longa, maxilla fuperiore longiore.

Graumam, intr. in hifor. nat, mamm. p. 89 . III. der Kleinauge.

Gatterer, brev. zool. I. p. 163. n. 3. Phyfeter (Miçrops) dorfo pinna longa, maxilla fuperiore longiore; das Kleinange.

Miiller, prodr. zoolog. dan. p. 7., n. 53. Phyfeter (Microps) dorfo pinna longa, maxilla fupe. riore longiore.

a. Mit gebogenen fachelförmigen Zähnen.

Boronfky, Thierreich; II. x. p. 29. n. 3. der kleimäugige Kafchelot.

Eberhard, Thiergefch. p. 145. die ziweyte Art. Schizeider, Abhandl. zur Zool. p. 227. die Cachso lotte mit gebogenen fichelforrmigen $Z$ ähnen. Hermanin, Comment. tab. affinit, anim. p. I24. Fibric. faun. groenland. p. 44. n. 27 . Phyfeter (Microps) dorfo pinnato, dentibus arcuatis, apice acuto,

P. Mit geraden Spitzen Zühnen.

Borousky, Thierreich, II, I. p. 33. der Kafchea lot mit Spitzignn Zühunens, 
Eberhaid; Thicigefchichte, p. 145. die erfte Art. Erxleben, Mammal. p. 617. **:*

Graumatm, intr. in hif, nat mamm. p. 90 . c.

\section{Tursio. Der Maftifth.}

Müller, Naturfynt. I. p. 503. n. 4. der Maftffch. Borowefky, Thierreich, II. I. p. 31. n. 4 . dev Maftifith.

Neuer Schaupl, d. Natur, VI. p. 702. IX. p. 493.

n. 4. der Majtffich.

Onomat. hift. nat. VI. p. 482 der Maft fisch.

Bat fch. Thiere, I. p. 262, der Maftfifch.

Eberhard, Thiergefch. p. I46. die dritte Art.

Schneider, Abhandl. zur Zoologie, p. 23I. Maft. fisch.

Goese, Allerley, neue Auflage, III. p. 479. der Maftiflch.

Erxleben, Mammal. p. 615. n. 4. Phýfeter (Tur. fio) dorfi pinna altiflima, apice dentiuin plano.

Linné, Syft. Nat. Edit. VI. p. 39. n. 2. Phyfeter pinna dorfa altiflina, apice dentium plano.

Limné, Synt. Nat. Edit. X. I. p. 77. n. 4. Phyfeter (Turfio) dorfi pinna altifima, apice dentium plano.

Graumianu, intr. in hift. nat. mamm. p. 89 IV. Maftficcio.

Gnttever, brev. Zoolog. I. p. 164. n. 4. Phyfeter (Turfio) dorfo pinna altiffina, apice dentium plano.

Miiller, zoolog. dan. prodrom. p. VIII. Phyfeter (Turfio) dorfi pinna altiflima, apice dentium plano.

Fapric. faun. groenl. p. 44. 11. 26. Phyfeter (Catodon) dorfo piunato, apice dentium plano. 
$V$ Verinderungen gegen die XIIte Edition und Ver. mehring dẹr Gattungen diefes Gefchlechts.

(-.) Die Anzahl der Gattungen diefes, Gefchlechts in nicht vermehrt; bey der zweyten Gattung aber find drey, und bey der dritten, zwey Varietäten aus einander géfetzt.

40. GESCHLECHT. DeLPhinus. Der Delphin.

Milller, Naturfyftem, I. p. 504. Gen. XL. Lefke, Naturgefch. p.' r94. Gen. XLVI. Boroweky, Thierreich, IL I. p. 34. Gen. IV. Bhmenbach, Handb. der Naturgefchichte, p. 132. Gen. XLVIII.

Bechftein, Naturgefch. Deutfchl. I. p. 782. Batfch, Thiere, I. p. 262.

Neuer Schauplatz der Natur, IX. p. 494.

Onomat. hift. nat. III. p. 588 .

Schineider, Abhandl. zur Zoologie, p. 245.

Erxleben, Mammalia, p. 618. Gen. L.

Graumann, intr. in hifor. nat. mamm. p. 90.

Gen. L.

Gatterer, brev. Zoolog. I. p. 164. Gen. L. Hermanin, tab. affin. animal. p. 127.

1. Ph ос A en A. Der Bramnfifch. ( $\left.{ }^{6}\right)$

Miiller, Naturfyft. I. p. 504. n. I. der Brawnfich. Lefke, Naturgefch. p. 194. n. I. das Meer Cchwein. Borow/ky,

(9) Schwimmt mit grofser Schṇelligkeit, und beugt dabey den Kopf und Schwanz immer, nach unterwarts. Kann 6 bis 8 Stua , den auffer dem Waffer am I.eben bleiben.- Lebr vom Raube anderer Fifche, und jagt und verfolge die Heringe in dic Bayen und Mearbufen. Die Begattung gefchiehet im Auguft. Das 
Borowelky, Thierreich, II. I. p. 34. n. I. dey Braunffich, das Meerfchwein.

Bhumenbach, Handb. der Naturgefch. p. I32. 11. I. Delphinus (Phocaena) corpore fubconiformi, dorfo lato pinnato, roftro fubobtufo; das Meerfchwein, der Braunfifch.

Bechftein, Naturgefch. Deutfchl. I. p. 783. n. x. der Braunfifch. p. 787. kleiner Delphin, kleines Meerfchuein, Tanmler, Tumler, Nife, Springer. Ebert, Naturlehre, II. p. I78. a'er Braunfifich. Funke, Naturgefch. I. p. 169. das Mleer /ch hwein. Gatterer, vom Nutzen und Schaden der Thiere, I. p. 44I. n. 24r. dev Braunfifch, Nifer, das Meerschwein.

Beckmann, Naturhift. p. 7r. c. das Meerfchwein. Neuer Schauplatz der Natur, IX. p. 494. n.,5. Braunifich.

Onomat. hift. nat. VI. p. 459. der Braunfifch, das Meerfchwein.

Batfch, Thiere, I. p. 263. der Braunfifch oder das Meer Cchwein.

Eberhard, Thiergefchichte, p. I47. Meerfchwein, Braunfifch.

Schneider, Abhandl. zur Zool. p. 246. der Braukfifch.

Bock, Naturgefchichte von Preuffen, IV. p. 25 I. der Braunfich, kleiner Delphin, kleines Meerfchwein, Taumler, Tumler, Springer.

Fivicher,

Weibchen trägt neun Monate, und bringt gewöhnlich Ein lunges, das, fo lange es fangt, der Mutter beftândig folgt. . Das Thier hat ein efsbares Fleifch, und vielen Speck, der auch zu Thran gefotten wird. Es lebt in Gefellfchaft, und zieht fich, zumal bey hevorftehendem Sturm, nach den Schiffen. - Die deutfchen fenennungen diefer und der folgenden Gattung werब̀en häufig verwechfelt. 
Fifcher, Zufätze zur Naturgefch, von Livland, p. 41. n. 486. Tumeler, kleines Meerschwein. Leem, Nachr. von den Lappen, p. 159. Meer. fchroeine oder Delphine.

Gunnerus, Schriften der Drontheim. Gefellfch.

II. p. 237 Delphin oder Nifen. p. 237. Tab.4. Cranz, Grönland, p. I38. n. I2. das Meer Cchwein. Egede, Grönland, p. 105. der Nifer oder das Meerfchwein.

Kalm, Schwed. Abhandl. X.p. 200. Mar/vin, Ifer. Fifcherftröm, Schwed. Abhandl. XXIII. p. 263. Meerfchweine.

Steller, Kamtfchatka, ' p. 148. Phocaenen oder

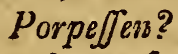

Schöpf, Keife durch Nordamerika, II. p. 409. Porpeffe?

Forfter, Bemerk. auf feiner Reife um die Welt, p. 169. das Meerfchwoin.

Linné, goth. Reife, p. 215. Meerfchweine.

Lizné, Natur-und Kunt-Hift. einiger fchwed.

Provinzen, p. 147. Delphine.

Hamb. Magazin, VI. p. 596. Delphin.

Iablonfky, allgem. Lex. p. 659. Meerfchwein.

Chandler, Reifen in Kleinafien, p. 4. Delphine.

Oekon. Zoologie, p. 60. n. 68. der Braunfich. Erxleben, Mammal. p. 6r8. n. I. Delphinus'(Phocaena) corpore fubconiformi, dorfo lato, roAro fubobtufo.

Linné, Syft. Natur. Edit. II. p. 59. Delphinus Phocaena.

Linné, Syft. N. Ed, VI. p.39. n. r. Delphinus corpore fubeoniformi, dorfo lato, roftro fubacuto. Linné, Syft. Nat. Edit. X. I. p. 77. n. x. Delphinus (Phocaena) corpore fubconiformi, dorfo lato, roftro fubobtufo. 
Limné, Fn. Suec.I. p. 99. n. 266. Delphinus corpo. re fubconiformi, dorfo lato, roftro fubacuto. Graumann, intr. in hif. nat. mamm. p. 90. I. Meerfchwein, Braunfffch.

Gatterer, brev. zool. 1. p. 164. n. x. Delphinus (Phocaena) corpore fubconiformi, dorfo lato, roftro fubobtufo; das Meer fchwein, der Branne fifch.

Charlevoix, nouv. Fr. III. p. 147. Marfouin. IViiller, zcol: dan. prodi: p. 7. n. 54. Delphinus (Phocaena) corpore fubconiformi, dorfo lato, roftro fubobtufo.

Fabric. faur. groenland. p. 46. n. 29. Delphinus (Phocaena) corpore fubconiformi, dorlo lato pinnato, roftro fubobcufo.

2. Dexpeis. Der Delphin. (7)

Milller, Naturfyft. I. p. 505. 11.2. der Tiimmler. Borowfky, Thierreich, II. I. p. 36. n. 2. dev Delphin, Tiimler.

Bhumenbach, Handb. der Naturgefch. p. 133. n. 2. Delphinus (Delphis) corpore oblongo fubtereti, dorfo

(7) Dief's ift der eigentliche Delphin der Alten, der durch die Gefchichte mit Arion, und wegen anderer vorgeblichen Proben feiner Menfchenliebe berühmt geworden it. Auf den alten Münzen von Grofs-Griechenland ift er genau abgebildet. Er gleicht dem vorigen ziemlich, fowohl im Aouisern als in dee Lebensart.' Nur hat er einen längern f́pitzigen Schnabel, der Braunfifch aber eine kurze fumpfo Schnauze. Die Benennung Springer hat ex davon, weil er bisweilen aus dem Waffer in die Höhe fpringet. Beyde krümmen fich beftandig zufammen, halten Kopf und Schwanz; niedergebogen, den Räcken aber in die Höhe. Auch der Delphin fchwimmt Fehr gefchwind, und fchwärmt truppenweife um die Schiffe; giebr anch eise Menge Speck und Thran, und entfernt fich, wie der Braunfith, ziemlich weit von Norden. 
dorfo pinnato, roftro attenuato acuto; der $D_{B}$. phin, Tiimmler:

Bechftein, Naturgefch. Deutfchl.I. p. 783.der Delphin, Tummler, Tïmler, Taumler, Springer, Saufich, Meerschwein, Seevarken.

Ebert, Naturlehre, II. p. 177. der Nifer oder dep eigentliche Delphin der Alten.

Gatterer, vom Nutzen und Schaden der Thiere,

I. p. 444. n. 242. der Delphin, Tümmler. Beckmann, Naturhift. p. 7r. b. der Tïmler. Neuer Schaupl:d. Natur, IX.p.494 n. 2. Tummler: Onomat. hift. nat. III. p. 588. das Meerschivein. Handb. d. Naturgefch. II. p. 256. der Delphin. Forfter, Bemerk: auf feiner Reife um die Welt, p. 169. der Delphin der Alten.

Batfch, Thiere, I. p. 263. der Tummler, oder eigentliche Delphin.

Eberhard, Thiergefchichte, p. r46. der Delphin, Meer:schwein, Tïmmler.

Schneider, Abh. zur Zool. p. 245. der Tiimler, od: Springer, Meer/chwein, der Delphin der Alten. $B o c k$, Naturgefch. von Preuffen, IV. p. 252. der Tummler, Deiphin.

Cranz, Grönl. p. I39.n. I3. der Delphin, Tiimmeler. Pontoppidan, Norwegen, II. p. 233. Springhval, oder der Springer.

Leem, Nachr. von den Lappen, p. 158. die Springer? Iablonfky, allgem. Lex. p. 264. Delphin. Éxxleben, Mainm. p. 621. 11.2. Delphinus (Delphis) corpore oblongo fubtereti, roftro attenuato, acuto. Linné, Syft. Nat, Edit.II. p. 59. Delphinus. Linné, Syf. N. Ed. VI. p. 39. n. 2. Delphinuscorpore oblongo fubtereti, roftro longo acuto.

Linné,S.N.Ed.X.I. p.77.n. 2. Delphinus (Delphis) corpore oblongo fubtereti, roftro attenuato, acuto. Ddd

Grazso 
Graumann, intr. in hift. nat. manim. p. go. II. Delphin, Meerschwein, Tummler.

Gatterer, brev. zool.1. p. 165. n.2. Delphinus (Del. phis) corpore oblongo fubtereti, roftro attenuato, acuto; der Delphin.

Yiiller, zcol. dan. prodr. p.7. n. 55. D. (Delphis) corpore oblongo fubtereti, roftro attenuato, acuto. Brown, Iam. p. 459. Delphinus corpore fubtereti oblongo, roftro producto, acuto.

Fabric. faun. groenland. p. 48. n. 30. Delphinus (Delphis) corpore oblongo fubtereti, dorfo pinnato, roftro attenuato acuto.

\section{Orca. Der Butzkopf. $\left({ }^{8}\right)$}

Miuller, Naturfyft. I. p. 506. n. 3. der Baitzkopf.

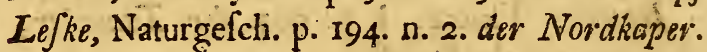
Borow Sky, Thierreich, II. I. p. 37. n.3. der Butzkopf. Tab. 4. C.

Blumenbach, Handb. der Naturgefch. p. 133, n. 3. Delphinus (Orca) pinna dorfi altifima, dentibus fubconicis, parum incurvis; der Nordkaper, Butzkopf.

Bechftein, N. G. Deutfchl, I. p. 785. der Butzkopf, Nordcaper, Pottfich, Buttkopf, Stuvmfifch.

Funke, Naturgefch. I. p. 167. der Nordkaper. (mit Balaena Mufculus verwechielt.)

Ebert, Naturlehre, II. p. I78. der Butskopf.

Gaitterer, vom Nutzen u. Schaden d. Th. I. p. 445. n. 243. der Butzkopf, Nordkaper, Sturmfich.

(8) Wird hâufig Nerdkaper genennt, und hat diele Benennung mit Balaena Mufculus gemein, fo wie Balaena Boops auch den Namen Butzkopf führt. Nach Schneider dïrften die Citata aus Gunnerus und Steller wohl auf den Sigedelpbin gehen. Treibt durch einen Schwung mit dem Schwanze die Heringe in einen Wirbel zufammen, uнd verfchlinge fie ronnenweife. 
Beckmann, Naturhiforie, p. yo. 2. der Nordka. per oder Butzkopf.

Neuer Schaupl.d. Natur, IX. p. 494. n. 3. Butkopf.

Onomatol. hilt. nat. II. p. I25. der Sturmfifch. Batfch, Thiere, I. p. 263. der Butzkopf. Eberhard, Thiergefch. p. 148. der Butskopf. Sihneider, Abhandl. zur Zool. pi 246. Butzkopf. Bock, Naturgefch. von Preuffen, IV. p. 253. der Buttkopf.

Cranz, Grönland, p. r38. n. Ir. der Butzkopf. Egede, Grönland, p. 98. Buittskopf.

Shaw, Reife, p. 169. der gezahnte Wallfifch, Orca. Chandler, Reifen in Kleinafien, p. 2. Gramhus oder Nordkaper.

Schöpf, Reife d. Nordamer. II. p. 409. Grampuffe. Forfler, Bemerk. auf feiner Reife um die Welt, p. 169. der Nordkaper.

Gunnerus, Schriften d. norwegifchen (drontheim.) Gefellfch. IV. 85. Stour - Vagnen.

Steller, Kamtfchatka, p. I04. Orcas; auf rulfisch Ko fatky genannt.

Beckmann, phyf. ökon. Bibl. I. p. 515. Orca. Abbild. d. Wallfilche; bey Homanns Erben. Fig. 3. Iabionfky, allgem. Lex. p. 186. Butzkopf. Erxleben, Mammal.p. 623. 1. 3. Delphinus (Orca) roftro furfum repando, dentibus latis ferratis. Linuté, Synt. Nat. Edit. II. p. 59. Orca. Linné, S. N. Ed. VI. p. 39. n. z. Delphinus roftro furfum repando, dentibus latis ferratis.

Linné, S. N. Ed. X. I. p. 77. n.3. Delphinus (Orca) roftro furfum repando, dentibus latis ferratis. Linné, Fn. Suec. I. p. 100. n. 267. Delphinus roftro furfum repando, dentibus latis ferratis.

Graumann, intr. in hifor. nat. mamm. p. 90. III. Butzkopf. 
Gatterer, brev. Zool. I. p. 165. n. 3. Delphinus (Orca) roftro furfum repando, dentibus latis ferratis; der Butzkopf.

Miuller, zool. dan. prodr. p. 8. n. 56. Delphinus (Orca) roftro furfum repando; dentibus latis ferratis.

Fabric. fn. groenl. p. 49. n. 3r. Delphinus (Turfio) corpore craffo, dorfo pinnato, roftro furfum repando, dentibus obtufis.

\section{B. Der Sïgedelphin. (?)}

IViiler, Naturfylt. I.p. 507. der Mürder, Killer. Miuller, Naturfyft. I. p. 507 . der Säbelfifich. Miiller, Naturfyftem, Supplem. p. 6r. der Butt. kopf.

Borvefky, Thierreich, II. I. p. 28. n. 4. Delphinus Serra; der Schwerdtfifch, Sïgefisch.

Ebert, Naturlehre, II. p. 179. der Sïbelfifch. Bat fch, Thiere, I. p. 263. der Schwerdt-oder Sïgedelphin.

Eberhard, Thiergefch. p. 147. n. 3. der Meerfäbel. (Gladius marinus.)

Schweifer, Abhandl. zur Zoologie, p. 247. der Speckhaner.

Cranz, Grönland, p. I39. n. I4. dev Scheverdt. fif $c h$.

Olaffen, Island, I. p. 290. b. Haa - Hirningm, Höfrungur. Delphinus (maximus) pinna in medio dorfo maiori acuminata.

Pontop.

(9) Die Benenuning Sigefifch künnte leicht Veranlafifung werden, das Thier mit Squalus priflis zu verwechfein, und durch den Namen Scbwerdffch künnte es wchl mit Xiphias Gladius verwechfelt werden. Ich habe daher die fchicklichfte Benennung Sägedelpbin gewählt, die ich nirgends_als beym Batfil gefun. den habe. 
Pontoppidan, Norwegen, II. p. 283. Speck-Hugger, oder Vahu. (Speckikauer oder W Wallfifchtödter.)

Goeze, Allerley; neue Aufl. III: p. 479. n1. 14. dev Killer oder Wallfifchtöater. p. 480 . die Säbelfinne.

Erxlebert, Mammalia, p. 625.

Grâumann, intr. in hiffor. nat. mamm. p. 90, der Schwerdtfifch.

Gatterer, brev. zool. I. p. 165. der Säbeliffch, Mür. der, Killer.

Fabric. faun. groenland. p. 46. n. 28. Delphinus (Orca) pinna dorfi altifima; dentibus fubconicis, parum incurvis.

4. Leucas, Der Weifsfific. $\left({ }^{10}\right)$

Schreber, Säugthiere, III. p. 315. die Bjeluga.

Pennant, arct. Zool. II. p. 176. Wittffich, Beliga. Pallas, Reife durch Rufsland, III. p. 85. SecBeluge.

\section{Ddd 3}

Pallas,

(10) Kommt in der Lebensart mit andern Delphinen tiberein, halt fich aber gern. bey dem Eife auf, wo er durch die Lücher Othem holen kann. Die Haut if einen Zoll diek, der Speck drey Zoll, das Fleifch roth. In Unterkieter fielien neunzehn kurze ftumpfe Zähne weit aus einander, die hleinern voran. Die in der nbern Kinnlade von gleicher Anzabl find fpitziger wnd ein wenig gebogen. Nährt fich von allerhand Fifchen, die ex in grofsen llauten vor fich her treibt und-verfolgt. Sein Schlund ift aber eng, fo dafs ihn eine allzugrofse Beure leicht erwürgt. Das Weibchen wirft im Frïhjahr Ein lunges, das erft blaulicht ausfieht, nachher aber weifs wird. Die fehr ftarke Haut verarbeitet man za Riemen. Das Fett kommt im Geichmacke dem Schweinefette, bey, und wird fammt dem Fleifche und den Eingeweiden gegeflen. Man fängt diefe Thiere in grofsen und Aarken, aus ikrer eignen Hsut verfertigten Netzen, 
Pallas, Reife, Ausz. III. p. 22. See - B̨̧luge, Weifsfifch.

Schneider, A bhandl. zur Zool. p. 250. Weifsfifch. Cranz, Grönland, p. 137. n. 1o. der Weifsfifch. Miiller, Sammlung. ruff. Gefch. III. 1. 2. 3. St. p. 253.

Fabric. fn. groenland. p. 50, n. 32. Delphinus (Albicans) dorfo impinnni, dentibus brevibus, apice plano.

I. G. Gmelin, Reife durch Sibirien, II. p: 439 . Beluga.

Erxleben, Mammal. p. 599. * (Sp. obfcur.)

Veraindernngen gegeñ die XIIte Edition, und Vere mehrung der Gattungen diefes Gefchlechts.

Bey dem Delphinus Orca in der Sügedelphin als eine Varietät vom Butzkopf getrennt. Dic vierte Gattung: D. Leucas in neu hinzuge. kominen. 


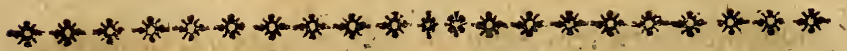

\section{WIEDERHOLUNG}

D E $\mathbf{R}$

NEU HINZUGEKOMMENEN GATTUNGEN.

In dem Gefchlecht Simia - - 15, Gattungen.

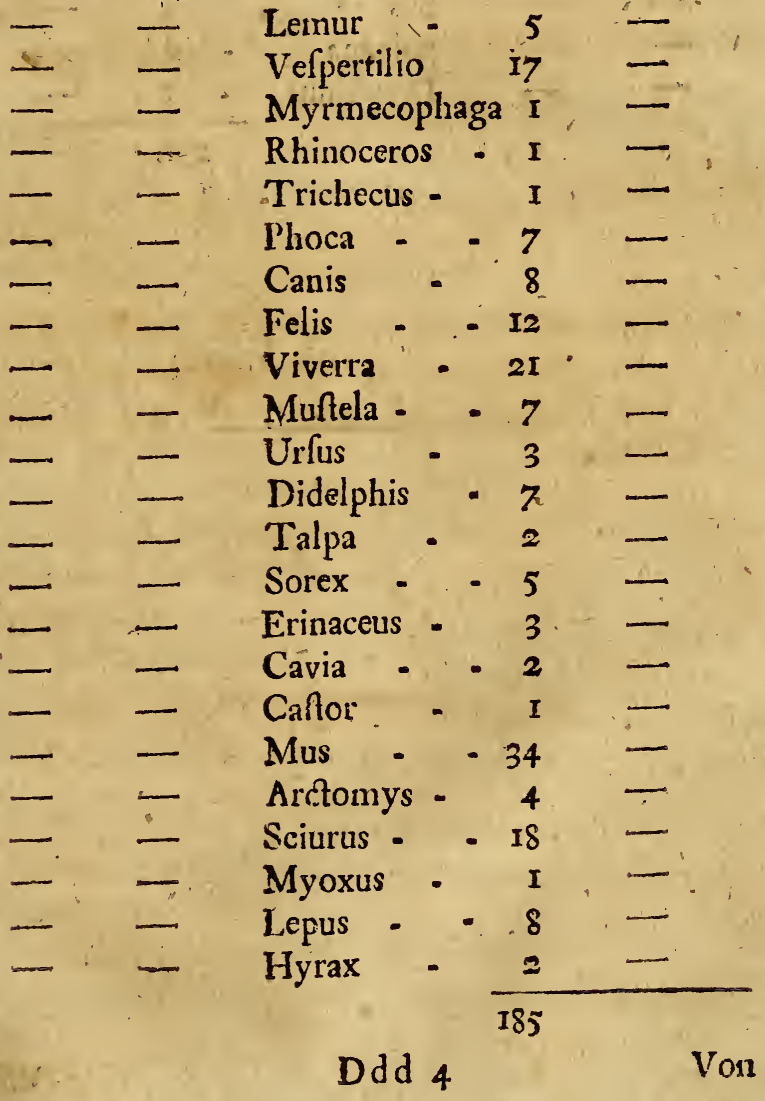


792 Wiederhol. d. neu hinzugek. Gattungen.

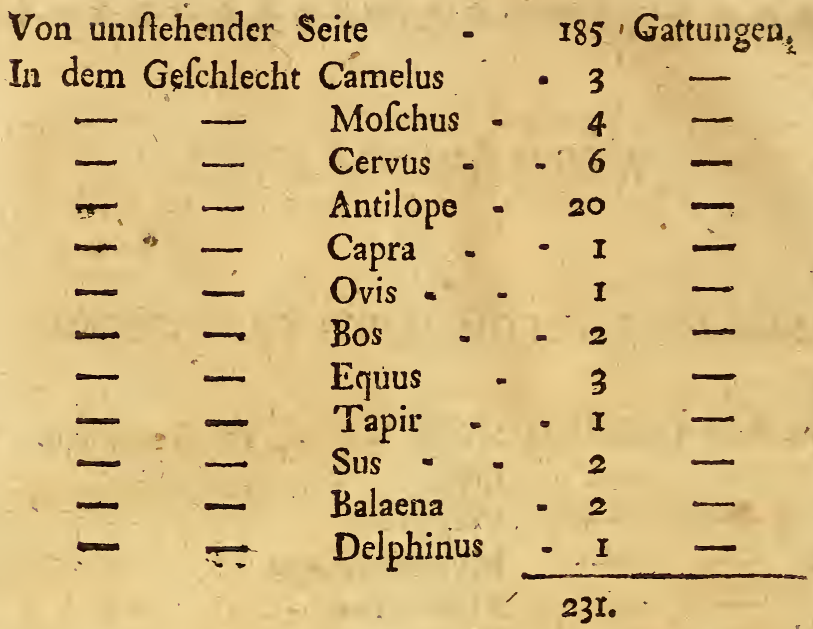




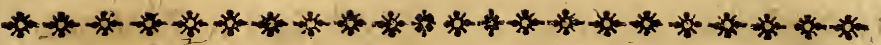

\section{VERZEICHNIS}

\section{A USL $\ddot{A} N D I S C H E R$ SYNONYMEN.}

2. Gefchlecht. Simia,

1. Satyrus.

Orang outang, malayifch, Sinfin, in China.

Pongo, Iocco, Enjocko, in Kongo.

2. Sylvanus.

Simia, Mona, in Itatien. Singe, Guenon, (das Weibchen) franz.

Simio, Ximio, IHono, (das Männchen) Simia, $X \bar{i}$. mia, Mona, (das Weib. cheir) fpanifch.

Bogio, Buıgio, (das Mäınchen) Bogia, Buigia, (das Weibclien) portugiefifch. Ape, englifch.

Aap, holländifch.

Obesjana, ruffifch. Majom, ungarifch.

6. Sphinx.

Babuino, italienifch. Baboivin, franzöfifch. Monazo,(das Männchen) 12. Beelzebul. Moncaza, (das Weibchen) fpanifch.
Bugio rabudo, portugief, Pavian, Bavian,"deut ch. Bavian, holländ. Baboon, engl. Babian, fchwed. Choakauma, Choakamina, hottentottifch. Pavian, (Paawiaan) Ku. tya majom (Kutja mas jomi) ungarifch.

9. Veter.

Elwandum, zeilanifch.

10. B. Silenus.

Wanduru, Rollway, in Zeilan. Slinger - aap, holländ.

15. Cynomolgus. Macaquo, in Kongo. Meerkatze, deutch. Matska - majom (Matfchka majom) ungarifch.

14. Diana.

Exquima, in Kongo.

- Guariba, Guereba, in Bra. filien.

Ddd 5

13. Seni- 
13. Seniculus.

Mono colorado, in Karthagena.

Alaubata, Singe rouge, in Guiana.

Arabata, am Oronoko.

$3 \mathbf{r}$, Sciurea.

Singe de mitit, in Cayenne. Sapajou aurore, Sapajou orange, Sapajou jaune, in Frankreich,

24. Iacchus.

Akarima, in Guiana.

Fonkes, äthịopifch.

Guereza, ambarifch.

\section{Gefchlecht. Veepertilio.}

6. Murinus.

Nottolo, Nottola, Sportiglione, Vifpiftrello, Vilpeftrello, Pipiftrello, italienifch.

Murcielago, Morcielago, Murciegaln, Mureguillo, fpanifch.

Morcego, portugief.

Chauve-Souris, franz. Fledermaus, Speckmans, deutCh.

Fledernuis, holländ.

Bat, Flittermouffe, Rear: anour $f_{e_{e}}$ Reremourese, englifch.
Löderlapp, Flädermus, fchwed.

Nattblacká, fmoländ.

Fiaggermunus, Aftenbah $k \theta$, dänifch.

Skind - Vinge, norweg.

Nietoper'sz, polnifch.

Netopyr, Letuczaga $m y \int c h$, ruff.

Tenderever, Pupperever, uingar.

Kanntle - TJkan, bey den cafanienfifchen 'Tataren. Fary-kanat, bey den Tavaren an den Flüffen Oby und Tfchulim,

Far-ganat, bey dep tfchatzenfifchení Tataren.

Omakiolot $/$ ch , tungufifch. Kut fchidu, bey den Tungufen, am Flufs Tungufca.

Irmifch, bey den Buräten. Thchongif chti cche - Kolja, bey den $T$ fcheremiffen. Sonatli Schiifchi, bey den Tfchuwafchen.

Kedi-Milai, morduanifch.

Knbort, firjänifch.

Bag̈bagai, kalmüekifch. Sakpdoofs, bey den Onia ken am Ienifey.

Togala, affanenfifch. Nahka . Sypo, finnifch. Indaga, 
Iudaga, japanifch.

Pellada, oder Sihkparple, in Lettland.

Nahthier, in Eftland.

Ataleph, hebräifch.

Atalepha, chaldäifch.

Parachadora, fyrifch.

Baphas, arabilch.

Anfeb, Perak, perfich.

15. Pictus.

Kiriwula, in Zeylan.

7. Gefchlecht. Bradypus.

\section{Tridactylus.}

$A i$, in Brafilien.

Ouaikaré, in Guiana.

Priguiza, portugief.

Hauti, Haut, perillo li.

gero, in dem fpanifchen

America.

Fanlthier, deutfch.

Leuy. aert, Luijaard, holländ.

Sloth, engl.

Refl-Kutya, ungar.

Lenivoy Pes, flavonifch.

2. Didactylus.

Potto, in Guinea.

Unau, Unaur gua Ju, bey den Anwohnern des Ma. ragnonifluffes.
8. Gefchlecht. Myrmeco. phaga.

Tamandra, brafilianifch.

Ouativi, in Guiana.

Achao, in Yucatan.

Hangya-Evö, ungar.

I. Didactyla.

Ouativi ouaon, in Guiana.

3. Iubata.

Onatiri ozaffu, in Guiana. Umbulu, in Kongo.

2. Gefchlecht. Manis.

r. Pentadactyla.

Alungu, auf Koromandel. Panggulling, in Iava.

Tchin, Chian Kiöpp, in china.

Quoggelo, in Guinea.

10. Gefchlecht. Dafypus.

Tatu, brafilianifch. Arșnadillo, fpanifch. Schildverken, holländ.

4. Sexcinctus.

Chirquinchum, in Mexiço. Armadillo, in Spanien. Encuberto, Encubertato portugief.

Schildverken, holländ.

6. Novem, 
6. Novencinctus,

Cachicamo, Atuco, Che de chuca, bey den Indianern am Oronoko.

Verdadeiro, in Portugal.

\section{Gefchlecht. Rhinocer os.}

s. Unicornis.

Chartis, Carcand, Corcadden, arabifch.

Carc, Gurg, Kerkedon, perfifch.

Lânduga, in Reiche Decan.

Kand a a mürrugam, malabarifch.

Abada, Numba, in Iava.

Tuabba, Nabba, bey den

\section{Hottentotten.}

Rinoceronte, italienifch.

Rhinoceronte, fpanifch.

Rhinoceros, porte comé, franzöfifch.

Nashorn, Na Jenhorn, deutfch.

Nozorozec, polnifch. Noforog, ruffifch. Reem, hebr. Kerkodon, perf. Abada, portugief.
Elephante, fpan.

Elephant, franz.

Eleplaant, dänifch.

Olyfant, Olyphant, hol. ländifch.

Elephant, engl.

Slon, ruflifch.

Elefant', (Elefaant) ungarifch.

Phill, türkifch.

Naeghe, äthiopifch.

Monzao, Mhanzo, in Kon-

go.

O. ond $_{\text {, }}$ in Guinea; bey den Sch warzen um Elmira.

Wâranam, malabar.

Anei, tamulifch.

Orang, malay.

Elffi, im Orient.

Twoba, Choà, bey den

Hottentotten.

$\mathrm{E} \lambda \varepsilon \varphi_{\alpha \text { ss, }}$, griechifch.

Elephantus, Barrus der Römer.

Cenalfa, arab.

Sion, illyr.

Elephant, Elefant, Helfant, deutfch.

Fill, perf.

Kassain, fanskritanifch.

Hati, indoftanifch.

\section{Gefchlecht. Elephas. \\ 6. Gefchlecht. Trichecus.}

r. Maximus.

Elfante, Leofante, ital.

I. Rosmarus.

Vache Marine, franz. 
Hors - hwoval, angelfächfifch.

Wallrofs, deutch.

Wallvus, holländ. fchwedifch.

Sea.Kow, englifch.

Hav - Heft, Hual-Ros,

Rosmar, norweg.

Mor.k, in Lappland.

Ro/t - Unger, Roftungr,

Rosmer, in Island.

Avec, Aurak, grönländ.

IMorfh, ruff.

Tinte, am Obyflufs.

2. Dugung.

Dugunn, Itkan Duginng, malayifch.

3. Manatus.

Manati, in Spanien.

Pezas Mouller oder Mul ger, bey den Portugiefen.

Lamantin, Lamentin, Va. che marine, bey den Fran. zofen.

Manati, Mariatee, engl. Söe - Koe, Hav - Nödd, dänifch.

Korowa morskaja, ruff. Kapuftuik, bey den Ruffen in Kamtfchatka.

Lereou, bey den Oualofen am Senegal.
Cojumero, in Guiana. Pege buey, am Amazo. nenfluffe.

Thousw, Châ-khoune, bey den Hottentotten.

Seekoejen, bey den Hollä̆sdern in Oftindien.

Seekuth, deutfch.

21. Gefchlecht. Phoca.

$\Phi \omega \dot{x n}$, griechifch. Robbe, Seehund, Sallhund, Meerkalb, deutfch.

Siäl, fchwed.

Sael Kaabe, dänifch.

$\mathrm{Sel}$, isländ.

Sea - calf, Seal, engl.

Moelrhon, britann. (Pen. nant.)

Phoque, Veaul marin, Loup marin, Chient mo. rin, Renard marin, franzöfifch.

$V$ echio marino, italien. Lobo marino, fpan. Tulen, ruff: (Steller.) Nerpen, in Sibirien. Pua, grönländ. (Cranz.)

2. Leonina.

Akomma, bey den Hot. tentotten.

Sea lion, bey den englifchen Seeleuten. 4. Iubą. 
4. Iubata.

Sireut cha, Sjutfcha, kurillifch.

3. Vitulina.

Labo marino, fpanifch.

Vecinio marino, italien.

Teaiu marin, franz.

Moelvhon, angelfächf.

Robbe, Seehund, Seckalb, deutfch:

Rob, Zee - Hond, holländ. Seal, Sea-calf, engl.

Sälhund, dänifch.

Selr, Landelir, Worfeluw, isländ.

Siall, Wikare-Siäl, fchwedifch.

Pies morski, polnifch.

Tjulen, ruff.

Kuma, tungufifch.

Häp; burätifch.

Nerpa, in Sibirien.

Purfe, Kafjgiak, in Grön. Jand.

Höutée, bey den Hotten-

\section{totten.}

Alg (das Mänuchen),

Lagy (das. W' eibchen),

Kut (das Iunge), in

Oefterbottn.

6. Groenlandica.

Sivartfiide, Attarfoak, in

Grönland.

Vadefelivi, in Islaud:
7. Hifpida.

Neitfek, Neitfoak', in Grönland.

8. Criftata.

Neitferfoak, in Grönland. Blaudruefeler, in Island. Nefaurfalik (M.), Kakor. lak (ein Iunges), Grönland.

9. Barbatà.

Ut fuk, in Gronland.

Utjelur. Wetrarfelur, in Island:

Lachtak, in Kạmtchatka

12. Gejchlecht. Casis.

I. Familiaris.

Perro, fpanifch.

Cam, portugiel.

Cane, italien.

Chieni (das Mänuchen),

Chienne (das Weibchen), franzöfifch.

$\mathbf{C i}_{i}$ (das Männchen), $\mathbf{G a f t}$ (das Weibchen), angelfächfifch.

Hond , holländ.

Dog, engl.

Hund, Rakke, Köter, Mysade, dänifch.

Köter, im Mecklenburgifchen.

Lubba, iṣländ. 
Hund, fchwedifch:

$P f i$, polnifch.

Pes, Sobaka (das Männchen), Suka (das Weib: chen), Pfitza (obfol.), Stzenja, Sczeriok (ein Iunges), ruff.

$E b$, Kutya, (Kutja); (das Männchen), Nöftenj-kutya, (Nöjchtenikutjia) (das Weibchen), ungarifch.

$I f t$, It , bey den cafanenfifchen Tataren.

It, bey den Tataren am Oby und Tfchulim.

$P_{i}$, bey den Tfcheremiffen.

Iida, bey den Tfchuivafchen.

$\boldsymbol{p}_{\text {umnu, bey den Wotjaken. }}$ $P$ inä, bey den Mordúanen.

Dfhiget, DShukel, (Nöfchtenohilo, das Weibchen) cingar.

Pon, firjänifch.

Kofha, Suka Guinookn; Ko. $h a$, in Kamtfchatka.

Kelb märre, in Aegypten. Nochoi , bey den Kămuk. ken.

It, bey den Bucharen. Kanang, bey den Tomenf. Oltiaken.
Tfchip, bey den Oniaken -D am Ieuifey.

Ninakin, bey den Tungu. fen an der Tungufka. Koira, finnifch, Küupek, türkifch. Nanakin, mongolifch. Lickhané, bey den Hot. tentotten.

Cotta, Kota, in Neuhol. land.

Keleb, hebraiifch.

Kalba, chaldäifch.

Kelbe, arabifch.

Kúwv, griechifch.

Keph oder Kolph, farac.

Sïg, oder Sig, perfifch.

Spaca, medifch.

Naji, tamulifch.

a. Domefticus.

Can di Paftori, ital. Chien de Berger, franz. Houshund, Schäfferhind, Schafried, Hirtenhand, deutch.

Huishoind, Herdershond, holländ.

Curre, Houfe-Dog, Shepherd's - Dog, engl.

Hushund, fehwed. Komondor, ungarifch.

3. Pomeranus.

Chien - Loup, franz. 
Pumi, ungar.

Ulfidur, Unglidur, isländifch.

\&. Aquaticus.

Perro de Aqua, Barbudillo, fpanifch.

Barbone, italien.

Barbet, franz.

Krulhond, holländ.

$W$ ater-Spaniel, engl.

Dogg, fchwed.

b. Melitaeus.

Perillo di falda, Spanifch. Cachorro, portugiel.

Cagnuolo, Cagmulino, ital.

Bichon, franz.

Lap.Dog, Shock, engl. Knöhund, fchwed.

\section{ข. Fricator.}

Mopse, Doguin, Dogue de Bologne, Dogue d'Al. lemagre, franz.

Mops, Mopper, Moppes:$l_{\xi}$, deutfch.

$\boldsymbol{p}_{\text {ug }}$ - Dog, engl.

Mops, fchwed.

Vetæere, (Wezere) ungarifch.

$\xi$. Moloffus.

Dogo, Perrogramade, โpanifch.
Dogue, franz.

Bullenbeifjer, Bärenteif:

fer, deutfch.

Wagthond, holländ.

Bull-dog, engl.

Blodhund, fchwed.

Sobaka medeljanskaja, ruffifch.

Szelérndek (Sseléndek) ungarifch.

o. Anglicus.

Can d'armi, italien.

Dogue de forte race, franz:

Englifche Dogge, deutfch. Maftiffe, engl.

Engel]k Dogg, fchwed.

$\pi$. Sagax.

$$
\text { Kopó, (Kopóo) ungar. }
$$

९. Gallicus.

Chien coumant, franz. Hound (ein grofser), Bea. gls (ein kleiner), engl.

$\sigma$. Scoticus.

Blood - hound, engl.

Sobaka vifhlaja, rufi.

ข. Avicularius.

Podenco, fpan.

Braceo, ital.

Braqus, chien coushant, franz.

Patryshond, holländ. 
Land-Spaniel, Harrier, Tumbler, Turnjpit, engl. englifch.

Rapphöns - hunzd, Fogel. hund, fchwed.

Hanse, fchived.

Sobaka ifpanskaja, ruft.

Vizsla, (Wifchla) ungar.

ax. Grajus:

Lebrel, Galgo, fpan.

Galgo, portugief.

Levriere, italien.

Leuron, Leurier, franz.

Windhond, holländ.

Greyhound, Grehound, engl.

Winthund, fchwed.

Sobaca gonczaja, ruff. Agár (Aghar) ungar.

88. Aegypticus.

Chien-turc, franz.

Naken-hund, fchwed.

ระ. Laniarius.

Maftin, Span.

Rafeiro, portugiel.

IMeagerhund, Schlächterhund, deutfch.

Can maftino, ital. Mátin, franz.

จิ.: Vertagus.

Perillo, Rapojero, Per. ro bako, fpan. Ba/fet, franz. Dashond, holländ.
Sinkurata kutya (Schin. kurata kutja) ungar

2. Lupus.

Lobo, fpan.

Lobo, portugief.

Lupo, italien.

Ulf, rlva, Ylgia, islän. difch.

Loup (das Männcben), Lonve (das Weibchen), Louveteau (ein Iunges), franz.

Wolf, holländ.

Wolf, engl.

Ulf, dänif ch.

Grabeen, Varg, norwegifch.

Warg, UIf, Gräben, fchwed:

Kumpi, Stolpe, Saibek: Gaine, Olgobutfh, lapp. länd.

Wilk, polnifch.

Wolk (das Männehen),

Wolczitza (das Weib: chen), ruffifch.

Kurt, türkifch.

Farkas. (Farkafch) ungarifch.

Bör, Aik, bucharifch.

Tfchono, kalınukkifch.

Boijuko, tungufifch.

Eee

TSchip. 
-T Tschipkáku, bey den Tungufen an der Tungufkà. Sithonnl, bey den Buräten.

Buire, bey den cafan. $T_{a}$. taren.

Biirjú, bey den Tataren am Oby, und bey den Tfchatzenf. Tataren.

Pirï, bey den Tfcheremifren.

Kafkar, bey den Tfchuwafchen.

Kión; bey den Wotiaken. Wjarges, bey den Morduanen.

Bóru, affanenf.

Susfi, finnifch.

Tiiimbune, bey den to. menf. Ofliaken.

Chÿyta, bey den Ofia- ken am Ienifey.

Köin, firjänifch.

Torqua, hottentottifch.

Wilks, Mefcha hunkis, in Lettland.

Hunt, in Enhland.

Zeeb, hebräifch.

- Kuorchn, in Kamtchatka. Lunumbengo, in Kongo. $\Lambda$ vxos, griechifch.

$D G i b, D i b, S a b b a$, ara. bifch.

Gürrk, perfifch.

$R u$, cingar.

Zfchefch, wogulifch.
8. Mexicantis.

Xoloitzcuintli, in Mexico.

3. Hyaena.

Hyaena, fpanifch. Hyena, portugies. Iena, italien. Hyena, engl. Ganmus, Belbus, bey den Römerin.

Dabbá, Dfabba, arab.

Dubbaih, in der Barbarey. Kaftaar, perfifch.

7. Aureus.

Skilachi, der jetzigen Griechen, von dem ächt griechifch. Worte oxy-

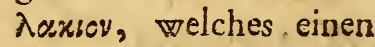
jungen Hund bedeutet. Schagall, Sjechaal perfich.

Schagall, kirgief. Schakall, tatarifch. Schakall, ruffifch, woraus Chacal, Siacalle, Siachal, Schachal, Siechal, Siacali, Iackal, Iackhals, und das

Chical der Türken, auch das

Zacalia, neu griechifch, entflanden find.

Deeb, Dib, Chatal, in der Barbarey. 
Waui, in Arabien.

Adibe, bey den Portugiefen in Indien.

Narî, d. i. Fuchs, und zwar infonderheit $K \vec{d} d$. tu: navi, d. i.' Strauchfuchs bey den Tamulern auf der Küfte Koroman. del.

Gôlâ, indoftanifch.

Thenfie, bey den Hotten. totten.

xr. Mefomelas.

Tennlie, Kénalie, bey den Hottentotten.

4. Vulpes.

Zorro (das Männchen), Zorra, Rapo Sa (das Weibchen) fpanifch.

Rapoza, portugief.

Volpe, italien.

Renard, franz.

Llwoynog (das Männchen),

Lheynoges (dás Weibchen), britannifch.

Vos, niederdeutfch.

Fox, engl.

Räv, Eiter . Unge, dänifch.

$R \ddot{a} f$, fchived.

Lis, Lif:ka, polnifcl. $L i s$ (das Männchen), Li. fitza (das Weibchen), ruffich.
Róka, (Rooka) ungar.

Rupfok, Raude, Zhiae. pok, Vielgok, lapplän. difeh.

Lap $a$, in Lettland.

Robbane, in Enthland.

Tiillki, bey den Türken.

Schulak, tungufifch.

Unagin, bey den Buräten.

Tfchafchea, Abfinges, bey den Kamtfchadalen.

Traleb, Dorén, ägypt.

Táleb, Abuthösni, arab.

Tulki, perfifch.

Nari, malabarifch, bey den Tamulern auf Ko. romandel.

Gjombuicaha, hochmala. barifch oder grendifch.

Quafis; in Guinea.

Schual, hebräifch. Keûlée, bey den Hotten. totten.

Terrianiak, grönländ,

5. Alopex.

Renard Charbonnier, in Burgund.

Brand-räf, fchwed. Brandfox, engl.

I3. Corfac.

Karfak, ruff.

Korfak, kirgiffech.
6. Lago. 
6. Lagopus.

Field-Rak, Mel-Rak, norweg.

Ficill-Racka, fchwed.

Nial, lappländ.

Pejez, rufí.

Arctic-fox, engl.

\section{Gefchlecht. Felis.}

I. Eeo.

Leon (das Männchen), Lecna (das Weibchen), fpanifch.

Leço, Lean (das Männchen), Leoa (das Weib. chen), portugief.

Lione, Leone (das Männcheni), Leona, Leonza, Lione Ja (d. Weibchen), italienifch.

Lion (das Männchen), Lionne (das Weibchen), franz.

Leetiv, holländ:

Law, meklenburg.

Lion| (das 'Männchen), Lione.s (das Weibchen), engl.

Léyon, fchiwed.

Lew (das Männchen), Lrvitza (das Weibchen), ruflifch.

Orofzláni (Orofslani), ungar.
Azed, arabifch.

Ino/chi/chi, japau.

Chamma, hottentott.

Sjir, Gehad, perf.

Gur (der neugeborne), Kephir (der Iunge), Arich (der grofse), Schachas, Labi (im beften und kräftigften Alter), Lebija (das Weibchen), Lais, Arich, Lâbbi (der veraltete), hebräifch.

Arjah, Arjavan, chaldäifch.

Arjo, fyrifch.

Sebey, bey den Saracenen.

2. Tigris.

Tigre, fpanifch.

Tigre, portugief.

Tigre (das Männchen), Tigra (das Weibchen), italien.

Tigre (das Männchen), Tigrese (d. Weibchen), franz.

Tyger, holländ.

Tiger, fchwed.

Tigr, ruff.

Tigris, (Tigrifch) ungarifch.

Paleng, perf.

Radja-outang, malayilch in Iava. 
Engri, in Kongo.

TqaJsonto. Kquassomo, hottentottifch.

Rako, in Iapan.

Lau - hus, chinee.

Hur - noun, bey den Chi- 4. nefern in Iava.

3. Pardus.

Pantera, fpanifch.

Panthera, portugief,

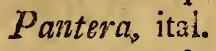

Panthere, franz.

Panther, engl.

Panter, fchwed.

Parantz(Pahrdutz)Tar.

ka Pardutz, ungar.

Nemr, arab.

9. Uncia

Faadh, arabifch.

Kod-hi bili, malabarifch. $P u$-pi, chinefífch. (Müller.)

$H_{i}$-rien-pao, chinefifch.

(Thevenot.)

Tigre d'Afrique, bey den franzöfifch. Rauchhändlern. (Büffon.)

Io. Leopardus.

Leopardal, Leopardo, fpanifch.

Leopardo, portugief.

Leoparda, ital.

Leopard, franz.
Lavipard, holländ.

Leopard, ruf:

Quelli, in Guinead

Engoi, in Kongo.

Onca.

Onca, portugief.

Bars', Babr, ruffifeh.

Kumik, tungufifch.

Ianu -ara, Iagu-ara, bra-

filianifch.

5. Pardalis.

Tlacoozlotl, Tlalocelotl, Tlatlauhgui ocelotl, mexicanifch.

II. Iubata.

Tiarou, Thlou, bey den

Hottentotten.

9. Concolor.

Cuguacu.ara, in Brafilien.

Puma, in Peru.

I3. Tigrina.

Maragua, Maragaia, in Brafilien.

6. Catus.

a. Ferus.

Gato montes, fpanifch. Gatto felvatico, ital. Chat fauvage, franz. Cath goed, britanm. Kot dziki, Zbik, polnifch. Eee 3 B. Do. 
B. Domefticus.

Gato, fpanifch.

Gato (das Männchen), Gata (das Weibchen), portugief.

Gatto, italien.

Chat, franz.

Gurcath (d: Männchen); Cath (das Weibchen), britannifch.

Kater (das Männchen), Kat (d. Weibchen), holländifch.

Cat, engl.

Cat, irrländ.

$K a t$, dänifch.

Katta, fchwed.

$K a t$, polnifch.

$K o t$ (M.), Kofchka (W.), ruff.

Bak-matska (Bak-matfch. ka, das M.) Matska, (Matfchka, d.W.) ungar. Kedi, bey den Türken. Matfch, Miitfch, Kot. Schach, bey den cafa. nenf. Tataren.

Myschy"k, bey den Ta. taren am Obyu. Tfchul. Prifs, bey den Tfcheremiffen.

Kotfchifch, morduanifch. Kon, firjänifch.

Mii, kalmukkifch. Mifchuk, buchar.
Kiffa, finnirch.

Choáï, hottentott.

$K o / c h k a$, illyr.

Ziinn, hebräifch.

15. Manul.

Manul, tatarifch, mogo. lifch.

Stepnaja Kofchka, ruff.

16. Serval.

Marcaputé, in Malabar.

Serval, bey den dafigen

l'ortugiefen.

17. Chaus,

Kyr - my fchak, tatarifch, Moes - gedu, tfchirkaft. Dikaja - ko ckika, ruff.

i8. Caracal.

Sigah-Ghush, perf. Kara-kulak, türk. Ainak-el-art, arab. Gat-el-Khalla, in der Barbarey.

7. Lynx,

Lince, Lynce, Lobocer. $v a l$, fpanifch,

Lince, Lobo cerval, por. tugief.

Lince, Lupo cervero, Lu. po cerviere, Luipo gatto, italien.

Lynx, Loup-cervier, fran. zöfifch. 
Los, holländ.

Lynx, Ounce, engl.

Los, Goup, norweg.

Warglo, fchwedifch.

Albos, lappländ.

Rys, Oftrowidz, polnifch, Rys, ruffifch.

Hiuz (Hius) ungarifch, Nondo, tungufifch. (Ge. orgi.)

Sylaufyn, tatar.

Potzchori, georgianifch. (Güldenft.)

Lu/Je, Lufe, in Lettland. Ileirs, in Eftland.

\section{Gefchlecht. Viverra.}

I. Ichneumon,

Tezerdea, in der Barba. rey.

Nemns, bey den Arabern in Aegypten.

Rat de Pharcon, franz.

7. Mungo.

Mungutia, javanifch. Mungo, bey den Portugiefen in Indien.

IMuncus; Moncos, Rotte: vanger, bey den Holländern dafelbn.

Chiri, Kirpele', in Malabar.

Lararangan, javanifch.
Sinifto, bengal. (Rumpf.)

Kurma, perfifch.

2. Nafua.

Coati, brafilianiffch.

Guache, in Mexico.

Quachy, in Caycnne.

Badger, bey den Englän-

veru in Guiana.

10. Vulpecula.

Yzquiepatl, in Mexico.

II. Quasje.

Quasje, in Surinam.

4. Putorius.

Skunk, in Neujork.

Pol -cat, bey den Englaindern in Amerika.

Pekan, Béte prtante, Enfant din diable, bey den Franzoferi dafelbft. Fifkatta, bey den Schweden in Penfylvanien.

I4. Zorilla.

Zorilla, bey den Spaniern in Amerika:

I6. Vittata.

Taguane, Maikél, b. d. Patagoniern?

5. Zibetha.

Qott-el-barr, arabifel. (Forskal.)

Eee 4

Sawâ- 
Sawádn puinei, malabar.

Can-can, äthiop.

Kouvóö, hottentott.

6. Genetta.

Geneitte, franz.

Genet-Kat, holländ.

Fuina di Conftantinopoli, in Italien.

21. Foffa.

Berbe, in Guinea.

Folja, Folfane, in Madagarkar.

15. Gefchlecht. Muffela.

I. Lutris.

Kalan, bey den Kamtfchadalen am Kamtfchatkaftrom.

Kaika, bey den Itelmänen dafelbft.

B. Brafilienfis.

Carigueibeiu, brafilian. Saricovicnne, am Plata. from.

Loutra, bey den Portugiefen in Südamerika.

3. Lutra.

Otter, holländ.

Otter, engl.

Odder, dänifch.

Otter, Slenter, in Norvegen.
Utter, fchived.

Dyfrgi, cambr.

Loutre, frauz.

Lodra, ital.

Nutria, fpan.

Wydra, poln.

- Wydra, ruff.

Schank, perf.

Sagif, türk.

Irgendir, tunguf.

Chalen, bey den Buräten.

Sagif, armen.

Zhievres, bey den Lap. pen.

Tovus, in Surinam.

Vidra, (Widra) ungar.

Uhdenis, Duppuris, Dukkeris, in Lettland.

Saarmas, Sarm, Kerl. Nirk, in Entland.

3. Lutreola.

Maink, fchwed.

Tuhcuri, finnländ.

Nurk, poln.

Norka, ruff.

Tackafch, an der Hudfonsbay?

Steinhnund, un Göttingen.

5. Vifon.

$V i$ fon, Foutrean, bey den Franzofen in Nordamerika.

Mink, Minix, b. d. dortigen Engländern u. Schweden. Iackasch, 
Iuckafch, bey den Eski- SamfJer, türk. mos? um die Hudfons- Lui, bey den Tfchere. bay.

12. Galera.

Voang, Shira, Vandfire, in Madagaskar.

14. Foina.

Fuina, fpanifch.

Foina, Fouina, ital.

Fonine, franz.

Bela graig, cambr.

Marter, holländ.

Martin, engl.

6. Martes.

Marta, fpan.

Marta, portugief.

Marta, Martura, IMorto. ra, Martorello, ital.

Marte, franz.

Belagoed, cambr.

Edlnarder, im Oefterreimifien.

Smofär, Surefàr, beyden Tfchuwafchen.

T56hinima, bey den Mor: duanen.

Tiılàn, firjäı.

Kuelna, grufifch.

Soofar, kalmuk.

Soofjar, buchar.

Akpa, affanenf.

Nïätä , finnländ.

Zauna, in Lettland.

Nuggis, in Efland.

Skalna, flavon.

9. Zibellina.

Marta Cebellina, Marta Cevellina, Spanifch.

Iforáo de Scytia, portugieffich.

Zibellino, italien. chifchen.

Marter, holländ.

Martin, Martlet, engl.

Maar, dän.

Mârd, fchwed.

Naette, lappländ.

Sabeldier , holländ.

Sable, engl.

Sabbel, fchwed.

Sobol, poln.

Sobol, ruff.

Sobol, morduan.

Kuna, poln.

Kunitza, Lido/Ja, ruff.

Sufár, tatar.

Iepka, Sutàr, hey den

Tataren am Oby und

Tfchul.

Kaltan, in Krasnojarsk.

Nyin zit, (Njufft) ungar.

Ky fch, bulgar.

Bulgàn, kalmukk.

Bulgàn, buchar.

Bula, burät.

Eé 5

Kymich 
Kymich Chym, Chym Huson, fpanifch. chymcha, in Kamtfchatka. Forćo, Foram, portugief. Kuivech, tatar. Lumuech, tfcheremin!.

$V$ is, Stör, wotiak.

Furetto, italien,

Si, bey den tomenf. Oftia-

Furet, franz.

Fret, holländ.

Ferret, engl. ken.

Eedfcha, bey den Oniaken am Ienifey.

$N_{i j} c h, U_{i} c_{c h}$, firjänifch.

Dinkjä, bey den' Tungufen an der Tunguska.

Tja, aflanenf.

Soboli, finnländ.

Sjafamuri, grufifch.

7. Putorius.

Putoro, fpanifch.

Foetta, Puzolo, Puzzolente, italien.

Putois, franz.

Ffwlbard, cambr.

Fritt, dänifch.

Nimise, bey den Arabern in der Barbarey.

15. Sarmatica.

Przewialka, poln.,

Peregnfna, ruf: -

16. Sibirica.

Kulon, tatar.

Kulonnok; Chorok, Choriok, rufr.

Nomo, bey den Tungufen.

Scholongo, bey den Buräten.

10. Erminea.

a. Aefliva.

Wezel, holländ.

Ilder, dänifch.

Boanid, Boitta, Goaaige (das Männchen), Gad-fe (das Weibchen, lappifich.

Tchorz, polnifch.

Lafitza, Lafotzka, ruff. Görény, ungar.

8. Furo.

Viverra, der alten Römer.
Stoat, engl.

Wesla, fchwed, in Smo. land.

Laska, Laficzka, poln.

B. Hyberna.

Armellino, italien.

Arminho, portugief.

Arminno, fpan.

Hexmelin, fchwed. 
Hermelin, dänifch.

Hermyn, holländ.

Lekatt, fchwed.

Carlwom, eambr.

Gornoftai, ruff.

Gronoftay, poln.

Pegymet, (Pedjmet) un.

gar.

Telek, b. d. Tungufen.

Ujing, b. d. Buräten.

Sehrmolihts, in Lettland, Nirk, in Eftland,

Sic - cuse-sue, in Nordamerika?

II. Vulgaris,

c. Aeftiva.

Donnola, Benuls, Ballotula, italien.

Donimnha, portugief.

Comadreia, fpan.

Belette, franz.

Weefel, engl.

Foumart, Fitchet, in

Yorkfhire,

Whitred, in Schottland.

Bromeen, cambr.

Laekatt, Vaefel, dänifch.

Röskatt, in Norwegen.

Lajka, ruff.

Unagin, brat?kifch.

Menyet, (Menjet) ungar. Faert el heile, in der Barbarey?
B. Nivalis.

Snömus, dänifch,

Snömus, fchwed.

Seibbfin, lapp.

Lasmizka, ruff.

16. Gejchlecht. Urfws.

I. Arctos.

Orfo, italien.

$O / \int_{0}$ (das Männchen), $O f$ fa (das Weibchen), fpa, nifch.

Urfo, UfJo (d. M.), Urv $\int a, U \iint_{a}(\mathrm{~d}, \mathrm{~W})$, portugiel:"

Ours, franz.

Beer, holläid.

Bear, engl.

Biörn, fchwed.

Biörn, dän.

Bams (d. M.), 'Bings

(d. W.), in Norwegen.

IVuriet, Kwoptza, Gnou: zia, lappifch.

Aenak (d. M.), Aefte (d.

W.), lappifch.

$K a r b u$, finnifch.

Medwed (d. M.), Mede wediza (d. W.), ruff.

Medve (Medwe), ungar:

Niedz'wiedz', pola.

Karhu, finnifch.

Aju, tatar.

Aju, tïrk. 
Chors, perfifch.

$D u b$, arab.

Kömnóptu, Amikan, tungufifch.

Kara-Gurfu, burät, Maskjä, tfcheremiff.

Obáh, tfchuwafch:

Gosidir, wotiak.

Oufta, Ohufta, morduan.

$O f c h$, firjän.

Gaas, Gafa, kamtfliadalifch.

Uiwnari, peruan.

Charagorefiin Etegö, kalmukkifch.

Ajik, buchar.

Korga, ofliak.

Korga, famojed.

Choija, bey den Oftiaken am Ienifey.

Kaltun, affanenf.

Datwi, grufifch.

Kamenof chi fchi, japan. Lahzis, in Lettland. Karro, in Efland.

5. Maritimus,

Nennok, in Grönland.

2. Meles.

Tafoo, ital.

Texon; Bivaro, fpan.

Texugo, Teixugo, por. tugief:

Blairean, Taiffon; Gri. fart, franz.
Das, holländ.

Badgi, Brock, Grey, engl.

Graevling, Brock, dän.

Gräf-Swin, fchwed.

Pryf Lhoyd, Pryf pen. frith, cambr.

Borfun, Iaswietz, poln. BaSfuk, Iaswetz, rufi: Bora (Bors), ungar.

Dorrakon, tunguf.

Ahp $\int c h a$, in Lettland.

Määr, in Enland.

Brok, Greving, Svinfok, norweg.

3. Lotor.

Attijhro, b. d. Irokefen.

Raccaon, b. d. Engländern.

Hefpan, Efpan, b. d. Holländern und Schweden in Amerika.

Mapach, in einigen amerikan. Gegenden.

Quachy, in Guiana.

Sjupp, b. d. Kürfchnern in Penfylvanien.

8. Gulo.

Iörf, fchwed.

Iaerv, Erv, in Norwegen.

Kola, um Drontheim. Fjällfras, Filfras, Eras, Snop, Snok, bey den fchwe- 
fchwedifch redenden Lappen.

Gieedk, b. d. norwegifch.

Lappen.

Rolfamaka, ruff:

RofJamaka, flavon.

Roffamaka, flavon.

Rafomaka, poln.

Tfchatak, tunguf.

Timuch, in Kamtfchatka.

Glutton, engl.

Glouton, franz.

Kumu, tatar,

Iungenda, oftiak.

Paria, firjän.

Peftap, alfanenf.

77. Gefchlecht. Didelphis.

3. Opoffum.

OpofJum, in Virginien.

Carigueyà, Iupatiima (das

Weibchen ), Tai - ibi

(das Männchen), in Brafilien.

Tai-ibi, in Paraguay.

Tlaquatzin, in Mexiko.

Cachorro do mato, bey. den Portugiefen in Brafio lien.

Bofchratte, holländ.

B. Molucea.

Cuffu-aru, Pelandor-arus, in $A$ mboina.
4. Murina.

Marmofa, in Brafilien.

9. Orientalis.

Cufcus in Amboina.

28. Gefchlecht. Talpa.

I. Europaea.

Talpa, italienifch.

Topo, fpan.

Toupeira, portugief.

Taupe, franz.

$M o l$, holländ.

Mole, Moldwarp, Want, engl.

Muldvarp, dän.

Vond, in Norwegen.

Mullvada, fchwed.

Surk, in Smoland.

Kret, poln.

Krot, ruff.

$V$ akondok, ungar.

Gwadd, Twerch dacar, cambr.

Thinfchemet, Hhodfed, hebräich.

Hhoulda, chald.

Cholda, fyr.

Topinara, bolognefifch.

Schaer, im fchweitzeri-

fchen.

Krtirze, illyr.

Kamennyi medred, Kamennaja fofedka, in Sibirien.

Kurvis, 

Kuirris, Kurmis, in Lett- Shrew, Shrewo - mouse,
land.

Mulh, Mugger, in EAland.

10. Gefchlecht. Sorex.

6. Mofchatus.

Wrichockol, Wuichuchols ruifr.

Chochul, in der Ukraine. Tfchirfin, an der Okka.

Dïsman, Däsmans rotta, fchwed.

7. Fodiens.

Gräber, um Berlin.

Souris d"ean, in Bour. gogne.

Blind - moues, engl.

Sljepuftchonka, Putara$k a$, in Rusland.

5. Araneus.

Mus araneus, Mus caecus, bey den Römern.

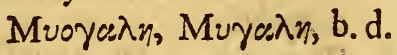
Griechen.

IMuzeraigne, Muserain, Mue et, Musetre, Sery, Sri, alt franz.

Mon faraigne, franz. Mufet, Mulette, in Savoyen.

Mufarring, in Bündten.

Toparagno, italien. Musgano, portugief. Murganho, fpan.

Llygoden goch, Chreift. lent, cambr.

Näbb-imus, fchwed.

Nebbe-mus, Muses"kiaer, norweg.

Angel-muus, dän.

Spitzmuis, holländ.

Bifammaus, in Schlefien. IMïtzer, in der Schweiz. Keret, polnifch.

Patkany (Patkaanj) un. garifch.

20. Gefchlecht. Erinaccus.

I. Europaeus.

Evinacens, Herinacens, der Römer.

Evinaceo, Riccio, Aizzo, italien.

Erizo, fpan.

Ourizo, portugief.

Heriffon, franz.

Eurchon, - alt franz.

Hledge - hog, engl.

Urchin, alt engl.

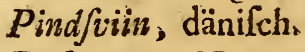

Buftivil, in Norwegen.

Igelkott, fchwed.

Iez, poln.

Iefch, ruff.

Tövis Difzno, ungar.

Drynog, Draen y coed.

cambr.

Hacrbe, 
Haerbe, Ganfud, bey

* Gefchlecht. Cavia. den Arabern in Aegy- 1. Paca.

pten.

Egel, Tzeren-Verken, holländ.

Kipod, helur.

Kopeda, chald.

Gejs Malak, Tzwijer. zatko, illyr.

$E / 2 s$, in Lettland. Siil, in Enland.

4. Auritus.

Io $h \hat{h}$, ruft.

Pak, Paka, in Brafilien. Pakivi, Ourana, in Guiana.

2. Acouchy.

Akouchy, in Cayenne. Aguti, auf den Infeln -. St. Lucie und Grenade.

3. Aguti.

Agouti, Acuti, in Guiana. Cotia, in Brafilien.

4. Aperea.

21. Gefchlecht. Hyftrix. Aperea, in Brafilien.

1. Criftata.

Spinofo, Porco fpinofo,

5. Cobaya.

Iftrice, italien.

E.jpin, Puerco e.jpino, fpanifch.

Porco efpinho, portugie. fifch.

Porc-epic, franz.

Porivipine, engl.

Steckelvarken, bolländ.

Dikobratz, riff.

Tzmban, arab. in der

Barbarey.

Qzieen - ja, in Südguinea.

Ghoukou, hottentost.

2. Prehenfilis。

Chionalu, in Brafilien.

Guinuea pig, engl. Cochon d' Inde, franz. Marfvin, fchwed.

Porcellino d' India, italienifch.

Cochino d" India, Cochino de Guinea, fpanifch.

Guineefch Biggetje, holländ.

Swinka Zamorska, poln. Cobaya, in Brafilien.

6. Capybara.

Capybara, Cabionara, Cabiai, Irabubo, bey verfchied. amerikan. $\mathrm{Na}$ tionen.

Capi- 
Capivard, bey den Franzofen in Amerika.

Capiiguara, bey den Quaraniern.

Atopehenra, b. d. Abipo. nern.

Caibai, Tybuarte, in Gu. iana.

23. Gefchlecht. Caftor.

1. Fiber.

Caftor, Fiber, bey den Römern.

Caftore, Bivaro, Bevero, italien.

Caftor, fpanifch.

Caftor, Bievre, franz.

Bever, holländ.

Bever, dänif́ch.

Bever, in Norwegen.

Baesver, fch swed.

Biur, in Smoland.

Beaver, englifch.

Bobr, ruff.

Bobr, poln.

Ko/chloki (ein Iunger), lartzy, (ein einjähriger), ruffich.

Bebris, lettifch.

Kobras, ehfnifch.

Hod, (Hood) ungar.

Muijeg, lappländ.

Lloftydan, cambr.

Chaly, burätifech.
24. Gefchlecht. Muss.

2. Ondathra.

Ondathra, bey den $\mathrm{Hu}$. ronen.

$M u f k \cdot r a t$, b. d. Engländern.

Rat - mufqué, b. d. Franzofen.

Desmans - rotta, bey den Schweden in Amerika,

12. Rattus.

Rata, fpanifch.

Ratto, ital.

Ratto, portugief.

Rat, engl.

Rat, franz.

Rotte, holländ.

Rotte, dänifch.

Rotta, fchwed.

Kryfa, rufi.

Kriffa, morduanifch.

Ulu TJkon, tatar.

Schonkfcha, tfchereminf.

$A s \cdot i \cdot \int c h u s c h i$, tfchuwa.

fchifch.

Budfchim-Schir, wotjak.

Wurdis, Burdiifch, firjä.

nifch.

Llygoden frengig, cam. brifch.

St fchurtech, polnifch.

Schurks, lettifch.

Rot, ehfnifch.

Göjii, Malonz• eger, ungar. 
Rotta, firnifcl.

Valska, isländ.

Surman, kalmukk.

Tava, bey den tomenf. Oftiaken.

Ora, aflanenf.

Wiftagua, grufifch.

13. Mufculus.

Muss, latein.

Mrius, fchwed.

Lille - Muns, Huns-Muns, dänifch.

Muis, holländ. Mouse, engl.

$M y \int_{\mathrm{c} h}, \mathrm{ruf}$.

$M y \int s$, polnifch.

Raton, Rata, fpan.

Ratinho, portugief.

Sorice, ital.

Souris, franz.

Llyguden, cambr.

Egér, ungarifh.

TJkan, tatar.

Schir, wotiakifch.

Schir, permifch.

Schir, firjänifch.

Hiir, eftrifch.

Tfchaor, morduan.

Koljä, tfcheremiff.

Pelle, lettifch.

Far, koptilch.

Huas "Mous, norweg.

Sölzafchan, bey den Türken.
Kuska, 'b. d. Tataren am

Oby u. Tf́chul.

$Z y z k a n$, b. d. tfchatzenf.

Tataren.

Houri, hottentottifch.

Chalgunz, kalmukk.

Satfchkan, buchar.

Uta, bey den Oliaken am Ienifey.

Kitrikon, tunguf.

Pen-rotta, finnifch.

Tagui, grufifch.

Achar, hebräifch.

Phar, Pfir, arabifch:

Achera, chaldäifch.

I7. Sylvaticus.

Mulot, Souris de terre, Rat Sauterelle, franz.

Ratte 'à la grande queue. in Bourgogne.

Bean - monse, in einigen Gegenden von England.

7. Agrarius.

Brandmaus, in Schlefien. Shitnik, rufr.

14. Vagus.

DJhilkis - Sitskan, tatar.

II. Amphibius.

Wodenoi krot, ruff,

Mataga, tunguf.

Kuter, jaksutifch. 
24. Rutilus.

Kultujach, jakutifch.

T)cheta mauftchu, kamt. fchadal.

25. Gregalis.

Niri-Katfchom, tunguf.

26. Oeconomus.

Kugutund, bey den Tun. gufen u. Buräten.

Kutugach, jakutifch.

Naulffchitfch, TegultSchitfch; kant fchadal.

36. Arvalis.

Campagniolo, ital. IMulot à courte queue; pe. tit rat de champs; cams. pagnol, franz.

Ratte coüttte, in Bourgogne.

29. Lagurus.

Dfhilkis - Thitskaiz, tatar.

5. Lemmus.

Leming, Laemen, Laemis, in Norwegen.

Luminite, b. d. fchwed. Lappen.

Fiällmuss. Sabelmas, fchwedilich:

Godde-Sapin, bey den dänifchen Lappen:

Pefrufchira, ralfifeh.
9. Cricetus:

Grent fch, Grutfchel, Erdwolf, in Schlefien.

Kriet fch, in Oeferreich. Skrzeczek, Chomik, poln. Hörtfchök, ungar.

Chomak, Karbufch, ruft.

37. Furunculus.

Orocht fchofchach, mon. golifch.

38. Talpinus:

Sljepufchonka, Semleroika, ruflifch.

Suchert fkan, tatar.

39. Capentis.

Blesinoll, bey den HolJändern am Kap.

40. Maritinus.

Kaun-howba, b. d. Hottentotten.

41. Afplialax.

Monon Zokor, bey den daurifchen Tungufen. Semlanaja Medivedka, bey den Rufen in Sibirien.

42. Typhlus.

Siepez, ruff:

Sinfkö̈ - Scht/chenjae, in der Ukraine.

$p_{\text {iefek ziemni, in Pohlen? }}$ SSóchor. 
Sfóchor.nómon, bey den wolgifchen Kalmukken.

* Gefchtecht. Arotomys.

I. Marmota.

Wurnelthier, in Deutfchland.

IInurmentle, IMiftbellerle, in der Schweitz.

Montanella, in Bündten. Niavnontana, im tridentifchen.

Illarmota, italien. Mlarmota, franifch. Marmotte, franz. IVarmot, engl.

3. Bobac.

Swiftch, Bobuk, poln. Baibak, in der Ukraine. Surók, ruft. Suinr, Sugur, Suwver, ta tarifch.

Tarbagän, mongolifeh. $B f c h d$, mongolifch.

6. Citellus.

"Sifel, böhmifch.

Sus $e l$, poln.

Suslik, ruff.

Avrafchka, bey den Kofaken.

Ievrafchka, bey den Ruf. fen in Sibirien.
Temuranka, b. d. Ruffen in der Gegend Barnaul. DShumburd, mongolifch. Dfiumburt, kalmukk. DShumbra, Suarma, bey den wolgifchen Kalmukken.

Sumura, burätifch.

DShymmuran, Dfinymron, tatar.

Simral, Inral, morduanifch.

Sakildau-t tkân, kirgifich. Tyrgdn, bey den krasnojarifchen Tataren.

Trka, koibalifch.

Thrugäh, jakutifch.

Syrith, kamtfchadalifch. Schilà, korätifch.

7. Gundi.

Gundi, arabifch.

25. Gefchlecht. Sciurus.

I. Vulģariș.

Eichkatz $l$, in Oefterreich. Ikorn, fchwed. lkorn, in Norwegen. Egern, dänifch. ¿xiovęos (Oppian.) Kap-

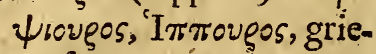
chifch.

$\mathbf{N} \eta \varepsilon \xi \leftrightarrow$, in Kappadocien. (Varin.) 
Efcureau, Efcurieu, Ecureuil, franz.

Schiarro, Schiratto, Schi. rattolo, Schirizolo, Scoi. attolo, ital.

Harda, Hardilla, Arda, Ardilla, Efquilo, fuan.

Ciuro, portugief.

Gwivecir, cambr. Feòrag, erfifch.

Bjelka, Wjekfcha, ruff,

Wewerka, illyr.

Wererka, böhm.

Wiewiorka, poln.

Mokus, Evet, ungar.

Uluk, tunguf.

Uru, morduan.

$U$, permäkifcl.

$U r$, tcheremiff.

Orawas, finuifch.

Orre, lappifch.

Kermang, burät.

Kermà, kalmukk.

Tien, Tiin, Tijin, tatar.

Tyiziz, buchar.

Tabjek, b. d. tomskifchen

Oltiaken.

$S_{a k}$, b. d. jenifeiskifchen

Oftiaken.

Schaga, affanifch. Bakfcha, tfchuwaft. Line, Leina, wogul. Wahwaris, lettifch. Orraw, eftnifch. Mela, grufifch.
3. Cinereus.

Ecurenil gris, ecureuil de Canada, de Virginie, b. d. Franzofen.

12. Hudfonius. Sikjik, eskimoifch?

I8. Macrourus.

Daudulana, Rukea, zeilonifch.

19. Maximus.

Grand rat de bois, auf der Küfte Malabar.

7. Striatus.

Burunduk, ruffifch.

Uldjuki, Ulbuki, tunguf.

Uhrda, wotiak.

Wahrtae, wogul.

D)julalà, bafchkir.

Dfchyryku, burät.

Dfhyraeki, mongol.

Schöpe, Schépek, oftiak. Kiigeriik, Köhrök, tatar. Kätho, indifch.

Denka, am Ienifey.

Pifeku, famojed.

Kurt chugaess, Mochotoy, jakut.

C. Americanus.

RöfJehvifsla, b.d. Schwe. den in Nordamerika.

Ohihoin, huronifch. 
25. Mexicanus.

Tlamototl, in Mexico.

26. Volucella.

Quimichpatlan, in Neufpanien.

Afrapanik, Sahouesquan$t a$, wildifche Namen in Virginien und Canada.

10. Volans.

Ljetaga, ruff.

Popyelycza Iätayacza, Wjewjorka Iätajacza. polu.

Saar-tien-kanat, tatar. Abarghán, b. d. gebirgifchen Samojeden.

Babàrchan, b. d, Tataren ain Ienifey.

Iluchön, jakutifch.

Tochlyng - langi, Initlanki, Iwe-laniki, Pailan-langi, oftiakifch bey den verfchiedenen Stämmen.

Toulm-leyn, Tauling. Lengen, wogulifch.

Tirta-tarek, famojed.

Poë $\int e$, bey den Oftiaken am Narym.

Bobontóll, b. d. werchonturifchen Wogulen.

Puloh, wotiakifch. Kiisstiiv, permäkifch.
Pall, firjian.

Olbo, mongol.

Uldjugi, tunguf.

$B \int c h a m a b \int c h i$, tangut.

28. Petaurifta.

Taquan, Taguan, auf den Philippinen.

* Geschlecht. Myoxus.

I. Glis.

Glis, der alten Römer. Ghiro, Gliero, italien. Liron, fpanifch, alt franz. Loir, franz. Arganciz, portugief. Dormouse, Rellmouse, engl.

Rell, Rellmuss; in der Schweitz.

puh, illyr.

Pouch, poln.

Semiljana bjelka, um Sa. mara, ruff.

3. Nitela.

Muscardino, italien. Mufcardin, Rat dort, Rat d'or, Croque noix, franzöfifch.

Dormoufe, Sleeper, engl.

Pathew, cambr.

Skogsmus, fchwed.

Hazelmuis, holländ.

Fff 3

26. $G$ :- 
26. Gefchlecht. Dipus.

1. Iaculus.

Alak-daagha, mongol.

Ialman, kalmukk.

Morin. Ialma (die grofse Spielart), Choin - Ialma

(die kleine Spielart), bey den Kalmukken.

Iratmon, Dfchjalmuan, Koi. rok, b. d. Bafchkiren.

Akkik, Malin, Tya Iel:

man, tatar.

Tajch, Arap, Trkan, kirgififch. (Falk.)

Semljanoï-Saё:z, ruff.

Tus chkantjócik, an Iaik.

Iemumant fchik (die mittle. re Spielart), ruff.

Therio.t $t u$, chinef.

Abalak, indifch. (Mefferfchmid.)

2. Ierboa.

Mus $\delta_{b} \pi$ ous, bey den al. ten Griechen.

Ierboa, arąb.

Djarbua, in Aegypten.

Tarbagantfchik, bey den Kofaken am Irtifch.

\section{Gefchlecht. Lepus.}

I. Timidus.

$\Lambda \alpha \gamma \omega \varsigma, \Pi \tau \omega \xi$, griech: Lepus, latein.
Lepre, Lievora, italien.

Liebre, fpanifch.

Lebre, portugief.

Lièvre, franzöfifch.

Haas, holländ.

Have, dänifch.

Häre, fchwed.

Hare, engl.

Saëz, ruff.

Zaiac, poln.

$\Upsilon \int$ gifarnog, Ceinach, cambrifch.

Nyul, poln:

Iafe, norweg.

Niaamel, lapp.

Tauecsham, türk.

Ufchnap, Ujchkan, fibir. Toufchak, Taufchakei, tunguf.

Schandalgan, burät.

Koich , bey den cafanenf. Tataren.

Kojan,, b. d. tfchatzenf: Tataren.

Kujan, b. d. Tataren am Oby.

Mercin, tfcheremiff.

Mholgât fch, tfchuwafch.

Luidket fch, wotiak.

Niimola, morduan.

Kït fch, perm.

$K o ̈ t / c h$, firjän.

Arnäh, arab.

Ufangi, japan.

Toofchkan, bucharifch. 
Njo, bey den tomenf: Krolik, poln.

Oftiaken.

Befs, b. d. Oftiaken am Ienifey.

Màngarà, affanenfifch.

I'́nix, funnifch.

Kurtgreli, grufifch.

$K_{c a} a, T_{c a}$, hottentutt.

Sakkis, lettilch.

Iönnis, eftnifich.

8. Tolai.

Tolai, Talai, ruff.

2. Cuniculus.

Conejo, Coneja, Coneguelo, Conejillo, Conejito, fpanịch.

Coelho, portugief:

Coniglio, ital.'

Lapin, Lapine, Lapreau, franz.

Lapin de Garenne (das wilde), Lapin de Cla. piers (das zahme), Cons. nin, Connil (obfol.), franz.

Cwninger, cambr.

Kiiniglhase, öfterreich.

Konnyn, holländ. Lain. preelen, die Iungen.

Coinin, irrländ.

Kanin, Mïne, dänifch.

Rabbet, Cony, eng!. Kanin, fchwed.
Krolik, ruff.

Tengeri-Nyul, ungar.

Kannikis , Kanninkentis, lettifch.

Koddojännes, chfnilch.

Saphan, hebr.

Thap $\int a$, chald.

Vebur, arabifch.

Befongergh, petf.

Cunicuinus, Isepufeulus, la. teinifch.

I1. Alpinus.

Pifcaulha, Sjenoftawetz, ruffifch?

$P i k a$, tungufifch?

12. Ogotona.

Ochodona, mungalifeh.

27. Gefchlecht; Camelus.

1. Dromedarius.

Dromedario, Dranedal, fpanifch.

Dromedario, portugief.

Dromedario, italien.

Dromedaire, franz.

Dromedary, englifch.

Werblisd Skoro bẽgajuf-

czii, ruff.

Egin, mohrenl.

Maihary, Asharry, in der Levante.

Schut- 
Schuttur, perfifch. a) Ner. 3. Glama.

b) Iurda Kaidam.c)Kokk. Glama, (das Weibchen),

d) Schutturhic Baad.

Geldowefi, türk. (Windkameel.)

Djämmel, im Orient.

2. Bactrianus.

Camello, italienifch.

Camello, fpan.

Camello, portugief.

Chamear, franz.

Cameel, dän.

Kemel, holländ.

Werblind, ruf:

Teve, ungar.

Deve, türk.

Dï̈̈, cafanenl.

Tjuja, tatar.

Tiiii, tichuwafchifch.

Tiie, tcheremiff:

Diii, wotiak.

Ifchim, Werblud, mor. duanifch.

Temjàm, kalmükk.

Tegö, buchar.

Kameli, finnifch.

Aklemi, grufifch.

Fymi, burätifch.

Gamal, hebr.

Gemal, alt arabifch.

Gimel, neu arabifch,

Gamala, chaldäifch,

Bócht, in Orient.
Urco (das Männchen', in Peru.

Carnero de la tierra, in Spanien?

5. Huanacus.

Pernichcatl, engl.

$P$ elon ichiatl ${ }^{\circ}$ quitli, in Mexiko?

Wiánaque, in Peru. Guanapo, in Peru.

6. Araucanus.

Hureque, Chillehueque, in Chili.

28. Gefchlecht. Mo/chus.

1. Mofchiferus.

Kabarga, in Krasnojarsk, ruft.

Honde, tungufifch.

Budek, burätifch.

$X e$, Hiang-tchang-tfe,

in China.

Saiga? in Irkutsk, Ilimsk, und an der Lena, ruff.

29. Gefchlecht. Cervus.

I. Pygargus.

Dikaja Koza, ruffifch.

Saiga, tatarifch?

Ahu, perfifch? 
2. Alces.

Alce, italien.

Alce, fpan.

Elan, franz.

Orignal, b. d. Franzofen in Amerika.

Elk, alt deut fch.

Eland, hollïnd.

$E l k$, engl.

MMoofe - deer, black Moofedeer, b.d. Engl. in Ame-' rika.

Elsdiur, dän.

Elg, Elsdyr, Ellgur, norweg.

Aelg, fchwed.

Los, poln.

Los, ruff.

Iavor, ungar.

Sochat, in Sibirien.

Took, tunguf.

Bogui, burät.

Bulin, tatar.

Buldan, tfchuwaafch.

Schórda, tfcheremifr.

Puf che', wotiak.

Sjärda, morduan.

Lö̈s, löra, firjän.

Hirwi, - finnifch.

Breedis, lettifch.

Pödder, ehfnifch.

Chandagai, kalmükk.

Bulan, buchar.

Pianga, b. d. tomenf. On iaken.
Cháajï, b. d. Oftiaken am Ienifey.

Alt fchang fch, aflanenf.

Iremi, grufifch.

Thanna, Tkumma, hot. tentott.

Han - ta-han, in Afien.

3. Elaphus.

Cervio (d. M.), Cervia (d. W.), italienifch.

Ciervo, Cierva, fpan.

Cervo, Cerva, portugiefifch.

$\operatorname{Cerf}$ (M.), Biche (W.), Fron (ein Iunges), $D a$. guel (Spiefshirfch), franzöfifch.

Carw (M.), Ewig (W.), Elain (ein Iunges), cam. brifch.

Hert, Hinde, holländ.

Hart, Red Deer (die ganze Gattung), Stag (M.), Hind (W.), Priket, (Spiefshirfch), engl.

Hiort, Kronhiort, Adelhiort, dänifch. Röd-Dyr (M.), Dyrkolle (W.), norweg. Hiort, Kronhiort (M.), Hint (W.), fchwed. Ielen, poln. Ifubr, in Irkutsk, und an der chinef. Grenze.

Iff 5 Marat, 
Maral', am Irtifch (M.), Lan' (W.), Olen' pramorogoi (Spiefshirfch), ruff:

Kunzaka, tunguf:

Gohu, burät.

Szarvas (M.), Nöftény, Szarvas, Suta (W.), Scarvas - borjú (ein Iunges), ungar.

Sö̈gïn, tưrk.

Thamma, hottentott.

Ayal, hebr.

Ajal, arab.

Gewazen, perf:

Ajela, chaldäifcl.

4. Tarandus.

Renne, franz.

Ren, Rens - Dyr, Hreine

Dyr, Graa-Dyr, Hals-

buk, norweg.

Rhen, fchwed.

Godde, dän. lappl.

Bö̈t foi, fchwed. lappl.

Olen²(M.), Oleniza'(W.) ruff.

Sagan, burăt.

Sägan, tunguf:

$P$ Pchi, tatar.

Piit fche, tfcheremifi:

Bulcin, ifchuwafch.

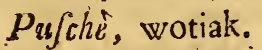

Olen, morduan.

Kitr: permifch.
Kör, firjäı.

Tukto, grönländ.

Oron, mongal. Buga, kalmikk.

Bugü, buchar. Adie, famojed. Adie, tomenf.

Serrga, of iakk.

Páettro, funich.

Schuoli, grufifch.

Sthóktetcho (witd), Oron (zahm) tunguf. Koja, affanenf.

Pangnck (M.), Kollanak

(W.), Norak (ein Iun. ges), in Gröuland.

IMeffi (im Iften Iahre), Orrya (das männ!. im zten), Whenial (das weibl.), Wubbers (das männl. in 3ten), Wattia (das trächtige), Whivnialrothe (das unbefruchtete), Koddutis (im 4 ten), Kolfitur (im 5ten), $M a$. chanis (im Gten), Nammatappatachis (im 7 ten), lappifch.

5. Dama.

Daino (M.), Damma (W.), italien.

Gamo (M.), Corza (W.), $V$ eniadito (ein Iunges), ¿panifcls.

Corza 
Coræ̧a (W.), Veado (ein Iunges), portagief.

Daim, Dain (M.), Daine (W.), franz.

Hydd (M.), Hyddes (W.) Elain (ein lunges) ${ }_{3}$ cambrifch.

Damhirfch, Tammkirfch, Tannhirfch, Tannbock, Tannwildpret, Tan ngeis, deutfch.

Desall, öfterreich.

Dein, Damhert, holländ. Buck (M.), Fallow-Deer, Doe (W.), engl.

Damhiort. Daa - Dyr, dän.

Dof, Doffiort, fchwed. Daniel, poln.

Serna, ruflifch.

Damuad.bak (M.), Suta. damval (W:), ungar. Kgóyes, hottentott.

6. Capreolus:

Caprizolo (M.), Caprizola (W.), italien.

Zorlito, Cabronzillo mon. tès, fpanifch.

Cabra montès, portugief. Chevreuil (M.), Chevrette. (W.), frauz. Iwerch (M.), Iyrchell(W.), cambr.

Rhee, holländ.
Roe, engl.

Raa, Raa.Dyr, Raa.Buk, - dänifch u. norweg. Radjutr, fchwed. Sarn, Sarna, Koza lesina poln.

Koza dikaja, ruf:

Gittschan, tungur.

Grichun, burät. Selldenus, türk. öz-bak (M.), öz- rö̈ttény. (W.), ungar.

Sáã, hottentott.

Eg (M.), Péngeg (W.), alfanenf:

* Gefchl. Camelopardalisa -

5. Giraffa.

Kemel Pardel, bolländ.

Camello. Pardo, ital.

Zomer, hebr.

Zuraphate, arab.

Deba, chaldäifch.

Seraphah, perf.

Liratakia zin, amhar.

* Gefchlecht. Antilope.

2. Lerwia.

La petite Vache brune, $\mathrm{b}$.

d. Franzofen am Senegal.

3. Rupicapra.

Camorcia, italienifch.

Caminza, Cabra montés. fpan 
Cabra montez, portugiefifch.

Chamois, (obfol.) rfard, Sarris, franz.

Geme, holländ.

Channois englifch. Koza Skalna, dzika, pol. nifch.

Dikaja koza, ruff. Havaji.Ketske, ungar.

\section{Dama.}

Nanguer, Naguer, atn Senegal.

Swift Antelope, engl.

8. Sahiga.

Suhak, poln.

Akim, türk.

Akkiik, tatar.

Margacz' (M.), Saiga (W.), ruft:

9. Gutturofa.

\section{Tzeiran, b. d. Türken?}

DSheren' (M.), Ona (W.), ruff, u. mongolifch?

14. Oryx.

Dabi, Ghazell, in Aegy. pten.

18. Scripta.

Oualofes, Zalofes, b, d. Aethiop.-am Senegall.
30, Gefchlecht. Capra.

B. Hircus.

Becco, Capra, Capretto, italienifch.

Cabron, Cabra, Cabrito, fpanifch.

Cabram, Cabra, Cabrito, portugiel.

Bouc, Cheure, Cheureau, franz.

Bwch, Gafr, Mynn, cambrifch.

Bock, Geit, Bokge, hol. ländifch.

Goat, engl.

$B u k$, Gied, dänifeh.

Buk, Gied, norweg.

Bock, Ged, Kiid, fchwed.

Koziel, poln.

Koza (W.), Kozel (M.), ruff.

Kafäh, tatar.

$K a \int a h h$, tfcheremiff. $K a \dot{j} a$, tfchuvafch.

Gurt-Ketfch, wotjak.

S $\ddot{a} \ddot{a}$, morduan.

Bothi, grufifch.

- Tanian, kalmükk.

Oet fchki, buchar.

Utfchku (M.), Tifchi-:

utfchku (W.), b. d. Tataren am Oby u. Tfchul. Otzkii (M.), T Tchi ötzkii (W.), b. d. tfchatżenf. Tataren.

Boikor, 
Boikon (W.), mongolifch. Ize, chaldäifch.

Gdhies, hebr.

Teayos, griech.

Mlaez, arab.

Anfe, faracen.

Bufan, perf.

2. Ibex.

Capra Selvatica, italien. Bouquetin, franz. (obf. Bouc - eftain.)

Takja, mongol.

Tau - Tökké, kirgif.

Baeden, in Aegypten.

Töghé, Himä, Bitfchin$j a ̈, b . ~ d . ~ B e r g t a t a r e n$.

Vat-kos, ungar.

Steenbock, hollärid.

rb/ch, in der Schweiz.

Kãoud a Schochokdema, hottentott.

31. Gefchlecht. Ovis.

I. Aries.

Montone, Ariete (M.),

Caftrone (ein Hammel), Pecora (W.), Agno, Agnello, Agna (Lamm), italieni.

Carnero, Carnero entero, Carnero cajudo, Morué. co (M.), Carnerio Ca. Arado, (Hammel), Oyle ja (W.), Cordero

(Lamm), fpanifch.

Carneiro (M.), Ovelha

(W.), Cordeiro(Lamm), portugief.

Belier (M.), Brebis (W.),

Mouton (H.), Agneanl

(L.), franz.

Hwrid, Maharen (M.),

Dafad (W.), Oen (L.), cambr.

$\operatorname{Ram}$ (M.), Schaep (W.), $\operatorname{Lam}(\mathrm{L}$.$) , hollä̀d.$

Ram, Scheep, engl.

Faar, Lam, Gimmer. Lam, dänifch.

Soud, Smale, Kaas, norwegifch.

Wadur (M.), Far (W.),

Lamb (L.), fchwed.

Owca, Owieczka, poln.

Baran, Owen (M.), Ow$z a(W)$, Kladenii ba$\operatorname{ran}(\mathrm{H})$, Agnetz, Iar$k a$ (L.), ruff.

Takih (M.), Saràk(W.),

Bäran (L.), tatar.

Tagàh (M.), Schórok (W.), Schorok Parán (L.), tfcheremiff.

Tagàh(M.),Sóroch(W.),

Potjak (L..), thchuwaffifch.

Tackah (M.), Ifch (W.),

If chpi (L.), wotiak. 
Barcin (M.), Bala (W.), $D$ chàla (L.), permäk. Mefch (M.), iifch (W:), Balápi; Baljapi (L.), firjän.

Coara; irrländ.

Goyn (W.), Kuffu (L.), türk.

Kós (M.), Iuh (W.), örit, Berbets (H:), Bárány, (L.), ungarifch.

Guza (M.), Goi (W.), Kojchy (L.), kaimükk. Kotjehkir (M.), Koy (W.), Kofchy (L.), bucharifch.

Kotfchar (M:), Koi (W:), Koofu (L.), b. d. Tatarèn am Oby.

Kotziar, Koi; Koofy, tfchatzenf.

Kor-koi (M.), Koi (W.), Kiiba-koi (L.) b. d. tomenf. Oftiaken.'

Oktfcha-fchivu (M.), Okiv cha (W.), Okt fchakan (L.), tunguf.

$K o i\left(M_{\text {. })}, K o i\left(\mathrm{~W}_{\text {:) }}, K o i\right.\right.$. zen $\left(\mathrm{Li}_{i}\right)$ affanenf. Péffi(M.), Laimmas (W.), Lammas-poika (L.), fin. nifch.

Tcha (M.), Zchwari (W.), Batkami (Li), grufifch.
Konin, mongolifch.

Ghoudie (die ganze Gato tung), Chaunia, (L.) hottentort.

GuJJo (W.), Geman (M.), Ihre ( $\left.\mathrm{H}_{\mathrm{i}}\right)$, burät.

Hrutur (M.), Aa, Aïr (W.), Soydur (H.), is. länd.

Dikerin (M.), Ana (W.), Imar (L.), chaldäifeh. Ail, El (M.), Zon, Zo. neb (W.), Kebes (L.), hebr.

Kabfa(M.), Genas (W:), Elg (L.), arab.

Nerameifich (M.), Go. fpand (W:), Barah (L:) perfifch.

Herman, in der Sch iveiz.

Oweze, Skop, illyr.

Wider (M.), Schaep

(W.), $\operatorname{Lam}($ L.), in Flandern.

Heirth, fchottifch.

Agaroine; in Madagafcar.

2. Ainmon.

Muffore, in Corfica und Sardinien.

Stepnoi Baran, Kamennoi Baran, Dikoi Baran. ruff:

Guldjhal (M.), Argali (W.), mongolifch. 
Kgr.taka , b. d. europäifchen Tataren.

Tfckúbbuku, jakutifch.

Dfholadfchin, lamutifch.

Goâdinacht fch, Goadinzadatfch, kamutfchadal.

Kytyp, korjätifch.

Kullem, Kulehm, ukinzifch. Kotfchkui, Kotfch - kohi, perfifch.

Dachkutfch, türkifch.

Stepnie Barani, in Sibirien.

Sibirian Goat, engl.

Chevre de Levant, franzöfifch.

3. Strepficerosi

Zackl, in Oefterreichifchen:

Magyar - Tuh, ungar.

Cretan Sheep, engl.

\section{Gefchlecht. Bos.}

I. Tauruss

a. Ferus,

a. Urus.

Tur, Wol dziki, poln.

Wild Oxe, dänifch.

Buizwol, Buil Lẽsnyi byk, ruffifch.

Belénd", Vad. Bika, un. garifch. c. Bifon.

Wifent, alt deutfch.

Zubr, poln.

Zimmbers in der Mol. dau.

ß. Domefticus.

Toro (Stier), Bue (Och. fe, Rind), Vacca (Kuh), Vitello (Kalb), italien. Toro (S.), Buey (O.), Vaca (Kh.), Ternera (Kb.), fpan.

Touro (S.), Boy (O.), Vaca (Kh.), Vitela (Kb.), portugief:

Taureau (S.), Boenf (O.), Vache (Kh.), Veau (Kb.), franz.

Tarw (S.), $Y_{c h}$, Eidion (O.), Buwch (Kh.), Llo (Kb.), cambr.

Stier (S.), Os (O.), Koe (K.), Kalf (Kb.), holländifch.

Bull, Steer (S.), Ox(O.), Cow (K.), Haifer (junge Kuh), Calf (Kb.), engl.

Tyr, Oxe (S.), Nid (O.), Koe (K.), dänifch. Tiztr (S.), Nöt (O.), Ko (K.), Kalf (Kb.), fchwedifch.

Wol, poln. 
Byk (S.), Wol (O.), Korowa (K.), Telja (Kb.), ruff.

Tunetz, Teletz (junger Stier), Telitza, Teluesh. $k a$, Innitza(jungeKuh), flavon.

Uggus (S.), $\operatorname{Sir}\left(\mathrm{K}_{.}\right), \boldsymbol{B}_{u \iota \text { - }}$ fau (Kb.), tatar.

iij chkiijsch(S.), iijschkál(K.), Prjefe (Kb.), tfchere. miffifch.

Wukor (S.), Inè (Kh.), Perit, Puriu (Kb.), tchuwaffifch.

$O \int c h(\mathrm{~S}),. 1 / k a l$ (K.), Kinn. gan (Kb.), wotiakifch. Búka (S.), îkal (K.), Wâs (Kb.), morduan.

$M i i j / s(\mathrm{~K}),. K u k a n$ (Kb.), permäkifch.

öfch (S.), Mos, Müfs (K.), Kukan (Kb.), firjän.

Bika (S.), Tehín (K.), Borju (Kb.), ökòr (O.), ungar.

Oeckiis (S.), Onneck (K.), türkifch.

Buká(S.), Sijir(K.), Bufait (Kb.), b. d. tfchatzenf. Tataren.

Tzar(S.), Eme-uker (K.),

Tugàl (Kb.), kalmükk. $U_{i}, U g \hat{i} \int_{s}(\mathrm{~S}),. \operatorname{Injak}\left(\mathrm{K}_{\mathrm{.}}\right)$, IMofó (Kb.), buchar.
Bugd (S.), Syr (K.), Buifui (Kb.), bey den Tataren am Oby und Tfchul.

Kor-Sir (S.), Sir (K.), Kïba - Sir (Kb.), b. d. tomenf. Oftiaken.

Nökdil-Schiru (S.), Nökdil (K.), Nökdil-itkon $(\mathrm{Kb}$.$) , tungufifch.$

Schar (S.), Tyg (K.), Tygwu (Kb.), affanenf. Härkä (s.), Lämä (K.), Wáfika (Kb.), finnifch. Hukur, mongol.

Durié-fa, Bubaa' (S.), Gojes (K.), Thona, Non$n a(\mathrm{~Kb}$.$) , hottentott.$ Bucha (S.), Uneng (K.), Irekir (O.), burätifich. Naut, isländ.

Chali (S.), Puri (K.), Ohbo (Kb.), grufifch.

4. Grunniens.

Sarlıı, kalmükk.

33. Gefchiecht. Equuts.

I. Caballus.

Cavallo (Hengft), Caval. la (Stute), Cavallo cafrato (Wallach), Polledro (Füllen), italicnifck.

Cavallo. 
Cavallo, Caballo (H.), Tegua (St.), Caballo caAtrado (W.), Potranka (dreyjähriges F.), Potro (vierjährig. Füllen), fpanifch.

Cavallo (H.), Egoa (St.), Cavallo caftrado (W.), Faca, Faquinha (F.), portugief.

Cheval (H.), Cavale, $\mathcal{O}_{\mathrm{u}}$. ment (St.), Cheval ongre (W.), Poulain (F.), franz.

March Ceffyl (H.), Cafeg (St.), Difpaiddfarich (W.), canubrifch.

Paardt, Paerdt (die gan. ze Gatt.), Heng $f_{t_{1}}$ (H.), $\operatorname{Mere}(\mathrm{St}$ ), holland.

Horfe (H.), Maré (St.), Gelding(W.), Colt (F.), englifeh.

$H e f t$ (die ganze Gattung), Hing $/ \mathrm{t}$ (H.), Hoppe $(S t$.$) , dänifch und nor-$ - vwegifch.

Hiaft, fchwed.

Kón, poln.

Kon' Lofchad (die ganze Gatt.), Sherebetz (H.), Kobyla (St:), Wieren, Wolosi, kladenoi kon" (W.), Scherebersok (F), ruff.
Alafehidh (dieganze Gatt.), Aiger (H.), Baitad, Bia (St.), Tini (F.), tatar. Immä̈ (die ganze Gattưng), ôfcha (H.), Wiillä (St.), Alafchih (W.), Tychat ma (F), tfcheremiff: $U t$ (die gauze Gatt.), Ir $j a$ (H.), Kfrjoih (St.), Tichäh (F.), tchiuwafch. Wal (die ganze Gattung), Ulofchò (H.), iurval (St.), TJchuni (F.), wotjakifch.

Ujchmäh (die ganze Gattung), Aiger (H.), Elda (St.), Parna, Wá fcinina (F.), morduar. Wiill (die ganze Gatt.), Tfchan (F.), permäkifch.

Wöll (die ganze Gatt.), Wöll (H.), Kobiilla(St.), Ujch (W.), Tfchan, (F.), firjärnifch.

Gaul (die ganze Gattung), Göule (diminutiv.), Han. kele, Heinzel (E.), um Nürnberg.

Adt, tïrkicch.

At (die ganze Gattung), Aigy'r (H.), Att (W.), $B_{z i}$ (Dt.), Krikin (T). bey den Tataren am Oby.u. Trchul. 
Iilkd (die ganze Gatt.), Bija (St.), Alafchà (W.), bey den tfchatzenf. Tataren.

Erè (die ganze Gattung), Erè-mórin (H.), Atziargà (W.), Gii'n (St.), Unagàn (₹.), kalmuikk. Lo (die ganze Gattung), Men-ló, Tjödör (H.), Paripa, Herélt - ló (W.), Kanza, Kabala-ló (St.), Tjkó, Venshe (F.), un. garifch.

At (die ganze Gatung), Aigir (H.), Erkek-at (W.), Bijà Bija (St.), Kulinn, Kuhunt - Schak (F.), bucharifch.

Künade (die ganze Gatt.), Kor-kinde (H.), Koptè (W.), Sirum (St.), Kúnding (F.), bey den to. menf. Oltiaken.

Miürin (die ganze Gatt.), Mnirin - Schiva (H.), Aktaki (W.), Njámi (St.), Minurintfchikan (F:), tunguffich.

Hufch (die ganze Gatt.), Ajkir (H.), Athich (W.), Pengufch (St.) Kuhiz (F.), aflanenf.

Héwoinenn (die ganze Gattung), Ori (H.), Ruma
(W.), Tómma, Märä (St.), War $\int a$ (F.), finnifch.

Zcheni (die ganze Gatt.), Lapfcha (W.), Adfchil$\mathrm{ga}(\mathrm{H})$, Tfchiki-tsgeni (St.), Kwizi (F.), grufifch.

Aferga (H.), Gu (St.), burätifch.

Hacqua (die ganze Gatt.), hottentott.

Sus, Sufah, hebr.

Suf fuatha, chaldaiifch:

Baiel, arabifch.

Asbecha, perf.

Kobyla, illyr.

'I Tros, griech.

2. Afinus.

a. Ferus.

Onagr, ruff.

Kulan, kirgif. Kuhh, perf.

B. Domefticus.

Afno IViccio (Männchen), Miccia (Weibchen), italienifch.

Afno, Borrico (M.), Bor rica (W.), fpanifch.

Burro (M.), Afna Bur$r a(W$.$) portugief.$

Ane (M.), Anefle (W.), franz. 
'Afyn (M.), Afen (W.), Beal, arabifch. cambrifch.

Eezel, Ezel, holländ.

$A \int s$, engl.

Afna, fchwed.

Ofel (M.), Oslitza(W.), ruff.

Mezeck, illyr.

'O

Mulus, Mulla, latein.

Ofzver, ungarifch.

3. Zebra.

Szamár (M.), SzamarKantza (W.), ungar.

Afal, irrländ.

Efcheck, türkifch.

Quciha, Ay, hottentottifch.

Chamor, hebr.

'Ovos, griech.

y. Mulus.

Mula, italien:

Zebra, Azebro, in Congo.

Burro do Matto, portu: giefifch.

Zeura, Zecora, in Abyffinien.

Zebre, Ane rayé, franz.

Efore, in Angola. (nach

Pyrard.)

Zeuera, Sebra, Benennun.gen in Congo.

IMulo, Mula, Macho, Azemiila, (d. i. Lafthier) fpanifch.

$\operatorname{Mu}$ (M.), Mula (W.), portugief.

Mutet, Mule, franz.

Mul (M.), Mules (W.), cambrifch.

Muuyl, Muylezel, holländ. Miule, engl.

inulaasna, fchwed.

Lafchak, Mul', Mefk, ruffifch.

Käter, türkifch.

Pered(M.), Pirdah(W.), hebr.

Cudana, chaldäifch.

34. Gefchlecht. Hippopo.

tamus.

I. Amphibius.

Foras lo bar, Abn mmer, in Aegypten.

Bihât, äthiopifch.

Gomari, anharifch.

Morfkaja tofchad', ruf. fifch?

Rivier-Paard, holländ.

River hor $s$, engl.

Hippopotane, Cheval ma rin, franz.

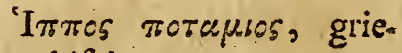
chifch.

Ippopotamo, ital.

Ggg 2

* Ge 
* Gefchlecht. Tapir.

I. Americanus.

Anta, portugiefifch. Maipuri, Manipuri, in Guiana.

Tapiira, in Brafilien.

Uagra, in Pert.

Tapiva, (nach dela Condamine),

Tapiierété, (nach Marcgr. und Pifo),

Topihire, (nach Thevet), Tapiroulfout, (nach de Lery), - in Brafilien.

Ent, (nach Rennefort), in Paraguay.

Danta, (uach de la Condamine, und d' Acunna), in Spanien u. Yurtugall.

Ante, (nach Herrera), Beori, in Neufpanien.

35. Gefchlecht. Siss.

1. Scrofa.

a. Ferus.

Puevro, Coríino (M.), Puerca, Cochina (W.), fpanifch. (die ganze Gattung.)

$p_{\text {ubrco montés, }} P_{\text {uleveco }}$ flueftre, Tabali, Tavali, Xavali (Haner), Iabali- na, Xavalina (Bache),

Lechon de Iabali (Frifchling), fpanifch.

Porco (M.), Porca (W.), portugiefifch. (die gan. ze Gattung.)

Porco montes, Iabali; Xavali (H.), Leitcío de porco montez (F.), portugiefifch.

Porco, italien. (die ganze Gattnng.)

Porco filvatico, Cinghiale (H.), italien.

Porc, Cochon, franzöfifch. (die ganze Gattung.)

Sangitier (H.), Laye (B.), $\operatorname{Marcafjaz}\left(\mathrm{F}_{\text {. }}\right)$, framzöfifch.

Zwinn, Vark̉en, holländifch. (die ganze Gattung.)

Wild Zieyn (H.), holländifch.

Hog, englifch, (die ganze Gatt.)

Wild Boor (H.), engl.

Sivin, fchwed. (die ganze Gatt.)

Will-Swin (H.), fchwedifch.

Sviin, dänifch, (die gar ze Gatt.)

Wiepra, polnifch, (die ganze Gatt.) 
Wieprz les'my, Dzik(H.), Leytría, Leitúo, Marpolinifch.

Sivinija, Porosja, ruft. (die ganze Gattung.)

IV epr, Dikaja, Sivin'ja, Dikii borov (H.), ruffifch.

Dontus, türk. (die ganze Gattung.)

Kinur (H.), in der Ukraine.

Tikalanda, tungufifch.

Chagni, Chagai, burätifch.

Vad-kan (H.), Vaddifz. mò, Vadeine (B); ứgarifch.

Chajer, hebr.

Chajirat, chaldäifch.

Kanifir, arab.

Mar, Buk, perf.

Werprz, illyr.

3. Domefticus.

Barraco, Berraco, Verruto, Cerdo (Eber, Kempe), Puerca, Cochino (Sau, Mutterfchwein), Cochinillo (Ferken), Lechòn, Lechoncillo, Lechomsito (Sog ferken, spanferkel), fpanifch.

Porco Barráo (E.), Porca (S.), Porquinho, Ba. coro, Bacoriztio (F.),

río (Sp.), portugief.

Verrat (F.), Thatye (S.),

Cochond de lait (F.), franzöfifch.

Verro (E.), Porca, Scrofa (S.), italien.

Batdd (E.), Hwch (S.), Mochyn (Bork), canibr. Beer (E.), Zeng (S.), Big, Speenuarteri (F.), holländ.

Boar (E.), Sow (S.), Pig (F.), eng!.

Rone, Orne (E.), Soe Privke (S.), Griis (F.), dänifch.

Swin, fchwed.

Wieprz, poln.

Borov (E.), Swin'ja (S.), Kaban (Bork), Porofenok (F.), ruff.

Kan, Artcinti (E.), Eme, Kotza, Gönye (S.), Malatz (F.), ungar.

Dongus (das crwachle-" ne), Dongus - ballas (F.), tatar.

Sisscih (erwachfen), Sis. sua-iga (tcheremillifch u. tfchurwaflifch.

Parfs (erwachfen), Pdr. ' pi (F.), wotiakifci. Tida(erwachfen), Pítrfos Tüläux (F.), mordwan. Ggg 3 Hakou 
Hakow (die ganze Gatt.), hottentott.

Gagai (erwachfen), Gagain-dfchuld-fagà (F.), kalmuikk.

Tongiis, Chyrss (erwachfen), Tongusuy, Balas$\int u(F$.$) , bucharifch.$

Sjika (erwachfen), porJas (F.), finnifch. Gotfchi (die ganze Gatt.), grufich.

\section{Tajaflu.}

Paquiras, in Amerika.

Tajajju, in Brafilien, (nach de Lery.)

Tajacu, (nach Marcgr. u. Pifo.)

Canigoris, (nach Marc(grav.)

Tajouffori, (nach dem Coreal.)

Qumathla coymatl, Quapizotl, Cayonatl, bey den Mexikanern.

Pelas, in der Bay aller Heiligen, (nach Dam. pier.)

Iovari, Paquire, auf der Infel Tabago, (nach Ro. chefort.)

Pcoquirä, im Amazonen. lande, (nach Gumilla.) Saino, Zaino, in den meh. reften Ländern von Ame. rika. (nach Acofta, )

Charinie, (nach Oviedo.)

Cojcui, (nach Coreal.)

4. Aethiopicus.

Hardlooper, bey den Holländern am Vorgeb. d. g. Hoifn.?

5. Babyrufia.

Babironffa, Babiroefa, in Oftindien.

Babi-rifa, malayifch.

37. Gefchlecht. IIIonodon.

I. Monoceros.

Narhual, Lighval, norwegifch. Narhval, iṣländifch. Tauvar, Killelluak, kermektok, tugalik, in Grö»land.

38. Gefchlecht. Balaena.

I. Myfticetus.

Wallvifch, holländ.

Whale, Greenlands Wha$l e$, engl.

Hualfijk, Sletbak, Gsörzlandfk Hval, norweg.

Grönlands . Hualfisk, fchíwedifch. 
Vatushalr, isländifch: Arbeik, Arbavirkfoak, grönländ.

Inngi, luni, koriät. Tkaka, hottentott.

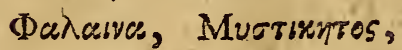
griech. ?

\section{B. Islandica.}

Sifh Q Qual, Lille - Hual, norweg.

2. Phyfalus.

Vinvisch, holländ.

Fin- fifh, engl.

Rör-Hual, Finne- Fijk, Stor-Hual, norweg.

Finn-fjk, fchwed.

Reider, lappifch.

Hunfubakr, isländ.

Tunnulik, Kepokak, Kepokarfoak, grönländ.

3. Boops.

Hrafi, Reydur, isländ. Keporkak, grönländ. .

5. Gibbofa.

a. Der Knotenfifch.

Knobbelvijch, Knabbelvifch, holländ.

Scrag - Whale, engl.

B. Der Pflockfích.

Penvif $c_{\hbar}$, holländ.
Bunch Humback - Wha: le, engl.

39. Gef.chlecht. Phyfoter.

I. Catodon.

Sviub- Huat, norweg.

Kigutilik, grönländifch?

2. Macrocephalus.

Trold-Hval, Hins. Hval, Sue-Hual, Buur.Hval, Bardhualir, norveg. Cachelot, Cachalat, franzöfifch.

Potvifch, Kaizilot , hol- " ländifch.

3. Microps,

Staur - Hyning, KobbeHerre, norweg.

Tikagulik, grönländ.

4. Turfio.

Maftuifch, holländ. Perrnak, grönländ.

\section{Gefchlecht. Delphinus.}

ז. Phocaena.

Marropa, fpan. Mitarfain, franz.

Brruinvifch, holländ. Porpus, Porpes, Porpafo Se, Porpoiffe, engl, Ggg 4 Marracosons 
- Marfowin, Tuwtier, dä. Huyfent, Heyjen, Hojnifich.

$N i \int e$, norweg.

rung, Leipter, islänMarfioin, Tumblare, Delfun, poln. fchwed.

Sreinia morfka; poln.

3. Orca.

Morkkaja, Swinja, ruí- a. Der Butzkopf.

fifcl.

Nija, grönländ.

2. Delphis.

Delfino, italien.

Durzplizin, franz.

Dolihyng, holländ.

Dolphin, Grampus, Por. pefe, engl.

Marfvin, dänifch.

Springer, norweg.

Botskop, holländ.

Grampus, engl.

North-Caper, fchottifch.

Hvyding, isländ.

Loppare, fchwed. Kojatiy, ruffich.

B. Der Sägedelphin.

Faian, lappifch. Arlo, Arlorfoit, Tiko gulik, grönländifch. 


\section{Regifter}

nach den seitenzalen.

$\mathrm{A}_{\text {a } 830}$ Abada 796 . Abalak 822. Abendwolf $187^{\circ}$ Abarghan 821. Abfetzferkel 74r. Ablinges 803 . Abspiuler 333 . Aboujer 749 . Abulhösni 803. Abu muer 835 . Achar 817. Achera 817. Ai 86. 87. 88. 89. Acanthion 383 . Achtziger 162. Achao 795. Ackermaus 440. 454. Acuti'405. Adarnefia 772. Adelhiort 825 . Adfchilga 834. Adibe 803 . Adie 826. Adil I 91 . Adimain 668. Adimari 668. Adive 190.
Adt 833 .

Aekich 250.

Aeneas 351 .

Aegagrus 647.

Aelg 825 .

Aér 830 .

Aefte 801.

Acthiops, Simia 30.

Aff 14 .

Affe 9 .

- der langarmige 13 .

- indianifche langarmige 13.

- gemcine 13. 14.

- der Alten 14.

- gemeine türkifche 14. 15\%

- init der Schweinsfchnauze 20.

- grofse brandfarbige 24.

- angolifche 25.

- der grïne 28.

- St. Jigo 29.

- blaunäulige 29.

- weifsäugige 30.

- weifsmäulige $3 \mathrm{r}$.

- der nickende $3 \mathbf{I}$.

- der rothe 33.

- fchwarznafige 35.

- weirsnafige 35 .

- kleiner ceylonifeher 35.

- der vierfingerige 38. Ggg 5 


\section{, Regifter}

Affe mit dem Fushsfchwanze Agnetz 829 .

43.

- langgefchwänzte von Para 47.

- der fehwarze 48. 52.

- mit grofsen Hundszähnen 49 .

— mit Flügelähnlichem Barte 49.

- der braungelbe 49 .

- grofse ungefchwänzte von benin 50.

- mit dem Ziegenbarte 50.

- mit geringeltem Schwanze 5०.

- der grofse von Malaeca 50.

- zist gelb und fchwarz gefprenil eltem Rücken 5 $\mathrm{x}$ :

Aguti 404 .

Ahergranraik 749.

Ahpicha 812.

Ahu 585.628. -

Aiger 833 .

Aigy'r 83.3 .

Ajal 826.

Ajela 826.

Ajik 812 .

Ail 830.

A.doupos 233.

Aju 8 rr.

Aizzo 814.

Akarima 794.

Akkiik 828.

Akkik 822 .

Aklemi 824 .

- der langgeftreckte röthliche 52 .

- ceylonifcher, mit einer Hafenfeharte 53.

Akoufchy 404 .

Akomma 797.

Akoim 828.

Akpa 809.

Aktaki 834.

Akuchi 404 .

Alactacha 528.

Alakdaga $62 \%$.

Alavata 794.

Albos 807 .

Alces 586.

Alg 798 .

Alopehenra 413 .

Alpaca 576 .

Altvater 22.

Aluate 38 .

Alpenhafe 558.

Alpenmurmelthier $47^{6}$.

Alpenmaus $477^{\circ}$.

Alpenratze 477.

Alafchah $833^{\circ}$

Algazel 638 .

Alikantifche Hund 162 .

Alko 173.

Alopex 199.

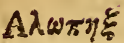




\section{nach den Sitenzahlen.}

$\mathrm{A} \lambda \omega \pi \eta \xi 198$.

Altfchangfeh 825 .

Alungu 100 .

Amboinefer 353 .

Amber 777 .

Ambrafích 777 .

Ameifenbär 319.

Amerikaner 4 .

Ameifenfreffer

- - der mexicanifche 259.

Ameifenjäger 94.

Ameifenbär 91. 97.

- - dritte Art 92.

- - grofse 93.94.

- - langhaarige 94.

- - erfte Art 95.

- - zwote Art 97.

- gepanzerte 104.

Ameifenfreffer $9 \mathrm{I}$.

- - der kleine 91. 93.

- - der zweyfingerige $9 \mathrm{I}$.

- - weifse americanifche 92.

- - zwozehige 92.

- - dreyfingerige 93.

- - brafilianifehe 93.

- - grösfte 94 .

- - mittlere 96 .

- - vierzehige 96.

- - kleine amerikani. fche 69.

- - afrikanifche 98.

Ameifenfchlucker 92 ,

Amikan 812.

Amfter 464 .

Ammion 678.

Ana 830.

Anarnak 760 .

Anei 796.

Ane 834 .

Ane rayé 835 .

Aneffe 834.

Angelmaus 376 .
Angel - muns 814.

Angorifche Hund 158.

Animal paradoxon 518.

Animal anonyme 20\%

Anfeb 795 .

Anfe 820.

Annoa 705 .

Anta 733 .

Ante 836 .

Antilope 6r8. 644. 645.

- - blau graue 6rg.

- - blaue 619.

- lichtgraue 6rg.

- - mit vorwärts geboge.

nen Haken 624.

- - weiffriifsige 625 .

- - feytifche 626 .

- - bunte 640.

- - angefchirrte, bemahlte

640.

- - grimmifche $64 \mathrm{I}$.

- - fchlangenhörnige 644 .

- - bengalifche 645 .

Apanigehak 224.

Apar 105. 106.

Apara 608.

Apedia. Simia 17.

Apella. Simia 40.

Aperea 408.

Aperkos 409.

Aquiqui 36.37 .

Arabata 37. 794.!

Arap 822.

Araneus 375 .

Arbavirkfoak 839:

Arbek 839 .

Arêtic - fox 804.

Arctomys 476 .

Arćtos 316.

Arda 820.

Ardilla 820.

Argali 678 .

Arganäz 82I. 


\section{$R \in g i, f \circ r$}

Argentata. Sim. 47.

Arjah 804.

Arich 804.

Arjo 804.

Armodillus ror.

Arnah 822.

Aries 660. .

Ariete 829.

Arkal 679.

A purog 323.

Arlo 840 .

Armadill 98. 102 .

- - afrikanifche $102,103$.

- - eingürtelige 103.

- 2wölfgürtelige 102.

- - achtzehngürtelige 104.

- mit drey Gürteln 105.

- orientalifche ro5.

- - mit vier Gürteln 106.

- - rechsgürtelige $10 \%$.

- - nit dem Halsfchilde

107.

- - mit fechs geflammen Gïrteln 108.

- fiebengürtelige 108.

Armelini $30 \%$.

Armellino 810.

Arminho 810.

Arminno 810.

Artoififche Hund 162 .

Afal 835.

Aferga 834.

Asbecha 834.

Alhaari 565 .

Afen 835 .

Afino 834 .

Afinus 717 .

Afiate 5 .

Afkir 834.

Ama 835 .

$x$ fpolax 471 .

Af5 $72 \% .835$.
Affaponik 5I1.821.

Afna 834 .

As - i - fchufchi 816 .

Aftrachanenfis, mus $457^{\circ}$

Afyn 835 .

Ataleph 795 .

Atalepha 795:

Att 833 .

Attarfoak 143.

Attijhro 812.

Atuco I I I. 296.

Atzargà 834.

Auak 797.

Auerochs 684. 697.

Auritus. Vefp. 70 .

Avee 797.

Avratchka 819.

Axis 607 .

Ayal 826 .

Aychhorn 503 .

Aygula. Simia 30.

Ay 835.

Aye. Aye 510.

Azebro 728.

Azed 804 .

Azemila 835 .

B.

Babarchan 82 r.

Babian 793.

Babiruffa 752 .

Babiroefa 752 .

Babirous 753 .

Baboon 18. 793.

Babr 80 ;.

Babyruffa 752 .

Bache 736 .

Bacorintio 837.

Bacoro 837.

Baetrianifche Kameel $56 \%$.

Badgei 8r2. 


\section{nach den Seitsniahlen.}

Badiger 807 .

Baedd 837.

Baeden 820.

Baesver 816 .

Bagbagai 794.

Bär 316.

- der weifse 318.324 .

- fchwarze 316.326.

- amerikanifche 334.326.

- kleiner ohne Mund 92.

Bärenbeifser 162 .

Bärenhund 162.

Bärenrobbe 133 .

Bärnenf cli 321 .

Baibak 482. 819 .

Bajeu 609.

Baital 833 .

Bakfcha 820.

Bak - matska $\$ 06$.

Bala 830.

Balaena 760.

Ralapi 830.

Baljapi 830 .

Ballutula 811 .

Banis 81 I.

Bambuc 482.

Bandeishhorn 509.

Baphas 795.

Barah 830.

Baran 829 .

Barbara (Muft.) 286.

Barbaresque 503.

Barbet I 56.800 .

Barbaftellus 77.

Rarbone 800 .

Bardhualir 839.

Bars 805.

Bartaffe, der weifse 22. 23.

- - der fchvarze 23.

Bartfirch $7 \sigma_{2}$.

Bartfledermaus 78 .

Bartkragen 56.

Bartmännchen 24
Bartmännerchen' 24.

Baftardinops 160. 16r.

Barany 830.

Barraco 837.

Barris 10.

Barfuk 329.

Baffet 801 .

Bafluk 812 .

Bauernhund 153.

Bauerpferd $7 \mathbf{1 2}$.

Bauerreckel 17 I.

Baumreuter 234.

Baumratze 438 .

Bavian 17. 18. 21.

Beal 835 .

Bean - moufe 817 .

Beagle $8 \mathrm{c} 0$.

Bear 811 .

Beaver 816 .

Bebris 816 .

Berker-el Wafh 634 .

Beelzehub 38.36 .

Beer 81 1.83 ?.

Behemoth 730.732,

Bei 833 .

Bejà 834 .

Piela graig 809 .

Belagoed 809.

Belbus 802.

Belette 8Ir.

Belénd' 83 I.

Belier 829 .

Belluae 706.

Belaga 789

Beori 836 .

Berbe 808.

Berbets 830.

Bergdachs 478.

Berggraber 558 .

Berghafe 537. 544. 545. 558 .

Berghirfch 597.

Uergkatze 226.

Bergkuh 734. 


\section{$R \in g i f t \in$}

Bergmaus 459. 530.

Bergochs 684 .

Bergratze 477 . 521. 530.532.

Bergziegen, wilde 647 .

Berraco $837^{\circ}$

Befangerah 822 .

Beftia 734.

Beftie 589.

Betze 150.

Befs 322 .

Beurelfledermaus 8r.

Beutelträger 81.82 .

Beutelratze $34 \mathrm{I}$.

Beutelthier $34 \mathrm{I}$.

- mausähnliches 350

- furinamifches $35 \mathrm{I}$.

- kurzfchwänzigte 353 .

Revero 816.

Bever 8 I6.

Bezoarantilope $\dot{\sigma}_{3} 6.645$.

Bezoarbock 338. 636.645 .

Bezoargazelle 636 .

Bezoarziege 647.

Biä 833 .

Biber 414 .

- kamtfchatkifche 278 .

Biberratze 370. 424.

Biche 825 .

Bichon 800 .

Bjelka 8ะ0.

Bjeluga 789.

Bienenbär 320.

Biefambiber 424 .

Biefanthier $577^{\circ}$

Biefamkatze 265.271 .272 .

Biefamratte 371 .:

Biefamratze 422.425 .

Big 837.

Biggel 625 .

Bihat 835 .

Bijà 834 .

Bika 832.

Bilchmaurs $484^{\circ}$.
Bilgmaus 484.

Billich 519.

Bings $8 \mathrm{I}$ r.

Biörn 8II.

Birkfuchs 194.

Birkmaus 443.

Bifamaffe 43 .

Bifamhirfch 578 .

Bifamreh 578 .

Bifambiber 37 .

Bifamthier $577^{\circ}$

Bifamziege 578

Bifammaus 376 .

Bifamochs 697. 698.

Bifamfchwein 745 .

Bifcafcha 537.

Bifon 687.

Bitfchinjä 829.

Bivaro 812. 816 .

Black Moofe - deer 825 .

Blafengazelle 633 .

Blaireau 812.

Blärsmaulwurf $47 \mathrm{I}$.

Blesmoll 470 .

Blindmaus $47 \mathrm{r}$.

Blind - moufe 814 .

Blodhund 800 .

Blood-hound 800 :

Blutfauger $60.63 \cdot 64 \cdot 85$.

Bobac 481 .

Bobontoll 82r.

Bobr 816.

Boccamele 315 .

Bocht 824 .

jock 648 .

- der blaue 6rg.

- himmelblaue afrikanifche 619.

- hochgehörnte kretifche 625.

- gefcheckte afrikanifche 633.

- geflechte 633 . 
Bock, weifsgeftreifte, rund- Brachyura (Didelph.) 353. hörnige 636 .

- bunte, bemahlte 640.

- wilder 647 .

- zahmer 648 .

- von Iuda 655 .

- orientalifche 679 .

Bockhirfch 625 .

Bocklamm 660 .

Bockskameel 625.

Bock-Cameel 625 .

Boëdfoi 826 .

Boeuf 831.

Böcklein, furinamifches 584 .

Bör 80 r.

Börgen 740 .

Bog $83 \mathrm{r}$.

Boggo 50.

Bogio 793.

Bogu 825.

Boijuko 80r.

Boik on 829.

Bokge 828 .

Bolle 688 .

Bolognefer 157. 158. 159.

Bonafus 685 .

Boops 767 .

Borica 834 .

Bork 740.

Borrico 834.

Borov 837.

Borfuk 812.

Bortl 802.

Borz 812.

Bos 682 .

Bosbock 643.

Bofchratte 344 .

Boshond 265.

Bothi 828.

Bouc 828 .

Bouc - eftain 829 .

Bouquetin 829.

Bracco 800.

Bradypus 86.

Bräcke 150.

Brandfox 803.

Brandfuchs r 99.

Brand - räf 803 .

Brandhirfels 596.

Brandmaus 440.

Braque 800 .

Braunfifch 78 r.

Braunfrett 258.

Braunfuchs 199.

Brebis 829.

Bredis 825 .

Breitmaul 770.

Brillnafe 66 .

Brock 812.

Bronwen 811 .

Bruinvifeh 839.

Bruchhafe 537 .

Brüllaffe, der fchwarze 36.

- - der rothe 37.

Brummochfe 698.

Brunii (Didelph.) 354 .

Bruta 86.

Bfcha 819.

Bfchamabfchi 82 r.

Bubaa 832.

Bubal 633 .

Bubalis 633 .

Pubalus 633 .

Bucha 832 .

Buchmarder 289. 291.

Buck 827 .

Buckelochs 687. 691. 697.

Buckelrücken 766 .

Budek 824.

Budel 155.157.

Budfchim-Schin 816 .

Bue 831.

Bueg 83r.

Büffel 699. 703 .

- grofse indianifche 691 . 


\section{$R \in g i f e r$}

Büfelel, klcine indianifhe 692. Bufchflisanzafe 39.

- tibetanifiche 698.

- mit den Pferdefchweif 699.

- afrikanifche 702.

Büffelochle 700.

Büffelsköpfe 701 .

Büffelsfchädel 701 .

Büre 802.

Bürju 802.

Bufalo 701 .

Buga 826. 832.

Bugio 793.

Bugio rabudo 793.

Bugu 826.

Buiel 834.

Buil Lesnvi byk 831 .

Buiwol 831.

Buka 832.

Bula 80g.

Bulan $825 \cdot 826$.

Bulgàn 809 . .

Bull $83 r$.

Bull - Dog 800.

Bulldiog 800 .

Bulle 683.

Bullenbeifser 162 .

Buntfiugel 78. 79 .

Buntfing 298.

Burdüfch 816 .

Burra 834.

Burro 834.

Burro do Matto 835.

Burunduk 820 .

Bufan 829.

Burchbock 643.

Bufchgott 15 .

Bufchhund $20 \mathrm{r}$.

Bufchmarder 29x.

Bufthinenfih 12.

Bufchratte 349.351 . 408 .

Bufchratze 350.
Butivil 854.

Burua 832:

Buttkopf 786 .

Butzk opf 786 .

Buwrhual 839 ,

Buwch 831 .

Bwch 828 .

Byk 832 .

C.

Caa 41 .

Caainora 838.

Caaigoara 748 .

Caballus 707 .

Caballo 843 .

Cabardyn 580 .

Cabra 828.

Cabra montez,828.

Cabram 827 .

Cabra montés 827.

Cabron 828.

Cabiai 412. 413 .

Cabrito 827 .

Cabrouzillo montés 827 .

Cachelot $773 \cdot 775 \cdot 776$.

Cachorro 800.

Cachorro do mato 813.

Cagnuolo 800.

Cagnuclino 800 .

Cagua - cuete 608 .

Cagnacuapara 608.

Cagui 43.45 .

Cahticuma 749:

Caji 4 r.

Caitaia 45.

Calf 83 I.

Callithrix 43 .

Cam 798 .

Cama 735.

Chameau 824. 


\section{nach den Seitenzahlen.}

Camehle 565.

Cameel 824.

Camello-Pardo 827.

Camelus 562.

Camelopardalis 615.

Cameelpardel 616 .

Camello 824 .

Camorcia 827.

Campagniolo 818.

Campagnol 455.

Canuza 827.

Can-can 808.

Cane 798.

Cancrivora 352.

Can d'armi 800.

Canguruo 355.

Canna 639.

Cannickel 551 .

Caninchen 550. $56 \mathrm{r}$.

Canis 149.

Can di Paftori 799.

Can - maftino 801 .

Capibara 412.

Capiiguara 413 .

Capivara 413 .

Capra felvatica 829.

Capra 646.

Cap - Hafe 554:

Capkatze 240.

Capretto 827. 828 .

Capriuolo 827.

Capricornus 656.657.

Capfohe Szavie 560.

Capuziner 40.

Capybara 412.

Caracal 242.

Carnero de la tierra 824.

Caraco 426.

Carcand 796.

Carcadden 796.

Caregueibeja 279.

Caribou 603.

Carigue 348.
Carigueia 347.

Carigueya 813.

Carw 828.

Carygueibeiu 279.

Carlum 8rI.

Carnero 829.

- entero 829.

- cajudo 829.

- caftrado 829.

Carneiro 820 .

Cartheuferkatze 240.

Cafeg 833.

Cafcui 838.

Caftor 4I 4 .

Caftore 816.

Caftrone 829.

Cat 806.

Catta 57.

Cath - goed 805.

Catodon 773.

Catus 232.

Caudivolvula 272 .

Cavalla 842.

Cavallo 842. 843 .

Cavale 843.

Cavia 401. 407. 414.

Cay 4I.

Cayoinatl 838.

Cayopollin 348 .

Cebra 727.

Cebus 18. 36. 46 .

Cebus Simiolus 3.5 .

Cebus tertius Guineenfis 2Z?

Ceffyl 833.

Ceilonifche Armadill 98.

Ceinach 822.

Cenalfa 796.

Cephalotes Vefp. 8I.

Cercopithecus 21. If, 27.

- Darbatus 24. 28.

- Faunus 27.

Cerda 207.

Cerf 825.

$\mathrm{Hbh}$

Cerva 


\section{$R \in g$ if $\in$ ?}

Cerva 825.

Cervicapra 644 .

Cervia 825 .

Cervio 825.

Cervo 825.

Cervus 585.

Cete 755 .

Chàa ja 825.

Chacal r9I.

Chacalthier Igr.

Châ - khoư $797^{\circ}$

Chaleu 808.

Chalguna 8I ?.

Chali 832 .

Chaly 816 .

Chamma 804 .

Chameau 566.

Chamois 828 .

Chamor 835 .

Chanus 221.

Chandagai 825 .

Charagorefín - Etegò 812.

- Chartis 796.

Chat 806 .

Chat fauvage 805 .

Chatal 802 .

Chauna 830.

Chaus 242.

Chauve Souris 794.

Chechul 8I4.

Che de chusa 796.

Cheval 843.

Chevre de Levant 831 .

Chevre 827.

Chevreau 827.

Cherrette 827 .

Chevreuil $609.82 \%$.

Chian. Kiupp 795 .

Chical 191. 802 .

Chichico 48 :

Chieche 263 .

Chien 798.

Chien der Berger $799^{\circ}$
Chien courant 800 .

Chien-loup 799.

Chien -marin 797.

Chien - ture 801.

Chien volant 62 .

Chilla 199.

Chilihueque 574 .

Chimpanfe IO.

Chimpanzee 10.

Chinche 262.275.

Chinchi 263.

Chinchilla 454.

Chine 253.

Chinefer Müzze 31 .

Chingha 263.

Chiri 807.

Chirofo 475 .

Choa 796.

Choáä 806.

Choakauma 793.

Choi .ja 812.

Choin - Jalma 822 .

Choiropithecos 20 .

Cholda 813.

Chologona 475 .

Chomak 18.

Chomik 818.

Choras 18. 19.

Choriok 810 .

Chorok 810.

Chors 812.

Choa kanmma 793.

Chuchie 838 .

Chunk 262 .

Chwiften 814.

Chym 809.

Chymchymcha 809 .

Chyres 838.

Chyyta 802.

Ci 798 .

Ciaro 820.

Cierva 825.

Ciervo 825. 
Cinghiale 836 .

Cirquinchum 104.

Cirquincon IO4.

Citellus 483 .

Civette 255.

Coara 830 .

Coale 258.

Coaci 356.333 .

Coati mondi $256.257^{\circ}$

Corita 39.

Coaito 33.

Coatithier 333.

- - das brafilifche 402 .

Cobaya 400.551 .

Cochina 836 .

Cochino 836 .

Cochino $\mathrm{d}^{5}$ India 815

Cochon 836 .

Cochon de bois 747.

Cochon de lait 837.

Cuchon d' Inde 815 .

Coesdoes 644.

Coinin 323 .

Cojumero 797.

Coelho 823.

Coticoes 354 .

Colt 833 .

Comadreia 8it.

Concolor 228.

Condoma 644 .

Coneguelo 823 .

Coneja 823 .

Conejillo 823 .

Conejito 823 .

Conejo 823 .

Conepatl 26r. 262.

Conejos 550 .

Conigion 823 .

Connil 823 .

Connin 823 .

Cony 823 .
Coquallin: 509 .

Cordero 829.

Cordeiro 829.

Cori 414.

Corinna 632.

Corfac 200.

Corza 825 . 827.

Corcui 748.

Cotta 799.

Coucang 52.

Cow 831.

Coyametl 748.

Coyotl 208 .

Coypu 423.

Cuati 334.

Cudus 639 .

Crabedago 209.

Cretan Sheep 83i.

Creutzbock 640 .

Creuzfuchs 205.

Cricetus $463^{\circ}$.

Crocuta 189.

Croque noix $82 \mathrm{r}$.

Cuaridu 396. 397.

Cucang 52.

Cudana 835.

Cudu 639 .

Cuguacu 608 .

Cuguar 229.

Cuguacuarana 229. 230 .

Culpeu 207.

Cuniculus 548.

Curre 799.

Curshint $6 \%$

Cufcus 8I3.

Cuffu - aru 813 .

Cuy 411.509 .

Cwningen 823 .

Cyanus 446 .

Cynocepthalus 16.22 .26 .28 .

Cynomolgus. Simia 25 .

Cynofuros. Simia $2 \mathrm{I}$.

Hhh 2

Cyper. 


\section{$R \in g i f \circ$}

Cyperhund 170.

Cyperkatze 240.

Crigetay 716 .

\section{D.}

Daa.Dyr $82 \%$

Dabbà 802 .

Dabh 320.

Dabi 828.

Dabuh 189.

Dachkutfeh $83 i$.

Dachmarder 289.

Dachs.327. 331.561 .

- in Guiana 256 .

- labradorifche $33 \mathrm{r}$.

- amerikanifche 333.

Dachsbär 328.

Dachshund 172.

Dachsfchliefer 172 .

Dachskriecher 172 .

Dachswürger 172.

Dachsfinder 172 .

Dafad 829.

Daim 82\%.

Dombock 604 .

Dämlein 604.

Dämling 604 .

Dämnhirfch 604 .

Däsman 814 .

Däsmans - rotta 816 .

Daguel 823 .

Dain 827 .

Daine 827 .

Daino 826 .

Dama 604. 623.

Daman lsrael 529.

Damantilope 624.

Damhert 827.

Damma 826.

Ḋamgeifs 604 .

Damhirfeh 604.

Dammhirfch 604.623.
Damunhirfch antilope 624.

Damthier 604.

Damvad bak 82\%.

Damwild 604 .

Damwildbrett 604 .

Dandulana 820.

Daniel 827.

Dant 693 .

Danta 734.

Dante 694. 703.

Das $56 \mathrm{r} .8 \mathrm{r} 2$.

Dashond 801.

Dafypus 102.

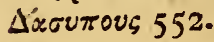

Datwi 812.

Deba 827.

Decumanus 426 .

Deeb 802.

Deer 60\%.

Degis 509.

Dein 827.

Delphinus $78 \mathbf{r}$.

Delphin 781. 784 .

- - kleiner 782 .

Delphis 784 .

Dendl 827 .

Denka 820.

Defman $37 x$.

Desmans - rotta 816.

Deve 150 .

Dhfib 8c2.

Diäna 27.28 .

Diämmel 824 .

Djarbua 822.

Dib Igr. 802.

Diberin 830.

Didelphis 341 .

Dikaja 836 .

Dikaja Koza 824.

Dikii borov 836 .

Dikoi Baran 830.

Dikobratz 815 .

Dirkjä 8 10. 

Dipus $527 \cdot 533$.
Dif́color 229 .
Difchon 628
Difpaiddfarch 833 .
Doe 827.
Dof 827 .
Dofhiort 827 .
Dog 163. 798 .
Dogg 800. $16_{3}$.
Dogo 800.
Dogue 800.
Doguin 800 .
Donus 836 .
Dormoufe $82 \mathrm{r}$.
Dongus 836 .
Dongus - ballas 836 .
Donninnha $8 \mathrm{I}$ I.
Donnola 811 .
Dorcas 630.634.
Drholadíchàn 831 .
Drhumbura 819.
Drhumbra 819.
Drhymmurain 819 .
Drhymron 8Ig.
Driggettai 716 .
Dfikketäi 7 I6.
Dfiulalà 820 .
Düä 824 .
Dubbah 187.
Duck 32.
Dudleji balaena 778 .
Dukkeris 808.
Diii 824 .
Duppuris 808.
Durié - fá 832.
Dytrgi 808 .
Dyrkolle 825.
Dzik 836 .

Dorrakon 812.

Dornfchwein 384. 394. 396.

Dorên 803 .

Draen y coed 814 .

Dromas 565 .

Dromedal 823 .

Dromedar 562.563 .565 .567 .

Dromedarien 565.568 .

Dromedario 823.

Dromedaire 823 .

Dromedary 823 .

Dromedarkameel 565 .

Dromthier 565.

Dryas 522.

Dry nog 814.

Dfabba 802 .

Drchàla 830 .

Dichjalınan 822 .

Dichyracki 820 .

Drehyryku 820 .

DCheren' 828.

Drhigel 799.

Dr'silkis - Sitskan 8I7.

Dfhukel 792.

\section{E.}

Eb 799.

Eber 740 .

Eberhirfch 753 .

Echinus 385 .

Exivos 385 .

Eckerchen 488.

Ecureuil 820.

Ectureuil gris 820 .

- - de Canada 820.

_- de Virginie 820.

Edelmarder 291.

Eedfcha 810.

Eezel 835 .

Eg 827.

Egel 814.

Eger 817 .

Egern 820.

Egerne 490.

Egin 823 .

Egoll 833.

Egret Ape 3 I.

Hhin 3
Eichel. 


\section{$R \in g i f t e r$}

Eichelmaus 52.

Eichenmaus 522 .

Eichhermelin 488 .

Eichhorn 487.

- das rothe 490.498. 499.

- - das weifsgraue 492.

- - fchwarze 493. 494.

- - veîrse 493.

- virginifche graue 495 .

- labradorifche 497.

- - vifenfarbige 497.

- carolinifche - perfi. fche 498 .

- - javanifche - rothbäuchige 499 .

- - langgefchwänzte - ceyg. lonifche 499.

- - gelbbäuchige 499.

- von Bopmbay - blondes - gelbes $52 \mathrm{I}$.

- - oftindif́ches - indiani. fches 521 .

- - afrikanifche -geftreif. te $50_{3}$.

- - bunte - brafilifche 507. 509.

- aus Dfchinfchi 598.

- - fiegende 510.

Eichhornaffe 57.

Eichhörquchen 489.506.

Eichhörnlein 49I.

Eichkatze 488 .

Eichkatzeri 488.

Eichfchläfer 522 .

Eidion 831.

Einhorn 756. 758 .

Einhornarfe $4 \mathrm{r}$.

Einharnfich 756 .

Eisbair 324 .

Eifenfchtein 394.

Eisfich 765 .

Eìsfuclas 204.
Eiferne Ferken 397.

Eiswallfifch 765 .

Eiter-Unge 803.

El 830.

Elain 825 .

Eland 639.640. 825

Elande 589.

E $\lambda_{2} x Q \circ G 596$.

Elaphus 59 I.

Elaurandus Ceb. 22.

Elbthier 298.

Elda 833 .

Elefant II 8 .

Elen 587.

Elend $587^{\circ}$

- das capfche 639.

Elendhirfeh 590.

Elendkuh 588.

Elenn 587.640.

Elenn antilope 640.

Elendthier 735 .

Elennthier $586,587.640$.

Elendsthier 589 .

Elent 587 .

Elephant 118.

Elephantenochre 699.

Elephas 118.

Elfante 796.

E!Gl 796.

Elg $825 \cdot 830$

Elk 825.591 .

Ellenkatze 298.

Ellgur 825.

Elsdiur 825.

Elsdyr 825.

Elf: 298 .

Eltis 298.

Eme 837.

Eme - Uker 832。

Émgalo 751 .

Fimpetra 483.

Empophos 639.

Encubert 107 . 


\section{nach ders Seitenzahlen.}

\begin{tabular}{|c|c|}
\hline $\begin{array}{l}\text { Engalla } 751 . \\
\text { Engoi } 805 . \\
\text { Engri } 805 . \\
\text { Ent } 836 . \\
\text { Equus } 706 . \\
\text { Erbsmaus } 441 . \\
\text { Erdbiber } 416 . \\
\text { Erdeichhorn } 504.50 \% \\
\text { Erdfahren } 454 \cdot \\
\text { Erdgräber } 470 . \\
\text { Erdhafe } 527.528 .530 . \\
\text { Erdhafe, der allerkleinfte } 557 . \\
\text { Erdmäufe } 470 . \\
\text { Erdmaus } 449.455 . \\
\text { Erdmenich } 5 \text {. }\end{array}$ & $\begin{array}{l}\text { Efpan 334. } \\
\text { Efpenmarder 291, } \\
\text { Efpin 814. } \\
\text { Esquilo } 820 . \\
\text { Efvre } 835 . \\
\text { Eulaffe } 30 . \\
\text { Eurchon } 814 \\
\text { Europäer 4. } \\
\text { Evet 820. } \\
\text { Ewfbard } 810 . \\
\text { Ewig } 825 . \\
\text { Exquima 27. } \\
\text { Erel 835. }\end{array}$ \\
\hline
\end{tabular}

Erdfchwein 98.

Erdratte 427.

Erdratze 357.

Erdichaf 572 .

Erdíchüffel 449.

Erdwolf $447 \cdot 464$.

Erdzeifel 484 .

Erdzeifelchen 484.

Erdzeift 455.

Ere - morin 834 .

Erinaceus 381.

- Doricatue xis.

Erizo 814.

Erkek - at 834.

Erminea 305 .

Erneb 539.

Faadh 221. 805

Faar 829.

Faca 833 .

Fähe 185 .

Färfe 688.

Fakan 840 .

Fallow Deer 82\%.

Faon 825 .

Faquinha 833 .

Fâr 829.

Far 817 .

Faras 344.

Farkas 800.

Fatuellus. Sitria 39.

Faule, amerikanifche 87.

Ertner 142 .

Erven 339 :

Ervetje 643.

Efchek 835 .

Efcurieux 820.

Efcureain 820.

Erel 717 .

- wilde 719.

-zahme 720 .

zeftreifte 726 .

Efelskuh -734.

Efis $8 \times 4$.

- des Marcgraws 87.

- afiatifche 90.

- kleinfte orientaliche 90.

- mit dem Hundskopf 52.

Faulthier 86.

- - das dreyfingerige 86.

87.

- - mit dres Klaten 86.

- - fchmächtige, weifsgraue

87.

- - amerikanifches 89.

- - das 2 weyfingerige 89 .

Hab 4

Faul. 


\section{$R \in g i f \in$}

Faulthier, Affe, verkleideter 90. Fin - filh 839.

-. - ceylonifches go.

Faulthieraffe 52.

Faunus. Simia 24.

Fech 429.

Fehwammen 490.

Feldefel 720 .

Feldfuchs 199.

Feldgeis $62 \mathrm{I}$.

Feldgott 39.

Feldhafe 537.

Feldinarder $29 \mathrm{r}$.

Feldmaus $437 \cdot 438.450 .455$.

- kleine 449. 454 .

- - grofse 464 .

- - felrfame Art 529.

Feldratte 427.449 .408$.

Felis 209. 233 .

Felfenantilope 62 .

Felfengeis 621 .

Felfengraber 558 .

Felfenfpringer 637.

Feorag 820.

Ferae 132.

Ferkelkaninchen 404 .

Ferkelmaus 410.

Ferkel 740.

Ferken 740 .

Ferret 8 IO.

Ferrum Equinum 82.

Fert el Haile $3 \mathrm{rz}$.

Fetrmaus 518.

Fetthier 559.

Fjallfras 812 .

Fiâllmus 818 .

Fiäll. Kacka 803.

Fiber $4 \mathrm{I} 5$.

Ficld. Rak 803.

Filander 354.

Filfras 812 .

Fill 795.

Finnfifch 765.768 .

Fin - Fiffch 765 .
Fifchbär 320 .

Fifcherwiefel 295.

Firchdieb 28I.

Fifchochfe 130 .

Fifchotter 280.

Fifchottermarder 28I.

Fifhtall 620.

Fif katta $80 \%$

Fitchet 8II.

Flädermus 794 .

Flaggerinuus 794 .

Flatterer 64.65 .66$.

Fleckmaus 468 .

Fledermaus 59: 65 .

- - die kleine von Ter. nate 62 .

- - die gröfste 63 .

- grofso von Madaga. fear 63 .

- Lurinamifche 65 .

- - gewiffe fehr grofse 65.

- - gemeine amerikani. fche 66.

- - ratzenartige 67.

- fpitzmausähnliche 69,

- - mit der Hafeufcharte 6 .

- katzenartige 69.

- langöhrige 70. 7 I.

- - kleinere gemeine $\mathbf{r}$.

- öhrigte $7 \mathrm{I}$.

- - gemeine 72.74.

- - die grofsen 73 .

- - die blaffe 75 .

$\perp$ - eine Art kleiner 77 .

- - fünfte Art kleiner

Zwergfledermäufe 77 .

- - mit bunten Fittigen 78.

- - gemahlte 79.

- buntgeflügelte 79 . 


\section{saih den Seitenzahlen.}

Fledermaus, hundsmäulige 80 . Founart $8 \mathrm{Ir}$.

- - grofsköpfige 81.

- - mit dem Hundskopf $8 \mathrm{r}$.

- - von Neuyork 83.

- - nordamerikanifche

83.

- - mit behaarten Fittigen 84 .

- - rauchfchwänzige 84 .

- - langhaarige 85 .

- grofse 85.

Flederinuis 794.

Flederratze 67 .

Fleifcherhund $I 7$ I.

Fliegende Hund 60. 61. 64.

- - von Ternate $6 \mathrm{r}$.

- - - suit rothem Halfe 62.

- - Füchfe 63.

- - gröfster mit Ohren 64 .

- - Ratze 67.68.

- Katze 69.

Fliegenfänger 36 .

Flieger 510 .

Flittermoufe 794 .

Flursochle 730े.

Flufsotter 281.

Flusspferd 730.

Foetta 810.

Fogelhund 800 .

Fohlen 707 .

Foina 288.

Fonkes 794 .

Forao 810.

Forao de Seytia 809.

Foram 810 .

Foras l'bar 835.

Furmoranifheg'

Forfa 271 .

Foffane $27 x$.

Foffe 272 .

Fonine 809 .
Foutreau 808.

Fox 803 .

Freffer 214.

Frett 30r.

- das melirte 271.

Frettbär 257 .

Frettel 301 .

Frettchen 301.

Frettele 301.

Frettmarder 301:

Fricator $16 r$.

Frifchling $73^{6}$.

Fritt 810.

Fuchs 194

- mexikanifcher 185 .

- furinamifchêr 186.

- der feluvarze 193.

- europäifcher 195.

- gemeine 196.

- braune 197.

- virginifche 202.

- graue ámerikanifche 202:

- blaue, weirse 203.

- arctifche 204.

- brafilianifche 23I.

Fuchsaffe 57 .

Fïchre, fliegende 63 .

Fuchseichhorn 493.

Füchfelınännchen $5 \%$

Füchshund 172 .

Füllen $70 \%$.

Fuina 809 .

Fuina di Conftantinopoli 808.

Furet 301:

Furetto 810.

Furo 301 .

Furunculus 469.

Fymi 824.

G.

Gaas 812.

Gabelhirfch 59t.

Hhb 5 


\section{$R \in g i f \circ r$}

Gämie 621.

Gäule 833 .

Gafr 828 .

Gagai 838 .

Gagain - drchuld - foga 838.

Gaine 80r.

Galera 287.

Galgo 801 .

Gama 572.

Gamal 824.

Gamala 824 .

Gamo 826.

Ganfud 815 .

Gangeshirith 607.608 .

Gannus 802.

Gartenmaus 455.

Gartenfchläfer 522 .

Garou, Gama 221.

Gafa 812.

Gaft 798 .

Gat - el Kalia 243.

Gat-el Khallah 243.

Gato 806.

Gato. montes 805 .

Gato felvatico 805 .

Gatto 806.

Gaul 708 .

Gauvera 705 .

Gazelle 6r8.630. 638 .

- - rothe 624 .

- chinefifche 627 .

- fpringende 629.

- - gemeine 630 .

- capifche 635 .

- - weirse 639 .

- amerikanifche 64I.

- - afrikaniliche 645 .

Gebirghirfch 597.

Gebirgmäufe 521 .

Gdhies 829.

Ged 828 .

Gehad 804.

Geis 650 .
Geis, eine indianifche 653 .

Geifs 600. 651 .

Gelding 833 .

Geldowefi 824.

Gemal 824 .

Geman 830.

Gemars 725 .

Gembis 622 .

Gems 620.

Geusbock 636 .

Gemfe 622 .

- afrikanifche 623 .

- amerikanifche 655 .

Genetta 269.

Genette 269. 271.

Genetha 270.

Genettkatze 260 .

Genithkatze 270.

Gepard 227.

Gerbo 530.

Gefs 815 .

Gefpenfterthier 52 .

Getulus 503.

Gewazen 826.

Ghazell 828 .

Ghiro 821 .

Ghoudie 830 .

Ghoukou 815.

Gjambûcaha $80_{3}$.

Gib 640.

Gibbar 767 .

Gieedk 813 .

Gied 828 .

Gierv 339.

Gigantea 354 .

Gimel 824.

Gimmerlam 829 .

Ginetta 271 .

Gin-hiang 32I.

Giraffe 615 .

Girnaffe 6 is.

Gift

Gladius marinus 788 . 


\section{nach den Soitewzahlen.}

Glama 571. 573 .

Ihxyes 189.

Glax 578.

Gliama 572 .

Gliero 821 .

Gliraria 52 r.

Glis $427.473 \cdot 519 \cdot 521$.

Glonton 813 .

Glutton 813.

Gmelins - Maus 475.

Gnometje 643.

Gnou 635 .

Gnouzia 811 .

Gnu 634.

Gnuthier 635 .

Goidinachtfch $83 i$.

Goadina datfeh 831 .

Goat 651.828 .

Godde 826.

Godde - Sapan 818.

Goldmarder 291.

Goldrattę 526 .

Gôlâ 803 .

Göfii 816,

Gohu 826.

Goi 830.

Gojes 832 .

Goldhund 192.

Goldmaulwurf 365 .

Goldfpitzmáus 366 .

Goldwolf 190.

Golock 13 .

Gomari 83.5.

Gönie 837 .

Gondir 812.

Gornoftai 8I I.

Gotfchi 838 .

Goyn 830.

Graa - Byr 826 .

Grabthier 187.

Gräber 373.557 .

Grabeen 801 .

Gräving 328 .
Gracvling 812.

Grät - fwin 812.

Gramhus 787.

Giampuffe 787.

Gramfelur 1.48 .

Grand rat de bois 820 .

Grafebär 319.

Grathier 621. 622:

Grauwerk 490. 492. 520.

Grefing 328.

Grehound 801.

Greis 37.

Grentfeh 464 .

Greuel 520 .

Greving 812.

Gréy 812.

Greyhound 80r.

Grichun 827 .

Grifart 812.

Griesfuchs 201 .

Grifon 264.

Gronoftay 811.

Grofskopf 8I.

Grofsohr 207.

Groundhog 480.

Grundhafe 537.

Grutfchel 464.

Guache 807.

Guachi 279.

Guallfifel $76 \mathrm{r}$.

Guanaco 571. $573 \cdot 574$.

Guanacos 572 .

Guanapo 824.

Guanque 446.

Guanico 57 I.

Guanicoes 574 .

Guanacu 574 .

Guariba 36.

Gubartes 767 .

Guenon 793 .

Guereba 36.

Guereza 794.

Giiib 640 . 
Guillino $42 \mathrm{r}$.

Guinea, pig. $8 \times 5$.

Guineefch Biggetje 815.

Gïrk 802.

Guemul 706.

Guevei 642 .

Guevi - Kagor 642.

Gün 834 .

Gïrtelthier 102 . fiche Arma. dill.

Guldfchah 830 .

Gulo 337.

Gundi 486.

Gur 804.

Gurt - Ketfch 828 .

Guffo 830 .

Guza 830.

Gwadd 813.

Gwiwair 820.

H.

Haa - Hirningur 788 .

Haafenkühlein 549.

Hacqua 834.

Häp 798 .

Häre 822.

Haerbe 814.

Härka 832 .

Häermlein 310 .

Haifer $83 \mathbf{I}$.

Haji 88.

Hakou 837.

Halbefel 717 .

Halbfuchs $256.328 \cdot 333$. 335 . 336.

Halbhafe 40 r. 406.

Halbkaninchen 401 .

Halsbuk 826 .

Hammel 660. 66و.

Hamadryas. Simia 2 r.

Hamfter $463 \cdot 480.484$.
Hamftermaus 468 .

Hamftermäufe 462 .

Hangga . Evö 795 .

Hankele 833 .

Hanfe 800 .

Han - ta - han 825.

Harda 820.

Hardillo 820.

Hardlooper 837.

Hare 822. .

Hari - mou 805.

Harlekin 160 .

Harrier 800.

Hart 825 .

Harte-Beeften 634 .

Has 539 .

Hafe $536.537 \cdot 539 \cdot 544 \cdot 546$.

547 .

- von Iava 406 .

- von Patagonien 414.

- veränderliche 543 .

- weifse 543. 544.

- der kleinfte 557.

- gehörnte 542.

- fchwarze 545 .

- halb weirs und halb graue 546.

- nordamerikanifche 546 .

- von der Hudfonsbay 546.

- von Californien 559 .

Hafelmaus $434 \cdot 484 \cdot 520.522$. 523. 524. 525 .

Hafelíchläfer 524 .

Hafenfledermaus 70 .

Hafenfurs 203.

Hafenferket 406 .

Hafenkillen $55 \%$.

Hafen - königlein 551 .

Hafenkühnlein $55 \mathrm{I}$.

Hafenmaus 406.

Hafenfcharte 69 .

Hafghiin 568 .

Haftatus Velp. 68. 


\section{nach den Seitenzahlen.}

Hati 796.

Haubenftachelfchwein 394.

Hauer 736 .

Haumaus 438.

Hauptbär 320.

Hausbock 6 19.

Haushund 152.17 I.

Hauskanincheri 552 .

Hauskatze 236 .

Hausmarder 288.

Hausmaus 429.433 .

Hauspferd 712 .

Hausratte 428.

Hausfchwein 742 .

Hausfpeicherwiefel 310.

Hauswiefel 311 .

Hausunke 298.

Havafi-Ketzke 828.

Hav - Nödd 797.

Hay-heft 126.796.

Hav - Säl 143 .

Hay 88.

Hazelinuis $82 \mathrm{x}$.

Heckenfehwein 384.

Hedige -hog 814.

Heermännchew 310.

Heerdenmaus 454.

Heermaus 438.

Heinzel 833 .

Heierth 830 .

Helfant 199.

Hemionus 716.

Hengft 707.

Herdershond 799.

Herelt - lò 834 .

Herinaceus 383 .

Heriffon 385.814 .

Herman 830.

Hermellanus' 308 .

Hermelin $305.308 .311,345$.

Hermelinwiefel 307 .

Hermyn 811 .

Hers 835 .
Herznafe 67.

Heft 833 .

Heulaffe $37 \%$

Héwoinen 834.

Heyfen 840.

Hhoulda 813.

Hhodfel 813.

Hiang - tehang - Se 579.

Hiir 817.

Hinde 591.825 .

Hindekalb 591 .

Hindin $59 \mathrm{r}$.

Hi-nen - pao 805.

Hinnus 724 .

Hingt 833.

Hint 825 .

Hiort 825 .

Hippelaphus 596.

Hippopotamus 730 .

Hircus 648 .

Hirnä 82.9.

Hirfch - 585. 591.603.

- wilde 586 .

- edle 593.597.

- korfikanifche 597 .

- kanadifche 598.

- eine feltzame Art 600.

- den man Rehe nennt 600.

- der grönlandifche 603 .

- der burgundifche 603.

- virginifche $60 \%$.

- dickleibigte 608 .

- grofse auf Zeilan 6r4.

- kleine chinefífche 6 r4.

Hirfchantilope 629.632 .645 .

Hirfchbock 644. 679. $59 \mathrm{I}$.

Hirf he 594.

Hirfcheber 752 .

Hirfchen 594 .

Hirfchkarneel $57^{2}$.

Hirfchgen, das fchmächtlge afrikanifche 582.

- - ungehörnte 582 . 


\section{$R \in g i$ fte}

Hirfchgen, mit rothem Haar Hbiutee 798.

582.

- - das furinamifche 584 .

- - das guineifche 613 .

Hirfchkalb 59r.

Hirfchkuh 59 r.

Hirfkuh, fardinifche $60 \%$.

Hirfshluchs 245 .

Hirfchrehe 642 .

Hirfchrehlèin 642.

Hirfchthiere 634 .

Hirfchwolf 245 .

Hirfchziege 638.645 .

Hirwi 825.

Hifpidus Vefp. 78. -

Hinz 807.

Hnufubabkr 766 .

Hod 816 .

Höckerochs 687.

Höfrumgen 788.

Hörtichök 818.

Höit 83.3.

Hofhund $153.17 \mathrm{I}$.

Hofpferd 712 .

Hofrung 840 .

Hog 743.836 .

Hoitzlacuatzin 398.

Holzhafe 537.

Holzmaus 522.

Homo I.

Hond 798.

Honiğbär 318. 319.

Honigdachs 273 .

Honigftucher 273 .

Hoppe 833 .

Hornaffe 39. 40.

Hornnafe II 5 .

Hornvieh 690.

Horfe 711.833 .

Hors hwal 796 .

Hound 800 .

Houri 817.

Houls - Dog 799.
Hrein. Dyr 826 .

Hrutur 830 .

Hualfifeh $7^{6}$ r. 762 .

Hualfik 837 .

Hual - Ros 796 .

Huals $76 r$.

Huanacus 573.

Huemul 706.

Hudfons - rat 462 .

Huenak 574 .

Hueque 574.

Hufeiren 82 .

Hufeifennafe 82 .

Hühnerhund $x 66$.

Huidobrius $42 \mathrm{I}$.

Huishond 799.

Hukur 832 .

Hund 149.154.

- fibirifche 154.

- kantrehatkirche 155.

- isländif́ne 155 .

- kleine dänifche 160 .

- angorifche 158.

- irrländifche 168.

- türkifche, nackte 170 .

- ägyptifche 170.

- barbarifche 170.

- von Burgos 173.

- mexikanifche 173 .

— wilde 173.208.

- grönländif́che 208.

- grofse dänifche 208.

- fliegender 60.64.

Hundsbeifser 25 .

Hundsigel 382.

Hundskopf 15. 18. 21. 26.61. 65.

Hundsmaul 80.

Hundspard 220.

Hundsfchnauze 53.

Hundefch wanz $2 \mathrm{t}$.

Hundstatu ro3. 


\section{mach den Seitsnzahlew.}

Hutaffe 31:

Hunt 802.

Huron 810.

Hurch 834.

Hushund 799.

Hutus - Muus 819.

Huyfen 840.

Hwch 837.

Hwrdt 829 .

Hyaena 186.

Hyaena Gefneri 18.

Hyäne 186.

- geftreifte 187.

- gefleckte 189.

Hydd 827:

Hyddes 827.

Hydrochaerus 413. 734.

Hurax 559.

Hyftrix 392.

\section{I.}

Iabali 836 .

Iabalina 836 .

Iacchus. Simia 43.

Iackal I9I.

Iackall 19 I.

Iackafch 808. 809.

Iackhal rgi.

Iaculus $355 \cdot 527 \cdot 529 \cdot 530$.

$$
534 .
$$

Jănnก 823.

Iaerv 812.

Iagdhund 164. 165 .

Iaguara 224 .

Iaguar 223 .

lagularéte 228 .

Iaikmaus 462 .

Ialman 822 .

Iaman 828 .

Ianuara 805 :

Jaques 251 .

lararangan $80 \%$
Iarboa 528. 531 .

Iar ganat 794 .

Iarka 829.

Iary - kanat 794.

Iafe 822.

Iaswetz 812.

Iaswietz 812 .

Iavali 749.836 .

Iavor 591.825 .

Iawari $224 \cdot 748$.

Ibex 656 .

Ichneumion 252. 254. 255 .

Iebraf́chken 485.

Ielek $81 x$.

Ielen 825 .

Iemuranka 819.

Iemurantf chik 82:

Iena 802.

Ienix 822 .

Iepka 809 .

Ieraffa 6 I 6 .

Ierboa 529.

Ierbuab 529.

Ierv 339.

Ierven 339.

Iefi II I.

Iefch 814.

Ievrafchka 484.485.

Iez 814 .

Igel 381.382 .

- ungeöhrte 386 .

- weifse amerikanifche 386 .

- furinamifche 386.

- malaccifche 387 .

- langöhrigte 388.

- kleinfte 389.

- oftindifehe 390 .

- Gibirifche 391.

- geöhrte 389.

Igelkott 814 .

Igelnafe 368 .

Iguavus 87.88 .

Ihre 830 . 


\section{$R \in g i f e r$}

Iida 799.

Iilkà 333 .

Iirataka Zin 827 .

Iird 534 .

liya 279.

Ikal 832 .

Ikhorn 490.

Ikorn 819.

IxT $\angle 5300.303$.

Ilachion 821 .

Ilk 298 .

Illing 298.

Iltis 297 .

- gepanzertes 100.

Iltnis 300.

Imel 564.

Imit - Lanki 321 .

Inumenbär 320.

Imnä $\$ 33$.

Impalankhe 646 .

Impangueza 646 .

Impumpes 208.

Imral 819.

Indri 54 .

I'ne 832.

Ingak 832 .

Inumus. Simia 15.

Inofchifchi SO4.

Infire 315 .

Jöra 825 .

Iocco 10, II.

$1 \pi \pi \circ 7^{11}$.

Ippoyotamo 835 .

Irabisto 815 .

Iremi 828 .

Irgendir 808.

Irjä 833 .

Iirataka 616.

Irmifch 794 .

Ifatis 203 .

Ifchaki 721 .

Jfchim 824 .

Ifchpi 829 .
Ifer 783 .

Ifkal 832 :

Iit 799 .

Iftrice 815 .

Ifubr \$25.

It 799 .

Iubata, Felis $22 \%$.

Itutz 315.

Iudaga 795.

Iuh $83^{\circ}$.

Iumar 724 .

Iumara 725 .

Iuwiarts 725 .

Iument 833 .

Iunetz 832 .

Iungfernbock 642 .

Iungfernhund 159 .

Iungenda 813 .

Iungi 839.

luni 839 .

Iupatiima 813.

Iunitza 832 .

Inpiterfifch 767 .

Jurda Kaidam 824.

Iwe -lanki 82 I.

Iwrch 827 .

Jyrchell $82 \%$.

Iyrgan 819.

Izquiepotl 258 .

Ize 829.

Kaas 829.

Kabala. Io 834.

Kaban 837.

Kabarga 579. 824.

Kabaffu 103.

Kabiai 413 .

Kabfa 830.

Kachelot 773. 775.776 .

- - der kleine 7.73 .

- - langköprige 774 . 


\section{nach den Seitenzahlen.}

Kachelot, weifsliche 778 . - von Nerrengland 778. Kachicame III.

Kadifchi 707.

Kâd-tu-nari 803.

Kälberlamm 606 r.

Känguruh 354 .

Käter 835 .

Kätho 820 .

Käuler 736 .

Kämmelthier 652 .

Kaftaar 802.

Katau 5 r.

Kahlbart 40.

Kahlohr 386.

Kaiko 808.

Kajopollin 349 .

Kakerlaken 9.

Kalan 808.

Kalb 688.689.

Kalblúchs $243 \cdot 248$.

Kalf 831 .

Kaltan 809.

Kaltun 812 .

Kamanofchifchi 812.

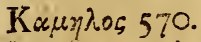

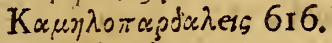

Kameel 502.

- einbucklichtes 562 .

- gemeines 563 .

arabifches 503 .

z wcybucklichte 567 .

türkifche 568 .

wollentragende 576 .

indianifche 6,6 .

Kaneelbosk 625.

Kameelpardel 615.

Kameelparder 615.

Kameelziege 571.572 .

Kameelzicgen 652.

Kamehlpardel 616.

Katrel 505 .
Kameli 824.

Kamel. Pard 617.

Kamelihier 865 .

Kamennaja. fodedka 8 I 3.

Kamennoi Baran 830.

Kamennyi medwed 813 .

Kammnafe 368 ,

Kaminerhund 163 ,

Kampagnol 454.

Kan $806.837^{\circ}$

Kanang 799.

Kanatle - Trkan 794.

Kand' á mürrugam 795.

Kanickelchen 552 .

Kanin 55 I.

Kaninchen 408. 414. 548.

- grofse brafilifche 402 .

- - brafilifches 405.408.

- von Iava 407.

- furinamifches 407 .

- - amerikanifches $40 \%$.

- indiarififhes 410 .

- mit fehr langemSchwan. ze 529 .

- - langgefchwänzte $547^{\circ}$

- Das gemeine 549.

- Das reiche 553 .

- angorifche 553 .

- rufifche 554 .

- ungefchrwänzte 555 .

- indianifches 556 .

- von Neukolland 559.

- von Californien 559 .

Kaninchenjäger 301 .

Kaninchenmans $4 \mathrm{I}$.

Kaninchenwiefel $30 \mathrm{r}$.

Kaningen 550 .

Kanifis 837.

Kannikenis 823 .

Kannihis 823.

Kantil 584 .

Kintza 834 a

Kâouda 829. 


\section{$R \in g i f \in$ r}

Kappen. Bichhorn 517.

Kapuftrik 797.

Karagan 201.

Kara.Gurfu 812.

Karakal 242.

IKarako 426.

Karburch 818 .

Karhu 8II.

Karibou 603 .

Karnickel 55 I.

Karnützchen 552.

Kartheuferkatze 239.

Karrah - Kulak 243.

Kafa 828.

Kafah 828 .

Karkar 802 .

IKafgam 796.

Kaffigiak I 39 .

Kaftor 416 .

IKat 806 .

Kater-806.

Katta 806:

Kattlu 249.

Katze 209.232.

- wilde 225. 233. 243 .

- brafilianifche 229.

- angorifche 238 .

- panirche 239.

- blate - rothe 240.

- die rauhe 243 .

- Pennants perfifche 243 .

- chinefifche mit fchlappen Ohren 250.

- kurzgefchwänzte von Iapan 250.

- wilde gefprenkelte 250 .

- von Neuf́panier 250.

- von Californien 251.

- wilde, mit fchwarzen Streifen 273.

- mit fpitzzulaufendem Kopfe 275 .
Katzenelckhorn 493.

Katzenluchs 248. 249 .

Katzenparder 206.

Katzentieger $23 \mathrm{I}$.

Kauw - howba 818.

Kavia $40 \mathrm{I}$.

Kayopollin 349.

Kebes 830 .

Kedi 806 .

Kedi - Milan 794.

Kelbe 799.

Kelbmärre 192. 799.

Kelcb 799.

Kemel 824 .

Kemel-Pardel 8270

Kenlie 803.

Kenguruh 354.

Kempe 740.

Keph 799.

Kephir 804.

Keporkak 769.

Kerb 808.

Keret 814.

Kerkodon 796 .

Kerma 820.

Kermang 820.

Kernektok 838 .

Kettenbund 175 .

Kevel 631 .

Kevella $6_{3} \mathrm{I}$.

Kgoyes 827 .

Kiefermarder 291 .

Kigulitik 777 .

Kiid 828.

Kilbe 558 .

Kilberlamin 66.

Killeluak 838 .

Killen $55 \mathrm{I}$.

Killer 788 .

Kinkajou 272.

Kiön 802.

Kipod 814. 


\section{nach den Seitenzahlen.}

Kiriwula 795 .

Kirmyfchak 242:

Kirpelé 808.

Kiffa 806 .

Kirriton 81\%.

Kladenï Baran 829.

Kladenoi Kon 833.

Klappmütze 145 .

Kleeblattnafe 68.

Kleinauge 778.

- - geflecktes 403.

- - grabendes 470 .

- - unterirdifches $47 \mathrm{x}$.

- - blindes 472.

Klippdachs 560 .

Klipdas 559.

Klippenhafe 558.

Klippmaus 445 .

Klippfpringer 637 .

Klippzeift 446.

Knabbelfifch 839.

Knähund 800 .

Knobbelfifch 759 .

Knoblatuchsmaus 451.

Knorchu 802.

Knotenfirch 769.770 .

Knurr $837^{\circ}$

Koa 823.

Kouti 256.258 .

Koatimondi 256.

Kob 630.

Koba 62g.

Kubras 816.

Kobülla 833.

Kobyla 833 .

Köchlani 70\%.

Kod - hi - bili 805.

Koddutis 826.

Koddojännes 823 .

Koedoe 644.

Koekebóe 315 .

Koger - Agan 314.

Kuha 799 .
Köhejle 70\%.

Koblbrenner 199.

Köhler 199.

Koi 830.

Koja 826 .

Koian 822.

Kojan 822 .

Köin 802.

Koira 799.

Koiti 333.

Koirok 822.

Koiwu 830.

Kok 824 .

Kokeboe 315 .

Kokobo 315.

Kola 339. 812.

Kolja 81 .

Kollanak 826.

Kolph 799 .

Komondor 799.

Kon 833.

König der Hirfche 643 .

Königshafe 553 .

Königswiefel 306.

Konin 830:

Konnyn 823.

Koofu 830.

Kopedo 814.

Kopo 800.

Koptè 834 .

Kör 826.

Korga 812.

Korin $6_{32}$.

Korine 632 .

Korinna 632 .

Kor - Kùnde 834 .

Kornferkel 464 .

Kornhamfter 464 .

Korowa 832.

Kor-koi 830.

Kührök 820.

Korfak 200.

Kor Sir 832 .

Ii i 2

Kos 
'Kos 830 .

Kofatky $787^{\circ}$

Kofchy 830.

Korchka 805.

Kotfchkar 830 .

Korha 799.

Kosina 155.

Koffitur 825.

Kot 806.

Kota 799 .

Kot-dziki 805.

Köther $17 \mathrm{I}$.

Kötfch y:2.

Kotfefifch 806 .

Kotfch - knohi $83 \mathrm{I}$.

Kotíchkui 831 .

Kotza 837.

Kotzkar 830.

Koulon 304.

Koupara 209.

Kouron 643 .

Kouvóö-808.

Koy 830.

Koza 828 .

Koza dikaja $827^{\circ}$

Koza lesna 827 .

Koza Skalna 828.

Kozel 828.

Koziel 828.

Kquariomo 805.

Krabbenfreffer 352.

Kragenzeift 458 .

Kranich 707.

Krebsotter 284.

Kret 813.

Kreutzbock 641.

Kreuzfuctis $20 \%$.

Krietich 45.484.

Kriffa 816 .

Krokute 189.

Krolik 823.

Kron - hiort 825 .

Kropfgazelle $62 \%$.
Krot 8 ! 3 .

Krtirze 813.

Krullinad 800.

Krummhorn 624 .

Krylatka I 44.

Kryfa 816.

Kfrjäh 833 :

Kuandu 396.

Küba-koi 830 .

Küiba-Sir 832.

Kubort 794.

Kudu 643.644.

Kuidus 640.

Kunduthier 644 .

Kuelna 809 .

Kügeriik 820.

Kuguar 228.

Kingutuna 818 .

Kuh 688 .

- die kleine braune 620 .

- grofse braune 629.

- aus der Barbarey 634.

Kuhantilope 633 .

Kuhr 834.

Kujan 822.

Kuifch 809 .

Kukan 832 .

Kulany 720 .

Kulchm 83\%.

Kulem $83 \mathrm{I}$.

Külle 551.552.

Kulon 304 .

Kuloninok 810.

Kultujach 8I8.

Kulún 833.834 .

Kulunt Schak 834

Kuma 798.

Kumpi 801.

Kumrah 725 .

Kuna 809 .

Kunaka 826.

Kundang 834.

Künde 834. 


\section{nach den Seitenzahlers.}

Kunele 551 .

Kiinelle 551.

Kuingan 832 .

Küngele 551 .

Küngrlin $55 x$.

Küniglein $410.550 .55 \mathrm{x}$.

Kuniglhafe $55 \mathrm{i}$.

Kunik 805.

Kunitza $8 \mathrm{cg}$.

Küniein $55 \mathrm{r}$.

Kunu 813.

Küpek 799.

Kür 826 .

Kurma 254.

Kurnis 814.

Kurris 814 .

Kurt 301.

Kurtgreli 823.

Kurtf́chligaefs 820 .

Kurzmaul $77 \cdot 78$.

Kurzfchwanz 17.

Kurka 817.

Kufkus 353.

Küinuir 821 .

Kufli 830.

Kut 798.

Kuter 817 .

Küttch 822 .

Kutfchida 794.

Kutugach 818.

Kusujach 454.

Kututhier 640.643.

Kutya 799.

Kutya-majom 793 .

Kwagga 729 .

Kwizi 834 .

Kwoptza 81 I.

Kymich 809.

Kyrmilchak 242.

Kyr - taka 831 .

Kytyp 83 .
L.

Labbi 804.

Labi 804 .

Labrador - Maus 462 .

Lacertus 100.102.

L.acha - Vacea 734.

Lachtak 147.

Läderlapp 794.

Lackati 8I I.

Lämä 832.

Läufer aus Midian und Epha 570.

Lagg 798 .

Lagopus 203.

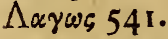

Lagurus 457 .

Lahzis 812.

Lais 804 .

Laktak 147.

Lama 571.572.

Lamantin 13 .

Lamb. 829.

Lam 829.

Lamm 660.

Línmas 830.

Lanmas - poika 830.

Lampreelen 823.

Lampreten $55 \mathrm{r}$.

Lampt 693.694.

Lan 825 .

Landbär 316 .

Landbiber 416 .

Landhippopotamus 733 .

Landotter 281 .

Landuga 796.

Landfelur 798.

Land - fpaniel 300 .

Langarm 13.

Langfurs 533 .

Langohr 48. 70. 71. 716 .

Langfamfchieicher 52 . 
Langfchleicher 52.

Langfchwang 399.400 .

Land - Selur 139 .

Lant 693.

Lanzenblatt 68.

Lap-1)og 800.

Lapin 556.823 .

Lapin de Garenne 823 .

- de Clapiers 823.

Lapine 823 .

Lapreau 823.

Lapriratraye 224.

Lapla 803.

Lapicha 834.

Lar. Simia 13.

Lafchak 835 .

Lafiopterus. Vefp. 84.

Lafitza 809 .

Lafiurus. Vefp. 84.

Lafka 81 I.

Lafotza 809 .

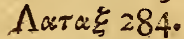

Laufhind 164.

Lauf kameel 564 .

Lau hu 805 .

Law 804 .

Leâo 804 .

Lebija 804 .

Lebre 822 .

Lebrel 80 .

Lechon $837^{\circ}$

Lechoncillo $83 \%$

Lechancito 837.

Lechon de labali 836 .

Leeuw 804.

Leina 820.

Leipter 840.

Leitzó 837.

Leitáa de Porco montez 836 .

Leithund 165 .

Lekatt 811 .

Lewender 460.

Leming 460 .
Leminger 460 .

Lemming 458 .

Lemur 52.

Lenivi - Pes 795.

Leo 210.

Leofante 796.

$\Lambda \varepsilon$ as $2 \mathrm{r} 3$.

Leor 804 .

Leone 804.

Leoniceps 46.

Leopard 2IT. 218. 220. 222.

230. $25 \mathrm{I}$.

- Kleine des Pennant 250.

Leopardal 805.

Leopardus 222.

Leporinus. Vefp. 69.

Lepre 822.

Lepus 536 .

- cornutus $54 \mathrm{I}$.

Leppard 218.

Lepturus. Vefp. 8I.

Lereou $797^{\circ}$

Lervi 620.

Lerwi 620.

Lerwia 620 .

Letuczaga inyfch 794.

Leue 212.

Levriere 800.

Levron 800.

Lew 804.

Leyon 804.

L.eytaa $837^{\circ}$

Lezard 100.102.

Lhama 572 .

Ljama 572. 573 .

Liberey.Eichhorn 503. 509.

Lichtmarder 291.

Lidme - Antilope $645^{\circ}$

Lidmi 645 .

Lidoffa 809.

Liebre 822.

Ljetaga 821 .

Lievora 822. 


\section{nach den Seitenzahlen.}

Lievre 822:

Lighval 838 .

Likhanée 799.

Lille Muus 817.

Lince 806.

Line 820 .

Lion 804 .

Lione 804.

Liron 821 .

Lis 803 .

Lifitza 803.

Lifzka 803.

Livreyiltis $26 \mathrm{r}$.

Llacma $57 \mathrm{r}$.

Llamaez $57^{2}$.

Llo $83 \mathrm{r}$.

Lloftlydam 8r6.

Llygoden 817.

Clygoden frengig 816 .

Llygoden goth 814 .

Lo 834 .

Lobo 80 r.

Lobo cerval 806.

Lobo marino $797 \cdot 798$.

Lodra 808.

Löming 460 .

Lömmer 460 .

Lörs 825 .

Löwe 210.230.

- gefleckter 221.

- der amerikanifche 229.

- der chilefifche 230 .

Löwenaffe 45.

Löweräfftchen 46 ..

Löweuhündchen 159.

Löwenkatze 146 .

Löwen - Meerkatze 46.

Löwenfchwanz 24.

Lominet 460 .

Longker 475 .

Loir 821.

Lopäre 840 .

Lori 52.
Loris 52.

Los 591.807 .825 .

Lotor 332.

Loup 801 .

Loup cervier 806 .

Loutra 808.

Louve 801.

Louvetcau 801 .

Lubba 798 .

Luchs 245.

- von Guiana 226.

- der perfifche 244.

- der weifse 248 .

- aus Canada $25 \mathrm{I}$.

Luchskalb 248 .

Luchskatze 242. 248.

Liickmaus 455 .

Lîdketfch 822 .

Luftipringer $514.51 \%$.

Luftepringergazelle 629 .

Lugubris. Sim. 5 I.

Lui 809 .

Luipard 805.

Lumınik 460 .

Lumufeh 809.

Lupo 801 .

Lupo cervero $89,6$.

Lupo gatto 806 .

Luppe 150.

Lupus 180.

Lupus Cervarius 248.

Lufch 150.

Lutfehe i $50^{\circ}$.

Lufeus 335 .

Lufe 807 .

Luffe $80 \%$.

Lutrenla 284.

Lutris 276.

Lutra 280.

Lutz 246.

Luumbengo 802.

Lux 246.

Lycaon 193:

Iii 4

Lyuce 


\section{$R \circ g i f t \circ r$}

Lynce 806.

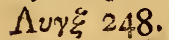

Lynx 50. 247. ff.

II.

Maar $3 \mathrm{C}$.

Macacso 25.

Macaco 55.

Macaquo 26.

Macaribo $60_{3}$.

Machanis 826 .

Macho 835 .

Macrotarfus 356 .

Madarogaitter. Simio 49.

Määr 812.

Maneftier 686.

Mäufe 454.

Maez 829.

Magazinmaus 454.

Magot 15.

Magu 42.

Magyar-Juh 83 r.

Maiaren 829.

Maihari 565.

Maikel 275.

Maimon i9. 20.

Maipuri 733.735 .

Maishund 253.

Mikack 25 .

Makauko 57.

Maki 52.

- der träge von Zeilan 52.

- der zweyfarbige 58.

- der wolligte 58.

- der fliegende 58.

- der kleinfe 59 .

Malak 815 .

Malatz $837^{\circ}$

Malbruc 24.

Malin 822.

Malon-eger 816 .

Maltheferhündchen 159 .
Mamberziege 653 .

Mambrine $653^{\circ}$

Mamusalia 1.

Manomet 20.

Manati 128. 130 .

Manatus 128.

Mandril 20.

Mangabey 30.

Màngarà 822 .

Mangarfahoe 729 .

Mangoufte 254 .

Manguife 254 .

Manicou 348.

Manipuri 836 .

Manipuris 734 .

Manis 98.99 . ror.

Mank 808.

Manul 241.

Manzao 796.

Manzo 796.

Mapach 33.4.

Mapurit 263.

Mapurito 264 :

Maracaja 23 I.

Maragéy 231.

Maragua $23 \mathrm{I}$.

Maral 826.

Marao 837.

Marcaflin 836 .

March 833.

Mârd 809 .

Märder 276. 289. 291. 296.

315.

Mare 833 .

Margacz' 828.

Margaja 231.

Margay 231.

Marmontana $8 \mathrm{rg}$.

Marmofe 349.

Marmota 476.

Marfiupial 34 r.

- Marfopa 839.

Marfvin 783.815 . 
Marta 809.

Marta cebellina 809.

Marter, f. Marder.

- - feytifcher 296.

Martes 29r.

Martin 809 .

Martlet 809 .

Martura 809.

Martorello 809.

Marke 82.

Masi jä 812.

Maltfifch 780 .

Maftiffe 800.

Matin 801 .

Maftochfe 688.

Mataga 817 .

Matrin 801 .

Matska 306 .

Matska - majom 793.

Maukanío 57 .

Aanlefel 721.724.

Maulinus 462 .

Maulochíen 725 .

Maulthier 721. 724. 734.

Maulwurf $357 \cdot 373$.

- - gefleckte $36 \mathrm{r}$.

- - veirse 362 .

- gelbe 363 .

- - graue 363 .

- - eifelifche 363 .

- - langgefchwänzte 364 .

- - rothe 364 .

- - grofse capifche 367 .

- von Canada 368.

- - virginifcher 369

Maulwurfsmaus 470 .

Manra. Simia 35.

Maus 422. 433 .

- die kleinfe 369 .

- grofse brafilianifche 402.

- kaninchenartige 405 .
Maus, indianifche $4 \mathrm{II}$.

- brafilianifche 408 .

- ancrikanifihe 412.

- groise 426 .

- norwegifche 429. 459 .

- veifse 437 .

- von Sitziliamma 444.

- rothe orientalifche 444 .

- geftreifte 445 .

- geftrichelte 445 .

- wilde 445.

- dreytingerige 445 .

- die rothe 452 .

- ökonomifche 453 .

- gefellige 456 .

- lichtgraue 457.

- mit der Halsbinde 458.

$\leftarrow$ indianifche 253 .

- bagdadifche 254.

- feytilche 296.

- polvifche 481.483 .

- langbeinigte 533. 535 .

- cafpifche 535.

Maufeeichhörnchen 5 19. $520 \%$

Maufeohr 73.

Mäufeplage 455 .

Maushund 253 .

Mazamen 608.

Mbaracaya 224.

Mebbia 208.

Mecatlchichiltie 608.

Medve 81 I.

Medwed $81 \mathrm{r}$.

Medwediza 8I 1.

Meerbär I 34 .

Meereinhorn 756.

Meer - Färklein $4 \mathrm{I} \mathrm{I}$.

Meerferkel 410.

Meerigel 395.

Meerkalb 132. 138 .

Meerkatzen 2 I. 25.

- - ganz weifse 23.

Iii 5

Meers. 


\section{$R \subset g i f \in \theta$}

Meerkatzen, bärtige 24. 27. Mico 47.

- te 26 . eigentlich fogenann. $\begin{aligned} & \text { Microuros, mus } 45 \% \\ & \text { Midas. Sim. } 48 .\end{aligned}$

- - grö́lsere angolenfi- Mii 805. fche 26.

- - ceylonifche 35 .

- fchwarze glatte 36.

- - eine andere 37.

Miko 47 .

Milfich 773 .

Mink 285.

Mifchuk 806.

riecht 45 .

- - unbärtige 46.

- - kleine von Para 47.

Meerkuh 129.

Meerochs 125 .

Meeroster 276. 278 .

Merpferd 125.

Meeríaluein 410.

Minbellerle $47 \%$.

Mitzli 230.

Mochyn 837.

Moelrhon 797.

Moes -gedu 806.

Mohr 28.

- Mohraffe 35 .

Mokoko $57 \cdot 58$.

Mokus 820.

Meerfchwein 394. 398. 409. Moldwarp 81 3.

4I. 38 I. 785 .

Meerfchweinchen 409.

Molgâtech 822.

Moll 357 .

- - - brafilifches 403 .

Meerwolf $18 \%$.

Meheri 565.

Mela 820.

Meles $327^{\circ}$

Melis 329 .

Melitaeus 158.

Mellivora 273.

Meminna 583.

Moloffe 80 .

Moloffus (Canis) 162.

Moloffus Verp. 80.

Molucea (Opoffum) 348.

Mona. Simia 32.

Monae 33.

Monachus 142.

Monax 480.

Monazo 793.

Mönch 42 .

Mönchsrobbe 140. I41.

Meních I.

Menfchenahuliche Thiere $\mathbf{x}$.

Mephitis 262.

Meràn 822.

Mere 833.

Mefch 830.

Mefcha lunkis 802 .

Mefomelas 192.

Merk 835 .

Meilix 826:

Metzgerhund $\mathbf{1} 7 \mathrm{x}$.

Mezek: 835.

Michuacaneas 174.

Moncus 254. $80 \%$.

Mone 32.

Mongoz 55.

Mongus 55 .

Monichus 33.

Monkje 42.

Mono 793 .

Monoceros 755 .

Mono colorado 794:

Monodon 755.

Monon - Zokor 818 .

Montanella 819.

Montene 
Montone 829.

Moofe: Deer 591. 825 .

Möppel r6r.

Moppel I6r.

Mops $16 \mathrm{I}$.

Mopshund rór.

Morcego 794.

Morcielago 794.

Mörder 788.

Morin - Ialma 822.

Mormon. Simia 18 .

Morfch 126.

Morfe 125.

Morfk 797 .

Morfkaja-lofchad' 835 .

Morskox $77^{2}$.

Morta. Simia 42.

Mortora 809 .

Mos 832 .

Mofchus 577.

Mofchusmaus 425 .

Mofchusochfe 698 .

Mofchusfchwein 748 .

Mofchusthier 577 .

- - das javanifehe 58\%

- - guineifche $58 \mathrm{r}$.

- - furinamifche 584,

Mofo 832.

Möfs 832.

Mouflon 679.

Mountain'226.

Moufe 817.

Mouftac 29.

Mouftache 29.

Mouton 820.

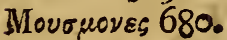

Mu 835.

Muffelthier 678 .

Muffoli 679.

Mufione 830.

Mution 678.

Müger 376 .

Mugger 814.
Mujeg 816

Muis $81 \%$.

Mula 835 .

Muldwarp 813.

Mulasna 835 .

Mule 835 .

Mules 835 .

Mulet 835 .

Mulh 814 .

Muillerefel $72 \mathrm{I}$.

Mulo 835.

Mülot 438 .

Mulot à courte queue 818 .

Mulus 721 .

Mullvada 813.

Muncts $80 \%$.

Muniftier 686 .

Mungutia 807 .

Mungo 252. 253. 254.

Muntjak 6r3.

Murciegalo 794 .

Murcielago 794.

Mureguillo 794 .

Murganho 874 .

Muriet $81 \mathrm{r}$.

Múrin 834.

Múrin -Schî ru 834'

Múri itfchikan 834.

Murinus. Vefp. 70 .

Murmelmaus 477 .

Murmelthier $476,48 \mathrm{r}$.

- - Arafsburgifches $464^{\circ}$

- t tehirkạfifches 474.

- - das podorifche 474.

- - anerikanifche 480 .

- - virginifche 480 .

- - rufifches 481 .

- - kanadifiche 482 .

- $\rightarrow$ das bereifte 483 .

- - capfches 560

Murmeutle $47 \%$.

Murzerchen 478 .

Mus 422. 


\section{$R \in g i f$ or}

Mus, ponticus 493.514 .

- aegyptius 531 .

- iaculus 531 .

- tamarifcinus 534 .

Mufarring 814.

Mufcardin 821 .

Mufcardino $82 \mathrm{r}$.

Mufefkiaer 814 .

Mufet 814.

Mufechier 583.

Mufetre 814.

Mufyano 814

Múf - rat 816 .

Murkratze 424.

Mufkusaffe, der ledergelbe 41 .

Murkasbock 578. ,

Murkus - Meerkatze 45.

Murkusochfe 697 .

Murkusfehwein 748 .

Mufliusratze 371 .

Murk.us - Siebenfchläfer 37 r.

Mufkusthier 578 .

Mufkusziege 579.

Musuion 680 .

Mufquafch 424 .

Muirs $83^{2}$.

Muntack 29.

Muftela 276 .

Müitzer 376 .

Muus 81\%.

Muyl 835.

Muylezel 835 .

Muzeraigne 8I4.

Muуผגว่ 378 .

Winn 828 .

Mynde 798 .

Myof́plax $47 \mathrm{I}$.

Ryarius 318 .

Hiygenter 95 .

Miyruezophaga gi.

-. $\quad$ fciurea 96.

$-\quad$ - myafura 97.

- ater 98.
Myfih 817.

Myrs 817.

Myficétus $76 z$.

N.

Naa. Hualen 756 .

Naar 755 .

Naarhual $755 \cdot 756$.

Nabba 796.

Nabelf chwein 749 .

Nabuna 6 r6.

Nachtfchatten 73 .

Nachtthier 69.

Näätä 809 .

Nächtling 75 .

Naeghe 796 .

Naette 809 .

Nago 565.

Nahthier 795 .

Naji 799.

Naka-Sypo 794.

Naken - hund 801 .

Nammatappatachis 826 .

Nanakin 799.

Nanger 623 .

Nanjuer 623 .

Nanodes. Sim. 5 r.

Nar - Hvual 756.

Nari 803.

Narica 257.

Narwal 755 .

Nafehorn 113.

Nalenfrett 256.257.

Nafua 256.

Nattblaka 794.

Nauttrchirch 818 .

Naut 832 .

Näbb-mus 814 .

Nebbe - mus 814.

Nebbe-Hival 772 .

Nieit fek 145.

Neitferipok 146. 


\section{nach den Seitenzahlen.}

Neitfoak 145.

Nemaeus. Simia 32.

Nemeftrina. Simia 16 .

Nems 275. 807 .

Ner 824 .

Nerpa 798.

Nerpen 797.

Nerz 284.

Netopgr 794.

Neufundländer 174.

Neujorker 83.

Niaanel 822.

Nial 804 .

Nielmaus 438.455.

Nieto perf 794 .

Nihiranak 224:

Nilgau 625 .

Nilgaut 626.

Nilpferd 730.

Nimfe 809.

Ninakin 799.

Njo 823.

Niri-Katfchati 818.

Nirk 808 .

Nira 840.

Nifh 809 .

Nife 782 .

Nifer 782 :

Nitela 522.

Nochoi 799.

Noctula. Veip. 75 .

Nortule 75 .

Nökuil 832.

Nökdil - Schiru 83\%.

Nökdil - ikton 832.

Nölling 293.

Nürz 284.

Nörz. Wiefelein 285.

Nüfch te nohilo 799 .

Nüfenj-kutya 799:

Nït 831.

Nondo 807.

Nouna 832.
Nonno 809.

Norak 826:

Nord-Caper 765.

Nordkaper 76.5. 770. 786 .

Norka 285. 308 .

Noforog 796 .

Nottolo 794 .

Nozorozec 796.

Nuggis 809 .

Niilmaus 455 .

Nüllmaus 455 .

Numba 796.

Nimola 822.

Nurek 235.

Nurk 803.

Nuísbeifser 524.

Nutria 808.

Nyul 822.

Nyulzt 809 .

Nyl.gau 625 .

Obah 812.

Obmaus 469.

Obnbär 320.

Obymatis 469 .

Ochodona 823.

Ochle 682. 683. 692.

- wilde 685.697.

- afrikanificher wilder 685.

- in Madagafear 69x.

- abylinifche 693 .

- mit hangenden-Hürnern 693.

- grunzende 698 .

- Kleine afrikanifie 703.

- vom Gebirge Nerever 704.

- von Dugueia 705.

Ochfenefel 725 .

Ochenpferd 725 . 
Odder 808.

Odobenus 124. 135.

Oecküs 832 .

Oedipus. Simia 45.

Oekör 832.

Oen 829.

Oefch 832.

Oetlihki 828 .

Oez - bak 827 .

Oez - nöfteny 827 .

Ogatona 558 .

Ohbo 832 .

Ohihoin 820 .

Ohnbart 46.

Oktficha 830 .

O theha kam 830.

Oktfcha . fchiru 830 .

Olbo 821 .

Olen' 825 .

Oleniza 825 .

Olen' pramorogoi 826 .

Olgobutich 8or.

Olulta 812.

Olyfant 796.

Olyphant 796.

Omokolotich 794 .

Ona 828.

Onager 719 .

Onca 223.

Once 227.

Ondathra 423 .

Onke 220.

Onneck 832 .

Onotatiri 725 .

Onza 224 .

Opoflum $345 \cdot 346$.

Ora 817 .

Orang 796.

Orang-Outang II.

Oran. Utan 12.

Uravas 820.

Orca 785 .

Oreotagus 637 .

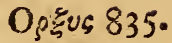

Ori 834 .

Orignal 59r.

Orne 837.

Orochtfchofchach 818.

Oron 825.

Orofzlani 804 .

Orre 820.

Orraw 820.

Orrya 826.

Oryx 636.639.

Orfo 81 I.

Ofch $8 \mathrm{r} 2$.

Ofcha 833 .

Ofel 835 .

Orlitza 835 .

Offo 81 I.

Offo hormiguero 95 :

Offon 796.

Oefzuer 835 .

Otter $276.28 \mathrm{r}$.

- ägyptifche 253 .

- brafilianifche 279 .

- kamt fchatkifche 276 .

- gemeine 281 .

- grofse - gelbliche von Guiana 313.

- von Siam - graue von Ba: tavia 314.

Otzkii 828:

Oualofes 828 .

Ouaikaré 795 .

Outiri 795 .

- onacil 795.

- onafiu 795 .

Oufta 812.

Ouifiti 44 .

Ouariri 95.

Ounce 807.

Ourana 815.

Ourizo 814.

Ours 81 r.

Ovelha 829. 


\section{nach den Seitenzahlen:}

Ovis 660.

Owca 829.

Owcze 830.

Owen 829 .

Owieczka 829.

Owza 829.

Ozelot 225.

Ozlot 225.

\section{p.}

Paardt 833 .

Paca 401 .

Paco 576 .

Pacnene 403.

Pacos 576 .

Paerdt 833.

Paettro 826.

Pag 402. 403.

Pagi 230.

Pague 402.

Pailan-langi $82 \mathrm{r}$.

Packian 171.

Palatinaffe 35 .

Paleng 804 .

Pall 821.

Palmen-Eichhorn 502.

Palmenratze 502.

Palmint 502.

Pan 15.

— der kleine 38.

Pangölin 99.

Panggulling 795 .

Pangnck 826 .

Pangolin 99.

Panifeus 38.

Panther 217.223 .225 .

- - der kleine 219. 220.

Panthera 219.220. 221 .

Pantherkatze 206.

Panterthier 218.220 ,

Panzerthier 102.
Panzerthier , wiefelähnlicheł 99.

- - mit dem Hundskopf 103.

-- hundsköpfige 113

Papio 16. 18.

Papion 18.

Papuine 838.

Parachadora 795.

Paran 829.

Pardalis 225.

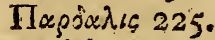

Pardel 218.

Pardelkatze $20 \%$.

Parder 218.

Pardis 217. 21.9.

Parduz 805.

Parforce.Hund 164 .

Paria 913.

Paripa 834.

Parna 833.

Parfpi 837.

Parfs 837.

Pafan 636 .

Pafeng 647 .

Pafquiros 749 .

Patas 33.

Pathew 82 .

Patira 749.

Patkany 814.

Patryshond 800 .

Pavian I6. 17.

- der kleine 7 .

- der braune 17.

- der gemeine 18.

- der graue 21.

- der fchwarze 49.

- ein neuer $5 \mathrm{r}$,

Pecaree 749.

Pecari 748.

Pecora 562.

Pegymet 81 .

Pekan 287: 
Pelandor-arn 813.

Pelas 838 .

pelladia 795.

Pelle 817 .

Pengeg 827.

Pengufch 834.

Pen-rotta 817.

Penvifch 839.

Perak 795.

Peregufna 8Io.

Pericnligero 88.

Perillo 801.

Perillo di falda 800 .

Perlunaus 444.

Pernak 839.

Pernichcatl 824.

Perro 798.

Perro baco 801 .

Perro de aqua 500.

Perrograndé 800 .

Perfpicillatus 65.

Pern 832.

Peruanifche Schaf 57.2.

Peruguenaife, der vierfingerige

$$
50 .
$$

pered 835.

Pes 799.

Pefez 804.

Péflii 530 .

Peftap 813.

Petaurifta 14.514 .516 .

Petze 150.

Pezze Mouller 797.

Pfeilmaus 530.

Pfeilchwanz 516.

Pferd 706. 707.

- wilde 711.727.

- herumirtende 7Ix.

- zahme 712.

- Arreifges 727 .

- indianiches 728 .

Pferdebär 319.

Pferdehirfcb $597^{\circ}$
Pfir 817.

Pflockfifch $769 \cdot 770$.

Phaeus 468 .

Phalanger 353.354 .

Phar 817.

Pharaonsmaus 252. 253. $41 \mathrm{I}$.

Pharaoratze 252. 253 .

Phatagin IOI.

Philander 341.344 .542$.

Phill 796.

Phoca I32.

- Lupina 148.

- Elephantina 135.

- Leporina 149.

- Fafciata 149.

Phocalna 781 .

Phoque 797 .

Phyfalus 765 .

Phyfeter 773 .

Pi 799.

Pian 347 .

Pianga, 825.

Picari 749.

Pickery 749.

Pictus. Vefp. 79.

Piculi 405.

Pies morski 798.

Piefek-ziemni 818 .

Pika 823.

Pillich $52 \mathrm{r}$.

Piloris 425 .

Pinä 799 .

Pinche $45 \cdot 46.48$.

Pindfviin 814 .

Pipiftrellus 76 .

Pirä 802 .

Pirdah 835.

Pifchtfchuga 485 .

Pifczucha 823.

Pifekn 820.

Pithecia. Simia 43.

Pithecke 14.

II. Anros 15 .

Pithe- 


\section{nach den Seitenzahlen:}

Pithekos 14.

Platogni 606.

Platthorn $63 \mathrm{I}$.

Plebun 772.

Poëfe 821 .

Pohano 603.

Pödder 825.

Podenko 800 .

Podje 356 .

Polarbär 324.

Polarfuchs 203.

Polecat 810. 221.

Polledro 832.

Pommer 153.

Pon 79ध.

Pongo 10. II.

Popyelycza lâtayacza 821 .

Pore 836 .

Porca 836 .

Porcaria. Simia 20.

Porcellino d'India 815.

Tore : epic 815.

Porc - epys 396.

Porco 836 .

Porco-Barráo 837.

Porco efpinho 815.

Porco montez 836 .

Porco filvatico 836 .

Porco fpinofo 815.

Porcupine 398.815.

Porcus 746.

Porosja 837 .

Porpes 839 .

Porpoiffo 839.

Porquinho 837.

Porofenok 837.

Polfenreiffer 43 .

Poffum 348.

Poftkaneel 564 .

Polatuche 5 I I $5 \times 3.514$.

Porpefien 783 .

Potfifch $773.774 \cdot 776.786$. Potjak 829 .
Potranka 833.

Potro 833 .

Potto 54. 90. 272.

Pottwallfifch 775.

Potvifch 839.

Potzchori $80 \%$.

Pouch 821 .

Pouh 521 .

Poulain 833.

Prjefe 832 .

Priquiza 795.'

Prikel 825.

Prifs 806.

Pryf , Llwgd 8I2.

Pryf-penfrith 812.

Przewiarka 810.

rfchi 826.

Pfi 799 .

Pfitza 799.

Pteropus 60.63.

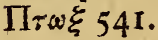

Pua 797 .

Puant 347.

Puccarara 406.409 .586$.

Pudel 156.

Pudu 680.

Puerca 836 .

Puerco 836.

Puerco efpino 815.

Puerco montes 836 .

Puerco filveitre 836 .

'Púffel 70r.

P'ug - Dog 800 .

Puh $82 \mathrm{r}$.

Puloh 821 .

Puina 229. 230.

pumi $8 \mathrm{co}$.

Purialio 444.

Pinu 799.

Pupi 805.

Hupperever 794.

Puri 832.

Purke 837.

I k k

Purpus. 


\section{$R \circ g$ if $\circ r$}

Purpurmaulwurf 365 .

Puirfchhund 167 .

Purfe 798.

Purfos 837.

Puru 832.

Pufché 825. 832 .

I'utois 810 .

Putorius 260. 264. 297.

Putoro 810.

Pütfche 826.

Puzolo 810.

Pygarg 628.

Pygmäe II.

Pyrame 157.

\section{Q.}

Qott -el - barr $80 \%^{\circ}$

Quacha 729.

Quogelo IOr.

Quagga 729.

Quagge 729 .

Quaiha 835 .

Quarthla. coyamotl 838 .

Quapizorl 838.

Ciafli 202. 803.

Ruasje 259.

Quati 39.

Quato 38.

Quautechallotl 493.

Queen.ja 815 .

Quelli 805.

Cuickhatch 335 .

Quimichpatlan 82I.

Quique 312.

Quinia 394.

Quota 39.

Quntto 38 .

R.

Ras 827.

Raa. Buk 827 .

Raa. Dyr 827.
Rabbet 480.823.

Rackun 333.

Raccoon 333.

Radjur 827.

Radja. outang 804.

Räv 803.

Rakle 798.

Rako 805 .

Ram 829.

Rammler 537. 551.

Rangifer 600. 603.

Rapofero 801.

Rapoza 803.

Rapphöns.hund $80 \mathrm{r}$.

Rata 817.

Rafomaka 812.

Raffeluaus 520.

Rat 8 í.

Rata $8 \mathrm{r} 6$.

Rat de bois 3.48 .

Rat de Pharaon Sos.

Rat dort 821 .

Rat d'or 821 .

Rutel 273 .

Ratelmaus 255.

Rat - musqué 816.

Rato 816 .

Raton $333.81 \%$.

Rat Sauterelle 8:7.

Ratte 428. 522. 531.

- grofse braflianifche 402.

- grofse 427.

- graufchwärzliche, norwe. gifiche $42 \%$.

- wilde 427.

- huipfende $42 \%$.

- weirse 433.522.

- oftindifche geftreifte 444.

- celbe 521 .

Ratte ì grande queve 817 .

Ratte couëtte 818.

Rattel 273 .

Rattenkönig 43 I. 


\section{nach den Seitenzahlen.}

Rattenmaus 429.

Ratto 816.

Rattus 428.

Ratze 298. 350. 429. 475 . $^{\prime}$ 520.

Ratze von Madagafcar 58.

Rauchfchwanz 457 .

Raude 803.

Ravale 344.

Rearnoufe 794.

Red Deer 825.

Reen 796.

Rehe 586.607 .609 .631 .

- guineifches 58r.

- ungẹfchwänzte 586 .

- mexikanifate 608 .

- brafilifche 608 .

Reh 609.

Rehbock 609.

Rehböcke 607.

Rebbücklein 582.

Rehehille 609.

Rchekätzchen 600 .

Rehialb 609.

Rehkuitzlein 6 c9.

Rehueque 574.

Rehziege 609.

Rehzicglein 582.

Reiner 60I.

Reismaus 468.

Reitmais 470.

Rellmaus 520.

Rellmoure $82 \mathrm{I}$.

Rellinurs 821 .

Renard 803.

Renard Charbonnier 803 .

Renard marin 797.

Renenioule 794.

Ren 826.

Renı 600.

Renne 825.

Renunhirsch 601.

Rens. Dyr 825.
Rennthier 598. 603.

Reft. Kutya 795 .

Reutmaus 376.

Rex Simiarum 38.

Rhee 827.

Rhen 826 .

Rhinoceros I I3. II 4.

Riccio 814.

Rieke 609.

Riefen 7 .

Rillow 32 .

Rind 688.

Rinder 588.

Rindvieh 688 .

- - abyininifches 693.

- - von Tinian 693.

- - aus der Barbarey 633:

Ringauge 55.

Ringelbär 319.

Pıvoxepars 116 .

River torfe $835^{\circ}$

Rivier-Paard 835 .

Kob 798.

Robbane 803 .

Robbe 132. 137. 139. f. See. hund.

- mit der Mahne 137.

- gemeine 538 .

- mit der Kappe 145 .

Roe 827.

Röd-Dyr 825.

Röffeisvifsla 820.

Rörqual 766 .

Rüskatt 8II.

Rola 803.

Rollmaus 520.

Roloway 35.36 .

Rone 837 .

Ropue 138.

Roquet 150.

Rofalia. Simia $4 \%$.

Rufenaffe 47.

Rofomak $33 \%$.

$\mathrm{Kkk}$ 


\section{$R \bullet g$ ifter}

Rors 707.

Rofshirfch 596.

Rofsmaul 125. 126.

Rofsmer 126.

Roftinger 127.

Roftunger 125.

Rot 816.

Rothfuchs 195. 199.

Rothhirfch 59 r.

Rothluchs 244.

Rothmaus 460.

Rotta 816. 817 .

Rotte 816 .

Ruttevanger 807.

Rougette 62 .

Rouffette 60.6r.

Ru 802.

Rubbe 139.

Rugette 62.

Rüf 803 .

Rïfelmaus 442 .

Riiffelf the Hund 162 .

Riufelträger $255^{\circ}$

- - der vierzehige 255.

- - der rothe 256 .

Rüffeträger $357^{\circ}$

Rukea 820 .

Ruma 834.

Rupfhafen 553 .

Rupicapra 620.

Rupfok 803 .

Ruffor $127^{\circ}$

Rys 807 .

5.

Sà ã 827 .

Saalhund 138 .

Saarmas 808.

Saar - tien - Kanat 821 .

Sabaea. Simia 28.

Sabbel 809 .

Sabeldier 809 .
Sabelinus 818.

Sable 809 .

Säbelfifch 788 .

Sïg 799 .

Sägedelphin 788.

Sael Kaabe 797.

Sälihund 132 .

Saez 822 .

Sagau 826 .

Sagif 808 .

Sagitta $515 \cdot 529$.

Sagoin $43 \cdot 44 \cdot 48$.

Sagoins 43 .

Sagouin 45.48 .

Sagouinchen 43.

Sagovin 43.45.

Sahouesquanta 821.

Saibch 80!.

saiga 626.

Saige 626 .

Saino 838 .

Saju 41 .

Sak 820 .

Sakiloors 794 .

Saki 43.

Sakildan - tflian 819.

Sakkis 823 .

Salhund 139 .

Samfier Sog.

Sandhafe 537. 544.

Sand-Klematige 470 .

Sandmanlivurf $47 \mathrm{I}$.

Sandmaus 467.474 .

Sandmoll 470.471 .

Sandziefel 468 .

Sanglicr 836 .

Sanglin 45 .

Sanguifuga 65.

Sapajis 36.

Sapaju, der braune 41 .

- der gelbe $4 \mathrm{I}$.

Sapajou aurore 794 .

- - jaune 794. 
Sapajou orange 794.

Saphan 823.

Saribur 336 .

Saricovienne 279.

Sarige 346.347 .

Sarluk 698 .

Sarm 808.

Sarmaticr 303.

Sarn 827.

Sarna 827.

Satfchkam 817.

Sattler 143 .

Satyrus. Simia II.

Sall $736 \sigma^{\prime} 743$.

Sambeller 17 I.

Saufich 785 .

Saufinder $17 x$.

Sauigel 385 .

Sauriiden I 7 I.

Savia 401.

Sawádu punei 808.

Scalopes 350 .

Scarvas. borjú 826.

Schaaf 66r. f. Schaf.

Schaafe 665. 667. 671. 673 .

Schachaz 804 .

Schaep 829 .

Schacr 813.'

Schär 359.

Schaf 660.

- fyrifches, mit lappigen Ohren 653 .

- englifches 663.

- hornlores 664 .

- nordifches 664 .

- rufirche 655 .

- fpanifche 665.

- gothländifche - isländi. fehe 665.

- afrikanifche $66 \%$.

- äthiopifche 657 .

- gaineifche 668.

- angolifche 608.
Schaf, fenegalifche 668 .

- in der Sahara 659.

- indianifche 669.

- fett feliwänzige 669. $67 \mathrm{r}$.

- arabifche 669.

- mit deni dicken Schwanze 675.

- breitfchwänzige 670.672.

- orientalifrhe 670.678 .

- kalmïckifche $67 \mathrm{I}$.

- kirgififche 67r.

- perfifche und tatarifche $67 \mathrm{I}$.

- abeffynifche $67 \mathrm{r}$.

- bucharifche 672 .

- langgefeliwänzte 672.

- ticherkaflifche 673.

- capiche 673.

- clementinifche 675 .

- wilde 678.679.

- cretifche 680 .

- hochgehörnte 680.

- wallachirche $68 \mathrm{I}$.

Schafdromedar 573 .

Schäferhund 150 .

Schafkameel 57 I. $575 \cdot 576$.

Schafried 799.

Schaga 820.

Sthagalf 802 .

Schaufelnafe 66. 67.

Sehakal rgo.

- - der capifche 192.

Schandalgan 822.

Schank 808.

Scharrmais 358. 449. 455 .

$47 \mathrm{I}$.

Sithaufelhirfch 604.

Schenfar 250.

Scheermaus 449 .

Scherrmauss 357.

Schepek 820.

Schiarro 820.

Schibbenlanm 66r.

$\mathrm{kkk} 3$ 


\section{$R \in g i f t e r$}

Schila 819.

Schildkrörenigel I0\%.

Schildkrötenfchwein 107.

Schildfchweinchen III.

Schildverkel ro5. II I.

Scbillke 66r.

Schimpanfe 10.

Schir 8:7.

Schiratto 820 .

Schiratiolo 820.

Sitiriuolo 820 .

Schlachtbiar 320.

Schlachtochfen 693.

Schiäfar 5 I8.

Schlaforer Ziefel 468.

Schlafmaus 443.

Schlafinäure 444 .

Schlafratte 518.519 .524 .527$.

Schlatratz 520.

Schläfrige in Amerika 88.

Schlaffichtige 87.

Schlangentödter 254.

Schleicher 53.

Schileuderaffe 13 .

Schmalricken 609.

Schmalthier 591.

Schnabelfifch $757: 77^{2}$.

Schneewiefel 31 r.

Schnorrmans 455 .

Schórdichu 826.

Scholongo 810.

Schonska 8r6,

Schont: 802:

Schöpe 820.

Schïps 660.

Schörinaus 455 .

Schorda 825 .

Schofshund 159 .

Schrauhhornfchat 68I.

Schrifrantilope 640.

Schrotman:s 520 ,

5 chual 803.
Schtualim 192.

Schulak 803 .

Schup 333 .

Schupp 332.

Schuppeneidechfe 98 .

Schuppenthier 98.

- - lurzgefcliwänzte 99.

- - fünffingerige 99 .

- langgeŕchwänzte ror.

- - rierfingerige IOI.

Schuppotter 286.

Schurks 816.

Schutturhic Baad 824 .

Schutur 824.

Sehwanzratte $35 \mathrm{I}$.

Schwarzohr 201.

Sihwein 736.744.

- wilde 736.749.

- zahme 740.

- cimhufige 743 .

- mit ungefpaltenen Klauen 743 .

- chinefilche 744 .

- famifche 744. 747.

- javanifche 744.

- guineifche 746 .

- langöhrige 746 .

- af̧ri!ranifche 750 .

- capfche 750.

- äthiopifche 750 .

- ungeheure wilde $75 \mathrm{I}$. 753.

- gehörntes 752 .

- oftindifehes fonderbares 763.

Schweinchen, indianifches 410.

Schweinhirfeh 752 .

Schweinfchwanzaffe I6.

Schweinigel 382.

Schweifshund 163.165 .

Schweitzer 506.

Schweli 826 .

Schwerdtdelphin 788 . 
Schwertelinaus 457 .

Schwulskopf 64 I.

Sciurea. Simia 40. 4I.

Sciurus 487.

Scoaffu 614.

Scoiattolo 820.

Scrag - Whale' 839.

Sczenja 799.

Sczenok 799 .

Sceaffe 131 .

Sea. Kow 797.

Sea-lion 797 .

Sebey 804 .

Sebra 835 .

Seebär I 32. 133.137.

Seebeluge 789.

Seebiber 278.279 .

Seeeinborn 755 .

Seehund 132. f. Robbe.

- der gemeine 137 .

- dritte Surte 139.

- graue I4I.

- fibirilche 142 .

- cafpifche 142.

- fchwarzfeitige 143.

- grönländif́che 143 .

- mit dem Mond I 43 .

- andere Sorte 143 .

- rauhe 144 .

- mit einer Haube 146 .

- grofse 146.

- geöbrte 147.

- hafenhaarige 149.

- bandirte 149.

Seekalb 134 .

Seekatzen 134 .

Seckocjen 797.

Seekuh 125. 129.

Seelöwe 134 .

- - der glatte 134 .

- - der zottige 136 .

Seeuchs 129:

Secotter 278 . 279 .
Seepferd 125. 126. 730. 731 .

Seerga 826 .

Seethiere, fäugende 755 .

Seevarken 785 .

Sehrnolihts 8II.

Seibbra $8 \mathrm{rr}$.

Seidenbudel 158.

Seidenhafe 553 .

Seidenhund 158 .

Seidenkaninchen 553.

Seiga 626.

Seigak 626 .

Sel 797.

Selldenus 827 .

Selr 798 .

Semivulpa 346.347 .

Semlanaja - Medwedka 818.

Semleroika 818.

Semljana bjelka 82 I.

Semlianaga Medwedka 47 r.

Semljanoï - Saëz 822 .

Seniculus. Simia 37.

Seraphah 827.

Serna 827.

Serotinus. Vefp. 75 .

Serpentum pabulum 534.

Serval $24 \mathrm{I}$.

Setzhafe 537.

Sghimel 565 .

Sheep 663 .

Shepherds - Dog 799 .

Sherebenok 833.

Sherebetz 833. :

Shira 809.

Shitnik 817.

Sfochor - nomon 819 .

Shock 800 .

Shrew 8r4.

Si 8 ro.

Síacali 802 .

Siacalle 802 .

Siachal 802.

Siäl 798 .

Kkk 4 
Sjafamuri 810 .

Sjàrda 825 .

Sibirian Goat $83 x$.

Sic - cufe fue 811 .

Siebenfchläfer 519.524 .525$.

- norwegifcher 427 .

Sjenaftawetz 823 .

Sifak 32 .

Siffleur 482 .

Sig 709.

Si gah: Ghurh 806.

Sihkfparne 795 .

Siil 315.

Sjir 804.

Sikfi: 820 .

Silberbär 318.

Silberfuchs 20 r. 202.

Sild: Qual 755.

Silcnus. Simia 23.

Sinita 9.

- callitrichus 33 .

Simio 793.

Simral 818.

Singe 793 .

Singe de nuit $794^{\circ}$

Singe rouge 794 .

Singes 37.56 .

Sinkurata. kutya 80 r.

Sinfin 793.

Sinfkoë.-cht!chenjae 818 .

Siraffa 615 .

Sirene 131 .

Sifel 819 .

Sisnà. iga 836 .

Sisnah 836 .

Sion 796 .

Sjum 834 .

Sjupp 333.

Sjutfcha 798 .

Siwutfcha 798.

Siyah ghufin 243 .

Skalna 809 .

Skilachi 802.
Eurovpos 492.

Skoghsmus 82 r.

Skop 830.

Skunk 260.

Skrzeczek 818.

Sleeper $82 \mathrm{I}$.

Slehbak 838 .

Slepez 470.472.

Sijepufchonka 818 .

Sijepunfchonka 814.

Slinger - asp 793 .

Slon 796 .

Sloth 795.

Smale 829 .

Smitten 50.

smofar 809 .

Snümus 81 I.

Snok 812.

Snop SI2.

Sobaka 799 .

- gonczaja 80 r.

- - meikeljanskaj3 800.

- - wirhlaja 800.

- ifpanskaja 8or.

Sobeln 296.

Sobol 809 .

Soboli 810 .

Sochat 825 .

Soëgûn 826 .

Soe 837 .

Süe-Koe 797.

Sölzficham 81 7.

Sogfchwein 742.

Soi $4 \mathrm{r}$.

Sonatli - Schüfchi 794.

Songarus 468 .

Song - Sehu 275.

Sooffar 809 .

Soofar 809 .

Sora 385 .

Sorex $36 \%$.

- aureus 366 .

- Mofrovitiens 372. 
Sorex carinatus 373.

- Daubentonii 373 .

- Tetragonurus 380.

- conftrictus $38 \mathrm{I}$.

Sorice 817 .

Soricinus. Vefp. 68.

Soroch 829.

Soud 829 .

Souris 817 .

Souris de terre $817^{\circ}$

Souris d'eau 814 .

Souslic 483 .

Suw 837 .

Soydur 830 .

Spaka 799.

Spalax 470.472 .

Späting 76 .

Spannferkel 74 ז.

spafuia 67.

spectrum 64 .

Specthatuer 788.789 .

Speck - Hugger 789 .

Spleckmans $72 \cdot 73 \cdot 75$.

- - die grofse 75 .

Speenvarken 837 .

spermafe 68.69

Speicherwiefel 310 :

Sphinx. Simia 17. I8.

Spiefsbücke 609 .

Spiéfshirfch 5) $\mathrm{x}$.

Spiefskalb 591 .

Spinofo 815.

Spitz 153 .

Spitzkopf 469 .

Spitzmaus 367 .

- - kleine ungefchwänzte 369 .

- - amerikanifehe 370.

- - grabende 373.

- javanifche 374 .

- - gemeine 374 .

- - furinamifche 378 .

- - perfífbe 378 .
Spitzmaus, brafilifche 378 .

- - kleinte gefchsänzte 379.

- eine Art unglanblich kleiner 379 .

- - mit vierfeitigeu Schwanze-380.

- - mit verkehrtem Sehwanze 380.

- - welche nach Mofchus riecht $38 \mathrm{I}$.

- - gröíste vom Cap 38r.

- - von der Hudronsbay 381.

Spitzmuis 814.

Spitzohr 746 .

Springbock 629 .

Springer 527. 529.532.535. 782.785 .

Springfuls 488.

Springhafe 527. 529. 533.

Springratte 427 .

Springratze 529 .

Spritzwall 756 .

Spürhund 164. 165. I66.

Spürwiefelchen 253.

Squafch 275 .

ธ̧ӓа 828 .

Stacheligel 389.

Stachelrücken 398.

Stacheithier 392. C. Stachelfchwein.

Stachelfchwein 388. 392.

- - von Malacca 387.

- - afrikanifche 392.

- - mit dem Bufche 393.

- - gefchwanzte 396 .

- - chilefifches 397.

- - verlarvtes 398.

- - amerikanifches 398.

Stänker 298.

Stänkermart 299.

$\mathrm{Kkk} 5$

Statir. 


\section{$R \in g i f \in r$}

Stänkerratze 298.

Stag 825 .

Stammocilen 689 .

Stanr-Hyning 839.

Steckelvarken 815.

Steenbock 829.

Steer 831 .

Steinbär 320.

Steilohr 193.

Steinbock 656 .

- - unbärtiger 626 .

- - afrikanifeher 631 .

- kaukafifcher 659.

Steindocke 161 .

Steingeis $62 \mathrm{I}$.

Steinfuchs 203.

Steinhafe 537.544. 558.

Steinhund 284.

Steinkatze 24I.

Steininarder 288.

Steinfchaf 679.

Steinziegen $62 \mathrm{I}$.

Steipe-Reydur $77 \mathrm{I}$.

Stier 683.

- wilde 684 .

- zahme 688.

- weirse madagafearifche 693.

Stierhirfch 633.

Stinkbinkfen 265.

Stinkdachs 265 .

Stinkthier 251. 258.260. 298.

Stépnaja Kof́chka 806.

Stepnie - Baranni 679. 831.

Stepnoi Baran 830.

Steppenfúchre 200. 201.

Steppenziege 626.

Srour. Vagnen 787 .

Strat 810 .

Stoc!maus 455 .

Stührla.nm 660 .

Stör 810.

Stolye 801.
Stofsmaus $454 \cdot 455$.

Straubfchaaf 620 .

Streifmaus 44I. 443.

Strepficeros 680. 681.

Stfchuitích 816.

Strumpfichiwanz 469.

Sturmfifch 786.

Stute 707.

Stutte 707 .

Sucheríkan 818.

Sue - Hual 839.

Sugur 819.

Suhac 626. 828. 834 .

Suka 799.

Suka Guinoka 799.

Sumpthafe 537.

Sumpfinaus 450 .

Sumpfotter 284 .

Sumpfotternarder 284 .

Sumpfratte $45 \mathrm{x}$.

Sumpfrchwein 412.

Sumura 819.

Sumxu 274 .

Sunfta 807.

Surikatte 255 .

Surk 813.

Surok 819 .

Surman 817.

Surmulot 426 .

Sus 736 .

Sufär 809 .

Sufel 819.

Suslic 484.

Suffi 802.

Sufuatha 834.

Suta 826.

Suur 819.

Suver 819.

Suwfár 809.

Svartrüde I 43.

Srimfok 812.

Sviin 836 .

Swift-Antelope 828. 
Swiffch 819.

Swin 836 .

Swinja 836 .

Swinka-Zamorska 815.

Sylaufyn 807 .

Sylvanus. Simia 13.

Syräth 810.

Syrichta. Simia 42.

Szamàr 835 .

Szamai kantza 835.

Szarwas \&26.

Szarvat 826.

Szavia 401 .

Szelendek 800.

T.

Taaleb 803 .

Tabjek 820.

Tache 150.

Tachs 329.

Tackah 8?9.

Taegulttchitfch 454 .

Tagala 794.

Tagfchläfer 520 .

Taguan 516 .

Tagui 817 .

Tai 833 .

Tajacu 749 .

Tagàh 829.

Tajafu 748.

Taibi 347 .

Tai . ibi 344.

Tajoufiu 838.

Talàh 829 .

Takja 829.

Talai 823.

Talapoin 34.

Taleb 803.

Talpa 357 .

Tamandua.I 96.97.

- - minor 87.

Tamandua, der kleine 92 .
Tamandua - guact1 94.95 .96

Tamanoir 94.

Tamari 48 .

Tamarin 46.47 .48$.

Tamarifkenmaus 534 .

Tamaríkenratze 535.

Tamendoa 95. 100.

Tamma 834.

Tanneninarder $29 \mathrm{r}$.

Tannenwildkalb 604.

Tannhirfch 604.

Tannkützle 604 .

Tanrec 390.

Tannki 315 .

Tapeti 555 .

Tapihire 836 .

Tapir 733.

Tapirete 733 .

Tapirouffou 836 .

Tapüre - ete 73.5 .

Taquan $82 \mathrm{I}$.

Tarandus 598.

Tarbagantfchik 822.

Tarpugannen 482.

Tarfer 356.

Tarfier 356.

Tartar 626.

Tarw 83i.

'Tafch 822.

Taflo 812.

Tatou IIO. II I.

Tatou - Kabaffu 112.

Tattu III.

Tatu I02. 103. III.

- muftellinus 99.

- Wiefel 104.

- apara 105.

- porcinus 105. III.

- mit drey Reifen 105.

- mit fechs Reifen 107.

- mit acht Reifen Ira

- haarigter $4 \mathrm{II}$.

Tatuette 108. 109. 1 ro. 
Tatupeb 107.

Tatupeba 107.

Tauling, Lengen $82 \mathrm{I}$.

Taumler 782.785.

Taupe 813.

Taureau 831.

Taureau-cerf 636 .

Tauifh 150.

Taufsham 822.

Taufthakki 822.

Tau. Tökiké 829.

Tauvar 838 .

Tava 817 .

Taxus 328.

Taijra 286. 287.

Tayac̀̀ 749 .

Tchin 795 .

Techichi 174 .

Tegõ 824.

Teguktfchitfch 454 .

Tehén 832.

Teixugo 812.

Teletz 832 .

Telja 832.

Telitza 832.

Telufchka 832.

Temamacama 608.

Temjàm 824 .

Tenderever 794 .

Tendrae 389.

'Tengeri - Nyul 823.

Tenlie 803.

Tepe Maxtlaton 231.

Ternera 83 .

Terrianiak 803.

Tenfel 19.

- der javanifche 99.

- formofanifche 98.99.

- chinefícher 99.

- tajovanifcher 100.

- der vierfache 688 .

Teufelskind 298.

Tere 185.824.
Texon 812.

Texigo 812.

Tezerdea 253 .

Tha 830.

Thaka 839.

Thapra 823 .

Théío-tu 822.

Thevang 53.

Thier $59 \mathrm{r}$.

- groises 633.

- im Rohr 732.

- das ritterlicke 737.

- das Pinkende 26I.

- ein gewiffes vierfüfsiges. $26 \mathrm{r}$.

- fo den Zibeth tragen 269.

- ein amerikanifches $334^{\circ}$

- ein namenlofes 352 .

- ein fonderlich rauhes 46\%.

- die aus den Wolken kom. men $46 \mathrm{r}$.

- " die zu den Feldmäufen gerechnet werden 474.

Thierkalb $59 \mathrm{r}$.

Thierwolf 246 .

Thinfchemet 813.

Thöle 150.

Thoes 192.

Thona 832.

Thos 190.

QWe 192.

Thous 186.

Tjäkko 30.

Tichâth 833 .

Tien 820.

Tieve 150.

Tiffe 185.

Tiger 214.224 .225$.

- königliche 2 I 4 .

- ceylonifche 215.

- afiatifcher 215 .

- junge ceylonifche 220.

- amerikanifche 224. 229. 
Tiger, mexil anifcher 224.

- von Guiana 224.

- der fchwarze 228.

- der gröfste 228.

- der rotise 229. 230.

- der rothbraune 229.

- von Patagonien $25 \mathrm{I}$.

Tigcrbufchkatze $24 \mathrm{I}$.

Tigr 804.

Tigre 804.

Tigre d'Afrique 805.

Tigerhund 106.

Tigcriltis 303 .

Tigerkatze 224. 23 I. 24 I.

- - amerikanifche $23 \mathrm{I}$.

Tiegerthier 214.

Tigerwolf 189.227.

Tijin 820.

Tiin 820 .

Tikagulik 839.

Timuch 339.

Tin $3 \times 5$.

Tirta-tarek 82 r.

Tif́hiutfchku 828.

'Tjuja 824.

Tiümbune 802 .

Tjulen 798.

Tiur 83 .

Tiute 797.

Tkamna 825 .

Tkanna 825.

Tkarou 805.

Thenfie 803.

Tklou 80 s.

Tkonw 79 ?.

Tkumma 825 .

THlacooulot $85 \%$.

Tlalocelotl 805 .

Tlamototl 821.

Tlaquatzin $344.347^{\circ}$

Thatlauhqui ocelot 805.

Tôa 823.

Torlilyng - lange $82 \mathrm{I}$.
Todtenkopf 42.

Todtenküpfchen $4 \mathrm{r}$.

Töghé 829 .

Töris Difzno 814.

Tolai 547.

Tonga 61 .

Tongùs 833.

Tongusny 838 .

Tonkong 614.

Took 825 .

Tooifchkan 822 .

Toparagno 814.

Topinara 813.

Topo 813.

Toro 83.

Torqua 802.

Toulin - leyn 82 r.

Touro $83 \mathrm{I}$.

Toupeira 813.

Toufchak 822 .

Tovis 808.

Tquafouiv 805.

Tragelaptus 596.

Tragocanclus 625.

Trampelthier $56 \%$.

Trepida. Simia 39.

Tretretretre 50.

Trichecus 124.

Trichternaie 64 .

Troglodyt 10. II.

Troglodytes. Simia IO.

Troid - Hual 839.

Troldqual 775.

Trollifich 775 .

Truye 837.

Tfchaar 877.

Tfchaklal $r 9 x$.

T́́ciarma 833 .

Tf́han 833.

Trchafcliea 803 .

Trehatak 8 I3.

Tfchelagatfchitfch $47 \%$.

Tfcherkatisus glis 473 . 


\section{$R \cdot g$ ifter}

Tfchefchchak 772 .

Tf́lieta nauftchu 818.

Thchigitai $7 \mathbf{1} 6$.

Tf́icò 834.

Trchinäma 809 .

Tfehingeroh 475 .

Trchiki - tsgeni 834.

T́́chi -ötzcii 828.

Trchip 799.

'Trchipkaku 802.

Tŕchirketiai 716 .

Tŕctongifchtifche - Kolga 799.

Tf́chono 801.

Tfchrfin 814.

Tŕnhíbbuku 83 r.

Tf́chùni 833.

Trkan 81 7. $\$ 22$.

Trödür 834 .

Túa 837.

Tüï 824.

Tuabba 796 .

Tuean 367 .

Tüe 824.

Tuequal $77^{\circ}$.

Tugal 832 .

Tugalik 837.

Tuhouri 808 .

Tukalanda 837.

Tuk to 826.

Tulan 8c9.

Tuläux 837.

Tulen 797.

Tüllki 803 .

Tulpermaus 456 .

Tumblare 839.

Tumbler 801.

Tümler 782.784 .785 .

Tur 83 ?.

Turbagan 482.

Turnpit 801 .

Turfio 780.

Tufchkantrchik 822 .

Twerch daear 813 .
Twoba 796.

Tya- Ielman 822.

Tybuarte 413 .

Tyg 832.

Tygexburchkatze 240 .

Tygerkatze 224. 231 .

Tygerpferd 727 .

Tygwu 832.

Tyiin 820 .

Tyr 831.

Tzar 833.

Tzebi 53 $\mathrm{I}$.

Tzeiran 629. 63x.

Tzurban 815 .

U.

Uabr $25 \mathrm{C} .56 \mathrm{I}$.

Uagra 836 .

Uarin 37.

Ucumari 812.

Uhrda $8=0$.

Uggus 832.

Uguîfs 832.

Uhdenis 808 .

Ui 832 .

Ujing 8II.

Uilch 810.830.

Uif́chlal 832 .

Uifch ufch 832.

Uiftiti 44 .

vibuki 820.

Ul!.jugi $82 \mathrm{r}$.

Ulüjuki 820.

Ulf 801 .

Ulfidur 800.

Uluk 820.

Ula. Trkan $8 \mathrm{r} 6$.

Ulofechó 833 .

Umbulu 795.

Unagàn 834.

Uniagin 803.81 I.

Unaul 89. 


\section{nach den Seitenzaklen.}

Uncia 219.

Uneng 832 .

Unglidur 800 .

Unguniayo 413 .

Unhe 298.

Unze 219.

Ur 820.

Uralmaus 458.

Urchin 814.

Urdar-Kettir 235.

Urigne 148.

Urochs 684 .

Urfo 811 .

Urfon 398.

Urfus 316.

Uru 820.

Urugundfche - Cholgona 454.

Unis 684 .

Urwal 833.

Ufangi 822 .

Ufeh 833 .

Ufchkan 822 .

Ufehmith 833 .

Ufchnap 822.

Ufio 811.

Ut 833 .

Uta 817 .

Uiter 803.

Utfchku 828 .

\section{V.}

Vacca 831.

Vache 83 I.

Vache brume 827 .

Vache marine $79 \%$

Vade - Sal 143.

Vah!t 739.

Vakiondok 813 .

Valrus 125 .

Valska 816.

Vanıpyr 60. 64. 65.66.

Vampyrus 60.
Vandfire 809 .

Vanfire 287.

Várg 801 .

Vari 56.

Varken 836 .

Vat - kos 829 .

Vatushalr 839 .

Veau 83 r.

Veaumarin 798.

Vebar 823.

Vechio marino 797.798.

Veh 492.

Vembe 834 .

Venadito 826 .

Vendo 827.

Verdadeiro 796.

Verken III.

Verföhnungsbock 668.

Vefpertilio 60. 72 .

- - Borfippae 61.

- - Cynocephahis 6r.

- _ caudatus 66.

- Marfupialis 8I.

- - Marmotte 79.

- - Equinus 82.

- Veter. Simia 22.

Vetzere 800 .

Vicugna 574 .

Vicunja 575 .

Vicunna 574.

Vicunnas 575.

Vicuune 575 .

Vicunias 575 .

Viehmarder 29 r.

Vielfrafs 188.336 .337$.

Viclgok 803 .

vigogne 573.575 .

Vigogne kameel 575 .

Vinfifh 839.

Vis 809 .

Vifcalcha 537.

1 ifealibos 537.

Vifon 286. 


\section{$R \in g i f t e r$}

Vitelo 83 .

Vitello 83 I.

Vittata 264 .

Vitulus marinus 148 .

Viverra $25 \mathrm{I}$.

Viverre 275.

Vizsla 801.

Voang 8c9.

Volpe 803.

Volucella 5 ro.

Vond 813 .

Vos 803 .

Vulpecula 258.

\section{W.}

Wabus 546.

Wachtelhund 157.158 .165$.

Wachthund 162 .

Wadur 829.

Währwolf 180 .

Wagthond 300.

Wahrtae 820.

Wahwaris 820 .

Wal 333 .

Waldantilope 643 .

Waldboch 641. 643.

Walderel 719.728 .735 .

Waldgeift 24 .

Waldgott 16.38 .

Waldhündin 609 .

Waidkater 235 .

Waldkatze 234 .

Waldmaus 437.438 .454 .524 .

- - amerikanifche lang.

fuifsige $350.351 .353 \cdot 354$.

- - grofse 452 .

- - rothe 524.

Waldmenfch, der afrikanifthe IO.

- - der offindifche II. Waldnymphe 21 .
Waldratze $343 \cdot 345 \cdot 346.427$. 438.

Waldrchweine $747 \cdot 75 \mathrm{I}$.

Waldtenfel I 3. 39.

Waldwiefel 302.

Waldziki 831 .

Wallach $70 \%$.

Wallfich 760 .

- - geıeine grönländifche $76 \mathrm{r}$.

- - dreyfiofsige 768: 771 .

- Langgefchnauzte 768 .

- amerikanifche 770 .

- mit rundew Unterkie. fer 771 .

- kleinfte 772 .

- Eezahnte 787 .

Wallfifchŕchwanz 128.

Wallfíchtödter 789 .

Wallrath 777 .

Wallrathfifch 777 .

Wallrots 124.730 .

Waldthier 261 .

Wanderratte 426 .

Wanderow 24.

Wanduru 24.

Want 8 I 3 .

Waranam 796.

Warree 735 .

Warrefchwein 738.

Warg 801 .

Warglo 807.

Wariroe 95.

Warfa 834.

Was 832 .

Wafchbär $333 \cdot 334$.

Wafchina 833 .

Wátika 832 .

Wafterferkel 413.

Wallerhafe 413 .

Waflerhund -156.167 .

Wallertuh 730 . 


\section{nach den Seitenzahlen.}

Waffermaus 369.371 .373 . $447.448 .45 \mathrm{I}$.

Waffermurmelthicr. $37 \mathbf{r}$.

Wafterochle $73^{\circ}$.

Waflerratte 447 .

- - wohlriechende $37 \mathbf{1}$.

Wafferfchwein 412. 730. 734.

Wafferfpitzmaus 369.377 .

Waffer-Zei凡 448.

Water - Spaniel 800 ,

Wattia 826 .

Waui 803 .

Wauwauwen $5 \mathrm{I}$.

Weidrinder 634 .

-Weifsarfeh 628 .

Weifsbart 23.

Weefel $8 \mathrm{rr}$.

Weifsfifch 773. 778. 789 .

Weirsnafe 34.

Weifsfch wanz 369 .

Weifszahn 381 .

Wepr 836 .

Werblud 824 .

Werblûd Skoro begajufczu 823.

Wefla 810.

Wetraríelur 798.

Wewerka 820.

Wezel 810.

Whale 838 .

Whenial 826 .

Whitred $8 \mathrm{II}$.

Whunialrothe 826 .

Wianaque 824 .

Wjarges 802.

Wickelfchwanz 272.

Widder 660. 669. 671.

- guineifeher 668 .

- cyprifcher wilder 679 .

Wiederkätende Thiere $56 \mathrm{r}$.

Wiekricha 820

W'ieprz 836.

Wiciel 276.286 .298 .301 .309$.
Wiefel, ägyptifche 253 .

- amerikanifche, ftinkende $26 \mathrm{r}$.

- der fchwarze 286.

- ruffifche - fibirifche 296. 304.

- grofse 305.

- kleine 308.

- javanifche 314.

Wiefeleichhorn 502 .

Wiewiorka 820 .

Wewjorka lätajacza 821 .

Wikare. Siäl 798 .

Wild Boar 836 .

Wild Oxe 83I.

Wildkalb 591 .

Wildmarder 291 .

Wilks 802 .

WVill. Swin 836 .

Wind 168 .

Windhond $80 \mathrm{r}$.

Windhund 168 .

Windhunt $80 \mathrm{r}$.

Windkamel 565 .

Windlpiel, irländifehes 167 ;

- - tïrkifches 168

- - gemines 168 .

- - cotiges 169.

- Kleines 169.

Winfelaffe 40.

Winterfchläfer 518 .

Wirrebocerra 534 .

Wiribiciri 584.

Wifent 697 .

Wintagua 817.

Wifon 283 .

Wittich $774.773,789$.

Whäenoi krot 81 7 .

Wöll 833 .

Woik 772 .

Wohlriechende Rehboek 579.

- - Gerafe 579. 
Wölfe, junge 185 :

Viol 831 .

Wötín 185.

Wolczicza $8 \mathrm{cr}$.

Wolf 180 .

- der mexikanifche 185.

- goldgelber I9r.

- fehwarzer 194.

- von Neuholland 209.

Wolfsbär 335 .

Wolf fuchs 209.

Wolftitund 200. 153.

Volfspard 220.

Wrilfstieger 227 .

Wolik 801 .

Wolorh 833.

Wolvarne 335 .

Woadchuck 480 .

Woudiack 480 .

Womlein. Wikfeiein 303.

Worfelur 798.

Wonwou $5 \mathrm{I}$.

Wubbers 826 .

Wrichochol SI4.

Wiuchuichol 370 .

Wuiblnaus 455 .

Wüll 833 .

Wüliä 833 .

Wikikor 832 .

Wundernafe 82.

Wunderthior 188.

Wurzelnans 452 .

Wirzelzein 454 .

Wychuchiol 37 I.

Wydra 808 .

Yaguạne 275.

Yaguareté 224.

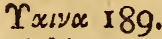

Ybrch 829 .

Ybrchen 658.

Ybfeh - Geyf 658.

Ych 83r.

Yerboa 531.

Yegua 843.

Yerbira 530.

Yhrugäh 819.

Ylf $80 \mathrm{r}$.

Ylgia 801.

Yly $80 \mathrm{r}$.

Yrka 8I9.

Yiart 828 .

Ýgifarnog 822.

$\Upsilon_{5 \rho 1 \xi} 306$.

Yzeren. Verken 815.

Yzer - verken 394.

Yzquiepatl 258.

\section{Z.}

Zacalia 802.

Zache 150 .

Zacl 68r. 83r.

Zïrtling 445 .

Zaiac 822.

Zaino 838 .

Zalofés 828 .

Zatze 150 .

Zaushe 150.

Zauna 809 .

Zaunigel 385 .

Zaupe 150 .

Zcheni 834.

Zchwari 830.

Zea. Hond 798.

Zebra 726.

Zebre 835 . 


\section{sach den Seiteinzahlen.}

Zebu 692.

Zecoa 728.

Zecora $72 \%$.

Zeeb 802 .

Zeidelbär 319.

Zeifel 484.

Zemni, Glis 474 .

Zenik 255.

Zerda $20 \%$.

Zeuera 835 .

Zeug 837 .

Zeura 835.

Zhiaepok 803 .

Zhievres 808 .

Zickelfchaf 681 .

Zibellina 294.

Zibellino 809 .

Zibeth 266. 268.

Zibetha 266.

Zibethratze 423.

Zibetkatze $265 \cdot 266.26 \%$

Zibethier 266. 268 .

Zibethfitzmaus 371 .

Ziege 646. 648 .

- blaué́riy.

- tatarifche 626.627

- wilde 627.

- wafferfcheue 628.

- gelbe 628 .

- afrikanifche 630 .

- libyfche 63I.

- grimmifche 64r.

- wilde, Govlongo 646.

- angorifche 651 .

- fyrifche 653 .

- levantifche 653.

- von Iuida 655.

- fuirifche 678 .

Ziegenantilope 639 .

Ziegenbock, ungarifcher 626 .

- wilder 647 .

- zabuer 648 .

- - afrikanifcher 654.
Ziegeneinhorn 626 :

Ziegenhaare 653 .

Ziegenochre 699 .

Ziefel 483.

- grauer 480 .

- polnifcher 481 .

- bunter 482 .

- géperlte, gefleckte 486 .

- gelbliche 486 .

Ziefelmaus 484 .

Ziefelratze 483.484 .

Zim 806.

Zimmber 831 .

Zippe 150.

- Zirafa 6 I6.

Zifel 484 .

Zismaus 376.

Zirsmaus 485

Zits - jam 529. 534 .

Zitteraffe 39 .

Zobei 294.

Zobeimanis 460 .

Zobelwiefelein 296.

Zomer 616 .

Zon 830.

Zo: co 830 .

Zovilla 263 .

Zorillo 263 .

Zorinno 264 .

Zorlito 327 .

Zor:0 803."

Zifehefch 802.

Zubr 831 .

Zugmaus 462 .

Zugzeilt 457.

Zugziefel 462 .

Zuraphate $82 \%$

Zuriaba 615 .

Zwerg 42 .

Zwergantilope 642 .

Zwergbocks 654 .

Zwergbiifiel 692.

Zwergbudel I 57. 


\section{Regifter nach den Seitenzahlen.}

Zwergeichhorn 503 .

Zwergfle.termaus 76.77 .

Zwerghare 551. 556.

Zwerghirfchchen $58 \mathrm{I} .582$.

583.584 .642$.

Zwergmaus 442.369 .

Zwergochfe 703 .

Zwergiege 654 .

Zweyzabn 756 .
Zwiebelmats 452 .

Zwiebelzeift 452 .

Zwin 836.

Zwitterefel 722.

Zyfel 484 .

Zyrelnaus 484 .

Zysinaus 484 .

Zyzkan 9 I 7.

\section{Einige Varbefferungen.}

S. 39. Z. 4. von unten 1. Monkey? - S. 73. Z. 7. von unten I. Ausz. I. - S. 89 . Z. I. von unten l. Guinea? S. 90 . Z. 18. 1. Botto? - S. 94. Z. 20. 1. Taulanoir. S. 146.1. Criftato. - S. 229. Z. 12. u. 13. 1. Cuguacuarana. - S. 51. n. 13. und 18. find durch einen Schreibfẹter als zwey verfehiedene Gattungen anfgefihhrt. Man ftreiche al. fo eine davon, welche iman will, weg, und nehme die Syno. nymen davon zu der andern. - Ehen fo ift durch einen Schreibfehler p. 128. bey n. It ein nnrichtiges Citatum aus Graumann und Gatterer angefiihrt. Dicfe beyden Stellen gehören zu p. 130. wo fie auch wirklich nochmals ftehen; p. 128. aber ift folgenderınafsen abzuändern:

Graumarn etc. p. $8 \%$. 11 I. Dugung.

Gatterer 1. c. p. 160. n. 3. Trichecus (Dugung) dentibus laniariis fuperioribus exfertis brevioribus: das indianifche Wailrofs.

S. 232. Atreiche man Zeile 22, weg. 




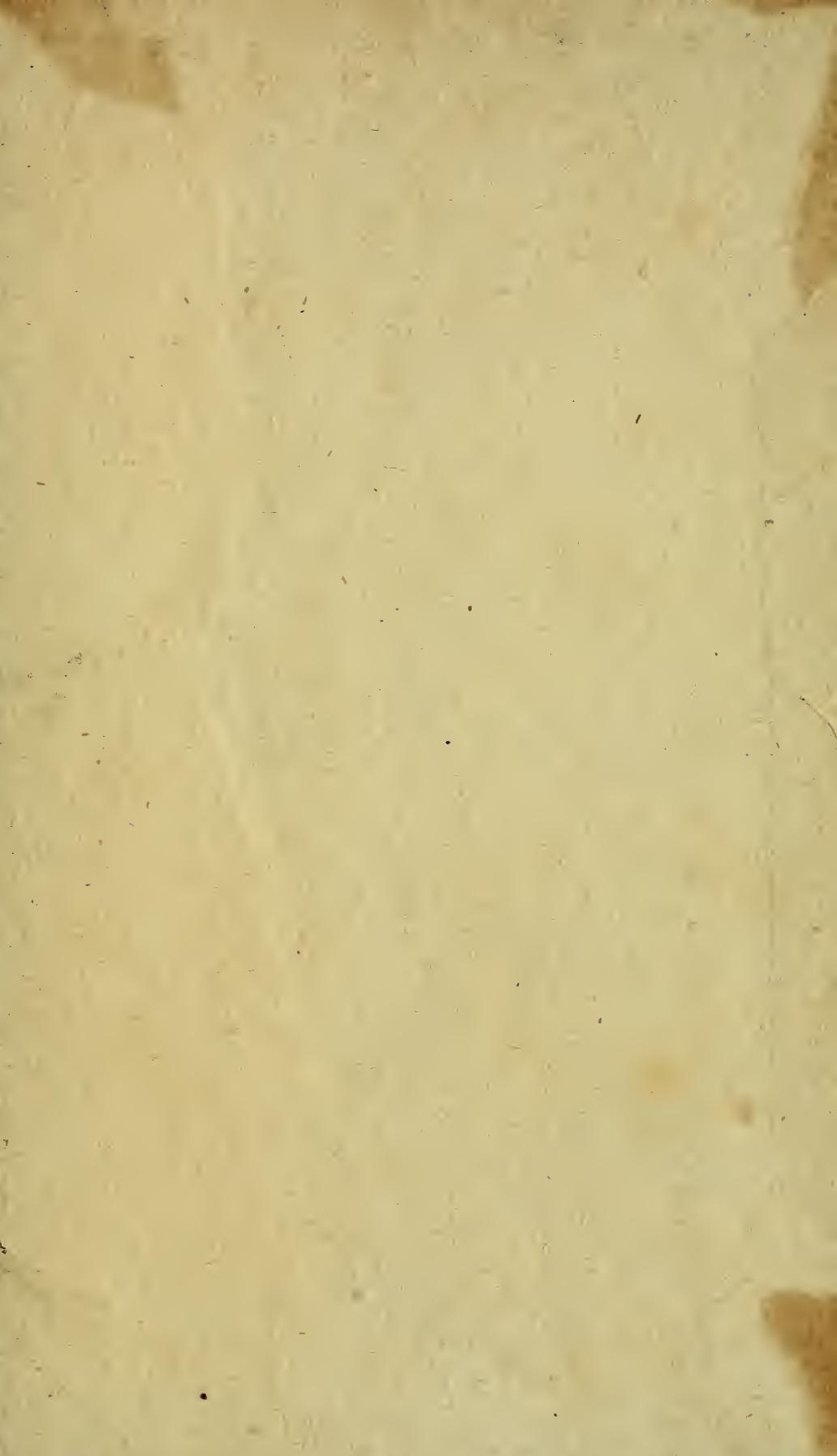




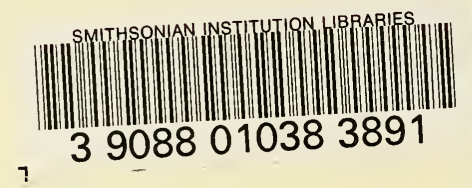

